\title{
32nd Annual Meeting of the European Association for the Study of Diabetes
}

\author{
Vienna, Austria 1-5 September 1996
}

\section{Abstracts}

\section{Index of Oral Presentations}

OP 1 Diabetic Foot-Clinical Studies ............ A 3

OP 2 Genetics of NIDDM .............. A

OP 3 Nutrition ................... A 7

OP 4 Beta-Cell Destruction and Defence........... A 9

OP 5 Insulin Action. . . . . . . . . . . . . . . . . A 11

OP 6 Glucose Toxicity ................ A 13

OP 7 Aetiology........................ A 15

OP 8 Aetiopathogenesis of Neuropathy. . . . . . . A A 16

OP 9 Pregnancy ............... A 18

OP 10 Transplantation and Engineered Beta Cells ....... A 19

OP 11 Endothelium Blood Flow and Diabetic Complications. A 21

OP 12 Glycation - induced Tissue Damage ........... A 22

OP 13 Insulin Analogues . . . . . . . . . . . . . . . . A 24

OP 14 Clinical Retinopathy ................ A 25

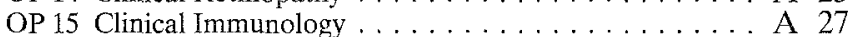

OP 16 Health Care ...................... A 28

OP 17 Beta-Cell Responses to Drugs and Novel Secretagogues A 30

OP 18 Insulin Processing . . . . . . . . . . . . . . . . . A 31

OP 19 Insulin Therapy: NIDDM . . . . . . . . . . . . A 33

OP 20 Treatment and Natural History of Neuropathy ...... A 34

OP 21 Hypertension ................... A 36

OP 22 Prediction of IDDM. . . . . . . . . . . . . . . A 37

OP 23 Mechanisms of Beta-Cell Stimulus-Secretion Coupling. A 39

OP 24 Signal Transduction .................... A 40

OP 25 Nephropathy ...................... 42

OP 26 New Drugs. . . . . . . . . . . . . . A A 43

OP 27 Insulin Resistance Syndromes ................ A 45

OP 28 Population Screening for IDDM. . . . . . . . . . A 46

OP 29 Endogenous Glucose Production .................. A 48

OP $30 \mathrm{~K}^{+}$-ATP Channels $\mathrm{Ca}^{2+}$ and Insulin Oscillations ..... A A 49

OP 31 Clinical Studies in Macrovascular Disease ........ A 51

OP 32 Insulin Therapy: IDDM ............... A 52

OP 33 Small Babies, Insulin Resistance and Free Fatty Acids . A 53

OP 34 Experimental Retinopathy ................ A 54

OP 35 Intra-Cellular Signalling . . . . . . . . . . . A 55

OP 36 Beta-Cell Dysfunction and Glucose Toxicity. ...... A 56

OP 37 Hypoglycaemia I . . . . . . . . . . . . . . . . A 57

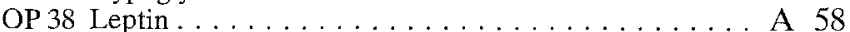

OP 39 Genetics of Complications. . . . . . . . . . . A 60

OP 40 Genetics of IDDM. . . . . . . . . . . . . . . . . . A 61

OP 41 Beta-Cell Differentiation and Replication ........ A 63

OP 42 Lipids .......................... A 64

OP 43 Hypoglycaemia II . . . . . . . . . . . . . . . A A 66

OP 44 Experimental Macrovascular Disease ........... A 67

OP 45 Insulin Resistance and Cardiovascular Risk . . . . . . A A 68

OP 46 Insulin Sensitizers . . . . . . . . . . . . . . . . . A 69

OP 47 Clinical Aspects of Glycation. . . . . . . . . . . A 70

OP 48 Islet Peptides and Insulin Secretion. . . . . . . . A 71

\section{Index of Poster Presentations}

PS 1 Genetics of NIDDM

PS 2 Genetics of IDDM.

A 72

PS 2 Genetics of IDDM.

A 79

PS 4 Prediction of IDDM

A 81

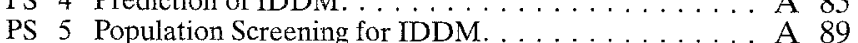

PS 6 Clinical Immunology . . . . . . . . . . . . . . A 92
PS 7 Animal Immunology................. A 97

PS 8 Cytokines, NO and Beta-Cell Integrity. . . . . . . . . A 100

PS 9 Development and Replication of Islets. . . . . . . . . A 108

PS 10 Glucose Recognition and Signal Transduction in

Beta-Cells.

A 111

PS 11 Ion Channels and Oscillation in Islet Cells . . . . . . . A 115

PS 12 Islet Responses to Drugs, Neurotransmitters and Peptides

A 121

PS 13 Mechanisms of Defective Islet-Cell Function. . . . . . . A 126

PS 14 Transplantation and Beta-Cell Engineering. . . . . . . A A130

PS 15 Glycation ........................ A 137

PS 16 Oxidative Stress . . . . . . . . . . . . . . . . . . . . A A139

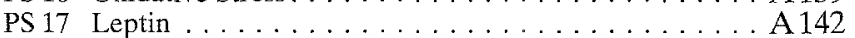

PS 18 Relatives of NIDDM . . . . . . . . . . . . . . . A147

PS 19 Insulin Resistance and NIDDM . . . . . . . . . . . . . A 149

PS 20 GLP-I . . . . . . . . . . . . . . . . . . . . . . . A 154

PS 21 Gastric Emptying. . . . . . . . . . . . . . . . A 156

PS 22 Kinetic Measurements in Vivo . . . . . . . . . . . . A 157

PS 23 Hepatic Metabolism . . . . . . . . . . . . . . . . . . A 160

PS 24 Muscle Metabolism. .................... A 162

PS 25 Adipose Tissue and Brain Metabolism . . . . . . . . . A 163

PS 26 Regulation of Insulin Secretion. . . . . . . . . . . . . . . A 164

PS 27 Insulin Receptors . . . . . . . . . . . . . . A 165

PS 28 Signal Transduction . . . . . . . . . . . . . . . . . A166

PS 29 Glucose Transporters . . . . . . . . . . . . . . . A 171

PS 30 Insulin Action in Vivo . . . . . . . . . . . . A 175

PS 31 Regulation of Metabolism . . . . . . . . . . . . . . . A 177

PS 32 Regulatory Peptides . . . . . . . . . . . . . . . A 181

PS 33 IGF and IGFBP ......................... A 184

PS 34 Stress Hormones in Diabetes - Catecholamines . . . . . . A 186

PS 35 Stress Hormones in Diabetes - Steroids . . . . . . . . . . . A 187

PS 36 Exercise . . . . . . . . . . . . . . . . . . . . . . . A 189

PS 37 Epidemiology . . . . . . . . . . . . . . . . . . . A 190

PS 38 Nutrition and Diet . . . . . . . . . . . . . . . . A A195

PS 39 Health Care, Education, Psychosocial Problems. . . . . . A 198

PS 40 Gestational Diabetes. . . . . . . . . . . . . . . A203

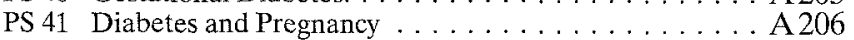

PS 42 Clinical Diabetes . . . . . . . . . . . . . . . . . . . . A210

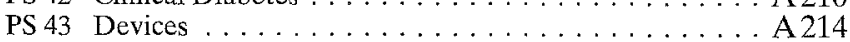

PS 44 Hypoglycaemia . . . . . . . . . . . . . . . . . . . . A217

PS 45 Insulin Analogues. . . . . . . . . . . . . . . . . . A221

PS 46 New Pharmacological Approaches . . . . . . . . . . A225

PS 47 Sulfonylureas and Metformin . . . . . . . . . . . . . A227

PS 48 New Antidiabetic Drugs . . . . . . . . . . . . . . . . . A232

PS 49 Coagulation Endothelium . . . . . . . . . . . . . . A A237

PS 50 Eye Complications . . . . . . . . . . . . . . . . . . . . . A 242

PS 51 Experimental Neuropathy . . . . . . . . . . . . . . A246

PS 52 Autonomic Neutopathy. . . . . . . . . . . . . . A250

PS 53 Somatic Neuropathy . . . . . . . . . . . . . . . A257

PS 54 Diabetic Foot. . . . . . . . . . . . . . . . A 261

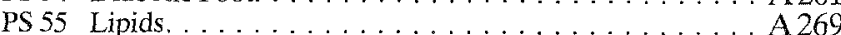

PS 56 Macroangiopathy Risk Factors . . . . . . . . . . . . A 274

PS 57 Macroangiopathy Pathogenesis. . . . . . . . . . . . . A279

PS 58 Macroangiopathy Diagnosis and Treatment. . . . . . A 282

PS 59 Hypertension. . . . . . . . . . . . . . . . . . . A287

PS 60 Clinical Nephropathy ... . . . . . . . . . . . . . . . A291

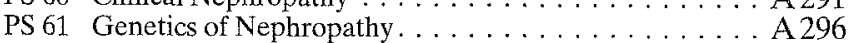

PS 62 Microalbuminuria and Hyperfiltration . . . . . . . . . A 300

PS 63 Experimental Nephropathy . . . . . . . . . . . . . . . . A 304

PS 64 Natural History and Treatment of Nephropathy . . . . . . A 306

PS 65 Other Complications. . . . . . . . . . . . . . . . . . . . A309 
OP 1

\section{Diabetic Foot - Clinical Studies}

\section{1}

The Effect of Diabetes, Neuropathy, Charcot arthropathy and ARTERIAL DISEASE ON THE FOOT MICROCIRCULATION.

A. Veves, C.M. Akbari, V.M. Donaghue, D. Zacharoulis, J.S. Chrzan, R. Freeman and F.W. LoGerfo. Deaconess-Joslin Foot Center, Boston, USA.

We studied the effect of neuropathy and hypoxia on the foot microcirculation in 5 groups: 31 diabetic neuropathic patients (DN), 17 with Charcot arthropathy (DA), 24 with diabetic neuropathy and peripheral vascular disease (DV), 10 diabetic patients without complications (DC) and 18 healthy controls (C). We employed single point laser Doppler and laser Doppler imaging scanner to measure vasodilatation on the dorsal foot in response to heating to $44.5^{\circ} \mathrm{C}$, and to iontophoresis of $1 \%$ acetylcholine (endothelium-dependent) and 1\% sodium nitroprusside (endotheliumindependent). The severity of neuropathy was similar in DN, DA, and DV groups but the Transcutaneous Oxygen Pressure was reduced in the DV $(37.0 \mathrm{~mm} \mathrm{Hg} \pm 4.5$, mean $\pm \mathrm{SE}$ ) compared to all other groups and in $\mathrm{DN}$ $(61.4 \pm 2.8)$ compared to DA $(71.1 \pm 3.1)$, DC $(75.2 \pm 3.8)$ and C $(77.8 \pm$ 2.2) $(p<0.001)$. The response to heat was reduced in the DN $(458 \pm 77, \%$ of increase over the baseline) and DV [335 \pm 69$]$ compared to DA (894 \pm $135), D C(991 \pm 212)$ and $C(1004 \pm 139, p<0.0001)$. Using the laser scanner the response to acetylcholine, which stimulates the production of Nitric Oxide (NO), was similarly reduced in the DN (18 \pm 3$)$, DA (22 \pm 6$)$ and DV $(19 \pm 4)$ compared to $D C(60 \pm 13)$ and $C(61 \pm 9)(p<0.001)$. The response to sodium nitroprusside (a NO donor) was lower in the DV $(10 \pm 4)$ compared to all other groups while it was similarly reduced in DN $(23 \pm 5)$ and DA (21 \pm 4$)$ compared to $D C(37 \pm 7)$ and $C(44 \pm 4), p<0.0001)$. Similar results were found using single point measurements. The indirect vasodilatation during acetylcholine iontophoresis, which depends on a normal nerve axon reflex, was reduced in DN $(29 \pm 8)$, DA $(41 \pm 13)$ and DV $(25 \pm 11)$ compared to DC $(286 \pm 99)$ and $C(268 \pm 62)(p<0.0001)$. We conclude that at the foot level neuropathy impairs the endotheliumdependent vasodilatation but spares the endothelium non-dependent response, while in patients with Charcot arthropathy the ability to increase the overall skin blood flow, as seen by the response to heat, remains intact.

3

MEETING ST VINCENT: CONSTRAINTS ON CASE ASCERTAINMENT FOR LOWER EXTREMITY AMPUTATION INCIDENCE

H J Bodansky', CM Airey ${ }^{2}$, I Dyas ${ }^{2}, S M$ Chell $^{2}, N$ Unwir $^{3}$ and D R R Williams ${ }^{2}$ The General Infirmary at Leeds', Nuffield Institute for Health University of Leeds', Department of Medicine University of Newcastle?

The St Vincent Deciaration called for a $50 \%$ reduction in amputation for diabetic gangrene within 5 years. This reduction in lower extremity amputation (LEA) is thought to be attainable through the provision of appropriate health care. An international study is cornparing LEA rates prospectively from July 1995 using multiple methods of case ascertainment and the 'capture-recapture' technique. This uses the degree of overlap between various data sources to estimate the 'tnue' incidence and thus, the extent of under-ascertainment. One centre undertook an additional retrospective study to assess data source quality using information recorded in operating theatre registers, hospital discharge summaries and by the regional prosthetic centre between July 1992 - Deceruber 1993. Over this 18 month period 291 cases of LEA were identified $(192,165$ and 66 respectively by the above overlapping sources), $36 \%$ of these being in people with diabetes. Annual LEA incidence in the general/diabetic population was $27 \times 10^{-5}(95 \% \mathrm{CI} 23,31) / 478 \times 10^{-5}$ $(378,604)$ respectively. 'Capture-recapture' analysis estimates a median 'true' incidence for the general population of $36 \times 10^{-5}(32,41)$ with a range of $31 \times 10^{-5}$ $(28,36)-39 \times 10^{-5}(35,44)$. The under-ascertainment of between 32 and 91 LEAs per annum in this study is attributable to constraints in the sources used and include; 1) incompleteness of the theatre record; 2) delays and inaccuracy in coding discharge data, particularly the absence of diabetes related details; 3) unavailability of hospital case notes: 4) few cases requiring prostheses; and 5) long interval between LEA and referral for prosthesis. In conclusion, if St Vincent requirements are to be seen to be met, accurate and up-to-date baseline information on LEA incidence is essential. In the light of the difficulties identified in this study, surveillance systems that are comparable cross-sectionally and longitudinally need to be established in order to measure this and other diabetes outcomes effectively.
A PROSPECTIVE STUDY OF NEUROPATHIC AND PSYCHOLOGICAL FACTORS IN FOOT ULCERATION L Vileikyte, JE Shaw, J Kincey, AL Carrington, C Abbott and AJM Boulton. Department of Medicine, Manchester Royal Infirmary, Manchester, UK.

Neuropathic foot ulceration is believed to result from a combination of neuropathy and inappropriate foot care. We studied four groups of subjects: D: diabetic controls ( $(n=51)$; DN diabetic neuropathic subjects ( $n=68)$; DNU: previous neuropathic ulceration $(n=36)$; $C$ : Charcot neuro-arthropathy $(n=17)$. Baseline tests included peroneal nerve conduction velocity (NCV), vibration, pressure and thermal perception thresholds (VPT, PPT, TPT). Questionnaires were used to assess foot care practice, attitudes to the feet, denial and belief in the efficacy of medical advice. During a mean follow up of 10.5 months, ulceration occurred in D - 4/51, DN - 9/68, DNU - 21/36, C 9/17. Within group $D N$, all neurological parameters were worse in subjects developing ulcers - VPT (mean) $36 \mathrm{~V} v 24 \mathrm{~V}$ p $<0.01$, PPT 5.8 v $5.0 \mathrm{p}<0.05$, TPT $8.3^{\circ} \mathrm{C}$ v $5.0^{\circ} \mathrm{C} p<0.05$, NCV $27.9 \mathrm{~ms}^{-1}$ v $36.1 \mathrm{~ms}^{-1}$ $p<0.01$. In groups DNU and $C$, no neurological tests differentiated subjects developing ulcers from those who did not. Psychologieal assessment showed a more negative attitude to the feet in DN subjects developing ulcers (median score $65 \mathrm{v} 76$ - $p<0.05$ ). Belief in the efficacy of advice was lower in those developing ulcers, but only reached significance in a combined DN and DNU group ( 15.5 v 17 . p<0.05). HbA1c was higher in DNU subjects developing ulcers (mean $10.4 \%$ $8.7 \%$ - $p<0.05$ ). Whereas neurological deficit and a negative attitude to the feet predicted first ulcers, only HbAlc predicted recurrent ulcers. This elevated HbA1c may reflect an aspect of self care, and in particular, a neglett of foot care.
4

\section{CAN MASS SCREENING AND EDUCATION PREVENT FOOT PROBLEMS?} THE NORTH WEST DIABETES FOOT CARE STUDY.

A.L. Carrington, C.A. Abbott, J. Kulkarni, E.R.E Van Ross and A.J.M. Boulton for the North West Diabetes Foot Care Strdy Group. Disablement Services Centre, Manchester.

Previous studies have shown that foot care and education can lower the incidence of diabetes related foot problems in small groups of high risk patients. Therefore, the aim of this population-based study was to examine this hypothesis in the general diabetes community. Adults with diabetes $(n=9710)$ were screened and educated in six districts, by highly trained research podiatrists and nurses over a two year period. The patients were seen in community, hospital and residential settings Education was based around a standard questionnaire and leaflet and also clear explanation of specific tests and results for neuropathy and peripheral vascular disease. Information was collected using a standard form and patients categorised as not at risk (33.15\%), moderate risk (15.64\%) and high risk (51.21\%) using a standard classification system based on previously determined risk factors. The overall prevalence of present foot ulceration was $1.61 \%$ and that of previous foot ulceration was $3.17 \%$. The prevalence of lower limb amputation at any level was $1.36 \%$. To determine the efficacy of the screening and education programme in the short term, the incidence of ulceration and amputation was determined for a six month period at the end of the study. Using accurate estimates of the total diabetes population of the six districts involved in the study, the percentage of new foot ulcers in the screened group $(0.41 \%)$ was compared to that of the non-screened group (1.24\%). This difference was found to be significant using the Chi Square test $(\mathrm{p}<0.0001)$. The incidence of amputation was also significantly lower in the screened group (screened $=0.12 \%, \mathrm{v}$ non-screened $=0.36 \%, p<0.001$ ). This study shows that, at least in the short term, mass screening of the general diabetes population by highly trained professionals can reduce the incidence of ulceration and amputation, with consequent health and economic savings. However, close follow up of these patients is required to examine the long-term benefits. 
FOLLOW-UP OF PATIENTS HOSPITALISED FOR DIABETIC FOOT

K.P.Bouter, I.M.Oving, R.R.M.de Groot, C.N.Lau, D.W.Erkelens, R.J.A.Diepersföot. Department of Internal Medicine, Bosch MediCentre, Dutch Centre for Health Care Information, Laboratory forPublic Health, Diakonessen Hospital Utrechi, Department of Internal Medicine, University Hospital Utrecht, The Netherlands.

The in-hospital mortality of patients hospitalized for diabetic foot lesions is approximately $10 \%$. Data on the prognosis after discharge however, are searce. In this study $256^{9}$ patients will be followed-up for a period of five years of untill death. Data he followed-up for a period of five years of untill death. Data on hospitalizations of patients with diahetes mellitus and ulcerative lesions of the limbs were obtained from the Dutch Infor-
mation System on Hosnital Care. Enquiry forms for 900 pamation System on Hosnital Care. Enquiry forms for 900 pa-
tients hopitalized in 1992 in 26 hospitals randomly distributed over The Netherlands were sent to the medical administration of the hospitals both in 1994 and 1905 . The forms were consecutively sent to the general practitioners (GP) of the patients. Ot the 26 hospitals 25 cooperated. A total of $345 \mathrm{GP}$ 's responded and 250 forms of which 91 patients died were evaluable in 1094 (136 men and 123 women, mean age $70.9 \pm 12.4$ years) and 125 forms of which 30 patients died $(74.4 \%$ follow un) in 1995 ( 64 men and 61 women, mean age $71.2 \%+12.8$ years). 1995 ( 04 men and 61 women, mean age $71.2+12.8$ years).
Most patients $(60.5 \%$, were on insulin therapy, $20.8 \%$ were on oral therapy and $2.7 \%$ were on a diet. A diagnosis of diahetic foot was made for the tirst time in 1992 in $44.4 \%$ of all cases. Within one year of follow-un $37.2 \%$ of all patients were readmitted to the hospital for diabetic foot lesions and $18.4 \%$ exira after a two year follow-up. The mortality after one year of tollow-un amounted to $35.5 \%$ and an extra of $24 \%$ after two year follow-un. The cause of death was acute myocardial ischacmia in $27.5 \%$ (resp). 26.7\%), CVA in $11 \%$ (resp. $10 \%$ ) and infections complications of diabetic foot in $18.7 \%$ (resp. $13.3 \%$ ) of all cases. Underlying cardiovascular disease was present in the majority $(60.8 \%$, resp. $68 \%)$ of the patients studied. From the preliminary results after two year of follow-up it is concluded that patients previously hospitalized for diabetic foot carry a high mortality risk and are prone to the development of new diăhetic foot lesions.
REDUCED TISSUE LEVELS OF TGF-\$1 MAY CONTRIBUTE TO POOR HEALIVG IN DIABETIC FOOT ULCERS JE Shaw, MJ Spencer, SE Herrick, MWJ Ferguson and AJM Roulton. Departments of Medicine and Biological Sciences, Manchester University, Manchester, UK.

Transforming growth factor beta (TGF-8) plays a key role in wound repair, and animal work has suggested that the application of TGF0 accelerates wound healing. However, no study has measured the amount and distribution of growth factors in human diabetic foot ulcers. This study, for the first time, compares biopsies from diabetic foot ulcers $(n=43)$ and sites distant to the site of ulceration (taken at the time of amputation) with biopsies from venous ulcers, nondiabetic ischaemic ulcers and normal skin. Biopsies were snapfrozen, sectioned and labelled for immunocytochemistry with specific growth factor antibodies. The sections were scored on a 1-5 scale for intensity of labelling. In diabetic ulcers and intact diabetic skin, TGF-B1 was found mainly in the epidermis, dermal cells (predominantly fibroblasts and leukocytes) and capillary walls, but at lower levels than in non-diabetic skin (both ulcerated and intact) (median score $2.0 \mathrm{vs} 3.5, \mathrm{p}<0.05$ ). Other areas (extra-cellular matrix, larger vessels) showed only minimal labelling with TGF-B1. In diabetic subjects, the ulcer and its immediate surround showed no increase in TGF-B1,2,3 or their receptors, when compared to sites distant to the ulcer (median seore $2.5 \mathrm{v} 2.0$, p>0.1). This was in contrast to TGF-B1 in non-diabetic ulcers and vascular endothelial growth factor in all ulcers, both of which showed distinct evidence of peri-wound up-regulation. We have found evidence of depressed levels of TGF-B1 in diabetic skin and a failure to increase TGF-B in response to ulceration. These features may be central to the delayed healing observed in diabetic foot ulcers.
COMPARISION OF THERMOREGULATORY AV-SHUNTFLOW AND NUTRITIVE CAPILLARY FLOW IN THE DIABETIC FOOT.

T. Forst, A Pfützner, T. Kunt, T. Pohlmann, U. Schenk, E. Küstner, J. Beyer III. Med. Clinic; Dept. Internal Medicine / Endocrinology; University Mainz Peripheral unmyelinated $\mathrm{C}$-fibre neuropathy plays an important role in the regulation of the neurogenic vasodilator responise and seemed to be implicated in the development of neurotrophic skin ulceration. We examined the response of the two major components of the microcirculation at the toe nailfold to intradermal injection of acetylcholine. Arteriovenous shuntflow was assesed using the technique of laser doppler flowmetry (LDF) and nutritive capillary blood flow was measured by the use of television microscopy (CBV. Diabetic neuropathy was assesed by examining vibration-, thermaland pain sensory threshoids and heart rate variability. Patients suffering from peripheral macrovascular disease were excluded from the study. Thittysix diabetic patients ( 19 non neuropathic; 17 neuropathic) were compared with 14 non diabetic control subjects. LDF-signal increased significantly from 38.3 \pm 9.9 to $99.2 \pm 19,8$ (mean \pm SEM $; p<0.001)$ in the control group and from $529+14.3$ to $117.4 \pm 28.5(\mathrm{p}<0.001)$ in diabetic patients without neuropathy. In the neuropathic diabetic group, only a slight increase in LDF from $42.5 \pm 10.6$ to $63.2 \pm 12.0(p<0.05)$ was observed. The absolute increase from basal LDF was significanty impaired in the neuropathic diabetic group compared with the non neuropathic diabetic group $(p<0.05)$ or the control group $(p<0.01)$. CBV increased from $2.3 \pm 0.13$ to $2.8 \pm 0.16$ $\mathrm{mm} / \mathrm{s}(p<0.05$ ) in the control group; from $2.4 \pm 0.14$ to $3.0 \pm 0.16 \mathrm{~mm} / \mathrm{s}$ ( $p$ $<0.01)$ in the non neuropathic diabetic group and from $2.5 \pm 0.15$ to $3.1 \pm$ $0.17 \mathrm{~mm} / \mathrm{s}(\mathrm{p}<0.05)$ in the neuropathic diabetic group. No significant difference in the total increase from basal CBV was found between the investigated groups. This study demonstrates an impaired neurovascular response in total skin microcirculation, while there is no evidence to support a diminished nutritive capillary flow or a capillary steal phenomenon following axon reflex stimulation.
8

AMBULATORY ALLOGENIC KERATINOCYTE CULTURE GRAFTING FOR THE TREATMENT OF DIABETIC FOOT WOUNDS.

I. Harman-Boebm' ${ }^{1}$ E. Boteach', H. Reuveni ${ }^{2}$, and N. Grossman ${ }^{2}$. Diabetic Foot Clinic' $^{1}$, and Skin Bank Laboratory ${ }^{2}$, Soroka Medical Center and Faculty of Health Sciences, Ben Gurion University of the Negev, Beer Sheva, Israel

Healing of wounds in the diabetic foot is a slow process that often results in chronic ulcers partial amputations and debilitation. During the last year, we have used cultured allogenic keratinocytes grafts $(\mathrm{KG})$ to stimulate healing of various cutaneous defects in 14 patients in the Diabetic Foot Clinic. The etiology of the defects was as follows: 13 patients have NIDDM, and one has IDDM; 7 patients had plantar ulcers associated with neuropathy and trauma ( 1 in the heel, 4 were metatarsal; and 2 in the toes or between the toes); 4 patients had defects following local surgical procedures or following local abscess drainage ( 2 amputated toes, 1 heel, and 1 midfoot amputation; ; patient had a nonhealing nail following onychonycosis; and 2 had leg ulcers due to trauma. Defects persisted and were treated for $12.25 \pm 15.09$ months by conservative treatments before grafting with $K G$, (range: 1.5 months to 5 years, median 5 months\}. Wound area was prepared for grating on an out patient basis by chemical and/or mechanical debridement, saline irrigations, and systemic or local antibiotics as required. KG were applied onto the wound bed in the out-patient clinic, followed by a shon rest at home. The average grafted area was $5.4+7.2 \mathrm{~cm}^{2}$, ranging from 0.7 to $24 \mathrm{~cm}^{2}$. Further treatments included irrigation with saline, with or without gentamicin. Follow-up showed that in $7 / 14$ of the patients, the wound area was reduced by $40 \%$ within the first 4 days following grafting. In others, the initial response was smaller. $2 / 14$ of the patients did not respond to KG. Complete healing was achieved in $8 / 14$ of the patients within $16 \pm 12$ weeks following treatment (range: 2 weeks to 9 months, median 10 weeks). Recurrences occurred in $5 / 14$ of the patients due to continuing shear stress or osteomyelitis. Our results demonstrate the beneficial effect of ambulatory application of KG in promoting healing of various cutaneous defects in diabetic feet, which otherwise may end with debilitating amputations. 
OP 2

\section{Genetic of NIDDM}

9

\section{TOTAL GENOME SCAN FOR IDENTIFICATION OF NIDDM SUSCEPTIBILITY FACTORS IN THE GK RAT}

Hong Jiao, Luo-Sheng Li, Anna Glaser, Joakim Galli, Hossein FakhraiRad, George Koike*, Howard J. Jacob*, and Holger Luthman. Dept. of Molecular Medicine, Karolinska Institute, Sweden, ${ }^{*}$ Cardiovascular Research Center, Massachusetts General Hospital, USA.

In order to investigate genetic susceptibility factors of NIDDM, we have arranged crosses between the diabetic Goto-Kakizaki rat and the normoglycemic Fischer-344 rat. Two crosses were performed, yielding 374 second-generation progeny which were subjected to an intraperitoneal glucose tolerance test. We have now selected 197 markers at an average distance of $9 \mathrm{cM}$, representing all chromosomes of the rat genome, and genotyped 235 progeny of the second-generation. This set of markers covers the rat genome with only three intervals larger than $40 \mathrm{cM}$. Quantitative trait locus (OTL) analysis has been performed with 25 phenotypes associated with diabetes (body weight, glucose and insulin levels, at fasting and different time points following glucose injection, as well as the ratio of insulin to glucose). In addition to the reported QTLs: Niddm 1,2,3, and Weight 1 (Nature Genetics, $12,31-37,1996)$, we have identified two new regions with LODs slightly less than $4(\mathrm{P}<0.0003)$ for weight and for the glucose level at 15 min, one QTL for the insulin to glucose ratio of $L O D=3.5$, and two regions for insulin levels with LODs $2.5(\mathrm{P}=0.003)$. In summary from the total genome scan, we have mapped 23 regions for at least one diabetes associated phenotype. Among them, 5 regions showed. LODs between 2 and 2.5; 9 with LODs 2.5-3: 2 with LODs 3-3.5, 7 with LODs $>3.5$. Fifteen regions showed suggestive evidence for more than one trait. These results demonstrate the feasibility to map traits for NIDDM susceptibility in the rat, and provide a means for identification of genes contributing to development of NIDDM in humans.

\section{0}

SIGNIFICANCE OF A MISSENSE MUTATION OF AMYLIN GENE (Ser20Gly) IN JAPANESE EARLY ONSET NIDDM S.Sakagashira, T.Sanke, T.Hanabusa, S.Ohagi, and K.Nanjo, The First Department of Medicine, Wakayama University of Medical Science, Wakayama, Japan

Amylin, a 37 amino acid peptide which is co-secreted with insulin from islet beta-cells, is a major constituent of islet amyloid deposits seen in NIDDM patients. Recent studies suggest that amylin may be involved in the pathogenesis of NIDDM. We therefore scanned the amylin gene for mutations in 294 Japanese NIDDM patients by SSCP analysis, and found a single heterozygous missense mutation (Ser to Gly at position 20 : Ser20Gly mutation) in 12 NIDDM patients. None of the 187 non-diabetic subjects and 59 IDDM patients had the mutation. Eight out of 12 patients carrying the mutation were diagnosed as having NIDDM before 35 years old, and they had severe diabetes (treated with insulin) and a strong family history of late onset NIDDM. The frequency of the affected patients in early onset ( $\leqq 35$ years) NIDDM was considerably high $(8 / 80: 10,0 \%$ ). Limited family studies disclosed that the mutation was transnitted not from the parent having NIDDM and a strong family history of diabetes but from the parent having very mild glucose intolerance (IGT). On the other hand, the remaining 4 affected patients were diagnosed as having NIDDM after 51 years old, and they had mild diabetes (treated with diet alone) without family history of diabetes. HPLC analysis revealed that affected patient's plasma contained small amount (16\%) of normal amylin and much larger amount of mutant amylin (84\%). This fact supports the notion that amylin is biologically active peptide and the mutation provides an important model to investigate a true action of amylin. We first report a missense mutation of anylin gene in Japanese NIDDM patients. This Ser20Gly mutation may play an important role in the pathogenesis of the early onset NIDDM in the Japanese population when it is combined with unknown susceptible genes for late onset NIDDM.
11

DECREASED INSULIN SECRETION IN HEALTHY SUBJECTS WITH SEQUENCE VARIANTS IN THE SULFONYLUREA RECEPTOR GENE A. M. Mølter ${ }^{1}$, T. Hansen ${ }^{4}$, K. Almind ${ }^{1}$ L. Hansen ${ }^{1}$, S. M. Echwald ${ }^{1}$, J. O. Clausen', S. A. Urhammer', H. Inoue ${ }^{2}$, J. Ferrer ${ }^{2}$, J. Bryan ${ }^{3}$, L. Aguilar-Bryan ${ }^{3}$, M. A. Permutt ${ }^{2}$ and $O$. Pedersen ${ }^{1}$ Steno Diabetes Center and Hagedorn Research Institute, Denmark'. Division of Metabolism, Diabetes and Endocrinology, Washington University School of Medicine, Saint-Louis, USA ${ }^{2}$. Departments of Cell Biology and Medicine, Baylor College of Medicine, Houston, USA ${ }^{3}$

In the gene encoding the high affinity sulfonylurea receptor (SUR) we examined two sequence variants, a silent polymorphism in exon 22 and an intron variant at position -3 of the intron/exon 24 boundary, for association to NIDDM and for a possible influence on insulin secretion after intravenous tolbutamide in a random sample of unrelated healthy young Danish Caucasians. The allelic frequency of the exon 22 variant was $0.051(95 \% \mathrm{Cl}$ $0.035-0.067)$ in NIDDM patients $(\mathrm{N}=386)$ and $0.027(0.013-0.041)$ in agematched control subjects with normal glucose tolerance $(\mathrm{N}=243)\left(\mathrm{X}^{2}=4.25\right.$, $p=0.039$; OR: $1.94(1.03-3.67))$. The allelic frequency of the intron variant was similar among NIDDM patients $(0.451(0.416-0.486))$ and control subjects $(0.440(0.396-0.484))$. Seventeen of 386 NIDDM patients had the combination exon 22 heterozygosity or homozygosity and intron heterozygosity $(0.044$ $(0.024-0.064))$ while 3 of 243 control subjects had the same combination $(0.012(0-0.026))\left(X^{2}=3.89, p=0.049\right.$, OR: $\left.3.69(1.07-12.71)\right)$. Ten of 380 unrelated healthy young Danish Caucasians $(0.026(0.010-0.042))$ had the combination exon 22 heterozygosity or homozygosity and intron heterozygosity. These individuals had significantly reduced serum $C$-peptide and insulin responses upon tolbutamide injection $(p=0.002$ and $p=0.05$ respective(y), but normal serum $C$-peptide and insulin responses upon glucose injection. In conclusion: A silent polymorphism in exon 22 of the high affinity SUR gene is associated with NIDDM. In combination with an intron variant at the intron/exon 24 boundary the association is higher, and young healthy carriers of the intragenic combination have reduced serum C-peptide and insulin responses upon a tolbutamide load.
12

A MISSENSE MUTATION AT CODON $326^{\text {Met } \rightarrow \text { lle }}$ IN THE GENE ENCODING THE PHOSPHATIDYLINOSITOL 3-KINASE p85 $\alpha$ SUBUNIT IS ASSOCIATED WITH DECREASED GLUCOSE TOLERANCE AND DECREASED GLUCOSE EFFECTIVENESS

T. Hansen, C.B. Andersen, S.M. Echwald, J.O. Clausen, S.A. Urhammer, L. Hansen and $\mathrm{O}$. Pedersen. Steno Diabetes Center and Hagedorn Research Institute, Copenhagen, Denmark

The phosphatidylinositol 3-kinase (PI3K) is an important regulator of both basal and insulin stimulated glucose metabolism and should be viewed as a candidate gene for insulin resistant disorders like NIDDM. Single strand conformational polymorphism (SSCP) scanning of the coding region of the regulatory PI3K p85 $\alpha$ suburit in cDNA isolated from human skeletal muscle tissue from 70 insulin resistant NIDDM patients and 12 control subjects revealed 3 silent polymorphisms and a missense mutation at codon 326 changing a Met to lie. Using allele specific oligohybridization we found a similar allelic frequency of the codon $326^{\text {met } \rightarrow \text { le }}$ variant in 404 NIDDM patients $(0.15(95 \% \mathrm{CI} 0.12-0.18))$ and 224 matched glucose tolerant control subjects $(0.16$ (0.13-0.19)). We also identified 263 wild type (Wt) individuals, 109 heterozygous $(\mathrm{He})$ and 8 homozygous $(\mathrm{Ho})$ for the codon 326 variant (allete frequency $=0.16$ $(0.13-0.19)$ ) in a random sample of unrelated healthy young Caucasians in whom we performed an IVGTT in combination with tolbutamide injection. Intravenous glucose tolerance $(\mathrm{Kg})$ and glucose effectiveness $(\mathrm{Sg})$ were significantly different between the groups $\left(\mathrm{Kg} ; \mathrm{Wt}: 2.3 \pm 1.1 \times 10^{-2} \mathrm{~min}^{-1}\right.$, He: $2.3 \pm 1.0$, Ho: $1.4 \pm 0.6$ $($ mean $\pm S D)(p=0.02)$; Sg; Wt: $2.1 \pm 0.7 \times 10^{-2} \mathrm{~min}^{-1}$, He: $2.2 \pm 0.6$, Ho: $1.7 \pm 0.6$ $(p=0.03)$ ). No significant difference was seen in insulin sensitivity ( $\mathrm{Si}$ ) (Wt: $15.3 \pm 9.5 \times 10^{-5} \mathrm{~L} \mathrm{~min}^{-1} \mathrm{pmol}^{-1}$, He: $15.1 \pm 8.6$, Ho: $\left.10.3 \pm 8.3(\mathrm{p}=0.20)\right)$. Both $\mathrm{Kg}$ and $\mathrm{Sg}$ were significantly reduced among Ho subjects compared to the combined values for $\mathrm{Wt}_{\mathrm{t}}$ and He carriers $(\mathrm{p}=0.004$ and $\mathrm{p}=0.03$, respectively). Moreover, in Ho carriers a $30 \%$ reduction was found in $S i(p=0.08)$. In conclusion: A common codon $326^{\mathrm{Met} \rightarrow \mathrm{ll}}$ variant in the gene encoding the PI3K p85 $\alpha$ regulatory subunit is in its homozygous form associated with decreased intravenous glucose tolerance and decreased glucose effectiveness in young healthy individuals. 
13

FAMILIAL DIABETES SECONDARY TO A NOVEL POINT MUTATION (1+709) OF mt DNA. IN THE REGION CODING FOR tRNA ${ }^{\text {Glu }}$

B. VIALETTES. V. PAQUIS-FLUCKINGER.P.SILVESTRE-AILLAUD

J.F.PELISSIER. H.NARBONNE. D.BENDAHAN. P.COZZONE.C.

DESNUELLE. UNIVERSITY OF MARSEILLE AND NICE,FRANCE

We report a pedigree with maternally inherited diabetes due to a novel point mutation $(14709)$ in the mt tRNA ${ }^{\mathrm{Glu}}$ gene and compare phenotypic expression to the classical MIDD form ( 3243 mutation). The proband was refered for diabeics and myopathy. Diabetes was diagnosed at 30 and insulin treated at $36 \mathrm{yT}(50 \mathrm{w} / \mathrm{d}$. HbAIC : $7.8 \%$ \%). Residual insulin secretion was low (PC $0.14 \rightarrow(0.23$ after glucagon) HLA DQ Bl Asp/n Asp and ICA negative. Myopathy was severe with muscle weakness and confirmed by biopsy (ragged red fibers. decrease of complexe IV). high lactate production during exercise test and ${ }^{31} \mathrm{P}$ SNMR (high phosphate inorganic/phosphocreatinine ratio. anomaly of $\mathrm{PH}$ ). Neurosensorial investigations showed the lack of macular pattern dystrophy and mild hearing impairement in high frequencies. The presence of mutations was confirmed by Southern blotting after NdeA digestion and show to be heteroplasmic in both blood cells and muscle Diabetes (NIDDM) was present in the mother, 2 brothers, 1 sister. Sensoriel defects were mild (impairement of hearing in bigh frequencies) or absent Some subjects were strickly normal despite presence of mutated $\mathrm{mt}$ DNA in blood. In comparison. in 3 pedigrees with MIDD by 3243 mutation, every diabetic exhibited macular pattern dystrophy. deafness and mild muscle involvement. Conclusion : this new type of diabetes secondary to mtDNA mutation has a more heterogencous clinical pattern and must be considered in the research of mitochondrial origin of maternaly inhcrital diabeies.

\section{5}

Separate contributions of IRS-1 and $\beta 3$-AR mutations to insulin resistance and obesity with no evidence for GSY and LPL mutations Y Zhang', N Wat', IM Stratton', MG Warren-Perry', M Orho ${ }^{2}$, L Groop², RC Turner ${ }^{11}$ Diabetes Research Laboratories, Radcliffe Infirmary, Oxford, UK, ${ }^{2}$ Department of Endocrinology, Lund University, S-21401 Malmö, Sweden

insulin receptor substrate-1 (IRS-1), $\beta 3$ adrenergic-receptor (B3-AR) and glycogen synthase (GSY) genes are candidate genes for NIDDM, insulin resistance (IR), dyslipidaemia (Dys) and obesity. We studied white Caucasians subjects with NIDDM, 227 being randomly selected, 49 NIDDM within the top 2 percentiles insulin resistance (IR); 54 with dyslipidaemia (Dys) in the top quintile triglyceride/insulin and the bottom quintile $\mathrm{HDL}$, and 166 non-diabetic control subjects. We examined the association of the simple tandem repeat DNA polymorphisms (STRPs) near the IRS-1 and GS genes, and the prevalence of mutations at codons of IRS-1 513 and $972, \beta 3-A R 64$ and GS 464 using restriction fragment length polymorphism (RFLP). The STRP alleles in IRS-1 were significantly different between NIDDM and controls $(\rho=0.015)$. The IRS-1 972 mutation was significantly different between 4 groups with increased prevalence in IR and Dys subjects (18\% and $26 \%$ compared with $11 \%$ in controls; $p<0.0005$ ). Those with or without IRS-1 mutations had similar other phenotypes. $\beta 3$-AR 64 mutation was not significantly different between 4 groups but BM: for subjects with homozygous, heterozygous or wild type had $p=0.05$ for linear trend. The GSY 464 mutation was not detected in any of the diabetic subjects or controls and the population association study using GSY STRP showed no difference in allelic frequencies between NIDDM patients and controls. A mutation in lipoprotein lipase (LPL) at codon 291, associated in the general population with low HDL cholesterol, was not at increased prevalence in the NIDDM patients with dyslipidaemia. In conclusion, IRS-1 972 had an increased prevalence in subjects with iR, with or withoul dystipidaemia. $33-A R 64$ was associated with increased obesity. These separate contributions to different features of NIDDM are examples of polygenic determinants.
14

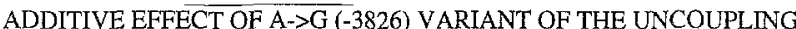
PROTEIN GENE AND THE TRP64ARG MUTATION OF THE 33 - ADRENERGIC RECEPTOR GENE ON WEIGHT GAIN IN MORBID OBESITY. K. Clément 1,2 J. RUIZ ${ }^{4}$, A.M Cassard-Doulcier ${ }^{3}$, F. Bouillaud ${ }^{3}$, D Ricquier $^{3}$, A. Basdevant 1 , Bernard Guy-Grand ${ }^{1}$ and Philipe Froguel ${ }^{2}$, Nutrition department Hôtel-Dieu, Paris ${ }^{1}$, CNRS EP10, Institut Pasteur de Lille et C.H.U Lille ${ }^{2}$, C.N.R.S, Meudon, France ${ }^{3}$, Division d'endocrinologie et du métabolisme, CHUV, Lausanne, Suisse 2,4 .

Obesity results from an imbalance between caloric intake and energy expenditure, which is partly genetically determined. Impaired thermogenesis might be involved in the development of obesity. Two genetic variants of the uncoupling protein (UC P) and the B3-adrenoceptor (B3-AR), which are major expressed proteins of the brown adipose tissue, were investigated as candidate genes for morbid obesity in a case-control study. Using PCR-RFLP assays, we have determined the prevalence of the $A \rightarrow G$ variant of the UCP gene in 238 morbidly obese subjects and control unrelated subjects. The presence of the Trp64Arg mutation was previously described in this population. A high prevalence $(42 \%)$ in French Caucasians of the $A->G$ change variation Jocated in the 5 flanking domain of the UCP gene was observed with no significant difference between morbidly obese and non obese subjects. However, the presence of the $A \rightarrow G$ allelic variant of the UCP gene was a predictive factor associated with high weight gain during adult life in morbidly obese Causasians (odd-ratio : $1.4, p=0.02$ ). This effect was previously described for the Trp64Arg mutation. Furthermore, an additive effect on high weight gain was also observed with both gene variants (Odd-Ratio : 4.95, Trend test : $p=0.05$ ). The attributable risks for UCP gene and B3-AR gene variants were respectively : $25 \%$ and $9 \%$. These data support the hypothesis of a possible link between energy balance, BAT in human and the development of overweight.
16

PUTATIVE ROLE OF MUSCLE GLYCOGEN SYNTHASE POLYMORPHISM IN THE DEVELOPMENT OF NIDDM

M. Orho*, M. Lehtovirta**, C. Forsblom**, K. Bengtsson***, C. SchalinJäntti**, and L. Groop*. Department of Endocrinology, Lund University*, Primary Health Care Centre in Skara, Skaraborg Institute***, Sweden and Helsinki University Hospital**, Finland.

Although muscle glycogen synthase locus on chromosome $19 \mathrm{y} 13.3$ has been associated with NIDDM in Finland, France, Japan and in Pima Indians, several studies have given negative results. However, in inbred strains of diabetes-prone (C57BL/6J) mice the development of diet-induced hyperglycemia was strongly linked to GYS1 locus. In order to replicate these findings, we undertook the current study where GYS1 polymorphism was determined in new larger groups of NIDDM patients and control spouses without a family history of NIDDM from Finland (Fin) (383 NIDDM patients: 156 males and 227 females, age 62.4 10.4 yrs, age at onset $51.2 \pm 15.8$ yrs, BMI $30.1 \pm 5.1 \mathrm{~kg} / \mathrm{m}^{2}$, fasting plasma glucose (FPG) $8.8 \pm 2.8$ mmolll and 270 control subjects: 120 males and 150 females, age $55.3 \pm 11.3 \mathrm{yrs}$, BMI $26.0 \pm 4.4 \mathrm{~kg} / \mathrm{m}^{2}$, FPG $\left.5.0 \pm 0.6 \mathrm{mmol} / \mathrm{l}\right)$ and from Skara in Sweden (Swe) (158 NIDDM patients: 78 males and 80 females, age $69.6 \pm 9.8 \mathrm{yrs}$ age at onset $60.1 \pm 10.4 \mathrm{yrs}$, BMI $28.3+4.5 \mathrm{~kg} / \mathrm{m}^{2}$, FPG $9.7 \pm 2.6 \mathrm{mmol} / \mathrm{l}$ and 114 control subjects: 54 males and 60 females, age $62.1 \pm 13.0$ yrs, BMI $25.1 \pm 3.6$ $\mathrm{kg} / \mathrm{m}^{2}$, FPG $\left.5.7 \pm 0.5 \mathrm{mmol} / \mathrm{l}\right)$. In both study groups, the frequency of allele 6 was increased in NIDDM patients compared to control subjects (Fin: NIDDM $28.0 \%$ controls $24.3 \%, \mathrm{p}=0.15$. Swe: NIDDM $29.8 \%$, controls $23.7 \%, \mathrm{p}=0.11$ ). When these two study groups were pooled, the frequency of allele 6 vs other alleles differed significantly between NIDDM patients $(28.5 \%$ vs $71.5 \%)$ and control subjects $(24.1 \%$ vs $75.9 \%$ ), p=0.039. Control subjects carrying allele 6 had significantly higher fasting plasma glucose concentrations compared to controls without this allele (Fin $5.03+0.55$ vs $4.89+0.55 \mathrm{mmol} / \mathrm{l}, \mathrm{p}=0.046$; Swe: $5.86 \pm 0.52$ vs $5.63 \pm 0.51 \mathrm{mmol} / \mathrm{l}, \mathrm{p}=0.021$, combined: $5.29 \pm 0.66$ vs $5.13 \pm 0.64 \mathrm{mmol} / \mathrm{l}$, $p=0.021$ ). Conclusions: We replicate and extend our previous findings by showing a weak but significant association between polymorphism in the GYS1 gene and NIDDM in this new Finnish-Swedish population. Of note, this polymorphism was associated with elevated fasting plasma glucose in nondiabetic subjects in both populations. 


\section{OP 3 \\ Nutrition}

17

DEVELOPMENTAL AND NUTRITIONAL CHANGES OF OB AND PPAR $\gamma$ GENE EXPRESSION.

D.J. Becker, V. Rousseau, L.N. Ongemba, J.C. Henquin and S.M. Brichard. Unité d'Endocrinologie et Métabolisme, University of Louvain, Brussels, Belgium

The ob gene encodes leptin, a satiety hormone, and the PPAR $\gamma$ gene encodes a transcription factor which controls adipocyte differentiation and expression of fat-specific genes. We have studied their regulation in white adipose tissue (WAT) during the suckling-weaning transition. Suckling rats ingest a high-fat diet (milk). Ob mRNA levels were very low in WAT of 15 day-old rats and rose $\sim 6$-fold until weaning at 21 days. When the rats were weaned onto a standard (high-carbohydrate) laboratory chow, ob mRNA kept increasing progressively and doubled between 21 and 30 days. This evolution contrasted with that of fatty acid synthase (FAS) mRNA which increased sharply, but only after weaning. To distinguish between the influence of developmental and nutritional factors on ob expression, a group of rats was weaned onto a highfat diet. This prevented the rise in glycaemia and insulinaemia, and the drop in plasma FFA which otherwise occurred at weaning. This also resulted in a slight $(10-15 \%)$ decrease in food intake and body weight gain. Under this high-fat diet, the rise of ob mRNA in WAT was larger (3.7-fold in 30 vs 21 day-old pups), whereas the normal rise of FAS mRNA levels was blunted. mRNA levels of PPAR $\gamma$, like those of ob, were low in WAT of 15 day-old suckling pups, doubled at 21 days, and reached a maximum already after 23 days. This evolution further differed from that of ob mRNA in being not influenced by the diet composition. In conclusion, ob expression markedly increases during the suckling-weaning transition, and this effect is accentuated by a high-fat diet. PPAR $\gamma$ also increased during suckling, but rapidly plateaued after weaning and no longer changed thereafter. PPAR $\gamma$ might control initial ob transcription, but does not seem to modulate subsequent ob expression.

\section{8}

EFFECTS OF A HIGH GLYCAEMIC INDEX STARCHY DIET ON THE ACTIVITY AND GENE EXPRESSION OF LIPID STORAGE-RELATED ENZYMES IN BOTH NORMAL AND DIABETIC RATS. M. Kabir, S. W. Rizkalla, A. Quignard-Boulange, J. Luo, N. Agheli, B. Ardouin, F. Bruzzo, G. Slama, Department of diabetes, INSERM U341, Hôtel-Dieu hospital, INSERM U177, Paris, FRANCE.

Previously we showed that 3 weeks of a hyperglycaemic index, vs low glycaemic index, starchy diet increased dramatically both basal and insulin stimulated lipogenesis in normal but not in diabetic rats. To better understand the underlying mechanisms, we decided to evaluate the effects of the same diets on the lipid storage-related enzymes activities such as fatty acid synthetase (FAS) and lipoprotein lipase (LPL), in the adipose tissue and the liver. Twenty four normal and 24 diabetic (postnatal Streptozotocin-n2) male Sprague Dawley rats were submitted to a diet containing $57 \%$ starch as either waxy corn starch (WCS) in the high glycaemic index diet (HGI) or Mungo bean starch (MBS) in the low glycaemic index diet (LGI). After the 3 week diet, body weight was comparable, as were liver and epididymal fat pad weights. In normal rats, the $\mathrm{HGl}$ diet increased plasma glucose $(159 \pm 9$ vs $133 \pm 7 \mathrm{mg} / \mathrm{dl}, \mathrm{p}<0.03$, one way Anova) without any change in plasma insulin. In diabetic rats, the $\mathrm{HG}$ ! diet increased only the plasma insulin levels ( $164 \pm 25$ vs $94 \pm 13 \mathrm{mU} / \mathrm{ml}, 0<0.01$ ). Plasma triglycerides were not significantly different in either normal or diabetic rats. Epididymal adipose tissue FAS activity was increased by $66 \%(\mathrm{p}<0.05)$ and that of the liver by $44 \%(p=0.056)$ after the HIG diet with LGI diet in normal rats but not diabetic ones. FAS gene expression was markedly increased after HGl diet in the adipose tissue of normal rats. By contrast, LPL activity was not significantly altered in the same tissue. We concluded that in normal rats the mere replacement of $57 \% \mathrm{LGl}$ starch by $\mathrm{HGl}$ one for 3 weeks led to inceased markedly FAS activity in both adipose tissue and the liver and inceased FAS gene expression markedly in adipose tissue. This was accompanied by an increase in plasma glucose levels. The LGI diet could be a new additive in dietary prescriptions in normal subjects to reduce risk factors.

\section{9}

\section{THE EFFECT OF MODIFIED FAT DIET ON INSULIN RESISTANCE AND} METABOLIC PARAMETERS IN TYPE II DIABETES.

M.Fanaian, J.Szilasi, L.Storlien and G.D.Calvert. Medical Research Unit, University of Wollongong, NSW, Australia.

This is a randomised prospective controlled study on the effectiveness of two diet programs, one an orthodox high carbohydrate low fat diet (High-CHO) and the other a high monounsaturated fat diet (HMUFA) on insulin resistance, serum lipids and other variables in 48 male and female $(M=25, F=23$, average age $44.2 \pm 0.9)$ patients with NIDDM (non-insulin-dependent diabetes mellitus) during a one year period. Patients were randomly allocated to the two dietary groups. The HMUFA group diet (using canola oil, containing both $\mathrm{n}-3$ and $\mathrm{n}-6$ fatty acids) were given a diet program to maintain body weight, aimed at providing $40 \%$ of energy intake as carbohydrate and $38 \%$ as fat (21\% monounsaturated). The High-CHO diet group were given a diet program containing $50 \%$ of energy intake as carbohydrate and $24 \%$ as fat, an equal dietary fiber content. Participants had frequent dietary consultation. A 4 day food record was analyzed every 3 months. Insulin resistance was measured in a subgroup of 28 participants by euglycemic clamp. After 12 months the following changes had occurred. The HMUFA group had $34 \%$ of energy intake as fat $(16 \%$ monounsaturated, $10 \%$ saturated), of the High-CHO group $(31 \%, 13 \%$ and $11 \%$ respectively). The HMUFA group had significant reductions in systolic $(\mathrm{p}=0.038)$ and diastolic blood pressure $(\mathrm{p}=0.009)$, fasting plasma glucose $(\mathrm{p}=0.026)$, triglyceride $(p=0.050)$, and significant increases in HDL-cholesterol $(p=0.011)$ and insulin sensitivity ( $p=0.005$ ) compared to High-CHO group. In HMUFA mean BP fell from $135 / 83$ to $127 / 23$ in 12 months: no change in High-CHO. Mean of glucose fell from 9.2 to $8.3 \mathrm{mmol} / \mathrm{L}$ in HMUFA, rose from 8.5 to $9.5 \mathrm{mmol} / \mathrm{L}$ in High-CHO. Mean of insulin sensitivity rose from 111 to $160 \mathrm{mg} / \mathrm{m}^{2} / \mathrm{min}$ in HMUFA, and fell from 130 to $91 \mathrm{mg} / \mathrm{m}^{2} / \mathrm{min}$ in High-CHO. This study concludes that after one year the monounsaturated-enriched diet program was associated with a better metabolic profile in NIDDM patients, and is more likely to be followed, than a High-CHO diet.

\section{0}

A REDUCED FAT DIET IMPROVES INSULIN SENSITIVITY COMPARED TO A MONOENE ENRICHED DIET IN COMBINED HYPERLIPIDEMIA

A.M. Louheranta, S.H. Lehtomäki, U.S. Schwab, T.M.L Ebeling, E.T. Voutilainen, A.K. Turpeinen and M.I.J. Uusitupa, Departments of Clinical Nutrition and Medicine, University of Kuopio, P.O. Box 1627, 70211 Kuopio, Finland

Patients with combined hyperlipidemia are relatively insulin resistant. Besides lipid metabolism dietary fats could modify glucose and insulin metabolism. Monoene enriched diet has been shown to be effective in lowering serum lipid concentrations. Furthermore, it may have a favourable effect on glucose tolerance as well. To investigate the effect of a reduced fat diet (National Cholesterol Education Program Step I type diet) compared with a monoene enriched diet on glucose and insulin metabolism 18 (13 men, 5 women) patients with combined hyperlipidemia were recruited. Subjects were middle-aged $(53 \pm 2$ years, mean \pm SEM) and moderately obese (BMI $29.6 \pm 0.8 \mathrm{~kg} / \mathrm{m}^{2}$ ). They followed each of the experimental diets for 4 weeks according to a randomized cross-over study design. Before and between the diet periods the subjects consumed an average Finnish diet ( $36 \%$ of energy ( $\mathrm{E} \%$ ) as fat) for 2 weeks. The reduced fat diet supplied $30 \mathrm{E} \%$ and the monoene enriched diet $36 \mathrm{E} \%$ as fat. The monounsaturated fatty acid content was $12 \mathrm{E} \%$ in the reduced fat diet and $20 \mathrm{E} \%$ in the monoene enriched diet. Frequently sampled intravenous glucose tolerance test was performed after both experimental diets. Insulin sensitivity index $\left(\mathrm{S}_{1}\right)$ was $40 \%$ higher after the reduced fat diet than after the monoene enriched diet $\left(2.42 \pm 0.42\right.$ vs. $\left.1.73 \pm 0.24 \cdot 10^{-4} \mathrm{~min}^{-1} / \mu \mathrm{U} / \mathrm{ml}, \mathrm{p}<0.02\right)$. Glucose effectiveness $\left(S_{\mathrm{G}}\right)$ did not differ between the diets $(0.0161 \pm 0.001$ vs. $0.0158 \pm 0.001 \mathrm{~min}^{-1}, \mathrm{NS}$ ). Compared to the baseline diet the reduced fat diet and the monoene enriched diet lowered significantly serum total and LDL cholesterol concentrations ( $-6.9 \%$ vs $-6.6 \%, p<0.03$ and $-7.4 \%$ vs $-8.0 \%, p<0.03$, respectively) whereas no changes could be observed in serum HDL cholesterol and triglyceride concentrations. In conclusion, the reduced fat diet and the monoene enriched diet were equally effective in lowering serum lipid values but the reduced fat diet resulted in better insulin sensitivity in patients with combined hyperlipidemia. 
EFFECT OF FISH OIL ON PLATELET FUNCTION,ERYTHROCYTE METABOLISM IN DIABETIC PATIENTS

A.A.Serhiyenko,L.M.Serhiyenko,I.S.Erin, M.R.Nesterovich,Y.M.Vendzylovi cn and A.M.Novosad. Department of Endocrinology, Lviv Medical

Institute, Lviv, Ukraine

High dietary intake of eicosapentaenoic acid (EPA) and docosahexaenoic acid (DHA) is of special interest in the possibility of influencing the metabolism of lipids and synthesis of eicosanoids. The aim of this study was to assess the long term effect of EPA and DHA on the activities of proteinkinase $\mathrm{C}$ (PK-C), $\mathrm{Na}^{+} \mathrm{K}^{+}$-ATPase, $\mathrm{Ca}^{2+} \mathrm{Mg}^{2+}$ - ATPase, levels of phospholipids and fatty acids in the membranes of erythrocytes and the levels of the ${ }^{125}\left[-6\right.$-ketoprostaglandin $F_{1 \%}\left(6-k e o_{0} P_{F} F_{1_{\alpha}}\right)$ and $125[-$ thromboxane $\mathrm{B}_{2} \quad\left(\mathrm{TXB}_{2}\right)$ in the blood plasma, activities of lactatedehydrohenase (LDF), hexokinase (HK), pyruvatkinase (PK) in the $\mathrm{RBCs}^{\prime}$ in NIDDM patients. It has been discovered that the progress of diabetic micro-and macrovessel disorders is accompanied by a decrease of the activities of $\mathrm{PK}-\mathrm{C}, \mathrm{Na}+\mathrm{K}^{+}-$ATPase and $\mathrm{Ca}^{2+}, \mathrm{Mg}^{2+}$-ATPase in the $\mathrm{RBC}^{\prime}$ s membranes. There is a considerable increase in the $\mathrm{TXB}$, level, activities of LDH, HK and PK in the RBC's and a decrease in serum 6-keto$\mathrm{PGF}_{1_{\alpha}}$, phospholipids and polyunsaturated fatty acids in the $\mathrm{RBC}$ : membranes. We have carried out an analysis of fish oil effects on the above indices in NIDDM patients. 41 patients ( $58 \pm 6$ years, $22 \mathrm{~m} / 19 \mathrm{f}$ ) werc allocated into two treatment groups. The lst group $(\mathrm{n}=22)$ was receiving cansules of fish oil every day $(1,75 \mathrm{~g}$ EPA, $1,75 \mathrm{~g}$ DHA and $0,1 \%(x-$ tocopherol acetate) and the 2 nd group $(n=19)$ was receiving placebo capsules of olive ofl. All patients were on the same diet. After 2 months of treatment there was a decrease in TXB2 level $(p<0,001)$, activitics of $\mathrm{LDH}$ HK, with simultaneous increases in EPA level, EPA/arachidonic acid ratio, activities of $\mathrm{PK}-\mathrm{C}\left(22,49 \pm 3,13 \mathrm{pmol}{ }^{32} \mathrm{P} / \mathrm{mg}\right.$ protein per $\left.1 \mathrm{~min}, \mathrm{p}<0,001\right)$ $\mathrm{Na}^{+}, \mathrm{K}^{+}$-ATPase and $\mathrm{Ca}^{2+}, \mathrm{Mg}^{2+}$-ATPase and the concentration of 6 ketoPGF $I_{\alpha}$ in the first group. In conclusion, EPA and DHA at moderate doses may exert antithrombic effects and may be used for prophylaxis and treatment of diabetic angiopathies.
THE EFFECT OF ETHANOL ON ENERGY PRODUCTION RATE IN WOMEN WITH TYPE 2 DIABETES MELLITUS

M.K. Larsen, H. Storm, C. Thomsen, C. Hansen and K. Hermansen. Dept of Endocrinology and Metabolism, Aarhus University Hospital, Tage-Hansensgade 2, DK-8000 Aarhus C, Denmark.

Type 2 diabetic subjects are often adyised to abstain from alcohol because of its high energy content and the positive association between type 2 diabetes and obesity. Howeyer, several epidemiological studies in non-diabetic subjects have shown a negative association between alcohol consumption and obesity. In addition, alcohol has been found to cause an energy independent increase in energy production rate (EPR) in normal women. The aim of the present study was to compare the acute effect of isoenergetic amounts of ethanol and glucose on EPR in 15 postmenopausal type 2 diabetic women. On 5 separate days patients drank in random order $250 \mathrm{~mL}$ non-caloric juice alone (a). with addition of 0.2 (b) or $0.4 \mathrm{~g}$ ethanol $/ \mathrm{kg}$ body weight (c) or isoenergetic amounts of glucose ( $\mathrm{d}$ and e). EPR was measured by indirect calorimetry (Deltatrac ${ }^{\mathrm{TM}}$ II) before, 15-40 minutes, and 75-100 minutes after the juice intake, respectively. Circulating levels of ethanol, glucose, insulin, free fatty acids, triacylglycerol and lactate were determined before the juice intake and during the subsequent $4 \mathrm{~h}$. The data (mean \pm SD) were analysed in a repeated measures fashion using RM-ANOVA or Friedman's test. We found that the peak level of ethanol was $16.4 \pm 2.5 \mathrm{mM}$ after the highest and $7.8 \pm 2.2 \mathrm{mM}$ after the lowest ethanol dose, respectively. The EPR before the 5 different juice drinks were identical $(p=0.97) . \triangle E P R(K J / m i n)$ after intake of $0.2 \mathrm{~g}$ ethanol $/ \mathrm{kg}$ body weight ( 75 to 100 minutes) increased by 30 times $(p<0.01)$ as compared to $\triangle E P R$ after the isoenergetic glucose load. In conclusion, the consumptiou of ethanol in a small quantity has a higher acute thermogenic impact than the isoenergetic amount of glucose in type 2 diabetic women without causing any acute aberration in the glycaemic control Consequently, small amounts of ethanol seem to be able to raise the energy production rate in an energy independent manner and may on a more long-term basis have a negative association with weight gain in type 2 diabetic women.

\section{3}

EFFECTS OF ALCOHOL ON POST EXERCISE METABOLIC RESPONSES IN TYPE 2 DLABETIC SUBJECTS.

13y) Rasmussen C Christiansen. OW Rasmussen and K Hermansen. Department of Endocrinology and Metaboism. Aarhus Lniversity Hospital.. Aarhus DK 8000 C. Denmark

Hypoglycaemia is a leared complication in type t diabetic subiects. the risk being increased by phrsical activity and alcohol. Previously we have found that alcohol had no acute effect on blood ghucose levels in trpe ? diabetic subjects. The question arises how an everydar situation eg. the combination of akohol incake and exercise affects the glucose and insulin responses in twpe? diabetic subjects. The aim was to stud $y$ ine combined metabolic effects of exercise and alcohol in 12 type 2 diabetic subjects. (on three test days the lasting type 2 diabetic subjects ingested a standard meal containing $50 \mathrm{~g}$ curbohydrate either followed bv l) rest. $2130 \mathrm{~min}$ exercise $1+0 \%$ of Vo2 max) or 3) alcohol intake $0 .+\mathrm{g} k \mathrm{~g}$ and $30 \mathrm{~min}$ exercise. We found that the blood glucose response area (mean $\pm S D$ ) to the standart meal was reduced following txercise $\left(2217 \pm 538\right.$ is. $20^{-} 1 \pm=502 \mathrm{mu} / 240$ $\min p<0.04)$. The comination of alcohol and exercise reduced the blond glucose responses

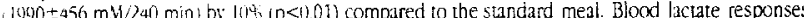
were signiticantly higher iter exercise and alcohol $(415 \pm 127 \mathrm{~m}(\mathrm{~V} / 2+0 \mathrm{~min})$ compared to the sandard meal $(203 \pm 5 \div \mathrm{mV} / 240 \mathrm{~min})(\mathrm{p}<0,003)$ and compared to the standard meal and exercise

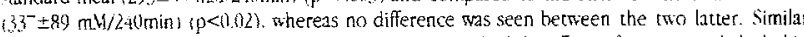
insulin response areas were obrained. Funhermore, we studied the effects of exercise and alcohol in - type 2 diabetic subjects mithout any meal. On four test davs the lasting twpe I diabecic subjects were either l) resting. 2) resting after drinking alcohol $0.4 \mathrm{~g} / \mathrm{kg}$. 3) exercising $30 \mathrm{~min}$. (40. of Vo maxy or 4 ) exercising arer drinking allohol. Sirrijar blood glucose response areas (mean \pm SD) were obtained in the four situations. Is compared to the resing dav $1221 \pm^{-8} 8 \mathrm{~m} / 240 \mathrm{~min}$ blood lactate responses aere signiticancly higher afer exercise $(363 \pm 37 \mathrm{~m} / 240 \mathrm{~min})(p<0.0 \mathrm{I})$ and after alcoho

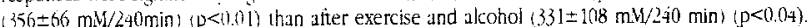
Simitar insulin response areas mere seen. Conctusion: The risk of acyte hypogivcaemia in type ¿liabetic subjects seems to be minimal after modemte chercise as well as after moderate exercise and alcohol intake. There seems to he no need for extra carbohwdrate. Statistics: RPYI Anowa. paired T test. Jiriedmans wwo wars Anora. Wikoxons paired rank test where appropriate.

\section{4}

GLUCOSE TOLERANCE HAS A CONTINUOUS RELATIONSHIP WITH TOTAL ENERGY EXPENDITURE.

N.J.Wareham ${ }^{1}$, S.H.J.Hennings ${ }^{1}$. N.E.Day 1 , C.N.Hales ${ }^{2}$ and A.M.Prentice ${ }^{3}$. Departments of Community Medicine and Clinical Biochemistry ${ }^{2}$ University of Cambridge and MRC Dunn Clinical Nutrition Unit ${ }^{3}$, Cambridge.

Imprecision in the measurement of total energy expenditure has led to underestimation of the importance of this exposure in the aetiology of the insulin resistance syndrome and NIDDM. This study was undertaken to investigate the relationship between glucose tolerance, physical fitness and total energy expenditure as assessed using the heart rate monitoring method. This technique has been shown to be accurate by comparison with measurements by the doubly-labelled water method and indirect calorimetry. 164 volunteers aged 30-40 years were randomly selected from a general pracrice register and underwent a standard $75 \mathrm{~g}$ OGTT. Resting energy expenditure and 4-day total energy expenditure were assessed using the heart rate monitoring method with individual calibration of the relationship between energy expenditure and heart rate. Energy expenditure was calculated as physical activity level (PAL), a weight independent measure of energy expenditure, where PAL $=$ total energy expenditure / basal metabolic rate. In the 74 male subjects the mean PAL was 1.89 (sd 0.40) and in the 90 women 1.76 (sd 0.31 ). In men the spearman rank correlation between PAL and 2-hour plasma glucose concentration was $-0.32(\mathrm{p}<0.01)$ and in women $-0.21(p<0.05)$. The 2 -hour plasma glucose was aiso negatively correlated with physical fitness as assessed by $\mathrm{VO}_{2 \max } \mathrm{kg}^{-1}(\mathrm{r}=-0.38, \mathrm{p}<0.001$ in men and $\mathrm{r}=-0.34, \mathrm{p}<0.001$ in women). We conclude that glucose tolerance has a continuous relationship with total energy expenditure and that this is demonstrable using the heart rate monitoring technique even in a small population-based cohort of young adults. 


\section{Beta-Cell Destruction and Defence}

\section{5}

IEF AND NEPHGE TWO-DIMENSIONAL GEL ELECTROPHORESIS "DATABASES" OF NEONATAL RAT ISLET PROTEINS: EFFECTS OF IL-1B H. U. Andersen, S. J. Fey', P. Mose Larsen', K. R. Hejnæs', T. MandrupPoulsen and J. Nerup. Steno Diabetes Center, Gentofte, 'Inst. of Med. Microbiol., Aarhus Univ., Aarhus, ${ }^{2}$ Novo Nordisk, Bagsværd, Denmark. Previous studies have shown that protein synthesis may play an important role in the intracellular mechanism of $\mathrm{LL}-1 \mathrm{~B}$ action and in the cellular defense against IL-1B. Two-dimensional (2-D) gel electrophoresis of pancreatic islet proteins may be an important tool facilitating studies of the molecular pathogenesis of IDDM. Therefore, the aim of this study was to create "databases" (DB) of all reproducibly detectable protein spots on $10 \%$ and $15 \%$ acrylamide 2-D gels of neonatal rat islets, labelled under standardized culture conditions. Islets were isolated from $4 \mathrm{~d}$ inbred WF rats. Following $4 \mathrm{~d}$ preculture and $24 \mathrm{~h}$ culture, the islets were labelled for $4 \mathrm{~h}$ with $\left[{ }^{35} \mathrm{~S}\right]$-methionine. From every sample, isoelectric focusing (IEF; $\mathrm{pH}$ 3.5-7) and non-equilibrium pHgradient electrophoresis (NEPHGE; pH 6.5-10.5) gels were prepared. Fluorographs were analyzed using the Biomage programme on a Sunsparc workstation. 1792 spots were present in 5 of 5 gels in the $15 \%$ DB, whereas 1373 spots were present in 5 of 5 gels in the $10 \%$ DB, yielding a spot detection reproducibility of $75.2 \%-91.7 \%$. In both DBs, the average coefficient of variation of the $\%$ integrated optical density (CV\% of $\% \mathrm{IOD}$ ) for spots present in all gels was between $42.4 \%$ and $45.7 \%$. When the same sample was analyzed in consecutive sets of gels on different days, the average CV\% of $\% 10 D$ was $35.5-36.1 \%$. When the same sample was analyzed repeatedly in one set of geis, the average CV\% of \%IOD was $30.2 \%-45.7 \%$. By visual analysis we have previously found 33 spots to be up-or downregulated by IL$1 B$ on WF rat isiet 2-D gels. Using \pm 2 SD of IOD \% of each DB spot as a cutoff level, comparison with the $10 \%$ DB confirmed 32 of these alterations and as expected identified several new protein changes. Thus, a total of 183 spots were upregulated, 113 downregulated and 34 synthesized de novo by IL-1B. in conclusion, we present the first $10 \%$ and $15 \%$ 2-D gel protein DB of islets of Langerhans and demonstrate its usage in detecting IL-1B-induced changes in protein levels.

\section{6}

IN VIVO INSULIN TREATMENT PROTECTS RAT ISLETS FROM CYTOKINE-INDUCED INHIBITION OF INSULIN SECRETION DESPITE INCREASED NITRIC OXIDE PRODUCTION

A. Dunger, D. Schröder, I. Klöting and U. Fischer, Gerhardt Katsch Institute of Diabetes, Karlsburg, Germany

Hyperactivity of beta cells has been hypothesized to render them more vulnerable against environmental factors while beta cell rest has been suggested to exert protective effects. To further test this hypothesis, islets obtained from 50-70 day-old normoglycemic diabetes-prone BB rats treated for 1-3 weeks with insulin (IT, $2 \mathrm{IU} / \mathrm{d})$ were exposed to cytokines (CY, 10 $\mathrm{U} / \mathrm{ml} \mathrm{ILl}-\beta+50 \mathrm{U} / \mathrm{ml} \mathrm{IFN}-\gamma+50 \mathrm{U} / \mathrm{ml}$ TNF- $\alpha$ ) for $20 \mathrm{hrs}$ or they remained $\mathrm{CY}$-untreated. Control islets were obtained from age- and gender-matched non-insulin-treated (NIT) BB rats. IT rats were characterized by a significantly lower non-fasting plasma glucose level as compared to NIT animals $(3.6 \pm$ SEM 0.5 vs $6.1 \pm 0.4 \mathrm{mmol} / / \mathrm{n}=6 \mathrm{ea} ., \mathrm{p}<0.01)$. Also, the insulin content of IT rat islets was significantly lower than that of NIT islets $(0.24 \pm 0.04$ vs $3.60 \pm 0.13 \mathrm{pmol} / \mathrm{sislet}, \mathrm{p}<0.01)$. No effect of $\mathrm{CY}$ exposure on insulin storage could be ascertained in both experimental groups. The CYinduced inhibition of glucose-stimulated insulin secretion seen in NIT rat islets (untreated: $51.87 \pm 1.42$ vs CY: $17.20 \pm 2.93$ fmol released insulin per pmol insulin content $/ 2 \mathrm{hrs}, \mathrm{p}<0.01$ ) was prevented by in vivo insulin treatment (untreated: $97.46 \pm 6.67$ vs CY: $95.32 \pm 8.09 \mathrm{fmol} / \mathrm{pmol}$ insulin in $2 \mathrm{hrs}, \mathrm{ns}$ ). Non-CY-treated islets of NIT and IT rats produced comparable amounts of nitric oxide as measured by means of nitrite accumulation in the culture medium. Following $\mathrm{CY}$ exposure, islets obtained from IT rats generated 4-5 times more nitric oxide than NIT rat islets did. It is concluded that beta cell rest due to in vivo insulin treatment prevents CY-induced inhibition of glucose-stimulated insulin secretion despite increased nitric oxide production, which may either indicate an uncoupling of the two processes, or point to insulin-mediated activation of mechanisms protecting the beta cells from detrimental effects of nitric oxide.

\section{7}

REGULATION OF THE INDUCIBLE CYCLOOXYGENASE IN PANCREATIC BETA CELLS BY IL-1 VIA NUCLEAR FACTORS NF- $\mathrm{kB}$ AND NF-IL-6.

C.H. Sorli, H.J. Zhang,Z. Liu, and R. P. Robertson, Minneapolis, MN.

Important biological mediators within the inflammatory beta cell microenvironment that may contribute to the initiation of Type I DM include cytokines (i.e., IL-1, TNF) and prostaglandins (PGE2), which can lead to pancreatic beta cell dysfunction and damage as well as modulate local immune responses. Inflammatory cytokines induce the transcription of several genes through the activation of specific transcription factors such as NF-kB and NF-IL-6. A potential target of these transcription factors in the beta cell is the inducible form of cyclooxygenase (COX-2) which has characteristics of an early phase protein and possesses consensus binding sequences for multiple transcription factors, notably NF-KB and NF-IL-6. We examined the expression pattern of both the inducible COX-2 and the constitutively expressed COX-1 in HIT cells and hamster islets to determine the enzyme responsible for PGE2 production by beta cells. Northern analysis revealed uniquely high levels of COX-2 mRNA that were not due to serum factors and were reduced by dexamethasone $(5 \mu \mathrm{M})$. In response to IL$1(5 \mathrm{ng} / \mathrm{ml})$. HIT cells showed a biphasic pattern of COX-2 mRNA expression with a transient increase followed by a dose and time-dependent decrease to levels $30 \%$ of baseline values. COX-2 promoter activity (assessed via transient transfections with a COX-2 promoter/CAT construct) mirrored mRNA expression with high baseline activity inhibited in a dose-dependent fashion by IL-1, indicating that regulation of COX-2 expression in HIT cells was likely at the level of gene transcription. The mechanism of high baseline expression of COX-2 mRNA was pursued using electromobility shift assays of nuclear extracts for NF-KB and NF-IL-6. NF-IL-6 binding activity to the COX-2 promoter was high at baseline and upon exposure of cells to IL-l exhibited a rapid reduction in activity mimicking mRNA levels. On the other hand, NF $* \mathrm{~KB}$ activity was low at baseline, dramatically increased within one hour after IL-1 exposure, and subsequently declined. It therefore appears that the uniquely high baseline expression of COX-2 is the result of NF-IL- 6 upregulation of COX-2 promoter activity. Using RT-PCR, we have identified the prostaglandin receptor EP3 in HIT cells and hamster islets supporting the responsiveness of these cells to PGE2. These data suggest that the interrelationships between IL-1, NF- KB, NF-IL-6 and COX-2 mediated PGE2 production may represent a novel regulatory mechanism for cytokine induced beta cell dysfunction within an inflammatory microenvironment.

\section{8}

INTERLEUKIN-1(IL-1) SIGNALLING IN RAT $\beta$-CELLS INVOLVES THE JNKSAPK AND THE 338 MITOGEN-ACTIVATED PROTEIN KINASE (MAPK)

C. M. Larsen ${ }^{\star}$, K. A. W. Wadt*, L. F. Juhlis, H. U. Andersen ${ }^{*}$ K. Seedorfa, J. Raingeaud\#, C.A. Dinarello\$ and T. Mandrup-Poulsen*. *Steno Diabetes Center and uhagedorn Research Institute, Gentofte, Denmark.\#University of Massachusetts Medical School, Worcester, USA and \$University of Colorado Health Sciences Center, Denver, USA.

IL-1 causes programmed cell death (PCD) in rat $\beta$-cells. Nitric oxide (NO) produced by IL-1 mediated expression of $B$-cell cytokine inducible NO-synthase has been implicated as second messenger for this effect. However since inhibition of NO synthesis only partially protects rat $B$-cells from IL-1 toxicity, other signals for $P C D$ may be involved. Sustained activation of the JNK/SAPK and the p38 MAPK has been implicated in the signalling pathways leading to PCD. IL-1 and other cellular stresses (endotoxin, TNF, UV-radiation, heat shock and extracellular hyperosmolarity) are known to activate JNK/SAPK and p38 MAPK in other cells. Activated JNKJSAPK phosphorylates the transcription factors ATF2, Elk-1 and c-Jun, whereas activated p38 MAPK phosphorylates ATF2 and via activation of MAPKAP kinase-2 the small heat shock protein (HSP) 25. The aim of this study was to investigate if JNKISAPK and $p 38$ MAPK are involved in $\mathrm{LL}-1$ signalling in isolated rat islets. 150 neonatal Wistar rat islets were incubated with $150 \mathrm{pg} / \mathrm{ml} \mathrm{IL-1}$ for $2.5 \mathrm{~min}$ to $24 \mathrm{~h}$. The phosphorylation of the substrates ATF2, Elk-1 and HSP25 by the lysates of IL-1 stimulated islets was investigated by [y$\left.{ }^{32} \mathrm{P}\right]$ incorporation in the substrate proteins separated by SDS-PAGE and visualized by autoradiography. $1 \mathrm{~L}-1$ exposure of rat islets resulted in phosphorylation of ATF2, Elk-1 and HSP25 within $5 \mathrm{~min}$., and this effect was sustained from between $160 \mathrm{~min}$ to $6 \mathrm{~h}$. To investigate if a known non-cytokine activator of the JNK/SAPK and p38 MAPK signalling pathways mimicked these effects islets were exposed to increasing extracellular osmolarity (285-525 mosM) by adding hyperosmolar saline to the incubation medium. Hyperosmolarity (525mosM) caused phosphorylation of ATF2, Elk-1 and HSP25 within $2.5 \mathrm{~min}$ and up to $12 \mathrm{~h}$. Hyperosmolarity $(285-525 \mathrm{mosM})$ mimicked the bimodal effect of $\mathrm{IL}-1$ on $24 \mathrm{~h}$ accumulated insulin release but not on NO production. in conclusion IL-1 causes a rapid and sustained activation of JNK/SAPK and p38 MAPK in rat islets, an effect mimicked by hyperosmolar stress. We suggest that JNK/SAPK and p38 MAPK activation is involved in signalling of NO independent effects of $I L-1$ in B-cells. 
29

\section{REGULATION OF CYTOPROTECTIVE ENZYME GENE EXPRESSION IN INSULIN-PRODUCING CELLS}

M. Tiedge, J. Drinkgern and S. Lenzen, Institute of Clinical

Biochemistry, Hannover Medical School, D 30625 Hannover Germany

It was the aim of this study to evaluate the expression and regulation of the cytoprotective enzymes superoxide dismutase (SOD), catalase (CAT) and glutathione peroxidase (GP) in insulin-producing cells under conditions of cellular stress. Thus we have performed Northern and Western Blot analyses in comparison to other tissues and after incubation of pancreatic islets and RINm5F cells (a) at high glucose concentrations, (b) at high oxygen concentrations and (c) after heat shock treatment. From all rat tissues pancreatic islets showed the lowest levels of cytoprotective enzyme gene and protein expression. When compared with the liver, mitochondrial Mn-SOD expression in islets was $50 \%$, whereas the expression of catalase and glutathione peroxidase was barely detectable in the range $<5 \%$. Incubation of islets at $30 \mathrm{mM}$ glucose for a period of $48 \mathrm{~h}$ did not affect the expression of SOD, CAT and GP. Islets which were cultured at $70 \%$ $\mathrm{O}_{2}$ for a period of $48 \mathrm{~h}$ showed a $50 \%$ increase of Mn-SOD expression. In RINm5F cells high oxygen pressure did not induce the expression of any cytoprotective enzyme. The activiation of cellular defense mechanisms by heat shock treatment of RNm5F cells at $41^{\circ} \mathrm{C}$ and $43^{\circ}$ $\mathrm{C}$ also failed to increase cytoprotective enzyme gene and protein expression. The low expression level of cytoprotective enzymes in insulin-producing cells, with emphasis on the enzymes CAT and GP for inactivation of hydrogen peroxide, may explain the extraordinary sensitivity towards reactive oxygen species. At variance from other tissues the experimental induction of oxidative stress did not result in a significant activation of cytoprotective enzymes. Our data indicate that pancreatic B-cells apparently cannot adapt their cellular defense mechanisms to various conditions of oxidative stress and th is may explain the susceptability during autoimmune diabetes.

\section{0}

OXYGEN RADICALS IMPAIR B-CELL FUNCTION LEADING TO NON-APOPTOTIC CELL DEATH

M.Di Matteo, J.Thorpe, *A.Loweth, *N.Morgan and I.Green, Univ. of Sussex, BN1 9QG. *Univ. of Keele, ST5 5BG. UK.

Superoxide, produced in the vicinity of islets by activated macrophages, is thought to be involved in B-cell cytotoxicity. The aim of this study was to determine the effects of oxygen radicals or cytokines on B-cell mitochondrial activity, protein synthetic ability, DNA laddering and $\beta$-cell morphology. The insulin-secreting cell line HIT-T15 or rat islets of Langerhans were treated with xanthine oxidase/hypoxanthine or combined cytokines (24h). Mitochondrial function was measured after $24 \mathrm{~h}$ treatment using the MTT assay . ${ }^{3} \mathrm{H}$-mixed amino acid incorporation into protein was determined during treatments. Xanthine

oxidase/hypoxanthine $(2.5 \mathrm{mU} / \mathrm{ml}+0.5 \mathrm{mM})$ significantly inhibited HIT-

T15 cell protein synthesis, but did not decrease mitochondrial function. Xanthine oxidase/hypoxanthine $(10 \mathrm{mU} / \mathrm{ml}+0.5 \mathrm{mM})$ decreased HIT-T 15 mitochondrial function to $18 \pm 3 \%$ of the value seen in control cells but did not cause significant DNA laddering as measured by gel electrophoresis. Low dose xanthine oxidase/hypoxanthine was sufficient to decrease mitochondrial activity in rat islets and caused loss of insulin from secretory granules and a high proportion of autolysis as revealed by electronmicroscopy. Combined cytokines IL $1 B+\mathrm{TNF} \alpha+\mathrm{IFN} \gamma$ (all $100 \mathrm{pM}$ ) decreased islet and HIT-T15 cell mitochondrial function to $54 \%$ and $73 \%$ of the respective controls and inhibited HIT-T15 cell protein synthesis. Electronmicroscopy of cytokine treated islets showed marked ultrastructural changes and minor apoptosis. Oxygen radicals and cytokines impair B-cell mitochondrial function and protein synthesis and cause marked ultrastructural changes. However, oxygen radicals do not cause DNA laddering which is characteristic of apoptotic cell death.

\section{1}

SIMILAR SENSITIVITY OF HUMAN AND RAT PANCREATIC ISLETS TO PEROXYNITRITE-MEDIATED CELL DYSFUNCTION AND DEATH C.A. Delaney ${ }^{1}$, B. Tyrberg ${ }^{1}$ and D.L. Eizirik ${ }^{1,2}{ }^{1}$ Dept of Medical Cell Biology, Uppsala University, Uppsala, Sweden, ${ }^{2}$ Dept of Metabolism and Endocrinology, Vrije Universiteit Brussel, Belgium.

Nitric oxide and reactive oxygen species may be concomitantly generated during insulitis. In addition to the individual toxic effects of these radicals, nitric oxide and superoxide can react to form peroxynitrite. Peroxynitrite may decompose at physiological $\mathrm{pH}$ into nitrogen dioxide and a highly toxic 'hydroxyl-like' intermediate. In this study we examined the sensitivity of rat and human islets to peroxynitrite both as acute exposure and after of rat and human islets to peroxynitrite both as acute exposure and after peroxynitrite $(200 \mu \mathrm{M})$ decreased islet glucose oxidation (by $41 \%$ and $31 \%$, respectively in rat and human islets; $p<0.01, n=5-6$ ) and induced DNA strand breakage as judged by an increase in mean comet length in the comet assay, from $51 \pm 3$ (control) to $74 \pm 6 \mu \mathrm{m}$ (peroxynitrite 200 $\mu \mathrm{M} ; \mathrm{p}<0.01, \mathrm{n}=3$ human islet preparations). Acute exposure to $1 \mathrm{mM}$ peroxynitrite induced widespread human islet destruction. Following a subsequent $24 \mathrm{~h}$ culture, glucose oxidation was still significantly decreased in the peroxynitrite-treated $(200 \mu \mathrm{M})$ rat and human islets (by $44 \%$ and $61 \%$ respectively; $p<0.01$ versus control, $n=5-6$ ) and there was a significant decrease in both human and rat islet retrieval. Islet cell viability was assessed using the trypan blue exclusion test and evidence of internucleosomal DNA degradation was quantified using the fluorophore propidium iodide (PI). Acute exposure to peroxynitrite tluorophore propidium iodide (Pl). Acute exposure to peroxynitrite
induced mainly an increase in islet cell lysis indicating toxic cell death. However, after $24 \mathrm{~h} 56 \%$ of peroxynitrite treated human islet cells were PI positive as compared to control $(7 \%)$, suggesting the presence of apoptosis. Exposure to a negative control (decomposed peroxynitrite i.e nitrite, hydrogen peroxide and sodium chloride) had no adverse effect on nitrite, hydrogen peroxide and sodium chloride) had no adverse effect on
rat and human islet function or survival. In conclusion, acute exposure to rat and human islet function or survival. In conclusion, acute exposure to a loss in islet cell survival. Moreover, this study provides evidence for the first time of a radical to which both human and rat islets appear to be equally susceptible.

\section{2}

TRIGGERING OF FAS (APO-l/CD95)-INDUCED APOPTOSIS $\mathbb{N}$ HUMAN PANCREATIC B-CELLS

C. Giordano, G. Stassi, M. Todaro, P. Richiusa, R. De Maria, M. Giordano, A. Mattina, M.S. Sbriglia and A. Galluzzo. Laboratory of Immunology, Endocrinology Section, University of Palermo, Italy.

Insulin-dependent diabetes (IDDM) is a T-cell mediated autoimmune disease resulting in the selective destruction of pancreatic $\beta$-cells. However, the mechanisms by which infiltrating T-cells and their secretory products (lymphokines) induce the death of $\beta$-cells are poorly understood. One mechanism of $\beta$-cell destruction could be apoptosis, a physiological mechanism of cell death regulating tissue development and cell mass in several organs that can be induced by the activation of the Fas (APO$1 / \mathrm{CD} 95$ ) antigen pathway. In the pancreas, Fas-ligand (FasL) positive infiltrating T-cells could induce apoptosis of those target cells expressing the Fas receptor. Although pancreatic islet cells do not normally express the CD95 receptor, we have recently found that human pancreatic $\beta$-cells purified by flow sorting do express the CD95 receptor following exposure to IL-1 $\beta$. Of note, IL-1 $\beta$ has been reported to be selectively toxic for pancreatic $\beta$-cells both in vitro and in vivo. In the present study we show that activation of the Fas pathway is followed by apoptosis of human pancreatic $\beta$-cells expressing the Fas receptor in vitro. Pancreatic $\beta$-cells were found to be highly apoptotic $(82 \pm 5.6 \%)$ after IL-1B treatment and activation of the Fas pathway induced by a $24 \mathrm{~h}$ incubation with anti-Fas $\mathrm{mAb}$. Apoptosis was not triggered in B-cells cultered in plain culture media (range $0.5-1.0 \%$ ). In conclusion, our findings support the hypothesis that the Fas pathway may play a role in determining the $\beta$-cell damage and death associated with IL-1 $\beta$. Further studies are necessary to investigate whether the Fas pathway is naturally activated in the pancreas during the prediabetic stage. 
OP 5

\section{Insulin Action}

33

PROFOUND DOWNREGULATION OF INSULIN RECEPTORS ON ISOLATED MONOCYTES DURING HYPOGLYCEMIC CLAMP. I. Koop, P. Preuss, U. Oltmanns, C. Loeffler, H. Lochs, R. Fussgaenger* and P. Jehle*. Depts. of Internal Medicine, Univ. Hospital Charité, Berlin and *Ulm, FRG.

Insulin receptor downregulation is observed during insulin resistance of type II diabetes, during intensified insulin therapy and prolonged hyperinsulinemia. The aim of this study was to investigate the effect of hypoglycemia on insulin receptor regulation on isolated human monocytes. Methods: Six healthy volunteers (3f, 3m, age: $22-26 y$, BMI: $22.3 \pm 1.2)$ were studied by a stepwise hypoglycemic hyperinsulinemic (1-2mU/kg body weight/min) clamp. At blood glucose levels of $4.9 \mathrm{mM}$ and $2.3 \mathrm{mM}$ monocytes were isolated and studied for competitive ${ }^{12.5} \mathrm{I}-$ A-14-insulin binding. Results: During hypoglycemia the total number of insulin binding sites per monocyte (IR) decreased by about $50 \%$ from $27,700 \pm 4600$ to $13,000 \pm 3600(p<0.05)$, and the specific insulin binding per $10^{6}$ monocytes (ISB) was lowered from $1.33 \pm 0.08$ to $0.83 \pm 0.08 \%$ $(\mathrm{p}<0.002)$. Insulin binding affinity $\left(\mathrm{IC}_{50}\right)$ did not change $(0.4 \pm 0.08 \mathrm{vs}$. $0.28 \pm 0.11 \mathrm{nM}, \mathrm{n.s}$.). No correlation was found between serum insulin or blood glucose levels and IR, ISB and $\mathrm{IC}_{50}$, respectively. Discussion: During an $8 \mathrm{~h}$ hypoglycemic hyperinsulinemic clamp a profound downregulation of insulin receptors on circulating monocytes was observed which exceeded that described under euglycemic hyperinsulinemic conditions. These data combined with the lack of correlation between serum insulin and receptor values suggest that other factors are at least in part involved in the modulation of the insulin receptor during hypoglycemia. Candidates are counterregulatory hormones like glucagon or epinephrin which have been shown to decrease insulin binding

\section{4}

INCREASED EXPRESSION OF INSULIN/IGF-I HYBRID RECEPTORS IN MUSCLE AND ADIPOSE TISSUE FROM SUBJECTS WITH NIDDM: RELATION TO IN VIVO INSULIN SENSITIVITY

M. Federici, O. Porzio, D. Lauro, L. Zucaro and G. Sesti. Dept. of Internal Medicine, University of Rome "Tor Vergata", Rome, Italy.

Evidence exists showing that insulin receptor (IR) and IGF-I receptor (IGF-IR) assemble as hybrids but their functional significance is still undefined To address this, we determined distribution of hybrid receptors (IR/GF-IR) in muscle and fat from 8 normal and 8 NIDDM subjects. 96-well microwells were coated with either MA-20 anti-IR antibody (ab) or an antipeptide $a b(C 2-a b)$ to the IGF-IR, and then incubated with muscle or fat lysates. Immobilized receptor were incubated with either ${ }^{125}$ I-insulin or ${ }^{125}$ I-IGF-I in the presence or absence of various amounts of the two unlabeled ligands. Hybrids were quantified as the fraction of ${ }^{125}$ I-IGF-I binding sites reactive with MA-20 ab and expressed as the percentage of total IGF-IR (type Ithybrids) immobilized with $\mathrm{C} 2-\mathrm{ab}$. In muscle the percentage of hybrid receptors was higher in NIDDM subjects than in control $(70.2 \pm 2.9 \%$ vs $44.0 \pm 4.6 \%$, respectively, $p<0.0003)$. The percentage of hybrids was negatively correlated with both IR number $(r=-0.7 ; p<0.03)$ and in vivo insulin sensitivity measured by insulin tolerance test (Kitt) $(r=-0.71, p<0.03)$. Insulin binding affinity, estimated as the concentration of unlabeled insulin reguired to inhibit $50 \%$ of maximal ${ }^{125}$ I-insulin binding $\left(\mathrm{IC}_{50}\right)$ was lower in NIDDM subjects than in controls $\left(\mathrm{IC}_{s 0}=1.31 \pm 0.08 \mathrm{nM}\right.$ and $0.68 \pm 0.16 \mathrm{nM}$, respectively, $p<0.004$ ) and was correlated with the percentage of hybrid receptors $(r=0.75, p<0.03)$. Similarly, in fat tissue the percentage of hybrids was higher in NIDDM subjects than in control $(70.0 \pm 3.9 \%$ vs $47.1 \pm 2.6 \%$, respectively, $p<0.0003$ ). The percentage of hybrids was negatively correlated with IR number $(r=-0.72 ; p<0.05)$, and Kitt ( $r=-0.70, p<0.05)$, whereas it was positively correlated with IGF-IR number $(r=0,7, p<0.007)$. IC $\mathrm{C}_{50}$ of insulin binding was lower in NIDDM subjects than in controls $\left(\mathrm{IC}_{50}=1.97 \pm 0.25 \mathrm{nM}\right.$ and $0.54 \pm 0.08 \mathrm{nM}$ respectively, $p<0.0001$ ) and was correlated with the percentage of hybrid receptors $(r=0.75, p<0.03)$. In both tissues, IGF-I binding affinity did not differ bewteen the two groups of subjects. Results demonstrate that: i) insulin/IGF-I hubrid receptors are overexpressed in two major target tissues of insulin action; ii) the proportion of hybrids is function of the number of IR, and, at least in adipose tissue, IGF-IR iii) increased expression of hybrid receptors which are known to bind IGF-I with higher affinity than insulin may contribute to diminish in vivo insulin sensitivity.

\section{5}

PREFERENTIAL ASSOCIATION OF THE ADAPTER PROTEIN GRB10 WITH INSULIN AS COMPARED TO IGF-I RECEPTORS. L. Laviola, F. Giorgino, H. Hansen, J.C. Chow, J.A. Baquero, J. Ooi, H. Riedel, and R.J. Smith. Joslin Diabetes Center, Boston, MA, USA. The biological effects of insulin and insulin-like growth factor-I (IGF-I) are mediated by interactions of the specific receptors for these two hormones with multiple intracellular proteins including IRS-1, IRS-2, Shc, and PI 3-kinase. Although insulin and YGF-I have different physiological functions, mechanisms for distinct signaling responses to the structurally related insulin and IGF-I receptors have not been demonstrated. The goal of this study was to identify insulin and IGF-I receptor-associated proteins that may define unique characteristics of insulin and IGF-I signaling through preferential interaction with receptors for only one of these hormones. The receptor intracellular domains were used as probes in yeast two-hybrid screening of a mouse embryo cDNA expression library. Multiple independent clones were identified that encoded the $\mathrm{SH} 2$ domain of the recently described Grb10 adapter protein. The receptor/Grb 10 interaction was then investigated in intact cells by co-precipitation analysis of receptor-deficient $\mathrm{R}$ - cells (mouse fibroblasts with homologous disruption of the IGF-I receptor gene) and transfected R-cells expressing similar numbers of either insulin receptors (R-IR cells) or IGF-I receptors $(\mathrm{R}+\mathrm{cell} s)$. Upon hormone stimulation, the phosphorylated insulin receptor in R-IR cells became associated with three proteins (approx. 65, 75, and $85 \mathrm{kDa}$ ), all recognized as Grb 10 isoforms by specific Grb 10 antibodies. Under the same conditions, essentially no Grb10 protein was detectable in IGF-I receptor immunoprecipitates from stimulated $\mathrm{R}+$ cells. In conclusion, we have identified Grbl0 as an adapter protein that interacts preferentially with insulin vs IGF-I receptors and, thus, may have a role in mediating insulin receptor-specific cellular responses.

\section{6}

LIPOIC ACID STIMULATES GLUCOSE UPTAKE IN MUSCLE AND FAT CELLS VIA PI3-KINASE AND TYROSINE-KINASES. A. Klip, T. Tsakiridis, S. Ewart, T. Ramlal, A Volchuk and $H$. Tritschler. The Hospital for Sick Children, Toronto, Ont., Canada. Lipoic/thioctic acid ( $R$-isoform) is a natural cofactor of pyruvate dehydrogenase. When added exogenously to isolated muscle preparations, lipoic acid stimulates glucose uptake. In vivo, lipoic acid supplementation improves glycemic control and reduces glycemia in diabetic animal models. We have recently shown that $\mathrm{R}$-lipoic acid (RLA) stimulates glucose uptake into L6 muscle cells in culture, with a time course similar to that of insulin. The purpose of this study was to compare the action of RLA with that of insulin in fat cells, and to explore the signalling pathways involved. In differentiated 3T3-L1 adipocytes, $2.5 \mathrm{mM}$ RLA stimulated glucose transport with a magnitude comparable to that of insulin ( 9 -fold in $1 \mathrm{~h}$ ). As in differentiated L6 myotubes, this stimulation of glucose uptake by RLA was almost completely blocked by $200 \mathrm{nM}$ wortmannin, suggesting involvement of PI3-kinase. In L6 myotubes RLA stimulated PI3kinase activity measured in p85 immunoprecipitates. In contrast, hypoxia did not stimulate PI3-kinase and stimulated glucose uptake in a wortmannin-insensitive manner. RLA caused time-dependent tyrosinephosphorylation of several proteins including IRS-1, as seen in cell lysates and PI3-kinase immunoprecipitates probed with anti-P-tyr antibodies. The phosphorylation was blocked by the tyrosine kinase inhibitor erbstatin $(10 \mu \mathrm{g} / \mathrm{ml})$. This drug was therefore tested on the ability of RLA to stimulate glucose uptake. In 3T3-Ll adipocytes, erbstatin reduced the stimulation of glucose transport by RLA by $35 \%$. We conclude that RLA stimulates glucose uptake into muscle and fat cells and that, like insulin, this process requires PI3-kinase activity. Moreover, the stimulation requires participation of tyrosine kinase(s). Thus RLA utilizes elements of the insulin signalling pathway, different from the hypoxia-utilized pathway. RLA may offer the opportunity to treat insulin resistance with RLA, a physiological compound. 
LEFECT IN GLUCOSE HOMEOSTASIS IN MICE LACKING A REGULATOR Y SUBUNIT OF PHOSPHATIDYLINOSITOL-3-KINASE $Y$. Terauchi, $K$. Tobe, $K$. Iwamoto ${ }_{2} H$. Nakajima*, T. Hanafusa ${ }^{*}, Y$. Matsuzawa ${ }^{*}, Y$, Yazaki, and T. Kadowaki. Tokyo Lniversity. *Osaka Lniversity. Japan

The aim of this study is to investigate the role of phosphatidylinositol-3kinase (PI3K) in glucose homeostasis by targeted disruption of a regulatory subunit of PI3K gene ( $\mathrm{p} 85 \alpha$ ) in the mice. PI3K activity associated with $\alpha P Y$ immunoprecipitates after an insulin injection in the homozygous mutant mice was reduced by $50 \%$ and $30 \%$ in the liver and skeletal muscle, respectively. Homozygotes were born alive and fertile. They had no defect in growth and food intake. Histological examinations revealed that they had no major abnormalities. Blood glucose levels in homozygotes were significantly lower than those in the wild-type (Nonstarved: $114 \pm 5$ v.s. $140 \pm 4 \mathrm{mg} / \mathrm{dl}, \mathrm{P}<0.01$ ), which were associated with decreased serum insulin levels and increased plasma glucagon levels. They showed a significantly smaller increase in both glucose and insulin levels when they were loaded with an intraperitoneal glucose. Glucagon loading test caused a significantly smaller increase in glucose levels, and insulin loading test revealed a marked delayed recovery from hypoglycemia in mice lacking p85a. After a $30 \mathrm{hr}-\mathrm{fast}$ blood glucose levels in the homozygotes remained significantly lower $(50 \pm 2$ v.s. $67 \pm 4 \mathrm{mg} / \mathrm{dl}$, $\mathrm{P}<0.01$ ) and they became ketotic rapidly. Serum levels of gluconeogenic precursors such as lactate, alanine, or glutamine after a fast were almost the same as those in the wild-type. Glycogen, glucose-6-phosphate, or fructose-1-phosphate stored in the liver was five to ten times less in homozygotes al ter a fast, although glycogen synthase activity in the liver was almost normal. Thus, the role of the liver in maintaining blood glucose levels, espectally gluconeogenic pathway, appears to be impaired. This study is the first to demonstrate that an $85 \mathrm{kD}$ subunit of PI3K plays a pivotal role in glucose homeostasis in vivo.
INSULIN SIGNALLING IN CARDIAC MUSCLE: EVIDENCE FOR A NOVEL FUNCTION OF P85ALPHA REGULATORY SUBUNIT OF PI 3-KINASE

M. Till, I. Uphures, A. Kessler, J. Niggemann \& J. Eckel, Molecular Cardiology, Diabetes Research Institute, Düsseldorf, Germany Phosphatidylinositol 3-kinase (PI 3-kinase) is a heterodimeric enzyme consisting of a $85 \mathrm{kDa}(\mathrm{p} 85$ ) regulatory and a $110 \mathrm{kDa}$ catalytic subunit, thought to play an essential role in insulin-stimulated GLUT4 recruitment. Two isoforms of p85 have been described, however, specific interaction with IRS-1 and subcellular localization in response to insulin in muscle tissue has not been investigated so far. Rat ventricular cardiomyocytes were stimulated with insulin $\left(10^{-7} \mathrm{M}\right.$ for 5 or $30 \mathrm{~min}$ ) followed by determination of [RS-1-associated and non-associated $\mathrm{p} 85$ protein by Western blotting using p85alpha monoclonal and p85general polyclonal antisera. p85alpha was readily detected in these cells, however, only minimal amounts (less than 1\%) associate to IRS.1. In contrast, about $30 \%$ of total $\mathrm{p} 85$ is recruited to IRS- 1 in response to insulin at both 5 and $30 \mathrm{~min}$. This correlates with a $2-3$ fold increase in IRS-1-associated p85 protein and a 5-10 fold increase in IRS-1-associated PI 3-kinase activity. Essentially no 885 protein was found associated to IRS-1 in cardiomyocytes contracting with $5 \mathrm{~Hz}$, despite max. stimulaticn of glucose transport under these conditions. On the other hand, p85alpha could be identified as a component of GLLTT-containing subcellular vesicles isolated from microsomal membranes by an immunoadsorption protocol. In four separate experiments p85alpha was found to increase in response to insulin ranging from 1.5 to 7.5 fold over basal. This was paralleled by an insulin-induced increase of p85alpha in the microsomal fraction.

In conclusion, our data show that in cardiac muscle p85alpha is not essential for insulin-induced activation of PI 3-kinase at the level of IRS-1. Further, contraction-induced activation of glucose transport does not involve the IRS-1 signalling system. Recruitment of p85alpha to GLUT4-vesicles may reflect direct involvement of PI 3-kinase in glucose transporter translocation. It is suggested that $\mathrm{p} 85$ isoforms participate in insulin signalling at several sites exerting selective, tissue-specific functions. (Supported by DFG, SFB 351 C2)

Do not use type size smaller than 10 pitch and

the text including title, author(s) and place's) of work must not exceed 30 lines
OKADAIC ACID EXERTS A FULL INSULIN-LIKE EFFECT ON GLUCOSE TRANSPORT AND GLUT4 TRANSLOCATION IN HUMAN ADIPOCYTES: EVIDENCE FOR A PI3-KINASE INDEPENDENT PATHWAY.

C.M. Rondinone and U. Smith. Lundberg Laboratory for Diabetes Research, Department of Internal Medicine, Sahlgrenska University Hospital, S-413 45 Göteborg, SWEDEN.

The effect of the serine/threonine phosphatase inhibitor, okadaic acid, and insulin on glucose transport activity, GLUT4 translocation to the plasma membrane and insulin's signaling pathway were examined in human adipocytes. Okadaic acid consistently produced a greater increase in the rate of glucose transport than that seen with maximally effective insulin concentrations. Furthermore, when the cells were incubated with both agents together a partial additive effect was seen. Both insulin and okadaic acid alone stimulated the translocation of GLUT4 to the plasma membrane while the combination of the two agents again produced an additive effect. Insulin, but not okadaic acid, stimulated PI3-kinase activity in antiphosphotyrosine immunoprecipitates. Wortmannin also completely inhibited the effect of insulin on glucose transport while the effect of okadaic acid remained essentially unchanged. When the cells were incubated with both agents, okadaic acid exerted an inhibitory effect on insulin-stimulated PI3-kinase activity but did not block the association of the $\mathrm{p} 85$ or $\mathrm{p} 110$ subunits of PI3kinase with IRS-1. Insulin-stimulated tyrosine phosphorylation of IRS-1 was only slightly reduced $(-20 \%)$ by okadaic acid.

Conclusion: Okadaic acid exerts a full insulin-like effect in terms of increasing glucose transport and GLUT4 translocation. However, this effect is independent of the activation of PI3-kinase. Thus, PI3-kinase lipid kinase is not essential for GLUT4 translocation in human adipocytes. Furthermore, these data show that different pathways exist which lead to GLUT4 translocation and increased glucose transport.

\section{A MECHANISM FOR GLUCOSE-INDUCED INSULIN RESISTANCE IN RAT ADIPOCYTES \\ A. Filippis, S.Clark* and J. Proietto. The University of Melbourne, Department of Medicine, Royal Melbourne Hospital, Parkville, 3050 and *The Institute of Human Nutrition, Deakin University, Malvern, 3144 Victoria, Australia}

Hyperglycaemia has been shown to cause insulin resistance, however its biochemical basis is not understood. Two possible mechanisms can be proposed: 1) an increase in diacylglycerol (DAG), via de novo synthesis from glucose, leading to activation of protein kinase $C$ (PKC) and 2) an increased flux of glucose through the hexosamine biosynthesis pathway (HBP). The aim of this study was to investigate the relative roles of these two mechanisms in glucose-induced insulin resistance. ${ }^{14} \mathrm{C}$-2-deoxyglucose uptake was measured in rat adipocytes pre-incubated with high glucose $(30 \mathrm{mM})$ in the presence of insulin $(0.7 \mathrm{nM})$. GLUT4 was measured in plasma (PM) and low density microsomal (LDM) membranes by Western blotting using a rabbit polyclonal antibody (R1159). Following a 2 hour pre-incubation with high glucose, basal glucose transport was inhibited ( $26 \pm 4 \%, p=0.035 n=7)$, as was insulin-stimulated glucose transport $(21 \pm 7 \%, p=0.022 n=7)$. High glucose reduced the basal level of GLUT4 in the PM by $50 \pm 9 \%,(\mathrm{p}=0.0046 \mathrm{n}=5)$ and increased the basal level of GLUT4 in the LDM, suggesting intracellular retention of GLUT4, while insulin-stimulated GLUT4 translocation to the PM was normal (410 $\pm 100 \%$ vs $350 \pm 60 \%, p=n s n=5$ ). The PKC-inhibitor Ro-31-8220, reversed the effects of HG on glucose transport but caused a further reduction in basal GLUT4 levels (67 \pm $8 \%, p=0.013 n=4$ ) in the PM, without affecting insulin-stimulated GLUT4 levels. There was a 2.4 fold increase in the de novo synthesis of DAG in cells pre-incubated with high glucose. Azaserine, an inhibitor of the first and rate-limiting enzyme of the HBP reversed the effect of high glucose on basal and insulin-stimulated glucose transport. Furthermore, azaserine reversed the effect of high glucose on basal GLUT4 levels. Ro-31-8220 and azaserine used in combination returned basal and insulin-stimulated glucose transport and GLUT4 levels to normal. It is concluded that 1) increased flux through the HBP causes retention of GLUT4 intracellularly in the basal state; an effect that is reversed by insulin. 2) The decrease in glucose transport is not due to changes in GLUT4 levels on the PM but to interference with GLUT1 function. 3) This interference is mediated by PKC activation. 
OP 6

\section{Glucose Toxicity}

41

PROLONGED GLUCOSE EXPOSURE INCREASES THE RELATIVE ABUNDANCE OF ISLET AMYLOID POLYPEPTIDE RELATIVE ABUNDANCE OF ISLET AMYLOID
PRECURSORS IN HUMAN AND RAT B-CELLS.

X. Hou, Z. Ling, E. Quartier, F. Schuit, D. Pipeleers and C. Van Schravendijk. Diabetes Research Center, Vrije Universiteit Brussel, Laarbeeklaan 103, B-1090 Brussels, Belgium.

Islet amyloid polypeptide (IAPP) is synthesized as a prepropeptide in islet $B$ cells and processed proteolytically at dibasic residues, similar to proinsulin. The aim of this study was to investigate whether chronically elevated glucose levels alter the cellular content in incompletely processed IAPP. Culture at 6 or $20 \mathrm{mM}$ glucose did not influence the molar ratio of total IAPP over insulin in human $(1.3 \%, n=3)$ and in rat $(0.6 \%, \mathrm{n}=6)$. Both human and rat $B$-cells contained three IAPP-like immunoreactive peptides with MW 7,6 and $4 \mathrm{kDa}$, as analysed in Western blots by laser scan densitometry. The presence of elevated glucose levels $(20 \mathrm{mM})$ increased the relative abundance in the $7 \mathrm{kDa}$ precursor (from $21 \pm 5$ to $33 \pm 7 \%$ in human and from $20 \pm 2$ to $34 \pm 3 \%$ in rat, $p<0.05$ ), with a concomitant decrease in the abundance of the mature $4 \mathrm{kDa}$ peptide (from $58 \pm 2$ to $40 \pm 5 \%$ in human and $58 \pm 6$ to $43 \pm 4 \%$ in rat) and without changes in the $6 \mathrm{kDa}$ peptide. Under basa glucose conditions, the cellular ratio of unprocessed over processed proinsulin was at least 10 -fold lower than that of unprocessed over processed IAPP. The observation that chronically elevated glucose levels can further increase the relative content in IAPP-precursors may be of pathogenic significance.

\section{3}

HYPERGLYCEMIA, BUT NOT HYPERINSULINEMIA, INHIBITS LIVER GLUCOSE-6 PHOSPHATASE ACTIVITY IN RAT. L. GUIGNOT and G. MITHIEUX, INSERM U. 449, Faculté de Médecine René Laënnec, F - 69372 Lyon Cédex 08.

It has been recently reported that liver glucose-6 phosphatase (Gic6Pase) activity is inhibited by 3 h-refeeding in rats. To study the respective roles of hyperglycemia and hyperinsulinemia in this inhibition, we have performed hyperinsulinic euglycemic and hyperglycemic clamps in rats. Five $h$ after food removal, anesthetized rats were perfused for $3 \mathrm{~h}$ with saline (S), or with insulin at $480 \mathrm{pmol} / \mathrm{h} \mathrm{(I)}$, or with glucose at $2.4 \mathrm{mmol} / \mathrm{h}$ (G1) and $3.9 \mathrm{mmol} / \mathrm{h}$ (G2). In group $\mathrm{l}$, the perfusion of $1.29+0.01 \mathrm{mmol} / \mathrm{h}$ glucose (meantS.E.M.,n=5) has been required to maintain glycemia at the basal level. Hepatic gluccise production (HGP) was assessed by $\left[3-{ }^{3} \mathrm{H}\right]$ glucose dilution and Glc6Pase assayed in homogenates from liver lobes freeze-clamped in situ. The results were:

\begin{tabular}{lccccc}
\hline Group & $\mathrm{n}$ & $\begin{array}{c}\text { Final glycemia } \\
\text { mmoll }\end{array}$ & $\begin{array}{c}\text { Final insulinemia } \\
\text { pmoh }\end{array}$ & $\begin{array}{c}\text { HGP } \\
\mu \mathrm{mol} / \mathrm{kg} / \mathrm{min}\end{array}$ & $\begin{array}{c}\text { Glc6Pase } \\
\text { U/g wet liver }\end{array}$ \\
\hline $\mathrm{S}$ & 5 & $8.6 \pm 0.5$ & $185 \pm 17$ & $77 \pm 9$ & $8.1 \pm 0.1$ \\
I & 5 & $7.7 \pm 1.4$ & $461 \pm 45^{*}$ & $21 \pm 12^{*}$ & $7.7 \pm 0.6$ \\
G1 & 4 & $12.6 \pm 0.1^{*}$ & $576 \pm 47^{*}$ & $27 \pm 2^{*}$ & $6.1 \pm 0.6^{\circ}$ \\
G2 & 4 & $17.3 \pm 0.9^{*}$ & $876 \pm 68^{*}$ & $0.3 \pm 6^{*}$ & $6.0 \pm 0.4^{*}$
\end{tabular}

The data are expressed as meantS.E.M.

$\because,:$ different from $S$ value, $p<0.05, p<0.01$, respectively.

Glc6Pase was not inhibited under euglycemic hyperinsulinic conditions (group I), and was significantly inhibited under hyperglycemic conditions (G1 and G2). Our data strongly suggest that hyperglycemia, but not hyperinsulinemia, could account for the inhibition of liver Gic6Pase activity during the postprandial period. This phenomenon might play an important role in the suppression of HGP in this situation.

\section{2}

GLUCOSE TOXICITY AND ALPHA CELL FUNCTION

G Sacchi, H-J Zhang and RP Robertson. University of Minnesota, Department of Medicine, Minneapolis, Minnesota.

High concentrations of glucose can impair beta cell function in vitro and in vivo, a phenomenon commonly termed glucose toxicity. We have found that beta cell lines chronically cultured in media containing supraphysiologic glucose concentrations exhibit glucose toxicity, i.e., they develop decreased levels of insulin gene transcription factors and gene expression, insulin content and insulin secretion. To determine if this phenomenon is specific for the beta cell or also applicable to the alpha cell we cultured a glucagon secreting line, alpha TC9, for twenty passages in media containing four different concentrations of glucose: $3,5.5,11.1,16.7 \mathrm{mM}$. Cells were split weekly and challenged every five passages with an aminoacid mixture in static and perifusion secretion studies. Static secretion studies utilized different concentrations of aminoacid mixture for one hour: $0,0.9,1.8,3.7,7.5$ and $15 \mathrm{mM}$. Perifusion studies were performed with aminoacid mixture $15 \mathrm{mM}$ for thirty minutes. No significant differences in secretion were observed over twenty passages (static secretion at $15 \mathrm{mM}$ aminoacid mixture: $40770 \pm 2532$ vs $40815 \pm 221$ vs $39175 \pm 364$ vs $42893 \pm 4502 \mathrm{pg}$ glucagon / $\mathrm{mg}$ protein for $3,5.5,11.1$ and $16.7 \mathrm{mM}$ glucose culture conditions, respectively; perifusion: $736 \pm 128$ vs $1165 \pm 289$ vs 820 \pm 39 vs $806 \pm 390 \mathrm{pg}$ glucagon / $\mathrm{mg}$ protein at $3,5.5,11.1$ and 16.7 $\mathrm{mM}$ glucose, respectively). Glucagon content was not significantly different : $1262 \pm 10$ vs $1545 \pm 15$ vs $1537 \pm 86$ vs $1587 \pm 365 \mathrm{ng}$ glucagon / $\mathrm{mg}$ protein at 3,5.5, 11.1 and $16.7 \mathrm{mM}$ glucose, respectively. We conclude that the adverse effect of supraphysiologic glucose concentration on function of the beta cell is specific and not likely to be applicable to the alpha cell.

\section{4}

LONG-TERM HYPERGLYCAEMIA DECREASES VASCULAR FRACTION OF EXTRACELLULAR SUPEROXIDE DISMUTASE

Ciechanowski K., Ciechanowicz A., Czekalski S.

University School of Medicine. Szczecin, Poland

Extracellular superoxide dismutase (EC-SOD) is the major SOD isoenzyme in human plasma. Upon affinity chromatography it was separated into 3 fractions with different affinity to heparin-Sepharose and heparan sulfate (in vivo). Intravenous injection of heparin leads to a prompt increase in plasma EC-SOD activity by release of vascular fraction.

The aim of this study was to evaluate the influence of long-term metabolic control of diabetes mellitus on vascular fraction of EC-SOD. Pre- and postheparinic plasma EC-SOD activity has been measured by spectrophotometric method in 22 healthy subjects (HS) and 38 diabetic patients. The diabetics were subdivided into 3 groups according to serumi fructosamine (SFRA) concentration: 15 subjects with $\$ F R A<3.0 \mathrm{mmol} / \mathrm{h}$ (DM1), 16 subjects with SFRA $3.0-4.0 \mathrm{mmol} / \mathrm{D}$ (DM2) and 7 persons with $\mathrm{sFRA}>4.0 \mathrm{mmol} / \mathrm{(DM} 3)$.

Preheparinic EC SOD activities in HS and DM1 groups were similar $(9.86 \pm 1.01 \mathrm{U} / \mathrm{ml}$ vs $9.47 \pm 0.61 \mathrm{U} / \mathrm{ml}, \mathrm{NS}$, respectively). The basal EC- SOD activities in DM2 $(8.79 \pm 0.73 \mathrm{U} / \mathrm{ml})$ and DM3 $(7.63 \pm 0.73 \mathrm{U} / \mathrm{ml})$ "were significantly lower $(p<0.001)$ compared to $H S$. Postheparinic EC=SOD activities in all diabetic groups (DM1 $17.69 \pm 1.63 \mathrm{U} / \mathrm{ml}$, DM2 13.10 \pm 2.11 $\mathrm{U} / \mathrm{ml}$ and DM3 $9.96 \pm 0.87 \mathrm{U} / \mathrm{ml}$ ) were significantly lower $(p<0.001)$ compared to control group $(20.33+1.68 \mathrm{U} / \mathrm{ml})$

Our results suggest that poor metabolic control of diabetes influences either basal or postheparinic activity of vascular fraction of EC-SOD in plasma. 
ACTIVATION OF HeXOSAMINE PATHWAY ABOLISHES INSULIN STIMULATED GLYCOGEN SYNTHESIS IN THE LIVER

A. Virkamäki, T. Utriainen, S. Hämäläinen, M.C. Daniels*, D. McClain* and H. Yki-Järvinen. University of Helsinki, Helsinki, Finland, and *University of Mississippi Medical Center, Jackson, MS, USA

Increased hexosamine metabolism causes insulin resistance in skeletal muscle and may mediate glucose induced insulin resistance. We examined how activation of the hexosamine pathway by glucosamine (GlcN) affects insulin sensitivity in tissues where the activity of the rate-limiting enzyme of the pathway, glutamine:fructose-6phosphate amidotransferase (GFA) is higher than in skeletal muscle. Chronically catheterized awake rats were infused with either GlcN $(30 \mu \mathrm{mol} / \mathrm{kg} \cdot \mathrm{min}, \mathrm{n}=8)$ or saline $(\mathrm{NaCl}, \mathrm{n}=8)$ during a 360 min euglycemic hyperinsulinemic (insulin 18 $\mathrm{mU} / \mathrm{kg} \cdot \mathrm{min}$ ) clamp (Ins). Whole body glucose uptake, glycolysis and glycogen synthesis was determined by infusing $\left[3-{ }^{3} \mathrm{H}\right]$ glucose for $360 \mathrm{~min}$. At $315 \mathrm{~min}$, [2${ }^{14} \mathrm{Cldeoxy}$-glucose was injected to determine tissue glucose uptake. GlcN reduced whole body glucose uptake by $49 \%(106 \pm 12$ vs $206 \pm 6 \mu \mathrm{mol} / \mathrm{kg} \cdot \mathrm{min}$, GlcN+Ins vs $\mathrm{NaCl}+$ Ins, $\mathrm{p}<0.001)$, glycogen synthesis by $37 \%(\mathrm{p}<0.01)$ and glycolysis by $21 \%$ (NS). GFA activity was highest in submandibular gland $(542 \pm 70 \mathrm{pmol} / \mathrm{kg} . \mathrm{min})$ followed by the liver $(181 \pm 15)$, fat $(62 \pm 13)$, soleus $(39 \pm 4)$, heart $(16 \pm 1)$, gastrocnemius (15 \pm 1$)$ and abdominous (15 \pm 1$)$ muscles. Glucose uptake decreased by GlcN $36 \%(p<0.05)$ in the submandibular gland, 34\% $(\mathrm{p}<0.05)$ in fat, $41 \%(\mathrm{p}<0.02)$ in soleus, $42 \%(\mathrm{p}<0.02)$ in the heart, $52 \%(\mathrm{p}<0.005)$ in the gastrocnemius and $31 \%$ $(p<0.05)$ in abdominal muscle. In the liver, the rate of glycogen synthesis averaged $(214 \pm 36 \mu \mathrm{mol} / \mathrm{kg}$ dry $\mathrm{min})$ during $\mathrm{NaCl}+\mathrm{Ins}$ infusions, but was completely abolished by GlcN $(6.3 \pm 2.8 \mu \mathrm{mol} / \mathrm{kg}$ dry min, $\mathrm{p}<0.001$, for $\mathrm{GlcN}+\mathrm{Ins}$ vs $\mathrm{NaCl}+\mathrm{Ins})$. Liver glycogen content was $91 \%$ lower during GicN+Ins $(19 \pm \mathbf{1 5} \mathrm{mmol} / \mathrm{kg}$ dry $)$ than during $\mathrm{NaCl}+$ Ins ( $206 \pm 29 \mathrm{mmol} / \mathrm{kg}$ dry, $\mathrm{p}<0.001$ ) while in e.g. abdominal muscle glycogen content was only $21 \%$ lower $(242 \pm 20$ vs $308 \pm 20 \mathrm{mmol} / \mathrm{kg}$ dry, GlcN+Ins vs $\mathrm{NaCl}+\mathrm{Ins}, \mathrm{p}<0.05$ ). These data demonstrate that amongst insulin-sensitive tissues, GlcN has its most profound effect in the liver suggesting that glucose induced insulin resistance may not be restricted to skeletal muscle.
GLUCOSAMINE INHIBITS GLYCOGEN SYNTHESIS AND SYNTHASE IN SKELETAL MUSCLE: POSSIBLE ROLE IN GLUCOSE TOXICITY

${ }^{1} A$. Giaccari, $2 \mathrm{~L}$. Morviducci, ${ }^{3} S$. Farrace, ${ }^{2} \mathrm{~L}$. Pastore, ${ }^{4} \mathrm{D}$. Zorretta, ${ }^{4} \mathrm{~A}$. Buongiomo, and ${ }^{2} \mathrm{G}$. Tamburrano.

${ }^{1}$ Dept. of Endocrinology, Catholic Univ.; 'Endocrinology, Clin. Med. 2 Univ. "La Sapienza"; ${ }^{3}$ D.A.S.R.S., Dept. of Medicine; ${ }^{4}$ Clin. Biochem. Ist. Sup. Sanità, Rome, Italy.

Aim of this study was to evaluate the effect of glucosamine (GluN) on skeletal muscle glycogen metabolism in presence of low, physiological insulin. Control and Diabetic rats ( $90 \%$ pancreatectomy) were studied with euglycemic clamp studies ( $6 \mathrm{~h}$ fast) with low insulin (4 $\left.\mathrm{mU} \cdot \mathrm{kg}^{-1} \cdot \mathrm{min}^{-1}, \sim 600 \mathrm{pmol} / \mathrm{l}\right)+$ GluN $\left(5 \mu \mathrm{mol} \cdot \mathrm{kg}^{-1} \cdot \mathrm{min}^{-1}\right)$ or saline. Before the studies, Diabetic rats were infused with phloridzin to start the infusions in presence of euglycemia (glycosuria). In Control rats GluN decreased muscle glycogen synthesis [3-3H]-glucose in

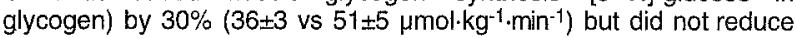
overall glucose uptake (127 \pm 4 vs $125 \pm 9$ ) although G6P (measured in

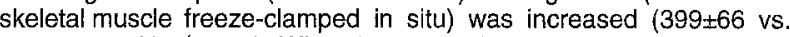
$232 \pm 45 \mu \mathrm{mol} \cdot \mathrm{kg}^{-1}$ w.w.). When kinetic analysis of glycogen synthase was performed, GluN determined a reduction of $F V_{01}$ (I/D form ratio: $18 \pm 3$ vs. $33 \pm 5 \%$ ), a small increase in $I-K_{m}(0.21 \pm .02$ vs. $0.15 \pm .03 \mathrm{mM}$ UDPG) and no changes in D- $K_{m}, I-V_{\max }$ and $D-V_{\max }$. In Diabetic rats glycogen synthesis and overall glucose uptake were decreased $(17 \pm 3$ and $\left.103 \pm 7 \mu \mathrm{mol} \cdot \mathrm{kg}^{-1} \cdot \mathrm{min}^{-1}\right)$, G6P was increased $\left(431 \pm 47 \mu \mathrm{mol} \cdot \mathrm{kg}^{-1}\right.$ w.w.) and remained unaffected by GluN. $F V_{01}$ was already reduced $(22 \pm 4 \%)$ with saline, and both $K_{m} s$ and $V_{m a x} s$ showed neither differences with saline nor changes after GluN. We conclude that glucosamine: 1) reduces skeletal muscle glycogen synthesis in Control rats, even in presence of $70 \%$ increased $\mathrm{G} 6 \mathrm{P}$, through a direct major reduction of glycogen synthase; 2) does not affect the rate of glucose transport/phosphorylation, and finally 3) has no effects in Diabetic rats, suggesting that increased hexosamine metabolism is already present.

\section{7}

MECHANISMS OF 'GLUCOSE TOXICITY' IN MAN. STUDIES WITH GLUCOSAMINE.

R.C. Bonadonna, T. Monauni, M.G. Zenti, G. Targher, B. Caruso, M. Caputo, E. Bonora, and M. Muggeo. University of Verona Medical School and Verona City Hospital, Verona, Italy.

In vitro and in vivo studies in the rat have shown that 'glucose (G) toxicity', i.e. G-induced impairments in insulin (I) secretion and action, is due to fructose-6-phosphate channeling through the hexosamine-phosphate (HP) pathway, a quantitatively minor metabolic fate of $G$. The flux through the HP pathway can also be increased by cell exposure to glucosamine (GMN) leading to defects in I action and secretion both in vitro and in vivo. Thus, GMN can be used to test the hypothesis that accelerating the HP pathway independently of $G$ impairs I secretion and action, which is a pre-requisite to advocate a pathogenetic role of the HP pathway in ' $G$ toxicity'. We performed 2 repeat studies in random order of both I secretion after an intravenous $G$ infusion (IVG: $1.67 \mathrm{mmol} / \mathrm{kg}$ of body weight) and I sensitivity (euglycemic I clamp, I 450 pmol//, in combination with $2-3 \mathrm{H}-\mathrm{G}$ infusion and indirect calorimetry) with a concomitant infusion of either normal saline (SLN) or GMN $(1.6 \mu \mathrm{mol} / \mathrm{min} / \mathrm{kg}$ of body weight, aimed to elevate GMN levels $\sim 10$-fold over baseline values) in 10 healthy, young, male subjects, $G$ areas $\left(0^{\prime}-10^{\prime}\right.$ and $\left.10^{\prime}-60^{\prime}\right)$ after IVG were similar with SLN (147 $\pm 3.6 \mathrm{mmol} / \mathrm{l}$ per $10^{\prime}$ and

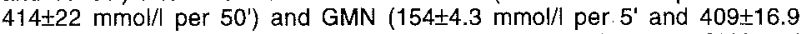
mmol// per 50') infusions ( $p=n$.s. for all comparisons between SLN and GMN). Early $\left(0^{\prime}-10^{\prime}\right)$ and late $\left(10^{\prime}-60^{\prime}\right)$ I secretion phases, as assessed by $C$ peptide (C-P) and I concentration curves, were similar with SLN (C-P and I $0^{\prime}-10^{\prime}: 10.9 \pm 1.3 \mathrm{nmol} / \mathrm{l}$ and $4.5 \pm 0.9 \mathrm{nmol} / \mathrm{l}$ per $10^{\prime} ; \mathrm{C}-\mathrm{P}$ and I 10'-60': $54.9 \pm 5.5$ nmol/I and $8.5 \pm 1.4 \mathrm{nmol} / /$ per $50^{\prime}$ ) and GMN (C-P and I $0^{\prime}-10^{\prime}: 11.2 \pm 1.4 \mathrm{nmol} / \mathrm{l}$ and $4.7 \pm 1.9 \mathrm{nmol} / / \mathrm{l}$ per $10^{\prime} ; \mathrm{C}-\mathrm{P}$ and $\mathrm{I} 10^{\prime}-60^{\prime}: 51.8 \pm 6.9 \mathrm{nmol} / /$ and $8.1 \pm 1.6$ $n$ mol/l per 50') infusions ( $\mathrm{p}=\mathrm{n} . \mathrm{s}$, for all comparisons between SLN and GMN) During the I clamp, GMN infusion affected neither peripheral $G$ disposal ( $3.18 \pm 0.28$ vs $3.14 \pm 0.35 \mathrm{mmol} / \mathrm{min})$, nor endogenous $G$ production $(211 \pm 42$ vs $192 \pm 64 \mu \mathrm{mol} / \mathrm{min})$, nor $\mathrm{G}$ oxidation $(0.94 \pm 0.079 \mathrm{vs} 1.02 \pm 0.12 \mathrm{mmol} / \mathrm{min})$, nor non-oxidative $\mathrm{G}$ metabolism (2.17 $\pm 0.24 \mathrm{vs} 2.05 \pm 0.25 \mathrm{mmol} / \mathrm{min}$ ) ( $\mathrm{p}=\mathrm{n} . \mathrm{s}$. for all comparisons between SLN and GMN). Therefore, these data do not support the contention that the HP pathway is to be included among the mechanisms of ' $G$ toxicity' in man.

\section{8}

PROLONGED SUPPRESSION OF GLUCOSE METABOLISM DURING EUGLYCEMIC CLAMPS CAUSES INSULIN RESISTANCE IN RATS J. H. Youn and J. K. Kim, USC, Los Angeles, California, USA

We previously showed that glucose metabolic impairment precedes insulin resistance during high fat feeding in rats. To determine whether glucose metabolic impairment causes insulin resistance, we examined the effects of suppression of glycolysis or glycogen synthesis on insulin-stimulated glucose uptake during $7.5 \mathrm{~h}$ euglycemic $(\sim 5.5 \mathrm{mM})$ hyperinsulinemic $(\sim 800 \mathrm{pM})$ clamps in rats. After the initial $2.5 \mathrm{~h}$ clamp to attain steady state insulin action, animals received a constant infusion of saline $(n=7)$, Intralipid $(0.9$ $\mathrm{m} 1 / \mathrm{h}$ ) and heparin (to suppress glycolysis; $\mathrm{n}=7$ ), or amylin $(50 \mathrm{pmol} / \mathrm{kg} / \mathrm{min}$; to suppress glycogen synthesis; $\mathrm{n}=7$ ) during the remaining $5 \mathrm{~h}$. Whole body insulin-stimulated glucose fluxes (i.e., glucose uptake, glycolysis, and glycogen synthesis), estimated using $\mathrm{D}-\left[3{ }^{3} \mathrm{H}\right]$ glucose infusion, were relatively constant during the clamps with saline infusion. In contrast, Intralipid infusion suppressed glycolysis by $\sim 32 \%$, and amylin infusion suppressed glycogen synthesis by $\sim 45 \%$ within $0.5 \mathrm{~h}$ after the start of Intralipid or amylin infusion $(\mathrm{p}<0.05)$. The suppression of metabolic fluxes, however, did not immediately affect insulin-stimulated glucose uptake despite increased glucose 6-phosphate content in skeletal muscle $(\mathrm{p}<0.05)$ due to compensatory increases in other metabolic fluxes (e.g., increased glycogen synthesis with Intralipid infusion). Insulin-stimulated glucose uptake started to decrease at $\sim 1 \mathrm{~h}$ and significantly decreased by $\sim 30 \%$ at the end of clamps $(p<0.05)$. Similar patterns of changes in insulin-stimulated glucose fluxes were observed in individual skeletal muscles in separate experiments. In conclusion, suppression of glucose metabolism caused decreases in insulinstimulated glucose uptake in skeletal muscle through a mechanism requiring a prolonged elevation of glucose 6-phosphate (possibly involving the hexosamine biosynthesis pathway), rather than the classic mechanism involving glucose 6-phosphate inhibition of hexokinase. 


\section{Aetiology}

49

THYMIC INSULIN-LIKE GROWTH FACTORS (IGFS) IN MAN AND IN AN ANIMAL MODEL OF AUTOIMMUNE IDDM

V. Geenen, I. Achour, O. Kecha, D.L. Greiner*, A. Rossini* and P.J. Lefèbvre

Department of Medicine, Divisions of Endocrinology and of Diabetes, Univ. of Liège, B-4000 Sart Tilman, Belgium and Department of Medicine, Univ. of Massachussets Worcester, MA 01655, USA.

This study aimed at identifying the major representative of the insulin hormone family in the thymic environment, as well as at investigating the bypothesis of a defect of thymic T-cell tolerance to the insulin family in the atiology of IDDM Immunocytochemistry (ICC) with antibodies (Abs) to epitopes of the insulin family revealed the presence of IR IGF-II in epithelial and nurse cells (TECITNC) of the human and rat thymuses. IR IGF-I was also identified in thymic stromal cells with a macrophage-like distribution and morphology. Neither IR proinsulin nor insulin could be detected by ICC with specific mAbs. The dominance of IGF-II over IGF-I in the human thymus was confirmed by specific RIAs after acid extraction and G-75 gel filtration in order to dissociate IGFs from their binding proteins. The close identity of thymic IR IGF-II with rec human IGF-II was demonstrated by HPLC. The association of IR IGF-II (but not IR IGF-I) with plasma membranes of cultured human TEC was demonstrated by confocal microscopy and ICC under non-permabilizing conditions. A comparative physico-chemical study was performed on the thymic acid extracts from control inbred WF rats, diabetes resistant (DR)-BB and diabetes prone (DP)-BB rats. A major difference appeared in the thymic IR IGF-II concentrations as well as in the G75 chromatographic profile of IR IGF-II with an almost complete absence of IR IGFII in the thymus from DP-BB rats. A dominant peak of IR IGF-II was found in the thymus extracts from control WF rats, as well as a smailer one in the thymus from DR-BB rats. Thymic IR IGF-I concentrations were very similar between the three groups and much lower than those of IR IGF-II. These observations confirm our previous studies that IGF-II is the major thymic self-antigen precursor of the insulin family. They provide further experimental evidence that a defect in the thymic IGF-II mediated $T$-cell education to the insulin hormone family may play a role in the pathophysiology of IDDM which remains to be further elucidated.

(Supported by the Juvenile Diabetes Foundation International and the National Fund for Scientific Research of Belgium.)

\section{0}

CLONING AND CHARACTERIZATION OF THE RAT GAD 65 PROMOTER K. Skak and B. K. Michelsen. Hagedorn Research Institute, Gentofte, Denmark. $\mathrm{GAD}_{65}$ is a major autoantigen in IDDM. The product of GAD, $\gamma$-aminobutyric acid (GABA) is the main inhibitory neurotransmitter in the brain. In the pancreatic islets GABA is cosecreted with insulin and is possibly involved in paracrine signaling. Metabolic activity in relation to insulin secretion has been shown to moctulate the expression of $\mathrm{GAD}_{65}$ in both human and rat islets. It has therefore been speculated that an increased metabolic load on the $\beta$-cell, e.g. during hyperglycemic episodes, might lead to a higher $G A D$ turnover resulting in an increased autoantigen presentation, thereby accelerating the $\beta$-cell destruction process. In this study we characterized the $\mathrm{GAD}_{65}$ promoter in order to clarify mechanisms of regulation of $\mathrm{GAD}_{65}$ expression. The rat $\mathrm{GAD}_{65}$ promoter was sequenced and the transcription initiation was determined by rapid amplification of cDNA ends to take place from multiple starting points (at -87 to -99 ) consistent with the finding that the promoter is TATA-less. The rat islet tumor cell line, MSL-G2, the mouse islet tumor cell line, $\beta \mathrm{HC}$, and the monkey kidney fibroblast cell line, COS-7 were transfected with successive 5' to $3^{\prime}$ deletion mutants of the promoter region fused to the coding region of a luciferase gene. The $\mathrm{GAD}_{65}$ promoter was able to direct the expression from the luciferase gene in the MSL-G2 and $\beta H C 9$ cells but not in the COS-7 cells demonstrating the specificity of the promoter. Deletion of a region of 90 bases proximal to the initiation site abolished promoter activity. This region contains two $\mathrm{Spl}$ binding sites and a PEA3 motif but no TATA box. Deletion of a region further upstream containing a PEA3 motif, a cAMP-responsive element (CRE), and an E-box (known to be necessary for insulin gene expression) resulted in a 5 fold reduction of the activity in MSL-G2 but not in $\beta H C 9$ cells. This might explain the low $\mathrm{GAD}_{65}$ expression in mouse islets as compared to rat islets. These reporter gene constructs will be useful to further investigate the regulation of islet $\mathrm{GAD}_{65}$ expression by factors known to influence $\beta$-cell viability, and might provide new tools to search for up- $\alpha$ downregulating compounds.

\section{1}

POPULATION AND FAMILIAL RISK OF B-CELL AUTOIMMUNITY IN THE FIRST TWO YEARS OF LIFE: DIABETES AUTOIMMUNITY STUDY IN THE YOUNG M. Rewers, G.S. Eisenbarth, J. Norris, H.A. Erlich, B. Beaty, G. Klingensmith and R.B. Elliott. University of Colorado, Denver, USA; Roche Molecular Systems, Alameda, USA and University of Auckland, New Zealand

It is unknown when autoimmunity preceding IDDM begins. To address this issue, we followed prospectively from birth 400 general population children with $10 \%$ - $2 \%$ lifetime risk of IDDM, i.e. those with the HLA- DQB1*0201/0302 genotype $(n=67), 0302 / 0302$ or $0302 / x(n=267)$ or $0201 / 0201(n=26)$ as well as those at risk for autoimmunity but not diabetes - with $\mathrm{DQB1}{ }^{\star 0602 / 0302}$ $(n=40)$. In addition, 118 offspring of IDDM mothers, 142 offspring of IDDM fathers and 181 siblings of IDDM children were studied under the age of 8 yrs, including, respectively, 30,32 , and 27 relatives younger than 2 yrs. The agespecific prevalence of autoimmunity (autoantibodies to insulin $>60 \mathrm{nU} / \mathrm{ml}$ $\mathrm{GAD}_{65}>0.05$ or ICA512 $>0.16$, by radioassays in the Barbara Davis Center laboratory or ICA $>5 \mathrm{JDF}$ in the University of Auckland laboratory is shown:

\begin{tabular}{|c|c|c|c|c|c|c|c|}
\hline \multirow[t]{2}{*}{ Age } & \multicolumn{4}{|c|}{ General population, HLA-DQB1 } & \multicolumn{2}{|c|}{ Offspring of IDDM } & \multirow[t]{2}{*}{ Siblings } \\
\hline & $\begin{array}{l}0201 / \\
0302\end{array}$ & $\begin{array}{l}0302 / \\
0302, \mathrm{x}\end{array}$ & $\begin{array}{l}0201 \\
0201\end{array}$ & $\begin{array}{l}0602 / \\
0302\end{array}$ & Mother & Father & \\
\hline $9 \mathrm{~m}$ & 0 & $2 \%$ & $4 \%$ & 0 & $20 \%$ & $4 \%$ & $18 \%$ \\
\hline $15 \mathrm{~m}$ & 0 & $1 \%$ & 0 & 0 & $8 \%$ & 0 & $22 \%$ \\
\hline $24 \mathrm{~m}$ & \multirow{2}{*}{\multicolumn{4}{|c|}{ incomplete follow-up, to date }} & $7 \%$ & 0 & $19 \%$ \\
\hline $3-7 \mathrm{yr}$ & & & & & $2 \%$ & $16 \%$ & $12 \%$ \\
\hline
\end{tabular}

In the first 15 months of life, general population chiddren with the susceptibility HLA alleles develop $\beta$-cell autoantibodies less often (2\%) than offspring ( $10 \%)$ or siblings $(20 \%)$ of IDDM persons. These results suggest that in the majority of IDDM cases autoimmunity is initiated after the age of 15 months. The absence of autoantibodies in the highest risk DQB1 *0201/0302 group and the initially higher prevalence of autoantibodies among offspring of diabetic mothers than fathers were unexpected and reauire further studies.

\section{2}

CONCORDANCE RATES FOR NON INSULIN-DEPENDENT DIABETES MELLITUS (NIDDM). A POPULATION BASED STUDY OF DANISH TWINS.

P. Poulsen, K.O. Kyvik, A. Vaag and H. Beck-Nielsen. Dept. of Endocrinology, Diabetes Research Centre, Odense University Hospital, Genetic Epidemiological Research Unit, Odense University, Denmark.

Previous twin studies have shown concordance rates between 0.45 and 0.96 for monozygotic (MZ) twin pairs, and considerably lower concordance rates for dizygotic (DZ) twin pairs, between 0.03 and 0.37 , thus demonstrating the imporfance of genetic factors for the etiology of non insulin-dependent diabetes mellitus. In this study we have been able to avoid, to a great extent, the potential biases characterizing former studies. Information on diabetes status and zygosity in all living Danish twin individuals born from 1921-40 and ascertained in the population based Danish twin register, have been done by postal questionnaire. All eligible twin pairs $(n=302)$, aged between 55 and 74 years, were subsequently examined with a standardized 2-hour oral glucose tolerance test (OGTT). Based on the OGTT we identified 62 twin pairs where one or both twins had NIDDM. The probandwise concordance rates were 0.50 for $M Z$ and 0.37 for $D Z$ twin pairs, $c \mathrm{hid}^{2}{ }_{1 \mathrm{~d}, 1}=0.40, \mathrm{p}>0.5$. The probandwise cumulative risk (interpretable as cumulative risk in relatives) to 74 years were estimated to 0.78 for $\mathrm{MZ}$ and 0.63 for $\mathrm{DZ}$ twin pairs, $\mathrm{chi}^{2}{ }_{1 \mathrm{~d} . \mathrm{f}}=0.003, \mathrm{p}>0.5$. We identified 155 pairs where one or both twins had an abnormal glucose talerance (IGT+NIDDM). The probandwise concordance rates were 0.63 for $M Z$ and 0.43 for $D Z$ twin pairs, chi ${ }^{2}{ }_{1, t}=4,45, p<0,05$. The fact that the concordance rates for NIDDM in MZ and DZ twin pairs were not statistical significantly different suggest that genetic factors are not as important for the development of NIDDM as previously anticipated. For development of abnormal glucose tolerance, however, genetic factors appear to play a role. We suggest that development of abnormal glucose tolerance is, partly genetically determined while progres-sion to NIDDM mainly is determined by environmental factors. 
SARDINIANS LIVING IN LAZIO RETAIN THEIR FIVEFOLD INCIDENCE OF TYPE 1 DIABETES COMPARED WITH THE HOST POPULATION Sa. Muntoni, M.T. Fonte, S. Stoduto, G. Marietti, A. Crinò, C. Bizzarri, P. Ciampalini, G. Multari, M.C. Matteoli, M.A. Suppa, L. Lucentini, M.L. Sebastiani, N. Visalli, P. Pozzilli, B. Boscherini and S. Muntoni, Centro per le Malattie Dismetaboliche e 1'Arteriosclerosi, Cagliari, Italy and Clinica Pediatrica, Università "Tor Vergata", Rome, Italy The aim of this migrant study was to assess whether the high IDDM incidence reported in Sardinia is reflected in Sardinian-heritage children born in a low-incidence region, Lazio, where a settlement of Sardinian ancestry totalled 48,628 people in 1991. Children 0-14-yr-old born in Lazio of at least one Sardinian parent were 6,946. All the hospitals and diabetes outpatient clinics in Lazio were our primary data source, whereas the Health Service files acted as the secondary, independent source. Between 1989 and 1994, IDDM had been diagnosed before age 15 in 16 children of Sardinian ancestry born in Lazio: of these, 3 were born of both Sardinian parents (A) and 13 had one Sardinian parent (B). The 16 cases $(\mathrm{AB})$ were also identified by the secondary source, the ascertainment probability being 100\%. Age-adjusted incidence (per 100,000 per year) was 37.4 (95\% CIs 7.7-108.4) in A, $36.6(19.4-62.6)$ in $B$ and $37.0(21.3-60.0)$ in $A B$, not different from the one of 34.4 (31.7-37.9) in Sardinia, but fivefold the rate of $7.9(7.1-8.8)$ in Lazio ( $p=0.000001$ ). Thus, children of two different ancestries exposed to the same environment have a fivefold difference in IDDM incidence, indicating a strong role for genetic factors.

\section{CHILDHOOD IDDM IS LINKED TO NITRATE LEVELS IN} DRINKING WATER

R.C. Parslow, G.R. Law, H.J. Bodansky, R. Williams and

P.A. McKinney. Paediatric Epidemiology Group, Centre for Health Services Research, University of Leeds, UK.

Standardised Incidence Ratios (SIRs) from the $97 \%$ completeYorkshire Register of Childhood Diabetes were used for a small-area ecological analysis of IDDM and nitrate in drinking water. In 1990-95 the nitrate content of 14,937 samples of drinking water were obtained. Samples related to 208 water supply zones (WSZ), geographically defined areas with homogenous water composition. Mean WSZ nitrate levels varied between $1.5 \mathrm{mg} \mathrm{l}^{-1}$ and $40 \mathrm{mg} \mathrm{l}^{-1}$ (maximum reading $59 \mathrm{mg} \mathrm{l}^{-1}$ ): EC recommendations are maximum levels of $50 \mathrm{mg}^{-1}$ and a target mean of $25 \mathrm{mg} \mathrm{I}^{-1}$. Research has linked high levels of dietary nitrate to increased incidence of IDDM. In the UK over $50 \%$ of dietary nitrate can come from drinking water. A Geographical Information System (ARC/INFO v.7.0) linked 10,430 small geographical areas each ascribing census data to the WSZs. WSZs were divided into four exposure categories by mean nitrate levels comprising approximately equal populations. Mean nitrate levels were significantly associated with $\operatorname{SIR}\left(\chi^{2}\right.$ test for trend $\left.=33.06, \mathrm{p}<0.001\right)$.

\begin{tabular}{lccllll}
\hline $\begin{array}{l}\text { Nitrate level } \\
\left(\mathrm{mg} \mathrm{I}^{-1}\right)\end{array}$ & O & E & SIR & $95 \% \mathrm{Cl}$ & $\begin{array}{l}\text { Population } \\
(0-16)\end{array}$ \\
\hline$\leq 2.7$ & 261 & 317.1 & $83.2^{*}$ & 73 & 93 & 168,748 \\
$>2.7-\leq 7.5$ & 284 & 324.0 & $87.6 *$ & 78 & 98 & 173,217 \\
$>7.5-\leq 19.1$ & 350 & 333.0 & 105.1 & 94 & 117 & 178,264 \\
$>19.1$ & 409 & 329.9 & $124.0 *$ & 112 & 137 & 175,804 \\
\hline
\end{tabular}

$* \mathrm{p}<0.05$

This is the first small-area ecological analysis performed in Europe showing a link between nitrate in drinking water and IDDM.

\section{OP 8}

\section{Aetiopathogenesis of Neuropathy}

\section{5}

INSULIN-LIKE GROWTH FACTOR-I (IGF-I) PROTECTS NEURONS FROM GLUCOSE-INDUCED CELL DEATH

J.W. Russell and E.L. Feldman, Department of Neurology, University of Michigan, Ann Arbor, MI

IGF-I has been proposed as a treatment for diabetic neuropathy, however its effects on sensory dorsal root ganglion neurons (DRG) have not been well-characterized. We studied the effect of IGF-I in reducing hyperglycemic changes in an in vitro rat DRG model. Control E15 DRG were cultured in defined medium, without serum, but containing 10 $\mathrm{ng} / \mathrm{ml} 2.5 \mathrm{~S}$ NGF to promote neuronal growth. Increasing concentrations of excess glucose $(20-300 \mathrm{mM})$, or IGF-I $(1-200 \mathrm{nM})$ were added to test DRG. Neurite growth was measured at 24-72 $\mathrm{h}$. To determine the presence of apoptosis, dissociated DRG were cultured in high glucose (HG) (150 mM-ED 50), in the presence or absence of IGF-I, and fluorescent labeling of fragmented DNA $3 \mathrm{OH}^{\prime}$ ends was measured. Cultured DRG neurons, exposed to high glucose for $24 \mathrm{~h}$, undergo dose dependent axonal degeneration. In HG, there is a dose dependent inhibition of neurite growth, even with a mildly elevated ( $20 \mathrm{mM}-315$ mOsm) glucose concentration ( $p<0.001$ ). When IGF-I is added to the HG medium, it is neuroprotective even at low concentrations ( $1 \mathrm{nM}$ ). With $10 \mathrm{nM}$ IGF-I, there is no significant difference between control and IGF-I/HG neurite growth, and only minimal improvement in neurite growth with IGF-I concentrations above $100 \mathrm{nM}$. After $48 \mathrm{~h}$ in culture the following percent of neurons were apoptotic: control $-5.5 \%$, HG$58 \%, 5 \mathrm{nM}$ IGF-I/HG-30\%, $100 \mathrm{nM}$ IGF-1/HG-10\%. The presence of NGF was not required for IGF-I to prevent neuronal apoptosis. Hyperglycemia inhibits neuronal growth, promotes axonal degeneration and programmed cell death in DRG neurons in a dose dependent fashion. IGF-I is able to prevent these changes even at low concentrations ( $1 \mathrm{nM})$, and potentially would be effective in ameliorating diabetic neuropathy. Supported by NIH grant R29 NS32843 and grants from the American Diabetes Association and the Juvenile Diabetes Foundation.

\section{6}

HEMODYNAMIC EFFECTS OF THERAPEUTIC POST-PRANDIAL HYPF RINSULINEMIA IN DIABETIC AUTONOMIC NEUROPATHY. r. rc.cellati, P. Bottini, S. Pampanelli, E. Scoscia, L. Scionti, G. Calabrese, P. Brunetti and G. B. Bolli, Di.M.I.S.E.M., University of Perugia, Italy To assess whether the therapeutic prandial s.c. insulin injection has appreciable hemodynamic effects in patients with diabetic autonomic neuropathy (AN) adapted to prolonged orthostatism, 6 diabetics without AN (DAN-), 5 with AN but no orthostatic hypotension (DAN $+\mathrm{OH}-$ ), 5 with $\mathrm{AN}$ and $\mathrm{OH}(\mathrm{DAN}+\mathrm{OH}+$ ) and 5 nondiabetics were studied. Subjects remained seated 2 hours before and $31 / 2$ hours after $0.2 \mathrm{U} / \mathrm{kg}$ insulin injected s.c. 30 min prior to ingestion of a mixed meal (peak plasma insulin $\sim 300 \mathrm{pM}$ at $90 \mathrm{~min}$ ) (insulin-meal study, I$\mathrm{M})$. As compared to a control study in which neither meal nor s.c. were given, but basal $(0.15 \mathrm{mU} / \mathrm{kg} / \mathrm{min})$ infused i.v., in the I-M study mean blood pressure (MBP) did not change neither in DAN-, nor in nondiabetics due to simultaneous increase in (forearm) peripheral vascular resistances (PVR) by $6.42 \pm 0.49 \mathrm{mmHg} / \mathrm{ml} / 100 \mathrm{ml} / \mathrm{min}$. In contrast, in $\mathrm{DAN}+\mathrm{OH}-$ and $\mathrm{DAN}+\mathrm{OH}+$ MBP decreased between $60-210 \mathrm{~min}$ by $6.6 \pm 0.4$ and $10.4 \pm 0.7 \mathrm{mmHg}$ (patients experienced symptoms of faintness), because PVR decreased by $2.17 \pm 0.52$ and $1.75 \pm 0.66 \mathrm{mmHg} / \mathrm{ml} / 100 \mathrm{ml} / \mathrm{min}$ ( $\mathrm{p}<0.05$ vs DAN-). Plasma noradrenaline (NA, nM) increased to $2.02 \pm 0.06$ in DAN-, $2.25 \pm 09$ in DAN+OH-, but only to $1.13 \pm 0.04$ in $\mathrm{DAN}+\mathrm{OH}+(\mathrm{CON} 1.79 \pm 0.08, \mathrm{p}<0.05)$. Heart rate $(\mathrm{HR})$ increased less in $\mathrm{DAN}+\mathrm{OH}-(1.9 \pm 0.4 \mathrm{~b} / \mathrm{min})$ and $\mathrm{DAN}+\mathrm{OH}+(4.7 \pm 0.54 \mathrm{~b} / \mathrm{min})$ than DAN- $(8.3 \pm 0.4 \mathrm{~b} / \mathrm{min})(\mathrm{p}<0.05)$ despite greater decrease in MBP in DAN+. In a 3rd study in DAN+OH+ where meal, but no s.c. insulin was given, MBP did not decrease. Thus, insulin, not the meal, causes arterial hypotension in DAN because release of NA to increase PVR is inappropriate. In patients with DAN, both $\mathrm{OH}+$ and $\mathrm{OH}-$, a therapeutic prandial s.c. insulin dose may cause symptomatic fall in blood pressure and faintness within 1-3 hours despite antecedent adaptation to orthostatism. 


\section{7}

\section{FASTING INCREASES HEART RATE VARIABILITY IN DIABETES}

J. Pumprla, K. Howorka and H. Thoma, Dept. of Biomedical Engineering and Physics, Univ. of Vienna, Austria

Spectral analysis of heart rate variability (HRV) permits the assessment of cardiovascular autonomic neuropathy (CAN) in diabetic patients, the evaluation of autonomic tone and the risk-stratification in patients with coronary heart disease. It is still not exactly known by which factors the HRV could be affected. Our aim was to evaluate the effects of at least $15 \mathrm{hrs}$ of fasting compared to a short postabsorptive period on HRV in a group of diabetic patients and controls. We examined HRV in 36 diabetic patients (IDDM: $n=32$, females: $n=20$, age $37,5 \pm 14,6$, diabetes duration $11,2 \pm 9,6$ years, $\left.B M l=23,4+2,9 \mathrm{~kg} / \mathrm{m}^{2}\right)$ with different degrees of CAN, using an on-line short-term spectral analysis of HRV (system VariaPulse TF $3^{\mathbb{Q}}$ ) telemetrically recorded in three positions (supine-standing-supine) each of 256 artifact-free heart beats. The measurements were made either in a common postabsorptive state (interval since the last meal $140+54 \mathrm{~min}$ ) or during the fasting day (interval since the last meal $1032 \pm 173 \mathrm{~min}$ ) on occassion of education in functional insulin treatment, discriminating between prandial, correctional and fasting insulin use. The intraindividual control measurements have been carried out on comparable time of the day and under comparable blood glucose levels $(121 \pm 40 \mathrm{mg} / \mathrm{dl}$ vs. $111 \pm 34 \mathrm{mg} / \mathrm{dl} ;$ N.S.). Fasting resulted in an increase of HRV and indices of parasympathetic activity in patients with all degrees of CAN (including its severe form): Cumulative Spectral Power (In $\left[\mathrm{ms}^{2}\right]=6,6 \pm 1,4$ vs. $\left.7,5 \pm 1,3 ; \mathrm{p}<0,001\right)$ and Power Spectral Density (In $\left[\mathrm{ms}^{2}\right]=9,4+1,5$ vs. $\left.10,1+1,4 ; p<0,0001\right)$ in High-Frequency-Band, as well as in an increase of Cumulative Total Spectral Power (In $\left[\mathrm{ms}^{2}\right]=8,7 \pm 0,8$ vs. $9,1 \pm 0,8 ; p<0,001)$. In a non-diabetic control group of 14 matched individuals for age, BMI and gender we detected an increase of a Cumulative Total Spectral Power $(p<0,01)$, but only a non-significant trend towards increasing of the vagal tone after $962+237$ min of fasting. Fasting in diabetic patients thus lead to a substantial increase of HRV, particularly of a vagal tone. The interpretation of short-term spectral analysis of HRV should take into consideration therefore the interval since the last meal. We propose a standardization of this interval.

\section{8}

SKIN MICROANGIOPATHY FAILS TO DISCRIMINATE DIABETIC PATIENTS WITH AND WITHOUT NEUROPATHY AND FOOT ULCERATION.

R.A. Malik ${ }^{1}$, D. Walker ${ }^{1}$, P. Brash ${ }^{2}$, A.J.M.Boulton ${ }^{1}$ and J.E.Tooke ${ }^{2}$. Dept. Medicine, Manchester Royal Infirmary, Manchester ${ }^{1}$, Dept. Vascular Medicine, Exeter $^{2}$, UK.

It has been suggested that the assessment of skin microangiopathy may act as a surrogate for microangiopathy in peripheral nerve and that a greater degree of skin microangiopathy in the foot skin of diabetic patients with neuropathy may determine the development of foot ulceration. Punch skin biopsies were taken from the dorsum of the foot in thitty diabetic patients subdivided into those without neuropathy (A) (Vibration perception threshold (VPT)- 11.1 \pm 3.5 . Cold threshold (CT)- $0.3+0.2$. Michigan Neuropathy Score (MNS) $-2.5+3.7$ ), with neuropathy but no foot ulceration (B) (VPT- $31.0 \pm 6.8$, CT $-3.7 \pm 2.1$, MNS- 18.9 \pm 6.9 ), and with neuropathy and foot ulceration (C) (VPT-33.5 \pm 4.2 , CT- $3.8=$ 2.4 , MNS- $21.2+2.4$ ). They were matched for age (Yrs) (A- $55.1+11.6, \mathrm{~B}-56.5$ $+9.6, \mathrm{C}-52.7+12.3)$ and duration of diabetes $(\mathrm{A}-22.5+11.5, \mathrm{~B}-22.1+11.5, \mathrm{C}-$ $22.7+11.1)$. Morphometric quantification was carried out employing detailed light and electronmicroscopy. We observed no significant difference between any of the groups for Epidermal thickness $(\mu \mathrm{m})(32.6+9.2$, B- $29.7+5.7, \mathrm{C}-34.6+$ 6.7); Capilary density (no.mm $\left.{ }^{2}\right)(A-33.4+17.2 . B-38.5+14.8 . C-42.7+24.4)$ Capillary luminal area $\left(\mu \mathrm{m}^{2}\right)(\mathrm{A}-7.9 \pm 2.9, \mathrm{~B}-7.7 \pm 2.6, \mathrm{C}-7.8 \pm 3.2)$, Basement membrane area $\left(\mu \mathrm{m}^{2}\right)(\mathrm{A}-85.9 \pm 25.2 . \mathrm{B}-92.7 \pm 33.5, C-99.2 \pm 25.4)$, Endothelial cell area $\left(u^{2}\right)(A-40.9+10 . \overline{3}, B-38.9+7.9, C-36.5 \div 9.7)$, Endothelial cell profile no. (A-4.7 $\pm 0.8 . \bar{B}-4.2 \pm 0.6, C-\overline{4} .6 \pm 0.7)$ and Pericyte Nuclear no. (A$0.8 \pm 0.2, \mathrm{~B}-0.7 \pm 0.3, \mathrm{C}-0.9 \pm 0.3)$. Thus skin capillary microangiopathy failed to differentiate between patients with and without diabetic neuropathy and therefore cannot be considered as a surrogate of microangiopathy in nerve. Furthermore it failed to explain the occurrence of foot ulceration in diabetic patients despite an equal severity of neuropathy.

\section{9}

MICROLIGHTGUIDE SPECTROPHOTOMETRIC MEASUREMENTS OF OXYGEN SATURATION AND BLOOD FLOW IN HUMAN SURAL NERVE. S Ibrahim, N Harris, M Radatz, J Jakubowski and J D Ward. Diabetes Research Unit, Department of Medical Physics and Department of Neurosurgery, Royal Hallamshire Hospital, Sheffield, UK.

Abnormalities of nerve blood vessels and circulation resulting in hypoxia have been implicated in the pathogenesis of diabetic neuropathy. We have applied microlightguide spectrophotometry to measure the intracapillary oxygen saturation $\left(\mathrm{HbO}_{2}\right)$ and blood flow in human sural nerve. We used a combination of the flexibie fibre optic lightguide and a rigid endoscope (diameter $1.9 \mathrm{~mm}$ ) which allows determination of the exact site of measurement over the surface of the nerve. The technique is minimally invasive using a $10 \mathrm{~mm}$ incision. 100 individual $\mathrm{HbO}_{2}$ measurements were taken at 3 sites with the endoscope $0.5-1 \mathrm{~mm}$ away from the surface. Patients were then given IV sodium fluorescein and nerve capillary blood flow was estimated from spectrophotometric measurements of nerve fluorescence. 7 patients with mild-moderate chronic sensory motor diabetic neuropathy and 7 matched healthy control subjects were studied. In the neuropathic group $\mathrm{HbO}_{2}$ was significantly lower than the control group $(66.5 \pm 5.7 \%$ Vs $77.3 \pm 6.1 \% \quad P=0.005)$ suggesting increased oxygen extraction to compensate for local hypoxia. The fluorescein rise time (RT), the time interval between $10 \%-90 \%$ of maximum fluorescence, was significantly slower in the neuropathic group $(48.7+21.7$ Vs $14.7+8.3$ $P=0.01$ ) indicative of impaired nerve blood flow. There was a significant correlation between $\mathrm{HbO}_{2}$ and $\mathrm{RT}(\mathrm{P}=0.0005)$ suggesting that low $\mathrm{HbO}_{2}$ in the neuropathic group is due to impairment of nerve blood flow. These results confirm the findings of other studies and highlight the importance of vascular factors in the aetiology of diabetic neuropathy.

\section{0}

MUSCULAR FATIGUE IN NIDDM WITH SUB-CLINICAL NEUROPATHY: MYOELECTRIC FEATURES. Piaggesi A., Rossi B., Schipani E., Chisari C., Ceraudo A., Navalesi R.. Pisa, Italy.

In 16 asymptomatic NIDDM [ 8 with (ND+; age $49.75 \pm 8.34$ yrs, DD $7.62 \pm 1.77 \mathrm{yrs}$ ) and 8 without (ND-; age $50.13 \pm 3.21 \mathrm{yrs}, \mathrm{DD} 8 \pm 2.39$ yrs) polyneuropathy] in stable metabolic control, and in 8 age-matched controls $(C)$, we measured resistance to stimulated isometric contraction of anterior tibial muscle.Stimulation was performed with a pulse train of $0.1 \mathrm{msec}$ at $35 \mathrm{~Hz}$ and supramaximal for $\mathrm{M}$-wave amplitude, while registration was obtained through a 4-bars electrode. Items considered were: Power Spectrum Median Frequency of Surface EMG Signal (MDF), Muscle Fiber Conduction Velocity (CV), Average Rectified Value of Power Spectrum of Surface EMG Signal (ARV), at rest and after 23" and $43^{\prime \prime}$ from the beginning of stimulation. In $N D+M D F, A R V$ and $C V$, either at rest, after $23^{\prime \prime}$, and after $43^{\prime \prime}$ all significanlty differed from NDand Controls, as shown in the Table:

\begin{tabular}{lcccc}
\hline & ND+ & ND+ & C & $p^{*}$ \\
\hline \hline MDF-Bas. (Hz) & $79.1 \pm 12.9$ & $100.2 \pm 22.3$ & $95.8 \pm 19.6$ & 0.05 \\
ARV-Bas. ( $\mu V)$ & $370.8 \pm 138.5$ & $575.5 \pm 215.5$ & $425.2 \pm 213.1$ & 0.05 \\
CV-Bas. (m/sec) & $4.07 \pm 0.51$ & $4.46 \pm 0.54$ & $4.63 \pm 0.31$ & 0.05 \\
MDF-23" (Hz) & $52.7 \pm 10.4$ & $66.5 \pm 13.3$ & $66.7 \pm 12.2$ & 0.05 \\
ARV-23" ( $\mu V)$ & $446.3 \pm 167.4$ & $732.5 \pm 267.6$ & $502.3 \pm 284.1$ & 0.01 \\
CV-23" (m/sec) & $3.27 \pm 0.53$ & $3.95 \pm 0.74$ & $3.68 \pm 0.34$ & 0.05 \\
MDF-43" (Hz) & $45.6 \pm 8.6$ & $59.1 \pm 11.9$ & $57.5 \pm 17.8$ & 0.01 \\
ARV-43" ( $\mu V)$ & $332.2 \pm 121.2$ & $501.6 \pm 206.1$ & $441.3 \pm 364.1$ & 0.01 \\
CV-43" (m/sec) & $2.89 \pm 0.37$ & $3.54 \pm 0.53$ & $3.28 \pm 0.26$ & 0.01 \\
\hline
\end{tabular}

*ANOVA: ND+ vs ND- and Controls
Our findings are consistent with a decrease in mean diameter of muscle fibres in ND+ respect to ND- and controls, indicative of PN-induced hypotrophy, even in non-denervated muscle fibres. 
OP 9

\section{Pregnancy}

61

COMBINED ISLET CELL ANTIBODY TESTING IN PATIENTS WITH GESTATIONAL DIABETES: A PROSPECTIVE FOLLOW-UP STUDY

K. Ferber, M. Füchtenbusch, M. Hummel, E. Standl, E. Albert*, A-G. Ziegler. Diabetes Research Institute and "Laboratory for Immunogenetics, LMU, Munich, Germany.

The aim of the present study was to evaluate the predictive value of islet antibody screening for the development of IDDM in 445 patients with Gestational Diabetes (GDM) in the following years postpartum. ICA was detected by immunofluorescence, GADA and IA2A by immunoprecipitation. The cut off for positivity of ICA, GADA, and IA2A during pregnancy was defined as the 99 . percentile of antibody values in 113 healthy pregnant women (ICA 10 JDFu, GADA $21 \mathrm{u}$ IA2A $23 \mathrm{u}$ ). $29 \%$ of the patients developed diabetes by 9 months, and $47 \%$ two years after delivery (GDM-Bg: $83 \%$, GDM-A: $20 \%$ ) and diagnosis was confirmed by OGTT. At delivery, $9.4 \%$ of all GDM patients were ICA+, 15.3\% GADA+ and $11 \%$ IA2A+. $7 \%$ were positive for more than one antibody (GDM-Bg 15\%, GDM-A $2.4 \%$, $\mathrm{p}<0.001$ ). Antibody positivity persisted during follow-up ( 9 months: $>1 \mathrm{ab}: 6.5 \%$ ). Of patients who were antibody positive during pregnancy, $69 \%$ developed diabetes two years postpartum as compared to $29 \%$ of ab- patients ( $p<0.001$ ). Thereby, antibody positivity in GDM was associated with an insulin requiring state, whereas $95 \%$ of antibody negative GDM patients remained free of insulin requiring diabetes. In addition to the mothers we investigated all their children for islet antibodies within the first two years of life. 10 children were identified to be positive for one or more antibodies ( 3 of GDM-Bg mothers, 7 of GDM-A mothers, 4 mothers being ab+) and $1 / 10$ developed IDDM at 5 years of age. We conclude that antibody screening may identify gestational diabetic women at risk for the development of IDDM thus allowing appropriate therapy.

IMPAIRED INSLLIN SECRETION AND ACTION IN WOMEN WITH A HISTORY OF GESTATIONAL DIABETES MELLITLS D. Romann, I. Winter, U. Lang*. H. Laube and T. Linn. 3rd. Med. Clinic \& Policlinic, Rodthohl 6; and *Clinic for Gynecology \& Obstetrics, Justus-Liebig-University, D-35385 Gießen, Germany.

Women with a history of gestational diahetes carry a high risk for the development of NIDDM in later life. The aim of this study was to determine the contribution of insulin action and insulin secretion to the impairment of glucose tolerance in women with a history of IGT or GDM (O'Sullivan criteria) during a pregnancy 2-5 years before. 34 women (age $33,5 \pm 0,9$ years, BMI $25,1 \pm 0,9 \mathrm{~kg} / \mathrm{m}^{2}$; mean $+\mathrm{SEM}$ ) underwent an oral glucose tolerance test $(75 \mathrm{~g}, 120$ minutes) and a undified intravenous glucose tolerance test (IVGT; Bergman's minimal model: $0,33 \mathrm{~g} / \mathrm{kg}$ glucose, tolbutamide protocol) to determine parameters of insulin secretion and insulin sensitivity. Using the WHO-criteria the patients were divided into 3 groups (A: normal; B: IGT; C: Diabetes) The IVGT showed a marked decrease regarding the glucose disappearance $\mathrm{K}_{G}(2,19(\mathrm{~A}) ; 1,08(\mathrm{~B})$ and $0,56(\mathrm{C}) \% / \mathrm{min}$; geometric means; variance analysis $p<0,001)$ and the first-phase insulin response $\mathrm{AIR}(198,0(\mathrm{~A}) ; 144,3(\mathrm{~B})$ and $35,9 \mathrm{pM}(\mathrm{C}) ; \mathrm{p}=0,0013)$. Glucose effectiveness $S_{0}(0,032(\mathrm{~A}) ; 0,016(\mathrm{~B})$ and $0,014 \mathrm{~min}-1(\mathrm{C}) ; \mathrm{p}=0,028)$ and insulin sensitivity index $S_{I}\left(6,75(\mathrm{~A}) ; 2,29(\mathrm{~B})\right.$ and $\left.2,23 \times 10^{-4} \mathrm{~min}^{-1} \mu \mathrm{U}^{-1} \mathrm{~m}\right]$ $(C) ; p=0,057)$ were also lower in glucose intolerant and diabetic women Covariance analysis could demonstrate no influence of age and BMI to these results. Furthermore, regression analysis showed a log-log-linear distribution between the glucose level $2 \mathrm{~h}$ after OGT and the minmod derived parameters implying a hyperbolic relationship. Thus these results underline the importance of both impaired insulin secretion and impaired insulin action for the development of IGT or NIDDM in the studied population of women with a history of GDM.

\section{2}

FOLLOW-UP OF WOMEN WITH PREVIOUS GESTATIONAL DIABETES MELLITUS: RECLASSIFICATIONAND FREOUENCY OF LATE COMPLICATIONS Zs. Kerényi, J. Tóth*, P. Stella, Gy. Bibok*, P. Kempler** and Gy. Tamás ${ }^{\star *}$, Szt. Imre Hospital, Haynal Imre ${ }^{\star}$ and Semmelweis** University, Budapest, Hungary Gestational diabetes mellitus (GDM) is a predictor of glucose intolerance (GD) later in life. Little is known about frequency of diabetic complications in this patients. Women with previous GDM insulinized during pregnancy (GDM-I; 113/146 [77\%]) were traced using postal questionnaires (mean age: $38.2 \pm 7.3$ [ $+\mathrm{SD}]$ yrs; time elapsed since diagnosing GDM: $6.9+2.6$ yrs; BMI: $26.8+6.2 \mathrm{~kg} / \mathrm{m} 2$ ), 80 of them investigated (2-step procedure). As controls served 25 of 49 traced GDM on diet during pregnancy

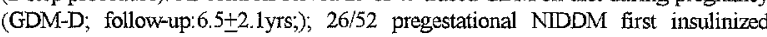
during gestation (preNIDDM; follow-up:6.3+2.2yrs; duration of diabetes: $9.2+2.9 \mathrm{yrs}$ ) from the same cohort together with 40 IDDM women matched for diabetes duration Fourty nine/ $80(61 \%)$ of GDM-I ( 43 diabetes [25 on insulin], 6 IGT), 11/25 (44\%) of GDM-D ( 6 diabetes [ 1 on insulin], 5 IGT; GDM-I vs GDM-D: $\mathrm{P}<0.05$ ) and all but one preNDDM had GI at follow-up ( $75 \mathrm{~g}$ oGTT, or fasting and/or postprandial blood glucose using a test-meal, $\mathrm{HbAlc}$ ). To screen for late complications blood pressure, proteinuria, microalbuminuria (MA, immuno-turbidimetric method), vibration perception threshold (VPT) were measured together with the standard battery of 4 cardiovascular autonomic tests and ophthalmoscony. Any retinopathy was found in 6 $(8 \%)$, lyppertension (treated or $\geq 160 / 95)$ in $18(23 \%)$ GDM-I; $4(15 \%)$ and $5 / 26$ $(19 \%)$ in NIDDM-I cases. One retinopathy and no hypertension were found in GDMD. MA was found in GDM-I: $16 \%$; GDM-D: $20 \%$; preNIDDM: $26 \%$; IDDM: $18 \%$; overt proteinuria (2/40) in IDDM only. Distribution of pathological cardiovascular tests in GI/normal women: 25/5 (GDM-I); 6/5 (GDM-D); 15/0 (preNIDDM); $\geq 2$ test +ve: $16 \%-25 \%-21 \%$; pathological VPT: $16 \%-8 \%-11 \%$ - none in IDDM According to our results 'mild' diabetes forms prone to diabetic complications at a rate at least similar to IDDM. Screening for GDM and caring for these patients regularly afterwards seem to be of utmost importance to prevent later $\mathrm{GI}$ and its complications.

\section{4}

DEFECTS IN INSULIN SECRETION AND INSULIN ACTION CHARACTERIZE GESTATIONAL DIABETES IN LATE PREGNANCY

M.Carvalheiro', I.Fagulha', A.Fagulha', L.Gomes', S.Paiva', E.Marta', E.Sobral' F.Leitäo, M.L.Pinto ${ }^{3}$, M.M.A.Ruas' and T.Buchanan'. 'Dept of Endocrinology ${ }^{2}$ Obstetric Clinic and ${ }^{3}$ Clinical Pathology, University Hospital Coimbra, Portugal ${ }^{4}$ University of Southem California, Los Angeles, USA

Our aim was to determine insulin sensitivity $\left(\mathrm{S}_{\mathrm{S}}\right)$, glucose effectiveness $\left(\mathrm{S}_{\mathrm{G}}\right)$, glucose tolerance $\left(\mathrm{K}_{\mathrm{G}}\right)$ and insulin secretion in 51 gestational diabetic women (GDM), 26 pregnant control (PGC) and 27 nompregnant control (NPC) women, with similar age (30 $\pm 4.6 ; 27.7 \pm 4.1 ; 28.9 \pm 6.2$ yrs, respectively) and $B M I(25.1 \pm 4.3 ; 23.6 \pm 4.9 ; 25 \pm 4.5$ respectively), in late pregnancy (28-38wks) or luteal phase of the menstrual cycle, during an insulin modified frequently-sampled intravenous glucose tolerance test (FSIVGTT: $300 \mathrm{mg}$ glucose/kg body weight, followed in $20 \mathrm{~min}$. by a 5 -min infusion of insulin $6 \mathrm{mU} / \mathrm{kg} / \mathrm{min}$ and blood sampled 14 times for $240 \mathrm{~min}) . \mathrm{S}_{\mid}\left(\times 10^{-4} \mathrm{~min}-\mathrm{per} \mu\right.$ $\mathrm{U} / \mathrm{ml})$ and $\mathrm{S}_{\mathrm{G}}\left(\mathrm{min}^{-1}\right)$ were estimated by Bergman's minimal model. $K_{\mathrm{G}}\left(\mathrm{min}^{-1} \mathrm{x} 100\right)$ was assessed between 8-19 mins after glucose injection. First phase of insulin secretion was expressed as the area under the insulin curve between 2-8 $\min (\mu$ $\mathrm{U} / \mathrm{m} \mid \mathrm{xmin}) . \mathrm{S}_{1}$ was significantly decreased in GDM vs PGC and vs NPC and in PGC vs NPC. Seven GDM women (13.7\%) had a not detectable $S$. $S$, was al so significantly decreased in GDM vs PGC and vs NPC and similar in PGC vs NPC. $K_{G}$ was not significantly different among groups. The first phase insulin sacretion was significantly smaller in GDM vs PGC but identical to NPC. On the contrary, in PGC vs NPC, the first phase was significantly higher ( 2.3 increment).

\begin{tabular}{llll} 
& \multicolumn{1}{c}{$\mathrm{NPC}$} & \multicolumn{1}{c}{ PGC } & \multicolumn{1}{c}{ GDM } \\
\hline $\mathrm{S}_{\mathrm{I}}$ (mean $\pm \mathrm{SD}$ ) & $4.99 \pm 2.41$ & $2.41 \pm 2.08^{*}$ & $1.07 \pm 1.01^{*+}$ \\
$\mathrm{S}_{\mathrm{G}}$ (mean $\left.\pm \mathrm{SD}\right)$ & $0.022 \pm 0.009$ & $0.023 \pm 0.008^{*}$ & $0.017 \pm 0.007^{*+}$ \\
First Phase (mean $\pm \mathrm{SD})$ & $322 \pm 165$ & $730 \pm 502^{*}$ & $449 \pm 324^{+}$ \\
$\mathrm{K}_{\mathrm{G}}$ (mean $\pm \mathrm{SE}$ ) & $1.96 \pm 0.3$ & $2.34 \pm 0.4$ & $1.55 \pm 0.2$ \\
\hline
\end{tabular}

${ }^{ \pm}<0.05$ vs NPC ${ }^{+} p<0.05$ vs PGC

Thus, unlike investigations with earlier versions of minimal model, we found significant reductions in $S_{\mid}$and $S_{G}$ in $G D M$ women vs normal pregnant women, but identical impaired B-cell function. These data indicate that defects in insulin secrection and action characterize GDM in late pregnancy in our predominantly lean patiens. 
65

DYSPRUPORTIONALLY ILLIVATED PROINSULIN CONCENTRATIONS IN GESTATIONAL DIABETES BEFORE AND AFTER DELIVERY

A. Kautzky-Willer, G. Pacini, B. Ludvik, P. Nowotny, W. Waldhäus! and R. Prager, Department of Medicine III, Division of Endocrinology \& Metabolism, University of Vienna, Austriat

Gestational diabeles mellitus (GDM) carries a high risk for later NIDDM and serves as a model for its early metabolic changes. Changes in proinsulin secretion are thought to represent early defects in beta-cell secretion in NIDDM and have been postulated to be involved in the pathogenesis of impaired glucose tolerance. Therefore, we assessed plasma concentrations of insulin, C-peptide and proinsulin both before and after delivery in GDM in comparison to pregnant women with normal glucose tolerance (NGT) and to non-pregnant healthy women (CS) during oral glucose tolerance tests $(100 \mathrm{~g})$. Kinetic analysis of insulin was performed with mathematical modeling providing indirect estimates of its secretion. Basal insulin secretion was raised in GDM $(48 \pm 5 \mathrm{pM} / \mathrm{min})$ vs. NGT $(37 \pm 3)$ and CS (36 \pm 3 ), while postprandial (total) insulin secretion was only elevated $(p<0.05)$ in

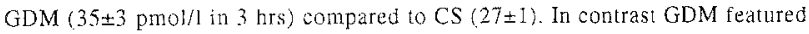
basal (bP) as well as postprandial ( $\mathrm{P}$ ) hyperproinsulinemia compared to both $\mathrm{NGT}$ and CS (oP: $2.8 \pm 0.3$ vs $1.7 \pm 0.1$ vs $1.4 \pm 0.2 \mathrm{nmol} / 1 ; \mathrm{P}: 10.7 \pm 1.5$ vs $7.6 \pm 0.8$ vs $6.0 \pm 0.9 \mathrm{nmol} / \mathrm{l} \times 3 \mathrm{hrs}, \mathrm{GDM}$ vs NGT vs $\mathrm{CS}, \mathrm{p}<0.05)$. The areas under the curve (AUC/180min) for the proinsulin/insulin ratio were higher $(\mathrm{p}<0.05)$ in GDM vs CS (basal AUC: $0.21 \pm 0.03$ vs $0.13 \pm 0.01$, total AUC: $0.11 \pm 0.01$ vs $0.08 \pm 0.01$, $\mathrm{p}<0.05)$ with only total AUC being increased vs NGT $(0.07 \pm 0.01, \mathrm{p}<0.05)$. After delivery insulin secretion and the proinsulin concentrations $(\mathrm{p}<0.01)$ decreased to the normal range in GDM, whereas the proinstlin/insulin ratio remained elevated. In summary, proinsulin hypersecretion and an elevated total proinsulin to insulin ratio is specific for GDM in the early post partum period (<3months). These data indicate (1) specific changes in the proinsulin availability during GDM with.(2) an increased proinsulin/insulin ratio serving as a potential marker for GDM potentiaily identifying stbjects at high risk for later development of NIDDM.

\section{6}

VITAMIN E TREATMENT DECREASES CONGENITAL MALFORMATION IN DIABETIC RAT PREGNANCY C. M. Simán, and U. J. Eriksson, Department of Medical Cell Biology, University of Uppsala, Uppsala, Sweden

Diabetic rats were supplemented with $2 \%$ of the antioxidant vitamin $\mathrm{E}$-acetate in the diet before and throughout pregnancy in an attempt to prevent congenital malformations in the offspring. After injection of $40 \mathrm{mg} / \mathrm{kg}$ i.v. of streptozotocin, female rats with a serum glucose level exceeding $20 \mathrm{mM}$ were mated, and the pregnancy was interrupted on gestational day 20 . A malformation rate of $23 \%$, and a resorption rate of $29 \%$ were decreased to $7 \%$ and $10 \%$, respectively, by vitamin $\mathrm{E}$ supplementation of the diabetic pregnant rats. The fetal weight in litters of diabetic rats increased from $2.7 \pm 0.1 \mathrm{~g}$ to $3.1 \pm 0.1 \mathrm{~g}$ by vitamin $\mathrm{E}$ treatment, compared to $3.8 \pm 0.03 \mathrm{~g}$ in control fetuses. HPLC-based analysis revealed very low fetal concentrations of liver $\alpha$-tocopherol (diabetic $1.9 \pm 0.2 \mu \mathrm{g} / \mathrm{g}$; control $0.7 \pm 0.1 \mu \mathrm{g} / \mathrm{g}$ ) as compared to the maternal liver (diabetic $35 \pm 4 \mu \mathrm{g} / \mathrm{g}$; control $27 \pm 3 \mu \mathrm{g} / \mathrm{g}$ ), suggesting a low antioxidant capacity in the fetus. Vitamin $\mathrm{E}$ treatment increased the $\alpha$-tocopherol concentration in fetuses of diabetic mothers to $9.2 \pm 0.8 \mu \mathrm{g} / \mathrm{g}$. Measurement of malonyldialdehyde (MDA) revealed an increased lipid peroxidation in fetuses of diabetic rats $(319 \pm 16 \mu \mathrm{M})$ compared to controls $(72 \pm 4 \mu \mathrm{M})$, which was normalized in the offspring of vitamin $\mathrm{E}$ treated diabetic rats $(89 \pm 6 \mu \mathrm{M})$. We conclude that supplementation of high doses of vitamin $\mathrm{E}$ to the diabetic mother has an antioxidative effect in the offspring and prevents fetal dysmorphogenesis.

\section{OP 10 \\ Transplantation and Engineered Beta-Cells}

\section{7}

INSULIN TREATMENT IMPROVES THE OUTCOME OF ISLET TRANSPLANTATION: EFFECTS ON BETA CELL MASS AND FUNCTION

J.F. Merino, V. Nacher, M. Raurell,O. Aranda,J. Soler and E. Montanya. Laboratori de Diabetis i Endocrinologia Experimental, Endocrine Unit. CSUB-Hospital de Bellvitge. Barcelona. Spain.

Insulin treatment may improve the outcome of islet transplantation. To determine the effect of insulin on transplanted (Tx) beta cells, 2 groups of streptozocin-diabetic C57BI/6 mice were Tx with 100 syngenic islets, an insufficient beta cell mass to achieve normoglycemia. Group $1(\mathrm{G} 1, n=14)$ was kept normoglycemic with insulin treatment from day 10 before $T x$ to day 14 after $\mathrm{Tx}$; when insulin was discontinued all G1 mice maintained normoglycemia. Group $2(G 2, n=14)$ was not treated with insulin and remained hyperglycemic throughout the study. Grafts were harvested 14 or 60 days after Tx. Beta cell mass was measured by point counting morphometry, beta cell area by planimetry and insulin content by RIA. 14 days after $T x$, the initially $\mathrm{Tx}$ beta cell mass $(0.22 \pm 0.02 \mathrm{mg})$ was reduced in both groups (G1: $0.09 \pm 0.01 \mathrm{mg}, \mathrm{p}<0.001$; G2: $0.14 \pm 0.02 \mathrm{mg}$, $p<0.01)$; beta cell area was lower in G1 grafts (G1: $219 \pm 16 \mu \mathrm{m}^{2}$; G2: $267 \pm 14 \mu \mathrm{m}^{2}, \mathrm{p}<0.05$ ), but insulin content was almost 10 times higher in $\mathrm{G} 1$ grafts (G1: $1.1 \pm 0.2 \mu \mathrm{g} ; \mathrm{G} 2: 0.15^{\prime} \pm 0.03 \mu \mathrm{g}$, $p<0.01$ ). After insulin withdrawal beta cell mass increased in $G 1$; in contrast it was progressively reduced in $\mathrm{G} 2$ (day 60 after Tx: G1: $0.23 \pm 0.04 \mathrm{mg}, \mathrm{G} 2: 0.08 \pm 0.02 \mathrm{mg}, \mathrm{p}<0.001)$. In summary, insulin treatment reduced the beta cell mass needed to achieve normoglycemia in islet Tx. Insulin treatment did not prevent an initial loss of beta cell mass, but preserved beta cell function after Tx.

\section{8}

INSULIN SECRETION IN PANCREAS TRANSPLANT RECIPIENTS: PITFALLS USING PERIPHERAL INSULIN AND C-PEPTIDE LEVELS.

E.Christiansen, A.Tibell, L.Kjems, CG.Groth, Aa.Vølund, C.Binder, S.Madsbad. Copenhagen, Denmark, and Stockholm, Sweden.

Accurate estimation of $B$-cell function following pancreas transplantation is important. However, peripheral insulin and/or C-peptide concentrations in the estimation of insulin secretion encounters several pitfalls mainly due to systemic insulin delivery and reduced kidney function. Therefore, we estimated insulin secretion rates from plasma $\mathrm{C}$-peptide levels using the deconvolution technique (two-compartment model for C-peptide distribution and after estimation of individual C-peptide kinetics) in six pancreas transplant recipients (Px), six kidney transplant recipients $(\mathrm{Kx})$ and six normal subjects (Ns). Insulin secretion was assessed during a four hour OGTT $(75 \mathrm{~g}$ ) by use of insulin or C-peptide and calculation of insulin secretion rates (ISR). C-peptide clearance rate was significantly reduced in $\mathrm{Px}(38 \pm 2 \mathrm{~min})$ and $\mathrm{Kx}(40 \pm 3 \mathrm{~min})$ compared to $\mathrm{N}_{\mathrm{s}}$ $(28 \pm 2 \mathrm{~min}, \mathrm{p}<0.05)$. Fasting C-peptide was significantly increased in $\mathrm{Px}(877 \pm 193$ $\mathrm{pmol} / \mathrm{L})$ and $\mathrm{Kx}(1470 \pm 202 \mathrm{pmol} / \mathrm{L})$ vs $\mathrm{Ns}(408 \pm 26 \mathrm{pmol} / \mathrm{L}, \mathrm{p}<0.05)$. Fasting insulin was $143 \pm 36 \mathrm{pmol} / \mathrm{L}$ in $\mathrm{Px}, 101 \pm 33 \mathrm{pmol} / \mathrm{L}$ in $\mathrm{Kx}$, and $32 \pm 6 \mathrm{pmol} / \mathrm{L}$ in Ns $(\mathrm{p}<0.05)$. Fasting ISR was similar in $\mathrm{Px} 13.9 \pm 2.0 \mathrm{pmol} / \mathrm{L} / \mathrm{min}$, and in Ns $13.8 \pm 1.8 \mathrm{pmol} / \mathrm{L} / \mathrm{min}$, but increased in $\mathrm{Kx}(28.3 \pm 4.6 \mathrm{pmol} / \mathrm{L} / \mathrm{min}, \mathrm{p}<0.05)$. The C-peptide response (incremental area under curve) during OGTT was $49 \pm 4 \%$ higher in $\mathrm{Px}, 145 \pm 12 \%$ higher in $\mathrm{Kx}$ compared to $\mathrm{Ns}(\mathrm{p}<0.05)$, whereas the insulin response was $92 \pm 5 \%$ higher in $\mathrm{Px}, 95 \pm 5 \%$ higher in $\mathrm{Kx}$ as compared to Ns $(p<0.05)$. In contrast, ISR was reduced by $13 \pm 3 \%$ in Px compared to Ns ( $p>0.05$ ) and increased by $85 \pm 6 \%$ in $\mathrm{Kx}$ compared to $\mathrm{Ns}(\mathrm{p}<0.05)$. Conclusion: Estimation of B-cefl function from insulin and C-peptide levels in pancreas transplant recipients leads to overestimation of insulin secretion due to absent first-pass hepatic insulin extraction and reduced clearance rate. Accurate estimation of insulin secretion and B-cell function after pancreas transplantation is possible using mathematical modelling. 
69

SERUM FREE MEDIA FOR CULTURE OF HUMAN ISLETS PRIOR TO TRANSPLANTATION

JA Kerr-Conte, F Pattou, YJ Xia, CAG Proye and J Lefebvre. Centre Hospitalier Universitaire de Lille. Lille, France.

In vitro culture of human islets prior to transplantation may allow pooling of several isolations, immunomodulation of islets, and quality controls of the preparation. CMRL 1066 containing $10 \%$ fetal calf serum (FCS) is considered the best available medium for human islet culture. Serum results in higher nitric oxide production implicated in decreased insulin secretion, and increased antigenicity of human islets. Moreover the use of animal sera in ex-vivo procedures appears legislatively undesirable for clinical islet transplantation. Aim of the study: Comparison of conventional CMRL 1066 media containing 10\% FCS or a serum substitute for primary human islet cultures at $37^{\circ} \mathrm{C}$. Methods: Islets isolated from 8 human pancreata by the technique of Ricordi were hand-picked and cultured for 14 days in CMRL 1066 (+pyruvate, nicotinamide, hydrocortisone and antibiotics, $\mathrm{pH} 7.2$ ) containing $10 \% \mathrm{FCS}$ or a serum substitute (SS: $2 \%$ Ultroser G (Gibco BRL), ethanolamine, transferrin, and sodium selenium. The function of islets was determined with: a static incubation in Kreb's Ringer Bicarbonate where stimulation index= insulin secretion with $16.7 \mathrm{mM} /$ mean basal insulin secretion with $3 \mathrm{mM}$, intracellular insulin content, and insulin and total protein biosunthesis after incubation with L- $\left(4,5-{ }^{3} \mathrm{H}\right)$ leucine. Results: Islets cultured +FCS remained responsive to glucose up to 7 days (D) culture at $37^{\circ} \mathrm{C}$ (D $1=3.5 \pm 0.5, D 7=2.6 \pm 0.3$ ). Their responsiveness was lost after 14 days (D14 $=1.5 \pm 0.2$, stimulated vs basal, $p>0.05$ ). Islets cultured with SS remained responsive to glucose even after 20 days of culture (D1 $=3.1+0.6, \quad \mathrm{D} 7=3.1+0.4, \quad \mathrm{D} 14=3.0+0.4, \quad \mathrm{D} 20=2.7+0.3) \quad(\mathrm{D} 1-\mathrm{D} 20$ stimulated vs basal, $\mathrm{p}<0.05$ ). After 20 days islets (SS) maintained $60 \%$ of original intracellular insulin YS $40 \%$ in islets (FCS) after 14 days. In 4-5 preparations, protein biosynthesis remained stable for 7D and subsequently diminished to $50 \%$ of initial values after 14D in FCS and SS cultures. Insulin biosynthesis decreased to $50 \%$ after 14 d culture (FCS) whereas levels increased to $160 \%$ with $\mathrm{SS}$. Conciusion: The use of a serum substitute for culture of human islets in CMRL 1066 allows the maintenance of intracellular insulin, insulin biosynthesis, and responsiveness to glucose for at least 2 weeks in culture. This serum free medium may be successfully used for human islet culture prior to tranplantation.

71

\section{MOLECULARENGINEERING OF INSULIN SECRETING CELLS} WITH GLUCOSE SENSORS

H. Kishikawa, S. Motoyoshi, T. Shirotani, E. Araki, K. Matsumoto, M. Shichiri. Department of Metabolic Medicine, Kumamoto University School of Medicine, Kumamoto, Japan

High capacity glucose transporter (GLUT2) and glucokinase (GK) are known to be involved in the regulation of insulin secretion from the pancreatic $\beta$ cells. To investigate the mechanism of glucose responsible insulin secretion, we first transfected AtT20 cells with human insulin gene(AtT2OHI cells). Then, AtT20HI cells were transfected with GLUT2 CDNA, GK cDNA and both cDNAs(AtT20HI-GT2,AtT2OHI-

GK,AtT2OHI-GT2+GK cells respectively), and studied thier insulin secretion pattern in static incubation and perifusion experiments. In static incubation experiments, the AtT20HI cells could secret mature insulin, but the secretion was not regulated by glucose. The AtT2OHI-GT2 and AtT2OHI-GK cells secreted insulin in glucose concentration-dependent fashion only less than $1 \mathrm{mmol} / \mathrm{l}$ of glucose. In contrast, the AtT20HIGT2+GK cells exhibited glucose concentration-dependent insulin secretion up to $25 \mathrm{mmol} / \mathrm{l}$ of glucose. In perifusion experiments, switching glucose concentration from $5 \mathrm{mmol} / \mathrm{l}$ to $25 \mathrm{mmol} / \mathrm{l}$ induced slight increase of insulin secretion in both AtT20HI-GT2 cells and AtT20HI-GK cells. On the other hand, the AtT20HI-GT2+GK cells showed an obvious insulin secretory response to $25 \mathrm{mmol} / 1 \mathrm{glucose}$, although those could not show biphasic insulin secretion pattern.

In conclusion, for the glucose responsible insulin secretion, both GLUT2 and GK could be necessary. However, for the typical biphasic insulin secretion observed in pancreatic $\beta$ cell might need other component(s) than GLUT2 and GK.
70

DEVELOPMENT OF MATURE HUMAN INSULIN PRODUCTION FROM ENGINEERED FIRROBLASTS AND PRIMARY RAT HEPATOCYTES. L. Falqui, S. Martinenghi, G.M. Severini, C. Berra, L. Monti, M.V. Taglietti, G. Pozza and C. Bordignon. Scientific Institute H.S. Raffaele, Milan, Italy. Autologous non-endocrine cells, engineered by gene transfer for glucoseregulated insulin secretion may represent a future tool for the treatment of IDDM. Non-endocrine cells lack the $\beta$-cell specific proteases for proinsulin cleavage, but are able to process proteins via the constitutive pathway. To exploit this property, we introduced two mutations in the human preproinsulin cDNA (HPI) by overlap extension PCR, to obtain a gene (HPIm) coding for a proinsulin molecule containing at the junctions Bchain/C-peptide and C-peptide/A-chain the cleavage sequences recognized by the constitutively expressed protease furin. The HPIm cDNA was then inserted into a retroviral vector under non-regulated transcriptional control. This vector also carries the CDNA encoding the truncated, non-functional receptor of the human nerve growth factor $(\triangle N G F r)$, for marking and selection of transduced cells. By this retroviral vector the HPIm cDNA was stably transduced into murine fibroblasts (NIH3T3) and human hepatoma cells (HepG2), resulting in mature insulin release (assessed by specific IRMA, cross reactivity with proinsulin $<0,001 \%$ ), ranging from 13.2 to 37.2 $\mathrm{pM} / 24 \mathrm{~h} / 10^{6}$ cells. Biological activity of this mutated insulin was assessed in diabetic athymic mice (plasma IRMA levels $<1.5 \mathrm{pM}$ ). I.p. injection of 6$10 \times 10^{7}$ transduced murine fibroblasts produced hypoglycemia in $60 \%$ of the recipients, and plasma IRMA levels of 26.4-73.2 pM. Primary cultures of rat hepatocytes were subsequently transduced. More than $50 \%$ hepatocytes could be transduced (measured by NGFr expression), when cultured with epidermal growth factor $(50 \mathrm{ng} / \mathrm{ml})$. As a consequence, mature insulin accumulated in culture media $\left(414 \pm 18 \mathrm{pM} / 24 \mathrm{~h} / 10^{6} \mathrm{cells}\right)$. These engineered hepatocytes may represent the first step in the development of primary cells secreting mature insulin in response to extra-cellular glucose variations.
72

STREPTOZOTOCIN-INDUCED SELECTION OF INSULIN ENRICHED CELLS IN A HETEROGENEOUS POPULATION OF RINM CELLS

K.Bloch and P.Vardi. The National Center for Childhood Diabetes, SCMCI, \& Felsenstein Research Center, Tel-Aviv University, Israel.

The use of RIN cells as model for pancreatic beta-cells is hampered by their heterogeneity. The aim of our study was to provide a method for RINm cells selection according to resistance to cell injury, morphology and function. Following streptozotocin (STZ) treatment we obtained STZ-resistant RINm cells (STZr) which differed from the naive cells (nRINm) in their subsequent resistance to STZ and alloxan (AL) treatment, as estimated by MTT (tetrazolium) colorimetric and $\left[{ }^{3} \mathrm{H}\right]-\mathrm{TdR}$ proliferation assays. The functional activity of $n R I N m$ and $S T Z r$ cells of the same passage was assessed by measurement of intracellular insulin content, insulin concentration in the culture medium, and glucoseinduced insulin secretion. We found that only a minority of $n R I N m$ cells survived STZ treatment, and such treatment selected for cells resistant not only to STZ, but also to AL. The cytotoxic effects of STZ (10-20 $\mathrm{mM})$ and $\mathrm{AL}(10 \mathrm{mM})$ were more pronounced in $n R I N m$ compared to $S T Z r$ cells $(\mathrm{p}<0.01)$. Following cryopreservation, only $S T Z r$ cells formed floating islet-like structures (pseudoislets) within 1-2 days after replating, while $n R I N m$ cells kept their monolayer characteristics. Both acid ethanol-extracted insulin content and the culture medium insulin concentration of $S T Z r$ cells were significantly higher when compared to nRINm cells: $293 \pm 10$ vs. $157 \pm 26 \mu U / 10^{6}$ cells $(\mathrm{p}<0.001)$, and $810 \pm 140$ vs. $270 \pm 16 \mu \mathrm{U} / 48 \mathrm{~h} / 10^{6}$ cells $(\mathrm{p}<0.01)$, respectively. Incubation of both cell populations with increasing glucose concentrations $(0,3,15 \mathrm{mM})$ showed a slightly higher insulin response in $S T Z r$ than in $n R I N m$ cells. In conclusion, our results suggest that RINm cells can be selected by STZ treatment, with a surviving cell subpopulation characterized by STZ and AL resistance, formation of pseudoislets, and a higher cell insulin content and secretion. 
OP 11

\section{Endothelium Blood Flow and Diabetic Complications}

\section{3}

INCREASED FOREARM BLOOD FLOW IN UNCOMPLICATED IDDM; NORMAL BASAL AND STIMULATED NITRIC OXIDE SYNTHESIS. G. Vervolt, J.F.M. Wetaels, J.A.Lutheman, J.H.M.Berden and P. Smirs. Depe of Medicine and Phamacolesy, Universiry Hexpital Nimegen. The Netherkands. Objective: There is considerable evidence that diahetic microngiopathy is preceded by a state of generalized capillary hyperperfusion. These observations have led to the hypothesis that chames in regronal hatemolynamies contribute to microvascular complications. Nitric oxide (NO) is a potent vasodilator and increases bood thow as well an sapillary permealbility. Therefore $\mathrm{NO}$ is a candidate for mediating incretses in houd flow and vasulat? permeability incluced hy diahetes. In vivo data in humilns with uncomplicated diabetes atre scarce and conflicting. We pertormed a study in which we investigated hasal (forearm) blood How and baseline as well an stimulited NO synthesis in uncomplicated type 1 diahetic patients (DP). Design and methods: We studied 47 controls (C) and 43 normodbuminuric DP. Foratm blood flow (FBF) was measured by plethysmography during brathial antery infusion of selline followed by infusion of $N^{6 \cdot}$-monomethyl-L-arainine (L-NMMA), noradenaline (NA) and acetylcholine $(\mathrm{ACh})$. The post-ocelusive reative hyperaemia test (PORH) was performed at the end of the sthdy. All subjects were in a fasting state. Glucose was not corrected to avoid confounding by the vasodiator effect of insulin. Data were analyzed by repented measures of ANOVA or lupatred t-test. Results are expressed its mean \pm SEM. Results: Basal FBF was significantly increased in DP compared to C $(2.9 \pm 0.2$ and $2.0 \pm 0.1 \mathrm{ml} / \mathrm{d} / \mathrm{mm}), \mathrm{P}<0.01$. L-NMMA calsed similar vasocontriction in DP ats in $C(29.9 \pm 1.7$ and $31.2 \pm 2.2 \%)$. Dose-response curves corrected for flow were identical in both groups. The valsowontricting effect of NA Wats not different $(44.8 \pm 2.5 \%$ in DP and $39.8 \pm 3.1 \%$ in $C)$. Although the relative increase of $\mathrm{FBF}$ during $\mathrm{ACl}$ was attentated in $\mathrm{DP}$ (703 \pm 72 and $980 \pm 97 \%)$, alnsilute FBF was not different $(23.5 \pm 2.0$ and $20.1 \pm 1.6 \mathrm{ml} / \mathrm{d} / / \mathrm{min})$. There were no differences in PORH (FBF $43.8 \pm 1.7$ versus $41.0 \pm 1.7 \mathrm{ml} / \mathrm{dl} / \mathrm{min}$ ). Conclusion: Batsal (forearm) hlood thow is incrased in uncomplicated type 1 diahetes mellitus. There is no evidence that hasal nitric oxide synthesis is increased. Other vasodilating factors may contribute to capillary hyperpertision. In uncomplicated IDDM stimulated NO syntlesis is not imprired. PORH is not atfenuated indicating ahenen of strucural changes of the atterial wall.

\section{5}

\section{EFFECT OF DIABETES ON THE ACTIVITY AND EXPRESSION OF MYOCARDIAL NO-SYNTHASES.}

P.Rösen, K.Stockklauser, T.Laufer, Th.Ballhausen

Diabetes Research Institute, Düsseldorf, FRG

Previously we presented evidence that the receptor mediated, NOdependent vasodilatation becomes progrediently impaired in the heart of diabetic rats. This defect could be prevented by antioxidants, but the underlying mechanisms remained unclear. To study whether changes in the NO generating system (NOS) are primary events, we determined the enzymatic activity of NOS (conversion of ${ }^{3} \mathrm{H}$-Arg into Citrullin) and the expression of the constitutive (cNOS) and inducible (iNOS) form of NOS by RT-PCR. Shortly after induction of diabetes (streptozotocin, $60 \mathrm{mg} / \mathrm{kg}$ body weight) the NOS activity became increased reaching a maximal activity after about 4 weeks ( $19 \pm 1$ vs $48 \pm 8 \mathrm{fmol} / \mathrm{mg}$ protein $/ \mathrm{min}, \mathrm{p}<$ 0.05 ). The increase in activity was associated with an elevation of mRNA encoding for cNOS- and iNOS. Thereafter, expression of both forms of NOS declined rapidly. After a diabetes duration of 10 weeks the mRNAs were reduced to about $50 \%$ of controls. NOS activity became diminished more slowly (normalised within 5 months) and then fell under control values. Treatment of the diabetic rats with tocopherol did not only prevent the increase, but reduced the activity in hearts of control and diabetic rats $(8 \pm 2$ vs $1 \pm 2 \mathrm{fmol} / \mathrm{mg}$ protein $/ \mathrm{min})$. The mRNA of both forms of NOS were diminished similarly. Thus, the changes in NOS activity and expression did not run in parallel to the impairment of the receptor mediated, NO dependent vasodilatation in diabetes. Our data suggest that diabetes leads to a short term increase in NOS activity and expression, which might reflect an initial activation by an increased shear stress and explain clinical observations of an hyperfusion at the onset of diabetes. The loss in activity and expression of NOS is assumed to indicate an irreversible loss of intact endothelium.
74

EFFECTS OF INSULIN AND ANTI-HYPERTENSIVE TREATMENT ON SOLUBLE ADHESION MOLECULES IN PATIENTS WITH TYPE II DIABETES.

M.P. Alberiche, G. Targher, R.C. Bonadonna, M. Muggeo, and E. Bonora. Division of Endocrinology and Metabolism, University of Verona Medical School, Verona, Italy.

Atherogenesis (ATG) is a complex, multi-step process, in which plaque formation can be schematically divided into an early phase (EP), characterized by mild oxidation/modification of lipids in low density lipoproteins (LDL), and a late phase (LP), characterized by extensive oxidation/modification of both lipids and apoprotein B in LDL. Each ATG phase is associated with the endothelial expression of specific cell adhesion molecules, which both play a pathogenetic role and may be used as markers of ATG phase and ATG activity. Anti-hypertensive therapy (T) exerts a protective action on cardiovascular complications in type 11 diabetes (NIDDM), whereas the role played by insulin (I) is still a controversial issue. We assessed the effects of acute insulinization and chronic (4-months) $T$ (lacidipine $4 \mathrm{mg} /$ day, $n=9$, or lisinopril $10 \mathrm{mg} /$ day, $n=7$ ) on serum levels of $P$ selectin (selectively expressed in the EP of plaque formation) and Intercellular Adhesion Molecule 1 (ICAM-1, selectively expressed in the LP of plaque formation) in 16 (11 males, 5 females) noninsulin treated, nonobese (BMI $=26 \pm 2 \mathrm{~kg} / \mathrm{m} 2$, age: $56 \pm 6 \mathrm{yrs}$ ) normotensive NIDDM patients. P-selectin and ICAM-1 were measured at baseline and at the end of a 4-hr euglycemic I ( $-300 \mathrm{pmol} / \mathrm{l})$ ciamp, both before and after chronic T. Basal P-selectin $(135 \pm 35 \mathrm{ng} / \mathrm{ml})$ and ICAM-1 $(269 \pm 20 \mathrm{ng} / \mathrm{ml})$ levels were positively correlated to both BMI $(r=0.30$ and $r=0.66$, respectively; $p<0.05-0.001)$ and waist girth ( $r=0.41$ and $r=0.40$, respectively; $p<0.05$ for both), but not to glycated hemoglobin, blood pressure, plasma lipids, or I sensitivity. T was associated with a fall in both P-selectin and ICAM-1 levels, but only the latter was statistically significant $(-13.8 \pm 6.3 \mathrm{ng} / \mathrm{ml}, \mathrm{p}<0.04)$. Similarly to and independently of $T$, I caused a consistent drop in ICAM $-1(-18.1 \pm 6.3 \mathrm{ng} / \mathrm{ml}$, $\mathrm{p}<0.01)$, but not $\mathrm{P}$-selectin concentrations. These data provide preliminary evidence that in nonobese, normotensive NIDDM patients both antihypertensive treatment and acute insulinization may favourably affect ATG and suggest that this beneficial action is primarily exerted at some unidentified step in the late phase of plaque formation.

\section{6}

THIAMINE DECREASES ADVANCEDGLYCOSYLATION-END PRODUCT FORMATION IN HUMAN ENDOTHELIAL CELLS CULTURED IN HIGH GLUCOSE.

E. Beltramo, M. La Selva, F. Pagnozzi, F. Pomero, E. Bena, M. Porta and G.M. Molinatti. Department of Internal Medicine, University of Turin, Turin, Italy. Aim of Study: We previously showed that thiamine (vitamin $B_{1}$ ) corrects delayed replication and increased lactate production, as caused by high glucose in cultured endothelial cells. The aim of this study was to verify if its effect on replication is due to a modulation of glycolysis leading to inhibition of Advanced Glycosylation End-product (AGE) formation. AGE increase in the presence of augmented concentration of both glucose and highly reactive glycolytic metabolites, in particular glyceraldheyde-3-phosphate (G3P). Thiamine acts as a coenzyme for transketolase, which allows G3P to enter the pentose-phosphate shunt, thus decreasing its concentrations in the cytosol. Materials and Methods: Human umbilical vein endothelial cells (HUVEC) were incubated in secondary culture with physiological $(5.6 \mathrm{mmol} / \mathrm{l})$ or high $(28.0 \mathrm{mmol} / \mathrm{l})$ glucose, with and without 150 $\mu \mathrm{mol} / \mathrm{l}$ thiamine, for 20 days. HUVEC were then lysed with $\mathrm{NaOH}$, the total protein concentrations were assayed by the Lowry method and spontaneous fluorescence detected at $370 \mathrm{~nm}$ excitation and $\mathbf{4 4 0} \mathrm{nm}$ emission by a fluorimeter. Results (fluorescence/total protein ratio, mean of 6 experiments \pm SD), expressed as percentages of control (5.6 mmol/l glucose), were: $241.5 \pm 163.2 \%$ for high glucose ( $p<0.05$ vs 5.6 glucose), $106.9 \pm 24.5 \%$ for 5.6 glucose + thiamine (NS vs 5.6 glucose, $p<0.05$ vs high glucose), and $117.5 \pm 21.2 \%$ for high glucose + thiamine (NS vs 5.6 glucose and $5.6+$ thiamine, $p<0.05$ vs 28.0 glucose). Conclusions: AGE production in HUVEC is prevented by thiamine in the presence of high glucose concentrations, possibly through the shift of excess G3P towards the pentose phosphate cycle. 
HIGH GLUCOSE INCREASES MONOLAYER PERMEABILITY TO MACROMOLECULES IN BOVINE RETINAL ENDOTHELIAL CELLS G. Romeo ${ }^{1}$, G. Pugliese ${ }^{1}$, F. Pricci ${ }^{1}$, G. Leto ${ }^{2}$, G. Galli ${ }^{3}$, C.M. Rotella ${ }^{3}$, G. Mazza ${ }^{2}, G$. Fuiano ${ }^{2}$, and U. Di Mario ${ }^{2}$ Univ. of ${ }^{1}$ Rome "La Sapienza", ${ }^{2}$ RC-Catanzaro and ${ }^{3}$ Florence, Italy

Vascular permeability was shown to be increased, together with peripheral blood flow, in tissues targets of diabetic complications, including the retina. In order to assess whether the diabetic milieu is capable of impairing retina endothelial cell barrier function directly, independent of the effect on the hemodynamics, bovine retinal endothelial cells (BREC) were cultured for 10 days under high glucose (HG) vs. iso-osmolat mannitol (M) and normal glucose (NG) conditions on permeable polyethylene terephthalate membranes inserted in 35- $\mathrm{mm}$ culture dishes. BREC were allowed to attain confluence, as assessed by contrast phase microscopy, then monolaye permeability was measured by the use of a radiotracer technique. Approximately $500,000 \mathrm{cpm} /$ well of 125 l-labelled horseradish peroxidase (HRP, MW 44,000), bovine serum albumin (BSA, MW 66,000), bovine lgG (MW 150,000) or fibrinogen (MW 400,000, to exclude an abnormal leakage of monolayers) were added to the upper chamber and $20 \mu \mathrm{l}$ aliquots of medium were sampled from both the upper and the lower chamber at 1,2,3 and 5 hours. BREC grown under $\mathrm{HG}$ conditions, but not those cultured in $M$, showed progressively increased protein leakage compared with NG control cells. At 5 hours, transendothelial passage expressed as percent of tracer appearing in the lower chamber, was $10.6 \pm$ 0.4 vs. $7.8 \pm 0.8(p<0.001)$ for HRP, $7.5 \pm 1.1$ vs. $5.2 \pm 0.6(p<0.001)$ for $B S A$ and $5.4 \pm 1.1$ vs. $3.4 \pm 0.7(p<0.01)$ for $\lg G$, whereas no significant leakage of fibrinogen was observed. DNA synthesis, as assessed by ${ }^{3} \mathrm{H}$-thymidine incorporation, and cell number, as assessed by crystal violet binding to cell nuclei, did not change in response to $H G$, thus ruling out the possibility that the increased permeability observed under these conditions was dependent on reduced cell density. These results show that the diabetic milieu directly impairs retinal endothelial cell barrier function, in addition to exerting an influence via changes in the retinal microcirculation.
MODULATION BY HIGH GIUCOSE AND TNF- $\propto$ OF $p 53$ AND CLUSTERIN MRNA EXPRESSION IN CULTURED ENDOTHELIAL CELLS

S.M. Baumgartner-Parzer, M. Artwohl and W. Waldhäusl. Dept. Med.III, Div. Endocrinology\&Metabolism, Währinger Gürtel 18-20, 1090 Vienna, Austria. The role and mechanisms of high glucose induced apoptosis in endothelial cells is still unknown, but may add to vascular disease in diabetes. This study was thus designed, to evaluate the effect of high ambient glucose and TNF- $\alpha$ on apoptosis in human umbilical vein endothelial cells (HUVECs) using ${ }^{3} \mathrm{H}$ thymidine release assays, in situ staining and FACS-analysis. Furthermore mRNA expression of $p 53$, a tumor suppressor gene involved in the regulation of apoptosis, and clusterin, originally described as marker of apoptosis, were assessed by Northern blot anlalysis. HUVECs were incubated with different concentrations of TNF- $\alpha(130-6600 \mathrm{U} / \mathrm{ml})$ for 4,24 or $48 \mathrm{~h} .{ }^{3} \mathrm{H}$-thymidine release assays revealed the most prominent effects after $24 \mathrm{~h}$ for $1300 \mathrm{U} / \mathrm{ml}$ TNF $-\alpha(124.8 \pm 18 \%$ of control, $p<0.0005, n=13)$. High glucose $(30 \mathrm{mM})$ long term culture $(13 \pm 1$ days increased p $53 \mathrm{mRNA}$ expression to $113 \pm 18 \%$ of control $(p<0.05, n=10)$, i.e. compared to cells in 5 mM glucose. P 53 mRNA upregulation after $24 \mathrm{~h}$ exposure to TNF- $\alpha$ was more pronounced in cells cultured in high glucose $(330 \mathrm{U} / \mathrm{ml}: 126 \pm 21 \%$ of control, $p<0.025, n=7 ; 1300 \mathrm{U} / \mathrm{ml}: 162 \pm 21 \%, p<0.005, n=5)$ than in cells in $5 \mathrm{mM}$ glucose $(330 \mathrm{U} / \mathrm{ml}: 104+25 \%$, $\mathrm{p}=\mathrm{ns}, \mathrm{n}=7: 1300 \mathrm{U} / \mathrm{ml}: 151+35 \%, \mathrm{p}<0.01$, $\mathrm{n}=5$ ) or $30 \mathrm{mM}$ mannose (330 $/ \mathrm{ml}: 118 \pm 18 \%, p<0.05, \mathrm{n}=6 ; 1300 \mathrm{U} / \mathrm{ml}: 128 \pm$ $16 \%, p<0.01, n=5$ ), used as osmotic control. Interestingly, TNF- $\alpha$ (330 and $1300 \mathrm{U} / \mathrm{ml}$ ) induced apoptosis was associated with reduced clusterin mRNA expression. In contrast to $p 53$ that effect was attenuated in cells cultured in high glucose $(330 \mathrm{U} / \mathrm{ml}: 95.5 \pm 13 \%$, of control, $p=n s, n=6)$ compared to cultures in $5 \mathrm{mM}$ glucose $(88.5 \pm 13 \%, p<0.05, \mathrm{n}=6)$ or $30 \mathrm{mM}$ mannose $(80.2 \pm 4 \%, p<0.0005, n=6)$. These data indicate differential regulation by high glucose and TNF- $\alpha$ of apoptosis specific or associated genes in human vascular endothelial cells.

\section{OP 12}

\section{Glycation - induced Tissue Damage}

79

\section{ADVANCED GLYCATION ENDPRODUCTS INDUCE CONTINOUS ACTIVATION OF THE TRANSCRIPTION FACTOR NF-KB} Angelika Bierhaus ${ }^{1}$, Thomas Illmer ${ }^{1}$, Thomas Luther ${ }^{1}$, Hans Tritschler ${ }^{2}$, Martin Müller ${ }^{1}$ and Peter P. Nawroth ${ }^{3} ; 1=$ Dept. of Pathol., TU Dresden, Germany; 2=Asta Medica, Frankfurt, Germany; 3=Dept: of Med. I, Univ. Heidelberg, Germany

Nuclear import of transcription factors belonging to the NF-kB family is controlled by cytoplasmic inhibitory proteins (IKBS). Activation and translocation of NF-KB from the cytoplasm into the nucleus follows a stimulation-dependent release of NF-kB from $1 \mathrm{kB}$. Recently, Advanced Glycation Endproducts (AGEs), supposed to play a major role in late diabetic complications, have been demonstrated to induce cellular oxidant stress resulting in NF-kB activation. To study the mechanism underlying AGE-dependent NF-KB activation, cultured endothelial cells $(E C)$ were incubated in the presence of $500 \mathrm{nM} \mathrm{AGE-}$ albumin. After initial stimulation with AGE-albumin, Western Blots and Electrophoretic Mobility Shift Assays (EMSA) revealed a 6 day lasting translocation of NF-kB-proteins ( $550, p 65, c-R e l$ ) into the nucleus. Translocation of NF- $\mathrm{kB}$ was accompanied by proteolytic degradation of $1 \times B-\alpha$ and $\mid \times B-\beta$. In addition, enhanced proteolytic processing of the NF-kB precursor p105 occured, as evidenced by the appearance of increasing amounts of NF- $\mathrm{KBp50}$ in the nucleus and IKB- $\gamma$ (the cleavage product of $\mathrm{p} 105$ ) in the cytoplasm. Transient transfection assays confirmed, that continuous AGE induced NF-KB activation resulted in the prolonged expression of target genes controlled by NF-KB. Activation of NF-KB was paralleled by an AGEdependent decrease in the level of glutathione, involved in cellular antioxidants defense mechanisms. Consistently, supplementation of the culture medium with the antioxidant thioctic acid reduced the AGE mediated NF-KB activation.

In summary AGEs might contribute to the development of late diabetic complications by inducing long lasting activation of NF- $\mathrm{kB}$, that can be reduced in the presence of thioctic acid.

\section{0}

UP.REGULATION OF MESANGIAL GROWTH FACTOR AND EXTRACELLULAR MATRIX SYNTHESIS BY ADVANCED GLYCATION ENDPRODUCTS VIA A RECEPTOR-MEDIATED MECHANISM

F. Pricci ${ }^{1}$ G. Pugliese ${ }^{1}$ G. Romeo ${ }^{1}$, F. Pugliese ${ }^{1}$, P. Menè ${ }^{1}, S$. Giannini 2, B. Cresci ${ }^{2}, G$. Galli ${ }^{2}$, C.M. Rotella 2 , U. Di Mario ${ }^{3}, \mathrm{H}$. Vlassara 4 Universities of ${ }^{1}$ Rome "La Sapienza", 2 Florence and ${ }^{3}$ RC-Catanzaro, Italy, and ${ }^{4}$ The Picower Institute, Manhasset, New York, USA

Enhanced nonenzymatic glycation with advanced glycosylation endproduct (AGE) formation may participate in the pathogenesis of diabetes-induced glomerular injury by modulating the synthesis, release and action of several growth factors, which in turn could mediate the increased extracelluiar matrix (ECM) and altered cell growth and turnover leading to mesangial expansion. We tested this hypothesis in an in vitro system using human mesangial cells (HMC) grown on (1) nonglycated or native bovine serum albumin (BSA); (2) glycated BSA with AGE formation (BSA-AGE); (3) glycated $B S A$ in which $A G E$ formation was prevented by the use of aminoguamidine (BSA-AM); and (4) BSA-AGE + antibodies directed against the AGE-receptor, OST/ $\alpha$-p60. HMC grown on BSA-AGE showed increased medium content of insulin-like growth factor $(I G F)-I(+78 \%)$ and transforming growth factor $($ TGF $)-\beta(+158 \%)$ and enhanced 1 GF-I $(+99 \%)$, IGF-II $(+76 \%)$ and TGF- $\beta(+174 \%)$ mRNA expression, compared with cells grown on BSA. Moreover, cells grown on BSA-AGE showed increased protein and mRNA levels of the ECM components fibronectin $(+124$ and $155 \%$, respectively) laminin $(+134$ and $142 \%)$ and collagen IV $(+71$ and $97 \%$ ) vs. cells cultured on BSA, whereas cell proliferation was unchanged. Growing cells on BSA-AM did not affect any of the above measurements. Co-incubation of BSA-AGE with $\alpha$-p60 antibody prevented AGE-induced increases in growth factor and ECM production and/or gene expression. These results suggest that mesangial IGF and TGF- $\beta$ synthesis is upregulated by glucose-modified proteins via an AGE-receptor-mediated mechanism. The parallelism with increased ECM production suggests that advanced nonenzymatic glycation may participate in mediating hyperglycemia-induced mesangial expansion. 
81

\section{ADVANCED GLYCATION ENDPRODUCT BINDING IN THE DIABETIC RAT KIDNEY}

S. Youssef. $T$. Soulis, and M.E. Cooper. Department of Medicine, University of Melbourne, Austin \& Repatriation Medical Centre, Repatriation Campus, West Heidelberg, VIC, Australia 3081.

Advanced glycation endproducts (AGEs) have been implicated in the pathogenesis of a number of diabetic complications. Aminoguanidine (AG) has been shown to decrease the accumulation of AGEs in the diabetic kidney. Several binding proteins specific fos AGEs have been isolated from various tissues. This study investigates changes in AGE binding associated with diabetes in the rat kidney using in vitro and in vivo autoradiographic techniques. AGEs (ribonuclease A (RNase) or bovine serum albumin (BSA) with $0.5 \mathrm{M}$ glucose for $4-6$ months) were radiolabelled with 125 I. Male Sprague-Dawley rats were randomised into control (con), con + AG, diabetic (diab) (streptozotocin $55 \mathrm{mg} / \mathrm{kg}$ ) and diab $+\mathrm{AG}$ and sacrificed after 3 weeks. Frozen kidney sections $(20 \mu \mathrm{m})$ were incubated with [ ${ }^{125}$ I]-AGE-RNase or [ $\left.{ }^{125} \mathrm{I}\right]$-AGE-BSA for 2 hours and exposed to X-ray film for 2 weeks. These studies have identified a low affinity binding site specific for AGEs in the kidney $\left(\mathrm{IC}_{50}=0.28 \mu \mathrm{M}\right)$. There was a significant increase in [ $\left.{ }^{125} \mathrm{I}\right]$-AGE binding in the diabetic kidney, which was decreased with $\mathrm{AG}$ treatment (mean data cortex, con, $265 \pm 6$; con $+\mathrm{AG}, 337 \pm 9$; diab, $491 \pm 17^{*}$; diab $+\mathrm{AG}, 290 \pm 8 \mathrm{dpm} / \mathrm{mm}^{2} ;{ }^{*}$ p $<0.01$ vs other groups) without a change in binding affinity. Studies using both $\left[{ }^{125} \mathrm{I}\right]$-AGE-BSA or $\left[{ }^{125} \mathrm{I}\right]-\mathrm{AGE}-$ RNase revealed similar binding characteristics and distribution in the kidney. To localise the AGE binding site, in vivo autoradiography was performed. [ $\left.{ }^{125} \mathrm{I}\right]$-AGEBSA $(15 \mu \mathrm{Ci})$ was injected into the abdominal aorta of the rat. Binding was observed in the renal cortex and was increased in diabetes (con, 565 \pm 50 ; diab, 1448 $\pm 56^{*}$ $\mathrm{dpm} / \mathrm{nm}^{2},{ }^{*} \mathrm{p}<0.01 \mathrm{vs}$ con). Emulsion autoradiography revealed that binding was localised to proximal tubules. Further studies are in progress to identify whether this binding site is receptor for AGE (RAGE), another AGE binding protein or an as yet undefined receptor. The increase in both AGE levels and AGE binding in diabetes may represent an important mechanism in the development of diabetic nephropathy.

\section{3}

\section{REDUCED BIOLOGICAL POTENCY OF MONO-GLYCATED INSULIN IN VITRO AND IN VIVO IN MICE}

A.C. Boyd, F.P.M. O'Harte, A.M. Gray, Y.H.A. Abdel-Wahab, H. McNulty, C.R. Barnett and P.R. Flatt. School of Biomedical Sciences, University of Ulster, Coleraine, BT52 1SA, U.K.

Insulin can be glycated in the pancreas in diabetes. This study investigates effects of glycated insulin on the regulation of glucose homeostasis. Glycated insulin was prepared in vitro under hyperglycaemic conditions in the presence of $\mathrm{NaBH}_{3} \mathrm{CN}(1000$ molar excess) and purified by RP-HPLC. Mass spectrometry confirmed a single glucitol adduct (mono-glycated insulin (MGI), Mw. 5971.8 Da.). Cellular 2-deoxy-D-[1-3H]glucose uptake and D-[U-14C]glucose oxidation activity was assessed in vitro using isolated abdominal muscle from weanling mice. Non-glycated insulin (NGI) stimulated a dose-dependent increase (32-77\%) in 2-deoxy-D- $[1-3 \mathrm{H}]$ glucose uptake at $10^{-9}$ and $10^{-8} \mathrm{~mol} / \mathrm{l}$ above basal (no insulin, $245 \pm 7 \mathrm{dpm} / \mathrm{mg} / \mathrm{hr}$ ). MGI displayed a reduced ability $(\mathrm{p}<0.05, \mathrm{n}=8)$ to stimulate glucose uptake at $10^{-9}$ and $10-8(19-23 \%)$ compared with NGI. MGI exhibited a 19-21\% reduction in $\mathrm{D}-[\mathrm{U}-14 \mathrm{C}]$ glucose oxidation activity compared to NGI at $10^{-8}$ and $10^{-7} \mathrm{~mol} / \mathrm{l}(\mathrm{p}<0.05, \mathrm{n}=12)$. Glucose lowering ability of MGI was examined in mice following intraperitoneal injection with $40 \%$ $(\mathrm{w} / \mathrm{v})$ glucose at a dose of $0.2 \mathrm{U} / \mathrm{ml} / \mathrm{kg}$. After $30 \mathrm{~min}$, injection of glucose alone raised $(\mathrm{p}<0.001)$ plasma glucose by $74 \%$ over basal $(7.5 \pm 0.4 \mathrm{mmol} / \mathrm{l})$. Simultaneous administration of NGI decreased plasma glucose by $24 \%$ to $5.7 \pm 0.2 \mathrm{mmol} / \mathrm{l}$ from basal $(p<0.01$ ). MGI evoked $20 \%$ less glucose lowering activity $(7.1 \pm 0.4 \mathrm{mmol} / 1, \mathrm{p}<0.01$, $\mathrm{n}=6$ ) compared with NGI. Preliminary observations using di-glycated insulin suggest a more pronounced reduction in glucose uptake $(\mathrm{p}<0.01)$. These observations indicate that glycation of insulin may contribute to insulin resistance observed in NIDDM.

\section{2}

EFFECTS OF GLYCATED AND NON-GLYCATED GLP-1[7. 36]AMIDE ON GLUCOSE METABOLISM IN MUSCLE

F.P.M. O'Harte, A.M. Gray, Y.H.A. Abdel-Wahab and P.R. Flatt. School of Biomedical Sciences, University of Ulster, Coleraine, UK.

The insulinotropic action of the gut hormone GLP-1[7-36]amide is well established, but its effects on glucose metabolism in peripheral tissue remain controversial. We examined the action of glycated and nonglycated GLP-1[7-36]amide on glucose uptake and oxidation in isolated muscle. Glycated GLP-1[7-36]amide (Mw. $3463.8 \mathrm{Da}$ ) was prepared under hyperglycaemic conditions in the presence of $\mathrm{NaBH}_{3} \mathrm{CN}(1000$ molar excess) and purified by RP-HPLC. Using isolated abdominal muscle strips from 3-5 weeks old mice, non-glycated GLP-1[736]amide $\left(10^{-10}\right.$ to $\left.10^{-8} \mathrm{~mol} / \mathrm{l}\right)$ stimulated (1.3-1.5 fold) 2-deoxy-D- 1 ${ }^{3} \mathrm{H}$ ]glucose uptake (mean $\pm \mathrm{sem}, 385 \pm 28$ to $449 \pm 37 \mathrm{dpm} / \mathrm{mg}$ tissue/h, $\mathrm{p}<0.001, \mathrm{n}=11$ ) during $30 \mathrm{~min}$ incubations at $30^{\circ} \mathrm{C}$, compared to control incubations without GLP-1[7-36]amide (294 $16 \mathrm{dpm} / \mathrm{mg}$ tissue/ $h, n=15$ ). This effect was reduced by $27-32 \%$ following glycation of the peptide at $10^{-10}$ and $10^{-8} \mathrm{~mol} / \mathrm{l}(267 \pm 22$ and $300 \pm 21 \mathrm{dpm} / \mathrm{mg}$ tissue/h, $\mathrm{p}<0.05, \mathrm{n}=5)$. Non-glycated GLP-1[7-36]amide (10-9 and $10-8 \mathrm{~mol} / \mathrm{l})$ induced a stepwise 1.4 and 1.7 fold increase $(p<0.01$, $\mathrm{p}<0.001$, respectively) in ${ }^{14} \mathrm{C}$-glucose oxidation in muscle $(0.46 \pm 0.04$ and $0.56 \pm 0.07 \mathrm{nmoles} \mathrm{CO}_{2} / \mathrm{mg}$ tissue $/ \mathrm{h}, \mathrm{n}=10$ ), as assessed by ${ }^{14} \mathrm{CO}_{2}$ production at $37^{\circ} \mathrm{C}$, compared to control incubations without GLP-1[736]amide $\left(0.32 \pm 0.02\right.$ nmoles $\mathrm{CO}_{2} / \mathrm{mg}$ tissue/ $\left.\mathrm{h}, \mathrm{n}=15\right)$. The stimulation of glucose oxidation by non-glycated GLP-1[7-36]amide was diminished by $28-32 \%$ following glycation (10-9 and 10-8 mol/l, $\mathrm{p}<0.05, \mathrm{n}=15)$. This study confirms that GLP-1[7-36]amide has a potent effect on glucose metabolism in vitro and that its action is impaired by glycation. The significance of extrapancreatic actions of GLP-1[7-36] amide and its glycation in vivo merit further investigation.

\section{4}

GLYCATION OF GLP-1[7-36]AMIDE BUT NOT GIP Y.H.A. Abdel-Wahab, F.P.M. O'Harte, A.M. Gray and P.R. Flatt. School of Biomedical Sciences, University of Ulster, Coleraine, UK.

Glycation of proteins in NIDDM can result in modification of biological potency. This study investigated the effects of glycation of the incretins GLP-1[7-36] amide and GIP on insulin secretion from glucoseresponsive BRIN-BD11 cells. Glycated GLP-1 and glycated GIP (confirmed by MALDI-MS) purified by RP-HPLC were prepared by incubation under hyperglycaemic conditions with a 1000 -fold molar excess of $\mathrm{NaBH}_{3} \mathrm{CN}$. Acute (20 min) incubations of BRIN-BD11 cells at $5.6 \mathrm{mmol} / \mathrm{l}$ glucose with non-glycated GLP-1 $\left(3 \times 10^{-10}, 10^{-9}\right.$ and $3 \times 10-9 \mathrm{~mol} / \mathrm{l})$ exerted a dose dependent $2.3-3.2$ fold increase of insulin secretion $(\mathrm{p}<0.05, \mathrm{n}=6)$. Non-glycated GIP $\left(3 \times 10^{-10}, 10^{-9}, 3 \times 10^{-9}\right.$ and $10^{-8} \mathrm{~mol} / \mathrm{l}$ ) induced 1.6-2.1 fold increases in insulin secretion but not in a dose dependent manner $\left(p<0.01\right.$ at $3 \times 10^{-10}, p<0.05$ at $10^{-9}$ and $\mathrm{p}<0.01$ at $10^{-8} \mathrm{~mol} / \mathrm{l}, \mathrm{n}=6$ ). The stimulatory action of non-glycated GLP-1 was decreased by $49-54 \%$ following glycation of the peptide $\left(p<0.05\right.$ at $3 \times 10^{-9}$ and $p<0.01$ at $\left.10^{-9} \mathrm{~mol} / \mathrm{l}\right)$. Conversely, glycation of GIP induced a $76-147 \%$ increase in insulin secretion when compared with the non-glycated peptide $\left(\mathrm{p}<0.05\right.$ at $3 \times 10^{-10}, \mathrm{P}<0.001$ at $10^{-9}$ and $3 \times 10^{-9}, \mathrm{P}<0.01$ at $\left.10^{-8} \mathrm{~mol} / \mathrm{l}, \mathrm{n}=6\right)$. Evaluation of the glucose dependency $(0,3,5.6,11.1$ and $16.7 \mathrm{mmol} / \mathrm{l})$ revealed a significant $35-75 \%$ decrease in insulin secretion induced by $10^{-9} \mathrm{~mol} / 1$ glycated GLP-1 at $11.1 \mathrm{mmol} / \mathrm{l}(\mathrm{p}<0.01)$ and $5.6 \mathrm{mmol} / 1(\mathrm{p}<0.001)$ compared with non-glycated GLP- 1 . In contrast, $10^{-8} \mathrm{~mol} / \mathrm{l}$ glycated GIP exerted a $73 \%$ increase in insulin secretion at $11.1 \mathrm{mmol} / 1$ glucose $(\mathrm{p}<0.01$, $\mathrm{n}=6$ ). These data indicate that GLP-1[7-36]amide and GIP can be glycated under hyperglycaemic conditions and that glycation of GLP-1 but not GIP impairs its insulinotropic action. 


\section{Insulin Analogues}

85

POSTPRANDIAL USE OF INSULIN LISPRO: A NEW THERAPEUTIC OPTION IN THE TREATMENT OF TYPE 1 DIABETIC PATIENTS?

G. Schemthaner ${ }^{1}$, W. Wein ${ }^{2}$, K. Sandholzer', S. Equiluz-Bruck' and M. Birkett ${ }^{3}$ Department of Medicine I, Rudolfstiftung Hospital, Vienna, Austria ${ }^{1}$. Eli Lilly, Austria $^{2}$; Eli Lilly Research Center, Erl Wood, UK ${ }^{3}$.

Only few data are available on the appropriate time of pre-prandial injection of regular insulin (reg) and postprandial use of insulin lispro (lis), providing optimal control of post-prandial blood glucose excursion. This randomized multiple cross-over study (latin square design) compared the glucodynamics of pre and postprandial lispro with pre prandial human regular related to start of the meal: regular: $-40 \mathrm{~min} /-20 \mathrm{~min}$ and 0 (start of the meal); lispro $-20 \mathrm{~min} / 0$ (start of meal) and +15 min after start of meal.

18 type I diabetics (HbAlc $\leq 8$, informed consent), were randomized to a sequence of 6 visits. The selected standard meal was identical for all patients and visits. Also insulin doses were identical. Blood glucose excursions 1-hour values in $\mathrm{mmol} / \mathrm{L}$ were:

\begin{tabular}{|c|c|c|c|c|c|}
\hline & $-40 \mathrm{~min}$ & $-20 \mathrm{~min}$. & $0 \mathrm{~min}$. & $+15 \mathrm{~min}$. & \multirow{3}{*}{$\begin{array}{l}\text { 1-hour } \\
\text { values }\end{array}$} \\
\hline Regular & 0.84 & 1.84 & 2.16 & & \\
\hline Lispro & & -1.12 & 0.18 & 2.07 & \\
\hline
\end{tabular}

Values were analysed in a pairwise fashion using the Neuman-Keuls method. 1-hour results: Lispro +15 was not different from reg $-20 \mathrm{~min}$ and reg 0 minutes. Lispro -20 was significantly different from all other groups but caused early hypoglycernia in some patients. Lis 0 and reg -40 were significantly different from reg $-20,0$ and lis +15 .

\begin{tabular}{l|c|c|c|c|c|}
\cline { 2 - 5 } & $-40 \mathrm{~min}$ & $-20 \mathrm{~min}$. & $0 \mathrm{~min}$ & $+15 \mathrm{~min}$. & $\begin{array}{l}\text { 2-hour } \\
\text { values }\end{array}$ \\
\hline Regular & 0.67 & 0.77 & 1.01 & & -0.49 \\
\hline Lispro & & -0.99 & -1.79 & \\
\hline
\end{tabular}

At 2-hour analysis, Lispro +15 values were numerically somewhat lower than al regular groups $(-40,-20,0)$, but not statistically different. Lis -20 and lis 0 were significantly different from all regular groups $(-40,-20,0)$, while lispro groups showed no inbetween differences. This study showed that the optimal time of reg injection is 20 to 40 minutes before the meal. Lispro given 15 minutes after start of the meal has a comparable glucodynamic pattem as regular human insulin given 0 or -20 minutes before the meal. The postprandial use of insulin lispro in type-1 diabetics is an attractive new therapeutic option, however, long term studies enrolling more patients are needed.

\section{6}

IMPROVED BLOOD GLUCOSE CONTROL WITH INSULIN ANALOGUE B28-ASP

P.M. Round ${ }^{1}$, K.J. Olsen' ${ }^{1}$ and P.D. Home ${ }^{2}$ for the B28-ASP UK Study Group. 'Novo Nardisk A/S, Denmark, and ${ }^{2}$ Freeman Diabetes Service, University of Newcastle upon Tyne, UK.

Previous clinical trials have failed to show improvement of overal blood glucose control with short-acting insulin analogues. The present study used dose adjustment guidelines to optimize blood glucose control in 90 Type 1 diabetic patients during 4-week treatment periods with human insulin analogue B28-Asp (B28Asp) compared to human insulin in a randomised double-blind design. Human NPH insulin was used throughout run-in and study periods for overnight insulin delivery. Serum fructosamine did not differ between insulin and analogue regimens (B28-Asp $3.76 \pm$ 0.05 vs insulin $3.82 \pm 0.06 \mathrm{mmol} / \mathrm{l})$ but glucose excursions outside the range $4.0-7.0 \mathrm{mmol} / \mathrm{l}$ were significantly lower with B28-Asp (AUC: B28-Asp $4699 \pm 452$ vs insulin $5275 \pm 346$ $\mathrm{mmol} / \mathrm{min}, \mathrm{p}=0.017$ ). 24 -hour profiles or 8 -point home profiles showed significantly lower glucose excursions after the three main meals by $1.3 \mathrm{mmol} / \mathrm{l}(\mathrm{p}=0.000-0.003)$, without deterioration of control before the next meal. Night-time glucose levels were higher with B28-Asp than human insulin (minima 6.0 \pm 0.5 vs $4.7 \pm 0.3 \mathrm{mmol} / \mathrm{l}, \mathrm{p}=0.021$ ), with no change in $\mathrm{NPH}$ insulin dose in this short study. There were 11 major hypoglycaemic events in the last 2 weeks of the B28-Asp regimen, and 22 on human insulin $(p=0.061)$. We conclude that it may be possible to improve both day and night-time blood glucose control with a B28-Asp insulin regimen; algorithm driven adjustment of night-time NPH doses may allow further improvements in blood glucose control in longer studies.
DURATION OF ACTION OF THE INSULIN ANALOGUE B28ASP IN COMPARISON TO REGULAR INSULIN

C. Weyer, T. Heise, C. Kapitza, A.A.R. Starke and L. Heinemann. Dep. of Metabolic Diseases a. Nutrition, Düsseldorf, Germany

We studied if the insulin analogue (IA) B28Asp besides its more rapid onset of action also had a shorter duration of action than regular human insulin. In order to characterise the complete time-action profile of the $1 \mathrm{~A}$ B28 Asp (Novo Nordisk, Bagsvaerd, Denmark) 24 healthy volunteers received a s.c. injection of $0.2 \mathrm{U} / \mathrm{kg}$ body weight by means of a pen into the abdominal region on one study day. On the second study day of this double-blind study they received an injection of regular human insulin $(R)^{\text {: }}$ Actrapid HM). Blood glucose was kept constant during euglycaemic glucose clamp experiments for 10 hours by means of a Biostator 15.0 mmol/l, baseline i.v. insulin infusion $0.15 \mathrm{mU} / \mathrm{kg} / \mathrm{min}$ ). Glucose infusion rates (GIR) necessary to keep blood glucose constant reflect the metabolic effect of the injected insulin. An exponential function was fitted to the individual GIR profiles in order to evaluate the summary measures. As we have shown in a previous study the half-maximal GIR and maximal $\mathrm{GIR}_{\max }$ were reached earlier and were higher after injection of B28Asp in comparison to Rl learly $t_{50} \% 44 \pm 12$ vs. $65 \pm 16$ min, $\mathrm{p}<0.001 ; \mathrm{t}_{\max } 105 \pm 18$ vs. $148 \pm 27 \mathrm{~min}, \mathrm{p}<0.001 ; \mathrm{GIR}_{\max } 10.2 \pm 2.3$ vs. $8.4 \pm 2.0 \mathrm{mg} / \mathrm{kg} / \mathrm{min}, \mathrm{p}=0.001$; after correction for baseline GIR). But not only the anset of action was more rapid, also the decline in metabolic activity after the maximal effect was more rapid after B28Asp than after RI. The half-maximal metabolic effect after the maximal effect was reached 80 min earlier (late $t_{50} \% 256 \pm 44$ vs. $337 \pm 56 \mathrm{~min}, \mathrm{p}<0.001$ ) After injection of Rl the metabolic effect was above the half-maximal level for $272 \pm 50 \mathrm{~min}$, thus, nearly $60 \mathrm{~min}$ longer than after injection of B28Asp $(213 \pm 42 \mathrm{~min} ; \mathrm{p}<0.001)$. The total area under the GIR profiles (AUC) after injection of B28Asp or RI was comparable (AUC $C_{0-10}$ n $2.48 \pm 0.59$ vs. $2.46 \pm 0.60 \mathrm{~g} / \mathrm{kg} / 600 \mathrm{~min} ; p=0.480$ ). The complete pharmacodynamic profile of IA B28Asp in comparison to RI showed, that it not only has a more rapid onset of action, but also considerably shorter duration of action, whereas the total metabolic effect is comparable.

\section{GLUCOSE TURNOVER PROFILES DURING CONTINUOUS}

INTRAVENOUS INFUSION OF LONG-ACTING INSULIN NN304. M. Hamilton-Wessler, M. Ader, L. Getty, J. Markussen, and R. N. Bergman. Dept. of Physiology \& Biophysics, U. of Southem California, Los Angeles, CA, USA and "Insulin Research, Novo Nordisk A/S, DK-2880 Bagsvaerd, Denmark.

The new prolonged-acting insulin analog, Lys $^{\mathrm{B} 29}$-tetradecanoyl, des(B30) human insulin (NN304), has been previously demonstrated with intravenous bolus injection to have a protracted action profile due to albumin binding in plasma, although specific temporal effects on glucose utilization (Rd) and production (HGO) were not examined. The aim of the present study was to investigate the effects of NN304 on glucose turnover during continuous intravenous hormone infusion. Euglycemic clamps (Hot GINF) with NN304 (3.6 pmol/min $/ \mathrm{kg}$ ) and somatostatin infusion $(0.8 \mu \mathrm{g} / \mathrm{min} / \mathrm{kg})$ for 8 hours were performed in normal dogs $(\mathrm{N}=6)$. Tracer $\left(3{ }^{3} \mathrm{H}-\right.$ glucose $)$ was infused throughout starting at $-120 \mathrm{~min}$. The requisite glucose infusion rate to maintain a mean plasma glucose of $6.4 \pm 0.1 \mathrm{mM}$ reached a steady state (SS) of $43.2 \mu \mathrm{mol} / \mathrm{min} / \mathrm{kg}$ by $310 \pm 8 \mathrm{~min}$. Plasma NN304 concentrations $(N=2)$ reached SS by $90 \mathrm{~min}$, with a halftime to $S S\left(t_{1 / 2}\right)$ of $25 \pm 2$ min. Rd increased significantly from basal $(13.4 \pm 1.1)$ to SS of $43.9 \pm 3.6$ $\mu \mathrm{mol} / \mathrm{min} / \mathrm{kg}$, with a $t_{1,2}$ of $202 \pm 17 \mathrm{~min}$. The $\mathrm{HGO}$ decreased significantly to SS of $0.7 \pm 0.8 \mu \mathrm{mol} / \mathrm{min} / \mathrm{kg}$, with a $\mathrm{t}_{1 / 2}$ of $166 \pm 20 \mathrm{~min}(p<0.001 \mathrm{vs}$. basal for $\mathrm{Rd}$ and $H G O)$. This is compared to native insulin effects with previously reported halftimes of $45 \pm 5 \mathrm{~min}$ for $\mathrm{Rd}$ and $43 \pm 5 \mathrm{~min}$ for HGO. It is notable, however, that the stimulation of $\mathrm{Rd}$ and suppression of $\mathrm{HGO}$ by NN304 followed similar dynamics ( $p=0.2, t_{1 / 2}$ Rd vs. HGO). The suppression of HGO was closely related to the analog-induced decline in plasma FFA from basal $(0.4 \pm 0.1)$ to $\mathrm{SS}$ of $0.1 \pm$ $0.02 \mathrm{mmol} / \mathrm{L}, \mathrm{r}^{2}=0.904$. In conclusion, these data 1 ) support the previously noted protracted action of the insulin analog NN304 and demonstrate similar dynamics for both glucose production and utilization, 2) extend the hypothesis of FFA as the signal for suppression of $\mathrm{HGO}$ to this insulin analog, and 3) show that the delayed insulin analog actions, apart from its protracted absorption, are most likely related to the analog profile in a compartment remote from plasma, i.e. interstitial fluid. 
89

TIME-ACTION PROFILE OF INHALED INSULIN

L. Heinemann, T. Traut and T. Heise; Dep. of Metabolic Diseases a. Nutrition, Düsseldorf, Germany

We studied the pharmacodynamic effect elicited by inhalation of $99 \mathrm{U}$ microcrystalline insulin in comparison to that of $10 \mathrm{U}$ soluble insulin injected subcutaneously and to that of $5 \mathrm{U}$ soluble insulin applied intravenously. The time-action profiles of the three insulin administrations were studied in 12 healthy volunteers lage $25 \pm 2$ years, BMI $22.1 \pm 1.4 \mathrm{~kg} / \mathrm{m}^{2}$ ) using the euglycaemic glucose clamp technique (blood glucose $5.0 \mathrm{mmol} / \mathrm{h}$; continuous i.v. insulin infusion $0.15 \mathrm{mU} / \mathrm{kg} / \mathrm{min}$ ). The insulins were administered to each volunteer on three separate study days in random order by use of a portable inhalation device or a syringe. The metabolic effect of the insulins was assessed as the glucose infusion rate measured during $6 \mathrm{~h}$ after insulin administration. Onset of action after insulin inhalation was substantially more rapid than after subcutaneous insulin injection and half-maximal action was reached earlier $(29 \pm 18$ vs. $52 \pm 13 \mathrm{~min} ; \mathrm{p}=0.0002)$. The maximal glucose infusion rate tended to be lower with the inhaled insulin than with the injected insulin $\left(6.5 \pm 2.5\right.$ vs. $8.7 \pm 2.8 \mathrm{mg} \mathrm{kg}^{-1} \mathrm{~min}^{-1}$; $p=0.053)$ and was reached earlier $(103 \pm 50$ vs. $146 \pm 51 \mathrm{~min}$; $p=0.011)$. The glucose infusion rates in the first $60 \mathrm{~min}$ after inhalation were significantly greater than after injection (area under the glucose infusion rate curve: $218.4 \pm 155.0$ vs. $123.9 \pm 73.1$ $\left.\mathrm{mg} \mathrm{kg} \mathrm{kg}^{-1} 60 \mathrm{~min}^{-1} ; p=0.035\right)$; the total metabolic effect was comparable $\left(1.49 \pm 0.67\right.$ vs. $1.83 \pm 0.50 \mathrm{~g} \mathrm{~kg}^{-1} 360 \mathrm{~min}^{-1}$; $p=0.131$ ). Bioeffectiveness of inhaled insulin was $9.7 \pm 4.0 \%$ vs. intravenous insulin and $15.7 \pm 10.7 \%$ vs. s.c. insulin application. With its rapid onset of action inhaled insulin might have potential for clinical use.

\section{OP 14 \\ Clinical Retinopathy}

\section{0}

NINE-YEAR STATUS OF DIABETIC RETINOPATHY AND PHOTOCOAGULATION IN THE UK PROSPECTIVE DIABETES STUDY.

S.J. Aldington ', I.M. Stratton ${ }^{2}$, R.R. Holman ${ }^{2}$, D.R. Matthews ${ }^{2}$, R.C. Turner ${ }^{2}$ and E.M. Kohner'. Royal Postgraduate Medical School, London UK'; University of Oxford, $\mathrm{UK}^{2}$.

We aimed to determine the status of diabetic retinopathy and retinal photocoagulation in a large group of Type II non-insulin dependent diabetic patients over a nine-year period from diagnosis of diabctes mellitus. Between 1978 and 1991 the UKPDS recruited newly diagnosed NIDD patients (median age 53) in 23 UK centres and randomly allocated them to conventional therapies of diet alone, oral agents or insulin. At entry and triennially thereafter subjects received a full clinical exanination. ophthalmic assessment and (since 1982) retinal photography, assessed using a modified Airlie House scheme. Of 3686 subjects assessed at diagnosis, 3785 at 3 years, 2978 at 6 years and 1561 at 9 years, retinopathy incidence increased from $36.4 \%$ at diagnosis. to $44.9 \%, 53.4 \%$ and $56.8 \%$ respectively. In a cohort of 3007 subjects assessed both at diagnosis and 3 vears, the proportion of subjects with retinopathy of more than just microaneurysms alone (i.e. level $3 \mathrm{I}<3 \mathrm{I}$ or worse) increased from $13.4 \%$ initially to $21.0 \%$ by 3 years. In a diagnosis to 9 -year cohort $(\mathrm{n}=972$ ) the proportion increased from $14.5 \%$ initially to $35.7 \%$ by 9 years. In the 9 -year cohort $596(61.3 \%)$ were initially free of retinopathy of whom $19 \quad(3.2 \%)$ subsequently required photocoagulation, $235(24.2 \%)$ initially had microaneurysms only of whom 19 $(8.1 \%)$ required photocoagulation during the 9 years, whilst of $141(14.5 \%)$ initially having retinopathy of more than microaneurysms alone ( $31<31$ or worse) $71(50.4 \%)$ subsequently received photocoagulation. We conclude that retinopathy status at diagnosis of NIDDM is a risk factor for subsequent progression and requirement for photocoagulation. Such findings may affect recommendations on follow-up and screening intervals in non-insulin dependent diabetes.

\section{1}

RISK FACTORS PREDICTING PROGRESSION OF DIABETIC RETINOPATHY OVER SIX YEARS IN 1579 NIDDM PATIENTS

I M Stratton, E M Kohner, D R Matthews, R R Holman, S Aldington, and R C Turner for UK Prospective Diabetes Study Group, Radcliffe Infirmary, Oxford, OX2 6HE, UK

1579 newly diagnosed patients with NIDDM with retinal photographs at diagnosis and 6 years later, were assessed for development or progression of diabetic retinopathy defined as $\geq 2$ step change in Modified Wisconsin Grading or photocoagulation. Baseline values following a three month dietary run-in, and associations with retinopathy progression when split into thirds are:-

\begin{tabular}{|c|c|c|c|c|c|c|}
\hline & \multicolumn{2}{|c|}{ Baseline } & \multicolumn{4}{|c|}{ Proportion progressing p value } \\
\hline & $\begin{array}{l}\text { Lower } \\
\text { tertile }\end{array}$ & $\begin{array}{l}\text { Higher } \\
\text { tertile }\end{array}$ & $\begin{array}{l}\text { Lowest } \\
\text { group }\end{array}$ & $\begin{array}{l}\text { Middle } \\
\text { group }\end{array}$ & $\begin{array}{l}\text { Highes } \\
\text { group }\end{array}$ & $\begin{array}{l}\text { for } \\
\text { trend }\end{array}$ \\
\hline Age (years) & 50 & 58 & $22 \%$ & $27 \%$ & $29 \%$ & 0.01 \\
\hline Body Mass Index $\left(\mathrm{kg} \mathrm{m}^{-2}\right)$ & 24.7 & 28.9 & $25 \%$ & $29 \%$ & $24 \%$ & n.s. \\
\hline Fasting plasma glucose (mmol I $\left.{ }^{-1}\right)$ & 7.2 & 9.0 & $12 \%$ & $22 \%$ & $43 \%$ & $<0.001$ \\
\hline $\mathrm{HbAlc}(\%)$ & 6.2 & 7.6 & $13 \%$ & $26 \%$ & $38 \%$ & $<0.001$ \\
\hline Fasting plasma insulin (mmol l-1) & 9.8 & 15.8 & $27 \%$ & $27 \%$ & $24 \%$ & n.s. \\
\hline Total cholesterol $\left(\mathrm{mmol} 1^{-1}\right)$ & 4.8 & 5.7 & $27 \%$ & $27 \%$ & $24 \%$ & n.s. \\
\hline LDL cholesterol (mmol 1 ${ }^{-1}$ ) & 2.96 & 3.87 & $25 \%$ & $29 \%$ & $25 \%$ & n.s. \\
\hline HDL cholesterol (mmol I $\left.{ }^{-1}\right)$ & 0.96 & 1.16 & $24 \%$ & $24 \%$ & $30 \%$ & 0.02 \\
\hline Systolic BP(mm Hg) & 125 & 143 & $20 \%$ & $27 \%$ & $31 \%$ & $<0.001$ \\
\hline Diastolic BP (mm Hg) & 79 & 87 & $23 \%$ & $24 \%$ & $31 \%$ & 0.004 \\
\hline Beta-cell function $(\% \beta)$ & 51 & 84 & $41 \%$ & $24 \%$ & $13 \%$ & $<0.001$ \\
\hline Insulin sensitivity (\%s) & 22 & 34 & $26 \%$ & $27 \%$ & $25 \%$ & n.s. \\
\hline
\end{tabular}

In multivariate logistic regression analyses the associations with fasting plasma glucose, systolic BP, HbAIc and beta-cell function remained significant. In conclusion, development of diabetic retinopathy in newly diagnosed NIDDM patients was associated with raised fasting plasma glucose, systolic blood pressure and $\mathrm{HbAlc}$ and with reduced beta-cell function. 
92

SHORT DURATION OF IDDM WITH MICROANGIOPATHY VS LONG DURATION WTTHOUT. RISK DETERMINANTS AND PROTECTIVE FACTORS.

B. Karamanos ${ }^{1}$, J.H. Fuller ${ }^{2}$, L.K. Stevens ${ }^{2}$ and the Eurodiab Study Group. Diahetes Center ${ }^{1}$, Hippokration Hospital, Athens, Greece, University College London? ${ }^{2}$ UK.

In IDDM patients glycaemic control and diabetes duration (DD) are major, but not the only, determinants of microangiopathy. To isolate other factors related with the development of complications or the protection from them, we compared IDDM patients with short DD and microangiopathy to others with long DD and no microangiopathy. In the Eurodiab Study a total of 3250 IDDM patients recruited from 31 European Centers were studied. From 300 patients with $\mathrm{DD}<6$, mean 3.2 years, $75(25 \%)$ had microangiopathy (Microalbuminuria and/or Retinopathy), and formed Group A, while from 1062 with DD $>14$, mean 19.5 years, $190(17.9 \%)$ had not microangiopathy and formed Group B. Group A compared to B, after age-adjustment, did not differ in BMI, 23.5vs23.2, Waist/Hip ratio, .85vs.82, Diastolic Blood Pressure, $76 v 573 \mathrm{mmHg}$ and HDL, $1.52 \mathrm{vs} 1.52 \mathrm{mmol} / 1, \mathrm{p}>.005$. Group A had higher HbAlc, 6.84vs6.07\%, Cholesterol, $5.5 \mathrm{vs} 5.1 \mathrm{mmol} / \mathrm{l}$, Triglycerides, $1.10 \mathrm{vs} 0.83 \mathrm{mmol} / 1, \mathrm{p}<.001$, LDL, $3.57 \mathrm{vs} 3.15 \mathrm{mmol} / 1$, von Willebrand Factor, 1.24vs1.04U/ml and Systolic BP(SBP), 121vs117, $p<.05$. Hypertension prevalence was, $9.3 \mathrm{vs5} .3 \%$, family history of Hypertension, $49 \mathrm{vs} 48 \%$ and family history of Diabetes, $22 \mathrm{vs} 21 \%$, not different between Groups A and B, while Group A had higher prevalence of Cardiovascular Disease, 14,7vs6.4\%, Smoking 42.7vs28.4\%, $p<.05$ and fewer Severe Hypoglyeaemic Episodes, $18.7 v s 37.9 \%, p<.01$. Conclusions: In IDDM patients: a) Microangiopathy is frequent (25\%), even with short DD, but also spares $20 \%$ of the patients after 20 years of diabetes b) Apart from glycaemic control, an atherogenic lipid profile, high SBP and endothelial dysfunction predispose to early microangiopathy, while when absent protect from it, even with long DD c) Smoking, an easy to abolish factor, is also important for the early development of microangiopathy.
93

High platelet derived growth factor $A B$ (PDGFAB) concentrations in vitreous fluid of patients with proliferative diabetic retinopathy are not due to vitreous bleeding.

H. Freyberger, E. Schifferdecker, R. Effert* , H. Yakut*, H. Schatz, K.-M. Derwahl // Med. Universitätsklinik Bergmannsheil Bochum, * Universitätsaugenklinik Essen

Introduction: As PDGF is presumed to play an important role in the pathogenesis of proliferative diabetic retinopathy, we continued a previously reported study measuring PDGFAB in the vitreous of patients with proliferative diabetic retinopathy, ischemic non-diabetic proliferative retinopathy and controls. Simultaneously we measured platelet factor IV as a stable thrombocyte marker in order to elucidate whether PDGFAB is produced by ocular cells or is due to vitreous bleeding.

Methods: In $0,5 \mathrm{ml}$ of native vitreous fluid obtained by vitrectomy from 23 patients with proliferative diabetic retinopathy, 4 of them with additional rubeosis iridis, 19 controls without retinal diseases and 4 patients with ischemic non-diabetic proliferative retinopathy we measured PDGFAB concentrations by Elisa-Biotracks RPN2162, simultaneously, platelet factor IV using a commercially available Elisa (Behring Enzygnost PF4).

Results: PDGFAB concentrations in proliferative diabetic retinopathy ranged from 32 to $290 \mathrm{pg} / \mathrm{ml}$, in additional rubeosis iridis from 140 and 280 $\mathrm{pg} / \mathrm{ml}$ (mean 100,05 $\pm 60,9$ ), in controls from 30 and $70 \mathrm{pg} / \mathrm{ml}$ (mean $41,7 \pm 15,8)(\mathrm{p}<0,05)$, in ischemic non-diabetic proliferative retinopathy from 50 to $170 \mathrm{pg} / \mathrm{ml}$. Platelet factor IV was not measurable in either of the samples.

Discussion: As platelet factor IV as a stable thrombocyte marker was not detectable in the vitreous of patients with proliferative diabetic retinopathy with higher PDGFAB levels compared to controls one might conclude that PDGFAB is derived from ocular tissue rather than from thrombocytes of vitreous bleeding

\section{4}

PLASMINOGEN ACTIVATOR INHIBITOR (PAI-1) ACTIVITY, ITS PROMOTER (4G/5G) GENOTYPE AND DIABETIC RETINOPATHY IN SUBJECTS WITH NIDDM.

Nagi DK, McCormack LJ", Mohamed-Ali $V^{b}$, Yudkin JS ${ }^{b}$, Grant $P J^{a}$ Knowler WC. NIH, Phoenix, USA. 'Diabetes and Thrombosis Research Group, Division of Medicine, University of Leeds and 'Department of Medicine, UCLMS, London, UK.

Disturbed fibrinolysis due to high levels of PAI- 1 is associated with NIDDM and macrovasular disease. Previous studies examining the relationship of fibrinolysis and diabetic microvascular disease have been inconclusive. To examine the relationship between fibrinolysis and diabetic retinopathy we examined 172 Pima Indians with NIDDM aged between $30-70$ years. PAI- 1 activity was measured by a chromogenic substrate method and PAI-1 $4 G / 5 G$ genotype was established using the allele specific primers and amplification of genomic DNA using the polymerase chain reaction. Retinopathy was asessed by ophthalmoscopy after pupillary dilation and classified as non-proliferative and proliferative. Any retinopathy was present in $70(41 \%)$ of subjects and $4(2.3 \%)$ subjects had proliferative retinopathy. PAl-1 activity was similar between subjects with and without retinopathy $(17.1 \pm 9.3$ vs $19.7 \pm 9.1 \mathrm{AU} / \mathrm{ml}, \mathrm{p}=0.09)$. PAl-1 activity was negatively correlated with duration of diabetes $\left(r_{s}=-0.18, p=0.001\right)$. In a logistic regression analysis controlled for age, sex, BMl, and duration of diabetes, retinopathy was significantly associated with fasting plasma glucose $(p<0.05)$ and $\mathrm{HbA} 1 \mathrm{c}(\mathrm{p}=0.008)$, but not with PAl-1 activity $(p=0.48)$. The prevalence of retinopathy in subjects with $4 \mathrm{G} / 4 \mathrm{G}, 4 \mathrm{G} / 5 \mathrm{G}$ and $5 \mathrm{G} / 5 \mathrm{G}$ genotype was $44 \%, 49 \%$ and $24 \%\left(x^{2}=8.2, \mathrm{df}=2, \mathrm{p}=0.016\right)$. These preliminary data indicate that subjects with $5 \mathrm{G} / 5 \mathrm{G}$ may be protected against diabetic retinopathy and that diabetic retinopathy in Pima Indians with NIDDM is not associated with high levels of PAI-1 activity and hence impaired systemic fibrinolysis. 
OP 15

\section{Clinical Immunology}

\section{5}

CAN BETA-CELL AUTOIMMUNITY BE INDUCED PRENATALLY?

A. Martikainen, P. Kulmala, P. Koskela, M. Knip, H.K. Akerblom and the Finnish TRIGR Study Group. Department of Pediatrics, University of Oulu, Oulu, the National Public Health Institute, Oulu, and the Children's Hospital, II Department of Pediatrics, University of Helsinki, Helsinki, Finland

We studied 104 mothers and their newborn infants from IDDM families in order to investigate the pattern of IDDM associated antibodies during pregnancy and to explore whether there are any signs of prenatal induction of beta-cell autoimmunity. Thirty-nine of the mothers, 45 of the fathers and 20 of the siblings had IDDM. Blood samples were obtained from the mother at the end of the first trimester and at the delivery, and in the newborn infant from cord blood. Nineteen (49\%) out of the 39 mothers with IDDM tested positive for ICA, $18(46 \%)$ had IA, while $22(60 \%)$ had detectable GADA at the end of the first trimester. The corresponding frequencies in nondiabetic mothers were $6 \%$ (4/65; $p<0.001), 0 \%(0 / 65 ; p<0.001)$ and $8 \%(5 / 65 ; p<0.001)$. Two mothers with IDDM turned negative for ICA during pregnancy, whereas two other mothers seroconverted to GADA-positivity. Eight mothers with IDDM became positive for IA during pregnancy, while one turned negative. Four nondiabetic mothers turned positive for $\mid A$ during pregnancy. A significant increase was seen in the IA levels during pregnancy from an initial median of $42 \mathrm{nU} / \mathrm{ml}$ to $89 \mathrm{nU} / \mathrm{ml}$ at delivery $(p<0.001)$. Three mothers, out of whom only one had IDDM became negative for GADA during pregnancy. Sixteen (41\%) infants of mothers with IDDM had ICA in their cord blood, $17(44 \%)$ were positive for $\mid A$ and $23(62 \%)$ for GADA. Five $(8 \%)$ infants with a nondiabetic mother had ICA, two $(5 \%)$ had IA and the same number GADA. There was a strong correlation between the ICA levels in the infants and those in the mothers $\left(r_{s}=0.98 ; p<0.001\right)$ and a somewhat weaker one for $I A\left(r_{s}=0.57 ; p<0.001\right)$ and GADA levels $\left(r_{s}=0.54 ; p<0.001\right)$. Altogether there were three infants testing positive for IAA and two infants positive for GADA without detectable maternal antibodies. Two had a father and three had a mother with IDDM. One of those with an affected father had ICA in cord blood, and his mother seroconverted to ICA positivity during pregnancy, and another with an affected mother had GADA in cord blood, and GADA appeared into the maternal circulation during pregnancy. These observations indicate that signs of beta-cell autoimmunity may be induced already during fetal life.

\section{7}

CELIAC DISEASE AND INSULIN-DEPENDENT DIABETES MELLITUS: A MULTICENTRIC STUDY: P.Cotroneo, I.De Vitis,, S.Addesa., G.Ghirlanda: coordinators. Partecipants:. A Manto, MR Pasqualetti, G Marietti*, Medicina Interna e Geriatria, * Pediatria, Univ. Cattolica, Roma; E Cardi, G Multari., MB Barbato., G Argo, Pediatria, Univ La Sapienza, Roma; G.Bottaro, N.Rotolo, M.Spina, Pediatria, Univ Catania; L.Cavallo, M Baldassarre, Pediatria, Bari ; S Bacci, M Liuzzi, Endocrinologia, IRCSS, S.Giovanni Rotondo, Italy.

In a previuos study we found that the prevalence of Celiac diasease (CD) in IDDM results higher than that reported in literature $(10 \%)$, either in Italy $(2.6 \%)$ than in Northern Europe $(4.1 \%)$. Aim of the present study was to verify the prevalence of $C D$ in italian diabetic population. 639 IDDM patients were screened for $C D$ using antigliadin (AGA) and antiendomisium antibodies(AEA). All positive patients underwent endoscopic biopsy of descending duodenum in order to verify the presence of villous atrophy. All these were considered celiacs and strated gluten-free diet and clinical follow-up. Statistical analysis was performed using Student T-test and Logistic Regression. 639 patients entered in the study. Positivity for the screening test was found in 101 cases $(15.8 \%) ; 36 / 101$ positive patients refused biopsy. 65 patients underwent endoscopy and in 50 of these villous atrophy was presented $(7.82 \%) .15$ subjects, positive for the serological screening $(10$ for AGA and 5 for both AGA and AEA) did not show villous atrophy $(2.2 \%) ; 1$ of these who repeated the biopsy one year later showed histologic signs of $\mathrm{CD}$. Classical signs of malabsorption were present in 15 patients, while 11 were asymptomatic. A significative correlation was found between the presence of villous atrophy and the duration of the earlier onset of IDDM. The overall prevalence of $C D$ in IDDM is higher than previously reported. Screening for $C D$ is mandatory in IDDM because of the frequent association between this two disorders and the increased risk of malignancy. Since the duration of IDDM is related with higher prevalence of $C D$, we suggest to performe serological tests in IDDM patients. AGA and AEA positive patients without villous atrophy could be in a "latent" phase of $\mathrm{CD}$.

\section{6}

Antibodies to IA-2 in IDDM, Stiff Man Syndrome and Autoimmune Endocrine Diseases: Evidence of High Specificity for IDDM N.G. Morgenthaler ${ }^{1}$, J. Seissler ${ }^{1}$, P. Achenbach ${ }^{1}$, D. Glawe ${ }^{1}$, M. Payton ${ }^{2}, H .-$ M. Meinck ${ }^{3}$, M.R. Christie ${ }^{2}$ and W.A. Scherbaum ${ }^{1}$. Department of internal Medicine [II. University of Leipzig. Germany, ${ }^{2}$ Department of Medicine, King's College School of Medicine, London, UK, ${ }^{3}$ Department of Neurology, University of Heidelberg, Germany.

Antibodies to the $40 \mathrm{kD}$ antigen (identified as tyrosine phosphatase IA-2) and glutamate decarboxylase (GAD65) are strongly associated with insulin dependent diabetes mellitus (IDDM). However, antibodies to GAD (GADA) can appear in the absence of IDDM, particularly in stiff man syndrome (SMS) and in some individuals with autoimmume polyendocine syndrome type 11 (APS II) and other organ specific autoimmune disease. The aim of this study was to compare the specificity of $\mid A-2$ antibodies (IA-2A) and GADA for IDDM by determining their frequency in different patient groups using in vitro translated ${ }^{35} \mathrm{~S}$-methionine labelled recombinant antigens in a radioligand assay on 96-well plates. IA-2A were present in 52/107 (49\%) IDDM patients, $9 / 19(47 \%)$ APS $\|$ patients with additional IDDM but in only $2 / 24(8 \%)$ SMS patients. $1 / 24(4 \%)$ APS II patient without IDDM and $1 / 113$ $(0.9 \%)$ patient with organ specific autoimmune disease had low level IA-2A. In contrast GADA were present in $73 / 107(68 \%)$ IDDM patients and $17 / 19$ (89\%) APS II patients with IDDM, but also in 20/24 (83\%) SMS patiens, 5/24 (21\%) APS II patients without IDDM and $22 / 113(19 \%)$ patients with organ specific autoimmune disease. The mean level of IA-2A was highest in the IDDM group whereas the mean GADA level was highest in the SMS group. Our results demonstrate that IA-2 is a major target antigen in IDDM. IA-2A may be more specific for IDDM than GADA, as the latter are also present in SMS, APS II without IDDM and organ specific autoimmune diseases. In this study $\mid A-2$ antibodies alone had a lower sensitivity for IDDM than GADA or ICA, but a combination of IA-2A and GADA proved to be a screening assay with high sensitivity and specificity. Using this approach, we detected $95 \%$ of IDDM patients with ICA levels $\geq 20$ JDF-U. This combined screening is a simple and sensitive method which may be of substantial benefit for population screening.

\section{8}

SYNCHRONOUS DECLINE OF SERUM SOLLBLE HLA CLASS I ANTIGEN AND B-CELL FUNCTION IN IDDM

K. Nakanishi, T. Kobayashi, N. Kogawa* and Y. Komatsu*. Toranomon Hospital, Tokyo, Japan; *Japan Energy Co., Saitama, Japan

Serum soluble HLA class I molecules (sHLA) have immunomodulatory functions and their serum levels correlate with the HLA class I phenotype We studied longitudinal changes of serum sHLA levels in insulin-

dependent diabetes mellitus (IDDM) in relation to autoantibodies (ICA and $\mathrm{GAD} A \mathrm{~b}$ ) and residual $\boldsymbol{B}$-cell function. A total of 191 serum samples were obtained from 40 IDDM patients (M/F: 19/21, mean [-SE] age at the onset $36 \pm 2$ vears) before and after the initiation of insulin therapy. sHLA was assayed by a sandwich ELISA. Residual B-cell function was assessed by the serum CPR response to a $100-\mathrm{g}$ oral glucose load. Serum silLA levels in IDDM patients at the initiation of insulin therapy (IDDM onset) were markedly reduced compared to those of normal controls (332.1 \pm 26.8 $\mathrm{ng} / \mathrm{ml}$ vs. $486.5 \pm 57.0 \mathrm{ng} / \mathrm{ml}, \mathrm{p}=0.0058$ ). They fell sharply during 6 months before and after the onset of IDDM (from 531.7 \pm 67.0 to $334.0 \pm$ $27.5 \mathrm{ng} / \mathrm{ml}, \mathrm{p}=0.028$ ) and remained low until 5 years later. After 6 years of IDDM onset, sHLA values again rose $(471.9 \pm 47.9 \mathrm{ng} / \mathrm{ml}, \mathrm{p}=0.013)$. This dynamic profile of sHLA and time course of B-cell loss were different between IDDM patients with and without HLA-A24. In those with HLAA24, sHLA levels were low for 2 years from the onset. Thereafter, they rose promptly and recovered at 3 years from the onset. In those without HLAA24, sHLA levels continued to decrease until 4 years after the onset and began to rise gradually thereafter. An early (within 18 months), complete loss of B-cell function occurred in 5 of 13 IDDM patients with HLA-A24 compared with 1 of 13 those without HLA-A24 $(\mathrm{p}=0.08)$. A late (more than 36 months after IDDM onset), complete loss of $B$-cell function occurred in 7 of 13 IDDM patients without HLA-A24 but in none of 13 those with HLA-A24 $(\mathrm{p}=0.0026)$. The positivities of ICA and GAD Ab were not different between IDDM patients with and without HLA-A24. These results indicate that the decline of SHLA is synchronous with the massive $B$-cell destruction, and that these events occur in acute phase in IDDM patients with HLA-A24, but in long period in those without HLAA.24. 
99

GAD-PEPTIDE INCREASES MRNA EXPRESSION FOR CYTOKINES IN MONONUCLEAR CELLS OF CHILDREN WITH IDDM. M. Karlsson and J. Ludvigsson. Department of Pediatrics, Faculty of Health Sciences, Linköping, Sweder

Insulin-Dependent Diabetes Mellitus (IDDM) is thought to be caused by an autoimmune destruction of the pancreatic $\beta$-cells. Antigens are presented to Thlymphocytes. When activated Th1- and Th2-lymphocytes produce different types of cytokines, Interferon- $\gamma$ (INF- $\gamma$ ) and Interleukin-4 (IL-4). Glutamic Acid Decarboxylase (GAD) is an autoantigen acting as a target: One major determinant of GAD recognised by persons at risk for IDDM is amino acids 247-279, a region which has significant sequence similarity to the P2-C protein of Coxsackie B virus, a virus that has been associated with IDDM both in humans and in experimental animals. The aim of the study was to elaborate a method for determination of messenger-RNA (mRNA) for IL -4 and INF- $\gamma$ to make it possible to identify if Th 1 and/or Th2 are activated by the Coxsackie-virus associated GADpeptide. Human peripheral blood mononuclear cells (PBMC) from healthy donors were isolated by Ficoll Paque and stimulated with tetanus toxoid. PBMC, from children with newly diagnosed IDDM, were stimulated with the specific GADpeptide. The first-strand $\mathrm{CDNA}$, achieved by reversed transcription, was diluted in different concentration. Reversed transcriptase coupled to the polymerase chain reaction (RT-PCR) was used to give a semi-quantitative mRNA expression of IL-4 and INF- $\gamma$.

The expression of mRNA for IL-4, and to a less degree INF- $\gamma$, seems to be semiquantitatively stronger in lymphocytes stimulated with tetanus toxoid than in lymphocytes incubated without antigen. Lymphocytes from children with IDDM, stimulated with the peptide of GAD known to have similar aminoacid sequence as Coxsackie B virus, shows increased INF- $\gamma$ mRNA expression compared with unstimulated lymphocytes from the same IDDM patients, whereas IL-4 shows a less pronounced but constant mRNA expression in both stimulated and unstimulated lymphocytes. Conclusion: Increased mRNA for INF- $\gamma$ in lymphocytes stimulated with a GAD-peptide suggests that Th1-cells play a role in the cellmediated response.

\section{OP 16 \\ Health Care}

100

INTERNATIONAL SURVEY ON HbA $\mathrm{A}_{\mathrm{Ic}}$ AND INSULIN TREATMENT IN CHILDHOOD DIABETES

H.B. Mortensen and P. Hougaard for the Hvidøre Study Group on Childhood Diabetes, Copenhagen, Denmark

The study was a cross-sectional investigation with participation of 22 pediatric departments, representing 18 countries in Europe, Japan and North America. The aim of the study was to evaluate the present level of metabolic control in children and adolescents with IDDM. Blood samples and information were collected March-August 1995 for all children with IDDM born 1977 or later. $\mathrm{The} \mathrm{HbA}_{\mathrm{c}}$ concentration was determined once and analyzed centrally. Normal range (2SD) is 4.4-6.3 (mean 5.4)\%. Further age, sex, duration of diabetes, height, body weight, number of daily insulin injections, types of insulin and insulin dosage were recorded. Reports from 2873 children were received. The $\mathrm{HbA}_{1}$ value for the population was $8.6 \pm 1.7 \%$ of total haemoglobin (mean \pm $\mathrm{SD}$ ). The age specific mean $\mathrm{HbA}_{4}$ values increased from around $12 \mathrm{yr}$ for girls and around 14-15 yrs for bovs. Adolescent girls (11-18 yrs, $\mathrm{n}=972)$ had significantly higher $\mathrm{HbA}_{1 \mathrm{c}}$ level $(9.1 \pm 1.9 \%)$ than adolescent boys $(8.7 \pm 1.7 \%$, $n=930, p<0.001$ ). The average insulin dose was constant at approximately 0.65 $\mathrm{U} / \mathrm{kg} / 24 \mathrm{~h}$ between 2 and $9 \mathrm{yrs}$ for both sexes, but there was a sharp increase during the pubertal years. The highest mean insulin dose for the girls was 0.98 $\pm 0.37 \mathrm{U} / \mathrm{kg} / 24 \mathrm{~h}$ at age $14 \mathrm{yrs}$, after which the dose plateaued and gradually decreased to $0.89 \pm 0.25 \mathrm{U} / \mathrm{kg} / 24 \mathrm{~h}(\mathrm{n}=77)$ at age 18. The mean insulin dose of the boys increased gradually between age 11 and $17 \mathrm{yrs}$ to $0.98 \pm 0.24 \mathrm{U} / \mathrm{kg} / 24 \mathrm{~h}$ and ending at $0.95 \pm 0.23 \mathrm{U} / \mathrm{kg} / 24 \mathrm{~h}$ at $18 \mathrm{yr}(\mathrm{n}=85)$. Insulin requirements and $\mathrm{HbA}$ were lower in patients with a diabetes duration less than 3 yrs probably due to a proportion having some residual \&-cell function. Sixty percent of the children $(n=1707)$ had two injections daily while a third $(n=1071)$ were on three or more injections daily. Thirty-seven per cent of the children on twiceand three times daily injections received a premixed form of insulin either alone or in combination with rapid acting and intermediate insulin. $\mathrm{Hb} \mathrm{A}_{1 \mathrm{c}}$ and insulin requirements increase during maturation of boys and particularly of girls with IDDM. Current treatment of childhood diabetes is inadequate despite the increased usage of intensified insulin treatment regimens in the paediatric centres involved across the world.
101

THE GLYCEMIC CONTROL IN PATIENTS WITH DIABETES IN FINLAND T. Valle', A. Reunanen ${ }^{1}$, V. Koivisto ${ }^{2}$. T. Kangas ${ }^{3}$ and A. Rissanen ${ }^{4}$. 'National Public Health Institute, ${ }^{2}$ Department of Medicine, Helsinki University Hospital, ${ }^{3} \mathrm{C}$ City of Helsinki, ${ }^{4}$ National Research and Development Centre for Welfare and Health, Helsinki, Finland.

In order to evaluate the quality of diabetes care in a national level, we studied the glycemic controf of Finnish diabetic patients aged over 15 years in 76 randomly selected clinics (59 primary care units, 17 hospitals) covering the whole of Finland. Physicians and diabetes nurses were asked to fill in a questionnaire based on the medical records in 1993 from a random sample of 50 (total $n=3800$ ) patients with diabetes. The response rate was $84 \%, 48 \%$ of the patients were mate. The mean age was 58.4 years (SD 16.7) and the mean duration of diabetes 11.3 years. The mean of body mass incex $(\mathrm{BM})$ was $27.9 \mathrm{~kg} / \mathrm{m}^{2} .13 \%$ of the patients were treated with diet only, $37 \%$ with oral hypoglycemic agents (OHA) alone, $39 \%$ with insulin alone, and $11 \%$ with a combination of $\mathrm{OHA}$ and insulin. Glycosylated hemoglobin had been measured in $67 \%$ of the patients. Only HBATc measurements with the normal method range as $4-6 \%$ were accepted. The mean of $\mathrm{HbA1c}$ was $8.61 \%(95 \% \mathrm{Cl} 8.53-8.69)$. $25 \%$ of the patients had $\mathrm{HbA} 1 \mathrm{c} 7.3 \%$ or lower while $25 \%$ had $\mathrm{HbA} 1 \mathrm{c} 9.7 \%$ or higher The mean value in diet treated diabetics was $6.45 \%(6.23-6.66)$. Diabetics who were treated with OHA alone had a mean value of $8.32 \%(8.18-8.47)$ and those with insulin alone $8.79 \%(8.68-8.90)$. Diabetics treated with the combination of $\mathrm{OHA}$ and insulin had the poorest glycemic controt - 9.45\% (9.21-9.70). Patients classified as type 2 diabetics (age-of-onset at 40 years or older) had a better metabolic control ( $\mathrm{HOA} 1 \mathrm{C}$ $8.52 \%, 8.41-8.63$ ) than type 1 diabetics (age-of-onset under 30 years) whose mean $\mathrm{HbA1c}$ value was $8.79 \%$ (8.64-8.94). There was no difference in the HbA1c level between sexes in type 1 diabetics. However, male type 2 diabetics had a better glycemic control than females $(8.26 \%$ vs. $8.77 \%, p<0.0001)$. This was partly due to the fact that male type 2 diabetics (mean of BMI $28.6 \mathrm{~kg} / \mathrm{m}^{2}$ ) were leaner than female patients $\left(\mathrm{BMI} 29.3 \mathrm{~kg} / \mathrm{m}^{2}\right)$. The difference of $\mathrm{HbA} 1 \mathrm{c}$ level between sexes was consistent in each treatment group. There was also a large regional variation in provinces $(n=11)$ ranging from $7.72 \%(7.40-8.04)$ to $9.22 \%(8.94-9.50)$. In conclusion, in spite of the well-organized diabetes care in Finland there is still a vast group of patients with a poor glycemic control and a large regional variation. 


\section{2}

POOR METABOLIC CONTROL IN 91 IMMIGRANTS AS COMPARED TO 804 GERMAN CHILDREN AND ADOLESCENTS WITH IDDM.

R.W. Holl, W. Hecker, B. Bartus, E. Heinze, M. Holder and W.M. Teller. Depart. Pediatrics I, Univ. of Ulm and Children's Hospital, Stuttgart, Germany The identification of factors associated with insufficient metabolic control in IDDM children and adolescents allows to target health care ressources in order to improve the longterm outcome. In France, î̉imitigrants have been identified as a high-risk group with specific problems. Using an electronic data collection system, $\mathrm{HbA}_{\mathrm{tc}}$-values were evaluated in $\mathbf{8 9 8}$ children and adolescents treated at two adjacent pediatric diabetes centers. Patients were stratified according to national descent: 91 patients who had immigrated into Germany were compared to 804 German patients. Neither the age at diagnosis $(7.7 \pm 0.2$ versus $8.2 \pm 0.4$ years) nor the gender ratio ( $\delta / q: 46 / 45$ versus $398 / 406$ ) differed significantly between German and foreign patients. Even during the first year, $\mathbf{H b A}_{\mathbf{~} \mathrm{c}}$ was significantly higher in foreign $(7.7 \pm 0.2 \%)$ compared to German patients $(7.3 \pm 0.1 \% ; \mathrm{p}<0.02)$. With the end of remission, a progressive rise in $\mathrm{HbA}_{\mathrm{Ic}^{-}}$ levels after 2 or more years of diabetes was observed. In foreign patients, the remission phase was shorter and less pronounced, while the subsequent rise in $\mathbf{H b A}_{\mathrm{Ic}}$ was markedly increased: After 5 years of diabetes, $\mathbf{H b A}_{\mathrm{lc}}$ averaged $8.5 \pm 0.4 \%$ in foreign compared to $7.2 \pm 0.1 \%$ in German patients $(\mathrm{p}<0.001)$. In patients beyond the remission phase (duration of diabetes $>2$ years), metabolic control was related to age: In German patients, $\mathbf{H b A}_{1 \mathrm{c}}$ was significantly higher in pubertal (11-15 years: $7.41 \pm 0.05 \%)$ compared to prepubertal $(<11$ years: $6.85 \pm 0.06 \%$ ) or postpubertal children ( $>15$ years: $\mathbf{7 . 3} \pm \mathbf{0 . 0 4} \%$ ). In foreign children, this difference was dramatically increased: prepubertal: $7.6 \pm 0.2 \%$, intrapubertal $10.5 \pm 1.4 \%$, postpubertal $8.4 \pm 0.25 \%$ $(\mathrm{p}<0.0001)$. Conclusion: Foreign children and adolescents display significantly higher $\mathbf{H b A}_{1 \mathrm{c}}$-values compared to German patients, especially during puberty. Education and treatment regimens have to be specifically tailored to foreign patients in order to achieve better control and prevent late complications.
103

Continuous quality management of structured treatment and teaching programmes for type-1-diabetes on the national level in Germany

U.A.Müller, ${ }^{*}$, K.M.Reinauer ${ }^{5}$ and M.Voss ${ }^{+}$for the German Working Group for Structured Diabetes Care (ASD) of the German Diabetes Association (GDA). "Dept. Internal Medicine II, Friedrich-Schiller-UniverAssociation (GDA). Dept. Internal Medicine 11 , Friedrich-Schiller-Univer-
sität Jena, Städisches Krankenhaus Sindelfingen, ${ }^{+}$Mathias-Spital Rheine; Germany.

Aim: In order to assure the quality management of treatment and teaching centers for type 1 diabetic patients on the national level a quality circle (ASD) was created. The centers have to fullifill the criteria of the GDA for structure quality. Process quality is continously evaluated by mutual hospitation of nurses and physicians in other centers (supervision) at least every two years. In an annual meeting observations during hospitations are presented and discussed. Outcome quality is controlled by an examination of at least 50 unselected type 1 diabetic patients 12 15 month after participation in the TTP (Evaluation), without newly diagnosed patients. The following parameters are evaluated: HbA1c, servere hypoglycemic events (glucose or glucagon injection), ketoacidosis and hospitalisation days per year (all causes) 12 months before and after participation in the TTP. Results: 51 out of 79 centers presented 70 evaluations in five annual meetings 1992-1996. Deficits in structure and process quality were detected and removed. The centers reevaluaand process quality were detected and removed. The centers reevalua-
ted 3673 type 1 diabetic patients. Mean $\mathrm{HbA} 1 \mathrm{c}$ was improved from 1.65 to 1.42 of the mean normal value. The incidence of severe hypoglycemic events (data of 2284 patients) decreased from 0.3 to $0.18 /$ patient/year and the incidence of ketoacidosis (data of 2005 patients) from 0.07 to $0.03 /$ patient/year. The hospitalisation rate (data of 1721 patients: $\min .0,18 ; \max .35,8$ days/patient/year) decreased from 5.69 to 4.56 days/patient/year. Consequences: Since 4 years the quality management of diabetes centers monitoring process and outcome quality represents an outstanding model for a nationwide quality circle. Better metabolic control was associated with a decrease in severe hypoglycemia in contrast to the DCCT which might be explained by better quality management.

\section{4}

Unnecessary high mortality in young adult patients with diabetes - a nationwide study covering 10 years

J. Östman 1), L. WibelI2), L. Nyström3), and the Group of Diabetes Incidence Study in Sweden (DISS).

Depts. of Internal Med., Huddinge Univ. Hospital, Karolinska inst., Stockholm1), Univ. Hospital, Uppsala2), and Dept. of Epidemiol. and Health Care Res., Univ. of Umeå3), Sweden In Sweden, all incident cases of diabetes in the 15-34-year age group have been registered since 1983. During 198392, 4115 patients (Type $173 \%$, Type $216 \%$, unclassifiable $8 \%$, and secondary diabetes $2 \%$ ) were reported, 58 of these died during the period, corresponding to 15496 person years. Autopsies $(n=45)$ and death certificates $(n=13)$ were evaluated together with clinical records. The Standard Mortality Rates were significantly higher in Type 1, Type 2 and secondary diabetes than in the reference population. In all types of diabetes, the mortality in males was 3-4-fold higher than in females. Hyperglycemia-ketoacidosis contributed to death in 11 cases $(19 \%)$ and hypoglycemia in 7 cases $(12 \%)$. From another angle, 21 cases were connected with over-consumption of alcoholic beverages, 4 cases with drug abuse and 7 cases with mental insufficiency and 8 were suicides. Only 18 patients had no such psychosocial factors. At most, 4 of the patients could fit in the category "dead in bed", which is lower than that reported in childhood-onset diabetes. Only 5 cases died because of ischemic heart disease.

Conclusions: In diabetes, alcoholism, mental problems and other forms of psychosocial maladjustment largely account for the mortality among young adults, particularly males, during their first 10 years after diagnosis. Diabetes entails a considerable risk for such patients, which must be identified early, if attempts to improve their prognosis are to be successful. 


\section{Beta-Cell Responses to Drugs and Novel Secretagogues}

\section{5}

PROTEIN KINASE C DOES NOT INFLUENCE THE TOLBUTAMIDE SENSITIVITY OF K-ATP CURRENTS

P. Proks, A. Moorhouse, P.A. Smith and F.M. Ashcroft. University Laboratory of Physiology, Parks Road, Oxford, OX1 3PT, UK

Sulphonylureas stimulate insulin secretion in pancreatic $B$-cells both by inhibiting $\mathrm{K}$-ATP channels and by direct interaction with the secretory machinery. The latter effect is abolished by protein kinase C (PKC) inhibitors and is absent if exocytosis is already stimulated by PKC (but not PKA) activation. This suggests that phosphorylation by PKC is required for the effect of sulphonylureas on exocytosis. The B-cell K-ATP channel is a complex of two proteins: an inwardly rectifying Kchannel subunit, Kir6.2, and a sulphonylurea receptor, SURI. SUR1 possesses 20 , and Kir6.2 six, putative PKC phosphorylation sites. We have therefore examined the effects of modulating PKC activity on whole-cell K-ATP currents, and their tolbutamide sensitivity, in single mouse pancreatic B-cells, using standard patchclamp methads. The dose-response curve for tolbutamide inhibition of K-ATP currents was half maximal at $2.8 \pm 0.4 \mu \mathrm{M}(\mathrm{n}=8)$ in control cells and was unchanged $(3.4 \pm 0.6 \mu \mathrm{M}, \mathrm{n}=8)$ in cells preincubated with $200 \mathrm{nM}$ phorbol ester (PMA) for $20 \mathrm{hr}$ to down-regulate PKC. The maximum K-ATP current was also unaffected ( $43 \pm 9 \mathrm{pA}, \mathrm{n}=18$ in control cells compared with $40 \pm 8, n=18$ in cells preincubated with PMA). Acute application of PMA, or the PKC inhibitor bisindolylmaleimide $(10 \mathrm{nM})$ had no consistent effect of the amplitude of the $\mathrm{K}$ ATP current or the inhibitory effect of $10 \mu \mathrm{M}$ tolbutamide. In the same cell preparations, acute application of PMA potentiated the increase in cell capacitance (a measure of exocytosis) elicited by depolarisation to $+20 \mathrm{mV}$ from $41 \pm 16 \mathrm{fF}$ to $146 \pm 30 \mathrm{fF}(\mathrm{n}=7)$. Following $20 \mathrm{hr}$ incubation with PMA, the mean capacitance increase was reduced to $12 \pm 5 \mathrm{fF}(\mathrm{n}=6)$ and subsequent application of PMA or tolbutamide was without effect. These data confirm that PKC potentiates exocytosis and further demonstrate that PKC is without effect on the amplitude or tolbutamide-sensitivity of K-ATP currents. We conclude that sulphonylureas potentiate exocytosis, and inhibit K-ATP channels, by different mechanisms.

\section{6}

GLIMEPIRIDE ENHANCES GLUCOKINASE AND GLUT2 mRNA EXPRESSION IN INSULINOMA CELLS

O.Porzio, R.Magnaterra, L.NJL.Marlier, R.Lauro and P.Borboni

Department of Internal Medicine-University of Rome "Tor Vergata" - Rome, Italy The glucase phosphorylating enzyme Glucokinase (GK) and the $\beta$-cell glucose transporter isoform Glut2 function as the glucose sensor unit of pancreatic $\beta$ cells. GK and Glut2 are regulated by either nutritional or hormonal factors and also Sulfonylureas have been involved in GK. regulation, determining an insulin-induced increase of pancreatic and liver GK activity. There are no data on regulation of Glut2 in $\beta$ cells by Sulfonylureas. We studied whether Glimepiride (Glim), a novel Sulfonylurea, is able to affect GK and/or Glut2 gene expression in $\beta$ TC6-F7 cells. Cells

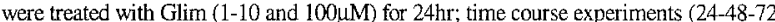
and $96 \mathrm{hr}$ treatments) were performed with $10 \mu \mathrm{M}$ Glim. At the end of the treatments cells were processed for GK and Glut2 mRNA quantitation by Competitive RT-PCR using two synthetic GK and Glut2 cRNA internal standards. For insulin release experiments, cells were incubated for $2 \mathrm{hr}$ at $37^{\circ} \mathrm{C}$ with 1 or $10 \mu \mathrm{M}$ Glim in the presence of increasing glucose concentration (from 0.1 to $16.7 \mathrm{mM}$ ). Supernatants were collected for insulin radioimmunoassay. Results demonstrate that Glim determines a dosedependent increase in GK mRNA expression $(9.08 \pm 0.96 \mathrm{pg} / \mu \mathrm{g}$ RNA in control cells: $10.00 \pm 2.98,11.52 \pm 1.42$ and $15.66 \pm 2.94 \mathrm{pg} / \mathrm{hg}$ RNA respectively at 1,10 and $100 \mathrm{\mu M}$ Glim). Glut 2 mRNA expression shows the same trend $(0.45 \pm 0.06 \mathrm{pg} / \mu \mathrm{g}$ RNA in control cells and 1.01 $\pm 0.19 \mathrm{pg} / \mu \mathrm{g}$ RNA at $100 \mu \mathrm{M}$ Glim). Time-course experiments showed an increase of both GK and Glut2 mRNA expression after $24 \mathrm{hr}$ exposure to $10 \mathrm{MM} \mathrm{Glim}$, as previously reported in dose-response experiments, whereas after 48 and $72 \mathrm{hr}$ both GK and Glut2 mRNA expression are reduced $(-18.85 \%$ and $-23.92 \%$ respectively at $48 \mathrm{hr}$ for GK and $-21.92 \%$ and $-25.36 \%$ respectively at $48 \mathrm{hr} 72 \mathrm{hr}$ for Glut2). GK and Glut2 mRNA levels returned to pretreatment values after 96hr. Insulin release is enhanced in a dose-dependent manner by 1 and $10 \mu \mathrm{M}$ Glim both at high glucose concentrations $(+45.08 \%$ and $+64.09 \%$ respectively) and at low glucose concentrations $(+10.30 \%$ and $+32.70 \%$ respectively). These data demonstrate that Glim determines coordinate modifications in GK and Glut2 mRNA expression; it improves glucose responsiveness and glucose sensitivity. It is possible to hypothesize that the increased insulin secretion induced by Glim is responsible for GK and Glut2 mRNA decrease at long term.
107

Clonidine-displacing substance (CDS), an endogenous imidazoline receptor ligand, is a potent insulin secretagogue SLF Chan \& NG Morgan. Cellular Pharmacology Group, Dept. of Biological Sciences, University of Keele, Keele, Staffs., ST5 5BG, U.K.

The insulin secretagogue activity of imidazoline compounds is mediated by an imidazoline binding site ( 1 -site) expressed in pancreatic $\beta$-cells, that is functionally associated with $\mathrm{K}_{\mathrm{ATP}}^{+}$channels. The natural ligand for I-sites is still unknown. One possible candidate is an endogenous "clonidine-displacing substance" (CDS), whose precise structure remains unclear. In this paper, we have used a methanolic extract of CDS from rat brain to determine whether CDS could be an endogenous insulin secretagogue acting at the islet I-site. One unit of CDS activity was assessed as the amount needed to displace $50 \%$ of specifically bound $\left[{ }^{3} \mathrm{H}\right]$ clonidine in rat brain membranes. This corresponds to approximately $5 \mathrm{pmole}$. Insulin secretory experiments were performed in 96 wells to minimise the amount of material used. CDS caused a dosedependent increase in insulin release from isolated rat islets, with an $\mathrm{EC}_{50}$ of $2 \mathrm{U} / \mathrm{ml}(=10 \mathrm{nM})$. The extent of potentiation was similar to that induced by $100 \mathrm{\mu M}$ efaroxan, a well-characterised imidazoline insulin secretagogue (control, $0.41 \pm 0.03 \mathrm{ng} / \mathrm{islet} / \mathrm{h}$; plus efaroxan, $0.82 \pm 0.07^{*}$; plus $25 \mathrm{nM} \mathrm{CDS \text {, }}$ $\left.0.79 \pm 0.07^{*}, p<0.001\right)$. CDS was also able to reverse the inhibitory effect of the $K^{+}{ }_{\text {ATP }}$ channel opener diazoxide on glucose-induced insulin secretion (diazoxide, $0.56 \pm 0.04$; plus $25 n M$ CDS, $1.32 \pm 0.19^{*}, p<0.001$ ). In addition, the imidazoline antagonists $R \times 801080$ and KU14R $(100 \mu \mathrm{M})$ were each able to antagonise the effects of CDS on stimulation of insulin release $(25 \mathrm{nM} \mathrm{CDS}$, $1.02 \pm 0.05$ vs $0.66 \pm 0.09(\mathrm{RX}) ; 0.56 \pm 0.05(\mathrm{KU})$ ) and reversal of diazoxide $(2.70 \pm 0.36$ vs $1.13 \pm 0.17(\mathrm{KU}), p<0.001)$. CDS was also active in isolated human islets. In conclusion, CDS behaves as a potent insulin secretagogue, at $\mathrm{nM}$ concentrations - doses at which an endogenous ligand would be expected to be active - and thus, the data suggests that CDS is an endogenous insulin secretagogue which acts through the islet imidazoline site.
108

Stimulation of insulin secretion by imidazoline compounds: inhibition of $\mathrm{K}_{\mathrm{ATP}}$ channels and interaction with the exocytotic machinery.

A.M. Efanov, S.V. Zaitsev, I.B. Efanova, O. Larsson, C.-G. Östenson, P.-O. Berggren and S. Efendić, The Rolf Luft Center for Diabetes Research, Department of Molecular Medicine, Karolinska Institute, Stockholm, Sweden

A novel compound with imidazoline structure, RX871024, was used to investigate the mechanisms by which imidazoline derivatives promote insulin secretion in pancreatic $\beta$-cells. RX871024 stimulated insulin release from rat pancreatic islets in a glucose-dependent manner. Low doses of the compound potentiated glucose-induced insulin secretion (10 $\mu \mathrm{M} \mathrm{RX871024}$ incerased insulin secretion in the presence of $3.3 \mathrm{mM}$ glucose from $10.8 \pm 1.2$ $\mu \mathrm{U} /$ islet $/ \mathrm{h}$ to $15.8 \pm 1.4 \mu \mathrm{U} / \mathrm{isle} / \mathrm{h}$ and in the presence of $16.7 \mathrm{mM}$ glucose from $37.6 \pm 7.6 \mu \mathrm{U} /$ islet/h to $112.3 \pm 12.5 \mu \mathrm{U} / \mathrm{isl}$ tet/h, $\mathrm{n}=6$ ), whereas high doses of the imidazoline induced insulin secretion at both basal and stimulatory glucose concentrations. This effect was not mediated by either $\alpha_{2}$-adrenergic or $I_{1}-$ and $\mathrm{I}_{2}$-imidazoline receptors. RX871024 blocked the activity of ATP-dependent $\left(\mathrm{K}_{\mathrm{ATP}}\right), \mathrm{Ca}^{2+}$-activated and delayed rectifier $\mathrm{K}^{+}$-channels and increased cytoplasmic free $\mathrm{Ca}^{2+}$ concentration $\left(\left[\mathrm{Ca}^{2+}\right]_{\mathrm{i}}\right)$. Moreover, under conditions when $\left[\mathrm{Ca}^{2+}\right]$, was clamped either by membrane depolarization with $55 \mathrm{mM} \mathrm{KCl}$ and 20 $\mathrm{mM}$ glucose or by the use of permeabilization technique, $\mathrm{RX} 871024$ was able to stimulate insulin secretion. This insulinotropic effect of the imidazoline was decreased by compounds inhibiting the protein kinase $\mathrm{A}$ and $\mathrm{C}$ activity. Thus, RX871024 promoted insulin release by at least two modes of action. One included an increase in $\left[\mathrm{Ca}^{2+}\right]_{1 ;}$ after blocking of $\mathrm{K}_{\mathrm{aTp}, \mathrm{p}}$-channels, membrane depolarization and activation of voltage-dependent $\mathrm{Ca}^{2+}$ channels and the other, a more distal elfect of the imidazoline directly affecting the exocytotic machinery. The sensitizing effect of low doses of RX871024 on glucose-induced insulin secretion suggests that imidazoline compounds of this type may constitute the basis for development of a new therapeutical concept in the treatment of diahetes. 
109

THE CREATINE ANALOGUE CYCLOCREATINE INCREASES INSULIN SECRETION IN INS-1 CELLS VIA A K CHANNELINDEPENDENT MECHANISM

K. Gempel ${ }^{1}$, D. Brdiczka ${ }^{2}$, R. Kaddurah-Daouk ${ }^{3}, T$. Wallimann ${ }^{4}, P$ Kaufhold and K-D Gerbitz, Insitute of Diahetes Research. Academic Hospital Schwabing. Munich. Germany: 2University of Konsianz. Germany: "Avicena Group Inc.. Cambridge. USA: ${ }^{3}$ ETH Zirich. Switzeriand

The rat insulinoma cell line INS-1 contains high amounts of the brain type of creatine kinase as well as lower amounts of mitochondrial creatine kinase We studied the influence on insulin secretion of creatine and its kinetically most active analogue cyclocreatine $(\mathrm{cCr})$. Creatine and even more $\mathrm{cCr}$ stimulated insulin secretion in a dose-dependent manner. Stimulation of secretion was paralleled by uptake and phosphorylation to cyclocreatine phosphate. Uptake and stimulation of insulin secretion could be inhibited by $\beta$-guanidino propionic acid, a known inhibitor of the plasma membrane creatine transporter. When insulin secretion was maximally stimulated by $\mathrm{cCr}$ it could not further be inhanced by glucose, but still could be strongly increased by forskolin plus phorbol myristate acetate. Two lines of evidence suggest that $\mathrm{CCr}$-induced insulin secretion is independent of the ATPsensitive $\mathrm{K}+$ channel: i) In the presence of diazoxide, which opens the $\mathrm{K}+$ channel (insulin seretion $45 \pm 18 \%$ of control), $\mathrm{cCr}(133 \pm 15 \%, \mathrm{p}<0.05$ vs diazoxide alone) but not glucose ( $49 \pm 9 \%$ ) stimulated secretion. ii) When the $\mathrm{K}+$ channel was bypassed and the plasma membrane depolarized by high external $\mathrm{K}+$ in the presence of diazoxide (insulin seretion $245 \pm 11 \%$ of control) $\mathrm{cCr}$ further increased insulin secretion ( $482 \pm 9 \%$ of control, $\mathrm{p}<0.05$ vs high $\mathrm{K}+$ /diazoxide). We propose that $\mathrm{cCr}$ may reduce the activity of plasma membrane $\mathrm{Ca} 2+$ export systems which rely on a high ATP/ADP ratio. Although the exact mechanism of $\mathrm{cCr}$ remains to be established it is a potent new insulin secretagogue.

\section{OP 18}

\section{Insulin Processing}

\section{0} NOVEL GLUCOSE RESPONSE ELEMENT IN THE DISTAL HUMAN
INSULIN PROMOTER.

M. Sander J. Wang and M.S German. The Hormone Research Institute, University of California at San Francisco, CA 94143-0534, USA

Insulin gene transcription is limited to the $\beta$-cells within the mammalian pancreas and, like insulin secretion, is regulated by glucose. To identify important regions within the human insulin gene promoter, we transfected truncated fragments of the promoter linked to a reporter gene into rat primary islet cells. Truncation of the region from -341 to $-260 \mathrm{bp}$ upstream from the transcription start site led to a marked drop in transcriptional activity. To determine what sequence elements within this region are responsible for activation of transcription in $\beta$-cells, we designed six small oligonucleotides (minienhancers) spanning this region and linked each to a minimal promoter driving a reporter gene. The minienhancer spanning the region between -290 and -241 bp of the promoter dramatically activated the reporter gene. The activity of the $-290 /-241$ minienhancer increased 4fold in response to high glucose concentration. The same minienhancer, when linked to the first $482 \mathrm{bp}$ of the rat glucagon promoter. also significantly enhanced transcription in primary islet cells, but was negatively regulated by glucose. Data from the other minienhancers demonstrated that function of the $-290 /-241$ minienhancer depends on at least two separate elements within this 50 base pairs. This same region has previously been shown to inhibit transcription in $\beta$-cell tumor cell lines. We confirmed this result when we found that, in contrast to its positive effects in primary islet cells, the $-290 /-241$ minienhancer acted as a negative regulator of transcription in primary non-islet-cells and in both $\beta$-cell and non-islet tumor cell lines. We here describe a novel glucose responsive positive regulatory region within the distal human insulin promoter. The evidence that the same element represses transcription in transformed $\beta$-cells demonstrates the importance of studying insulin gene expression in normal $\beta$-cells. Since the activity is limited to primary islet-cells, we conclude that this region is important for islet specificity of insulin gene transcription and contributes to the transcriptional response to glucose.

\section{1}

ANALYSIS OF GLUCOSE RESPONSIVE ELEMENT OF HUMAN INSULIN GENE PROMOTOR USING MIN6 CELL

N. Furukawa, T. Shirotani, K. Kaneko, E. Araki, H. Kishikawa, and M. Shichiri, Department of Metabolic Medicine, Kumamoto University School of Medicine, Kumamoto, Japan

Transcription of insulin gene in pancreatic $\beta$-cell is regulated by glucose. Using MIN6 cell line which secreted insulin in response to glucose concentration, we first analyzed the enhancer element of human insulin gene responsible for glucose dependent expression by CAT assay. Secondly, we characterized trans-acting factors which bound to the element by electrophoretic mobility shift assay (EMSA) and UV crosslink assay. Finally, in order to test for glucose dependent phosphorylation-status of its trans-acting factor, we performed EMSA using nuclear extract treated with phosphatase. By CAT assay using various deletions of the insulin gene promotor, we identified the enhancer element responsible for glucose. The element was located between at 230 and -200 of the insulin gene promotor, which contained homeodomain protein (IPF1) binding site termed A3 element. In EMSA analysis, three bands termed CT1, CT2, and CT3 composed of DNAprotein complexes were detected. EMSA analysis using a probe with mutation at TAAT sequence demonstrated that CT1 and CT2 complexes were composed of nuclear proteins which bound to TAAT sequences of A3 element. UV crosslink assay demonstrated that each molecular weights of nuclear proteins responsible for CT1, CT2, and CT3 were $36 \mathrm{kDa}, 48 \mathrm{kDa}$, and $152 \mathrm{kDa}$, respectively. When nuclear extracts were treated with phosphatase all three bands reduced significantly.

In conclusion, these results suggest that TAAT sequences of A3 element of insulin gene and phosphorylation of trans-acting factors are necessary for glucose responsible transcription of human insulin gene in MIN6 cells. 
112

THE $5^{\prime}$ UNTRANSLATED REGION ( $5^{\prime}$ UTR) OF INSULIN MRNA CONTAINS A GLUCOSE RESPONSIVE SEQUENCE.

H. Cragg, B. Wicksteed, K.I.J. Shennan and K. Docherty. Department Of Molecular and Cell Biology, University of Aberdeen, Aberdeen, AB9 1AS, U.K.

The $\beta$-cells of the pancreatic islets of Langerhans are exquisitely sensitive to circulating blood glucose concentrations. This sensitivity is dependent on both the uptake and metabolism of glucose, and response is mediated in the short term through effects on the rate of translation of preexisting insulin mRNA, and secretion of insulin from the cell. Glucoseinduced translational effects on proinsulin biosynthesis occur within twenty minutes of exposure, and are mediated primarily through a stimulation of the rate of translational initiation, in addition to the transfer of initiated insulin mRNA from free to membrane bound ribosomes, and a decrease in the extent of ribosomal pausing. The present study investigates the role of the insulin gene $5^{\prime}$ UTR in these events. Two plasmid vectors were constructed. One (pTKpLUC) contained a firefly luciferase gene (LUC) under the control of the thymidine kinase promoter (TKp) from Herpes Simplex virus. In the other construct (pUTRLUC) the $5^{\prime}$ UTR sequences between the TKp and LUC were replaced with the $5^{\prime}$ UTR from the mouse II insulin gene. These contructs were transfected into the mouse $\beta$-cell line MIN- 6 , and the cells incubated for short periods of time $(20-60$ minutes) in low $(0.5 \mathrm{mM})$ or high $(16 \mathrm{mM})$ glucose. There was no effect of glucose on the luciferase activity in cells transfected with pTKpLUC, however a $2-5$ fold increase in activity was observed in cells transfected with pUTRLUC. This effect was inhibited by mannoheptulose, and mimicked by the leucine metabolite 2- keto-isocaproic acid. We conclude that the $5^{\prime}$ UTR sequence contains a glucose responsive element that may be important in the translational regulation of preproinsulin biosynthesis.

\section{3}

PROINSULIN CAN BE CONVERTED TO INSULIN IN LIVING CELLS BY
EITHER OF THE CONVERSION ENDOPROTEASES PC2 AND PC3

J. Kaufmann, J.-C. Irminger and P.A. Halban. U. Geneva, Switzerland.

B-cell granules contain two conversion endoproteases, $\mathrm{PC}_{2}$ and $\mathrm{PC} 3$. Based upon this and studies in vitro, it has been suggested that each enzyme cleaves proinsulin at only one of its junctions (PC2: C-peptide/Achain; PC3: B-chain/C-peptide) and that both are needed for complete conversion to insulin. We have now studied proinsulin conversion by just $\mathrm{PC} 2$ or $\mathrm{PC} 3$ in living cells. To this end human proinsulin and/or $\mathrm{PC} 2$ or PC3 were expressed by infection of cells with the corresponding recombinant adenovirus. Proinsulin conversion was studied by pulsechase followed by immunoprecipitation and HPLC. Over-expression of endoproteases was monitored by Western blot. Conversion by $\mathrm{PC} 2$ alone was studied in $\mathrm{GH} 3$ cells. The $\mathrm{GH} 3$ cells used in this study do not express detectable levels of either $\mathrm{PC} 2$ or $\mathrm{PC} 3$. Conversion to insulin in such native $\mathrm{GH} 3$ cells was limited to $2 \%$ by $60 \mathrm{~min}$. When $\mathrm{PC} 2$ was co-expressed with proinsulin, conversion was increased to $44 \%$ at $60 \mathrm{~min}$ and $64 \%$ at 120 min, with $9.7 \%$ (60 min) and $9.2 \%$ (120 min) des-64,65-split proinsulin $\mathrm{PC} 3$ was also active in $\mathrm{GH} 3$ cells when over-expressed by recombinant adenovirus infection. AtT20 cells express very high endogenous levels of PC3 with little detectable PC2. Proinsulin conversion in AtT20 cells led to $40 \%$ and $74 \%$ insulin at 60 and $120 \mathrm{~min}$ chase respectively, with $13 \%$ and $6 \%$ des-31.32-split proinsulin. Over-expression of PC2 in these cells did not accelerate proinsulin conversion. It is concluded that both PC2 and PC3 are able to cleave human proinsulin at both its junctions when overexpressed in regulated secretory cells. Although they display a preference for the site of initial cleavage (reflected by generation of des-31.32- or des64.65-split proinsulin for $\mathrm{PC} 3$ and $\mathrm{PC} 2$ respectively) the conversion enzymes, at least when expressed at high levels, are less fastidious in their substrate specificity than previously supposed.

\section{4}

EXPRESSION IN SINGLE CLONAL $\beta$-CELLS OF A RECOMBINANT CHIMAERA BETWEEN INSULIN AND FIREFLY LUCIFERASE: TOWARDS REAL-TIME DIGITAL IMAGING OF INSULIN TRAFFICKING AND RELEASE.

Aristea Pouli, Lara Mitra, J. George Schofield and Guy A. Rutter. Department of Biochemistry, School of Medical Sciences, University of Bristol, Bristol, BS8 ITD, U.K

A means of monitoring intracellular trafficking and release of insulin in single pancreatic islet $\beta$-cells would provide a powerful tool to dissect the molecular basis of the regulation of these processes. We have constructed a plasmid in which human preproinsulin cDNA is fused in-frame and upstream of cDNA encoding firefly luciferase. Preproinsulin cDNA was amplified from a template plasmid (T71, Prof K. Docherty, University of Aberdeen, U.K.) using PCR primers to engineer a $5^{\prime}$ Hind $\mathrm{III}$ and $3^{\prime} \mathrm{NcoI}$ site. The $3^{\prime}$ primer also incorporated the coding sequence ( 42 nucleotides) for a c-myc epitope tag between insulin and luciferase. The amplified 346 base-pair product was restricted and ligated into plasmid pGL3 (Promega) containing luciferase cDNA which lacks the wild-type peroxisomal targeting sequence. The 2.1 kilobase preproinsulin.luciferase fusion product was subcloned via flanking Xhol and Xbal sites into vector pcDNAlneo (Invitrogen) under cytomegalovirus promoter control. This construct was microinjected into clonal B-cells (INS-1) which were then cultured for $24 \mathrm{~h}$ (11 mM glucose) to allow the expression of the chimaeric protein. During incubation with the cofactor, luciferin, photon release from the chimeric product could be imaged in real-time in single living cells using an intensified charge-coupled device camera (Hamamatsu Photonics) and inverted microscope (Zeiss Axivert TV100, 10x objective) Immunocytochemistry with a monoclonal anti-myc antibody revealed localisation as expected to the endoplasmic reticulum, Golgi apparatus, and secretory granules. These data suggest that the fusion of insulin with luciferase does not interfere with the proper targeting of the mature hormone and should provide a method for monitoring insulin secretion from single living $\beta$ cells. 
OP 19

\section{Insulin Therapy: NIDDM}

115

COMPARISON OF BEDTIME INSULIN REGIMENS IN NIDDM: METFORMIN PREVENTS INSULIN-INDUCED WEIGHT GAIN

H. Yki-Järvinen, Kati Nikkilä, Leena Ryysy, Timo Tulokas, Raimo Vanamo and M. Heikkilä. Jorvi, Kymenlaakso, Lappi and Etelä-Saimaa Central Hospitals, and Department of Medicine, University of Helsinki, Finland. Combination therapy with bedtime NPH improves glycemic control similarly but induces less weight gain than other insulin treatment regimens. In a new Finnish placebo-controlled multicenter trial we compared 4 bedtime insulin regimens: bedtime $\mathrm{NPH}+$ either glibenclamide (SU, $10.5 \mathrm{mg}$ ), metformin (MET, 2 grams), both (SU + MET) or a second injection of NPH in the morning $(B I+M I), 60$ patients with NIDDM, all previously treated with SU only, were centrally randomized and treated in $\mathbf{4}$ centers. Results after 3 months:

\begin{tabular}{|c|c|c|c|c|}
\hline & $\begin{array}{l}\mathbf{B I}+ \\
\mathbf{S U}\end{array}$ & $\begin{array}{l}\text { BI+ } \\
\text { MET }\end{array}$ & $\begin{array}{l}\mathbf{B I}+ \\
\mathbf{S U}+\mathbf{M E T}\end{array}$ & $\begin{array}{l}\text { BI+ } \\
\text { MI }\end{array}$ \\
\hline Number & 15 & 17 & 15 & 13 \\
\hline Age (yrs) & $59 \pm 3$ & $58 \pm 3$ & $56 \pm 2$ & $57 \pm 2$ \\
\hline BMI, 0 mos & $27 \pm 1$ & $28 \pm 2$ & $28 \pm 1$ & $28 \pm 1$ \\
\hline $\mathrm{HbA}_{\mathrm{Ic}} 0 \mathrm{mos}$ & $9.4 \pm 0.5$ & $10.2 \pm 0.6$ & $9.6 \pm 0.4$ & $10.2 \pm 0.5$ \\
\hline $\mathrm{HbA}_{\mathrm{ic}} 3 \mathrm{mos}$ & $7.8 \pm 0.2$ & $8.1 \pm 0.4$ & $7.5 \pm 0.3$ & $8.8 \pm 0.4$ \\
\hline $\mathrm{HbA}_{\mathrm{xc}}$ change & $-1.6 \pm 0.2$ & $-2.0 \pm 0.4$ & $-2.1 \pm 0.2$ & $-1.3 \pm 0.4^{x}$ \\
\hline \multirow{2}{*}{$\begin{array}{l}\text { Weight change, kg } \\
\text { BI/MI dose, IU }\end{array}$} & $2.1 \pm 0.0$ & $-0.5 \pm 1.0^{\mathrm{xx}}$ & $1.7 \pm 0.5$ & $1.8 \pm 0.9$ \\
\hline & $15 \pm 3 /-$ & $34 \pm 11^{x x} /-$ & $15 \pm 2 /-$ & $19 \pm 2 / 23 \pm 2$ \\
\hline
\end{tabular}

${ }^{x} p<0.05$ or less vs other groups, ${ }^{x_{p}}<0.05$ vs BI + MET and BI+SU+MET.

Conclusions: Bedtime NPH combined with metformin improves glycemic control equally to other combination therapy regimens but effectively prevents insulin induced weight gain in patients with NIDDM.
116

SVALUATION OF OPTMAL THERAPY IN TYPE-2 DIABETIC PATIENTS WSUTFICIENTLY TREATED WTTH SULFONYLUREAS: THE AUSTRIAN INSULIN INTERVENTION STUDY. Ch.Schnack, G.Biesenbach, Q.Kacerovsky, R. Mihaljevic, I.Pecrilk, Th.Pieber, and G.Schernthaner. Rudolfstiftung Hospital, Vienna, Austria The effects of four different therapeutic regimens with insulin and/or metformin on metabolic control and body weight are evaluated in a multicenter prospective intervention trial in type-2 diabetic patients (at present $n=127$ ) with insufficient metabolic control on sulfonylurea treatment $\left(\mathrm{Hb}_{\text {ic }}\right.$ 8-11\%). The patients (mean age 63,3 yrs, diacetes duration 11,3 yrs) randomly receive metformin plus sulfonylureas(SM), a bedtime insulin injection plus sulfonglureas (SI), two mixed insidin injections daily (I) or two mixed insulin injections per day plus netformin (MI). Data for 6 months therapy are available for 74 patients (A: baseline, B: 6 months treatment).

\begin{tabular}{|c|c|c|c|c|c|}
\hline \multicolumn{2}{|c|}{ Therapy Group } & \multirow{3}{*}{$\begin{array}{c}\operatorname{SM}(n=15) \\
9,7+0,3\end{array}$} & \multirow{2}{*}{$\begin{array}{c}S I(n=20) \\
9,5 \pm 0,2\end{array}$} & \multirow{2}{*}{$\begin{array}{c}I(n=19) \\
9,7+0,2\end{array}$} & \multirow{2}{*}{$\begin{array}{r}\mathrm{BI}(\mathrm{n}=20) \\
10,0+0,2\end{array}$} \\
\hline \multirow{2}{*}{\multicolumn{2}{|c|}{$\mathrm{HoA}_{1 \mathrm{C}}(\%)$}} & & & & \\
\hline & & & $8,3+0,4 *$ & $8,0+0,3^{*}$ & $8,4 \mp 0,4^{*}$ \\
\hline \multirow{2}{*}{\multicolumn{2}{|c|}{$\begin{array}{c}\Delta(B-A) \\
\text { Body weight } A\end{array}$}} & $-0,6$ & $-1,2$ & $-1,7+0,3^{+}$ & $-1,6+0,4^{+}$ \\
\hline & & 74,3 & 73,9 & $81,1+3,7$ & $77,2+2,5$ \\
\hline \multirow[t]{2}{*}{ (kg) } & dy weight $A$ & $75,8+3,4$ & $76,5+2,4^{*}$ & $83,4+3,4$ & $78,3+2,4$ \\
\hline & $\Delta(B-A)$ & $1,5+0,8$ & $2,6+0,8$ & $2,3+1,3$ & $1,1+0,7$ \\
\hline
\end{tabular}

At 6 months the mean ( + SEM) insulin dosages were 14+1,7 IE (SI), 29+3,2 IE (I) and $22+1,7$ IR (MI) respectively. A slight increase of body weight was seen in all treatment groups, however reached statistical significance only in patients treated with a single bedtime insulin injection in addition to sulfonylureas. Our data emphasize the positive metabolic effects of insulin therapy compared to metformin in type-2 diabetic patients insufficiently treated with sulfonylureas.

\section{7}

COMBINED BEDTIME INSULIN - DAYTIME SULPHONYLUREA VS INSULIN THERAPY ALONE IN SECONDARY FAILURE OF NIDDM PATIENTS

J. Fövényi, E. Thaisz, L. Lehotkai, A. Grosz, T. Sallai and G. Kocsis. Péterfy Teaching Hospital, Budapest, Hungary

The aim of the study was to compare the efficacy of the continuation of oral sulphonylurea therapy + bedtime insulin treatment with conventional insulin therapy in secondary failure of NIDDM patients over a period of minimum 12 months. Two hundred and ninety patients -118 males, 164 females, age: $60,2+11,1$ yr, diabetes duration: $10,4 \pm 6,6$ yr, BMI: $28,6 \pm 1,5$ - were enrolled in the study. The patients were randomly selected into two groups. In Group A (145 patients) the daytime treatment with sulphonylureas ( $96 \%$ glibenlamide, $4 \%$ gliclazid) was continued, and the bedtime application of 8-10 IU NPH insulin (Humulin N or Insulatard HM) was started. The insulin dose was stepwise increased in order to achieve normal $(4-7 \mathrm{mmol} / 1)$ fasting blood glucose levels with blood glucose values below $10 \mathrm{mmol} / 1$ during the day. In 84 cases (Group Al) the combined therapy was succesful over a period of minimum 12 months (19,2 4,8 months), in 57 failure cases (Group A2) the treatment with oral agent was stopped within 6 months and the patients were switched over to twice daily insulin regimens. In another 145 patients (Group B) the sulphonylurea treatment was stopped and twice daily insulin regimen was started. Results were evauted 12 months after initiation of the therapies. $\mathrm{HbA}_{1 \mathrm{c}}$ values decreased significantly in all of three goups: Group A1: $9,24 \pm 1,26$ vs 7,44 $\pm 0,80 \%, p<0,001$, Group A2: 9,48 $\pm 1,36$ vs $7,82 \pm 0,88 \%, p<0,001$, Group B: $9,42 \pm 1,18$ vs $8,03 \pm 0,92 \%, p<0,001$. The final $\mathrm{HBA}_{1 \mathrm{c}}$ results of Group A1 were significantly lower $(\mathrm{p}<0,01)$ than that of other two groups. In Group A1 18 among 84 succesfully treated patients the NPH insulin was replaced by long-acting crystalline-zinc insulin (Humulin $U$ or Ultratard HM) to avoid daytime hyperglycaemia. Insulin doses: In Group A1 one month after initiation of the combined treatment the NPH insulin doses were 9,8 $81,8 \mathrm{IU}, 6$ months later the NPH insulin doses increased to $16,1 \pm 1,4 \mathrm{IU}$ and the crystalline-zinc insulin doses to $18,0 \pm 1,8 \mathrm{IU}$. In Group A2 the insulin doses were: $44,6 \pm 3,6 \mathrm{IU}$, in Group B: $43,6 \pm$ $4,2 \mathrm{IU}$ respectively. On the basis of our experiences the combination therapy daytime sulphonylurea - bedtime insulin seems to be a treatment of choice in NIDDM patients with secondary failure to sulphonylureas.
118

CAUSES OF WEIGHT GAIN DURING INSULIN THERAPY: ROLE OF LEPTIN, INSULIN, AND ENERGY BALANCE S. Mäkimattila, K. Nikkilä*, S.-L. Karonen and H. Yki-Järvinen. University of Helsinki, Helsinki, "Jorvi Hospital, Espoo, Finland The etiology of weight gain during insulin therapy is unclear. We searched for factors predictive of weight gain during 6 months of bedtime insulin therapy in 23 NIDDMs (BMI $27 \pm 1 \mathrm{~kg} / \mathrm{m}^{2}$ ). Resting energy expenditure (EE), energy intake (EI), glucose calories lost in urine $\left(E_{\text {urine }}\right)$, serum leptin and insulin concentrations were determined at 0 and 6 months. Fasting glucose decreased from $14.4 \pm 1.0$ to $8.2 \pm 1.0 \mathrm{mmol} / \mathrm{l}$ during 6 months $(p<0.001)$. Weight gain averaged $3.3 \pm 0.8 \mathrm{~kg}(\mathrm{p}<0.001)$ over 6 months (range $-2.9-17.0)$. $E_{\text {urine }}$ averaged $278 \pm 68 \mathrm{kcal} /$ day (range $1-1200$ ) at 0 and $26 \pm 9$ (range $0-207$ ) at 6 months $(p<0.01)$. Serum insulin increased from $11 \pm 1$ to $17 \pm 2 \mathrm{mU} / \mathrm{l}(p<0.005)$, and leptin from $8 \pm 1$ to $11 \pm 1 \mathrm{ng} / \mathrm{ml}$ $(p<0.005)$. The change in $E_{u r i n e}$ but not changes in EE, EI, or leptin or insulin concentrations explained $47 \%$ of weight gain during 6 months $(p<0.005)$. Serum leptin correlated with insulin $(r=0.49$, $p<0.02)$ and fat mass $(r=0.74, p<0.001)$. The change in leptin was significantly $(62 \%)$ determined by changes in fat mass $(p=0.001)$ and insulin $(p<0.001)$; it was not related to changes in EE or EI. We conclude that failure to reduce $E I$ in proportion to $E_{\text {urine }}$ is the most significant determinant of weight gain during insulin therapy in NIDDM. The individual tendency for weight gain can be estimated by determining diurnal glucose excretion before insulin therapy. Serum leptin closely parallels the degree of insulinemia and fat mass but does not explain weight gain during insulin therapy. 
VA COOPERATIVE STUDY ON GLYCEMIC CONTROL AND COMPLICATIONS IN TYPE II DIABETES (VACSDM): MICRO AND MACROVASCULAR EVENTS J. Colwell, C. Abraira, W. Henderson, N. Emanuele, S. Levin, F. Nuttal, C. Sawin, J. Comstock and I. Pacold. VACSDM Study Group, Hines, IL.

The VACSDM was a feasibility study designed to determine whether a significant difference in HgbAlc could be achieved with intensive (INT) vs. standard (STD) insulin therapy in 153 male type II diabetic patients who had elevated HgbAlc levels while on drug therapy. Secondarily, the study determined the prevalence of micro and macrovascular disease and the effects of intensive insulin management on these endpoints. Baseline findings: $(n=153)$

\begin{tabular}{|c|c|c|c|c|}
\hline Retinopathy & $\%$ & Albuminuria & $\%$ & CVD \\
\hline None & 38 & $<30 \mathrm{mg} / \mathrm{d}$ & 46 & By History \\
\hline $2 N P D R$ & 62 & $>30 \mathrm{mg} / \mathrm{d}$ & 54 & $\begin{array}{l}\text { By Holter, MUGA } \\
\text { Total }\end{array}$ \\
\hline
\end{tabular}

From 9-30 months, HgbAIc (STD) was maintained at $9.2 \%$, (INT.) $7.1 \%(\mathrm{P}<.001)$ Data at 24-27 months:(P=N.S. for STD vs. INT.)

\begin{tabular}{|c|c|c|c|c|c|}
\hline pathy & $\mathrm{N}$ & le & orse & vents & No \\
\hline STD & 67 & $67 \%$ & $33 \%$ & STD $(n=78)$ & 16 \\
\hline INT. & 63 & $67 \%$ & $33 \%$ & INT. $(n=75)$ & 24 \\
\hline
\end{tabular}

CV history was a significant predictor of new CV events $(P=037)$. Treatment, insulin dose, hypoglycemia, lipids, blood pressure, microalbuminuria, or smoking had no correlation. HgbA Ic was of borderline significance $(\mathrm{P}=.057)$. Patients who entered without microalbuminuria (INT) had no progression at 12 months ( $11 \pm 1.5$ to $37 \pm 18$ $\mathrm{mg} / 24 \mathrm{hr})$ when compared to STD $(14.1 \pm 1.4$ to $66 \pm 19 \mathrm{mg} / 24 \mathrm{hr} ; \mathrm{P}=008)$. STD with microalbuminuria had a significant increase at 24 months ( $135 \pm 19$ to $470 \pm 17 \mathrm{mg} / 24 \mathrm{hr}$ )

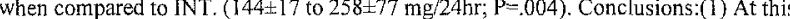
stage of type II diabetes there is a very high prevalence of albuminuria and macrovascular disease. (2) Short term intensive insulin therapy has no effect on macrovascular events or on retinopathy, but retards the progression of albuminuria. (3) A full-scale, long term trial comparing STD to INT insulin therapy is planned to assess the benefit/risk ratio of insulin treatment strategies at this stage of type II diabetes.

\section{OP 20 \\ Treatment and Natural History of Neuropathy}

120

$\alpha$-Lipoic acid Prevents the Deficit in Glucose Uptake in Experimental Diabetic Neuropathy, Low PA, Schmelzer JD, Zollman PJ, Tritschler H-J.

Mayo Foundation, Rochester, MN, U.S.A, \& Asta Medica, Frankfurt, Germany

Endoneurial glucose is increased in the ischemic and hypoxic peripheral nerve of experimental diabetic neuropathy (EDN), which has an increased dependence on anaerobic metabolism. Insulin treatment, by reducing energy substrate stores in these ischemic nerves, results in an energy crisis and the development of axonal degeneration, a situation akin to "insulin neuritis" and the temporary worsening of neuropathy in human subjects when tight insulin-induced glycemic control is imposed. Since $\alpha$-lipoic acid (LA) has been shown to improve glucose uptake in other tissues, we evaluated its effect on glucose uptake in peripheral nerve of EDN. Four groups of rats $(\mathrm{N}=10$ per group) were studied: Controls (Con); Controls + LA (ConLA); EDN (STZ); EDN + LA (STZ-LA). Duration of diabetes was 1 month, and LA, $100 \mathrm{mg} / \mathrm{kg}$, was administered IP 5 times per week for 1 week prior to the terminal experiment. Glucose uptake was evaluated using 2-reoxy-D $\left[{ }^{14} C\right]$-glucose (2-DG) autoradiography of mid-sciatic nerve, L.5 dorsal root ganglion (DRG), and superior cervical ganglion (SCG). The blood glucose of diabetic rats (STZ, 435 \pm 21 ; STZ-LA, $441 \pm 34$ ) was significantly increased $(\mathrm{P}<0.01$ ) over controls (Con, 97 \pm 3 ; Con-LA, $90 \pm 2$ ). 2-DG uptake (in $\mathrm{mmol} / 100 \mathrm{~g} / \mathrm{min}$ ) was significantly different among groups $(\mathrm{P}<0.001)$. It was significantly reduced $(\mathrm{P}<0.01)$ in the sciatic nerve of STZ (STZ: Con: Con-LA; $10.7 \pm 0.6: 17.8 \pm 0.4: 16.2 \pm 0.5$ ), and completely prevented by LA pretreatment in EDN (STZ-LA, 17.5 \pm 0.6 ). Uptake in L5 DRG was significantly reduced

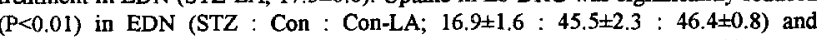
completely prevented with LA pre-treatment in EDN (STZ-LA, 51.1 \pm 2.9 ), Corresponding values for SCG showed a significant reduction $(\mathrm{P}<0.01)$ in EDN (STZ Con : Con-LA; $17.0 \pm 1.5: 55.8 \pm 3.7: 54.8 \pm 2.5$ ) and completely prevented by LA (STZ-LA, 61.2 \pm 3.0 ). Since LA administration is associated with a prevention of the nerve blood flow (BF) and conduction deficits, we suggest that this improvement is associated with improved glucose utilization, and not an increase of polyol pathway hyperactivity (which is associated with worsening conduction and $\mathrm{BF}$ deficits).
121

EFFECTS OF TREATMENT WITH THE ANTIOXIDAN $1 \alpha$ TLIPOIC ACID ON CARDIAC AUTONOMIC NEUROPATHY IN NIDDM PATIENTS A 4-MONTH RANDOMIZED CONTROLLED TRIAL (DEKAN STUDY)

F. A. Gries ${ }^{1}$, F. Conrad ${ }^{2}$, H. Ulrich ${ }^{2}$, G. Reichel ${ }^{3}, H$. Schatz ${ }^{4}$, and D. Ziegler'; 'Diabetes-Forschungsinstitut, Universität Düisseldorf; ${ }^{2}$ ÁSTA Medica AG, Frankfurt/Main; ${ }^{3}$ Paracelsus-Klinik, Zwickau; ${ }^{4}$ Medizinische Klinik \& Poliklinik, Krankenanstalten Bergmannsheil, Bochum, Germany

There is accumulating evidence suggesting that free radical-mediated oxidative stress is implicated in the pathogenesis of diabetic neuropathy. The efficacy and safety of the antioxidant $\alpha$-lipoic acid were studied in randomized, double-blind placebo-controlled multicentre trial (Deutsche Kardiale Autonome Neuropathie Studie) (DEKAN Study) in NIDDM patients with cardiac autonomic neuropathy (CAN) assessed by reduced heart rate variability (HRV) who were randomly assigned to treatment with a daily oral dose of $800 \mathrm{mg} \alpha$-lipoic acid (ALA) $(n=39)$ or placebo $(n=34)$ for 4 months. Parameters of HRV at rest included the coefficient of variation (CV), root mean square successive difference (RMSSD), and spectral power in the low-frequency (LF: $0.05-0.15 \mathrm{~Hz})$ and highfrequency (HF: $0.15-0.5 \mathrm{~Hz}$ ) bands. There were 17 drop-outs (ALA $n=10$; placebo: $n=7$ ). Mean blood pressure, heart rate, and $\mathrm{HbA}_{1}$ levels did not differ between the groups during the study. RMSSD increased from baseline to 4 months by $1.5(-37.6-77.1) \mathrm{ms}$ (median [min-max]) in the group treated with ALA and decreased by $-0.1(-19.2-32.8) \mathrm{ms}$ in the placebo group $(\mathrm{p}<0.05$ for ALA vs placebo). Power spectrum in the LF band increased by $0.06(-0.09-0.62) 10^{-4} \mathrm{~Hz}^{2}$ in ALA, whereas it declined by $-0.01(-0.48-1.86) 10^{4} \mathrm{~Hz}^{2}$ in placebo ( $p<0.05$ for ALA vs placebo) Furthermore, there was a trend toward a favorable effect of ALA vs placebo for the $C V$ which increased from baseline to 4 months by 0.19 $(-3.16-12.45) \%$ in ALA, but only by $0.008(-1.48-4.47) \%$ in placebo
$(\mathrm{p}=0.097$ for ALA vs placebo). A similar trend was noted for the HF band power spectrum showing an increase by $0.016(-1.051-0.356)$ $10^{-4} \mathrm{~Hz}^{2}$ in ALA, but a reduction by $-0.002(-0.730-0.483) 10^{-4} \mathrm{~Hz}^{2}$ in placebo ( $\mathrm{p}=0.094$ for ALA vs placebo). No differences between the groups were noted regarding the rates of adverse events. These findings (1) $800 \mathrm{mg} / \mathrm{day}$ for 4 months may improve CAN in NIDDM. 


\section{2}

RESULTS OF A PHASE II MULTICENTER STUDY OF ZOPOLRESTAT IN PATIENTS WITH PERIPHERAL DIABETIC POLY NEUROPATHY D.A. Greene, J. C. Arezzo, S. S. Klioze, M. J. Peterson, M. Y. Lakshminarayanan and the Zopolrestat Phase II Neuropathy Study Group. Univ. of Michigan Med. Ctr., Ann Arbor, MI; Albert Einstein Coll. of Med, Bronx, NY; and Pfizer Ceniral Research, Groton, CT, USA

The safety and efficacy of zopolrestat were evaluated in a 12-week, double-blind, multicenter study. A total of 291 patients with painful symmetrical diabetic polyneuropathy were randomized to groups receiving either $1000 \mathrm{mg}$ of the aldose reductase inhibitor zopolrestat $(\mathrm{N}=146)$ or placebo ( $\mathrm{N}=145)$. A composite measure of peripheral neuroelectrophysiology (NE parameter) and the clinical cvaluation of neuropathic signs and symptoms were the two primary efficacy endpoints. For both the intent-to-treat and evaluable paticnt analyses, the NE parameter was significant in favor of zopolrestat ( $p=0.001$ and 0.002 , respectively). Treatment was associated with an improvement in nerve conduction velocity in all six nerve segments evaluated (range 0.7 to $1.2 \mathrm{~m} / \mathrm{sec}$ ); significant differences comparcd to placebo were achieved for four individual nerve segments (i.e., median motor, distal and proximal median sensory, and peroneal motor). Among the evaluable paticnts, mean changes in all sensory and motor amplitudes trended in favor of zopolrestat with responses in the ulnar nerve nearly reaching significance $(p=0.065)$. Clinical signs and symptoms of painful peripheral diabetic neuropathy were assesscd using ten parameters. There were no significant differences between the treatment groups. Baseline and final sural nerve biopsies were obtained from a small subset of 17 patients ( 9 zopolrestat, 8 placebo). Biochemical evaluation of these samples confimed a significant reduction in the sorbitol/glucose ratio in the zopolrestattreated group. Treatment with zopolrestat was generally well tolerated. Overall, $17.8 \%$ of zopolrestat-reated patients and $11.7 \%$ of placebo-treated patients reported adverse events which included headache, flatulence, nausea, and abdominal pain. Eighteen patients ( 17 zopolrestat, 1 placebo) were discontinued from the study due to increased plasma transaminases; all but one patient were asymptomatic, and the abnormalities reversed upon drug discontinuation. The results support a possible role for zopolrestat in the treatment of diabetic polyneuropathy.
123

A CONTROLLED TRIAL OF TOPICAL GLYCOPYRROLATE AS THE FIRST SPECIFIC TREATMENT FOR DIABETIC GUSTATORY SWEATING C.A. Abbott, J. Shaw, K. Tindall and A.J.M. Boulton. Department of Medicine, Manchester Royal Infirmary, Manchester M13 $9 \mathrm{WL}$, U.K.

Gustatory sweating (GS), a manifestation of autonomic neuropathy, is particularly common amongst diabetic patients with multiple complications, especially nephropathy. To date, however, there has been no specific treatment available for this condition. Topical application of the anti-muscarinic compound glycopyrrolate has been found to be effective in a single case report. To determine efficacy we thus performed a double blind crossover study of $0.5 \%$ topical glycopyrrolate versus placebo Fourteen diabetic subjects with a history of GS were recruited. Each treatment was given for 2 weeks separated by a one week washout. GS was measured by a sweat test performed at baseline, 2 and 5 weeks. Absorbent dressings were placed on the forehead, while the patient consumed a food previously shown to cause sweating. Sweating was measured by the increase in weight of the dressing over 20 minutes. Patients also kept a diary, in which GS was rated on a 0-10 scale for every meal by a diary score. The mean $\pm S D$ value for the sweat tests was significantly lower on glycopyrrolate $\left(1.77 \pm 2.18 \mathrm{mg} \mathrm{cm} \mathrm{cm}^{-2}\right)$ than at baseline (7.29 \pm 6.11$), p<0.02$, with no effect of placebo $(7.30 \pm 7.26)$. The mean $\pm S D$ diary score on glycopyrrolate $(0.72 \pm 0.57)$ was significantly lower than or placebo $(2.11 \pm 1.73), p<0.02$, as was the mean $\pm S D$ number of sweating episodes (diary score $>0$ ) (16.17 \pm 4.86 vs $27.75 \pm 14.16, p<0.01$. We conclude that topical glycopyrrolate is the first effective treatment for gustatory sweating.

\section{4}

THE NATURAL HISTORY OF DIABETIC NEUROPATHY IS GOVERNED BY THE DEGREE OF GLYCAEMIC CONTROL. A 10-YEAR PROSPECTIVE STUDY IN IDDM

D. Ziegler, R. Piolot, and F. A. Gries; Diabetes Research Institute at the Heinrich Heine University, Düsseldorf, Germany

There is little information available on the natural history of peripheral and autonomic neuropathy in patients with IDDM. In a prospective study we examined 32 newly diagnosed IDDM patients aged 12-36 years who were followed over 10 years. Motor and sensory nerve conduction velocity (MNCV, SNCV), coefficients of variation of heart rate variability at rest $\left(C V_{R}\right)$ and during deep breathing $\left(C V_{B}\right)$, and clinical assessment were performed at diagnosis, after 3 months as well as after $1,2,4,5,8$, and 10 years. Thermal discrimination and vibration perception thresholds (TDT, VPT) were measured from the second year onward. Mean $\left( \pm\right.$ SEM) $\mathrm{HbA}_{1}$ levels over 10 years within the normal range of $<8.3 \%(7.8 \pm 0.1 \%)$ were observed in 11 patients (Group 1), while mean $\mathrm{HbA}_{1} \geq 8.3 \%(9.9 \pm 0.3 \%)$ was found in 21 patients (Group 2). After 10 years, SNCV was significantly faster in Group 1 than in Group 2 in the median $(55.4 \pm 1.7$ vs $48.3 \pm 1.7 \mathrm{~m} / \mathrm{s}$ ), ulnar $(54.0 \pm 2.1$ vs $47.7 \pm 2.0 \mathrm{~m} / \mathrm{s})$, and sural nerve $(46.9 \pm 1.7$ vs $39.9 \pm 1.0 \mathrm{~m} / \mathrm{s})$ and $\mathrm{MNCV}$ was faster in the peroneal nerve $\left(48.9 \pm 1.0\right.$ vs $42.8 \pm 1.2 \mathrm{~m} / \mathrm{s}$ ) (all $p<0.05$ ). Likewise, $\mathrm{CV}_{\mathrm{R}}$ and $\mathrm{CV}_{\mathrm{B}}$ as well as TDT and VPT in the foot deteriorated significantly in Group 2 as compared with Group 1 (all $p<0.05$ ). The Neuropathy Disability Score was $0.56 \pm 0.24$ in Group 1 and $3.41 \pm 0.75$ in Group 2 after 10 years $(p<0.05)$. For most parameters, significant differences between the groups were noted first after only 1-2 years. Thereafter these differences increased continuously. After 10 years, none of the patients of Group 1, but 14 patients of Group 2 developed asymptomatic (stage $1 ; n=12$ ) or symptomatic (stage $2 ; n=2$ ) neuropathy $(p<0.05$ ). In conclusion, during the first decade after diagnosis of IDDM poor glycaemic control constitutes an essential determinant in the early development of neuropathy, but this process may be prevented by near-normoglycaemia. 


\section{5}

HYPERDYNAMIC CIRCULATION IN NORMOTENSIVE, INSULIN-RESISTANT OFFSPRING OF PARENTS WITH TYPE 2 DIABETES

K. Rett, F. Knerr, B. Balletshofer, E. Maerker, A. Burtscher, S. Feilmeier, M. Wicklmayr and H.U. Häring. University of Tübingen, IV. Med. Dept., Diabetes Research Institute at the Schwabing Hospital, Munich, Germany

Aim of the study: Ir the prospective MUPPED siudy (MUnich Primary Prevention and Early Detection) on insulin resistance, healthy subjects with parental history of type 2 diabetes are screened for symptoms of the insulin resistance syndrome (IRS) Subjects and methodss: 74 male and female subjects aged between 18 and 50 years (median 34) with at least one type 2 diabetic parent were selected. Entry criteria were normal casual blood pressure $(<140 / 90 \mathrm{mmHg}$ ) and non-diabetic oral glucose tolerance ( $2 \mathrm{~h}$ blood glucose $<11.1 \mathrm{mmol} / 1$ after $75 \mathrm{~g}$ oligosaccharides). 72 glucose tolerant subjects were enrolled and underwent the measurement of insulin sensitivity (isoglycemic hyperinsulinemic glucose-clamp). In 39 subjects, a complete $24 \mathrm{~h}$ ABPM profile was available. Results: $65 \%$ of the relatives were insulin-resistant (metabolic glucose clearance rate $\mathrm{MCR}<5.0 \mathrm{ml} \mathrm{kgLBM}^{-1} \cdot \mathrm{min}^{-1}$ ). Compared with the insulin-sensitive subjects, body mass index and body fat were higher. The metabolic pattern included higher fasting and postglucose serum insulin (AUC), and higher postglucose blood glucose (AUC). Mean systolic and diastolic ambulatory blood pressure (24h, daytime and nighttime) was not different between groups and was not cor-

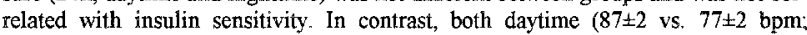
$\mathrm{p}<0.05)$ and nighttime heart rate $(75 \pm 2$ vs. $65 \pm 2 \mathrm{bpm} ; \mathrm{p}<0.05)$ as well as the nocturnal decline in diastolic pressure $(15.1 \pm 1.3$ vs. $9.7 \pm 1.7 \%, p<0.05)$ and daytime pulse pressure $(40 \pm 1$ vs. $34 \pm 2 \mathrm{mmHg}, \mathrm{p}<0.05)$ were higher in resistant offspring and correlated with insulin sensitivity. Conclusion: In young offspring of subjects with type 2 diabetes, prevalence of clamp-derived insulin resistance is surprisingly high. In addition to the known metabolic differences, insulin-resistant offspring already differ from their insulin-sensitive counterparts in ABDM-derived cardiovascular parameters like heart rate, puls pressure, and nighttime dipping. This pattern reveals a hyperdynamic circulation that might precede established hypertension

\section{6}

HYPERTENSION IS A PROMINENT FEATURE OF DIABETIC AUTONOMIC NEUROPATHY: A 10-YEAR FOLLOW-UP STUDY B. Krahulec, L. Strbová and I. Balažovjech. 2nd Department of Internal Medicine, Comenius University School of Medicine, Bratislava, Slovak Republic

Hypertension seems to have a close relationship to diabetic autonomic neuropathy (DAN). This abstract presents 10 -year data on blood pressure (BP), cardiovascular reflexes and QT dispersion (QTD /an indicator of ventricular arrhythmias/) in a cohort of 72 IDDM patients ( $38 \mathrm{M}, 34 \mathrm{~F}$; mean of age: 36.5 , confidence limits: 34.1-38.9 yrs.; diabetes duration 14.4, 11.9-16.9 yrs.) in comparison with the pertinent parameters at their entry into the study. The initial investigations with a battery of cardiovascular reflexes (deep breathing, active orthostasis, Valsalva maneuver) revealed 21 patients $(29.2 \%)$ with DAN. Patients with DAN had higher systolic BP $(149.6,140.7-158.5$ vs. $135,131.2-138.8 \mathrm{mmHg}, \mathrm{p}<0.005)$, higher heart rate $(92.8,85.7-99.8$ vs. $78.4,74.6-82.1$ beats $/ \mathrm{min}, \mathrm{p}<001$ ), prolonged QTc interval (462, $451-473$ vs. $435,443-447 \mathrm{~ms}$, $\mathrm{p}<0.005)$ and higher QTD $(50.9,41.6-60.4$ vs. $39.6,32.7-46.5 \mathrm{~ms}$, $\mathrm{p}<0.05$ ). $86.7 \%$ diabetics with DAN had hypertension or borderline hypertension in comparison to $36.1 \%$ of patients without DAN $(\mathrm{p}<0.05)$. QTD in hypertensive diabetics was significantly higher than in non-hypertensive patients $(63.3,46.4-80.1$ vs. 36.04, 29.6$42.5 \mathrm{~ms}, \mathrm{p}<0.001$ ). Double product (systolic BP x heart rate) correlated linearly with QTD $(r=0.43 ; n=42 ; \mathrm{p}<0.005)$. 42 diabetics were evaluated 10 years later. 8 of diabetics with DAN $/ 38.1 \%$ / and 1 diabetic without DAN $/ 2 \%$ / died during the observation period. All of them were hypertensive. The rest of the patients did not respond to our call. Systolic and diastolic BP, heart rate, QTc interval and QTD did not deteriorate significantly within the 10 year period. In summary, diabetics with DAN have a poor prognosis, which may be related to hypertension. As treatment of DAN is rather elaborate, effective lowering of blood pressure should be the main goal of pharmacological intervention in these patients.

MAGNETIC RESONANCE ANGIOGRAPHY AND RENAL ARTERY STENOSIS IN HYPERTENSIVE PATIENTS WITH TYPE 2 DIABETES

A.C.J.Robinson, C.Kong, C. Henzen, P.Pacy, P.Chong, W.Gedroyc ${ }^{a}$, R.S.Ekkeles and S.Robinson. Unit of Metabolic Medicine \& Department of Radiology ${ }^{a}$. St. Mary's Hospital Medical School, London

Patients with diabetes are at increased risk of renal artery stenosis (RAS) although the prevalence is unknown. Treatment with ACE inhibitors has proved beneficial in cardiovascular disease, the major cause of mortality in Type 2 diabetes (NIDDM). They are also usefu in diabetic nephropathy and hypertension. Thus many individuals with NIDDM would benefit from this class of drugs. However, ACE inhibitors may precipitate acute renal failure in the presence of RAS. We have used the previously validated technique of magnetic resonance angiography (MRA) to estimate the prevalence of RAS in hypertensive patients $(36 \mathrm{M}, 14 \mathrm{~F}$ : age $60 \pm 10$ years) with NIDDM who were considered for $A C E$ inhibition. All underwent $3 D$ phase contrast MRA with a 1.5 Tesla Signa scanner (General Electric Milwaukee). RAS was demonstrated in $14 \%(7 \mathrm{M})$. There were no significant differences between those with and without RAS with respect to age ( $64 \pm 11 \vee 59 \pm 10$ years), $\mathrm{HbA} 1 \mathrm{c}(8.7 \pm 1.9 \vee 7.6 \pm$ $1.2 \%)$, total cholesterol $(5.1 \pm 0.5 \times 5.7 \pm 1.6 \mathrm{mmol} / \mathrm{l})$, triglyceride $(1.7$ $\pm 0.8 \vee 2.4 \pm 1.9 \mathrm{mmo} / /)$, HDL $(1.2 \pm 0.6 \vee 1.1 \pm 0.3 \mathrm{mmol} / /)$ or creatinine $(153 \pm 43 \vee 131 \pm 61 \mu \mathrm{mol} / \mathrm{l})$. We conclude that RAS is relatively common in hypertensive patients with NIDDM. ACE inhibitors must be used with caution and appropriate monitoring in this group of patients.
INTRACELLULAR PARTITIONING OF GLUCOSE METABOLISM IN TYPE 2 DIABETIC SUBIECTS WITH AND WITHOUT HYPERTENSION BEFORE AND AFTER TREATMENT WITH LACIDIPINE OR LISINOPRIL. E. Bonora, G.Targher, M. Alberiche, R. Bonadonna, S. Uleri and M. Muggeo. Department of Endocrinology and Metabolism, University of Verona, Italy. Both type 2 diabetes mellitus and arterial hypertension are insulin resistant: states. In this study we quantitated total, oxidative and non-oxidative rates of glucose disposal, glycogen synthesis, total and non-oxidative glycolysis, as well as hepatic glucose production, Cori cycle and lipid oxidation, by using a 4 -h euglycemic $(-5 \mathrm{mM})$ insulin clamp $(-300 \mathrm{pM})$ in combination with dual tracer infusion $\left(1^{3} \mathrm{HI}-3\right.$-glucose and $I^{14} \mathrm{Cl}-\mathrm{U}$-glucose $)$ and indirect calorimetry in 20 normotensive diabetic (D) and 20 sex-, age- and BMImatched hypertensive diabetic (HD) nonobese $(B M I<30)$ patients. Subjects were studied twice: following a 4-week wash-out period and after a randomized, double-blind, double-dummy, 4-month period of treatment with $4 \mathrm{mg} /$ day lacidipine (a calcium-channel blocker) or $10 \mathrm{mg} /$ day lisinopril (a ACE-inhibitor) $(n=10$ for each treatment in each parallel group). Before treatment, no differences were found between the two groups in the basal rates of glucose metabolism, while significar.tly $(p<0.05)$ lower rates ( $\mu \mathrm{mol} / \mathrm{min} \cdot \mathrm{kg} \mathrm{FFM}$ ) of total $(17.4 \pm 0.9$ vs $28.5 \pm 3.0)$ and non-oxidative $(7.7$ \pm 0.9 vs $16.3 \pm 3.0$ ) glucose disposal and glycogen synthesis $(3.5 \pm 2.0$ vs 12.4 \pm 3.3 ) were found in $H D$ vs $D$ subjects during insulin infusion. In the whole group of subjects $(n=40)$ anti-hypertensive treatment resulted in a significant increase in total $(26.9 \pm 1.9$ vs $23.0 \pm 1.8)$ and non-oxidative $(16.7+1.7$ vs 12.2 $\pm 1.7)$ glucose disposal rates during insulin clamp $(p<0,01)$. The increase in glucose disposal rates was not substantially different in HD vs $D$ subjects, and in patients treated with lacidipine vs lisinopril. These results suggest that i) insulin resistance is more severe in hypertensive than in normotensive nonobese diabetic subjects, due to a more pronounced defect in non-oxidative glucose metabolism; ii) anti-hypertensive agents improve insulin-mediated non-oxidative glucose disposal, regardless of the presence of hypertension, and without any difference between lacidipine and lisinopril. 


\section{9}

EFFECT OF AMLODIPINE ON INSULIN SECRETION AND GLUCOSE TOLERANCE IN NIDDM: A DOUBLE BLIND STUDY. G. Meregalli, R. Mangili, R. Ghelardi, S. Scotto, A. Caumo, G. Pozza and A.E. Pontiroli. Istituto Scientifico San Raffaele, Università di Milano, Italy.

Amlodipine is a calcium channel blocker with a long-acting antihypertensive effect, without known major effects on glucose and insulin levels in normotensive or hypertensive patients. The aim of this study was to explore the effect of treatment with amlodipine on the glucose and insulin response to intravenous glucose tolerance test (IVGTT) in 15 patients ( 8 men, 7 women) with NIDDM and essential hypertension, receiving treatment with oral hypoglycaemic agents and/or diet. Their age was $62 \pm 4$ years, known duration of diabetes was $9 \pm 6$ years, and glycaemic control was stable. The study had a randomized, double-blind, placebo-controlled cross-over design, where each treatment phase lasted for 6 weeks. A 3-week washout was undertaken before each treatment phase. An IVGTT was perfomed before and at the end of each treatment period. Calculated Conard constants, reflecting glucose tolerance, remained unchanged during amlodipine ($0.00175 \pm 0.00012$ vs placebo $-0.00185 \pm 0.00020 \log \left[\mathrm{mg}^{\mathrm{d}} \mathrm{dl}-1\right] \cdot \mathrm{min}^{-1}$, mean $\pm \mathrm{SEM}, \mathrm{p}=\mathrm{ns}$ ). Insulin secretion, as evaluated by the calculation of areas under curve was also unaffected by amlodipine $(3,452 \pm 711$ vs placebo 3,481 $\left.\pm 605 \mathrm{~min} \cdot \mu \mathrm{U} \cdot \mathrm{ml}^{-1}, \mathrm{p}=\mathrm{ns}\right)$. Major blood lipids, glycacmic profiles and HbAlc levels also compared among treatments, whereas blood pressure was lower during amlodipine as expected (systolic: 132.2 \pm 19.7 vs $140.2 \pm 15.6 \mathrm{~mm} \mathrm{Hg}$, diastolic: $76.4 \pm 7.8$ vs $85.4 \pm 7.7 \mathrm{~mm}$ $\mathrm{Hg}, \mathrm{p}<0.05$ for both). We suggest that amlodipine represents a safe and effective antihypertensive treatment also in NIDDM patients, with no adverse effects on insulin secretion and glucose tolerance. This may be a perspectively relevant characteristic in clinical practice.

\section{OP 22 \\ Prediction of IDDM}

\section{0}

\section{ANTIBODIES TO TWO DISTINCT TYROSINE PHOSPHATASE-LIKE PROTEINS IN IDDM.}

C.J. Hawkes, C. Wasmeier, M.R. Christie and J.C. Hutton. Dept. of Medicine, King's College School of Medicine and Dentistry, London, UK; Dept. of Clinical Biochemistry, Addenbrooke's Hospital, Cambridge, UK; and Barbara Davis Center for Childhood Diabetes, Denver, Colorado, USA.

Antibodies in Type 1 diabetes (IDDM) recognize islet antigens detected as $40 \mathrm{kD}$ and $37 \mathrm{kD}$ tryptic fragments (40k- and $37 \mathrm{k}$-antigens) and the former of these has been identified as a protein tyrosine phosphatase (PTP)-like protein, IA-2/ICA512. A closely related PTP phogrin, was recently cloned by screening an insulinoma cDNA library with antibodies raised to insulin secretory granules. In this study we have analyzed reactivity of antibodies in IDDM to recombinant phogrin expressed either in E. coli or by in vitro transcription/translation of cDNA, and determined the relationship of the protein to the $37 \mathrm{k}$ antigen. Antibodies in IDDM bound to the cytoplasmic domain of phogrin, but not to the region of the molecule localized within the granule lumen. Trypsin treatment of immunoprecipitated phogrin generated $37 \mathrm{kD}$ fragments and the cytoplasmic domain of the protein blocked antibody binding to the $37 \mathrm{k}$-antigen expressed in insulinoma cells. Antibodies to the cytoplasmic domains of the two PTPS were determined in 60 recent onset IDDM patients, aged $0-14$ years. Of these $28(47 \%)$ were positive for antibodies to phogrin and $40(67 \%)$ for IA-2 antibodies; all but one of the phogrin antibody positive patients also possessed antibodies to IA-2. Sera from 43 age-matched control individuals were all negative for antibodies to both proteins. Addition of recombinant IA-2 did not completely block autoantibody binding to phogrin, indicating the presence of antibody epitopes unique to phogrin. The results demonstrate that two distinct PTP-like proteins are recognized by antibodies in IDDM and identify phogrin as the islet $37 \mathrm{k}$-antigen, antibodies to which has previously been shown to be strongly associated with IDDM in a number of populations.

\section{1}

GAD65-AUTOANTIBODIES ARE PREFERENTIALLY ASSOCIATED WITH THE HIGHEST HLA-DQ-LINKED RISK IN SIBLINGS OF IDDM-PATIENTS $C$ Vandewalle, J Vanacker, $K$ Ver Elst, $C$ Semakula, $C$ Van Schravendijk, $B$ Van der Auwera, $F$ Gorus and the Belgian Diabetes Registry; Diabefes Fesearch Center, Vrije Universiteit Brussel, BDR, Brussels, Belgium.

We have previously shown that GAD65-Ab are associated with HLA-DQlinked risk in adult, but not in young IDDM-patients. We investigated whether such age-restriction also existed in 485 Caucasian siblings of IDDM-patients, aged 0-39 yr. GAD65-Ab were assayed by a modified radiobinding assay using purified human recombinant 35 S-GAD65 for tracer. This allowed to express GAD65-Ab activity as \% tracer bound. Because autoantibody titers were not normally distributed in 387 healthy control subjects, age- and sexindependent cut-off values for GAD65-Ab-and IAA-positivity were calculated by ROC-curve analysis. Overall, $9 \%$ of siblings were positive for GAD65-Ab ( $\geq 1.7 \%$ tracer bound), $6 \%$ for IAA ( $20.7 \%$ tracer bound), $6 \%$ for low titer ICA ( $\geq 12$ JDF units) and $3 \%$ for high titer ICA ( $\geq 50$ JDF units). GAD65-Ab were positively associated with IAA and ICA, but not with age and sex. As previously shown in siblings for IAA and for high titer ICA, GAD65-Ab occurred most frequently in presence of the high risk DQA ${ }^{*} 0301$ DQB1 ${ }^{*} 0302 / D Q A 1{ }^{*} 0501-D Q B 1{ }^{*} 0201$ genotype $(23 \%)$, compared to $12 \%$ for DQA1*0301-DQB1*0302/non(DQA1*0501-DQB1*0201),6\% for DQA1*0501DQB1*0201/non(DQA1*0301-DQB1*0302) and $4 \%$ in absence of both risk haplotypes $(p<0.001)$. Siblings with the high risk genotype had significantly higher GAD65-Ab titers than the other subjects $(p<0.001)$. By analogy to previous results for IAA, GAD65-Ab were associated with DQA1*0301DQB ${ }^{*} 0302 / D Q A 1^{*} 0501-D Q B 1^{*} 0201$ in siblings under age 10 (33 vs $8 \%$ without, $\mathrm{p}=0.002$ ). At variance with IAA, but in accordance with findings in adult IDDM-patients, GAD65-Ab were also most frequently present in adult siblings (10-39 yr) with the high risk genotype (16 vs $7 \%$ without, $p<0.05$ ). Multivariate analysis confirmed that both GAD65-Ab prevalence and titers were associated with IAA, ICA and DQA1*0301-DQB1*0302/DQA1*0501 DQB1*0201, but not with age and sex. In conclusion, both in young (0-9 yr) and in adult siblings (10-39 yr), GAD65-Ab are preferentially associated with the high risk DQA1*0301-DQB1*0302/ DQA1*0501-DQB1*0201 genotype. 
COMBINED TESTING FOR ANTIBODIES TO GAD AND PTP-IA-2 IN FAMILY MEMBERS: CAN WE REPLACE ISLET CELL ANTIBODIES? PJ Bingley, AJK Williams, T Collins, M Shattock, GF Bottazzo, EAM Gale, St Bartholomew's and the Royal London School of Medicine, London, UK.

Islet cell antibodies (ICA) provide the basis for assessment of risk of IDDM in family members and recruitment into intervention trials, but are more difficult to assay on a large scale than antibodies to glutamate decarboxylase (GADA) and protein tyrosine phosphatase IA-2 (IA-2A). We compared the performance of GADA and IA-2A measured by radiobinding assay with that of conventional ICA in 1219 non-diabetic relatives of children with IDDM in a population-based prospective study. Antibodies were measured in initial samples collected from 737 parents and 482 siblings (median age at screening $32 \mathrm{yr}$ (range, 1.6-61) with median follow-up $6.6 \mathrm{yr}(0.2-10.5) .13$ relatives developed IDDM after 0.7-7.5 yr. Antibody levels were classified as low $(<97.5$ th centile of schoolchild controls), intermediate (97.5th-99.5th centile) or high ( $\geq 99.5$ th centile). ICA were intermediate in $3.5 \%$ and high in $1.7 \%$ relatives; GADA intermediate in $9.9 \%$ and high in $2.8 \%$, and IA-2A intermediate in $3.0 \%$ and high in $2.4 \%$. The cumulative risks of IDDM within 10 years in those with intermediate and high ICA were $7.5 \%$ ( 2 cases) and $49 \%$ ( 6 cases). The risks with intermediate and high GADA were $12 \%$ ( 3 cases) and $43 \%$ ( 8 cases), and with intermediate and high IA-2A were $2.1 \%$ ( 2 cases) and $26 \%$ ( 6 cases). The risk with low antibody levels was always $<1 \%$ ( $p<0.0001$ in logrank test for all antibodies). The risk in family members with 2 antibodies $\geq 97.5$ th centile was $21 \%$ ( 3 cases) and $67 \%$ in those with elevated levels of three antibody markers ( 6 cases). 56 family members had high levels of GADA or 1A-2A and 12 of this group developed IDDM giving overall risk $35 \%$. ICA $\geq 20 \mathrm{JDF}$ units gave the same risk but was found in only 32 family members, 7 of whom developed IDDM. We conclude that GADA offer the hest overall performance but sensitivity is improved without loss of specificity by testing in combination with ICA or IA-2A. A strategy combining GADA and IA2A should enhance the overall efficiency of screening and has the potential to replace ICA in the identification of family members at high risk of IDDM.
DEVELOPMENT OF A COMBINED GAD/A2-ANTIBODY-TEST SYSTEM IN VENOUS AND CAPILLARY BLOOD SAMPLES OF TYPE 1 AND PRE-TYPE-1 DIABETIC PATIENTS

J. Dittler, M. Hummel, U. Mollenhauer and A.-G. Ziegler. Diabetes Research Institute, Academic Hospital Schwabing, Munich, Germany

In order to facilitate population screening for islet cell antibodies we have developed a combined test system for the detection of GAD and IA2 Antibodies (GADIA2-combi) in venous and capillary blood samples. For this we tested 115 patients with newly diagnosed IDDM, 55 Antibody-positive $(\mathrm{Ab}+)$ relatives of IDDM patients and 101 normal controls. For the capillary blood measurement we collected and centrifuged 200ul blood out of the fingertip and used $6 \mu 1$ for each assay. The proportion of GAD and IA2 antigen was 1:1. Of 101 controls the normal range (defined as 99 . percentile) of our combi-test was 15 units (99. percentile of single GAD-test: 13 ; 99 percentile of single IA2-test: $5 \mathrm{u}$ ). Of 115 newly diagnosed patients (either $\mathrm{GAD}+$ or IA2 + or $\mathrm{GAD}+$ and IA2+) $90.4 \%(\mathrm{n}=104)$ were detected positive in the GADIA2-combi; $7.8 \%(\mathrm{n}=9)$ were tested false negative. In $\mathrm{Ab}+$ relatives $100 \%$ were correctly diagnosed. Thereby the combi-results correlated highly with single $\mathrm{Ab}$-titers of GADA ( $\mathrm{R}=0.41 ; \mathrm{p}<0.001)$ and IA2A $(\mathrm{R}=0.47$ $p<0.001)$. Interestingly combi-Ab-titers were significantly higher in patients and relatives who exhibeted both IA2A and GADA (mean $\pm \mathrm{SD}=78 \pm 30 \mathrm{u}$ $\mathrm{p}<0.006$ ) than in subjects who were positive for either GADA or IA2A (mean $\pm S D=41 \pm 21 ; p<0.006$ ). Furthermore we compared combi-test results of venous blood samples with results obtained in capillary blood (GADIA2-capcombi). We found a striking highly significant correlation between capillary and venous blood for positive and negative Antibody-titers $(R=0.956$ $\mathrm{p}<0.001$ ). This new screening approach may help to realise general population screening at an early age where venous blood sampling is difficult for prediction and prevention of type 1 diabetes.

\section{4}

GAD AND IA-2 ANTIBODIES MAY REPLACE ICA IN PRIMARY SCREENING FOR IDDM RISK ASSESSMENT IN RELATIVES E. Bosi E. Bazzigaluppi, M.R. Pastore, A. Balini, R. Bonfanti, N. Dozio, C. Belloni, S. Genovese, F. Meschi, and E. Bonifacio. San Raffaele Scientific Institute, University of Milan, Milan, Italy

slet cell antibodies (ICA) are the established marker for IDDM risk assessment. Several antigen-specific humoral markers have been identified and could replace the relatively labour-intensive ICA screening test. The aim of this study was to compare the effectiveness of antibodies to glutamate decarboxylase (GADA), insulin (IAA) and the protein tyrosine phosphatase-like IA-2 molecule $(\mid A-2 A)$ as screening tests in first degree relatives of IDDM patients. ICA detected by immunofluorescence and quantified in JDF units, GADA and IA-2A measured by immunoprecipitation of ${ }^{35} \mathrm{~S}$-labelled in vitro translated proteins and IAA, measured by a radiobinding assay were determined in 806 first degree relatives (470 parents, 336 siblings) participating in the prospective San Raffaele IDDM family study within the Lombardy region, and in 100 new onset IDDM cases from the same region. Threshold for antibody assays was calculated at the 99 th centile of control samples. Elevated levels of ICA were detected in $24(3 \%)$, GADA in $41(5.1 \%)$, IA-2A in $17(2.1 \%)$ and IAA in $30(3.7 \%)$ relatives. Each antibody was more prevalent in siblings than in parents. At least one antibody was detected in $83(10.3 \%)$ relatives: $67(8.3 \%)$ had one, $7(0.9 \%)$ had two, $5(0.6 \%)$ had three and $4(0.5 \%)$ had all four antibodies. Of the 16 relatives having two
or more antibodies, 13 were siblings. Over a maximum follow-up of 7 years, 5 relatives ( 4 siblings and 1 parent) developed IDDM: all had GADA 4 had ICA 2 had IA-2A and 2 had IAA; 4 had two or more antibodies. Amongst new onset IDDM cases, ICA were detected in $88 \%$, GADA in $70 \%$, IA-2A in $54 \%$, and either GADA or IA-2A in $90 \%$. The findings suggest that GADA in combination with IA-2A may replace ICA as the primary screening test for IDDM risk assessment in first degree relatives. 


\section{Mechanisms of Beta-Cell Stimulus - Secretion Coupling}

\section{5}

ISLET GLYCOLYSIS ENSURES AN EFFICIENT COUPLING OF PYRUVATE OXIDATION TO OXIDATIVE PHOSPHORYLATION. J. Tamarit-Rodriguez, E. Giné and $O$. Alcázar. Department of Biochemistry, Complutense University, Madrid-28040, Spain.

Pyruvate is substantially metabolized in islets but it does not stimulate secretion. This raises questions about the specific role of mitochondrial ATP-production and the absolute need of a glycolytic mediator in glucoseinduced B-cell activation. Islet metabolism of glucose and pyruvate were comparatively studied: Their mitochondrial oxidation rates (pmol/islet $x$ hour) were measured as the rotenone $(10 \mu \mathrm{mo} / \mathrm{l})$ sensitive production of ${ }^{14} \mathrm{CO}_{2}$ from the corresponding $\left[{ }^{14} \mathrm{C}\right]$ substrates and the respective degree of coupling to oxidative phosphorylation was evaluated by the percentage decrease induced by oligomycin $(2 \mu \mathrm{g} / \mathrm{ml})$. Their rates of anaerobic metabolism were calculated according to the lactate accumulated in the medium, measured with a HPLC-method. Glucose oxidation was 4-fold higher at 20 compared to $3 \mathrm{mmol} / \mathrm{l}$ and it was decreased within $60-70 \%$ by oligomycin and rotenone $(9.3 \pm 0.5, n=28 ; 4 \pm 0.4, n=9 ; 2.8 \pm 0.2, n=7)$. Pynuvate oxidation increased dose-dependently in the range 1 to $20 \mathrm{mmo} / \mathrm{l}$ and it was decreased by rotenone within $65 \%$. However, oligomycin did not affect the oxidation rate at $1 \mathrm{mmol} / 1(1.2 \pm 0.1, \mathrm{n}=9$ vs. $0.9 \pm 0.1, \mathrm{n}=6$; NS $)$ and reduced it within only $30 \%$ at $10 \mathrm{mmol} / 1(7 \pm 0.3, \mathrm{n}=10$ vs. $5 \pm 0.2, \mathrm{n}=11$; $\mathrm{P}<0.001$ ). Leucine-, but not glutamine-,oxidation was also sensitive to oligomycin. Whereas $20 \mathrm{mmol} / \mathrm{l}$ glucose was converted to lactate $(26.6 \pm 1,1, \mathrm{n}=11)$, pyruvate did not increase it above the level recorded at 0mmol/l ( $4.8 \pm 1.3, n=7$ vs. $6.5 \pm 0.5, n=7$; NS). Glucose, but not pyruvate, stimulated insulin secretion. Exogenous pyruvate is metabolized differently than that endogenously derived from glucose : Islet glycolysis might ensure a correct channelling of pyruvate into well coupled mitochondria.

137

\section{CYTOCHROME P450 CYP4A EXPRESSION IN PANCREATIC ISLETS OF LANGERHANS}

C.R Barnett, J. Clarke and P.R. Flatt. School of Biomedical Sciences, University of Ulster, Coleraine, Northern Ireland, U.K.

Male Wistar rats $(n=40)$ were treated with sodium clofibrate $(100 \mathrm{mg} / \mathrm{kg}$, intraperitoneal injection, 3 days), a specific inducer of CYP4A proteins. CYP4A expression within pancreatic islets was determined by immunocytochernistry and Western blotting. CYP4A protein levels were inducible ( 2 -fold) by sodium clofibrate pretreatment. The effects of CYP4A expression on insulin secretory responsiveness was investigated using collagenase isolated islets. Insulin release from control islets incubated with $3 \mathrm{mmol} / \mathrm{l}$ glucose (basal) was $1.2 \pm 0.05$ $\mathrm{ng} / \mathrm{islet} / \mathrm{hr}$ (mean sem, $\mathrm{n}=6$ ). Incubation with $16.7 \mathrm{mmol} / \mathrm{l}$ glucose, $25 \mathrm{mmol} / \mathrm{l} \mathrm{KCl}$ or $100 \mu \mathrm{mol} / \mathrm{l}$ arachidonic acid caused a $4.9,9.0$, and 4.8 fold increase in insulin release $(\mathrm{p}<0.001)$. Forskolin $(25 \mu \mathrm{mol} / \mathrm{l})$ or phorbol 12 - myristic 13 -acetate $(10 \mathrm{nmol} / \mathrm{l})$ potentiated glucose-induced insulin release 1.3 and 1.6 fold $(p<0.01)$ whereas adrenalin $(1 \mu \mathrm{mol} / 1)$ caused a 4.3 fold decrease $(\mathrm{p}<0.001)$. Islets from sodium clofibrate pretreated animals displayed similar responsiveness except for arachidonic acid. Insulin release in response to arachidonic acid at $3 \mathrm{mmol} / \mathrm{l}$ glucose was enhanced 6.0 fold by sodium clofibrate pretreatment ( $\mathrm{p}<0.001$ compared to control islets). Sodium clofibrate pretreatment did not cause any significant differences in plasma glucose (control : $8.2 \pm 0.2$; sodium clofibrate : $8.8 \pm 0.3$ ) or total insulin content (control : $81.8 \pm 1.2 \mathrm{ng} /$ islet; sodium clofibrate: $79.7 \pm$ $2.4 \mathrm{ng} / \mathrm{islet}$ ). Preincubation of islets in a range of concentrations of sodium clofibrate $(1 \mathrm{mmol} / \mathrm{l}-10 \mathrm{mmol} / \mathrm{l})$ did not alter insulin output in response to $3 \mathrm{mmol} / \mathrm{l}$ glucose or $16.7 \mathrm{mmol} / \mathrm{l}$ glucose $(1.4 \pm 0.1$ or 5.3 $\pm 0.1 \mathrm{ng} / \mathrm{islet} / \mathrm{hr})$. These studies demonstrate CYP4A expression within the pancreatic islets alters insulin secretory responsiveness.
136

INACTIVATION OF B-CELL PROTEIN PHOSPHATASES AND CARNITINE PALMITOYLTRANSFERASE 1 BY GLYCOLYTIC AND KREBS CYCLE INTERMEDIATES, ATP, GTP, GLUTAMATE, AND SULFONYLUREA.

$\AA$. Sjöholm 1, G.A. Cook2, and R.E. Honkanen ${ }^{3}$. 1) Dept. of Molecular Medicine, Karolinska Institute, Stockholm, Sweden. 2) Dept. of Pharmacology, University of Tennessee, Memphis TN, U.S.A. 3) Dept. of Biochemistry, University of South Alabama, Mobile AL, U.S.A.

The two aims of this study were: 1) To investigate effects of glucose metabolites on $\beta$-cell serine/threonine protein phosphatase (PP) activities; and, 2) to study effects of sulfonylurea on B-cell carnitine palmitoyltransferase 1 (CPT-1) activity. PP and CPT-1, whose expression we demonstrate in B-cells, may be key enzymes controlling B-cell insulin secretion through their effects on protein phosphorylation and fatty acid oxidation, respectively. 1) Fructose-1,6-bisphosphate, 3-phosphoglycerate phosphoenolpyruvate, oxalate, citrate, glutamate, ATP and GTP dose-dependently inhibited B-cell PPase activities when added to homogenates of clonal $\beta$-cells (RINm5F). 2) Addition of glibenclamide to homogenates of rat islets produced a dose-dependent inhibition of CPT-1 activity, mimicking that of malonyl-CoA. We suggest 1) that certain glucose metabolites generated in glycolysis and the Krebs cycle may directly activate the stimulus-secretion coupling by inhibiting PP, thereby contributing to inducing a hyperphosphorylated state of $B$-cell proteins; and 2) that the insulinotropism of glibenclamide may in part be explained by inactivation of 1 -cell CPT-1 with the attendant switch from fatty acid s-oxidation to generation of long-chain acyl-CoA esters that may lead to protein kinase $C$ activation.

\section{8}

NITRIC OXIDE (NO) MEDIATES THE FIRST PHASE OF GLUCOSE STIMULATED INSULIN SECRETION FROM PANCREATIC ISLETS.

H. Laffranchi', C. Richter ${ }^{2}$ and G. A. Spinas' ${ }^{1}$ 'Div. of Endocrinology and Metabolism, University Hospital, 8091 Zürich; ' Lab. of Biochemistry I. ETH Zürich, 8092 Zürich, Switzerland.

Nitric oxide (NO) acts as second messenger in a variety of celi types. There is substantial evidence to suggest that NO could also be involved in the insulin secretory pathway of pancreatic $\beta$-cells. In this study the effect of NO on acute glucose-stimulated insulin secretion from isolated islets of Langerhans was investigated. Islets were isolated from the pancreas of newborn rats (Zur-SD) and precultured in RPMI-1640 for 5-6 days. Islets were then transferred to a perifusion chamber $(300 \mu)$ ) and perifused with Krebs-Ringer buffer (KRHB) at a flow rate of $600 \mu \mathrm{l} / \mathrm{min}$. After an equilibration period of $45 \mathrm{~min}$ in $\mathrm{KRHB}+1.6 \mathrm{mM}$ glucose, $0.5 \mathrm{mM}$ L-methylarginine (LNMMA), 0.5mM D-methylarginine (D-NMMA), $10 \mu \mathrm{M}$ carboxy-PTIO, or $3 \mu \mathrm{M}$ $O D Q$, respectively, was added to the buffer. Glucose concentration in the perifusion medium was kept at $1.6 \mathrm{mM}$ for another $10 \mathrm{~min}$, was then raised to $16 \mathrm{mM}$ for $40 \mathrm{~min}$ and was finally reduced to $1.6 \mathrm{mM}$ for $15 \mathrm{~min}$. The increase in glucose concentration resulted in a typical biphasic insulin secretion from control islets. The NO synthase inhibitor L-NMMA caused a marked decrease of the first phase insulin secretion (FPIR): area under the curve (AUC) $12.9 \pm 2.8 \mathrm{pg} /$ islet $\times$ min vs. $25.9 \pm 3.6 \mathrm{pg} /$ islet $x$ min in control islets $(p<0.01)$. The second phase was not influenced by L-NMMA: AUC $24.7 \pm 3.1 \mathrm{pg} / \mathrm{islet} x \mathrm{~min}$ and $26.0 \pm 8.3 \mathrm{pg} / \mathrm{islet} \mathrm{x}$ min in control and L.NMMAexposed islets, respectively. Perifusion with the inactive enantiomer D-NMMA showed no significant difference as compared with control islets. Addition of carboxy-PTIO, a NO scavenger, to the perifusion medium significantiy inhibited FPIR without affecting the second phase: FPIR AUC $33.7 \pm 6.5 \mathrm{pg} / \mathrm{islet} x \mathrm{~min}$ in controls vs. $21.8 \pm 8.0 \mathrm{pg} / \mathrm{sislet} \mathrm{x}$ min with carboxyPTIO $(p=0.05)$; second phase AUC $41.7 \pm 23.4 \mathrm{pg} / \mathrm{islet} x$ min in controls vs. $40.3 \pm 21.9 \mathrm{pg} /$ islet $x \min$ with carboxy-PTIO. Incubation with the guanylate cyclase inhibitor ODQ had no influence on glucose-stimulated insulin release. These results show that endogenously formed NO is at least par'; involved in the signal transduction of FPIR and that NO does not stimulate insulin secretion via the activation of guanylate cyclase. 
CYCLIC AMP STIMULATES EXOCYTOSIS IN MOUSE B-CELLS BY BOTH PKA-DEPENDENT AND PKA-INDEPENDENT MECHANISMS.

L.Eliasson', E.Renström², C.Ämmälä' and P.Rorsman'2, 'Department of Medical Biophysics,

University of Göteborg, Sweden and ${ }^{2}$ Islet cell physiology, Novo Nordisk A/S, Denmark

Cyclic AMP (cAMP) potentiates glucose-stimulated insulin secretion through a PKAdependent pathway. To further investigate the properties of CAMP-dependent modulation of insulin secretion, we have used whole-cell capacitance measurements. Under control conditions a $500-\mathrm{ms}$ voltage-clamp depolarisation from $-70 \mathrm{mV}$ to $0 \mathrm{mV}$ elicited a capacitance increase of $32 \pm 17 \mathrm{fF}$, corresponding to the exocytosis of 15 secretory granules. Inclusion of Sp-cAMPs $(0.1 \mathrm{mM})$ in the pipette solution dialyzing the cell interior potentiated depolarization-evoked exocytosis and a capacitance increase of 313 $195 \mathrm{fF}$ $(\mathrm{P}<0.02)$ was observed. This effect of Sp-cAMPs could not be prevented by the PKAinhibitor Rp-cAMPs and in the simultaneous presence of $0.1 \mathrm{mM} \mathrm{Sp-cAMPs}$ and $0.5 \mathrm{mM}$ Rp-cAMPs the capacitance increase amounted to $246 \pm 126 \mathrm{fF}$. By contrast, Rp-cAMPs effectively prevented forskolin-stimulated exocytosis evoked by a train of $6-8$ depolarisations. The dynamics of the cAMP action was further investigated in experiments using photorelease of caged cAMP and $\mathrm{Ca}^{2+}$. Simultaneous release of both $\mathrm{CAMP}$ and $\mathrm{Ca}^{2+}$ resulted in biphasic stimulation of exocytosis: a rapid $(<1 s)$ stimulation followed by a secondary acceleration seen $>10$ seconds after the application of cAMP. The late effect of cAMP was further investigated in experiments where $\left[\mathrm{Ca}^{2+}\right]_{i}$ was clamped at $2 \mu \mathrm{M}$ by infusion of $\mathrm{a} \mathrm{Ca}^{2+}$-EGTA buffer. Under these conditions, addition of $\mathrm{CAMP}$ had a strong stimulatory action and the average rate of capacitance increase rose from $4 \pm 1 \mathrm{fF} / \mathrm{s}$ to $47 \pm 13$ $\mathrm{fF} / \mathrm{s}(\mathrm{P}<0.01)$. This effect was effectively prevented by Rp-cAMPs and in the presence of the antagonist, the rate of exocytosis had fallen to $5 \pm 2 \mathrm{fF} / \mathrm{s}(\mathrm{P}<0.01)$. These data suggests that CAMP stimulates exocytosis in B-cells by dual mechanisms: 1) by a PKA-independent mechanism exerted at a distal level, possibly involving the interaction between CAMP and a receptor; and 2) by an $\mathrm{PKA}$-dependent effect involving acceleration of granule mobilization thus promoting the refilling of the readily releasable pool of granules.

\section{OP 24 \\ Signal Transduction}

\section{0}

$\alpha_{2}$-HSG IS AN ALLOSTERIC INHIBITOR OF THE INSLILIN RECEPTOR TYROSINE KINASE.

G. Grunberger, D.D. Deutsch, M.A. Leon and P.R. Srinivas

Wayne State University School of Medicine, Detroit, Michigan, U.S.A.

Human plasma $\alpha_{2}-\mathrm{HS}$ glycoprotein (HSG) inhibits insulin-stimulated insulin receptor tyrosine kinase activity (IR-TKA) and DNA synthesis. It does not inhibit insulin binding nor insulin-stimulated metabolic responses. To characterize the molecular basis for these findings, we explored whether the inhibitor binds directly to IR and the nature of its inhibition. IR were autophosphorylated with $\left[{ }^{33} \mathrm{P}\right]-\mathrm{ATP}$ in presence or absence of $\alpha_{2}-\mathrm{HSG}$ and immunoprecipitated with anti- $\alpha_{2}$-HSG Ab. Autoradiography showed a single band at $95 \mathrm{kD}$, corresponding to the IR $\beta$-subunit. Next, $\left.{ }^{125} \mathrm{I}\right]$-labeled $\boldsymbol{\alpha}_{2}$-HSG was incubated with $I R$, separated by gel-sieving chromatography, and radiochromatographs were constructed from the elution profiles. The binding data again suggest that $\alpha_{2}$-HSG binds to the IR. Kinetic studies were done to measure the inhibitory effect of $\alpha_{2}$-HSG on IR-TKA as the substrate concentrations (ATP or poly GT) were varied. Neither the velocity $v$ s. substrate concentration curves of human IR-TKA (in the presence or absence of $\alpha_{2}$-HSG) nor Lineweaver-Burke plots were linear. The data fit well to the Monod Wyman Changeaux equation for an allosteric dimer. The kinetic data, linearly transformed using the logarithmic form of the Hill equation, show that $\alpha_{2}-\mathrm{HSG}$ does not alter the Hill coefficient. $\alpha_{2}-\mathrm{HSG}$ inhibits the insulin effect on IRTKA by reducing the $V_{\text {max }}$ of the IR-TKA in a concentration-dependent manner and by increasing the insulin-stimulated $S_{0.5}$ for ATP and poly GT. It has been hypothesized that insulin-stimulated IR serine kinase activity (SKA) might mediate insulin's metabolic responses. $\alpha_{2}$-HSG did not affect IR-SKA in contrast to its complete inhibition of IR-TKA. This finding is consistent with our data in that $\alpha_{2}$-HSG inhibits the insulin-induced mitogenic but not metabolic signaling of IR. In conclusion, $\alpha_{2}-H S G$ may bind directly to IR and function as an allosteric inhibitor of IR-TKA.

\section{1}

REAL-TIME IMAGING OF THE ACTIVATION BY GLUCOSE OF THE LPYRUVATE KINASE PROMOTER IN SINGLE CLONAL $\beta$ CELLS.

Guy A. Rutter and Axel Kahn*. Department of Biochemistry, School of Medical Sciences, University of Bristol, Bristol, BS8 1TD, U.K. *Institute Cochin de Génétique Moléculaire, 24 rue de Fauberg St-Jacques, 75104, Paris, France

The L-pyruvate kinase (L-PK) gene is expressed solely in the liver and in islet $\beta$ cells. Elevated glucose concentrations increase the expression of the gene in both tissues, though the intracellular signalling mechanisms involved are unclear ${ }^{1}$. An LA box consisting of two $5^{\prime}$ CACGGG3' motifs and located at -157 base pairs upstream of the transcriptional start site, has been implicated in the effects of glucose. Mutations within this motif, which binds a transcription factor termed USF (Upstream Stimulatory Factor) prevent induction of the gene. In order to investigate the intracellular signalling pathways which may participate in the transciptional activation of the L-PK gene we have developed a non-invasive technique for analysing the expression of the gene in single living cells. Signals are normalised to the expession of a second, independent, gene within the same cell. We subcloned the $-183-+10$ region of the L-PK gene upstream of the firefly luciferase $\left(\mathrm{luc}_{\mathrm{C}}\right)$ gene. This construct was co-microinjected into clonal B-cells (INS1) with a second plasmid in which the cytomegalovirus (CMV) promoter drove expession of Renilla reniformis luciferase $\left(\mathrm{luc}_{\mathrm{ren}}\right)$. Cells were then cultured for $24 \mathrm{~h}$ at varying glucose concentrations. Firefly and renilla luciferase activities were monitored independently by sequential addition of the cofactors of these distinct enzymes, luciferin and coelenterazine. Photon release from single living cells was imaged and quantified with an intensified charge-coupled device camera (Hamamatsu Photonics) attached to an inverted microsope (Zeiss TV100; $10 \mathrm{x}$ objective). In three independent experiments an increase in glucose concentration from 3 to $30 \mathrm{mM}$ increased the ratio of luc :luc ren $_{\text {from }} 0.025 \pm 0.003$ (mean \pm S.E.M., $n=56$ cells) to $0.27 \pm 0.08$ ( $n=$ 62 cells), corresponding to an 11-fold activation of the L-PK gene promoter. By allowing the microinjection of a wide variety of molecules (CDNA, antisense RNA, antibodies, etc.) this single cell approach will facilitate the dissection of the signalling pathways responsible for L-PK gene induction by glucose. 'Vaulorth S. and A. Katn. 1994. FASEB Joumal 8:28-35. 
142

THIS ABSTRACT HAS BEEN WITHDRAWN BY THE AUTHOR.
143

INSULIN-INDUCED ACTIVATION OF MAP KINASE INVOLVES GRB-2, SHPTP2, PI-3 KINASE AND PLC $\gamma$

K. Seedorf, L. Juhl, L. Hansen, L. Nielsen and L. Mosthaf, Department of Molecular Signaling, Hagedorn Research Institute, Niels Steensens Vej 6, DK-2820 Gentofte. Treatment of cells with insulin results in activation of signal transduction pathways involved in metabolic as well as mitogenic responses. The MAP kinase (MAPK) pathway is in this context of considerable importance. Several signalling cascades can result in MAPK activation, the exact pathway of insulin induced MAPK activation is however unclear. In this study we investigated whether GRB2, SHPTP2, PI-3 kinase, PLCY and various PKC isoforms contribute to insulin-mediated activation of MAPK. Using transiently transfected A-293 fibroblasts overexpressing the respective wildtype (wt) or mutant (mu) proteins as a model system, MAPK mediated phosphorylation of GST-Elk substrate was determined using a PhosphoImager software.

Insulin treatment of untransfected 293 cells results in a transient activation of MAPK which peaks at $10 \mathrm{~min}$ and declines within $60 \mathrm{~min}$ to almost basal levels. To study the involvement of GRB2 and SHPTP2 in insulin mediated activation of MAPK we determined the effect of the GRB2 SH2 domain mutant R86A and the catalytically inactive SHPTP2 mutant C459A on MAPK activity. Both mutants reduce MAPK activation dramatically, Overexpression of wt GRB2 has no effect, while SEPTP2 positively regulates MAPK activation in an expression level dependent manner. Overexpression of wt PLCy results in a minor inhibition, while the lipase inactive mutants PLC $\gamma \mathrm{H} 335 \mathrm{~F}$ and $\mathrm{H} 380 \mathrm{~F}$ inhibit MAPK activity to a much further extend. We next constructed a p85 PI-3 kinase subunit mutant that fails to interact with the catalytic p110 subunit. This mutant inhibits MAPK activity in a dose-dependent manner. Since the effects of PI-3 kinase and PLC $\gamma$ are most likely mediated by certain PKC isoforms, we constructed kinase negative mutants of certain PKC isoforms. While overexpression of kinase inactive PKC $\zeta$ clearly has a positive influence on MAPK activation, PKC $\alpha, \beta 1$ and $\beta 2$ mutants are only slightly inhibitory. The wt PKCs $\alpha, \beta 1$ and $\zeta$ show no effect, while PKC 22 enhances the insulin mediated activation of MAPK 2-fold. In summary, our data indicate that MAPK is regulated by various signaling components, which include GRB-2, SHPTP2, PI-3 kinase and most likely PLC $\gamma$. How PI-3 kinase, PLCy and the various PKC isoforms are involved in insulin-mediated MAPK activation is currently under investigation.

\section{4}

INSULIN ACTIVATES MAP KINASE IN 3T3-L1 ADIPOCYTES VIA RAS-INDEPENDENT PATHWAY.

B. Draznin, K. Carel, and J.W. Leitner, UCHSC and VAMC, Denver, CO, USA.

Insulin and other growth factors are potent activators of the p21RasMAP kinase pathway. We have recently observed that in

differentiated 3T3-L1 adipocytes (A), but not in 3T3-I 1 fibroblasts (F), PI-3 kinase and PKC constitutively inhibit p120 GTPase activating protein (GAP) allowing insulin to stimulate p21Ras $\bullet$ GTP loading via its activation of SOS. To further characterize tissue-specific differences in insulin signaling, we compared the mechanisms of MAP kinase activation by insulin in the mitogenically active $F$ with the metabolically active $A$. In both cell lines, insulin significantly increased p21Ras $\bullet$ GTP loading ( $\approx 2-2.5$ fold) and activated ERKI/ERKII and $\mathrm{p} 38 \mathrm{MAP}$ kinases ( 3 fold in $\mathrm{F}$ and 5 fold in A). Inhibition of Ras farnesylation with lovastatin prevented activation of p21Ras in both $F$ and $A$. In $F$, this was accompanied by an inhibition of the stimulatory effect of insulin on MAP kinase. In contrast in A, despite an inhibition of activation of p21Ras by lovastatin, insulin continued to stimulate MAP kinase activity ( 7 fold). Also, despite a complete inhibition of activation of p21Ras achieved by PKC depletion, (a maneuver that activates GAP that hydrolyses GTP in A), insulin stimulated MAP kinase in these cells. Transfection of $F$ with a dominant-negative Ras completely blocked insulin's ability to activate MAP kinase. These results demonstrate an important distinction between the mechanism of insulin signaling in the metabolically and the mitogenically active cells. Insulin activates MAP kinase by the Ras-dependent pathway in the fibroblasts and by the Ras-independent pathway in the adipocytes. 


\section{Nephropathy}

\section{5}

RENAL HAEMODYNAMIC FACTORS AND INCIPIENT UROPATHY IN IDDM PATTENTS WTTHOUT NEPHROPATHY : A 7-YEAR PROSPECTIVE STUDY

J.Y Poirier A Moisan, J. Le Cloirec, D. Maugendre, P. Bourguet, H. Allannic. Service des Maladies Métaboliques, Service de Médecine Nucléaire- Rennes- France.

Aim : To determine the predictive value of glomerular filtration rate (GFR), 24h.albumin excretion rate (AER), blood pressure (BP) for diabetic nephropathy. Methods Prospective study (mean follow-up: $7 \pm 1.6 \mathrm{yrs}$ ) including 90 IDDM patients (age: $33 \pm 9$ yrs duration of diabetes: $11 \pm 7 \mathrm{yrs}$ AER: $6.5 \pm 8 \mu \mathrm{g} / \mathrm{mn}$ $\mathrm{BP}<15090$ ). AER was determined cach year; GFR (99mTc-DTPA), Renal Plasma Flow (RPF, $131_{\mathrm{I}}$-Hippuran), Filtration Fraction (FF $=$ GFR/RPF) and separate kidney function were measured at baseline, two or three years later and at the endpoint. Results $1^{\circ}$ ) At baseline, 38 patients were hyperfiltering with GFR $\geq 158$ $\mathrm{ml} / \mathrm{mn} / 1.73 \mathrm{~m}^{2}$ (mean GFR $+2 \mathrm{SD}$ of 42 controls). As compared to the 52 normofiltering patients, their age, duration of diabetes, HbA1c, AER were not different, while RPF and FF were higher $\left(p<10^{-3}\right)$. A correlation between GFR and RPF was only found in the normofiltering group $\left(t=0.56 \mathrm{p}<10^{-4}\right)$. Slow intrarenal excretion was observed in 53 diabetic patients and 2 controls ; asymmetric $99 \mathrm{~m} \mathrm{Tc}$ renal uptake was found in 10 diabetics but not in controls. $2^{\circ}$ ) At the end-point : Six patients had developed permanent microalbuminuria. Their initial BP was higher $(\mathrm{p}<0.01)$ but their initial GFR was not different from those with permanent normoalbuminuria. In the hyperfiltering group, GFR and FF decreased (173 \pm 13 at baseline vs $137 \pm 24$ and $29 \pm 4.36$ vs $23.51 \pm 3.56 ; p<10^{-4}$ ) without relationship with mean HbA1c; RPF was unchanged and correlated with GFR $\left(\mathrm{r}=0.61 \mathrm{p}<10^{-4}\right)$. In the normofiltering group, GFR and RPF did not changed ( $130 \pm 15$ vs $129 \pm 23$; $521 \pm 88$ vs $546 \pm 121$ ). Slow intrarenal excretion and asymmetric renal uptake were strickly unchanged. Conclusions. In this 7 -year prospective study a) Hyperfiltration does not predict permanent microalbuminuria b) Increased GFR returns to normal values irrespective of the mean $\mathrm{HbAl} \mathrm{c}$ c) Elevated blood pressure may predict permanent microalbuminuria d) "Incipient" uropathy is frequent and more constant than hyperfiltration.

\section{7}

THE EFFECT OF LISINOPRIL ON ALBUMIN EXCRETION RATE IN NONHYPERTENSIVE INSULIN DEPENDENT DIABETES (IDDM) PATIENTS.

The EUCLID Study Group. EUCLID Study Group Co-ardinating Centre, EURODIAB, Department of Epidemiology and Public Health, University College London, WC1E 6BT, UK

ACE inhibitors slow the decline of renal function in macroaibuminuric IDDM patients, but their role in early renal disease is less clear. We performed a randomised, double-blind placebo controlled trial of lisinopril (10-20 mg daily) in 530 men and women with IDDM (20-59 years) with normo- or microalbuminuria. Patients, recruited from 18 European centres, were not on anti-hypertensive treatment, and had a resting blood pressure between $\geq 75$ and $\leq 90 \mathrm{mmHg}$ diastolic (DBP), and $\leq 1.55 \mathrm{mmHg}$ systolic at entry. Primary endpoint was change in albumin excretion rate (AER), assessed by two overnight urine collections at baseline, $6,12,18$ and 24 months. There were no differences in patient chas 7.4 $\mu \mathrm{g} / \mathrm{min}$ and $13 \%$ (placebo), and $7.0 \mu \mathrm{g} / \mathrm{min}$ and $17 \%$ (lisinopril). After one month, $\mathrm{DBP}$ was $3 \mathrm{mmHg}$ lower on lisinopril $(\mathrm{p}=0.0001$ ). At two years, AER was $18.4 \%$ lower on lisinopril ( $95 \%$ CI 1.1,32.6, $\mathrm{p}=0.04$, adjusted for baseline AER and centre), absolute treatment difference $2.13 \mu \mathrm{g} / \mathrm{min}$. Treatment effect differed by baseline albuminuric status ( $\mathrm{p}=0.04$ for interaction). Lisinopril reduced AER over two years in microalbuminurics by $46 \%(95 \% \mathrm{CI}-24.0,76.5, \mathrm{p}=0.1)$, in normoalbuminurics by $13.1 \%(95 \% \mathrm{CI}-1.7,25.7, \mathrm{p}=0.1)$, absolute treatment differences 27.97 and $1.09 \mu \mathrm{g} / \mathrm{min}$. For patients who completed 24 months, final AER for those on lisinopril was $34.7 \mu \mathrm{g} / \mathrm{min}$ lower in those with microalbuminuria at baseline $(\mathrm{p}=0.003)$, and $0.43 \mu \mathrm{g} / \mathrm{min}$ in those with normoalbuminuria at baseline $(p=0.4)$. Adverse events were similar in both groups, including hypoyglycaemic events (placebo 11, lisinopril 10), but excepting cougl (placebo 7 , lisinopril 18). We conclude that lisinopril slows progression of renal disease in non-hypertensive IDDM patients, with greatest benefit in those with microalbuminuria
146

CYCLOSPORINE NEPHROTOXICITY IN INSULIN-DEPENDENT DIABETIC PATIENTS (IDDM): AN EIGHT YEARS FOLLOW-UP

H.-H. Parving, L. Tarnow, F.S. Nielsen, P. Rossing, T. Mandrup-Poulsen, R. Østerby, J. Nerup, Steno Diabetes Center, Gentofte Denmark

The aim of the present study was to evaluate kidney function 8 years after treatment with CsA $(9.3$ to $7.0 \mathrm{mg} / \mathrm{kg} / \mathrm{day}$ ) in young patients (mean $20 \mathrm{yr}$ ) with newly diagnosed IDDM participating in a randomized double-blind placebo-controlled CSA trial. Twenty-one patients received CSA for (mean (SD)) 12.5 (4.0) months and 19 patients received placebo for $14.4(3.8)$ months. The two groups were similar (CsA vs placebo) with regard to mean arterial blood pressure (MABP), urinary albumin excretion rate (UAE), serumcreatinine and glomerular filtration rate (GFR) at baseline. $\mathrm{HbA} \mathrm{A}_{10}$ (mean (SD) during 8 yrs follow-up was also the same $8.9(1.5) \%$ vs $8.4(1.5) \%$ in the CSAgroup and the placebo-group, respectively. During the eight years after cessation of study medication 3 (CsA) and 2 control patients were lost to follow-up. Four out of $18 \mathrm{CsA}$ treated patients developed persistently elevated UAE above $30 \mathrm{mg} / 24 \mathrm{~h}(\mathrm{n}=3$ with microalbuminuria), while all the 17 placebo treated patients had normal UAE (below $30 \mathrm{mg} / 24 \mathrm{~h}$ ), except one patient with a biopsy proven IgA nephritis. The CSA group had a more pronounced raise in UAE (follow-up/baseline $(95 \% \mathrm{Cl}) 2.7(1.4-5.0)$ vs $1.1(0.6-1.8)$ in the placebo treated group, $p<0.05$. GFR ( $\mathrm{m} / / \mathrm{min})$ declined (baseline-1994) with 6.3 (SEM 6$)$ in the former CSA group while it rose with $7.4(5)$ in the placebo group $p=0.05 .24$ hour blood pressure was nearly the same in 1994: CsA: $131 / 77(4 / 2) \mathrm{mm} \mathrm{Hg}$ vs placebo: $127 / 75(2 / 2) \mathrm{mm} \mathrm{Hg}$, respectively. Five randomly selected CSA treated patients had a kidney biopsy performed shortly after the treatment was stopped. Cyclosporine-induced nephropathy (interstitial fibrose/tubular atrophy and/or arteriolopathy) was present in 4 subjects, two of whom had elevated UAE. Our 8 year follow-up study suggests that short lasting CsA treatment in young newly diagnosed normoalbuminuric IDDM patients accelerates the loss in kidney function and the rate of progression in UAE. Longer term follow-up are mandatory to clarify whether the CSA treated patients are at increased risk of developing clinical nephropathy.

\section{8}

White coat hypertension in NIDDM patients with and without incipient and overt diabetic nephropathy.

P Gæade, P. Vedel, O. B. Pedersen, F. S. Nielsen and H.-H. Parving. Steno Diabetes Center, Gentofte, Denmark.

Early data has suggested a high prevalence of white coat hypertension (approximately $50 \%$ ) in NIDDM patients. To study this phenomenon further we determined the prevalence of white coat hypertension (office hypertension with normal blood pressure values at home) by comparison of office blood pressure (Hawksley Random sphygmomanometer) and the ambulatory day ( 7 am to $11 \mathrm{pm}$ ) blood pressure (A\&D TM2420) in hypertensive (repeated office blood pressure $\geq 140 / 90 \mathrm{mmHg}$ ) NIDDM patients with normoalbuminuira (urinary albumin excretion (UAE) $<30 \mathrm{mg} / 24 \mathrm{~h}, \mathrm{n}=30$, age 61 (SD 7) years, 20 males, group 1), with microalbuminuria (UAE $30-300 \mathrm{mg} / 24 \mathrm{~h}, \mathrm{n}=51$, age 55 (7) years, 35 males, group 2) and with diabetic nephropathy (UAE $>300$ mg/24h, $n=47,62(7)$ years, 36 males, group 3). All previous antihypertensive medication was withdrawn 2 weeks before the study, if given $(35 \%)$. The office blood pressure was $155 / 86$ (SE 3/2) $\mathrm{mmHg}, 156 / 89(2 / 1) \mathrm{mmHg}$ and $171 / 90(3 / 2) \mathrm{mmHg}$ in group 1,2 and 3 , respectively $(p<0.05$ comparing group 3 to groups 1 and 2). Applying established criteria white coat hypertension was confirmed if the day blood pressure was lower than 136/87 $\mathrm{mmHg}$ in males and 131/86 in females. The prevalence of white coat hypertension was significantly higher in group 1 as compared to group 2 and group 3,23 (95\% confidence interval, $10-42) \%$ vs $8(2-19) \%$ and $9(2-20)$ $\%,(p<0.05)$ with no difference between the latter two groups. In conclusion the prevalence of white coat hypertension in normoalbuminuric NIDDM patients resembles that observed in non-diabetics with essential hypertension whereas the prevalence is significantly lower in NIDDM patients with incipient or overt diabetic nephropathy. 


\section{9}

LOSS OF CAPILLARY BASEMENT MEMBRANE HEPARAN SULPHATE IN IDDM PATIENTS WITH NEPHROPATHY: A UNIVERSAL ALTERATION?

P. Mørk Hansen', P.E. Høyer', H. Yokoyama', J. van den Born ${ }^{3}$,

$T$. Jensen ${ }^{1}, J$. Berden ${ }^{3}, T$. Deckert' and C. Garbarsch' ${ }^{2}$. 'Steno Diabetes Center. Gentofte. Denmark, 'Institute of Medical Anatomy, Section A, University of Copenhagen, Denmark, ${ }^{3}$ Division of Nephrology, University Hospital St. Radboud, Nijmegen, The Netherlands.

We have hypothesised that in IDDM patients with nephropathy loss of heparan sulphate (HS) is not only seen in glomeruli, but also within the vessel walls of extrarenal tissue. The aim of the present study was to quantify the amount of HS, heparan sulphate proteoglycan (HSPG) and collagen IV within skeletal muscle capillary basement membranes. Skeletal muscle biopsies were taken from 9 healthy controls $(\mathrm{C})$ and 20 IDDM patients: 7 with normal urinary albumin excretion (UAE) (UAE $<30 \mathrm{mg} / 24 \mathrm{~h})\left(D_{0}\right), 5$ with incipient nephropathy (UAE between $30-$ $300 \mathrm{mg} / 24 \mathrm{~h})\left(\mathrm{D}_{1}\right)$ and 8 with clinical nephropathy $(\mathrm{UAE}>300 \mathrm{mg} / 24 \mathrm{~h})\left(\mathrm{D}_{2}\right)$. After immunohistochemical staining the absorbance of HS, HSPG and collagen IV were measured by a scanning and integrating microspectrophotometer. Data were analysed using the unpaired Kruskal-Wallis test. A significant difference in the absorbance of HS and collagen IV was found between subgroups: $0.477 \pm 0.082$ (mean \pm SD) (C); 0.627 $\pm 0.031\left(D_{2}\right): 0.542 \pm 0.098\left(D_{1}\right): 0.371 \pm 0.118\left(D_{2}\right): p=0.006$ and 0.836 \pm 0.111 (C): $0.838 \pm 0.300\left(D_{0}\right) ; 0.970 \pm 0.173(D,) ; 0.512 \pm 0.248\left(D_{2}\right): p$ $=0.02$ for HS and collagen IV respectively. Within the diabetic groups HS was inversely correlated to albuminuria $(r=-0.76, p=0.003)$. No statistically significant difference in the absorbance of HSPG was found between groups. These results correspond to what has previously been demonstrated in kidney biopsies. We conclude that alterations of the content of heparan sulphate and collagen IV within the extracellular matrix are present, not only in the glomerular filtration barrier, but also in the skeletal muscle capillary basement membrane of patients with IDDM and albuminuria. We suggest that this might reflect a universal change.

\section{0}

TGF-B1 RENAL OVEREXPRESSION IN LONG-TERM DIABETIC RATS AND IN HIGH GLUCOSE MESANGIAL CELL CULTURES: INHIBITION BY HEPARIN

E Schleicher ' , M Ceol 1,3, U Sauer ', A Nerlich 2, B Baggio ${ }^{3}, F$ Anglani ${ }^{3}$ and G Gambaro ${ }^{3}$ 1 Institute of Diabetes Research, 2 Institute of Pathology, University of Munich, Murich, Germany, ${ }^{3}$ Institute of Int. Medicine, Dept. of Nephrology, University of Padova, Italy. Previous investigations have shown that treatment with a chemically modified, low anticoagulant heparin prevents morphological and functional changes of the glomerular basement membrane and mesangial matrix deposition and overexpression of type IV collagen. Since TGF- $\beta$ is now recognized as a potent fibrogenetic growth factor, it was our aim to investigate the effect of heparin treatment on TGF- $B 1$ expression in streptozotocin diabetic rats, 12 months duration, and on mesangial cell cultures in high glucose medium Normal (C), diabetic (D), heparin-treated (12 $\mathrm{mg} / \mathrm{kg} /$ day) healthy (C/hep) and diabetic (D/hep) rats were studied. Porcine mesangial cells were treated with increasing glucose concentrations for 24 and $48 \mathrm{hrs}$ with or w/o the same heparin as above, $10 \mathrm{mg} / \mathrm{l}$. In situ hybricization of TGF-B1 was performed on cultured mesangial cells and on kidney sections with an antisense CRNA probe and visualized with silver staining. TGF- 11 expression was quantified by counting the grains per cell. Glomenular mesangial cells were recognized by immuno-histochernistry with anti-Thy 1.1 serum on sequential sections. For a better quantitation of RNA, RNase protection assay for TGF-B1 was performed in mesangial cell extracts. An enhanced mRNA signal of TGF-B1 was detected in glomerular mesangial $(43.0 \pm 7.0$ grains/cell), Bowman $(24.7 \pm 6.8)$ and tubular cells $(41.0 \pm 5.6)$ of the $D$ group in respect to group $C(20.7 \pm 3.8,23.0 \pm 2.0,18.0 \pm 2.8$ respectively) which was decreased to control levels in the D/hep group. Heparin had no effect in C/hep rats (12.5 $22.1,13.0 \pm 4.2$ $15.5 \pm 3.5$ respectively). Statistical significances were $F=11.7 ; p=0.002$ and $F=6.08 ; p=0.026$ and $F=16.8 ; p=0.000$ respectively. In cell cultures, an enhanced expression of TGF- $B 1$ was observed, which was time- and glucose-concentration dependent. In conclusion, this study shows that 1) TGF-B1 is overexpressed in the kidney, both at the glomenular and tubular level, in long-term diabetic animals; 2) TGF-B1 is overexpressed in high glucose mesangial cell cultures; 3 ) at least in diabetic conditions, heparin is a potent TGF- $B 1$ inhibitor both ,in viro" and "in vitro". This suggests that in diabetic nephropathy, the renoprotective effect of heparin is mediated through a TGF- $\beta$ inhibitory mechanism.

\section{OP 26}

\section{New Drugs}

\section{1}

GLP-1 TABLET A NEW THERAPEUTIC ALTERNATIVE FOR NIDDM. M. Gutniak, H. Larsson, S. Sanders, O. Juneskans, JJ Holst and B. Ahren. Vällingby Med. Center, Karolinska Institute, Stockholm, Dept. of Medicine, Lund Univ., Malmö, TheraTech Inc. Salt Lake City, USA, Karolinska Pharmacy, Stockholm, Sweden, Dept. of Medical Physiology, PANUM Institute, Copenhagen, Denmark.

Glucagon-Like Peptide (7-36)amide (GLP-1) is a promising alternative anti-diabetogenic agent for the treatment of diabetes. Previously, the effects of the peptide have been studied following i.v and s.c injection. Now, a mucoadhesive, biodegradable buccal GLP-1 tablet $(9 \mathrm{~mm}$ ) containing $119 \mathrm{nmol}$ has been developed as a possible alternative to injection treatment. Eight healthy volunteers and 7 NIDDM patients received a single tablet under fasting conditions and before a standard meal in this randomized, placebo controlled study. In healthy subjects the mean peak GLP-1 concentration was $132+17 \mathrm{pmol} / \mathrm{L}$ and occurred $30 \pm 4 \mathrm{~min}$ following application. The mean placebo adjusted AUC was $8145 \pm 873 \mathrm{~min}{ }^{\circ} \mathrm{pmol} / \mathrm{L}$ consistent with a relative bioavailability of $7 \%$ vs i.v injection and $47 \%$ vs s.c injection. The levels of active peptide measured using N-terminal RIA increased in parallel with the total GLP-1 immunoreactivity measured using C-terminal RIA. Half-life of total peptide activity after buccal administration was $27 \mathrm{~min}$. The active peptide half-life was $24 \mathrm{~min}$. Placebo adjusted glucose concentrations decreased $1.3 \pm 0.2 \mathrm{mmol} / \mathrm{L}$, plasma insulin increased to peak of $252 \pm 57 \mathrm{pmol} / \mathrm{L}$ and glucagon decreased $17 \pm 3 \mathrm{ng} / \mathrm{L}$ consistent with the increase in plasma GLP-I concentrations. In NIDDM patients GLP-1 pharmacokinetics was similar to that of healthy subjects. In fasting state GLP-1 lowered the blood glucose levels from $8.1 \pm 0.6$ to $5.9 \pm 0.3 \mathrm{mmol} / \mathrm{L}$ (placebo $8.1 \pm 0.8$ to $6.7 \pm 0.8 \mathrm{mmol} / \mathrm{L}$ ) after $270 \mathrm{~min}$. The $T_{\max }$ of GLP-I induced insulin release was $20 \mathrm{~min}$. In prandial experiments the meal induced glucose rise was significantly reduced by GLP-1 $(8.1 \pm 1.2$ vs $12.6 \pm 1.6$ at 60 $\mathrm{min})$. The blood glucose lowering effect of the peptide was maintained for 2 hours. No adverse effects were reported during the study. We conclude that therapeutic plasma levels of GLP-1 were achieved after a single buccal tablet in humans. The peptide had marked glucose lowering effect in NIDDM patients. This new alternative treatment form may be feasible in the future for NIDDM.

\section{2}

MODULATION OF $\mathrm{K}_{\triangle T P}$ CHANNELS AND CYTOSOLIC Ca ${ }^{2+}$ IN ISOLATED INSULIN-SECRETING CELLS BY BTS 67582 , A NOVEL HYPOGLYCAEMIA -INDUCING AGENT.

"Jones, R.B., Shepherd. R.M. Kane. C.. Hashmi. M.N., A. Moores, Harding, E.A. and Dunne. M.J. "Knoll Pharmaceuticals. Nottingham, UK and The Deparment of Biomedical Science. Shcfficld University, Shefficld, S10 2TN.

BTS 67582 (BTS) is a nont-sulphonylurea. anti-diabetic agent with blood glucose-lowcring propertics. We studied the mechanisms of action of BTS on the function of insulin-secreting cells by patch-clamp techniques and microfluorimetry with fura- 2 . In intact rat $\beta$-cells, BTS $(100 \mu \mathrm{M})$ closed $\mathrm{K}_{\mathrm{ATp}}$ channeis, depolarized the cell membrane and initiated the appcarance of action potentials $(n=8)$. The concentration-response profile for BTS was studied in excised inside-out patches: BTS consistently inhibited $\mathrm{K}_{\mathrm{ATP}}$ channels and the $\mathrm{K}_{1}$ for half-maximal inhibition of was approximatcly $5 \mu \mathrm{M}(\mathrm{n}=20)$. Intact glucoseresponsive mouse islets were used to establish the effects of BTS on cytosolic $\mathrm{Ca}^{2+}$ levels $\left(\left[\mathrm{Ca}^{2+}\right]_{i}\right)$; the average glucose-induced increase in $\left[\mathrm{Ca}^{2+}\right]_{i}$ was $130 \pm 11$ nmol and the time to half maximal rise $\left(\mathrm{t}_{1 / 2}\right)$ was $219 \pm 31$ seconds $(\mathrm{n}=12)$. In the presence of $2 \mathrm{mM}$ glucose, $100 \mu \mathrm{M}$ BTS raised $\left[\mathrm{Ca}^{2+}\right]_{\mathrm{i}}$ by $85 \pm 20 \mathrm{nM}$, and the $\mathrm{t}_{1 / 2}$ was $573 \pm 40$ seconds $(\mathrm{n}=8)$. Similarly, $500 \mu \mathrm{M}$ BTS elevated $\left[\mathrm{Ca}^{2+}\right]$ by $73 \pm 10 \mathrm{nM}$, $t_{1 / 2}=594 \pm 55$ scconds $(n=18)$. However. when either $5-7 \mathrm{mM}$ or $10-15 \mathrm{mM}$ glucose was present, BTS had rapid effects on $\left[\mathrm{Ca}^{2+}\right]_{i}$ and these actions occurred without any significant delay in onset. Under these conditions BTS potentiated the rise in $\left[\mathrm{Ca}^{2+}\right]$ induced by glucose through either an elevation of the mean cytosolic $\mathrm{Ca}^{2+}$ Ievcl. an increase in the rate of glucose-induced oscillations or by prolonging the duration of the peaks $(n=17)$. Increases in internal $\mathrm{Ca}^{2+}$ were dependent upon $\mathrm{Ca}^{2+}$ influx since, (i) removal of the external $\mathrm{Ca}^{2+}$ or (ii) the presence of the hypcrpolarizing $K_{\text {ATp }}$ channel agonist diazoxide $(250 \mu \mathrm{M})$ attenuated the BTSinduced rise in $\left[\mathrm{Ca}^{2+}\right]_{1}(n=18)$. These data indicate that BTS is an effective nodulator of $K_{\text {ATP }}$ channels in $\beta$-cells and that channel inhibition leads to the clevation of $\left[\mathrm{Ca}^{2+}\right]$ and insulin secretion. 
BTS 67582 IMPROVES 24H PLASMA GLUCOSE CONTROL AND POSTPRANDIAL INSULIN SECRETION IN NIDDM PATIENTS

W.D. Byrom', A. Weil', T.J. Brown ${ }^{1}$ and J.R. Bratty', 'Clinical Development, Knoll Pharmaceuticals, Nottingham, UK, ${ }^{2}$ Apex Research, Munich, Germany.

BTS 67582 (1,1-dinethyl-2-[2-morpholinophenyl] guanidine monofumarate) is a novel oral anti-diabetic agent. We examined $24 \mathrm{~h}$ glycaemic control following 500 mg BTS 67582 bid and placebo for 7 days in a double-blind two-way crossover study in 16 diet-treated NIDDM patients (Age: $59.1 \pm 8.9$ years (mean \pm sd); BMI: $31.1 \pm 2.5 \mathrm{~kg} / \mathrm{m}^{2}$; Sex: 14 male, 2 female). Patients were hospitalised from mid-day on day 6 to 10 p.m. on day 7. Meals ( $50 \%$ carbohydrate) were standardised.

\begin{tabular}{|c|c|c|c|c|}
\hline Plasma gltcose $(P G)$ (means) & BTS 67582 & Placebo & $\Delta(\%)$ & $\bar{p}$ \\
\hline AUC $(0-24 \mathrm{~h})(\mathrm{mmol} . \mathrm{h} / \mathrm{l})$ & 207.6 & 237.8 & $-13 \%$ & 0.01 \\
\hline Post-breakfast AUC $(0-3 \mathrm{~h})(\mathrm{mmol} \cdot \mathrm{h} / \mathrm{l})$ & 31.5 & 35.5 & $-11 \%$ & 0.04 \\
\hline Post-lunch AUC $(0-3 \mathrm{~h})(\mathrm{mmol} \cdot \mathrm{h} / \mathrm{l})$ & 27.6 & 32.6 & $-15 \%$ & 0.001 \\
\hline Post-tea AUC(0-3h) (mmol.h/l) & 25.3 & 30.3 & $-17 \%$ & 0.01 \\
\hline Day 7 fasting PG $(\mathrm{mmol} / \mathrm{l})$ & 8.7 & 9.6 & $-8 \%$ & 0.07 \\
\hline Nocturnal trough PG $(\mathrm{mmol} / \mathrm{l})$ & 8.1 & 8.5 & $-5 \%$ & 0.25 \\
\hline Plasma insulin (INS) (medians) & BTS 67582 & \multicolumn{2}{|c|}{ Placebo } & $p$ \\
\hline Post-breakfast AUC $(0-3 \mathrm{~h})(\mu \mathrm{IU} \cdot \mathrm{h} / \mathrm{ml})$ & 172.9 & \multicolumn{2}{|l|}{97.8} & $<0.001$ \\
\hline Post-lunch AUC $(0-3 \mathrm{~h})(\mu \mathrm{IU}, \mathrm{h} / \mathrm{mI})$ & 101.5 & \multicolumn{2}{|l|}{97.8} & 0.02 \\
\hline Post-tea AUC $(0-3 h)(\mu I U . h / m l)$ & 102.5 & \multicolumn{2}{|l|}{76.6} & $<0.001$ \\
\hline Day 7 fasting INS $(\mu \mathrm{IU} / \mathrm{ml})$ & 10.6 & \multicolumn{2}{|l|}{10.4} & 0.32 \\
\hline
\end{tabular}

BTS 67582 significantly reduced $24 \mathrm{~h}$ PG and post-prandial PG with corresponding significant enhancements in post-prandial INS secretion. INS returned rapidly to pre-meal levels, mirroring PG profiles. Fasting INS was unchanged, with no evidence of nocturnal hypoglycaemia. In conclusion, short-term treatment with BTS 67582 improved PG control and enhanced INS secretion in response to glycaemic stimuli in NIDDM patients. BTS 67582 may offer advantages over some long acting sulphonylureas.

\section{5}

EFFECT OF LONG-TERM ACARBOSE AND GLIBENCLAMIDE TREATUENT ON VISCERAL FAT ACCUMLLTTION IN PATIENTS NITH NIDDN S. Katoh"1, Y. Mori"1, J. Yokoyama*1, N. Tajima*1, K. Ikeda*2 and Y. Ikeda* ${ }^{*}$ *1 Jikei Univ. Sch, of Med., Tokyo, *2 Kawaguchi Municipal Medical Center, Saitama

Recently, the intra-abdominal(visceral) fat accumulation has been reported to be associated with insulin resistance and glucose intolerance in obese NIDDH. In the present study, we examined the effect of acarbose $(A)$ and glibenclamide(G) on fat distribution and insulin resistance in patients with NIDDM. 41 NIDDM patients who had not received any oral hypoglycemic agent were divided into two groups. 14 mild cases were treated with $A(150 \sim 300 \mathrm{mg} / \mathrm{day})$, the others were treated with $G(1.25 \sim 7.5 \mathrm{mg} / \mathrm{day})$ and followed for 24 weeks. Ve evaluated yisceral fat area(V), subcutaneous fat area(S) and V/S ratio by the abdominal CT of the umbilical level. Ne evaluated insulin resistance by the oral glucose tolerance test. In A-treated group, the body mass index(BMI) af ter the treatment decreased significantly $(\mathrm{p}<0.05) \mathrm{com}-$ pared with that before the treatment, but BMI did not change significantly in G-treated group. In G-trated group, the $V\left(139.4 \pm 67.0 \mathrm{~cm}^{2}\right)$ and $\mathrm{V} / \mathrm{S}$ ratio( $1.000 \pm 0.428)$ after the treatment increased significant$l y(p<0.05)$ compared with $V\left(102.4 \pm 52.3 \mathrm{~cm}^{2}\right)$ and $V / S$ ratio $(0.770 \pm 0.444)$ before the treatment. Those decreased in $\mathrm{A}$-treated group but they were not significant. An oral glucose tolerance test after the treatment showed a significant decrease in plasma glucose levels $(\mathrm{PG})$ in both groups, and showed a significant increase in plasma insulin level(PI) after glucose load in G-treated group, and a decrease in PI in $\mathrm{A}$ treated group compared with those before the treatment. In conclusion, al though glibenclamide treatment resulted in visceral fat accunulation and significant aggravation in insulin resistance, acarbose treatment did not.
EFFECT OF GLIBENCLAMTE ON INSULIN RELEASE AT MODERATE AND HIGH HYPERGLYCEMIA IN NORMAL MAN. JJM Ligtenberg ${ }^{1}$, WJ Sluiter', WD Reitsma', TW Van Haefter ${ }^{2}$. Groningen University Hospital', Utrecht University Hospital ${ }^{2}$, The Netherlands. There are differences in sulphonylureas in stimulating first and second phase insulin release. We studied the effect of glibenclamide on insulin secretion at submaximally- and maximally stimulating blood glucose levels. 12 healthy non-obese subjects were studied. Glibenclamide $10 \mathrm{mg}$ or placebo (randomized, double blind) was taken $30 \mathrm{~min}$ before a $4 \mathrm{hr}$ hyperglycaemic glucose clamp (blood glucose $8 \mathrm{mmol} / \mathrm{l}$ during the first $2 \mathrm{hr}$ and $32 \mathrm{mmol} / \mathrm{l}$ in the next 2 hr). Hyperglycaemic clamp 8 mmol/l: Blood glucase $7.9 \pm 0.02$ and $8.0 \pm 0.02 \mathrm{mmol} / 1$ (with and without glibenclamide, NS). Areas under the $\Delta$ insulin curve $\left(\mathrm{AUC}_{\Delta \text { inssiris }}\right)$ from $0-10 \mathrm{~min}$ (first phase): $1007 \pm 235$ vs $1059 \pm 261 \mathrm{pmol} / \mathrm{l} .10$ min (with and ; without glibenclamide, NS), but a significantly larger increase with glibenclamide from 30-120 min (second phase): $16087 \pm 4489$ vs $7107 \pm 1533 \mathrm{pmol} / 1.90 \mathrm{~min}$ (with and without glibenclamide, $P<0.03)$. $\mathrm{AUC}_{\Delta C \text { peppite }}$ from $0-10$ min: $6.3 \pm 1.1$ vs $5.4 \pm 1.1$ nmol/1.10 min (NS), but from $30-120 \mathrm{~min}$ significantly higher with glibenclamide: $188.3 \pm 27.3$ vs $84.3 \pm 6.9 \mathrm{nmol} / \mathrm{L} .90 \mathrm{~min} \quad(P<0.01)$. Hyperglycaemic clamp $32 \mathrm{mmol} / \mathrm{l}$ : Blood glucose $31.6 \pm 0.1$ and $31.1 \pm 0.2$ $\mathrm{mmol} / \mathrm{l}$ (with and without glibenclamide, NS). $\mathrm{AUC}_{\text {simulin }}$ from 120-130 min were not different: $2411 \pm 640 \mathrm{vs} 3193 \pm 866 \mathrm{pmol} / 1.10$ min (with and without glibenclamide, $P=0.29$ ). AUC sirsulin $_{\text {from }}$ 150-240 min showed no difference: $59623 \pm 8735$ vs $77389 \pm 15161$ pmol/1.90 min (with and without glibenclamide, $P=0.24$ ). Surprisingly, $A U C_{A C \text {.pptide }}$ from 120-130 min and from 150-240 min were slightly lower with glibenclamide. In conclusion, glibenclamide increases second phase insulin release at submaximally stimulating blood glucose levels without enhancement of the first phase, and has no effect on insulin secretion at maximally stimulating blood glucose levels. This could explain the well-known failure of therapy with SU in NIDDM patients, presenting with high blood glucose values.

\section{6}

GLYCAEMIC IMPROVEMENT OVER ONE YEAR IN A DOUBLE-BLIND TRIAL OF ACARBOSE IN 1.946 NIDDM PATIENTS

RR Holman, CA Cull and RC Turner RC.

Diabetes Research Laboratories, University of Oxford, UK.

1.946 NIDDM subjects from the UK Prospective Diabetes Study werc randomised to additional double-blind therapy with acarbose or placebo, titrating to a maximum dose of $100 \mathrm{mg}$ three times daily. Of 1,308 subjects with data at one year $62 \%$ were nale with mean (SD) age 60 (9) y, weight 84 (17) $\mathrm{kg}$, diabeles duration 7.6 (2.9) $\mathrm{y}$, median (incerquartile range) fasting plasma glucose (ipg) 8.5 (6.7 to 11.1 ) mmol $\mathrm{l}^{-1}$ and haemoglobin $\mathrm{A}_{\mathrm{k}}\left(\mathrm{HbA}_{\mathrm{k}}\right) 7.9(6.7$ to 9.5$) \%$, with no significant differences between subjects randomised to acarbose or placebo. $14 \%$ of subjects were treated concurrently with dict alone, $52 \%$ with monotherapy ( $26 \%$ sulphonylurea, $20 \%$ once daily insulin, $6 \%$ metformin) and $34 \%$ with conbined therapy (16\% sulphonyfurea/metiormin, $14 \%$ multiple insulin, $4 \%$ sulphonylurea/insulin). At one year, a lower proportion of patients were taking acarbose compared with placebo $(50 \%$ v $72 \%, \quad p<0.00001)$ with reasons for non compliance being tlatulence $(22 \% \vee 6 \%, p<0.0001)$, diarthoea $(13 \% \vee 5 \%, \quad p<0.05)$ or other problems $(14 \% \vee 17 \%$, n.s.). Intention to treat analysis at one year showed that subjects allocared to acarbose, compared with placebo, had significantly lower median $\mathrm{HbA}_{\mathrm{k}}$, greater $\mathrm{HbA}_{w}$ reduction, lower median fpg and greater mean weight reduction. These differences werc larger in subjects remaining on their allocaled therapy (308 acarbose, 477 placebo) at one year.

\begin{tabular}{|c|c|c|c|c|c|c|}
\hline & \multicolumn{3}{|c|}{ By inlention to treal } & \multicolumn{3}{|c|}{ By actual therapy } \\
\hline & acarbose & placebo & $\mathrm{p}$ & acarbose & placebo & p \\
\hline $\mathrm{HbA}(\%)$ & 7.5 & 7.9 & $<0.0006$ & 7.1 & 7.9 & $<0.00001$ \\
\hline$\Delta \mathrm{HbA}_{1}(\%)$ & -0.4 & -0.1 & $<0.00001$ & -0.7 & -0.1 & $<0.00001$ \\
\hline$\Delta$ fipg $\left(\mathrm{mmod} \mathrm{I}^{1}\right)$ & -0.1 & 0.5 & $<0.000$ & -0.2 & 0.5 & $<0,00001$ \\
\hline$\Delta$ Weigfle $(\mathrm{kg})$ & -0.4 & 0.0 & $<0.03$ & -0.7 & 0.2 & $<0.002$ \\
\hline
\end{tabular}

These acarbose related differences did not differ significantly with respect to concurrent antidiabetic therapy.

Conclusion: Acarbose improved glycaemic control over one year in trealed NIDDM subjects although the non-compliance rate for acarbose was greater than for placebo. 


\section{Insulin Resistance Syndromes}

\section{7}

THE LACK OF INSULIN SECRETORY RESPONSE TO GLUCOSE IS A VERY EARLY DEFECT IN THE GK RAT. IN VIVO AND IN VITRO STUDIES IN FETUSES.

M.-H. GIROIX, P. SERRADAS, M.-N. GANGNERAU and B. PORTHA

Lab. Physiopathology of Nutrition, CNRS URA 307, University Paris 7, Paris, France.

We have previously shown that diabetes in the adult GK rats is characterized by an impaired glucose-induced insulin secretion. To study the development of this defect, we have examined in vivo and in vitro the GK B-cell secretory function at fetal age. In vivo B-cell reactivity to glucose was studied in the 21 day-old GK fetuses and compared to that in the Wistar fetuses (controls, W), using an hyperglycemic clamp protocol applied to the mothers during $60 \mathrm{~min}$. This technique allowed to obtain a steady-state hyperglycemia in the corresponding fetuses. Under these conditions, after 5 min hyperglycemia, fetuses increased their plasma insulin level by $45 \%$ compared to the basal level before hyperglycemia. By contrast, plasma insulin in the GK tetuses remained at the basal level despite hyperglycemia similar to that in $W$ fetuses. Lack of reactivity to glucose in the GK fetuses was also found altered after $60 \mathrm{~min}$ hyperglycemia. Two sets of studies were performed in vitro. First, we evaluated the secretory riposte to glucose and other stimulatory agents by GK fetal islets as obtained after a one week-tissue culture. The basal insulin release was lower in the GK islets; however, when expressed as percentage of insulin content, it became not different from the $W$ islets. Insulin output in response to each one of the tested secretagogues was lower in the GK islets. However, the fold increase in the responses relative to basal value was similar to that in the $W$ islets. Second, insulin response of letal B-cells to glucose and to glucose plus forskolin was assessed using perifusion of pancreatic tissuc fragments. The kinetic of insulin release by perifused fetal fragments was similar in GK and W. When expressed relative to the corresponding mean basal value, the insulin release patterns were superimposed in the two groups. In conclusion: 1) a lack of pancreatic reactivity to glucose is detectable in vivo in the GK rat as early as fetal age; 2) such a defect represents a primary feature of this rat model of NDDM; 3) this defect is not intrinsic to the GK fetal B-cell.

\section{8}

A MUTATION OF MUSCLE GLYCOGEN SYNTHASE GENE IS RELATED WITH INSULIN RESISTANCE IN JAPANESE NIDDM

H.Shimomura, T.Sanke, K.Ueda, M.Nakamura, and K.Nanjo, The First Department of Medicine, Wakayama University of Medical Science, Wakayama, Japan

Glycogen synthase (GYS) is a key enzyme of non-oxidative pathway of glucose metabolism which has been reported to be related with insulin resistance in the patients with NIDDM. It has also been considered that impaired GYS activity in the skeletal muscle is an inherited trait in patients with NIDDM. We therefore scanned the GYS gene for mutation in Japanese NIDDM patients. Genomic DNA obtained from non-obese late onset NIDDM patients $(n=180)$ and non-obese non-diabetic subjects without family history of diabetes ( $>60$ years, $n=121$ ) were subjected to single strand conformational polymorphism analysis. Only one abnormal conformer was found in the DNA fragment corresponding to the exon 10 . DNA sequencing revealed one point missense mutation (Met to Val at the position 416 : M416V mutation). The mutant allele frequency in the NIDDM patients $(12,5 \%)$ was slightly high but not statisticaily significant compared with that in non-diabetic subjects $(10.7 \%)$. However, the insulin sensitivity index (SI) estimated by the Minimal Model analysis in the NIDDM patients carrying the mutation was significantly lower than that in the NIDDM patients without the mutation $(1.16 \pm 0.28, n=21$ Vs $2,20 \pm 0,20, n=60$, Mean $\pm \mathrm{SE}, \mathrm{p}<0,01$ ). The glucose effectiveness (SG), age, body mass index, and levels of HbAlc and serum lipids were not significantly different between the two groups. These findings suggest that the M416V mutation of the muscle GYS gene is one of the pathogenesis of insulin resistance seen in non-obese Japanese patients with NIDDM.

\section{IS MUSCLE AND RENAL BLOOD FLOW ALTERATIONS IN NIDDM DURING ACUTE HYPERGLYCEMIA HAS COMPENSATIVE FEATURES?}

U.Kasimov and J.Tourakulov. Department of Diabetology of institute of Endocrinology, Tashkent, Uzbekistan.

In recent years it has been shown that ability of insulin to increase blood flow is linked to glucose uptake in sceletal muscle. This study aimed to elucidate alterations of renal and muscle blood flow in connection with insulin secretion in NIDDM patients. Results: Blood vessel response to short-term hyperglycemia during intravenous glucose tolerance test (IVGTT, $0.5 \mathrm{~g}$ glucose $/ \mathrm{kg}$ ) in NIDDM patients without proteinuria (9 mild, controlled by diet only, and 15 severe diabetics), 18 subjects with impaired glucose tolerance (IGT) and 8 control ones was studied using simultaneous evaluation of renal blood flow (RBF, expressed in $\mathrm{ml} / \% / \mathrm{min}$ ) by ${ }^{131} \mathrm{f}$ hyppurate and muscle blood flow of arm (MBF, expressed in $\mathrm{ml} / \mathrm{min} / 100 \mathrm{~g}$ muscle tissue) by ${ }^{133} \mathrm{Xe}$. Increase of MBF (Mean $\left.\pm \mathrm{SD}\right)(1.75 \pm 0.66$ vs $2.15 \pm 0.70, p<0.05$, Wilcoxson's T-criteria) with decrease of RBF $(19.2 \pm 4.0$ vs $17.7 \pm 3.4, p<0.05$ ) was observed after glucose load in NIDDM patients. In mild diabetics RBF decreased significantly, but MBF had a tendency to increase. On the contrary, in severe diabetics MBF increased significantly

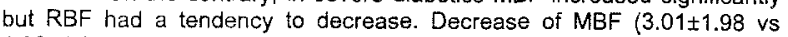
$1.96 \pm 1.25, p<0.05)$ and a tendency of RBF to decrease was observed in IGT subjects. In control subjects MBF decreased $(2.90 \pm 1.80$ vs $1.59 \pm 0.36$, $p<0.05)$ and RBF increased $(15.6 \pm 5.9$ vs $18.2 \pm 6.1, p<0.05)$. Area under curve of first phase $(0-5 \mathrm{~min}$ ) of insulin secretion (AlS, $\mathrm{mkU} / \mathrm{ml} / \mathrm{min}-1$, by

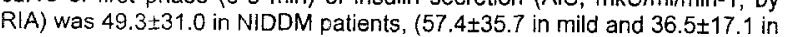
severe diabetics), $133.8 \pm 67.8$ in (GT subjects and $179.7 \pm 92.1$ control ones, repectively. RBF $(r=+0.59, p<0.01)$ and $\mathrm{MBF}(r=-0.44, p<0.05)$ after glucose load correlated with AIS in NIDDM patients. In conclusion: Acute increases of MBF during IVGTT in NIDDM may be as compensatory mechanism to increase glucose uptake in muscle and decreases RBF - as result of blood flow redistribution.
DIRECT QUANTITATION OF REgIONAL MUSCLE GLUCOSE UPTAKE AND BLOOD FLOW USING ${ }^{18} \mathrm{FDG}$ AND $\mathrm{H}_{2}{ }^{15} \mathrm{O}$ IN NIDDM $T$ Utriainen, $P$ Nuutila, $T$ Takala, $T$ Rönnemaa, $M$ Raitakari, $T$ Tolvanen, U Ruotsalainen, O Kirvelä, $\boldsymbol{H}$ Yki-Järvinen.

Universities of Turku and Helsinki, Finland.

Data regarding the contribution of defects in insulin stimulation of blood flow vs glucose extraction to insulin resistance have been controversial in NIDDM. PET combined with $\left[{ }^{18} \mathrm{~F}\right]$-2-deoxy-glucose and $\mathrm{I}^{15} \mathrm{OI}-\mathrm{H}_{2} \mathrm{O}$ allows simultaneous quantitation of blood flow and glucose uptake within several regions in a given individual in skeletal muscle. We employed this technique to study 8 NIDDM subjects $\left(\mathrm{HbA}_{10} 8.2 \pm 0.4 \%\right)$ and 7 normal subjects (CTRL) matched for age $(43 \pm 2$ vs $43 \pm 2$ yrs $), B M I(27 \pm 1$ vs $26 \pm 1$ $\left.\mathrm{kg} / \mathrm{m}^{2}\right)$ and maximal oxygen uptake ( $\left.32 \pm 2 \mathrm{vs} 36 \pm 3 \mathrm{mi} / \mathrm{kg} \cdot \mathrm{min}\right)$. Femoral muscle blood flow and glucose uptake were measured before and after $60 \mathrm{~min}$ of euglycemic hyperinsulinemia (insulin infusion $5 \mathrm{mU} / \mathrm{kg} \cdot \mathrm{min}$ ). In NIDDM subjects both whole body $(39 \pm 4 v s 51 \pm 3 \mu \mathrm{mol} / \mathrm{kg}$ body weight $\cdot \mathrm{min}, \mathrm{p}=0.02$ ) and skeletal muscle glucose uptake $(68 \pm 5$ vs $93 \pm 7 \mu \mathrm{mol} / \mathrm{kg}$ muscle $\cdot \mathrm{min}$, $\mathrm{p}=0.02$ ) were significantly decreased. Insulin increased muscle blood flow by $45 \pm 12 \%$ from $2.0 \pm 0.4$ to $2.6 \pm 0.4 \mathrm{ml} / 100 \mathrm{~g}$ muscle $\min (p<0.01)$ in NIDDM and by $53 \pm 10 \%$ from $2.2 \pm 0.2$ to $3.3 \pm 0.4 \mathrm{ml} / 100 \mathrm{~g}$ muscle $\mathrm{min}(\mathrm{p}<0.01)$ in CTRL (NIDDM vs CTRL, NS). Muscle blood flow was not correlated with muscle glucose uptake either interindividually or within muscle regions. In conclusion, insulin resistance in skeletal muscle in NIDDM can be attributed to a defect in cellular glucose extraction, not blood flow. Furthermore, the lack of correlation between glucose uptake and flow within regions of skeletal muscle in the same individual imply that insulin stimulation of glucose uptake and blood flow are not closely coupled phenomena. 
161

THE PHENOTYPE OF NIDDM IS RELATED TO EARLY DEFECTS IN GLUCOSE METABOLISM IN THE OFFSPRING OF NIDDM PATIENTS I.Vauhkonen ', L. Niskaner', M. Uusitupa ${ }^{2}$ and M. Laakso', Departments of Medicine ${ }^{1}$ and Clinical Nutrition ${ }^{2}$, Kuopio University Hospital and University of Kuopio, Finland

To investigate whether the phenotype of NIDDM patients is related to glucose metabolism of their offspring, we measured insulin sensitivity and insulin secretion in the offspring of NIDDM patients with low fasting C-peptide $(\leq 0.45$ nmolil) reflecting impaired insulin secretion (group $A, n=21$ ) and the offspring of diabetic patients with high fasting $C$-peptide $(\geq 0.88$ nmol/l) reflecting insulin resistance (group $B, n=17$ ). The probands were followed-up to ten years from the time of diagnosis, their phenotype was well characterised and they did not have glutamic acid decarboxylase or islet-cell antibody positivity. Whole-body glucose uptake measured by the hyperinsulinaemic euglycaemic clamp technique was higher in group A than in group B $(51.6 \pm 1.9 \mathrm{vs} .39 .2 \pm 2.1 \mu \mathrm{mol} / \mathrm{kg} / \mathrm{min}$, $p=0.002$ ), which was due to both low oxidative and nonoxidative glucose metabolism $(23.7 \pm 1.2$ vs. $19.2 \pm 1.0 \mu \mathrm{mol} / \mathrm{kg} / \mathrm{min}$; $\mathrm{p}=0.006$, and $27.9 \pm 1.9 \mathrm{vs}$. $20.0 \pm 2.1 \mu \mathrm{mol} / \mathrm{kg} / \mathrm{min} ; \mathrm{p}=0.007$, respectively). The first-phase insulin secretion (insulin area under the curve during the furst 10 minutes) measured by an intravenous glucose tolerance test was lower in group A than in group B (3480 I $360 \cdot \mathrm{min} \gamma \mathrm{s} .5100 \pm 570 \mathrm{pmol} / \mathrm{l} \cdot \mathrm{min}, \mathrm{p}=0.02$ ). Furthermore, during the hyperolycaemic clamp the maximal insulin secretion at blood glucose level $\sim 20$ $\mathrm{mmol} / \mathrm{l}$ was constantly lower at all time points in group A compared to group B (plasma insulin at the end of the hyperglycaemic clamp: $972 \pm 126$ vs. $1836 \pm$ $348 \mathrm{pmol} / \mathrm{h}, \mathrm{p}=0.008$ ). To conclude, our novel finding is that the phenotype of NIDDM patients characterises also their non-diabetic offspring. Therefore, it is likely that these two groups of non-diabetic offspring have different genetic background predisposing to different early defects in glucose metabolism.

\section{2}

$\beta_{3}$-ADRENERGIC RECEPTOR GENE MUTATION (TRP64ARG) AND INSULIN SENSITIVITY IN NON-OBESE NIDDM

K.Ueda, T.Sanke, M.Nakamura, G.Matsumoto, and K.Nanjo,

The First Department of Medicine, Wakayama University of Medical Science, Wakayama, Japan

$\beta_{3}$-adrenergic receptor is expressed in the visceral fat in humans and is considered to play an important role for fat metabolism. Recently, a mutation of the $\beta_{3}$-adrenergic receptor (Trp64Arg) has been reported in relation to obesity, syndrome $\mathrm{X}$ and early onset NIDDM, In order to investigate the relationship between insulin sinsitivity and this Trp64Arg mutation, we sereened the mutation in 89 non-obese $\left(B M I<28.0 \mathrm{~kg} / \mathrm{m}^{2}\right)$ Japanese patients with NIDDM and 39 non-diabetic subjects by Mva I RFLP method combined with PCR. The mutant allele frequency was similar between NIDDM patients and non-diabetic subjects $(17.4 \%$ Vs $19.0 \%$ ). However, the insulin sensitivity index (SI) estimated by the Minimal Model analysis of NIDDM patients with the mutation $(n=15)$ was significantly higher than that of NIDDM patients without the tmutation $(\mathrm{n}=38)\left[2.91 \pm 0.48 \mathrm{Vs} 1.91 \pm 0.24 \times 10^{-4} / \mathrm{min} \cdot(\mu \mathrm{U} / \mathrm{ml})\right.$, mean $\left.\pm \mathrm{SE}\right]$. Age, duration of diabetes, BMI, HbAlc, blood pressure and glucose effectiveness $(\mathrm{SG})$ were not significantly different between the two groups. We also compared serum lipids levels. Although serum levels of total cholesterol and HDL cholesterol were similar, serum triglyceride level in the patients group with the mutation was significantly higher than that in those without the mutation $(115.3 \pm 11.8$ Vs $79.1 \pm 7.9 \mathrm{mg} / \mathrm{dl}, \mathrm{p}<0.05)$. These results suggest that the Trp64Arg mutation of the $\beta_{3}$-adrenergic receptor gene may be associated with insulin sensitivity through lipids metabolism in NIDDM patients.

\section{OP 28 \\ Population Screening for IDDM}

\section{3}

THE 'SARDINIAN SCHOOL CHILDREN-IDDM' (SSI) STUDY: PREVALENCE AND CORRELATION OF ISLET CELL (ICA) AND THYROID ANTIBODIES IN 8387 HEALTHY SCHOOL CHILDREN

A Loviselli, M A Cambosu, F Velluzzi, P Mele, M F Atzeni, M Shattock*, A Balestrieri, M Songini**, S Mariotti, G F Bot ${ }^{-} 7 \mathrm{ZO}^{*}$ and SSI Study Group. Endocrinology, University of Cagliari, Cagliari; *Dept of Immunology, St Bartholomew's and the Royal London School of Medicine \& Dentistry, London; **Centre for Metabolic Diseases, Ospedale San Michele, Cagliari.

IDDM is often associated with thyroid autoimmunity. Data on the prevalence of thyroid antibodies (TA) in young populations at large have not been systematically obtained and their correlation with ICA rarely attempted. We have measured ICA and TA in 8387 school children (aged 6-14) recruited from 26 towns of the 4 Provinces in Sardinia, ie Nuoro [Nu], Cagliari [Ca], Oristano [Or] and Sassari [SS], as part of a study (1986-1993) to design maps of pre-IDDM and to assess the extent of endemic goitre in the Island. ICA were measured by indirect IFL $(\geq 5 \mathrm{JDF}$ units) and TA either by passive hemagglutination $(\geq 1: 400)$ or by RIA $(\geq 200 \mathrm{U} / \mathrm{ml})$. The results are summarised in the Table.

$\begin{array}{ccccccc}\text { Provinces } & \text { Towns (n) } & \text { School children (n) } & \text { ICA (n) } & \text { TA (n) } & \% \text { ICA } & \% \text { TA+ } \\ \text { SS } & 3 & 1416 & 1287 & 1299 & 5.0 & 1.4 \\ \text { NU } & 11 & 1948 & 1701 & 1713 & 3.7 & 2.8 \\ \text { CA } & 7 & 3059 & 2629 & 2762 & 5.7 & 2.6 \\ \text { OR } & 5 & 1964 & 1755 & 1752 & 2.5 & 4.0\end{array}$

The overall prevalence of ICA was higher than that of TA $(4.22 \%$ vs $2.7 \%$, respectively). SS and $\mathrm{CA}$ had the highest prevalence of ICA and the lowest of TA, while the reverse applied to OR. ICA and TA were neither correlated in individual towas nor related to iodine intake and goitre prevalence. 17/7372 $(0.2 \%)$ children were positive for ICA and TA. They had higher subclinical hypothyroidism $(2 / 17,11.7 \%)$ compared to the rest of the cohort $(69 / 7872,0.8 \%$ $\mathrm{p}<0.001$ ). Of the 1114 children with the longest follow-up (median $4.7 \mathrm{yrs}$ ), 5 develop IDDM. All had ICA, but none had TA. In conclusion, Sardinian school children have a rather high prevalence of ICA and TA, but their presence is not geographically related. Longitudinal studies will clarify the role of ICA and TA as risk factors for the development of thyroid autoimmune disease and/or IDDM.

\section{4}

OCCURRENCE AND PERSISTENCE OF IDDM-ASSOCIATED AUTOANTIBODIES IN UNAFFECTED SCHOOL CHILDREN

M. Knip. J. Rahko, P. Kulmala, P. Vähäsalo, and J. Karjalainen. Department of Pediatrics, University of Oulu, Oulu, Finland

To study the frequency of single and multiple autoantibody-positivity in children from the Finnish background population, we analyzed 3661 unaffected school children aged 7-16 years for ICA and GAD antibodies (GADA). In addition all children found positive for ICA and/or GADA were tested for IAA. The subjects positive for at least one antibody in the first sample were reanalyzed about 2 years later Initially 102 children $(2.79 \%)$ tested positive for ICA, while only $19(0.52 \%)$ had GADA. After adding IAA, $91(2.49 \%)$ had one detectable autoantibody, $14(0.38 \%)$ two antibodies and four $(0.11 \%)$ three antibodies. Accordingly there were altogether 109 children $(2.98 \%)$ positive for at least one autoantibody. Two of these one with two and the other with three autoantibodies, presented with clinical IDDM before the second sample. 104 subjects were available for retesting. Seroconversion to autoantibody-negativity occurred in five cases for ICA, in one case for GADA and in two cases for IAA, while two, 19 and nine turned positive for ICA, GADA and IAA, respectively. Out of those 88 initially positive for one autoantibody, two turned negative, 61 remained single positive, and 25 turned positive for two antibodies. Out of those 13 retested individuals initially positive for two antibodies three turned single positive, six remained double positive and four became triple positive. All three retested subjects initially positive for three antibodies remained so. When considering that the cumulative risk of a Finnish child to progress to IDDM before the age of 20 years is about $0.6 \%$, these data indicate that signs of beta-cell autoimmunity is about five times more common than clinical disease in the general population. Beta-cell autoimmunity seems to be persistent in most children, at least over a short period of ob-servation. The observed frequency of $0.5 \%$ for initial positivity for multiple autoantibodies (at least 2 antibodies) suggests that the use of such an approach in the prediction of IDDM at the population level may be highly relevant, especially, when we know from studies in Finnish children with newly diagnosed IDDM that the sensitivity of multiple antibodies is approximately $77 \%$. Taking together, this information implies that the positive predictive value of multiple autoantibodies may come close to $100 \%$ in the general population. 


\section{5}

A SIMPLE, SMALL VOLUME ASSAY FOR THE MEASUREMENT OF COMPETITIVE INSULIN AUTOANTIBODIES.

A.J.K. Williams, P.J. Bingley, M.L Bruley and E.A.M. Gale.

Dept. of Diabetes and Metabolism, St.Bartholomew's Hospital, London UK

Measurement of insulin autoantibodies (IAA) may improve prediction of IDDM, especially in children less than 10 years old. Established assays require relatively large amounts of serum, and in young children this may be a limiting factor. We have developed a radio-binding assay, suitable for screening large numbers of samples, that requires only small amounts of serum. $5 \mu$ l of serum are incubated in duplicate with ${ }^{125}$-I labelled insulin for three days. Immune complexes are precipitated using Protein A Sepharose and after washing, bound label is counted in a gamma counter. Results are expressed in arbitrary units read from a standard curve. To obtain competitive IAA (CIAA) levels, sera found to have an IAA level above the 95 th percentile of 522 schoolchild controls (median age 11.3 years, range 9.1-13.7 yrs) are re-assayed in the same manner, but with and without addition of unlabelled insulin. Using this strategy, $53 \%$ of samples taken from 230 IDDM patients within 1 week of diagnosis (median age 10.2 years, range $1.3-20.7 \mathrm{yrs}$ ) and $1 \%$ of the schoolchild controls had CIAA levels above 0.75 units, while $67 \%$ of cases and $2 \%$ of controls were above 0.27 units. The prevalence of CIAA above 0.27 units was similar in male and female patients at diagnosis ( $95 / 136$ vs $59 / 94, p=N S$ ), but greater in patients aged less than 10 years $(90 / 111$ vs $64 / 119, p<0.0001)$. The median levels were also higher in the younger age group (2.68 units vs 0.35 units, $\mathrm{p}<0.0001$ ). In conclusion, we believe that it is feasible to measure CIAA with $30 \mu \mathrm{l}$ of serum using this simple assay, and that good sensitivity and specificity can be achieved.

\section{6}

LADA IS GENETICALLY DIFFERENT FROM DDM.

AL. Carlsson, T. Tuomi, H. Li, C. Forsblom, L. Groop and the Botnia Research Group, Diabetes and Endocrine Research Laboratory, Dept. Endocrinology. Malmö University Hospital, Sweden and Helsinki University Hospital, Finland.

Susceptibility to IDDM is conferred through several genes, of which the most important are HLA Class II (IDDM1) and a VNTR in the insulin gene promoter region (IDDM2), which is in linkage-disequilibrium with $\mathrm{Hphl-polymorphism.}$ The aim of the study was to investigate whether GADab positive NIDDM or LADA (latent autoimmune diabetes of adults) share susceptibility genes in common with IDDM. Since 1989 we have been recruiting all patients with NIDDM from a restricted area in Finland with a population of 250000.97 of 971 (10\%) NIDDM patients were GADab+ (index >5). Compared with 99 GADab- NIDDM patients (46M/53F), the LADA group (36M/46F) had a lower BMI ( $27.1 \pm 4.7$ vs. $30.1 \pm 2.9)$. lower age at onset of diabetes $(58.1 \pm 13.2$ vs. $62.0 \pm 8.3 \mathrm{yrs} ; \mathrm{p}=0.02), 30 \mathrm{~min}$ insulin response to oral glucose tolerance test $(43.2 \pm 31.7$ vs. $61.0 \pm 44.3 \mathrm{mU} / \mathrm{f} ; \mathrm{p}=0.018)$ and were more often treated with insulin ( 43 vs. $26 \%, p=0.02$ ). These two groups and a control group consisting of spouses without a family history of diabetes (CONT; 44M/52F; age 64.12 \pm 7.6) were typed for HLA DQB 1*0201,*0302, and *0602(3) by PCR followed by dot-blot hybridization with oligonucleotide probes, and for $\mathrm{Hphl}$ polymorphism in the insulin gene by PCR and RFLP.

Of the DQB1 genotypes (0201/0302; 0201/X; 0302/X; 0201/0602(3); 0302/0602(3);0602(3)/X; X/X), 0302/X was increased in the LADA vs. both NIDDM and CONT $(24.4 \%$ vs. $9.1 \%$ \& $9.7 \%, \mathrm{p}=0.0075$ and 0.014$)$. The IDDM protective genotype 0602(3)/X was decreased in both LADA and NIDDM groups compared with the CONT $(20.7 \%$ \& $21.2 \%$ vs. $36.6 \%, p=0.03$ and 0.047$)$. There was no significant difference in the genotype frequencies of Hph1 polymorphism between the three groups $(+1+: 49,53,54 \%$; +1 - or $-1-: 51$, $47,46 \%)$, except for a decrease in the IDDM associated +/+ genotype in the LADA patients with high GADab levels (25\% in GADab $>70$ compared with $55 \%$ in GADab $5-70 ; \mathrm{p}=0.052$ ).

We conclude that except for DQB $1 * 0302$, the LADA did not show an increased frequency of the IDDM susceptibility genotypes supporting a concept of a diabetic subgroup separate from IDDM.

\section{7}

RELATIONSHIP BETWEEN IMMUNE MARKERS AND CHARACTERISATION OF DIAGNOSED TYPE 2 DIABETIC PATIENTS A. Pié, M. Pié and E. Faure. Endocrinology, University Hospital, Zaragoza, Spain.

561 NIDDM patients, 328 women and 233 men, 30 to 75 years old, have been tested for ICA and GADA to study if there was any relationship whit their age, sex, age at diagnosis, years of evolution of the disease, $B M I$, waist/hip ratio $(W / H)$, family history of diabetes $(\mathrm{FH}+)$, residual $\mathrm{B}$ cell function determined by c-peptide 6 ' after $1 \mathrm{mg}$ of i.v. glucagon and other autoantibodies. ICA was measured by indirect immunofluorescence extended incubation on human pancreas sections, GADA by immunoprecipitation of in vitro translated $35 S$ recombinant GAD 65, $6^{\prime}$ c-peptide $\left(6^{\prime} \mathrm{c}\right.$-p) by radioligand assay, parietal cell antibodies(PCA) by indirect immunofluorescence and thyroglobulin(TGA) and thyroid microsonal antibodies(TMA) by haemoaglutination. Mean \pm SD are: for agc $62,2 \pm 10,1$; age at diagnosis $(\mathrm{dg}) 52,1 \pm 10,5$; years of evolution $10 \pm 8,3 ;$ BMI $29,3 \pm 5$ $\mathrm{Kg} / \mathrm{m} 2 ; \mathrm{W} / \mathrm{H} 0,94 \pm 0,09 ; 6^{\prime} \mathrm{c}$-peptide $4,2 \pm 2,1 \mathrm{ng} / \mathrm{ml}$. Age, age at dg and vears of evolution have been distributed in intervals of 5 years, $B M I$ in $\leq 25$ and $\leq 30 \mathrm{Kg} / \mathrm{m} 2$, $W / \mathrm{H}$ in $\leq 0,85$ and $\leq 0,95,6^{\prime} \mathrm{c}-\mathrm{p}$ in $\leq 1,92 \mathrm{ng} / \mathrm{ml}$. 53 patients $(9,53 \%)$ are ICA $>10 \mathrm{JDF}$ $\mathrm{U} . ; 41$ patients $(7,8 \%)$ are GADA $>$ Mean +3 SD of a control population $n: 173$. Chi Square test has been performed with the following P-Values: GADA $>M+3 S D$ for age p:0,0004; age at dg p:0,0002; BMI $\leq 25 \mathrm{p}:<0,0001 ; \mathrm{W} / \mathrm{H} \leq 0,85 \mathrm{p} ;<0,0001 ; 6^{\prime} \mathrm{c}-\mathrm{p} \leq 1,92$ ng/ml p: $<0.0001$; PCA positives(+) p:0.0498, TGA+ p:0,0010; TMA+ p: $<0,0001$. ICA $>10 \mathrm{JDF} U$. for age at dg p:0,0103; BMI $30 \mathrm{p}: 0,0184 ; \mathrm{W} / \mathrm{H} \leq 0,95 \mathrm{p}: 0,0337 ; 6^{\prime} \mathrm{c}-$ $\mathrm{p} \leq 1,92 \mathrm{ng} / \mathrm{ml}$ p:0.0219; TMA + p:0,0058. Non significant differences were observed for GADA $>M+3 S D$ and ICA $>10$ JDF $U$. for sex, years of evolution and $F H+$ and for ICA $>10$ JDF $U$. also for age, PCA+ and TGA+. GADA $>M+3 S D$, has an observed frequencies greater than the expected values: for age, from 30 to 50 and 61 to 65 ; age at dg from 30 to 40 and 61 to $65 ; \mathrm{BMI} \leq 25 ; \mathrm{W} / \mathrm{H} \leq 0,85 ; 6^{\prime} \mathrm{c}-\mathrm{p} \leq 1,92 \mathrm{rg} / \mathrm{ml} ; \mathrm{PCA}+$; TGA+ and TMA+. ICA>10 JDF U., has an observed frequencies greater than the expected values: for age at dg from 35 to 45 and 61 to 70 ; $\mathrm{BMI} \leq 30$; W/H $\leq 0,9^{\circ} 5^{\circ} \cdot 6^{\prime} \mathrm{c}$ $\mathrm{p} \leq 1,92 \mathrm{ng} / \mathrm{ml}$ and TMA + . We conclude that NIDDM patients that are GADA $>\mathrm{M}+3 \mathrm{SD}$ and ICA $>10$ IDF $\mathrm{U}$. present different anthropometric parameters, residual $B$ cell function and different distribution ages at diagnosis.

\section{8}

DISTINCT GENETIC AND IMMUNOLOGICAL FEATURES IN PATIENTS WITH INSULIN-DEPENDENT DIABETES BELOW AND ABOVE AGE 40 AT ONSET T. Lohmann, H..J. Verlohren, S. Schröder ${ }^{1}$, J. Seissler, N. Morgenthaler, J. Rötger, K. Dähn and W.A. Scherbaum. Dept. of Internal Medicine III, and Institute of Clinical Immunology, University of Leipzig, 04103 Leipzig, Germany.

Young age at onset is a relevant parameter associated with a rapid onset of insulindependent diabetes (IDDM). We investigated the correlation between islet-reiated antibodies (islet cell antibodies [ICA], antibodies [Ab] to glutamic acid decarboxylase [GAD] and the tyrosine phosphatase IA2), T cell responses to a panel of immunodominant GAD peptides and HLA class II isotypes in 20 IDDM patients 12-35 years at onset (group 1), 20 patients with DDM above 40 years at onset (group 2), 10 patients with non-insulin-dependent diabetes (NIDDM) and 11 healthy controls. All patients were less than 2 years from diagnosis without diabetic complications and well treated as estimated by $\mathrm{HBA}_{\mathrm{lc}}$ values below $8 \%$. ICA were measured by immunofluorescence test (limit of detection $5 \mathrm{JDF}-\mathrm{U}$ ), GAD-Ab (sensitivity 82\%) and IA2$\mathrm{Ab}$ (sensitivity $72 \%$ ) by immunoprecipitation techniques. T cell responses were tested in 5 days proliferation assays against GAD peptides identified in previous experiments as typical for IDDM. HLA class II alleles were typed by polymerase chain reaction. ICA and GAD-Ab were more prevalent in IDDM patients compared to NIDDM patients and healthy controls ( $p<0.01$ ), but only IDDM group 1 had IA2-Ab ( $p<0.05$ to IDDM group 2, NDDM and controls). Moreover, antibody profiles differed between IDDM patients of groups 1 and 2 since only IDDM group 2 showed i) $\mathrm{GAD}-\mathrm{Ab}+/ \mathrm{ICA}$ - and ii) GAD-Ab-/IA2-/ICA+ Ab-profiles ( $\mathrm{p}<0.05$ to IDDM group 1) suggesting i) different $A b$-epitopes or ii) distinct antigens in these patients. T cell responses to GAD peptides were seen in $75 \%$ of IDDM group 1 ( $\mathrm{p}<0.02$ to NIDDM and controls) and in 56\% of IDDM group 2 compared to $33 \%$ of NIDDM patients and $36 \%$ of the controls. IDDM patients of group 1 were more frequently DR3/DQ2+ and DR4/ DQ8+ and less DR2+ compared to IDDM patients of group $2(p<0.05)$. In conclusion, our data provide strong evidence for humoral and cellular autoimmunity in IDDM patients with onset below and above 40 years of age. However, late onset differs from young onset IDDM in respect to $\mathrm{T}$ cell responses, HLA class II types and a lack of IA2-Ab. These findings have consequences for the diagnostic strategy to identify slow onset IDDM in individuals above 40 years of age in that Ab-screening needs combination of ICA and GAD-Ab but not IA2-Ab. 


\section{Endogenous Glucose Production}

169

INCREASED RENAL GLUCOSE PRODUCTION IN INSULIN DEPENDENT DIABETES (IDDM)

A.MITRAKOU,D.PLATANISIOTIS,A.TOLLIAS,L. VLACHOS,D.MOURIKIS, V. NADKARNI,C.MEYER.M.KREIDER,J.GERICH and S.RAPTIS.University of Rochester N.Y. and 2nd Dept of Internal Medicine Propaedeutics,Athens University, Greece

In IDDM increased release of glucose into the systemic circulation (SGR) in the postabsorptive state is largely due to gluconeogenesis (GN). Although liver and kidney are both capable of $G N$, it has been generally assumed that the increased $G N$ is solely due to hepatic GN. However since renal GN is increased in streplozocin diabetic rats and since most studies have failed to demonstrate increased splachnic glucose release in IDDM ,we postulated that there may be increased renal GN in IDDM. To test the hypothesis we assessed SGR.renal glucose release (RGR) and hepatic glucose release (HGR.calculated as SGA - RGR) in postabsorptive IDDM subjects after overnight withdrawal from insulin and after insulin infusion $(0.8 \mathrm{mU} / \mathrm{kg} / \mathrm{min}$ for $180 \mathrm{~min}$ ) using a combination of balance (renal vein catheter) and isotope (6-3H glucose) techniques. Compared to normal volunteers SGA

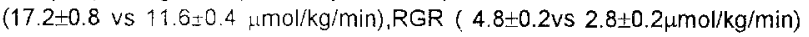
and HGR (12.4 $1.0 \mathrm{VS} 8.8 \pm 0.6 \mu \mathrm{mol} / \mathrm{kg} / \mathrm{min})$ were all increased in IDDM subjects (all $P<0.05$ ). RGR was increased by $70 \%$ versus $40 \%$ for $H G R$ and accounted for a greater proportion of SGA in IDDM subjects ( $28 \pm 1.5 \mathrm{vs}$ $24 \pm 0.5 \%$ ). Infusion of insulin suppressed HGO by $80 \%$ but reduced RGR only by $50 \%$ in the IDDM subjects $(p<0.05)$. We conclude that in humans renal glucose release is increased and makes a substantial contribution to glucose overproduction and finally that one can no longer assume that systemic glucose appearance is synonymous with hepatic glucose output.

\section{1}

HEPATIC RESPONSE TO INTRAPORTAL GLUCOSE DELIVERY AND CONCOMITANT GLUCONEOGENIC AMINO ACID INFUSION M.C. Moore, P.J. Flakoll, P.-S. Hsieh, C. Venable, M.T. Monahan, D.W. Neal, and A.D. Cherrington, Vanderbilt University, Nashville, TN, USA

This study compared the effect of concomitant peripheral $(\mathrm{Pe})$ or intraportal (Po) gluconeogenic amino acid (gngAA; ala, gly, gln, glu, ser, thr) infusion on net hepatic glucose uptake (NHGU) and hepatic glycogen synthesis during Po glucose infusion. Three groups ( $n=6 /$ group) of conscious, $42 \mathrm{~h}$-fasted dogs were studied. A primed $(40 \mu \mathrm{Ci})$, continuous $(0.4 \mu \mathrm{Ci} / \mathrm{min})\left[3{ }^{3} \mathrm{H}\right]$ glucose infusion was begun at time 0. After an 80-min equilibration period and a 40-min control period, each dog received contimuous infusions of somatostatin, Po insulin (4-fold basal), Po glucagon (basal), and Po glucose $\left(19.4 \mu \mathrm{mol} \mathrm{kg}^{-1} \mathrm{~min}^{-1}\right)$ during a $4 \mathrm{~h}$ experimental period. During the experimental period, 1 group (NoAA) received no gngAA, 1 group (POAA) received a mixture of gngAA Po at $7.6 \mu \mathrm{mol} \mathrm{kg}^{-1} \mathrm{~min}^{-1}$, and 1 group $(\mathrm{PeAA})$ received the gngAA mixture $\mathrm{Pe}$ at a rate which produced the same hepatic load of gngAA as observed in PoAA. Pe glucose was infused as needed to increase the hepatic glucose load to $150 \%$ of basal. Glycogen synthesis was calculated as DPM ${ }^{3} \mathrm{H}$ in glycogen $\div$ mean arterial and portal vein specific activity. Experimental period results (mean \pm SEM in $\mu \mathrm{mol} \mathrm{kg}^{-1} \cdot \mathrm{min}^{-1} * *<0.05 \mathrm{vs}$ the other 2 groups):

\begin{tabular}{lccccc}
\hline Group & $\begin{array}{c}\text { Hepatic } \\
\text { glucose load }\end{array}$ & $\begin{array}{c}\text { Net hepatic } \\
\text { glucose } \\
\text { uptake }\end{array}$ & $\begin{array}{c}\text { Net hepatic } \\
\text { gngAA load }\end{array}$ & $\begin{array}{c}\text { Net hepatic } \\
\text { gngAA } \\
\text { uptake }\end{array}$ & $\begin{array}{c}\text { Hepatic } \\
\text { glycogen } \\
\text { synthesis }\end{array}$ \\
\hline NoAA & $\mathbf{1 6 2 . 1 \pm 2 0 . 0}$ & $9.7 \pm 3.2$ & $26.6 \pm 2.8^{*}$ & $4.9 \pm 0.6^{*}$ & $9.1 \pm 1.6$ \\
PoAA & $180.3 \pm 12.7$ & $\mathbf{1 . 8 \pm 0 . 7 *}$ & $56.3 \pm 5.8$ & $15.6 \pm 2.5$ & $3.6 \pm 0.4^{*}$ \\
PeAA & $176.3 \pm 19.0$ & $9.1 \pm 2.4$ & $54.9 \pm 7.3$ & $11.5 \pm 2.1$ & $7.7 \pm 1.4$ \\
\hline
\end{tabular}

Conclusions: Concomitant portal, but not peripheral, infusion of gluconeogenic amino acids blunts net hepatic glucose uptake and hepatic glycogen synthesis during portal glucose infusion. A sensor for the route of amino acid delivery must exist, and it causes diversion of glucose from the liver to muscle and adipose tissue during portal amino acid delivery.

\section{0}

DIRECT ASSESSMENT OF SECRETION, KINETICS AND CLEARANCE OF BCELL PEPTIDES DURING HEPATIC VEIN CATHETERIZATION

B. Ludvik, J. Nolan, K. Thomaseth, G.Pacini, M. Clodi, R. Prager and J. Oletsky, San Diego, CA; Vienna, Austria; and Padova, Italy

Little is known about the secretion, kinetics, and clearance of the endogenously released B-cell peptides during a dynamic test. To directly evaluate the secretion and clearances of C-peptide, insulin and islet amyloid polypeptide (IAPP), we performed hepatic vein catheterization experiments (HVC) in 6 lean, healthy controls (C), 6 obese, insulin resistant subjects $(O)$ and 6 patients with NIDDM (D). Splanchnic blood flow was assessed by infusion of indocyanine green dye. Following glucose administration $(75 \mathrm{gms})$ the respective peptides and glucose were measured every 15 min over a period of $240 \mathrm{~min}$, and the parameters were calculated by ineans of a general circulatory model. Arterial glucose was $98 \pm 4 \mathrm{mg} / \mathrm{dl}$ at basal, $180 \pm 17$ at 60 and $152 \pm 10$ at $120 \mathrm{~min}$ in $\mathrm{C}, 100 \pm 1,19 \mathrm{I} \pm 8$ and $158 \pm 10$ in $\mathrm{O}$, and $202 \pm 28,343 \pm 29$ and $358 \pm 26$ in $D(p<0.05$ vs. $O$ and $C$ ). By $240 \mathrm{~min} C$ and $O$ returned to preload values while glucose of $D$ was still higher $(27 . \pm 29)$. C-peptide secretion rate was in C $84 \pm 42 \mathrm{pmol} / \mathrm{min}$ at basal, $721 \pm 325$ at $60 \mathrm{~min}$, and $324 \pm 173$ at $120 \mathrm{~min}$, in $\mathrm{O}$ $493 \pm 141,1858 \pm 622$, and $1875 \pm 443$ ( $p<0.05$ vs. $C$ and $D$ ), in D $158 \pm 41,584 \pm 230$, and $630 \pm 125$, by 240 min all returned to basal values. Cumulative insulin secretion calculated for the portal vein was $131 \pm 14 \mu \mathrm{M} / 3 \mathrm{~h}$ in $\mathrm{C}, 386 \pm 98$ in $\mathrm{O}(\mathrm{p}<0.05$ vs. $\mathrm{C}$ and D), and $92 \pm 18$ in D. Hepatic extraction of insulin was estimated $55 \%$ of the secreted hormone in $\mathrm{C}, 61 \%$ in $\mathrm{O}$, and $78 \%$ in $\mathrm{D}$ ( $\mathrm{p}<0.05$ vs. C). Our analysis showed that IAPP and C-peptide are proportionally secreted, and the co-secretion factors were $0.006 \pm 0.003$ in $\mathrm{C}, 0.0025 \pm 0.0003$ in $\mathrm{O}$ ( $\mathrm{p}<0.05$ vs. $\mathrm{C}$ ), and $0.002 \pm 0.0001$ in $\mathrm{D}$ $(\mathrm{p}<0.05$ vs. D). In conclusion, this method directly quantifies prehepatic B-ceil hypersecretion in $O$. The increased hepatic extraction of insulin in $D$, the mechanism of which has to be elucidated, could further contribute to the low peripheral insulin levels in these patients. Finally, the reduction of the IAPP/C-peptide cosecretion factor in $O$ and $D$ confirms the results in animal experiments that LAPP secretion is impaired prior to that of insulin during the transition from normal to inaired glucose tolerance.

\section{2}

FREE FATTY ACID DECREASES INITIAL HEPATIC UPTAKE OF PORTALY INFUSED GLUCOSE

M.Kubota, Y. Shiba, I.Nakahara, T.Tomita, M.Ikeda, M.Matsuhisa, T Watarai, R.Kawamori*, and Y Yamasaki Osaka University School of Medicine, Osaka, and *Juntendo University School of Medicine, Tokyo, Japan

Recent studies have suggested that high plasma free fatty acid (FFA) observed in diabetics decreases peripheral glucose uptake, however, its effect on hepatic glucose uptake has not been investigated. The aim of this study is to clarify the effect of high plasma FFA level on glucose metabolism such as hepatic glucose production (HGP), initial hepatic glucose uptake (IHGU), and glucose metabolic clearance rate (MCR). The study was conducted using dual stable tracer method $\left(6,6-{ }^{2} \mathrm{H}_{2}\right.$-glucose, U-

${ }^{13} \mathrm{C}$-glucose) in SD rats $(\mathrm{n}=7)$ during basal state and during portal glucose $/$ insulin infusion $(9.0 \mathrm{mg} / \mathrm{kg} / \mathrm{min}, 9 \mathrm{mU} / \mathrm{kg} / \mathrm{min})$, with or without concomitant infusion of triglyceride emulsion at two doses [HI $(\mathrm{n}=8)$ : $15 \mathrm{mg}$, LO (n=7): $5 \mathrm{mg} / \mathrm{kg} / \mathrm{min}$ ] to establish different high plasma FFA level. Plasma FFA in $\mathrm{HI}$ and $\mathrm{LO}$ were higher than N/C $(n=7)$ during both basal $(1.70 \pm 0.72,0.55 \pm 0.08,0.34 \pm 0.03 \mathrm{mmol} / \mathrm{l})$ and glucose loading period $(1.11 \pm 0.48,0.47 \pm 0.07,0.18 \pm 0.10 \mathrm{mmol} / \mathrm{)})$. In accordance with plasma FFA level, HGP in $\mathrm{HI}$ and LO was significantly higher than that in LO both during basal (HI: $0.063 \pm 0.016, \mathrm{LO}: 0.047 \pm 0.007, \mathrm{~N} / \mathrm{C}$ : $0.038 \pm 0.004 \mathrm{mmol} / \mathrm{kg} / \mathrm{min}$ ) and portal glucose loading period. Plasma insulin level in $\mathrm{HI}$ and LO was higher than N/C because of FFA induced insulin secretion. During portal glucose loading, IHGU in HI and LO was significantly lower than $\mathrm{N} / \mathrm{C}(6.5 \pm 2.0,8.4 \pm 2.9,16.6 \pm 1.0 \%)$. MCR in HII $(29$ $\mathrm{ml} / \mathrm{kg} / \mathrm{min}, \mathrm{P}<0.05$ ) and $\mathrm{LO}$ were similar to that in N/C. In summary, the infusion of triglyceride emulsion not only increased HGP but also reduced hepatic uptake of portaly infused glucose. Thus, plasma FFA level is one of the determinants of hepatic glucose uptake. 
173

EVIDENCE THAT "AUTOREGULATION" OF HEPATIC GLUCOSE OUTPUT IS MEDIATED BY FREE FATTY ACIDS.

M. Ader and R.N. Bergman. Department of Physiology and Biophysics, University of Southem California, Los Angeles, CA, USA.

Hyperglycemia per se can inhibit hepatic glucose output (HGO), termed giucose "autoregulation". The mechanism by which hyperglycemia inhibits $\mathrm{HGO}$ is unknown. In light of recent evidence implicating free fatty acids (FFA) as the link in suppression of HGO by insulin, we examined the role of FFA in suppression of HGO by glucose itself. Glucose clamps were performed ( $n=8 \mathrm{dogs}$ ), in which glucose was raised to 3 successive levels $(90-$ min each) by variable labelled glucose during somatostatin and basal intraportal insulin and glucagon. Glucose, FFA, and HGO were measured at basal, and during final 30 min of each hyperglycemic period (steady state: SS).

\begin{tabular}{lcccc} 
& Basal & SS \#1 & SS \#2 & SS \#3 \\
\cline { 2 - 5 } GLUCOSE (mM) & $5.4 \pm 0.1$ & $8.5 \pm 0.2$ & $12.5 \pm 0.2$ & $16.6 \pm 0.2$ \\
NSUULIN ( $p M)$ & $105 \pm 15$ & $47 \pm 5$ & $64 \pm 10$ & $86 \pm 13$ \\
HGO (mg/min $/ \mathrm{kg})$ & $2.54 \pm 0.20$ & $2.06 \pm 0.17$ & $1.17 \pm 0.19$ & $0.52 \pm 0.33$ \\
$\quad$ (\% basal) & & $82.4 \pm 5.2$ & $44.5 \pm 6.0$ & $27.2 \pm 4.9$ \\
FFA (mmol $/$ L) & $0.46 \pm 0.07$ & $0.31 \pm 0.05$ & $0.18 \pm 0.13$ & $0.13 \pm 0.03$ \\
(\% basal) & & $74.3 \pm 14.0$ & $42.8 \pm 8.6$ & $31.6 \pm 8.1$
\end{tabular}

Hyperglycemia per se caused a progressive fall in FFA during clamps, which strongly paralleled the concomitant fall in HGO. There was a tight relationship between FFA and HGO at SS $(r=0.976 ; p<0.0001)$ and during dynamic changes in FFA $(r=0.887 ; p<0.007)$. These data reveal the effect of hyperglycemia to suppress free fatty acids in the absence of secreted insulin. These results suggest that suppression of glucose production by glucose per se may be mediated by a similar peripheral signal as that which mediates its suppression by insulin: free fatty acids.
174

Hepatic perivenous glucose metabolism is altered in offspring of protein-restricted rat dams

M. Desai, S. P. Burns*, R. A. Iles*, R. D .Cohen*, and C. N Hales. Department of Clinical Biochemistry, University of Cambridge, Addenbrookes Hospital, Cambridge CB2 2QR, U.K., and "Medical Unit, The Royal London Hospital, (School of Medicine, Queen Mary \& Westfield College), London E1 1BB, U. K.

Using a novel mapping technique we studied zoned hepatic enzyme activities associated with glucose homeostasis in the ex-vivo perfused liver in female offspring of rat dams fed either a $20 \%$ (C) or $8 \%$ (LP) protein diet during pregnancy and lactation. All offspring were weaned on a normal $20 \%$ protein diet and studied at 6 months of age. Gluconeogenesis from $6 \mathrm{mM}$ lactate was greater in LP than C rats (1.59 \pm 0.14 vs $1.18 \pm 0.10$, means $\pm S E M$ of $n=9$ per group; $p<0.05$ ). The LP rats had overall reduced glucokinase activity compared to the controls $(0.92 \pm 0.07$ vs $1.34 \pm 0.06 \mathrm{U} / g$ liver; $p<0.002)$ with no change seen in the hexokinase activity $(0.29 \pm 0.03$ vs $0.29 \pm 0.02 \mathrm{U} / \mathrm{g}$ liver, respectively). The effect of small digitonin-induced perivenous destructions (up to $30 \%$ of lobular volume) in $\mathrm{C}$ rats resulted in a loss of $40 \%$ of the total glucokinase activity, indicating a major concentration of glucokinase in this zone; this perivenous emphasis was absent in LP rats. Consistent with this, there was an increase in glucose output after digitonin in $C$ rats $(24 \%, n=11 ; p<0.005)$, an effect which was absent in LP rats ( $2.7 \%$ increase, $n=9 ; p=0.66$ ). This means that in $\mathrm{C}$ rats unlike in LP rats, the glucose produced in periportal zone from lactate is partially consumed in the perivenous zone. Although the relative liver weights are comparable, the LP rats had significantly larger liver lobules. Thus maternal low protein alters liver anatomy in adult female offspring, and these livers have an altered perivenous zone with respect to glucose metabolism.

\section{OP 30}

\section{$\mathrm{K}^{+}$-ATP Channels $\mathrm{Ca}^{2+}$ and Insulin Oscillations}

\section{5}

THE CLONED $\beta$-CELL $K_{\text {ATP }}$ CHANNEL: TOLBUTAMIDE-, DIAZOXIDE- AND ATP-SENSITIVITY.

C Ämmälä and FM Ashcroft University Laboratory of Physiology, Parks Road, OXford OXI $3 P T, U K$

In pancreatic $\beta$-cells the inhibition of ATP-sensitive $\mathrm{K}^{+}$-channels ( $\mathrm{K}_{\mathrm{ATP}}$-channels) plays a key role in glucose-mediated and sulphonylurea-mediated insulin secretion. Sulphonylureas stimulate insulin secretion by binding to a high affinity receptor (SUR1) thereby inhibiting the $\mathrm{K}_{\mathrm{ATP}}$-channel. The $\beta$-cell $\mathrm{K}_{\mathrm{ATP}}$ channel is hetero-multimeric channel complex formed by at least two subunits: SUR1 - a member of the ATP Binding Casette-transporter family, and $\mathrm{K}_{\mathrm{ir}} 6.2$ - an inwardly rectifying $K^{*}$-channel. Coexpression of SUR1 with $K_{i}, 6.2$ produces a channel resembling the native $K_{\text {ттр }}$-channel. $K_{\mathrm{in}} 6.2$ does not form a functionai channel alone and it is therefore difficult to discriminate between properties endowed by SUR1 and those residing with $\mathrm{K}_{\mathrm{ir}} 6.2$. We used $\mathrm{K}_{\mathrm{i}} 6.1$, a channel $74 \%$ homologous to $\mathrm{K}_{\mathrm{ir}} 6.2$, to investigate the interaction of $\mathrm{K}_{\mathrm{i}}$-channels with SUR1. cDNA encoding $K_{i r} 6.1$ (or $K_{i} 6.2$ ) was transfected into HEK293 cells either alone or together with SUR1. Whole-cell currents were studied $48-72 \mathrm{~h}$ after transfection, using the patch-clamp technique with $40 \mathrm{mM}$ external $\mathrm{K}^{*}$. Cells transfected with K 6.1 expressed larger current than mock transfected cells $\left(2.9 \pm 0.5 \mathrm{nA}, \mathrm{n}=6\right.$ and $0.2 \pm 0.05 \mathrm{pA}, \mathrm{n}=19$ respectively). The $\mathrm{K}_{\mathrm{i}} 6.1$ current did not increase with time when dialysed with $0.3 \mathrm{mM}$ ATP and was not blocked by $0.5 \mathrm{mM}$ tolbutamide or activated by $0.34 \mathrm{mM}$ diazoxide. When cells coexpressing Kir6.1 and SUR 1 were dialysed with $0.3 \mathrm{mM}$ ATP the current increased from $1.6 \pm 0.2 \mathrm{nA}$ to $2.0 \pm 0.2 \mathrm{nA} \quad(\mathrm{n}=13, \mathrm{p}<0.002)$ within $15 \mathrm{~min}$. No time-dependent increase was seen in cells dialysed with $5 \mathrm{mM}$ ATP. This suggests the $\mathrm{K}$ 6.1/SUR 1 current is sensitive to ATP. Tolbutamide blocked the current by $40 \pm 6 \%(n=29 p<0.0001)$ and diazoxide produced an activation of $159 \pm 14 \%$ $(n=17, p<0.0005)$. We therefore conclude that SUR1 endows $K_{i} 6.1$ with sulphonylurea-, diazoxide- and ATP-sensitivity. Our results further suggest that SUR1 may confer tolbutamide, diazoxide and ATP-sensitivity on the native $\beta$ cell channel complex.

\section{6}

IDENTIFICATION AND VOLTAGE-DEPENDENCE OF Na/Ca EXCHANGE CURRENT IN MOUSE PANCREATIC B-CELLS

D. Gall, K. Bokvist", P. Rorsman ${ }^{*}$ and A. Herchuelz. Lab. of Pharmacology, Brussels University School of Medicine, Brussels, Belgium and "Islet Cell Physiology, Novo Nordisk A/S, Copenhagen, Denmark.

The existence of a $\mathrm{Na} / \mathrm{Ca}$ exchange current was investigated in single pancreatic $\beta$-cells by combining the techniques of whole-cell voltage clamp and intracellular perfusion. Ionic currents that could interfere with the measurement of the $\mathrm{Na} / \mathrm{Ca}$ exchange current were blocked by using caesium and nifedipine, $\mathrm{Na} / \mathrm{Ca}$ exchange was activated by the presence of $21 \mu \mathrm{M}$ calcium in the pipette solution. The membrane current $\left(\mathrm{I}_{\mathrm{m}}\right)$ was recorded during a $1 \mathrm{~s}$ ramp pulse from $-70 \mathrm{mV}$ to $+30 \mathrm{mV}$. The observed current was significantly reduced by suppressing the transmembrane sodium gradient or by adding $10 \mu \mathrm{M}$ of the well known exchange inhibitory peptide (XIP) in the intracellular solution. At a membrane potential $\left(\mathrm{V}_{\mathrm{m}}\right)$ of $-70 \mathrm{mV}, \mathrm{I}$ was $-3.92 \pm 0.31 \mathrm{pA} / \mathrm{pF}(\mathrm{n}=21)$ in the presence and $-1.95 \pm 0.18 \mathrm{pA} / \mathrm{pF}(\mathrm{n}=16, \mathrm{p}<0.001)$ in the absence of a transmembrane sodium gradient ( $\mathrm{Na}^{+}$replaced by sucrose). Similar results were obtained with $\mathrm{Na}^{+}$replaced by choline-Cl $\left[\mathrm{I}_{\mathrm{m}}=-1.46 \pm 0.16 \mathrm{pA} / \mathrm{pF}\right.$ $\left.(\mathrm{n}=13, \mathrm{p}<0.001), \mathrm{V}_{\mathrm{m}}=-70 \mathrm{mV}\right]$. In the presence of $10 \mu \mathrm{MXIP}$ in the pipette solution and of a transmembrane $\mathrm{Na}^{+}$gradient, $\mathrm{I}_{\mathrm{m}}$ was $-1.73 \pm$ $0.12 \mathrm{pA} / \mathrm{pF}(\mathrm{n}=16, \mathrm{p}<0.001), \mathrm{V}_{\mathrm{m}}=-70 \mathrm{mV}$. The dependence of $\mathrm{I}_{\mathrm{m}}$ on the transmembrane sodium gradient together with its sensitivity to XIP suggests the existence of a $\mathrm{Na} / \mathrm{Ca}$ exchange current. The reversal potential of this exchange current was in agreement with the expected theoretical model for the $\mathrm{Na} / \mathrm{Ca}$ exchange with a stoechiometry of $3 \mathrm{Na}^{+}: 1 \mathrm{Ca}^{2+}$. In conclusion, the present work demonstrate the existence of a voltage-dependent $\mathrm{Na} / \mathrm{Ca}$ exchange current in the $\beta$-cell. 
FIRST PHASE ELEVATION OF $\mathrm{Ca}^{2+}$ IN GLUCOSE-STIMULATED $\beta$-CELLS IS INDEPENDENT OF INTERNAL $\mathrm{Ca}^{2+}$ SEQUESTRATION AND RELEASE

E. Gylfe and Y.-J. Liu. Department of Mecical Cell Biology, Uppsala University, BMC Box 571, S-751 23 Uppsala, Sweden

It has been proposed that the prolonged initial elevation of cytoplasmic $\mathrm{Ca}^{2+}$ in glucose-stimulated $\beta$-cells involves a combination of intracellular $\mathrm{Ca}^{2+}$ release and influx of the ion through voltagedependent channels, whose open state is partially maintained by an inward current activated by $\mathrm{Ca}^{2+}$ store depletion. Using the indicator fura- 2 we have evaluated this possibility by measuring cytoplasmic $\mathrm{Ca}^{2+}\left(\left[\mathrm{Ca}^{2+}\right]_{i}\right)$ and $\mathrm{Sr}^{2+}\left(\left[\mathrm{Sr}^{2+}\right]_{i}\right)$ in individual mouse $\mathrm{B}$-cells exposed to the respective cation. Elevation of glucose from 3 to $11 \mathrm{mmol} / \mathrm{I}$ induced large amplitude oscillations of $\left[\mathrm{Ca}^{2+}\right]_{i}$ or $\left[\mathrm{Sr}^{2+}\right]_{i}$. In most cases the first oscillation had higher amplitude and/or longer duration than the subsequent ones. Exposure to hyperpolarizing diazoxide during glucose stimulation caused lowering of $\left[\mathrm{Ca}^{2+}\right]_{i}$ to basal levels but the oscillations reappeared upon diazoxide omission. Also the first of the reappearing oscillation tended to reach a higher amplitude and the duration was sometimes increased. The intracellular $\mathrm{Ca}^{2+}$-ATPase inhibitor thapsigargin, which abolished sequestration and release of intracellular $\mathrm{Ca}^{2+}$ and $\mathrm{Sr}^{2+}$, did not prevent a glucose response in terms of $\left[\mathrm{Ca}^{2+}\right]_{i}$ or $\left[\mathrm{Sr}^{2+}\right]_{i}$ oscillations with a more pronounced initial elevation. In fact the first $\left[\mathrm{Ca}^{2+}\right]_{i}$ but not $\left[\mathrm{Sr}^{2+}\right]_{i}$ oscillation tended to be further proionged. The results indicate that intracellular sequestration and mobilization of $\mathrm{Ca}^{2+}$ are not required for the glucose-stimulated individual $\beta$-cell to respond with oscillations initiated by a more pronounced first phase elevation of $\left[\mathrm{Ca}^{2+}\right]_{\text {i }}$.

\section{9}

TEMPORAL AND QUANTITATIVE CORRELATIONS BETWEEN CYTOPLASMIC $\mathrm{CA}^{2+}$ IN ISLET CELLS AND INSULIN SECRETION J.C. Jonas, P. Gilon and J.C. Henquin. Unité d'Endocrinologie et Métabolisme, University of Louvain, Brussels, Belgium.

An increase in cytoplasmic $\mathrm{Ca}^{2+}\left(\left[\mathrm{Ca}^{2+}\right]_{\mathbf{i}}\right)$ in $\mathrm{B}$ cells is essential for stimulation of insulin secretion. However, whether $\left[\mathrm{Ca}^{2+}\right]_{i}$ changes directly regulate secretion remains disputed. This question was addressed here by investigating the relationship between $\left[\mathrm{Ca}^{2+}\right]_{i}$ and insulin secretion in the same islets. Both events were measured simultaneously in single mouse islets loaded with fura-PE3 and perifused with a medium containing diazoxide, to prevent any effect of glucose on the membrane potential, and either 4.8 or $30 \mathrm{mmol} / 1 \mathrm{~K}^{+}$. Continuous depolarization with $30 \mathrm{mmol} / 1 \mathrm{~K}^{+}$in the presence of 15 $\mathrm{mmol} / \mathrm{l}$ glucose induced a sustained rise in $\left[\mathrm{Ca}^{2+}\right]_{\mathrm{i}}$ and insulin release (no oscillations of secretion were detected). In contrast, alternating between 30 and $4.8 \mathrm{mmol} / 1 \mathrm{~K}^{+}(1 \mathrm{~min} / 2 \mathrm{~min}$ or $2.5 \mathrm{~min} / 5 \mathrm{~min})$ triggered synchronous $\left[\mathrm{Ca}^{2+}\right]_{i}$ and insulin oscillations of regular amplitude in each islet. The amplitude and the duration of insulin oscillations were correlated with those of $\left[\mathrm{Ca}^{2+}\right]_{i}$ oscillations. This quantitative correlation between $\left[\mathrm{Ca}^{2+}\right]_{\mathrm{i}}$ and insulin secretion was confirmed by experiments in which extracellular $\mathrm{Ca}^{2+}$ was raised stepwise in the presence of $30 \mathrm{mmol} / 1 \mathrm{~K}^{+}$. This resulted in parallel stepwise increases in $\left[\mathrm{Ca}^{2+}\right]_{\mathrm{i}}$ and insulin secretion. However, while the successive $\left[\mathrm{Ca}^{2+}\right]_{\mathrm{i}}$ levels were unaffected by glucose, each plateau of secretion was much higher in 20 than $3 \mathrm{mmol} / \mathrm{l}$ glucose. In conclusion, there exists a close temporal and quantitative relationship between $\left[\mathrm{Ca}^{2+}\right]_{\mathrm{i}}$ and insulin secretion at a given glucose concentration. Only the quantitative relationship is affected by changing the glucose concentration.

\section{PULSATILE INSULIN RELEASE AT STABLE CYTOPLASMIC Ca ${ }^{2+}$}

J. Westerlund, R. Brown, S. Dryselius, E. Mörtberg and P. Bergsten; Dept of Medical Cell Biology, University of Uppsala, Uppsala, Sweden

The role of the cytoplasmic $\mathrm{Ca}^{2+}$ concentration $\left(\left[\mathrm{Ca}^{2+}\right]_{i}\right)$ for pulsatile insulin release was studied in isolated pancreatic islets from ob/ob-mice. $\left[\mathrm{Ca}^{2+}\right]_{\mathrm{i}}$ was measured with dual wavelength fluorometry in parallel with insulin release by ELISA. In the presence of $3 \mathrm{mmol} / 1$ glucose insulin release was pulsatile with a frequency of $0.42 \pm 0.03$ /min despite a stable and low $\left[\mathrm{Ca}^{2+}\right]_{\mathrm{i}}$. Depolarization by raising $\mathrm{K}^{+}$to $31 \mathrm{mmol} / \mathrm{l}$ resulted in a stable elevation of $\left[\mathrm{Ca}^{2+}\right]_{\mathrm{i}}$. However, insulin release remained pulsatile without change in frequency. An initial marked increase of the pulse amplitudes was followed by gradual decline. By collecting perifusate every $3 \mathrm{sec}$ the slow pulses could be resolved into rapid ones with a frequency of $3.8 \pm 0.5 / \mathrm{min}$. Blocking the ATPsensitive $\mathrm{K}^{+}$channels with $1 \mathrm{mmol} / \mathrm{l}$ tolbutamide, which in most cases causes sustained elevation of $\left[\mathrm{Ca}^{2+}\right]_{\mathrm{i}}$, also resulted in pulsatile release of insulin $(0.38 \pm 0.04 / \mathrm{min})$. In an attempt to examine the role of intracellular ATP on pulsatile insulin release, ouabain was used to block the ATP consumption by the $\mathrm{Na} / \mathrm{K}$ pump. In islets exposed to $11 \mathrm{mmol} / \mathrm{l}$ glucose addition of 0.1-1 mmol/1 ouabain caused a concentrationdependent increase in the amplitude of the glucose-induced insulin pulses without effect on the frequency. Although $\mathrm{Ca}^{2+}$ is an important regulator of insulin release the data indicate that secretion remains pulsatile also when $\left[\mathrm{Ca}^{2+}\right]_{i}$ is stable. It is likely that oscillatory generation of ATP is a major determinant of pulsatile insulin release. Apart from being a factor regulating $\left[\mathrm{Ca}^{2+}\right]_{\mathrm{j}}$, ATP availability may be rate-limiting for the energyrequiring exocytosis of insulin.

\section{0}

EXPRESSION OF RYANODINE RECEPTOR GENES IN ISLETS AND EFFECTS OF RYANODINE RECEPTOR-AGONISTS ON CYTOPLASMIC FREE $\mathrm{Ca}^{2+}$ CONCENTRATION IN $\beta$-CELLS.

M. S. Islam, I. Leibiger, B. Leibiger, G. Jacobsson, D. Rossi, D. Luc-Webb, V. Sorrentino, B. Meister, and P.-O. Berggren. The Rolf Luft Center for Diabetes Research, Department of Molecular Medicine, Karolinska Institute, Karolinska Hospital, 17176 Stockholm.

Several groups have reported conflicting results concerning the involvement of a ryanodine receptor intracellular $\mathrm{Ca}^{2+}$ release channel $(\mathrm{RyR})$ and its putative activator, cyclic adenosine diphosphate ribose (CADPR), in glucose-induced increase in the cytoplasmic free $\mathrm{Ca}^{2+}$ concentration $\left(\left[\mathrm{Ca}^{2+}\right]_{i}\right)$ in $\beta$-cells. To resolve these controversies, we studied expression of RyR genes in pancreatic islets and examined $\mathrm{Ca}^{2+}$ release by pharmacological activators of RyRs. In mouse or human $\beta$-cells loaded with fura-2, and perifused with $\mathrm{Ca}^{2+}$-free medium, no increase in $\left[\mathrm{Ca}^{2+}\right]_{i}$ was caused by caffeine. Beta alanyl ryanodine, a potent RyR agonist did not increase $\left[\mathrm{Ca}^{2+}\right], 2,2^{\prime}$-dithiodipyridine $\left(2,2^{\prime}-\mathrm{DTDP}\right)$ a reactive disulfide that activates sarcoplasmic reticulum RyR, released $\mathrm{Ca}^{2+}$ from intracellular stores in $\beta$-cells. However, this $\mathrm{Ca}^{2+}$ release was abolished when intracellular stores were depleted by prior application of carbachol. 2,2-DTDP-induced $\mathrm{Ca}^{2+}$ release was not inhibited by ryanodine $(50 \mu \mathrm{M})$. Microinjection of $8-\mathrm{NH}_{2}$ - $\mathrm{CADPR}$, a cADPR antagonist, did not inhibit glucose-induced increase in $\left[\mathrm{Ca}^{2+}\right]_{\text {. }}$. We used RNase protection assay and specific probes for the three mouse RyRs to detect mRNAs of respective RyRs in the total RNA prepared from islets. In these assays, a faint band was detected with the probe for RyR2. In immunofluorescence studies, islets were labelled with anti-RyR and anti-insulin antibodies. No RyR-like immunoreactivity (-LI) was detected in the insulin-containing cells but strong signal was detected from cells associated with islet microvasculature. The presence of $\mathrm{IP}_{3}$ receptor (TP3R) in $\beta$-cell is established from functional studies but immunofluorescence staining of islets with anti-IP3R antibody failed to detect IP3R-LI in $\beta$-cells. These data suggest that expression of RyR in $\beta$ cells is very low and is difficult to detect by immunofluorescence studies using available antibodies. Furthermore, effect of $\mathrm{CADPR}$ on a RyR-like channel does not seem to be involved in glucose-induced increase in $\left[\mathrm{CC}^{2+}\right]$. Pancreatic islets however, express RyR2 and the major cell types expressing it appear to be the cells associated with islet microvasculature. 
OP 31

\section{Clinical Studies in Macrovascular Disease}

\section{1}

Intima-media thikness of common carotid artery is higher in siblings of NIDDM patients than in subjects without family history of diabetes. G. De Pergola, M. Sciaraffia, N. Pannacciulli, A. Minenna, E. Laudadio, M. Tarallo and R. Giorgino. Institute of Medical Clinic, Endocrinology and Metabolic Diseases, School of Medicine, University of Bari, Bari, Italy

The quantification of the artery intima-media thickness by high resolution ultrasound color-doppler has been shown to truly reflect the anatomical intima-media thickness. Therefore, this method may provide information on the early clinically silent stages of atherosclerosis. The objective of the present study was to investigate whether subjects with 1 or both parents affected by NIDDM have a different intima-media thickness of common carotid artery as compared to individuals without family history of diabetes until the third generation. To this aim, 91 normotensive premenopausal women with normal glucose tolerance were enrolled in the study. They were divided in 4 groups: group 1, represented by 22 normal weight women with family history of NIDDM (BMI: $22.0 \pm 2.14$, age: $30.4 \pm 7.28$ ); group 2 , represented by 16 normal weight women without family history of NIDDM (BMI: $21.1+1.88$, age: $28.8+6.40$ ); group 3 , represented by 26 obcsc patients with family history of NIDDM (BMI: $32.7 \pm 5.44$, age: $30.1 \pm 7.47$ ) and group 4, represented by 27 obese patients without family history of NIDDM (BMI: $32.7 \pm 6.27$, age: $29.2 \pm 8.88$ ). The intima-media thickncss of common carotid artery was higher in group 1 than in group $2(0.93 \pm 0.10$ vs $0.75+0.17 \mathrm{~mm}, \mathrm{P}<0.05)$ and in group 3 than in group $4(1.12+0.26 \mathrm{vs}$ $0.91 \pm 0.23 \mathrm{~mm}, \mathrm{P}<0.05$ ). The present study suggests that premenopausal women with 1 or both parents affected by NIDDM have an carlicr development of atherosclerosis as compared to age-matched women without family history of diabetes. This effect seems to be independent of the influence of obesity.

\section{2}

THALLIUM SCINTIGRAPHY: HIGH PREDICTIVE VALUE OF CARDIOVASCULAR EVENTS IN ASYMPTOMATIC DIABETICS S.Halimi, J. Machecourt, P. Y. Benhamou, R.Boizel and D. Fagret D.Cordonnier, University Hospital,Grenoble, FRANCE. Aims:1)-to determine the annual rate of CV mortality and events,2)to assess the value of myocardial scintigraphy (T1-SPECT) and particularly the number of pathological segments.Methods:204 diabetic patients (158 NIDDM and 46 IDDM, age 59 \pm 11 yrs) prospectively screened with stress-RI-T1-SPECT (exercise $n=104$, dipyridamole $n=100) ; 163$ patients $(76 \%)$ had no CV history, but all had 2 risk factors or more (i,e: age $\geq 60$ years, duration of diabetes $>$ 15 years, CV events, micro or macro albuminuria \pm reduced GFR, current or past smoking and hypertension). Our population represents a high risk group of asymptomatic diabetic pts, $56 \%$ had albuminuria (> $30 \mathrm{mg} /$ day). Follow-up(systematic clinical and ECG) average period : 25 months (range 3-89 months). Kaplan-Meier survival analysis was periormed.CV events occurred in 60 of 204 pts: Major $C V$ events: $n=10$ pts died from CV causes, $n=18$ pts = non fatal Q-wave myocardial infarction (MI).Odd Ratios were: 17 $(\mathrm{p}<0.001)$ for BMl<25, $7.6(\mathrm{p}<0.002)$ for age $>60$ years; $9.8(<0.005)$ for albuminuria $>30 \mathrm{mg} /$ day; $5.2(0.001)$ for GFR $<0.7 \mathrm{ml} / \mathrm{sec}$ and $7.9(p<0.001)$ for $>2$ pathological segments at T1 SPECT. Other $C V$ events (unstable angina, congestive heart failure, need for myocardial revascularization) $n=32$ pts.Alburninuria ( $>30 \mathrm{mg} /$ day) and $>2$ pathological segments remain highly predictive. The annual rate of mortality is very high $(2.4 \%)$ vs to the general population $(0.98 \%)$ By contrast with non diabetics, the negative predictive value of $\mathrm{T} 1$ SPECT is $89 \%$ vs $99 \%$ suggesting that it may fail to predict CV events in a few patients. However, T1-SPECT provides an interesting tool with a high additive value for the prediction of future CV events in asymptomatic diabetics with a high CV risk (mainly albuminuria).

\section{3}

ARRHYTHMIAS AND MORTALITY AFTER MYOCARDIAL INFARCTION IN DIABETIC PATIENTS: RELATIONSHIP TO DIABETES TREATMENT

T.M.E. Davis, R.W. Parsons, R. Broadhurst, M. Hobbs and K. Jamrozik, University of Western Australia, Departments of Medicine and Public Health, Fremantle and Nediands, Western Australia.

Data from previous, largely animal, studies suggest that diabetes treatment at presentation influences outcome following acute myocardial infarction (AMI). In particular, arrhythmogenicity may differ between types of oral hypoglycaemic drugs. To assess the relationship between arrhythmias and mortality post-AMI and diabetes treatment, we retrospectively analysed data from all patients admitted to hospital in Perth, Australia between 1985 and 1993 who satisfied international Classification of Disease (9th version) criteria for AMI. 5.715 patients aged 25 to 65 years were identified of whom $745(12.9 \%)$ were diabetic. Short (28-day) and long term survival and complication rates in the non-diabetic and diabetic groups were compared. For diabetics, survival, atrial and ventricular fibrillation, ventricular tachycardia, heart block and pulmonary oedema were treated as outcomes, and factors related to eacit were assessed using multiple logistic regression. Diabetes treatment type was added to the model to assess its significance. Long term survival was compared by means of a Coxproportional hazards model. Mortality at 28 days was $12.0 \%$ and $28.1 \%$ for non-diabetic and diabetic patients respectively; there were no significant drug effects in the diabetic group. The rate of ventricular fibrillation amongst diabetic patients taking glibenclamide $(11.8 \%)$ was similar to that of non-diabetic patients $(11.0 \%)$ but was lower than that for those taking either gliclazide $(18.0 \%)$ or insulin $(22.8 \% ; P<0.05)$; there were no other treatment-related differences in complications. Long term survival in diabetic patients was reduced in those taking digitalis and/or diuretics. Consistent with known cardiac effects of different generations of sulphonylurea drugs, there appear to be drug-dependent effects on the frequency of ventricular fibrillation after AMt in diabetic patients, but this is not associated with reduced short or long term survival.

\section{4}

RISK OF TIASTROKE IS BIPHASICALLY RELATED TO METABOLIC CONTROL IN NON-INSULIN DEPENDENT DIABETES MELLITUS

R.Anichini. G.Seghieri. G.Meucci. S.E.Giustini. P.Lattari. G.Pettinà and V Mundula. Diabetes Unit. Pistoia. Italy.

Whether and to what extent the risk for stroke/transient ischaemic attack (TIA) is independently related to metabolic control in paticnts with non-insulin-dependen diabcics mellitus (NIDDM) is matter of debate. The present cross-sectional study presents dati from a large population including 1365 patients affected with NIDDM ( 641 males and $72+$ females. whose mean age $( \pm S D)$ was $6+ \pm 11$ vears). evaluating the prevalence of TIA/stroke. as compared with the prevalence of coronary heart disease (CHD) and of peripheral vascular disease(PVD). all of which diagnosed by standardised clinical and instrumental criteria. in relation to the levels of glycated haemoglobin ( $\mathrm{HbAlc}$ ). Percentage of patients affected with TIA/stroke was slightly decreased across quartiles of $\mathrm{HbAlc}(10 \% .10 \% .9 \%$ and $7 \%$ ) while prevalence of CHD and of PVD were weakly higher in upper HbAlc quartiles (21\%. $23 \% .24 \%$. $24 \%$ and $18 \%$. 19\%. 21\%. 22\% respectively). The relative risk for TIA/stroke. adjusted for sex age duration of diabetes. blood pressure. proteinuria, therapy and HbAlc was significantly correlated to age. hypertension. male sex proteinuria. insulin therapy and showed a biphasic correlation with HbAlc. according to a parabolic model where the odds ratio for the risk of TIA/stroke rose by about $30 \%$ for $\mathrm{HbAlc}$ values of $4.4 \%$ to $5.6 \%$ and conversely decreased for values of $5.7 \%$ to 9. $7 \%(\mathrm{p}=0.00 \mathrm{I})$. According to this same analysis odds ratio for $\mathrm{CHD}$ and for PVD were related to the same variables and increased by about the $40 \%$ for $\mathrm{HbAlc}$ values ranging from $5.6 \%$ to $9.7 \%$. In conclusion, within the observational limits of this transversal study. a) the relative risk of TIA/stroke in NIDDM is associated with age. hypertension, male sex. proteinuria. and insulin therapy. while b) it is not linearly related to metabolic control. differently from what happens for risk of $\mathrm{CHD}$ and of PVD which appear directly associated to metabolic control throughout the whole $\mathrm{HbAlc}$ range. 


\section{Insulin Therapy: IDDM}

\section{5}

HIGH-DOSE INTRAVENOUS INSULIN INFUSION IN NEWLYDIAGNOSED IDDM: EFFECTS ON BETA-CELL FUNCTION ${ }^{1}$ B.Eisfelder, ${ }^{1,2}$ A.G.Ziegler, ${ }^{1,2}$ E. Standi and ${ }^{1,2} \mathrm{O}$.Schnell. ${ }^{1}$ Diabetes Research Institute and ${ }^{2}$ Schwabing City Hospital, Kölner Platz 1, 80804 Munich.

High-dose intravenous insulin infusion at the onset of insulin-dependent diabetes mellitus is thought to improve subsequent beta-cell function. To study this hypothesis, we assigned newly diagnosed IDDM patients to receive either an experimental high-dose intravenous insulin infusion for two weeks $(n=9$, maintenance of blood glucose levels between 60 and $100 \mathrm{mg} / \mathrm{dl}$ ) or an intensified conventional insulin therapy ( $\mathrm{n}=11$, four injections per day). The clinical characteristics of the two groups were: $\mathrm{HbAlc} 10.5 \pm 2.0$ vs $12.1 \pm 2.9 \%$, age $25 \pm 7$ vs $28 \pm 7 \mathrm{yr}$, BMI $20.6 \pm 3.0$ vs $21.9 \pm 2.6 \mathrm{~kg} / \mathrm{m}^{2}$. Beta-cell function, as assessed with fasting plasma Cpeptide and C-peptide after mixed-meal stimulation (60 min), was similar in both groups: $1.2 \pm 0.4 \mathrm{vs} 1.1 \pm 0.7 \mathrm{ng} / \mathrm{ml}$ and $2.0 \pm 1.0 \mathrm{vs} 2.1 \pm 1.1 \mathrm{ng} / \mathrm{ml}$. During the first two weeks, the experimental-therapy group obtained three times more insulin than the conventionally treated group $(1.2 \pm 0.4$ vs $0.4 \pm 0.1 \mathrm{U} / \mathrm{kg} / \mathrm{day}, \mathrm{p}<0.001)$. After the first two weeks, both groups received intensified conventional insulin therapy. At one year, daily insulin dose and BMI were comparable between the two groups $(0.5 \pm 0.2$ vs $0.5 \pm 0.1 \mathrm{U} / \mathrm{kg} / \mathrm{day}$ and $22.8 \pm 2.6$ vs $24.2 \pm 2.6 \mathrm{~kg} / \mathrm{m}^{2}$ ). Fasting and stimulated $(60 \mathrm{~min}) \mathrm{C}$-peptide levels suggest no difference between the two groups $(0.6 \pm 0.3$ vs $0.8 \pm 0.7 \mathrm{ng} / \mathrm{ml}$ and $3.7 \pm 1.9$ vs $4.5 \pm 2.3 \mathrm{ng} / \mathrm{ml}$ ). Metabolic control, as evidenced by $\mathrm{HbAlc}$ was similar between the two groups ( $5.4 \pm 0.6$ vs $6.0 \pm 1.1 \%$ ). We conclude that high-dose intravenous insulin infusion in the initial treatment of IDDM is not superior in preserving beta-cell function over a one-year period when compared with intensified conventional insulin therapy.

\section{7}

Risk of Adverse Effects of Intensified Treatment IDDM:

A Meta-Analysis

M. Egger, G Davey Smith and P. Diem

Bern, Switzerland and Bristol, UK.

Intensified insulin treatment aiming at near-normal glycaemia is the recommended therapy of IDDM. While the benefits of intensified treatment are well recognised, the countervailing risks have been poorly characterised. We examined the risk of severe hypoglycaemia, ketoazidosis (DKA) and death in a meta-analysis of randomised trials comparing conventional and intensified treatment regimens in IDDM.

Fifteen randomised controlled trials including 1028 patients allocated to intensified and 1039 patients allocated to conventional treatment were identified. Logistic regression was used to calculate odds ratios (OR) and $95 \%$ confidence intervals (CI). The combined OR ( $95 \% \mathrm{CI}$ ) for severe hypoglycaemia was $2.99(2.45-$ $3.64)$, for ketoazidosis $1.74(1.27-2.38)$ and for death from all causes $1.40(0.65-3.01)$. The risk of hypoglycaemia was determined by the degree of normalisation of glycaemia achieved $(\mathrm{p}=0.005$ for interaction). The risk of DKA depended on the type of intensified treatment used. ORs were 7.20 (2.95-17.58) for exclusive use of pumps and $1.28(0.90-1.83)$ for multiple daily injection. Mortality was significantly $(\mathrm{p}=0.007)$ increased for causes potentially associated with acute complications (7 vs. 0 deaths, 5 deaths attributed to DKA and 2 sudden deaths).

There is a substantial risk of severe adverse effects associated with intensified insulin treatment, and mortality from acute metabolic causes may be increased. Multiple daily injection regimens appear to be safer than treatment with insulin pumps. The likely benefits and risks must be considered carefully for each patient with IDDM.
186

INSULIN INJECTION AT MEAL TIME OR 30 MINUTES BEFORE; Effects on Metabolic Control and Postprandial Glyceamic Excursions in IDDM Patients.

A M Rosenfalck, O Christensen, A Dejgaard and $\mathbf{J}$ Hilsted.

Department of Internal Medicine and Endocrinology, Hvidovre University Hospital, Copenhagen, Denmark.

IDDM patients are advised to inject insulin 30 minutes before the meal. The purpose of the present study was, in a randomized crossover design, to investigate the effect of Insulin Actrapid ${ }^{\circledR}$ on metabolic control and postprandial glycaemic excursion, when given at meal time or 30 minutes before in IDDM patients on basal bolus regimen. After a 4 week run in period patients were allocated to inject their meal related insulin at either time 0 or -30 minutes before meal, for the next two 6 weeks trial periods. At the end of each period patients were admitted to the hospital for a meal test. 17 IDDM patients $\left(110^{\circ}\right.$ and 6o ), age $34.8 \pm 5.7 \mathrm{yrs}($ mean $\pm \mathrm{SD})$, diabetes duration $8.5 \pm 7.9 \mathrm{yrs}$ were included. Meal test blood glucose (BG) $\mathrm{mmol} / \mathrm{l}$ profiles:

\begin{tabular}{|c|ccccccccc|}
\hline Inj & -30 & 0 & 10 & 20 & 40 & 90 & 120 & 180 & 240 \\
\hline-30 & 5.9 & 5.4 & 4.8 & 5.1 & $6.7^{*}$ & 8.8 & 8.2 & 6.3 & 5.8 \\
0 & 5.6 & 5.5 & 5.5 & 6.1 & 8.5 & 9.8 & 8.9 & 6.1 & 5.8 \\
\hline
\end{tabular}

Injection of insulin 30 minutes before the meal was associated with a decrease in $B G$ of $1.1 \mathrm{mmol} / 1$ resulting in significantly lower $B G$ values $30-50$ minutes after the meal compared with injection at time $0 \mathrm{~min}$. AUC was not significantly different. HbAlc at inclusion $8.7 \pm 0.9 \%$. During study period $\mathrm{HbAlc}$ decreased in total $0.59 \%$, of this $0.3 \%$ in the run-in period; no significant difference between periods.

In conclusion: Insulin injection 30 minutes before the meal leads to a reduced postprandial BG compared with injection at meal time. However, we were not able to demonstrate any effect of injection time on the metabolic control.

\section{8}

EFFECT OF MENSTRUAL CYCLE ON INSULIN DEMAND IN MDM WOMEN

Gy. Tamás, Á. Gy. Tabák, P. Vargha and Zs. Kerényi*, Semmelweis University and Szt. Imre Hospital ${ }^{\star}$, Budapest, Hungary

A hypergiycaemic tendency or ketoacidosis before menstruation and hypoglycaemic episodes in the first half of the menstrual cycle in DDDM women were observed To study this phenomenon diaries (blood glucose [BG] values and insulin dose given) of 32 wellcontrolled IDDM women (A; mean age: 36.2 [range 19-49]yrs; diabetes duration: 19.3 [4 36lyrs; BMI: $23.6 \pm 3.1[\mathrm{SD}] \mathrm{kg} / \mathrm{m} 2$; HbAlc: $6.7 \pm 1.3 \%$ ) with regular periods (mean length: $28.4[25-35]$ days; $n-462$ ) were evaluated. All patients were on intensified insulin therapy and educated to modify insulin dosage to avoid hypo-and hyperglycaemic tendency caused by the menstrual cycle. Postmenopausal IDDM women $(\mathrm{B} ; \mathrm{n}=8$; age: $58.3+2.1[56-60] \mathrm{yrs}$; duration: $23.3 \pm 7.5[19-32 \mathrm{lyrs}$, BMI: $28.1 \pm 3.2[25.5-31.6] \mathrm{kg} / \mathrm{m} 2$; HbAlc: $7.9 \pm 1.1 \%$ ) and male IDDM patients (C) n=8; age: $39.8+18.0$ [25-52]yrs; duration: $18.0+5.8$ [13-23lyrs; BMF $230 \div 11 \mathrm{~kg} / \mathrm{m} 2 ; \mathrm{HbAlc} 7.2+1.0 \%)$ served as controls. In control groups calendar months were used as analogues to the cycles $(B: n=63 ; C: n=101)$. To assess possible premenstrual increase and pxstmenstrual decrease in insulin demand and to avoid disturbing effect of different cycle lengths insulin dose given and BG (fasting and preprandial) were statistically analysed on days 1-4 and 10-13 a) 'forwards' after menstruation (follicular phase) as well as b) calculated 'backwards' from the last day of the cycle (luteal phase), resp.

\begin{tabular}{|c|c|c|c|c|c|}
\hline & \multicolumn{3}{|c|}{ Insulin dose (IU/day) } & \multicolumn{2}{|c|}{$\overline{B G(m m o l l l})$} \\
\hline & day $1-4$ & day $10-13$ & $P$ & day $1-4$ & day $10-13 P$ \\
\hline Forward $A$ & $42.7 \pm 10$. & $40.5 \pm 10.4$ & $<0.001$ & $7 \pm 2.6$ & \begin{tabular}{|l|l|l}
$7.1 \pm 2.9$ & $\mathrm{NS}$
\end{tabular} \\
\hline & $45.4 \pm$ & 6.3 & NS & $8.6 \pm 3.0$ & $8.9 \pm 3.4 \mathrm{NS}$ \\
\hline & $51.9 \pm 8.2$ & $51.8+8.1$ & NS & $8.9 \pm 3.3$ & $9 \pm 3.0 \mathrm{NS}$ \\
\hline ackward A & $43.3 \pm 11.0$ & $41.4 \pm 10.7$ & 0.001 & $7.3 \pm 2.9$ & $7.3 \pm 2.8 \mid \mathrm{NS}$ \\
\hline
\end{tabular}

Weil-fittod polinomials (person, as grouping factor and time [cycles with 28 days] as covariate; ANCOVA: $\mathrm{P}<0.001$; 3rd degree) in menstruating IDDM women proved a mean decrease in insulin dose from 46.7 to 43.3 U/day on day 11 of the cycle (BG 5.7+0.9 mean decrease in insulin dose from 46.7 to 43.3 U/day on day 11 of the cycle (BG $5.7+0.9$
vs $6.0+1.1 \mathrm{mM} / \mathrm{L}$; PS). Changes in insulin demand as an effect of the menstrual cycle have also to be taken into consideration in educating patients on intensified therapy. 
OP 33

\section{Small Babies, Insulin Resistance and Free Fatty Acids}

189

EVIDENCE FOR INSULIN-RESISTANCE DEVELOPING IN YOUNG ADULTS WITH INTRA-UTERINE GROWTH RETARDATION.

J.Léger. C.Léry-Marchal, J.Block, A.Pinet. K.Benali. D.Porquet. D.Collin P.Czernichow-INSERM CJF 93-13, Paris and Centre Hospitalier, Haguenau -France lncreased risk for development of insulin-resistance (IR) and NIDDM has been reported from various populations in subjects born with a low birthweight (BW). In a well-defined Caucasian cohort, factors of IR and syndrome $X$ was compared in young adults between subjects born with an intra-uterine growth retardation (IUGR) and controls. Gestational age was $37-42$ wk in the 2 groups. The population base was made of all individuals included between 1971-78 in the same city maternity registry $(n=12000)$. IUGR was defined as $<3^{\text {rd }}$ perc $(n=236: B W=2550 \pm 334 \mathrm{~g}:$ age $=20.5 \pm 2 y)$ and controls (CTRL) were randomly selected among individuals between $25-75^{\text {th }}$ perc $(n=280 ; B W=3410 \pm 215 \mathrm{~g}$; age $=20.8+2$. lyr) of the birthweigth distribution in this population. OGTT were performed according to WHO recommendations. None of the subjects was diabetic. Five IUGR and three CTRL were glucose intolcrant $(p=0.2)$ "True" plasma insulin (INS) was measured using IRMA and proinsulin (PINS) using a standard RIA. Comparisons of means were tested after adjustment for gestational age, sex and current BMI, and log transformation for INS and PINS:

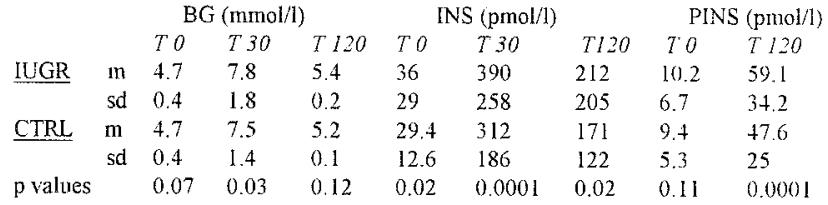

No significant effect of the etiology of IUGR was demonstrated on the rcsults of OGTT. There was no significant difference between IUGR and CTRL in the mcan blood pressure values, nor in fasting lipids profile.These results show that the "small baby syndrome" was defined by the early development of IR without the ollicr elements of the syndrome $\mathrm{X}$ in this not-ethnically diabetes-prone cohort.

\section{0}

INSULIN-RESISTANCE WTHOUT INSULIN SECREION DEFECT IS ASSOCLATED WTTH LOW BIRTH WEXGHT IN YOUNG ADULTS AT RISK FOR NDDM

C.Lévy-Marchal, DJ.Pettitt, RE.Pratley, C.Bogardus - National Institute of Diabetes and Digestive and Kidney Diseases, Phoenix, AZ, USA

Risk for NIDDM in Pima Indians shows a U-shaped relationship with birth weight. In this population insulin-resistance (IR) is a major risk factor for NDDM and a low acute insulin response to glucose (AIR) contributes to a lesser extent to the development of the disease. The aim of the present study was to test the effect of birth weight on IR and AIR in young Pima adults (age:18-30 yr.) before the appearance of overt diabetes. 261 non-diabetic subjects were enrolled in this study and classified according to birth weight: low birth weight (LBW) $\leq 2500 \mathrm{~g}(\mathrm{n}=8)$, high birth weight $(\mathrm{HBW}) \geq 4500 \mathrm{~g}(\mathrm{n}=6)$ and intermediate (IBW) for the remaining individuals $(\mathrm{n}=247)$. Glucose uptake was measured using the euglycemic clamp technique at low insulin dose. Results are corrected for the estimated metabolic body size (EMBS) after underwater weight measurement. AIR was measured as the mean increment of plasma insulin from 3-5 min. after IV glucose load in subjects with a normal oral glucose tolerance ( $\mathrm{nLBW}=5, \mathrm{nMBW}=117$ and $\mathrm{nHBW}=4$ ). Results for glucose uptake rate and AIR were compared after logarithmic transformation using ANOVA to adjust for body fat and sex.

mean \pm SD

Age (yr.)

Body Fat (\%)

2hr-OGTT Glucose

$(\mathrm{mmol} / \mathrm{l})$

Glucose Uptake Rate

(mg/min.kgEMBS)

AIR (pmol/1)

$\begin{array}{lll}\text { LBW } & \text { IBW } & \text { HBW } \\ 24.1 \pm 2.9 & 24.9 \pm 3.1 & 25.4 \pm 4.7 \\ 27 \pm 12 & 32 \pm 9 & 31 \pm 7 \\ 6.4 \pm 1.9 & 6.8 \pm 1.7 & 6.2 \pm 1.2 \\ 2.64 \pm 0.6 & 3.06 \pm 1.3 & 2.95 \pm 1.1 \\ 2106 \pm 1506 & 1602 \pm 1062 & 1278 \pm 408\end{array}$

Despite the small sample size, the difference in mean glucose uptake rates was of borderline significance $(p=0.07)$ between LBW and IBW, but did not differ significantly between $\mathrm{HBW}$ and IBW. There was no statistically significant difference in mean AIR between the three groups. In a population with a high risk for NIDDM. these results indicate that a low birth weight may be associated with IR but not with reduced insulin secretion early in aduithood.

\section{1}

FREE FATTY ACIDS AND THE INSULIN RESISTANCE SYNDROME A. Khan, S. Jinagouda, S. Damani, R. Boyadjian, M. R. Gabriel, K. ElTawil, V. Kamdar, and M. F. Saad, University of Southern California Medical school, Los Angeles, CA, USA

Free fatty acids (FFA) may contribute to the pathogenesis of the insulin resistance syndrome. We studied the relation between insulin-mediated FFA suppression and components of this syndrome in 124 Asian-Indians (60 men, 64 women) aged $46 \pm 0.8$ yrs (mean $\pm S E$ ) with body mass index (BMI) $24.4 \pm 0.3$ $\mathrm{kg} / \mathrm{m}^{2}$. Forty-two subjects had normal gtucose tolerance (NGT), 46 impaired glucose tolerance (IGT), and 36 NIDDM (WHO criteria). None was receiving any medications. During the first 30 minutes of the oral glucose tolerance test, FFA were suppressed by $29 \pm 5,18 \pm 4$, and $6 \pm 4 \%$ in women with NGT, IGT, and NIDDM respectively $(\mathrm{p}<0.001)$. Men showed a similar trend $(15 \pm 4$, $7 \pm 4$, and $1 \pm 5 \%$ respectively, $p<0.001)$, but the degree of suppression was significantly lower $(\mathrm{p}=0.002$, two-way ANOVA). In the whole group, the percent of FFA suppression was significantly $(p<0.05)$ correlated with fasting $(r=-0.56)$ and $2 h$ glucose $(r=0.28)$, fasting insulin $(r=-0.27)$, the waist/hip ratio $(r=-0.33)$, high-density lipoprotein $(r=0.32)$, triglycerides $(r=-0.40)$, and mean blood pressure $(1 / 3$ systolic $+2 / 3$ diastolic, $r=-0.28$ ), but not with age, BMI, or $2 \mathrm{~h}$ insulin. After controlling for age, sex, BMl, and the waist/hip ratio, FFA suppression continued to correlate significantly with fasting $(r=-0.52)$ and $2 h$ glucose $(r=-0.32)$ levels, fasting insulin $(r=-0.24)$, triglycerides $(r=-0.34)$, and mean blood pressure ( $r=-0.22)$. Thus, impaired insulin-mediated FFA suppression is associated with several components of the insulin resistance syndrome. The role of FFA in the pathogenesis of this syndrome needs further exploration. The sex difference in insulin-mediated FFA suppression may contribute to the decreased expression of the insulin resistance syndrome in women.

\section{2}

THE FREE FATTY ACID CONCENTRATION PREDICTS WORSENING TO DIABETES FROM IMPAIRED GLUCOSE TOLERANCE

M.A. Charles ${ }^{1}$, E. Eschwège ${ }^{1}$, B Balkau ${ }^{1}$, N. Thibult ${ }^{1}$, J.R. Claude ${ }^{2}$, J.M. Warnet ${ }^{2}$ and J. Girard ${ }^{3}$. IINSERM U21, Villejuif; 2 DASES, Paris; ${ }^{3}$ CNRS, Meudon, FRANCE

Increased levels of free fatty acid may worsen glucose tolerance by decreasing glucose uptake and/or impairing insulin secretion ( $\beta$-cell lipotoxicity). The aim of this study was to evaluate the epidemiological evidence for this hypothesis. From the Paris Prospective Study (working men aged 44-55 years) the 2-year incidence of diabetes, as defined by a glucose level of $\geq 11.1 \mathrm{mM} 2 \mathrm{hr}$ after a $75 \mathrm{~g}$ OGTT, was evaluated in 409 IGT men. Nineteen became diabetic. Comparing baseline values of those who became diabetic with those who did not, there were differences between mean age $(49.6 \pm 1.2$ vs $48.7 \pm 1.8$ (mean $\pm S D) ; p<0.005)$, central adiposity, measured by the iliac thigh ratio $(1.87 \pm 0.12$ vs $1.79 \pm 0.14 ; \mathrm{p}<0.02)$, plasma glucose $(m M)$ fasting $(6.4 \pm 0.8$ vs $5.9+0.6 ; \mathrm{p}<0.03)$ and $2 \mathrm{hr}(9.3 \pm 1.0$ vs $8.8 \pm 0.8 ; p<0.008)$, plasma free fatty acids $(m M)$, fasting $(46 \pm 15$ vs $40 \pm 16$; $\mathrm{p}<0.13)$ and $2 \mathrm{hr}(17 \pm 6$ vs $14 \pm 7 ; \mathrm{p}<0.03), 2 \mathrm{hr}$ insulin $(p M)$ (402 [108-1500] vs 510 [129-1960] (geometric mean [95\% CI]); $p<0.13$ ); factors not predictive $(\mathrm{p}>0.2)$ were BMI, blood pressure, family history of diabetes, cholesterol, triglyceride and fasting insulin levels. After adjustment for age, the variables predictive of diabetes in a multivariate logistic model were the fasting and $2 \mathrm{hr}$ glucose concentrations $(\mathrm{p}<0.0002 ; \mathrm{p}<0.03)$, the iliac-thigh ratio $(p<0.007)$, the $2 \mathrm{hr}$ levels of both free fatty acids $(\mathrm{p}<0.05)$ and insulin $(\mathrm{p}<0.003)$. We have shown, prospectively, that in the IGT, central adiposity and $2 \mathrm{hr}$ after a glucose load, increased free fatty acids associated with decreased insulin levels, are predictive of worsening glucose tolerance. 


\section{Experimental Retinopathy}

193

GLYCOLYTIC PATHWAY, REDOX STATE OF NAD-COUPLES, AND ENERGY METABOLISM IN LENS IN RATS WITH SHORT-TERM DIABETES

P.J.Oates 1, T.Nakano2, J.M.Petrash2 2 J.R.Williamson' ${ }^{2}$, and I.G.Obrosova ${ }^{2}$, IPfizer Inc, Groton,CT and 2Washington University, St.Louis, MO, USA

The study was aimed at evaluating glycolysis, redox state of free cytosolic NAD. couples, and adenine nucleotide system in lenses of rats with short-term (10 d) streptozotocin-diabetes(D). Glycolytic intermediates, $\alpha$-glycerophosphate (GP) ATP ADP, and AMP were assayed in individual lenses spectrofluorometrically by enzymatic methods; sorbitol and myo-inositol were quantified by GCMS. Free cytosolic NAD+/NADH ratios were calculated from the lactate dehydrogenase system. Sorbitol (Mean $\pm \mathrm{SD}$ ) increased to $23.6 \pm 2.6 \mathrm{in} \mathrm{D}$ (vs $0.115 \pm 0.026 \mu \mathrm{mol} / \mathrm{g}$ in controls $(\mathrm{C}))$ while myo-inositol decreased. The profile of glycolytic intermediates (increase in G6P and F6P, decrease in FDP, PEP, pyruvate, and lactate) suggests inhibition of glycolysis at the sites of phosphof ructokinase, enolase and pyruvate 12.7 in $\mathrm{D}, \mathrm{p}<0.001$. Cytosolic redox imbalance was partially prevented by tolrestat an aldose reductase inhibitor, at a dose of $100 \mathrm{mg} / \mathrm{kg}$ bwt/day, as well as by CP 166,572 , a sorbitol dehydrogenase inhibitor, at a dose of $200 \mathrm{mg} / \mathrm{kg} \mathrm{bwt} /$ day, in spite of opposite effects of the two drugs on sorbitol accumulation per se. A dramatic decrease in $\mathrm{GP}$ in $\mathrm{D}+\mathrm{ARI}$ (to $0.51 \pm 0.04$ vs $1.70 \pm 0.15$ in $\mathrm{D}$, and $0.30 \pm 0.03$ $4 \mathrm{~mol} / \mathrm{g}$ in $\mathrm{C}$ ), as well as the favourable catalytic efficiency of AR for GA3P ( -500fold higher ys glucose) suggests the possibility of GA3P reduction to GP in lens in vivo, and the contribution of this step to diabetes induced redox imbalances. ATP are were reduced in $\mathrm{D}$, compared to $\mathrm{C}$. The results indicate early changes in lens glucose utilization, redox state of NAD-couples, and energy metabolism in D. Depressed glycolysis is consistent with decreased NAD $+\mathrm{NADH}$ and adenine nucleotide phosphorylation. Redox changes associated with energy deficiency and decreased phosphorylation potential may result in impairment of ATP-dependent transport mechanisms, synthesis of glycoproteins/proteoglycans, thus contributing to sugar cataractogenesis.

\section{5}

DECREASED EXPRESSION OF INSULIN-LIKE GROWTH FACTOR I AND ITS RECEPTOR IN THE RETINA OF DIABETIC PATIENTS

C.Gerhardinger, M.Mizutani and M.Lorenzi, Schepens Eye Research Institute and Harvard Medical School, Boston, MA, USA

We are investigating events and mechanisms involved in the development of the vaso-obliteration that represents the trigger for proliferative retinopathy. Having detected accelerate death of microvascular cells in the retina of patients with background retinopathy, we compared in these patients the retinal expression of insulin-like growth factor I (IGF-I) and its receptor (IGF-IR), since IGF-I is known to be a growth/survival factor for multiple cell types and to protect from apoptosis. Total retina RNA was isolated within $30 \mathrm{~h}$ of death from 6 diabetic patients (age $63.8 \pm 7.6 \mathrm{y}$, duration of diabetes 7 $\pm 4.8 \mathrm{y})$ and 6 age and sex-matched nondiabetic controls. Northern blot analysis showed in the diabetic patients decreased levels of both IGF-I (IGFI/actin ratio $1.7 \pm 1.0$ vs $5.0 \pm 2.1$ in controls, $p=0.005$ ) and IGF-IR (IGFIR/actin ratio $13.3 \pm 5.0$ vs $36.9 \pm 16.8$ in controls, $p=0.008$ ). IGF-I was localized by immunohistochemistry to the Müller cells of the retina, and semiquantitative analysis of signal intensity in 4 diabetic and 4 nondiabetic retinas showed a trend toward decreased IGF-I levels in the retinas of diabetics patients $(0.88 \pm 0.85$ vs $2.16 \pm 1.06$ in the control retinas, $p=0.1)$ Immunoreactivity for IGF-IR as intense in discrete structures at the pedunculus of cones ad less intense in the inner plexiform layer. Retinal microvessels in trypsin digests also showed a positive staining reaction for IGF-IR. Comparison of IGF-IR abundance in diabetic and control patients are in progress. These findings indicate that the synthesis of a factor with growth/survival properties is decreased in the retina of patients with background diabetic retinopathy, and that Müller cells are affected by diabetes.

\section{4}

ENDOTHELIN RECEPTOR SUBTYPES IN RETINA OF DIABETIC RATS. EFFECT OF INSULIN TREATMENT.

J.A. de Juan, F.J. Moya, A. Rípodas, A. Fernandez-Cruz. J.M. Arencibia and R. Fernandez-Durango. Lab. Diabetes. Dpto. Medicina Interna III. Hospital Universitario San Carlos. Madrid Spain.

We investigate the characteristics of Endothelin (ET) receptor subtypes in retinal membranes of streptozotocin (STZ)-diabetic rats and the effect of insulin treatment by using radioligand binding assay. We have previously identified ETA and ETB receptor subtypes in rat retina using the subtype-selective ligands BQ123 and Sarafotoxin 60 (S6c), respectively. Male Wistar rats (180-200 g) were divided into three groups: control rats (C Group), STZ-induced diabetic rats (D Group) and insulin-treated diabetic rats (I-T Group). Rats were killed 12 weeks after injection of STZ. The competitive experiments were performed with $12 \mathrm{pM}$ of ${ }^{12} \mathrm{I}$-ET-1. Results are expressed in the Table.

\begin{tabular}{|c|c|c|c|c|c|c|c|c|}
\hline & \multicolumn{4}{|c|}{ SATURATION } & \multicolumn{3}{c|}{ DISPLACEMENT } \\
\hline & \multicolumn{2}{|c|}{${ }^{125}$ I-ET-1 } & \multicolumn{2}{|c|}{125} & \multicolumn{3}{c|}{ I-ET-3 } & \multicolumn{2}{c|}{ S6C } & \multicolumn{2}{c|}{ BQ123 } \\
\hline & Kd & Bmax & Kd & Bmax & \%ETA & $\%$ ETB & \%ETA & $\%$ ETB \\
\hline C & $63 \pm 3$ & $131 \pm 15$ & $41 \pm 1$ & $54 \pm 7.1$ & $55 \pm 3.2$ & $45 \pm 3.2$ & $46 \pm 4.9$ & $54 \pm 4.9$ \\
\hline D & $51 \pm 4$ & $141 \pm 25$ & $40 \pm 4$ & $110 \pm 20^{a}$ & $34 \pm 3.9^{a b}$ & $66 \pm 3.9^{a b}$ & $24 \pm 6.1^{a b}$ & $76 \pm 6.1^{a}$ \\
\hline I-T & $57 \pm 3$ & $171 \pm 21$ & $52 \pm 6$ & $66 \pm 9.6$ & $47 \pm 1.8$ & $53 \pm 1.8$ & $34 \pm 4 . I$ & $66 \pm 4.1$ \\
\hline
\end{tabular}

Values are means \pm SEM from 5 experiments ${ }^{a}$ significant $(p<0.05)$ difference for $D$ Group vs. C Group $;^{b}$ significant $(p<0.05)$ difference for D Group vis. I.T Group. $K d$ and Bmax values are expressed in pM and finol/mg protein, respectively.

Saturation experiments using ${ }^{125} \mathrm{I}-\mathrm{ET}-3$ demonstrated a higher density of ETB receptors in retina of $\mathrm{D}$ Group, in agreement with the results obtained with the competitive inhibition of ${ }^{125}$ T-ET-1 by the ligands. Retinal membranes of I-T Group showed a proportion of ETA and ETB receptors similar to C Group. In conclusion, there is a higher proportion of ETB receptors and a lower proportion of ETA receptors in retinal membranes from diabetic rats compared with those of control rats. Insulin treaiment increased the proportion of ETA receptors and decreased the proportion of ETB receptors in comparison to the diabetic rats.

This work was supported in part, by a grant from DGICYT (No.PB92-0737).

\section{6}

ROLE OF ADVANCED GLYCATION ENDPRODUCTS IN THE DEVELOPMENT OF DIABETIC RETINOPATHY

S. Yamagishi, T. Kawakami, Y. Yamamoto, M. Nomura, S. Harada, C.C. Hsu and $H$. Yamamoto. Department of Biochemistry, Kanazawa University School of Medicine, Kanazawa 920 , Japan

Using endothelial cell-pericyte co-culture systems, we have previously show'n that both the growth and function of endothelial cells are under the control of neighboring pericytes, explaining why retinal endothelial cells deteriorate consequent to pericyte loss, the earlicst histopathological hallinark in diabctic retinopathy. Morcover, we have recently found that advanced glycation endproducts (AGE) not only retard pericyte growth but also exert an immediate toxicity to pericytes through intcractions with a receptor for AGE (RAGE), proposing a novel mechanism underlying pericyte loss. In the present study, we investigated effects of $A G E$ on endothelial cells. We show first that AGE prepared with bovine scrum albumin and glucose stimulate the growth of endothelial cells cultivated from human umbilical vein or skin microvessels, but inhibit their prostacyclin-producing ability through their interaction with RAGE. Further, we demonstrate that AGE stimulate tube formation of microvascular endothelial cells and uprcgulatc mRNA for vascular cndothelial growth factor (VEGF). Monoclonal antibody auainst human VEGF were found to neutralize both the AGE-induced DNA svnthesis and tulo formation. The results indicatc that AGE may promote angiogenesis on one hand through the mediation by autocrine VEGF. and thrombogenesis on the other, thereby leading to the development of diabetic proliferative retinopathy. 
OP 35

Intra-Cellular Signalling

\section{7}

ACTIVATION OF PROTEIN KINASE C (PKC) IN HUMAN THROMBOCYTES IN VIVO BY GLUCOSE AND INSULIN: RESPONSES IN NIDDM V. Pirags, R. Assert, H. Schatz and A. Pfeiffer; Medizinische Universitätsklinik Bergmannsheil, D-44789 Bochum, Germany and Medizinische Akademie Riga, Latvia

PKC directs the gene expression of TGFB, bFGF and TGF alpha, of ECM proteins and others and appears to participate in the pathogenesis of diabetic complications in experimental diabetes. PKC is activated by hyperglycemia due to synthesis of its second messenger diacylglycerol. Since data in man are lacking we investigated whether hyperglycemia causes an activation of PKC in humans. Methods: The activation of PKCB was determined in thrombocytes during insulin sensitivity tests (octreotide + glucose + insulin) after 0,60 and $150 \mathrm{~min}$. Thrombocytes were rapidly isolated by differential centrifugation and $\mathrm{PKCB}_{2}$ was determined by quantitative immune blots relative to recombinant standards by laserdensitometry. Results: During infusion of insulin/glucose BG increased by $128 \mathrm{mg} / \mathrm{dl}$ and insulin levels were $40 \mu \mathrm{U} / \mathrm{ml}(n=27)$; membrane $\mathrm{PKCB}_{2}$ increased by 20 and $31 \%$ after 60 and $150 \mathrm{~min}(\mathrm{p}<0.05)$. The analysis of subgroups showed that only $10 / 19$ NIDDM showed a marked increase of $\mathrm{PKCB}_{2}$ while $9 / 19$ did not $(<20 \%$ ). Triglycerides were significantly higher in non-activators but not other parameters of diabetes control or treatment. Conclusions: $1 . \mathrm{PKCB}_{2}$ is activated by combined increases in insulin + glucose in human thrombocytes. 2 . $\mathrm{PKCB}_{2}$ responses in NIDDM patients differ which is not related to the parameters of diabetic control or duration. In view of the association of PKCB with diabetic complications differences in its activation may correlate with differences in the development of complications.

\section{9}

SPECIFIC INCREASE IN $p 85 \alpha$ BY DEXAMETHASONE LEADS TO INHIBITION OF INSULIN AND IGF-I STIMULATED PI 3-KINASE ACTIVITY. F. Giorgino', M. T. Pedrini'2, L. Matera', and R. J. Smith'2. 'Istituto di Clinica Medica, Endocrinologia e Malattie Metaboliche, Bari, Italy and ${ }^{2 J} J$ slin Diabetes Center, Boston, MA

Activation of phosphatidylinositol (PI) 3-kinase represents a key signaling event for insulin and IGF-I regulation of cellular metabolism. Two highly homologous isoforms of the $85-\mathrm{kDa}$ regulatory subunit ( $\mathrm{p} 85$ ) of PI 3-kinase have been cloned, p $85 \alpha$ and $\mathrm{p} 85 \beta$, which are encoded by distinct genes and form each a complex with the 110-kDa catalytic subunit (p110) of the enzyme. The objective of this study was to investigate the effects of the glucocorticoid dexamethasone on the expression of $p 85 \alpha$ and p $85 \beta$ isoforms of PI 3-kinase in L6 skeletal muscle cells, their coupling with the p110 catalytic subunit, and their association with IRS-1. Dexamethasone treatment induced a 4 -fold increase in $p 85 \alpha$ protein content, but had no effect on p $85 \beta$ and only modestly increased p 110 content by $38 \%$. The increase in p850 protein was associated with a coordinate increase in $p 85 \alpha$ mRNA, indicating that dexamethasone enhances p $85 \alpha$ gene expression. Stimulation of cells with insulin or IGF-I induced the association of both p85 isoforms and of p110 with IRS-1 and markedly increased PI 3-kinase activity in IRS-1 immunoprecipitates. Greater amounts of $p 85 \alpha$ and lower amounts of $p 85 \beta$, respectively, were found in IRS-1 immunoprecipitates in cells treated with dexamethasone, such that the $\alpha \beta$ ratio was markedly higher than in control cells both in the basal state and in response to hormone stimulation. In spite of the increase in both total and IRS-1-associated p $85 \alpha$, IRS-1-associated PI 3-kinase activity was decreased by $45 \%$, which was explained by lower levels of the catalytic subunit p1 10 associated with IRS-1. In conclusion, dexametbasone induces a specific increase in the expression of the p85 $\alpha$ regulatory subunit of PI 3-kinase that is not associated with a coordinate increase in the p110 catalytic subunit. Thus, in dexamethasone-treated cells, a substantial amount of p $85 \alpha$ is "free" (i.e., not coupled with $\mathrm{p} 110$ ) and competes with both $\mathrm{p} 85 \alpha / \mathrm{p} 110$ and $\mathrm{p} 85 / \mathrm{\beta} / \mathrm{p} 110$ complexes for the association with IRS- 1 , leading to increased $\mathrm{p} 85 \alpha$ but decreased p853, p1 10, and PI 3-kinase activity in IRS-1 immunoprecipitates. These results suggest a novel PI 3-kinase regulatory mechanism capable of inhibiting cell signaling through this protein.

\section{8}

Insulin-induced rearrangement of actin stress fibres involves active Phosphotyrosine Phosphatase 1D and Focal Adhesion Kinase

J.A. Maassen, D.M. Ouwens, A. Ulirich, and G.C.M. van der Zon. Dept. of Medical Biochemistry, University of Leiden. The Netherlands, and Dept. of Molecular Biology, Max Planck Institut für Biochemie, Martinsried, Germany.

Insulin evokes a rapid reorganization of the actin cytoskeleton in Chinese Hamster Ovary (CHO) and NIH3T3-derived cells overexpressing the human insulin receptor. These changes include a reduction in the amount of cytosolic actin fibres, the occurrence of cortical actin polymerization, and the formation of protruding structures from the cell surface. The rearrangement of the actin cytoskeleton was accompanied by dephosphorylation of tyrosine phosphorylated Focal Adhesion Kinase (ppl25 $5^{\mathrm{FAK}}$ ).

We observed previously that tyrosine dephosphorylation of $\mathrm{pp} 125^{\mathrm{FAK}}$ induced by insulin requires active phosphotyrosine phosphase 1D (PTP ID). Expression of a dominant-negative mutant of PTP 1D (Cys 463 Ala substitution) leads to a high number of stress fibres in quiescent cells. In response to insulin, no dephosphorylation of pp $125^{\mathrm{FAK}}$ was observed and the number of cytosolic stress fibres was unaffected, while protruding structures at the cell surface were normally induced. This suggests that active PTP ID is required for the reduction of cytosolic actin fibres, but not for membrane ruffling. Binding of PTP 1D to the activated insulin receptor proceeds to tyrosine phosphorylated Tyr1158. The contribution of this interaction to celfular responses was studied in CHO-derived cells expressing the mutant insulin receptor in which Tyr 1158 is replaced by Phe (FYY). In FYY cells no insulin-induced pp $125^{\mathrm{FAK}}$ dephosphorylation and disappearance of cytosolic stress fibres was observed. We conclude that the insulin-induced rearrangements of cytosolic actin proceed via a pathway involving active PTP $1 D$ and dephosphorylation of pp125 $5^{\text {AKK }}$. This response is initiated by phosphorylation of Tyr 1158 of the insulin receptor. The formation of protruding structures from the cell surface is mediated by another signaling pathway.
200

AMINO ACIDS (AA) STIMULATE PROTEIN ANABOLISM VIA P7O S6 KINASE PHOSPHORYLATION

E. Brambilla, M-E. Patti, I. Terruzzi, C.R. Kahn and L. Luzi. Brigham and Women's Hospital and Joslin Diabetes Center, Boston, U.S.A

AA have been shown to activate protein synthesis and inhibit proteolysis. To define whether AA stimulate protein anabolism via activation of p70 $S 6$ kinase, male Sprague-Dawley rats with chronic catheterization of the carotid artery and jugular vein were studied with a primed-continuous infusion of $\left[1-{ }^{14} \mathrm{C}\right]$-leucine and $\mathrm{AA}$ (balanced amino acid solution) and/or the euglycemic insulin clamp. After 90 minutes of tracer equilibration, somatostatin (SS, 10 $\mu \mathrm{g} / \mathrm{kg} / \mathrm{min}$ ) was infused for additional 120 minutes to inhibit the endogenous insulin secretion. Study 1 : a) SS; b) SS + AA ( $x 4$ of basal); c) SS +AA (x8); d) $\mathrm{SS}+\mathrm{AA}(\times 8)+$ insulin $(20 \mathrm{mU} / \mathrm{kg} / \mathrm{min})$ maintaining euglycemia; e) $\mathrm{SS}+$ ins $(20 \mathrm{mU} / \mathrm{kg} / \mathrm{min})$ at euglycemia. Study 2 : SS + AA (x8) time-course 0, 15 , 30, 60, and 120 minutes. At sacrifice, liver and gastrocnemius and quadriceps muscles were collected for the assay of fractional synthetic rate and p70 S6 kinase. ELF=endogenous leucine flux; MPS=muscle synthetic rate; $L P S=$ jiver synthetic rate. Below are represented data at 60 minutes.

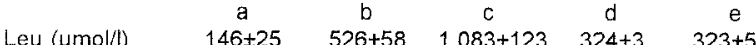

$\begin{array}{lccccc}\text { Ala }(\mu \mathrm{mol} / \mathrm{l}) & 279 \pm 26 & 1,265 \pm 198 & 1,556 \pm 221 & 817 \pm 30 & 126 \pm 11\end{array}$

$\begin{array}{llllll}\text { BCAA (umol/l) } & 669 \pm 69 & 1,933 \pm 345 & 3,241 \pm 689 & 1,294 \pm 20 & 279 \pm 8\end{array}$

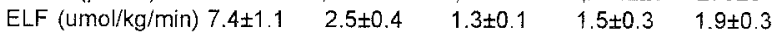

$\begin{array}{llllll}\text { LPS (\% of bas) } & 100 & 259 & 488 & 219 & 79\end{array}$

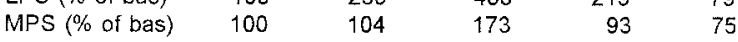

p70 S6 (\% of bas) $\quad 100 \quad 175 \pm 6 \quad 269 \pm 111 \quad 149 \pm 41 \quad 114 \pm 5$ In summary: 1) An increase of plasma amino acid concentration is associated with a dose-dependent increase in p70 S6 kinase (4-fold at $60 \mathrm{~min}, p<0.03$ ) and protein synthesis in liver but not in muscle; 2) AA dose-dependently inhibit proteolytic rate; 3) p70 S6 k and LPS peak at 60 minutes of AA infusion; 4) the addition of insulin to $A A$ does not result in further stimulation of p70 S6 kinase or protein synthesis in liver. Conclusion: AA selectively activate LPS and inhibit ELF; this effect is correlated ( $r=0.90$ and -0.94 respectively) with the phosphorylation of p70 S6 kinase. 
OP 36

\section{Beta-Cell Dysfunction and Glucose Toxicity}

201

ALTERED HUMAN B-CELL FUNCTION RESULTING FROM A SULPHONYLUREA RECEPTOR GENE MUTATION AND SUBSEQUENT LOSS OF $K_{\text {ATP }}$ CHANNEL ACTIVITY

M.J. Dunne, C. Kane. R.M. Shepherd. P.R.V. Johnson", R.F.L. James", K.J. Lindley", A. Aynslcy-Grecn", J.P. Clement IV ${ }^{\infty}$, J. Bryan ${ }^{\infty}$, and L. Aguilar-Bryan ${ }^{\infty}$ Biomedical Science. Sheffield University, Sheffield. UK, *Department of Surgery, Leicester University, Leicester, UK. Institute of Child Health, London, UK and Baylor College of Medicine. Houslon, USA

$\mathrm{K}_{\mathrm{ATP}}$ channels in $\beta$-cells are a heleromultimeric complex of a $\mathrm{K}^{+}$channel inward rectificr $\left(K_{\mathrm{R}} 6.2\right)$ and the sulphonylurea receptor (SUR). The channel has been localized to chromosome $11 \mathrm{p} 15.1$ and this site has been linked to Persistent Hyperinsulinemic Hypoglycemia of Infancy (PHHI), the most common cause of hypoglycenia in newborn. Here, we have examined the properties of $\beta$-cells isolated from a PHHI patient - the 10th child of consanguineous parents and the third offspring affected with PHHL. Her excessive glucose requirement and refractoriness to medical therapy (glucagon, diazoxide and nifedipine) led to a $95 \%$ pancreatectomy taking place aged 5 wecks. Intact islets were then isolated and electrophysiological (patch-clamp) studies undertaken. On-cell recordings and cell-free patch data failed to identify the presence of $K_{\text {ATP }}$ channels $(n=27)$, and there were no effects of diazoxide $(200-500 \mu \mathrm{M})$ on $\mathrm{K}^{+}$currents $(\mathrm{n}=18)$. Blood samples were taken and DNA extracted to be analyzed for the exon 5 mutation. This point mutation has been described in PHHI patients of Arab cxtraction and it causes loss of a Mspl restriction site. Afterwards, the PCR product was sequenced and the $G$ to $A$ mutation was identified. This mutation is predicted 10 lead to the truncation of the second nuclcotide-binding fold of the SUR protein. Thus, in reconstitution experiments a parallel mutation was engineered in the hamster SUR, which when co-expressed with $\mathrm{K}_{\mathrm{IR}} 6.2$ in COS cells failed to reconstitute functional $\mathrm{K}_{\mathrm{ATP}}$ channels as assayed by ${ }^{86} \mathrm{Rb}^{+}$efflux; (i) there were no effects of metabolic poisoning ( $\left.\mathrm{n}=4\right)$ nor (ii) diazoxide $(200.500 \mu \mathrm{M})$ on the eflnux of ${ }^{86} \mathrm{Rb}^{4}(\mathrm{n}=4)$. Our data show for the first time how a point mutation in the SUR, identified in a patient, leads to the altered function of human $\beta$-cells and this provides the molecular basis for the onset of PHHI.
202

NITRIC OXIDE CONTRIBUTES TO IMPAIRED INSULIN RELEASE IN THE SPONTANEOUSLY DIABETIC GOTO-KAKIZAKI RAT C.-G. Östenson, A. Guenifi, M. Sundén and S. Efendic, Dept of Molecular Medicine, Karolinska Hospital and Institute, Stockholm, Sweden Indirect evidence suggests that nitric oxide (NO) suppresses insulin secretion. Thus, the competitive inhibitor of NO synthase, $N^{c}$-nitro-L-arginine methylester (L-NAME), increases insulin release stimulated by arginine or glucose. To study whether NO plays a role for the impaired insulin secretion in NIDDM, the effect of L-NAME on insulin release was assessed in isolated islets and perfused pancreas of the spontaneously diabetic Goto-Kakizaki (GK) rat, a model of NIDDM, and control (C) Wistar rat. Incubation of isolated islets in $16.7 \mathrm{mM}$ glucose as compared to $3.3 \mathrm{mM}$ glucose did not stimulate insulin release in GK rats (6.7 \pm 0.9 vs $5.2 \pm 1.0 \mu \mathrm{U} / \mathrm{islet} / \mathrm{h})$ but increased the release 4-fold in $C$ rats $(23.3 \pm 1.8$ vs $6.0 \pm 1.0 \mu \mathrm{U} /$ islet $/ \mathrm{h}$, $\mathrm{p}<0.001$ ). At $3.3 \mathrm{mM}$ glucose, addition of 5 or $10 \mathrm{mM} \mathrm{L-NAME}$ did not affect the insulin release, either from GK or $\mathrm{C}$ rat islets. At $16.7 \mathrm{mM}$ glucose, however, 5 and $10 \mathrm{mM} \mathrm{L}-\mathrm{NAME}$ augmented the insulin release from GK rats islets approximately two and three times as compared to the response to $16.7 \mathrm{mM}$ glucose alone $(14.1 \pm 1.7$ and $20.1 \pm 3.5 \mu \mathrm{U} /$ islet $/ \mathrm{h}$ respectively, vs $6.7 \pm 0.9 \mu \mathrm{U} /$ islet $\mathrm{h}, \mathrm{p}<0.01$ ). In $\mathrm{C}$ rat islets $5 \mathrm{mM} \mathrm{L-NAME}$ did not enhance the insulin response at $16.7 \mathrm{mM}$ glucose, but $10 \mathrm{mM}$ of the inhibitor induced an almost two-fold increase $(45.8 \pm 6.7$ vs $23.3 \pm 1.8$ $\mu \mathrm{U} /$ islet $\mathrm{h}, \mathrm{p}<0.01$ ). In the perfused pancreas, $5 \mathrm{mM} \mathrm{L-NAME}$ at $16.7 \mathrm{mM}$ glucose induced a similar acute insulin response in GK $(2,097 \pm 178 \mu \mathrm{U} / 4$ $\min )$ and $\mathrm{C}(2,421 \pm 491 \mu \mathrm{U} / 4 \mathrm{~min})$ rats. In conclusion, $\mathrm{L}-\mathrm{NAME}$ enhances insulin release in the rat pancreas at stimulating but not at basal glucose levels. This enhancing effect by L-NAME is more pronounced in GK than in $\mathrm{C}$ rat islets, suggesting that NO may contribute to the impaired insulin secretion in the GK rat.

\section{3}

HIGH GLUCOSE INCRIASIS ISIII AMYLOD POIYPFPTIDH CONSTITUTIVT SHC RETORY PATHWAY IN HUMAN PANCRIAATK ISI.ETS R. Gasa A Novials.' R. Casamitjana .I. Sanchez and R. Gomis Fndocrinology and Nutrtion Unit,!' Inrmones L.aboratory. Hospital C linw. University of Barcelona. Barcelona. Spain

A proportion of Islet Amvlord Polypepide (IAPP) nat be released by a constitulivelaki valcum-independent pathway m human pancreatic islets as it has been previonsly thon n in neonatal rat $\beta$-cells. To test this bypothesis and to examine whether hyperglycaemia may affect IAPP secretory pattern. human pancreatic islets were cultured in RPMI- 1640 with 5.5 or $24.4 \mathrm{mM}$ glueose for 8 days At the end of culture islet peptide secretion was studied during 90 min-incubation in the absenco of glucose (G) or in the presence of : 1$)(\% 16.7 \mathrm{mM}$. 2) \& $16.7 \mathrm{mM}$ plus the anstiturive release inhibitor Brefeldin $\mathrm{A}$ (BFA) or 3 ) $(316.7 \mathrm{mM}$ plus the calcium chelator EGTA In islets cultured at low glucose. glicose-stimulated release of both IAPP and insulin was blocked by ECTTA but unaffected by BFA. In islets cultured at $\mathrm{G} 24.4 \mathrm{mM}$ in contrast BFA inhibited by $21 \%$ glucose-induced IAPP secretion $(\mathrm{P}<0.05)$ whereas increased insulin secretion (22\%). Addition of EGTA reduced by $50 \%$ both IAPP and insulin release ( $P<0.001$ ). To evaluate the role of nonenromatic glvosilation in this IAPP sorting into the constitutive pathway, aminoguanidine was added to the $24.4 \mathrm{mM}$ i culture. The proportion of IAPP released through the BFA-sensitive pathway remained unchanged $(29 \%, P<0.05)$ and removal of calcium decreased by $50 \%$ IAPP release $(P \propto 0.001)$. Insulin secretion of the same islets was again stimulated by BFA (44\%) and inhibited by EGTA $(50 \%, \mathrm{P}<0.001)$ addition. We conclude that in the absence of calcium . glucose does not stimulate IAPP secretion in human pancreatic islets. However. prolonged exposure to high glucose results in an increment of the proportion of IAPP . but not of insulin, which is released by the constitutive (BFA-scnsitive) pathway. Moreover. this does not appear to be related to non-enzymatic glycosylation mechanisms.

\section{4}

GLUCOTOXICITY - A REGULATOR OF GLUT2 AND INSULIN GENE EXPRESSION AS WELL AS INSULIN SECRETION IN INS-1 CELLS B Brock, S Gregersen and K Hermansen. Dept. of Endocrinology and Metabolism, Aarhus University Hospital, 8000 Aarhus, Denmark.

We have previously shown that longterm exposure to high glucose downregulates the expression of GLUT2 mRNA and insulin mRNA in the glucose sensitive beta cell-line INS-1. The aim of the present investigation was to determine 1) if this downregulation is reversible and can be prevented by altemate exposure to low and high glucose, 2) how the glucose mediated insulin secretion is altered. Cells were cultured in a modified RPMI 1640 medium containing different glucose levels. Total RNA was analysed by northem blotting and insulin secretion analysed by RIA. Students unpaired one-tailed t-test was used to test the degree of significance. We found 1) that the downregulation of GLUT2 mRNA and insul in mRNA in cells cultured in $26.6 \mathrm{mM}$ glucose for 4 days could be totally counteracted by subsequent culture in $6.6 \mathrm{mM}$ glucose. In cells exposed to changing glucose concentrations $(6.6 \mathrm{mM}$ and $26.6 \mathrm{mM}$ ) for 8 days the amount of GLUT2 mRNA was reduced by $50 \%$ of that found in cells cultured entirely in $6.6 \mathrm{mM}$ glucose and the double of that found in cells cultured entirely at 26.6 $\mathrm{mM}$. Interestingly, the downregulation of insulin mRNA was not concomtantly counteracted by culturing alternately in high and low glucose. Furthe rmore 2) we detected a significant increase in the glucose mediated insulin release in cells cultured 4 days in either $6.6 \mathrm{mM}$ glucose $(\mathrm{p}<0.01$ ) or $6.6 \mathrm{mM}$ glucose +10.1 $\mathrm{mM}$ fructose $(\mathrm{p}<0.01)$ or galactose $(p<0.01)$ as compared to the release after only 1 days culture. In contrast, the glucose mediated insul in release in cells cultured for 4 days in $16.7 \mathrm{mM}$ glucose was significant decreased $(p<0.01)$. Conclusions: The downregulation of GLUT2 gene expression and insulin gene expression found after longterm exposure to high glucose is reversible and could partly be counteracted by altemating berween low and high glucose. The glucose mediated insull in secretion is concomitantly changed by exposure to high glucose. The glucotoxicity is not mediated via an unspecific osmotic effect. 
OP 37

\section{Hypoglycaemia I}

\section{5}

\section{INTRAVENOUS LACTATE PROTECTS CEREBRAL FUNCTION DURING HYPOGLYCAEMIA.}

I,A. Macdonald, P. King, H. Parkin, M.F. Kong, C. Barber and R.B.

Tattersall. Queen's Medical Centre, Nottingham, UK.

The hypothesis that intravenous lactate prevents cerebral dysfunction during hypoglycaemia was tested in $9(6 F)$ healthy and 9 IDDM (6M) subjects. Changes in Four Choice Reaction Time (RT), Auditory Brain Stem Response (ABR) and $\mathrm{P}_{300}$ latency were used as measures of cerebral function. Subjects were studied twice, 4 weeks apart using a $60 \mathrm{mu} / \mathrm{m}^{2} /$ min hyperinsulinaemic clamp to achieve blood glucose (BG) concentrations of $4.5,3.3$ and 2.5 $\mathrm{mmol} /$. At one visit, $40 \mu \mathrm{mol} / \mathrm{kg} / \mathrm{min}$ sodium lactate was infused; at the other normal saline. Cerebral function tests were performed at each blood glucose concentration. IDDM subjects showed no significant changes in any of the measures of cerebral function at $B G$ of $3.3 \mathrm{mmol} / 1$, but in healthy subjects, RT increased by $14.1+/-4.34 \mathrm{~ms},(\mathrm{p}<0.02)$ with saline but not during lactate $(-1.1+/-3.48 \mathrm{~ms})$ infusion. At BG of $2.5 \mathrm{mmol} / \mathrm{l}$, RT and $\mathrm{P}_{300}$ latency increased in IDDM subjects with infusion of saline $(30.6+1-9 \mathrm{~ms}$, $\left.\mathrm{p}<0.01 \mathrm{RT} ; 27.6+/-9.9 \mathrm{~ms}, \mathrm{p}<0.05 \mathrm{P}_{300}\right)$ but not lactate. $(-5.4+/-3.9 \mathrm{~ms}$ $\mathrm{RT}$; $-5.3+/-8.4 \mathrm{~ms} \mathrm{P}_{300}$ ) No changes occurred in ABR latency. In healthy subjects, at BG of $2.5 \mathrm{mmol} / 1$ all three tests deteriorated with saline $(35.1+/$ $5.42 \mathrm{~ms} p<0.001 \mathrm{RT} ; 10.2+/-3.69 \mathrm{~ms}, \mathrm{p}<0.05, \mathrm{P}_{300}$ and $0.06+/-0.02 \mathrm{~ms}$, $\mathrm{p}<0.001 \mathrm{ABR})$ but not lactate. $\left(2.8+/-3.96 \mathrm{~ms} \mathrm{RT} ; 7.14+/-7.05 \mathrm{~ms} \mathrm{P}_{300}\right.$ and $0+/-0.04 \mathrm{~ms} A B R$ ). Thus the hypothesis that intravenous lactate prevents cerebral dysfunction during hypoglycaemia is supported in IDDM as well as healthy volunteers. The lack of deterioration in $\mathrm{RT}$ at $3.3 \mathrm{~mol} / 1$ and $\mathrm{ABR}$ at $2.5 \mathrm{mmol} / 1$ in IDDM subjects suggests cerebral adaptation due to previous hypoglycaemia.

\section{6}

A POST-HYPOGLYCAEMIC INCREASE OF LACTATE CAN RESCUE COGNITIVE FUNCTION AND COUNTERREGULATORY

RESPONSES TO HYPOGLYCAEMIA

A. Maran, C. Crepaldi, T.Lucca, I. Macdonald, S.Del Prato, A. Avogaro and A.Tiengo. Cattedra di Malattie del Metabolismo Padova, Italy \& Queen's Medical Centre, Nottingham, England.

Substrates other than glucose may delay and reduce counterregulatory responses to hypoglycaemia. To investigate whether lactate may function as an alternative substrate in the presence of low blood glucose we studied 7 volunteers $(6 \mathrm{M}, 1 \mathrm{~F}, 25 \pm 1$ years) using a stepped hypoglycaemic clamp $(1.5 \mathrm{mU} / \mathrm{kg} / \mathrm{min}$ intravenous insulin: plasma glucose (PG) reduced to $50 \pm 1 \mathrm{mg} / \mathrm{dl}$ by $160 \mathrm{~min}$. and restored using exogenous glucose) on two different occasions. In one study Na-lactate $(30 \mu \mathrm{mol} / \mathrm{kg} / \mathrm{min})$ was infused at the onset of symptoms of hypoglycaemia, in the other saline. Adrenaline responses started at similar glucose levels (PG $58 \pm 2$ vs 58 $\pm 1 \mathrm{mg} / \mathrm{dl}$ ) but were markedly reduced by lactate (AUC $165 \pm 28$ vs 314 $\pm 55 \mathrm{pmol} / \mathrm{ml} / 180 \mathrm{~min}, \mathrm{p}=0.01$, peak $2.6 \pm 0.5$ vs $4.8 \pm 0.7 \mathrm{pmol} / \mathrm{ml}$, $p=0.005$ ) with no differences in norepinephrine responses ( $P G 56 \pm 3$ vs $55 \pm 2, p=0.6$, AUC $544 \pm 125$ vs $500 \pm 75 \mathrm{pmol} / \mathrm{ml}, p=0.5$ ). Similarly, symptoms were experienced at similar glucoses (autonomic $54 \pm 1$ vs $54 \pm 2 \mathrm{mg} / \mathrm{dl}$, neuroglycopenic $50 \pm 1$ vs $53 \pm 2 \mathrm{mg} / \mathrm{dl}$ ) but were significantly diminished by lactate (score at $160 \mathrm{~min} 2.6 \pm 1$ vs $8.8 \pm 1$, $p=0.02$ and $0.4 \pm 0.4$ vs $4.8 \pm 1, p=0.01$ ). Cognitive function deteriorated in both studies (slowing of responses at PG $55 \pm 2$ vs $54 \pm 2$ ) but a more profound impairment was prevented by lactate ( $\Delta$ msec 4-choice reaction time at $160 \mathrm{~min} .22 \pm 12$ vs $77 \pm 31, p=0.02$ ). We conclude that a rise in lactate after the development of hypoglycaemic symptoms acutely reduces counterregulatory and symptomatic responses to hypoglycemia and prevents a further deterioration of cognitive function during hypoglycaemia in man.

\section{7}

COGNITIVE DYSFUNCTION COINCIDES WITH THE ONSET OF HYPOGLYCAEMIA AND ONLY IMPROVES AFTER GLUCOSE RECOVERY M.L. Evans, J. Lomas, A. Pernet, D. Hopkins, S. Joseph and S.A. Amiel. Dept of Medicine, King's College School of Medicine \& Dentistry, London, UK.

We set out to investigate current thinking that impairment of brain function during hypoglycaemia takes time to develop and may improve, as may symptoms, during sustained hypoglycaemia, as this has implications both for clinical practice and for experimental design in investigations of counterregulation. 5 non-diabetic men (aged $21-33$ years) underwent a hyperinsulinaemic ( $1.5 \mathrm{mU} / \mathrm{kg} \cdot \mathrm{min}$ ) glucose clamp on two occasions. One study was a euglycaemic control. In the other, arterialised plasma glucose was kept at $5 \mathrm{mmol} / \mathrm{l}$ for $60 \mathrm{~min}$, lowered rapidly to $2.6 \mathrm{mmol} / 1$ for $90 \mathrm{~min}$ and then restored to $5 \mathrm{mmol} / \mathrm{l}$. Cognitive function was assessed by a small test battery of 4 choice reaction time (4CRT), Trail making B (TMB) and the Colour and ColourWord (CW) Stroop subtests. This takes 15 minutes, allowing repeated administration. Coonitive function, symptoms and catecholamines were measured every 20 minutes. There were no significant changes in any measurement during euglycaemia. After 20 $\mathrm{min}$ at $2.6 \mathrm{mmol} / \mathrm{h}$, adrenaline rose from $0.18 \pm 0.03$ to $3.59 \pm 0.17 \mathrm{nmol} / \mathrm{h}, \mathrm{p}<0.01$; roradrenaline from $1.88 \pm 0.14$ to $3.19 \pm 0.25 \mathrm{nmol} / 1, \mathrm{p}<0.01$; total symptom scores from $16.5 \pm 0.4$ to $22.8 \pm 1.9, p<0.05$ and autonomic symptom scores from $8.8 \pm 0.3$ to $15.2 \pm 1.5, \mathrm{p}<0.01$. All then remained high (peaks $5.2 \pm 0.9,3.2 \pm 0.3,30.9 \pm 5.4,21.3 \pm 2.5$ (respectively) with no evidence of adaptation. In contrast, $4 C R T$ increased immediately at $2.6 \mathrm{mmol} / \mathrm{l}$ by $16.8 \pm 9.4 \%$ (coefficient of variation at $5 \mathrm{mmol} / 12 \%$ ), as did CW (from $95.8 \pm 5$ to $113.8 \pm 3.5$ secs, $p<0.01$ ). Neither $4 C R T$ nor $C W$ recovered during sustained hypoglycaemia (peaks $34.6 \pm 11.6 \%$ and $116.6 \pm 2.3$ ). A trend to impaired TMB was not significant., During recovery, all parameters returned to baseline except $4 C R T$. We conclude that during hypoglycaemia, impairment of cognitive function is immediate, is shown by CW and 4CRT and may precede counterregulation, with no adaptation over $90 \mathrm{~min}$. These data help in the rational design of experiments in this field and have implications for hypoglycaemia in clinical practice.

\section{8}

REGIONAL CEREBRAL DIFFERENCES IN GLUCOSE UTILISATION - A POSSIBLE MECHANISM FOR HYPOGLYCAEMIA UNAWARENESS? I Cranston, PK Marsden, M Evans, J Lomas and SA Arniel. Department of Medicine, Kings College \& Clinical PET Centre, St Thomas' Hosp LONDON. If we are to understand the diversity of cerebral responses to hypoglycaemia, we must first seek to determine the mechanisms underlying cerebral glucose utilisation. In vitro, intracellular phosphorylation $\left(\mathbf{k}_{3}\right)$ can occur at rates many times that of transport from the plasma $\left(k_{1}\right)$. However in vivo, hexokinase activity is suppressed such that phosphorylation may become rate-limiting. Observation of cerebral function during hypoglycaemia has led to the suggestion that there may be regional differences in the brain's ability to maintain metabolism in the face of glucose deficiency. In order to determine if this is due to regional mechanistic differences in glucose metabolism, we have studied 10 male right-handed IDDM patients using Positron Emission Tomography (PET) and the tracer ${ }^{18} \mathrm{~F}$-2-deoxyglucose $\left({ }^{18} \mathrm{FDG}\right)$ to measure the actual in vivo rates of $k_{1}$ and $k_{3}$ regionally within the brain under controlled conditions.

Subjects underwent euglycaemic clamping for 90 minutes prior to intravenous bolus injection of $185 \mathrm{MBq}$ of ${ }^{18} \mathrm{FDG}$ and quantitative dynamic PET scanning with on-line analysis of arterial tracer levels over 60 minutes. Analysis of volumetric regions of interest placed on a co-registered MRI image was undertaken using a standard 3-compartment model (after Sokoloff).

Rates of glucose transport $\left(k_{1}\right)$ were fairly constant in all cortical regions, mean $0.00176 \pm 0.000028 \mathrm{sec}^{-1}$, whilst in the cerebellum rates were consistently higher $0.00217 \pm 0.00004 \mathrm{sec}^{-1} \quad(p=0.0004)$. By contrast, $k_{3}$ values were consistently higher in cortical areas $0.00254 \pm 0.00007$ than cerebellum $0.00161 \pm 0.00003(p=0.001)$. Expressed as a ratio between $k_{1}: k_{3}$, this gives values of 0.743 in cortex vs 1.451 in cerebellum $(p=0.0002)$. This suggests that the rate-limiting step for glucose utilisation may be regionally variant. Further investigations of these parameters during hypoglycaemia may help to explain symptom and cognitive differences seen in different groups of diabetic patients in response to hypoglycaemia occurring in daily life. 
209

RECOVERY OF HYPOGLYCEMIA UNAWARENESS IN IDDM WITH AUTONOMIC NEUROPATHY.

C. i anelli, C. Lalli, S. Pampanelli, M. Lepore, L. Bartocci, P. Brunetti and G. B. Bolli. Di.M.I.S.E.M., University of Perugia, Italy.

To establish whether hypoglycemia unawareness $(\mathrm{HU})$ is similarly reversible in IDDM patients without and in those with, overt autonomic neuropathy with orthostatic hypotension (DAN- and DAN+), 12 patients with $\mathrm{HU}(\mathrm{N}=7 \mathrm{DAN}-$, $\mathrm{N}=5 \mathrm{DAN}+$ ) were studied with the hypoglycemic clamp (plasma glucose, $\mathrm{PG}$, decreased stepwise from 5 to $2.2 \mathrm{mM}$ ) before and after 3 month of a programme of meticulous prevention of hypoglycemia $(\mathrm{H})$. Prevention of $\mathrm{H}$ decreased frequency of $\mathrm{H}$ (defined as any blood glucose $<4 \mathrm{mM}$ ) from $0.42 \pm$ 0.06 to $0.05 \pm 0.01$ episodes/patient-day, and increased HbAlc from $6.1 \pm 0.3$ to only $6.9 \pm 0.4(\mathrm{p}<0.05)$. After prevention of $\mathrm{H}$, responses of autonomic and neuroglycopenic symptoms (AS, NS) to $\mathrm{H}$ improved both in DAN- (AS score. from 4.5 \pm 0.2 to $9.0 \pm 0.5$, NS from $4.0 \pm 0.7$ to $7.2 \pm 1$ ) and in DAN+ (AS from $3.9 \pm 0.4$ to $10.5 \pm 1.1$, NS from $5.1 \pm 0.6$ to $7.6 \pm 0.9)(p<0.05)$. Cognitive function (CF, expressed as z-score of 12 tests) at $2.2 \mathrm{mM}$ deteriorated more after prevention of $\mathrm{H}$ (DAN- from $-5 \pm 0.4$ to $-9.1 \pm 0.8$, DAN+ from $-1.1 \pm 0.1$ to $-6.2 \pm$ $0.5)(\mathrm{p}<0.05)$. In contrast, responses of adrenaline (EPI) and pancreatic polypeptide (PP) improved only in DAN- (EPI from $1.1 \pm 0.2$ to $2.1 \pm 0.3 \mathrm{nM}$, PP from $45 \pm 8$ to $112 \pm 16 \mathrm{pM}, \mathrm{p}<0.05$ ) not in DAN+ (EPI from $0.72 \pm 0.07$ to $0.84 \pm$ $0.08 \mathrm{nM}, \mathrm{PP}$ from $31 \pm 5$ to $28 \pm 3 \mathrm{pM}, \mathrm{p}=\mathrm{NS}$ ). Glycemic thresholds of AS, NS, and EPI responses decreased after prevention of $\mathrm{H}$ both in DAN+ and DAN- by $0.6 \pm 0.02 \mathrm{mM}(\mathrm{p}<0.05)$. In conclusion, meticulous prevention of $\mathrm{H}$ improves recognition of $\mathrm{H}$ in IDDM, but counterregulation recovers only in DAN. Intensive therapy with improved prognosis for $\mathrm{HU}$ and $\mathrm{H}$ is feasible in $\mathrm{DAN}+$.

\section{0}

CATECHOLAMINE RESPONSE AND SYMPTOM RECOGNITION DURING HYPOGLYCEMIA AETER PANCREAS TRANSPLANTATION

DM Kendall, DP Rooney, YFC Smets. L Salazar-Bolding,

RP Robertson. University of Minnesota, Department of Medicine, Minneapolis. Minnesota.

Impaired neurohormonal responses and symptom unawareness during hypoglycemia are characteristic of patients with long-standing Type I diabetes (IDDM). Pancreas transplantation ( $\mathrm{P} \times \mathrm{Tx})$ establishes and maintains long-term normoglycemia and eliminates severe hypoglycemia in patients with IDDM. Whether pancreas transplantation provides significant improvement in epinephrine (EPI) and/or symptom (SX) response during hypoglycemia is not known. We performed stepped hypoglycemic clamps in 14 PxTx recipients (duration IDDM $25 \pm 2$ yr, $40 \pm 10$ months post-transplant), $18 \mathrm{IDDM}$ patients (duration $28 \pm 3 \mathrm{yr}$ ), 12 healthy control (CONT) and 5 kidney transplant (KID TX) recipients to determine whether EPI secretion ànd/or symptom awareness are improved by pancreas transpiant. Both PX

TX and IDDM had a history of severe hypoglycemia and hypoglycenic unawareness and established autonomic dysfunction as determined by RR variation and Vulsalvid ratio. Sequential 45 minute glucose plateaus of $70,60.50$ and $40 \mathrm{mg} / \mathrm{dl}$ werc achicved and mean $( \pm S E)$ EPI and SX score were determined.

\begin{tabular}{ccccc} 
EPI (pg/mi) & $\mathbf{7 0}$ & $\mathbf{6 0}$ & $\mathbf{5 0}$ & $\underline{\mathbf{6 0}}$ \\
\hline Px Tx & $68 \pm 10$ & $191 \pm 29 *$ & $369 \pm 63^{*}$ & $465 \pm 58 *$ \\
IDDM & $72 \pm 9$ & $119 \pm 21$ & $182 \pm 26$ & $262 \pm 38$ \\
CONT & $68 \pm 11$ & $307 \pm 36$ & $547 \pm 45$ & $777 \pm 63$ \\
KID TX & $118 \pm 11$ & $230 \pm 48$ & $451 \pm 48$ & $715 \pm 87$ \\
Sx SCORE & & & & \\
\hline Px Tx & $0.2 \pm 0.1$ & $2.8 \pm 0.78$ & $5.7 \pm 0.8 \S$ & $8.1 \pm 0.58$ \\
DDMM & $0.1 \pm 0.1$ & $0.8 \pm 0.2$ & $2.2 \pm 0.6$ & $4.1 \pm 0.6$ \\
CONT & $0.3 \pm 0.2$ & $2.1 \pm 0.5$ & $5.2 \pm 0.8$ & $7.3 \pm 0.5$ \\
KID TX & $1.2 \pm 0.4$ & $4.0 \pm 1.1$ & $6.8 \pm 1.2$ & $9.2 \pm 0.5$
\end{tabular}

Mean EPI was significantly greater in PX TX than in IDDM at 60.50 and $40 \mathrm{mg} / \mathrm{dl}$ $\left({ }^{*} \mathrm{p}<0.05\right)$, although it remained lower in PX TX than in CONT $(\$ p<0.02)$. SX scores at all times during hypoglycemia were not significantly different between PX TX and CONT and were higher in PX TX than IDDM ( $<<0.01)$. KID TX did not differ from CONT in any of the measured responses. In conclusion, sustained normoglycemia after pancreas transplantation in patients with long-duration diabctes and established autonomic dysfunction results in improved EPI responses and normalization of symptom recognition during insulin-induced hypoglycemia.

\section{OP 38 \\ Leptin}

\section{1}

IS LEPTIN THE LINK BETWEEN OBESITY AND INSULIN RESISTANCE?

M. R. Gabriel, S. Damani, A. Khan, S. Jinagouda, R. Boyadjian, W. W. Kades, M. A. Ayad, M. F. Saad, University of California Medical School, Los Angeles, CA, USA.

Obesity leads to the development or worsening of insulin resistance. It is also associated with increased plasma concentration of leptin, the obese $(o b)$ gene product. The relation between leptin and insulin resistance was studied in 37 nondiabetic men aged $39 \pm 2$ yrs [mean $\pm S E$ ] with a body mass index (BMI) of $26.9 \pm 0.8 \mathrm{~kg} / \mathrm{m}^{2}$. Insulin sensitivity was determined with the hyperinsulinemic euglycemic clamp. Average fasting leptin level was $4.5 \pm 0.62 \mathrm{ng} / \mathrm{ml}$ (range 0.8-22) and was higher in the obese $\left(B M l>27 \mathrm{~kg} / \mathrm{m}^{2}\right)$ than in the nonobese $(7.2 \pm 1.3$ vs. $2.9 \pm 0.3 \mathrm{ng} / \mathrm{ml}$; $\mathrm{p}=0.006)$. Leptin levels correlated with body weight $(\mathrm{r}=0.71)$, BMl $(r=0.84)$, the waisthip ratio $(r=0.42)$, and fasting insulin $(r=0.54$; $p<0.01$ for each), but not with age or fasting glucose. Leptin levels were also related to basal glucose disposal, glucose infusion rate (GINF) and the insulin sensitivity index $(r=-0.67,-0.76,-0.72$ respectively; $p<0.001$ for each), but not to $\%$ suppression of hepatic glucose output $(-0.14, p=0.42)$. After adjusting for age and $\mathrm{BMI}$, leptin continued to be significantly correlated with fasting insulin, basal glucose disposal GINF, and the insulin sensitivity index $(r=0.35,-0.33,-0.52,-0.66$ respectively; $p<0.03$ for each), but not the waisthip ratio. In conclusion, leptin is related to peripheral (skeletal musc $(e)$ insulin resistance independent of adiposity. Elevated plasma leptin concentration in obesily may contribute to the pathogenesis of the associated insulin resistance through a central (hypothalamic) or a peripheral mechanism.

\section{2}

IN VITRO REGULATION OF OB GENE EXPRESSION BY HORMONES AND NUTRIENTS IN WHITE ADIPOSE TISSUE

B. Reul, D.J. Becker, V. Rousseau, L.N. Ongemba, J.C. Henquin and S.M. Brichard. Unité d'Endocrinologie et Métabolisme, University of Louvain, Brussels, Belgium.

Leptin is a satiety factor encoded by the ob gene in adipose tissue. In this study, we investigated the regulation of the ob gene by culturing $12-24 \mathrm{~h}$ in MEM) pieces of epididymal fat pads from fasted rats in the presence of various hormones and substrates. Very low levels of ob mRNA were detected under control conditions. However, addition of dexamethasone to the medium increased ob expression in a concentration-dependent manner ( $\mathrm{EC}_{50} \sim 10$ $\mathrm{nmol} / \mathrm{l})$. With $1 \mu \mathrm{mol} / \mathrm{l}$ dexamethasone, the ob mRNA levels were similar to those in fresh fat pads from fed rats. The effect of dexamethasone was blocked by actinomycin $\mathrm{D}$, which indicates an action on transcription. Unlike dexamethasone, insulin, even when combined with high glucose concentrations, did not induce ob expression, although it strongly increased the accumulation of fatty acid synthase and GLUT $_{4}$ mRNA in the presence of glucose. Unexpectedly, insulin dose-dependently inhibited dexamethasoneinduced ob mRNA accumulation. This effect was already observed at low concentrations of insulin $\left(\mathrm{EC}_{50} \sim 1 \mathrm{nmol} / \mathrm{l}\right)$ and was mimicked by the insulinlike agent, vanadate $(25 \mu \mathrm{mol} / \mathrm{l})$ or by $\mathrm{IGF}_{1}(100 \mathrm{nmol} / \mathrm{l})$. This inhibition by insulin required a minimum amount of glucose or of lactate/pyruvate. Several other hormones (triiodothyronin, rat growth hormone or glucagon), alone or in the presence of dexamethasone, had no specific effect on ob gene expression. However, the $\beta_{3}$-adrenoceptor agonist, BRL 37344 inhibited by $50 \%$ the induction of $o b$ by dexamethasone. In conclusion, we provide evidence for a reciprocal regulation of ob gene expression by dexamethasone (positive effector), and insulin and $\beta_{3}$-adrenoceptor agonist (negative effectors) in cultured white adipose tissue. 
213

PLASMA LEPTIN IS RAPIDLY REDUCED BY INTRAVENOUS INSULIN SECRETAGOGUES IN NON-DIABETIC SUBJECTS.

B. Ahren and H. Larsson, Dept of Medicine, Lund University, Sweden

Leptin, the ob/ob gene product, has recently been suggested to regulate food intake by a negative feedback from the adipose tissue. We have previously shown that plasma leptin correlates to plasma insulin independently of body fat content. In this study we examined the effect of insulin and iv insulin secretagogues on plasma leptin in non-diabetic women aged 58-64 years, using the following methods: 1. hyperglycemic clamps at blood glucose (BG) levels of mean \pm SE $13.2 \pm 0.4$ and 26.5 $\pm 0.6 \mathrm{mM}\left(\mathrm{n}=9, \mathrm{BM} 127.3 \pm 2.1 \mathrm{~kg} / \mathrm{m}^{2}\right), 2$. iv arginine $(5 \mathrm{~g})$ at fasting $\mathrm{BG}, 13.2$ and $26.5 \mathrm{mM} \mathrm{BG}\left(\mathrm{n}=9, \mathrm{BMI} 27.3 \pm 2.1 \mathrm{~kg} / \mathrm{m}^{2}\right)$, 3. iv glucose bolus $(0.3 \mathrm{~g} / \mathrm{kg}$ body weight, peak BG $17.0 \pm 0.9 \mathrm{mM}, \mathrm{n}=10$, BMI $\left.26.6 \pm 0.6 \mathrm{~kg} / \mathrm{m}^{2}\right)$, 4. euglycemic, hyperinsulinemic clamps (BG $5.2 \pm 0.04 \mathrm{mM}$, steady state insulin $689 \pm 41 \mathrm{pM}, \mathrm{n}=10 . \mathrm{BM}$ $25.1 \pm 0.5 \mathrm{~kg} / \mathrm{m}^{2}$ ) and 5 . hypoglycemic, hyperinsulinemic clamps (BG nadir $2.1 \pm 0.2$ $\mathrm{mM} 45 \mathrm{~min}$ after start of insulin infusion, $\mathrm{n}=7$, BMI $29.3 \pm 2.1 \mathrm{~kg} / \mathrm{m}^{2}$ ). We found that plasma leptin was significantly reduced by increasing BG to $13.2(28.8 \pm 7.5 \mathrm{ng} / \mathrm{ml}$. $\mathrm{p}<0.01)$ and $26.5(26.9 \pm 6.7 \mathrm{ng} / \mathrm{ml}, \mathrm{p}<0.01)$ vs at fasting $B G(32.5 \pm 8.7 \mathrm{ng} / \mathrm{ml})$. Arginine decreased leptin levels 2 and $4 \mathrm{~min}$ after injection at fasting $B G(p=0.01)$ but not at BG 13.2 or BG $26.5(p=N S)$. Iv glucose reduced plasma leptin already after 4 $\min (25.9 \pm 4.5 \mathrm{vs}$ fasting $28.6 \pm 4.9 \mathrm{ng} / \mathrm{ml}, \mathrm{p}<0.0 \mathrm{l})$, and the reduction remained at 60 $\min (27.1 \pm 4.7 \mathrm{ng} / \mathrm{ml}, \mathrm{p}=0.04 \mathrm{vs}$ fasting). In contrast, plasma leptin levels were unchanged during the euglycemic, hyperinsulinemic clamp (19.1 \pm 2.9 vs $18.3 \pm 2.5$ $\mathrm{ng} / \mathrm{ml}$ at fasting and after $120 \mathrm{~min}$ clamp, $\mathrm{p}=\mathrm{NS}$ ), as well as during the hypoglyce mic, hyperinsulinemic clamp (28.5 $46.3 \mathrm{vs} 28.7 \pm 6.3 \mathrm{ng} / \mathrm{ml}$ at fasting and after 45 $\min$ insulin infusion, $p=N S$ ). Thus, hyperinsulinemia at eu- or hypoglycemia does not affect plasma leptin, while lryperglycemia in combination with hyperinsulinemia reduces plasma leptin. Arginine injection further decreases leptin at fasting. but not at higher $\mathrm{BG}$ where plasma leptin is already reduced. Thus, plasma leptin is rapidly altered by iv insulin secretagogues, independently of insulin. This suggests that hyperglycemia is of importance in the rapid regulation of plasma leptin in humans.

\section{4}

\section{LEPTIN AND THERMOGENESIS IN MAN}

J.A. Tuominen, P. Ebeling, M.L. Heiman, T. Stephens and V.A. Koivisto. Helsinki University Hospital, FIN-00290 Helsinki, Finland and Lilly Recearch Laboratories, Indianapolis, IN 46285, USA

We examined changes in serum leptin concentrations (RIA) and its relation to thermogenesis (indirect calorimetry) in 12 healthy men $\left(39 \pm 2\right.$ yrs, BMI $\left.22.8 \pm 0.3 \mathrm{~kg} / \mathrm{m}^{2}\right)$ during a $4 \mathrm{hr}$ euglycemic hyperinsulinemia performed on a control day and after a day of a competitive marathon run. As compared to the control day, after the marathon day baseline FFA concentration $(543 \pm 0.73 \mu \mathrm{mol} / \mathrm{A}$ vs $955 \pm 96 \mu \mathrm{mol} / 1, \mathrm{p}<0.05)$, lipid oxidation rate $(0.8 \pm 0.1 \mathrm{mg} / \mathrm{kg} / \mathrm{min}$ vs $1.2 \pm 0.1 \mathrm{mg} / \mathrm{kg} / \mathrm{min}, \mathrm{p}<0.01)$ and energy expenditure $(5.2 \pm 0.1 \mathrm{vs}$. $5.5 \pm 0.1 \mathrm{~kJ} / \mathrm{min}, \mathrm{p}<0.01$ ) were elevated. Baseline serum leptin concentrations were similar on the control and postexercise day and increased during insulin infusion by $35-45 \%$ on both days ( $p \leq 0.01$ ). Baseline serum leptin concentration correlated directly with serum insulin $(r=0.65, p<0.05)$ and cortisol $(r=0.64, p<0.05)$ and inversely with serum growth homone concentration $(r=-0.66, p<0.05)$. In the postexercise study, the rise in energy expenditure during insulin clamp correlated with serum leptin concentration as determined before $(r=0.61, p<0.05)$ or at the end of insulin infusion $(r=0.55, p<0.05)$. Thus, in healthy individuals with normal body weight: 1) Hyperinsulinemia increases serum leptin concentrations. 2) A rise in energy expenditure correlates with serum leptin concentration. These data suggest that leptin is involved in the regulation of energy expenditure in man.

\section{5}

LEPTIN SYNTHESIS IS RESISTANT TO INSULIN IN IDDM P. Ebeling ${ }^{\prime}$, J.A. Tuominen ${ }^{1}$, U-H. Stenman ${ }^{2}$, M.L. Heiman ${ }^{3}$, T.W. Stephens ${ }^{3}$, and V.A. Koivisto ${ }^{1}$.'Helsinki University Central Hospital, Department of Medicine, SF-00290 Helsinki, Finland, ${ }^{2}$ Helsinki University Central Hospital, Department of Clinical Chemistry, SF-00290 Helsinki, Finland, ${ }^{3}$ Lilly Research Laboratory, Indianapolis, IN 46285, USA

The $o b$ gene product, leptin, is synthesized in the adipose tissue. Insulin stimulates the $o b$ gene expression and increases serum leptin concentrations in mice and in healthy man. Obese women have had higher ob gene mRNA levels than obese men, suggesting that sex hormones are involved in the regulation of leptin synthesis. We studied the relationship of leptin, insulin and sex hormones in $15 \mathrm{men}$ with IDDM (age $29 \pm 2 \mathrm{yrs}$, BMI $22.7 \pm 0.5 \mathrm{~kg} / \mathrm{m}^{2}$, insulin dose $44 \pm 4$ units/day, $\mathrm{HbA}_{1 c} 8,1 \pm 0.3 \%$, diabetes duration $13 \pm 2 \mathrm{yrs}$ ) in the fasting state. In addition, the effect of a $4 \mathrm{~h}$ euglycemic hyperinsulinemia $(625 \pm 34 \mathrm{pmol} / 1)$ on plasma leptin concentration was determined. Fasting leptin concentration was positively related with body fat $\%(\mathrm{r}=0.67, \mathrm{p}<0.01)$ and negatively to serum testosterone $(r=-0.55, p<0.05)$. There was no significant correlation between fasting plasma leptin and serum cortisol, growth hormone or insulin level or daily insulin dose. In the regression analysis body fat percentage alone accounted for $62 \%(p<0.001)$ and together with fasting glucose concentration for $71 \%$ of the variation in fasting leptin concentrations $(\mathrm{p}<0.001)$. Fasting plasma leptin level $(2.7 \pm 0.2 \mathrm{ng} / \mathrm{ml})$ remained unchanged $(2.7 \pm 0.2 \mathrm{ng} / \mathrm{ml})$ during hyperinsulinemia. Conclusions: 1$)$ In non-obese IDDM patients leptin synthesis is resistant to insulin. 2) While the data suggest a decreased sensitivity to hormonal regulation of leptin synthesis in IDDM patients, body fat and testosterone may contribute to the regulation.

\section{6}

INSULIN LIKE EFFECTS OF LEPTIN: STIMULATION OF GLUCOSE UPTAKE AND GLYCOGEN SYNTHESIS IN $\mathrm{C}_{2} \mathrm{C}_{12}$ MYOTUBES

L. Berti, M. Kellerer and H. Häring, Medizinische Klinik und Poliklinik (IV. Abteilung), Eberhard-Karls-Universität, Otfried-Müller-Str. 10, 72076 Tübingen, Germany

The recently identified ob gene product leptin which is secreted only from white fat cells, is thought to act as a satiety factor by binding to specific sites in the hypothalamus. Recent data suggest that administration of recombinant leptin is effective in reduction of body weight, total body fat, and serum concentrations of glucose and insulin resulting in normalization of the diabetic metabolism. Since leptin seems to play a role in glucose metabolism we investigated whether leptin stimulates glucose uptake and glycogen synthesis. Mouse $\mathrm{C}_{2} \mathrm{C}_{12}$ myotubes were incubated for $60 \mathrm{~min}$ with recombinant leptin $(1-500 \mathrm{ng} / \mathrm{ml})$ and then 2-deoxyglucose uptake was measured. Leptin $(1-25 \mathrm{ng} / \mathrm{ml})$ induces glucose uptake to $86 \%+/-14(\mathrm{n}=3)$ of maximal insulin stimulation. Insulin had only a small additive effect $(20 \%$ $+/-20 ; n=3)$. In addition incubation of myotubes with leptin $(1-500 \mathrm{ng} / \mathrm{ml})$ over several hours $(3$ to $18 \mathrm{~h}$ ) stimulates glycogen synthesis to $93 \%+1-15$ $(n=7)$ of maximal insulin stimulation at leptin concentrations between lng $/ \mathrm{ml}$ and $100 \mathrm{ng} / \mathrm{ml}$. Short term incubation has smaller effects $(40 \%+1-8$ of maximal insulin stimulation; $n=3$ ). Insulin stimulation after leptin incubation was additive on glycogen synthesis which increases about $95 \%$ $+/-17(\mathrm{n}=4)$ at $10^{-10}$ and $10^{-9} \mathrm{M}$ insulin. In summary the data show that leptin mimicks insulin effects at concentrations between $1-100 \mathrm{ng} / \mathrm{ml}$ with regard to glucose uptake and glycogen synthesis. This might contribute to nomalization of hyperglycemia in animal modells after administration of leptin. 


\section{Genetics of Complications}

\section{7}

SUSCEPTIBILITY TO DIABETIC NEPHROPATHY IN IDDM IS STRONGLY ASSOCIATED. WITH THE ALDOSE REDUCTASE GENE A.E. Heesom, M.L. Hibberd, B.A. Millward and A.G. Demaine. Plymouth Postgraduate Medical School, University of Plymouth, Plymouth, England

We have investigated the role of the aldose reductase (ALR2) gene in the susceptibility to diabetic nephropathy (DN) and retinopathy (DR) in patients with IDDM. DNA from 173 British caucasoid patients with IDDM and 102 normal healthy controls were typed for a (CA) dinucleotide repeat polymorphic marker in the 5' region of ALR2 (5'ALR2) using the polymerase chain reaction. Seven alleles (Z-6 to $Z+6)$ were detected in the patients and controls. The patients were categorised according to the presence or absence of nephropathy or retinopathy. There was a highly significant increase in the frequency of the Z-2 5'ALR2 allele in patients with nephropathy - "nephropaths' $(\mathrm{n}=54)$ compared to those with no microvascular disease after 15 years of diabetes - 'uncomplicated' $(\mathrm{n}=45)\left(35.5 \%\right.$ vs. $12.2 \% \chi^{2}$ $=14.2, p<0.0001$ ). There was a corresponding increase in the frequency of the $\mathrm{Z}+2$ allele in the uncomplicated group compared to the nephropaths $\left(38.9 \%\right.$ vs. $\left.8.6 \% \chi^{2}=25.2, p<0.0001\right)$. No significant differences were found in patients with retinopathy without nephropathy - 'retinopaths' $(n=39)$ or patients who have had diabetes for less than 5 years -'short duration' $(n=37)$. The nephropaths had an increased frequency of the Z/Z-2 5'ALR2 genotype compared to the uncomplicated group $(44.2 \%$ vs. $8.9 \% \times 2=14.9, p<0.0001$, Odds Ratio 8.1) with a decreased frequency of the $Z / Z+2$ genotype $(11.5 \%$ vs. $46.7 \%)$. This genotype was also decreased in the retinopaths $(12.8 \%$ vs. $46.7 \%)$. These results strongly suggest that the ALR2 or an adjacent locus is implicated in the susceptibility to diabetic nephropathy but not retinopathy.

\section{9}

DIFFERENCES IN ALBUMIN EXCRETION RATE RESPONSE TO LISINOPRIL BY ACE GENOTYPE IN INSULIN DEPENDENT DIABETES (IDDM).

The EUCLID Study Group. EUCLID Study Group Co-ordinating Centre, EURODIAB, Department of Epiderniology and Public Health, University College London, WC1E 6BT, UK

The association between ACE genotype and diabetic renal disease is controversial, and the effects of ACE inhibitors on this relationship are unclear. We examined the effects of ACE gene insertion/deletion (I/D) polymorphism on rate of change of albumin excretion rate (AER) in a randomised, double-blind placebo controlled trial of lisinopril in 530 men and women with IDDM (20-59 years) with normo- or microalbuminurja. Patients, recruited from 18 European centres, were not on antihypertensive treatment. There were $77(15 \%)$ patients with the II polymorphism, $296(58 \%)$ with ID, and $137(27 \%)$ with DD. There were no significant differences in baseline characteristics, or in prevalence of microalbuminuria (19\% in II, $13 \%$ in $\mathrm{ID}$, and $17 \%$ in $\mathrm{DD}, \mathrm{p}=0.3$ ). At two years in the placebo group, AER had increased by $15.6 \mu \mathrm{g} / \mathrm{min}$ in the II group, $11.5 \mu \mathrm{g} / \mathrm{min}$ in the ID group, and 8.4 $\mu \mathrm{g} / \mathrm{min}$ in the DD group $(\mathrm{p}=0.2)$. The treatment effect differed by ACE genotype $(\mathrm{p}=0.1$ for interaction). At two years, AER was $57 \%$ lower on fisinopril in the II group ( $p=0.02), 19 \%$ lower in the ID group $(p=0.1)$, and $19 \%$ higher in the DD group ( $p=0.4$ ) compared with placebo (adjusted for baseline AER and centre). The absolute trearment difference (placebo-lisinopril) at two years was $8.85 \mu \mathrm{g} / \mathrm{min}$ in the II group, $2.22 \mu \mathrm{g} / \mathrm{min}$ in the ID group, and $-1.56 \mu \mathrm{g} / \mathrm{min}$ in the DD group. We conclude that progression of AER is greatest in patients with the II polymorphism, and least in those with the DD polymorphism. People with the II polymorphism appear to benefit most from treatment with lisinopril.

\section{8}

ACE GENE POLYMORPHISM IN HYPERTENSIVE NIDDM PATIENTS

K. Bengtsson*, M. Orho**, U. Lindblad*, Erik B $ø$ g-Hansen*, Jonas Ranstam***, L. Råstam**** and L. Groop**. Primary Health Care Centre in Skara, Skaraborg Institute*. Department of Endocrinology** and Department of Community Medicine***, Lund University, Sweden.

Deletion-insertion polymorphism of the ACE gene has been associated with myocardial infarction in non-diabetics and with myocardial infarction in NIDDM patients without other riskfactors. Despite the putative role of altered ACE activity in the pathogenesis of hypertension (HT) no association has been found between the $\mathrm{ACF}$ gene and hypertension. Our aim was to study whether the ACE gene polymorphism is a riskfactor for hypertension in patients with NIDDM. From the outpatient clinic for hypertension and diabetes in Skara we drafted NIDDM patients with HT (n=91. 41 males and 50 females, age $69.6 \pm 9.1 \mathrm{yrs}, \mathrm{BMI} 28.9 \pm 4.5 \mathrm{~kg} / \mathrm{m}^{3}$ ) patients with HT $\left(\mathrm{n}=206,79\right.$ males and $127 \mathrm{females}$, age $64.1 \pm 13.0 \mathrm{yrs}$, BMI $\left.26.0 \pm 4.6 \mathrm{~kg} / \mathrm{hr}^{1}\right), \mathrm{NIDDM}$ patients without HT ( $\mathrm{n}=74,39$ males and 35 females, age $69.0 \pm 10.8$ yrs, BM1 27.7 \pm 4.4 $\left.\mathrm{kg} / \mathrm{m}^{2}\right)$ and healthy controls $(n=188,94$ males and 94 females, age $60.3 \pm 13.0 \mathrm{yrs}, \mathrm{BM}$ $\left.25.5 \pm 3.9 \mathrm{~kg} / \mathrm{m}^{2}\right)$. Diagnosis of hypertension was based on national guide-lines effective during study period, diastolic blood pressure repeatedly $90 \mathrm{mmHg}$ or above, or ongoing treatment. Criteria for NIDDM followed WHO recommendations. The deletion (D) or insertion (I) of a $287 \mathrm{bp}$ fragment in intron 16 of the ACE gene was determined by polymerase chain reaction followed by agarose gel electrophoresis. The frequency distribution of DD, ID and II genotypes in NIDDM patients with HT $(30.8 \%, 50.6 \%, 18.7 \%)$, in HT patients $(28.2 \%, 47.1 \%, 24.8 \%)$, in NIDDM patients without HT $(29.7 \%, 39.2 \%, 31.1 \%)$ and in controls $(25.5 \%, 45.2 \%, 29.3 \%)$ did not differ significantly between the study groups. However, when we analysed all the patients and control subjects with $\mathrm{BMI}<27$, a significant distortion of $\mathrm{ACE}$ genotype was observed in NIDDM patients with HT $(35.3 \%, 61.8 \%, 2.9 \%)$ vs controls $(23.5 \%$, $48.5 \%, 28.0 \% ; \mathrm{p}<0.008)$, and vs NIDDM patients without HT $(30.0 \%, 36.7 \%, 33.3 \%$; $\mathrm{p}<0.005)$ and vs HT patients $(27.2 \%, 50.7 \%, 22.1 \% ; \mathrm{p}<0.04)$. The genotype frequency distribution differed significantly also between lean (BMI<27) NIDDM patients with HT $(35.3 \%, 61.8 \%, 2.9 \%)$ compared to obese (BMI>27) patients in the same group $(29.1 \%, 41.8 \%, 29.1 \% ; \mathrm{p}<0.009)$. The genotype frequency distribution in patients and control subjects with BMT>27 did not differ significantly between the study groups. Conclusions: ACE polymorphism is associated with hypertension in nonobese Swedish NIDDM patients. This data suggests that II genotype might protect lean NIDDM patients from hypertension.

\section{0}

ANGIOTENSIN I CONVERTING ENZYME AND ANGIOTENSINOGEN POLYMORPHISMS INTERACT FOR DIABETIC NEPHROPATHY DEVELOPMENT.

M. MARRE*, on behalf of the GENEDIAB (Génétique de la Néphropathie Diabétique) Study Group, (* Angers) France and Belgium.

Renal action of angiotensin II may favour Diabetic Nephropathy (DN) development in IDDM patients, and some components of the reninangiotensin system are genetically determined. We selected 494 patients with high risk for DN (IDDM before age of 35 years and proliferative retinopathy) in 17 diabetic clinics in France and Belgium : $56 \%$ men, age $44+/-12$ years, diabetes duration $29+/-10$ years, classified as 1 : no nephropathy $(n=157), 2$ : incipient $(n=104), 3$ : established $(n=126), 4$ : advanced nephropathy $(n=107$; creatinine $>150 \mu \mathrm{M}$ or renal replacement). The following DNA polymorphisms were analyzed : Angiotensin Converting Enzyme (ACE) Insertion / Deletion (I/D), Angiotensinogen (AGT) M235T and T174 M, Angiotensin II subtype 1 receptor (AT1R) T573C and A1166C.ACE I/D polymorphism was related to nephropathy : D allele frequency in stage $1: .525,2: .586,3$ : $.599,4: .612$ (X2 for trend $5.135, \mathrm{p}=.023$ ). In a logistic polychotomous regression analysis using age at IDDM onset, diabetes duration and time to retinal proliferation, the $\mathrm{D}$ allele was an independent risk factor for $\mathrm{DN}$ : adjusted odds ratio $1.881(95 \%$ CI $1.204-2.289 ; \mathrm{p}=.0055)$, but not the I allele (odds ratio 1.034 (.722-1.482)), suggesting a dominant effect of D allele on DN development. When introduced in this model, AGT M235T interracted significantly with ACE I/D (odds ratio 2.156(1.174$3.958)$ ) : in the 404 patients with ACE ID or DD genotypes, AGT MC235T was related to nephropathy stages : MM / MT / TT in 1: $34 / 50 / 16 \% ; 2: 41 / 43 / 16 \% ; 3: 36 / 38 / 26 \% ; 4: 20 / 54 / 26 \%$ (X2 14.13; $\mathrm{p}=.0282 ;$ codominant effect). Other polymorphisms were not contributive. Interaction between $A C E$ I/D and AGT M $235 \mathrm{~T}$ polymorphisms contributes to the development of DN in IDDM subjects. 
ASSOCLATION BETWEEN ACE GENE POLYMORPHISM, THICKNESS IN YOUNG TYPE 1 DIABETIC PATIENTS

D. Frost ${ }^{*}$, M. Pfohl ${ }^{\star *}$, P. Clemens $s^{\star *}$, H.-U. Häring ${ }^{* *}$ and W. Beischer* *Bürgerhospital, Medizinische Klinik 3, Stuttgart (Germany) and

* *Medizinische Universitätsklinik, Abteilung IV, Tübingen (Germany) The insertion-/deletion (I/D) polymorphism of the angiotensin converting enzyme (ACE) gene is discussed to be an independent, genetically determined risk marker for cardiovascular events. The deletion polymorphism results in an increased ACE plasma activity and thus influences the regulation of blood pressure. We studied possible associations between ACE genotype and hypertension, early atherosclerosis and albuminuria in 70 young type 1 diabetic patients, 30 men and 40 women, age $29.9 \pm 6.4$ y, diabetes duration $13.6 \pm 8.9 \mathrm{y}$ (mean \pm SD). The ID polymorphism of the ACE gene was determined by polymerase chain reaction. For evaluation of early atherosclerosis, we measured the intima-media thickness (IMT) of the carotid arteries by high resolution ultrasound. Patients being homocygous for ACE DD had hypertension $(\mathrm{RR}>140 / 90 \mathrm{mmHg}$ in repeated measurements or established antihypertensive therapy) significantly more often $(\mathrm{p}<0.05)$ than patients with ID or II and had the highest albumin excretion rates (AER). The IMT of patients with DD was significantly $(p<0.05)$ greater than that of patients with ID and slightly greater than the IMT of patients with II. In the latter, the proportion of smokers was unusually high, increasing their vascular risk (see table).

\begin{tabular}{|c|c|c|c|c|c|}
\hline $\begin{array}{c}\text { ACE } \\
\text { genotype }\end{array}$ & $\mathrm{n}$ & $\begin{array}{c}\text { hypertension } \\
(\%)\end{array}$ & $\begin{array}{c}\text { IMT } \\
(\mathrm{mm})\end{array}$ & $\begin{array}{c}\text { AER } \\
(\mu \mathrm{g} / \mathrm{min})\end{array}$ & $\begin{array}{c}\text { smokers } \\
(\%)\end{array}$ \\
\hline DD & 37 & 32.4 & $0.64 \pm 0.13$ & $444.6(0-4872)$ & 32.8 \\
\hline D & 17 & 5.9 & $0.58 \pm 0.11$ & $246.9(0-2190)$ & 11.8 \\
\hline II & 16 & 6.3 & $0.62 \pm 0.16$ & $63.4(0-405)$ & 62.5 \\
\hline
\end{tabular}

Young type 1 diabetic patients who are homocygous for the deletion polymorphism of the ACE gene (DD) have an increased risk for the development of hypertension and early atherosclerosis. Hypertension and albuminuria are possible determinants for the carotid artery wall thickening.
RELATIONSHIP BETWEEN ANGIOTENSINOGEN I-CONVERTING ENZYME (ACE) GENE POLYMORPHISM AND DIABETIC. RETINOPATHY AND NEPHROPATHY IN IDDM

M. Pfohl, D. Frost*. M. Koch, P. Clemens. A. Patzies. R.M. Schmuilling. W Beischer*, and H.-U. Häring. Medizinische Lniversitälsklinik Tübingen. and *Bürgerhospital Stuttgart. Germany

The insertion/deletion (I/D) polymorphism of the ACE gene has been teported to be associated with nephropathy in IDDM. We studied the relationship between the ACE V/D gene polymorphism and diabetic retinopathy and nephropathy in 210 IDDM subjects ( 107 male. 103 female). aged between 18 and 70 years. The DNA of the subjects was analysed using the polymerase chain reaction (PCR) to type for the presence (1) or absence (D) of the $287 \mathrm{bp}$ fragment in intron 16 of the ACE gene, an additional Iallele specific PCR was performed in DD to prevent mistyping of ID subjects. The relative frequency of the different genotypes was $33.8^{\circ} \circ$ (DDi. $43.8 \%$ (ID) and $22.4 \%$ (II). There were no significant differences between the three genotypes in age. body-mass-index. blood pressure. plasma total and LDL-cholesterol, and triglycerides. The prevalence of diabetic retinopathy as assessed by retinal photographs was $33.8 \%$ (DD). $30.4 \%$ (ID), and $27.7^{\circ}$ o (II), the prevalence of mictoalbuminuria (albumin excretion rate (AUR) > $20 \mu \mathrm{g} / \mathrm{min}$ ) or nephropathy (AUR $\geq 200 \mu \mathrm{g} / \mathrm{min}$ ) was $23.9 \%$ (DD). $16.30 \%$ (ID), and $17.0 \%$ (II). Detailed analysis of the data revealed. that the higher percentage of microalbuminuria or nephropathy in the DD genotypes may in parts be due to the higher frequency of DD genotypes in the IDDM subjects with a duration of diabetes of $\geq 30$ years, while the mean duration of diabetes was only slightly higher in the DD group $(15.7+1.2$ y vs. $13.5+0.9 \mathrm{y}$ (ID) and $14.6+1.4 y(\mathrm{II}), \mathrm{p}<0.05$ ). We conclude that in this sample of IDDM subjects the DD genotype is weakly associated with diabetic retinopathy and nephropathy, but this association is partly caused by the higher prevalence of the DD genotypes in subjects with long duration of diabetes

\section{OP 40}

\section{Genetics of IDDM}

\section{3}

CTLA-4 GENE POLYMORPHISM CONFERS SUSCEPTIBILITY TO IDDM INDEPENDENTLY FROM GENETIC AND IMMUNE DISEASE MARKERS B. Van der Auwera, C. Vandewalle, F. Schuit, I. De Leeuw ${ }^{1}$, J.A. Todd ${ }^{2}$, F. Gorus and the Belgian Diabetes Registry. Diabetes Research Center, Vrije Universiteit Brussel, Brussels, Belgium, University of Antwerp-UIA, Antwerp, Belgium, and ${ }^{2}$ The Wellcome Trust Centre for Human Genetics, University of Oxford, Oxford, UK

Apart from genes in the MHC complex (IDDM1) and the $5^{\prime}$ insulin gene polymorphism ( $5^{\prime}$ INS, IDDM2), several other genes have been proposed to contribute to IDDM susceptibility. Recently, linkage has been shown between the cytotoxic T-lymphocyte-associated protein 4 (CTLA-4) gene on chromosome $2 \mathrm{q}$ and IDDM. In the present study we wanted to confirm the diabetes association of a CTLA-4 gene A/G transition polymorphism by studying a registry-based group of 525 recent-onset IDDM patients under age 40 years and 530 healthy control subjects. In addition, we investigated associations between this CTLA-4 marker and established genetic (HLA-DQ, $5^{\prime}$ INS) and immune disease markers (IAA, ICA, GAD65-Ab), determined within the first week of insulin treatment. The CTLA-4 GG and AG genotypes occurred more frequently in patients ( $14 \%$ and $51 \%$ respectively) than in control subjects $(10 \%$ and $46 \%$ respectively). The presence of $G G$ conferred a RR of $1.6(95 \% \mathrm{Cl} 1.1-2.3 ; \mathrm{P}=0.02)$ and the presence of at least one $\mathrm{G}$ allele conferred a RR of $1.5(95 \% \mathrm{Cl} 1.2-2.0 ; \mathrm{P}<0.001)$, confirming linkage analysis in other populations. Since the genotype distribution was not age- or gender-dependent, possible interactions with other IDDM markers were studied without stratification for these variables. There was no preferential association between the GG genotype or the $G$ allele on the one hand and the presence or absence of HLA-DQA1*-DQB1* risk haplotypes or genotypes (0301-0302 and/or 0501-0201), the 5 ' INS 1/1 risk genotype, or autoantibodies (IAA, ICA, GAD65-Ab) on the other hand. For 151 patients, thyrogastric autoantibodies (anti-thyroid peroxidase, anti-TSH receptor, antiparietal cell, anti-intrinsic factor) were available but association between CTLA-4 risk alleles or genotypes and markers of polyendocrine autoimmunity could not be demonstrated. In conclusion, the G-containing CTLA-4 genotypes confer a moderate but significant RR for IDDM, independently from age, gender, genetic or immune disease markers.

\section{4}

GENETIC DISSECTIOY OF COMPLEX DISEASES BY QTL ANALYSIS IN (BB X SHR)F2 HYBRIDS

Vogt L. Voigt, B., Kovacs, P. and I. Klöting „Gerhardt Katsch“ Institute of

Diabetes, Eniversity of Greifswald, Karlsburg

Experimental crosses are a powerful tool for genetic dissection of complex diseases as diabetes or hypertension because of the ability to dissect quantitative traits, e.g. blood glucose insulin, body weight or blood pressure into discrete factors. so-called QTL's. This prompted us to use our phenotypically well-characterized [(BB/OK SHR Mol)F $1 \times$ BB $/ O K]$ and $[(B B / O K \times S H R / M o l) F 1 \times$ SHR $]$ first backcross hybrids combined to an $F 2$ population $(n=230$ ) for linkage analysis and search for QTI 's. At first we selected 40 hybrids with the mose extreme phenotypes for body weight. glucose tolerance, serum triglycerides. cholesterol. total protein urea. $\mathrm{Ca}$ and $\mathrm{P}$ which were gerotyped using PCR-analyzed microsatellite markers. Regions showing evidence for linkage to one of the traits studied were subsequently examined in the full set of 230 hybrids. The data were analyzed with the Mapmaker/QTL computer programme. The threshold for significance in a F2 population is a lod score of 4.3 corresponding to a $\mathrm{P}$-value of $5 \times 10^{-5}$. Using these criteria, for body weight we detected significant linkage to a region between $l l 4$ and $A b p$ on chromosome 10 (lod score 11.8), between ( $r l$ and Tilp on chromosome 18 (lod score 9.1). between $\mathrm{Ig} / 2$ and DIMgh/2 on chromosome 1 (lod score 7.6) and between Npy and Spr on chromosome 4 (lod score 7.2). Interestingly, the same region between Npy and Spr on chromosome 4 was also significantly linked with a lod score of 9.1 for serum total protein. A second locus for total protein was detected between $O$ lf and DI8Mit 9 on chromosome 18 (lod score 8.1). A region linked to basal nonfasting glucose was not found. however after an i.p.GTT a significant linkage was found between olf and DI\&Mit9 on chromosome 18 with lod scores of 4.9 and 4.7 , respectively for glucose leveis at 10 and 60 minutes after glucose load. Furthermore, a region right of the $G h$ locus on chromosome 10 was linked with serum urea (lod score 5.2) and a locus between $A r$ and Mycs on chromosome $\mathrm{X}$ was linked with serum P (lod score 5.7). These findings reveal not only novel results but also indicate the genetic complexity of traits. Despite the genetic complexity we were able to map loci affecting traits which play an important role not only in the development of diabetes but also in the development of hypertension. 
THE HLA-A2,CW1,B56,DR4,DQ8 HAPLOTYPE - A GENETIC CONTRIBUTION TO THE HIGH AND INCREASING INCIDENCE OF IDDM IN FINLAND

E. Tuomilehto-Wolf, J.Tuomilehto, G. Vidgren and the DiMe Study Group. Diabetes and Genetic Epidemiology Unit, Nationa! Public Health Institute, Helsinki, Finland

The incidence of IDDM has steadily increased in Finland by $2.8 \%$ per year since the 1950s. It was in 1991 (40/100,000/yr in the age group 0-14 years) 3.2 times higher than in 1953. In the first population-based prospective HLA family study in IDDM carried out nationwide in Finland (758 IDDM families and 110 population-based HLA genotyped control families) we have found a new IDDM susceptibility haplotype HLA-A2,Cw1,B56,DR4,DQ8 only seen in Finnish IDDM patients. it carries the highest absolute risk susceptibility to IDDM which is now $260 / 100,000 / y r$ and was confirmed in an ongoing prospective Offspring Study comprising 150 Finnish families ascertained through a parent with IDDM. Both preferential zygotic assortment of this haplotype and its more frequent occurrence $(p>0.009)$ in the younger age group $(0-4$ years in which the incidence increased by $4 \%$ /year between 1985 . 92) are genetic reasons why Finland has the highest incidence in the world. In order to study preferential zygotic assortment all IDDM probands were excluded as they had been consecutively ascertained for the presence IDDM. We analyzed how often the A2,CW1,B56,DR4,DQ8 haplotype was transmitted compared to the alternative parental haplotype to 99 siblings in 75 informative families. Mothers transmitted the A2,CW1,B56,DR4, DQ8 haplotype $23 \%$ more often than the alternative maternal haplotype. A sex-associated effect in the transmission of this haplotype was also seen as $62 \%$ of the siblings were of the same sex as the parent from whom they had inherited it. Our data provide empirical evidence that the $A 2, C W 1, B 56, D R 4, D Q 8$ haplotype which has been found by detailed molecular DNA analysis to consist of $A^{*} 0201$, $\mathrm{C}^{*} 0101, \mathrm{~B}^{*} 5601$, TNFalpha6,BFS,C4A3,C4B0,DRB1 ${ }^{*} 0401, \mathrm{DQA1}{ }^{*} 0301$, $\mathrm{DQB} 1^{*} 0302, \mathrm{DPB}^{*} 10401$ contributes to the high and increasing incidence of IDDM in Fintand by enlarging the pool of susceptible individuals through preferential zygotic assortment especially in the younger age group.

\section{7}

ASSOCIATION OF THE HLA CLASS I REGION WITH IDDM IS RELATED TO GENDER AND AGE AT ONSET OF DIABETES

A. Hodgkinson, B.A. Miliward and A.G. Demaine. Plymouth Postgraduate Medical School, University of Plymouth, Plymouth UK. Recent studies have suggested that the HLA class I region may play a role in determining the age at onset of IDDM. We have investigated the role of the class I region (HLA-B to HLA-E) by molecular typing the P3A, 28L, 66R, P3B loci in 311 caucasoid patients with IDDM and 196 normal healthy caucasoid controls. The patients separated into three groups depending upon the age at onset of IDDM $-<10$ years, $10-20$ years or $>20$ years. There was a highly significant increase in the frequency of the $1.5 / 1.5$ kilobase $(\mathrm{kb})$ P3B genotype (detected using the enzyme Pst-1) in the patients compared to the controls $(\chi 2=33.1, p<0.00001)$. The frequency of the P3B genotypes differed substantially with age at onset - the $1.5 / 1.5 \mathrm{~kb}$ genotype was reduced in the $<10$ years group $(n=101)$ and increased in the $10-20$ years group $(n=126)$. In addition, these differences were related to gender. The $1.5 / 1.5 \mathrm{~kb}$ P3B genotype was found in only $6.6 \%$ of the females in the $<10$ years but was present in $30.1 \%$ of the $10-20$ years $(\chi 2=15.3, p<0.0001)$. Conversely, the $1.8 / 1.8 \mathrm{~kb}$ P3B genotype was found in $36.1 \%$ of females in the $<10$ years and $14.3 \%$ in the $10-20$ years. In addition, $1.8 / 1.5 \mathrm{~kb}$ P3B genotype differed between the males in the $<10$ years and $10-20$ years groups $(30.0 \%$ vs $73.0 \%$, $\chi^{2}=18.3, p<0.00001$ ). There were no significant associations with age at onset or gender with the $\mathrm{P} 3 \mathrm{~A}, 28 \mathrm{~L}$ and $66 \mathrm{R}$ loci. These results suggest that the susceptibility gene is localised telomeric of HLA-X and P3B and may be regulated by sex hormones.
LINKAGE OF TYPE 1 DIABETES TO $15 q 26$ (IDDM3) IN THE DANISH POPULATION

F. Pociot ${ }^{1}$, M. Zamani ${ }^{2}$ P. Raeymaekers ${ }^{2}$, J. Nerup ${ }^{1}$ and J.-J. Cassiman'. 'Steno Diabetes Center, Gentofte, Denmark and ${ }^{2}$ Center for Human Genetics, University of Leuven, Leuven, Belgium.

Type 1 diabetes is a polygenic disorder as demonstrated both in man and animal módels. Whole-genome searches have identified a number of chromosomal regions wich demonstrated putative linkage to IDDM. However linkage results must be replicated to be credible. In the present study we investigated the IDDM 3 locus mapping to chromosome $15 \mathrm{q} 26$ in a nationwide, population-based sample of Danish IDDM multiplex families ( $n=81$ families) and in 82 healthy controls by affected sib pair and linkage analysis, and intrafamilial and case-control association studies. The results confirm the linkage of D15S107 to IDDM in these families $(p=0.010)$ and provide strong evidence for linkage when the data were combined with those of previous studies $(p=0.0005)$. Our analysis shows that the $D 15 S 107^{\star} 130$ allele is a susceptibility allele $(p=0.02 ; R R=3.55)$ and further demonstrate that $D 15 \$ 107$ contributes for up to $16 \%$ of the familial clustering of IDDM $\left(\lambda_{\mathrm{s}}=1.55\right)$ assuming a pure multiplicative model. In the present data set affected sib pair analysis suggests a possible independent action of HLA by D15S107.

\section{8}

CTLA4 EXON 1 VARIANTS IN PATIENTS WITH IDDM AND WITH NIDDM.

K. Badenhoop, H. Donner, H. Rau, J. Braun, T. Siegmund, J. Herwig and K.H. Usadel.- Med. Dept. I, University Hospital Frankfurt,

Theodor-Stern-Kai 7, D 60590 Frankfurt am Main, FRG A large part of the genetic susceptibility to type 1 (insulindependent) diabetes mellitus (IDDM) is conferred by genes in the human leukocyte antigen (HLA) region on the short arm of chromosome 6 . Amongst other candidate genes is the cytotoxic $T$ lymphocyte antigen 4 (CTLA4) located on chromosome $2 q 33$ in man. Any IDDM susceptibility locus may also predispose to type 2 (non-insulin dependent) diabetes (NIDDM). We therefore

investigated the distribution of the CTLA4 exon 1 polymorphism 149 $A / G)$ in IDDM and NIDDM. This dimorphism at codon 17 results in an aminoacid exchange (Thr/Ala) in the leader peptide of the expressed protein and was analysed by PCR, SSCP and RFLP analysis in 168 patients with IDDM, 174 with NIDDM and 242 controls. Patients with IDDM had more A(Thr) alleles than controls both as homozygotes $(17 \%$ vs. $14 \%$ ) and heterozygotes (50\% vs. $45 \%)$ and less G(Ala) as homozygotes $(33 \%$ vs. $41 \%)$. The difference between patients with IDDM and controls positive for the predisposing HLA DQA $1{ }^{*} 0501$ was more pronounced: $20 \%$ of patients $(n=93)$ with IDDM were $A(T h r)$ homozygous (vs. $11 \%$ of 102 controls), $53 \%$ heterozygous $(47 \%$ controls) and $27 \%$ G(Ala) homozygous $(42 \%)$ controls, $p<0.04 ; 73 \%$ patients were $A(T h r)$ positive compared with $58 \%$ controls, $p<0.04$. The distribution of CTLA4 alleles between patients with NIDDM and controls was not different. In conclusion. CTLA4 alteles may contribute to genetic susceptibility in a subgroup of IDDM patients with HLA

DOA ${ }^{*} 0501$, where we have observed such an association also with Graves' disease, another $T$ cell mediated autoimmune endocrine disorder. In these patients threonine-17 of CTLA4 and HLA DOA 1*0501 appear to predispose in a synergistic manner. 


\section{OP 41}

\section{Beta-Cell Differentiation and Replication}

229

\section{KERATINS AS MARKERS OF DUCT-TO-ISLET CYTODIFFERENTIATION IN FETAL PANCREAS}

L. Bouwens, W.G. Lu, R. De Krijger ${ }^{*}$ and D. Pipeleers. Diabetes Research Center, Vrije Universiteit Brussel, Brussels, Belgium, and "Department of Pathology, University Hospital Dijkzigt, Rotterdam, The Netherlands.

Although it is generally assumed that islet cells originate from ductal epithelium during ontogenesis, direct evidence for this is lacking. We examined fetal rat, porcine and human pancreas by immunohistochemistry for the appearance of transitional cells co-expressing islet hormones and the ductal markers keratin- $7,-19$ or -20 . These keratins are specific markers for ductal cells in pig, man, and rat, respectively. The rat pancreatic primordium (day 13) consisted entirely of keratinpositive cells. Islet morphogenesis (day 17-21) was characterized by the formation of large keratin-positive cell clusters which were continuous with ducts or ductules and differentiated via hormone/keratin double-positive transitional cells into hormone-positive/keratin-negative islets. In porcine end-gestational pancreas hormone/keratin-double positive transitions were seen as individual cells or as clusters budding from ductules or ducts. After birth the endocrine cells were keratin-negative. In human midgestational pancreas initially (12-14 weeks) all epithelial cells were keratin-positive. Keratin/hormone-double positive cells were found in clusters budding from ducts and ductules. Hormone-expressing cells lost keratin expression during the course of differentiation (1640 weeks). It is concluded that keratins are good markers for islet morphogenesis. Our observations give direct evidence for the ductal epithelial nature of islet stem cells.

\section{0}

PANCREATIC DUCT CELLS EXPRESS GASTRIN AND TGF- $\alpha$ DURING DUCT TO ISLET-CELL DIFFERENTIATION IN DUCT-LIGATED ADULT RATS

R. N. Wang and G. Klöppel. Institute of Pathology, University of Kiel, Michaelisstr. 11, D-24105 Kiel, Germany

In adult rats islet-cell neogenesis can be stimulated by partial duct ligation. Duct to islet-cell differentiation is thought to be regulated by growth factors such as gastrin and TGF- $\alpha$. To test this hypothesis, we examined the expression of gastrin and TGF $-\alpha$ at the mRNA and protein level in pancreatic tissue following partial duct ligation. Pancreatic specimens were investigated on days 3, 5, 7 and 14 after duct ligation by means of non-isotopic in situ hybridisation, immunocytochemistry and Western blotting. Gastrin mRNA was strongly expressed in newly developed duct-like cell structures of the ligated tail part of the pancreas before the period of pronounced islet cell neogenesis (day 3 and 5), while immunostaining for gastrin remained negative. In the non-ligated head portion and control pancreases, gastrin mRNA was not expressed. Expression of TGF- $\alpha$ mRNA and protein was found to be increased in the ligated tail portion of the pancreas at day 3 and, particularly, at day 5 , while there was no enhanced signal in the non-ligated part. Western blotting revealed two different TGF- $\alpha$ isoforms (18 kDa and 42 $\mathrm{kDa}$ ). The induction of gastrin and TGF- $\alpha$ expression preceded the increase in BrdU pulse labelling index of beta and alpha cells, which had its peak at day 7 . Our study thus demonstrates that pancreatic duct cells which give rise to new islet cells following duct ligation overexpress gastrin mRNA, TGF- $\alpha$ mRNA and protein. This suggests that these putative growth factors are involved in islet neogenesis.
ACQUIRED SENSITIVITY TO THE TOXIC EFFECT OF INTERLEUKIN 1-BETA FOLLOWING INSULIN PROMOTER FACTOR 1 MEDIATED DIFFERENTIATION FROM ALPHA TO BETA CELL PHENOTYPE

K. Nielsen, P. Senup, OD. Madsen, J. Nerup, and AE. Karisen, Steno Diabetes Center \& Hagedorn Research Institute; Niels Steensensvej, DK-2820 Gentofte, Denmark.

Interleukin-1-beta (L-1) is specifically toxic to the beta-cell in the islets of Langerhans, a phenomenon which has been assaciated with cytokine induced nitric oxide (NO) formation. The transcription factor Insulin Promoter Factor 1 (IPF1) is required for pancreas-development and is expressed in the beta- but not in the a:pha-cells. Transfection and stable expression of IPF1 in a MSL-AN cell-line of glucagon expressing alpha-cell phenotype was followed by a phenotype-change into amylin and insulin-producing beta-cells. AlM: To determine if the IPF1 induced change from alpha- to beta-cell phenotype would render the cells sensitive to the toxic effect of IL-1. METHODS: Following the IPF1 transfection, two clones containing the expression vector were selected. One without expression of the IPF1 gene staying insulin negative (IPF1.) and the other with IPF1 expression, tuming insulin and amylin positive (IPF1+). The cell-lines were exposed to IL-1 at different concentrations A): $150 \mathrm{pg} / \mathrm{mt}(60 \mathrm{U})$, B): $1500 \mathrm{pg} / \mathrm{ml}$, and C): $3000 \mathrm{pg} / \mathrm{ml}$, and induced NO and cytotoxicity were determined after 3 days of culture. RESULTS: The IL-1 induced NO (measured as $\mu$ mol/h accumulated nitrite) was in the same range in both cel-lines; A): $0,95 \pm 0,18$ in the IPF1- vs. 2,73 $\pm 0,24$ in IPF1+; B): $19,91 \pm 1,66$ vs. $16,96 \pm 1,21$; and C): $20,76 \pm 2,09$ vs. $17,96 \pm 1,72$. Despite similar induction of NO in both cell-Iines, the high IL-1 concentrations were specifically toxic to the IPF1+ beta-cell phenotype (measured as Lactate DeHydrogenase (LDH)-release in percent of control cells), whereas the IPF1- alpha phenotype was only slightly affected: A): $-0.6 \pm 3.7$ in the IPF1 - vs. $3,12 \pm 1.9$ in the $(P F 1+; B): 2,9 \pm 0.8$ vs. $18,5 \pm 2,9$; and C): $3,6 \pm 2.1$ vs. $24,2 \pm$ 3,1. CONCLUSION: The IPF1 induced change from an alpha- to a beta-cell like phenotype is associated with an acquired sensitivity to the toxic effect of $1 \mathrm{~L}-1$, despite equal amounts of NO is induced in both cell-lines. This system will be used (by 2-D-gel electrophoresis and massspectrometry) to identify factors responsible for the IPF1 induced beta-cell maturation and acquired sensitivity to the toxic effect of IL-1.
NICOTINAMIDE AND SODIUM BUTYRATE HAVE POTENT SYNERGISTIC EFFECTS ON FETAL PORCINE Bi-CELL DIFFERENTIATION

T. Otonkoski, J. Ustinov. M.-A. Huotari, E. Kallio and P. Häyry, Transplantation Laboratory, University of Helsinki, Finland

Our aims are: 1) to elucidate mechanisms controlling pancreatic is-cell growth and regeneration and 2) 10 generate $\beta$-cells for transplantation from the fetal porcine pancreas. Pancreases were obtained from 80-95-day pig fetuses and digested with collagenase into small cell clusters which were then maintained in suspension tissue culture for up to 4 weeks. In the standard medium (containing 5\% human serum) the number of cells and the amount of insulin decreased by $90 \%$ in 3 weeks. Addition of porcine growth hormone or prolactin $(0.5 \mathrm{\mu g} / \mathrm{ml})$, retinoic acid $\left(10^{-7} \mathrm{M}\right)$, D-vitamin analogue $\mathrm{CB} 1093$ $\left(10^{-7} \mathrm{M}\right)$ or $\beta$-mercaptoethanol $(50 \mu \mathrm{M})$ into the culture medium had no effects. In the presence of $10 \mathrm{mM}$ nicotinamide (NIC) or I mM sodium butyrate $(\mathrm{NaB})$ the cell number was also decreased (by 80 and $90 \%$, respectively). However, the insulin content of the cultured tissue increased 2 . fold. When NIC and NaB were combined, the total insulin content increased 12,5-fold, and the insulin content per cellular DNA increased 100-fold. The proportion of insulin-positive B-cells increased 5-fold (from 10 to 50\%) with these differentiation factors. NIC-cultured cells still proliferated at 3-4 weeks after plating to culture whereas with $\mathrm{NaB}$ proliferation was almost nonexistent after 2 weeks (BrdU labelling index $2.7 \pm 0.8$ vs. $0.3 \pm 0.2 \%$, $\mathrm{p}<0.001)$. The vast majority of proliferating cells were insulin-negative; double-labelled cells represented maximally $0.2 \%$ of total cells under any condition. Cells cultured for 3 weeks with NIC or NaB responded to glucose (2-fold) whereas the combination of NIC and $\mathrm{NaB}$ was associated with glucose-insensitive insulin release. The potent synergism of NIC and NaB has proven useful for the study of mechanisms controlling B-cell differentiation. This approach may also prove valuable for the generation of pure and functional porcine $B$-cells for transplantation. 
GROWTH AND DIFFERENTIATION FACTORS IN PANCREATIC B CELLS: INS-1 RAT INSULINOMA CELL LINE AS A MODEL M.-A. Huotari, J. Ustinov, E. Aavik, S. Pakkala and T. Otonkoski, Transplantation Laboratory, University of Helsinki, Finland

Several growth factors have been shown to regulate pancreatic B-celis. In order to obiain a more complete picture of the growth regulation of these cells, we have started to characterize the responses of the relatively differentiated INS-1 rat insulinoma cell line to a wide variety of factors. For proliferation studies the cells $\left(\mathrm{J}, 5 \times 10^{5} \mathrm{mI}\right)$ were cultured in medium containing $0.5 \%$ FCS for $24 \mathrm{~h}$ and then stimulated for a $24-\mathrm{h}$ period with the factor to be tested. DNA synthesis was determined by incorporation of $\left({ }^{3} \mathrm{H}\right)$ thymidine during the last 4 $h$. All results are expressed in the order of decreasing potency $(p<0.001$ for all effects). DNA synthesis was stimulated by growth hormone ( $\mathrm{GH}, 184 \pm 3 \%$ of control $\pm S E$ ), insulin-like growth factor-I (IGF-I, 164 2 ) and vascular endothelial growth factor (VEGF, $148 \pm 5$ ), and inkibited by the D-vitamin analogue $\mathrm{CB} 1093(38 \pm 7)$, sodium butyrate (NaB, $50 \pm 4)$, nicotinamide (NIC, $65 \pm 7)$, activin-A $(67 \pm 5)$ and retinoic acid $(70 \pm 2)$. Insulin content per DNA was increased by NIC $(147 \pm 3)$ and $\mathrm{NaB}(241 \pm 3)$. The combination of $10 \mathrm{mM}$ $\mathrm{NIC}$ and $1 \mathrm{mM} \mathrm{NaB}$ was the most potent differentiation factor resulting in 3,4-fold inhibition of DNA synthesis and 4,7-fold increase in insulin content per DNA. Epidermal growth factor (EGF), platelet-derived growth factor (PDGF), nerve growth factor (NGF), basic fibroblast growth factor (bFGF), angiopeptine, follistatin, neuregulin (NDF), bone morphogenic protein-2 (BMP-2) and transforming growth factor- $\beta$ (TGF- B) bad no significant effects. Exogenous hepatocyte growth factor (HGF) was also inactive. However transfection of human HGF cDNA into INS-1 cells resulted in markedly stimulated growth and decreased insulin synthesis. From our results, VEGF emerges as a novel potential $B$-cell growth factor. Vitamin $D$, nicotinamide and sodium butyrate are potent factors useful for the identification of genes responsible for $\beta$-cell differentiation.

\section{OP 42}

\section{Lipids}

\section{5}

PARAOXONASE GENE POLYMORPHISM IN NON-DIABETIC RELATIVES OF NON-INSULIN DEPENDENT DIABETIC FAMILIES

M. Armstrong, C.L. Birch, V. Jones, M.W. Stewart, D.B.Humphriss, D.M. Turnbull and M. Walker. University of Newcastle upon Tyne, UK.

Paraoxonase is linked to HDL-cholesterol and has the potential to limit the formation of atherogenic LDL oxidation products. Two alleles of the paraoxonase gene result from a glutamine (A allele) to arginine (B allele) exchange at position 192. The $B$ allele was associated with decreased HDLcholesterol levels in a population study and ischaemic heart disease (IHD) in non-insulin dependent diabetes (NIDDM). We examined the relationship between the $B$ allele and HDL-cholesterol levels in non-diabetic first degree relatives of families with 2 or more living NIDDM patients. Relatives ( $n=131)$ and control subjects ( $\mathrm{n}=131$ ) with no family history of diabetes were matched for age and sex, and none had clinically detectable IHD. Genotyping was performed using the change in a $\mathrm{A} 1 \mathrm{wI}$ restriction endonuclease site associated with the 192 polymorphism. Serum HDL-cholesterol levels were lower in the relatives $(1.25 \pm 0.31$ [mean $\pm S D$ ] vs $1.41 \pm 0.41, \mathrm{p}<0.001)$, although genotype frequencies were comparable in the 2 groups $(\mathrm{A} / \mathrm{A}, \mathrm{A} / \mathrm{B}, \mathrm{B} / \mathrm{B}: 0.48,0.44,0.08$ and $0.50,0.46,0.04$; relatives and controls, $\left.\mathrm{Chi}^{2}=1.58, \mathrm{NS}\right)$. In addition, there were no differences in HDL-cholesterol levels between relatives with the A/A genotype $(\mathrm{n}=63,1.29 \pm 0.30 \mathrm{mmol} / \mathrm{l})$ and those with the $\mathrm{B} / \mathrm{B}(\mathrm{n}=11,1.22 \pm 0.29 \mathrm{mmol} / \mathrm{l})$ and $B / B+A / B$ combined $(n=68,1.22 \pm 0.30$ mmol/l) genotypes, before and after correcting for important co-variates. In conclusion, decreased HDL-cholesterol levels in non-diabetic relatives of NIDDM families are not telated to the allelic forms of the paraoxonase gene.

\section{PROGESTERONE STIMULATES PANCREATIC CELL REPLICATION} IN VIVO

A.G. Nieuwenhuizen, G.A. Schuiling, S.M.S. Liem, G.C.J. van der SchaafVerdonk, L.G. Hilbrands and T.R. Koiter

Dept. Obstetrics \& Gynaecology, University of Groningen, the Netherlands

During the second half of the rat pregnancy a temporary increase in islet-cell proliferation occurs. Based on in vitro evidence, Sorenson et al. suggested that progesterone is involved in the suppression of islet-cell proliferation towarcls the end of gestation (Endocrinology 133: 2227-2234, 1993). In this study, we investigated the effects of progesterone on pancreatic cell proliferation in vivo. Progesterone was administered s.c. for 7 days to pregnant (from day 7 until 14 of gestation) and to nonmpregnant rats, by means of 3 silastic implants (length 3.8 $\mathrm{cm}$, inner diameter $3.35 \mathrm{~mm}$, outer diameter $4.64 \mathrm{~mm}$ ) containing the crystallized hormone. Controls received 3 empty implants. Over the last 24 hours of the progesterone treatment islet-cell proliferation, as measured by BrdU incorporation, was significantly enhanced in pregnant (126 \pm 19 vs $84 \pm 5$ divisions/1000 islet cells) and non-pregnant rats $(26 \pm 4$ vs $15 \pm 3$ divisions $/ 1000$ islet cells). Progesterone treatment also increased replication rates in exocrine pancreas tissule of pregnant ( $19 \pm 2$ vs $13 \pm 1$ divisions/area unit) and of non-pregnant $(23 \pm 1$ vs $7 \pm 1$ divisions/area unit). As plasma coneentrations of progesterone were within the physiological range, these observations do not support the hypothesis of Sorenson and coworkers.

To investigate this phenomenon further we also studied the etfects of progesterone in 14 days ovarjectomized (OVX) rats and male rats. In OVX rats progesterone was ineffective $(11 \pm 2$ vs $15 \pm 1$ divisions/1000 islet cells; $4 \pm 1$ vs $6 \pm 1$ divisions/area unit exocrine tissue), In male rats, on the other hand, progesterone increased pancreatic cell proliferation both in endocrine $(29 \pm 3$ vs $15 \pm 2$ divisions/ 1000 islet cells) and in exocrine tissue $(36 \pm 3$ vs $27 \pm 3$ divisions/area unit). We concluded that progesterone stimulates pancreatic cell proliferation regardless of sex. The mode of action of the hormone is obscure, but the presence of gonads appears to be required. As neither glucose tolerance nor basal- and glucose-stimulated insulin secretion were affected by the steroid in any of the experimental groups, the physiological impact of this progesterone-effect remains unclear.

\section{6}

\section{DIABETES, OBESITY AND LIPAEMIA: MUTATIONS AT THE LIPOPROTEIN LIPASE (LPL) GENE LOCUS}

Qiuping Zhang ${ }^{1}$, Elisabeth Cavallero ${ }^{2}$, Julian Cavanna' ${ }^{1}$, Andrea Kay ${ }^{1}$, Aline Charles $^{2}$, Sylvie Braschi ${ }^{2}$,Leon Perlemuter ${ }^{2}$,Bernard Jacotot ${ }^{2}$, David J. Galton ${ }^{1}$.

'Department of Human Metabolism and Genetics, St Bartholomew's Hospital, London EC1A 7BE; 'Department of Internal Medicine and Department of Endocrinology, Hôpital Henri Mondor, Creteil, France.

Background: The common association of diabetes $m$., obesity and hyperlipidaemia may have a primary aetiological basis. Insulin resistance has been postulated as possible cause; but defects in the plasma transport of triglyceride or fatty acids could also be primary determinants. We have therefore studied 18 patients with diabetes $\mathrm{m}$., obesity and severe hypertriglyceridaemia for defects of a key protein involved in the clearance of plasma triglycerides, lipoprotein lipase.

Methods: DNA was prepared from leucocytes of 18 patients with the above syndrome and all exons of lipoprotein lipase were amplified by PCR. The products were sequenced using the dideoxy chain termination method.

Findings: Eight of the subjects were found to possess genetic variants at the lipoprotein lipase gene locus. These were :

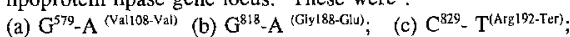

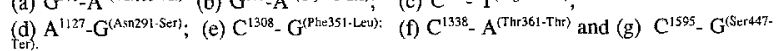

Three of these, (c), (e), and (f) have not hitherto been described. (f) appears to be a population polymorphism whose allele frequency in normolipidaemic diabetics was found to be 0.12 out of 162 chromosomes studied. The others are all rare at frequencies of $<0.01$ and may contribute to the phenotype by impairing clearance of plasma triglycerides.

Interpretation: We conclude that genetic variants at the lipoprotein lipase locus occur commonly in subjects with this syndrome and may affect the individual's response to obesity and diabetes $\mathrm{m}$. for the development of lipaemia. 
237

HYPERGLYCEMIA-INDUCED HYPERINSULINEMIA ACUTELY LOWERS PLASMA APOLIPOPROTEIN B BUT NOT LIPOPROTEIN(A) IN MAN.

S.C. Riemens, J.J.M. Ligtenberg and R.P.F. Dullaart. Department of Endocrinology, University Hospital Groningen, The Netherlands.

Acute hyperinsulinemia suppresses hepatic apolipoprotein B (apo B) and triglyceride production in human subjects. Lipoprotein (a) $(\operatorname{Lp}(\mathrm{a}))$ is formed by attachment of apo B to apolipoprotein (a) (apo (a)), which is also synthesized in the liver. Since the effect of acute hyperinsulinemia on the plasma $\mathrm{Lp}$ (a) level is unclear, we measured plasma triglycerides, cholesterol, apo B and $\mathrm{Lp}$ (a) in response to 3 hour hyperglycemia-induced hyperinsulinemia (blood glucose clamped at $10 \mathrm{mmol} / \mathrm{l}$ ) in 16 subjects (8 women and $8 \mathrm{men}$ ). In a control experiment saline was infused in another group of 7 men. Plasma triglycerides decreased by $29 \pm 14 \%$ (mean \pm SD) from $1.43 \pm 0.86$ to $1.07 \pm 0.81 \mathrm{mmol} / 1(\mathrm{p}<0.001)$ after 3 hour of hyperinsulinemia and this fall was greater than the unchanged triglyceride level during saline infusion $(p<0.001)$. Plasma cholesterol fell by $8 \pm 5 \%$ from $5.16 \pm 1.05$ to $4.77 \pm 1.00 \mathrm{mmol} / \mathrm{l}(\mathrm{p}<0.001)$, which was different from the unchanged cholesterol during saline infusion $(\mathrm{p}<0.02)$. Plasma apo $\mathrm{B}$ decreased from $0.73 \pm 0.19$ to $0.66 \pm 0.18 \mathrm{~g} / 1$ or by $9 \pm 8 \%$ $(\mathrm{p}<0.001)$, which was again different from the minor fall in apoB $(-3 \pm$ $3 \%$ ) during saline infusion $(\mathrm{p}<0.02)$. However, plasma $L p(a)$ (geometric mean, 95\% Cl) remained unchanged during hyperinsulinemia (69 (37-128) $\mathrm{mg} / \mathrm{l}$ vs. $74(40-138) \mathrm{mg} / \mathrm{l}$ after 3 hour; change $8 \pm 15 \%$, N.S.), as well as during saline infusion (change $5 \pm 15 \%$, N.S.). Moreover, the $\%$ fall in apo B was greater than the \% change in Lp(a) during hyperinsulinemia $(\mathrm{p}<0.05$ ). In conclusion, this study demonstrates that acute hyperglycemia - induced hyperinsulinemia has a different effect on the plasma apo B and $L p$ (a) level in healthy subjects. It is hypothesized that $L p(a)$ formation is not influenced by acute hyperinsulinemia in contrast to the insulin-induced inhibition of apo $\mathrm{B}$ production.

\section{9}

MEAL INTAKE DOES NOT AFFECT HDL2 AND HDL3 APOPROTEIN KINETICS IN HUMANS.

P Lucidi, E. Volpi, F. Monacchia, G. B. Bolli and P. De Feo. Di.M.I.S.E.M., Perugia (Italy).

Meal absorption decreases plasma HDL-cholesterol concentrations; at present, it is unclear whether this effect is mediated through a decrease of intravascular rates of appearance (IRA) or an increase of intravascular rates of disappearance of HDL. To answer this question, the IRA of HDL2 and HDL 3 apoproteins were measured in 8 healthy volunteers, randomized in two groups (CTRL and MEAL) matched for age (CTRL 26 \pm 3 , MEAL 24 \pm 1 years) and BMI (CTRL 22 \pm 1 , MEAL $23 \pm 1 \mathrm{Kg} / \mathrm{m}^{2}$ ). All subjects were studied for 8 hours. The first part of the study (overnight fasting, $0-240 \mathrm{~min}$ ) was used to assess apoprotein kinetics in the post absorptive state; the second part (240-480 min) was used to compare apoprotein kinetics between the fasted (CTRL group, $n=4$ ) and the fed state (intragastric infusion of a $632 \mathrm{Kcal}$ glucose-lipid meal, MEAL group, $\mathrm{n}=4$ ). Plasma apoprotein IRA were measured using sequential ultracentrifugation and plasma $\mathrm{KIC}$ specific activity (SA) as precursor pool SA during the infusion of $\left[1-{ }^{14} \mathrm{C}\right]$ leucine. Plasma $\mathrm{HDL}$ cholesterol decreased by $10 \%$ after meal ingestion $(\mathrm{p}<0.05)$. In the postabsorptive state $(0-240 \mathrm{~min})$ the IRA $\left(\% \cdot \mathrm{h}^{-1}\right)$ of HDL 2 and HDL 3 apoproteins did not differ between the CTRL and the MEAL groups (HDL2: $1.2 \pm 0.4,1.1 \pm 0.1 ; \mathrm{HDL} 3: 0.9 \pm 0.2,1.1 \pm 0.1$; respectively). Meal ingestion did not affect the IRA of HDL2 (MEAL 0.9 \pm 0.2 vs CTRL $1.1 \pm 0.2, \mathrm{p}=\mathrm{NS}$ ) and HDL3 apoproteins (MEAL 1.0 0.1 vs CTRL $0.8 \pm 0.2, p=N S$ ). In conclusion, during meal absorption, the plasma HDL-cholesterol decrement is not due to a decrease of HDL intravascular rates of appearance, but to an increase of their disappearance rates. Since the mixed meal absorption induced a physiologic increase of insulinemia, we can conclude that insulin does not affect the intravascular rates of appearance of HDL.
238

DEFECTIVE SUPPRESSION OF YLDLI APO B PRODUCTION BY INSULIN IS AN INHERENT FEATURE OF DYSLIPIDEMIA IN NIDDM

R.Malmstrom', C.J.Packard ${ }^{2}$, M.Caslake ${ }^{2}$, D.Bedford ${ }^{2}$, P.Stewart ${ }^{2}$, J.Shepherd ${ }^{2}$ and MR. Taskinen'. 'Department of Medicine, University of Helsinki, 00290 Helsinki, Finland and ${ }^{2}$ Department of Pathological Biochemistry, Royal Infirmary, Glasgow G4 OSF, Scotland, UK.

In healthy subjects acute insulin administration suppresses VLDLI (Sf 60-400) apo B production without any effect on VLDL2 (Sf 20-60) apo B metabolism. To test the hypothesis that the suppression of VLDL I apo $B$ production is impaired in NIDDM we studied 7 men with NIDDM (age $54 \pm 2 \mathrm{yr}$, BMI $27.0 \pm 0.9 \mathrm{~kg} / \mathrm{m}^{2}$, triglycerides $1.82 \pm 0.20$ minol/t, mean \pm SE) during A) saline infusion and B) 8.5 hrs hyperglycemic (P-gluc $10.8 \pm 0.7 \mathrm{mmol} / 1)$ hyperinsulinemic $(1.0 \mathrm{mU} / \mathrm{kg} / \mathrm{min})$ clamp. 5 subjects participated also in euglycemic (P-gluc $5.4 \pm 0.0 \mathrm{mmol} / \mathrm{t}$ ) hyperinsulinemic clamp after a low-dose overnight insulin infusion (study $\mathrm{C}$ ). A bolus injection of $[3-2 \mathrm{H}]$-leucine was given on each occasion to trace apo B kinetics in VLDLI and VLDL2 subclasses. The kinetic constants were derived with SAAM 30 program using a non-steady state multicompartmental model. In study B after $30 \mathrm{~min}$ insulin infusion VLDLl apo B synthesis was $12 \%$ lower compared to saline infusion ( $752 \pm 76$ vs $663 \pm 98 \mathrm{mg} / \mathrm{d}$, NS) and after $510 \mathrm{~min}$ infusions $16 \%$ lower ( $701 \pm 86$ vs $590 \pm 114 \mathrm{mg} / \mathrm{d}$, NS), while FFA were suppressed by $85 \%$. In study C VLDL 1 apo B synthesis ( $646 \pm 118$ and $603 \pm 145$ $\mathrm{mg} / \mathrm{d}$ at 30 and $510 \mathrm{~min}$ ) did not differ significantly from study A and B. VLDL2 direct input was $160 \pm 14,188 \pm 54$ and $213 \pm 45 \mathrm{mg} / \mathrm{d}$ and input from VLDL1 $644 \pm 89$, $608 \pm 107$ and $616 \pm 135 \mathrm{mg} / \mathrm{d}$ in study $\mathrm{A}, \mathrm{B}$ and C. VLDL/ FCR was $7.6 \pm 1.1,8.3 \pm 2.9$ and $11.0 \pm 4.4 \mathrm{pools} / \mathrm{d}$, VLDL2 FCR was $5.8 \pm 0.5,5.9 \pm 0.6$ and $6.2 \pm 0.7 \mathrm{pools} / \mathrm{d}$, resp. The data indicate that the suppression of VLDL 1 apo B production seen in healthy controls $(35 \%$ at $30 \mathrm{~min} 51 \%$ at $510 \mathrm{~min}$ ) is impaired in NIDDM. The defect was observed despite efficient suppression of FFA by insulin, indicating that the site of impaired insulin action is located in the liver. We suggest that the inability of acute hyperinsulinemia to inhibit the secretion of VLDLI particles is a component of insulin resistance and the major cause of hypertriglyceridemia in NIDDM.

\section{0}

ELEVATED FREE FATTY ACIDS ARE ASSOCIATED WITH THE PRESENCE OF VENTRICULAR PREMATURE COMPLEXES IN NONINSULIN DEPENDENT DIABETICS.

P.Gualdiero,D.Manzella,F.Saccomanno,MR.Tagliamonte,F.Turano,G.Paolisso, F. D'Onofrio. Dept of Geriatric Medicine- 2nd University-Naples-Italy Elevated free fatty acids (FFA) have been shown "in vitro" to have pro-arrhythmic effects. We aim at investigating the association between elevated fasting plasma FFA concentrations and the presence of ventricular premature complexes (VPCs) in non-insulin dependent diabetic (NIDDM) patients. 89 NIDDM patients were enrolled. In all patients 24-hours ambulatory electrocardiograph recordings, twodimensional controlled M-mode Echocardiographic recording, limited treadmill exercise testing with Bruce protocol and dypiridamole myocardial tomoscintigraphy perfusion with Tallium -201 were made. Furthermore, in all patients fasting blood samples were drawn for metabolites determinations. Patients were non-ischemic, slightly overweight a with an upper body fat distribution, normotensive and without left ventricular hypertrophy. Univariate analysis demonstrated that patients at the 3rd tertile for fasting plasma FFA concentrations had the strongest increase in VPCs. VPCs and FFA were significantly correlated $(r=0.27 \mathrm{p}<0.01)$ independently of age, sex, BMI, waist/hip ratio, left ventricular mass index, glycated haemoglobin, fasting plasma insulin and triglycerides concentrations and, daily physical activity. In the multiple logistic regression analysis the relationship between FFA and VPCs was independent of age, sex, BMI, waisthip ratio, left ventricular mass index, mean arterial blood pressure and ejection fraction $(\mathrm{OR}=2.8 ; 95 \% \mathrm{Cl}=1.4-3.8)$. Finally , after addition to this model of glycated haemoglobin, fasting plasma insulin, triglycerides and potassium concentrations, fasting plasma LDL/HDL cholesterol ratio and, daily physical activity the association of FFA and VPCs was still significant $(\mathrm{OR}=1.295 \% \mathrm{CI}=1.0$ 2.3). In conclusion, elevated fasting plasma FFA concentrations are associated with a greater number of VPCs in NDDM patients. Such a relationship seems independent of the main metabolic parameters present in our patients. 


\section{OP 43}

\section{Hypoglycaemia II}

241

Reduced cardiac parasympathetic tone during acute hypoglycaemia

Thomson $\mathrm{GA}^{a}$, Galloway $\mathrm{P}^{2}$, Julu $\mathrm{POO}^{\mathrm{b}}$, Hansen $\mathrm{S}^{\mathrm{b}}$, Burns L, Fisher $\mathrm{BM}^{\mathrm{a}}$, Semple $\mathrm{CG}^{\mathrm{a}}$

a. The diabetes unit, The Southern General Hospital NHS Trust, Glasgow (UK) b. The peripheral nerve and autonomic unit. The Southern General Hospital NHS Trust. Glasgow (UK)

The parasympathetic response to hypoglycaemia is poorly understood. The present study was performed to assess cardiac parasympathetic responses to hypoglycaemia. Cardiac vagal tone (CVT) was measured continuously in 6 male control subjects (age $32 \pm 2.4$ years; mean $\pm \mathrm{SEM}$ ) and 6 male diabetic patients (age $35 \pm 1.6$ years) using a technique based on microcomputer analysis of ECG R-R variability. All subjects were relaxed (non-sleeping) and supine throughout the study. Continuous recordings of heart rate (HR) and CVT were made from 10 minutes prior to the administration of intravenous soluble insulin $\left(0.15 \mathrm{u} \mathrm{Kg}^{-1}\right)$. until 4 hours after the autonomic reaction to hypoglycaemia (R). Basal CVT was higher in diabetic patients $(11.4 \pm 1.3$; $[p<0.05])$ than in control subjects. Basal HR was lower in diabetic paticnts $\left(61.7 \pm 2.4 \mathrm{~min}^{-1} ;[\mathrm{p}<0.05]\right)$ than in control subjects. CVT fell in response to hypogkycaemia by $58 \pm 5.7 \%(p<0.001)$ in control subjects and by $70.3 \pm$ $7 \%(p<0.001)$ in diabetic patients to reach a nadir at R. CVT increased subsequently to reach apparently suprabasal levels in both groups by $R+60$ minutes although significance only occurred in diabetic patients at $R+120$ minutes $(34 \pm 8.1 \%$ increase; $p<0.05$ ). Significant increases in HR accurred in control subjects $(25.5 \pm$ $6.2 \% ; \mathrm{p}<0.01)$ and diabetic patients $(50 \pm 16.7 \% ; \mathrm{p}<0.05)$ at $\mathrm{R}$. This study suggests that acute hypoglvcaemia causes significant changes in synpathovagal balance with reflex suprabasal cardiac vagal tone following restoration of normoglycaemia

\section{3}

IMPAIRED PERIPHERAL PHYSIOLOGICAL RESPONSES TO HYPOGLYCAEMIA IN INSULIN TREATED TYPE II DIABETES

S.R. Peacey, C. Bedford, S. Marlow, N. Harris, C.A. Hardisty, I.A Macdonald and S.R. Heller. University Department of Medicine and Department of Diabetes, Northern General Hospital and Department of Medical Physics, Roval Hallamshire Hospital, Sheffield. University Department of Physiology, Queens Medical Centre, Nottingham, Eugland.

It is unclear whether tightly controlled patients with type II diabetes acquire impaired physiological responses to hypoglycaemia or if this is affected by treatment type. We therefore studied 15 patients with type II diabetes who had been randomised to receive either insulin (group $\mathrm{I}, \mathrm{n}=7$ ) or sulphonylurca (group $S, n=8$ ) therapy as part of the UKPD study. The groups were matclicd for age, sex, BMI, $\mathrm{HbAl}$ and duration of diabetes. We exanined the physiological, symptomatic and counterregulatory responses to hypoglycaemia, using a stepped hyperinsulinaemic hypoglycaemic clamp. Peak tremor response (mean \pm sem) $(0.14 \pm 0.04$ vs $0.33 \pm 0.06$ volts; $p<0.02)$ and peak sweating response [median (range) $16(-2$ to 157$)$ vs 171 (20 to 376$\left.) \mathrm{g} / \mathrm{m}^{2} / \mathrm{hr} ; \mathrm{p}=0.02\right]$ was reduced in group I compared to group $\mathrm{S}$. There were no differences in peak glucagon rise (I vs S, $52 \pm 23$ and $41 \pm 7$ $\mathrm{ng} / \mathrm{l} ; \mathrm{p}=0.62)$, glycaemic threshold for glucagon release $(2.7 \pm 0.1$ and $2.6 \pm$ $0.1 \mathrm{mmol} / \mathrm{l} ; \mathrm{p}=0.53)$, peak adrenaline rise $(4.1 \pm 1.2$ and $4.9 \pm 1.1 \mathrm{nmol} / /$; $p=0.65)$ or glycaemic threshold for adrenaline release $(3.4 \pm 0.1$ and $3.9 \pm 0.2$ mmol/t; $p=0.07$ ). There were no differences in the peak symptom response nor the glycaemic threshold for symptoms or deterioration in cognitive function. We conclude that insulin treatment results in an impaired peripheral response to hypoglycaemia although other components of the physiological response to hypoglycaemia appear unaffected. This could increase the risk of severe hypoglycaemia when those patients with type If diabetes are treated with insulin.

\section{2}

EFFECTS OF NOCTURNAL POSTHYPOGLYCEMTC HYPERGLYCEMIA ON RESPONSES TO MORNING HYPOGLYCEMIA IN HUMANS.

S. Pampanelli, C. Lalli, P. Del Sindaco, M. Ciofetta, P. Brunetti and G.B. Bolli, Di.M.I.S.E.M., University of Perugia, Italy

To establish the effects of nocturnal post-hypoglycemic hyperglycemia (PHH) on responses to morning hypoglycemia $(\mathrm{H}), \mathrm{H}$ (stepped hypoglycemic clamp where plasma glucose, $\mathrm{PG}$, was decreased from 5.0 to $2.2 \mathrm{mM}$ ) was induced between $08: 00-14: 00 \mathrm{~h}$ in 6 nondiabetic subjects on 3 occasions (S1, S2, S3, random order). In S1, morning H was induced after nocturnal euglycemia; in $\mathrm{S} 2$, after nocturnal $\mathrm{H}$ (PG, $2.5 \mathrm{mM}$ between 22:00-01:00 h) but subsequent euglycemia between 02:00-09:00 h; in S3, after nocturnal $\mathrm{H}$ as in $\mathrm{S} 2$, but hyperglycemia (PG $\sim 14 \mathrm{mM}$ ) between 02:00-09:00 h. In $\mathrm{S} 2$, at $2.2 \mathrm{mM} \mathrm{PG}$, responses of adrenaline and other counterregulatory hormones (CRH), and symptoms (S) decreased by $\sim 15 \%$ vs S1 ( $p<0.05$ ), but cognitive function (CF) deteriorated $\sim 50 \%$ less than in $\mathrm{S} 1(\mathrm{p}<0.05)$. Glycemic thresholds (GT) of CRH, $\mathrm{S}$ and $\mathrm{CF}$ did not change ( $\mathrm{S} 1$ vs S2 $\mathrm{p}=\mathrm{NS}$ ). In S3, PHH did not affect neither responses nor GT of $\mathrm{CRH}$ and $\mathrm{S}$. In contrast, $\mathrm{CF}$ deteriorated more than in $\mathrm{S} 2(\mathrm{p}<0.05)$ and was no longer different from $\mathrm{S} 1$ ( $\mathrm{p}=\mathrm{NS}$ ). In conclusion, the effects of recent, antecedent $\mathrm{H}$ on $\mathrm{CRH}$ and $\mathrm{S}$ responses to $\mathrm{H}$ are not affected by short-term $(\sim 7 \mathrm{~h})$ hyperglycemia occurring between the two $\mathrm{H}$ episodes. However, $\sim 7 \mathrm{~h}$ of PHH selectively affects CF. These findings may be relevant to IDDM patients who exibit interval hyperglycemia between two close $\mathrm{H}$ episodes.

\section{4}

THIS ABSTRACT HAS BEEN WITHDRAWN BY THE AUTHOR. 


\section{OP 44}

\section{Experimental Macrovascular Disease}

\section{5}

REDUCED VENOUS COMPLIANCE, BUT NORMAL ADRENOCEPTOR ACTIVITY, IN PATIENTS WITH NON INSULIN DEPENDENT DIABETES MELLITUS (NIDDM) WITH MICROALBUMINURIA

Zinkweg Jl, Huvers FC, de Haan CHA, Wolffenbuttel BHR, Schaper NC; Dept. of Int. Medicine, University Hospital Maastricht and Zuiderziekenhuis Rotterdam. The Netherlands

Introduction: both loss of visco-elastic properties and increased (nor)adrenergic contrictor responses of the vascular wall could contribute to the development of hypertension in NIDDM with microalbuminuria $(\mathrm{M}+$ ). Therefore, the constrictor responses of the dorsal hand vein to a local stepped infusion of noradrenalin (NA, $0.3-160 \mathrm{ng} / \mathrm{min}$ ) was determined with the LVDT technique in normotensive $\mathrm{M}+$ and M- NIDDM patients and in healthy controls (C). Furthermore, forearm venous compliance (Vcomp) was determined by strain gauge plethysmography. Patients: 10 (7M/3F) M + , age (mean \pm SD) $59 \pm 6$, RR $136 \pm 15 / 79 \pm 12 ; \underline{12}$ (8M/4F) M-, age $59 \pm 5$, RR $122 \pm 12 / 83 \pm 5 ; \underline{12} \mathrm{C}(8 \mathrm{M} / 4 \mathrm{~F})$, age $57 \pm 7, \underline{R R}$ $125 \pm 15 / 82 \pm 7$. In each patient the maximal contrictor effect of NA (Emax) and the log dose inducing $50 \%$ of this effect (ED50) was calculated. Results: Vcomp (median, interq.in $\mathrm{ml} / 100 \mathrm{ml} / \mathrm{mmHg}$ ) was reduced in the $\mathrm{M}+$ patients in comparison to the C subjects: $0.044(0.037 / 0.050)$ vs $0.058(0.046 / 0.063), \mathrm{p}<0.05 ; \mathrm{M}$ patients were not different from $C$ subjects: $0.050(0.038 / 0.054)$. The ED50 of NA (mean $\pm \mathrm{SD}$ in $\log \mathrm{ng} / \mathrm{min}$ ) was not different between the groups; $\mathrm{M}+: 0.83$ $\pm 0.38 ; \mathrm{M}-: 0.92 \pm 0.52 ; \mathrm{C}: 0.86 \pm 0.45$. Emax was not different between the groups. Conclusions: Peripheral venous compliance is reduced in M+ NIDDM patients. This reduction could, by increasing cardiac pre-load, contribute to the development of hypertension. Venous adrenoceptor activity is undisturbed in both M- and M+ NIDDM patients. In IDDM the reverse was observed in earlier studies ( $M+$; unchanged Vcomp and increased adrenoceptor activity), suggesting differences in the pathobiology of the vascular wall in microalbuminuric NIDDM and IDDM patients.

\section{6}

HYPERINSULINEMIA AND FIBRINOLYSIS IN THE ELDERLY. R.P. Stolk, P.B. Leurs, ${ }^{2}$ B.H.R. Wolffenbuttel ${ }^{2}$ and D.E. Grobbee.' Dept. of Epidemiology \& Biostatistics, Erasmus University Medical School, Rotterdam; and Dept. of Internal Medicine, University Hospital Maastricht, The Netherlands.

Subjects with a history of cardiovascular disease have increased glucose and insulin levels, while hyperinsulinemia, in contrast to hyperglycemia, seems not a risk factor for atherosclerosis. A possible explanation for this discrepancy is that insulin is related to fibrinolysis. Therefore we studied this association in the Rotterdam Study, a population-based prospective cohort study in the elderly. The population consisted of a random sample of 144 men and women (mean age 67.3, SD 6.2), not using anticoagulants, stratified by glucose intolerance at follow-up. Glucose metabolism was assessed at baseline using a non-fasting OGTT; insulin was measured in the post-load samples only. After 2.5 years (range $1.3-5.1$ ) a second, fasting, OGTT was performed; insulin was measured in both fasting and post-load samples. At follow-up fibrinolytic activity was assessed by activity of plasminogen activator inhibitor-1 (PAl-1) and antigen levels of tissue plasminogen activator (TPA), which includes PAl-1/tPA complexes.

In subjects with NGT $(n=50)$, IGT $(n=48)$, and DM $(n=46)$ the level of PAl-1 was 14.0 (SD 8.3), $22.1(12.1)$, and 20.3 (12.6). For tPA these values were $10.7(3.5), 14.0(4.2)$, and $15.4(5.8)$. Both PAl-1 and IPA (ng/ml) were significantly associated with fasting and post-load insulin levels (per $10 \mathrm{mU} / \mathrm{l}$ ): ageadjusted coefficients of linear regression were 5.43 (SE 0.86), p<0.001) and $2.75(0.38, p<0.001)$ for the fasting insulin level, respectively, whereas 0.13 $(0.05, p=0.01)$ and $0.09(0.02, p<0.001)$ were found for the post-load insulin level. These associations did not change after further adjustment for serum glucose, HbA1c, body mass index, systolic blood pressure, and degree of atherosclerosis, or after excluding subjects with diabetes mellitus. Higher postload insulin levels at baseline were associated with increased PAl- 1 and PA levels at follow-up: $0.50(0.15, p=0.001)$ for PAI-1, and $0.33(0.06, p<0.001)$ for $\mathrm{PAA}$. These associations remained also the same after further adjustment.

These results suggest that higher insulin levels are associated with impaired fibrinolysis, in elderly subjects with and without diabetes mellitus.

\section{7}

EPINEPHRINE INDUCES VON WILLEBRAND FACTOR RELEASE FROM CULTURED ENDOTHELIAL CELLS: ROLE OF CAMP IN EXOCYTOSIS.

UM Vischer and CB Wollheim, Division de Biochimie Clinique, CMU, I rue Michel Servet, 1211 GENEVA 4, Switzerland.

Plasma von Willebrand factor (vWf) levels are increasingly recognized as a risk factor for cardio-vascular disease. This adhesive glycoprotein is released from endothelial cell (EC) storage granules after stimulation with thrombin, histamine and other agents, an effect mediated by an increase in cytosolic free calcium $\left(\left[\mathrm{Ca}^{2+}\right]_{i}\right)$. In vivo epinephrine and the vasopressin analog DDAVP increase vWf plasma levels, although they are thought not to induce vWf release from cultured EC. Since these agents act via a cAMP-dependent pathway in responsive cells, we examined the role of cAMP in endothelial secretion. vWf release from cultured EC increased in response to forskolin ( $3 \mu \mathrm{M}$ or higher), which activates adenylate cyclase (AC) (from $100 \pm 27$ to $149 \pm 9 \%, n=6, p<0.05$ ). The response to forskolin was much stronger when cAMP degradation was blocked with IBMX $(100 \mu \mathrm{M})$, an inhibitor of phosphodiesterases $(298 \pm 19 \%, \mathrm{p}<0.01)$, whereas IBMX alone had no effect. vWf release could also be induced by the cAMP analogs dibutyryl-cAMP $(142 \pm 14 \%, p<0.01)$ and 8-bromo-cAMP $(125+10 \%, p<0.05)$; although their effect was weak, they clearly potentiated the response to thrombin. Epinephrine (together with IBMX) caused a small, dose-dependent increase in vWf release, maximal at $10^{-6} \mathrm{M}(149 \pm 11 \%, \mathrm{p}=0.001, \mathrm{n}=15)$, and also potentiated the response to thrombin. This effect is mediated by AC-coupled $\beta$-adrenergic receptors, since it is inhibited by propranolol and mimicked by isoproterenol. In contrast to thrombin, neither forskolin nor epinephrine caused an increase in $\left[\mathrm{Ca}^{2+}\right]_{i}$ as measured with the calcium indicator fura- 2 . Our data suggest that cAMP-dependent signalling events are involved in the control of exocytosis from endothelial cells. The direct stimulation of vWf release by epinephrine and other cAMP-raising agents may be implicated in the increases in rWf plasma levels in atherosclerosis and diabetic nephropathy.

\section{8}

INFLUENCE OF RHEOLOGICAL DISORDERS ON CARDIAC HYPERTROPHY IN DIABETIC RATS.

D. Mesangeau, J. Pariès, F. Zkhiri, M. Garnier, P. Valensi. Nutrition Laboratory, Paris-Nord University, Bondy and LIPHA Institute, Chilly-Mazarin. France.

In diabetic patients, left ventricle hypertrophy is not always due to hypertension. We have investigated the onset of an eventual cardiac hypertrophy in the Goto-Kakisaki $(G K)$ rats (a model of non-obese genetically-determined diabetes) and the influence of blood pressure (BP) and red blood cells (RBC) rheological disorders. BP was monitored by a telemetric method after implantation of an aortic catheter from 4 to 15 months. The filtration index of RBC (FI) was measured on Hanss hemorheometer and RBC aggregation (MEA) on the Myrenne aggregometer. After sacrifice of the animals, at $3, \cdot 6, .12$ or 18 months, heart was weighed and biochemical parameters were measured. In the GK rats BP was very similar as in the control rats (Wistars), FI was significantly higher at 6, 12 and 18 months, MEA and RBC sodium and magnesium were also significantly higher, FI correlated significantly with plasma cholesterol, triglycerides and phospholipids and negatively with RBC magnesium $(\mathrm{p}=0.002)$. Cardiac weight was significantly higher at $3,6,12$ and 18 months and correlated significantly with FI $(\mathrm{p}=0.044)$ and MEA $(\mathrm{p}=$ 0.005 ). Heart proteins, triglycerides, $\mathrm{Na}, \mathrm{K}, \mathrm{Ca}$ and $\mathrm{Mg}$ concentrations were significantly lower than in the controls. In these diabetic rats, this study shows that 1) cardiac hypertrophy occurs as an early complication of diabetes, independent of hypertension, 2) it seems to consist mainly of fibrosis and to be strongly influenced by rheological disorders, 3) plasma lipids may contribute to these disorders, RBC magnesium may have a protective effect. 


\section{Insulin Resistance and Cardiovascular Risk}

\section{9}

HYPERINSULINEMIA DOES NOT EXPLAIN THE HIGH RISK OF CARDIOVASCULAR DISEASE (CVD) IN MAURITIUS J. Tuomilehto, A. Tamosiunas, G. Dowse, H. Gareeboo, D. Fareed, KGMM Alberti and P. Zimmet on behalf of the Mauritius NCD Group. Department of Epidemiology and Health Promotion, National Public Health Institute, Helsinki, Finland.

It has been proposed that hyperinsulinaemia may explain the excess coronary heart disease (CHD) in Asian Indians. The evidence is, however, indirect and based on cross-sectional data. Mortality from CHD and the prevalence of NIDDM are high in Mauritians where most of the people are of Asian Indian origin. We followed prospectively a cohort of 2116 randomly chosen subjects free of CHD at baseline. The baseline examination comprised a large number of CHD risk factors, serum insulin and resting ECG. During a 5-year follow-up fatal and non-fatal CVD events were registered and the survivors were re-examined at 5 years. There were 99 deaths ( 45 of CVD) and 441 new CHD events (54 "hard" CHD). In univariate analysis abnormal glucose tolerance and high fasting blood glucose (FBG) were significant predictors of all these endpoints. Neither fasting nor 2-hr post-load serum insulin were associated with these endpoints. Of total and CVD deaths $36 \%$ and $34 \%$ in men and $26 \%$ and $48 \%$ in women, respectively, occurred in diabetic subjects. In multivariate Cox regression analysis FBG or diabetes remained significant predictors. High serum creatinine was related to all-cause mortality. In conclusion, there was no evidence that hyperinsulinaemia is a risk factor for CHD incidence or CVD mortality in Mauritius, whereas hyperglycaemia and abnormal glucose tolerance increase the risk
250

PLASMA CONCENTRATIONS OF AMYLIN REFLECT INSULIN SENSITIVITY IN RELATIVES OF PATIENTS WITH NIDDM AND IN HEALTHY SUBIECTS

J.E. Koda', B. Nybolm', M.S. Fineman!, K.Y. Hove ${ }^{2}$ and O. Schmitz'. 'Amylin Pharmaceuticals, Inc., San Diego, CA, USA and 'Medical Department M (Endocrinology and Diabetes), University Hospital of Aarhus, Aarhus, Denmark

Amylin, cosecreted with insulin from the pancreatic beta cell, circulates at concentrations below $100 \mathrm{pM}$ in humans. Animal studies have suggested that anylin concentrations above $4 n M$ can generate insulin resistance; however treatment of subjects with IDDM with an amylin agonist failed to affect insulin sensitivity at plasma levels of $350 \mathrm{pM}$. To explore the relationship between physiological amylin concentrations and insulin resistance, we measured plasma amylin and insulin sensitivity in insulin resistant healthy first degree relatives of NIDDM patients $(R)$ and a matched healthy control group (C). To do so, we used two newly developed research assays that measure either amylin or amylin plus glycosylated amylin (total amylin). $40 \mathrm{R}$ and $35 \mathrm{C}$ were examined by an oral glucose tolerance test (OGTT) and by a hyperinsulinaemic (insulin infusion rate $0.6 \mathrm{mU} / \mathrm{kg} / \mathrm{min}$ ) euglycaemic clamp combined with tracer dilution technique ( $\left(3-{ }^{3} \mathrm{Hglucose}\right)$. All subjects had a normal OGTT, but insulin stimulated glucose uptake (Rd) was decreased in R compared to $\mathrm{C}(4.55 \pm 0.24$ vs. $5.83 \pm 0.24 \mathrm{mg} / \mathrm{kg} / \mathrm{min}, \mathrm{p}<0.001)$. AUCs for plasma giucose $(816.1 \pm 19.9$ vs. $732.0 \pm 23.0 \mathrm{mM} \cdot 120 \mathrm{~min}, \mathrm{p}<0.01)$ and serum insulin $(31,347 \pm 2,597$ vs, $25,372 \pm 1,829$ pM. 120 min, $0.05<\mathrm{p}<0.10$ ) during the OGTT were higher in $R$ compared to C. Although the concentrations of plasma total amylin and plasma amylin tended to be higher in $\mathrm{R}$ than in C (AUC: total amylin: $2415 \pm 281$ vs. $2025 \pm 173$ pM 120 min; amylin $1108 \pm 150$ vs. $903 \pm 121 \mathrm{pM} \cdot 120 \mathrm{~min}$ ) the difference did not reach statistical significance. However, in both groups an inverse correlation was found between AUC for plasma total amylin and Rd (R: $\mathrm{r}=-0.49, \mathrm{p}<0.01 ; \mathrm{C}: \mathrm{r}=-0.42, \mathrm{p}<0.05)$ as well as plasma amylin and $\mathrm{Rd}(\mathrm{R}: \mathrm{r}=-0.35, \mathrm{p}<0.05$; $\mathrm{C}: \mathrm{r}=-0.41, \mathrm{p}<0.05$ ). Furthermore, a correlation was observed between anylin and insulin in all subjects $(r=0.54, p<.001)$. In conclusion, employing newly developed immunoassays, this study demonstrated an inverse correlation between $\mathrm{Rd}$ and the circulating concentrations of amylin and total amylin in both $\mathrm{R}$ and $\mathrm{C}$, following an OGTT

\section{1}

INSULIN RESISTANCE IS A RISK FACTOR FOR IGT, NIDDM, HYPERTENSION AND CHD IN A PROSPECTIVE POPULATION STUDY. I. Zavaroni, L. Bonini, A. Zuccarelli, P. Gasparini, A.L. Barilli, E. Dall'Aglio and G.M. Reaven Clinica Medica Generale, Parma University, Italy and Stanford University, USA

Insulin Resistance Syndrome (Syndrome $X$ ) is a condition characterized by multiple abnormalities including abnormal glucose tolerance, dislipidernia, increased arterial blood pressure, associated with insulin resistance and compensatory hyperinsulinemia. It has been hypothesized that this condition might predispose individuals to an increased risk of cardiovascular disease. To test this hypothesis a prospective analysis of a population of 720 healthy volunteers, factory workers, has been performed. They were divided into 4 quartiles on the basis of their levels of insulin area after OGTT at baseline . Insulin area was considered as an index of insulin resistance. A comparison has been made between the subjects who completed the follow up after 11 years belonging to the 1 st and the 4 th quartile, respectively normoinsulinemic (117 subj.) and hyperinsulinemic(118 subj). The hyperinsulinemic group, at baseline, differed for age $(39.7 \pm 0.9$ vs $43.3 \pm 0.9 \mathrm{p}<0.05$ ), gender ( $53 \mathrm{M} / 64 \mathrm{~F}$ vs $86 \mathrm{M} / 32 \mathrm{~F}$ ) and $\mathrm{BMI}$ ( 25.03 vs 26.9 $p<0.001$ ). After adjusting for age, gender and BMI they showed higher levels of plasma glucose $(p<0.0001)$, TG $(p<0.001)$, Uric acid $p<0.001)$, lower levels of $\mathrm{HDL}$ cholesterol $(p<0.002)$ and higher values of systolic $p<0.05$ ) and diastolic $(p<0.001)$ blood pressure. After 11 years all these abnormalities persisted in the hyperinsulinemic group. Furthermore they presented a higher incidence of NIDDM (0 vs $4.24 \%$ p $<0.05$ ), IGT ( 2.27 vs $10.27 \% \quad p<0.01$ ). Hypertension (7.69 vs $24.58 \% p<0.001)$ and $\mathrm{CHD}(0$ vs $5.08 \% p<0.05$ ). The multiple logistic regression analysis indicated that insulin area, as a quartile index, significantly predicted NIDDM, IGT, hypertension and CHD, independently of age, gender and BMI. This study gives longitudinal evidence of the insulin resistance syndrome and document that in this population insulin resistance is a risk factor of impaired glucose tolerance, diabetes, hypertension and coronary heart disease.

\section{2}

CLUSTERING OF INSULIN RESISTANCE, DYSLIPIDEMIA, AND HYPERTENSION AND THE RISK OF ACUTE MYOCARDIAL INFARCTION IN MEN

H-M Lakka, T.A. Lakka, J. Tuomilehto and J.T. Salonen, Research Institute of Public Health, University of Kuopio, Kuopio, Finland Hyperinsulinemia, hyperglycemia, a decreased serum high density lipoprotein (HDL) cholesterol concentration, hypertriglyseridemia, and hypertension each have been found to increase the risk of coronary heart disease (CHD). We investigated the association of the cluster of insulin resistance (serum insulin of $\geq 19.0 \mathrm{mmol} / \mathrm{l}$, blood glucose of $\geq 6.7 \mathrm{mmol} / \mathrm{l}$, or oral medication for diabetes), dyslipidemia (serum HDL cholesterol of $<1.0 \mathrm{mmol} / \mathrm{l}$ or serum triglycerides of $\geq 2.3 \mathrm{mmol} /$ ), and hypertension (systolic blood pressure of $\geq 160 \mathrm{mmHg}$, diastolic blood pressure of $\geq 95 \mathrm{~mm} \mathrm{Hg}$, or antihypertensive medication) to the risk of acute myocardial infarction (AMI) in 2,537 randomly selected men in Eastern Finland aged 42-60 years. The relative risks of AMI in the men with one, two or three of insulin resistance, dyslipidemia, and hypertension, those who had none of these conditions as a reference group, were 2.1 (95\% Cl 1.5 $3.0, \mathrm{p}<0.001), 2.9(95 \% \mathrm{CI} 1.9-4.2, \mathrm{p}<0.001$ ), and 3.6 ( $95 \% \mathrm{Cl} 2.0-6.3, \mathrm{p}<$ 0.001 ), respectively, after adjustment for age, the year of examination, history of CHD, family history of CHD, smoking, and serum total cholesterol. The respective relative risks for the cluster of traditional coronary risk factors (current smoking, serum total cholesterol of $\geq 6.5 \mathrm{mmol} / \mathrm{l}$, or hypertension) were $1.8(95 \% \mathrm{Cl} 1.1-2.8, \mathrm{p}=0.02), 3.2(95 \% \mathrm{CI} 2.0-5.1, \mathrm{p}<0.001)$, and 3.5 $(95 \% \mathrm{Cl} 2.0-6.7, \mathrm{p}<0.001)$ when adjusting for insulin-resistance-associated coronary risk factors. The cluster of insulin resistance, dyslipidemia, and hypertension had an equally strong independent association with the risk of AMI as the combination of traditional coronary risk factors. We suggest that the measurement of fasting blood glucose, serum HDL cholesterol, and serum triglycerides, in addition to serum total cholesterol and blood pressure should be included as routine measures to evaluate the total coronary risk of a person. 
OP 46

\section{Insulin Sensitizers}

253

EFFECTS OF PIOGLITAZONE ON ADIPOCYTE-SPECIFIC GENE EXPRESSION OF ZUCKER ( $f$ a/fa) RATS.

L.Doare $^{1}$, S.Hallakou ${ }^{1,2}$,M.Kergoat ${ }^{1}$; F.Foufelle ${ }^{2}$, J.Morin ${ }^{2}$, M.Guerre-Millo ${ }^{3}$,P.Ferré ${ }^{2}{ }^{1}$ LIPHA Laboratories,91380, ChillyMazarin. ${ }^{2}$ U342,INSERM, 75014,Paris. ${ }^{3}$ U177,INSERM, 75005, Paris.

Pioglitazone (PIO) is a thiazolidinedione which lowers hyperglycemia by enhancing peripheral insulin sensitivity. However, a stimulant effect on adipocyte differenciation is described in vitro for these drugs. Recently, it had been reported that thiazolidinediones was ligands of a nuclear factor, PPAR $\gamma$, that induced adipocyte differentiation. We have investigated, in vivo, the effects of PIO in the cbese Zucker rats (fa/fa) characterized by a strong insulin resistance. Four week-treatment with PIO $(10 \mathrm{mg} / \mathrm{kg})$ markedly improved insulin sensitivity and induced a 4 fold increase of glucose uptake in deep or subcutaneous adipose tissue (IVGTT and glucose utilization measured by injection of $2 \mathrm{DG}^{14} \mathrm{C}$ ). By northern blot analysis, we have also observed a 4 to 6 fold overexpression of the adipocyte genes involved in glucose utilization: glucose transporter GLUT4 and fatty acid synthase (FAS). A 48h treatment with PIO increased the expression of nuclear factors of adipocyte differentiation, C/EBPo, pOb24 and the genes characteristic of terminal adipose differentation (FAS, GLUT4, PEPCK); by contrast, in primary culture of adipocytes, there is no change, in response to PIO ,of FAS, GLUT4 and C/EBP $\alpha$ mRNA levels whereas PEPCK mRNA level was increased. These effects of pioglitazone on FAS and GLUT4 expression seems to be due to an induction of adipocyte differentiation while the effect on PEPCK expression seems to be linked to a direct gene regulation. In conclusion, PIO improves insulin resistance but also induces adipocyte differenciation and may thus worsen the obesity state.

\section{4}

\section{TROGLITAZONE ACUTELY INHIBITS INSULIN-STIMULATED GLYCOGENESIS IN ISOLATED RAT SKELETAL MUSCLE.}

S.Neschen, C.Noe, M.Roden, C.Vogl, W.Waldhäusl, and C.Fümsinn. Dept.Med.III, Div.Endocrinol.\&Metab., Univ.Vienna, Vienna, Austria. The direct short-term effect of troglitazone (TRO) exposure on glucose handling of isolated rat soleus muscle was investigated in vitro. Exposure of insulin-stimulated muscle obtained from healthy Sprague-Dawley rats to troglitazone resulted in increased rates of glucose transport (cpm ${ }^{3} \mathrm{H}-2$-deoxyglucose/mg/h: control, 536 \pm 32 , vs. $3250 \mathrm{nM}$ TRO, 728 $\pm 52 ; \mathrm{p}<0.05)$ and glycolysis ( $\mu \mathrm{mol}$ lactate released/g/h: control, $12.1 \pm 0.4$, vs. $3250 \mathrm{nM}$ TRO, $25.6 \pm 1.1 ; p<0.0001)$. Insulin-stimulated rates of glycogen synthesis were distinctly reduced by TRO in normal muscle (umol glucose incorporated into glycogen/g/h: control, 3.65 $\pm 0.24 ; v s .325 \mathrm{nM}$ TRO, 2.81 $\pm 0.21, p<0.005$; and vs. $3250 \mathrm{nM}$ TRO, $2.34 \pm 0.27, \mathrm{p}<0.005$ ), in muscle rendered insulin-resistant by high glucose pretreatment in vitro (control, $2.74 \pm 0.28$; vs. $325 \mathrm{nM}$ TRO, $2.12 \pm 0.30, p<0.05$; and vs. $3250 \mathrm{nM}$ TRO, 1.26 $\pm 0.12, p<0.001)$, and in muscle obtained from insulin resistant obese Zucker rats (control, 2.37 \pm 0.33 ; vs. $325 \mathrm{nM}$ TRO, 1.18 $\pm 0.10, \mathrm{p}<0.05$; and vs. $3250 \mathrm{nM}$ TRO, $0.84 \pm 0.12$, $p<0.005$ ). The results demonstrate a catabolic response of skeletal muscle glucose metabolism to short-term TRO exposure, which must be distinguished from insulin-sensitizing effects of chronic TRO administration. The findings argue against an acute insulin-sensitizing or insulin-mimetic potential of TRO, as it has been hypothesized by others.

\section{5}

Preservation of beta-cell function with Metformin or Troglitazone in the Zucker Diabetic Fatty Rat.

S. Sreenan, J. Sturis, W. Pugh, C.F. Burant and K.S. Polonsky. Pritzker School of Medicine, University of Chicago, Chicago, IL

The aim of this study was to determine if $\beta$-cell function could be preserved in the Zucker Diabetic Fatty (ZDF) rat by treatment with metformin (MF) or troglitazone (TZ). Zucker lean controI (LC), Zucker fatty (ZF) and ZDF rats received either MF, TZ or no treatment from 6 weeks (before diabetes onset) until 12 weeks of age. Fasting glucose, triglyceride (TG), free fatty acid levels (FFA) and incorporation of radiolabelled glucose into glycogen in soleus muscles in the presence or absence of insulin were measured. Insulin secretion rates (ISR) by the perfused pancreas in response to an increase in perfusate glucose concentration from 2 to $10 \mathrm{mM}$ were determined. Both drugs prevented hyperglycemia in the ZDF rats (mean \pm SEM fasting glucose $-12.6 \pm 1.4 \mathrm{mM}, 7 \pm 0.3 \mathrm{mM}$ and $5.7 \pm 0.5 \mathrm{mM}$ in untreated, MF and $\mathrm{TZ}$ treated $\mathrm{ZDF}$ rats respectively, $\mathrm{p}<0.05$ ). In $\mathrm{ZDF}$, TGs and FFAs were reduced 55 and $50 \%$ respectively by $M F(p<0.005)$ and 71 and $31 \%$ by $T Z(p<0.05)$. TZ but not MF improved muscle insulin sensitivity in ZDF (mean glucose incorporation into glycogen $=2.35 \pm 0.22 \mathrm{pmol} / \mathrm{mg}$ wet weight $/ \mathrm{min}$ vs $1.97 \pm 0.11$ in untreated $Z D F s, p<0.005)$ and $Z F$ rats. ISR at $2 \mathrm{mM}$ glucose was elevated in untreated $Z F$ and $Z D F$ animals $(2.3 \pm 0.8$ and $1.8 \pm 0.2$ compared to $0.02 \pm 0.003 \mathrm{pmol} / \mathrm{min}$ in LC, $\mathrm{p}<0.01$ ). The 2 to $10 \mathrm{mM}$ increase in glucose, stimulated substantial increases in ISR in untreated LC and ZF( $>4$ fold), but less than a 2 -fold rise in ZDF animals. Basal ISR (at $2 \mathrm{mM}$ ) was reduced to 2 and $6 \%$ of that in untreated rats by MF and 2 and $15 \%$ by TZ in ZF and ZDF rats respectively $(p<0.04)$. Improved responsiveness to glucose stimulation was observed (a 22 fold (MF) and 10 fold (TZ) increase in ISR between 2 and $10 \mathrm{mM}$ glucose) in ZDF rats. In summary, MF and $\mathrm{TZ}$ prevent onset of hyperglycemia, improve the lipid profile, significantly reduce basal insulin secretion and enhance glucose stimulated insulin secretion in the ZDF rat. Only TZ improves muscle insulin sensitivity. In conclusion preservation of $\beta$-cell function contributes to the reduction in glycemia with $\mathrm{MF}$ or TZ treatment in the ZDF rat.

\section{6}

Troglitazone but not gLIBEnClamide Reduces PROINSUlin-like MOLECULES, RISK FACTORS FOR CARDIOVASCULAR DISEASE, IN NIDDM PATIENTS E.A. Foot*, Z.V. Williams*, V. Mohamed-Ali ${ }^{+}$, J.S. Yudkin ${ }^{+}$and D.J.A. Eckland* *GlaxoWellcome Research \& Development Ltd., Greenford Road, Greenford, Middlesex. UB6 OHE and ${ }^{+}$Department of Medicine, University College, London Medical School, Whittington Hospital, London. UK.

Increasing evidence links raised proinsulin levels with a greater risk of cardiovascular disease and to factors predisposing to atherogenesis. Sulphonylureas have been shown to increase both insulin and proinsulin levels. In contrast, troglitazone, an insulin action enhancer under development for the treatment of NIDDM, has been shown reduce both fasting and post-prandial insulin in NIDDM . patients, in addition to improving glycaemic control. In this double-blind, parallelgroup, placebo-controlled trial we showed that intact and des 31,32 proinsulin levels are also decreased. Twenty-five NIDDM patients (mean age 61 years; range 48-69) on stable glibenclamide therapy (fixed dose during and for at least 4 months prior to study), with two fasting blood glucose values $\geq 7$ and $\leq 12 \mathrm{mmol} / 1$ and $\mathrm{HbA} 1 \mathrm{C} \leq 10 \%$, were randomised to additional therapy with troglitazone $600 \mathrm{mg}$ od or placebo for 6 weeks. Fasting and 24 hour blood samples were taken at the beginning and end of the study. Insulin, intact and des 31,32 proinsulin were measured by a two-site immunometric assay. Analysis was performed on $\log$ transformed data using analysis of covariance allowing for effects due to baseline (covariate) and treatment. Results are presented as adjusted geometric mean at week $6(\%$ reduction compared to placebo):

Fasting des 31,32 proinsulin (pmol/1) Fasting intact proinsulin (pmol/) Fasting derived specifïc insulin (pmol/l)

Fasting total proinsulin/insulin ratio

24 des 31,32 proinsulin (weighted mean) 13.5

$24 \mathrm{~h}$ intact proinsulin (weighted mean) $\quad 16.9$

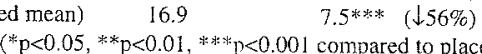

\begin{tabular}{ll} 
Troghitazone \\
\hline $3.4^{*}$ & $(\downarrow 56 \%)$ \\
$3.4^{* * *}$ & $(\downarrow 54 \%)$ \\
66.7 & $(\downarrow 34 \%)$ \\
$0.115^{*}$ & $(\downarrow 33 \%)$ \\
$8.8^{* * *}$ & $(\downarrow 35 \%)$ \\
$7.5 \% * *$ & $(\downarrow 56 \%)$
\end{tabular}
$\left({ }^{*} \mathrm{p}<0.05, * * \mathrm{p}<0.01,{ }^{*} * * \mathrm{k} p<0.001\right.$ compared to placebo)
In conclusion, in contrast to glibenclamide therapy alone, the addition of troglitazone reduced both fasting and total 24 hour exposure to proinsulin like molecules. This may be indicative of troglitazone's potential to reduce the risk of cardiovascular disease in troglitazone treated NIDDM pateints. 


\section{Clinical Aspects of Glycation}

257

IMPAIRED CLEARANCE OF TOXIC FOOD-DERIVED ADVANCED GLYCATION ENDPRODUCTS AS A RISK FOR DIABETIC NEPHROPATHY. T.Koschinsky*, C.He, T.Mitsuhashi*, C. Liu. C. Buenting, K.Heitmann, and H. Vlassara*, Düsseldorf, Germany and Manhasset, NY

Advanced glycation endproducts (AGEs) include chemically and biologically active species, shown to contribute to diabetic complications. The infusion of exogenous AGEs to normal animals can cause vascular and renal pathology. Chemically crosslinking AGEs are found in cooked foods and once absorbed, remain active in the circulation, posing an added risk for toxicity. We have now compared the oral uptake, and renal cleanrance of dietary AGEs in 22 diabetic (DB) patients without renal disease (RD), with micro-, or macroalbuminuria, after a single meal consisting of egg white ( $45 \mathrm{~g}$ protein) cooked at $90^{\circ} \mathrm{C}$ for 3 hrs with fructose $(90 \mathrm{~g})$ (AGE-diet) or without fructose (CL-diet). Diet, serum and urine AGE content was quantitated by ELISA. Oral uptake correlated significantly with serum AGE levels $(r=0.8)$. The area under the curve (AUC) in serum (AGE U/ml.hr) correlated inversely with urinary AUC ( $r=-$ $0.6, p<0.05)$. Compared to non-DB individuals $(n=4), D B$ patients with early or late RD exhibited significantly decreased as well as delayed (by ca. $10 \mathrm{hrs}$ ) urinary AGE excretion after oral AGE uptake. Compared to Non-DB and DB without RD who excreted $30 \%$ of orally absorbed AGEs, DB with overt nephropathy (creatinine clearance $20-40 \mathrm{mg} / \mathrm{dl}$ ) excreted $<5 \%$ of that in the urine, suggesting markedly prolonged half life of food-derived AGEs in these patients. Orally absorbed AGEs from serum or urine exhibited marked crosslinking activity upon exposure to native serum ${ }^{125} \mathrm{I}$-albumin, forming high $\mathrm{mw}$ complexes, as evidenced by SDS-PAGE, suggesting these were chemically active after absorption.

Summary: (1) Renal clearance kinetics of food AGEs are markedly impaired in diabetics, even those with early renal disease, prolonging their half life in circulation and tissues. (2) Diet-AGEs include reactive species, posing diabetic patients, especially those with early renal disease at increased risk for AGEtoxicity. Restricted dietary intake of AGE-rich foods may delay onset and progression of diabetic nephropatby.

\section{9}

AMINOGUANIDINE ATTENUATES MESENTERIC VASCULAR HYPERTROPHY AND TGF-B1 mRNA IN DIABETIC RATS, J.R.Rumble, M.E.Cooper, T.Soulis, A.Cox, M.Jasik, G.Jerums and R.E.Gilbert. University of Melbourne, Department of Medicine, Austin and Repatriation Medical Centre, Heidelberg, Australia. Extracellular matrix (ECM) expansion and vascular remodelling bave been implicated in the microvascular complications of diabetes. Previous studies in our laboratory have demonstrated mesenteric vascular hypertrophy after 3 weeks of experimental diabetes with evidence of ECM expansion, intimal proliferation and medial hypertrophy. These changes may be secondary to the effects of advanced glycation end products, which have been shown in a non-diabetic model to increase cytokine and growth factor gene expression. The aim of this experiment was to examine the effect of aminoguanidine (AG) on mesenteric vascular hypertrophy and gene expression of the prosclerotic cytokine TGF-B1 and type IV collagen ( $\alpha$ I(IV)) in experimental diabetes. Male Sprague-Dawley (SD) rats were randomised to control (C) and diabetic (D) (STZ-treated) groups (with and without $A G, 1 \mathrm{~g} /$ in drinking water), $n=8-12$ rats per group. Rats were sacrificed at 3 weeks and 8 months after STZ injection. Northern analysis of RNA from mesenteric arteries was performed using probes for TGF-B1, $\alpha 1$ IV collagen and 18SrRNA. Diabetes was associated witb increased mesenteric vascular weight at 3 weeks and 8 months which was attenuated by AG treatment. Diabetes was also associated with increased TGF- $B 1$ and aIIIV) mRNA levels which were reduced by AG treatment. Data are shown as mean \pm SEM.

\begin{tabular}{l|l|l|l|l|l|l}
\hline & & 3 weeks & & & 8 mths & \\
\hline Grp & Mes wt & TGF-BI mRNA & $\alpha 1(\mathrm{IV}) \mathrm{mRNA}$ & Mes wt & TGF-B1 mRNA & $\alpha 1(\mathrm{IV}) \mathrm{mRNA}$ \\
\hline $\mathrm{C}$ & $34 \pm 1$ & $1.0 \pm 0.01$ & $1.0 \pm 0.2$ & $50 \pm 4$ & $1.0 \pm 0.1$ & $1.0 \pm 0.1$ \\
\hline $\mathrm{D}$ & $60 \pm 1 \dagger \dagger$ & $2.1 \pm 0.2 \dagger \dagger$ & $2.3 \pm 0.3 \dagger \dagger$ & $90 \pm 4 \dagger \dagger$ & $1.8 \pm 0.3 \dagger$ & $1.7 \pm 0.3^{*}$ \\
\hline $\mathrm{DA}$ & $40 \pm 1 \dagger \S$ & $1.3 \pm 0.1 \S$ & $1.7 \pm 0.2^{*}$ & $63 \pm 6 \S$ & $0.8 \pm 0.1 \mathrm{9}$ & $1.0 \pm 0.2 \#$ \\
\hline
\end{tabular}

In conclusion, $\mathrm{AG}$ may act to decrease diabetes-associated vascular hypertrophy and ECM expansion by decreasing TGF-B1 gene expression.

\section{8}

ADVANCED GLYCOSYLATION END PRODUCTS (AGE'S) IN SERUM ARE INCREASED IN CHILDREN AND ADOLESCENTS WITH IDDM T.J. Berg ${ }^{1,2}$, K . Dahl-Jørgensen ${ }^{1}$, P.A. Torjesen ${ }^{2}$, R. Bucala ${ }^{3}$, K.F. Hanssen ${ }^{1}$, Aker Diabetes Research Centre ${ }^{1}$ and Hormone Laboratory ${ }^{2}$, Aker University Hospital, Oslo, Norway and Picower Institute for Medical Research ${ }^{3}$, Manhasset, New York

We have developed a sandwich fluoremetric-immunoassay for measuring AGE's in serum utilizing polyclonal antibodies made from rabbit immunized with AGE-RNase. Using this assay we have earlier shown that AGE's in serum predict the progression of morphological pathology in the kidney of diabetic patients with microalbuminuria.

In the present study we aimed to investigate whether the serum levels of AGE's in a cohort of diabetic adolescent patients were different from normal subjects IDDM patients ( $\mathrm{n}-69)$ were compared with healthy non-diabetic control subjects $(n=24)$. Age was $12.7 \pm 4.3$ (mean, \pm SD) years in the diabetic group and $12.5 \pm 2.2$ years in the control group; sex distribution (male/female) was $36 / 33$ and 14/10, respectively; Tanner stage (pubic hair) (I/II-V) was $24 / 42$ vs. $14 / 10$. HbAlc was $8.4 \pm 2.2$ vs. $4.9 \pm 0.7 \%$ and blood glucose $13.1 \pm 6.3$ and $5.1 \pm 1.0 \mathrm{mmol} / \mathrm{l}$, respectively. Diabetes duration was $5.2 \pm 3.7$ years.

The serum levels of AGE's were significantly elevated in the diabetic group when compared to controls $(14 \pm 3.4$ vs. $11.5 \pm 2.8 \mathrm{U} / \mathrm{ml}, \mathrm{p}<0,0005$. A significant correlation $(r=0.25, p<0.04)$ was found between the levels of AGE's and HbA Ic in the diabetic group but not in controls. No significant correlation was found between AGE's in serum and diabetes duration in the diabetic group or serum AGE's and blood glucose or age in either of the groups. There were no differences in levels of AGE's between males and females or between prepubertal or pubertal diabetic patients or controls. We are now investigating the dynamics of serum AGEs in newly diagnosed IDDM children.

We conclude that AGE's are increased in young patients with diabetes already after a short duration of diabetes. Since AGE's are linked to the pathogenesis of vascular complications, this finding might indicate that the pathological processes leading to diabetic late complications start even before puberty.

THE MOLECULAR BASIS OF THE LINK BETWEEN DIABETES MELLITUS AND PANCREATIC CANCER Y. Yamamoto ${ }^{1,2}$, S. Yamagishi ${ }^{1}$, C.C. Hsu ${ }^{1,3}$, K. Kobayashi' ${ }^{2}$, H. Yamamoto 1

${ }^{1}$ Department of Biochemistry and ${ }^{2}$ First Department of Internal Medicine, Kanazawa University School of Medicine, Kanazawa 920, Japan. ${ }^{3}$ Chung Shan Medical and Dental College, Taichung, Taiwan, Republic of China.

Epidemiologic studies have established a frequent association between diabetes mellitus and pancreatic cancer. Although hyperglycemia may account for this association, its molecular mechanism remains to be clarified. In this study, we investigated effects of advanced glycation endproducts (AGE) on the growth of Mia $\mathrm{PaCa}-2$ cells, a human pancreatic cancer cell line. AGE prepared with bovine serum albumin and glucose were found to significantly stimulate $\mathrm{Mia} \mathrm{PaCa}-2$ cell synthesis of DNA as well as viable cell number in a dose-dependent manner. Evidence that platelet-derived growth factor-B (PDGF-B) mainly mediates this growth promotion was obtained; $A G E$ upregulated the level of PDGF-B mRNA, and antibodies against PDGF-BB completely neutralized the AGE-induced DNA synthesis. Moreover, antisense oligodeoxyribonucleotides complementary to mRNA encoding a cell surface receptor for AGE (RAGE) were found to reverse both the AGE-induced DNA synthesis and the upregulation of PDGF-B mRNA. These results thus indicate that the AGE action would seem to be receptor-mediated and that hyperglycemia-deriven AGERAGE interactions could play an active part in the development and progression of pancreatic cancer in diabetic subjects through the mediation by autocrine PDGF-B. 


\section{Islet Peptides and Insulin Secretion}

261

REGULATION OF GABA-RELEASE FROM RAT ISLET B CELLS
A. Smismans, A. De Vos, I. Smolders, F. Schuit, D. Pipeleers

Diabetes Research Centre \& Pharmaceutical Institute, Vrije

Universiteit Brussel, Laarbeeklaan 103, B-1090 Brussels, Belgium.

$\gamma$-Aminobutyric acid (GABA) is known to be produced and secreted by pancreatic islets. The compound may play a role in the local regulation of the endocrine pancreas. In order to investigate the factors which influence GABA release from islet $B$ cells, we measured GABA content in purified rat $B$ cells and their medium following 24 hour culture under different conditions. At $10 \mathrm{mM}$ glucose without glutamine, medium GABA content $\left(34.4 \pm 2.9 \mathrm{pmol} / 10^{3} \mathrm{cell} / 24 \mathrm{~h}\right)$ was 5 -fold higher than in the cells $\left(7.5 \pm 1.2 \mathrm{pmol} / 10^{3}\right.$ cells $)$; similar values were measured in the presence of low $(3 \mathrm{mM})$ or high $(20 \mathrm{mM})$ glucose levels. Addition of glutamine (up to $2 \mathrm{mM}$ ) dose-dependently increased GABA content in medium and cells $(p<0.01)$ without modifying the ratio; this stimulatory effect was slippressed by the presence of $20 \mathrm{mM}$ glucose. Addition of IBMX did not influence, in itself, the GABA values but prevented the suppressive effect of 20 $\mathrm{mM}$ glucose. Regression analysis showed a strong linear correlation between medium and cellular GABA content $(r=0.9, p<0.01)$. The variations in medium GABA content were not related to those in insulin release - which were independent of the glutamine levels and increased with the glucose concentration or the presence of IBMX. In tracer experiments, ${ }^{14} \mathrm{C}$ incorporation into GABA was clearly detected in the presence of $1 \mathrm{mM}\left[\mathrm{U}-{ }^{14} \mathrm{C}\right]$ glutamine, but not with 10 $\mathrm{mM}\left[\mathrm{U}-{ }^{14} \mathrm{C}\right] g$ lucose. These experiments indicate that $\mathrm{GABA}$ release from islet $B$ cells depends on its rate of biosynthesis; production and release of the compound are glutamine-dependent and occur independently of the regulation of insulin release.

\section{3}

C-PEPTIDE STIMULATES $\beta$-CELL $\mathrm{Na}^{+}, \mathrm{K}^{+}$-ATPase ACTIVITY: A NEGATIVE FEEDBACK MECHANISM ON INSULIN SECRETION?

J. Wahren, S. Owada, B-L. Johansson, P-O. Berggren and A. Bertorello

Depts of Clinical Physiology and Molecular Medicine, Karolinska Institute, Karolinska Hospital, Stockholm, Sweden.

Insulin secretion requires $\beta$-cell depolarization and increase in cytoplasmic free $\mathrm{Ca}^{2+}$ concentration. Factors that inhibit these processes would be expected to retard insulin secretion. Since membrane potential is maintained at least in part by activity of the $\mathrm{Na}^{+} \mathrm{K}^{*}$ ATPase, it is possible that compounds that negatively affect secretion also increase $\mathrm{Na}^{+}, \mathrm{K}^{+}$-ATPase activity. Therefore, we have examined whether C-peptide, released in equimolar concentrations with insulin and found to negatively modulate secretion of insulin, may do so by stimulating $\beta$-cell $\mathrm{Na}^{+}, \mathrm{K}^{+}$-ATPase activity. Single microdissected islets from adult normoglycemic ob/ob mice were used, which are made up almost exclusively of $\beta$-cells. $\mathrm{Na}^{+}, \mathrm{K}^{+}-\mathrm{ATP}$ ase activity was determined from the hydrolysis of ${ }^{32} \mathrm{P}-\mathrm{ATP}$ in the presence and absence of ouabain. Rat C-peptide $\left(10 \mathrm{~min}, 37^{\circ} \mathrm{C}\right)$ elicited a concentration dependent stimulation of islet $\mathrm{Na}^{+}, \mathrm{K}^{+}$-ATPase activity $\left(\mathrm{EC}_{\overline{5} \emptyset \approx 1 \mathrm{nM}}\right)$. Equal concentrations of insulin had no effect. $90 \%$ of the C-peptide effect was achieved within $3 \mathrm{~min}$. Human C-peptide elicited $\mathrm{a} \approx 50 \%$ greater stimulation of $\mathrm{Na}^{+}, \mathrm{K}^{+}$-ATPase activity. Randomly scrambled C-peptide had no effect. $\mathrm{Na}^{+}, \mathrm{K}^{+}$-ATPase activity of isolated $\beta$-cell plasma membranes was not stimulated by C-peptide, suggesting that its action is mediated via a defined intracellular signalling system. The inhibitory action of glucose ( $15 \mathrm{mM}, 15 \mathrm{~min})$, on islet $\mathrm{Na}^{+}, \mathrm{K}^{+}$-ATPase activity was abolished by $100 \mathrm{nM}$ of C-peptide. In conclusion, our results indicate that C-peptide, released in response to glucose may provide a physiological negative feedback mechanism on insulin secretion by stimulating $\mathrm{Na}^{+}, \mathrm{K}^{+}$-ATPase activity of the $\beta$-cell.

\section{2}

EFFECTS AND LOCALIZATION OF THE NEUROPEPTIDE PACAP AND PACAP RECEPTOR EXPRESSION IN INSULIN PRODUCING TISSUES K. af Klinteberg ${ }^{3}$, A. Naser Al-Amin ', J. Hannibal ${ }^{3}$, F. Sundler ${ }^{2}$ and B. Ahrén', Depts of ${ }^{1}$ Medicine, Malmö, ${ }^{2}$ Physiology and Neuroscience, Lund University, Sweden, ${ }^{3}$ Clinical Chemistry, Bispebjerg Hospital, Copenhagen, Denmark. We have previously demonstrated that the neuropeptide pituitary adenylate cyclase-activating polypeptide (PACAP) stimulates insulin secretion from the clonal cell line HIT-T15 through an effect involving formation of cyclic AMP. In the present study, we used immunocytochemistry and in situ hybridization to localize the peptide and its receptors in the rat and mouse pancreas, as well as in the two clonal insulin producing cell lines HIT-T15 and RINm5F. We also characterized the insulinotropic effect of PACAP27 and PACAP38 in these tissues. PACAP immunoreactivity was localized to intrapancreatic ganglia and single nerve terminals in both rat and mouse pancreas. The PACAP-fibers were distributed both in the exocrine and the endocrine parenchyma, as well as in ganglia. In contrast, PACAP was not expressed in the islet cells or in HIT-T15 or RINm5F cells. Furthermore, in situ hybridization using probes for the PACAP receptor mRNA revealed a strong signal for both the type I and the PACAPR-3 receptors in both cell lines. PACAP27 and PACAP38 were equipotent at stimulating insulin secretion from both HIT-T15 and RINm $5 \mathrm{~F}$ cells. The lowest stimulatory dose in HTT-T15 cells for both peptides was $1 \mathrm{nM}(\mathrm{P}<0.05)$ whereas in $\mathrm{RINm} 5 \mathrm{~F}$ cells the insulinotropic effect was obtained at $0.1 \mathrm{nM}$ by the two peptides $(p<0.001)$. In mouse islets the dose-response pattern for the insulinotropic effect of the peptides was biphasic with a slight effect already at 0.1-1 pM. A marked effect was then seen at doses above $1 \mathrm{nM}$. To conclude, 1) PACAP is expressed in pancreatic nerves, but not in endocrine cells, 2) both type I and PACAPR-3 receptors are abundant both in HIT-T15 and RINm5F cells, 3) PACAP27 and PACAP38 are equipotent insulinotropic peptides, and 4) a slight stimulatory effect is seen already at low dose-levels $(0.1 \mathrm{pM})$ in islets.

\section{4}

SELECTIVE LOSS OF C-PEPTIDE FROM GRANULES IS THE
CONSEQUENCE OF ITS TRUNCATION AND DEGRADATION.

M. Paoletta, C.B. Verchere, M. Neerman-Arbez, K. Rose, S.E. Kahn and P.A. Halban. U. Geneva, Switzerland and U. Washington, Seattle, USA.

C-peptide and insulin are produced in equimolar amounts by proinsulin conversion. Recent studies using both primary and transformed B-cells have shown a selective loss of C-peptide (CP) relative to insulin from granules resulting in a secreted insulin/CP ratio $>1$. To characterise further such loss, transformed (INS) B-cells were pulse-labeled $\left(20 \mathrm{~min}\right.$; [ $\left.\left.{ }^{3} \mathrm{H}\right] \mathrm{Leu}\right)$ and chased. At set times, secretion was stimulated for $15 \mathrm{~min}$ and labeled $\mathrm{CP}$ and insulin in the media (representing secretory granule content) analysed by HPLC. Such analysis revealed a novel form of granular $\mathrm{CP}$. This product was shown by microsequencing and mass spectrometry to be des-(27-31)CP, a truncated CP molecule lacking its $5 \mathrm{C}$-terminal residues. Granular truncation of $\mathrm{CP}$ was both rapid and extensive, with des-(27$31) \mathrm{CP}$ accounting for $35.2 \pm 8.1$ and $72.0 \pm 4.3 \%$ of total granular labelled $\mathrm{CP}$ [intact plus des-(27-31)] at $20 \mathrm{~min}$ and $6 \mathrm{~h}$ of chase respectively. Intact $\mathrm{CP}$ was also lost from granules due to more extensive degradation. Such degradation was apparent as from $80 \mathrm{~min}$ of chase, and increased with time. At 6 h of chase, labelled insulin/CP in stimulated media (granules) was $9.1 \pm 2$ reflecting the combination of $32.1 \pm 1.8 \%$ truncation and $54.8 \pm 4 \%$ degradation of $C P . C P$ is also lost from granules of primary B-cells due to truncation and degradation, but both processes are more pronounced in transformed cells. Degradation, but not truncation, was completely inhibited by $25 \mathrm{mM} \mathrm{NH}_{4} \mathrm{Cl}$, indicating that the two processes are separate and that the former is dependent upon organelle acidification. In conclusion, $\mathrm{CP}$ is much less stable than insulin in secretory granules (presumably due to crystallisation of the latter). It may be lost from granules due either to limited truncation, leading to secretion of a novel $B$ cell secretory peptide [des-(27-31)CP], or more extensive degradation. 


\section{Genetics of NIDDM}

\section{5}

IDENTIFICATION OF DIFFERENTIALLY EXPRESSED GENES

IN NIDDM BY CDNA DIFFERENTLAL DISPLAY

X Huang, L Koranyi, M Lehtovirta and L Groop. Wallenberg Laboratory, Department of Endocrinology, Malmö General Hospital, Lund University, 20502 Malmö, Sweden

Since skeletal muscle insulin resistance is a key feature of NIDDM we assumed that genes of importance for the development of insulin resistance and NIDDM may show abnormal expression in muscle. The aim of the study was therefore to isolate differentially expressed genes in skeletal muscle of patients with NIDDM by using cDNA. differential display. Total RNA preparations were isolated from muscle biopsies from one NIDDM patient (male, age 45 years, BMI $24.7 \mathrm{~kg} / \mathrm{m}^{3}$ ) and one healthy control subject ( male, age 49 years, BMI $24.5 \mathrm{~kg} / \mathrm{m}^{2}$ ). Sixteen differential candidate clones were identified after screening up to $4000 \mathrm{mRNA}$ species. Partial DNA sequence analysis demonstrated that 8 out of these were distinct genes, including clones $2 \mathrm{~A} 1,2 \mathrm{~A} 2,2 \mathrm{~T} 1,2 \mathrm{~T} 2$, showing $100 \%$ sequence similarity to the mitochondrial gene of NADH dehydrogenase subunit 1; clone $2 \mathrm{~T} 3$ and $2 \mathrm{~T} 4$, with $100 \%$ sequence similarity to the mitochondrial cytochrome $\mathrm{C}$ oxidase subunit 1 gene; clone $2 \mathrm{~A} 3$, with $100 \%$ sequence similarity to the mitochondrial gene of tRNA-Leu; and clone $2 \mathrm{~A} 4$, with $100 \%$ sequence similarity to the mitochondrial DNA D-loop region. The cDNA bands of all these 8 mitochondrial clones were only observed in the cDNA pools of the NIDDM patient, suggesting an elevated mRNA expression of these clones in NIDDM. Taken together, these results suggest that altered expression of mitochondrial genes may be involved in the development of NIDDM. Moreover, cDNA differential display can be used to identify novel genes with altered mRNA expression in NIDDM.

\section{7}

SIB-PAIR LINKAGE AND ASSOCIATION STUDIES OFTHESULFONYLUREA RECEPTOR GENE LOCUS IN NIDDM AND MORBID OBESITY IN FRENCH CAUCASIANS

E. Hani ${ }^{1}$, N. Vionnet ${ }^{1}$, K. Clément ${ }^{1,2}$, G. Velho ${ }^{3}$, J. Hager ${ }^{1}$, M.A. Permutt ${ }^{4}$ and P. Froguel ${ }^{1}$.CNRS EP 10, Institut Pasteur and C.H.R.U, Lille 1; Department de Nutrition, Hôtel-Dieu, Paris ${ }^{2}$, INSERM U-358, Hôpital Saint-Louis, Paris ${ }^{3}$, France; Division of Metabolism, Diabetes and Endocrinology, Saint-Louis, Missouri, USA 4.

A primary pancreatic $\beta$-cell defect may be involved in hyperglycaemia and weight gain. Here we report results of sib-pair linkage studies of the pancreatic sulfonylurea receptor gene locus (SUR) in NIDDM and morbid obesity as well as results of a case-control study of three sequence variants of SUR gene in two populations with non-insulin dependent diabetes mellitus (NIDDM) or extreme obesity. Linkage studies with the microsatellite markers D11S902 and D1 1S92 gave no evidence for linkage of the SUR locus to NIDDM. In contrast, one of the markers studied in the obese pedigree set, D11S419 which is in the vicinity of the SUR gene revealed some evidence for linkage to obesity among the siblings with $\mathrm{BMI} \geq 27 \mathrm{~kg} / \mathrm{m} 2$ ( $\mathrm{p}$-value $=0.007$ ). In the association study, the exon $22 \mathrm{~T}$-allele was more common in patients with NIDDM or morbid obesity than in controls $(\mathrm{p}<0.02)$ This variant was associated with morbid obesity (odds ratio: $2.15, \mathrm{p}=0.04$ ) and with NIDDM in overweight diabetic subjects (odds ratio:2.32, $\mathrm{p}=0.04$; association dependent on the BMI). For the SUR exon 7 variant, there was no significant difference in the genotypic frequencies between the NIDDM or the obese patients compared to the controls. Although the frequencies for intron the 24 variant were similar in all groups, the homozygous morbidly obese patients had a more deleterious form of obesity. We conclude that the SUR locus, may contribute to the genetic susceptibility in some forms of NIDDM and in a stronger way to obesity in the French population.

\section{6}

THE IDDM2 GENE CONFERS AN INCREASED RISK FOR IMPAIRED BETA-CELL FUNCTION IN NIDDM.

T. Tuomi, ÅL. Carlsson, L. Groop and the Botnia Research Group, Diabetes and Endocrine Research Laboratory, Dept. Endocrinology, Malmö University Hospital, Sweden and Helsinki, Finland.

Several chromosomal regions are known to increase susceptibility for IDDM. One of them (FDDM2) is located in the polymorphic promoter region of the insulin gene on chromosome $11 \mathrm{p} 15$. Homozygozity for short alleles of the VNTR (Class I), which are in linkage-disequilibrium with a Hph1-site in the insulin gene, confers an increased risk for IDDM. It is not known whether this IDDM risk genotype is associated with impaired $\beta$-cell function in NIDDM. To test this hypothesis we measured the insulin response to oral glucose tolerance test in 112 Finnish NIDDM patients [71 GADab- (GAD-antibody negative)/41 GADab+]. 58 (40/18) were homozygous for the IDDM-predisposing $\mathrm{Hph} 1$-allele (INS $+/+$ ), 42 (24/17) were heterozygous (INS+/-) and 12 (7/5) homozygous for INS- - as typed by PCR followed by RFLP.

In GADab- patients the INS $\%$ genotype was associated with a marked reduction in the insulin response to OGTT (INS- $/$ vs. INS $+/+\&+/-$ : incremental area under curve $2313 \pm 799$ vs. $6168 \pm 4547 \mathrm{mU} /$; $\mathrm{p}=0.0097$ ). In contrast, in GADab+ patients the presence of neither the INS- - - nor the IDDM susceptibility genotype INS $+/+$ influenced insulin secretion. Frequencies of the 3 genotypes did not differ between the GADab+ or GADab- NIDDM groups and the control group: INSt $/$ : $40 \%, 52 \%, 51 \%$; INS $+1-36 \%, 36 \%, 38 \%$; INS $-1-6 \%, 11 \%, 6 \%$.

We conclude that the IDDM2 gene confers an increased risk for impaired $\beta$-cell function in NIDDM. Therefore, the IDDM2 gene should be considered a susceptibility gene for NIDDM characterized by impaired $\beta$ cell function.

\section{8}

UNCOUPLING PROTEIN POLYMORPHISM AND $\beta 3$ ADRENERGIC RECEPTOR MUTATION IN SWEDISH NIDDM PATIENTS.

M. Carlsson. M. Orho, M. Lehto and L. Groop. Department of Endocrinology, Malmö University Hospital, Malmö, Sweden.

A low metabolic rate (BMR) is a risk factor for weight gain, which in turn increases the risk for NIDDM. Therefore, genes influencing BMR, as the genes encoding the $\beta 3$ adrenergic receptor $(\beta 3 R$ ) and the uncoupling protein (UCP), could be putative candidate genes for obesity-linked NIDDM. To test this hypothesis we studied whether the recently described $\mathrm{A} / \mathrm{G}$ polymorphism in the UCP gene promotor region or Trp64 $\rightarrow$ Arg64 mutation in the $\beta 3 R$, could influence obesity and basal metabolic rate in Swedish subjects from NIDDM families. The polymorphisms were analyzed by PCR-RFLP using restriction enzyme Bcll (UCP) or BSTOI ( $\beta 3 R$ ) followed by agarose gel electrophoresis. UCP gene polymorphism was determined in 399 NIDDM, 49 IGT and 189 nondiabetic subjects. The genotype frequency (GG, GA, AA) did not differ between NIDDM and IGT/rondiabetic subjects (GG=5\%, $\mathrm{GA}=40 \%, \mathrm{AA}=56 \%$ vs $\mathrm{GG}=6 \%, \mathrm{GA}=37 \%, \mathrm{AA}=57 \%$ ), and no differences in sex, age. body mass index (BMI), waisthip ratio (w/h), blood pressure (BP) or lipids were observed herween the different genotype carriers. Basal metabolic rate (BMR) was measured with indirect calorimetry in 128 subjects. No difference in BMR between the carriers of the GG or GA genotypes compared to AA genotype carriers were seen in NIDDM $(29.22 \pm 4.2$ vs $29.4 \pm 4.2 \mathrm{cal} / \mathrm{kgFFM} \mathrm{min})$ or nondiabetic $(28.0+3.2$ vs $28.8+2.9$ ) subjects. In addition 32 NIDDM sibpairs ( 19 females, 13 males), matched for sex and discordant for the UCP allele were studied. No significant differences in BMI (27.8 vs $\left.29.1 \mathrm{~kg} / \mathrm{m}^{2}\right)$, w/h $(0.92$ vs $0.92 \mathrm{~m})$, trigl $(1.71$ vs $1.80 \mathrm{mmol} / \mathrm{l})$ or BP (142 vs $140 \mathrm{mmHg}$ ) were observed between the different genotype carriers. 410 subjects (211 males, 199 females) were tested for $\beta 3 \mathrm{R}$ mutation. 32 individuals ( 4 nonNIDDM, 3 IGT and 25 NIDDM) were heterozygous for the Arg64 allele. Subjects with Arg64 allele had higher triglycerides (2.67 vs $1.87 ; p=0.013)$ and $w / h(0.95$ vs $0.90 ; p=0.03)$, than the Trp64 homozygotes. No Ara64 homozygotes were found. In the present study no association between UCP Bcll polymorphism and NIDDM, BMR or obesity was observed. However the study confirms that presence of the $\beta 3 R$ Arg64 allele is associated with features of the insulin resistance syndrome. 


\section{9}

A NEW MISSENSE MUTATION IN THE GLUCOKINASE GENE IN AN ITALIAN MODY FAMLY Y

^M.Maioli ${ }^{*}$ C. Bertini, ${ }^{*}$ M. Maioli, ^P.Fresu, ^ G. Tonolo, *M.Pirastu,

*Istituto di Ricerea sulle Talassemie e Anemie Mediterranee CNR-Cagliari-Italy

^Servizio di Diabetologia, Istituto di Clinica Medica, University of Sassari, Italy Maturity Onset Diabetes Mellitus of the Young (MODY), a form of rion insulin dependent Diabetes Meilitus (NIDDM), is characterised by early age of onset, autosomal dominant mode of inheritance and high penetrance. Mody is genetically heterogeneous, but in some cases is associated to mutations in the locus of the glucokinase (GCK) gene. We identified nine multigenerational Italian families with early onset type 2 diabetes and a pattern of inheritance consistent with a diagnosis of Maturity Onset Diabetes Mellitus of the Young (MODY). The glucokinase gene was screened in at least one affected subject of each family. The GCK was studied by SSCP (Single Strand Conformation Polymorphism) analysis and direct sequencing of PCR products in one affected member from each family. From these rine Italian families studied so far, we found only two subjects, belonging to different families, with missense mutations in the GCK gene. One, (A188T) found in a Continental Italian family, confirm a mutation previously reported in the Japanese population, which is genetically different from the Caucasian, the other is a new one found in a Sardinian family, which genetic structure is different from the rest of Italy and Europe (T206M). Plasma fasting glucose levels in the affected kindreds of this last family were higher than the mean plus three standard deviations of plasma glucose concentration in the 7 control subjects of our study. One subject alone in this family, although having a mutation in the glucokinase gene, showed high basal plasma insulin levels and the first phase insulin secretory response to an intravenous glucose challenge was biunted. In conclusion our data: 1-confirm in an Italian family the presence of a GCK gene mutation previously found in a population, with a different genetic background indicating the $\mathrm{CpG}$ dinucleotide as a possible "hot spot" for mutation, 2- report for the first time a new missense mutation in the GCK gene in a Mody family belonging to the Sardinian population; 3-suggesis that the prevalence of GCK mutations in Italian MODY families is lower than that previously described in France

\section{0}

IN SEARCH FOR DIABETES SUSCEPTIBILITY GENES: ASSOCIATION OF IDDM FAMILIAR TRAIT WITH RAISED NaHEXCHANGE ACTIVITY.

O. Giampietro, C. Bertoni, F. Ruberti, F. Piazza, M. Casini, E. Boldrini and E. Matteucci. Ist. Clinica Medica II, Università di Pisa, Italy.

To search for recurrent features which distinguish type 1 insulindependent diabetes mellitus (IDDM) and its familiar background, we studied 33 healthy subjects (37 $\pm 12 \mathrm{y}$, range 23-68), 42 type 1 diabetics $(33 \pm 12 \mathrm{y} ; 12$ with incipient nephropathy, 10 with retinopathy) and their 64 relatives (26 siblings, $30 \pm 7 \mathrm{y}$, and 38 parents, $56 \pm 8$ y) by measuring hemato-urinary analytes and erythrocyte $\mathrm{Na}-\mathrm{H}$ antiport. Compared with matched healthy controls, type 1 diabetics had higher glycemia, antiport activity and urinary total protein, albumin and AAP. The rate of $\mathrm{Na}-\mathrm{H}$ exchange did not distinguish nephropathic from non-nephropathic subjects, while the highest activities occurred in the retinopathic ones. Siblings of type 1 diabetics had raised antiport, apart from proband's renal status. Siblings of nephropathic probands $(n=10)$ had also higher serum triglycerides and albuminuria than matched controls. Parents of type 1 diabetics had borderline glycaemia and higher antiport than matched controls. Parents of nephropathic diabetics $(n=12)$ presented hyperactive antiport as well elevated serum total cholesterol and albuminuria. Parents of retinopathics $(n=6)$ showed raised antiport activity, mean blood pressure and proteinuria. Hence, increased antiport rate seems a common feature in IDDM families; in addition, in relatives of nephropathic probands, it clusters with cardiovascular risk factors, such as dyslipidemia and albuminuria. Just as Nhe- 1 is a likely candidate locus associated with disease susceptibility in NOD mice, we suggest that the higher prevalence of hyperactive $\mathrm{Na}-\mathrm{H}$ exchange in diabetic and non diabetic members of families with IDDM, apart from proband's renal diabetic complications, does implicate the antiporter in the development of IDDM itself, rather than of diabetic nephropathy.

\section{1}

INSULIN SECRETION AND SENSITIVITY IN DIABETIC AND NON DIABETIC CARRIERS OF MODY-3 SUSCEPTIBILITY HAPLOTYPES G. Velho, M.E. Pueyo, M. Vaxillaire, K. Clément, P. Froguel and J.-J. Robert. INSERM U358 and U30, Paris and CNRS EP10, Lille, France. A third susceptibility locus to MODY was localised to a 5 centimorgan interval of chromosome 12q. We here report the assessment of insulin secretion (IVGTT, hyperglycemic clamp, arginine test) and insulin sensitivity (euglycemic hyperinsulinemic clamp) in 5 NIDDM and 4 normoglycemic carriers of the diabetes susceptibility haplotype $(\mathrm{M}+)$, and 8 unrelated controls. C-peptide response to an IVGTT was decreased and delayed in M+ NIDDM subjects when compared controls $(0-10 \mathrm{~min}$ area under the curve: $1.13 \pm 1.54$ vs. $13.02 \pm 6.22$ $n M . \min , m \pm S D, p=0.002$; time of $C$-peptide peak: $8: 15 \pm 3: 30$ vs. $1: 15 \pm 0: 43 \mathrm{~min}, p=0.0002$ ). Responses to a $10 \mathrm{mM}$ glucose clamp (average $60-120 \mathrm{~min}$ value: $0.67 \pm 0.22$ vs. $2.70 \pm 0.43 \mathrm{nM} ; \mathrm{p}=0.0001$ ) and intravenous arginine test $(0-10 \mathrm{~min}$ area under the curve: $4.51 \pm 0.62$ vs. $12.49 \pm 4.95 \mathrm{nM}$.min; $p=0.01$ ) were also reduced in $M+$ NIDDM subjects. A between-subject as well as a within-subject heterogeneity was observed in the responses to these tests in the 4 $\mathrm{M}+$ normoglycemic individuals. Two subjects had abnormal responses to the 3 tests (IVGTT and arginine test < mean-1SD of controls, and hyperglycemic clamp < mean-2SD of controls). One subject had an abnormal response to the hyperglycemic clamp only (< mean-2SD of controls), and one subject presented normal range responses to the 3 tests. Evaluations of other $M+$ normoglycemic individuals are currently being performed. Insulin resistance was observed during the euglycemic hyperinsulinemic clamp only in the more severely affected M+ NIDDM subjects. In conclusion, MODY 3 affected individuals present a severe insulin secretory defect in response to both glucose and arginine, that can be detected in normoglycemic carriers of the diabetes susceptibility haplotype. Beta-cell failure is the primary defect associated with this form of diabetes.

\section{2}

\section{SIBPAIR COLLECTION STRATEGIES IN NIDDM}

MI MCCarthy, L Kruglyak and ES Lander. Whitehead Institute for Biomedical Research, Cambridge, MA and Imperial College School of Medicine at St Mary's, London, UK. When planning a sibpair collection to be used in a genome-wide search for NIDDM susceptibility loci, two questions arise: (1) which family types will be most informative for detection of linkage? (2) shrould recruitment extend beyond the index sibpair (to parents \& unaffected sibs)? We expect the optimal pedigree structure and collection strategy to depend on the genetic architecture but the true segregation model for NIDDM is unknown. To assess the consequences of misspecification of the genetic model on the power of altemative collection strategies, we modelled 2 populations (representing ethnic groups with low and high rates of NIDDM). They were defined by their population prevalences $\left(k_{p}\right)$ and sibling recurrence rates $\left(k_{\mathrm{sib}}\right)$ : (a) " $\mathrm{L}$ " $\left(\mathrm{k}_{\mathrm{p}}=0.05, \mathrm{k}_{\mathrm{sib}}=0.25\right),(b) " H$ " $\left(\mathrm{k}_{\mathrm{p}}=0.2, \mathrm{ksb}_{\mathrm{sb}}=0.4\right)$. For each, we derived $\sim 200$ alternative two-locus model solutions: these covered a wide range of values for parameters such as dominance, allele frecuency, phenocopy rate and cene interactions which are unlikeiy to be known accurately. Under each model, we derived the identity-by-descent distribution at the trait locus when sibpairs were ascertained from different pedigree structures and collection strategies. The number of sibpairs needed to obtain a pointwise lod score of 2.6 was taken as a measure of power. We could identify no universal "ideal" pedigree siructure. Densely-affected sibships were more powertul than sparsely-affected when phenocopy rates were high: the reverse held when phenocopias were rare (for " $\mathrm{H}$ ", the power of pairs from sibships with 2 of 5 affected was between 0.25 and 30 times that from sibships with 4 of 5 affected). Collection of "random" sibpairs (ascertained without regard to the status of parents or other sibs) proved surprisingly effective (for "L" the number of sibpairs varied from 0.93 to 1.44 times that needed when specifying a sibship with exactiy 2 of 3 affecteds). Conditioning on parental status had highly variable effects - for "L" the relative power of conditioning on 1 vs 0 affected parents ranged $>400$-fold. In NIDDM, most parents are unavailable and subclinical affection is frequent: fuil enumeration of the affection status of all 1st-degree relatives is costly and slows recruitment. Our study indicates that this effort produces only a modest increment in power when compared with the more convenient approach of ascertaining all sibpairs. 
FAMILIALITY OF QUANTITATIVE METABOLIC TRAITS IN A FINNISH POPULATION.

R.M. Watanabe, E.R. Hauser, T. Valte, J. Eriksson, R.N. Bergman, and M. Boehnke for the Finland-United States Investigation of NIDDM Genetics (FUSION) Study. University of Michigan School of Public Health, Ann Arbor. MI, USA; National Public Health Insitute, Helsinki, Finland; and University of Southern California School of Medicine, Los Angeles, CA, USA.

Non-insulin dependent diabetes mellitus (NIDDM) is a complex disorder encompassing multiple metabolic defects. To date there has not been convincing evidence for linkage between genetic markers along the genome and NIDDM per se or NIDDM-related quantitative traits (QT). As part of the FUSION study, we sought to determine whether individual QTs which determine glucose tolerance exhibit familiality in this Finnish cohort. Tolbutamide-modified frequently-sampled intravenous glucose tolerance tests (FSIGT) were performed in offspring $(n=369)$ and spouses $(n=141)$ of affected sibling pairs identified for the FUSION study. Minimal Model analyses were performed on FSIGT data to obtain quantitative measures of insulin sensitivity $\left(S_{1}\right)$, glucose effectiveness $\left(S_{G}\right)$, and insulin secretion assessed as the acute insulin response to glucose (AIR). The disposition index (DI), a measure of insulin resistance-corrected $\beta$-cell function, was also derived as $\mathrm{S}_{\mathrm{I}} \times \mathrm{AIR}$. For each QT, one-way analysis of variance was performed on values obtained from offspring using families with more than one offspring, adjusting for age, gender, and body mass index. Significant familial aggregation was observed for each trait in offspring of the 126 families tested (See Table). However, significant correlations were also noted among the QTs examined. When analyses were

\begin{tabular}{|c|c|c|c|}
\hline rait & ralue & $\mathrm{p}$-value & \multirow{5}{*}{$\begin{array}{l}\text { repeated further adjusting for the other } 2 \text { traits (e.g., } \\
\mathrm{S}_{\mathrm{G}} \text { adjusting for } \mathrm{S}_{\mathrm{I}} \text { and } \mathrm{AIR} \text { ), evidence for familiality } \\
\text { persisted. We conclude there exists familiality for } \\
\text { Minimal Model derived quantitative traits in the } \\
\text { offspring of our Finnish affected sibling pairs. }\end{array}$} \\
\hline$S_{t}$ & 1.40 & 0.010 & \\
\hline $\mathrm{S}_{1}$ & & & \\
\hline IIR & & & \\
\hline & & & \\
\hline
\end{tabular}

\section{5}

SCREENING FOR MUTATIONS IN THE KIR6.2 SUBUNIT OF THE B-CELL K-ATP CHANNEL

H. Sakura, N. Wat, V. Horton, R. Turner and F.M. Ashcroft. University Laboratory of Physiology, Parks Road, Oxford, OX1 3PT, UK and Diabetes Research Laboratories, Radcliffe Infirmary, Oxford, UK.

The $B$-cell ATP-sensitive K-channel (K-ATP channel) plays a key role in insulin secretion elicited by both glucose and sulphonylureas. This channel consists of two subunits: a sulphonylurea receptor, SUR1, and an inwardly rectifying K-channel subunit, Kir6.2. The aim of this study was to determine whether mutations in the Kir6.2 subunit are associated with NIDDM. We screened $>100$ NIDDM patients and 80 normal individuals for mutations in the Kir6.2 gene by single stranded conformational polymorphism (SSCP) analysis. We identified two silent mutations (A190A, S363S) and five missense mutations (E23K, L270V, I337V, L355P and S385C) in both normal and NIDDM individuals. The E23K and $\mathrm{I} 337 \mathrm{~V}$ variants were completely linked, all individuals possessing $E$ at 23 also having $I$ at 337 , while those having $\mathrm{K}$ at 23 also had $\mathrm{V}$ at 337 . The variant $\mathrm{E} 23 \mathrm{~K} / \mathrm{I} 337 \mathrm{~V}$ was also highly polymorphic: in NIDDM subjects, 56 individuals (41\%) were homozygous for E/I, 17 individuals (13\%) homozygous for $\mathrm{K} / \mathrm{V}$ and 62 individuals ( $46 \%$ ) were heterozygous (EI/KV). In the case of $L 270 \mathrm{~V}, 101$ NIDDM patients were homozygous for $\mathbf{L}, 1$ was homozygous for $\mathrm{V}$, and 11 were heterozygous. Both of these highly polymorphic variants (E23K/1337V, L270V) were found with equal frequency in NIDDM and normal subjects. We conclude that mutations in NIDDM are unlikely to be a major cause of diabetes mellitus.

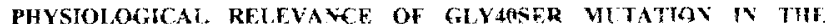
GLUCAGON RECEPTOR GEAF IN THE SARDINLAN POPILATION

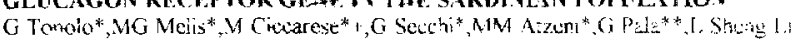

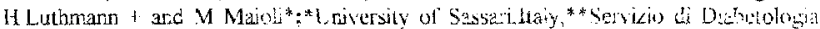

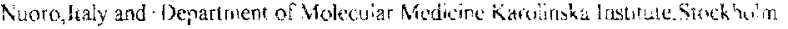
Background $A$ Glytoser mulation $(M)$ in the g'ucagen receptor genc has been

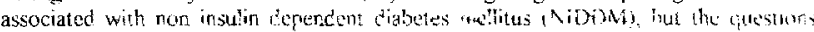
raised about its physio'ogical implizations have no been resolved Aim of this stur'y was to screen the Glyasoser mulation in a large Sardinian population and to investigate the

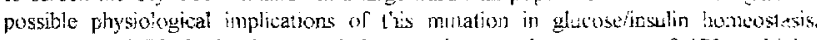
Patients and Methods (Jur poridation consists, al the moment, of 170 nunitiper NIDDM Sardinian farnities (selected on the basis of at least two sitrs havirns VIDDM grandparents burn in Sardinia and homogerencously distributed accruss dit Sardinia) with 4.38 siblings. So far the $\mathrm{V}$ was screeved in 256 siblings and addionally :0 93 non

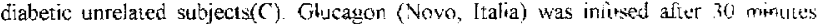
run-in, at 1,3, 9 and $27 \mathrm{ng} / \mathrm{Kg}$ imin (30min each), fo."owed by a recovery ferisd $(60 \mathrm{~min})$, in two groups of 6 unrelated subjects well matched for diabetic state. BMI age,sex etc and differing only for the presence of the mutation (M) or its absence $(V)$ Every $15 \mathrm{~min}$ blood for glycemia (Giv, $\mathrm{mmo} / \mathrm{l}$ ), glucagon (pg/ml), and insuline:ria (IRI, uU/oul) was taken from a contralateral catheter. Results $9 / 256$ (3 5\%\% NWOM sibting and $4 / 93(4.3 \%) \mathrm{C}$ were mutant. Plasrna glucagon increased significantly in a dosedependent manner during the infusion (starting from the lowest dose) without any significant difference between the groups of $6 \mathrm{M}$ and the 6 weth matched $\mathrm{N}$. Mutants had a lower glycemic increase in response to glucagon infusion comparec to $N$ at any time durirg glucagon infusion being signiffeant $(0<0.05)$ at $45,60,75$ and $90 \mathrm{~min}$.

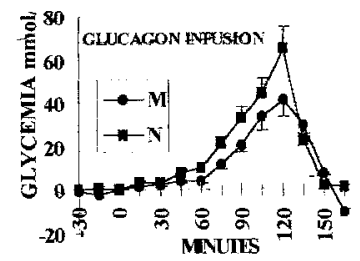
IRI increase during glucagon infusion was not significantly different in our two groups at any time. Conclusion: Our preliminary results indicate that the Gly40Ser mutation may not be invariably associated with NIDDM but in-vivo has an importanit physiological role in the glucose/insulin homeosthasis under glucagon control.
276

ANAIYYSIS OF ACE POLYMORPHISM IN SBB-PAIRS CONCORDANT FOR NIDDM AYD MICROALBUMINURIA

A. Solini*, A. Sfriso, E. Duner, M. Trevisan, S. Inchiostro, A. Buetio, F. Santeusanio ${ }^{\circ}$, P.P. Faronato, M. Maioli^, G.C. Tonolo , G. Crepaldi and R. Nosadini. Universities of Padova, Ferrara*, Perugia ${ }^{\circ}$, Sassari ${ }^{\wedge}$, Italy

Recent case-control studies have observed an association between deletion allele (D) of angiotensin-converting enzyme (ACE) polymorphism and nephropathy in IDDM and NIDDM, while other studies have denied this association. We lave used the sib-pairs model, which permits to evaluate the presence of a linkegedisequilibrium in I'D polymorphism without "a priori" hypothesis on transmission and penetrancy of genes. We studied 286 sib-pairs from North-East of Italy (NE) and Sardinia (S), concordant for NIDDM (age at diagnosis $>40$ years, no insulin therapy in the first three years of the disease). Proband was defined as sib with longer duration of disease. Sib-pairs were either concordant for nornoalbuminuria (188 NIDDM subjects with albumin excretion rate (AER) $<20 \mu \mathrm{g} / \mathrm{min}:$ MA-) or for micro-macroalbuminuria (98 NIDDM subjects with AER $>20 \mu \mathrm{g} / \mathrm{min}: \mathrm{MA}+$ ) ACE genotypes werc determined using the polymerase chain reaction (PCR) technique on DNA isolated from peripheral leukocytes with alternative primers that prevent misclassification of insertion aflele (I). We used the identicy by state (IBS) method of Bishop et al. with $X^{2}$ test to compare the observed allelc frequencies with the expected values. There were not significant differences between expected and observed frequencies of ACE genotype in the two groups (MA-: observed II $10.6 \%$, ID $54.2 \%$, DD $35.1 \%$ vs expected $1114.0 \%$, ID $47.3 \%$, DD $38.7 \%$; MA+: observed II $32.6 \%$, ID $41.8 \%$, DD $25.5 \%$ vs expected II $28.7 \%$, ID $49.7 \%$, DD $21.5 \%$ ). In a case-control approach, we also studied two groups of non diabetic subjects (C) coming from the same areas, observing a difference in II genotype of $C$ from $S$ vs NE \{S ( $=96)$ : II 4\%, ID 57\%, DD 39\%; NE ( $\mathrm{n}=181)$ : II 14\%, ID $39 \%, \mathrm{DD} 47 \%, \mathrm{p}<0.01\}$. We conclude that: i) the presence of allele $\mathrm{D}$ is not associated with micro/macroalbuminuria in sib-pairs with NIDDM; ii) possible differences in I frequency could be accounted for by genctic differences in the two populations. 


\section{7}

A GENOME-WIDE SCREEN FOR NIDDM-SUSCEPTIBILITY GENES IN JAPANESE

N. Iwasaki!, M.Ogatal. S. Karibe', H. Ohgawaral.2, V. Boriraj', L.E. Mereu ${ }^{3}$, N.J.Cox ${ }^{3}$ and Y.Omoril.

Diabetes Center and 2Medical Research Institute. Tokyo Women's Medical College. Tokyo, Japan; and 'Howard Hughes Medical Institute. The University of Chicago, Chicago, USA

Genetic factors contribute to the development of NIDDM. We have begun a genome-wide screen to Iocalize the genes that contribute to the development of this form of diabetes mellitus in Japanese. The study population consisted of 194 affected sibpairs. $(112$ sibships with 2 affected, 16 with 3 affected, 4 with 4 affected. and I with 5). A diagnosis of NIDDM was made using the criteria of WHO. The age at diagnosis was $43.5 \pm 11.7$ years. duration of diabetes was $13.5 \pm 9.2$ years. BMI at the time of study was $23.0 \pm 3.2$, and current treatment was $29 \%$ diet. $40 \%$ oral hypoglycemic agent, $31 \%$ insulin. Subjects have been genotyped with polymerase chain reaction-based microsatellite markers. Evidence tor linkage has been evaluated using two different two-point linkage statistics. a maximum likelihood of odds score (MLOD) computed using the estimated identity-by-descent (IBD) probabilities and a chi-square statstic comparing the observed and expected distribution of alleles identical-bystates (IBS). In addition, multipoint analysis has been used to maximize the information from each chromosome and to provide a bias for exclusion. Of the 94 markers typed to date. DI5S112 showed the best evidence, although nominal ( suggestive and significant evidence for linkage require MLOD values greater than 2.6 and 4.0. respectively). for linkage with NIDDM. Additional markers are being typed on chromosome 15 to determine whether this represents a true linkage and thus describes: the location of a NIDOM-susceptibility genes in Japanese, of is a chancit deviation. The genome-wide screen is continuing in order to localize all the NIDDM-susceptibility loci segregating in this population.

Table 1. Linkage of D15S112 with NIDDM in Japanese affected sibpairs IBS Observed (Expected)

$\begin{array}{cccccc}0 & 1 & 2 & \chi^{2} & \text { MLOD } \\ \mathrm{D} 15 \mathrm{~S} 112 & 3(6) & 51(66) & 10.3(84) & 9.25(2 \mathrm{df})(\mathrm{p}<0.01) & 1.78(\mathrm{p}<0 .(105)\end{array}$ $(\mathrm{PIC}=0.497)$

\section{8}

(hHTg $\mathrm{x}$ BN) $\mathrm{F}_{2}$ HYBRIDS FOR GENETIC DISSECTION OF THE INSULIN RESISTANCE SYNDROME

I. Klimeš, D. Gašperíková, E. Šeböková, D. Ježová, R. Kvetňanský and I. Klöting. Institute of Experimental Endocrinology, Slovak Academy of Sciences, Bratislava, Slovak Republic and 'Department of Laboratory Animal Science, Greifswald University, Germany

The non-obese hereditary hypertriglyceridemic, insulin resistant and hypertensive rat (known as the hHTg rat) develops symptoms of the insulin resistance syndrome closely resembling the human diseases. Experimental animal crosses offer an ideal setting for genetic dissection of such complex diseases into discrete genetic factors (QTLs). Therefore, male and female hHTg rats were crossed with healthy Brown-Norway (BN) rats to generate (hHTg x BN) $F_{2}$-hHTg and (BN x hHTg) $F_{2}$-BN hybrids. To avoid sex influences 123 male $F_{2}$ hybrids were studied for diabetes and hypertension related traits at the age of 4 months. Although the parental traits were changed, no significant differences were found between both the $F_{2}$-hHTg and $\mathrm{F}_{2}-\mathrm{BN}$ hybrids in any trait studied. However, comparing the distribution and mean values of traits between male hHTg and $\mathrm{BN}$ rats and male $\mathrm{F}_{2}$ hybrids, interactive gene effects were observed in fasting plasma insulin $(0.5 \pm 0.3$, $0.5 \pm 0.2$ vs. $\left.1.9 \pm 1.0 \mathrm{ng} \cdot \mathrm{ml}^{-1}\right)$ and glucose $\left(5.5 \pm 0.7,5.5 \pm 0.6\right.$ vs. $\left.4.3 \pm 0.8 \mathrm{mmol} . \mathrm{I}^{-1}\right)$ levels. A dominant action of hHTg genes in $\mathrm{F}_{2}$ hybrids were seen in basal plasma adrenaline $\left(312 \pm 233,68 \pm 60\right.$ vs. $\left.311 \pm 374 \mathrm{pg} \cdot \mathrm{ml}^{-1}\right)$ and noradrenaline $\left(437 \pm 344,232 \pm 143\right.$ vs. $\left.451 \pm 336 \mathrm{pg} \cdot \mathrm{ml}^{-1}\right)$ levels, and of $\mathrm{BN}$ genes in body weight $(293 \pm 22,344 \pm 25$ vs. $327 \pm 34 \mathrm{~g})$ and fasting plasma triglyceride $(1.5 \pm 0.2,1.2 \pm 0.2$ vs. $1.3 \pm 0.4 \mathrm{mmol}^{\left.-\mathrm{I}^{-1}\right)}$ levels. Intermediate gene effects were seen in systolic and diastolic blood pressure, and in the sympathoadrenal hormone responses to 20 and 120 minutes of immobilization stress, respectively. In summary, these phenotypic findings indicate to the high potential of this $\mathrm{F}_{2}$ hybrid cohort for search of QTLs, and consecutively for identification of genes acting within biochemical and/or physiological network comparable to that of patients with the insulin resistance syndrome. (Supported in part by EURHYPGEN, GAT and $G A V)$.

\section{9}

ASSOCIATION OF THE GLY4OSER VARIANT OF THE GLUCAGON RECEPTOR WITH FEATURES OF THE INSULIN RESISTANCE

L.-S. Lit, R. Reneland ${ }^{\dagger \dagger}, M$. Grangvist ${ }^{\dagger}, H$. Lithell ${ }^{\dagger \dagger}$, and H. Luthmant. TDept. of Molecular Medicine, karolinska Institute, Stockholm, †tDept. of Geriatrics, uppsala University, Uppsala, Sweden

A glucagon receptor variant, Gly40-->Ser, was recently reported to be associated with non-insulin dependent diabetes mellitus (NIDDM). To examine the possible role of the variant in the development of NIDDM, we determined the prevalence of the variant and the association with clinical parameters in 112970 -year-old men living in Uppsala, Sweden. An oral glucose tolerance test and a hyperinsulinemic-euglycemic clamp were performed in all subjects. We examined the frequency of the Gly4oser variant in the 1129 men by restriction-enzyme digestion with BstE II. In 160 patients with NIDDM, $1.88 \%$ possessed the Gly40Ser variant compared to $0.91 \%$ in 662 healthy controls, and in 307 patients with IGT, $1.30 \%$ had the Gly40ser variant. No homozygates for the variant were detected. The frequency of the variant was not significantly different between the three groups. The thirteen males with the Gly40Ser variant exhibited higher fasting plasma insulin levels (19.32 $\pm 2.17 \mathrm{vs}$. $11.86 \pm$ $0.24 \mathrm{mU} / \mathrm{l} ; p=0.0006$ ), decreased rate of glucose disposal $(1.91 \pm 0.6$ vs. $3.16 \pm 0.06 \mathrm{mg} / \mathrm{kg} / \mathrm{min} ; p=0.04)$, and higher night systolic blood pressure (137.24 \pm 6.6 vs. $119.5 \pm 0.70 \mathrm{~mm} \mathrm{Hg} ; \quad p=0.008$ ) compared to non-carriers. No significant differences were found for mean serum triglyceride or mean free fatty acid. We conclude that people with the Glyalaser variant of the glucagon receptor display features of insulin resistance in the population of 70 -year-old men.

\section{0}

MUTATIONAL ANALYSIS OF THE INSULIN RECEPTOR SUBSTRATEIN NON-INSULIN DEPENDENT DIABETES.

S. Mammarella, D.L. Esposito, B. Creati, A. Di Valerio, F. Della Loggia ${ }^{\circ}, F$. Capani $^{\circ}$, A. Consoli ${ }^{\circ}$, R. Mariani-Costantini, F.G. Caramia*, P. Battista and A. Cama. Istituto di Patologia Umana, ${ }^{\circ}$ Centro Antidiabetico and Cattedra di Patologia Medica, Università "G. D'Annunzio" - Chieti; *Dipartimento di Medicina Sperimentale, Universita "La Sapienza" - Roma.

The insulin receptor substrate-1 (IRS-1) is an intracellular protein which is phosphorylated by the insulin receptor and mediates the insulin action. Its possible involvement in the pathogenesis of NIDDM is being debated. Two molecular variants of IRS-1 that respectively result in the substitutions of Gly 972 with Arg (Arg 972 -allele) and of Ala 513 with Pro (Pro $513_{\text {-allele) }}$ have been detected in NIDDM patients as well as normoglycemic individuals. We have analyzed the IRS-1 gene in 63 NIDDM patients and in 47 normoglycemic subjects. Using SSCP, PCR direct-sequencing and restriction enzyme analysis we found Arg ${ }^{972}$-allele in 9 out of 63 unrelated NIDDM patients and in 7 out of 47 normoglycemic controls. The Pro 513 allele was detected in one normoglycemic control. In addition to common allelic variants of the IRS-1 gene, we detected a novel deletion of Gly 723 in one NIODM patient. This is not a common polymorphism since it was observed in 1 out of 220 chromosomes analyzed. Furthermore, this amino acid is conserved in human, mouse and rat IRS-1 proteins. Moreover, the Gly 723 deletion creates an additional consensus sequence for protein kinase $C$ phosphorylation which could play a role in regulating IRS-1 function. In an additional NIDDM patient, we detected a novel amino acid substitution Ser $\rightarrow$ Tyr at codon 1043. Intriguingly, this amino acid substitution abolishes the consensus sequence for a glycogen synthase kinase 3 (GSK3) phosphorylation site. GSK3 could also play a role in regulating IRS-1 function. (The financial support of Telethon - Italy - Grant no. E.295 - is gratefully acknowledged) 
281

PHENOTYPE CHARACTERISTICS OF TWO DANISH MODY FAMILIES WITH LINKAGE TO CHROMOSOME 12

S. A. Urhammer', T. Hansen', H. Eiberg ${ }^{2}$ and O. Pedersen'

Steno Diabetes Center and Hagedorn Research Institute'. University Institute of Medical Biochemistry and Genetics, Department of Medical Genetics², Copenhagen, Denmark.

Maturity onset diabetes of the young (MODY) is a heterogenous form of diabetes. At the genetic level three different subsets are known. The objective of the present investigation was to study the pancreatic $\beta$-cell function as well as the insulin sensitivity ( $\mathrm{Si}$ ) and glucose effectiveness $(\mathrm{Sg})$ of marker-positive MODY family members with linkage to chromosome 12 (MODY3). Seven affected subjects (with impaired glucose tolerance (IGT) or diabetes (DM)) and one normal glucose toleran (NGT) individual with the disease haplotype, and 8 matched non-related control subjects underwent a frequently sampled intravenous glucose tolerance test (FSIVGTT) with addition of tolbutamide after 20 minutes. Data from the FSIVGTT were analyzed by Bergmans Minimal Model method. The MODY3 affected subjects had normal sensitivity to insulin and normal glucose effectiveness compared to control subjects (Si: $13.3+0.7 \times 10^{-5} \mathrm{~L} \mathrm{~min}^{-1}$ pmol $^{-1}$ versus $15.0+0.6$ (mean $+\mathrm{SD}$ ); Sg: $1.5 \pm 0.5 \times 10^{-2} \mathrm{~min}^{-1}$ versus $2.2 \pm 0.7$ ). However, their acute insulin response to an intravenous glucose load was severely reduced (incremental insulin response 0-10 min: $208 \pm 245$ versus $1941 \pm 334(p<0.005)$ ). The insulin secretion response following tolbutamide was normal (incremental insulin response 19-35 min: $2555 \pm 2650$ versus $2858 \pm 913$ ). The NGT carrier of the MODY3 haplotype had high acute serum insulin responses (0-10 min: $3517 ; 19-35$ min: 7079), normal insulin sensitivity (Si: $11.0 \times 10^{-5} \mathrm{~L} \mathrm{~min}^{-1} \mathrm{pmol}^{-1}$ and normal glucose effectiveness ( $\mathrm{Sg}$ : $2.6 \times 10^{-2} \mathrm{~min}^{-1}$ ). In conclusion, MODY caused by a locus at chromosome 12 seems to involve a deficient insulin secretion upon glucose stimulation at an early stage of disease development. MODY3 affected subjects had normal insulin secretory capacity upon tolbutamide stimulation as well as normal insulin sensitivity and glucose effectiveness.

\section{2}

GENETIC MANIPULATION OF HUMAN ENDOCRINE CELLS: THE PRODUCTION OF A VIABLE MITOCHONDRIAL DNA DEPLETED CELL LINE. J.C. Alcolado, E.J. Sherratt and A.W. Thomas. Department of Medicine, University Hospital of Wales, Heath Park, Cardiff, CF4 4XN, Wales, UK.

Mitochondrial gene defects have been implicated in the pathogenesis of NIDDM. Firstly, both human and animal models of NIDDM show an excess of maternal transmission; Secondly, mitochondrial toxins including streptozotocin result in diabetes; thirdly, mitochondrial DNA mutations have been identified in patients with the disease. In order to investigate the role of mitochondrial DNA (mtDNA) in diabetes our aim is to establish a phenotypic expression system by depleting cells of total mtDNA followed by the production of clonal cytoplasmic hybrids (cybrids harbouring diabetogenic mtDNA mutations. Rat insulinoma (RIN) cells and a human thyroid cell line, transformed with SV40, were separately maintained in RPMI 1640 media supplemented with $10 \% \mathrm{FCS}$. Ethidium bromide ( $50 \mathrm{nM}$ ) was then added to the media together with pyruvate, glucose and uridine. Ethidium bromide intercalates with DNA and since mtDNA is intracytoplasmic, this technique allows preferential depletion of mtDNA. After a total of 22 cell divisions in ethidium bromide supplemented media, total DNA was extracted from both the RIN and humant thyroid cell lines (Nucleon kit). Using appropriate PCR primers it was not possible to detect any mtDNA fragments in the thyroid cell tine, although nuclear genes were easily amplified. Removal of pyruvate, uridine or glucose from the media caused cell death. These results confirm that all mtDNA has been removed from the thyroid cell line and these cells are effectively mtDNA "free". They are kept viable by anaerobic glycolysis. The requirement for uridine results because dihydrooratate dehydrogenase, an enzyme in the pyrimidine biosynthetic pathway, requires a functional electron transport chain for its activity. Similar experiments with the RIN cell line suggest that these cells are relatively resistant to ethidium bromide treatment in that total mtDNA depletion has yet to be achieved. This is the first report of a viable mtDNA depleted endocrine cell line. Future studies will investigate the effect of specific miDNA. mutations inserted into these cells by cytoplasmic fusion techniques.
283

MITOCONDRIAL DNA DEPLETED ENDOCRINE CELLS DIFFERENTIALLY EXPRESS GENES INVOLVED IN VESICLE TRANSPORT.

A.W. Thomas, E.J. Sherratt and J.C. Alcolado. Department of Medicine, University Hospital of Wales, Heath Park, Cardiff, UK, CF 4 XN.

Mitochondrial gene defects have been implicated in the pathogenesis of NIDDM. Previous studies suggest impaired insulin secretion is a feature of mitochondrial diabetes and, in order to study the effects of mitochondrial DNA (mtDNA) on hormonal secretion further, we have developed a phenotypic expression system in which a human thyroid cell line (Ori3) has been totally depleted of mtDNA. Wildtype (control) and mtDNA depleted $\left(\right.$ rho $\left.^{\circ}\right)$ cells were studied by the technique of differential display. Total RNA was extracted from both cell lines and, following DNAse treatment, a reverse transcriptase reaction was carried out using a variety of polyT anchored primers (eg. $\mathrm{T}_{12} \mathrm{AG}$ or $\mathrm{T}_{12} \mathrm{CG}$ ). The resulting cDNAs were amplified in radio-labelled PCR reactions employing arbitary 10 mers and the relevant anchored primers. PCR products from control and tho ${ }^{\circ}$ cells were run on $6 \%$ denaturing polyacrylamide gels followed by autoradiography and comparison for differentially expressed bands. Such bands were cut from the gels, cloned into T-overhang vectors and sequenced. Using this powerful technique we have identified 12 clones which are differentially expressed between control and rho cells. Differential expression was confirmed by Northern blotting. One of the clones was $100 \%$ homologous with the recently described RNAse L inhibitor and another shows strong homology with a gene encoding a protein of importance in vesicle transport. We conclude that mtDNA depletion results in a variety of changes within endocrine cells that could contribute to impaired bormonal secretion
284

AUTOANTIBODIES IN NIDDM: ASSOCIATION WITH LOW RISK MHC CLASS I| ALLELES IN LATE-ONSET PATIENTS V.A. Horton', I.M. Stratton', P.Z. Zimmet', I.R. Mackay², G.F. Bottazzo ${ }^{3}$, M. Shattock, R.R. Holman ${ }^{1 *}$, and R.C. Turner ${ }^{1 \cdot}$ for the UK Prospective Diabetes Study Group' Oxford, UK ${ }^{2}$ Melbourne, Australia ${ }^{3}$ London, UK

In a study of 718 randomly selected patients with NIDDM and 200 normoglycaemic controls, the presence of islet cell antibodies (ICA), glutamic acid decarboxylase antibodies (GADA), HLA DR3, DR4, and DQß57nonAsp genotypes were assessed. The overall prevalence of ICA and GADA in patients age $25-65$ was $9 \%$ and $15 \%$ respectively. In those age $<35$ years, $50 \%$ of patients had autoantibodies and, compared with those without, were less obese (mean BMl of $24 \vee 31 \mathrm{~kg} \mathrm{~m}^{-2}$ ) and more hyperglycaemic (haemoglobin Atc 9.7 v. $7.8 \%$ ). In those age $55-65$ years, patients with autoantibodies were not as different as in younger patients from those without antibodies: BMi $\left(26 \vee 28 \mathrm{~kg}^{-2}\right)$ and haemoglobin $A 1 \mathrm{c}$ $(10.4 \vee 9.3 \%)$. Whereas $97 \%$ of autoantibody positive patients age $<35$ years required insulin by 6 years, only $50 \%$ did in those $>55$ years. In those age $<45$ years, DR3/4, DR4, and nonAsp/nonAsp were more common than in the general population (odds ratios $2.9,1.9$, and 1.7 ) and $68 \%$ of those with autoantibodies also had DR3/DR4 or DR4; in contrast, only $31 \%$ with antibodies had DR3/4 or DR4 in those $55-65$ years, similar to $30 \% \mathrm{DR} 3 / 4$ or DR4 in controls. In conclusion, we can infer either that in some older patients with NIDDM, ' $\beta$-cell-related' autoantibodies may be secondary to non-autoimmune beta cell destruction, or in those without high risk $H L A$ genotypes, autoimmune $\beta$-cell destruction can be a slow process that leads to NIDDM 


\section{5}

DNA POLYMORPHISMS AND MAPPING ON CHROMOSOME 6q OF PLASMA-CELL ANTIGEN I (PC-1), AN INSULIN SIGNAL INHIBITOR. A. Doria, S. Federman, S.S. Rich, J.H. Warram, A.S. Krolewski, Joslin Diabetes Center and Harvard Medical School, Boston, MA, USA

PC-1 (5'-nucleotide phosphodiesterase) is a membrane glycoprotein expressed in several tissues, including skeletal muscle, where it inhibits insulin signaling. Overexpression of $P C-l$ has been recently reported in fibroblasts of NIDDM patients (Maddux et al, Nature 1995), and may have a genetic origin. Investigating $P C-I$ as a candidate gene for NIDDM, we screened this locus for restriction fragment melting polymorphisms (RFMP) by denaturing gradient gel electrophoresis (DGGE) blotting. Genomic DNA from 10 non-diabetic and 10 NIDDM subjects was digested with frequent cutting endonucleases, separated by DGGE, blotted on nylon membranes, and hybridized with overlapping $P C-1$ cDNA probes. RFMPs were identified in AluI (PC1/AluI) and DdeI (PC1/DdeI) digests by PC-1 CDNA bp 2144-2303 and 1842-2144, respectively. Both RFMPs have two alleles (at 14 and $14.5 \%$ of denaturant concentrations for $\mathrm{PC} 1 / \mathrm{Alu}$, at 21 and $22 \%$ for $\mathrm{PCl} / \mathrm{Dde}$ ). While PCl/Alul was not associated with NIDDM, PCl/Ddel minor allele was twice as frequent among 187 NIDDM cases (5.3\%) than 73 non-diabetic controls $(2.7 \%)(\mathrm{p}=0.20)$. By linkage analysis of $\mathrm{PCl} / \mathrm{Alu}$ relative to other polymorphic markers in 8 multigenerational families, $P C-1$ was mapped on chromosomal band 6q22-23 at a recombination fraction of 0.08 from marker d6s270 (LOD score $=3.75$ ). Genotyping is in progress to ascertain whether linkage exists between NIDDM and this chromosomal region in 28 multigenerational families (397 individuals) with multiple members $(n=127)$ affected by NIDDM with an autosomal dominant pattern of inheritance.

\section{6}

THE HUMAN GLUCAGON RECEPTOR GENE POLYMORPHISM AND NIDDM IN THE UK

E.J. Sherratt, A.W. Thomas and J.C. Alcolado. Department of Medicine, University Hospital of Wales, Heath Park, Cardiff, CF4 4XN, Wales, UK.

Patients with NIDDM have a raised plasma concentration of glucagon and it bas been postulated that this may play a role in the failure to suppress hepatic gluconeogenesis in these patients. An abnormality in the glucagon receptor could underlie such a defect. Recently a missense mutation in the human glucagon receptor gene was discovered in exon 2; it changes a glycine at position 40 to a serine residue. The mutation was found to be highly associated with NIDDM in a pooled set of French and Sardinian patients and showed some linkage in French pedigrees. However, the mutation could not be detected in any NIDDM subjects from a group of patients recruited in Finland. In view of this conflicting data, we have searched for the Gly40Ser mutation in 98 unrelated patients with NIDDM and a strong family history for the disease and 101 control subjects. All subjects were British caucasoids. DNA was extracted from peripheral leukocytes and exon 2 of the glucagon receptor was amplified by the polymerase chain reaction. The presence of the Gly40Ser mutation resulted in the loss of a BstE II restriction site. We found the mutation in 4 of the patients with NIDDM and only $l$ of the control subjects ( $p>0.1$, Yates corrected) The control subject with the mutation was teviewed at the age of 89 years she had no evidence of NIDDM. Our data suggest that. although the Gly40Ser mutation is more common in British patients than in those from Finland, there is little evidence to support the association with NIDDM.

\section{7}

MUTATIONAL ANALYSIS OF THE GENE ENCODING A HUMAN $\beta$ CELL INWARDLY RECTIFYING POTASSIUM CHANNEL (BIR).

L.Hansen, S.M.Echwald, T.Hansen, and O.Pedersen. Steno Diabetes Center and Hagedorn Research Institute, Copenhagen, Denmark.

A new member of the inwardly rectifying potassium channel family (Kir) called BIR was recently cloned. The BIR gene is located $3.5 \mathrm{~Kb}$ downstream of the human sulfonylurea receptor (SUR) gene on chromosome 11, locus 11p15.1, and coexpression of these cDNAs in pancreatic $\beta$-cells and glucose-responsive insulin-secreting cell lines shows that they form a complex of at least two subunits inhibited by sulfonylureas and stimulated by diazoxide. Recently, an inherited form of inappropriate insulin secretion, persistent hyperinsulinemic hypoglycemia of infancy ( $\mathrm{PHHI}$ ) was shown to be caused by mutations in the SUR subunit of this complex. These genes are the first since the discovery of the glucokinase mutations in the MODY families to be involved in an inheritable form of pancreatic $\beta$-cell dysfunction. Since insulin secretion is impaired in noninsulin dependent diabetes mellitus (NIDDM) patients these genes are obvious candidate genes to explain the genetic contribution to this multifactorial disease. We therfore SSCP-scanned the coding region from genomic DNA in 57 NIDDM patients and found a total of 6 different polymorphisms: Codon 23 (GAG/AAG), Glu $\rightarrow$ Lys, allelic frequency 0.38 ; codon 190 (GCT/GCC), Ala $\rightarrow$ Ala, allelic frequency 0.33; codon 267, (CTC/CTG), Leu $\rightarrow$ Leu, allelic frequency 0.03 ; codon 269 (CTG/GTG), Leu $\rightarrow$ Val, allelic frequency 0.03 ; codon 336 (ATC/GTC), Ile $\rightarrow$ Val, allelic frequency $0.38 ; \operatorname{codon} 381$ (AAG/AAA) Lys $\rightarrow$ Lys, allelic frequency 0.03 . When 70 control subjects were analyzed, there was no significant difference in the allelic frequencies between the group of controls and the group of NIDDM patients for any of the polymorphisms. We conclude: That the BIR gene is not a major gene for NIDDM. However, the missense polymorphisms identified, if influencing the normal function of $\beta$. cells, may well act together with other yet unidentified disrupted genes that impair $\beta$-cell function in NIDDM.

\section{8}

\section{A COMMON AMINO ACID POLYMORPHISM IN IRS-1 CAUSES IMPAIRED INSULIN SIGNALING:} EVIDENCE FROM TRANSFECTION STUDIES

K. Almind. G. Inoue. O. Pedersen and C. R. Kahn, Joslin Diabetes Center, Boston. MA and Steno Diabetes Center. Gentofte. Denmark Insulin receptor substrate-1 (IRS-1) is the major cytoplasmic substrate of the insulin and $[G F-1$ receptors. Recent studies have identified multiple sequence variants of IRS-1. especially in patients with non-insulin-dependent diabetes mellitus. In the present study, we have examined insulin stimulated processes in $32 \mathrm{D}(\mathrm{IR})$ cells, a myeloid progenitor cell stably overexpressing the insulin receptor. transfected with wildtype human-IRS-1 or the most common human variant of IRS-1 in which glycine 972 is replaced by arginine. As compared to wildtype IRS-1, insulin stimulation of cells transfected with mutant IRS-1 exhibited a $32 \%$ decrease in incorporation of ['H]thymidine into DNA $(\mathrm{p}=0.002)$, a $36 \%$ decrease in IRS-1 associated PI 3-kinase activity $(p=0.004)$ and a $25 \%$ decrease in binding of the regulatory subunit p85 of PI 3 -kinase to IRS-1 $(p=0.002)$. There was also a tendency for a decrease in Grb2 binding to IRS-1 and insulin-stimulated MAP kinase activity, however, these were not statistically significant. The changes occurred with no change in insulin receptor or IRS-I tyrosine phosphorylation. Furthermore, we were able to demonstrate by a gel shift assay, that p70 S6 kinase is phosphorylated after insulin stimulation in cells expressing either normal IRS-I or mutant IRS-I.

These data indicate that the mutation in codon 972 in IRS-1 impairs insulin stimulated signaling, especially along the PI 3-kinase pathway, and may contribute to insulin resistance in normal and diabetic populations. 
289

SEARCH FOR TRINUCLEOTIDE REPEAT EXTENSIONS IN MUSCLE AND THEIR POTENTIAL ROLE IN NIDDM

M. Lehto*, Z. C. Xie*,$M$. Lehtovirta**;, and L. Groop*. Dept. of Endocrinology, University of Lund, Malmö, Sweden*, and Helsinki University Hospital, Finland***

Extension of expressed trinucleotide repeats has been associated with earlier onset and unequal parental transmission in neurodegenerative disorders Segregation pattern in some NIDDM farnilies shows similar features. Since skeletal muscle insulin resistance is a key feature in NIDDM, we screened a human muscle cDNA library with CAG-and CGG-oligoprobes in order to find mRNA transcripts carrying long polymorphic trinucleotide repeats. Totally 4 CAG- and 6 CGG-repeat positive CDNA-clones were isolated, subcloned and seguenced. Five of them represented known gene products (EMBL: HSDB 11, HSUO7857. HSPCAR, HUMZFHPA. HSRGM). CDNA sequences containing more than 7 repeats were tested with PCR from genomic DNA samples $(50$ NIDDM patients and 50 control subjects from Finland). Two of the CAG-repeat containing genes, CAGl (novel gene) and CAG4 (HSDB11), showed 5 and 3 alleles, respectively. No major extensions of these repeats were observed in either study group. One of the CGG-repeat positive cDNA-clones was identified as new human Rab-isoform encoding a 225 aminoacid protein. The specific function of this GTP-binding protein is yet unknown, but it could be involved eg. in vesicle transport of GLUT-4 in muscle. A mutation $\mathrm{Ser}^{224} \rightarrow \mathrm{Phe}^{224}$ was found in the carboxyterminal end of the protein. This aminoacid substitution is located two aminoacids apart from the prenylation site, which has been shown to be vital for protein function. Screening of the mutation was performed in 554 NIDDM patients and 308 control subjects from Sweden and Finiand. The allele frequency of this mutation was low in control subjects (Swe; 0.6\%, Fin; 0.3\%), but was about 3 times higher among NIDDM patients (Swe; 2.2\%, Fin; $1.0 \%$ ) $(p<0.05)$. No homozygous carriers for the mutation has been found. Conclusions: The data suggest that NIDDM is unlikely to be caused by expanded CAG- or CGG-trinucleotide repeats in muscle. We, however, also report a new Rab-isoform and four previously unknown gene products, which could be candidate genes for NIDDM.

\section{0}

SYMPATHOADRENAL AND CARDIOVASCULAR FUNCTION IN FIRST DEGREE RELATIVES OF NIDDM PATIENTS

M Elam, PA Jansson, F Waagstein and JW Eriksson, Depts of Neurophysiology, Cardiology and Medicine, Sahlgrenska Hospital, 41345 Göteborg, Sweden Insulin resistance is a prominent component in the pathogenesis of NIDDM but the initial mechanisms are not identified. Since stress, catecholamines and cAMP produce a marked insulin resistance, we examined sympathoadrenergic activity in 10 healthy subjects with two first-degree relatives with NIDDM (R) and 10 control subjects without family history of NIDDM (C). They were pairwise matched for sex $(\mathrm{F} / \mathrm{M} 6 / 4)$, age ( $35 \pm 1$ vs $35 \pm 1$ for $\mathrm{R}$ and $\mathrm{C}$ ) and BMI (23.6 \pm 0.6 vs $23.2 \pm 0.4)$. Fasting blood glucose $(5.1 \pm 0.1$ vs $4.9 \pm 0.1$ $\mathrm{mmol} / \mathrm{L})$, plasma insulin $(7.7 \pm 0.8$ vs $7.4 \pm 1.6 \mathrm{mU} / \mathrm{L})$, C-peptide $(0.44 \pm 0.04$ vs $0.4 \pm 0.05 \mathrm{nmol} / \mathrm{L}$ ) were similar, whereas HbAlc was higher in $\mathrm{R}$ vs $\mathrm{C}$ $(4.8 \pm 0.1$ vs $4.5 \pm 0.1 \% \mathrm{p}<0.02)$. Resting muscle sympathetic activity (MSA) assessed by microneurography from the peroneal nerve was similar in $\mathrm{R}$ and $\mathrm{C}(25.6 \pm 4.0$ vs $29.9 \pm 4.2$ bursts $/ \mathrm{min}, \mathrm{NS})$ as were plastna noradrenaline (NA) and glycerol, but adrenaline (A) and FFA tended to be lower in R $(p<0.1)$. Following stress induced by inmersion of one hand in icecold water and then arithmetic tasks, FFA and glycerol (reflecting lipolysis) increased more in $\mathrm{R}$ than $\mathrm{C}(\mathrm{p}<0.05)$ but NA and $\mathrm{A}$ were similar. In maximum ergometer cycle test, $\mathrm{VO}_{\max }$ was nearly identical $(33.8 \pm 2.4$ vs $34.9 \pm 2.8 \mathrm{ml} / \mathrm{kg} / \mathrm{min}$ for $\mathrm{R}$ vs C) as was maximum working capacity ( $191 \pm 12$ vs $193 \pm 17 \mathrm{~W}$ ), anaerobic threshold and resting and maximum exercise heart rates and blood pressures. Linear regression showed no association beween $\mathrm{VO}_{2 \max }$ or MSA and insulin sensitivity (IS; glucose disposal) assessed with euglycemic clamp at two insulin levels $(-20$ and $\sim 90 \mathrm{mU} / \mathrm{L})$. IS was slightly reduced in $R$ vs $C(p<0.05)$. Conclusion: In these NIDDM-prone individuals insulin resistance is not due to altered muscle sympathetic activity or oxygen uptake capacity. Instead, the sensitivity of the adipose tissue to sympathoadrenergic stimuli may be enhanced contributing to early metabolic perturbations in the development of NIDDM.

\section{1}

LINKAGE ANALYSIS AND MOLECULAR SCANNING OF THE PC2 GENE IN NON-INSULIN DEPENDENT DIABETES K.Kalidas, E.Dow', S.V.Gelding ${ }^{1}$ and D.G.Johnston'. ' ${ }^{1}$ St. Mary's Hospital Medical School, ${ }^{2}$ London Hospital Medical School, London, UK

Defective insulin secretion in Non-Insulin Dependent Diabetes (NIDDM) has been attributed to pancreatic Beta-cell dysfunction. Hyperproinsulinaemia is an aspect of this dysfunction in NTDDM patients. A possible cause is an impairment in the conversion of proinsulin to mature insulin. Insulin is produced from its precursor molecule, proinsulin, by the action of two endopeptidase enzymes, prohormone convertase 2 (PC2) and prohormone convertase 3 (PC3) (also known as $\mathrm{PCl}$ ). The study of the $\mathrm{PC2}$ gene as a candidate gene has been prompted by the association of NIDDM with hyperproinsulinaemia. We have used a simple tandem repeat polymorphism (STRP) in intron 2 of the PC2 gene to screen 26 pedigrees which include Caucasian, Afro-Caribbean, Asian and two MODY families. Standard two-point linkage analyses were performed using several models. Cumulative negative LOD scores of -9.8 , with other models of analysis having LOD scores of less than -2 , have excluded linkage, indicating that mutations in the $\mathrm{PC} 2$ gene are unlikely to have a major role in the predisposition of NIDDM. As a minor role in a polygenic model cannot be excluded, we have used heteroduplex analysis to screen the 12 exons of the PC2 gene for mutations. There was no evidence of mutations in the 60 affected individuals that were screened, confirming that the PC2 gene does not contribute to NIDDM in these individuals. 
PS 2

\section{Genetics of IDDM}

292

CTLA-4 REGION PREDISPOSES TO IDDM AND GRAVES' DISEASE

L. Nistico', 5, R. Buzzetti ${ }^{1}$, L.E. Pritchard ${ }^{2}$, M.T. Martinez ${ }^{3}, M$. Serrano-Rios ${ }^{3}$, A. H. Barnett ${ }^{4}$, R. Tosi ${ }^{5}$, P. Pozzilli ${ }^{1}$ and J.A. Todd ${ }^{2}$ Ist. Clinica Medica II, Univ. Roma La Sapienza and Inst. Cell Biology CNR, Rome, Italy, ${ }^{3}$ Facultad de Medicina, Univ. Complutense Madrid, Spain, ${ }^{4}$ Dept. of Medicine, Univ. Birmingham and ${ }^{2}$ The Wellcome Trust Centre for Human Genetics, Univ. Oxford, UK.

The identification of several chromosome non-HLA regions linked to insulin-dependent diabetes mellitus (IDDM) demonstrates the polygenic inheritance of this disease. We focussed on chromosome $2 q 31-33$ region that shows homology with murine chromosome 1 , to where Idd 5 has been mapped and contains the autoimmune disease candidate genes CTLA-4 and CD28. Performing multipoint linkage analysis on 48 multiplex families we detected maximal evidence of linkage $\left(p=6.5 \times 10^{-5}\right)$ in the D2S72-CTLA-4-D2S116 region. An A/G transition in position 49 of exon 1 of CTLA-4, which encodes a Thr/Ala substitution in the leader peptide, was tested for transmission disequilibrium to IDDM offspring in five indipendent family datasets. The $\mathrm{G}$ allele was preferentially transmitted in two datasets $(n=231, p=0.0001)$ and a positive trend $(p=0.074)$ was observed in a third dataset $(n=180)$. There was no evidence of preferential transmission in the last two datasets $(n=407)$. The transmission of the $G$ allele to unaffected siblings did not deviate from the expected $50 \%$. Since an association of the CTLA-4 microsatellite with Graves' disease was reported in a previous case-control study, we analysed a panel of Graves' patients $(n=94)$ and controls $(n=77)$ and we found the frequency of the $G$ allele to be significantly higher in patients $(p=0.037)$ compared to controls. Overall, there is convincing data for an association of the CTLA-4 gene region with IDDM. Family-based studies will be required to confirm the association with Graves' disease.

\section{4}

THE ALLELE DISTRIBUTION OF THE DOB1 AND DOA1 IN THE BULGARIAN POPULATION AND AMONG CASES WITH IDDM

Gavrilov D., M. Konstantinova, R. Savova, D. Popova, K. Koprivarova, V. Hristov, I. Kremensky

\section{RESUME}

We investigated the association between MCA - DQA1 and DQB1, and insulin Dependent Diabetes Mellitus (IDDM) in a Bulgarian population. A sample of 110 children with IDDM and 152 controls have been tested. Those groups were formed on the basis of the WHO criteria and consisted of children below 14 years of age from the North - Western region of Bulgaria. PCR amplification of the second exon of MCA - DQA1 and $D Q B 1$ region has been performed and genotyping has been done by Slot - blot hybridization with specific probes ( 9 for MCA - DQA1 and 15 for DQB1).

Collected data about the MCA - DQA1 gene reveals that individuals having the $D Q A 1 * 0301$ allele $(R R=7.9, p<0.001)$ are with the highest risk for developing IDDM. The DQA ${ }^{\star} 0103(\mathrm{RR}=0.28, p, 0.025)$ and $D Q A 1^{\star} 0201(R R=0.17, p<0.005$ ) alleles play a statistically significant protective role.

From the data about the DQB1 gene individuals with the $D Q B 1 * 0302$ allele $(R R=6.14, p<0.001)$ have the highest risk in comparison with the whole population. The alleles DQB1*0301 $(R R=0.269, p<0.001), D Q B 1 * 0602(R R=0.1, p<0.001)$ and $D Q B 1 * 0603$ $(R R=0.327, p<0.05)$ may have a protective role.

The collected data in that investigation confirms the role of the aminoacid residue at the 57th place in the DQB chain for the development of IDDM. Individuals that are heterozygous for Asp57-/Asp57 have the highest risk for IDDM (RR=10.93, $p<0.001)$, and those that are homozygous for Asp57+/Asp57 have the lowest $(R R=0.197, p<0.001)$

Certain differences in the observed frequencies and association of some allelic forms with IDDM in comparison with other populations are discussed.

\section{3}

INTERLEUKIN-1 GENE CLUSTER POL YMORPHISMS AND IDOM: LINKAGE STUDIES IN A LARGE DANISH IDDM FAMILY MATERIAL

O.P. Kristiansen', R. Bergholdt'. F. Pociol', J. Johannesen!, C.A. Dinarello', J Nerup'. T. Mandrup-Pouisen' and The Danish Study Group of Diabetes in Childhood, 'Steno Diabetes Center. Denmark. "Health Science Center, University of Colorado, Colorado, USA. IDDM susceptibility loci and the IL-1 gene cluster have been mapped to chromosome 2q12$\mathrm{q} 33$ and $2 \mathrm{q} 12-\mathrm{q} 21$, respectively. Polymorphisms of the $\mathrm{LL}-1 \mathrm{~B}$ (Taq) RFLP), the IL-1 receptor antagonist (Aa) and the $[L-i$ receptor type 1 (RTI) genes have been shown to be assoclated with IDDM in a Danish population. Aim: To test for haplotype sharing (HS) and/or transmission disecuilibrum (TD) of 1) an IL-1B Taql RFI.P. 2) an IL-1B Aval RFLP. 3) an IL1Ra 2 nd intron polymorphism, and 4) an IL-1RTI Pstl RFLP Material: 138 IDDM sib-pair families (SF) with a total of 165 sibpairs (both parents in 100 families, one parent in 38 families and all offspring genotyped) and 105 vertical IDDM tamilies (VF) (one affected parent, both parents genotyped, and one aflecled offspring). Methods: PCR followed by enzymatic cleavage (when appropriate) and electrophoresis. Analysis: TO tests (TDT) and HS as described by Spelman. Results: $1 \mathrm{~L}-1 B$ Taq RFLP: HS analysis showed significant non-sharing in 55 intormative SF comprising 71 affected sibpairs, 23 shared vs. 48 nonshared alleles, (chi ${ }^{2, h s}=8.8 ; i d f, p=0.003$ ). $71 \mathrm{SF}$ and $67 \mathrm{VF}$ were informative for the TDTs. permitting 186 and 87 TDTs, respectively. No TD was demonstrated in SF: $101 \mathrm{~B}^{*} 1$ alleles and $85 B^{*} 2$ alleles were transmitted to affected offspring $(p=0.24) B^{* 1}$ and $B^{*} 2$ allele transmission to affected offspring in VF were 31 and 56 ( chi $\left.^{2 \text { id }}=7.18 ; 1 \mathrm{df}, p=0.007\right) . \mathrm{B}^{*} 1$ and $\mathrm{B}^{*} 2$ allele transmission to non-affected offspring in $V F$ were 48 and $45(\mathrm{p}=0.76)$. Comparison of affected $v s$ non-affected offspring in $V F$ revealed significant different transmission in the two groups $(\mathrm{p}=0.04$ ). LL-1B Aval RFLP: TDT in 71SF and 72VF, and HS in 48 SF did not demonstrate TD or HS. IL-1Ra and $\angle L-1 R T I$ polymorphisms: TDTs were periormed in 65 SF/64 VF and 65 SF/61 VF, respectively. HS was tested in 52 (IL-1Ra) and 50 (IL-1RTI) SF No TD or HS could be demonstrated. Conciusion: Overali we found no evidence tor linkage of the tested markers. However in a subset of the material, the VF, the IL-1ß TagI RFLP B'2 allele was found be transmitted significantly more often than expected to affected offspnng whereas random transmission was observed for the non-aftected offspring in VF.

\section{5}

A COMPARATIVE STUDY OF DR/DQ ALLELES IN JAMAICAN TYPE ! DIABETIC PATIENTS IN TWO COUNTRIES

J.M.Hewarda, C.H.Mijovic a, E.Morrison "s and A.H.Barnett ${ }^{\text {a }}{ }^{\text {a }}$ Department of Medicine, University of Birmingham, England, B15 2TH and 'Department of Biochemistry, University of West Indies, Jamaica.

Genetic susceptibility to Type I diabetes is associated with both genetic and environmental factors. The aim of this study was to compare the HLA-DR/DQ genotypes of diabetic patients $(n=49)$ and controls $(n=97)$ born and resident in Jamaica with a previously typed group of Jamaican diabetic patients $(n-39)$ and controls $(n=97)$ born and resident in the UK to assess the effect of environmental factors on susceptibility to Type I diabetes. All diabetic subjects were diagnosed at $<30$ years of age, were ketosis prone and absolutely dependent on insulin from diagnosis. The controls were healthy with no family history of Type 1 diabetes. They were $H L A$ typed using the polymerase chain reaction with sequence specific primers (PCR-SSP). The DR3-DQ2 and DR4-DQ8 haplotypes were positively associated and the DR2-DQ6 haplotype was negatively associated with Type I diabetes in theJamaica-resident population as previously shown in the UK group. Comparison of the Jamaica-resident diabetic patients with those resident in the UK showed an increased frequency of the following categories: DR3-DQ2 and/or DR4-DQ8 (Jamaica: $39 / 49(79.5 \%$ ) vs UK:22/39(56.4\%);p<0.01); DR3DQ2/DR4-DQ8 (Jamaica: $15 / 49(30.6 \%)$ vs UK:2/39(5.12\%);p<0.005) and DR4-DQ8 ( Jamaica :23/49(46.9\%) vs UK:9/39(23\%); ; $<0.025)$. There was no significant difference in distribution of MHC alleles between the two control populations. The differences between the diabetic populations in the two countries may be accounted for by the different environments leading to varying contributions from $\mathrm{MHC}$ and/or non-MHC encoded disease susceptibility genes. Alternatively there may be undetected heterogeneity between the $\mathrm{MHC}$ haplotypes identified in the two populations eg. at the DRB1 locus. Further investigation into these possibilities will allow better understanding of the multifactorial influences on susceptibility to Type I diabetes. 
296

A NOVEL TYPING METHOD FOR TRANSPORTER ASSOCIATED WITH ANTIGEN PROCESSING 2 ALLELES EVALUATING THEIR ROLE IN IDDM

CL Perry, CH Mijovic, CS Cockram, D Jenkits and AH Bamett. Deparment of Medicine, Birmingham University, Birmingham, England, B15 2TH

The MHC encoded transporter associated with antigen processing (TAP) genes encode subunits of a transporter which carries endogenous peptides to nascent HLA class I molecules. The TAP2 alleles TAP2*0101 and TAP2*(1201 have been positively and uegatively associated with IDDM respectively in white caucasian case/control studies, This has led to the hypothesis that TAP2 alleles are directly implicated in susceptihility to IDDM. Caucasian family studies, however, suggest that this association is due to linkage disequilibrium with HLA-DR/DQ suscentibility alleles. An altentative approach to identifying any role for the TAP2 alleles is to study different ethnic groups; these show varying linkage disequilibrium relationships witlin the MHC. The ain of this study was to compare the TAP2 alleles of Chinese diabetic patients and healthy etluically matched controls. Only $75 \%$ of the population could be unambiguously typed using conventional TAP2 allele specitic polymerase chain reaction (PCR). A new technique was established using allele specific, medium range PCR which allows typin of all the heterozygous individuals. In contrast to white caucasians neither TAP2*(0) (0) $(25 / 47(53 \%)$ diabetic patients vs $49 / 69(71 \%)$ controls, $\mathrm{p}=\mathrm{NS})$, nor TAP2*(1)201 (21/47 (44.6\%) diabetic patients vs $36 / 69(52 \%)$ controls, $\mathrm{p}=\mathrm{NS})$ were significantly associated with IDDM. These results do not reflect white Caucasian case/control data hut support data from family studies, indicating that TAP2 associations with IDDM are secondary to associations with HLA-DR/DQ alleles. Analysis of different ethmic groups continues to be an important tool in dissecting out true diabetogenic genes in gentic regions where there is strong linkage disequilibrium.

\section{8}

GENOTYPES POSITIVE FOR IILA DQBI*U301 ANI NEGATIVE FOR DQA1*U3O1 ALLLLES CONFER TIIE STRONGEST PROTEC TION AGAINST IDDM IN HI NGARLAN CHILDREN

R. Hermann, G. Soltész, C. Mijovic*, A. H. Barmett*: Departmemt of Paediatrics, University Medical school of Péess. Hungary: Department of Nedicine. University of Birminghamll. Q'nited Kingdom*

('sing the Hungaraan National (hildhood Diabetes Registry (ascertainmen! $\% 0^{\circ}$ 。 Diab Med 7:111-114, 1990) all consecutive incident IDDM cases (aged (2-14 vears) diagnosed between 1st of January. 1980 and 31 st of December, 1995 in a well detined region of Hungary have been HI.A DQA.I and DQBI genotyped ( $\mathrm{n}=108)$. Randomly selected healthy schoolchildren from the same region were used as controls $(\mathrm{n}=[\% \cdot 4)$

Polymerase (hain Reaction - Seyuence-Specific Oligonucleotide Probing method was applied for HLA typing. Both DQ2 (DQA $1 * 0501 / \mathrm{DQB} 1 * 0201)$ and DQ8

(DOA $1 * 0301 / \mathrm{DOB} 1 * 0302$ ) haplotypes conferred significant risk (Relative Rısk /RR) $433,95 \%$ Confidence Interval /CI $2252-7.47$ and $\mathrm{RR}: 17.67 . \mathrm{CI} \cdot 214-34.52, \mathrm{p}^{\times} \cdot 10^{-7}$ ) for IDDM. The highest positive disease associatioi, however, was shoun by the DQ2/DQ8 heterozygous genotype (RR:93 01, Cl: $14.87-3796.09, p<10^{-7}$ ). Strong negative association of the $D O B 1^{*} 0.301$ allele was observed with IDDN (RR:0.09. C1:0.03-0.21, $<<10^{-7}$ ). Genotypes with DOB $1 * 0301$ alleles were negatively associated with the disease even in the presence of the diabetogenic $D Q B$ ) ${ }^{*} 0201$ allele (RR 0.08 $\mathrm{Cl}: 0.00-0.53, \mathrm{p}=0.005$ ), but the negative disease association of the $\mathrm{DQBI} * 0301$ allete was neutralised in those genotypes which were also positive either for the diabetogenic DQA $1 * 0301$ or DQB $1 * 0302$ alleles. All $(6 / 108)$ diabetic patients who were $\mathrm{DOB1}{ }^{*} 0301$ positive carried also the DQA $* 0301$ allele. Genotypes positive for DQB $1 * 0301$ and negative for DQA ${ }^{*} 0301$ allele (63/194 among controls) conferred the strongest negative association with IDDM (RR:0.02, Cl:0.00-0.5., $\mathrm{p}=0,005)$. In conclusion, the hirhest risk for IDDM is conferred by the DQ2/DQ8 genotype in the Hungarian population and the $\mathrm{DQB} 1 * 0301$ allele may represent strong protection against IDDM in Hungarian children, however its effect can be neutralised in genotypes where the strongly diabetogenic $\mathrm{DQA} 1 * 0301$ and $\mathrm{DQBI} *_{0} 302$ alieles are also present.

\section{7}

ROLE OF AMINO ACIDS LOCATED IN THE ANTIGEN BINDING GROOVE OF HLA CLASS II MOLECULES IN THE RISK FOR IDDM

M. Zamani, R. Vlietinck and J.-J. Cassiman - Center for Human Genetics, University of Leuren, Leuven, Belgium

We have reanalysed the data on the association of the HLA DRB1, DQB1 and DQA1 with IDDM in different ethnic groups from the literature to determine whether different amino acids in the HLA class II alleles play a preponderant role in the susceptibility for IDUMi and whether some of these amino acid(s) provide a major risk in all populations and ethnic groups. In the Belgian population lysine at position 71 of the DRB1 chain which is encoded by the DRB1*0301, ${ }^{*} 0302,{ }^{\star} 0303,{ }^{*} 0401,{ }^{\star} 0409,{ }^{*} 1303$ alleles, provides a major risk for IDDM. These findings were confirmed even with higher risks in the Danish population. Reanalysis of the literature shows that the DRB1 ${ }^{\text {Lys7\%+ }}$ allele: provides the highest risk for IDDM in the Belgian, Danish, Greek, Taiwanese and Chinese population while this is not the case in Nonwegians, Sardinians and Algerians. Indeed in the Sardinian and Algerian population the DRB1*0401 allele encoding Lys ${ }^{71+}$ is very rare. Nevertheless, the few cases are always in the patient group.

We measured the sensitivity, specificity and a modified positive and negative predictive value (PPV) (NPV) of tests for the DRB1/45714, DQB1 $1^{\text {Asp57. }}$ and $\mathrm{DQ} \alpha 1^{\mathrm{Ang} 52+}$ alleles. Results revealed that the sensitivity of the test for $\mathrm{DR} B 1^{1 \text { ys } 71+}$

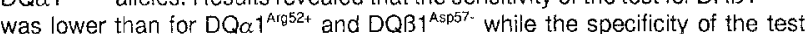

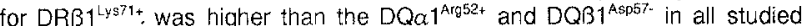
populations. The results also show that the DRR $1^{\text {Lys71* allele has a higher PPV }}$

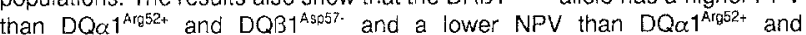
DQß31 $1^{\text {Asp } 57 .}$ in all studied populations. The PPV for DR $11^{\text {Lys } 71+}$ in Belglans was increased in the homozygous genotypes DRB $1^{\text {Lys } 71+1+\text {. Th }}$ The was also higher

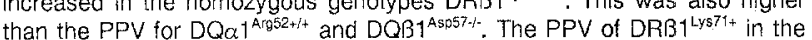
Danish was also increased in the homozygous genotype DRB $1^{\text {Lys } 71+t+}$ (we had only data on DRB1 $1^{\text {Ly } 71+t+}$ in Beigians and Danish).

These results therefore show that DRß1 $1^{\text {Ls } 81+}$ is a major susceptibility allele with high specificity and PPV for IDDM in different populations.
299

\section{INSULIN DEPENDENT DIABETES MELLITUS (TYPE 1): ANALYSIS OF HLA DQA $1^{52}$ AND HLA DQB1 ${ }^{57}$ CODONS IN POLISH POPULATION}

Witas H., Bodalski J.,Jẹdrychowska-Dańska K., Różalski M., Młynarski W., Cedzyńska D., Brzeziańska E

Molecular Biology Unit, Institute of Pediatrics, Medical University of Lodz, 91-738 Lodz, Poland

Allelespecific amplification PCR method (ASA-PCR) has allowed us to genotype possible codons at HLA-DQA $1^{52}$ and HLA-DQB1 ${ }^{57}$ locations what in turn has given the possibility to find respective amino acid expressed. Frequency analysis of codons was performed on IDDM children $(n=50)$ and control subjects $(n=50)$ of Polish origin. Obtained results revealed that aliphatic and hydrophobic amino acid residues (phenotypes: Val/Ala and Ala/Ala) are present at significantly higher frequency at DQ $\beta^{57}$ in IDDM children $(74 \%)$ that in control subjects (18\%). We have found dominant protective effect of DQ $\beta \mathrm{Asp}^{57}$. It has been shown that Asp in heterozyoous state was present in $28 \%$ IDDM patients versus $70 \%$ control subjects $\mathrm{RR}=0.167$. Homozygous Asp was not found in IDDM children while it was present in $28 \%$ of controls ( $\mathrm{RR}=0.025$ ), which suggests its stronger protective effect. Simultaneous typing of both studied codons revealed that $D Q \alpha A g^{52+} / D Q \beta A s p^{57}$ accompany IDDM phenotype at much higher frequency $(68 \%)$ that the physiological one (22\%); $R R=7.53$. The correlation between IDDM

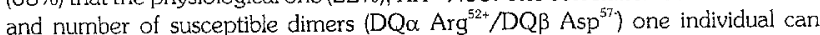
assemble has been observed in dose respective manner. The highest relative risk ( $\mathrm{RR}=4.47$ ) was obtained for individuals who can express IV (100\%) susceptible dimers ( $28 \%$ of IDDM patients versus $8 \%$ controls). Presented results are the first qualitative and quantitative analysis of studied polymorphisms in Polish IDDM children. These preliminary studies should lead to detailed analysis of allelic variants in Polish population what is necessary in the evaluation of children which belong to the group of the highest risk.

Supported by KBN grant 4 PO5E053 08 
300

THE TRANSPORTER-ASSOCIATED WITH ANTIGEN PROCESSING (TAP2) GENE AND TYPE I DIABETES IN MULTIPLEX FAMILES

MA Kelly. CH Mijovic. KH Jacobs. CL Perry. D Jenkins and AH Barnetı Department of Medicine. University of Birmingham. B15 2TH. UK.

Polymorphisms of the MHC-encoded TAP2 gene on chromosome six have shown associations with IDDM in Caucasian case/control studies. The alleles TAP2 *(1)101 and TAP2*(1)201 were positively and negatively associated respectively. This may either reflect a direct role for TAP2 alleles in predisposition to IDDM or be a result of linkage disequilibrium with HLA-DR/DQ susceptibility alleles. The aim of this study was to determine whether TAP2 alleles confer a risk of disease independent of that associated with HLA-DRDQ alleles by studying transmission of HLA-DR/DQ-TAP2 haplotypes in multiplex families. Families from the British Diabetic Association (BDA) Warren repository were typed for DR.DQ and TAP2 alleles using allele-specific PCR. Haplotypes inherited by diabctic offspring werc defined as transmitted (T) and those occurring only amongst unaffected famils members as non-transmitted (NT). HLA-DR/DQ-TAP2 $\mathrm{T}$ (number $\mathrm{N}=325$ ) and NT $(N=191)$ hapiotypes were unambiguously determined in 129 families. HLADR3-DQ2 and DR4-DQ8 haplotypes were increased and DR2-DO1 haplotypes reduced in frequency in the T group vs the NT group ( $112(34.5 \%)$ vs $2.3(12 \%)$ $118(36.3 \%)$ vs $13(6.8 \%)$ and $\left(5(1.5 \%)\right.$ vs $23(12 \%)$ respectively. $\left.p_{c}<10^{-6}\right)$. $\mathrm{TAP} 2 * 010) 1$ atleles were increased in frequency in the T $(255(78.5 \%)$ vs NT $\left(81(42.4 \%)\right.$ group $\left(\mathrm{p}_{c}<10^{-6}\right) * 020 \mathrm{I}$ alleles were not significantly decreased in the T group (T:38(11.6\%) vs NT:33 (18.8\%)). The distribution of the TAP2 alleles on T DR.3-DQ2 haplotypes did not however, differ significantly from the distribution on NT DR3-DQ2 haplotypes. This was also the result for DR4-DQ8 and DR2DQI haplotypes. These data indicate that there is no significant independent effect of TAP2 allele specificity on the risk associated with IDDM as determined by HLA-DRDQ haplotypes. A large repository of multiplex families is a useful resource for assessing whether candidale MHC-encoded susceptibility alleles have a primary role in determining susceptibility to IDDM .

\section{PS 3}

\section{Aetiology of IDDM}

\section{1}

GAD65 ANTIBODIES IN INSULIN-DEPENDENT DIABETES (IDDM). MALNITRITION-RELATED DIABETES (MRDM) AND NON-INSELIN DEPENDENT DIABETES (NIDDM) PATIENTS FROM EASTERN INDIA.

CB. Sanjeevi. A. Kanungo K.C. Samal. B.B. Tripathy. A. Lernmark. and A. Falorni. Dept of Molecular Medicine. Stockholm; Dept of Endocrinology. Cuttack: Dept. of Medicine. Seatle: Dept. of Internal Medicine and Endocrine \& Metabolic Sciences. Perugia. Sweden. India, ISA and Ital: Antibodies to glutamate decarboxylase 65 , GAD 6.5 ) are present in $80 \%$ of Caucasians patients with IDDM. The prevalence of (AADos antibodies $(A b)$ in IDDM ( $n=47)$, MRDM $(n=78)$, and NIDDM $(n=154)$ patients and 122 healthy controls from Cuttack in Eastern India. was determined. MRI) MI was subdivided into Protein deficient diabetes mellitus (PDDM)(n=52) and Fibrocalculous panereatic diahetes (FCPD) $(n=26)$. MRDM patients are typically young at onset with low body mass index. require insulin for glycemic control, have insulin resistance and do not develop ketosis on withdrawal of insulin. FCPD. but not PDDM, patients have abdominal pain and calculi in the pancreas. CAD65Ab was evaluated using a RIA with invitre translated recombinant human 35S-GAD65. We found GAD65Ab in $9 / 47(19 \%)$ IDDM $(p<0.001$ vs controls $;: 11.154$ i $7 \%$ ) NIDDM $(p=0.0 \%$ vs controls); 9/52 (17\%) PDDM ( $\mathrm{p}<0.001$ vs controls): $0 / 26(0 \%) \quad \mathrm{F}(\mathrm{PP})$ patients and $3 / 122(2 \%)$ healthy controls. The frequency of ( $\mathrm{AD} 65 \mathrm{Ab}$ in PDDM patients was not influenced by disease duration $(5 / 28.18 \%$, in 0.2 years: $1 / 6,17 \%$, in $3-4$ years: $2 / 12.17 \%$, in $5-10$ years and $1 / 6,17 \%$ in $>10$ years). In contrast. in IDDM, the frequency of GAD65A $\mathrm{b}$ was higher in patients with short-term $(0-2$ years $)(5 / 19,26 \%)$ than with fong-term duration $(>2$ vears) $(2 / 28,14 \%)(\mathrm{p}=0.45)$. In NIDDM patients diaunosed $<30$ vears. GAD65Ab were present in $\mathbf{5 / 2 8}(18 \%)$ as compared $7 / 76(9 \%)$ in $>30$ years old $(p=0.29)$. We conclude that patients from Cuttack in Eastern India. have GAD65Ab in IDDM at a low but significant frequency compared to Caucasians. Furthermore. GAD65Ab positive NIDDM patients $<30$ years duration are presumably slow-onset IDDM. The absence of GAD65Ab in FCPD suggest that it represents secondary diabetes. In contrast. an autoimmune pathogenesis may be important in PDIDM suggesting that protein deficiency may induce p-cell autoreactivity in susceptible individuals.

\section{2}

DEVELOPMENT OF DIABETES IN SUBJECTS MTH TROPICAL CALCIFIC PANCREATITIS (TCP)

A.K. Azad Khan', L. Ali', N.S. Chowdhury', Z Hassan', H. Rahman², S.K Saha ${ }^{2}$, A.Q.M. Mohsin ${ }^{1}$ and JMA Hannan'. 'Research Division, BIRDEM ${ }^{2}$ Department of Gastroenterology, IPGMR, Dhaka, Bangladesh.

As a part of an attempt to elucidate the etiopathogenesis of fibrocalculus pancreatic diabetes (FCPD), the serum levels of glucose, insulin and C-peptide were estimated in $24 \mathrm{TCP}$ (age: $21.83+5.91, \mathrm{M}+\mathrm{SD}$ ) and $15 \mathrm{FCPD}$ (age: $21.33+5.61$, $\mathrm{M} \pm \mathrm{SD}$ ) subjects. Islet Cell Antibodies (ICA) and Insulin Autoantibodies (IAA) were measured in 20 TCP and 8 FCPD cases. ERCP was performed in 19 TCP and 8 FCPD cases. Serum insulin, C-peptide and autoantibodies were estimated by ELISA. Highly significant difference was found regarding the $\mathrm{BM}$ of the two groups (TCP: $17.53 \pm 2.94$ Vs FCPD: $15.02 \pm 3.04, M \pm S D, p<0.015$ ). FCPD had about 2 times less fasting C-peptide (TCP: $1.27+0.60 \mathrm{Vs}$ FCPD: $0.69+0.50 \mathrm{ng} / \mathrm{ml}, \mathrm{M}+\mathrm{SD}$ ), 4 times higher fasting glucose (TCP: $4.42+0.99$ Vs FCPD: $14.89_{ \pm} 10.15$ mmoll, $\mathrm{M} \pm \mathrm{SD}$ ) and 3 times higher $2 \mathrm{hr}$ postprandial blood glucose (TCP: $7.13+2.19 \mathrm{Vs}$ FCPD: $23.09 \pm 10.53$ ) levels. The fasting C-peptidelglucose ratio also showed a marked difference (TCP: $0.30 \pm 0.15$ Vs FCPD: $0.09+0.11$ ). 5 out of 20 TCP $(25 \%)$ and 1 out of 8 FCPD $(12.5 \%)$ cases showed ICA positivity and 7 of the TCP $(35 \%)$ and 1 of the FCPD (12.5\%) subjects showed IAA positivity. When either of the two tests was considered $12(60 \%)$ TCP and $2(25 \%)$ FCPD subjects showed antibody positivity. No significant difference was found between the two groups regarding the autoimmune status. The percent of subjects showing mild, moderate and severe changes of chronic pancreatitis in ERCP scored by Axon criteria were $1(5.3 \%), 2$ $(10.5 \%)$ and $16(84.2 \%)$ in TCP and 0,0 and $8(100 \%)$ respectively in FCPD cases; these differences are not statistically significant. The results suggest that FCPD should not be considered simply as a secondary diabetes consequent to generalized pancreatic damage in TCP. 


\section{IDDM CLUSTERING: IMPLICATION FOR UNDERLYING} AETIOLOGICAL MODELS

G.R. Law, P.A. McKinney, R. Williams, F.E. Alexander, M.A. Kelly, H.J. Bodansky. Paediatric Epidemiology Group, University of Leeds, UK. Insulin-dependent diabetes mellitus (IDDM) in children has both a genetic and environmental actiology. The environmental factors involved are unidentified but one possible hypothesis suggests viral infection as a risk factor. Statistical methods detecting disease clustering will lead to better understanding of the underlying pattern of incidence of IDDM and may support viral associations. Three distinct methods of detecting disease clustering have been applied to a $97 \%$ complete population-based register of children (0-16) with IDDM in Yorkshire (Northern England) as follows 1. The Knox-Mantel (KM) test to detect space-time clustering.

2. The Potthoff-Whittinghill (PWa) test to detect spatial clustering between geographical areas in all risk strata.

3. The Potthoff-Whittinghill (PWb) test to detect spatial clustering between areas and within risk strata (age groups).

All three tests applied to 1490 children diagnosed 1978-90 provide evidence for significant clustering (test statistic standard normal deviate; $\mathrm{KM} \mathrm{z}=2.54$ $p=0.005, P W a z=1.92 p=0.028, P W b t=2.11 p=0.017$ ). Furthermore very significant clustering occurs in the youngest age group $(0-4$ year olds, $\mathrm{KMz}=3.31 \mathrm{p}<0.001, \mathrm{PWaz}=2.31 \mathrm{p}=0.010, \mathrm{PWbt}=3.20 \mathrm{p}=0.001)$. These results show increased incidence of IDDM occurring in the same area at the same time more frequently than expected by chance, particularly in the $0-4$ year olds. The application of the selected statistical tests is a novel approach to discern an underlying aetiological model. A viral hypothesis remains consistent with these results which show clear non-random patterns of distribution. Infections perinatally would provide a good model for explaining such clustering.

\section{5}

REGIONAL DIFFERENCES IN THE INCIDENCE OF INSULIN. DEPENDENT DIABETES MELLITUS (IDDM) AMONG CHILDREN IN FINLAND, 1987 TO 1991

Karvonen $M^{i}$, Rusanen $J^{\prime}$, Sundberg $M^{i}$, Virtala $E^{l}$. Colpaert $A^{2}$, Naukkarinen $A^{2}$, Tuomilehto $J^{\prime}$ for the DiMe Study Group

'National Public Health Institute, Mannerheimintie 160. FIN-00300 Helsinki

${ }^{2}$ Department of Geography, University of Oulu, FIN-90570 Oulu

The regional pattern of IDDM incidence in Finland was assessed applying several methods. The incidence and the Bayes relative risk (RR) for IDDM were calculated by municipality, by functional area, and (employing the GIS technique) by area of equal size population at risk among 1728 children aged 14 years or under diagnosed to have IDDM during the years 1987 to 1991 . The association of IDDM incidence with the degree of urbanization was assessed by the Spearman non-linear correlation analysis using the population density as a criterion of the urban/rural area status.

The overall mean incidence was 35 per 100,000tyear and the incidence within 460 municipalities varied from $4(95 \% \mathrm{CI} 0.1 .24 .6)$ to 245 ( $95 \% \mathrm{CI}$ $29.7 ; 885.4)$ per $100000 /$ year, without any regional pattern in incidence. The RR for IDDM ranged among municipalities from 0.82 to 1.34 and showed a clear spatial pattern in incidence. Among 20 functional areas the incidence of IDDM varied from $26(95 \%$ CI $15.8 ; 39.9)$ to 43 (95\% CI 36.3:50.3) per $100,000 /$ year and the RR for IDDM varied from 0.73 (95\% CI $0.47 ; 1.14)$ to $1.27(95 \% \mathrm{Cl} 0.90 ; 1.41)$. The incidence evalualed in four zones (south-north direction) was highest in the central part of the country. An inverse correlation between population density and the incidence of IDDM $\left(r_{\text {and }}=-\right.$ $0.73, p=0.05$ ) and also between the childhood population density and the incidence $\left(r_{\text {raak }}=-0.85, p=0.01\right)$ indicated that the incidence was higher in the less densely populated rural areas than in the more densely populated suburban and urban areas.

\section{4}

DETECTION OF ENTEROVIRUS RNA IN ADULT ONSET INSULIN DEPENDANT DIABETES MELLITUS.

C. Hober', D. Hober ${ }^{2}$, L. Andréoletti ${ }^{2}$, C. Allienne', S. Belaich', M.C. Vantyghem', D. Wattre ${ }^{2}$ J Lefebure'. (1) Service d'Endocrinologie t Maladies Métaboliques, USN-A, (2) Laboratoire de Virologie. CHRU, 59037 Lille Cedex, France

Enterovirus infection have been recently linked to childhood-onset insulin-dependant diabetes mellitus (IDDM). We evaluated the prevalence of enterovirus infection at onset of adult IDDM by using the detection of enteraviral RNA carried out in whole blood by a reverse transcription and a semi-nested polymerase chain reaction (Semi-nected RT-PCR). The set of primers used in enzymatic amplification step was selected for an annealing in the 5' untranslated genomic region which is highly conserved among the enterovirus group. The EDTA-treated whole blood was taken from 1) 12 newly diagnosed IDDM adult patients admitted to the hospital with ketosis including 7 patients with diabetic keto-acidosis (DKA) (age $28.2 \pm 10.4)$, 2) 12 patients with metabolic ketosis decompensation in the course of IDDM, including $7 \mathrm{DKA}$ (age $32.6 \pm 10.6)$; 3) 10 patients with metabolic decompensation in the course of NIDDM (age : $57.9 \pm 13.3$ ) and 4) 13 healthy adults (28.2 \pm 10.4). In patients with onset of IDDM, enterovirus was detected in 5 out of 12 patients ( $42 \%$ ) and 4 of these 5 patients had DKA (4 among 7 patients with DKA (57\%)). By contrast, enterovirus sequences detection was positive in only 1 out of 12 IDDM patients with ketosis décompensation $(8 \%)$ and negative in all NIDDM patients and healthy adults. This findings suggest a relationship between enterovirus infection and adult-onset IDDM

\section{6}

COW MILK AND BREAST FEEDING INVOLVEMENT IN THE DEVELOPMENT OF IDDM IN ISRAEL

A.Arbel, E.Sprecher, Z.Josefsberg, N.Weintraub, M.Karp and P.Vardi, SCMCI, Petah-Tikva, Israel.

In addition to major genetic effects, environmental factors seem to play a crucial role in triggering the process of beta cell autoimmunity in IDDM Recently, it was suggested that early introduction of cow milk and duration of breast feeding (BF) are involved in IDDM development Since in Israel the incidence of IDDM varies among the different ethnic groups $\left(8.1 \times 10^{5}\right.$ in Ashkenazi Jews, $5.7 \times 10^{5}$ in Sephardic Jews and $3.4 \times 10^{5}$ in $A r a b s$ ), we have initiated a large study in order to evaluate the role and interaction of genetic and non-genetic factors in this disease. As a part of a questionnaire, we obtained data concerning $B F$ and cow milk introduction in over 170 DDM patients (mean age 18 yrs \pm 12 s.d.) and in over 100 healthy controls $(16 \pm 7)$. We applied a logistic regression model to the group of Jewish patients ( $n=213,140$ patients) in order to control for the influence of ethnic subgroups (Sephardi and Yemenite in contrast to Ashkenazi), examine the influence of BF duration, time of cow milk introduction, and the interaction of ethnicity with feeding management. No significant difference emerged concerning any of these factors in this model. A second logistic regression model included and contrasted Arabs ( $\mathrm{n}=72,35$ patients) with Jews ( $\mathrm{n}=213,140$ patients) as a single group. No interaction of ethnicity with feeding factors emerged, however, this model, perhaps due to the larger number of subjects, indicated that earlier introduction of cow milk was significantly associated with development of IDDM (Wald chi-square $p<0.03$, Odds Ratio $=0.86 \mathrm{I}$ per month delay of first exposure to cow milk). In conclusion, our preliminary results suggest that in Israel, there is evidence that early introduction of cow milk induces susceptibility to IDDM across ethnic groups. No evidence was found to indicates that BF duration is associated with IDDM protection, or that ethnic groups differ in regard to this protective effect.

Supported in part by Camp N.Y.D.A. 
307

SUPLUS CONSUMPTION OF NITROSOAMINES IN MICE WITH GESTATIONAL DIABETES DECREASES SENSIBILITY TO STREPTOZOTOCIN IN OFFSPRING

J.Lementa, V.Poltorack. Ukrainian Researsh Institute of Endocrine Diseases Pharmacotherapy, Kharkov, Ukraine

We reported that gestational diabetes (GD) in mothers induced heightened reactivity to streptozotocin (STZ) in offspring. The aim of this study was to estimate the influence of the suplus consumption of nitrosoamines ( $\mathrm{SCN}$ ) by mice with GD on the sensibility to STZ in their offspring. Parental mice (CBA $\times$ C $57 \mathrm{BL} / 6 \mathrm{~J}) \mathrm{F}_{1}$ received $0.025 \%$ solution of sodium nitrite and amidopyrine in drinking water ad libitum the whole study. From the 46th day of the experiment the females were treated with STZ (40 mg/kg/day x 5 days i.p.) and 15 days after the $1 \mathrm{st} \mathrm{STZ}$ injection the mice were coupled. To characterize the sensibility to STZ in offspring of mice with GD treated (the 1st group) and nontreated with SCN (the 2nd group) the i.p.GTT was performed at 2 monthes of age (a month after a single injection a low dose STZ-35 mg/kg b.wt., i.p.). Our data revealed the decreased basal glycemia and the improved glucose tolerance in $(\mathrm{SCN}+\mathrm{GD})$ mice as compared to GD mice $(\mathrm{p}<0.001)$. The sensibility to STZ was enhanced in offspring of GD mice as compared to offspring of (SCN+GD) mice. The glycemic pattern in offspring of $(\mathrm{SCN}+\mathrm{GD})$ mice during i.p.GTT was like to control animals $(\mathrm{p}<0.001)$ in contrast to impaired one's in offspring of GD mice (blood glucose levels at $0,30,60,120 \mathrm{~min}$ after glucose load were respectively $3.2+0.4$ $\mathrm{mmol} / \mathrm{l}, 7.9+1.7 \mathrm{mmol} / \mathrm{l}, 5.3+0.3 \mathrm{mmol} / \mathrm{l}, 4.0+0.6 \mathrm{mmol} / \mathrm{l}$ in the $1 \mathrm{st}$ group and $6.8+0.6 \mathrm{mmol} / \mathrm{l}, 12.3+1.2 \mathrm{mmol} / \mathrm{l}, 10.6+0.7 \mathrm{mmol} / \mathrm{l}, 6.8+0.2 \mathrm{mmol} / \mathrm{l}$ in the 2nd group, $p<0.001)$. The diabetic index was decreased in offspring of the ist group in comparison with the 2 nd group $(1.8+0.5 \mathrm{vs}$. $5.1+0.5, p<0.01)$. We suggest that the suplus consumption of nitrosoamines in mothers with GD protects from the heightened sensibility to STZ development in offspring

\section{8}

INTRAUTERINE GROWTH PATTERN AND RISK FOR CHILDHOOD ONSET TYPE 1 (INSULIN-DEPENDENT) DIABETES

G Dahlquist, S Sandberg Bennich, B Källen

Department of Pediatrics, Umeá University, Umeå; Department of Internal Medicine. Uppsala University, Uppsala; Tornblad Instifute, University of Lund, Sweden.

The aim of the present study was to investigate weather prenatal growth affects the risk to develop childhood onset insulin-dependent diabetes mellitus (IDDM). Data from a total of 4585 diabetic children recorded in a nationwide childhood case registry and born after 1973 were linked with data from the nationwide Swedish Medical Birth Registry. For each IDDM child three control children were randomly selected among infants born the same year and at the same hospital as the proband. For all children data on birth weight, gestational duration, maternal age and parity, maternal diabetes diagnosis during pregnancy, number of previous spontaneous abortions, maternal smoking in early pregnancy and sex specific birth weight by gestational week expressed as a standard deviation score (SDS) were recorded. There was a clear trend in the odds ratio (OR) for childhood onset diabetes according to birth weight deviation in SDS. The OR ( $95 \%$ confidence intervals) for small gestational age $(<2 \mathrm{SD})$ after stratification for maternal age, parity, smoking habits and maternal diabetes was $0.81(0.65-0.99)$ and for large for gestational age ( $>2$ SD) after similar stratification was $1.20(1.02-1.42)$. High maternal age was associated with ant increased risk for childhood diabetes in the absence of maternal diabetes whereas young maternal age increased the risk for the child when the mother had diabetes. It is concluded that the intrauterine conditions affecting prenatal growth seem to also affect the risk to develop childhood diabetes in the way previously described for postnatal growth: a poor growth decreases and access growth increases the risk.

\section{9}

GUILLAIN-BARRE-LIKE SYNDROME AND DIABETES IN MICE INFECTED WITH ENCEPHALOMYOCARDITIS (EMC-M) VIRUS

T. Bock, U.J. Weber. B. Pakkenberg, P. Fredman, K. Buschard. Bartholin Instituttet, Kommunehospitalet, Copenhagen, Denmark, and Department of Neurochemestry, University of Göteborg, Göteborg, Sweden.

The aim was to examine the neurological manifestations in mice of infection with the diabetogenie EMC-M virus. Like polio virus, the EMC-M virus is an enterovirus of the picorna group. $105 \mathrm{BALB} / \mathrm{c}$ mice were infected and followed clinically. In 4 paretic and in 4 control mice the total number of alpha motor neurons in the spinal cord was estimated stereologically by the optical fractionator, and the size distribution was measured using the rotator in a vertical design. Diabetes in the form of hyperglycemia (20 $20 \mathrm{mmol} / \mathrm{L})$ and giucosuria was found in $33 \%$ of the animals, but $90 \%$ of the mice developed paresis beginning in the hind legs and sometimes progressing to the front legs like a second neuron paresis. The paresis developed within 4 to 10 days atter the virus inoculation, and the animals were sacriticed it badly attacked. Otherwise, the paresis remitted in about a week. The clinical, neurological manifestations in the mice resembled Guillain-Barré syndrome in humans. Nude mice did not develop diabetes or paresis, after EMC-M virus treatment. Hence, as presumed in humans, the Guillain-Barrélike syndrome in mice is dependent on a functional thymus-derived immune system even though it is virus-induced. Anti-sulphatide antibodies are seen in $65 \%$ of the human cases and were also found in the virus-infected mice. The motoric neurons of the anterior horn of the spinal cord were not reduced in number in the paretic mice and the distribution according to size was almost identical with that of control mice. indicating that the paresis may be due to changes in the peripheral part of the neuron, which again tits well with the common belief respecting the human disease. Thus. we have described an animal model which simultaneously displays diabetes and a Guillain-Barré-like syndrome.

\section{0}

METABOLIC AND CLINICAL EVENTS PRECEDING DIABETES MELLITUS ONSET IN CYSTIC FIBROSIS

D.Cucinotta, A.Di Benedetto, T.Arrigo, R.Scoglio, E.Di Cesare, F.De Luca and G.Squadrito.Departmentof InternalMedicine and Institute of Pediatrics, University of Messina, Italy.

Out of a cohort including 30 cystic fibrosis (CF) patients with baseline normal fasting blood glucose, annually checked for glucose tolerance and insul in secretion with the oral glucose tolerance test (OGTT), 7 subjects ( 3 males, mean age $21 \pm 3.9$ years) developed diabetes mellitus $5.1 \pm 1$ years after the beginning of the observation period. Metabolic and clinical parameters were retrospectively evaluated in these patients (study group) and in 7 age-matched subjects ( 4 males, mean age $21.1+2$ ), belonging to the same cohort, who did not develop diabetes during a superimposable (5.2 \pm 0.9 years) follow-up period (control group). In the study group glucose tolerance was impaired in all but one patients since the first OGTT and glucose areas progressively increased over time (from $18.5+2$ to $24+5.3$ $\mathrm{mM}, \mathrm{p}<0.025$ ), whereas in the control group glucose areas remained stable (from $15.5 \pm 2.4$ to $15.6 \pm 2.1 \mathrm{mM}$ ). A progressive blunting of insulin secretion occurred during the observation period in both groups (from $750 \pm 114$ to $354 \pm 138 \mathrm{pM}$ in the study group, from $930 \pm 320$ to $342 \pm 180 \mathrm{pM}$ in the control group; $\mathrm{p}<0.005$ ). Neither modification of glycosylated hemoglobin levels over time nor serum islet cell antibodies at diabetes diagnosis were observed in the study group. The overall clinical course of the disease (body mass index, nutritional status, pulmonary and pancreatic function tests) was not different in both groups and remained stable during the observation period. These results indicate that in CF diabetes mellitus onset is preceded by a long-standing deterioration of glucose tolerance whilst insulin secretion progressively declines over time, irrespectively of glucase tolerance status. The prediabetic worsening of glucose tolerance is not linked to a worsening of $C F$ clinical conditions. 
311

ASSOCIATIONS BETWEEN INFECTIONS DURING THE YEAR PRECEDING DIAGNOSIS AND ICA OR IAA IN DIABETIC CHILDREN AND SIBLINGS P.Vähäsalo, M.Knip, J.Karjalainen, R.Lounamaa, H.K.Akerblom and the Study Group on Childhood Diabetes in Finland. Department of Pediatrics, University of Oulu, Oulu, the Children's Hospital, II Department of Pediatrics, University of Helsinki, Helsinki, Finland

To study the relation between infections during the year preceding the diagnosis of IDDM in the index case and the occurrence of islet cell antibodies (ICA) or insulin autoantibodies (IAA) in IDDM families we analysed 781 probands ( $<15$ years) for ICA and 755 for IAA and 610 of their non-diabetic siblings (3-19-years) for ICA and IAA upon diagnosis of the proband. Data on the occurrence of infections were collected by means of a questionnaire. Infections during the preceding year were observed in $73.4 \%$ of the diabetic children and in $55.9 \%$ of their siblings, the frequency of respiratory infections being $57 \%$ and $54 \%$ and that of gastroenteritis $21.5 \%$ and $20.2 \%$, respectively. ICA were observed in $84.1 \%$ and IAA in $46.8 \%$ of the probands, and in $7.5 \%$ and $2.6 \%$. of the siblings. The index cases with a history of ane or more respiratory infections during the year preceding their diagnosis more often had detectable ICA $(86.9$ vs. $81.3 \%$; Cl for the difference $-7.8-189 \%$; $\mathrm{p}=0.04), \mathrm{ICA} \geq 20$ JDFU ( 69.8 vs. $61.3 \% ; \mathrm{Cl}$ for the difference $-3.2-20.3 \% ; \mathrm{p}=0.02)$ and IAA ( 51.0 vs. $42.6 \% ; \mathrm{CI}$ for the difference $-1.7-18.5 \% ; \mathrm{p}=0.03$ ) than did the others. Likewise the index cases with at least one episode of gastroenteritis during the preceding year more often tested positive for ICA $\geq 20$ JDFU ( 74.9 vs. $64.1 \%$; CI for the difference $-4.3-25.9 \% ; p=0.01$ ) than the remaining probands. ICA and IAA also occurred together more often among those index cases with one or more respiratory infections than among those without (44.8 vs. $36.3 \%$; $\mathrm{Cl}$ for the difference $-0.7-17.7 \% ; \mathrm{p}=0.02$ ). The siblings with one or more respiratory infections during the preceding year tested positive for IAA more often than those without ( 3.5 vs. $1.1 \% ; \mathrm{Cl}$ for the difference $0.0-4.9 \% ; \mathrm{p}=0.05$ ). The findings indicate that infections, especially respiratory infections during the preceding year increase the frequency of both ICA and IAA in diabetic children at diagnosis and that respiratory infections increase the prevalence of IAA in the siblings. These observations support the view that viral infections may induce and/or promote $\beta$-cell destruction in individuals with an increased risk of progressing to IDDM.
312

ENVIRONMENTAL TOXINS AFFECT THE REACTIVITY OF IMMUNE CELLS FROM NON-OBESE DIABETIC MICE

Radons. J., Burkart, V. and Kolb, H., Diabetes Research Institute at the University of Düsseldorf, D-40225 Düsseldorf, Germany

Environmental toxins such as superantigens have been implicated in the pathogenesis of type I diabetes mellitus. We therefore tested the hypothesis of an abnormal reactivity of the immune system towards superantigens during the prediabetic phase. For this purpose, splenocytes from diabetes prone female NOD mice were exposed to two well characterized superantigens, staphvlocaccal aureus enterotoxin-B (SEB) and toxic shock syndrome toxin-1 (TSST-1). Cells from $\mathrm{BALB} / \mathrm{c}$ and $\mathrm{C} 57 \mathrm{BL} / 6$ mice were used as normal controls. After $72 \mathrm{~h}$ of coculture with the superantigens or the mitogen concanavalin A (Con A), the proliferative response and mitochondrial activity were determined. In the culture supernatants the cytokines IFN- $\gamma$ and IL-10 were measured. At $40 \mathrm{~d}$ of age NOD splenocytes displayed a severely impaired response to $S E B$, a partially response to TSST-1, but a normal reactivity to Con A. At $70 \mathrm{~d}$ of age NOD splenocytes had retained complete refractoriness to SEB but had converted to an abnormally high IFN- $\gamma$ and IL-10 response towards TSST-I, despite a normal proliferative and metabolic response. The cytokine response to Con $A$ was biased towards IFN- $\gamma$. Since IFN- $\gamma$ and IL-10 are crucial disease promoting or protecting mediators in prediabetic NOD mice, any contact with environmental superantigens will affect disease progression. It is important to note that immune cells from prediabetic NOD mice respond differently to common environmental stimuli than immune cells from control strains.

\section{3}

THIS ABSTRACT HAS BEEN WITHDRAWN BY THE AUTHOR. 
PS 4

\section{Prediction of IDDM}

\section{4}

THE TYROSINE PHOSPHATASE-LIKE DOMAINS OF IA-2 AND A RELATED MOLECIUE ICA37 ARE TARGETS OF ISLET ANTIBODIES

$V$. Lampasona, M. Bearzatto, and E. Bonifacio. Departments of Laboratory Medicine and Interr al Medicine, Istituto Scientifico San Raffaele, Milan, Italy

The protein tyrosice phosphatase(PTP)-like molecule IA-2 is a major autoantigen in insul $n$ dependent diabetes (IDDM). The aim of this study was to characterise autoantibody reactivity against the $\mid A-2$ molecule and to determine whether reactivity extends to other PTPS. We tested serum reactivity in 80 new onset IDDM patients against the full length IA-2 protein, the IA-2 ectodomai , juxtamembrane(JM) region, and PTP-like domain, the PTP-like domain 0 a novel islet protein ICA37, and the PTP domains of HPTP $\beta$, HPTPd. A tibody reactivity was determined by immunoassay of ${ }^{35}$ S]methionine-labilled in vitro transcribed and translated proteins. Reactivity against 1 7-2 was confined to epitopes in the JM and the PTP-like domains: $17 / 80$ ney onset IDDM sera had antibodies to both the IA-2 PTP. like and JM domains, $18 / 80$ to only the PTP-like domain, and $8 / 80$ to the JM region only. The PTP-like domain of ICA37 was cloned from human islet RNA. DNA sequence analysis showed $>80 \%$ homology to IA-2. Antibodies to the PTP-like domain of ICA37 were found in sera from $26(32 \%)$ patients, including 25/35 wit! IA-2 PTP-like domain antibodies and one serum with antibodies against the JM region, but not the PTP-like domain of IA-2. Reactivity against 'he PTP-like domain of ICA37 was not found in IDDM sera without antiboties to IA-2 or control sera. No reactivity was observed against the PTP dcmains of HPTP $\beta$ or HPTPS. These data suggest that 1 autoantibodies in I[IDM recognise determinants which are shared between IA-2 and ICA37; 2 . both IA-2 and ICA37 have unique autoantibody epitopes within their PTP-lik 3 domains; and 3 . islet autoantibody reactivity does not extend to less homologous PTPs.

\section{6}

MAPPING OF EPITOPES FOR AUTOANTIBODIES TO IA-2 IN TYPE I DIABETES.

E.C.I. Hatfield, M.A. Payton and M.R. Christie. Dept. of Medicine, King's College School of Medicine and Dentistry, London, U.K. Patients with Type 1 diabetes possess antibodies to the cytoplasmic domains of two closely related tyrosine phosphatase-like proteins, IA2 and phogrin, previously detected as $40 \mathrm{kD}$ and $37 \mathrm{kD}$ tryptic fragments, respectively. The majority of patients with IA-2 antibodies also have antibodies to phogrin, but a proportion are positive for IA-2 antibodies alone. Since immune recognition of phogrin might arise through cross-reactivity with the highly homologous IA-2, we have investigated the major regions of $I A-2$ recognized by antibodies in phogrin antibody positive $(n=26)$ and negative $(n=12)$ IDDM patients. Analysis of antibody binding to in vitro transcribed and translated polypeptides representing different regions of the cytoplasmic domain of IA-2 identified five different patterns of reactivity with antibodies in IDDM. Protein footprinting analysis, where polypeptide fragments generated on protease treatment of immune complexes are studied, also indicated considerable heterogeneity in antibody recognition of IA-2, particularly at the C-terminus of the molecule. The majority (62\%) of phogrin antibody positive patients were able to bind a polypeptide representing amino acids $643-937$ of IA-2, whereas only $16 \%$ of phogrin antibody negative patients bound this fragment. Almost all $(90 \%)$ of phogrin antibody negative patients bound epitopes either in the region immediately adjacent to the transmembrane domain, or at the $\mathrm{C}$ terminus of the molecule. The results demonstrate that regions recognized by IA-2 antibodies in IDDM are heterogeneous and that there are differences in antibody recognition of IA-2 between phogrin antibody positive and negative IDDM patients. Cross-reactive epitopes on IA-2 and phogrin may be localized predominantly within the central part of the cytoplasmic domains of these molecules.

\section{5}

IFNช-SECRETION, HLA-DQB 1 * ALLELES AND REACTIVITY TO GAD65 IN PATIENTS WITH AUTOIMMUNE POLYENDOCRINE DISEASE TYPE 1 . P.Klemetti, P.Björses, T.Tuomi, J.Ilonen, A.Hinkkanen, J.Perheentupa, O.Vaarala. Children's Hospital, University of Helsinki, Department of Biochemistry, National Public Health Institute, Helsinki, Turku Immunology Centre and the Department of Virology, University of Turku, FINLAND. Humoral immunity to GAD65 is frequently seen in patients with autoimmune polyendocrine disease type I (APD I) although insulin-dependent diabetes mellitus (IDDM) is found only in a small subgroup of patients. We tested proliferation of peripheral blood mononuclear cells (PBMC) from patients with APD I to GAD65 and its relation to GAD65 antibodies, to HLA$\mathrm{DQB}^{*}$ risk alleles for IDDM, and to the secretion of IFN $\gamma$ by GAD65 stimulated PBMC. IDDM had been diagnosed in 4 of 44 patients. Cellular response (SI>3) to GAD65 $(10 \mu \mathrm{g} / \mathrm{ml})$ was found in 15 of 44 patients with APD I (34\%) and in 3 of 28 controls $(11 \%)\left(\mathrm{p}=0.03 ; \chi^{2}\right.$ test). GAD65 antibodies were seen in 14 of 44 patients $(32 \%)$. Negative correlation ( $r=-$ $0.4338, p=0.03$ ) was found between the levels of antibodies and the SIs against GAD65 among patients who responded to GAD65 (SI>3 or Ab>5). Cellular immunity to GAD65 was associated with HLA DQB $1 * 0201$ allele ( $p=0.03 ; \chi^{2}$ test), whereas GAD65 antibodies did not associate with HIADQB1* alleles. IFN $\gamma$-secretion was measured in the supernatants of GAD65 (1 $\mu \mathrm{g} / \mathrm{ml}$ ) stimulated PBMCs. Increased IFN $\gamma$-secretion ( $>50 \mathrm{pg} / \mathrm{ml}$ ) was found in 16 of 28 patients tested $(57 \%)$ of whon 9 patients did not have proliferative response to GAD65. In controls, low IFN $\gamma$-secretion was detectable only in two of 19 tested subjects $(11 \%)$. Coexistence of high levels of antibodies and the proliferative response to GAD65 or increased IFNy secretion was rare. Our results confirm the previous findings that autoimmunity to GAD65 may be reciprocally regulated between cellmediated and humoral immunity.

\section{7}

AUTOANTIBODIES TO TYROSINE PHOSPHATASES IA-2 AND IA-2B IN INSULIN-DEPENDENT DIABETES MELLITUS

J. Seissler, N.G. Morgenthaler, M.S. Lan', A.L. Notkins', W.A. Scherbaum. Department of Internal Medicine III, University of Leipzig, Germany and 'Laboratory of Oral Medicine, NIH, USA.

Autoantibodies to 40,000 and $37,000 \mathrm{Mr}$ tryptic islet cell fragments are major target antigens in insulin-dependent diabetes mellitus (IDDM). Recently, the antigens have been identified as members of the tyrosine phosphatase family, IA-2/ICA512 (40kD) and IA-2ß/phogrin (37kD), which are highly homologeous in the immunoreactive intracytoplasmic domains. Autoantibodies were studied in patients with new-onset IDDM (age 1-48 years), prediabetic individuals and in patients with other autoimmune endocrinopathies (Hashimoto's thyroiditis, Graves'disease, Addison's disease, pernicious anaemia) using in vitro translated ${ }^{35}$-methionine labelled proteins (intracytoplasmic human recombinant IA-2 and mouse recombinant $I A-2 B$ ) Using radioligand assays antibodies to IA-2 and antibodies to $I A-2 B$ were detected in $78(67,8 \%)$ and $54(45,8 \%)$ of 120 patients with new-onset IDDM Of 7 prediabetic individuals studied 7-30 months before the onset of IDDM all were positive for anti-IA-2 and 6 subjects had also anti-IA-2B. In contrast, only one of 100 patients with other organ-specific autoimmune diseases had anti-IA2 and anti-IA-2B and 100 normal controls were negative for both antibodies. A strong association was found between the presence and the levels of antibodies to IA-2 $\beta$ and antibodies to IA-2 $(p<0.001)$. Only one individual had antibodies which exclusively react with the IA-2 $\beta$ molecule. Interestingly, 17 double positive sera were observed in which the binding to IA-2B were considerably higher compared to the binding to IA-2.

The study demonstrates a heterogeneous humoral autoimmune response directed to tyrosine phosphatases IA-2 and IA2 $\beta$ in IDDM. Both anti-IA-2 and anti-IA2 $\beta$ represent diabetes-specific markers which may be useful for the prediction of IDDM. 


\section{8}

QUANTITATIVE DETERMINATION OF GLUTAMATE DECARBOXYLASE (GAD65) IN SERUM AS AN EARLY MARKER FOR ß-CELL DESTRUCTION? M. Schlosser, B. Ziegler, P. Augstein and M. Ziegler. Institute of Diabetes "Gerhardt Katsch", Karisburg, University of Greifswald, Germany

Autoantibodies to the $65 \mathrm{kD}$ isoform of glutamate decarboxylase (GAD-AAbs) are detectable of high prevalence in sera at recent onset IDDM and in prediabetic stage. It is proposed that the GAD-AAbs are secondarily generated against released B-cell antigens, so that the detection of GAD65 in sera might be an earlier marker for the $\beta$-cell destruction than the GAD-AAbs. Thus the study was aimed to investigate the in vitro half time $\left(t_{1 / 2}\right)$ of GAD65 in human serum and the in vivo $t_{1 / 2}$ in the circulation of LEWIS rats as well as to detect GAD65 in sera of rats after ß-cell destruction by streptozotocin. GAD65 was measured by a sensitive ELISA based on two monoclonal antibodies with different epitope specificities to GAD65 which allows to quantify $30 \mathrm{pg} / \mathrm{ml}$ (i.e. $0.023 \mathrm{fmol} /$ well) corresponding to 3,000 rat beta cells only. The GAD65 content of 1,000 human islets amounted to 78 ng compared to $43.7 \mathrm{ng}$ in LEWIS rat islets and $37.4 \mathrm{ng}$ in $\mathrm{BB} / \mathrm{OK}$ rat islets. To determine $\mathrm{t}_{1 / 2}$ of GAD65 in human serum, a pig cerebellum extract $(2,772 \mathrm{ng}$ GAD65/mal) was preincubated with serum, plasma and biood at $37^{\circ} \mathrm{C}$ and $4{ }^{\circ} \mathrm{C}$ and assayed by the ELISA. In serum, plasma and blood $t_{1 / 2}$ was comparable and amounted to $90 \mathrm{~min}$ at $37^{\circ} \mathrm{C}$. After $24 \mathrm{~h}$ incubation at $37^{\circ} \mathrm{C}$ only $3.9 \%(108 \mathrm{ng} / \mathrm{ml})$ were detectable in contrast to $702 \%(1,947 \mathrm{ng} / \mathrm{ml})$ after incubation at $4{ }^{\circ} \mathrm{C}$. Furthermore, after i.v. application of $2,000 \mathrm{ng}$ of human recombinant GAD65 into LEWIS rats $(\mathrm{n}=3) \mathrm{t}_{1 / 2}$ was reached after $3 \mathrm{~h}$ in all animals. After $24 \mathrm{~h}$ no GAD65 was detectable in the rat sera The data were confirmed by i.v. application of the pig cerebellum extract. Additionally, adult LEWIS rats $(\mathrm{n}=4)$ received one application of $50 \mathrm{mg}$ streptozotocin / $\mathrm{kg}$ body weight and the serum GAD65 content was analyzed after 8,24 and $48 \mathrm{~h}$. However, no GAD 65 could be detected in the circulation of animals although $B$ cells are completely destroved. In conclusion, it is unlikely to quantify GAD65 as an additional and earlier marker of B-cell destruction. Furthermore, it is questionable to quantify GAD65 in patients after islet transplantation as a marker for the rejection of the transplant.

\section{9}

SENSITIVTTY OF GAD65 AND ICAS12 AUTOANTIBODIES AS DISEASE MARKERS IN A POPLIATTON OF YOUNG NNSULIN-DEPENDENT DIABETIC PATIENTS

M.M. Zanone, M. Pierropaolo*, M. Peakman*, S. James*, C. Sacehetti**, R. Quadri, L. Chiandussi, M. Trucco* and F. Cenutti**. Depts. of internal Medicine and **Paediatrics, Univ. of Torino, Italy; *Dept. of Immunogenetics, Children's Hospital, Pittsburgh, USA.

Autoantibodies to islet autoantigens, glutamic acid decarboxylase (GAD65) and tyrosine phosphatase ICA512, measured in combination, have been proposed as predictive markers of IDDM. To analyse the potential for screening children with these markers, we examined their sensitivity in identifying IDDM in a population of 37 children at diagnosis of IDDM (mean (SD) age 7.4 (4.1) years). We also examined the natural history of the autoantibodies amongst childhood diabetics in 91 adolescents with IDDM (age 14.7 (1.6) years; mean duration of IDDM 7 (3.5) years). 30 normal adolescents (age 14.4 (1.9) years) without family history of IDDM were the control group. GAD65 and ICA5I2 autoantibodies were assessed by a quantitative radioimmunoprecipitation assay, using in vitro transcribed/translated proteins and expressed as indices derived using standard positive and negative sera. GAD65 and 1CA512 autoantibodies were detected in $49 \%$ and $62 \%$ of newly diagnosed IDDM children. $76 \%$ had at least one autcantibody, but the autoantibodies occurred together in only $35 \%, 1 / 30$ of controls (3\%) had GAD65 autoantibodies and another control (3\%) had ICA512 autoantibodies. GAD65 and ICA512 autoantibodies were present in $44 \%$ and $45 \%$ of diabetic adolescents, but the mean ICA512 index was significantly higher amongst the newly diagnosed patients $(p<0.001)$. At diagnosis, levels of GAD65 autoantibodies correlated with age $(\mathrm{p}=0.05)$, and in the adolescent patients levels of both autoantibodies declined with diabetes duration $(\mathrm{p}<0.001)$. Only the presence of ICA512 was significantly associated with ICA ( $\mathrm{p}<0.05)$, and mean ICA512 index was higher in ICA positive than in ICA negative patients, in both diabetic groups ( $\mathrm{p}<0.05$ for newly diagnosed patients, and $\mathrm{p}<0.005$ for diabetic adolescents). Our findings indicate that positivity for either GAD65 or ICA512 autoantibodies is a highly sensitive marker of IDDM in the paediatric age group, while positivity for both has a diagnostic sensitivity of $35 \%$

\section{0}

DIABETIC PATIENTS WITH HIGH GADG5 INDEX

$M$ Landin-Olsson. A Falorni. $\AA$ Lemmark and the DISS-group (Diabetes Incidence Study in Sweden). Dept of Medicine, University Hospital, Lund. Sweden

At onset most IDDM patients have antibodics against GAD65. It is not clear whether these antibadies are directed against one or several different epitopes on the GAD65 protein. The aims were to study the reactivity of high GAD65Ab sera against GAD67. a GAD65/67 chimer and the association with ICA. During two years, all diabetic patients in the ages $15-34$ years were sampled at diagnosis $(n=798)$ on a population based level in Sweden. Antibodies against human GAD65 were measured in a radioimmunoprecipitation assay. All samples were analysed in dilution 1:25 and an index was calculated in relation to a positive and a negative control. All samples with an index $>1 \quad(n=73)$ were further analysed in dilution 1:250 and 1:1000. The GAD65/67 chimer consisted of the main part of GAD67 (a) 1-445) and the 149 last aminoacids in the C-terminal end of GAD65 (aa 437-585). The antibody levels against the GAD65/67 chimer and GAD67 were analysed in all the high GAD samples $(n=73)$. The results show that median GAD65 index rose significantly with each difution from 1.2 to 6.7 to 13.4 , in dilution $1: 25,1: 250$ and $1: 1000$ respectively. All except two of the 73 samples were highly positive for the GAD65/67 chimer but mostly negative for GAD67. Despite the high GAD levels in these samples. $18 \%$ were negative for ICA. In conclusion, this study shows that among newly diagnosed young adult diabetic patients about $10 \%$ have very high levels of GAD65-antibodies. A main epitope in the C-terminal end of the GAD65 protein seems to be common for diabetic patients. With modification of the antigen of the GAD 65 assay, the specificity of the GAD65 antibody test maybe increase. The concordance of GAD65 antibody and ICA positivity was not influenced by the GAD65-antibody level.

\section{1}

A HIGHLY SENSITIVE AND SPECIFIC IMMUNORADIOMETRIC ASSAY FOR HUMAN INSULIN USING MONOCLONAL ANTIBODIES

E. Siohan!'. S. Allauzen'? A Demuynck', M. Rousset'. M. Laprade'. M. Bouanani". B. Pau'? P.Y. Marquet' ('SANOFI Diagnostics Pasteur, 3 R. Poincaré. 92430 Marnes La Coquette. France / 'CNRS UMR 9921. UFR Pharmacie, 34060 Montpellier, France)

A panel of highly specific monoclonal antibodies (MAbs) directed against human insulin was producing via a novel immunization procedure. Seven MAbs were purified and caracterized. The antigenic sites on the insulin molecule recognized by these MAbs were mapped, using different immunoanalysis methods (RIA, ELISA. BIAcore ${ }^{T M}$ ). Three or four antigenic regions were defined on the antigenic surface of insulin. presumably depending on the presentation of the molecule in the different immunoassay procedures. Three overlapping regions were defined on the adsorbed form of the molecule. using a competitive inhibition ELISA. Region I and III were found to be distinct, whereas region II overlapped the two other regions. Four overlapping regions were defined on the molecule in solution using a sandwich ELISA. Region I and III were found to be distinct, as observed when the insulin was adsorbed onto the microplate, but the reactivity of group II Mabs was dependent on the presentation of the molecule. These same results have been obtained with the BIAcore ${ }^{\mathrm{TM}}$ method. Moreover, a synergistic effect was observed between MAbs of group I and Mabs of group III when binding to insulin. Therefore, these MAbs are particularly suited for use in an ultrasensitive immunoradiometric assay (IRMA)

Method : $50 \mu \mathrm{l}$ of sample or standard insulin solution and $300 \mu \mathrm{l}$ of ${ }^{125}$ I-labelled $\mathrm{MAb}$ (19) were incubated in polystyrene tube coated with the capture MAb (PlO). After two incubating hours at room temperature with agitation, the tubes were washed and the radioactivity was measured. The dilution recovery and the evaluation of teproducibility have demonstrated the efficacy of the test. The use of synergistic monocional antibodies results in a very low detection limit for this assay. This detection limit is $0.2 \mu \mathrm{UI} / \mathrm{ml}(8 \mathrm{pg} / \mathrm{ml})$. No cross reactivity was observed with proinsulin $\{0$ to $200 \mathrm{\mu g} / \mathrm{ml}$ ) and with C-peptide $(0$ to $200 \mathrm{ng} / \mathrm{ml}$ demonstrating the specificity of this IRMA. In conclusion, this highly sensitive and specific immunoassay could greatly improve our ability to assess diabetic status. 
AN AUTOMATED IMMUNO-CHEMILUMINOMETRIC INSULIN ASSAY FOR THE ACCESS® IMMUNOASSAY SYSTEM J.P. Saintouil ${ }^{1}$, G. Klee ${ }^{2}$, B. Furlow ${ }^{2}$, C. Shellum ${ }^{3}$ and T. Smith ${ }^{3}$

Sanofi Diagnostics Pasteur, 3 R. Poincaré, 92430 Mames La Coquette, France

Mayo Clinic and Mayo Foundation, Rochester, MN 55905, USA.

${ }^{3}$ Sanofi Diagnostics Pasteur, Chaska, MN 55318, USA.

We have developed a sensitive and specific two-site insulin immunoassay for the Access(e) automated immunoassay system. The assay is standardized to the WHO $66 / 304$ insulin standard. A $20 \mu \mathrm{l}$ serum sample is combined with monoclonal antiinsulin antibody coupled to paramagnetic particles and a second monoclonal antiinsulin antibody conjugated to alkaline phosphatase. The mixture is incubated for 30 minutes followed by particle washing and addition of chemiluminescent enzyme substrate. The time to the first result is 40 minutes and 100 samples can be assayed per hour.

We studied precison by calibrating the instrument and running Biorad Lyphochek controls in duplicate twice per day for two weeks. We obtained overall CVs of 5.1, 4.3 and $3.9 \%$ at levels of $3.4,31$, and $69 \mu \mathrm{JU} / \mathrm{ml}$ respectively. We studied withinrun precision at low concentration by running 10 replicates at concentrations of $0.25,0.13$, and $0.063 \mu \mathrm{IU} / \mathrm{ml}$. The CVs at the respective levels were $7.2,6.7$, and $12.5 \%$. The analytical sensitivity based on $2 \mathrm{SD}$ from the zero calibrator was 0.03 $\mu \Pi U / \mathrm{ml}(0.21 \mathrm{pmol} / \mathrm{l})$. Comparison of the test results obtained from 95 serum sarnples measured concurrently on the ACCESS $($ ) (y) and the Abbott IMx $(x)$ yielded a linear regression of $\mathrm{y}=1.10 \mathrm{x}-1.3 \mu \mathrm{IU} / \mathrm{ml}, \mathrm{r}=0.97$. Dilution recovery was studied by diluting serum samples into the zero calibrator. The average recovery for six patients was $103 \%$ (range of $89-111 \%$ ). Spike recovery was studied by spiking insulin into serum samples at levels of $25,50,100$ and 200 $\mu \pi \mathrm{J} / \mathrm{ml}$. The average recovery for 5 patients was $102 \%$ (range of $98-104 \%$ ). Cross reactivity to proinsulin was studied by spiking 5 patient sera having endogenous insulin concentrations ranging from $20-50 \mathrm{pmol} / \mathrm{l}$ with $140,000 \mathrm{pmol} / \mathrm{l}$ of proinsulin. The proinsulin cross reactivity was less than $0.03 \%$. The combination of good performance characteristics and full automation make this assay an excellent tool for insulin analysis.

\section{3}

ISLET CELL AUTOANTIBODIES (GADab, ICA, IAA) IN RELATION TO HLA-TYPES IN THE DANISH DIABETIC TWIN REGISTER

K.O.Kywik, H. Beck-Nielsen, P.J. Bingley, T. Dyrberg, E.M.A. Gale, A Green, J.S. Pedersen. Odense University, Denmark, St. Bartholomews Hospital, UK. Zymo Genetics, USA.

In a study based on the young cohort of the Danish population based twin register we have previously shown that approximately $70 \%$ of both monozygotic and dizygotic healthy twin partners of twins with insulindependent diabetes are positive for at least one islet cell autoantibody (GADab, ICA or IAA). Furthermore, the prevalence of each antibody was at least twice as high as in ordinary first degree relatives. Antibody positivity thus seems to be a function of the twin status and possibly environmentally rather than genetically determined. To further elucidate this we have analysed the relationship between antibody status and HLA types. For fifty-four twin pairs (19 monozygotic and 35 dizygotic pairs) both antibody status and HLA types (DRB1, DQA1, DQB1) were available and the twin individuals were stratified according to whether they had zero, one or two high-risk HLA-types (defined as the haplotypes DR3-DQA1*0501-DQB1*0201 and DR4-DQA1*0301$D Q B 1 * 0302$ ). The analysis was done for healthy vs diabetic twin individuals, both pooled and stratified for zygosity. There were no relationship between either islet cell antibody alone and having zero (only dizygotic pairs), one or two high risk HLA haplotypes. The relationship between being positive for one, two or three islet cell antibodies were not related to number of high risk haplotypes either. Thus there is no dose relationship between number of high risk HLA haplotypes and islet cell autoimmunity. Our previous suggestion that autoimmunity is environmentally determined is further supported by this finding.

\section{4}

COMBINED DETECTION OF GAD65 AND IA2 AUTOANTIBODIES IN IDDM: A MAJOR STEP TOWARDS REPLACEMENT OF THE HISTOCHEMICAL ISLET CELL ANTIBODY (ICA) TEST

W. Richter, R. Hartmann, U.W. Wiest, , U. Hartmann and B.O. Böhm; Department of Internal Medicine 1, University of Ulm, UIm, Germany

Despite the technical difficulties encountered by the classical ICA test of indirect immunofluorescence on human pancreatic tissue and the uncertainty which antigens compose the targets of ICA, most studies on serological diagnosis and prediction of IDDM were based on this marker. Autoantibodies to GAD65 (GAD65-A) and IA2 (IA2-A) were shown to contribute to the ICA staining seen on tissue sections and we asked whether combined detection of these two subspecificities of ICA can replace the histochemical ICA test. We established radioimmunoassays for separate detection of autoantibodies to GAD65, full length $\mid A 2$, the cytoplasmic part of IA2 $\left(I A 2_{c}\right)$ and for combined detection of GAD65-A and $\mid A 2_{-}-A$ in one step. Characterization of sera from patients with IDDM, other organ-specific autoimmune diseases, and with polyendocrine autoimmune syndrome by the separate tests demonstrated a completely different antibody profile regarding frequency and titre of GAD65- and IA2-A in these autoimmune diseases. $\mid A 2_{c}-A$ appeared in much closer correlation with IDDM than GAD65-A and were, therefore highly specific for IDDM. GAD65-A and ICA, in contrast, were of higher sensitivity for IDDM. $94.6 \%$ of the ICA-positive and $53 \%$ of the ICA-negative IDDM sera but only $0.08 \%$ of the 695 normal control sera were scored positive by combined detection of GAD65-A and $\mid A 2_{c}-A$. This quantitative, reproducible and generally available assay, therefore, improved the diagnostic sensitivity and specificity compared to ICA detection and represents a major step towards replacement of the classical ICA test. Analysis of sera from 204 first degree relatives of IDDM patients demonstrated that this test allows cost-saving and most effective large scale screening of sera for prediciton of IDDM.

\section{5}

COMPARISON OF ICA TITERS OF NEW-ONSET DIABETIC PATIENTS USING TRFI- OR CONVENTIONAL ICA ASSAY.

M.Rulli, H.Kojola and O.Simell, Department of Pediatrics and MediCity Research Laboratory, University of Turku and Wallac Oy, Turku, Finland

Conventional immunohistochemical ICA assay uses serum dilutions and classical fluorochromes, where tissue autofluorescence and subjective judgement of the titration end point cause problems. In the new time-resolved fluorescence imaging (TRFI) assay the $\mathrm{Eu}^{3+}$-chelate labeled anti-human IgG and time-resolved fluorescence imaging of the specific signal are used. The $\mathrm{Eu}^{3+}$-chelate signal, which has longer decay time than the conventional fluorochromes $(1000 \mu \mathrm{s}$ vs. $100 \mathrm{~ns})$, is detected after decay of the autofluorescence. Serum dilution is unnecessary as the islet specific fluorescence intensity is digitally measured and analyzed. The sera having close ICA titers in conventional assay, had different specific fluorescence intensities of undiluted sera in TRFI assay and slopes of the dilution curves varied markedly. Fourty six sera of new-onset diabetic children were analyzed used TRFI and conventional ICA assay. One child in TRFI assay $(2.2 \%)$ and 6 children $(13.0 \%)$ in conventional assay had negative ICA titer. The ICA titers measured by the new assay were on the average 66 JDFU higher than the titers of the conventional assay $(p=0.0001)$. We conclude that the TRFI assay is more specific and objective compared to the conventional ICA assay. 
AUTOANTIBODIES TO GLUTAMIC ACID DECARBOXYLASE AND ICA512/IA-2 DISPLAY DISTINCT HLA ASSOCIATIONS

TB Rambrand, BK Michelsen, F Pociot, M Knip, KS Rønningen, T Dyrberg, JS Petersen, J Nerup, P Kuimala, J Ilonen, HK Åkerblom, the Childhood Diabetes in Finland Study Group, and the Danish Study Group of Diabetes in Childhood. Hagedorn Research Institute, Denmark; Steno Diabetes Center, Denmark; University of Oulu, Finland; The National Hospital, Norway; NovoNordisk A/S, Denmark

Autoantibodies to glutamic acid decarboxylase (GAD) and ICA512/A-2 are serologic markers of insulin-dependent diabetes mellitus (IDDM), while major genetic contributions are mediated by HLA-DQ genes. To investigate the association between HLA-DQ alleles and autoimmunity to GAD65 and ICA512/1A-2, we examined sera from 427 Danish IDDM multiplex family members, 92 Finnish siblings with new onset IDDM, and 44 healthy subjects in a radioligand immunoprecipitation assay using in vitro translated human recombinant ${ }^{35}$ S-methionine labeled GAD65 or ICA512//A-2 as antigens. Median duration of IDDM in Danish families was 12.3 years at the time the samples were obtained. 61\% (121/198) of Danish IDDM patients had autoantibodies to GAD65, while $48 \%$ (95/198) immunoprecipitated ICA512/IA-2. Of the Finnish diabetic siblings 62\% (57/92) had GAD65 autoantibodies while $80.3 \%$ (74/92) were positive for ICA512/IA-2. The prevalence of GAD65 autoantibodies decreased significantly in individuals with a duration of IDDM exceeding 5 years $(\mathrm{P}<0.002)$, and was higher in patients with IDDM onset after 10 years. Significantly more Danish sib pairs than expected were concordant for ICA512/IA-2 ( $\mathrm{P}=0.02)$. This is probably due to the contribution of the HLADOB $1 * 0302$ allele, which was highly increased in patients with autoantibodies to ICA512/IA-2 $(\mathrm{P}<0.0001)$. In contrast, the HLADQB1*0201 allele was associated with autoimmunity to GAD65 $(\mathrm{P}=0.04)$, but negatively associated with $\mathrm{ICA} 512 / \mathrm{IA}-2$ antibodies $(\mathrm{P}=0.0002)$.

\section{8}

RE ACTIVITY TO GLYCOLIPID IS NEGATIVEL Y REL ATED TO ISLET CELL AVTIBODY TITRES AND THE PRESE VCE OF GAD E Cabrera-Rode', J Morales', O Diaz-Horta', S Piquer', I Viñets', $M$ Puig-Domingo and $A$ de Leiva' 'National lnstituie of Endocrinologv la Havana, Cuba -Endocrinology Dept Hospital Sant Pau, Barcelona, Spain

Glycolipids, mainly GM-1, are one of the most important deteminants of islet cell autoantibody (ICA) reactivity $\mathrm{X}_{\mathrm{e}}$ aimed to investigate the glycolipid reactivity of IC A positive sera and its relationship with glutamic acid decarboxylase antibodies (GADA) and insulin autoantibodies (IAA) in 43 type 1 diabetic subjects (IDDM) 20 first degree relatives (FDR) and 11 FDR which became diabetic at follow-up (Pre-IDDM) ICA detection was carried out by indirect immunofluorescence and GADA and IAA by radiobinding assay. Total (TR), partial (PR) or null reactivity (NR) to glycolipids, was quantified according to the degree of inhibition of ICA reactivity when ICA positive sera were incubated with high concentration human pancreatic glycolipid extracts. TR, PR and NR were similar for the three groups $58 \%, 28 \%$ and $14 \%$ in IDDM, $54 \%, 27 \%$ and $18 \%$ in preIDDM and $65 \%, 25 \%$ and $10 \%$ in FDR A negative correlation was found between ICA titres and TR in all groups. TR was higher among GADA negative sera $(80 \%$ vs. $53 \%$ ), while no differences were found in relation to IAA. In conclusion, the majority of ICA reactivity is conferred by glycolipidic epitopes, the decrease of TR to glycolipids in the GADA positive sera indicates that $\mathrm{GAD}$ accounts for at least part of ICA reactivity.
IA2 AND GAD65 ANTIBODIES COMPLEMENT ISLET CELL ANTIBODIES IN IDDM PREDICTION

J Morales ${ }^{1}$, M. Puig-Domingo', D. Mauricio ${ }^{1}$, S. Piquer ${ }^{1}$ I. Viñets ${ }^{1}$, V.

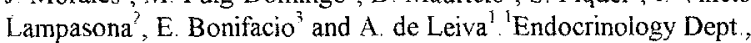
Hospital Sant Pau, Barcelona, Spain. ${ }^{2}$ Laboratory of Medicine and "Dept. of Internal Medicine, Istituto Scientifico San Raffaele, Milan, Italy.

Despite the fact that islet cell antibodies (ICA) have been demonstrated to be a robust IDDM predictor, the addition of new immunological markers may enhance the prediction of the disease. We aimed to determine the prevalence of IA2 antibodies (IA2-Abs), GAD65 antibodies (GAD65-Abs) and their relationship with ICA titre, among subjects with islet cell antibody (ICA) positivity: 43 newly onset IDDM subjects (n.o.IDDM), 30 first degree relatives of IDDM subjects (FDR) (follow-1p $4.9 \pm 2.3 \mathrm{yrs}$ ) and 10 relatives who developed IDDM (preIDDM) within $3.0 \pm 2.4$ yrs follow-up. ICA were measured by indirect immunofluorescence. Combined detection of IA2-Abs and GAD65-Abs was done using a simple radio-binding assay. Frequency for these antibodies were $65 \%, 50 \%$ and $27 \%$ for IA-2, and $65 \%, 50 \%$ and $20 \%$ for GAD65, in n. .IDDM, pre-IDDM and FDR groups, respectively. Positivity and negativity for both IA2-Abs plus GAD65-Abs was: $44 \%$ and $14 \%$ in the n. IDDM group, $30 \%$ and $30 \%$ in the pre-IDDM group and $10 \%$ and $63 \%$ in the FDR group. Percentage of high ICA titres $(\geq 20$ IDFu) in subjects positive for one or both antibodies (IA2-Abs and GAD65-Abs) was: $70 \%, 100 \%$ and $45 \%$ among n.o.IDDM, pre-IDDM and FDR, respectively $(\mathrm{p}<0.05)$. We conclude that although high ICA titre remains as a strong predictive marker, $\mathrm{IA2}$ and GAD65 Abs are associated with a higher risk of IDDM in ICA positive subjects.

\section{9}

DIABETES-RESISTANCE ALLELE DQB1*0602 PROTECTS FROM LOSS OF IMMUNE TOLERANCE TO GM2-1 BUT NOT TO OTHER DIABETES-ASSOCIATED AUTOANTIGENS. S. Dionisi, R. Gianani, B. Carabba, L. Farilla, GS. Eisenbarth. U. Di Mario and F. Dotta. Dept. of Endocrinology, University of Rome: Dept. of Clinical and Exp. Medicine, University of RC, Catanzaro, Italy: Barbara Davis Center, Denver, CO, USA.

Recent data have shown that the $\mathrm{DQB} 1{ }^{* 0602}$ allele confers dominant protection from type 1 diabetes even in presence of circulating ICA. The islet-specific GM2-1 ganglioside is target of autoantibodies strongly associated with progression to type 1 diabetes in ICA+ relatives. In the present study we aimed to determine the influence of this protective allele on the occurrence of anti-GM2-1 and of other diabetes-associated autoantibodies (anti-insulin, anti-GAD65, anti-ICA512) in ICA+ relatives of type 1 diabetic subjects. Fourty-six prospectively evaluated $I C A+$ relatives were analyzed for the occurrence of IAA, of anti-GAD65 and of anti-ICA512 (all determined by fluid phase RIA) and of anti-GM2-1 (by indirect immunoperoxidase on TLC plate) autoantibodies; 21/46 subsequently developed type 1 diabetes. Expression of the $\mathrm{OQB1} 1^{\star} 0602$ ailele was found in $7 / 46$ individuals; none of them progressed to overt disease. Among the 46 relatives, 29 were $\mid A A+, 43$ were anti-GAD65+, 23 were anti-lCA512+ and 32 were anti-GM2-1+. Among 0602+ relatives, all seven expressed GAD65 autoantibodies, while a reduced frequency of IAA and anti-ICA512 (2/7 and $1 / 7$ respectively) was observed. Interestingly, anti-GM2-1 autoantibodies were not detected in any of the $0602+$ relatives, being the only autoantibody not observed in this diabetes-resistant group of individuals. In conclusion, in $1 C A+$ relatives, the $D Q B 1^{*} 0602$ allele, in addition to confer a dominant protection from progression to disease, appears to determine an absolute resistance to the loss of immune tolerance against the GM2-1 islet ganglioside. This is in accordance with previous findings showing that autoimmunity to this ganglioside is highly associated to diabetes development in this group of individuals 
330

ISLET CELL SURFACE ANTIBODIES (ICSA) BIND TO HUMAN ISLETS. VALIDATION OF RIA WITH ELECTRON MICROSCOPY. B. Kniola and J. Ludvigsson, Department of Pediatrics, Faculty of Health Sciences, Linköping, Sweden.

Islet Cell Surface Antibodies (ICSA) have been demonstrated significantly more often in serum of patients with IDDM and their relatives than in healthy controls, but some investigators have been unable to show binding to human islets. It has therefore been suggested that ICSA is an artefact.

Aims: To localise and quantify ICSA attachment to different specimens, including human pancreas, using ICSA-pos and ICSA-neg patients' serum determined by a RIA-method.

Material and Methods: Ten ICSA-positive sera were identified with a RIA-method (RIN5AH-cells as substrate, $125 \mathrm{~J}-\mathrm{IgG}$ as tracer), and compared with 10 ICSA-negative sera from IDDM children. Sera were incubated with ultrathin sections of RIN5AH-cells, stained with Goatantihuman IgG conjugated with $10 \mathrm{~nm}$ gold particles and viewed in Transmission electron microscopy (mag 30.000). Image analyses was used. To test cell specificity we used rat tumour cells (GH3), human normal pancreas, human insulinoma, and mice liver.

Results: Sections showed good morphology. ICSA-pos sera gave 5 times higher immunolabelling density than ICSA-neg sera on RIN5AH sections and immunolabelling also on hunan normal pancreas and human insulinoma, but not on GH3-cells or mice liver cells. The colloidal gold technique could confirm the RIA results but had higher sensitivity .

Conclusion: ICSA is not an artefact and the antibodies bind to human islet cells.

\section{1}

B.R.A.H.M.S ANTI-GAD65 TEST - A SIMPLE ONE-STEP ASSAY WITH SUBMOLECULAR SPECIFICITY

${ }^{1}$ M. Ziegler, ${ }^{2}$ A. Bergmann, ${ }^{2}$ R. Weckermann, 'M. Strebelow, 'M. Schlosser and 'B. Ziegler 'Institute of Diabetes "Gerhardt Katsch" Karlsburg, Universitiy of Greifšwald; 'B.R.A.H.M.S Diagnostica GmbH, Berlin, Gernany

Autoantibody formation accompanies the process of 1 -cell destruction by insulitis often resulting in IDDM. A major antoantigen for both humoral and cellular autoimmunity in IDDM is glutamic acid decarboxylase (GAD), GAD autoantibodies (GAD-AAb) are an important marker of the autoimmune process of IDDM, they occur in the majority of cases with new-onset IDDM and in the prediabetic stage. However, GAD-AAbs are also found in some probands without IDDM, including those with stiff-man-syndrome (SMS) or with B-cellspecific islet cell autoantibodies (ICA), having a low risk of developing IDDM. Differences in the GAD-AAb profile between these nondiabetic individuals and those with IDDM suggest that disease-specific epitopes may exist. Our aim was to develop an monoclonal antibody-based GAD-AAb test detecting the AAbs to the conformation-dependent epitope region (aa 221-442) targeted by IDDM sera. Monoclonal GAD-Abs were generated from Balb/c mice by hybridoma technique and characterized regarding their epitope specificity. In addition, the competitive inhibition of their 125I-GAD65 binding by IDDM sera was compared. In the B.R.A.H.M.S anti-GAD test - a simple solid phase tube assay two mc-GAD-Abs form via native porcine GAD preparation a sandwich complex whose formation is inhibited by patient ' $S$ GAD-AAbs of identical epitope specificity. Test tubes are coated with the me-GAD65-Abl directed to the conformational epitope region aa 221-442 and the indicator antibody mc-GAD65-Ab2 125I-labelled, shows maximum tube binding via $G A D$ in the absense of $G A D$ autoantibodics of IDDM sera. For the B.R.A.H.M.S anti-GAD test the diagnostic sensitivity was detected to be $74.9 \%(176 / 235)$ of new-onset IDDM, the specificity was amounted to $1.8 \%(7 / 398)$ both in accordance with results of the labelled-GAD precipitation assay. All high risk individuals of developing IDDM of a general schoolchildren population were highly positive by the B.R.A.H.M.S-anti-GAD test.

Prediction of risk for developing IDDM by combined analysis of GAD-AADs. LAAs, ICA512-AAbs and ICAs in a general population of schoolchildren M. Strebelow. P. Augstein. U. Jacobt, B. Zicgler. M. Schlosser and M. Zirgk'r Institute of Diabetes "Gerhardt Katsch" Karlsburg. University of Greifswald Identification of subjects at risk for developing IDDM is based on detcition of AAbs, because the destruction of limedls during the prediabetic stage is acconpanied by formation of AAbs to B-cell antigens. More than $9\left(\mathrm{H}^{\prime \prime}\right.$ of new-onset IDDM patients are without diabetic relatives and with a peak incidence during pubcrty. Thus the aim of our study was the screening of a gencral school-children population for B-cell autommunity. Up to now we have screened in capillary blow 4239 schoolchildren for GAD-AAbs. IAAs. ICA512-AAbs. using 125I-latwllid antigens (ICA512 was a kindly gitt from D. U. Ribin. Bayer corp. and 1251Insulin from Hocelst $A G$ ) and compared with ICAs with following results:

\begin{tabular}{|c|c|c|c|}
\hline$-L+h$ & meralune? & mean age & $\operatorname{sic} x$ \\
\hline GAlD-AAb (-ths to ghrtamic acid decarboxylase) & $205 \%$ & $9.8 \pm 2.1$ & $4111 \mathrm{~V} / 40$ \\
\hline LA Insulinanountibodies & $1.53^{\circ} \mathrm{o}$ & $10.8 \cdot 3.1$ & $35 \mathrm{~m} 301 \mathrm{l}$ \\
\hline ICA512-AAh (AAbs aguims islet cell anrigen 5t? & $1.46^{\circ} \%$ & $11.1: 3.6$ & $27 m 35 t^{\circ}$ \\
\hline 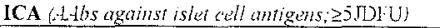 & $2.47 \%$ & $11.5 \pm+.5$ & $5 . \mathrm{t} 11 / 521^{\circ}$ \\
\hline $\mathrm{OAD}-\mathrm{AAB}$ andor $\mathrm{IAA}$ & 3.370 & $10.2 \pm 2.7$ & $7 \ln / 721$ \\
\hline ( $A D D-A A B$ andion $1 C A$ & $4.220_{10}$ & $10.6 \cdot 5.1$ & $\sin \varphi(1:$ \\
\hline GAD-AAb and or $1 C A 512$ & $3.40 \%$ & $10.3: 2.7$ & $68 \mathrm{~m} 790^{\circ}$ \\
\hline 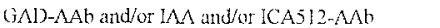 & $+60 \%$ & $10.5 \pm 32$ & 0 m/ $10 \mathrm{st}$ \\
\hline OnV GAD-AAb & $1.58 \%$ & $9.9 \pm 2.1$ & $28 \mathrm{~m} / 390$ \\
\hline mly $1 \mathrm{~A}$ & $1.88 \%$ & $12.2: 5.4$ & $\operatorname{thm} 390^{\circ}$ \\
\hline only IC $\triangle 512-A A b$ & $1.200^{\circ}$ & $126 \cdot 16$ & 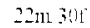 \\
\hline (mly ICA & $1.220 \%$ & $11.0 \cdot+8$ & $25 \mathrm{~m}=-1$ \\
\hline
\end{tabular}

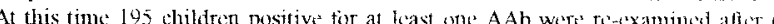
nonths to differentiate the risk of developing IDDM. For GG. I "i the AAt positivity was confirmod. 6 children were positive for all lour AAbs. all with at high GAD-AAb titre. These sera revealed a striking hinding to an IDDMassociated contormational epitope region of GADo5. Onc senum. nicgative for ICA was positive for the confomational GAD cpitope. ICA512 and inswin. These children were classitied as high risk individuals and netabolic characterizations were recommended as soon as possible to decide aboul utervention. 
PREDICTION OF IDDM IN SCHOOLCHILDREN

U Samuelsson and J Ludvigsson, Dept of Pediatrics, Central Hospital, Karlskrona and University Hospital, Linköping, 5 weden

Islet Cell Antibodies (ICA), Insulin Autoantibodies (IAA) and C-peptide were 1987 determined in 1031 healthy schoolchildren aged $7-8$ years and 12-13 years. 14 were ICA-positive and 40 were IAApositive. In 1994 we traced $98,4 \%$ of the children. 4 children had got diabetes. 3 boys and 1 girl (incidence 55/100000/year). All boys were ICA-positive 1987: 192,14 and 9 JDF-units respectively. The cirl was ICA-negative but GADA-positive (bloodsample stored). None had IAA. They had normal serum C-peptide values 1987 but low C-peptide values in urine $(3,2,5,4$ and $6,3 \mathrm{nmol} / 1$ respectively; mean for the 1031 chiddren=17,82 nmol/1). New bloodsamples were in 1994 taken from 133 still non-diabetic children (antibodypositive 1987 +negative controls)and we analyzed ICA, IAA and GADantibodies(GADA) together with C-peptide in serum and urine. Among them 6 were GADA-positive 1994 $4,5 \%)$. They had somewhat 1 ower $C$-peptide values than GADA negative children both in serum(0,86 n$\mathrm{mol} / \mathrm{l} \mathrm{resp} 1,19 \mathrm{nmol} / 1, \mathrm{n} . \mathrm{s})$ and in urine $(9,65 \mathrm{nmol}$ resp $20,17 \mathrm{nmol} / 1, p=0,07)$. 2 girls were both ICApositive and CADA-positive both in 1987 and 1994. Both were IRA-negative 1987, one was positive 1994, C-peptide in their urine was rather low $1987(12,0$ resp 12,65$)$ and extremely low $1994(4,5$ resp 1,35 nmol/1).0ur results show that ICA and GADA together with C-peptidevalues can be used topredict IDOM in the general childhood population. C-peptide in urine seems to be more sensitive than [-peptide in serum.

\section{6}

PREDICTORS OF INSULIN REQUIREMENT IN PATIENTS WITH SLOWLY PROGRESSIVE TYPE I DIABETES

I.Satman, M.T.Yilmaz, K.Karsidag, N.Dinccag, Y.Altuntas, and S.Karadeniz. Istanbul Faculty of Medicine, Istanbul University, Turkey. Slowly progressive type 1 diabetes (SPTID) is an atypical form of.JDDM which usually appears as young-onset NIDDM, but mostly requires insulin within five years of diagnosis. To determine the predictors of insulin requirement, a consecutive sample of 64 SPTID patients were followed-up for (a period of $3-8$ years. Patients were divided into two groups according to the time to insulin requirement, and compared retrospectively. Group I (insulin requirement $>1$ year) consisted of $34(19 \mathrm{~F}, 15 \mathrm{M})$ and Group II (insulin requirement $\leq 1$ year) $30(17 \mathrm{~F}, 13 \mathrm{M})$ patients. Age at onset in Group I was younger than Group II $(35.3 \pm 8.4$ vs. $42.6 \pm 12.7$ yrs, $\mathrm{p}<0.005)$. Insidious-onset and post-gestational presentation were more common in Group I ( 53 vs. $27 \%, p<0.001$ and 16 vs. $0 \%, p<0.005$ ), whereas acute-onset and incidental diagnosis were more frequent in Group II $(53$ vs. $27 \%, p<0.001$ and 13 vs. $3 \%, p<0.01$ ). Family history of diabetes was more frequent in the first than the second group (56 vs. $40 \%$, $p<0,02$ ). Group [1 patients had a mean initial FBG higher than Group I $(p<0.002)$, although there were no difference in $\mathrm{HbAlc}, \mathrm{BMI}$, and frequency of obesity at baseline. Both groups had a similar beta cell reserve (Basal and stimulated C-peptide: $0.9 \pm 0.6$ and $1.6 \pm 0.8 \mathrm{ng} / \mathrm{ml}$, and $1.1 \pm 0.7$ and $1.4 \pm 0.9 \mathrm{ng} / \mathrm{ml}$ in groups $\mathrm{I}$ and II). No difference was found in the frequency of ICA and IAA, but high-titre ICA ( $\geq 20 \mathrm{JDFu})$ was more prevalent in Group li (52 vs $21 \%, p<0.001$ ). Initial insulin requirement and the prevalence of long-term complication on follow-up were comparable in both groups. In conclusion, our data suggest that middle age-onset, acute presentation and high-titre ICA may predict earlier insulin requirement in SPT1D.
THE 'SARDINIAN SCHOOL CHILDREN-IDDM' (SSI) Study. PREDICTIVE VALUE OF ICA, GADA AND IA-2iCA IN 1114 HEALTHY SCHOOL CHILDREN. $\checkmark$ Sepe, A Loviselli*, S Al-Temimi, R Foxon, M Shattock, F Velluzzi", P Mele*, A Balestrieri*, S Mariotti*, M Songini**, G F Bottazzo \& the SSI Study Group. St Bartholomew's and The Royal London School of Medicine \& Dentistry, UK. *istituto di Medicina Interna, Universita' di Cagliari \& "*Centre for Metabolic Diseases and Atherosclerosis, Cagliari, Italy.

The EURODIAB data have confirmed that Sardinia and Finland are the regions with the highest incidence of IDDM in Europe. In order to identify pre-IDDM individuals in Sardinia, sera from over 10,000 school children were randomly collected. Of them, 1114 with the longest follow-up (median 4.7 years) were tested for ICA, GADA and IA-2icA. Positivity was defined as ICA $>5$ JDF-units, whereas GADA and IA-2icA were considered positive for values falling beyond 2 steps over the 75 th percentile [ 1 step $=1.5 \times(75$ th -25 th $)], 12$ and 6 LH units respectively. Sensitivity (Se), Specificity $(\mathrm{Sp})$ and Positive Predictive Value (PPV) were related to pre-IDDM sera. Results are summarized in the Table.

\begin{tabular}{|c|c|c|c|c|}
\hline \multirow{2}{*}{ Test } & Positive & $\overline{\mathrm{Se}}$ & $s p$ & $\overline{\text { FPV }}$ \\
\hline & No $(\%)$ & $\%$ & $\%$ & $\%$ \\
\hline $1 C A>5$ & $59(5.3)$ & 100 & 95 & 8 \\
\hline $\mid C A>20$ & $18(1.6)$ & 100 & 98 & 28 \\
\hline GADA & $16(1.5)$ & 40 & 99 & 13 \\
\hline AA-2iaA & $27(2.4)$ & 80 & 98 & 15 \\
\hline $\mid C A=20 \& G A D A$ & $4(0.4\}$ & 40 & 100 & 50 \\
\hline $\mid C A>20 \& 1 A-2 i C A$ & $7(0.6)$ & 80 & 99 & 57 \\
\hline GADABIA-2icA & $3(0.3)$ & 40 & 100 & 67 \\
\hline$|C A=20 \& G A D A \&| A-2 i \subset A$ & $3(0.3\}$ & 20 & 100 & 33 \\
\hline
\end{tabular}

Eight $(15 \%)$ out of $59 \mathrm{ICA}^{+}$sera recognized the ' $37 \mathrm{~K}$-antigen' of them, 6 reacted with $37 \mathrm{kD} / 40 \mathrm{KD}$ fragments and $\mid A-2 i c$, and 2 sera recognized only the $37 \mathrm{kD}$ fragment, and but one of them was positive for IA-2icA. Five children developed IDDM between 2 and 60 months from the enrollment into the study, all were $I C A^{+}$and had

family history of diabetes. Four reacted to the $37 \mathrm{kD}$ and $40 \mathrm{kD}$ fragments and the IA-2ic. Two were positive for all three autoantibodies. In conclusion: a) high correlation was found between ' $37 \mathrm{~K}$-antigen' autoantibodies and IA-2icA b) combination of ICA $>20$ JDF-units \& IA-2icA showed high Se and PPV and are promising tests to identify pre-IDDM indjviduals from the background of the general population.

\section{7}

INCIDENCE AND PREVALENCE OF TYPE 1 DIABETES MELLITUS AMONG ADULT POPULATION IN LITHUIANI A 1991-1995

ROgirauskas, RZ̆Zabinkevičius, A.Norkus and the Lithuanian Collaborative Group for the Epidemiology of IDDM. Institute of Endocrinology, Kaunas Medical Academy, Kaunas, Lithuania

The aims of the study were to determine the incidence and of IDDM in a prospective study of 15.39 year aged young adults and prevalence among $\geq 15$. year age population during the years 1901-1995. Two sources of information were used: urgent reports of onset of and annual reports from all physicians tesponsible for diabetes care of all out-patient clinics of Lithuania. The incidence ascertainment was assessed by the capture-rexapture method. During the registry period 512 cases ( 330 males and 182 females) were identified. The average 5 -year incidence rate was 9.296 per $100,000 /$ year $(95 \%$ Poisson confidence interval was 8.345-10.355) for males and 5.221 (CI 4.515 . 6.037) for females, respectively (Chi square sex difference 32.262 , $\mathrm{df}=4$, $\mathrm{p}<005$ ). Male/fernale ratio was 1.813 . The highest age-specific risk of IDDM was observed around 25.29 years of age Results of the regression models showed that the incidence of IDDM had tendency to increase. Age-adjusted 15. 39 curmulative incidence rate was 0.313 for males and 5.231 for fenales, $p<0.05$. The largest proportion of LDDM was diagnosed during full winter $(29.5 \%)$ and full auturn $(29.5 \%)$ and the lowest in summer $(14.8 \%)$. No significant geographical variation was observed. On 31.12.1991 there were 2526 adult ( 215 -year) patients with IDDM or 87.0 per 100,000 inhabitants (CI 83.7-90.5) in 31.12.1995 - 3046 or 104.9 (CI 101.3-108.7), respectively, p<0.05. Age-adjusted prevalence rates in 1991 were 97.6 for males and 77.4 for females $(p<0.05)$ in $1995-120.2$ and $90.9(p<0.05)$, respectively. In 1095 the domination of males was especiaily prominent in 35-39-year age group $(226.4 / 100,000)$ and the domination of females - in 25-29-year age group (143.8). During 5 -year period the mean increase of number of patients was $3.4+0.3 \%$ /year. Our data shows that the number of adult patients with IDDM in Lithuania in the year 2000 should be approximately 3610 , and in 2010 about 5300 . 


\section{8}

PREVALENCE AND PREDICTIVE VALUE OF GAD-ANTIBODIES IN DIABETES MELLITUS DIAGNOSED ABOVE 40 YEARS OF AGE. $M$ Seubert, JJJ de Sonnaville, L Moser, J Seissler and RJ Heine. Laboratory for General Practitioners Amsterdam (ATAL), Department of Internal Medicine, University of Leipzig and Department of Endocrinology, Free University Hospital, P.O Box 7057, 1007 MB Amsterdam, The Netherlands.

The aim of the study was to assess the prevalence of glutamic acid decarboxylase autoantibodies (GADA) in patients with type II diabetes mellitus diagnosed above 40 years of age, and its predictive value with respect to insulin therapy after 2 and 3 years of follow-up. GAD-antibodies, a marker for autoimmune diabetes, were determined using a radioligand assay with $\left[{ }^{35}\right.$ SI-methionine labelled human recombinant GAD by in vitro translation/transcription in 776 patients above 40 years of age participating in a regional shared care project since 1992 . Of these patients $42.5 \%$ were men, mean age was $64.9 \pm$ std 12.6 years and mean diabetes duration $4.3 \pm 5.8$ years. At entry blood glucose lowering therapy consisted of insulin in $4.1 \%$, oral agents in $53.4 \%$ and diet only in $42.4 \%$. No GADA were found in $60.2 \%$ of the patients, while 10.7 , 26.7 and $2.4 \%$ had GADA-titers of $>0.1-1.0,>1.0-10.0$ and $>10.0$ $\mathrm{U} / \mathrm{ml}$ respectively. Insulin therapy was initiated in $45 / 158(28.5 \%)$ of the GADA-positive (titer $>0.1 \mathrm{U} / \mathrm{ml}$ ) and in $52 / 199(26.1 \%)$ of the GADA-negative patients followed for 2 years. After 3 years of follow-up, 21/47 (44.7\%) of GADA-positive (titer $>0.1 \mathrm{U} / \mathrm{ml}$ ) and $16 / 50(32.3 \%)$ of GADA-negative patients became insulin requiring. Five of the $6(83 \%)$ patients with high GADA-titers $(>10 \mathrm{U} / \mathrm{ml})$ and $32 / 91(35.2 \%)$ with lower titers had to use insulin within 3 years. In conclusion, GADA are present in an unexpectedly high percentage of the elderly patients, formerly diagnosed as having type II diabetes mellitus. Only presence of high GADA-titers predicts for insulin requirement within the next 2-3 years.

\section{9}

WHY DO IDDM AND NIDDM PATIENTS DIFFER IN THEIR IMMUNE RESPONSES TO INSULIN THERAPY?

N S. Fineberg, S. E Fineberg, J A Galloway, J H Anderson, Indiana University and Lilly Laboratories, Indianapolis, IN USA

Although we have consistently observed that IDDM patients develop insulin immune responses more readily, we have previously been unable to differentiate the effects of age and insulin secretion on the immune response. To resolve these issues, we pooled two large data bases from patients newly treated with insulin and studied for 1 year (randomized trials of human, pork and mixed beef pork (MBP) insulins, $n=254$ and pork and MBP insulins, $n=566$ ). Age, insulin secretion as indicated by fasting c-peptide (FCP) and insulin origin were studied. Bound insulin ( $\mathrm{Bl}=$ total-free $>0)$ and $\%$ specific binding $(\mathrm{SB}>4 \%)$ were used as indices of a significant insulin immune response. Patients with significant SB were younger $(41.6$ vs $50.6 ; p<0.001)$ and had lower levels of $F C P(0.23$ vs $0.37 \mathrm{pM} ; p<0.001)$. When separated into nonsecretors ( $F C P<0.1$ pmol) vs secretors ( $F C P \geq 0.1$ pmol), patients with significant $S B$ were younger in both subgroups $(p<0.001)$. In addition, patients on MBP were more likely to have significant $S B$ (odds ratio $12.9, p<0.001)$ regardless of age or FCP level. Stepwise logistic regression was used to assess whether these effects were independent. Source, FCP and age all entered the equation at significant levels with age and FCP being inversely related to antibody development whereas use of MBP predisposed to the development of antibodies. Similar results were seen with $\mathrm{BI}$. Both younger age and lower levels of FCP independently increase the insulin immune response and heip explain the tendency for IDDM patients to develop antibodies and for NIDDM patients to resist antibody development.

\section{0}

NO SPREADING OF IMMUNE RESPONSE AGAINST GLUTAMIC ACID DECARBOXYLASE IN LATENT AUTOLMMUNE DIABETES B. Ziegler, I Rjasanowskil M. Strebelow, M. Schlosser, A. C. Powers2 and M. Ziegler. Institute of Diabetes and 1Clinicum Karlsburg. University Greifswald Germany, 2Vanderbilt University, Nashville USA

Autoantibodies against glutamic acid decarboxylase (GAD-AAbs) are an important marker of the autoimmune-mediated B-cell destruction in IDDM GAD-AAbs are also found in patients with Stiff-man syndrome (SMS), however both disease-associated GAD-AAbs are described to differ in their reactivity with linear and conformational GAD epitopes. Recently it could be demonstrated that patients with an initial diagnosis of NIDDM frequently become insulin dependent refered as latent autoimmune diabetes of adults (LADA). The aim of our study was to evaluate whether LADAassociated GAD-AAbs more resemble those of SMS patients who develop IDDM also in the adult age. For this, the displacement of murine monoclonal GAD antibodies (mc-GAD-Abs) from their 125I-GAD65 binding by senum autoantibodies was measured by immunoassay. Only the sera of SMS patients substantially reduced the GAD binding of the mcGAD-Abs recognizing a $N$ - and C-terminal linear GAD epitope region as well as a middle conformational GAD65 region. Mc-GAD65-Abs targeting this conformational GAD region (amino acid 221-442) were displaced by the GAD-AAbs of the IDDM (25/30) and all LADA patients (20/20). In addition, about $15 \%$ of LADA patient's sera were reactive with linear epitope regions. In summary our results show that linear epitopes located in the $\mathrm{N}$-terminal and $\mathrm{C}$-terminal region are not immuno-dominant for the GAD-AAbs of the most patients with early and late onset of IDDM Thus, the LADA-associated immune response more closely resemble to those of LDDM than SMS patients and is seldom spread throughout the length of GAD

\section{1}

GAD 65 ANTIBODY BUT NOT ICA POSITIVITY IN ADULT-ONSET DIABETIC PATIENTS IS ASSOCIATED WITH EARLY PROGRESSION TO CLINICAL INSULIN DEPENDENCY

E. Hatziagelaki, C. Jaeger, E. Maeser. R.G. Bretzel and K. Federlin. Third Medical Department and Policlinic, Justus-Liebig University, Giessen, Germany.

Correct classification of diabetic patients in adulthood at the time of diagnosis is often difficult. The aim of the present study was to determine the significance of GAD $65 \mathrm{Ab}$ as an autoimmune marker in patients with aduitonset diabetes associated with early insulin dependency. In the present study, 150 patients aged between $30-60$ years from our diabetes outpatient clinic participated with the initial diagnosis of NIDDM according to WHO Though treatment was started with diet or diet plus oral hypoglycaemic agents, insulin therapy had to be introduced in a subset of patients within two years after diagnosis. In all cases, serum obtained at the time of the initial diagnosis was analysed for islet-cell (ICA) and GAD $65 \mathrm{Ab}$. Neither ICAAntibody status nor body mass index (BMI) were significantly associated with early insulin requirement (<2yrs after diagnosis). In contrast, GAD $65 \mathrm{Ab}$ were significantly associated with the occurence of insulin dependency within 2yrs after the initial diagnosis $(p<0.01$ ), thus identifying a substantial proportion of patients who would initially have been misclassified as NIDDM. In conclusion, determination of GAD $65 \mathrm{Ab}$ in patients with adult-onset of diabetes appears to be highly recommendable and it may contribute to the correct classification and adequate treatment of patients with onset of diabetes in adulthood. 


\section{Clinical Immunology}

\section{2}

GAD65 ANTIBODIES PERSISTENCE IS ASSOCIATED TO A DECREASED B CELL FUNCTION IN NEWLY-DIAGNOSED TYPE I DIABETES.

G.Carreras, D.Mauricio,J. Morales, A. Pérez, M.Puig-Domingo and A. de Leiva. Hospital Sant Pau. UAB. Barcelona. Spain.

Our group previously reported that persistence of ICA one year after type I diabetes (IDDM) diagnosis was associated to lower deterioration of $B-c e l l$ function than non persistence. Here we reported the relationship between the profile of serum GAD65 antibodies (GAD65-Abs; mesured by a simple radio-binding assay) and stimulated plasma c-peptide levels in 72 IDDM subjects ( 47 males, 25 females; mean age $24.5 \pm 9$ years) followed for one year after diagnosis. Percentage GAD65-Abs positivity at 0 , 3,6 and 12 months from diagnosis were $65 \%, 71 \%$, $61 \%$ and $57 \%$, respectively. At clinical onset, GAD65-Abs $(+)$ versus GAD65-Abs $(-)$ groups did not differ by age, sex, BMI, $\mathrm{HbA}_{1 c}$, clinical simptoms or C-peptide levels. However, C-peptide levels for GAD65-Abs (+) subjects were lower at 6 months of follow-up when compared to the GAD65-Abs (-) group ( $609 \pm 297$ vs $427 \pm 280 \mathrm{pmol} / 1 ; \mathrm{p}<0.05)$. Integrated mean value for stimulated c-peptide during the whole annual investigated period displayed diminished value for subjects showing persistence of GAD65-Abs as compared with GAD65Abs (-) subjects $(319 \pm 185$ vs $453 \pm 228$ pmol/1; p $0.05)$. We conclude that persistence of GAD65-Abs is associated to more severe damage of the beta cell function.

\section{3}

GAD AND IA-2 ANTIBODIES IN RELATION TO DISEASE PRESENTIATIOX AND C-PEPTIDE SECREIION

M. Cassone Faldetta, N. Visalli, M.G. Cavallo, D. Andreani, R.D.G Leslie, M. Hava and P. Pozzilli. Clinica Medica II, Policlinico Umberto I, University of Rome "La Sapienza", Rome, Italy and * Department of Diabetes and Metabolism, St Bartholomew's Hospital Medical College, London.

We evaluated the integrated parameters of metabolic control and the autoantibody frequency at onset of insulin dependent diabetes mellitus (IDDM) to characterize the disease according to the age at diagnosis.

Two hundred and thirty five consecutive cases of recent onset $(<4$ weeks) IDDM patients (age $5-45$ years) were studied. Presentation of the disease and integrated parameters of metabolic control including basal and stimulated C-peptide (1mg i.v. glucagon), glycosylated haemoglobin and insulin dose required at diagnosis, were analysed in patients stratified according to their age at diagnosis. Five age groups were considered including patients diagnosed between 5-7 years of age $(n=10), 7-10$ years $(n=38), 10-17$ years $(n=94), 17-20$ years $(n=17)$ and $20-45$ years $(n=76)$ Autoantibodies to glutamic acid decarboxylase (GAD) and to tyrosine phosphatase like molecule (IA-2) were measured by imnunoprecipitation assays

Patients diagnosed before puberty had significantly reduced $C$-peptide secretion compared with patients diagnosed at a later age $(p<0.02)$. Overall there was an inverse correlation between baseline (but not stimulated) C-peptide secretion and age at diagnosis $(p=0.007)$. Glycosylated haemoglobin did not differ at diagnosis between different age groups. GAD antibodies were found in $65 \%$ and $1 \mathrm{~A}-2$ antibodies in $59 \%$ of patients. GAD antibodies tend to be more frequent in patients diagnosed over 17 years compared to younger patients $(p=0.05)$, whilst IA-2 antibodies were not age related.

These data suggest that beta cell damage and the consequent time of insurgence of hyperglycaemia in IDDM differ between patients diagnosed before or after puberty, the process being more destructive only in the very young children.

\section{4}

GAD65 ANTIBODY ISOTYPES IN FIRST DEGREE RELATIVES SUGGEST A TH2 IMMUNE RESPONSE IN NON-PROGRESSORS.

J. S. Petersen, P. Kulmala, M. Knip, T. Dyrberg and the Childhood Diabetes in Finland Study Group. Hagedorn Research Institute, NovoNordisk, Denmark and University of Oulu, Finland.

In the NOD mouse progression to disease has been associated with a Th1 response whereas protection has been associated with a Th2 response. The isotype profile of antigen specific antibodies have been demonstrated to represent the Th1/Th2 response. Determination of isotypes of GAD65 autoantibodies could therefore distinguish progressors from non-progressors. We measured GAD65 autoantibody isotypes in GAD65 autoantibody positive first degree relatives, 6 at low risk (ICA neg.) and 7 progressors (ICA pos) before and at clinical onset of IDDM and in 5 controls. GAD65 antibody isotypes were measured in a radioligand assay using human GAD65 and anti-humanIg isotype specific antibodies for immunoprecipitation. The rank order of Ig isotypes was similar in the 7 progressors before and at clinical onset of IDDM, i.e. IgG $1>\operatorname{IgG} 4>\operatorname{IgM}>\operatorname{IgE}>\operatorname{IgA}>\operatorname{IgG} 3>\operatorname{IgG} 2$. By contrast, the rank order, $\operatorname{IgG} 1>\operatorname{IgM}>\operatorname{IgE}>\operatorname{IgG} 4>\operatorname{IgG} 3>\operatorname{IgA}>\operatorname{IgG} 2$, and isotype levels in the low risk siblings reflected a more immature (IgM) and Th2 like (IgE) response compared to the progressores, i.e. the level of IgM and IgE was significantly ( $\mathrm{p}=0.01)$ higher 1612 vs. 225 and 981 vs. 203 (mean cpm above the mean 2 SD of the 5 controls) respectively. In conclusion, detection of the isotype profile of GAD65 autoantibodies may help refine prediction further.

\section{5}

CHARACTERISTICS OF CHILDREN WITH NEWLY DIAGNOSED DIABETES NEGATIVE FOR IDDM-ASSOCIATED AUTOANTIBODIES E. Sabbah, P. Kulmala, R. Veijola, P. Vähäsalo, H. Reijonen, J. llonen, H.K. Akerblom, M. Knip and the Childhood Diabetes in Finland Study Group. Department of Pediatrics, University of Oulu, Oulu, Turku Immunology Centre and Department of Virology, University of Turku, Turku, and the Children's Hospital, II Department of Pediatrics, University of Helsinki, Helsinki, Finland. To characterize autoantibody-negative children with diabetes we analyzed 747 newly diagnosed subjects younger than 15 years of age (mean 8.4 years) for antibodies to islet cells, the $65 \mathrm{kD}$ isoform of glufamic acid decarboxylase and insulin. We compared those children testing negative for all three autoantibodies to the remaining ones for demographic data, clinical presentation natural course of the disease over the first 2 years and HLA DQB1 defined genetic markers. Close to $5 \%(36 ; 4.8 \%)$ of the children had no autoantibodies at clinical diagnosis. Their mean age (10.4 years) was older than that of the other subjects ( 8.3 years: $p=0.002$ ), but there were no differences between the two groups in sex distribution, clinical signs, blood glucose levels or frequency of ketoacidosis at diagnosis. The autoantibody-negative children had higher serum C-peptide concentrations at $12(\mathrm{p}=0.005), 18(\mathrm{p}<0.001)$, and 24 months $(p<0.001)$, and lower daily insulin doses at $6(p<0.001), 12(p<0.001)$ $18(p=0.02)$, and 24 months $(p<0.001)$ than those positive for at least one antibody after adjustment for age and multiple comparisons. A higher proportion of the autoantibody-negative children were in clinical remission at 12 months $(43.5 \%$ vs. $14.1 ; p=0.001)$ and 18 months $(14.0 \%$ vs. $2.4 \% ; p<0.001)$. No dif ferences were seen in the frequency of familial IDDM and the distribution of HLA DQB1 genotypes between the two groups. Accordingly children testing negative for IDDM-associated autoantibodies at disease presentation are characterized by an older age at diagnosis, prolonged preservation of endogenous insulin secretion, decreased requirement of exogenous insulin over the first few years of the disease, and an increased frequency of clinical remission. On the other hand we could not detect any differences in symptoms and signs at diagnosis or in the frequency of genetic risk markers. These data indicate that children testing negative for disease-associated autoantibodies at diagnosis do represent classical IDDM and not a young-onset subtype of NIDDM. 
346

ANTICARDIOLIPIN, ANTINUCLEAR AND ANTITHYROID AUTOANTIBODIES IN CHILDREN AND ADOLESCENTS WITH TYPE 1 DIABETES MELLITUS P. Ciampalini, L. Giovannelli ${ }^{*}, D$. Gianni, S. Corbi and A. Crinó - Dept. of Endocrinology and "Lab. of Immunology, "Bambino Gesù" Hospital, Rome, ITALY Type 1 diabetes mellitus (IDDM), like other endocrinopathies, is characterized by an increased prevalence of organ and non-organ-specific autoantibodies (Ab) Recently, high levels of anticardiolipin antibodies $(\mathrm{aCL})$ have been found in IDDM and autoimmune thyroid disease. We evaluated the prevalence of aCL-lgG and aCL-lgM (by ELISA assay) in 56 children and adolescents with IDDM (32 f, $24 \mathrm{~m}$; age $11.8 \pm 5$ yrs with duration of disease $3.9 \pm 2.4 \mathrm{yrs}$ ), and verified a possible correlation with antithyroid $A b(T A A)$ and antinuclear $A b$ (ANA). All patients were divided into 3 groups based on IDDM duration: Group I (n. 20, at onset - HDA1C: 8.8+2.2); Group II (n. 22, 1-5 yrs - HbA1c: $5.5 \pm 1.9$ ); Group III (n. 14, $>5$ yrs HbA1c: $5.8 \pm 2.1) .102$ age and sex-matched healthy subjects served as control group. aCL-IgG and aCL-IgM were positive respectively in $5 / 56(9 \%)$ and $1 / 56$ (2\%) out of diabetc patients, without any significant difference to the control group. ANA were positive in 12 patients out of $56(21 \%)(p<0.0001$ vs. control group) and TAA (HTG and/or TMS) in $11(20 \%)(\mathrm{p}<0.0001) .4$ TAA positive patients $(3 \mathrm{f}, 1 \mathrm{~m})$ resulted affected by Hashimoto's thyroiditis and subclinical hypothyroidism $(7 \%)$, while the other 8 patients had normal thyroid function. The frequencies of $\mathrm{aCL}$ ( $\mathrm{lgG}$ and $\mathrm{ggM}$ ). TAA and ANA according to the duration of the disease are reported in the table ( ${ }^{*} p<0.005$ and ${ }^{*} p<0.0001$ vs. control group).

\begin{tabular}{|c|c|c|c|c|}
\hline AUTOANTIBODIES & Group I & Group II & Group III & Control group \\
\hline - aCL-IgG (>20 GPL) & $2(10 \%)$ & $3(14 \%)$ & 0 & $8(8 \%)$ \\
\hline - aCL-IgM (>1OMPL) & $1(5 \%)$ & 0 & 0 & $2(2 \%)$ \\
\hline - HTG and/or TMS & $2(10 \%)$ & $3(14 \%)$ & $6(43 \%)^{* *}$ & $2(2 \%)$ \\
\hline - ANA & $3(15 \%)$ & $5(23 \%)^{* *}$ & $4(29 \%)^{*}$ & $3(3 \%)$ \\
\hline
\end{tabular}

No correlation was found between $\mathrm{aCL}$ (IgG and IgM) and sex, chronological age, metabolic control, and duration of the disease. In $3 / 5$ positive a-CL patients, ANA were positive too, but none of them had TAA. In conclusion, except aCL, TAA and ANA are significantly more frequent in young diabetic population and therefore the screening for these autoantibodies, especially for TAA, should be periodically repeated right after the onset of diabetes. Good metabolic control, short duration of IDDM and asbence of microvascular complications could explain the low prevalence of a- $\mathrm{Cl}$ in our patients.

\section{7}

PREVALENCE OF CELIAC DISEASE IN A COHORT OF CHILDREN AT ONSET OF TYPE 1 DIABETES.

R. Bonfanti, M. Viscardi, E. Bognetti, MC. Riva, P. Macellaro, F. Meschi, G. Barera, C. Bianchi and G. Chiumello.

Department of Pediatrics, Scientific Institute H San Raffaele, University of Milan, Italy.

The prevalence of celiac disease in Insulin Dependent Diabetes Mellitus (IDDM) patients has been reported between 1 to $4 \%$. Antiendomysial antibodies (EMA) detected by indirect immunofluorescence on section of monkey's distal esophagus, are a sensitive and specific marker of celiac disease. The aim of the study was to screen with EMA a cohort of newly diagnosed IDDM patients for celiac disease. We studied since $1 / 93$ to $12 / 95174$ patients $(72 \mathrm{~F}$, $102 \mathrm{M}$, mean age $8.7 \pm 4.7 \mathrm{yrs}$ ), consecutively referred to our Department for IDDM onset. All of them were tested for EMA and total IgA (to exclude selective IgA deficit); patients with positive EMA were indicated for the intestinal biopsy. We also evaluated: metabolic data ( $\mathrm{HbAlc}$, fructosamine, venous blood gas analysis), and auxologic parameters (height standard deviation score, relative body weight). All patients studied had normal IgA values; $11 / 161(6.8 \%$, 4F/7M) had positive EMA antibodies. The jejunal biopsy was performed in 8 of them ( 3 EMA positive patients refused) and a subtotal villar atrophy was found in 7 patients (confirmed celiac disease prevalence $=4 \%$ ). All patients with celiac disease at biopsy were HLA DR3/ DQ2. There were no significative differences for all the parameters studied at onset of IDDM between EMA positive and negative patients, but EMA positive patients were younger than EMA negatives : $4.86 \pm 2.3$ yrs vs $8.83 \pm 4.7$ yrs ( $p=0.01$ Mann Whitney $U$ test). In conclusion these results show that the increased prevalence of celiac disease is already present at clinical onset of IDDM. Clinical parameters, apart from age, are not different between patients with or without markers for celiac disease. We can speculate that a common immune-mediated mechanism is involved in both diseases.
348

NATURAL HISTORY OF IDDM IN PATIENTS WITH ENDOCRINE AUTOIMMUNITY: 'THE UK AND PADUA POLYENDOCRINE PROSPECTIVE STUDY'

M Lai, C Betterle*, R Foxon, R Zanchetta*, A C Spadaccino*, Christie M**, E Bonifacio**** and G F Bottazzo. Dept of Immunology, St Bartholomew's and the Royal London School of Medicine \& Dentistry, London, UK; *Istituto di Semeiotica Medica, University of Padua, Padua; **Dept of Medicine, King's College Hospital, London; ***Istituto Scientifico San Raffaele, Milan.

Autoimmunity (AI) to islets is associated with $\mathrm{AI}$ to other endocrine organs and IDDM can develop in patients with AI polyendocrinopathy (PE). We have followed $145 \mathrm{PE}$ patients, who were originally identified as ICA positive $(\geq 5$ JDF units) but non-diabetic, for up to 15 years for development of IDDM. 27 of the patients developed IDDM and in 12 of these IDDM was preceded by a period of $>1$ year of non-insulin requiring diabetes (slow onset IDDM). In this study, we have analysed sera from these patients for GADA and IA-2icA, using recombinant human in vitro transcribed and translated proteins. The Table summarises the results.

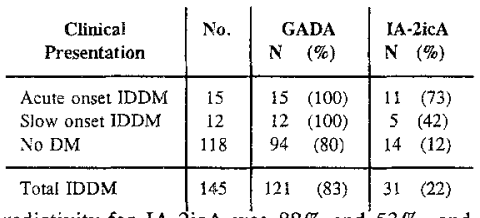

Specificity and predictivity for IA-2icA was $88 \%$ and $53 \%$, and for GADA $20 \%$ and $22 \%$, respectively. 48 sera were tested for $37 \mathrm{kD} / 40 \mathrm{kD}$ antibodies and for $I \mathrm{~A}$ 2icA. In the majority, there was a good correlation for positive and negative results, but in a few instances IA-2icA were detected in the absence of the other antibodies, indicating a higher sensitivity of the IA-2icA assay. In addition, a few sera had antibodies only to the $37 \mathrm{k}$ antigen, recently identified as phogrin. The results demonstrate an unusual high prevalence of GADA in absence of IDDM in PE patients and show that the presence of IA-2icA can identify most PE patients at risk for acute onset IDDM.
349

IN RECENT-ONSET INSULIN-DEPENDENT DIABETIC PATIENTS GAD65 POSITIVITY IS NOT ASSOCIATED WITH A SIGNIFICANT INCREASE OF THYROID AND GASTRIC AUTOIMMUNITY

I. De Leeuw, P. Abrams, J. Daems and the Belgian Diabetes Registry, Antwerp, Brusseis, Belgium

Previous studies have shown that at onset of type I diabetes significant levels of anti-thyroid peroxidase antibodies (anti-TPO) were more present in those patients positive for islet-cell auto antibodies (ICA) and anti-insulinauto antibodies (IAA) Giutamine acid decarboxylase autoantibodies (GAD65 Ab) are actually considered as good markers for type I diabetes and are also elevated in the Autoimmune Polyendocrine Syndrome 1 and in the Stiffman-syndrome. The purpose of this study consists of estimating if GAD $65 \mathrm{Ab}$ positivity $(\geq 0.026 \%)$ at onset of the disease is also associated with a higher incidence of thyroid (anti-TPO $>100 \mathrm{Um}^{-1}$ ) and gastric antiparietal-cell (APA $>1 / 20$ ) autoimmunity. Blood samples from 135 newonset IDDM (47 women and 88 men, median age $25 \mathrm{y}$, range 10-39y), collected from the Belgian Diabetes Registry, were assayed for GAD65 Ab, anti-TPO and APA with recognized methods. GAD65 AD were positive in $78.5 \%$ of the patients, anti-TPO in $19.3 \%$ and APA in $10.4 \%$. Women had a significantly higher positivity than men for GAD65 Ab $(91.5 \%$ versus $71.5 \%, p<0.01)$, for anti-TPO (34\% versus $11.4 \%, p<0.01)$ and for APA $(19.2 \%$ versus $5.7 \%, p<0.02)$. However only $16 \%$ of the patients who were GAD65 positive were also anti-TPO positive (NS) and $9.6 \%$ of the GAD65 Ab positive showed a significant titer of APA (NS). While GAD65 AB had the same prevalence in the younger (10-25y) as in the older group (26-39y) there was a non-significant trend for higher titers of anti-TPO and APA in the older group as well in men as in women. From this data is looks that in contrast to ICA and IAA positivity, GAD65 Ab positivity at onset of type I diabetes does not permit to speculate on the coexistence of thyroid and gastric auto-immunity and could belong to another auto-immune epitope. 
ANTIBODY RIESPONSE TO EXOGINOUUS ILUMAN INSULIN IN DIABETES: PREFERIENTIAL ASSOCIATTION WITH MARKIRRS OF AUTOIMMUNTTY? K. Decochez, B. Keymeulen, L. Van Gial*, F. Gorts and G.Somers. Depls of Endocrinology, Frec University of Brussels and University of Antwerp*, Belgium.

Ahout $40 \%$ of IIDDM paticuts ( $<40$ yrs) present insulin autoantibodies (IAA) before the staut of insulin treatment. In more than $95 \%$ of IDDM patients, human insulin s.c. increases or induces insulin antibodics $(\mathrm{I} A)$. To investigate whether this IA response reflects an inherent propery of ilurimmune diabetes or rather the antigenicity of human insulin, I $\Lambda$ titer was measured before and after 6 months of treatment with human (neutral/protamine) insulin in 55 NIDDM patients with secondary failure to oral hypoglycemic agents (age 40-75 yrs, diahetes duration: 2-19 yrs, fasting plasma C-peptide: $0.7 \pm 0.1 \mathrm{fmol} / \mathrm{l}$, mean $\pm S D$ ) and in $32 \mathrm{IDDM}$ patients $(\geq 40 \mathrm{yrs:} n=12$, age: 41-74 yrs,C-peptide: $0.2 \pm 0.1$ cmoll/; $<40$ yrs: $n=20$, age: $2-39$ yrs, C-peptide: $0.1 \pm 0.1$ finol/l). All subjects were tested for the presence of autoantibodies (IAA, ICA and GAD65-Ab) before insulin treatmenl. Prevalenee of IA $\Lambda$ positivity $(\geq 0.7 \%$ tracer bound) was significantly lower in NIDDM paticnts (2/55) compared in IDDM patients $<40$ yrs $(8 / 20, p<0.001)$ but not in comparison to IDDM patients $\geq 40 \mathrm{yrs}(1 / 12)$. At least one type of autoantihody was detecled in $32 / 32(100 \%)$ IDDM patients but only in $8 / 55(15 \%)$ NIDDM natients $(\mathrm{n}<0,001$ ). The range of diabetes duration in NIDDM patients wals 2.8-16.6 yrs in those with autoimmune markers compared to 2.0-19.4 yrs in those withou (NS). I $\mathrm{A}$ responsiveness alter 6 months of insulin treatment was detined as an increase above the I $\mathrm{A}$ cut-off vilue or of $0.3 \%$ above the already elevated pretreatment IAA valuc and was noted in all IDDM patients but only in $18 / 55(33 \%)$ of the NIDDM paticnts $(\mathrm{p}<0.001)$. The median change in IA titer was $0.1 \%$ (range: $-0.3-21.0 \%$ ) in NIDDM patients versus $4.8 \%(0.3-15.6)$ in IDDM patients $\geq 40 \mathrm{yrs}$ $(p<0.001)$ and $9.2 \%(0.6-38.3)$ in IDDM patients $<40 \mathrm{yrs}(\mathrm{p}<0.001)$. Prevalence of IA. esponsiveness in NIDDM was signilicantly greater in the presence of 3 -cell untoimmunity than in its ahsence (6/8 versus $12 / 47,0<0.015)$. No cotrelation between Ige, diabetes duration or insulin dose and IA responsiveness was found in NIDDM. In zonclusion, the immune response to exogcnous insulin seens related to preexisting uutoimmunity rather than 10 intrinsic attigenicity of recombinant hormone per se.
ANTIOXIDATIVE DEFENCE IN ICA-POSITIVE FIRST-DEGREE RELATIVES OF TYPE $I$ DIABETIC PATIENTS

M. Vučić, B. Ročić, J. Knežević-Ćuća, V. Profozić, I. Pavlić-Renar, *A Radica, *J. Pasini and $\check{Z}$. Metelko, Vuk Vrhovac Institute and *Rebro Clinical Hospital, Zagreb, Croatia

It has been suggested that oxidative stress might be involved in the pathogenesis of type 1 (insulin-dependent) diabetes mellitus, taking part in initiating and/or augmenting an autoimmune destruction of the pancreatic $\beta$-cell.

The aim of this study was to investigate the antioxidative defence system in ICA-positive first degree relatives of insulin-dependent diabetic (IDD) patients (prediabetic subjects) by evaluating total plasma antioxidant status (TAS) and $\mathrm{Cu}, \mathrm{Zn}$-superoxide dismutase ( $\mathrm{Cu}, \mathrm{Zn}$-SOD) activity in isolated polymorphonuclear leukocytes. The study was performed in 10 prediabetic subjects (mean age $=30.8 \pm 9.2$ years, ICA-range $=6-85 \mathrm{JDF} U$ ), 12 recently diagnosed IDD patients (mean age $=34.3 \pm 6.1$ years, mean $\mathrm{HbAlc}=9.76 \pm 2.98 \%$ ) and 14 age- and gender-matched ICA-negative healthy controls. TAS and $\mathrm{Cu}, \mathrm{Zn}-\mathrm{SOD}$ activity were determined by the colorimetric and xanthine/xanthine-oxidase assays, respectively.

Plasma TAS was significantly lower in prediabetic subjects, when compared to healthy controls, whereas no difference was found in respect to IDD patients $(1.64 \pm 0.08$ vs $1.80 \pm 0.12$ vs $1.71 \pm 0.20 \mathrm{mmol} / 1 ; \mathrm{p}=0.02$ and 0.49 , respectively). Cu, Zn-SOD activity in polymorphonuclears of prediabetic subjects was unchanged in comparison to healthy controls, but significantly higher than in IDD patients $(0.94 \pm 0.33$ ws $1,06 \pm 0.43$ vs $0.58 \pm 0.39 \mathrm{U} / \mathrm{mg}$ proteins; $\mathrm{p}=0.79$ and 0.04 , respectively).

These data indicate that a disturbance of overall extracellular antioxidative defence in prediabetes could be related to decreased polymorphonuclear $\mathrm{Cu}, \mathrm{Zn}-\mathrm{SOD}$ activity in clinically manifest IDDM.

\section{2}

THE SIGNIFICANCE OF HUMAN IgGI KAPPA IN PREDICTION FOR AUTOIMMUNE ACTIVATION IN TYPE 1 DIABETES

i.Yaylım(1), B.Güvener(1), E.Özkök(1), I.Satman (1,2) and M.T. Yılmaz(1,2) (1) Istanbul University, institute for Experimental Medicine, (2) Istanbul Medical Faculty, Div. of Diabetes, Turkey.

Circulating immune complexes (cIC) are elevated in autoimmune activation period in Type 1 diabetes. In this study we investigated the predictive value of fractions of human $\operatorname{lgG}$ by precipitation and purification of $\mathrm{ClC}$ in three different clinical stages (preclinical, early, late) in 29 Type 1 diabetics and in 8 non-diabetic controls. The precipitation of $\mathrm{clC}$ is accomplished by caprilic acid-ammonium sulphate method, purification by agarose gel filtration electrophoresis and fractioning of igG using protein $A$ sepharose column in HPLC. $\mathrm{CIC}$ is determined with complement fixation technique, ICA by indirect immunofluorescence and insulin antibody with RIA. There were 7 diabetics in preclinical stage (mean chr. age $25 \pm 10.6 y$ rs, ICA<20.JDF $7 / 7$, IAA $5.1 \pm 1.3 \%$ ), 9 in early clinical stage (mean chr. age 17.3 $\pm 6.6 y r s$, duration of diabetes $2.8 \pm 1.1 \mathrm{mo}, I C A(+) 6 / 9$, AlA $11.8 \pm 6.5 \%)$, 13 in late clinical stage (mean chr. age $35.5 \pm 14 y r s$, dur. of diab. $5.2 \pm 6.7 y r s$, ICA $(+) 6 / 13$, AlA $23.7 \pm 18.5 \%)$. The control group consisted of 8 subjects (mean chr. age $24.4 \pm 11.9 y r s$, ICA $(+)$ 0/8, IAA $0 \%$ ). In Type 1 diabetic group $\mathrm{ClC}$ rate was significantly elevated compared to the control

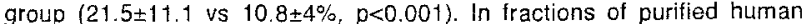
IgG, IgG1 $\mathrm{k}$ was found to be most elevated. Purified total $\mathrm{lgG}$ and $\lg \mathrm{G} 1 \mathrm{k}$ $(\mathrm{mg} / \mathrm{dl})$ levels were as follows: In control group $1.72 \pm 0.4$ vs $0.98 \pm 0.2$, in preclinical stage $3.9 \pm 1.0$ vs $2.03 \pm 0.9$, in early clinical stage $3.77 \pm 1.8$ vs $1.8 \pm 0.91$, in late clinical stage $5.1 \pm 1.8$ vs $1.86 \pm 0.6$. As a result, in pre- and early clinical stages of Type 1 diabetes, in which autoimmune activation is augmented, IgG1k levels are found to be significantly elevated; in late clinical stage these levels show a decrease although total IgG levels are elevated as a consequence of interfering antibodies like AlA. Changes in $\lg G 1 \kappa$ and positivity of ICA are correlated in different stages of diabetes.

\section{3}

High proinsulin levels in lace predianetes stage C Rodríouez-Viliar, I Conget, R Casamitjana*, J Vidal, M Fernândez ard R Gomis. Endocrinology anci Nutrition Unit, Homonology Unit ${ }^{x}$. Hospital Clínic, Barcelona, spain.

Aim: to analyze the fasting proinsulin levels in first degree relatives of IDDM patiencs. Subjects and Methods: We studiec and followed 41 first degree relatives of IDDM patients separated into three groups: $1^{\circ}$ / 18 first degree relatives with normal fasting g'ucose and negative ICA (1BM/BF] aged from 7-60 vears; 20$) 17$ Eirst degree relatives with normal fasting glucose and positive ICA (1OM/7F) aged from $8-31$ years; $\left.3^{\circ}\right) 6$ first degree relatives with normal fasting glucose and positive ICA (5M/LT), aged froil $14-52$ years, who developed IDDY after 32 months. he tested fasting proinsul in (DRO) and insulin (IRI) levels by radioinmunoassay (RIA) and the PRo/iRI ratio. Results: On comparison of the ICA positive groun who developea IDDM with the ICA positive without IDDM groud, the former group showed significantly higher fro tastino levels $\mid \mathrm{Z} \pm \mathrm{BS}$ : $24+3$ pH/L vs $13.5: 1.3, p<0.001)$. The PRO fasting levels in the ICa + with IDOK group were also higher than those in the ICA negative without IDDY group (15.69 +2.20 , D $<0.051$. No differences were observed in DRo fasting leveis between the ICA positive and the ICA negative group without IDDM. Nevertheless, no differences were observed in Easting IRI levels in the three groups: $1^{\circ} 9$ ICA with IDCM $\left.(98.9+25.05 \mathrm{mM} / 1), 2^{3}\right)$ ICA without IDDM (101.2+21.8) and $\left.3^{\circ}\right)$ ICAwithout IDOM $\{178,4 \pm 47,7\}$. When we analyzec the RRO/IRI tatio we observed higher PRO/IRI ratio in the ICA+ with IDDM groug as compared with the ICA. group $10.32+0.08$ vo $0.15+0.01, p<0.0011$, although we did not observe any differences in PRo/IRI rat io in the ICA + with IDDM as compared with the ICA+ without IDDM group $10.19 \pm 0.03)$. Conclusion: In spite of not observing differences in the basal insulin sectetion in first degree relatives of IDDM patients, those who developed the disease showed figh proinsulin secretion. These data indicate that fasting PRO levels and the PRO/CRI rario may be used as additional markers for defining the late prediabetes srage. 


\section{4}

\section{ANALYSIS OF THE T-CELL RECEPTOR AV REPERTOIRE IN TYPE 1 DIABETES \\ Heike Naserke, Ivana Durinovic-Bello; Patrick Ott and Anette-G. Ziegler. Diabetes Research Institute and Academic Hospital München-Schwabing, Munich, Germany.}

Type 1 diabetes results from a T-cell-mediated destruction of insulin-producing $B$-cells. The $T$ cell receptor (TCR) plays a centrale role in antigen recognition and immune regulation and is postulated to contribute to the development of autoimmune disorders. We analyzed the TCRAV repertoire of peripheral blood $T$ cells by polymerase chain reaction in 8 patients with newly diagnosed type 1 diabetes, 9 non-diabetic antibody positive relatives at high risk of type 1 diabetes and 9 healthy control subjects. We found increased frequency of T-cells expressing TCR AV17 and AV11 families in antibody positive relatives (mean \% of internal standard: AV17: $87 \%$ and AV11: $88 \%$, respectively; $p=0.006$ and $p=0.04$ ) and increased $A V 17$ expression in type 1 diabetic patients (mean $38 \%$; $=0.04$ ) compared to control individuals (mean AV17: $8 \%$ and AV11: $24 \%$, respectively). Expansion of single TCRAV families in our patients may be generated through an antigen-driven stimulation of $T$ cells. This is supported by our previous findings that $T$ cells reactive with multiple islet cell antigens exist in the preclinical and clinical stage of autoimmune diabetes.

\section{5}

THE ROLE OF CYTOKINES IN PATHOGENESIS OF IDDM AND ITS COMPLICATIONS

I.A.Bondar, V.I.Masicheva , S.D.Oreshkova, L.D.Sidorova. Medical Institute Internal Diseases Dpt. Krasn.Prospect 52, 630091, Novosibirsk, Russia.

The present study we aimed at investigation of the expression cytokine genes and the level of cytokines in blood of IDDM patiens. To evaluate the level of expression of cytokine genes (IL-1 1 , IL- 6 , TNF- $\alpha$ ) in blood hybridization test was used while level of IL-1 $\beta$ and TNF- $\alpha$ in serum was measured by ELISA analys. The group of IDDM patiens consists of 62 and control group - of 50 persons. All IDDM patients had the frequency and level of expression of all determined cytokine genes reliably higher then in control group $(44 \%, 20 \%, 40 \%$ patients and $26 \%, 0 \%, 13 \%$ control for IL-1 $\beta$, IL- 6 , TNF- $\alpha$ correspondingly). The analys of IDDM dynamics have revealed the lack of expression of the same cytokines during recently appeared, slowly and/or favourable developing of the desease. During long-term IDDM the cytokines expression was observed for patients with progressive microangiopathy, under insuline dosage changing and under infection. The low content of TNF- $\alpha$ in blood serum registrated in $31 \%$ IDDM patients are thought to play an important tole in beginning of desease and infection. While the high level of IL-1 1 revealed in $29,3 \%$ patients is the marker of fast developing of vascular complications and of generalization of autoimmunity. The increasing of TNF- $\alpha$ and decreasing of IL-1 $\beta$ levels during treatment coincided with positive dynamic of IDDM. Carried out correlation analys have shown that the amount of serum IL-1 $\beta$ and TNF- $\alpha$ and expression of cytokine genes are linked to metabolic disorder, are dependent of insuline, cortizol, duration of IDDM and age. Dependence of some immunological (CD3, CD4. HAE3, HAE9, IgA) and biochemical factors (cholesterol, albumine, seromucoid and potassium) on the level of cytokines in serum and cytokine mRNAs in blood cells was revealed. Presented data have clearly indicated that cytokines are important for pathogenesis IDDM and its complication and for prediction of IDDM dynamic.

\section{6}

$T$ LYMPHOCYTES SUBSETS DISTURBANCES IN THE BLOOD OF SUBJECTS AT HIGH-RISK OF INSULIN-DEPENDENT DIABETES.

A. Krętowski, M. Szelachowska, M. Pietruczuk ${ }^{*}$, I. Kinalska, Department of Endocrinology and Department of Clinical Biochemistry*, Medical Academy Białystok, Poland

It is suggested that the key role in the process of autoimmune destruction of pancreatic B-cells play activated T-cells. They were found in the human and animal islets at the onset of IDDM. There are also a few studies of the lymphocytes subsets disturbances in the peripheral blood at the diabetes diagnosis. However these findings could not reflect the changes at the early stage of B-cells autoimmune destruction, which begins even years before the clinical onset. The aim of this study was the estimation of the lymphocyte subpopulations in peripheral blood of ICA positive first degree relatives of IDDM subjects, since the abnormalities of humoral immunity were established as an early marker of the autoimmune process. The study was performed in 39 first degree relatives with persistent ICA $>10$ JDF (ICA titers were determined by the ENDIT central laboratory, London) and 34 healthy, age and sex matched control subjects. Leukocytes preparation from whole blood were performed in the Q-Prep EPICS Immunology Workstation. T cell subsets were measured using the combinations of monoclonal antibodies: $\mathrm{CD} 2, \mathrm{CD} 3, \mathrm{CD} 4, \mathrm{CD} 8$, CD14, CD45, CD45RO, CD56 and flow cytometry techniques (EPICS $\mathrm{XL}$, Coulter). In the group at the prediabetic stage we observed statistically significant increase of the percentage of CD45RO+ memory cells $(p<0.01)$ and decrease of NK cells $(p<0.05)$ in peripheral bloód in comparison to the control. We suggest that activated CD8+ memory $T$ cells and NK cells could be involved at the initial stage of autoimmune process, probably in the antigen presentation in pancreatic B-cells.
357

HISTOPATHOLOGICAL AND IMMUNOHISTOCHEMICAL FEATURES OF THE LABIAL SALIVARY GLANDS IN CHILDREN WITH TYPE I DIABETES.

A.MARKOPOULOS* A.GALLI- TSINOPOULOU * M.BELAZI. "Dentistry Department "and 4 th Pediatric Department " Aristotes University of Thessaloniki Greece

The aim of this study was to investigate the morphological alterations of labiai salivary glands in children with type I diabetes. For this purpose labial salivary glands under local anesthesia were excised from the lower lip of ten diabetic children at the onset of diabetes (mean age 10 vears). Additionally ten similar biopsies were obtained from ten healthy children (mean age 9 years). The tissues were conventionally processed and embedded in paraffin wax. The first section was stained with hematoxylin-eosin, while two additional sections were stained immunohistochemically using the indirect immunoperoxidase avidin - biotin method in order to detect $T$ and $B$ cells. The results of this study showed that a mononuclear infiltration was apparent in all diabetic children Lymphocyte aggregations were randomly seen throughout the salivary gland section. Lymphocytes were particularly seen around various ducts. In contrast the salivary glands of healthy controls were free of infiltrates and were characterized normal. The immunohistochemical analysis for the characterization of the infiltrates showed that the majority of these cells were $T$ cells. B cells were also observed to a lesser extend and were predominantiy found on the periphery of infiltrates. These findings indicate that a destruction of labial salivary glands takes place in children with type I diabetes. This phenomenon is mainly T cell mediated. The similarity of this condition with the well - known destructive process of the pancreas of diabetics (insulitis ) suggests that the labial salivary glands and pancreas may share a common antigen that might be the target of the autoimmune process in typel diabetes 


\section{8}

GAD65 REACTIVE LYMPHOCYTES FROM PATIENTS WITH IDDM EXPRESS GUT-SPECIFIC HOMING RECEPTOR $\alpha 4 \beta 7$-INTEGRIN O.Vaarala, P.Klemetti, J.Paronen, J.M. Kantele, E. Savilahti and H.K. Åkerblom Children's Hospital, University of Helsinki, Department of Biochemistry and Department of Bacteriology, National Public Health Institute, Helsinki, Finland. Migration of lymphocytes to the pancreas is a prerequisite for insulitis in insulindependent diabetes mellitus (IDDM). Mucosal vascular addressin (MAdCAM-1). involved in the recirculation of lymphocytes to the gut, is found in the inflamed islets in nonobese diabetic (NOD) mice. In humans, triggers of the gut immune system such as exposure to cow milk proteins or to enteroviral infections are associated with the risk of IDDM. We tested the role of gut-specific lymphocyies expressing $\alpha 4 \beta 7$ integrin, which is a ligand for MAdCAM- 1 , in the cellula: immunity to glutamate decarboxylase (GAD65). Proliferation to GAD65 in peripheral blood mononuclear cells (PBMC) and in the $\alpha 4 \beta 7$-depleted population of PBMC was studied in 7 patients with IDDM and in one subject at bigh risk of IDDM (258 JDF units of ICA). $\alpha 4$ 4 7+ PBMC were depieted by immunomagnetic separation using monoclonal antibody to $\alpha 4 \beta 7$ (ACT-1). A marked decrease $(>30 \%)$ in proliferation to GAD65 was found in 4 patients and in the subject with high ICA levels (No.6 in Figure) after depletion. In contrast, depletion of $\alpha .4 \beta 7$ +lymphocytes resulted in increased reactivity to a parenteral antigen, tetanus toxoid, indicating that these two antigen-specific T-cell populations show distinct homing properties.

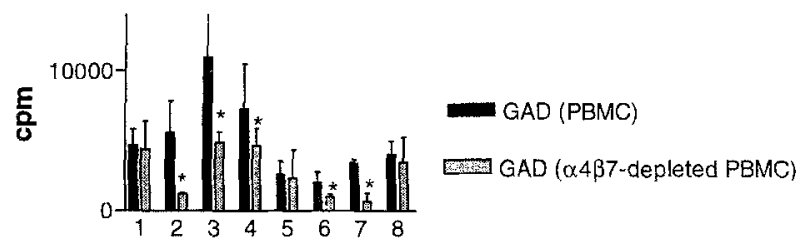

These findings demonstrate that islet cell reactive lymphocytes express a gut-specific homing receptor, which emphasizes the role of gut immunity in IDDM.

\section{0}

ANTI-INSULIN ACTIVITY IN NEW-BORN SERUM IS NOT IgG-MEDIATED L. Castaño, J.R. Bilbao, B. Calvo and I. Urrutia, Endocrinology \& Diabetes Research Group, Hospital de Cruces, Barakaldo-Bizkaia, Spain INTRODUCTION: Autoantibodies to insulin (IAA) are a major immunological marker of progression to type I diabetes (IDDM), being present in more than $50 \%$ of new onset diabetics and among first degree relatives at high risk. Biochemical characterisation of diabetes-related IAA has shown that they are IgG immunoglobulins. It has been shown that IAA may be found in some new-borns from the general population. We have studied this activity among general population new-borns, and attempted to determine its nature.

MATERIAL. \& METHODS: We analysed sera from 116 new-borns without family history of type I diabetes as well as from 21 of their mothers at delivery. Anti-insulin activity and other diabetes-related autoantibodies (islet cell -ICA-, and anti-GAD) were determined by IDW standardised methods. In 12 of the new-born samples, the IAA assay was also performed after lgG separation with either protein $A$ or $G$ agarose.

RESULTS: Anti-insulin activity was detected in the serum of $91 \%$ of the newborns (control population at other ages $<2 \%$ ), while anti-GAD and ICA levels did not differ from those found in the general population. IAA were present in $19 \%$ of the mothers at delivery, but at lower levels than in their children. All protein A/G purified IgG fractions from new-born sera were negative for IAA, and the activity remained in the unbound fraction. In the case of IAA positive new onset diabetics $(n=5)$ the activity was bound to the protein $A / G$ resin. CONCLUSION: Highly sensitive/specific IAA-RIA methods are able to detect anti-insulin activity in the majority of new-born sera. However, this activity is not IgG related, which together with the absence of other autoantibodies, suggests that there is no islet specific autoimmune response. Biochemical studies are needed to determine the nature of this insulin-binding molecule present at birth.
359

PREDICTORS OF LONG-TERM RESIDUAL BETA-CELL FUNCTION IN IDDM. M. Fernánitz-Castañer, J.Gonzálbez, M.J.Carrera, V.Nacher, R.Insa. E.Montanya and J.Soler. Endocrine Unit. CSU Bellvitge. Barcelona. Spain.

Residual B-cell function can be present several years after the diagnosis of IDDM in some patients. To study the possible influence of clinical, metabolic and immunological parameters at diagnosis and during the first two years of the evolution of IDDM on the long-term preservation of a significant residual B-cell function, we analysed the data of 57 IDDM patients diagnosed from March 1986 to March 1990 that have completed a five year follow up period in 1995 . The influence of individual characteristics at diagnosis (age, sex, duration of symptoms, ketoacidosis, islet-cell antibodies) and during the first two years of follow-up (residual B-cell function, metabolic control and insulin requirements) on residual B-cell function at five years was evaluated by multiple linear regression (stepwise method). Differences between groups were evaluated by non-parametric tests.

18 patients had a significant residual B-cell function at five years ( post-glucagon C. peptide $\geq 0.15 \mathrm{mmol} / 1$ ). When compared to patients without residual $\mathrm{B}$-cell function $(\mathrm{n}=39)$, they showed minor significant differences in age (22.7 vs 20.0 years), sex (77.7 vs $48.7 \%$ of males), duration of symptoms (12.9 vs 7.2 weeks), ketoacidosis at diagnosis (23.3 vs $46.1 \%$ ), and ICA positivity at diagnosis (41.1 vs $69.4 \%$ ). They also had a better metabolic control ( 8.8 vs $10.8 \%$ of total HBA1C) with less insulin $(0.48 \mathrm{vs} 0.71 \mathrm{uj} / \mathrm{kg}$ ) during the first two years of evolution. Initial B-cell function was similar between the two groups. In the multivariate analysis, only metabolic control during the second year of evolution $(\mathrm{p}=0.008)$, ketoacidosis at diagnosis $(p=0.026)$ and $\operatorname{sex}(p=0.026)$ had an independent influence on B-cell function at five years of diagnosis.

We conclude that a good metabolic control in the first years of evolution of IDDM can have a positive influence in the long-term preservation of residual B-cell function.

\section{1}

Binding specificity of IAA in cord blood. The EarlyBird study.

TS Galloway, BA Millward, D Fewster and TJ Wilkin, Department of Medicine, Plymouth Postgraduate Medical School, University of Plymouth, Drake Circus, Plymouth PL4 8AA

We have previously reported insulin binding in 7-10\% cord blood of infants born to non-diabetic mothers, which is displaceable with excess insulin and occupies a distribution apart from the remaining $90 \%$. We show here that these IAA are predominanty immunoglobulin $G$ and show variable cross-reactivity with the related molecules proinsulin and IGF-1, but none with myoglobin. cord sera from the offspring of non-diabetic mothers were radioassayed for binding to insulin, proinsulin and IGF-1 using a cold ligand displacement step and PEG and anti-human IgG separately as phase separants. Immunoprecipitation identified as IAA positive the same sera as $d i d P E G$, and in the same rank order of binding. The molarity of cold ligand required to displace 50 : binding (a measure of affinity) was, for insulin $0.105-$ $0.298 \mathrm{nmol} / 1$, for proinsulin $2.60-4.32 \mathrm{nmol} / 1$ and for IGF-1 $8.96-81.9 \mathrm{nmol}$. IAA positive sera from both newly diagnosed and treated IDDM patients showed similar cross-reactivity to proinsulin but none at all to IGF-1, suggesting idiotype differences. The data suggest that class-switched IAA are present in cord sera where they may represent a pool of natural insulin antibodies. The idiotype differences we have noted may reflect antigendriven maturation from birth to diabetes onset. 


\section{2}

AUTOIMMUNITY TO PANCREATIC BETA CELLS IN CONGENITAL DIABETES MELLITUS.

F. Dotta, C. Cilio, L. Farilla, C. Moretti, A. Bosco, N. Sulli, P. Colarizi, C. Tiberti, E. Anastasi, G. Multari and U. Di Mario. Dept. of Endocrinology and Dept. of Peidatrics, University of Rome; Dept. of Experimental and Clinical Medicine, University of Catanzaro, Italy.

Congenital diabetes is an extremely rare form of the disease and very little is known about the pathogenetic mechanisms involved in such a condition. We report a case of a neonate who developed IDDM in the first day of life, followed by diarrhea and eczematous diffuse skin lesions. He died at 29 days of age because of necrotizing enterocholitis. He had no family history for type 1 or type 2 diabetes, while 6 other male neonates in his mother's family died within the first year of life (one with diarrhea and skin lesions). At day 4 of life he and his mother were studied for the presence of ICA, IAA, anti-GAD65, anti-GM2-1 and anti-KCA512 autoantibodies; circulating levels of $C$ peptide and $T$ lymphocyte subsets were also determined. We could also perform immunohistochemical and morphological studies on the pancreas and on several other organs including heart, kidney, fiver, thyroid, thymus, spleen and brain obtained at autopsy in the neonate. Plasma $\mathrm{C}$ peptide was undetectable in the neonate and was normal in the mother; $T$ cell subsets were normal in both subjects, while the baby, but not the mother, was positive at high titer both for IAA and for anti-GAD65 autoantibodies indicating an autoimmune basis of the diabetic syndrome in this case. Both subjects were negative for other autoantibodies. Histological studies showed the presence of a massive mononuclear cell infiltrate destroying the endocrine pancreas and leaving the exocrine portion of the gland intact. Such infiltrate contained CD4, CD8 and NK cells. By immunohistochemistry, glucagon-positive but not insulin-positive cells were found among infiltrating mononuclear cells, indicating the $B$ cell specificity of the destructive process within the pancreas. Interestingly, a similar infiltrate was found in the heart, while other organs examined were normal. In conclusion, we have demonstrated that $B$ cell autoimmunity can be involved in the development of congenital IDDM and, therefore, that loss of immune tolerance to islet components can aiready start in intrauterine life. The presence of myocarditis together with insulitis may suggest that a viral infection may be the cause or at least the precipitating event leading to $B$ cell destruction in this case.

\section{PS 7}

\section{Animal Immunology}

\section{3}

IN THE BB/PFD RAT AUTOANTIGEN IS NECESSARY FOR MAINTENANCE OF AUTOIMMUNE MEMORY

C. Mathieu, K. Casteels, J. Laureys and R. Bouillon, LEGENDO, KULeuven,

Belgium

The BB/Pfd rat colony distinguishes itself from other BB rat colonies by the fact that it develops type I diabetes spontaneously, but does not show disease recurrence after syngeneic islet transplantation, when this transplantation is performed several days after diabetes diagnosis. BB/Pfd rats transplanted on the day of diabetes diagnosis do show diabetes recurrence. The only difference between rats at the two timepoints of transplantation is the amount of $B$ cells still present in the native pancreas of the transplanted rats: in diabetic BB/Pfd rats all $B$ cells have disappeared within 8 days after diabetes diagnosis. We proposed therefor the hypothesis that in these BB/Pfd rats autoimmune memory is not sustained due to the disappearance of autoantigen. The aim of the present study was to confirm or refute this hypothesis. BB/Pfd rats were screened for diabetes daily and on the day of diabetes diagnosis, rats were randomised in 2 groups: group $I(n=6)$ received insulin until day 12 and was then transplanted with 2000 neonatal BB/Pfd islets under the kidney capsule. Group II $(n=6)$ received not only insulin but was injected daily from the day of diabetes diagnosis until day 12 with 100 neonatal BB/Pfd islets intraperitoneally, in order to maintain the presence of islet autoantigens. On day 12 also these rats were transplanted using the same protocol as described above. After a follow up period of already 60 days, only $1 / 6$ rats in group I showed disease recurrence, while $4 / 6$ rats in group II destroyed the syngeneic islet graft within 10 days. These data confirm the hypothesis that in the BB/Pfd rat model autoimmune memory is only sustained in the presence of autoantigen. Recently in the NOD mouse similar conclusions could be drawn as demonstrated by Larger et al. These observations may not only have implications for the understanding of the pathogenesis of type I diabetes and its recurrence after islet transplantation, but also for clinical islet transplantation itself.

\section{4}

VASCULAR CELL ADHESION MOLECULE 1 MEDIATES ISLET LYMPHOCYTE ADHESION IN DIABETIC INSULITIS IN MICE

M. Enghofer, R. Ludwig, J. Bojunga, K.H. Usadel and K. Kusterer Dept. of Medicine I, J.W. Goethe-University, Frankfurt a. M., FRG

The role of the interaction between the adhesion molecule VCAM- 1 on recipient islet endothelium and VLA-4 on circulating donor lymphocytes in the transfer model of low-dose streptozotocin (STZ) diabetic insulitis was investigated. Diabetes was induced in C57BL/6 mice by daily i.p. injections of $40 \mathrm{mg} / \mathrm{kg} \mathrm{STZ}$ over $5 \mathrm{~d}$. On day 21 donor lymphocytes were prepared by Ficoll gradient centrifugation of a splenic homogenate. The cells were stained by the fluorochrome acridine red in vitro. $2 \times 10^{7}$ of these cells were infused into the $V$. cava of anesthetized recipient mice within $5 \mathrm{~min}$. Recipient mice had been pretreated with a subdiabetogenic dose of STZ (5 mg/kg i.p.) $24 \mathrm{~h}$ before the transfer. Endothelial adhesion of stained donor lymphocytes (DLA) in islets and adjacent exocrine pancreatic tissue was measured for $1 \mathrm{~h}$ after cell transfer under a fluorescence microscope. In the experimental group ( $n=5) 400 \mu \mathrm{g} / \mathrm{kg}$ monoclonal anti-VCAM-1 was given i.v. 10 min before the transfer. Control animals $(n=10)$ received PBS i.v. In the control group islet DLA was $8.6 \pm 5.8$ cells/islet $x h$ and DLA in exocrine tissue was $3.4 \pm 3.3$ cells/region of interest (ROI, same size as islet) $\times h$. Anti-VCAM-1 reduced islet DLA to $2.4 \pm 0.7$ cells/islet $x h(p \leq 0.05$, unpaired $t$-Test), whereas DLA in exocrine tissue was unchanged in this group $(3.2 \pm 7.1 \mathrm{cells} / \mathrm{ROI} \times \mathrm{h}, \mathrm{n.s}$. $)$. Blood flow velocity and donor lymphocyte flux were similar in both groups. We conclude that the adhesion molecule VCAM-1 on islet endothelium mediates an important step in the initial phase of lymphocytic infiltration of islet tissue in this transfer model of diabetic insulitis. 


\section{5}

THE ONSET OF DESTRUCTIVE INSULITIS IN NOD MICE IS CHARACTERIZED BY IGIF AND IL-12p40 GENE EXPRESSION

V. Burkart, H. Kolb and H. Rothe. Diabetes Research Institute at the University of Düsseldorf, D-40225 Düsseldorf, Germany

The acceleration of diabetes in NOD mice by cyclophosphamide (CY) treatment induces a shift of the T-helper lymphocyte type (Th) from Th2 to Th1. This shift occurs with the onset of a destructive form of insulitis and is characterized by an increased expression of interferon $\gamma$ (IFN- $\gamma$ ). In order to study the mechanisms involved in this process we examined the expression of the regulatory cytokines interleukin 12 (IL12) and IFN- $\gamma$-inducing factor (IGIF) in NOD mice. By reverse transcriptase polymerase chain reaction (RT-PCR) we found a 6fold increase of IL-12p40 mRNA in pancreas and spleen within $3 \mathrm{~d}$ of $\mathrm{CY}$ treatment. In parallel we observed a 5 fold increase of IGIF mRNA in the pancreas of NOD mice. Compared to the nonadherent spleen cell fraction we found a 1000 fold higher expression of IL-12p40 mRNA and $a 10$ fold higher expression of IGIF mRNA in the adherent fraction of the spleen cells. This finding indicates that macrophages are the major source of IL-12 and IGIF in CY-treated NOD mice. In normal $\mathrm{BALB} / \mathrm{c}$ mice no signal of IL-12p40 mRNA and only a weak signal of IGIF mRNA was detected. In conclusion, upregulation of IL-12- and IGIF-expression in macrophages appears to drive the differentiation of naive $T$ cells towards $\mathrm{IFN}-\gamma$ producing Thl cells which mediate destructive insulitis.

\section{6}

SOLUBLE FORMS OF INTERCELLULAR ADHESION MOLECULE-1 (ICAM-1) INTERVENE IN PATHOGENESIS OF IDDM IN NOD MICE

S. Martin, E. Heidenthal, B. Schulte, H. Kolb - Clinical Department, Diabetes Research Institute at the Heinrich-Heine-University, Düsseldorf, Germany

Adhesion molecules play an important role in the migration of white blood cells from vessels into tissue and in mediating costimulatory signals during $T$-cell activation. Soluble forms of adhesion molecules are detectable in sera of healthy persons at low levels, and in increased levels in a variety of pathological conditions. We described elevated levels of naturally circulating interceltular adhesion molecule 1 (cICAM-1) in subjects at genetic risk but without any clinical or immunological signs of IDDM, compared to IDDM patients and healthy controls. Recently, we demonstrate that autoreactive T-cell proliferation could be suppressed by recombinant soluble ICAM-1 and that multivalent ICAM-1-Ig fusion proteins were approximately 1000 -fold more effective. Aim of this study was to investigate the therapeutic effect of soluble ICAM-1 and multivalent ICAM-1-Ig fusion proteins in the pathogenesis of IDDM in NOD mice. Recombinant proteins were constructed using the murine amino acid sequence of ICAM-1 and IgG1. Large scale production was performed using the bacculovirus expression system and purification by immunoaffinity chromatography. Beginning of age 35 days NOD mice received $5 \mu \mathrm{g}$ recombinant soluble ICAM-1 or multivalent ICAM-1-Ig by intraperitoneal injections three times a week until the age of 170 days. Histological examinations were performed at $120 \mathrm{~d}$. Diabetes development was monitored by measuring urine glucose and in case of positivity by blood glucose determination. Blood glucose values above $200 \mathrm{mg} / \mathrm{dl}$ were considered as diabetic. In comparison to sham treated animals diabetes development was significantly reduced in NOD mice treated with soluble ICAM-1 or ICAM-1-Ig (control $45 \%$, ICAM-1 $8 \%$ and ICAM-1-Ig $8 \%$ diabetes incidence at 170 days). After termination of therapy animals treated with multimeric ICAM-1-Ig were longer protected than animals treated with monomeric ICAM-1 (control $71 \%$, ICAM-1 $38 \%$ and ICAM-1-Ig $21 \%$ diabetes incidence at 220 days). Histological examination of pancreata at $120 \mathrm{~d}$ showed marked reduction of advanced stage insulitis in treatment groups. These data suggest that soluble monomeric ICAM-1 and ICAM-1-Ig prevent infiltration and development of IDDM in NOD mice and thus provide novel means for therapy of immune mediated diseases.

\section{7}

EFFECT OF INTRATHYMIC ADMINISTRATION OF MYCOBACTERIAL HEAT SHOCK PROTEIN 65 AND PEPTIDE p227 ON THE DEVELOPMENT OF DIABETES IN NOD MICE: CAUTION REQUIRED IN VACCINATION STUDIES

D. P. Funda ${ }^{1,2}$, M-L. Hartoft-Nielsen ${ }^{1}$, A. Kaas ${ }^{1}$ and K. Buschard ${ }^{1}$

${ }^{1}$ Bartholin Instituttet, Kommunehospitalet, Copenhagen, Denmark, ${ }^{2}$ Department of Immunology and Gnotobiology, Institute of Microbiology, Czech Academy of Sciences, Prague, Czech Republic.

Heat shock protein 65 (hsp65) and a derived peptide, p227 are reported autoantigens in IDDM I $p$ injection of hsp 65 reduced diabetes incidence in NOD mice and administration of p227 cured already diabetic NOD mice. On the other hand, glutamic acid decarboxylase (GAD65) has been shown as the key target antigen in murine IDDM and its intrathymic administration led to a lower incidence of diabetes in NOD micc. The aim of this study was to evaluate, whether the intrathymic injection of mycobacterial hsp65 or the 2227 can prevent diabetes in NOD mice. 3-week-old NOD female mice were injected intrathymically with $50 \mu \mathrm{g}$ of hsp65 $(\mathrm{n}=30), 5 \mu \mathrm{g}$ of $\mathrm{p} 227(\mathrm{n}=30)$, and PBS $(\mathrm{n}=28)$. The incidence of diabetes was observed for the following 280 days and the degree of insulitis was scored by computer image analysis. No significant differences in diabetes incidence were observed among the three groups of mice. Interestingly, hsp65 treated mice developed diabetes at $177 \pm 6$ days compared to $202 \pm 8$ days $(\mathrm{p}=0.015)$ for the p277 treated group and $197 \pm 7$ days $(p=0.033)$ for controls. The degree of insulitis and average islet size did not significantly differ among the three groups of diabetic mice. Thus, our data show an earlier onset of diabetes in hsp65 treated mice and no improvement of the incidence by neither hsp65 nor p227, suggesting that hsp65 as an autoantigen in IDDM acts in a different way from that observed with the GAD65. The results pose the question of the potential danger of hsp65; caution should therefor be taken in future vaccination studies.

\section{8}

EFFECT OF ANTAGONIST OF B. BRADYKININ RECEPTOR IN EXPERIMENTAL DIABETES IN RATS

M A Ortiz, C. Xaus*, J. Roselló* and J.M ${ }^{a}$ Pou. Dept. Endocrinology. Hosp. Sant Pau. Autonomous University of Barcelona. *C.S.I.C. University of Barcelona.

The present study was designed to elucidate the effect of a $\mathrm{B}$ bradskinin receptor antagonist (HOE-140) in the pancreatic endothelin sunthesis and prostacyclin production and their relationship with plasma insulin levels in a model of Streptozotocin (STZ) induced experimental diabetes. Wistar male rats were distributed in 4 groups: A: controls. B: STZ rats $(100 \mathrm{mg} / \mathrm{kg}$ once). C: STZ rats intraperitoneaily treated with NOsinthase inhibitor (NAME. $1.0 \mathrm{mg} / \mathrm{k} / 5$ days) and D: STZ with HOE- 140 $(1.32 \mathrm{mg} / \mathrm{kg} / 5 \mathrm{dars})$. Pancreatic endothelin and 6-Keto-PGF $1 \alpha$ (6-K-PGF) were measured by ELISA (pg/mg.protein). Plasma insulin (by RIA) and glycaemia levels were also determined(basal $\mathbf{l}_{i} / \mathbf{G}_{\mathrm{l}}$ index).

Results: In STZ rats, NAME increased endothelin pancreatic production and HOE-140 did not. (group A: $8.6 \pm 2.1$, B: $6.02 \pm 1.36$, C: $21.7 \pm 3.5^{*}$, D: $10.7 \pm 1.48 . * 0<0.04), 6-\mathrm{K}-\mathrm{PGF}$ was stimulated by the NAME and HOE-140 (group: A: $162.5 \pm 24.3$, B: $119.4 \pm 19.5$, C: $241.8 \pm 62.4^{*}$, D:243.3 $\pm 61.5^{*}$ $\left.{ }^{*} \mathrm{p}<0.04\right)$. The pancreatic relation between $6-\mathrm{K}-\mathrm{PGF} /$ endothelin was higher in STZ treated with HOE-140 than with NAME $(p<0.05)$. HOE-140 partially protected $\mathrm{I}_{0} / \mathrm{G}_{0}$ in $\mathrm{STZ}$ rats ( $40 \%$ vs controls).

Conclusions: We can conclude that the $\mathrm{B}_{2}$ bradykinin receptor antagonist (HOE-140) protect $\beta$ pancreatic cell from the STZ effect by an important increase of 6-K-PGF without endothelin modification and it was suggested a mechanism of yasodilation in islet microcirculation secondary to the HOE140 effect. 
369

EFFECTS OF NICOTINAMIDE ON THE DEVELOPMENT OF DLABETES IN NON OBESE DIABETIC (NOD) AND C57BL/KsJ DIABETIC (db/db) MICE. N.C. Tumer and V.Piercy. SmithKline Beecham Pharmaceuticals, The Frythe, Welwyn, Hetrfordshire AL6 9AR. U.K.

Nicotinamide (NIC), an inhibitor of poly (ADP-ribose) synthase, is currently in clinical trials for the prevention of IDDM. In this study we compared the effects of NIC on the development of diabetes in NOD and $d b / d b$ mice, models of IDDM and NDDM respectively, 4-6 week old mice were treated with NIC, $5 \mathrm{~g} / \mathrm{kg}$ in the diet, for 12 weeks $(d b / d b)$ or 24 weeks (NOD). Untreated mice served as baseline (day 0 ) and time-matched controls. Untreated NOD mice did not become hyperglycaemic, but pancreatic bistology showed evidence of insulinitis and there was a significant $(p<0.05)$ reduction in both senum $(s)$ and pancreatic $(p)$ insulin (ins) levels ( $\mathrm{s}$ ins $0.99 \pm 0.3 \mathrm{ng} / \mathrm{ml}$; $\mathrm{p}$ ins $112 \pm 31 \mathrm{ng} / \mathrm{mg}, \mathrm{n}=10$ with standard error of the mean) compared to baseline controls ( $\mathrm{s}$ ins $2.49 \pm 0.4 \mathrm{ng} / \mathrm{mI}$; $\mathrm{p}$ ins $317 \pm 64$ ng/mg, n=6). Both serum $(2.8 \pm 0.5 \mathrm{ng} / \mathrm{ml})$ and pancreatic $(363 \pm 104 \mathrm{ng} / \mathrm{mg})$, insulin levels were preserved in NOD mice treated with NIC $(n=9 ; p<0.05$ compared to untreated controls). In $d b / d b$ mice baseline blood glucose and serum and pancreatic insulin levels were $10.5 \pm 1 \mathrm{mM}, 127 \pm 37 \mathrm{ng} / \mathrm{ml}$ and $85 \pm 16 \mathrm{ng} / \mathrm{mg}$ respectively $(\mathrm{n}=7)$. Mice became markedly hyperglycaemic $(28.2 \pm 1.2 \mathrm{mM}, \mathrm{n}=7)$ with an associated fall in serum insulin levels to $11.7 \pm 1.8 \mathrm{ng} / \mathrm{ml}$; neither were affected by NIC treatment. Pancreatic insulin content however, was significantly lower in NIC treated $(17 \pm 5 \mathrm{ng} / \mathrm{mg}, \mathrm{n}=7)$ compared witb untreated mice ( $30 \pm 2.6$ $\mathrm{ng} / \mathrm{mg}$ ). An increase in water consumption and urine output was evident by week 2 in treated mice, but not until week 10 in untreated mice. Urinary glucose excretion was greater in treated than untreated mice from weeks 1 to 4 . NIC reduced body weight gain. In conclusion, although nicotinamide treatment preserved insulin secretion and secretory capacity in an animal model of IDDM, the accelerated development of polydypsia, polyuria and glycosuria together with a lowering of pancreatic insulin content in $d b / d b$ mice indicates, that in a model of NIDDM nicotinamide accelerated the development of diabetes.

\section{1}

CELLULAR INFILTRATION PATTERN IN RAT ISLET ALLOGRAFTS CAUSING PROTECTION AGAINST IDDM IN BB RATS.

T. Sparre, U. B. Christensen, A. Cooke', T. Mandrup-Poulsen and J. Nerup. Steno Diabetes Center, Gentofte, Denmark. Department of Pathology, Division of Immunology, University of Cambridge, Cambridge, UK.

IDDM is characterized by mononuclear ceil infiltration in the islets and immune-mediated selective destruction of B-cells. BB-DP rats receiving istet allografts but not syngrafts are protected against IDDM development normaly occurring on day $69 \pm 25$ in our colony. To investigate the difference in the pattem of mononuclear cell infiltration in allogeneic or syngeneic grafted islets to BB-DP rats, 200 neonatal Wistar Kyoto (WK) or diabetes prone BB (BB-DP) rat: islets were transplanied under the kidney capsule of 30 day-old BB-DP rats. The rats were killed on days $7,12,23,37,48,90$ and 173 after transplantation or at onset of diabetes. The grafts and pancreata were stained for MHC class I and II, $\alpha \beta-T C R, C D 4, C D 8, E D 1$ and insulin. The insulin content (percentage of stained area of the total graft area) was $15-25 \%$ lower on days 7,12 , and 23 and $10-20 \%$ higher on days $37,48,90$ and 173 after transplantation in allografts than in syngrafts.

\begin{tabular}{||l|l|l|l|l|l|l|}
\hline \multicolumn{7}{|c|}{ Infiltrating cells expressed as cell/mm ${ }^{2}$ in allogratts $(\mathrm{n}=3 /$ syngrafts $(\mathrm{n}=6)$} \\
\hline Day & MHC 1 & MHC II & $\alpha$ - $-T C R$ & CD4 & CD8 & ED1 \\
\hline 37 & $721 / 796$ & $343 / 700$ & $77 / 106$ & $131 / 399$ & $11 / 114$ & $174 / 213$ \\
\hline 48 & $109 / 337$ & $226 / 249$ & $11 / 42$ & $550 / 240$ & $3 / 9$ & $40 / 84$ \\
\hline 173 & $36 / 775$ & $116 / 966$ & $91 / 246$ & $113 / 318$ & $18 / 127$ & $102 / 315$ \\
\hline
\end{tabular}

A similar infiltration pattern for MHC I, $\alpha \beta-T C R$ and ED1 positive cells was seen in the native islets in situ and in the graft in the syngraited BB-DP rats. In the allografted rats the native islets in situ showed similar pattem for MHC Il as in the graft, but only scarce infitration of $\alpha B-T C F$ and $\mathrm{ED} 1$ positive cells. In conclusion, islets ailografts to BB-DP rats reduce cellular infiltration in the native islets and prevents IDDM, and allograft rejection is inhibited. Fewer inilitrating cells are seen in the allografts at corresponding timepoints when compared to syngrafts. Further studies are needed to investigate if allograting has immunomodulating effects in BB-DP rats.
370

PREVENTION OF TYPE I DIABETES BY LATE INTERVENTION WITH ANALOGUES OF VITAMIN $D_{3}$ COMBINED WITH CYCLOSPORIN A. K. Casteels, M. Waer, R. Bouillon, K. Allewaert, J. Laureys and C. Mathieu Lab. Experimental Transplantation and LEGENDO, KULeuven, Leuven, Belgium In animal models, type I diabetes can be prevented by chronic use of immunosuppressants, i.e. Cyclosporin A (CyA), but this approach is unacceptable in humans. In NOD mice, diabetes can also be prevented by non-calcemic analogues of vitamin $D_{3}, 1,25(\mathrm{OH})_{2} D_{3}$, when treatment is started early, i.e. before insulitis is present. A more relevant question for the human situation is however, whether these substances can arrest progression to clinically overt diabetes when administered in a more advanced disease stage, when active $\beta$ cell destruction is present. The aim of the present study was to evaluate the protective potential of a non-calcemic analogue of $1,25(\mathrm{OH})_{2} \mathrm{D}_{3}, \mathrm{MC1} 288$, both alone and in combination with a short induction course of $\mathrm{CYA}$, in NOD mice that already show active $B$ cell destruction. To confirm the presence of insulitis histologically in each individual mouse pancreatic biopsies were performed, 15 days prior to therapy. Subsequently these mice were randomised into 3 treatment groups, receiving respectively CyA $7.5 \mathrm{mg} / \mathrm{kg} / \mathrm{d}$ from d85 to d105 $(\mathrm{n}=19) ; \mathrm{MC} 12880.1 \mu \mathrm{g} / \mathrm{kg} / 2 \mathrm{~d}$ from $\mathrm{d} 85$ to $\mathrm{d} 200(\mathrm{n}=20)$ or the combination of both regimens $(\mathrm{n}=20$ ). Before treatment was administered (day 70 ), insulitis was present in $69 \%, 83 \%$ and $71 \%$ of mice in the CyA, the MC1288 and the combination group respectively (NS). Diabetes outcome by 200 days of age was $74 \%(14 / 19)$ in the CyA-treated group, which is comparable to diabetes incidence in the stock colony. Treatment with MC1288 did not reduce disease incidence $(70 \% ; 14 / 20)$, but the combination therapy reduced diabetes incidence to $30 \%(6 / 20)(p<0.01 v s$ CyA group, $\mathrm{p}<0.025$ vs $\mathrm{MC} 1288$ ). All treatments were well tolerated, without any side effects on calcium and bone metabolism. Immunological screening could not detect immunosuppression. At present we could not demonstrate circulating suppressor cells (in co-transfer experiments), but local immunoregulation seems to be present as is suggested by PCR analysis of pancreatic mRNA. In conclusion, we propose a role for non-calcemic analogues of $1,25(\mathrm{OH})_{2} \mathrm{D}_{3}$ in prevention of type $I$ diabetes. Even late intervention when the autoimmune disease is already active, can achieve disease protection, when this therapy is combined with a short induction course of an immunosuppressant such as CyA.

\section{2}

DIFFERENT INSULIN STRATEGIES ACT DIFFERENTLY ON DIABETES PREVENTION IN THE LOW-DOSE STZ MOUSE MODEL.

${ }^{1}$ E. Ponte, ${ }^{2} \mathrm{E}$. Anastasi, ${ }^{2} \mathrm{C}$. Tiberti, ${ }^{2} \mathrm{E}$. Vecci, 'G. Ghirlanda and ${ }^{3} \mathrm{U}$. Di Mario. Universities "Sacro Cuore", "La Sapienza" of Rome and "RCCatanzaro, Italy.

At present, insulin treatment in animal models of diabetes is considered to be an interesting approach in attempting to prevent or delay beta cell destruction. The objective of this work was to evaluate an appropriate prevention strategy using insulin in the low-dose streptozotocin (STZ) mouse model. Diabetes has been induced in male mice C57Bl6/J with STZ (50 $\mathrm{mg} / \mathrm{Kg}$ bw for five consecutive days). Insulin (1U/day) was injected in STZ mice (Group A, n.21) for ten consecutive days before the induction of diabetes and for a further ten days: control non-diabetic mice (Group B. n.16) were treated using the same protocol. Control groups, normal mice (Group C. n. 10) and diabetic mice (Group D, n.25) have been included in the study. The animals of each group were killed at two intervals: half of them at day 12 and the remainder at day 24 from the beginning of the treatment. Blood glucose levels $(\mathrm{mmol} / 1)$ and insulitis calculated as percentage of infiltrated islets multiplied by the degree of infiltration (score) were evaluated at 12 and 24 days. At day 12 we observed the following glycaemic values (mean $\pm \mathrm{SD})$ : $\mathrm{A} 1(1.9 \pm 0.6)$, $\mathrm{Bl}(7.8 \pm 2.7), \mathrm{Cl}(7.0 \pm 0.4), \mathrm{D} 1(12.5 \pm 4.0)$ and insulitis score: A1 $(37 \%), \mathrm{B} 1(0), \mathrm{Cl}(0)$, D1 $(67 \%)$. At day 24 the glycaemic values were: $\mathrm{A} 2(13.0 \pm 4.3), \mathrm{B} 2(8.0 \pm 1.8), \mathrm{C} 2(10.2 \pm 2.2), \mathrm{D} 2$ $\{17.0 \pm 6.5)$, while the insulitis scores were $\mathrm{A} 2(30 \%), \mathrm{B} 2(0), \mathrm{C} 2(0), \mathrm{D} 2$ $(77 \%)$. At day 12 and 24 differences in mean blood glucose levels and insulitis scores between Group A and Group B were statistically significant: $(p<0.001, p<0.05) ;(p<0.01, p<0.01)$. In conclusion, this work suggests that the dose of insulin administered is able to reduce insulitis at day 12 and 24 but not to normalise glycaemia over time. In the early phases of islet damage, treatment with insulin should be prolonged over time in order to avoid the depletion of its protective action. 
ORAL INSULIN FOR THE PREVENTION OF IDDM IN NOD MICE: POTENTIATION BY BACTERLAL ADJUVANT

B. Hartmann, K. Bellmann, R. Kleemann and H. Kolb; Clinical Department of the Diabetes Research Institute at the Heinrich-Heine-University, Düsseldorf

Oral treatment with insulin is about to be tried in clinical studies for prevention of type I diabetes. However, beneficial effects of oral insulin on diabetes development were only limited in NOD mice and absent in BB rats. Hence, potentiation of clinical effects appear desirable. We show here that an orally administered benign bacterial adjuvant significantly enhanced diabetes suppression by oral insulin in NOD nice. 42d-old female NOD mice were treated in groups of 36 animals perorally with a) normal saline b) $1 \mathrm{mg}$ insulin (porcine insuline, Hoechst $A G$ ) c) $200 \mathrm{mg} / \mathrm{kg}$ adjuvant (endotoxin free protein extract of E. coli) d) $200 \mathrm{mg} / \mathrm{kg}$ adjuvant $+1 \mathrm{mg}$ insulin weekly $1-3$ times for 3 months. Weight, urine glucose and blood glucose were measured as parameters of metabolic function. On day 162 five animals per group were sacrified and pancreas and gut were obtained for immunohistochemical and mRNA analysis. The remaining animals were followed for determination of diabetes incidence. Kinetics of diabetes development were similar in the control group and in mice receiving adjuvant only, reaching $70 \%$ at $250 \mathrm{~d}$ of age. Oral insulin delayed diabetes onset but did not decrease diabetes incidence. The group receiving oral insulin plus adjuvant showed a significantly reduced incidence of $40 \%$ on day 250 . The insulin treated groups showed a reduction of insulitis and an increased expression of the diabetes-protective cytokine interleukin- 4 by islet infiltrating celis. Treatment with the bacterial adjuvant upregulated Th2 cytokine expression in the gut and this probably potentiated the induction of Th2-type "suppressor T cells" by oral insulin. Clinical trials of oral insulin in pre-type I diabetes should consider the use of bacterial adjuvant for improving clinical outcome.
REPEATED BCG VACCINATION IS MORE EFFECTIVE TIIAN SINGLE DOSE IN PREVENTING DIABETES IN NOD MICF, N. Shehadeh, A. Etzioni, A. Cahana, G. Teninboum, B Gorodetsky, D. Barzilai and E. Karnieli. Juvenile Diabetes Unit-Fndocrine Institute and Pediatric Department, Rambam Medical Center and the B.

Rapapport Faculty of Medicine- Technion, Haifa.

Stimulation of the NOD mouse immune -system with single BCC; vaccination can inhibit the development of diabetes. The optimal dose, the time and number of vaccinations is still to be clarified In this study we evaluated the protective effect of repeated $\mathrm{BCG}$ vaccinations on preventing diabetes in NOD mice. $17 / 32(53 \%)$ of the control group, $8 / 31(26 \%)$ of the single vaccine (at age 35 days) treated mice, $7 / 2.3$ $(30 \%)$ of the single vaccine (at age 90 days) treated mice developed diabetes, and none of the repeated BCG vaccination (at age $35 \& 90$ days, $n=14$ ) animals developed the disease, up to 250 days of age $(\mathrm{P}<0.05$-compared with controls and each of the single vaccination groups). While, the severity of insulitis was lower in repeatedly $\mathrm{BCC}$ treated mice, at age 120 days, compared with controls and single $B C G$ vaccination groups, we could not detect significant deference's in the Intracellular adhesion molecule-1 (IC.AM-1) expression in the various groups. There were no differences in weight gain and blood hematocrit between the different groups. Our report demonstrates that repeated BCG vaccination is safe and more effective than a single dose in preventing type I diabetes in NOD mice. This data should be considered in planing new human trials with BCG

\section{PS 8}

\section{Cytokines, NO and Beta-Cell Integrity}

\section{5}

NEITHER NITRIC OXIDE NOR PGE SYNTHESIS MEDIATE THE EFFECTS OF CYTOKINES ON FETAL ISLETS

Garcia C, Alias-Diaz J, Villa N, Hemandez J, Balibrea IL, Vara E; D. Biochemistry (F Medicine) and Surgery (HUSC), Madrid, SPAIN

It has been previously shown that both interlenkin 1 (IL-1) and tumor necrosis factor (TNF $\boldsymbol{\alpha}$ ) inhibit the insulin secretory response to glucase of fetal islets. On the other hand, some effects of cytokines on target celis have been found to be mediated through the production of either prostaglandin $\mathrm{E}_{2}$ ( $\mathrm{PGE}$ ) or nitic oxide (NO). This study was designed to investigate the role of $\mathrm{PGE}_{2}$ and $\mathrm{NO}$ in modiating the effect of cytokines on fetal islets. The pancreata of 21.5-day-old Wistar rat fetuses were partially digested with collagenase After overnight culture, the islets were separated from exocrine tissue and cultured for 6 days. Purified isiets were then incubated for 18 hours in the presence or absence of $I L-1$, $\mathrm{TNF} \alpha, \mathrm{PGE}_{2}$, sodium nitroprusside (NP), indonethacin (INDO), $N^{\omega}$-nitro-L-arginine (NAME), aminoguanidine ( $\mathrm{AG}$ ), and combinations. The $\mathrm{NO}$ and $\mathrm{PGE} \mathrm{F}_{2}$ release, and the insulin, somatostain, lipid peroxides (LPO), cGMP, and $\mathrm{PGE}_{2}$ content of the islets were measured. Insulin and somatostatin secretion were sludied in batch-type incubations in $\mathrm{KRB}$ ( 5 or 20 mmollL glucose). Both IL-I and TNF $\alpha$ increased the production of PGE (39.7 76.3, IL -1 and $15.6 \pm 4.2, \mathrm{TNF} \alpha$, vs. $6.2 \pm 1.0, \mathrm{pg} / \mathrm{siset}$, medium, $\mathrm{n}=14, \mathrm{p}<.01)$ and NO $(52.7 \pm 4.5, \mathrm{IL}-1$, and $56.1 \pm 3.8$, TNF $\alpha$, vs. $35.5 \pm 1.9, \mathrm{pmol} / \mathrm{islet}, \mathrm{n}=20, \mathrm{p}<.01)$. The increase in $\mathrm{PGE}_{2}$ was blocked by INDO, and that of NO was blocked by either INDO, NAME, or AG. However, neither INDO nor NAME or AG were able to modify the inhibitory effect of IL-1 and TNF $\alpha$ on islet insulin secretion and content. In addition, neither NP (a NO donor) nor exogenous PGE did modify the insulin response of fetal islets. We conclude that, although IL-I and TNF $\alpha$ stimulate the production of both PGE and NO by fetal rat islets, these mediators are nol responsible for the cytokine-induced inhibition of the insulin secretory response to glucose.

\section{6}

CYTOKINE RELEASE DURING CO-CULTURE OF HUMAN LYMPHOMONONUCLEAR CELLS WITH FRESH OR CULTURED ALLOOR XENOISLETS.

$R$ Giannarelli, P. Marchetti, M. Ferdeghini, C. Arvia, C. Tellini, A. Coppelli, S. Del Guerra, R. Lupi, R. Consolini, C. Prontera, M. Carmellini, F. Mosca, R. Navalesi. Cattedra Malattie Metabolismo, Istituto Clinica Medica II, Università di Pisa, Italy

According to the pattern of cytokines they release, activated T-helper (Th) lymphocytes can be subdivided into two subpopulations, Th1 and Th2. Differential activations of these Th subsets are likely to occur during allo- vs xeno-recognition. In this study we evaluated whether isolated human (HI) and bovine (BI) islets, either fresh (Fr) or cultured for 4 weeks ( $4 w$ ) activate Th1 and Th2 lymphocytes differently. We prepared human lymphomononuclear cells (LMC) from peripheral blood, and cocultured $1 \times 10^{6}$ LMC with 100 hand-picked islets, either fresh or cultured, for 1 to 5 days. Soluble interleukin-2 receptor (IL-2R, U/L), interferon $\gamma$ (IFN, U/mI), and interleukin-10 (IL-10, pg/ml) were measured by sandwich enzyme linked immunoadsorbent assay. As shown in the table, after 1 day of co-culure, compared to controls (C, LMC without islets), Fr-HI and Fr-BI caused a similar release of IL-2R and IFN, whereas IL-10 concentrations increased with Fr-BI, but not Fr-HI. After 5 days of co-culture. IFN levels were significantly higher than at day 1 with both FT-HI $(4.6 \pm 0.2)$ and $\mathrm{Fr}-\mathrm{BI}(4.6 \pm 0.3)$, whereas IL-10 concentrations remained constant with Fr-HI, and decreased significantly with Fr-BI $(57 \pm 12)$. After 4 weeks of culture, a decreased release of $\mathrm{IL}-2 \mathrm{R}$ and IFN was observed, whereas $\mathrm{IL}-10$ remained stable (see table).

\begin{tabular}{lcllll}
\hline & $\mathrm{C}$ & $\mathrm{FI}-\mathrm{HI}$ & \multicolumn{1}{c}{ FR-BI } & $4 \mathrm{w}-\mathrm{HI}$ & $4 \mathrm{w}-\mathrm{BI}$ \\
\hline $\mathrm{IL}-2 \mathrm{R}$ & $12.9 \pm 1.9$ & $34.2 \pm 2.3^{*}$ & $28.7 \pm 5.6^{*}$ & $14.9 \pm 2.8$ & $7.9 \pm 3.3$ \\
IFN & $0.39 \pm 0.04$ & $1.22 \pm 0.05^{*}$ & $1.25 \pm 0.06^{*}$ & $0.51 \pm 0.08$ & $0.60 \pm 0.08$ \\
IL-10 & $42.9 \pm 5.2$ & $47.2 \pm 11$ & $151.5 \pm 38.2^{*}$ & $62.2 \pm 6.7$ & $102.8 \pm 16.4^{*}$ \\
\hline
\end{tabular}

Thus, Th1 activation (increased IL-2R and IFN) occurred similarly with fresh alloThus, Th1 activation (increased IL-2R and IFN) occurred similarly with fresh allo-
and xenoistets, and was more marked at day 5 than day 1; Th2 activation (increased IL-10) occurred with the xenoislets, and was more marked at day 1 than at day $5 ; 4$ week culture decreased Th1, but not Th2 activation. 


\section{7}

PARTICIPATION OF TY $\delta$ CELLS FROM TYPE I DIABETIC CHILDREN IN INHIBITION OF INSULIN SECRETION IN VITRO. N: Kvirkvelia, I. Vturina, N. Porakishvili, N.Gachechiladze, M.Kiknavelidze and R.Vasilov, Tbilisi State University, Tbilisi, Georgia, Institute of Biotechnology, Moscow, Russia

The effective mechanism responsible for autoimmune beta cell destruction remain elusive. In order to investigate, whether $T$ lymphocytes bearing $\gamma \delta$ Tell receptor could be involved in this process, we studied the role of $T \gamma \delta$ cells in inhibition of insulin secretion in vitro. As is known peripheral blood lymphocytes (PBL) from Type I diabetics inhibit insulin secretion by culturing rat beta cells. Inhibition is mediated by Tcells and is specific for Type I Diabetes. In our experiments after coculturing of PBL from Type I diabetic children and rat islet cells, islet cells loss the ability to respond to stimulatory media. Islet cell function was assessed by net insulin release in the presence of basal or stimulatory media and was expressed as insulin release index-IR. IR index in the group of diabetic children was $1.04 \pm 0.05$, while IR index in nondiabetic control subjects was $1.08 \pm 0.09(\mathrm{P}<0.01)$. In order to elucidate if $T \gamma \delta$ cells are responsible for this inhibiton we: 1)treated PBL from diabetic children with anti $\mathrm{T} \gamma \delta$ antibodies -TCR $\delta$ I (which recognises all $T \gamma \delta$ cells ) prior to coculturing with rat islet cells. Treatment of PBL with TCR $\delta$ I antibodies suppressed the inhibition of insulin secretion-IR index was increased to $1.82 \pm 0.15$, $(\mathrm{p}<0.01) ; 2$ ) isolated the fraction of large granular lymphocytes ( $L G L$.), enriched for T $\gamma \delta$ celis $(65 \%)$. The fraction of LGL inbibited insulin secretion (IR index was $1.09 \pm 0.02)$ as unfractionated PBL of diabetic children. The conclude that the suppression of inhibition of insulin secretion by anti $-T \gamma \delta$ antibodies and blocking of insulin secretion by LGL enriched for $T \gamma \delta$ cells indicate the possible involvement of $\mathrm{T} \gamma \bar{\delta}$ cells in anti-islet cellular immunity in vivo.

\section{8}

INTERFERON- $\gamma$ AND GLUCOSE STIMULATE MHC CLASS I EXPRESSION BY HUMAN AND RAT PANCREATIC $\beta$-CELLS

D. Pavlovic, M. Van de Winkel, B. Van der Auwera, M. C. Chen, E. Schuit, L. Bouivens, and D. Pipeleers. Diabetes Research Center, Vrije Universiteit Brussel, 1090 Brussels, Belgium.

Both increased MHC class I expression and aberrant MHC class II expression have been considered as potential $\beta$-cell triggers to their autoimmune destruction. Alterations in $\beta$-cell MHC expression are attributed to locally released cytokines, in particular interferon- $\gamma$. The present study used flow-cytometry and competitive reverse transcription - polymerase chain reaction to examine the effect of recombinant interferon- $\gamma$ upon $\mathrm{MHC}$ class I and class II expression by rat and human islet cells cultured at 6 and $20 \mathrm{mM}$ glucose. At both glucose concentrations, interferon- $\gamma$ induced a 3 to 4 -fold increase in surface MHC class I expression of rat and human $\beta$-cells; a similar effect was observed in the endocrine $\alpha$ cells and in the non-endocrine ductal cells. Interferon$\gamma$ elevated the cellular content in MHC class I mRNA; this stimulatory effect was 2-fold amplified in the presence of $20 \mathrm{mM}$ glucose. In the absence of interferon- $\gamma$, glucose $20 \mathrm{mM}$ also increased the $\beta$-cell content in MHC class I mRNA. Rat and human islet endocrine cells were negative for MHC class II, both in the absence and presence of interferon- $\gamma$. These data indicate that the increase in $\beta$-cell MHC class I expression - which was described in pancreata of diabetic patients - may result from exposure to locally released interferon- $\gamma$ and/or from the presence of a hyperglycemic state.
379

INTERFERON- $\alpha$ INDUCED ENZYME ACTIVITIES ARE DIFFERENTIALLY EXPRESSED IN $\beta$ AND $\alpha$ CELLS

$V$. Bonnevie-Nielsen, $K$. Buschard and T. Dyrberg. Dpt. of Medical Microbiology, Odense University, DK-5000 Odense. The Bartholin Institute, DK-1399 Copenhagen and Novo Nordisk, DK-2880 Bagsværd, Denmark.

In the etiopathogenesis of type 1 diabetes virus infections may play a significant role. However, it is largely unknown how viruses specifically attack the pancreatic $B$ cells. In any event, the presence of lymphocytic infiltrates in the islets of Langerhans is suggestive of an autoaggressive cytotoxicity, upregulated by different cytokines. We examined IFN- $\alpha$ dependent enzyme activities in BTC3 and $\alpha$ TC3 cells and in isolated rat $\beta$ and non- $\beta$ cells. IFN- $\alpha$ induces the expression of $2^{\prime}, 5^{\prime}$-oligoadenylate synthetase $\left(2^{\prime}, 5^{\prime} \mathrm{AS}\right)$ a family of enzymes that converts ATP into oligoadenine nucleotides $\left(2^{\prime}, 5^{\prime} \mathrm{A}\right)$. These enzyme products mediate mRNA degradation and thereby inhibit virus replication. In the presence of 500 $\mathrm{U} / \mathrm{ml}$ IFN- $\alpha$, the 2',5'AS activity amounted to $700 \%$ in BTC3 but did not increase significantly in $\alpha$ TC3 cells. In isolated rat $\beta$ celis the 2,,5'AS activity increased by $90 \%$ but not in non- $\beta$ cells. Enzyme activity was determined by the production of radioactive 2',5'A from $\alpha^{32} \mathrm{P}-\mathrm{ATP}$ and separated by ascending TLC. Western blotting of $\alpha \mathrm{TC} 3$ and BTC-cell homogenates confirmed the presence of at least two isoforms of the 2',5'As enzyme. We suggest that IFN- $\alpha$ induced 2',5'As activity may have a detrimental effect on $B$ cells.

\section{0}

LYMPHOCYTE INDUCED ICAM-1 EXPRESSION ON PANCREATIC BETACELLS OBTAINED FROM DIARETES-PRONE BB/OK RATS

H. Wanka, B. Kuttler and H.J. Hahn; Institute of Diabetes "Gerhardt Katsch" Karlsburg, Ernst-Moritz-Arndt-University of Greifswald, Germany

The destruction of pancreatic beta-cells leading to insulin-dependent diabetes is mediated by T-lymphocytes. To investigate the relationship between effector and target cells we used a 4 d syngeneic mixed lymphocyte (effector) islet (target) coculture (MLIC). After culture we measured the cytokine (IFNy, TNFa, MIP-2) release into the medium, the cytolytic capacity of $3 \times 10^{5}$ splenocytes from $90 \mathrm{~d}$ old $\mathrm{BB} / \mathrm{OK}$ rats against syngeneic pancreatic islets $\left({ }^{51} \mathrm{Cr}\right.$-release assay) and the expression of MHC class-I, MHC class-II and ICAM-1 antigens on beta-cells. Freshly isolated lymphocytes caused a specific ${ }^{51} \mathrm{Cr}$-release of $2.6 \pm 1.1 \%$. Cell cultivation without any stimulation resulted in a significant decrease of reactivity $(0.2 \pm 0.2 \%, \mathrm{ps}$ 0.05 ). Stimulation with syngeneic islets caused a significant increase of cytotoxicity $(6.2 \pm 1.8 \%, p \leq 0.05)$. During the MLIC islets induced a significantly enhanced release of IFNy $(62.0 \pm 14.7 \mathrm{vs}, 12.8 \pm 2.4 \mathrm{pg} / \mathrm{ml}, \mathrm{p} \leq 0.01)$ and MIP-2 $(2443.0 \pm 300 \mathrm{vs}$. $992.0 \pm 126.8 \mathrm{pg} / \mathrm{ml}, \mathrm{p} \leq 0.01)$. In contrast, TNFa secretion remained unchanged. Freshly isolated islets were composed of $85.7 \pm 3.0 \%$ beta-cells, from which $2.7 \pm 0.4$ $\%$ expressed MHC class-II antigen, $70.9 \pm 5.1 \%$ MHC class-I antigen and $5.8 \pm 1.0 \%$ ICAM-1 molecules, respectively. Independent on the presence of lymphocytes cultivation of islets caused a significant reduction of beta-cell number to about $74 \%$. The lymphocytes did not alter the expression of MHC class-I or MHC-class-II antigens on beta-cells. But they increased dramatically the ICAM-1 expression on beta-cells. Thus, the adhesion molecule was observed on $56.3 \pm 5.9 \%(\mathrm{p} \leq 0.01)$ of betacells compared to $20.4 \pm 4.6 \%$ of beta-cells obtained from control islets. This effect could not be found in a syngeneic MLIC using splenocytes from healthy LEW. IA rats. The results demonstrate a mutual relation between syngeneic lymphocytes and pancreatic beta-cells in vitro. Durind the MLIC lymphocytes of diabetes-prone $\mathrm{BB} / \mathrm{OK}$ rats became activated by syngeneic beta-cells and beta-cells reacted with an enhanced expression of ICAM-1 molecules probably dne to cytokine release. Thus, the results underline that target cells accelerate its own destruction by expression of specific epitops which probably enhance the target-effector cell binding. 


\section{1}

GLUCOSE STIMÜLATED ISLET CELLS ARE MORE SENSITIVE TO MONONUCLEAR DESTRUCTION THAN ISLET CELLS AT REST

A.Ekblond, M.Schou and K.Buschard, Bartholin Instituttet, Kommunehospitalet, DK-1399 Copenhagen K,Denmark

It has been shown that prophylactic insulin treatment prevents or delays IDDM in BB rats, NOD mice as well as in open humans studies. Dependent on the dosage, this treatment decreases endogenous insulin production and beta cell activity, and it may thereby restrain the autoimmune lysis. By the use of a chromium release assay we have investigated whether beta cell activity plays a role in in vitro islet cell destruction by mononuclear spleen cells (MNC). Mean differences in \% specific lysis \pm SEM $(\% S A \pm S E M)$ between unstimulated and stimulated $\mathrm{BB}$ rat islet cells with diabetic $\mathrm{BB}$ rat MNC as effector cells at effector:target $(E: T)$ ratios $25: 1 ; 50: 1 ; 100: 1$; and $150: 1$ were $0.6 \pm 1.9 ; 5.2 \pm 1.7 ; 10.6 \pm 2.3$ and $9.0 \pm 2.1$ respectively $(P=0.003)$. Using non-diabetic $\mathrm{BB}$ rat MNC against stimulated and unstimulated $\mathrm{BB}$ rat islet cells we found no significant difference in specific lysis $(P=0.9)$. Activity dependent cytotoxicity was also detected in cases with $\mathrm{BB}$ rat MNC effectors and non-diabetes prone Wistar Furth (WF) rat islet cells as targets; differences in \%SR + SEM at E:T ratios from $25: 1$ to $150: 1$ were $4.0 \pm 3.1$; $5.8 \pm 2.6 ; 12.2 \pm 5.9$ and $18.2 \pm 7.2$ respectively $(P<0.001)$. Also $W F$ rat MNC towards $\mathrm{BB}$ rat islet cells showed activity dependent cytotoxicity; differences in $\% S R \pm S E M$ were $8.2 \pm 3.6,7.8 \pm 4.2,9.6 \pm 3.1,17.6 \pm 9.2$ with increasing effector target ratios $(P=0.007)$. WF effectors to WF targets did not show activity dependent cytotoxicity $(P=0.4)$. The functional dependency of the islet cell destruction is most likely a product of both a mononuclear cell dysfunction and a specific islet cell expression as it can be initiated by both diabetes prone $\mathrm{BB}$ rat islet cells and $\mathrm{BB}$ rat mononuclear cells - one without the other. Thus, results show that in vitro glucose stimulated pancreatic islet cells are subjects to a higher cellular immunologic destruction by mononuclear spleen cells than unstimulated islet cells.
382

RECOVERY FROM TYPE 1 DIABETES IN THE BB/S-R RAT INVOLVEMENT OF ALTERED SENSITIVITY TO IL-1B? P. R. Hitchcock, D. J. Gwilliam, J. Barley* and A. J. Bone Dept. of Pharmacy, University of Brighton, Brighton, U.K., " Biomedical Research Facility, University of Southampton, Southampton, U.K. The spontaneously diabetic BB/S rat, exhibits a disease process closely resembling that in man. We report here a subgroup of the BB/S diabetes prone rat which demonstrate the ability to recover from IDDM (BB/S-R). We have studied 13 animals, all of which developed IDDM (hyperglycaemia, weight-loss), subsequently recovering a state of normoglycaemia, insulin therapy being withdrawn. Animals were diagnosed as diabetic at a mean age of $115 \pm 16$ days, receiving insulin therapy $(1,49 \pm 0.1$ lu/day) for a tota $19.7 \pm 3$ days, which was administered during 1 to 4 episodes of IDDM. istets were isolated from BB/S-R rats (mean age $245 \pm 22$ days) following a prolonged symptom-free period $(90 \pm 8$ days) and their glucose-stimulated insulin response profile compared to Wistar control islets (control $2 \mathrm{mM}$ glucose $1.54 \pm 0.19,20 \mathrm{mM}$ glucose $4.68 \pm 0.32$; BB/S-R $2.23 \pm 0.45$ and $4.47 \pm 0.81$ respectively: mean $\pm S E M, n=5$ ). Calcein AM/ethidium bromide staining confirmed the structural integrity of the BB/S-P islets, which also had comparable insulin contents to control islets. Cultured islets were treated with $\mathrm{IL}-1 \mathrm{~B}$ in a range of concentrations. BB/S-R islets maintained a significant secretory capacity after $24 \mathrm{hr}$ exposure to $\mathrm{LL}-1 \mathrm{~B}(0.021 \mathrm{U} / \mathrm{ml})$ (basal, $1.27 \pm 0.09$. Stimulated, $4.63 \pm 1.53 \mathrm{ng} / \mathrm{islet} / \mathrm{hr}$ mean \pm SEM $\mathrm{p}<0.01$ ) in contrast to Wistar controls in which the response was inhibited. Nitrite production in control islets was induced by $\mathrm{IL}-1 \mathrm{~B}(0.021 \mathrm{U} / \mathrm{ml})$ (untreated $-2.77 \pm 0.79$, treated $-4.75 \pm 0.28 \mu \mathrm{m} / 100$ islets $/ \mathrm{ml}$ mean \pm $\mathrm{SD}$, however there was no significant elevation in the BB/S-R islets $(8.15$ $+2.35 \mu \mathrm{M}$ and $7.74+0.81 \mu \mathrm{M}$ respectively). This lack of induction may partly refflect the significantly elevated basal level of nitrite production $(p<0.05)$ in these islets. Thus, preliminary results indicate that $\mathrm{BB} / \mathrm{S}-\mathrm{R}$ islets may provide a useful insight into the mechanisms of islet cell defence and repair.

\section{3}

SUPPRESSIVE EFFECTS OF INTERLEUKIN-12 ON MOUSE AND RAT PANCREATTC ISLET FUNCTION

J. Sternesjö and S. Sandler, Department of Medical Cell Biology, Uppsala University, Uppsala, Sweden.

When administered in vivo the cytokine interleukin-12 (IL-12) accelerates development of autoimmune diabetes in nonobese diabetic mice. It has been suggested that this effect is due to expansion of Th1-cells and enhanced production of Th1-type cytokines (e.g. interferon- $\gamma$ ). In this study we examined the effects of $\mathrm{KL}-12$ on isolated islets from the NMRI mouse and SpragueDawley rat. Islets were cultured in medium RPMI $1640+10 \%$ fetal calf serum and exposed for $48 \mathrm{~h}$ to recombinant mouse IL-12 $(0$ $0.1,0.5,1,10 \mathrm{ng} / \mathrm{ml})$. The islet glucose metabolism, as measured by the glucose oxidation rate (pmol/10 islets $\times 90 \mathrm{~min}$ ), was $422 \pm$ 31 in the mouse control group and decreased to $306 \pm 28$ in mouse islets cultured with $10 \mathrm{ng} / \mathrm{ml} \mathrm{IL-12}(\mathrm{p}<0.05)$. Lower concentrations of $\Pi$ - 12 did not induce any signicant suppressive effect on glucose oxidation. In rat islets the glucose oxidation rate was $615 \pm 58$ in the control group and $488 \pm 62$ after addition of $0.1 \mathrm{ng} / \mathrm{ml}$ IL- 12 $(p<0.01)$. There were no significant changes in the other experimental groups. Islets cultured with $10 \mathrm{ng} / \mathrm{ml} \mathrm{IL}-12$ showed a decrease in medium insulin accumulation, (\% compared to control), both in mouse $(80 \pm 7.5)$ and $\operatorname{rat}(72 \pm 4.1)(p<0.05$ and $p<0.01)$. In mouse islets IL-12 did not induce any nitric oxide production compared to control as measured by nitrite formation(pmol/islet $\mathrm{x}$ h). In control rat islets nitrite formation was $0.37 \pm 0.08$ in control and decreased to $0.03 \pm 0.03$ with $10 \mathrm{ng} / \mathrm{ml} \mathrm{IL}-12$ added $(\mathrm{p}<0.01)$. The present study showed that IL-12 has suppressive effects on mouse and rat pancreatic islet function without inducing nitric oxide formation.

\section{4}

CYTOKINES INDUCE EXPRESSION OF INTERFERONREGULATORY FACTOR-1 (IRF-1) IN INSULIN-PRODUCING CELLS

M. Flodström ${ }^{1,2}$ and D.L. Eizirik ${ }^{2}{ }^{1}$ Department of Medical Cell Biology, Uppsala University, Uppsala, Sweden and 'Department of Metabolism and Endocrinology, Vrije Universiteit Brussel, Brussels, Belgium.

Activation of the enzyme nitric oxide synthase (iNOS) may be an important mechanism of $\beta$-cell damage in IDDM. IL- $\beta$ alone induces iNOS expression in rodent islets, but several cytokines are required to iNOS expression in rodent islets, but several cytokines are required to with electromobility shift assay (EMSA) that activation of the transcription factor NF-KB is necessary but not sufficient for iNOS induction in human islets. In the follow up of these experiments we studied the expression of IRF-1 in insulin-producing cells. IRF-1 binds to interferon-regulatory element, present in the mouse and human iNOS promoter, and it is necessary for iNOS induction in murine macrophages. In a time course experiment $\operatorname{RINm} 5 \mathrm{~F}$ were exposed to $\operatorname{IFN} \gamma(1000 \mathrm{U} / \mathrm{ml})$ and IL-1B $(25 \mathrm{U} / \mathrm{ml})$, alone or in combination. After 30 min there was already a clear increase in IRF-1 mRNA expression, as evaluated by Northern blot analysis, with peak after $1 \mathrm{~h}$ and decrease after $2 \mathrm{~h}$. After $1 \mathrm{~h}$ IL-1B and IFN $\gamma$ induced respectively a 6and 17-fold increase in IRF-1 mRNA expression $(n=6 ; P<0.05$ vs controls), while a combination of IL-1 3 + IFNy induced a 30 -fold increase $(\mathrm{P}<0.001)$. Exposure of rat pancreatic islets to IL-1 + IFNy for $2 \mathrm{~h}$ induced a 4.5 fold increase in IRF-1 protein expression $(\mathrm{n}=4$; $\mathrm{P}<0.05)$, as judged by Western blot analysis. In parallel experiments IL-1B $(50 \mathrm{U} / \mathrm{ml})+\mathrm{IFN} \gamma(1000 \mathrm{U} / \mathrm{ml})$, but not IL-1B or IFN $\gamma$ alone, induced a 2-300\% increase in nitrite production by human pancreatic islets. As a whole, these data suggest a role for IFN $\gamma$ and IRF-1 on the regulation of islet iNOS expression. 
385

\section{S.METHYL-L-THIOCITRULLINE PROTECTS RAT PANCREATIC ISLETS AGAINST INTERLEUKIN-1 $\beta$ INDUCED SUPPRESSION}

S. Sandler, E. Forsbeck and Nils Welsh. Department of Medical Cell Biology, Uppsala University, Uppsala, Sweden.

Nitric oxide, induced by pancreatic islet exposure to cytokines, has been implicated in $\beta$-cell destruction in insulin-dependent diabetes mellitus. In this context it could be worthwhile to characterize inhibitors of the nitric oxide generating enzyme. For this prupose rat pancreatic islets were cultured for $48 \mathrm{~h}$ in medium supplemented without or with 10,100 or $500 \mu \mathrm{M}$ of $S$ methyl-L-thiocitrulline (MTC), in the absence or presence of 25 $\mathrm{U} / \mathrm{ml}$ of interleukin-1 $\beta$. MTC alone did not affect the islet glucose oxidation rate, but all concentrations of MTC prevented interleukin-1 $\beta$ induced suppression of the islet glucose metabolism. Moreover, MTC $(100 \mu \mathrm{M})$ completely protected against cytokine mediated inhibition of medium insulin accumulation, glucose stimulated insulin release and (pro)insulin biosynthesis. Interleukin-1 $\beta$ caused a more than 10 -fold increase in medium nitrite production, an indication of nitric oxide production, which was blocked by MTC. Acutely in the absence of interleukin-1 $\beta$ islet glucose stimulated insulin release was enhanced by MTC $(100 \mu M)$. The efficacy of MTC,

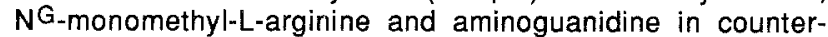
acting interleukin-1 $\beta$ induced nitrite formation was also compared. When estimating the half-maximal inhibitory concentration values for this effect, it was approximately $10 \mu \mathrm{M}$ for MTC and about $1000 \mu \mathrm{M}$ for $\mathrm{NG}_{\text {-monomethyl-L-arginine and }}$ aminoguanidine. Thus the present study shows that MTC can potently block cytokine induced activation of nitric oxide synthase in rat pancreatic islets.

\section{7}

EBSELEN DECREASES NF-kB ACTIVATION AND REDUCES CYTOKINE-INDUCED NITRIC OXIDE FORMATION BY INSULINPRODUCING CELLS

D. L. Eizirik, M. Flodström and M.A.R. de Mello. Department of Medical Cell Biology, Uppsala University, Uppsala, Sweden and Department of Metabolism and Endocrinology, Vrije Universiteit Brussel, Brussels, Belgium.

Interleukin-1 (IL-1) may be a mediator of B-cell damage in insulin dependent diabetes mellitus (IDDM). IL-1 mechanism of action in rodent insulin-producing cells probably includes activation of the transcription factor NF-kB, increased transcription of the inducible form of nitric oxide synthase (iNOS) and subsequent production of nitric oxide (NO). Reactive oxygen intermediates, specially $\mathrm{H} 2 \mathrm{O} 2$, have been proposed as second messengers for NF-kB activation. In the present study we tested whether ebselen (2-phenyl-1,2-benzisoselenazol-3(2H)-one), a glutathione peroxidase mimic compound, could counteract the effects of $\mathrm{ML}-1 \mathrm{~B}, \mathrm{H} 2 \mathrm{O} 2$ and alloxan in rat pancreatic islets and in the rat insulinoma cell line RINm5F (RIN-cells). Some of these experiments were also reproduced in human pancreatic islets. Ebselen $(20 \mathrm{mM})$ prevented the increase in nitrite production by rat islets exposed for $6 \mathrm{~h}$ to IL-1B (90\% reduction in nitrite production; $\mathrm{P}<0.05)$. It also induced significant $(\mathrm{P}<0.05)$ protection against the acute inhibitory effects of alloxan or $\mathrm{H} 2 \mathrm{O} 2$ exposure, as judged by the preserved glucose oxidation rates. Ebselen prevented the increase in nitrite production by human islets exposed for $14 \mathrm{~h}$ to a combination of cytokines (IL-1 1 + tumor necrosis factor- $\alpha+$ interferon- $\gamma)(80 \%$ reduction in nitrite production; $\mathrm{P}<0.05$ ). In RIN-cells, ebselen counteracted both the expression of iNOS mRNA (evaluated by Northern blot analysis; $P<0.01$ ) and the increase in nitrite production induced by $6 \mathrm{~h}$ exposure to IL-1B $(P>0.01)$. Ebselen also partially inhibited ( $30 \%$ inhibition) IL-1B-induced NF-kB activation, as evaluated by electrophoretic mobility shift assay. As a whole, these data indicate that ebselen partially counteracts cytokineinduced nitric-oxide synthase activation in pancreatic B-cells, an effect associated with inhibition of NF-kB activation.

\section{6}

CILIARY NEUROTROPHIC FACTOR (CNTF) AND IL-6 POTENTIATE THE $\beta$-CELL INHIBITORY EFFECT OF IL-1 $\beta$ VIA INCREASED NO SYNTHESIS

Karin A. W. Wadt, Claus M. Larsen, Henrik Ulits Andersen, and Thomas Mandrup-Poulsen, Steno Diabetes Center, Gentofte, Denmark

The functional receptor complexes for the IL-6 family of cytokines consisting of IL-6, CNTF, leukaemia inhibitory factor, IL-11, cardiotrophin-1 and oncostatin $\mathrm{M}$ use $\mathrm{gp} 130$ as signal transducing element. $\beta$-cells express receptors for several nerve growth factors and can undergo a neurotypic response to neuronal differentiation factors. The islets of Langerhans are richly innervated, CNTF is present in peripheral nerves and may exert paracrine actions on non-neuronal cells. The aim of this study was therefore to examine if CNTF modulated the action of IL-1 13 on insulin release and NO synthesis and if so whether this action was shared by other cytokines signalling via gp 130, i.e IL-6. Neonatal Wistar rat islets were precultured for $24 \mathrm{~h} \pm 20 \mathrm{ng} / \mathrm{ml}$ CNTF or $10 \mathrm{ng} / \mathrm{ml} \mathrm{IL-6,} \mathrm{followed} \mathrm{by}$ a $24 \mathrm{~h}$ culture period with $0-150 \mathrm{pg} / \mathrm{ml} \mathrm{IL}-1 \beta \pm 20 \mathrm{ng} / \mathrm{ml}$ CNTF or $10 \mathrm{ng} / \mathrm{ml}$ IL-6. The islets were then exposed to a $2 \mathrm{~h}$ glucose challenge. $20 \mathrm{ng} / \mathrm{ml}$ of CNTF had no effect by itself but potentiated $\mathrm{LL}-1 \beta$ mediated NO synthesis by $31-116 \%$ ( $p<0,05$ at 5,10 and $150 \mathrm{pg} / \mathrm{ml}$ of $\mathrm{LL}-1 \beta)$, inhibition of accumulated insulin release by $34-49 \%(p<0,02$ at 10 and $50 \mathrm{pg} / \mathrm{ml}$ of $\mathrm{IL}-1 \beta$ ) and of insulin response to $2 \mathrm{~h} 16,7 \mathrm{mM}$ glucose challenge by 31 $36 \%(\mathrm{p}<0,05$ at 5,10 , and $50 \mathrm{pg} / \mathrm{ml}$ of IL- $1 \beta) .10 \mathrm{ng} / \mathrm{ml}$ of IL- 6 potentiated $15 \mathrm{pg} / \mathrm{ml} \quad \mathrm{IL}-1 \beta$ mediated NO synthesis by $45 \%(\mathrm{p}<0,05)$, inhibition of accumulated insulin release by $46 \%$ and of insulin response to a $2 \mathrm{~h} 16,7$ $\mathrm{mM}$ glucose challenge by $41 \%$. In conclusion CNTF potentiates the $\beta$-cell inhibitory effect of IL-1 $\beta$ via increased NO synthesis an effect shared by IL-6. These findings indicate that signal transduction via gp130 influences islet NO synthesis and underline the importance of neuro-immunoendocrine interactions in the pathogenesis of IDDM.

\section{8}

CYTOKINE INHIBITION OF PROTEIN SYNTHESIS IN RINm5F CELLS IS NITRIC OXIDE MEDIATED AND REVERSED BY TGFB N.John, J.Mabley and I.Green, Univ. of Sussex, Brighton. BN1 9QG.

This study examined nitric oxide dependency of cytokine-mediated inhibition of protein synthesis. RINm5F cells were treated for 24 hours with cytokines in RPMI medium with or without arginine, the substrate for nitric oxide production, or with TGF3 pretreatment to prevent induction of nitric oxide synthase. Protein synthesis was determined by measuring tritiated mixed amino acid incorporation for 24 hours coincident with cytokine treatment; control incorporation was $4 \times 10^{3}$ $\mathrm{dpm} / 10^{5}$ cells in 24 hours. Nitric oxide production was assessed by measuring medium nitrite levels. Induction of nitric oxide synthase was observed by Western blotting.

\begin{tabular}{|c|c|c|c|c|c|}
\hline Treatment & \multicolumn{3}{|c|}{ Amino Acid Incorporation (\% -TGFß control) } & Nitr & Nitrite $(\mu \mathrm{M})$ \\
\hline & targinine & $+\mathrm{TGFB} 1$ & -arginine & +arginine & -arginine \\
\hline Control & $100.1 \pm 1.0$ & $86.2 \pm 2.6$ & $99.9 \pm 2.4$ & $0.9 \pm 0.2$ & $0.4 \pm 2.0$ \\
\hline $\mathrm{IL}-1 \beta(100 \mathrm{pM})$ & $82.0 \pm 2.4 * f$ & $75.5 \pm 4.1 *$ & $95.5 \pm 4.3$ & $23.5 \pm 2.6 * * 5$ & $0.6 \pm 0.2$ \\
\hline TNF $(100 \mathrm{pM})$ & $99.4 \pm 1.4$ & $76.5 \pm 3.9 *$ & $102.2 \pm 3.4$ & $1.2+0.1$ & $1.5 \pm 0.2$ \\
\hline IFN (100pM) & $98.2 \pm 2.4$ & $86.3 \pm 3.0$ & $103.7 \pm 3.6$ & $1.0 \pm 0.5$ & $0.1 \pm 0.0$ \\
\hline 3 Cytokines & $58.7 \pm 5.6 * * \mp$ & $83.1 \pm 3.0$ & $88.9 \pm 4.5$ & $41.7 \pm 0.8$ & $0.8 \pm 0.1$ \\
\hline
\end{tabular}

IL-1B and combined cytokines inhibited protein synthesis in arginine-containing medium. Synergistic inhibition of protein synthesis by combined cytokines was restored to the level of TGFB alone by 24 hour TGFB pretreatment. Removal of arginine from the medium and addition of arginine analogues reversed both the inhibitory effects of IL-1B alone and the synergistic inhibition of combined cytokines. Removal of arginine or TGFB pretreatment prevented production of nitrite by IL- $1 B$ and combined cytokines. Western blotting demonstrated that TGFß prevented the induction of iNOS by IL-1B or combined cytokines in RINm5F cells. In conclusion, cytokine-mediated inhibtion of protein synthesis is largely dependent on nitric oxide. 
INTERLEUKIN-1 (IL-1) INDUCED NITRIC OXIDE (NO) PRODUCTION IN ISOLATED RAT ISLETS OF LANGERHANS IS REGULATED BY GLUTATHIONE (GSH)

M Nikulina, HU Andersen, V Poltorack, J Nerup, T Mandrup-Poulsen. Steno Diabetes Center, Gentofte, Denmark and Ukranian Scientific Research Institute of Endocrine Diseases Pharmacotherapy, Kharkov, Ukraine.

IL-1 inhibits insulin secretion and is selectively cytotoxic to rat $\beta$-cells by causing expression of inducible NO synthase (iNOS) leading to the formation of toxic NO radicals. Nuclear factor $k B(N F K B)$ seems to be the nuclear transcription factor responsible for iNOS induction by cytokines. Oxidation by free oxygen radicals (FOR) have been implicated in NFKB activation. GSH may have opposing effects on cytokine induced iNOS expression. Thus, by participating in the antioxidant enzymatic defence system, GSH may inhibit cytokine mediated FOR dependent iNOS expression whereas reduction of NFKB cysteines by thiols, e.g. GSH is necessary for DNA binding of NFKB. Further, thiols are required as cofactor for iNOS activity. The aim of this study was to investigate the role of GSH in IL-1 induced NO production in rat islets of Langerhans. The effect of buthionine sulfoxamine (BSO), an inhibitor of the key enzyme in GSH synthesis gamma-glutamylcysteine synthetase on IL-1 stimulated NO formation from isolated rat islets was investigated. Islets from 4-7-day-old newborn Wistar rats were preincubated for 6 hours with $0.1,0.5,1.0$ or $2.0 \mathrm{mM}$ BSO prior to culture for $24 \mathrm{~h}$ with or without $10 \mathrm{pg} / \mathrm{ml} \mathrm{IL}-1$. NO production was measured by nitrite accumulation in the medium determined by the Griess reaction. BSO dose dependently inhibited IL-1 induced NO production with a maximal effect at $1 \mathrm{mM}(39 \%, \mathrm{p}=0.01)$. We conclude that depletion of islet GSH attenuates IL-1 induced NO production and that GSH is important in the regulation of islet NO synthesis.
NO SYNERGISM BETWEEN IL1 $\beta$ AND TNF $\alpha$ ON NITRIC OXIDE PRODUCTION AND INHIBITIONOF INSULIN SECRETION OF ISOLATED RAT ISLETS S.H. Fu, B.R.S. Hsu, Y.Y. Huang and F.H. Chang Chang-Gung Medical Center and National Taiwan University, TAIWAN

The synergistic action of IL $1 \beta$ and TNF $\alpha$ has been demonstrated on several cell types. However, conflicted data were noted on isolated islets. In order to study the effect of TNF $\alpha$ on beta-cell function, 30 islets were incubated with murine TNF $\alpha .(20 \mathrm{ng} / \mathrm{mL})$ and murine $\operatorname{IL} 13$ ( $1 \mathrm{ng} / \mathrm{mL}$ ) simultaneously or sequentially for an initial $14 \mathrm{hrs}$ and subsequent $48 \mathrm{hrs}$. Nitrite and glucosestimulated insulin secretion $(360 \mathrm{mg} / \mathrm{dL} \times 60 \mathrm{~min})$ were determined daily Mouse IL-1 receptor antagonist peptide (IL-1ra, $1000 \mathrm{ng} / \mathrm{mL}$ ) was used to block endogenous IL $1 \beta$. The result was listed as below:

\begin{tabular}{|c|c|c|c|c|c|c|c|c|c|c|}
\hline & \begin{tabular}{|l|l|} 
Initial \\
$14 \mathrm{hr}$
\end{tabular} & \multicolumn{3}{|c|}{$\begin{array}{c}\text { Next } \\
0-48 \mathrm{hr}\end{array}$} & \multicolumn{3}{|c|}{$\begin{array}{c}\text { Insulin secretion } \\
(u \mid \cup / i s l e t) \times 60 \mathrm{~min} \\
n=6\end{array}$} & \multicolumn{3}{|c|}{$\begin{array}{c}\text { Nitrite production } \\
\text { (pmole/islet) } \\
n=10\end{array}$} \\
\hline & $\begin{array}{ll}\text { IL } & T N \\
\beta & F a\end{array}$ & & $\begin{array}{l}\mathrm{TN} \\
\mathrm{Fa}\end{array}$ & $\begin{array}{l}\mathrm{L} 1 \\
\mathrm{ra}\end{array}$ & $\begin{array}{l}\text { Post } \\
\text { initial } \\
14 \mathrm{hr}\end{array}$ & $\begin{array}{l}\text { Post } \\
\text { next } \\
24 \mathrm{hr}\end{array}$ & \begin{tabular}{|c|} 
Post \\
next \\
$25-48 \mathrm{hr}$
\end{tabular} & $\begin{array}{l}\text { Initial } \\
\text { period } \\
(14 h r)\end{array}$ & $\begin{array}{c}\text { Next } \\
0-24 \mathrm{hr}\end{array}$ & $\begin{array}{c}\text { Next } \\
25-48 \mathrm{hr}\end{array}$ \\
\hline & 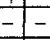 & 4 & & $=$ & $4,9 \pm 1.6^{9}$ & $4.7 \times 0.7^{\circ}$ & $3.6 \div 0.3^{9}$ & $0.7 \pm 0.8^{1}$ & $9.2+2$ & $3.1 \pm 4.1$ \\
\hline & 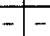 & - & + & + & $5.1 \pm 0.8^{2}$ & $4.6 \pm 0.6^{\circ}$ & $3.6 \pm 0.6^{9}$ & $0.4 \div 0.9^{\prime}$ & $9.1 \pm 3.0^{\mathrm{m}}$ & $\sqrt{2.9 \pm 3.7}$ \\
\hline & - & - & + & + & $3.9 \pm 0.5^{n}$ & $3.8 \pm 0.2^{\circ}$ & $28 \pm 0.5^{n}$ & $5.3 \pm 0.9^{k}$ & $14.9 \pm 3.8^{n}$ & $12.3 \pm 2.7^{9}$ \\
\hline & - & - & & $t$ & $3.5 \pm 0.4^{b}$ & $3.1 \pm 0.3^{\circ}$ & $28 \pm 0.5^{\mathrm{n}}$ & $5.8 \pm 2.3^{k}$ & $12,6 \pm 4.1^{n}$ & $10.2 \pm 1.4$ \\
\hline & & + & & & $40 \pm 0.5^{c}$ & $3.5 \pm 0.2^{\prime}$ & $3.0 \pm 0.3^{\prime}$ & $5.6 \pm 3.2^{1}$ & $17.7 \pm 5.3^{\circ}$ & $21.5 \pm 3.7^{\prime}$ \\
\hline & + & +1 & + & -1 & $3.6 \pm 0.5^{\circ}$ & $3.3 \pm 0.4^{\top}$ & $3.0 \pm 0.2^{\prime}$ & 5.4 & $17.5+4.55^{5}$ & $22.7 \pm$ \\
\hline
\end{tabular}

Data were expressed as mean \pm SEM. Student's $t$-test was used to analyze difference of means.

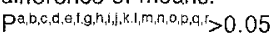

Our data suggested that TNF $\alpha$ is not effective in itself or synergism with IL $1 \beta$ to induce nitric oxide production or suppress beta-cell function of isolated rat islets.

\section{1}

EVIDENCE FOR THE INVOLVEMENT OF CGMP IN NITRIC OXIDE-INDUCED APOPTOSIS IN HIT-T15 CELLS

A.C.Loweth, J.H.Scarpello*, G.T.Williams and N.G.Morgan. Cellular Pharmacology Group, Depts of Biological Science and Medicine,* Keele University, ST5 5BG, UK.

Nitric oxide (NO) may mediate the cytokine-induced cytotoxicity thought to effect autoimmune destruction of islet B-cells in IDDM. Since NO is known to elevate cGMP levels in islets and clonal B-cells, we have examined the possibility that increased CGMP levels, hitherto having no ascribed function in islets, propagate NO signalling leading to apoptosis. The NO donor, GSNO, dose-dependently increased cell death in HIT-T15 cells, cultured for $24 \mathrm{~h}$ (Dead cell counts: Control: $0.87 \times 10^{4} \pm$ 1328: $50 \mu \mathrm{M}$ GSNO: $0.75 \times 10^{4} \pm 910 ; 75 \mu \mathrm{M}$ GSNO: $1.09 \times 10^{4} \pm 1389 ; 100 \mu \mathrm{M}$ GSNO: $1.77 \times 10^{4} \pm 1859^{*} ; 200 \mu \mathrm{M}$ GSNO: $4.79 \times 10^{4} \pm 4056^{* ;} ;{ }^{*}<0.001$, compared to control). Nuclear morphology characteristic of apoptosis was confirmed in the detached, dead cells by DNA electrophoresis and electron microscopy. The specific guanylyl cyclase (GC) inhibitor, ODQ, significantly inhibited GSNO-induced cell death ( Dead cell counts: Control: $1.26 \times 10^{4} \pm 2828 ; 200 \mu \mathrm{M}$ GSNO: $6.81 \times 10^{4} \pm 8068^{*} ; 200 \mu \mathrm{M}$ GSNO $+10 \mathrm{mM}$ ODQ: $3.32 \times 10^{4} \pm 1465^{* *} ;{ }^{*} \mathrm{p}<0.001$ compared to control, ${ }^{* *} \mathrm{p}<0.001$ compared to GSNO). Cell permeant cGMP analogues also significantly increased cell death (Dead cell counts: Control: $1.85 \times 10^{3} \pm 353 ; 100 \mu \mathrm{M}$ 8-bromo-cGMP-5.47 $\times 10^{3} \pm$ 931( $\mathrm{p}=0.01)$. Control: $0.53 \times 10^{4} \pm 1187 ; 300 \mu \mathrm{M}$ dibutyryl cGMP: $1.21 \times 10^{4} \pm 775$ $(\mathrm{p}<0.001)$. Furthermore, both cGMP analogues were able to reverse ODQ's inhibition of NO-induced cell death (Dead cell counts:(1)Control: $0.71 \times 10^{4} \pm 1563 ; 200 \mu \mathrm{M}$ GSNO: $15.96 \times 10^{4} \pm 4374^{*} ; 200 \mu \mathrm{M}$ GSNO $+10 \mu \mathrm{M}$ ODQ: $5.79 \times 10^{4} \pm 8296^{* *} ; 200 \mu \mathrm{M}$

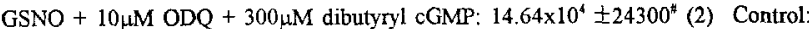

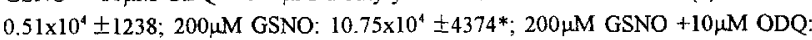

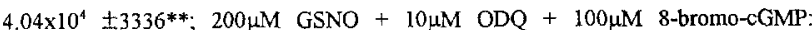
$8.99 \times 10^{4} \pm 13300^{*},{ }^{*} \mathrm{p}<0.001$ compared to control, ${ }^{* *} \mathrm{p}<0.001$ compared to $200 \mu \mathrm{M}$

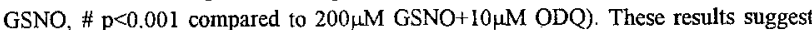
that the mechanism of NO-induced apoptosis in HIT-T15 cells includes GC activation, leading to elevated CGMP levels.
PEROXYNITRITE, NITRIC OXIDE AND SUPEROXIDE DECREASE CELL VIABILITY AND PROTEIN SYNTHESIS IN RINm5F CELLS. S.Thomas, M.DiMatteo, J.M.Sheehan and I.C.Green. Biochemistry department, University of Sussex Brighton, BN19QG, UK.

We have previously shown that cytokine-induced and chemicallyderived free radicals result in a decrease in cellular viability and a reduction in protein synthesis in insulin-secreting cells. Peroxynitrite, which has a shorter half life than nitric oxide and superoxide, may be formed via a reaction between these two free radicals and is a potentially more injurious species in pathological states such as IDDM. In this study we compared the effects of peroxynitrite, nitric oxide and superoxide on cell viability and protein synthesis in RINm5F cells as measured by cellular reducing ability (3-(4,5-dimethylthiazol-2-yl)25-diphenyltetrazolium bromide (MTT) reduction) and de novo protein synthesis. Cells were treated for $24 \mathrm{~h}$ with nitric oxide derived from s-nitrosylated glutathione (GSNO), superoxide from xanthine oxidase/hypoxanthine $(\mathrm{XO} / \mathrm{HX})$ and peroxynitrite from the reaction of ozone with sodium azide (quenched in sodium hydroxide). All compounds showed a dose-dependent decrease in MTT reduction. GSNO $(1 \mathrm{mM})$, peroxynitrite $(1 \mathrm{mM})$ and $\mathrm{XO}(5 \mathrm{mU} / \mathrm{ml}) / \mathrm{HX}(0.25 \mathrm{mM})$ inhibited MTT reduction to $60.0 \pm 5.0 \%(\mathrm{p}<0.001), 74.9 \pm 4.1 \%(\mathrm{p}<0.05)$ and $60.1 \pm 3.7 \%(\mathrm{p}<0.001)$ of control values $(100 \%)$ respectively. A similar dose-dependent decrease in de novo protein synthesis was seen with each of the three compounds GSNO $(0.5 \mathrm{mM}) 72.0 \pm 6.0 \%(\mathrm{p}<0.05)$, peroxynitrite $(1 \mathrm{mM}) 33.1 \pm 6.0 \%(\mathrm{p}<0.01)$ and $\mathrm{XO}(5 \mathrm{mU} / \mathrm{ml})$ /HX $(0.25 \mathrm{mM}) 68.1 \pm 2.9 \%(\mathrm{p}<0.001)$ versus control $(100 \%)$. Protein synthesis is more sensitive to inhibition by nitric oxide and peroxynitrite than MTT reduction. We observe that insulin-secreting cells may be compromised equally by nitric oxide, superoxide and peroxynitrite. However, the damaging effects of exogenously applied peroxynitrite may be masked by its extremely short half life in physiological medium; its potential toxicity when produced within the cell may be much greater. 


\section{3}

REGULATION OF RINMSF CELL ARGINASE BY NITRIC OXIDE SYNTHASE REACTION PRODUCTS

J.M. Cunningham, J. G. Mabley and I. C. Green. Department of

Biochemistry, University of Sussex, Brighton BN19QG, UK.

IL-1B-induced nitric oxide (NO) generation in islets of Langerhans inhibits islet function. A number of factors including the intracellular concentration of arginine substrate may control the rate of NO generation by induced nitric oxide synthase (NOS). Here we have identified the arginine-utilising enzyme arginase in the insulin secreting cell line RINm5F and investigated the effects of IL-1B stimulation and a NOS reaction intermediate and product on its activity. Cells were cultured for 2 days $\left(4 \times 10^{6} / 60 \mathrm{~mm}\right.$ dish in RPMI $+2 \%$ FCS $)$ and treated \pm IL- $1 B(0.1 \mathrm{nM})$ for $18 \mathrm{~h}$. Cytosol preparations were incubated with U-14C-arginine $(37 \mu \mathrm{M})$ for 45 min. at $37 \mathrm{C}^{\circ}$ and radiolabelled products (14C-citrulline from NOS, 14C-ornithine and 14C-urea from arginase) separated by C18 RPHPLC or Dowex AG 50 ion-exchange chromatography. IL-1B increased citrulline production (from $0.01 \pm 0.002$ to $0.58 \pm 0.03 \mathrm{pmol}$ $\mu \mathrm{g}^{-1}$ cell protein) and decreased ornithine production (from $4.60 \pm 0.20$ to $\left.3.40 \pm 0.20 \mathrm{pmol}^{-1} \mathrm{~g}^{-1}\right)(\mathrm{P}<0.001)$. We then investigated whether the IL-1ß-induced NOS reaction intermediate hydroxyarginine and reaction product NO could be responsible for inhibition of arginase activity. Arginase from untreated cells was inhibited by hydroxyarginine $\left(\mathrm{IC}_{50}=50 \mathrm{uM}\right)$, and by the $\mathrm{NO}$ generating compounds S-nitrosoglutathione $(500 \mu \mathrm{M}: 69 \pm 7 \%$ of control) and 3morpholinosydnonimine (1mM: $57 \pm 7 \%$ of control) $(\mathrm{P}<0.05)$. We conclude that during cytokine-directed B-cell assault NOS-catalysed production of hydroxyarginine and NO may directly inhibit arginase activity thereby increasing the availability of arginine substrate for further NO production.

\section{4}

OXIDATIVE STRESS CAUSES DEPOLARIZATION AND INSULIN SECRETION IN RINm5F-CELLS: MECHANISM AND LOCALIZATION OF ACTION

R. R. Schaich, P. Krippeit-Drews, G. Drews, I. Koopmann and H.P.T. Ammon, Department of Pharmacology, Institute of Pharmaceutical Sciences, University of Tuebingen, Germany.

Thiol oxidants have been shown to inhibit but also to cause insulin release in vitro. Here the effects of membrane permeant thiol reagent tertbutylhydroperoxide ( $\mathrm{t}-\mathrm{BHP}$ ), diazene dicarboxylic acid bis-( $\mathrm{N}^{\text {- }}$ methylpiperazide) (DIP) and/or the non membrane permeant analog of DIP (bis- $\mathrm{N}^{\prime}$-methyliodide; DIP-salt) on $\mathrm{K}^{+}{ }_{\text {ATP }}$ channel current (whole cell mode of the patch clamp technique), depolarization (bisoxonol method), cytosolic calcium (quin 2-method) and insulin secretion of RINm5F-cells were tested. All compounds induced a marked depolarization of the cells in a concentration dependent manner. Accordingly, the $\mathrm{K}_{\text {ATP }}^{+}$current (measured by a $10 \mathrm{mV}$ step from the holding potential of $-70 \mathrm{mV}$ ) was reduced from $238 \pm 29 \mathrm{pA}$ (control) to $136 \pm 26 \mathrm{pA}$ and $13 \pm 1 \mathrm{pA}(\mathrm{n}=4)$ with $0,1 \mathrm{mM}$ and $4 \mathrm{mM}$ DIP, respectively. DIP and DIP-salt have been previously shown to inhibit the $\mathrm{K}_{\text {ATP }}^{+}$current in mouse pancreatic $\mathrm{B}$ cells, too. The depolarization elicited by $\mathrm{t}$-BHP was associated with an increase of cytosolic calcium. T-BHP, DIP and DIP-salt also induced insulin secretion when added for two or ten minutes to the incubation medium. Our data suggest, that thioloxidants by oxidizing thiolgroups of the $\mathrm{K}_{\text {ATP }}^{+}$ channel cause inhibition of potassium efflux with subsequent depolarization leading to calcium uptake and insulin secretion. Since this effect was also achieved with the nonpermeating thioloxidant DIP-salt it is possible, that the thiols of the $\mathrm{K}_{\text {ATP }}^{+}$channel in question are accessible from outside the cell.

\section{5}

\section{$\mathrm{H}_{2} \mathrm{O}_{2}$ INFLUENCES ION CURRENTS, INSULIN SECRETION AND [ $\left.\mathrm{Ca}^{2+}\right]_{i}$ OF MOUSE PANCREATIC B CELLS \\ M. Dröge*, G. Drews*, G. Zempel, H.P.T. Ammon*, F. Lang, and P. Krippeit-Drews} Depts. of Pharmacology* and Physiology, D-72076 TUBINGEN, FRG

Macrophages, infiltrating pancreatic islets, contribute to $B$ cell death in the development of IDDM by secretion of reactive oxygen species, e.g. $\mathrm{H}_{2} \mathrm{O}_{2}$. We have shown that $\mathrm{H}_{2} \mathrm{O}_{2}$ hyperpolarizes $\mathrm{B}$ cells, thereby inhibiting glucose induced electrical activity. The hyperpolarization is owing to an increase in $K_{A T P}$ current, an effect dependent on an intact cell metabolism. Here the underlying factors and effects on $B$ cell function were studied. The cellular ATP concentration (IATP], measured by bioluminiscence) is decreased by $\mathrm{H}_{2} \mathrm{O}_{2}$ ( $1 \mathrm{mM}$ throughout) from $66 \pm 11 \mathrm{pg} /$ islet to $52 \pm 9 \mathrm{pg} / \mathrm{sis}$ et and $32 \pm 5 \mathrm{pg} / \mathrm{sis}$ et $(\mathrm{n}=6)$ within $5 \mathrm{~min}$ and $30 \mathrm{~min}$ of treatment, respectively. This decrease in [ATP]; readily explains the $\mathrm{H}_{2} \mathrm{O}_{2}$ induced increase in KATP current. Accordingly, insulin secretion elicited in vitro by $15 \mathrm{mM}$ glucose, decreased in response to $\mathrm{H}_{2} \mathrm{O}_{2}$ within 2 min from $42 \pm 3$ pgislet.min to $26 \pm 3$ $\mathrm{pg} / \mathrm{is}$ let min. Insulin secretion from $\mathrm{H}_{2} \mathrm{O}_{2}$ treated islets remained continuously approx. $20 \mathrm{pg} /$ islet min lower than from controls, even $10 \mathrm{~min}$ after washout of $\mathrm{H}_{2} \mathrm{O}_{2}$. This effect is in accordance with the electrophysiological data. The intracellular $\mathrm{Ca}^{2+}$ concentration $\left(\left[\mathrm{Ca}^{2+}\right]_{i}\right)$ was measured by the fura-2 technique as the ratio of emitted light at $340 \mathrm{~nm} / 380 \mathrm{~nm}$ excitation wavelength. Basal $\left[\mathrm{Ca}^{2+}\right]_{\text {i }}$ at $0.5 \mathrm{mM}$ glucose exhibited a ratio of $0.17 \pm 0.02(n=12, \approx 60 \mathrm{nM})$. Addition of $15 \mathrm{mM}$ glucose augmented $\left[\mathrm{Ca}^{2+}\right]_{i}$ to $0.84 \pm 0.07(n=12, \approx 300 \mathrm{nM})$ in the first phase, sometimes followed by oscillations of $\left[\mathrm{Ca}^{2+}\right]$. Surprisingly, $\left[\mathrm{Ca}^{2+}\right]$, was not decreased in parallel to the $\mathrm{H}_{2} \mathrm{O}_{2}$ induced suppression of electrical activity, but increased biphasically in response to $\mathrm{H}_{2} \mathrm{O}_{2}$. A first transient peak to a ratio of $1.21 \pm 0.24(\mathrm{n}=4)$ was tollowed by a sustained increase to $2.29 \pm 0.15(n=3)$. When cells were treated with $\mathrm{Ca}^{2+}$-free solution in $15 \mathrm{mM}$ glucose, $\mathrm{H}_{2} \mathrm{O}_{2}$ elicited only the first transient peak. It amounted to a ratio of $0.45 \pm 0.07 \quad(n=8)$. In summary, although $\mathrm{H}_{2} \mathrm{O}_{2}$ increases $\left[\mathrm{Ca}^{2+}\right]_{\text {i }}$ it inhibits insulin secretion by reducing $[A T P]_{\text {, }}$, thus activating $K_{A T P}$ current and thereby suppressing electrical activity.

\section{6}

METALLOTHIONEIN: IN-VITRO INDUCTION PREVENTED STREPTOZOTOCIN-INDUCED B-CELL IMPAIRMENT

$P$. Ohly and H. Gleichmann. Diabetes Research-Institute, HeinrichHeine-University, Düsseldorf, Germany.

Metallothionein(s) (MT) are cellular antioxidant protein(s) regarded to scavenge in particular hydroxyl radicals, which have been reported to be the most toxic of the group of reactive oxygen species (ROS). ROS are regularly generated and during inflammatory reactions. Physiologically, cells are protected against ROS toxicity by their system of antioxidants. ROS have also been implicated to be effector molecules involved in the pathogenic pathway of B-cell destruction in autoimmune diabetes. Furthermore, streptozotocin (STZ) has been reported to generate ROS mediating fragmentation of nuclear DNA.This laboratory has reported that MT is constitutively expressed and significantly ( $<<0.001)$ induced with zinc in pancreatic islets of mice in vitro and in vivo. Our aim was to study in vitro, if MT induction can protect islets from STZ-induced functional impairment as determined by basal and glucose-stimulated immune-reactive insulin (IRI) release. We found that MT induction with zinc prior to incubation with subtoxic concentrations of STZ rescued ß-cell function from STZ toxicity, i.e. the glucose-stimulated IRI release was comparable to or exceeded the value measured in solvent-treated control cultures. Thus, the index of glucose-stimulam tion was 0.9 after incubation of islets with STZ compared to 4.7 obtained in control cultures. Pretreatment with zinc prior to incubation with STZ resulted in an index of 4.1. Currently, we analyse the effect of $M T$ induction on the development of multiple-low-dose STZ diabetes. in a pilot study $\mathrm{Zn}$ induced MT induction significantly prevented hyperglycemia. 
397

LONG-TERM EFFECTS OF NICOTINAMIDE ON THE SYNTHESIS OF NO BY IL-1-STIMULATED RIN CELLS.

F.J.Bedoya ${ }^{1}$, J.Andrade', M.Conde', R.Ramírez', J.Monteseirín ${ }^{2}$, J.Conde ${ }^{2}$ and F.Sobrino ${ }^{1,2}$. 'Departamento de Bioquímica Médica y Biología Molecular. Universidad de Sevilla and ${ }^{2}$ Servicio de Alergia e Inmunología. Hospital Universitario Virgen Macarena. Sevilla. Spain.

We have studied the long-term effects of nicotinamide (NIC) on the synthesis of NO by insulin producing cells. NIC delayed the formation of nitrite by $\mathrm{IL}-1 \beta-(\mathrm{LL}-1,25 \mathrm{U} / \mathrm{ml})-$ stimulated RINm5F cells and previous exposure of cells to $\mathrm{LL}-1$ for $15 \mathrm{~h}$ prevents this effect. The delay is associated with a lack of cytokine-induced iNOS enzyme activity in cell extracts and can be overcome by removal of NO with oxyhemoglobin. NIC $(20 \mathrm{mM})$ directly inhibits NOS activity in extracts from cells incubated with $\mathrm{IL}-1$ for $6 \mathrm{~h}$ and $24 \mathrm{~h}$ and oxyhemoglobin counteracts this inhibition. NIC removes efficiently $\mathrm{O}_{2}-$ generated by the xanthinexanthine oxidase system. Hence, $\mathrm{NIC}$ could act by scavenging $\mathrm{O}_{2}$. generated during $\mathrm{IL}-1$ stimulation and then allowing NO to inhibit iNOS. In intact cells, protection from NIC is associated with IL-1induced expression of MnSOD activity and pyrrolidine dithiocarbamate (PDTC), an inducer of MnSOD mRNA in this cell system, counteracts the inhibitory effect of NIC on NO formation. In addition, the stimulation by NIC of IL-1-induced nitrite production in PDTC-treated cells is a novel action that should be considered when the drug is proposed as potential agent for the prevention of IDDM.
398

GLUCOSE TRANSPORTER 2: PREFERENTIAL REDUCTION OF PANCREATIC ISLET IRNA AND PROTEIN EXPRESSION BY MULTIPLE-LOW-DOSE STREPTOZOTOCIN

Z. Wang and H. Gleichmann. Diabetes Research-Institute, Heinrich-Heine-University, Düsseldorf, Germany.

An experimental model of autoimmune diabetes is that which is induced by multiple-low-dose of streptozotocin (MLD-STZ), a naturally occuring B-cell toxin. MLD-STZ induces B-cell subtoxicity and T-cell-dependent immune reactions. Since the glucose transporter 2 (GLUT2) is required for glucose homeostasis, our aim was to analyse if MLD-STZ exerts damage on B-cell GLUT2 protein and mRNA expression. By using Western blot analysis and relative quantitative RT-PCR, a gradual significant reduction of GLUT2 protein and mRNA was found with the number of STZ injections. Interestingly, when the GLUT2 protein was reduced below $10 \%$ of the control, the mouse had hyperglycemia. Moreover, the MLD-STZ effect on GLUT2 mRNA expression was preferential. Thus, the mRNA expression of B-actin, a house-keeping gene, and glucokinase, a pivotal enzyme in glucose metabolism, remained completely unaffected by MLD-STZ. The PCR products were scanned by laser densitometer. The following ratios were obtained. For GLUT2 over B-actin, the ratio for solvent-treated mice was 1.7. Seven days after the fifth STZ injection, however, the ratio was reduced to 0.35. In contrast, the ratio of glucokinase to B-actin remained constantly unchanged compared to the control value. In conclusion, GLUT2 appears to be a key target for MLD-STZ toxicity, and might be the pivotal lesion initiating the autoimmune pathway of $\beta$ cell destruction. Interestingly, MLD-STZ-induced GLUT2 decrement and hyperglycemia was prevented by pretreatment with 5-thio-Dglucose.

\section{9}

EXPRESSION OF GLUT-2 AND GLUCOKINASE IN B-CELLS OF NEW ONSET AND INSULIN-TREATED DIABETIC BB RATS

K.D. Kohnert, B. Hehmke, I. Klöting and W. Besch, Institute of Diabetes, Karlsburg, University of Greifswald, Germany

The question posed by the present study was whether direct contact with mononuclear cells infiltrating the pancreatic islets, exposure to inflammatory cytokines or the hyperglycemia caused reduction of GLUT-2 glucose transporter and comparable changes in the glucokinase (GK) expression in insulin-dependent diabetes. Diabetes-prone $\mathrm{BB} / \mathrm{OK}$ and diabetes-resistant congenic BB.1A/OK rats were used to study the expression of the GLUT-2 and GK protein by immunostaining of pancreas sections and by Western blotting of islet cell lysates. In pancreas sections from non-diabetic BB and diabetesresistant BB.1A rats both islet GLUT-2 and GK were fully expressed, which was associated with intense insulin immunostaining. GLUT-2 and GK expression was variably reduced in diabetic pancreases with established insulitis and reduced $\beta$-cell insulin staining. In diabetic BB rats, at disease onset and after long-term insulin treatment, the ratio of GLUT-2 to insulinimmunoreactive cells was decreased by $62 \%$ and $75 \%(p<0.001)$, respectively, and the B-cells had lost their GK immunoreactivity. Westem blot analysis of lysates from these islets revealed reduced expression of the major $55 \mathrm{kDa}$ GLUT-2 and the $56 \mathrm{kDa}$ GK protein. Exposure of isolated islets from nondiabetic BB rats to the cytokine combination of $\Pi \mathrm{L}-1 \beta(50 \mathrm{U} / \mathrm{m} 1)$, TNF- $\alpha\left(10^{3}\right.$ $\mathrm{U} / \mathrm{ml})$ and $\left[\mathrm{FN}-\gamma\left(10^{3} \mathrm{U} / \mathrm{ml}\right)\right.$ for $24 \mathrm{~h}$ at $11 \mathrm{mmol} / \mathrm{l}$ glucose stimulated GLUT-2 and GK expression. Treatment with 10-fold higher concentrations did not affect GLUT-2 but reduced GK expression by $25 \%$. Glycemic improvement in the diabetic animals by insulin treatment was not able to restore GLUT-2 and GK expression or the pancreatic insulin content. These data suggest that depletion of pancreatic insulin and necrosis of $\beta$-cells rather than cytokine action play a significant role in the decreased expression of the islet GLUT-2 and $\mathrm{GK}$ protein in diabetes of the BB rat.
400

INTERLEUKIN-6 STIMULATES INSULIN mRNA EXPRESSION BY $\mathrm{Ca}^{2}+$ DEPENDENT MECHANISM.

H. Shimizu, K. Ohtani, Y. Tanaka, N. Sato, Y. Kato and M. Mori, 1st Dept. Intern. Med., Gunma Univ. Schl. Med., Maebashi 371, Japan.

We have demonstrated that interleukin (IL)-6 stimulates insulin secretion, but the exact mechanism has not been established. The insulin gene has a consensus sequence of an IL-6 responsive element in its promoter region. The present studies were undertaken to examine a hypothesis that IL-6 may stimulate insulin gene expression. Human recombinant IL-6 (10 and $100 \mathrm{pg} / \mathrm{ml})$ was added in HIT-T 15 cells and changes of insulin secretion for 2 hours were measured. The cells were immediately sonicated and total RNA was extracted by ISOGENE. Insulin and $\beta$ actin mRNA expressions were measured by RT-PCR method. In the second experiment, the effects of nifedipine $(10,100 \mathrm{nM})$ addition on the IL-6-induced insulin secretion and insulin mRNA expression were investigated in HIT-T 15 cells. The addition of IL- 6 dosedependently stimulated insulin mRNA expression, resulting in an increase of insulin secretion. The addition of nifedipine significantly antagonized the IL-6-induced stimulation of insulin secretion. Nifedipine completely deleted an increase of insulin mRNA expression by IL-6. It is concluded that the addition of IL-6 stimulates insulin gene expression by a $\mathrm{Ca}^{2+}$-dependent mechanism. 


\section{1}

NATURAL CERAMIDE INDUCES APOPTOTIC DNA FRAGMENTATION IN CULTURED PANCREATIC BETA-CELLS

\footnotetext{
N. Ishizuka, A. Kanatsuka, K. Yagui, K. Yamada, Y. Saito and H. Makino

Department of Internal Medicine II, Chiba University School of Medicine,

Chiba 260, Japan
}

Cytokines induce apoptotic DNA damage in the pancreatic beta-cells, however, the exact mechanisms and sequence of events are not clear. Here we investigate a role of ceramide for the apoptotic signaling pathway in pancreatic beta-cells. The apoptotic cell death was detected by nuclear staining with Hoechst 33342 dye and apoptotic DNA fragmentation was analyzed by in situ end-labeling immunocytochemistry and agarose gel electrophoresis. C2-ceramide, a synthetic cell-permeable ceramide analog, and natural ceramides (type III and IV) dispersed in ethanol did not induce any morphological changes, however, the natural ceramides dispersed in a solvent mixture of ethanol and dodecane induced time- and dosedependently chromatin condensation and DNA tragmentation in pancreatic beta-cells, MIN6. The induction of apoptosis was detected as early as 3 hours after the addition of both ceramides. The effective concentration of the ceramide is as low as $200 \mathrm{nM}$. Tumor necrosis factor- $\alpha$ (TNF- $\alpha$ ) time- and dose-dependently caused chromatin condensation and DNA fragmentation in the cells. Ceramide, $200 \mathrm{nM}$, and $T N F-\alpha, 10 \mathrm{nM}$, induced internucleosomal DNA fragmentation. These results suggest that ceramide may be implicated as mediators of apoptosis in the pancreatic beta-cells.

\section{3}

NICOTINAMIDE INDUCED APOPTOSIS IN RINTIFF CELLS IS ASSOCIATED WITH ACTIVATION OF PIICE AND IS PREVENTED BY BCl-2 EXPRESSION

J. Saldeen and N. Welsh. Department of Medical Cell Biology, Uppsala University, Uppsala, Sweden.

The aim of the study was to determine the effect of inhibition of the DNA repair enzyme poly(ADP-ribose) polymerase (PARP) on RINm5F cell apoptosis. ADP-ribosylation activities in intact cells, as determined using $\left[2{ }^{3} \mathrm{H}\right]$ adenine labeling of cellular $\mathrm{NAD}^{+}$, were decreased by incubation of RINm5F cells for $16 \mathrm{~h}$ with $10-50 \mathrm{mM}$ nicotinamide (NA) or $20 \mathrm{mM}$ 3-aminobenzamide. A $24 \mathrm{~h}$ exposure to $50 \mathrm{mM} \mathrm{NA}$ or $10 \mathrm{mM} 3$-aminobenzamide both resulted in massive apoptosis in RINm5F cells as demonstrated by propidium iodide staining of fragmented nuclei and by the occurence of a DNA ladder following gel electrophoresis of low molecular weight DNA. Moreover, $50 \mathrm{mM}$ NA also induced the appearance of the $85 \mathrm{kD}$ proteolytic PARP fragment on immunoblots indicating activation of a protease resembling ICE (interleukin-1 $\beta$ converting enzyme). PARP fragmentation could, however, not be prevented by non-toxic concentrations of the ICE inhibitor Z-Val-Ala-Aspfluoromethylketone. Apoptosis induced by NA was not paralleled by stabilization of the p53 protein as assessed by immunoblot analysis. The expression of the anti-apoptotic protein $\mathrm{Bcl}-2$ and its mRNA was undetectable in RINm5F cells, both before and after exposure to NA. However, stable clones expressing human $\mathrm{Bcl}-2$ were generated by transfecting RINmF cells with the bel-2 containing BMGNeo construct and selection with $\mathrm{G} 418$. Clones expressing high levels of $\mathrm{Bcl}-2$ did not become apoptotic when exposed to as much as $80 \mathrm{mM}$ of NA. It is concluded that inhibition of PARP in the rapidly dividing RINm5F cell line appears to induce apoptosis and that this process can be prevented by $\mathrm{Bcl}-2$ expression.
402

PROTEIN SYNTHESIS SUPPRESSES A CONSTITUTIVE APOPTOTIC PROGRAM IN RAT PANCREATIC B CELLS.

A. Hoorens, M. Van de Casteele and D. Pipeleers. Diabetes Research Center, Vrije Universiteit Brussel, B-1090 Brussels, Belgium.

It is unknown whether pancreatic $B$ cells can undergo apoptosis and whether this process contributes to the development of diabetes. We demonstrate that rat islet $B$ cells constitutively express an apoptotic program which is activated when formation of new mRNA or proteins is blocked. Apoptotic $B$ cells were detectable by electron microscopy following treatment with actinomycin $D$ or cycloheximide. In addition to the typical ultrastructural features of apoptosis, these cells exhibit severe degranulation and myelinoid bodies. A fluorescence microscopic method was developed to quantify the percent $B$ cells dying from apoptosis during culture. Both actinomycin D and cycloheximide caused a dose- and timedependent increase in the number of apoptotic $B$ cells, up to 70 percent after 7 days of culture; virtually no apoptotic $B$ cells were detected in control preparations or in conditions leading to primary necrosis such as treatment with the mitochondrial poison antimycin $A$ Survival of $B$ cells seems thus dependent on the synthesis of proteins which suppress an endogenous suicide program. We observed that the intercellular heterogeneity in protein synthesis correlates with a different individual sensitivity to apoptotic conditions. Cells with a low threshold for glucose seem to contain higher levels of protective factors than those with a high threshold. This experimental model allows to further investigate the regulation of apoptosis in $B$ cells and to identify agents which induce or prevent $B$ cell death.

\section{4}

APOPTOTIC CELL DEATH OF BETA-CELLS TRIGGERED BY GLIBENCLAMIDE

S. Kagimoto, Y. Yamada, A. Kubota, H. Ishida and Y. Seino

Department of Metabolism and Clinical Nutrition, Kyoto University Faculty of Medicine, 54 Shogoin-Kawahara-cho, Sakyo-ku, Kyoto 606, Japan

[AIM] Sulfonylureas (SU) are well established and useful agents in treatment of non-insulin-dependent diabetes mellitus. However, considering that $S U$ act by stimulating pancreatic $B$-cells through elevation of the cytosolic calcium concentration, concern that they might contribute to the deterioration in NIDDM, by impoverishment of pancreatic $B$-cells, is appropriate. [METHODS] We have investigated the viability and DNA fragmentation of RINm $5 F$ cells and isolated rat pancreatic islets using MTT assay and the electrophoresis of genomic DNA, after exposure to various concentrations of glibenclamide. [RESULTS] The exposure to glibenclamide of as low a concentration as $10 \mathrm{nM}$ for 8 hours significantly decreases cell viability both in isolated rat pancreatic islets and RINm5F cells. The toxicity of glibenclamide is dose dependent and is inhibited significantly by pretreatment with $10 \mu \mathrm{M}$ cyclohexamide or coexisting nitrendipine at a concentration of $50 \mu \mathrm{M}$. Electrophoresis of genomic DNA shows the fragmentation typical of apoptosis, and the quantity of fragmented DNA also is decreased by cyclohexamide pretreatment or coexisting nitrendipine.

[CONCLUSION] Glibenclamide can cause apoptotic cell death both in pancreatic islets and RINm5F cells, which is mediated, at least partially, through influx of calcium ion. 


\section{Development and Replication of Islets}

405

EFFECT OF GROWTH FACTORS ON PANCREATIC DUCTAL CELL PROLIFERATION AND DIFFERENTIATION IN VITRO

I. Rooman and L. Bouwens, Department of Experimental Pathology, Vrije Universiteit Brussel, Brussels, Belgium.

It is generally recognized that islet $\beta$-cells originate from ductal epithelium during ontogeny. In adult animals neogenesis of B-celis can be induced from ductal epithelium by various experimental conditions in vivo. The growth factors (GFs) regulating neogenesis remain unknown but recently TGF- $\alpha, H G F, N G F$ and VEGF have been proposed to be involved. We studied the effect of these GFs on proliferation and differentiation of ductal cells isolated from adult rat pancreas. Primary, organotypic duct cell cultures were exposed to these GFs in the absence of serum for a period of 3 days and BrdU was added prior to harvesting. The BrdUlabelling index (LI) of ductal cells characterized by keratin-20, was analysed by double immunocytochemical staining on paraffin sections. The results demonstrate that from the $4 \mathrm{GFs}$ tested, only VEGF induced a statistically significant increase in BrdU-incorporation by ductal cells $(p<0.002 ; n=6)$. VEGFtreated duct cells showed a nearly threefold increase in LI. Immunocytochemical staining revealed that these cells express the high affinity VEGF-receptors Flk-1 and Flt-1. By immunocytochemical staining for insulin, we analysed a possible endocrine differentiation of the cultured ductal cells. However, none of the tested GFs induced an increase in number of insulin-positive cells within these cultures. It is concluded that VEGF exerts potent mitogenic activity on pancreatic duct cells but is not solely responsible for regulating endocrine differentiation.

\section{7}

SYNERGISTIC ACTIVATION OF THE INSULIN GENE BY IPFI AND BETA2/NEUROD IN PANCREATIC EXOCRINE CELLS.

Helle V. Petersen, Frank G. Andersen, Jan Jensen, Ole D. Madsen, and Palle Serup. Hagedorn Research Institute, Niels Steensensvej 6, DK-2820, Gentofte, Denmark.

Using polyclonal antisera raised against GST-IPF1 and GSTBeta2 fusion proteins we have analysed expression of IPF1 and Beta2 in adult rat pancreas. Whereas IPF1 expression is restricted to the $\beta$-cells and a small fraction of $\delta$-cells. Beta2 appears to be expressed in all islet cells. Neither protein could be detected in exocrine cells. Previous studies have shown that IPF1 can activate insulin reporter genes in transient transfections in glucagon producing cell lines. We now show that IPF1 can activate insulin reporters in pancreatic duct-like ARIP cells only if a cDNA for the helix-loop-helix factor Beta2/NeuroD is co-transfected. In the pancreatic acinar-like cell line AR42J IPF1 alone can acticvate insulin reporters but co-transfection of Beta2/NeuroD potentiates this activation. We conclude that the cell specific transcription of the insulin gene is involving co-operativity between IPF-1 and Beta2/NeuroD.

\section{6}

IDENTIFICATION OF TRANSCRIPTION PRODUCTS IN MOUSE PANCREATIC B-CELLS DEPENDING ON TRANSCRIPTION FACTOR IPF-1

1.B. Leibiger ${ }^{1,2}$, A. Witt ${ }^{2}$, B. Leibiger ${ }^{1,2}$

'Rolf Luft Center. Dept. Molec. Medicine. Karolinska Hospital. Stockholm. Sweden: ${ }^{2}$ Inst. Biochemistry, University of Greifswald. Greifswald. FRG

In pancreatic islets of adult rodents the transcription factor IPF-1 is mainly expressed in B-cells and to a lesser extend in D-cells. In these cells IPF-I has been shown to be involved in transcriptional control of insulin and somatostatin genes. Since we found in DNA/protein binding studies that IPF-1 is able to bind to promoter elements of other B-cell expressed genes (GLUT-2, amylin, glucokinase) we became interested in the identification of additional genes controlled by IPF-1 $2.5 \times 10^{5}$ phage plaques of a mouse pancreatic islet cDNA library on two identical sets of filter lifts were analysed by the "differential screen" technique in two hybridization rounds. As probes we used radioactive cDNAs from $\beta$ TC -3 cells transfected either with an IPF-1 expression construct or with a vector generating IPF-1 anti-sense mRNA. 86 phage plaques were identified by this approach and analysed by DNA-sequencing. To verify the IPF-1-dependence we amplified the cDNA inserts of the isolated phages by PCR, separated them by gel electrophoresis and generated two identical blots on nitrocellulose. Again, filters were hybridized using as probes radioactive cDNAs from $\beta T C-3$ cells transfected either with pRSVIPFl or with $\mathrm{pRSV}-\mathrm{IPF} 1_{\text {anti-sense. }}$ The hybridzation signals were measured by phosphorimaging and normalized by co-expressed CAT and $\beta$-galactosidase genes Beside expected transcripts of mouse insulin genes I and IL, we identified the transcription products of the following genes to be directly or indirectly dependent on IPF-1: amylin, pancreatic stone proteine, mitochondrial presumptive protein-2. mitochondrial cytochrome oxidase subunit 1 , and one transcript of an unknown gene.

408

INDUCTION OF INSULIN AND IAPP PRODUCTION IN GLUCAGONOMA CELLS BY INSULIN PROMOTER FACTOR 1.

Palle Serup, Jan Jensen, Frank G. Andersen, Mette C. Jørgensen, Niels Blume $\dagger$, Jens J. Holst $\#$, and Ole D. Madsen. Hagedorn Research Institute, Niels Steensensvej 6, DK-2820, Gentofte, Denmark. †Present address: Strang-Cornell Cancer Research Laboratory, Rockefeller University, Smith Hall, 2nd floor, 1230 York Avenue, New York, New York 10021. \#Department of Medical Physiology, The Panum Institute, University of Copenhagen, $2200 \mathrm{~N}$ Copenhagen, Denmark.

Insulin Promoter Factor-1 (IPF1), a member of the homeodomain protein family, serves an early role in pancreas formation as evidenced by the lack of pancreas formation in mice carrying a targeced disruption of the IPFI gene, In adults IPF1 expression is restricted to the $\beta$ cells in the islets of Langerhans. We report here that IPF1 induce expression of a subset of $\beta$-cell specific genes (insulin and islet amyloid polypeptide [IAPP]) when ectopically expressed in clones of transformed pancreatic islet $\alpha$-celis (AN \#697). In contrast, expression of IPF1 in rat embryo fibroblasts (NEDH REF) failed to induce insulin and IAPP expression. This is most likely due to the lack of at least one other essential insulin gene transcription factor, the basic helix-loop-helix (bHLH) protein Beta2/NeuroD which is expressed in AN \#697 but not in NEDH REF cells. Other $\beta$-cell specific genes such as those encoding GLUT2 and the GLP-1 receptor were not activated. We conclude that IPF 1 is a potent transcriptional activator of endogenous insulin genes in non- $\beta$ islet cells, which suggest an important role of IPF1 in $\beta$-cell maturation. 


\section{9}

MATURATION FROM A PRE-BETA TO A BETA-CELL PHENOTYPE IS ASSOCIATED WITH AN ACQUIRED SENSITIVITY TO THE TOXIC EFFECT OF STREPTOZOTOCIN AND INTERLEUKIN 1 .

AE. Karlsen, K. Nielsen, Jan Jensen, HU. Andersen, T. Mandrup-Poulsen, OD. Madsen and J. Nerup. Steno Diabetes Center \& Hagedom Research Institute, Niels Steensensvej, DK-2820 Gentofte, Denmark.

Interieukin 1-beta (IL-1) and strepotzotocin (STZ) have been shown to be specifically toxic to the beta-cells in rat isieis of Langerhans. This effect has for IL-1 and other cytokines been associated with an induced nitric oxide production, and for STZ with release of NO from this nitrosourea derivative of glucose following up-take through the GLUT-2 glucose transporter.

AIM: To determine the value of a piuripotent MSL-tumour cell line for identification and characterisation of factors involved in cytokine, NO and STZ induced beta-cell destruction. The MLS-cell-line can dependent on culture condition obtain either a pre-beta or beta-cell like phenotype, determined by PCR and histochemical analysis. METHODS: The pre-beta and beta-cells are exposed to different concentrations of $I L-1$ or $S T Z$ and induced NO and cytotoxicity determined after 3 days of culture. RESULTS: Whereas NO fomation (measured as $\mu$ mol// accumulated nitrite) is not induced by any of the IL-1 concentrations in the pre-beta cell-line, a dose-dependent NO production is seen in the beta-cell phenotype: $150 \mathrm{pg} / \mathrm{ml} / \mathrm{L}-1=$ $1,72 \pm 0,59 ; 1500 \mathrm{pg} / \mathrm{ml}=5.0 \pm 0,7 ;$ and $3000 \mathrm{pg} / \mathrm{ml}=10,6 \pm 1,14$. Furthermore an association between the amount of NO and frequency of apoptotic cells was found. Addition of STZ results in a dose-dependent cytotoxicity only in the beta-cells (measured as Lactate DeHydrogenase (LDH) release in percent of control cells): $1 \mathrm{mM}$ STZ $=18.9+\% 2,8 \%$ cytotoxicity; $2,5 \mathrm{mM} \mathrm{STZ}=25,1 \pm 4,7 \% ; 5 \mathrm{mM} \mathrm{STZ}=30,5 \pm 4,5 \% ;$ and $7,5 \mathrm{mM} \mathrm{STZ}=69,4 \pm$ $10,5 \%$. No effect was seen on the pre-beta-cells. PCR analysis demonsirate that both phenotypes express GLUT-2, which has recently been suggested to mediaie STZ uptake. CONCLUSION: This pluninotent cell-line represent a valuable toof in identifying factors involved in beta-cell differentiation/maturation, as weil as in cloning and characterisation of genes/proteins involved in cytokine and STZ mediated beta-cell cytotoxicity.

\section{0}

INDUCIBLE GENE EXPRESSION IN B-CELLS BY USE OF AN ESTROGEN RESPONSTVE RETROVIRAL VECTOR

M. Möhlig, P. Horn, J. Hofmann", C. Stocking", H. Schaz, A Pfeiffer

Med. Klinik und Poliklinik Bergmannsheil, Ruhr-Universităl Bochum, Germany

* Heinrich Pette Institut fir experimentelle Virologie, Hamburg, Germany

The stable genetic manipulation of B-cells is of great interest for therapeutic and experimental purposes. Retroviral techniques allow the stable insertion of single copies of foreign genes. In contrast, lipofection, electroporation or $\mathrm{CaP} 04$ cause the incorporation of large concatemerizeu and frequenily rearranged DNA-fragments. Since litte is known about the efficiency of retroviral gene expression in B-celis we tested the potent retroviral Myelo_Proliferative Sarcoma Vinus (MPSV) promoter and an estrogen receptor (ER) responsive inducible retroviral construct. METHODS: Two replication defective retroviral constructs were produced and used to infect RINm5F-cells: (a) a retrovirus in which the lac $Z$ gene is driven by 3

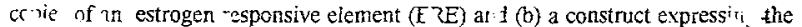
es'roren receptor under the control of the MPSV-promoter. Transient :-ing expression of the ER was achieved by electroporation with an ER constnuct under the control of a CMV-promoter (HEGO, kindly supplied by P. Chambon). Expression of the ER was also assessed using a 2-copy vitellogenin ERE promoterluciferase construct. RESULTS: Stable expression of the ER driven by the MPSVpromoter resulted in no basal gene expression and an about 4-fold E2-induction of either the stably integrated ERE-driven lac- $Z$ gene or a transiently expressed EREdriven luciferase construct. Transient maximal expression of the ER under control of a CMV-promoter (a multicopy expression) resulted in 6-8-fold higher levels of E2-responses representing an about 40-fold increase over basal expression. CONCLUSION: 1. An estrogen responsive retroviral construct allows ERcontrolled gene expression in B-cells essentially without basal expression. 2 . The response depends on the number of ER expressed in the cells. Multiple copies of the MPSV-promoter controlled ER-gene are required for strong inducible expression which may be achieved by multiple infections. 3. The use of an inducible retroviral vector in B-cells represents a promising way to obtain stable and high level controllable gene expression.

Supported by the DFG.

\section{1}

PROLIFERATIVE ACTIVITY IN THE HUMAN FETAL ENDOCRINE PANCREAS

W.G. Lu, R. De Krijger ${ }^{\star}$ and L. Bouwens. Department of Experimental Pathology, Free University Brussels (V.U.B.), Brussels, Belgium, and "Department of Pathology, University Hospital Dijkzigt, Rotterdam, The Netherlands.

Our objective was to evaluate the proliferative potential of endocrine cells in the human fetal pancreas. We analysed 20 human fetal pancreata between 12 and 41 weeks of gestational age by immunohistochemical double staining for the proliferative marker Ki-67 (MIB-1) and the endocrine marker synaptophysin or islet hormones. Tissues from legal or spontaneous abortions were collected with permission of the local ethical committee. Ki-67 nuclear labelling was detected in all four hormone-expressing cell types $(\alpha, \beta, \delta$ and $P P$ ) and in duct epithelial cells, the latter being identified by the keratin-19 marker. With increasing gestational age (1241 weeks), the Ki-67 labelling index (LI) of ductal cells and of synaptophysin-positive endocrine cells decreased in parallel from $17 \%$ to $4 \%$ and from $9 \%$ to $1 \%$, respectively. The LI of ductal cells was always statistically significantly higher than the LI of synaptophysin-positive endocrine cells. The LI of insulin-positive cells was significantly lower than that of the synaptophysin-positive cells and was very low $(<0.18 \%)$ after 19 weeks. These results suggest that the proliferative potential of human beta-cells is minimal and that their ontogenesis largely depends on the differentiation from hormone-negative islet precursor cells.

\section{2}

THE EFFECT OF POOR FOE TAL AND NEONATAL NUTRITION ON ISLET FUNCTION AND GLUCOSE TOLERANCEIN HIGH FAT FED-RATS MR Wilson \& SJ Hughes, Department of Physiology, St. Mary"s Hospital Medical School, Imperial College, London.

We have investigated whether malnutrition during the foetal-neonatal period impairs pancreatic B-cell function and glucose homeostasis in aduit animals consuming a high fat diet. Pregnant and lactating rats were fed control or low-protein diet (to induce protein-energy malnutrition) and offspring returned to normal diet for 13 weeks. Glucose tolerance and islet function were then assessed in adults fed normal or high fat diet $(30 \%$ fat $)$ for 4 weeks. Feedino low-protein diet impaired weight gain in neonates $(10.4 \pm 0.4$ vs $15.5 \pm 0.7 \mathrm{~g}$ in controls at 7 days old, $\mathrm{n}=50, \mathrm{p}<0.001)$. Islets from previously malnourished rats consuming high fat diet exhibited high basal insulin release $(34.2 \pm 5.0$ vs $14.7 \pm 2.5$ $\mu \mathrm{U} /$ islet/hr in controls, $\mathrm{n}=20, \mathrm{p}<0.001$ ) and impaired responses to 10 $(287 \pm 42$ ys $454 \pm 57 \mu \mathrm{U} /$ islet/hr in controls) and to $16 \mathrm{mmol} / \mathrm{l}$ glucose $(318 \pm 28$ vs $448 \pm 46 \mu \mathrm{U} / \mathrm{islet} / \mathrm{hr}$ ) $\mathrm{n}=20, \mathrm{p}<0.05$. In perifused islets a delayed and impaired secretory response to $10 \mathrm{mmol} / \mathrm{l}$ glucose was seen $(1.40 \pm 0.37$ vs $4.53 \pm 0.64 \mathrm{mU} / 40 \mathrm{~min}$ in controls, $\mathrm{p}<0.01)$ although islet insulin content was unchanged $(2.75 \pm 0.47 \mathrm{vs} 2.62 \pm 0.30 \mathrm{mU} / \mathrm{islet}$ in controls). High fat-feeding tended to impair glucose tolerance in control rats (area under GTT: $594 \pm 58$ vs $463 \pm 41$ mmoles/ 120 min, NS) and in previously malnourished rats (area under GTT: $686 \pm 82$ vs $505 \pm 63$, NS). In the latter the deterioration in glucose tolerance induced by high fat feeding was significant when compared to controls $(\mathrm{p}<0.05$, $\mathrm{n}=7$ ). Plasma insulin levels (at $60 \mathrm{~min}$, GTT) were also lower than in controls $(88 \pm 24$ vs $203 \pm 56 \mu \mathrm{U} / \mathrm{ml})$. These studies suggest that poor foetal-neonatal nutrition impairs the functional development of pancreatic islets such that in the presence of (high fat-induced) insulin resistance in adult rats, glucose tolerance is markedly impaired. 
413

ISLET CAPILLARY BLOOD PRESSURE IS INCREASED IN AN ANIMAL MODEL OF NON-INSULIN-DEPENDENT DIABETES MELLITUS. P-O Carlsson ${ }^{1}, L$ Jansson ${ }^{1}, C-G$ Östenson ${ }^{2}$ and Ö Källskog ${ }^{3}$. Departments of Medical Cell Biology ${ }^{1}$ and Physiology and Medical Physics ${ }^{3}$, Uppsala University, Uppsala, Sweden and Department of Endocrinology 2 , Karolinska Institute and Hospital, Stockholm, Sweden.

The aim of the present study was to measure pancreatic islet capillary pressure during basal conditions and after an acute glucose-stimulation of insulin release in normal rats, and in GK rats, an animal model of non-insulin-dependent diabetes mellitus (NIDDM). Hydrostatic pressures in single pancreatic islet capillaries were determined in vivo by direct measurement using micropuncture technique. The pancreatic islets were visualized by injection of Neutral Red. This intravital staining had no effect on islet function, whole pancreatic and islet blood flow or capillary pressure in the exocrine pancreas. Islet capillary blood pressure in normoglycemic rats was estimated to $3.1 \pm 0.3 \mathrm{~mm} \mathrm{Hg} \quad(n=15)$. Administration of D-glucose $(1 \mathrm{~g} / \mathrm{kg})$ doubled this value $(6.3 \pm 0.6 \mathrm{~mm} \mathrm{Hg}$; $\mathrm{n}=8 ; \mathrm{P}<0,001$. Student's paired $\mathrm{t}$-test), whereas no effect was seen after injection of an equimolar dose of the non-metabolizable glucose derivative 3-0 methyl glucose. In GK rats, basal islet capillary blood pressure was increased compared with controls $(5.7 \pm 0.4 \mathrm{~mm} \mathrm{Hg} ; n=10 ; P<0.001$ Student's unpaired t-test). A chronically increased islet capillary blood pressure may contribute to the pertubation of islet function seen in long-standing NIDDM, by causing damage to the islet vasculature.

\section{4}

GASTRIC INHIBITORY POLYPEPTIDE POTENTIATES GLUCOSESTIMULATED ISLET BLOOD FLOW INCREASE IN ANESTHETIZED, RATS

A. M. Svensson 1 , S. Efendic 2, C-G. Österison 2 and L. Jansson ${ }^{1}$. 1 Department of Medical Cell Biology, Uppsala University, Uppsala, Sweden. 2The Rolf Luft Center for Diabetes Research, Department of Molecular Medicine, Karolinska Institute, Karolinska Hospital, Stockholm, Sweden.

The aim of the study was to evaluate to what extent the incretin candidate hormone gastric inhibitory polypeptide (GIP) affects splanchnic blood flow, and especially pancreatic islet blood flow, in anesthetized rats. For. this purpose, male Sprague-Dawley rats were injected intravenously with either saline or GIP ( 5 or $15 \mu \mathrm{g} / \mathrm{kg}$ body weight) $10 \mathrm{~min}$ before blood flow. measurements with a microsphere technique. Furthermore, $1 \mathrm{ml}$ of either saline or $30 \%$ D-glucose was given intravenously $3 \mathrm{~min}$ before the blood flow measurements. The lowest dose of GIP decreased colonic blood flow in the normoglycemic rats, but did not affect the blood perfusion of the other investigated organs. The highest dose of GIP decreased whole pancreatic, duodenal and colonic blood flow, whereas islet blood flow was unaffected. However, fractional islet blood flow was increased. In the hyperglycemic rats, in which the islet blood flow was increased compared with saline-pretreated animals, both doses of GIP further enhanced islet blood flow. No effect on pancreatic, fractional isiet or duodenal blood flow was seen after GIP administration, whereas colonic blood flow was decreased. It is concluded that GIP can augment the glucose-stimulation of islet blood flow, and it may be that this helps to increase the availability of secreted insulin. GIP decreased colonic blood flow in both normo- and hyperglycemic rats, but the functional importance of this is unknown at present.

\section{5}

PANCREATIC BLOOD FLOW AND INSULIN RELEASE IN ADULT RAT FED A LOW PROTEIN DIET PRE AND POSTNATALLY

V. Iglesias-Barreira, M.T. Ahn, B. Reusens, S. Dahri, C. Remacle and J.J. Hoet, Univ. Catholique de Louvain, Louvain-la-Neuve, Belgium

The pancreatic islet blood flow and insulin secretion of adult rats submitted to an isocaloric low protein diet ( $8 \%$ vs $20 \%$ ) during pre and post-natal life was investigated by non-radioactive microspheres technique. In basal state, female rats chronically exposed to a low protein diet (LP) display a specific marked decrease in the islets blood flow $(10 \pm 2$ vs $29 \pm 5 \mathrm{ml} / \mathrm{min}$.g pancreas; $\mathrm{P}<0.01)$ and in overall pancreatic blood flow $(\mathrm{P}<0.01)$. The plasma insulin level was however not affected. During a glucose challenge, the control animals enhanced their blood flow 2.3-fold whereas the LP group reached control values with a 6-fold increase. Insulin plasma level rose similarly in the control and the LP animals. A third group of animals named recuperation (R), exposed to a low protein diet only in utero, displayed comparable values for islet blood flow to control, but an increased basal insulin (2.0 \pm 0.3 vs $1.2 \pm 0.1 \mathrm{ng} / \mathrm{ml}, \quad P<0.05$ ). These data are consistent with the possibility of the dissociation of islets blood flow and insulin secretion in basal condition, which may not be the case after glucose challenge. Furthermore, the low blood flow values in LP group may reflect either an impaired pancreatic vasculogenesis or a dysfunction of the endothelial cells. 


\section{Glucose Recognition and Signal Transduction in Beta-Cells}

416

SIMILAR INSULINOTROPIC EFFECTS OF ALFA- AND BETA-DGLUCOSE ON LARGE MAMMAL AND HUMAN ISOLATED PANCREATIC ISLETS.

R. Lupi, P. Marchetti, C. Tellini, A. Coppelli, S. Del Guerra, R. Giannarelli, $P$ Masiello, M. Carmellini, F. Mosca, R. Navalesi. Cattedra Malattie Metabolismo, Istituto Clinica Medica II, Università di Pisa, Italy

Under physiological conditions, D-glucose (DG) is the most important controller of insulin secretion (IS). In solution, DG is a mixture of approximately $1 / 3$ alfa- and $2 / 3$ beta-DG. In rodent islets the alfa-DG anomer is more effective than the beta anomer in triggering IS. We evaluated the effect of alfa- and beta-DG on IS from isolated large mammal and human islets, and compared the results with those from rat islets. Islets of Langerhans from four Wistar rats, two adult pigs and two human multiorgan donors were isolated by collagenase digestion and density gradient purification. Insulin secretion studies were performed at $37^{\circ} \mathrm{C}$ in albumin-containing Krebs-Ringer bicarbonate solution (KRB). After a preliminary incubation period of $45 \mathrm{~min}$ in KRB with $3.3 \mathrm{mmol} / 1 \mathrm{DG}$, batches of 10 islets of comparable size were incubated for $5 \mathrm{~min}$ with $2 \mathrm{ml}$ of KRB containing 5.5 or $16.7 \mathrm{mmol} / 1$ of either DG (prepared $60 \mathrm{~min}$ before the beginning of the experiments, and kept at $37^{\circ} \mathrm{C}$ ), or the pure alfa- or betaanomer, which had been rapidly dissolved in the medium (pre-warmed at $37^{\circ} \mathrm{C}$ ). The results obtained are summarized in the table, showing that indeed alfa-DG is more potent than beta-DG in triggering insulin release from rat islets at $5.5 \mathrm{mmol} / \mathrm{l}$ secretagogue concentration.

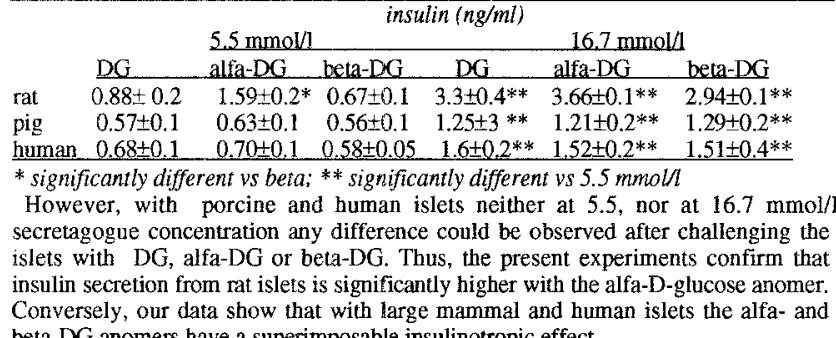
beta-DG anomers have a superimposable insulinotropic effect.

\section{7}

ENZYME-TO-ENZYME CHANNELLING OF KREBS CYCLE INTERMEDIATES IN PANCREATIC ISLET CELLS

W.J. Malaisse, L. Ladrière, T.-M. Zhang, I. Verbruggen* and R. Willem*. Laboratory of Experimental Medicine, Brussels Free University and *High Resolution NMR Centre, Vrije Universiteit Brussel, Brussels, Belgium. Attention was recently drawn to the relevance of protein-to-protein interaction to the regulation of nutrient metabolism in islet cells. For instance, it was proposed that glucokinase must interact with a suitable binding site to become activated. Likewise, hexose phosphates were reported to undergo enzyme-toenzyme channelling in the early steps of glycolysis. The present study concerns a third process of enzyme-to-enzyme tunnelling. It indeed documents the oriented transfer of symmetric Krebs cycle intermediates in islet cells. Groups of $0.8-1.010^{8}$ RINm5F cells were incubated for $120 \mathrm{~min}$ in $10 \mathrm{ml}$ of a Hepes-bicarbonate buffered medium containing $5 \mathrm{mmol} / \mathrm{l} \mathrm{D}$ glucose and $10 \mathrm{mmol} / 1\left[2-{ }^{13} \mathrm{C}\right]$ propionate. The amount of $\left[1-{ }^{13} \mathrm{C}\right]$ lactate and $\left[3-{ }^{13} \mathrm{C}\right]$ lactate released by the incubated cells, as measured by ${ }^{13} \mathrm{C}$ NMR, averaged, respectively, $29 \pm 18$ and $64 \pm 7 \%(n=3)$ of the paired amount of $\left[2-{ }^{13} \mathrm{C}\right]$ lactate. This indicates that symmetric Krebs cycle intermediates, such as succinic and fumaric acid, undergo oriented transfer in the sequence of reactions catalyzed by succinate thiokinase, succinate dehydrogenase and fumarase. These findings are relevant to the interpretation of radioactive data, such as the production of ${ }^{14} \mathrm{CO}_{2}$ resulting from the oxidation of ${ }^{14} \mathrm{C}$-labelled acetyl-CoA in the Krebs cycle. More importantly, the kinetics of the concerned enzymes may well differ when they are integrated in a single metabolon, rather than acting independently of one another. This is especially relevant to models of type- 2 diabetes, in which one of these enzymes, i.e. succinate dehydrogenase, may become a rate-limiting step in the Krebs cycle.

\section{9}

Mitochondrial aNA content and respiratory chain activity of purified pancreatic islet cells

I Conget, A Barrientos*, JM Manzanares, R. Gomis and F Cardellacht. Bndocrinology Unit, * Internal Medicine Unit. Hospital Clínic, Barcelona, Spain.

Interest has recently been focused on the putative role of alterations in mitochondrial activity and mutations in the mitochondrial genome for the development of NIDDM. Aim: to investigate the normal mitochondrial respiratory chain activity of non-purified and purified islet cells in order to further explore whether some diabetic states can be explained in terms of alterations of nitochondrial oxidative process. Methods: Pancreatic islets were isolated from Wistar rats, Unpurified islet cells were obtained in the presence of trypsin and DNase, purified $B$ and non- $\$$ cells being prepared by autofluoresecence activated sorting using a flowcytometer. Intact cell respiration and the substrate oxidation in digitonine permeabilized cells were neasured polarographycally. Specific activity of the indivioual complexes of the respiratory chain was performed spectrophotometrically in unpurified islet celis. The relative amounts of nitochondrial and nuclear DNB in all three cell populations, as well as, in rat brain and skeletal muscle, were stimated by dot blotting. Resuits: Intact cell respiration (Cell resp), oxidation of different substrates: pyravate-nalate

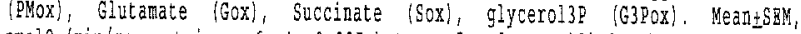
$\mathrm{amolO} / \mathrm{min} / \mathrm{mg}$ protein, $\mathrm{n}: 6$, ${ }^{2} p<0.005$ between $B$ and unpurified cells.

$\begin{array}{lcll} & \text { Jnpurified cells } & B \text { cells } & \text { non- }- \text { cells } \\ \text { Cell resp } & 17.92 \pm 1.58 & 16.53 \pm 2.20 & 19.45 \pm 5.97 \\ \text { PM ox } & 20.48 \pm 2.72 & 17.51 \pm 2.28 & 25.95 \pm 7.95 \\ \text { G ox } & 24.00 \pm 3.95 & 16.48 \pm 2.61 & 22.46 \pm 5.29 \\ \text { S ox } & 50.08 \pm 4.93 & 25.71 \pm 5.01 * & 34.30 \pm 2.98 \\ \text { G3P ox } & 21.22 \pm 2.70 & 19.45 \pm 3.45 & 26.48 \pm 1.22\end{array}$

The relative arount of ntDNA with respect to the nuclear DNA was not different between unpurified islet cells, $B$ and non- 8 cells, and was also similar to that obtained from brain and skeletal muscle. In sumbary, we have described mitochondrial respiratory chain activity in unpurified, 8 and non- $B$ islet cells. In our opinion, our results may represent the prior step to investigating the potential patogenic role that alterations in oxidative phosphorilation could play in some diabetic states. 
420

CASTANOSPERMINE, ISLET GLUCAN-1,4- $\alpha$-GLUCOSIDASE ACTIVITY AND GLUCOSE STIMULATED INSULIN RELEASE H. Mosén, A. Salebi, M. Linell and I. Lundquist, Department of Pharmacology, University of Lund, Sweden.

Castanospermine, an indolizine alkaloid formed in the seeds of Castanospermum australe, is known to inhibit different types of glycosidases. In view of a recent proposal that glucose-induced insulin release involves activation of islet $\alpha$-glucosidehydrolases, we set out to investigate the action of castanospermine on insulin release and islet lysosomal enzyme activities by a combined in vitro and in vivo approach. Castanospermine markedly and dosedependently suppressed glucose stimulated insulin secretion from isolated mouse islets, the $\mathrm{EC}_{50}$ being as low as $10^{-7} \mathrm{M}$. Similarly, castanospermine dose-dependently inbibited acid $\alpha$-glucosidehydrolase activities in islet homogenates, the $\mathrm{EC}_{50}$ for inhibition of the acid glucan-1,4- $\alpha$-glucosidase activity being approximately $10^{-7} \mathrm{M}$. In contrast, castanospermine did not influence other lysosomal enzyme activities such as acid phosphatase and Nacetyl- $\beta$-D-glucosaminidase. Neutral $\alpha$-glucosidase (endoplasmic reticulum) was only moderately inhibited. Moreover, after incubation of intact islets with castanospermine the same pattern of lysosomal enzyme activities was revealed; i.e. the acid $\alpha$-glucosidehydrolases were profoundly suppressed, whereas other lysosomal enzyme activities were unaffected. Castanospermine did not influence insulin release induced by the phosphodiesterase inhibitor isobutylmethylxanthine (IBMX) in isolated islets. Further, complementary in vivo experiments showed that pretreatment of mice with castanospermine markedly suppressed the insulin response to an i.v. glucose load, whereas the response to IBMX was unaffected. The results strongly suggest that an activation of the acid glucan-1,4- $\alpha$-glucosidase may serve as an important transduction signal for insulin release stimulated by glucose but not for insulin secretion induced by direct stimulation of the cyclic AMP system.

\section{1}

Cis-ELEMENTS INVOLVED IN TRANSCRUPTIONAL CONTROL OF THE RAT GLUCOKINASE GENE BY VTTAMIN $D_{3}$. RETINOIC ACID AND THYROID HORMONE $T$, IN HIT M2.2.2 CELLS

B.Leibiger. I.B. Leibiger:

The Rolf Luft Center for Diabetes Research. Department of Molecular Medrcine. Karolinska Hospital. S-171 76 Stockholm. Sweden

The correct expression of the glucokinase gene in pancreatic B-cells is a prerequisite to glucose-dependent insulin secretion. Initial studies have been undertaken to characterize cis- and trans-acting elements involved in the transcriptional control of the glucokinase gene in insulin producing cells. The nutrient- and hormonedependent transcriptional regulation of this gene is poorly understood: data concerning the role of glucose are contradictory, while other studies have shown the impact of glucocorticoid and thyroid hormones on glucokinase gene transcription. The aim of the present study was to analyse cis-elements of the rat glucokinase upstream promoler (rBGK) which contribute to transcriptional control in insulin producing HIT cells when stimulated by vitamin $D_{3}$. thyroid hormone $T_{3}$ and retinoic acid (RA). On the basis of the high honology of the two patindromic sequences 5'-TGGTCACCA-3'. located at positions -168 bp (TGGT2) and -9) bp (TGGTI) to reported binding motifs for members of the nuclear receptor superfamily. we decided to study the role of TGGT1 and TGGT2 in stimulusdependent expression of a rBGK-promoter/CAT fusion gene in HIT M2,2,2 cells. Following transfection with a $\mathrm{rBGK}-180 \mathrm{CAT}$ construct. HIT celts werc incubated with either $10 \mathrm{nM}$ vitamin $\mathrm{D}_{3}, 1 \mu \mathrm{M}$ RA. or $100 \mathrm{nM} \mathrm{T}$, for 24 hours. Treatmon with vitamin $\mathrm{D}_{3}$ and $\mathrm{RA}$ led to an increase in CAT activity (1.33- and 1.45-fold. respectively), whereas incubation with $\mathrm{T}_{3}$ caused a drop of CAT expression $1067 \%$ Knocking-out the TGGT1-motif led to a decrease in CAT expression to $34 \%$ when compared to its wild type counterpart and did not allow stimulation by vitamin D, and RA. The effect of T3 remained unaffected and caused a similar-fold decrease in CAT activity. On the other hand, mutation in the TGGT2-rnotif had no effect on either stimulation by vitamin $D_{3}$ and $R A$ or inhibition by $T_{3}$ but calsced a decrcasc in CAT expression to $28 \%$ of the wild type construct. Knocking-out both TGGT-motifs almost abolished CAT expression in HIT M2.2.2 cells (2.5\%).

\section{2}

THIS ABSTRACT HAS BEEN WITHDRAWN BY THE AUTHOR.

\section{3}

PERMEABILIZATION OF ISLET CELLS IDENTIFIES POOLS OF ADENINE NUCLEOTIDES WITH DISTINCT CHARACTERISTICS

P. Detimary, J.C. Jonas and J.C. Henquin. Unité d'Endocrinologie et Métabolisme, University of Louvain, Brussels, Belgium.

Adenine nucleotides are thought to serve as second messengers in the control of $\beta$ cell function by glucose, e.g. by regulating the activity of ATPdependent $\mathrm{K}^{+}$channels. However, their localization in different intracellular pools may mask the biologically relevant changes and complicate the interpretation of measurements in whole cells. In this study, the plasma membrane of mouse islet cells was permeabilized by the $\alpha$-toxin from Staphylococcus Aureus to compare diffusible (cytoplasmic) and more stable (organelle) pools of nucleotides. After permeabilization of fresh cells, $\sim 70 \%$ of ATP and $-50 \%$ of ADP and AMP rapidly diffused out of the cells, whereas the insulin content did not vary by more than $5-10 \%$. The remaining pool(s) were stable for at least $90 \mathrm{~min}$ at $4^{\circ} \mathrm{C}$. Incubation of the cells in 20 instead of $2 \mathrm{mmol} / \mathrm{h}$ glucose prior to permeabilization did not affect the stable pools, which suggests that the increase in the ATP/ADP ratio measured in intact cells occurred in the diffusible pool. In contrast, the pool resistant to permeabilization varied with the degree of $\beta$ cell granulation. Its size was proportional to that of insulin stores when these were progressively decreased by stimulating secretion to different degrees before permeabilization. From these results, it can be calculated that, depending on the ambient glucose concentration and the degree of $\beta$ cell degranulation (e.g. cultured vs. fresh cells), -10 to $30 \%$ ATP, $\sim 30$ to $40 \%$ ADP and $\sim 30$ to $50 \%$ AMP of intact cells are contained within insulin secretory granules. When these figures are taken into account, it emerges that the ADP variations brought about by glucose in a metabolically active (cytoplasmic) pool are large and compatible with a role in stimulus-secretion coupling. 


\section{4}

\section{N a + INVOLVEMENT IN GLUCOSE AND NEUTRAL AMINO ACID-INDUCED INSULIN SECRETION}

N.H. McClenaghan, C.R. Barnett and P.R. Flatt. School of Biomedical Sciences, University of Ulster, Coleraine, BT52 1SA, N.Ireland, UK.

Involvement of $\mathrm{Na}+$ in insulin secretory responses to glucose and to amino acids known to be co-transported with $\mathrm{Na}^{+}$, were examined using islet derived BRIN-BD11 cells. Acute 20 min tests $(n=6)$, with 16.7 $\mathrm{mmol} / \mathrm{l}$ glucose caused a 2 -fold stimulation $(\mathrm{p}<0.001)$ of insulin release above $1.1 \mathrm{mmol} / \mathrm{l}$ glucose $\left(0.89 \pm 0.07 \mathrm{ng} / 10^{6} \mathrm{cells} / 20 \mathrm{~min}\right.$, mean \pm SEM). Incubation with $10 \mathrm{mmol} / \mathrm{l}$ of L-alanine, $\alpha$-aminoisobutyric acid (AIB) or L-proline induced respective 10.4, 1.3 or 3-fold increases in insulin output $(\mathrm{p}<0.01)$ compared with $16.7 \mathrm{mmol} / \mathrm{l}$ glucose $(1.83 \pm$ $0.09 \mathrm{ng} / 10^{6}$ cells $/ 20 \mathrm{~min}$ ). These effects were significantly greater than those observed with $10 \mathrm{mmol} / \mathrm{l}$ amino acid at $1.1 \mathrm{mmol} / 1$ glucose $(p<0.01)$ and were effectively removed by either replacement of extracellular $\mathrm{Na}+$ with equimolar $\mathrm{N}$-methylglucamine ${ }^{+}$or $\mathrm{Ca}^{2+}$ omission. Blocking $\mathrm{Na}+$ channels with tetrodotoxin $(4 \mu \mathrm{mol} / \mathrm{l})$ at either 1.1 or 16.7 $\mathrm{mmol} / \mathrm{l}$ glucose exerted no significant effect on glucose or amino acidinduced insulin release. However, at $16.7 \mathrm{mmol} / \mathrm{h}$ glucose, $\mathrm{Na} / \mathrm{K}$ pump blockade with $1 \mathrm{mmol} / 1$ ouabain, evoked 1.6-2.3-fold stimulation $(\mathrm{p}<0.05)$ of insulin release over that recorded with $10 \mathrm{mmol} / \mathrm{L}$-alanine, AIB or L-proline. Although ouabain exerted no effect on glucoseresponsiveness, it enhanced the stimulatory effects of $\mathrm{L}$-alanine and $\mathrm{L}$ proline (1.6 and 1.3-fold; $\mathrm{p}<0.05$ ), and evoked AIB-induced insulin release $(1.9$-fold, $\mathrm{p}<0.001)$ at $1.1 \mathrm{mmol} / 1$ glucose. The results indicate these metabolizable and non-metabolizable amino acids enter BRINBD11 cells to evoke insulin secretion dependent on glucose, co-transport with $\mathrm{Na}^{+}$and $\mathrm{Ca}^{2}+$ influx.

\section{5}

PANCREATIC NITRIC OXIDE SYNTHASE INHIBITION AND ARGININE INDUCED INSULIN SECRETION.

R. Gross, M. Roye, M. Manteghetti, *P. Masiello and G. Ribes. Lab. Pharmacol. Fac. Med., Bd Henri IV, UMR 9921 CNRS, Montpellier, France; *Ist. Patologia Generale, Univ. Pisa, Italy.

Nw-Nitro-L-Arginine Methyl Ester (L-NAME), a competitive inhibitor of Nitric Oxide Synthase (NOS) is known to potentiate arginine-induced insulin secretion; this suggests that NOS activity exerts an important negative control on $\mathrm{B}$ cell response to the amino acid. In order to determine if Nitric Oxide (NO) generated by the enzyme is indeed responsible for this modulation, we explored the ability of substitutive treatments with Sodium Nitroprusside (SNP), a chemical NO donor, to suppress L-NAME effect. In the isolated perfused rat pancreas, in presence of a $5 \mathrm{mmol} / 1$ glucose, arginine ( $5 \mathrm{mmol} / \mathrm{l})$ induced a transient monophasic stimulation of insulin release $(96 \pm 10 \mathrm{ng} .20 \mathrm{~min}$ ) which was converted, by the addition of L-NAME $(5 \mathrm{mmol} / \mathrm{l})$, into a significantly greater biphasic one (354 \pm 43 ng. $20 \mathrm{~min} ; \mathrm{p}<0.001)$. SNP in the range $(3 \mathrm{nmol} / \mathrm{l}$ $300 \mu \mathrm{mol} / \mathrm{l})$ was unable, at any concentration, to counteract this $\mathrm{L}$ NAME effect. Conversely, the NO donor, at 3 and $300 \mu \mathrm{mol} / 1$, further increased the effect of L-NAME plus arginine (722 $\pm 52 \mathrm{ng}$ and $733 \pm 49.20$ min respectively; $p<0.001)$. On the other hand, citrulline $(5 \mathrm{mmol} / \mathrm{l})$, the co-product of NOS activity provoked a $50 \%$ reduction of the second phase of arginine plus NAME induced insulin release (114 \pm 7 vs $259 \pm 24 \mathrm{ng} 15 \mathrm{~min}, \mathrm{p}<0.001$ ) as well as that recorded in the presence of arginine plus NAME plus SNP $3 \mu \mathrm{mol} / 1$. In conclusion, 1) NO does not seem implicated in NOS negative control of B cell response to arginine; 2) Citrulline, the co-product of NOS activity, could at least partly be responsible for it.

\section{6}

NO DONORS, IONIC CHANNELS AND INSULIN RELEASE

M.-H. Antoine, R. Ouedraogo, A. Herchuelz and P. Lebrun. Department of Pharmacology, Université Libre de Bruxelles, Brussels, Belgium.

Recent studies have suggested the involvement of nitric oxide (NO) in pancreatic B-cell function. Few investigations, however, have addressed the question whether modifications in transmembrane ionic movements might play a role in the physiological response to NO. The present study was undertaken to assess the effects of NO donors on ionic and secretory events in rat pancreatic islets. Hydroxylamine, sodium nitroprusside (SNP), 3-morpholinosydnonimine (SIN-1) and S-Nitroso- $\mathrm{N}$-acetylpenicillamine (SNAP) provoked a concentration-dependent inhibition of the glucoseinduced insulin release. This inhibitory action was counteracted by glibenclamide. Hydroxylamine, SNP and SIN-1 increased the rate of ${ }^{86} \mathrm{Rb}$ outflow from prelabelled and perifused rat pancreatic islets. The stimulatory effect of hydroxylamine and SNP persisted in the absence of external $\mathrm{Ca}^{2+}$ but was impaired by glibenclamide; a hypoglycemic sulfonylurea reported to selectively block the ATPsensitive $\mathrm{K}^{+}$channels. Hydroxylamine, SNP and SIN-1 also decreased ${ }^{45} \mathrm{Ca}$ outflow from glucose-stimulated pancreatic islets. Moreover, hydroxylamine and SNP inhibited the rise in $\left[\mathrm{Ca}^{2+}\right]_{1}$ induced by $16.7 \mathrm{mM}$ glucose but not that evoked by $\mathrm{K}^{+}$depolarization. These experimental results suggest that the negative insulinotropic action of the NO donors results, at least in part, from the direct or indirect activation of ATP-sensitive $\mathrm{K}^{+}$channels leading to a decrease in $\mathrm{Ca}^{2+}$ influx and $\left[\mathrm{Ca}^{2+}\right]_{\mathrm{i}}$.

\section{7}

DIFFERENTIAL EFFECTS OF CIS AND TRANS FATTY ACIDS ON INSULIN RELEASE FROM ISOLATED NOUSE ISLETS OF LANGERHANS.

K.K Alstup, S.Gregersen and K.Hermansen. Deparment of Endocrinology and Metabolism. Aarhus University Hospital. Гage-Hansen gade 2, DK-8000 Aarhus C, Denmark.

Both in vitro and in vivo studies in animals have reveated that elevated levels of free latty acids (FFA) induce impaired $\beta$-cell functions corresponding to the abnormalitics heing present in NIDDM. It is well establised that consurnption of high amounts of rans fatty acids is associated with an unfarourable lipid and lipoprotein profile and schaemic heart disease. The spatial configuration of fatty acids has an important impact on different metabolic and physical functions. However. it is not known, in what way the spatial configuration of fatty acids influences $\beta$-cell function. The r resent study was conducted to clucidate a possible different intluence on insulin release of the trans and cis forms of C18:1 fatty acids. Overnight incubated mouse islets of Langerhans were used: insulin secretion were measured with RIA: Students unpaired t-test was used for comparison. In the presence of $16.7 \mathrm{mmol} / \mathrm{l}$ glucose the addition of cisitrans vacoenic acid ( $\mathrm{C}$ 18:1(position 11) and oleic acid/elaidic acid (C 18:1 (position 9)) caused a dose dependent increase in the insulin secretion with a maximum of the fatty acids at 3.0 mmolli. Trans raccenic acid elicited a 2 -fold (p<1.0001) higher insulin response than cis vaccanic acid. The same tendency was sien with a cis/trars contiguration in position n-9 (oleic acid and elaidic acid) although the difference was not significant. Furthermore. both cis and trans forms of the $C 18: 1$ clicited a glucose dependent $\beta$-cell secretion. At low glucose $(3.3 \mathrm{mmot} / \mathrm{l})$ all the fatty atids did not change insulin release. In conclusion: We have demonstrated. that trans vaccenic acid stimulated insulin secretion more potently than cis vaccenic acid. Our indings suggest, that not only the chain length and amount of double bindings but aiso the spatial contiguration of fatty acids is or imponance tor the regulation of $\beta$ cell secretion in the mouse. 
428

FATTY ACIDS INHIBIT ACETYL-COA CARBOXYLASE GENE EXPRESSION IN THE PANCREATIC B-CELL LINE INS-1.

T. Brun, F. Assimacopoulos-Jeannet, and M. Prentki. Department of Nutrition, University of Montreal, Canada and Departement de Biochimie Médicale, University of Geneva, Switzerland.

Acetyl-CoA carboxylase (ACC) may act as a "nutrient sensor" in the B-cell and an "anaplerotic/malonyl-CoA pathway" has been suggested to be implicated in glucose-induced insulin secretion. We have studied the long term expression of ACC by nutrients in INS-I cells. Glucose, from 5 to $20 \mathrm{mM}$ caused a marked accumulation of ACC mRNA and protein. The long chain fatty acids (FFA) myristate, palmitate, oleate, and linoleate, as well as the short chain FFA butyrate and caproate, inhibited both basal and glucose-stimulated ACC transcript induction. The inhibition was detected by $4 \mathrm{~h}$ and a maximal $70 \%$ effect occurred at $12 \mathrm{~h}$ following cell exposure to FFA. In contrast, 2-bromopalmitate, an inhibitor of the B-oxidation of fatty acids, had no effect, suggesting that FFA must be metabolized to change ACC gene expression. The action of nutrients likely occurs at the transcriptional level since linoleate and glucose did not modify the half life of the ACC transcript. Linoleate also inhibited ACC protein accumulation at low and high glucose. Prolonged exposure of INS-1 cells to FFA markedly altered the glucose-induced insulin response, resulting in high basal insulin release and a suppression of glucose-induced insulin secretion. This was associated with an exaggerated rate of FFA oxidation at all tested glucose concentrations. The data strengthen the view that ACC is a key enzyme in 15 -cell signaling and that alterations in its expression by fatty acids may contribute to defective insulin secretion in type 2 diabetes.
429

INHIBITION OF GLUCOSE-INDUCED INSULIN SECRETION BY RHC 80267 AND ACA IS MEDIATED BY AN INCREASE IN K $\mathrm{K}^{+}$PERMEABILITY.

P. Thams and K. Capito. Department of Medical Biochemistry \& Genetics, Biochemistry Laboratory A, University of Copenhagen, Copenhagen, Denmark.

The effects of the diacylglycerol lipase inhibitor 1,6-bis-(cyclohexyliminocarbonylamino)-hexane (RHC 80267) and the phospholipase $A_{2}$ inhibitor $\mathrm{N}$-(p-amylcinnamoyl)anthranilic acid (ACA) on insulin secretion, glucose metabolism and ${ }^{86} \mathrm{Rb}^{+}$efflux in mouse pancreatic islets were studied. RHC $80267(35 \mu \mathrm{mol} / \mathrm{L})$ and ACA $(100 \mu \mathrm{mol} / \mathrm{L})$ inhibited giucose $(16.7 \mathrm{mmol} / \mathrm{L})$-induced insulin secretion, but did not inhibit insulin secretion induced by $\mathrm{K}^{+}(40 \mathrm{mmol} / \mathrm{L})$ or the phorbol ester TPA $(0.16 \mu \mathrm{mol} / \mathrm{L})$. Glucose utilization and glucose oxidation were not impaired by RHC 80267 or ACA. $\mathrm{K}^{+}$or TPA potentiated glucose-induced insulin secretion, and prevented inhibition of glucoseinduced insulin secretion by RHC 80267 and ACA. In comparison, potentiation of glucose-induced insulin secretion by arachidonic acid (AA; $200 \mu \mathrm{mol} / \mathrm{L}$ ) failed to counteract inhibition of glucose-induced insulin secretion by RHC 80267 or ACA, suggesting that inhibition by these agents was not mediated by a decrease in AA accumulation in islets. Thus glucose $(16.7 \mathrm{mmol} / \mathrm{L})$-induced insulin secretion, which was potentiated by AA $(200 \mu \mathrm{mol} / \mathrm{L})$ from $3.2 \pm 0.6(8)$ to $7.0 \pm 1.2(8) \mathrm{ng} / \mathrm{h} / 5$ islets $(\mathrm{P}<0.025)$, was reduced by RHC $80267(35 \mu \mathrm{mol} / \mathrm{L})$ to $0.8 \pm 0.2(6)(\mathrm{P}<0.025)$ in the absence and to $0.9 \pm 0.2(6) \mathrm{ng} / \mathrm{h} / 5$ islets $(\mathrm{P}<0.025)$ in the presence of $\mathrm{AA}(200 \mu \mathrm{mol} / \mathrm{L})$. Both RHC 80267 and $\mathrm{ACA}$ stimulated ${ }^{86} \mathrm{Rb}^{+}$efflux from islets. AA also stimulated ${ }^{86} \mathrm{Rb}{ }^{+}$effiux, suggesting that stimulation of ${ }^{86} \mathrm{Rb}^{+}$efflux by $\mathrm{RHC} 80267$ and $\mathrm{ACA}$ was not due to a decrease in AA accumulation. This stimulation by $A A$ was not dependent on $A A$ metabolism. In contrast to RHC 80267 and ACA, AA stimulation of ${ }^{86} \mathrm{Rb}^{+}$efflux was attenuated in $\mathrm{Ca}^{2+}$ free medium. Parallel experiments with diazoxide $(100 \mathrm{\mu mol} / \mathrm{L})$ revealed that $\mathrm{RHC} 80267$ and ACA mimicked the effects of diazoxide, a specific activator of ATP-sensitive $\mathrm{K}^{+}$channels in islets, on both insulin secretion, glucose metabolism and ${ }^{86} \mathrm{Rb}^{2}$ efflux. In conclusion, therefore, it is suggested that RHC 80267 and ACA, independently of their action on AA release, tnay inhibit glucose-induced insulin secretion by opening of ATP-sensitive $\mathrm{K}^{+}$ channels in islets.

\section{0}

BINDING TO RECEPTORS FOR ACTIVATED C KINASE DEFINES FUNCTIONAL SPECIFICITY OF PKC ISOENZYMES IN ISLET CELLS M. Yedovitzky ${ }^{1}$, D. Mochly-Rosern ${ }^{2}$, J.A. Johnson ${ }^{2}$, M.O. Gray ${ }^{2}$, D. Ron', E. Abramovitch ${ }^{1}$, E. Cerasi ${ }^{1}$ and R. Nesher'. ${ }^{1}$ Department of Endocrinology and Metabolism, Hebrew University - Hadassah Medical center, Jerusalem IL and ${ }^{2}$ Department of Molecular Pharmacology, Stanford University Schoool of Medicine, CA, USA.

Protein Kinase C (PKC) is known to participate in B-cell signal transduction. At least six PKC isoenzymes have been identified thus far in islet $\beta$-cells. This work is aimed at delineating the specific role of each isoenzyme in $\beta$-cell activation. Immunohistochemical confocal microscopy of adult rat islets revealed that stimulation with glucose $(20 \mathrm{mM})$ or phorbol $12-$ myristate, 13-acetate (PMA, $100 \mathrm{nM}$ ) resulted in translocation of $\alpha \mathrm{PKC}$ and $€ P K C$ to sites corresponding to $\beta$-cell membrane, $\zeta P K C$ to a perinuclear region, whereas $\delta P K C$ concentrated in a polar, perinuclear region, believed to correspond to the Golgi apparatus. Activated PKC binds to isoenzymespecific anchoring proteins, RACKs, (Receptors for Activated C Kinase), of which two have been already identified. Short synthetic peptides corresponding to the isoenzyme-specific RACK-binding site on PKC have been synthesized. $\beta C 2-4$, a nine amino acid peptide derived from the PKC's C2 domain, was previously found to block translocation and function of $\mathrm{C} 2$-containing isoenzymes in neonatal cardiac myocytes. In islet cells, $\mathrm{BC} 2-4$ blocked translocation of $\alpha \mathrm{PKC}$ and reduced the insulin response to glucose or to PMA by $40 \%(\mathrm{P}<0.002)$. $\varepsilon V 1-2$, an eight amino acid peptide analogue of RACK-binding site on $E P K C$, specifically inhibited translocation of $\varepsilon P K C$ and reduced insulin response to glucose or PMA by $45 \% \quad(\mathrm{P}<0.004)$. The effect of the two translocation inhibitors was additive. Therefore at least two PKC isoenzymes, the calcium-dependent type $\alpha$ and the calcium-independent type $\varepsilon$, are involved in the regulation of insulin secretion. Using these and additional isoenzyme-specific inhibitors of PKC binding to RACKs, the role of individual PKC isoenzymes in insulin secretion by islet cells can be determined.
431

HOW DOES MYOSIN LIGHT CHAIN KINASE (MLCK) CONTROL INSULIN RELEASE FROM THE PANCREATIC $\beta$ CELL?

I. Niki, M. Hisatomi, Y. lida and H. Hidaka. Department of Pharmacology, Nagoya University School of Medicine, Nagoya 466, Japan

The aim of this study is to investigate the roles of calmodulindependent processes in the control of insulin release from the pancreatic B cell by biological and biochemical approaches. We observed movement of the quinacrine-sensitive granules in the living $\beta$-cells under a phase-contrast microscope. This movement was abolished by an intracellular Ca chelator, BAPTA, and significantly reduced by $\mathrm{W}-7$, the calmodulin antagonist. We raised anti-MLCK monoclonal antibodies, which inhibited $\mathrm{Ca}^{2+}$-induced insulin release from streptolysin-O permeabilized islets. Inhibition of the release by these antibodies were proportional to their inhibitory actions on the MLCK activity in vitro. The antibody consistently inhibited insulin release caused by the C-kinase activator 12-O-tetradecanoyl-phorbol 13acetate or the stable GTP analogue, GTP $\gamma$ S, even at a substimulatory concentration $(0.1 \mu \mathrm{M})$ of $\mathrm{Ca}^{2+}$. Such inhibition, however, disappeared when the concomitant $\mathrm{Ca}^{2+}$ concentration was lowered to $0.01 \mu \mathrm{M}$. $32 \mathrm{P}$ incorporation into the myosin light chain peptide by the islet homogenate demonstrated that phosphorylation of the peptide occurs in $\mathrm{a} \mathrm{Ca}^{2+}$-dependent manner with a threshold between 0.01 and $0.1 \mu \mathrm{M}$ $\mathrm{Ca}^{2+}$. These findings suggest that MLCK activated by a substimulatory concentration of $\mathrm{Ca}^{2+}$ may control insulin release via acting on a proximal step in the secretory pathway, presumably translocation of the insulin granules. 
432

SUBCELLULAR LOCALISATION AND ROLE OF SYNAPTOTAGMIN IN INSULIN-SECRETING PANCREATIC $\beta$-CELL LINES Lang, J., Fukuda, $\mathrm{M}^{+}{ }^{+}$, Zhang, $\mathrm{H}_{\text {., }}$

Kiraly, C.,Mikoshiba, K. ${ }^{+}$, and C. B. Wollheim

Div. de Biochimie Clinique, Departement de Médécine, CMU, Université de Genève, Genève, $\mathrm{CH}$ and ${ }^{+}$Dep. of Molecular Neurobiology, Inst. of Medical Science, Univ. of Tokyo, Tokyo, Japan

Various approaches have demonstrated the importance of the calciumsensing and phospholipid-binding protein synaptotagmin in neurotransmitter release from small synaptic vesicles. We investigated whether this protein may also be an effector for calcium in the exocytotic secretion of insulin from Large Dense Core Vesicles (LDCV) in the endocrine pancreatic $\beta$-cell.

The synaptotagmin isoforms I, and II could be detected in the $\beta$-ceil lines RINm5F, HIT-T15 and INS-1 by Northern and Western blot analysis, although expression levels were considerably lower than in brain. Subcellular fractionation and confocal microscopy of INS-1 cells demonstrated that synaptotagmin I comigrates mainly with fractions enriched in insulin-containing secretory granules (LDCV), whereas only small amounts are present in fractions containing the synaptic vesiclelike microvesicles. In SL-O permeabilized INS-1 and HIT-T15 cells antibodies or Fab-fragments directed against the first calcium-binding domain of synaptotagmin I or II inhibited calcium-induced exocytosis up to $60 \%$, but not GTPyS-stimulated exocytosis. Control IgG or Fab were inactive. In addition using a transient cotransfection system in HIT-T15 cells we found that synaptotagmin II mutants which are deficient in calcium-dependent phospholipid binding ( $\Delta$ 180-183 D231S) decreased KCl-induced secretion by 60 and $30 \%$, respectively. Overexpression of wildtype synaptotagmin I or II did not alter secretion. In conclusion, our findings suggest that synaptotagmin constitutes an important effector for calcium also in the exocytosis of LDCV containing endocrine hormones and stresses the difference between calcium- and G-protein induced secretion.

\section{PS 11 \\ Ion Channels and Oscillation in Islet Cells}

\section{3}

EXPRESSION OF THE CLONED BETA-CELL ATP-SENSITIVE POTASSIUM CHANNEL IN XENOPUS OOCYTES

FM Gribble, R Ashfield and FM Ashcroft. University Laboratory of Physiology, Parks Road, Oxford, OX1 3PT, UK.

Closure of the ATP-sensitive potassium channel in pancreatic $\beta$-cells plays a key role in both glucose-mediated and sulphonylurea-mediated insulin release The $\beta$-cell K-ATP channel is a complex of the sulphonylurea receptor (SUR1) and an inwardly-rectifying potassiun channel (Kir6.2), which can be heterologously expressed in manmalian cells. The aim of this study was to determine whether the Xenopus oocyte could also act as a heterologous expression system. Xenopus oocytes were defolliculated and injected with either $10 \mathrm{mM}$ Tris (control) or with mRNA encoding SUR1, Kir6.2 or a 1:1 mixture of SUR1 and Kir6.2. After 2-4 days, whole-cell currents were measured using the 2-electrode voltage clamp in $90 \mathrm{mM}$ external $\mathrm{K}^{+}$. Under these conditions, small currents were observed in both mRNA-injected and control cells. Application of $3 \mathrm{mM} \mathrm{Na}$ azide, a metabolic inhibitor, resulted in the appearance of large K-selective currents in oocytes coinjected with SUR1 and Kir6.2 (currents at $-100 \mathrm{mV}$ increased from $0.26 \pm 0.03$ to $11.5 \pm 1.5 \mu \mathrm{A}$ : mean \pm sem, $\mathrm{n}=17$ ), but not in control, SUR1-injected or Kir6.2-injected oocytes. Currents started to increase 5 minutes after addition of azide, reached a maximum after 15 minutes and were reversible on removal of azide. The azide-activated currents were further increased by the K-channel openers: $179 \pm 12 \%$ increase by $340 \mathrm{MM}$ diazoxide, $139+6 \%$ by $1 \mathrm{mM}$ pinacidil, and $105+2 \%$ by $0.5 \mathrm{mM}$ cromokalim $(n=5$ of each). The currents were blocked by the sulphonylureas tolbutamide ( $0.5 \mathrm{mM}, 96.0 \pm 0.5 \%$ block), glibenclamide $(100 \mathrm{nM}, 94.9 \pm 0.8 \%)$

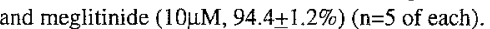

In conclusion, the cloned $\beta$-cell K-ATP channel can be expressed in Xenopus oocytes and is activated by the metabolic inhibitor azide. The expressed currents show pharmacological properties characteristic of the native $\beta$-cell $\mathrm{K}$ ATP channel.

\section{4}

DIADENOSINE-POLYPHOSPHATES ARE EFFECTIVE INHIBITORY LIGANDS OF ATP DEPENDENT POTASSIUM CHANNELS IN THE PANCREATIC $\beta$-CELL. Juan Manual Rovira, Cristina Ripoll and Bernat Soria, Dept. of Physiology and Institute of Neurosciences, University of Alicante School of Medicine, Aptdo, 374, Alicante 03080 (Spain)

We have investigated the effects of diadenosine tetraphosphate (Ap4A) on unitary currents present in $\beta$-cell membranes. Dinucleotide polvphosphates have recently been demonstrated in the cytosol of mammalian cells, but the intracellular function of these compounds remains unknown. Due to the structural similarity with ATP, it has been proposed that these compounds could act as inhibitors of the ATP regulated potassium channels $\left(\mathrm{K}_{A T P}\right)$. In this regard, it has been recently shown that diadenosine polyphosphates, such as diadenosine tetra-, penta- and hexaphosphate (Ap4A, Ap5A, Ap6A) can inhibit cardiac $K_{A T P}$ channels when applied on the inner side of the membrane. We tested the effect of Ap4A, ApSA and Ap6A on mouse pancreatic B-cell using the inside-out configuration of the patch clamp technique finding that all of them were effective inhibitors of the $\mathrm{K}_{\mathrm{ATP}}$ channel activity. Ap4A inhibits the $K_{\text {ATP }}$ channel in a dose-dependent manner (half-maximal concentration is in the order of $20 \mu \mathrm{mol} / \mathrm{l})$. Ap4A is as potent inhibitor of the $K_{\text {ATP }}$ channel as ATP and, although reversible, has a slower dissociation rate. In addition to intracellular inhibition, ATP also "refreshes" the channel from spontaneous run-down. Contrariwise to ATP. Ap4A $(40-200 \mu \mathrm{mol} / \mathrm{l})$ was inneffective as $\mathrm{K}_{\mathrm{ATP}}$ "refresher". Moreover, these compounds do not affect the conductance or the kinetic properties of other potassium channels of the $\beta$-cell, such as the calcium-activated potassium channel. According to these results. we propose that diadenosin polyphosphates could be implicated in the modulation of ATP interaction with the inhibitory nucleotide site of the $\mathrm{K}_{4 T p}$ channel 
435

EFFECT OF $\mathrm{Ca}^{2+}$-CHANNEL INACTIVATION ON BURSTING MOUSE PANCREATIC B-CELLS

M.A. Wahl, C. Schuhmacher and H.P.T. Ammon. Department of Pharmacology, Institute of Pharmaceutical Sciences, Eberhard-KarlsUniversität, Auf der Morgenstelle 8, D-72076 Tübingen

Bursting electrical activity is typical for the pancreatic B-cell challenged by glucose. Duration of bursts, which is related to insulin release, is abolished by repolarizations. It was recently shown that the repolarizations depend on the influx of $\mathrm{Ca}^{2+}$ through voltage-dependent $\mathrm{Ca}^{2+}$-channels. It was our aim to study the effect of additions of $\mathrm{Sr}^{2+}$ or $\mathrm{Ba}^{2+}$, which are know to inhibit the inactivation of voliage-dependent $\mathrm{Ca}^{2+}$-channels on the burst-pattern of glucose-stimulated mouse pancreatic $B$-cells by intracellular recording of electrical activity. B-cells within islets displayed a regular bursting pattern at $16.7 \mathrm{mmol} / \mathrm{l}$ glucose in a buffer containing 2.5 mmol// $\mathrm{Ca}^{2+}$. Increasing the extracellular $\mathrm{Ca}^{2+}$ concentration by addition of 1 or $5 \mathrm{mmol} / \mathrm{produced}$ a concentrationdependent reduction of the burst-phase duration to 83 or $50 \%$ of the control $\left(p<0.001\right.$ for $\left.5 \mathrm{mmol} / / \mathrm{Ca}^{2+}\right)$. In the presence of the $\mathrm{Ca}^{2+}$-channelbjocker isradipine, the repolarizing potency of an increase in extracellular $\mathrm{Ca}^{2+}$ was abolished. Addition of 1 or $5 \mathrm{mmol} / \mathrm{S} \mathrm{Sr}^{2+}$ decreased the electrical activity by about 87 or $68 \%\left(p<0.01\right.$ for $5 \mathrm{mmol} / \mathrm{S \textrm {Sr } ^ { 2 + }}$ ), the effects being less pronounced as with $\mathrm{Ca}^{2+}$. In contrast, the addition of $\mathrm{Ba}^{2+}(0.25$ to $2 \mathrm{mmol} / \mathrm{)})$ depolarized the B-cells in a concentrationdependent manner. Since an increase in extracellular $\mathrm{Ca}^{2+}$-concentration is known to increase $\mathrm{Ca}^{2+}$-channel inactivation, $\mathrm{Sr}^{2+}$ to reduce this inactivation, but $\mathrm{Ba}^{2+}$ to suppress it, almost completely, our data present evidence that the induction of repolarizations in the glucose-challenged pancreatic $B$-cell is related to the inactivation of the voltage-dependent $\mathrm{Ca}^{2+}$-channel.

\section{6}

HYPERPOLARIZING EFFECT OF INACTIVATION OF $\mathrm{Ca}^{2+}$. CHANNELS IN MOUSE PANCREATIC B-CELLS

C. Schuhmacher, H.P.T. Ammon and M.A. Wahl. Department of Pharmacology, Institute of Pharmaceutical Sciences, Eberhard-KarlsUniversität, Auf der Morgenstelle 8, D-72076 Tübingen

Electrical activity of pancreatic B-cells is closely related to insulin secretion induced by nutrients. This activity is characterized by depolarization - hyperpolarization cycles. To further clarify the mechanism of hyperpolarization, we elucidated the possible contribution of $\mathrm{Ca}^{2+}$-channel inactivation to this event. Membrane potentials from mouse pancreatic $\beta$-cells were measured by intracellular recording. To open voltage-dependent $\mathrm{Ca}^{2+}$-channels, cells were clamped to a membrane potential of $-40 \mathrm{mV}$ using $\mathrm{KCl}(12-19 \mathrm{mmol} / \mathrm{l})$. Under these conditions, increasing the extracellular $\mathrm{Ca}^{2+}$-concentration from 2.5 to 10.0 or $20.0 \mathrm{mmol} / \mathrm{l}$ hyperpolarized the cells by about 5 to $10 \mathrm{mV}$.

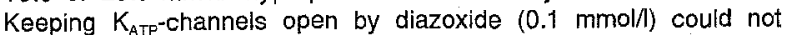
prevent this hyperpolarization. Using penitrem $A$ (10 or $100 \mathrm{nmol} / \mathrm{l})$, or TEA ( $1 \mathrm{mmol} / \mathrm{l})$, which block the large conductance $\mathrm{K}_{\mathrm{Ca}}^{+}$-channels, or apamin, which blocks the small conductance $\mathrm{K}_{\mathrm{ca}}$-channels was also without effect on $\mathrm{Ca}^{2+}$-induced hyperpolarization. The hyperpolarizations as induced by $\mathrm{Ca}^{2+}$ also occurred when $\mathrm{Sr}^{2+}(1 ; 2$ or 5 $\mathrm{mmo} / \mathrm{l})$ was added to the perifusate. Using $\mathrm{Ba}^{2+}(1 \mathrm{mmol} / \mathrm{h})$, which prevents inactivation of voltage-dependent $\mathrm{Ca}^{2+}$-channels, in contrast, did not induce hyperpolarization but induced occurrence of spikes. Our data show that the hyperpolarization based on an increase in $\mathrm{Ca}^{2+}$-influx can not be prevented by drugs affecting the function of $\mathrm{K}^{+}$-channels but only by suppression of inactivation of $\mathrm{Ca}^{2+}{ }^{2+}$ channels. Therefore, the $\mathrm{Ca}^{2+}$-induced inactivation of $\mathrm{Ca}^{2+}-$ channels is a mechanism being active at the threshold potential of the pancreatic $\beta$-cells which could bo responsive for the occurrence of hyperpolarizations.

\section{7}

AN ENERGY-REQUIRING STEP DISTAL TO $\mathrm{CA}^{2+}$ INFLUX IN STIMULUS-SECRETION COUPLING IN PANCREATIC B-CELLS.

T. Grimmsmann and I. Rustenbeck. Institute of Pharmacology, University of Gottingen, Germany

The inhibitors of mitochondrial ATP production, CCCP $(10 \mu \mathrm{M})$ and and sodium azide $(5 \mathrm{mM})$ induced a prompt and marked increase of the cytoplasmic $\mathrm{Ca}^{2+}$ concentration $\left(\left[\mathrm{Ca}^{2+}\right]_{\mathrm{j}}\right.$ ), as measured by $\mathrm{Ca}^{2+}$ imaging with Fura-loaded single B-cells from ob/ob mice. However, this increase of $\left[\mathrm{Ca}^{2+}\right]_{i}$ was not accompanied by insulin secretion. Rather, these agents completely suppressed the secretory response to $20 \mathrm{mM}$ glucose in accordance with the fuel hypothesis of insulin secretion. Since both agents induced a mitochondrial depolarisation (registered as an increase of fluorescence of rhodamin 123-loaded Bcells) and since the increase of $\left[\mathrm{Ca}^{2+}\right]_{i}$ was not (CCCP) or only partially (azide) inhibited by the $\mathrm{Ca}^{2+}$ channel blocker, $\mathrm{D} 600$, a mobilization of $\mathrm{Ca}^{2+}$ from internal stores must be assumed. Thus the internal source of $\mathrm{Ca}^{2+}$ could be the reason for the lack of secretion in response to increased $\left[\mathrm{Ca}^{2+}\right]_{\mathrm{i}}$. Like CCCP and azide, the inhibitor of the mitochondrial $F_{0} / F_{1}$-ATPase, oligomycin $(5 \mu \mathrm{M})$, suppressed glucose-induced insulin secretion, but this compound did not induce internal $\mathrm{Ca}^{2+}$ mobilization and mitochondrial depolarisation. However, when insulin release was induced by $\mathrm{Ca}^{2+}$ influx through voltage-dependent $\mathrm{Ca}^{2+}$ channels, oligomycin suppressed the secretory response, but not the increase of $\left[\mathrm{Ca}^{2+}\right]_{\mathrm{i}}$. We conclude that a decrease of ATP in pancreatic B-cells inhibits secretion not only by opening $\mathrm{K}_{\mathrm{ATP}}$ channels but also at a step distal to $\mathrm{Ca}^{2+}$ influx. Consequently, any increase of $\left[\mathrm{Ca}^{2+}\right]_{i}$ which is linked to cessation of ATP supply, fails to elicit an insulin secretion.

\section{8}

EMPTYING OF INTRACELLULAR CA ${ }^{2+}$ POOLS STIMULATES TWO MODALITIES OF $\mathrm{CA}^{2+}$ INFLUX IN PANCREATIC B-CELLS P. Gilon, Y. Miura and J.C. Henquin. Unité d'Endocrinologie et Métabolisme, University of Louvain, Bnsssels, Belgium.

In B-cells, $\mathrm{Ca}^{2+}$ influx occurs mainly through voltage-dependent $\mathrm{Ca}^{2+}$ channels. In non-excitable cells, depletion of intracellular $\mathrm{Ca}^{2+}$ pools triggers a membrane potential-independent $\mathrm{Ca}^{2+}$ influx (capacitative $\mathrm{Ca}^{2+}$ entry). In the present study, we have investigated how emptying of these pools by thapsigargin (TG), an inhibitor of the endoplasmic reticulum $\mathrm{Ca}^{2+}$-ATPase, influenced $\mathrm{Ca}^{2+}$ influx in B-cells. Cytoplasmic $\left.\mathrm{Ca}^{2+}\left(\mathrm{Ca}^{2+}\right]_{\mathrm{i}}\right)$ was monitored in clusters of mouse B-cells loaded with fura-2. To test the effect of TG independently of changes in membrane potential, the latter was clamped with various $\left[\mathrm{K}^{+}\right]$in the presence of diazoxide. In $4.8 \mathrm{mmol} / / \mathrm{K}^{+}$, changing from a $\mathrm{Ca}^{2+}$-free medium to a medium containing $2.5 \mathrm{mmol} / \mathrm{C} \mathrm{Ca}{ }^{2+}$ caused a minor rise in $\left[\mathrm{Ca}^{2+}\right]_{\mathrm{i}}(10-15 \mathrm{nmol} / \mathrm{l})$. This rise was larger after emptying intracellular $\mathrm{Ca}^{2+}$ pools with TG, but it did not exceed $\sim 30-80 \mathrm{nmol} / \mathrm{l}$ and was resistant to blockade of voltage-dependent $\mathrm{Ca}^{2+}$ channels by $\mathrm{D} 600$. This demonstrates the presence of a small capacitative $\mathrm{Ca}^{2+}$ entry. When the membrane potential was clamped at depolarized levels with 10,20 or $45 \mathrm{mmol} / \mathrm{K} \mathrm{K}^{+},\left[\mathrm{Ca}^{2+}\right]_{\mathrm{i}}$ was raised to increasing plateau levels tanging between 100 and $900 \mathrm{nmol} / \mathrm{h}$. TG increased the plateau level of $\left[\mathrm{Ca}^{2+}\right]_{\mathrm{i}}$ marginally $(35 \mathrm{nmol} / 1)$ in $10 \mathrm{mmol} / / \mathrm{K}^{+}$, and was without effect in $20-45 \mathrm{mmol} / / \mathrm{K}^{+}$. In contrast, when the membrane potential was not clamped $\left(4.8 \mathrm{mmol} / 1 \mathrm{~K}^{+}\right), 15 \mathrm{mmol} / \mathrm{l}$ glucose induced $\left[\mathrm{Ca}^{2+}\right]_{\mathrm{i}}$ oscillations which were potentiated by TG. This potentiation was much larger than expected from the capacitative $\mathrm{Ca}^{2+}$ entry and was prevented by D600, which suggests an effect on the membrane potential. In conclusion, emptying of intracellular $\mathrm{Ca}^{2+}$ pools in B-cells induces a small capacitative $\mathrm{Ca}^{2+}$ entry and potentiates glucose-induced $\mathrm{Ca}^{2+}$ influx through voltage-dependent $\mathrm{Ca}^{2+}$ channels. 
439

GLUCOSE-MEDIATED Ca ${ }^{2+}$ SIGNALLING IN THE NOVEL INSULIN SECRETING CELL LINE BRIN-D 11

A.P. Salgado ${ }^{1}$ R.M. Santos', A.R. Tomé ${ }^{1}$, A.P. Fernandes', P.R. Flatt ${ }^{2}$ and L.M. Rosario ${ }^{1}$ Center for Neurosciences and Dept. of Biochemistry University of Coimbra. Coimbra. Portugal; 2 School of Biomedical Sciences, University of Uister, Northern Ireland, UK.

A novel insulin-secreting cell line, BRIN-DI1, was recently established following electrofusion of RINmSF cells with rat pancreatic $\beta$-cells. We have now characterized the effects of metabolizable (glucose and $\alpha$ ketoisocaproic acid, $\mathrm{KIC}$ ) and non-metabolizable (high $\mathrm{K}^{+}$and tolbutamide) insulin secretagogues on the cytosolic free $\mathrm{Ca}^{2+}$ concentration $\left(\left[\mathrm{Ca}^{2+}\right]_{i}\right)$ of monolaver and single BRIN-D1l cells, aiming at assessing the responsiveness of the cells to physiological stimuli and its underlying mechanism. The $\left[\mathrm{Ca}^{2+}\right]_{\text {i }}$ was monitored by conventional spectrofluorometry and ratiometric fura-2 fluorescence microscopy (photomultiplier-based and imaging techniques). An increase in glucose concentration caused a dosedependent $\left[\mathrm{Ca}^{2+}\right]_{\text {increase (up to }} 16.7 \mathrm{mM}$ with a threshold around $3 \mathrm{mM}$ ), which was suppressed by mannoheptulose. Exposure to nifedipine, $\mathrm{Ca}^{2+}$. free medium or diazoxide suppressed the glucose-evoked $\left[\mathrm{Ca}^{2+}\right]$ transients The depolarizing agents tolbutamide and high $\mathrm{K}^{+}$evoked a dose-dependent increase in $\left[\mathrm{Ca}^{-1}\right]_{\text {. }}$. Direct activation of the TCA cycle with KIC or exposure to the aminoacids glycine, arginine and serine proved to be very effective in raising the $\left[\mathrm{Ca}^{2+}\right]_{i}$. The data indicate that: 1) the $\left[\mathrm{Ca}^{2+}\right]_{i}$ rises evoked by the different secretagogues in BRIN-D1I cells are similar to those recorded from pancreatic $\beta$-cells; 2) glucose-generated $\mathrm{Ca}^{2+}$ signals in BRIN-D1l cells are strictly dependent upon regular metabolic function, blockade of ATP-sensitive $\mathrm{K}^{+}$channels and $\mathrm{Ca}^{2 *}$ influx through voltagesensitive $\mathrm{Ca}^{2+}$ channels. By responding to glucose in the normal physiological range BRIN-DII cells represent an effective model for biochenical and biophysical studies of glucose-dependent processes potentially relevant for type II diabetes.

This work was supported by JNICT.

\section{0}

DEPOLARIZATION ELEVATES INOSITOL 1,4,5-TRISPHOSPHATE AND MOBILIZES $\mathrm{Ca}^{2+}$ IN MOUSE B-CEŁLS SENSITIZED BY CAMP

Y.J. Liu., E. Grapengiesser, B. Heliman and E. Gylfe Department of Medical Cell Biology, Uppsala University, BMC Box 571, S-751 23 Uppsala, Sweden

Depolarization-dependent mobilization of intracellular $\mathrm{Ca}^{2+}$ was studied by measuring inositol 1,4,5-trisphosphate $\left(\mathrm{IP}_{3}\right)$ and cytoplasmic $\mathrm{Sr}^{2+}$ and $\mathrm{Ca}^{2+}$ in individual mouse $\mathrm{B}$-cells exposed to the respective cation. Glucagon and other agents raising CAMP induced repetitive and pronounced $\mathrm{Sr}^{2+}$ and $\mathrm{Ca}^{2+}$ transients superimposed upon the large amplitude oscillations found in glucose-stimulated B-cells. Both the large amplitude oscillations and the superimposed transients disappeared after hyperpolarization with diazoxide, while $\mathrm{IP}_{3}$-mediated mobilization of the cations by carbachol persisted. The carbachol effect was sensitive to elevation of CAMP, which considerably amplified the response. The $\mathrm{Ca}^{2+-A T P a s e}$ inhibitor thapsigargin prevented the appearance of the transients evoked by carbachol and CAMP but not the glucose-induced large amplitude oscillations. The $\mathrm{Ca}^{2+}$ channel blocker methoxyverapamil exhibited opposite specificity in inhibiting the large amplitude oscillations under conditions when the transients often remained. In glucose-stimulated B-cells exposed to both diazoxide and methoxyverapamil transients from a basal level could be restored by $\mathrm{K}^{+}$depolarization. Depolarization of B-cell-rich pancreatic slets under such conditions resulted in a $45.2 \pm 9.1 \%(p<0.005)$ increase in the $\mathbb{I P}_{3}$ content. The results indicate that $B$-cells respond to depolarization with a small elevation of $\mathbb{I P}_{3}$. In the presence of agents raising CAMP, which apparently sensitize the $\mathbb{P}_{3}$ receptors, depolarization causes intracellular $\mathrm{Ca}^{2+}$ mobilization. This mechanism may be important for the electrophysiological burst activity in B-cells exposed to endogenous glucagon in intact islets.

\section{1}

EVIDENCE FOR A ROLE OF INTRAMITOCHONDRIAL $\mathrm{Ca}^{2+}$ IN NUTRIENT-STIMULATED INSULIN SECRETION

P. Maechler, E.D. Kennedy, and C.B. Wollheim. Division de Biochimie Clinique, Centre Médical Universitaire, Geneva, Switzerland. An increase in cytosolic $\mathrm{Ca}^{2+}$ concentration is a key event during nutrient-induced insulin secretion. We investigated the role of mitochondrial metabolism in insulin secretion by measuring increases in the mitochondrial $\mathrm{Ca}^{2+}$ concentration $\left(\left[\mathrm{Ca}^{2+}\right] \mathrm{m}\right)$, which are known to activate Kreb's cycle dehydrogenases. The mitochondrial substrate succinate (Suc) was tested in clones of INS-1 cells stably expressing the $\mathrm{Ca}^{2+}$-sensitive photoprotein aequorin targeted to the mitochondria. Intact or staphylococcus $\alpha$-toxin permeabilized cells were studied. In intact cells, $5 \mathrm{mmol} / \mathrm{L}$ of the cell permeant methyl-Suc caused an increase in $\left[\mathrm{Ca}^{2+}\right] \mathrm{m}$ and a concomitant induction of insulin secretion ( 10 fold above baseline) when measured in the same cells. Repeated stimulations of methyl-Suc at $5 \mathrm{~min}$ intervals resulted in a marked desensitization. The response was restored within $30 \mathrm{~min}$. This desensitization phenomenon was also observed in rat islet cells transiently transfected with the same aequorin plasmid. In permeabilized INS-l cells, in which extramitochondrial $\left[\mathrm{Ca}^{2+}\right]$ was clamped to $2 \mu \mathrm{mol} / \mathrm{L}$, the marked $\left[\mathrm{Ca}^{2+}\right] \mathrm{m}$ rise generated by $1 \mathrm{mmol} / \mathrm{L}$ Suc was greatly attenuated when repeated stimulations were applied. This latter phenomenon points to the role of mitochondria in desensitization. As in intact cells, resensitization occurred after 30min. Glucose $(10 \mathrm{mmol} / \mathrm{L})$ caused similar alterations in $\left[\mathrm{Ca}^{2+}\right] \mathrm{m}$ and insulin secretion as methyl-Suc in intact $\mathbb{I N S}-1$ cells. In conclusion, it is suggested that the $\left[\mathrm{Ca}^{2+}\right] \mathrm{m}$ rise evoked by mitochondrial substrates plays a key role in glucose-stimulated insulin secretion.

\section{2}

STIMULATION OF CALCIUM UPTAKE AND INSULIN SECRETION BY NON PENETRANT PHOSPHATASE INHIBITOR MICROCYSTIN-LR

Thomas Leiers and Hermann P.T. Ammon

Department of Pharmacology, Institute of Pharmaceutical Sciences, University of Tübingen, Germany

Okadaic acid and calyculin A, cell-penetrating inhibitors of protein phosphatases PP-1 and $\mathrm{PP}-2 \mathrm{~A}$, have been shown to produce transient rise in cytosolic calcium $\left(\left[\mathrm{Ca}^{2+}\right.\right.$, ) but to inhibit depolarization-induced $(\mathrm{KCl} 30 \mathrm{mM})$ elevation of $\left[\mathrm{Ca}^{2+}\right]_{i}$ and insulin release. As far as distribution of phosphatases is concerned, PP-1 is found in the membrane, whereas PP-2A and PP-1 are present in the cytosol.

Microcystin- $L R$ is a non-cellmembrane-penetrating inhibitor of PP-1 and PP-2A.

In RINm5F cells the effect of Microcystin-LR in concentrations between $100 \mathrm{nM}$ and $2 \mu \mathrm{M}$ on $\left[\mathrm{Ca}^{2+}\right]_{i}$ and insulin release was studied. Cytosolic calcium concentrations were estimated using the fura- 2 method.

In control experiments $\mathrm{KCl}(30 \mathrm{mM})$ produced the known rise in $\left[\mathrm{Ca}^{2+}\right]_{\mathrm{i}}$. MicrocystinLR in a concentration range between $750 \mathrm{nM}$ and $2 \mu \mathrm{M}$ for 10 minutes also increased cytosolic calcium in a concentration-dependent manner. Inhibitory effects on $\mathrm{KCl}-$ mediated elevation of $\left[\mathrm{Ca}^{2+}\right]_{i}$ could not be observed.

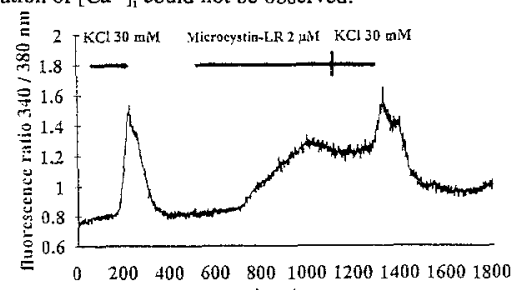

Application of Microcystin-LR $(2 \mu \mathrm{M})$ for 60 minutes produced significant insulin release $[5.72 \pm 0.81$ vs $15.57 \pm 1.59 \mu \mathrm{U}$ IRI $/ \mu$ g protein (mean $\pm S E M, n=3$ )]

Our data indicate that a non-cell-permeable inhibitor of protein phosphatases, probably by interfering with membrane-PP-1, causes calcium-uptake and insulin secretion. It appears that membrane-permeant and non-membrane-permeant phosphatase inhibitors act in a different way on $\left[\mathrm{Ca}^{2+}\right]_{;}$and insulin secretion. 


\section{3}

INHIBITION OF INSULIN RELEASE BY SYNTHETIC PEPTIDES SHOWS THAT THE H3 REGION AT THE C-TERMTNAL DOMAIN OF SYNTAXIN-1 IS CRUCIAL FOR CALCIUM BUT NOT FOR GTP- $\gamma$-S INDUCED SECRETION.

F. Martín", E. Salinas , J. Vazquez", B. Soria" and J. A. Reigt. "Department of Physiology and Department of Neurochemistry, Institute of Neurosciences, University of Alicante, Aptdo. 374, 03080 Alicante; " $\mathrm{Center}$ of Molecular Biology, School of Sciences, University Autonoma of Madrid, Cantoblanco, 28049 Madrid, Spain.

Recent studies show that syntaxin-1 is implicated in the calcium-dependent secretory pathway in pancreatic endocrine $\beta$-celis. In the present study, we have examined the functional role of a singular region of the syntaxin- 1 molecule by using two synthetic peptides (Syn-1 and Syn-2) corresponding to two not overlapping segments of 23 residues of the $\mathrm{H3}$ region (amino acids 191-265) at the C-terminal domain of the protein. Peptides Syn-1, Syn-2 and two additional peptides, with the same amino acid composition but in random sequence to be used as controls (Syn-1C and Syn-2C, respectively) were synthesized by t-boc chemistries using an authomated solid-phase peptide synthesizer. Peptides were purified by RP-HPLC. Functional experiments were carried out in digitoninpermeabilized cells incubated for $10 \mathrm{~min}$ in the presence of the different synthetic peptides. Basal or stimulated secretion were measured in media containing 5 mmol/ EGTA, $10 \mu \mathrm{mmo} / / \mathrm{Ca}^{2+}$ and 100 ummol/ GTP-y-S. Insulin was determinated by RIA. Peptides Syn-1 and Syn-2 provoked a dose-dependent inhibition of $\mathrm{Ca}^{2+}$-dependent insulin release $\left(\mathrm{IC}_{\mathrm{s}_{0}} \sim 46\right.$ and $32 \mu \mathrm{mmol} / /$ for Syn-1 and Syn-2, respectively). For both peptides, maximum inhibitory effect was achieved at $200 \mu \mathrm{mmol} / \mathrm{Syn}-1=73 \%$ and Syn-2 $=84 \%$ ). Control peptides (Syn$1 \mathrm{C}$ and Syn-2C) had not inhibitory effect at $200 \mu \mathrm{mmol} / \mathrm{l}$. By contrast, 200 $\mu \mathrm{mm}$ / $/ 1$ of Syn-1 or Syn-2 did not prevent $100 \mu \mathrm{mmo} / \mathrm{GTP}-\gamma-\mathrm{S}$-stimulated insulin secretion. These results demonstrate that specific structural determinants of the $\mathrm{H} 3$ region of syntaxin-1 selectively control $\mathrm{Ca}^{2+}$-mediated insulin secretion but are not required for I GTP- - -S-stimulated insulin release.

\section{4}

$\mathrm{Ca}^{2}+$ SIGNALLING IN SOMATOSTATIN-PRODUCING CELLS FROM THE HUMAN PANCREAS

B. Hellman, A. Berts, Y-J. Liu and E. Gylfe. Department of Medical Cell Biology, Uppsala University, Biomedicum, Box 571, S-751 23 Uppsala, Sweden

So far studies of $\mathrm{Ca}^{2+}$ regulating pulsatile release of the human islet hormones have been restricted to the insulin-producing $\beta$-cells. We have now examined $\mathrm{Ca}^{2+}$ signalling in human somatostatin-producing $\delta$-cells obtained from three female cadaveric organ donors $34-45$ years old. The cytoplasmic concentrations of $\mathrm{Ca}^{2+}$ and its analogue $\mathrm{Sr}^{2+}$ were monitored with ratiometric Fura-2 technique in the presence of respective cation in $\delta$-cells subsequently identified by immunostaining for somatostatin. Similar observations were made with $\mathrm{Ca}^{2+}$ and $\mathrm{Sr}^{2+}$. The $\delta$-cells exhibited spontaneous rhythmic activity in terms of slow (frequency $0.1-0.4 / \mathrm{min}$ ) oscillations arising from close to basal levels. These oscillations were transformed into sustained elevation in the presence of $20 \mathrm{nmol} / 1$ glucagon or $1 \mathrm{mmol} / 1$ tolbutamide. During perifusion with $20 \mathrm{mmol} / \mathrm{l}$ glucose the addition of glucagon resulted also in the appearance of short $(<30 \mathrm{~s})$ transients, which disappeared upon exposure to 100 $\mathrm{nmol} / \mathrm{l}$ of the intracellular $\mathrm{Ca}^{2+}-\mathrm{ATP}$ ase inhibitor thapsigargin. Analysing small aggregates of islet cells it was evident that the $\mathrm{Ca}^{2+}$ signals in the $\delta$-cells can be synchronous with those in adjacent $\beta$-cells. It is concluded that secretion of pancreatic somatostatin in man depends on similar $\mathrm{Ca}^{2}+$ signals as those regulating the pulsatile release of insulin.

\section{5}

\section{PHYSIOLOGIC REGULATION OF GLUCAGON SECRETION BY} CALCIUM IN CLONAL PANCREATIC ALPHA CELLS

\author{
A.S.Rajan, Division of Endocrinology, Baylor College of Medicine,
} One Baylor Plaza, Houston, Texas 77030, USA

Diabetes is universally associated with a relative or absolute increase in the circulating levels of ghucagon, which contributes to hyperglycemia and the tendency to ketosis. The intraceliular mechanisms that control the release of glucagon from pancreatic $\alpha$-cells are poorly understood. In part, this is due to the difficuity in isolating normal $\alpha$-cells and the lack of good alternate in vitro models to study $\alpha$-cell secretion. The objective of this study was to investigate the utility of a clonal cell line, $\alpha$ TC-6 (Powers et al, Diabetes 39(4):406, 1990) as a normal $\alpha$-cell surrogate. I characterized the $\alpha$ TC- 6 cells by immunocy tochemistry and radioimmunoassay, monitored glucagon secretion in static incubation assays and measured cytosolic $\left[\mathrm{Ca}^{2+} \mid\right.$ levels using fura-2 spectroscopy. I established that the $\alpha$ TC -6 cell line is a homogenous population of $\alpha$-cells that secrete pancreatic ghucagon and produce no insulin. At physiologic doses, the $\alpha$-cell secretagogues arginine and $\mathrm{K}^{+}$enhanced glucagon secretion from $\alpha \mathrm{TC}-6$ cells. $\mathrm{K}^{+}(5.9-55 \mathrm{mM})$ stimulated a dosedependent increase in glucagon release with a peak elevation of $282 \%$ over basal levels ( $\mathrm{n}=6$, peak at $45 \mathrm{mM} \mathrm{K}^{+}$). Arginine (10) $\mathrm{mM}$ ) stimulated a $20-\mathbf{2 5 \%}$ increase in glucagon secretion over basal levels $(n=3)$. Unlike basal glucagon release, $\mathrm{K}^{+}$or arginine-stimulated sceretion was dependent on external $\mathrm{Ca}^{2+}$ (peak effect at $2-2.5 \mathrm{mM} \mathrm{Ca}{ }^{2+}$ ). $\mathrm{K}^{+}$or arginine-stimulated secretion was paralled by a rapid 3-4 fold increase in cytosolic $\left[\mathrm{Ca}^{2+}\right]$. Both the rise in cytosolic $\left[\mathrm{Ca}^{2+}\right]$ and glucagon secretion were attenuated by the $\mathrm{Ca}^{2+}$ channel blocker nimodipine $(1 \mu \mathrm{M})$. I conclude that $\mathrm{Ca}^{2+}$ is an important intracellular regulator of glucagon secretion. These data demonstrate that $\alpha$ TC- 6 cells are a useful practical tool for studying the physiologic regulation of $\alpha$-cell glucagon secretion and its dysregulation in diabetes.

\section{6}

THE RATE OF $\mathrm{Ca}^{2+}$ INFLUX IS A MAJOR DETERMINANT FOR SLOW $\mathrm{Ca}^{2+}$ OSCILLATIONS IN PANCREATIC $\beta$-CELLS.

E. Grapengiesser, M. Eberhardson and A. Tengholm. Department of Medical Cell Biology, Uppsala, Sweden.

In individual pancreatic $\beta$-cells glucose-induced rise of the cytoplasmic $\mathrm{Ca}^{2+}$ concentration $\left(\left[\mathrm{Ca}^{2+}\right]_{\mathrm{i}}\right)$, is manifested either as oscillations (0.2-0.5 $\left.\mathrm{min}^{-1}\right)$ or as a sustained elevation. The role of the plasma membrane permeability to $\mathrm{Ca}^{2+}$ for the establishment of these slow oscillations was investigated by dual wavelength microfluorometric measurements of $\left[\mathrm{Ca}^{2+}\right]_{i}$ in individual mouse $\beta$-cells loaded with fura-2. Promoting the entry of $\mathrm{Ca}^{2+}$ by raising the extracellular $\mathrm{Ca}^{2+-}$ concentration from 1.3 to $10 \mathrm{mmol} / 1$ or adding the $\mathrm{Ca}^{2+}$ channel agonist BAY K $8644(1 \mu \mathrm{mol} / 1)$ or the $\mathrm{K}+$ channel blocker tetraethylammonium $+(T E A ; 10-20 \mathrm{mmol} / \mathrm{l}$ ) caused steeper rises and higher peaks of the glucose-induced oscillations. However, when extracellular $\mathrm{Ca}^{2}+$ was lowered to $0.5 \mathrm{mmol} / \mathrm{l}$ the oscillations were transformed into a sustained suprabasal level. It was possible to transform a glucose-induced sustained elevation of $\left[\mathrm{Ca}^{2+}\right]_{\mathrm{i}}$ obtained in the presence of $1.3 \mathrm{mmol} / \mathrm{I} \mathrm{Ca}{ }^{2}+$ into slow oscillations by promoting the entry of $\mathrm{Ca}^{2+}$ either by raising the extracellular $\mathrm{Ca}^{2+}$ concentration to $10 \mathrm{mM}$ or adding TEA or BAY $\mathrm{K} 8644$. The results indicate that glucose-induced slow oscillations of $\left[\mathrm{Ca}^{2+}\right]_{i}$ require that $\mathrm{Ca}^{2+}$ influx exceeds a critical rate and that $\left[\mathrm{Ca}^{2+}\right]_{i}$ has a negative feed-back effect on the entry of $\mathrm{Ca}^{2+}$. It is likely that variations in the consumption of ATP related to the extrusion of $\mathrm{Ca}^{2+}$ affect the voltage-dependent entry of $\mathrm{Ca}^{2+}$ required for the slow oscillations of $\left[\mathrm{Ca}^{2+}\right]_{i}$. 


\section{7}

EFFECT OF ANTISENSE INHIBITION OF Na/Ca EXCHANGE ON CYTOSOLIC $\mathrm{Ca}^{2+}$ OSCILLATIONS IN PANCREATIC B CELLS F. Van Eylen ${ }^{1}$, J. Albuquerque ${ }^{2}$ and A. Herchuelz ${ }^{1}$. Laboratory of Pharmacology ${ }^{1}$ and Laboratory of Applied Genetics ${ }^{2}$, Brussels Free University, Belgium.

In the pancreatic $B$ cell, $\mathrm{Na} / \mathrm{Ca}$ exchange $(\mathrm{NCX})$ displays a quite high capacity but has remained difficult to investigate because of the absence of specific inhibitors. In order to better characterize the role of NCX in the B cell, phosphorotioated antisense oligonucleotides (AS-oligos) targeted against the $\mathrm{Na} / \mathrm{Ca}$ exchanger were used to repress the expression of the exchanger in pancreatic islet cells. NCX activity was evaluated by measuring cytosalic free $\mathrm{Ca}^{2+}$ concentration $\left[\mathrm{Ca}^{2+}\right]_{i}$ in single $\mathrm{B}$ cell using fura 2. Exposure of pancreatic $B$ cells to $500 \mathrm{nM}$ of the AS-oligos for 20 to $24 \mathrm{hrs}$ inhibited NCX activity by about $55 \%$ as measured by the inhibition of the increase in $\left[\mathrm{Ca}^{2+}\right]_{1}$ provoked by extracellular $\mathrm{Na}^{+}$removal. In contrast, control oligos (non-sense and mismatched) did not affect NCX activity. In the absence of AS-oligos pretreatment, $82 \%$ of islet cells exposed to $11.1 \mathrm{mM}$ glucose displayed increases in $\left[\mathrm{Ca}^{2+}\right]_{\mathrm{i}}$, the increases being either oscillatory ( $55 \%$ ) or sustained $(45 \%)$. After AS-oligos pretreatment, only $31 \%$ of islet cells responded to glucose and $33 \%$ of the responding cells displayed $\left[\mathrm{Ca}^{2+}\right]_{i}$ oscillations that, in addition, were altered quantitatively and qualitatively. The present study shows that AS-oligos may specifically inhibit $\mathrm{Na} / \mathrm{Ca}$ exchange in pancreatic $B$ cells. Inhibition of $\mathrm{Na} / \mathrm{Ca}$ exchange expression markedly alters glucose-induced $\left[\mathrm{Ca}^{2+}\right]_{i}$ increases and oscillations.

\section{8}

VOLUME OSCILLATIONS IN PANCREATIC $\beta$-CELLS.

A. Tengholm, E. Gylfe and B. Heilman. Department of Medical Cell Biology, University of Uppsala, Sweden.

In pancreatic $\beta$-cells glucose induces oscillations of cytoplasmic $\mathrm{Ca}^{2+}\left(\left[\mathrm{Ca}^{2+}\right]_{i}\right)$ resulting in pulsatile insulin secretion. Using either conventional or confocal fluorescence microscopy of single pancreatic $\beta$-cells we have investigated whether these oscillations are related to variations in cell volume. Dual wavelength recordings with fura- 2 or $\mathrm{BCECF}$ were applied to measure $\left[\mathrm{Ca}^{2+}\right]_{\mathrm{i}}$ or cytoplasmic $\mathrm{pH}$ during hypotonic challenge or glucose stimulation. Changes in cell volume were simultaneously monitored by measuring the concentrations of the indicators in a limited part of the cell by excitation at an ion-insensitive wavelength. Reduction of medium osmolarity from 300 to $200 \mathrm{mosm} / \mathrm{l}$ resulted in $19 \pm 1 \%$ increase of the cell volume within 1-3 min, followed by a decrease within 10 min to a steady-state value of $13 \pm 3 \%$ above the control value. The swelling was associated with intracellular acidification by $0.20 \pm 0.07$ $\mathrm{pH}$ units and with an increase of $\left[\mathrm{Ca}^{2+}\right]_{\mathrm{i}}$ by $126 \pm 8 \mathrm{nmol} / \mathrm{l}$ which required the presence of $\mathrm{Ca}^{2+}$ in the extracellular medium. Using confocal microscopy and the $\mathrm{Ca}^{2}+$ indicator fura red, cell volume changes were reflected as variations in the cross sectional area of the cells. This sensitive approach made it sometimes possible to unmask small oscillations in volume during the hypotonic challenge. Similar volume oscillations paralleling those of $\left[\mathrm{Ca}^{2+}\right]_{\mathrm{i}}$ were occasionally seen in the absence of hypotonic stress when the $\beta$-cells were stimulated with $11 \mathrm{mmol} / \mathrm{l}$ glucose. It is concluded that volume regulation during hypotonic stress of the $\beta$-cells is associated with changes of cytoplasmic $\mathrm{Ca} 2+$ and $\mathrm{pH}$ and that volume oscillations may be present both under hypotonic conditions and during glucose stimulation.
449

REAL-TIME SIMULTANEOUS MONITORING OF INSULIN (5-HT) SECRETION AND CYTOSOLIC $\mathrm{Ca}^{2+}$ FROM SINGLE ISLETS OF LANGERHANS

R. M. Barbosa ${ }^{1,2}$, A. M. Silva', I. A. Stamford ${ }^{3}$, R. M. Santos ${ }^{\prime}$ and L. M. Rosário'. 'Center for Neurosciences and Dept. of Biochemistry; ${ }^{2} \mathrm{Lab}$. of Instrumental Analysis; Faculty of Pharmacy, University of Coimbra, Coimbra, Portugal; ${ }^{-3}$ London Hospital Medical College, UK.

It has been previously demonstrated that, under especially favourable experimental conditions (supraphysiological glucose and above-normal extracellular calcium levels). insulin is released from individual islets in a pulsatile fashion and that this process is coincident with bursting electrical activity and oscillations in the cytosolic free $\mathrm{Ca}^{2+}$ concentration $\left(\left[\mathrm{Ca}^{2+}\right]\right.$ ) . In those experiments, insulin was collected by periodically sampling the perfusate and later assayed using radioimmunoassay techniques, thus drastically limiting the time resolution of the analysis. We have now used microamperometric approach (tissue-implanted carbon-fibre microelectrodes) to monitor on-line the release of an electroactive insulin tracer, 5-hydroxytryptamine (5-HT), from beta-cell territories localized to intact mouse pancreatic islets. Intra-islet insulin (5-HT) release was found to be pulsatile in the presence of regular extracellular $\mathrm{Ca}^{2+}$ levels and 11-20 $\mathrm{mM}$ glucose. Underlying these periodical fluctuations were synchronous oscillations in $\left[\mathrm{Ca}^{2+}\right]_{i,}$ as revealed by whole islet fura-2 microfluorometry. Raising glucose concentration in the stimulatory range increased the duration (but not the amplitude) of the $\left[\mathrm{Ca}^{2+}\right]_{i}$ oscillations and both the duration and the amplitude of the secretory signals (hence the secretion per oscillation, as assessed by the integrated amperometric current per oscillation). Glucose-evoked release of an important labile pool of the hormone is seemingly under moment-to-moment control by cytosolic free calcium, with calcium burst duration encoding for the graded secretory response to the hexose. Oscillatory insulin output is an essential feature of the native beta-cell network and has a strong potential to underlie the regulation of glucose homeostasis in healthy subjects.

This work was supported by JNICT.

\section{0}

INSULIN SECRETION AND CYTOPLASMIC Ca ${ }^{2+}$ ACTIVITY IN MOUSE PANCREATIC B-CELLS SHOW ANION SELECTIVITY

G. Larsson-Nyrén and J. Schlin. Department of Histology and Cell Biology, University of Umeå, Sweden

Previous data have shown that perchlorate $\left(\mathrm{ClO}_{4}^{-}\right)$potentiates stimulated insulin secretion, probably by affecting the gating of voltage-activated L-type $\mathrm{Ca}^{2+}$ channels. The aim of this study was to analyse the anion selectivity of pancreatic B-cells by studying the effects of chaotropic anions $(12 \mathrm{mM})$ close to $\mathrm{ClO}_{4}{ }^{-}$in the Hofmeister series $\left(\mathrm{SCN}^{-}, \mathrm{NO}_{3}{ }^{-}, \mathrm{I}^{-}, \mathrm{Br}^{-}\right.$as well as $\left.\mathrm{Cl}^{-}\right)$on the dynamics of insulin sectetion in isolated islets and $\left[\mathrm{Ca}^{2+}\right]_{\mathrm{i}}$ in single $\mathrm{B}$-cells from non-inbred $a b / o b$ mice. All anions, except $\mathrm{Cl}$, significantly amplified glucose-stimulated $\left(20 \mathrm{mM}\right.$ ) insulin secretion in an anion-selective manner (anion sequence: $\mathrm{SCN}^{-}$ $>\mathrm{NO}_{3}>{ }^{-}>\mathrm{Br}^{-}>\mathrm{Cl}^{-}$) during both the first and second phases of secretion. The chaotropic anions also markcdly reduced the lag-time for secretion in a selective way $\left(\mathrm{SCN}^{-}>\mathrm{I}>\mathrm{NO}_{3}>\mathrm{Br}>\mathrm{Cl}^{-}\right)$. The glucose-stimulated level of cytoplasmic $\mathrm{Ca}^{2+}$ activity (Fura 2) was amplified $\left(\mathrm{SCN}>\mathrm{I}>\mathrm{NO}_{3}>\mathrm{Br}^{-} \approx \mathrm{Cl}^{-}\right)$and the lag-time for glucose-induced elevation of cytoplasmic $\mathrm{Ca}^{2+}$ activity was reduced by the anions ( $\mathrm{SCN}>\mathrm{I}^{-}>\mathrm{Br}>\mathrm{NO}_{3}>\mathrm{Cl}-\mathrm{Cl}^{-}$). The results show that the pancreatic $\mathrm{B}$-cells are anion selective with the selectivity order found in 'thyroidlike systems'. The strikingly simnilar anion selectivity of amplified secretion and calcium level on the one hand and shortened lag-phases of secretion and calcium level on the other hand suggest that both types of anion effects may be caused by action at a single site. That the anion selectivity of both glucose-stimulated insulin secretion and intracellular calcium activity were very similar supports of the idea that this site is associated with the B-cell L-type calcium channel. 
451

\section{INSULIN PULSE AMPLITUDE CORRELATES WITH IRREGULARITY OF INSULIN SECRETION}

M. Żarković, J. Ćirić, M. Stojanović, Z. Penezić, B. Trbojević, M. Drezgić and M. Nešović, Institute of Endocrinology, Dr Subotića 13, 11000 Belgrade, Yugoslavia

In non insulin dependent diabetes mellitus (NIDDM) pulsatile insulin secretion is irregular. Therefore, we wanted to quantitate insulin secretion regularity in NIDDM patients, in obese patients with low insulin concentrations $(<20 \mathrm{mU} / \mathrm{l}$, OLI group) and in obese patients with high insulin concentrations ( $>20 \mathrm{mU} / \mathrm{l}$, OHI group). Study group consisted of 36 patients (12 OLI, 13 OHI, 11 NIDDM). After 12 hours fasting, insulin was sampled for 90 minutes with 2 minutes intersample interval. Insulin secretion regularity was expressed as approximate entropy (ApEn). ApEn was calculated according to algorithm by Pincus et al. Higher ApEn values signify less regular pulses. Pulsatility analysis was done using PulsDetekt program. Statistical analysis was done by Kruskal-Wallis test and Spearman correlation. Data are expressed as median, minimum and maximum. There was no difference between groups in calculated insulin half life (OLI 7.67, 3.20-11.33; OHI 10.01, 4.13-17.88; NIDDM 8.33, 3.28-13.21 min) and number of insulin pulses per 90 minutes (OLI 11, 6-16; OHI 11, 5-14; NIDDM 12, 5-14). Pulse amplitude (OLI 3.05, 1.96-11.52; OHI 10.25, 5.1022.05; NIDDM 14.37, 4.61-21.99 mU/L) and ApEn (OLI 0.82, 0.35-1.38; OHI 1.23, 0.55-1.50; NIDDM 1.25, 0.70-1.48) were significantly lover in OLI group. ApEn was correlated with pulse amplitude (all patients $\mathrm{R}=0.67$, $\mathrm{p}<0.01$; non-diabetic (OLI+OHI) $\mathrm{R}=0.59, \mathrm{p}<0.01$, NIDDM $\mathrm{R}=0.73$, $p=0.011$ ). It seems that irregularity of insulin secretion is characteristics of insulin resistance, and that it is not unique to NIDDM.
452

PREDIABETES IS CHARACTERIZED BY LOWER FREQUENCY IN INSULIN OSCILLATIONS

H. Carlqvist, J. Pigon, A. Clark, K. Sundquist, O. Larsson, S. Zeitsev, CG. Östenson and S. Efendic, Department of Molecular Medicine, Karolinska Institute, Sweden.

In healthy man, insulin secretion is characterized by oscillations with a period of 10-16 min, whereas this regular oscillatory pattern is lacking in patients with non-insulin dependent diabetes mellitus (NDDM). In the present study, oscillations in basal insulin secretion were investigated in healthy subjects with a low insulin response (LIR) to glucose (8 subjects aged $30-65$ years, BMI $24 \pm 1.8 \mathrm{~kg} / \mathrm{m}^{2}$ and fasting glucose $4.5 \pm 0.4 \mathrm{mM}$ ) which we consider to be prediabetic. As controls were included subjects with a high insulin response (HIR) to glucose (7 subjects aged 28-46 years, BMI $22 \pm 3.8 \mathrm{~kg} / \mathrm{m}^{2}$ and fasting glucose $4.5 \pm 0.2 \mathrm{mM}$ ). Subjects with impaired glucose tolerance (IGT) (6 subjects aged $39-51$ years, BMI $27 \pm 2.1 \mathrm{~kg} / \mathrm{m}^{2}$ and fasting glucose $5.1 \pm 0.6 \mathrm{mM}$ ) and patients with NIDDM (7 patients aged $39-59$ years, BMI $27 \pm 3.0 \mathrm{~kg} / \mathrm{m}^{2}$ and fasting glucose $6.3 \pm 1.0 \mathrm{mM}$ ) were also studied. Plasma insulin was analyzed in quadruplicate in samples drawn every second minute for 2 hours. In HIR autocorrelation analysis revealed a clear oscillatory pattern with a periodicity of $10-18 \mathrm{~min}(\mathrm{p}<0.001)$. Also following Fourier transformation a 10-18 min peak in the spectral density function was obtained. In LIR slower oscillations were present, with a periodicity of $20-22 \mathrm{~min}$ (autocorrelation $\mathrm{p}<0.001$ ). IGT subjects as well as NIDDM patients lacked periodic insulin oscillations. In conclusion, insulin secretion in subjects with normal glucose tolerance demonstrates an oscillatory pattern, while this periodicity is absent in subjects with decreased glucose tolerance. In prediabetes, insulin release is decreased and characterized by a prolonged periodicity in oscillations.

\section{3}

RESTORATION OF PULSATILE INSULIN SECRETION

P.C. Butler, T.W. Laedtke, O. Schmitz, N. Pørksen, and J. Veldhuis, Edinburgh, Scotland, Rochester, Minnesota, Aarhus, Denmark, Charlottesville, Virginia.

Pulsatile insulin secretion has been reported to be irregular and diminished in NIDDM. This may be due to depletion of the immediately secretable insulin pool or abnormalities in the pacemaker and/or inter-islet communication system. Repletion of immediately secretable insulin stores (by overnight inhibition of insulin secretion but not synthesis with somatostatin and hyperglycemia) would transiently correct the former but not the latter defects. To address this we studied 12 patients with NIDDM twice by one minute (intense) plasma sampling for 80 minutes (7:00 am$8: 20 \mathrm{am})$ following an overnight $(8: 00 \mathrm{pm}-7: 00 \mathrm{am})$ hyperglycemic clamp $(\sim \mathrm{mmol} / \mathrm{l})$ and either saline (SAL) or somatostatin (SMS) $60 \mathrm{ng} / \mathrm{kg} / \mathrm{min}$ infusion. Saline or somatostatin were discontinued at 7:00 am prior to intense sampling, but the hyperglycemic clamp was maintained $(9.2 \pm 0.3$ vs $9.3 \pm 0.4 \mathrm{mmol} / \mathrm{l}$ ) which required a greater glucose infusion rate after SMS $(p<0.01)$ consistent with increased insulin stores and secretion. Plasma insulin concentrations were assayed by a 2 site immunochemical assay and deconvolved to quantify pulsatile insulin secretion. Pulsatile insulin secretion was $\sim 800 \%$ greater after SMS versus SAL $(\mathrm{p}<0.01)$. The latter was achieved by a $\sim 800 \%$ increase in pulse mass ( $30 \pm 7$ vs $288 \pm 40$ $\mathrm{pmol} / \mathrm{L} / \mathrm{min}, \mathrm{p}<0.05)$ and a $\sim 30 \%$ increase in pulse frequency $(7.2 \pm 0.6 \mathrm{vs}$ $9.2 \pm 0.8 \mathrm{pulses} / \mathrm{hr}, \quad \mathrm{p}<0.05$ ) after SMS. We conclude, (1) during hyperglycemia using a sensitive insulin assay regular high frequency insulin pulses of diminished mass are detected in NIDDM, and (2) after discontinuation of overnight SMS, there is a marked increase in pulsatile insulin secretion primarily due to enhanced pulse mass. These data imply that a depletion of immediately secretable insulin stores rather than defects in the pacemaker or inter-islet communication system is the mechanism causing impaired pulsatile insulin secretion in NIDDM. 


\section{Islet Responses to Drugs, Neurotransmitters and Peptides}

\section{4}

Modulation of Tolbutamide Sensitivity of K-ATP Currents in Mouse Pancreatic $\beta$-Cells by Intracellular Nucleotides and Magnesium M. Faehling*, P. A. Smith and F. M. Ashcroft

University Laboratory of Physiology, Parks Rd., Oxford, OX1 3PT, UK *present address: Dept. of Internal Medicine II, University Ulm, R.-Koch-Str. 8, 89081 Ulm, Germany

Sulphonylureas, which are widely used in the treatment of NIDDM, inhibit ATP-sensitive $\mathrm{K}$-channels (K-ATP channels). The exact mechanism of action remains unclear. We have used the standard whole-cell configuration of the patch-clamp technique to investigate the effect of magnesium and intracellular adenine nucleotides on the inbibition of whole-cell K-ATP-currents in single mouse pancreatic $\beta$-cells produced by the sulphonylurea tolbutamide. In dose-response curves, the tolbutamide concentration producing half-maximal inhibition of the K-ATP current $\left(\mathrm{K}_{\mathrm{d}}\right)$ was $4.2 \pm 1.1 \mu \mathrm{M}(\mathrm{n}=4)$ with $2 \mathrm{mM}$ intracellular $\mathrm{Mg}^{2+}$ but increased to $258 \pm 114 \mu \mathrm{M}(\mathrm{n}=7)$ when intracellular magnesium was reduced to $<30 \mathrm{nM}$. Addition of $0.3 \mathrm{mM}$ ATP, ADP, AMP-PNP or $A T P \gamma S$ partially restored the efficacy of tolbutamide in $\mathrm{Mg}^{2+}$-free solution $\left(\mathrm{K}_{\mathrm{d}}=17.7 \pm 2.3 ; 9.3 \pm 2.3 ; 30.9 \pm 16.2\right.$ and $7.3 \pm 5.7 \mu \mathrm{M}$, respectively $\left(\mathrm{n}=3-4\right.$ in each case)) but was without effect in the presence of $\mathrm{Mg}^{2+}$. The nucleotide effect therefore does not involve hydrolysis; it was, however, closely related to the potency of the nucleotide to inhibit K-ATPchannel activity. These results suggest that $\mathrm{Mg}^{2+}$ and adenine nucleotides are involved in coupling tolbutamide binding to inhibition of K-ATPchannel activity.

\section{6}

SYNTHESIS AND CHARACTERISATION OF KUTAR A NOVEL ANTAGONIST OF THE PANCREATIC ISLET IMIDAZOLINE BINDING SITE

N.G. Morgan, A.L. Pallett, M. Mourtada, S.L.F. Chan, J. Clews* and C.A. Ramsden* Departments of Biological Sciences and Chemistry*, Keele University, Staffs ST5 5BG, UK.

A number of imidazoline compounds have been found to stimulate insulin secretion by inducing closure of ATP-sensitive potassium (K-ATP) channels in pancreatic B-cells. This results from binding to an imidazoline receptor site associated with K-ATP channels but the receptor has eluded full characterisation. We have undertaken a systematic structure-activity analysis of imidazoline insulin secretagogues and have synthesised a novel antagonist, KU14R, based on the structure of the agonist efaroxan (Efx). KU14R did not, itself, stimulate insulin secretion from isolated rat islets nor did it modify glucose-induced secretion $20 \mathrm{mM}$ glucose: $2.1 \pm 0.36 \mathrm{ng} / \mathrm{islet} / \mathrm{h}_{i} 20 \mathrm{mM}+$ $100 \mu \mathrm{M}$ KU14R: $2.3+0.291$. However, the compound completely prevented the stimulation of insulin secration in response to Efx (control: $0.92 \pm 0.19$ ng/islet/h; $100 \mu \mathrm{M} \mathrm{Efx:} 1.65 \pm 0.21 ; \mathrm{Efx}+100 \mu \mathrm{M} \mathrm{KU}: 0.78 \pm 0.18$; $p<0.0011$. In the presence of KU14R, the ability of Efx to prevent the inhibition of glucose-induced insulin secretion mediated by diazoxide was also inhibited $120 \mathrm{mM}$ glucose: $2.82 \pm 0.33 \mathrm{ng} / \mathrm{islet} / \mathrm{h} ; 20 \mathrm{mM}+200 \mu \mathrm{M}$ Dz: 1.33 $\pm 0.13 ; 20 \mathrm{mM}+\mathrm{Dz}+\mathrm{KU}: 1.28 \pm 0.23 ; 20 \mathrm{mM}+\mathrm{Dz}+\mathrm{Efx}: 2.88 \pm 0.32$ $20 \mathrm{mM}+D z+E f x+K U: 1.55 \pm 0.25 ; p<0.0011$. This effect was dosedependent over the range $1 \mu \mathrm{M}-100 \mu \mathrm{M} \mathrm{KU14R}$ (EC 50 approximately $40 \mu \mathrm{M}$ ) and KU14R also inhibited the secretory response to other imidazolines including phentolamine, RX821002 and midaglizole. Despite the change in its functional activity at the imidazoline receptor compared to efaroxan, KU14R retained the a2-antagonist properties of the parent compound and reversed the inhibition of insulin secretion mediated by noradrenaline. In conclusion, we have synthesised a novel antagonist of the islet imidazoline receptor which should be useful in the further characterisation of this binding site.

\section{5}

MECHANISM OF INSULINOTROPIC ACTION OF Ca $\mathrm{Ca}^{2+}$ SENSITIZER PIMOBENDAN--A NEW CARDIAC INOTROPE

S. Kato, H. Ishida, Y. Okamoto, Z. Huang, S. Fujimoto and Y. Seino. Dept. of Metabolism \& Clinical Nutrition, Kyoto Univ. School of Medicine, Kyoto, JAPAN

A newly developed cardiac inotropic agent pimobendan, (Boeringer Ingerheim, Germany) has recently been clinically used for patients with heart failure in Japan. It has been demonstrated that this agent augments the $\mathrm{Ca}^{2+}$ sensitivity of troponin $\mathrm{C}$ and also inhibits phosphodiesterase III activity in the cardiomyocytes. Since intracellular $\mathrm{Ca}^{2+}$ is known to be the key signal for the exocytotic process of insulin secretory granule including actin-myosin system, we tested the effect of pimobendan on the pancreatic $\beta$ cell function. Insulin secretory capacity during $30 \mathrm{~min}$ batch incubation and islet content of CAMP after the incubation were determined. In addition, intracellular $\mathrm{Ca}^{2+}$ concentration was measured by video image analysis using fura- 2 , and the $\mathrm{Ca}^{2+}$ sensitivity of exocytotic apparatus was assessed using the electrically permeabilized islets. Insulin released by $6.6 \mathrm{mM}$ glucose alone $(1.17 \pm 0.15 \mathrm{ng} / \mathrm{islet} / 30 \mathrm{~min})$ was significantly augmented by $25 \mu \mathrm{M}$ pimobendan and $100 \mu \mathrm{M} \mathrm{IBMX}$ to the values of $3.94 \pm 0.40$ and $8.70 \pm 1.08$, respectively $(P<0.01)$. Co-application of pimobendan and IBMX evoked still larger insulin release (12.50 \pm 0.89$)$. However, pimobendan influenced neither on cAMP content nor on intracellular $\mathrm{Ca}^{2+}$ concentration. In permeabilized islets, released insulin was $2.45 \pm 0.30$ at $1 \mu \mathrm{M} \mathrm{Ca}^{2+}$, which was significantly augmented by $25 \mu \mathrm{M}$ pimobendan to the value of $4.15 \pm 0.45(\mathrm{P}<0.05)$. Thus, pimobendan is an effective insulin secretagogue which sensitizes the exocytotic apparatus in the pancreatic $\beta$ cells without affecting c AMP content.

\section{7}

BINDING SITES OF IMIDAZOLINE- AND GUANIDINIUMCOMPOUNDS AND FUNCTIONAL RELEVANCE C. Herrmann P. Ratzka, I. Rustenbeck and A. Hasselblatt. Institute of Pharmacology, University of Göttingen

The enhancement of glucose-induced insulin secretion by imidazoline compounds is related to an inhibitory action of these compounds on $K_{A T P}$ channel activity. To test whether this action is mediated by binding to an imidazoline receptor, we compared the effects of selected imidazoline compounds on $\mathrm{K}_{\mathrm{ATP}}$ channel activity with their binding to HIT T15 cell membranes. Competition binding experiments were performed using $5 \mathrm{nM}\left[{ }^{3} \mathrm{H}\right]$ clonidine and masking $\alpha$ receptors with noradrenaline. Clonidine, which binds preferentially to $I_{1}$ receptors was chosen because it binds also to $I_{2}$ sites and in contrast to the $I_{2}$ ligand, idazoxane, stimulates insulin secretion when $\alpha$-receptors are blocked. Clonidine displacement revealed two different binding sites: The $\mathrm{K}_{\mathfrak{i}}$ values of $2.6 \mathrm{nM}$ for guanabenz, 20.0 $\mathrm{nM}$ for idazoxane, $37.6 \mathrm{nM}$ for clonidine and $105 \mathrm{nM}$ for rilmenidine indicate that the high-affinity site is an $\mathrm{I}_{2}$ receptor, while the low affinity site was characterized by $K_{\mathrm{i}}$ values of $0.6,4.8$, and $39.6 \mu \mathrm{M}$ for guanabenz, clonidine and idazoxane, respectively. This rank order parallels the efficiency of these compounds to inhibit $K_{\text {ATP }}$ channels in inside-out patches from B-cells. Amiloride which in contrast to the above compounds displaced clonidine only incompletely from HIT cell-membranes, did not inhibit $\mathrm{K}_{\mathrm{ATP}}$ channels in the inside-out mode. No displacement of clonidine was seen with tolbutamide and diazoxide. Conclusions: 1) The high-affinity imidazoline/guanidinium binding site in insulin secreting cells can be classified as an $\mathrm{I}_{2}$ site which was recently identified as monoaminooxidase. 2) Binding to the low affinity site which is related to the inhibitory action on $K_{A T P}$ channels, but the site is probably neither identical with the $\mathrm{K}^{+}$channel nor the sulfonylurea receptor. 
458

EFFECTS OF $\alpha_{2}$-AGONISM, IMIDAZOLINES AND GiPROTEIN ON INSULIN SECRETION IN BETA CELLS

H. Hirose, H. Maruyama, Y. Seto, K. Kido and T. Saruta. Keio University School of Medicine, Tokyo 160, Japan

It is well known that alpha-2 adrenergic receptor agonism inhibits insulin secretion and stimulates glucagon secretion both in animal and human studies. Recently, alpha-2 inhibitors DG-5128, SL 84.0418 and MK-912 have been studied in type 2 diabetic patients. To clarify the action mechanisms of these agents, we investigated the effects of alpha-2 agonists and antagonists $\left(10^{-10}\right.$ to $\left.10^{-4} \mathrm{M}\right)$ and pretreatment by pertussis toxin (PTX) on glucose-stimulated insulin secretion in hamster insulinoma cell lines, HIT-T15. Alpha-2 agonists clonidine and oxymetazoline as low as $10^{-8}$ $M$ significantly inhibited glucose-stimulated insulin secretion (53 and $59 \%$ of control). These inhibitory effects were completely abolished by 18 -hour pre-incubation of HIT-T15 cells with $100 \mathrm{ng} / \mathrm{ml}$ PTX. Imidazoline derivative alpha-2 antagonist DG-5128 at $10^{-4} \mathrm{M}$ doubled insulin secretion $(\mathrm{P}<0.01)$. Clonidine and oxymetazoline are also imidazoline derivatives and they stimulated insulin secretion at $10^{-4} \mathrm{M}$ in PTX-treated group ( $P<0.05$ for both). These results indicate that glucose-stimulated insulin secretion is inhibited by alpha2 agonists through PTX-sensitive GTP-binding protein in HITT15 cells. It is also suggested that imidazoline derivatives at high concentration directly stimulates insulin secretion
459

ACTION OF IMIDAZOLE ANTIMYCOTICS ON ION CHANNEL CURRENTS IN MOUSE PANCREATIC B-CELLS

S. Welker and G. Drews, Pharmazeutisches Institut, Abt. Pharmakologie, Auf der Morgenstelle 8,72076 Tübingen, Germany

Recent studies with non-excitable cells reported that imidazole antimycotics influence the activity of ion channels which are regulated by the filling state of intracellular $\mathrm{Ca}^{2+}$ stores. This capacitive $\mathrm{Ca}^{2+}$ entry has been shown to be influenced by inhibitors of the P450-system, antimycotics with imidazole structure. There is evidence for the existence of a capacitive inward current in Bcells, too. However, the physiological significance of this current is still unclear. To test whether imidazole antimycotics are useful as a tool to separate this current from others, we first studied whether these agents affect the "classical" ion currents in mouse B-cells using the patch-clamp technique. Whole-cell $\mathrm{Ca}^{2+}$ and $\mathrm{K}^{+} \mathrm{ATP}$ currents were elicited by 70 and $10 \mathrm{mV}$ voltage-steps from the holding potential of $-70 \mathrm{mV}$, respectively. $1 \mu \mathrm{M}$ and $10 \mu \mathrm{M}$ clotrimazole decreased L-type $\mathrm{Ca}^{2+}$ channel current from - $105 \pm 6 \mathrm{pA}$ to $-77 \pm 6 \mathrm{pA}(\mathrm{n}=4$, $p<0.02)$ and $-12 \pm 3 p A(n=4, p<0.001)$, respectively. Clotrimazole also affected the $\mathrm{K}^{+}$ATP current. $0.1 \mathrm{\mu M}$ Clotrimazole reduced the current amplitude from $135 \pm 18 \mathrm{pA}$ to $85 \pm 14 \mathrm{pA}(\mathrm{n}=7, \mathrm{p}<0.01)$ and $1 \mu \mathrm{M}$ further reduced it to $22 \pm 7 \mathrm{pA}$ $(n=4, p<0.02)$. Accordingly, clotrimazole depolarized $B$-cells from $-82 \pm 0.4 \mathrm{mV}$ to $-45 \pm 3 \mathrm{mV}(n=8, p<0.001)$ and induced irregular spike activity measured in the current-clamp mode at $0.5 \mathrm{mmol} / \mathrm{l}$ glucose. Ketoconazole and econazole also inhibited K+ ATF whole-cell current, but higher concentrations were needed. Clotrimazole at a concentration of $10 \mu \mathrm{M}$ almost completely blocked single channel $\mathrm{K}^{+}$ATP current in cell-free membrane patches suggesting a direct effect on the channels. Clotrimazole $(10 \mu \mathrm{M})$ also reduces an inward current which is elicited by hypotonic conditions from $-108 \pm 11$ pA to $-17 \pm 3 p A$ ( $n=8$, $p<0.001)$. In conclusion, imidazole antimycotics appear to have effects on different B-cell ion currents and thus may influence insulin secretion, particularly during oral administration of the drugs.

\section{0}

INTRACELLULAR $\mathrm{Ca}^{2+}$ SIGNALLING EVENTS INITIATED BY IMIDAZOLINES IN MOUSE ISLETS OF LANGERHANS

R.M. Shepherd, M.N. Hashrni and M.J. Dunne. The Department of Biomedical Science, Sheffield University. Shefficld, S10 2TN, UK.

Several imidazoline compounds will act as insulin secretagogues by mechanisms that involve $\mathrm{K}_{\mathrm{ATP}}$ channel inhibition. We studied the effects of three imidazolines efaroxan (EF), phentolamine (PHE) and idazoxan (IDZ) on cytosolic $\mathrm{Ca}^{2+}\left(\left[\mathrm{Ca}^{2+}\right]_{i}\right)$ in mouse islets loaded with fura-2, at $37^{\circ} \mathrm{C}$. $\mathrm{EF}$ and PHE both stimulate insulin secretion. In the presence of $2 \mathrm{mM}$ glucose, $\mathrm{EF}(200 \mu \mathrm{M})$ initiated a sustained and reversible increase in $\left[\mathrm{Ca}^{2+}\right]$; average rise $=180 \pm 10 \mathrm{nM}(\mathrm{n}=10)$, and the time to half maximal rise $\left(\mathrm{t}_{1 / 2}\right)$ was $150 \pm 20$ seconds $(\mathrm{n}=10)$. PHE had little effect on $\left[\mathrm{Ca}^{2+}\right]$ at $200 \mu \mathrm{M}$, but consistently elevated $\left[\mathrm{Ca}^{2+}\right]_{i}$ at $500 \mu \mathrm{M}$; average rise $127 \pm 17$ $\mathrm{nM}, \mathrm{t}_{1 / 2} 857 \pm 45$ seconds $(\mathrm{n}=6)$. The actions of PHE were not as readily reversible. In comparison to tolbutamide $(500 \mu \mathrm{M})$-induced rises in $\left[\mathrm{Ca}^{2}{ }^{2}\right]$, both $\mathrm{EF}$ and PHE produced similar peak responses (tolbutamide elevated $\left[\mathrm{Ca}^{2+}\right]_{j}$ by on average $146 \pm 15 \mathrm{nM}, \mathrm{n}=12$ ), but their actions were invariably much slower in onset $\left(t_{1 / 2}\right.$ for tolbutamide $=15 \pm 5$ seconds, $n=12$ ). Interactions between $E F$ and glucose were also studied. Two effects were observed; (i) EF potentiated nutrientinduced increases in $\left[\mathrm{Ca}^{2+}\right]$, and (ii) the onset of the EF-induced rise in $\left[\mathrm{Ca}^{2+}\right]$, was now immediate and occurred without any significant delay $(n=8)$. These data suggest that glucose may facilitate the actions of the imidazolines. Elevation of $\left[\mathrm{Ca}^{2+}\right]$ by both $\mathrm{EF}$ and PHE was dependent upon $\mathrm{Ca}^{2+}$ influx since there was a prompt inhibition of $\left[\mathrm{Ca}^{2+}\right]_{i}$ following removal of external $\mathrm{Ca}^{2+}(\mathrm{n}=5$ and 4 , respectively), and the responses were attenuated by the hyperpolarizing $K_{\mathrm{ATP}}$ channel agonist diazoxide $(250 \mu \mathrm{M})(\mathrm{n}=5$ and 11 , respectively). Finally, IDZ an imidazoline which does not affect insulin release at relatively low concentrations $(200 \mu \mathrm{M})$, had no effect on $\left[\mathrm{Ca}^{2+}\right],(\mathrm{n}=5)$. Our data indicate for the first time that the hypoglycaemia-inducing imidazolines PHE and EF will elevate $\left[\mathrm{Ca}^{2+}\right]_{i}$ in pancreatic islets. These findings provide a key link between $\mathrm{K}^{+}$channel inhibition and the initiation of insulin secretion by these agents.
461

ADRENALINE INHIBITS INSULIN SECRETION INDUCED BY VOLTAGE PULSES IN RAT $\beta$ - AND INS-1 CELLS.

S. Uilrich, U. Fröbe and S. Lehr, Physiologisches institut der AlbertLudwigs-Universität, Hermann-Herder Str. 7, D 79104 Freiburg, Germany.

Adrenaline inhibition of insulin secretion is accompanied by repolarisation of the plasma membrane and inhibition of action potentials. The aim of the present study was to investigate whether adrenaline inhibits the capacitance increase which reflects exocytosis induced by depolarizing voltage pulses in rat B cells and the INS-1 cell line. Capacitance measurements were performed using the perforated patch clamp technique with amphotericin $B$ and the standard whole cell configuration. In perforated patch clamp experiments depolarisation of rat $B$ cells by 4 successive voltage pulses each of $0.3 \mathrm{~s}$ duration from a holding potential of $-70 \mathrm{mV}$ to $0 \mathrm{mV}$ induced increases of capacitance by $51 \pm 14 \mathrm{fF}(\mathrm{n}=15)$ under control conditions $(0.5 \mathrm{mmol} / / \mathrm{glucose})$ and by $107 \pm 17 \mathrm{fF}(n=15)$ under stimulation with $16.7 \mathrm{mmol} / \mathrm{l}$ glucose, $5 \mu \mathrm{mol} / \mathrm{l}$ forskolin and $50 \mu \mathrm{mol} / \mathrm{h}$ tolbutamide. The addition of $5 \mathrm{mmol} / \mathrm{EGTA}$ abolished the increase in capacitance almost completely. Adrenaline $(1 \mu \mathrm{mol} / \mathrm{l})$ reduced the increase of capacitance by half $(n=10)$. Pertussis toxin pretreatment of the cells abolished the adrenaline effect. In standard whole cell configuration where the pipette solution was in free communication with the cell cytosol similar changes in capacitance were observed in rat $B$ cells and in INS-1 cells. Therefore, capacitance measurements allow to follow the dynamic changes in exocytosis and to measure effects of adrenaline in individual cells interfering with the cytosolic composition via the patch pipette. The present results suggest that adrenaline inhibition of insulin secretion involves a regulatory site distal to repolarisation and inhibition of $\mathrm{Ca}^{2+}$ influx. 


\section{2}

ADRENALINE STIMULATES SECRETION IN RAT PANCREATIC $\alpha$-CELLS BY PKADEPENDENT ACCELERATION OF Ca ${ }^{2+}$. INFLUX THROUGH L-TYPE $\mathrm{Ca}^{2+}$. CHANNELS. J. Gromada, W.G. Ding, K. Buschard ${ }^{*}$, E. Renström, P. Rorsman. Islet Cell, Physiology, Novo Nordisk and *Bartholin Instituttet, Copenhagen, Denmark.

The regulation of exocytosis in glucagon-secreting $\alpha$-cells is largely unknown. Here we have applied patch-clamp techniques and fluorimetry to elucidate the mechanism behind adrenergic stimulation of exocytosis in rat pancreatic $\alpha$-cells purified by FACS $\approx 80 \% \alpha$-cells and $3 \%$ B-cells). The cells were stimulated by $500 \mathrm{~ms}$ voltage-clamp depolarizations from .70 to $0 \mathrm{mV}$ applied at 2 min interval. Under control conditions, membrane depolarization was associated with activation of voltage-gated ( $\mathrm{L}$-type) $\mathrm{Ca}^{2+}$-currents $\left(225 \mathrm{pA}\right.$ in $2.6 \mathrm{mM}$ external $\mathrm{Ca}^{2+}$ ), increased cytoplasmic free $\mathrm{Ca}^{2+}\left(\left[\mathrm{Ca}^{2+}\right]_{\text {; }}\right.$ from a basal $0.2 \mu \mathrm{M}$ to a peak $\left.0.8 \mu \mathrm{M}\right)$ and produced a capacitance increase of $30.90 \mathrm{fF}$. Stimulation with adrenaline produced a 3 -fold stimulation of exocytosis. This effect is mediated by $\beta$. adrenergic receptors as it was mimicked by isoprenaline and prevented by propranolal but unaffected by yohimbine (an $\alpha_{2}$-adrenergic antagonist). The stimulatory action on exocytosis was abolished by pretreatment with $\mathrm{Rp}-8 \cdot \mathrm{Br}$. cAMPS: a specific inhibitor of protein kinase A (PKA). Potentiation of exocytosis was associated with an approx. doubling of the integrated $\mathrm{Ca}^{2+}$-current $\left(\mathrm{O}_{\mathrm{Ca}}\right)$ and increased the amplitude of the depolarization.evoked $\left[\mathrm{Ca}^{2+}\right]_{\mathrm{i}}$ transient by $30 \%$. Increasing $\mathrm{O}_{\mathrm{Ca}}$ to the same extent with the $\mathrm{Ca}^{2+}$-channel agonist BAYK8644 11 $\mu \mathrm{M})$ produced a comparable stimulation of exocytosis. Photolytic release of caged cAMP during whole-cell recordings when $\left[\mathrm{Ca}^{2+}\right]_{\bar{i}}$ was clamped at $0.2 \mu \mathrm{M}$ failed to potentiate exocytosis. These data suggest 1) that exocytosis in the $\alpha$-cell is $\mathrm{Ca}^{2+}$. dependent; and 2) that adrenaline stimulates exocytosis by promoting $\mathrm{Ca}^{2+}$.entry with resultant acceleration of $\mathrm{Ca}^{2+}$-induced secretion.

\section{4}

Characterization of the Molecular Subtype of Muscarinic Receptors in Rat Islets of Langerhans and RINm5F Cells

H. Rochlitz, R. Mura, J. Sieradzki, H. Schatz, A. Pfeiffer Medizinische Universitätsklinik Bergmannsheil, Bochum

Acetylcholine is thought to induce the priming of $\beta$-cells in the vagal phase, which enhances first phase insulin secretion and prevents insulin resistence. Acetylcholine acts via muscarinic receptors, of which 5 molecular subtypes are known and which activate different signal transduction pathways. The aim of this study was the identification of the muscarinic receptor subtype in isolated rat pancreatic islets and in the rat insulinoma cell line RINm5F, to obtain further insigths in the signal processing of these cells. Methods: Islets were isolated from the pancreas of male Wistar rats following the protocol of Lacy and Kostianovsky. RNA was extracted with guanidinium thiocyanate by the method of Chomczynski and Sacchi, then treated with DNase (required, because the muscarinic receptor genes have no intron) and reverse transcribed into $\mathrm{CDNA}$. PCR was performed with 5 subtype specific primer pairs and analyzed by gel electrophoresis and DNA sequencing. Results: $c D N A$ of $m 3$ receptor was detectable in isolated rat pancreatic islets and RINm5F cells, whereas none of the other isoforms $\mathrm{m} 1, \mathrm{~m} 2, \mathrm{~m} 4$ and $\mathrm{m} 5$ were shown. The detection of $\mathrm{m} 3$ receptor cDNA indicates the existence of $m 3$ receptor $m R N A$ and therefore the presence of $m 3$ receptors in rat pancreatic islets and RINm5F cells. These findings are consistent with pharmacological studies which are unable to distinguish $\mathrm{m} 4$ and $\mathrm{m} 5$ receptors. This molecular characterization is the basis for possible therapeutic trials with selective $m 3$ receptor agonists and mutation screening of the $\mathrm{m} 3$ receptor gene.
463

POSSIBLE ROLE OF ENDOGENOUS ISLET CATECHOLAMINES IN THE PARACRINE CONTROL OF INSULIN SECRETION.

J.J. Gagliardino, M.I.Borelli and M.Rubio. CENEXA (UNLP-CONICET), La Plata, and Instituto de Investigaciones Farmacológicas, Buenos Aires, Argentina.

The aim of this work was to establish the possible role of endogenous islet catecholamines (EICAs) upon the control of insulin secretion in adult normal rats. For this purpose, tirosine hydroxylase (TH) activity, epinephrine (E) content and insulin secretion elicited by glucose were measured in islets isolated from normal male Wistar rats fed either a standard commercial diet (SD) or a diet composed only by carbohydrates (CHD) during a week. As control of the possible contribution of neurons and nerve terminals to TH activity and $\mathrm{E}$ content, both parameters were aiso assessed in islets isolated from rats previously submitted to surgical solarectomy (S). TH activity was estimated measuring $\mathrm{CO}_{2}$ production by the islets from ${ }^{14} \mathrm{C}-\mathrm{L}$ tirosine, and E content by HPLC. Insulin secretion was measured in islets incubated for $60 \mathrm{~min}$ in KRB buffer with 3 or $16 \mathrm{mM}$ glucose. Insulin in the medium was measured by RIA. TH activity and $\mathrm{E}$ concentration measured in islets isolated from intact or $\mathrm{S}$ rats were similar. $\mathrm{TH}$ activity was significantly higher in islets isolated from CHD compared to SD rats $0.60 \pm 0.06 v s 0.33 \pm 0.04 \mathrm{pg} / \mathrm{mg}$ protein; $\mathrm{p}<$ 0.001 ). The $E$ content of islets from CHD was significantly lower than that measured in islets from $\mathrm{SD}(0.018 \pm 0.001 v s 0.031 \pm 0.005 \mathrm{pg} / \mathrm{mg}$ protein; $\mathrm{p}<0.05)$. CHD islets released significantly less insulin in response to glucose than SD islets

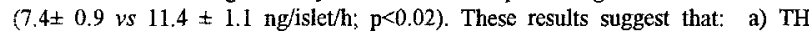
activity and $\mathrm{E}$ content measured in the islets have an endogenous rather than a neural origin; b) islet Th activity can vary according to food composition; c) the association of high $\mathrm{TH}$ activity with low $\mathrm{E}$ content measured in islets from $\mathrm{CHD}$ rats might indicate that the enzyme activity in the islets is controlled by a CA-endproduct as in other tissues; and d) changes in TH activity and E content are accompanied by significant changes in the amount of insulin released by isolated islets in response to glucose. Altogether, our results might indicate that EICAs participate in the paracrine control of insulin secretion.
465

ROLE OF MUSCARINIC RECEPTORS ON INSULIN AND GLUCAGON SECRETION IN PERFUSED RAT PANCREAS $H$. Maruyama, $H$. Hirose, K. Ito K. Koyama, K. Kido and $T$. Saruta. Keio University School of Medicine, Tokyo 160. Japan

We investigated the modulating effects of acetylcholine $(A C h)$ on insulin and glucagon secretion in response to changes in glucose concentration, using perfused rat pancreas preparations. Pre-perfusion with $10^{-3} \mathrm{M}$ ACh for $5 \mathrm{~min}$ potentiated the first phase of glucose-stimulated insulin secretion compared with control group $(5.77 \pm 1.87$ vs $2.04 \pm 0.26 \mathrm{nmol} / \mathrm{min}, \mathrm{p}<0.05$ ), but did not affect glucagon secretion in response to glucose concentration changes. Continuous addition of $10^{-5} \mathrm{M}$ atropine to the perfusate suppressed the first phase of glucose-stimulated insulin secretion to $1.15 \pm 0.14 \mathrm{nmol} / \mathrm{min}(p<0.05)$. Atropine also suppressed low-glucose induced glucagon secretion $(0.59 \pm 0.09$ vs $1.22 \pm 0.19 \mathrm{ng} / \mathrm{min}, p<0.01)$. Addition of $10^{-4} \mathrm{M}$ hexamethonium did not influence high- or low-glucose induced secretion of either insuiin or glucagon. Muscarinic receptor antagonists p-F-HHSiD $\left(M_{1}+M_{3}\right)$ and DAMP methiodide $\left(M_{3}\right)$ at concentrations of $10^{-8} \mathrm{M}$ to $10^{-5} \mathrm{M}$ suppressed $\mathrm{ACh}$ induced insulin and glucagon secretions. Changes in glucose concentration did not modify pancreatic polypeptide (PP) secretion from perfused rat pancreas. Histopathological examinations revealed markedly high acetylcholinesterase activities in islets than in exocrine tissue. We conclude that ambient ACh has local effects on islet beta- and alpha-cells, potentiating insulin and glucagon secretion in response to glucose concentration changes, probably via $M_{3}$ receptors. 
466

EXPRESSION OF CHOLECYSTOKININ-A RECEPTORS IN RAT ISLETS AND THE RAT INSULINOMA CELL LINE RINM5F S. Karlsson, B. Ahrén and F. Sundler, Departments of Medicine, Malmö and Physiology and Neuroscinces, Lund University, Sweden Cholecystokinin (CCK) is known to stimulate insulin secretion under in vivo conditions and in isolated rat islets of Langerhans. Whether specific CCK-receptors are expressed in the islet B-cells is, however, not known. We therefore investigated, by in situ hybridization, the expression of CCK-A and -B receptors in islet cells of fresh frozen sections of rat pancreata as well as in the rat insulinoma cell line RINm5F. For each receptor a mixture of three different $35 \mathrm{~S}-\mathrm{dATP}$ labelled oligodeoxyribonucleotide probes were used. Furthermore, the effects of CCK-8 on insulin secretion were studied in the RINm5F cells. It was found that rat islets expressed CCK-A but not CCK-Breceptors. The expression of CCK-A receptors was concentrated to the central parts of the islets corresponding to the islet B-cells. Also in RINm5F cells CCK-A-receptors were expressed. In RINm5F cells Dglyceraldehyde $(10 \mathrm{mmol} / \mathrm{l})$ stimulated insulin secretion from $2660 \pm 542 \mathrm{pmol} / 1$ to $7883 \pm 1007 \mathrm{pmol} / 1(\mathrm{p}<0.001, \mathrm{n}=8)$. However, CCK-8 (0.01 to $100 \mathrm{nmol} / \mathrm{l})$ was without effect on insulin secretion in the presence of $10 \mathrm{mmol} / \mathrm{L} \mathrm{D}$-glyceraldehyde $(\mathrm{n}=10-11)$. Also at 1 $\mathrm{mmol} / 1$ of D-Glyceraldehyde CCK-8 $(1-100 \mathrm{nmol} / \mathrm{l})$ was without effect. This is in contrast to the dose-dependent insulinotropic effect of CCK-8 in normal rat islets. It is concluded that CCK-receptors of the CCK-A subtype are expressed both in normal rat pancreatic islets and in the rat insulinoma cell line RINm5F. However, whereas, CCK-8 stimulates insulin release from normal rat islets, the CCK-receptors expressed in RINm5F cells seem not to be coupled to the secretion of insulin.
467

EFFECT OF VTAMMN $D_{3}$ DEFICIENCY ON GLUCOSE METABOLISM WTTHIN RAT PANCREATIC ISLETS.

B. Billaudel, L. Barakat, B.Ch.J. Sutter and A. Faure-Dussert. Lah Endocrinology, University Bordeaux I, F - 33405 Talence Cedex, France.

Vitamin $D_{3}$ deficiency reduced glucose-induced insulin release, as well in humans as in experimental animals, in relation to an altered calcium handling within B-cells. As glucose metaholism plays a crucial role in calcium handling the purpose of this study was to investigate whether vitamin $D_{3}$ deficiency affects some aspects of $B$-cells glucose metabolism in the rat. To this end, islets were isolated from either a control group (C) raised on a normal diet or an experimental group (-D) receiving a vitamin $D_{3}$ depleted diet for five weeks after weaning. Both the utilisation of $D$ $\left[5-{ }^{3} \mathrm{H}\right]$ glucose and oxidation of $\mathrm{D}-\left[6{ }^{14} \mathrm{C}\right]$ glucose were severely affected at $16.7 \mathrm{mmol} / /$ glucose (respectively : $-D=190 \pm 12$ (21) vs $C=247=$ $25(18) \mathrm{pmol} / \mathrm{h} / \mu \mathrm{g}$ protein $(-29 \%)$ and $-\mathrm{D}=11.0 \pm 1.2(21)$ vs $\mathrm{C}=28.1 \pm$ $3.7(18) \mathrm{pmol} / \mathrm{h} / \mathrm{\mu g}$ protein $(-60 \%), \mathrm{p}<0.001)$. No significant effect could be seen with $4.2 \mathrm{mmol} / /$ glucose. Moreover, the preferential stimulation of $D-\left[6-{ }^{14} \mathrm{C}\right]$ glucose oxidation versus $D-\left[5-{ }^{3} \mathrm{H}\right] \mathrm{glucose}$ utilisation, currently observed in (C) rats during glucose stimulation $(11.4 \pm 0.2 \%)$ as judged from the ratio of these two parameters, was severely impaired $(p<0.001)$ in $-D$ rats $(7.0 \pm 0.4 \%)$. These results were in favour of a primary impaiment of oxidative responsiveness in islets from $-D$ rats. This hypothesis was confirmed using islets perifusion experiments in which D-glyceraldehyde stimulus showed dynamic insulin secretion profiles similar to D-glucose ones. In conclusion, the present data are compatible with an alteration of glucose metabolism of B cells preferentially located at a distal site of the oxidative events.

\section{8}

ORAL VANADYL SULPHATE DOES NOT CORRECT DEXAMETHASONEINDUCED HYPERGLYCAEMIA IN AGING RATS BUT MODIFIES INSULIN RESPONSIVENESS IN THE ISOLATED PERFUSED PANCREAS.

V. De Tata, M. Bombara, V. Fierabracci, M. Barbera, M. Novelli, P. Masiello and E. Bergamini. Istituto di Patologia Generale, University of Pisa, Italy.

Both vanadate and the vanadyl forms of vanadium have insulin-like effects on glucose metabolism, including the capability to correct hyperglycaemia and other metabolic alterations in experimental diabetes. We have shown that dexamethasone $(0.125 \mathrm{mg} / \mathrm{kg} / \mathrm{die}$ s.c. for 13 days) can induce insulin resistance, as indicated by the significant rise of plasma insulin and NEFA levels, in both young and old rats, which is however associated with hyperglycaemia only in aged animals. The aim of this study was to investigate whether oral vanadyl sulphate $\left(\mathrm{VOSO}_{4}\right)$ was able to correct the observed dexamethasone-induced hyperglycaemia in old rats. Our results show that while in young (3-mo-old) rats plasma glucose levels were unchanged in all the experimental groups, in aged (18-mo-old) rats VOSO administration $(0.5 \mathrm{mg} / \mathrm{ml}$ in the drinking water), starting 4 days after dexamethasone treatment, did not correct the increase in glycaemic values induced by glucocorticoids. Preparations of isolated perfused pancreas showed that in 18-mo-old rats (differently from 3-mo-old animals) $\mathrm{VOSO}_{4}$ treatment caused a significant increase of both phases of glucose-stimulated insulin release with respect to age-matched controls. Interestingly, the perfused pancreata of animals treated with both $\mathrm{VOSO}_{4}$ and dexamethasone showed a glucose-stimulated insulin secretion blunted respectful to that observed with the one or the other substance alone albeit higher than that of untreated controls. Indeed, IRI release in response to $16.7 \mathrm{mM}$ glucose was $65 \pm 21,399 \pm 24,438 \pm 124,206 \pm 20 \mathrm{ng} / 15 \mathrm{~min}$ in untreated control, VOSO treated, dexamethasone-treated and dexamethasone plus $\mathrm{VOSO}_{4}$-treated rats, respectively, In conclusion, oral vanadyl sulphate treatment in aging rats influences the insulin response to glucose of the isolated perfused pancreas but is unable to correct dexamethasone-induced hyperglycaemia.

\section{9}

EFFECTS OF AGE AND DEXAMETHASONE TREATMENT ON INSULIN SECRETION FROM ISOLATED PERFUSED RAT PANCREAS.

M. Novelli, M. Barbera, V. Fierabracci, M. Bombara, V. De Tata, E Bergamini and P. Masiello. Ist. Patologia Generale, University of Pisa, Italy.

We have previously shown that secretory function of isolated islets of Langerhans is impaired in aging animals. This study was aimed at comparing the insulin secretory capabilities of the isolated perfused pancreata of young (3-mo-old) and aged (18-mo-old) dexamethasone-treated Sprague-Dawiey rats and untreated controls. Dexamethasone $(0.125 \mathrm{mg} / \mathrm{kg} / \mathrm{die}$ s.c. for 13 days) induced a significant rise in plasma insulin and NEFA levels in both young and old rats. However, plasma glucose did not change in young rats. whereas significantly increased in 18-mo-old animais $(120 \pm 6,177 \pm 27$, $264 \pm 43$ and $246 \pm 59 \mathrm{mg} / \mathrm{dl}$ after $0,4,9$ and 13 days of treatment, respectively). Preparations of isolated perfused pancreas from 3- and 18-moold dexamethasone-treated rats and age-matched controls showed that basal insulin secretion was significantly increased by dexamethasone treatment in old rats only. Both the first and the second phase of glucose-stimulated insulin release were reduced (by 73 and $65 \%$, respectively) in old controls respectful to young ones. Nevertheless, dexamethasone treatment enhanced the insulin response to $16.7 \mathrm{mM}$ glucose more in the pancreas of old rats than in that of young rats (438 $\pm 124 \mathrm{vs} 65 \pm 21 \mathrm{ng} / 15 \mathrm{~min}$ in old, and $300 \pm 100$ vs $202 \pm 40 \mathrm{ng} / 15 \mathrm{~min}$ in young treated and untreated rats, respectively). A subsequent stimulation with $16.7 \mathrm{mM}$ glucose plus $1 \mathrm{mM}$ IBMX also elicited significantly different responses in the perfused pancreas from different animal groups. In conclusion, our results indicate that: 1) dexamethasoneinduced insulin resistance is higher in aging than in young rats; 2) there is no correlation between plasma glucose and NEFA levels; 3 ) in spite of the impaired insulin response to glucose in the isolated perfused pancreas of aging rats, the remarkable enhancement of this response upon dexamethasone treatment indicates a still preserved compensatory flexibility, nevertheless usually insufficient to restore homeostasis in vivo. 
470

INSULINOTROPIC ACTION OF THE MONOETHYL, MONOPROPYL, MONOISOPROPYL, MONOALLYL AND DIALLYL ESTERS OF SUCCINIC ACID

J.A. García-Martínez, T.-M. Zhang**, M.L. Villanueva-Peñacarrillo, I. Valverde, F. Björkling* and W.J. Malaisse** Fundación Jiménez Díaz, Madrid, Spain, *Leo Pharmaceutical Products, Ballerup, Denmark and **Laboratory of Experimental Medicine, Brussels Free University, Brussels, Belgium.

The methyl esters of succinic acid are potent insulin secretagogues. They also potentiate the secretory response of the B-cell to other insulinotropic agents that are or could be used in the treatment of non-insulin-dependent diabetes, such as hypoglycaemic sulphonylureas, meglitinide analogues or GLP-1. The in vivo admininistration of these methyl esters may lead, however, to the undesirable generation of methanol. We have investigated, therefore, whether other esters of succinic acid also display insulinotropic action. In the presence of $7 \mathrm{mmol} / \mathrm{l} \mathrm{D}$-glucose, several esters of succinic acid $(10 \mathrm{mmol} / \mathrm{l})$ augmented insulin release from incubated rat islets, with the following order of potency: monopropyl $\sim$ monoethyl $\geq$ monoisopropyl $\sim$ monoallyl $\geq$ diallyl. Likewise, in anaesthetized fed rats, the intravenous administration of the esters (2 $\mu \mathrm{mol} / \mathrm{g}$ body wt.) caused, within $2 \mathrm{~min}$, paired increments in plasma insulin concentration averaging $8.0 \pm 0.5(\mathrm{n}=6), 4.6 \pm 0.3(\mathrm{n}=10), 4.7 \pm 0.5(\mathrm{n}=6)$, $2.2 \pm 1.1(\mathrm{n}=3)$ and $1.5 \pm 0.3(\mathrm{n}=3) \mathrm{ng} / \mathrm{ml}$ in the case of the monopropyl, monoethyl, monoisopropyl, monoallyl and diallyl ester, respectively. These findings thus indicate that several succinic acid esters that are not susceptible to lead, through intracellular hydrolysis, to the undesirable production of methanol, remain potent insulin secretagogues both in vitro and in vivo. It now remains to be investigated whether some of these new esters may, like the dimethyl ester of succinic acid, also stimulate insulin release when given enterally rather than parenterally.

\section{1}

IMPORTANCE OF DYNORPHIN FOR INSULIN SECRETION FROM ISLETS OF LANGERHANS

L. Sørensen 1 , K. Buschard ${ }^{1}$, R. Ekman ${ }^{2}$, J. Efanova ${ }^{3}$, P.O. Berggren ${ }^{3}$, M. Birkenbach', and K. Josefsen'. 'Bartholin Instituttet, Copenhagen, Denmark; ${ }^{2}$ Mölndals Sjukhus, Göteborg and ${ }^{3}$ Karolinska Institutet, Stockholm, Sweden; ${ }^{4}$ University of Chicago, Chicago, USA.

Transcription of the dynorphin gene is initiated shortly after glucose stimulation of starving MIN6 cells, an insulin producing cell line that responds to glucose at physiological concentrations. Likewise, dynorphin secretion increases from MIN6 cells upon glucose stimulation. To investigate if dynorphin could be an intra-islet regulator of insulin secretion we examined the effect of dynorphin and naloxone, a kappa-opioid receptor antagonist, on insulin secretion from isolated rat islets of Langerhans. Batches of 25 islets were incubated in microtiter wells in $2.8,4.5,11$ or $20 \mathrm{mM}$ glucose and dynorphin $1-18$ or naloxone was added in concentrations ranging from $10^{-12} \mathrm{M}$ to $10^{-9} \mathrm{M}$ and $10 \mathrm{ng} / 1$ to $120 \mu \mathrm{g} / 1$, respectively. The insulin secretion did not change following dynorphin addition, neither when incubated for 30 min nor for 3 days. Likewise, addition of dynorphin A 1-18 did not change the concentration of intraceilular calcium in small clusters of beta cells as recorded by calcium imaging with fura- 2 . Addition of naloxone, however, increased the insulin secretion in a concentration dependent manner in media containing $4.5 \mathrm{mM}$ glucose and above $(\mathrm{p}=0.00018)$, but not in unstimulated islets $(2.8 \mathrm{mM}$ glucose $)$. Although it cannot be excluded that naloxone could directly stimulate insulin secretion from islets, our results suggest that an inhibitory endogenous opioid tonus exists in islets and that this tonus can be suspended by naloxone. The study emphasizes the importance of opioids in the regulation of insulin secretion from islets of Langerhans.
STEVIOSIDE AND STEVIOL STIMULATE INSULIN SECRETION FROM ISOLATED MOUSE ISLETS.

P.B. Jeppesen, S. Gregersen and K. Hermansen. Dept. of Endocrinology and Metabolism. Aarhus University Hospital, Tage-Hansen Gade 2, DK-8000 Aarhus C. Denmark.

Plants provide a vast resource of compounds with potential for the development of new antidiabetic drugs. Interestingly, leaves of Stevia rebaudiana Bertoni $(\mathrm{SrB})$ have been used as traditional treatment for diabetes in Brazil. The aim of this study was to investigate the effects of to compounds found in $\mathrm{Sr} B$ - the diterpene glycoside stevioside and the aglucon diterpene carboxylic alcohol steviol- on the release of insulin from isolated mouse islets using batch and perifusion experiments. Student's unpaired one-tailed t-test and ANOVA one way analyses were used to test the degree of significance. We found that both stevioside $\left(5.0 \times 10^{-3}-10^{-9} \mathrm{M}\right)$ and steviol $\left(10^{-3}-10^{-9} \mathrm{M}\right)$ potently and dose dependently increased insulin secretion in the presence of $16.7 \mathrm{mM}$ glucose $(\mathrm{P}<0.05)$. Furthermore. both compounds stimulated $\beta$-cell secretion in a glucose-(3.3-16.7 $\mathrm{mM})$ dependent manner. The maximal insulin responses were obtained at concentrations of $10^{-5} \mathrm{M}$ stevioside $(\mathrm{P}<0.01)$ and $10^{\circ}$ ${ }^{6} \mathrm{M}$ steviol $(\mathrm{P}<0.01)$, respectively. It is noteworthy, that the effect of the two substances on insulin secretion is still prominent in the absence of extracellular $\mathrm{Ca}^{2+}$. In conclusion, the extracts from leaves of SrB-stevioside and steviol-potently stimulate insulin secretion from mouse islets and may be a source as new hypoglycaemic drugs. 


\section{Mechanisms of Defective Islet-Cell Function}

\section{3}

GLUCOSE-SENSITIVE B-CELL TRANSCRIPTION FACTOR IUF1 (INSULIN UPSTREAM FACTOR 1) IS ABSENT FROM A. HUMAN NESIDIOBLASTOMA (PHHI) CELL LINE

W.M. Macfarlane, H. Cragg, *R.F.L. James, H.M. Docherty, and K. Docherty. Department Of Molecular and Cell Biology, University of Aberdeen, Aberdeen, AB9 lAS, U.K., and ${ }^{*}$ Department of Surgery, University of Leicester, Leicester, LE2 7LX, U.K.

The $\beta$-cell homeodomain transcription factor IUF1 (IPF 1/STF1/ADXI) binds in a glucose-responsive manner to four sites within the human insulin gene promotor, transactivating transcription of the insulin gene. In the present study, activity of IUFI was analysed in the human nesidioblastoma clonal cell line NES2Y. Persistant Hyperinsulinemic Hypoglycemia of Infancy (PHHI, or nesidioblastosis) is an autosomal recessive disorder of glucose homeostasis, characterised by unregulated secretion of insulin and profound hypoglycemia. $\beta$-cell dysfunction includes a defect in glucose-regulated insulin secretion, which is linked to mutations in the sulphonylurea receptor gene. In the analysed cell line, IUF1 DNAbinding activity was undetectable. The binding activity of all other transcription factors analysed was normal. Immunocytochemical staining and Western Blot analysis with a specific IUF 1 antibody were unable to detect the IUF 1 protein. IUF 1 mRNA was detectable at low levels by RTPCR and the sequence of this mRNA was normal. The cells stained positively for insulin, and showed a blunted secretory response to glucose. In transfection studies using the -50 to -250 region of the human insulin gene enhancer (upstream of a luciferase reporter gene), the nesidioblastoma cells lacked a functional glucose responsive transcriptional activity. IUF1 plays a critical role in islet cell ontogeny, $\beta$-cell specific and glucose-sensitive expression of the insulin gene. We propose that loss of expression of the glucose-sensitive IUF1 protein may contribute to $\beta$-cell dysfunction in PHHI (nesidioblastosis).

\section{4}

IN VITRO STUDIES OF FUNCTIONAL ISLETS FROM A TYPE 1 HUMAN DIABETIC PATIENT

C. Kane, R.M. Shepherd, M.N. Hashmi, S.M. Swift", P.R.V. Johnson", D Olojugba", R.F.L. James", A.L. Pallett", N.G. Morgan " and M.J. Dunne, Biomedical Science, Sheffield University, Shefficld, "Department of Surgery, Clinical Sciences Building, Leicester University, Leicester, and "Biological Sciences, Keele University, Keele, UK

The functional regulation of islets of Langerhans from a Type 1 diabetic have been studied. The patient (16 years old) received insulin therapy for 10 months prior to organ donation and death was unrelated to diabetes. Immuno-histochemical inspection of the pancreas revealed the presence of intact islets and cells staining positive for insulin. Isolated islets were poorly responsive to $8 \mathrm{mM}$ glucose and to the sulphonylurea glibenclamide (GLIB) $(1 \mu \mathrm{M}) ; 8 \mathrm{mM}$ glucose elcvated secretion only transiently to a peak $225 \%$ above basal ( $n=3$ perifusions of islets), GLIB by $150 \%(n=3)$. In contrast, $20 \mathrm{mM}$ glucose produced a more marked release of insulin $(325 \%(n=3))$, which was inhibited by the $\mathrm{K}_{\mathrm{ATP}}$ channel agonist diazoxide $(200 \mu \mathrm{M} ; 57 \%(\mathrm{n}=3))$ and reversed by efaroxan (EF) $(100 \mu \mathrm{M} ; 237 \%$ vs basal). The ionic basis for these effects was investigated through patch-clamp techniques. In intact cells, $\mathrm{K}^{+}$channels were activated by diazoxide $(200 \mu \mathrm{M}, \mathrm{n}=14 / 26)$, and inhibited by GLIB $(100 \mathrm{nM}, \mathrm{n}=5)$ and $\mathrm{EF}(200 \mu \mathrm{M}, \mathrm{n}=5)$. In isolated patches three types of $\mathrm{K}^{+}$channels were characterized; (i) an ATP-inhibited channel (non-linear current (I)-voltage (V) relationship, with an inward current conductance of approximately $66 \mathrm{pS}$ ), (ii) a $\mathrm{Ca}^{2+}$ - and voltage-gated $\mathrm{K}^{+}$channel (linear I-V, 210pS) and (iii) non-selective cation channel (linear I-V, 20pS). The ATP-sensitive $\mathrm{K}^{+}$ channel was blocked by ATP $(0.5-1 \mathrm{mM})$ and but was relatively unaffected by ADP $(0.5-1 \mathrm{mM})$ in the presence of ATP ( $\mathrm{n}=11$ patches). These channels were also more sensitive to $E F(100 \mu \mathrm{M}$, which inhibited channels to $4.6 \pm 2 \%$ of control, $n=9$ ) than either GLIB (100nM) or tolbutamide $(100 \mu \mathrm{M}) ; 33 \pm 14 \%$ and $48 \pm 10 \%$, respectively, $\mathrm{n}=7$. These data indicate that altered sensitivity of $\mathrm{K}^{+}$channels to internal ADP and glibenclamide could underlie the poor glucose and sulphonylurea secretory responses of $\beta$-cells from this diabetic patient.

\section{5}

IS THE AGE-DEPENDENT DECLINE OF PANCREATIC ISLET FUNCTION A REVERSIBLE PHENOMENON?

R. Perfetti, Y. Wang, J.M. Egan. Diabetes Unit, LCP, NIA, NIH, Baltimore, MD 21224

Aging is an etiologic factor in non-insulin-dependent diabetes mellitus. We recently characterized the $\beta$-cell abnormalities which occur with age. Using isolated rat islets, we demonstrated that normal aging is associated with a progressive diminution of the insulin mRNA levels, a decline of the total pancreatic insulin content, a reduction in the number of glucose-responsive cells and a diminution of the amount of insulin released per cell. The aim of this study was to identify "factor(s)" capable of reversing those abnormalities. We infused $1.5 \mathrm{pmol} / \mathrm{kg}$ glucagon-like peptide-1 (7-36) amide (GLP-1)in 22-month-old Wistar rats $(n=6)$ for $48 \mathrm{~h}$. Treated and control rats $(n=6)$ were fasted overnight. A catheter was inserted into the femoral artery for blood sampling. Glucose $(1 \mathrm{~g} / \mathrm{kg}$ i.p.) was given and blood assayed for glucose and insulin levels every fifteen minutes for ninety minutes. Fifteen minutes after glucose injection GLP-1 treated rats showed significantly lower plasma glucose levels $(\mathrm{mM})$ compared to untreated controls $(9.04 \pm 0.92$ vs. $11.61 \pm 0.23, p<0.01)$. At thirty minutes, the two animal groups still showed significantly different glucose levels $(8.61$ \pm 0.39 vs. $10.36 \pm 0.43, p<0.05$ ). The profile of insulin levels was also dramatically different between the two animal groups. For example, at fifteen minutes, plasma insulin levels $(\mathrm{pM})$ were $936 \pm 163$ vs. $395 \pm 51$ $(p<0.01)$. Finally, GLP-1 treatment increased insulin mRNA levels as determined from ex vivo pancreatic RNA extracts. GLP-1 increased insulin mRNA levels 3 fold when compare to untreated controls, reaching levels present in 6-month-old rat pancreas. In conclusion isolated islets from old rats show multiple defects in their activity, these defects can in part be reversed by GLP-1 treatment.

\section{6}

DYSFUNCTION OF ISLET LYSOSOMAL ENZYME ACTIVITIES AND INSULIN RELEASE IN YOUNG GK RATS.

R. Henningsson, A. Salehi, C-G Östenson, S. Efendic and I. Lundquist. Department of Pharmacology, University of Lund and Department of Molecular Medicine, Karolinska Institute, Stockholm, Sweden. Recent evidence links one of the multiple signals involved in glucose stimulated insulin release with activation of the islet lysosomal glycogenolytic enzyme acid glucan-1,4- $\alpha$-glucosidase. We have performed a detailed analysis of different lysosomal enzyme activities in pancreatic islets of young 6 weeks old spontaneously diabetic GK rats and Wistar control rats in relation to the insulin secretory response. The in vivo and in vitro (isolated islets) insulin response to glucose was markedly impaired in the GK rat as compared to the Wistar controls. This impairment could be fully restored by addition of the adenylate cyclase activator forskolin to the incubation medium. Islet activities of several lysosomal enzymes such as acid phosphatase, $N$-acetyl- $\beta$-Dglucosaminidase, $\beta$-glucuronidase and cathepsin D were all reduced by $20-35 \%$ in the GK rat compared to Wistar controls. In contrast, the activities of the lysosomal $\alpha$-glucosidehydrolases, i.e. acid glucan-1,4- $\alpha$-glucosidase and acid $\alpha$-glucosidase were increased by $40-50 \%$. The activity of the neutral $\alpha$ glucosidase (endoplasmatic reticulum) was unaffected. On the other hand, in liver tissue, these enzyme activities were of the same magnitude in GK and Wistar rats with the exception of $\beta$-glucuronidase which was lower $(-35 \%)$ in the GK liver. Notably, when comparing the different enzyme activities in islet tissue vs liver tissue in the control rats, the activities of acid glucan-1,4- $\alpha$ glucosidase and acid $\alpha$-glucosidase were 15 fold higher in the islets than in the liver. No such impressive difference was observed with regard to the other lysosomal enzyme activities recorded. The results recorded indicate that the defective insulin response to glucose in the GK rat is probably multifactorial in origin, and in addition to the previously observed impairment of islet glucose metabolism; e.g. increased glucose utilization and glucose cycling and decreased activity of glycerophosphate dehydrogenase as well as a defective closure of ATP sensitive $\mathrm{K}^{+}$channels, it also includes disturbances in the regulatory function of the islet lysosome-acid glucan-1,4- $\alpha$-glucosidase system. 
477

THE ROLE OF ENDOGENOUS NITRIC OXIDE IN GLUCOSE-INDUCED INSULIN SECRETION IN CONTROL AND DIABETIC GK RATS

Y.Tsuura, H.Ishida, T.Shinomura, M.Nishimura and Y. Seino: Kyoto University, Kyoto, Japan

Nitric oxide (NO) is well known to play a important role in intracellular signal transduction system in various tissues. In pancreatic $\beta$-cells, exogenous NO reportedly inhibits glucose -induced insulin secretion, and we have shown recently that this mechanism is due to disturbed intracellular glucose metabolism at phosphofructokinase (PFK) level, resulting in open of ATP-sensitive $\mathrm{K}^{+}$channels (J Gen Physiol 104: 1079, 1994). In the present study, we have examined the functional role of endogenous NO on insulin secretion and its possible role in the pathogenesis of impaired glucose-induced insulin secretion in non-insulin dependent diabetes mellitus (NIDDM). NO generated from 100 islets of control and GK rats and one liberated from sodium nitropurnuside (SNP) were measured by chemiluminescence method, and presence of NO synthase (NOS) in extracts of control and GK rats islets were deternined by Western blotting. NO liberated from $1 \sim 100 \mu \mathrm{M}$ SNP inhibited glucose-induced insulin release dosedependently, being paralleled by inhibition of PFK activity. Only brain-type NOS (bNOS) was expressed in islets of control and GK rats to the same extent. NO formed from 100 control islets in the presence of $16.7 \mathrm{mM}$ glucose $(134 \pm 18 \mathrm{pM} / 30 \mathrm{~min} / \mathrm{ml})$ was significantly larger than that with $2.8 \mathrm{mM}$ glucose $(83 \doteq 12 \mathrm{pM})$. The formation of NO from GK rat islets in the presence of $2.8 \mathrm{mM}$ glucose was considerably higher than that in controls $(138 \pm 24 \mathrm{pM})$, and NO generation was almost identical when incubated with $16.7 \mathrm{mM}$ glucose $(156 \pm 22 \mathrm{pM})$. The bNOS inhibitor, 7-nitro indazole (7-NI) enhanced glucose-, but not glyceraldehyde-induced insulin secretion. Other NOS inhibitors including aminoguanidine and $\mathrm{N}$-iminoethyl-L-ornithine also enhanced exclusively glucose-induced insulin secretion. These results suggest that NO has a physiologically inhibitory function in glucose-induced insulin release through suppressing PFK activity. In addition, the higher NO generation under basal glucose concentration in GK rats could be a causative factor of selectively impaired glucoseinduced insulin release in NDDM.

\section{8}

Kreb's cycle substrate $\alpha-\mathrm{KIC}$-induced insulin release is impaired in GK but not $F_{1}$ hybrids of GK-Wistar rats

Amel Guenifi, Samy M. Abdel-Halim, Suad Efendić, Claes-Göran Östenson Endocrine \& Diabetes Unit, Department of Molecular Medicine, Karolinska Institute, Stockholm, Sweden.

The GK rat is a model of non-obese non-insulin dependent diabetes mellitus (NIDDM). While it is generally accepted that glucose-induced insulin release is markedly impaired in GK rats, contradicatory data was reported concerning the insulinogenic effect of substrates directly metabolised in Kreb's cycle such as leucine and $\alpha$-ketoisocaproic acid $(\alpha-K I C)$. In this report we have studied the effects of glucose or $\alpha-K I C$ on insulin release in GK rats, $F$, hybrids of GKWistar rats and normal Wistar rats. In contrast to GK rats, hybrid rats are characterised by near normal fasting blood glucose levels and a glucose tolerance intermediate between control and GK rats. As expected, glucoseinduced insulin release was markedly impaired in GK and hybrid rats irrespective whether experiment were performed in the isolated perfused pancreas or isolated islets. Similarly, $\alpha$-KIC-induced insulin release was decreased by approximately $50 \%$ in GK islets ( $P<0.005$ vs controls) and isolated perfused pancreas ( $p<0.002$ vs controls) but preserved in hybrid islets (24.6 \pm 3.9 in hybrids vs $28.7 \pm 2.0 \mu \mathrm{U} /$ /islet in controls). The slope of potentiation by glucose $(5.5-16.7 \mathrm{mM})$ of arginine $(10 \mathrm{mM})$-stimulated insulin secretion was impaired in hybrid islets. However, the slope of potentiation of arginine-stimulated insulin secretion by $\alpha-K I C(5-20 \mathrm{mM})$ was preserved in hybrid islets. In conclusion, both glucose and $\alpha$-KIC-induced insulin release is impaired in GK islets while the $\alpha$-KIC effect was preserved in hybrid islets. This suggests that in hybrid rats, which represents a model of impaired glucose tolerance, the defect in the glycolytic pathway constitutes the molecular basis behind the impaired insulin response to glucose. The defective insulin release in response to $\alpha-K I C$ in the GK rat may reflect a gene-dose response effect and/or a glucotoxic action of chronic pronounced hyperglycaemia.

\section{9}

The strong insulin release induced by forskolin in GK rats is accompanied by enhanced generation of cAMP in the diabetic islets Samy M. Abdel-Halim, Amel Guenifi, Claes-Göran Östenson, Suad Efendić Endocrine \& Diabetes Unit, Department of Molecular Medicine, Karolinska Institute, Stockholm, Sweden.

Glucose-induced insulin release is markedly impaired in isolated islets from the GK rat model of NIDDM. We recently demonstrated that forskolin exerts strong insulinotropic responses at $3.3 \mathrm{mM}$ glucose in GK but not control rats by mechanisms independent of a rise in $\left[\mathrm{Ca}^{2+}\right]_{\text {; }}$ or changes in the $\mathrm{K}_{\text {(RTP) }}$ channel and largely involving direct interaction with the exocytotic machinery in the B-cell. To further study the signals mediating these responses in the GK rat, we investigated concurrent insulin release and cyclic adenosine monophosphate (CAMP) generation from isolated control and GK islets in response to $5 \mu \mathrm{M}$ forskolin at 3.3 and $16.7 \mathrm{mM}$ glucose. The islets were cultured for $48 \mathrm{~h}$ in RPMI-1640 with $11 \mathrm{mM}$ glucose. Insulin release was impaired in $\mathrm{GK}$ rat islets in response to $3.3 \mathrm{mM}$ ( $\mathrm{p}<0.002$ vs controls) and $16.7 \mathrm{mM}$ glucose ( $<<0.0001 \mathrm{vs}$ controls). Stimulation with forskolin increased insulin release by approximately 4-fold in control islets at $3.3 \mathrm{mM}(5.9 \pm 0.5 \mathrm{vs}$ $27.2 \pm 4.3 \mu \mathrm{U} /$ islet with forskolin, $p<0.0001)$ and $16.7 \mathrm{mM}$ glucose $(25.8 \pm 2.5$ vs $104.5 \pm 11.3 \mu \mathrm{U} /$ islet with forskolin, $p<0.0001$ ). Forskolin however induced a strong 9-fold increase in insulin release in GK islets at $3.3 \mathrm{mM}(3.9 \pm 0.6 \mathrm{vs}$ $36.9 \pm 2.9 \mu \mathrm{U} /$ islet with forskolin, $p<0.0001$ ) and an 18-fold increase at 16.7 $\mathrm{mM}$ glucose $(6.3 \pm 0.6$ vs $110.3 \pm 12.1 \mu$ U/islet with forskolin, $p<0.0001)$. Cyclic AMP levels in GK islets was similar to control islets at $3.3 \mathrm{mM}$ glucose and significantly elevated at $16.7 \mathrm{mM}$ glucose ( $p<0.0001 \mathrm{vs}$ control). Forskolin, however, induced an enhanced CAMP generation in GK islets at both $3.3 \mathrm{mM}(279 \pm 14$ in GK vs $148 \pm 14$ fmol/islet in control, $p<0.0001)$ and $16.7 \mathrm{mM}$ glucose ( $243 \pm 14$ in GK vs $85 \pm 10$ fmolislet in controls, $p<0.0001)$. In conclusion, our results demonstrate that forskolin fully restored insulin release at both low and high concentrations of the sugar in GK islets. The strong insulin responses induced by forskolin in GK islets is probably mediated by concurrently enhanced generation of CAMP in the diabetic Bcells.
480

Correction of hyperglycemia with phlorizin does not normalize glucose metabolism and insulin release in GK rat islets.

ZC Ling, A.Khan, C-G Östenson, S.Efendic. Department of Molecular Medicine, Karolinska Hospital, Stockholm, Sweden.

We previously reported abnormal glucose metabolism and insulin release in islets from GK rats, a non-obese, spontaneous model of NIDDM. To investigate whether these defects are due to hyperglycemia or not, we have normalized the glycemia of GK rats by treatment with phlorizin (phl) dissolved in propylene glycol (pg) for 30 days. I.P. glucose tolerance test after 21 days of treatment showed a normalization of morning blood glucose level in phl-treated but not in pgtreated rats ( $4.03 \pm 0.6$ vs $11.3 \pm 0.9 \mathrm{mM})$. In addition, glucose tolerance was normal in phl-treated rats. Glucose oxidation and utilization (pmol/ngDNA/h) were similar in phl and pg treated GK rat islets at both 5.5 and $16.7 \mathrm{mM}$ glucose (Table). There was no difference in $\mathrm{GO} / \mathrm{GU}$ ratio $(\%)$ between these two groups. Insulin release $(\mu \mathrm{U} /$ islet $/ \mathrm{h}$ ) was also similar in phl and pg-treated GK rats at both 5.5 and $16.7 \mathrm{mM}$ glucose.

\begin{tabular}{lcrcr} 
GK rats & \multicolumn{2}{c}{ phl treated $(\mathrm{n}=8)$} & \multicolumn{2}{c}{ pg treated $(\mathrm{n}=8)$} \\
Glucose & 5.5 & 16.7 & 5.5 & 16.7 \\
GO & $0.44 \pm 0.1$ & $1.32 \pm 0.3$ & $0.48 \pm 0.1$ & $1.32 \pm 0.2$ \\
GU & $1.83 \pm 0.2$ & $3.97 \pm 0.7$ & $2.31 \pm 0.3$ & $4.8 \pm 1.0$ \\
GO/GU & $21.3 \pm 2$ & $30.8 \pm 4$ & $23.1 \pm 2.4$ & $33.5 \pm 5.1$ \\
Insulin & $18.9 \pm 4$ & $34.5 \pm 5$ & $19.9 \pm 2.2$ & $38.8 \pm 5.8$
\end{tabular}

In conclusion, normalization of glycemia by phl did not improve glucose metabolism and insulin release in islets from GK rats. This indicate that the defect in the glucose signalling pathway in the $B$-cell is a primary feature of diabetes in GK rats. 


\section{1}

MECHANISMS INVOLVED IN HUMAN PANCREATIC ISLET GLUCOSE INSENSITIVITY.

A. Andersson1, D.L. Eizirik1,2, M. Flodström1, D.G. Pipeleers2, C. Hellerström1 and L. Jansson1. 1Department of Medical Cell Biology, Uppsala University, Uppsala, Sweden and 2Department of Metabolism and Endocrinology, Vrije Universiteit Brussel and B-cell Transplant, Brussels, Belgium.

We recently showed that human pancreatic islets grafted into alloxandiabetic nude mice, and thereby exposed to a diabetic environment for 4 or 6 weeks, developed a secretory defect that was independent of the islet insulin stores (f Clin Invest 96:721, 1995). In order to investigate this impairment of glucose-induced insulin release more in detail we perfused the graft-bearing kidney $(\approx 300$ human islets per graft) with another insulin secretagogue, $10 \mathrm{mM}$ arginine, 6 weeks after implantation. Furthermore, human islet grafts were harvested from the subcapsular renal space and examined with regard to their rates of (pro)insulin biosynthesis and glucose oxidation. Northern blots were used to estimate the insulin mRNA contents of the transplanted human islets. It was found that the insensitivity to glucose stimulation was accompanied by a similar impairment to acute arginine stimulation. Rates of glucose oxidation of the grafted human islets were decreased $(38 \% ; \mathrm{P}<0.05)$ after exposure to hyperglycemia for 6 weeks, whereas the rates of glucose-stimulated (pro)insulin biosynthesis were unaffected. In support of this, the contents of insulin mRNA were similar in islets exposed to normo- or hyperglycemia. We conclude that the secretory defect of human islets induced by long-term hyperglycemia is not associated with a defect in the biosynthetic machinery of insulin. An impairment of the metabolic handling of the glucose molecule might, however, play a role in this context.

\section{2}

\section{ISLET AMYLOID POLYPEPTIDE AND INSULIN GENE EXPRESSION ARE REGULATED IN PARALLEL BY GLUCOSE IN VIVOIN RATS}

H. Mulder ${ }^{1}$, B. Alurén ${ }^{2}$ and F. Sundler. Departments of Physiology and Neuroscience', Lund, and Medicine, Malmö University Hospital, University of Lund; Sweden.

Islet amyloid polypeptide (IAPP) is a putative islet hormone, that has been implicated in diabetes pathogenesis due to its capacity to form islet amyioid in NDDM and its metabolic effects antagonizing insulin action and release. LAPP is co-stored with insulin in $\beta$-cell granules and, thus, under most conditions released in paraltel with insulin. We have previously shown that IAPP and insulin are differentially expressed under certain conditions, such as experimental diabetes in rodents. To study the role of gluonse in the regulation of IAPP expression and whether it is differentiated from that of insulin, we fasted 15 rats for $48 \mathrm{~h}$. Then, five rats received $0.5 \mathrm{~g}$ glucose ip four times with a $4 \mathrm{~h}$-interval, five rats received $0.5 \mathrm{~g}$ glucose twice with a $4 \mathrm{~b}$ interval and five rats received saline ip four times with a 4 h-interval. In addition, five rats fed ad libitum were given saline four times with a 4 h-interval, thus serving as controls. Plasma glucose was $5.6 \pm 1 \mathrm{mM}$ in fasted rats vs $9.6 \pm 0.6 \mathrm{mM}$ in rats fed ad libitum $(\mathrm{P}<0.001)$, while two and four gluocse injections raised plasma glucose to $8.6 \pm 0.2$ and $11.4 \pm 1 \mathrm{mM}$, respectively ( $\mathrm{P}<0.05$ vs fasted rats for both). IAPP and insulin mRNA levels in islets were determined by quantification of the optical density of in situ hybridization labelling. Fasterl rats reduced their LAPP and insulin mRNA levels to $43 \pm 2$ and $51+3 \%$ of controls, respectively $(\mathrm{P}<0.001$ for both). In rats given two glucose injections, IAPP and insulin mRNA levels were $78 \pm 4$ and $70 \pm 4 \%$ of controls. respectively ( $\mathrm{P}<0.01$ vs fasted rats for both), and in those receiving four glucose injoctions, $85 \pm 4$ and $83 \pm 6 \%$ of controls, respectively $(\mathrm{P}<0.001$ vs fasted rats for both). In a repeated experiment, concomitant administration of actinomycin D blocked the gluoose-induced increase of IAPP gene expression. Thus, in vivo, expression of LAPP and insulin are altered in parallel in fasted rats and in response to gluonse given to such animals, being bighly correlated ( $r=0.97 ; P=0.033$; the effect of glucose is most likely due to increased IAPP and insulin gene transcription, since it was blocked by actinomycin $\mathrm{D}$. The expression of IAPP and insulin therefore appear to be regulated in parallel by gluoose, which is consistent with a role for IAPP in glucose homeostasis. It is likely that in NIDDM, hyperglyoemia will increase IAPP expression and this may be harmful, due to the amyloid-forming capacity and the insulin-antagonizing effects of IAPP.

\section{3}

REDUCTION OF AMYLIN CONTENT IN RAT ISLETS BY ADENOVIRUS-MEDIATED DELIVER OF RAT AMYLIN ANTISENSE cDNA

Novials, A., Jiménez-Chillarón*, J.C., Franco, C., Casamitjana, R., Gomis, R., Guinovart, J.J.* and Gomez-Foix, A.M*. Department of Endocrinology and Diabetes, Hospital Clínic, University of Barcelona. *Departament of Biochemistry and Molecular Biology. School of Chemistry. University of Barcelona. Spain.

An increased production/secretion of IAPP (islet amyloid polypeptide) or alternatively the alteration of IAPP/insulin ratio might be involved in the pathogenesis of NIDDM (non insulin-dependent diabetes). The aim of this work was to test whether amylin $\beta$-cell content could be reduced by the delivery of IAPP antisense CDNA mediated by recombinant adenovinus. We have used recombinant adenovirus containing the $\beta$-galactosidase reporter gene (AdCMV- $\beta \mathrm{Gal}$ ) and viruses including a rat IAPP antisense cDNA (AdCMV- $\alpha$ IAPP). Transduction of freshly isolated rat islets with AdCMV$\beta \mathrm{gal}$, at a moi of 50 , resulted in the expression of $\beta$-galactosidase activity in about $50 \%$ of the islets, 48 -h post-infection. Exposition of islets to AdCMVaIAPP, under the same conditions, caused a 30\%-reduction ( $<<0.001$ ) in the amylin content $(101 \pm 29 \mathrm{fmol} / \mathrm{silet})$ versus untransduced islets $(145 \pm 35$ $\mathrm{fmol} /$ islet), as measured by RIA, whereas transduction with AdCMV- $\beta$ Gal did not cause any significant effect. In the same islets insulin content was also determined, and no significant differences were observed in AdCMV-oIAPP or AdCMV-BGal with respect to untransduced cells. Northern blot analysis showed that IAPP mRNA levels were decreased by $50 \%$ in AdCMV- $\alpha$ IAPP islets compared to controls and that insulin mRNA levels were unchanged. These data suggest that recombinant adenovirus may be a useful tool to mediate the blocking of IAPP expression in islets cells. Therefore, this system might have an application in a potential gene therapy treatment for NIDDM.

\section{4}

EXPOSURE TO HIGH GLUCOSE INCREASES PROINSULIN CONTENT AND RELEASE IN HUMAN ISLETS.

F. Bertuzzi, K. Saccomanno* ${ }^{*}$ C. Socci, C. Berra, M.V. Taglietti, E. Dalcin, G. Pozza and A.E Pontiroli, Istituto Scientifico San Raffaele, and "Institute of Endocrine Sciences, Ospedale Maggiore di Milano IRCCS, Milan, Italy.

Hyperglycemia can impair islet insulin responsiveness to glucose. The aim of this study was to assess, in an in vitro model of hyperglycemia, the effects of a culture in high glucose concentrations on islets content and release of insulin (1) and proinsulin-like molecules (PLM). Human islets from 6 pancreas were cultured overnight in CMRL $1066(10 \%$ FCS, $1 \%$ L-glutamine, $1 \%$ antibiotics), and then were divided into two groups and culfured for 48-h in standard CMRL (control islets), or CMRL at a final glucose concentrations of $16.7 \mathrm{mM}$ (experimental islets). After the 48-h experimental culture, istets were perifused and acutely stimulated for 20 minutes with glucose $16.7 \mathrm{mM}$. followed by glucose $3.3 \mathrm{mM}$ for $20 \mathrm{~min}$. As expected, islets cultured in high glucose released more I under basal conditions than control islets; in addition these islets lost the ability to release $I$ in response to an acute glucose stimulation, and showed a paradoxical I response to low glucose levels. The PLM/l ratio was higher in islets cultured in high glucose than in control islets, after high glucose $\left(0.47 \pm 0.10\right.$ vs $0.23_{ \pm} .04$ of control islets; $\left.n=12 ; p<0.05\right)$, and low glucose $(0.92 \pm 0.24$ vs $0.24 \pm .04$ of control islets; $n=8 ; p<0.05)$. By electron microscopy a morphologic analysis showed an increased ratio of $\mathrm{Pl}$ to 1 -containing granules $(0.4 \pm 0.04$ in control islets and $1.2 \pm 0.19$ in test islets: $\mathrm{p}<0.01$ ). Morphological analysis were confirmed by a double gold immunolabelling using specific monoclonal antibodies against pro-insulin (P) or insulin (I). The evidence that culture in high glucose can increase the content and release of PLM and Pl by isolated human islets suggests that a high PLM is a secondary effect of increased secretory demand on $\beta$ cells. 
485

DIFFERENTIAL EFFECT OF CHRONIC HIGH GLUCOSE ON ISLET PROINSULIN IN DIABETES- PRONE AND -RESISTANT ANIMALS

N. Kaiser, G. Leibowitz, E. Cerasi and D.J. Gross. Dept. of Endocrinology Metabolism, Hebrew Univ.-Hadassah Medical Ctr., Jerusalem, ISRAEL

Patients with Type 2 diabetes show increased relative levels of plasma proinsulin and proinsulin-conversion intermediates, which are corrected by strict glycaemic control. This suggests that glucose per se may be implicated in the generation of the aberrant secretory profile. The question remains, however, whether the presence of intrinsic $\beta$-cell defects underlie the failure of proinsulin processing upon a prolonged increase in secretory demand. The study was conducted in vitro in cultured islets from the diabetes-prone Psammomys obestus and normal diabetes-resistant rats. Islet monolayer cultures were exposed to RPMI 1640 medium ( $11.1 \mathrm{mM}$ glucose), or to medium containing $33.3 \mathrm{mM}$ glucose; insulin-related peptides (IRP) were resolved by HPLC and analyzed by insulin RIA. Prolonged exposure (10-14 days) of rat islets to high glucose resulted in 30-50\% reduction in insulin content without increase in the proinsulin/insulin ratio. When subjected to a similar protocol, islets from pre-diabetic Psammomys reduced their insulin content by $50 \%$ within 3 days of exposure to high glucose and increased the relative level of proinsulin-related peptides (PRP) -2 fold. In the in vivo situation, pancreatic extracts from non-fasted diabetic Psammomys contained $36 \pm 4 \%$ PRP in contrast to $17 \pm 3 \%$ and $<10 \%$ in pancreatic extracts of pre-diabetic Psammomys and rats, respectively. Thus, prolonged exposure of pre-diabetic Psammomys islets in vitro to high levels of glucose could reproduce the modified $\beta$-cell IRP profiles observed in vivo in the diabetic animal. These results support the hypothesis that hyperproinsulinaemia is secondary to the inadequate ability of $\beta$-cells of diabetes-prone individuals to meet a sustained increase in insulin demand whereas normal individuals may tolerate a similar increase in demand without changing the profile of $\beta$-cell peptides.

\section{6}

HEXOKINASE SHIFT TO MITOCHONDRIA IS ASSOCIATED TO THE INCREASED SENSITIVITY TO GLUCOSE OF PANCREATIC ISLETS EXPOSED TO HIGH GLUCOSE

M.Anello, AM. Rabuazzo, G. Patanc̀, R.Vigneri and F.Purrello. Instilute of Int. Medicine, Endocrinology and Metabolism, Univ. of Catania, Italy.

Rat pancreatic islets chronically cultured at high glucose $(16.7 \mathrm{mM})$ become desensitized to a further glucose stimulation. In vivo, before desensitization, an increased sensitivity to glucose may be observed. To better understand the mechanism of this effect, we incubated rat pancreatic islets in CMRL medium containing 5.5 or $16.7 \mathrm{mM}$ glucose and then measured insulin release in static incubation. Islets exposed for $3 \mathrm{~h}$ to Gl6.7 had an increased sensitivity to glucose, as indicated by a shift to the left of the glucose dose-response curve ( $\mathrm{EC}_{50} 6.0 \pm 09$ vs. 11.6 \pm 1.2 , in islets exposed to $G 16.7$ or $G 5.5$, respectively; $n=5, p<0.05$ ). We then investigated in these islets the glucose phosphorylating activity, a major component of the glucose sensor of B cells. The low-Km phosphorylating activity

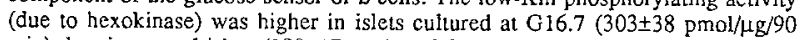
min) than in control islets $(180 \pm 17, n=4, p<0,05$ ). Since hexokinase, upon binding 10 mitochondria is more active and less susceptible to inhibition by glucose-6phosphate, we measured mitochondrially bound hexokinase activity and found that it was increased in islets cultured at G16.7 (123 \pm 3 vs $58 \pm 10 \mathrm{pmol} / \mu \mathrm{g} / 90 \mathrm{~min}$, $p<0.01)$. Furthermore, the mitochondrial/cytosolic distribution of the enzyme was altered after islet exposure to high glucose, the mitochondrially bound enzyme being increased to $47 \pm 6 \%$ vs. $19 \pm 4 \%$ in control islets $(p<0.01, n=3)$. When, during islet exposure to G16.7, hexokinase binding to mitochondria was inhibited by the addition of $20 \mathrm{nM}$ dicyclohexylcarbodiimide (DCC), no increase of sensitivity to glucose was observed (EC50 10.9 $\pm 04, n=3$, similar to that of control islets). In contrast to the low- $\mathrm{Km}$, hexokinase-dependent phosphorylation, the high- $\mathrm{Km}$ glucose phosphorylating activity (due to glucokinase) was similar in islets cultured at $\mathrm{G} 16.7$ or $\mathrm{G} 5.5$ ( $87 \pm 15$ vs. $76 \pm 8 \mathrm{pmol} / \mathrm{\mu g} / 90 \mathrm{~min}$, respectively, $\mathrm{n}=4)$.

In conclusion, the present data confirm in a fully in vitro system that an increased sensitivity to glucose precedes pancreatic islets glucose desensitization. When islets become more sensitive to glucose, their mitochondrially bound hexokinase activity increases. The increased secretory activity of islets exposed at G16.7 in response to glucose concentrations lower than the normal secretory threshold may be, therefore, a consequence of the cell hexokinase shift from the cytosolic to the more active mitochondrially bound form.

\section{7}

AN ALDOSE REDUCTASE INHIBITOR PARTIALLY PREVENTS CHRONIC HIGH GLUCOSE-INDUCED IMPAIRMENT OF HIT CELL FUNCTIONS M.Kishimoto, Y.Kajimoto, H.Watada, T.Matsuoka, T.Watarai, M.Kubota and Y.Yamasaki First Department of Medicine, Osaka University School of Medicine, Osaka, Japan.

Chronic exposure of HIT cells to high glucose conditions has been shown to lead to decreased insul in mRNA, insulin content and insulin secretion. These changes can be prevented by culturing the cells under low glucose conditions. In this study, we sought to determine whether such glucose-induced changes imposed upon HIT cells is associated with alteration in polyol pathway or not. Using innovative trifluoroacetyl derivatization and gas chromatography / mass spectrometry methods, we identified the polyol synthesizing activity in HIT cells. The polyol synthesizing activity in the cells, which revealed high $\mathrm{Km}$ for glucose (Km: $1450 \pm 330 \mathrm{mM}, V \max : 330 \pm 58 \mathrm{pmol} / \mathrm{min} / \mathrm{mg}$ protein) was almost completely blocked by SNK860(0.1 mM), an aldose reductase inhibitor. HIT cells kept for 5 weeks under $11.1 \mathrm{mM}$ glucose revealed impairment of glucose responsive insulin secretion and low insulin content. However, when cells were cultured with $0.1 \mathrm{mM}$ SNk860, the impaired responsiveness to glucose was recovered (41.2 to $62.9 \mathrm{pmol} / \mathrm{min} / \mathrm{mg}$ protein at $2 \mathrm{mM}$ glucose) and the insul in content was increased significantly. These data strongly suggested that impaired cell function of HIT cells kept under high glucose conditions is relevant to the polyol pathway. The effectiveness of aldose reductase inhibitor provides support for the potential usefulness of this agent in preventing beta cell glucose toxicity observed in hyperglycemic NIDDM cases.

\section{8}

DIFFERENTIATION OF GLUCOSE TOXICITY FROM BETA CELL EXHAUSTION IN THE PANCREATIC ISLET CELL LINE, HIT-T! 5 Moran A, Zhang, H-J, Olson LK, Poitout V, and Robertson RP. University of Minnesota, Department of Medicine, Minneapolis. Minnesota.

We have previously shown that chronic culture of HIT-T 15 cells in high glucose concentration leads to decreased insulin secretion, content, mRNA levels, gene transcription, and two important transcription factors, STF- 1 and RIPE-3bl activator. All of these effects are prevented by chronically culturing cells in low concentration. We postulated glucose toxicity rather than beta-cell exhaustion as the mechanism for these findings. Wo designcd two experiments to differentiate these two possibilities: 1) Late passage (p99) HIT cells which were begun at p72 and had lost insulin gene expression during prolonged culture in $11.1 \mathrm{mM}$ glucose were switched to $0.8 \mathrm{mM}$ glucose and carried on to p146. 2) HIT cells were cultured in $0.8 \mathrm{mM}$ or $11.1 \mathrm{mM}$ glucose with and without $10^{-6} \mathrm{mM}$ somatostatin (SRIF), a potent inhibitor of insulin secretion, from $\mathrm{p} 74$ to $\mathrm{p} 100$. In the first experiment, insulin mRNA expression returned to $10-15 \%$ of control levels by p 125 . In the second experiment. SRIF successfully suppressed insulin levels in cells grown in $11.1 \mathrm{mM}$ glucose, thereby preventing beta cell exhaustion. Insulin mRNA leve's in HIT cells cultured under the four experimental conditions were compared by slot blot analysis and insulin gene promoter activity was measured by transient transfections with a CAT reporter gene. Insulin mRNA abundance and insulin gene promoter activity were both markedly decreased in cells grown under high glucose conditions with and without SRIF compared to cells grown under low glucose conditions $(n=4, p 92-94)$ :

$\begin{array}{lcc} & \text { Insulin mRNA/ } \beta \text {-actin RNA } & \text { INSCAT/RSVCAI } \\ 0.8 \mathrm{mM} \text { glucose } & 0.46 \pm 0.06 & 0.50 \pm 0.11 \\ 0.8 \mathrm{mM} \text { glucose }+ \text { SRIF } & 0.51 \pm 0.03 & 0.54 \pm 0.12 \\ 11.1 \mathrm{mM} \text { glucose } & 0.08 \pm 0.00^{*} & 0.16 \pm 0.01^{*} \\ 11.1 \mathrm{mM} \text { glucose }+ \text { SRIF } & 0.22 \pm 0.02^{*} & 0.17 \pm 0.02^{*}\end{array}$

p $<0.0001$ compared to $0.8 \mathrm{mM}$ glucose

These data demonstrate that the effects induced by prolonged culture of HIT cells in supraphysiologic glucose concentrations are not only preventable by culturing cells in low glucose, but also are partially reversible. Since the evolution of this defect in insulin gene expression is not prevented by chronic inhibition of insulin secretion with SRIF, glucose toxicity rather than beta cell exhaustion is likely as the mechanism for the defect in insulin gene expression. 
489

LDL-BINDING AND UPTAKE BY ISLET $\beta$ CELLS.

A. Grupping, M. Cnop, C. Van Schravendijk, T. Van Berkel* and D. Pipeleers. Diabetes Research Center, Vrije Universiteit Brussel, Belgium. *Division of Biopharmaceutics, Sylvius Laboratory, University of Leiden, The Netherlands.

Abnormalities in lipoproteins are common in diabetes. It is unknown whether variations in form or concentration of lipoproteins influence the function of pancreatic $\beta$ cells. This study investigates whether low density lipoproteins (LDL) interact with rat and human $\beta$ cells. Islet cell preparations were examined for the presence of LDL-receptors. At $4^{\circ} \mathrm{C}$, binding of ${ }^{125} \mathrm{I}-\mathrm{LDL}$ to human cells was characterized by a $\mathrm{K}_{d}$ of $(4.8 \pm 0.5) \mathrm{nM}$ and a receptor number of $2 \mathrm{x}$ $10^{4}$ sites per cell; rat islet cells exhibited a higher $\mathrm{K}_{d}((9.4 \pm 2.1) \mathrm{nM})$ and higher receptor number $\left(2 \times 10^{5}\right.$ sites per cell). Binding of LDL to islet cells is representative for that to purified $\beta$ cells; no significant binding level was detected on endocrine non- $\beta$ cells. At $37^{\circ} \mathrm{C}$, the cells were found to incorporate and degrade $125 \mathrm{I}-\mathrm{LDL}$ in a chloroquine $(50 \mu \mathrm{M}-100 \mu \mathrm{M})$ and ammonium chloride $(10 \mathrm{mM})$ sensitive compartment, presumably the lysosomes. Uptake of LDI was also demonstrated with fluorescently labeled DiI-LDL. The presence of very low density lipoproteins (VLDL) competed with LDL binding and uptake; this was not the case for high density lipoproteins (HDL) or acetylated low density lipoproteins (AcLDL). We conclude that human and rat islet $\beta$ cells express LDL-receptors with high affinity and specificity for their ligand. Further work should assess to which extent uptake of LDL (dys)regulates the pancreatic $\beta$ cells.
490

\section{LDL-TOXICITY IN RAT $\beta$ CELIS.}

M. Cnop, A. Grupping and D. Pipeleers Diabetes Research Center, Vrije Universiteit Brussel, Belgium.

Human and rat $\beta$ cells exhibit low density lipoprotein (LDL)-receptors which appear responsible for the uptake of the lipoproteins. The present study examines whether exposure to LDL reduces the viability of rat islet $\beta$ cells. Culture during 48 hours resulted in a dosedependent cytotoxicity in single $\beta$ cell preparations, reaching 100 percent cell death at $10 \mu \mathrm{g}$ LDL protein $/ \mathrm{ml}$. Only a minor toxicity was observed in suspended $\beta$ cell aggregates or islets. Cell death was prevented by the simultaneous presence of antioxidative compounds (glutathion, vitamins A, C, and E and dithiothreitol), EDTA, butylated hydroxytoluene (BHT) and Probucol. The cytotoxic action of LDL was associated with an increase in its negative charge and TBARS content, indicating the occurrence of oxidative reactions. These particle modifications were not observed in the presence of Probucol, BHT and vitamin C. Oxidation of LDL prior to culture with $\beta$ cells did not reproduce the LDL-cytotoxicity suggesting that LDL intermediates or unstable side products are responsible for the cytotoxic effects rather than the oxidized end-product. It is concluded that LDL can be toxic for rat islet $\beta$ cells during its oxidative modification.

\section{PS 14}

\section{Transplantation and Beta-Cell Engineering}

491

RENAL HEMODYNAMICS IN KIDNEY AND PANCREAS TRANSPLANTED PATIENTS. ROLE OF KIDNEY MASS REDUCTION AND CYCLOSPORINE

E. Bianchi, L. Luzi, A. Secchi, E. La Rocca. D. Ciurlino, E. De Pascaie, M. Giordano C. Pluvio, P. Castellino, G. Pozza. Departmenes of Medicine San Raphael Hospital, Milano, Napoli, Catania, Italy.

The regulation of renal hemodynamics in kidney and pancreas transplanted patients is not completely understood yet. Aim of the study was to study renal hemodynamics in basal state and after aminoacid i.y. infusion (Solamin Forte $7.5 \%$, Pierrel, 3.3 $\mathrm{mg} / \mathrm{Kg} / \mathrm{min}$ lasting $150 \mathrm{~min}$ ) to measure renal functional reserve in: I) 7 kidney and pancreas transplanted patients (KPTx) ( $35 \pm 5$ years, $21 \pm 1 \mathrm{BM}, 1.26 \pm 0.5 \mathrm{mg} / \mathrm{dl}$ serum creatinine, $23 \pm 8$ months from transplantation); II) 16 kidney transplanted patients (KTx) (38 \pm 3 years, $25 \pm 3 \mathrm{BMI}, 1.25 \pm 0.1 \mathrm{mg} / \mathrm{dl}$ serum creatinine, $23 \pm 4$ months from transplantation): III) 7 Cyclosporine $A$ treated patients for chronic uveitis (CsA) ( $38 \pm 3$ years, $30 \pm 3 \mathrm{BMI}, 1.05 \pm 0.04 \mathrm{mg} / \mathrm{dl}$ serum creatinine, $5 \pm 2$ months of treatment): IV) 6 patients who had a kidney removed for various reasons $(\mathrm{Nx})(45 \pm 6$ years, $30 \pm 3$ BMI, $1.02 \pm 0.1 \mathrm{mg} / \mathrm{dl}$ serum creatinine, $82 \pm 16$ months from nephreciomy); V) 9 healthy voluriteers (C) (34 \pm 4 years, $25 \pm 3$ BMI, $0.6 \pm 1.2 \mathrm{mg} / \mathrm{dl}$ serum creatinine). During hyperaminoacidemia, GFR increased in $C$ (from $80 \pm 6$ to $91 \pm 6 \mathrm{ml} / \mathrm{min}$ ) and $\mathrm{Nx}$ (from $57 \pm 3$ to $68 \pm 7 \mathrm{ml} / \mathrm{min}$ ) (P<0.01 vs. basal), while did not change significantly in KPTx (from $48 \pm 9$ to $48 \pm 2 \mathrm{ml} / \mathrm{min}$ ), KTx (from $39 \pm 3$ to $37 \pm 3 \mathrm{ml} / \mathrm{min}$ ) and CsA (from $58 \pm 3$ to $53 \pm 4 \mathrm{ml} / \mathrm{min}$ ). Similarly RPF did not change in KPTx (from $175 \pm 35$ to $172 \pm 25 \mathrm{ml} / \mathrm{min}$ ) and KTx (from $243 \pm 27$ to $235 \pm 25$ $\mathrm{ml} / \mathrm{min}$ ) while it increased significantly in other groups (from $257 \pm 17$ to $344 \pm 33$ $\mathrm{ml} / \mathrm{min}$ in $\mathrm{Nx}$, from $364 \pm 61$ to $441 \pm 55 \mathrm{ml} / \mathrm{min}$ in $\mathrm{CsA}$ and from $412 \pm 49$ to $472 \pm 72$ $\mathrm{ml} / \mathrm{min}$ in $\mathrm{C})$. Renal vascular resistance (RVR) was higher in KPTx $(0.38 \pm 0.09$ $\mathrm{mmHg} / \mathrm{ml} \cdot \mathrm{min})$ and in $\mathrm{KTx}(0.29 \pm 0.04 \mathrm{mmHg} / \mathrm{ml} \cdot \mathrm{min})$ than in $\mathrm{Nx} \quad(0.21 \pm 0.01$ $\mathrm{mmHg} / \mathrm{ml} \cdot \mathrm{min}), \mathrm{CsA}(0.23 \pm 0.04 \mathrm{mmHg} / \mathrm{ml} \cdot \mathrm{min})$ and $\mathrm{C}(0.13 \pm 0.02$ mmHg/ml-min).

These data demonstrate that in kidney and pancreas transplanted patients RFR is lacking and renal hemodynamics is similar in these patients and in kidney transplanted patients. These results seem to be related to CsA treatment and not to the reduction of renal mass.

\section{2}

METABOLIC CHARACTERIZATION OF SUCCESSFUL PANCREAS TRANSPLANTS IN TYPE I DIABETES.

RP Robertson, DER Sutherland, and DM Kendall, University of Minnesota, Departments of Medicine and Surgery, Minneapolis, MN.

Pancreas transplantation is the most consistently reliable method of achicving normal glycemia and normal $\mathrm{HbAlc}$ levels in Type I diabetic patients. Although ample documentation of the immediate beneficial metabolic effects has been reported, no published information exists that addresses their long-term stability. To ascertain long-term stability of metabolic function of 96 recipients, cross-sectional data obtained through five years post-transplantation were analyzed. Values $=\overline{\mathrm{x}} \pm \mathrm{SE}$

\begin{tabular}{l|c|c|c|c} 
Yr Post-Transplant & 1 & 2 & $31 / 2$ & 5 \\
\hline Insulin response & $135 \pm 15$ & $123 \pm 13$ & $111 \pm 15$ & $116 \pm 16$ \\
to glucose, $\mu \mathrm{U} / \mathrm{m}]^{\mu}$ & $\mathrm{n}=48$ & $\mathrm{n}=39$ & $\mathrm{n}=22$ & $\mathrm{n}=23$ \\
\hline C-peptide response & $1.13 \pm 0.11$ & $0.98 \pm 0.14$ & $0.90 \pm 0.16$ & $1.30 \pm 0.25$ \\
to glucose, nmol/L & $\mathrm{n}=41$ & $\mathrm{n}=31$ & $\mathrm{n}=14$ & $\mathrm{n}=17$ \\
\hline Glucagon response & $292 \pm 22$ & $293 \pm 26$ & $302 \pm 32$ & $281 \pm 29$ \\
to arginine, $\mathrm{pg} / \mathrm{m} \mathrm{l}^{\mathrm{c}}$ & $\mathrm{n}=44$ & $\mathrm{n}=36$ & $\mathrm{n}=18$ & $\mathrm{n}=22$ \\
\hline FPG, $\mathrm{mg} / \mathrm{dl}$ & $85 \pm 1$ & $84 \pm 2$ & $87 \pm 2$ & $88 \pm 2$ \\
& $\mathrm{n}=50$ & $\mathrm{n}=37$ & $\mathrm{n}=20$ & $\mathrm{n}=24$ \\
\hline HbAlc, \% (nl<6.0) & $5.3 \pm 0.1$ & $5.3 \pm 0.1$ & $5.4 \pm 0.1$ & $5.2 \pm 0.1$ \\
& $\mathrm{n}=58$ & $\mathrm{n}=48$ & $\mathrm{n}=23$ & $\mathrm{n}=24$ \\
\hline Normal control subjects: $\mathrm{a}=58 \pm 6, \mathrm{n}=19 ;$ & $\mathrm{b}=1.06 \pm 0.10$,
\end{tabular}

Normal control subjects: $\mathrm{a}=58$
$\mathrm{n}=15 ; \mathrm{c}=156 \pm 23, \mathrm{n}=18$

In addition to these cross-sectional data, prospective data over the five year period in patients receiving at least two and up to five separate assessments revealed no downward trend in metabolic function or maintenance of normal glucose levels in patients who maintained their graft. Conclusion: Successful pancreas transplantation provides pancreatic islet function that results in normal or nearly normal glycemic control for up to five years post-operatively in Type I diabetic recipients receiving no exogenous insulin or oral hypoglycemic agent therapy. 
493

METABOLIC EFFECTS OF ISLET GRAFT: COMPARISON WITH SEGMENTAL AND WHOLE PANCREAS GRAFT

A Secchi, MV Taglietti, P Maffi, C Socci, R Caldara, E La Rocca, F Bertuzzi, G Ferrari, R Castoldi, L Piemonti, P De Nittis, V Di Carlo and G Pozza.

San Raffaele Scientific Institute, University of Milan, Milan, Italy Endocrino-metabolic responses to insulinogenic stimuli were studied in patients undergoing intrahepatic islet transplantation ( $T x)$, segmental or whole pancreas Tx. Patients: I Tx group: 2 IDDM patients who received islet and kidney $(\mathrm{K})$ tx (clinical effects: 7 cases: insulin independence, 2 cases: reduction of insulin requirement, 3 cases: serum $C$. Peptide $>1 \mathrm{ng} / \mathrm{ml}$ ). S Tx group: 16 IDDM patients submitted to segmental pancreas and $\mathrm{K} t x . \mathrm{W}$ Tx group: 16 patients submitted to whole pancreas and K Tx. Methods: i.v. Glucose Tolerance Test (IVGTT, $0.5 \mathrm{~g} . / \mathrm{kg}$ ), and Arginine infusion test $(30 \mathrm{~g}$. over $30 \mathrm{~min}$, Area under the curve -Delta AUC- for serum insulin -IRI- calculated with the trapezoidal method over $60 \mathrm{~min}$ ). Results: IVGTT 3-6 mos: IRI peak was achieved at the first minute after glucose infusion in all the groups. It was $121 \pm 18(\mathrm{~W}), 40 \pm 6(\mathrm{~S})$ and $16 \pm 5 \mathrm{uU} / \mathrm{ml}$ (I: 5 patients). IVGTT 3 yrs: IRI peak was maintained in the pancreas transplanted groups $(104 \pm 17,43 \pm 12$, respectively), while it was lost in the I pts $(6 \pm 5,2 \mathrm{pts})$, despite evidence of good islet function (HbA1c: 3.4 and 6.3\%, respectively). Arginine test, 3-6 mos: the IRI peak at 5 minutes was $57 \pm 8(\mathrm{~W}), 37 \pm 7$ (S) and $34 \pm 4$ (I) $\mathrm{uU} / \mathrm{ml}$. The second phase of insulin secretion during arginine infusion $(30 \mathrm{~min})$ was present in all the patients (W: $69 \pm 10, \mathrm{~S}: 41+7$, I: $37+5 \mathrm{uU} / \mathrm{ml}$ ). Delta AUC was lower although not statistically, in I tx $(1192 \pm 224 \mathrm{uU} / 60 \mathrm{~min})$ than in $\mathrm{S}$ and $W$ groups $(1414 \pm 307$ and $2097 \pm 1052 \mathrm{uU} / 30 \mathrm{~min}$, respectively). Arginine test, 2 yrs: the IRI peak was maintained (S: $36 \pm 7, \mathrm{I}$ tx: $32 \pm 6 \mathrm{uU} / \mathrm{ml}$ ) in both groups. Delta AUC was lower in I tx than in S tx $(720 \pm 182$ vs $1303 \pm 315$ $\mathrm{uU} / 30 \mathrm{~min}$ ). Conclusions; in conclusion a prompt insulin release after i.v. glucose or arginine is observed 3-6 mos after islet transplantation. After 23 years it is maintained only after Arginine, while it is lost after i.v. glucose, despite good metabolic function.

495

BONE AND MINERAL METABOLISM IN IDDM PATIENTS AFTER SUCCESSFUL PANCREAS-KIDNEY TRANSPLANTATION

Y.F.C.Smets, J.W. vd Pijl, J. Ringers, J.W. de Fijter, H.H.P.J. Lemkes and N.A.T. Hamdy. University Hospital, Leiden, The Netherlands.

Low bone turnover is prevalent in IDDM patients in whom bone mass is reported to be reduced. Simultaneous pancreas-kidney transplantation (SPK) successfully restores normoglycaemia and corrects uraemia, but little is known about its long-term effects on bone and mineral metabolism particularly in view of the polential deleterious effects of the relatively higher doses of corticosteroids used on bone. To address this question, we studied 27 patients (19 male and 8 female), at least 12 months after successful SPK (max. 99 months). Age at the time of SPK: $40 \pm 8$ years, duration of diabetes 24.5 56 years and mean $\mathrm{HbA}_{1} \mathrm{C} 8.5 \% .13$ of the 27 patients required renal replacement therapy before SPK (HD:7; CAPD: 6). At a median time of 34 months after SPK, all patients were insulin-independent and mean creatinine clearance was $62 \pm 22 \mathrm{ml} / \mathrm{min}$ with 6 patients having clearances of $<50$ but $>25 \mathrm{ml} / \mathrm{min}$. Serum calcium was normal in all patients but 4 ( 2 decreased and 2 mildly increased), phosphate was normal in all patients and there was a mild increase in alkaline phosphatase activity without a concommitant increase in liver enzymes in 5 patients. Serum osteocalcin was increased in 9 of the 20 patients in whom it was measured. There was no increase in the $24 \mathrm{hr}$ urinary excretion of calcium or hydroxyproline. There was a significant relationship between degree of renal impairment and serum PTH, the mean value of which was $8.4 \pm 5.8 \mathrm{pmol} / \mathrm{l}$ (normal $<6.5 \mathrm{pmol} / \mathrm{A}$ ). Bone mass was measured using dual energy $\mathrm{X}$-ray absorptiometry (DEXA). At the lumbar spine, 8 patients had normal $t$-scores, 11 had $t$-scores between -1SD and -2.5SD and 8 had tscores $>-2.5 \mathrm{SD}$. At the femoral neck, all patients had a $t$-score of $<-1 \mathrm{SD}$, with 18 showing $\mathrm{t}$-scores of $<-2.5 \mathrm{SD}$ suggesting a more marked cortical than trabecular cancellous bone loss in these patients, contrary to the expected had this bone loss been solely induced by corticosteroid therapy. Our findings suggest that trabecular bone loss is less prevalent than expected after successful pancreas-kidney transplantation, and that the cortical bone loss frequently reported in IDDM patients before transplantation persists after successful establishment of adequate pancreatic and renal function, possibly exacerbated by the presence of persistent hyperparathyroidism.

\section{4}

EFHCT OF COMIBINED KIDNLY-PINCREAS-TRANSPLATATION ON TISSE OXYGEN TENSION IN SKE] ET AL MLSCLE OF TYPE-F-DI.ABETIC P.TTIENTS

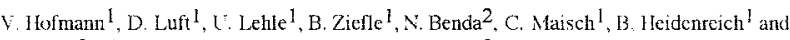
$\therefore$. Hopt ${ }^{3}$. ${ }^{1}$ Department of Internal Medicine IY, ${ }^{2}$ Institute of Medical Biometrics, ${ }^{3}$ Department of Surgery, Eberhard-Karls-Lniversity, Tuebingen, Germany

Combined kidney-pancreas-transplantation ( $\mathrm{KP} \times \mathrm{Tx})$ in type-[-diabetic patients with end-stage diabetic nephropathy improves metabolic control and quality of life. The beneficial effect on advanced late complications is not evident. To investigate the effect on skeletal muscle microcirculation we used polarography to measure tissue oxygen tension $\left(\mathrm{tpO}_{2}\right)$ in the anterior tibial muscle at rest, during a cold-pressor-test and after nifedipine $10 \mathrm{mg} \mathrm{p.0.}$ ingestion. We compared 10 patients before KPTx to 12 patients $4,3 \pm 1,8$ years after successful KPTX. Both groups had comparable mean age, gender distribution, body-mass-index, duration of diabetes and hemodialysis before transplantation, smoking habits, severity of retinopathy, macroangiopathy, peripheral and autonomic neuropathy and oxygen tension in arterialised capillary blood. At rest tpO2 in patients before KPTx $(25,8 \pm 9,1 \mathrm{mmH})$ did not differ significantjy from $\mathrm{tpO}_{2}$ in patients after $\mathrm{KPTx}(25,6 \pm 7,4 \mathrm{mmHg}$. $\mathrm{p}=0,97)$ and was comparable to $\mathrm{tpO}_{2}$ in patients with short duration of diabetes $(28,3 \pm 10,4 \mathrm{mmHg})$ and in normal controls $(28,6 \pm 4,9 \mathrm{mmHg}: \mathrm{p}=0,47)$. During the cold-pressor-test ssstolic biond pressure and $\mathrm{tpO}_{2}$ rose significantly in both groups: before $\mathrm{KPTx} \mathrm{RR}_{\mathrm{rest}}=1+9 \pm 24 \mathrm{mml}$. $\mathrm{RR}_{\mathrm{cpt}}=160 \pm 30 \mathrm{mmHg}(\mathrm{p}=0,006), \mathrm{tpO}_{2 \mathrm{rest}}=25,8 \pm 9,1 \mathrm{mmHg}, t \mathrm{t}_{2 \mathrm{cpt}}=33,0 \pm 9,5 \mathrm{mmlg}$ $(\mathrm{p}=0,001)$, and after $\mathrm{KPTx} R R_{\text {rest }}=147 \pm 22 \mathrm{mmHg} . R_{\mathrm{cpt}}=163 \pm 24 \mathrm{mmHg}(\mathrm{p}=0,0003)$, $\mathrm{tpO}_{2 \mathrm{rest}}=25,6 \pm 7,4 \mathrm{mmHg}, \mathrm{tpO}_{2 \mathrm{cpt}}=31,7 \pm 7,8 \mathrm{mmHg}(\mathrm{p}=0,013)$. After nifedipine ingestion diastolic blood pressure fell significantly in both groups: before $\mathrm{KPTx}^{\mathrm{R} R} \mathrm{rest}_{\text {res }}=88 \pm 13 \mathrm{mmHg}$, $\mathrm{RR}_{\text {nifedipine }}=80 \pm 10 \mathrm{mmHg}(\mathrm{p}=0,006)$ and after $K P T x \quad R R_{\text {rest }}=86 \pm 11 \mathrm{mmHg}$. $\mathrm{RR}_{\text {nifedipine }}=73 \pm 9 \mathrm{mml} \mathrm{g}(\mathrm{p}=0,0001)$. But no nifedipine-induced $\mathrm{pO}_{2}$ increase was detected before KPTx $\left(\Delta t \mathrm{PO}_{2}=3,2 \mathrm{mmHg}, \mathrm{p}=0,12\right)$ or after KPTx $(\Delta \mathrm{tpO}=4,2 \mathrm{mmlg}, \mathrm{p}=0,07)$ as opposed to normal controls ( $\Delta \mathrm{tpO}_{2}=7.7 \mathrm{mmHg}$ ) and diabetic patients with short duration of disease ( $\Delta \mathrm{tpO}_{2}=8,3 \mathrm{mmHg}$ ). Conclusion: Despite normal values at rest tpO $\mathrm{O}_{2}$ in patients with long-standing diabetes did not increase significantly after nifedipin ingestion. This indicates an impaired myogenic vasodilatation that has not been restored $4,3 \pm 1,8$ years after KPTx.

\section{6}

PANCREATIC VENOUS THROMBOSIS IN SIMULTANEOUS KIDNEY AND PANCREAS TRANSPLANTATION: COAGULATION STUDY IN 26 TYPE I DIABETIC PATIENTS.

L. Kessler ${ }^{*}$, M.L. Wiesel ${ }^{* *}, K$. Boudjema***, J.P. Cazenave ${ }^{* *}, L$. Grunebaum $^{\star *}$, Ph. Wolf ${ }^{\star \star *}, \mathrm{Ph}$. Réville* and ' $M$. Pinget*. * Service d'Endocrinologie, Hôpital Central. ** Service d'Hémostase ef E.T.S. *** Service de Transplantation, 67000 Strasbourg, FRANCE

The principal causes of failure of a pancreas transplant are rejection and vascular thrombosis. Thus the aim of this work was to compare coagulation and fibrinolysis in groups of kidney and pancreas transplanted type 1 diabetic patients having or not experienced thrombosis of their pancreatic graft vein. From December 1990 to June 1995, simultaneous kidney and duodenopancreas transplantation with bladder drainage was performed in 26 uremic type 1 diabetic patients (age $=38 \pm 6.7$ years, duration of diabetes $=23.6 \pm 5.9$ years). During the first week, methylprednisolone, azathioprine and antilymphocyte serum are given as immunosuppressive therapy. Acute thrombosis of the pancreatic vein was observed among 5 patients during the first postoperative day (group A) whereas 21 patients did not develop thrombosis (group B). In each group, at least 6 months after transplantation, we analyzed : haemostatic factors (fribrinogen, thrombin time, prothrombin time), coagulation inhibitors (C and $S$ proteins), fibrinolysis (plasminogen inhibitor activator) and endothelial cell abnormalities (Von Willebrand factor). Micro and macro vascular complications were evaluated using a score ranging from 0 to 12 . Haemostatic factors, coagulation inhibitors, and fibrinolysis were similar in the two groups. In contrast, Von Willebrand factor differed significantly: group $A: 3.29 \pm 1.15$ versus group $B 2.07 \pm 1 \mathrm{Ul} / \mathrm{ml}(p<0.05)$. Though the vascular score increased in both groups, it was significantly higher in group A $(8 \pm 0.94)$ than in group $B(4.87 \pm 1.3), p<0.001$. Furthermore, Von Wil!ebrand factor and vascular score were highly correlated in group $A(R=0.939)$. Conclusion : Von Willebrand factor was significantly increased in kidney-pancreas transplantated type 1 diabetic patients presenting pancreatic thrombosis of the graft and exibited a strong correlation with the severity of vascular complications. 
497

EFFECT OF LONG-TERM GLUCOSE NORMALIZATION ON LASER DOPPLER BLOOD FLOW IN IDDM PATIENTS

S. Dachauer, N. Eberl, W. Piehlmeier and R. Landgraf Dept. of Internal Medicine "Innenstadt", University of Munich, Germany

To assess the effect of a long-term glucose normalization on peripheral blood flow a cross-sectional study was started. 23 IDDM patients with severe secondary complications were enrolled: Group $\mathbf{A}(n=7)$ : patients prior to combined pancreaticrenal grafting (age $39.1 \pm 1.5 \mathrm{yrs}$, duration of diabetes $21.8 \pm 1.0 \mathrm{yrs}$, duration of dialysis $16.3 \pm 8.8 \mathrm{~m}$, HbA1c $7.1 \pm 0.3 \%)$; Group $B(n=11)$ : patients after successful simultaneous pancreas/kidney grafting (age $42.7 \pm 2.5$ yrs; duration of diabetes $26.5 \pm 1.1 \mathrm{yrs}$, duration of dialysis $29.9 \pm 7.1 \mathrm{~m}$, time since transplantation $54.6 \pm 11.2$ $\mathrm{m}$, HbAlc $5.0 \pm 0.3 \%$ ); Group C ( $=5)$ : posttransplant patients with functioning kidney graft, under insulin therapy (age $31.4 \pm 1.2 \mathrm{yrs}$, duration of diabetes $22.6 \pm 3.4$ yrs, duration of dialysis $30.4 \pm 6.1 \mathrm{~m}$, duration of renal graft functioning $60.6 \pm 16.4 \mathrm{~m}$, HbAlc $7.0 \pm 0.5 \%$ ). Data so far were compared to 4 control persons (Group D): age $24.3 \pm 1.4$ yrs, HbAlc $5.2 \pm 0.7 \%$ (mean \pm SEM). Only with respect to age groups A and $B$ differed significantly from $C$ and $D$ (each $p<0.01$ ) and group $C$ from $D(p>0.05)$. Laser Doppler blood flow was measured in supine position on the dorsum of the foot using Periflux 4001 Master. Perimed AB, Sweden and Perisoft 4.4 software. At room temperature of $22 \pm 20 \mathrm{C}$, mean local skin temperature was very similar in the groups. Basal blood flow was taken as mean of a 2 min measurement. After arterial occlusion using $220 \mathrm{mmHg}$ for $3 \mathrm{~min}$ maximum byperemia was assessed. The biological zero flow was subtracted from measured values. Basal blood flow was lowest in Group B (6.1 \pm 1.1 Perfusion Units) and Group D (6.9 \pm 2.3 P.U.); Group C: $7.7 \pm 2.9$ P.U.; Group A: $11.7 \pm 2.1$ P.U. (n.s.). Maximum hyperemia was highest in Group D (61.6 \pm 1.3$)$. Group B: $30.1 \pm 3.6$ P.U., Group A: $24.4 \pm 4.1$, Group C: $14.6 \pm 4.4$ P.U. (Significance: D vs. A and C. p $<0.05 ; D$ vs. B, p $<0.01 ; A$ vs. C, $p<0.05$ ). Since an impaired microcirculation due to diabetes is characterized by an elevated basal blood flow and an impaired hyperemic response, these preliminary results suggest that longterm glucose normalization has a positive influence on diabetic microangiopathy.

\section{8}

IMPROVEMENT OF LIPID AND LIPOPROTEIN PROFILE AFTER SEGMENTAL AND WHOLE PANCREAS TRANSPLANTATION E. La Rocca, A. Secchi, G. Ruotolo, M. Pacchioni, V. Di Carlo, G. Pozza San Raffaele Scientific Institute, University of Milan, Italy

Hyperinsulinemia, insulin resistance, immunosuppressive treatment and diet are common risk factors for lipid disorders in transplanted patients. Aim of our study was to compare, before transplantation (TX) and during a 3 years follow-up period, the effects of glycometabolic control on lipid and lipoprotein profile in recipients of kidney and segmental (KPS) or whole (KPW) pancreas transplant. Cholesterol (Cho), triglycerides ( $\mathrm{Tg}$ ), apoliprotein (apo) A and B in plasma and in major liporotein fractions (VLDL, LDL and HDL), fasting serum free insulin (F IRI), glycosilated hemoglobin (HbA1c), creatinine and whole blood cyclosporine were evaluated in all patients. One, 2 and 3 yrs after TX, creatinine levels were similar in both groups (KPW vs KPS creat. $\mathrm{mg} / \mathrm{dl} 3$ yrs: $1.4 \pm .1$ vs $1.3 \pm .1$ ). HbAlc values were similar 1 yrs after $\mathrm{TX}$ and were statistically lower in KPW than in KPS 1 yrs after TX (KPW vs KPS HbA1c $\%: 1$ yrs $5.3 \pm .1$ vs $6 \pm .2 p=.03 ; 2$ yrs $5.5 \pm .1$ vs $5.7 \pm .1 ; 3$ yrs $5.7 \pm 2$ ys $6 \pm .1$ ). One and 2 yrs after TX F IRI was higher in the former than in the latter group (KPW vs KPS F IRI uU/ml: 1 yrs $19 \pm 10$ vs $12 \pm 4$; 2 yrs $23 \pm 12$ vs $15 \pm 8 ; 3$ yrs $20 \pm 3$ vs $20 \pm 5$ ). total Cho, Tg, LDL and apo B levels and Cho, $\mathrm{Tg}$ and apo B levels on LDL, were statistically lower in KPW than in KPS 1 yrs after TX (KPW vs KPS 1 yrs: plasma tot $\mathrm{mg} / \mathrm{dl}$ Cho $177 \pm 46$ vs $225 \pm 41 \mathrm{p}=.04$; Tg $109 \pm 49$ vs $193 \pm 66 \mathrm{p}=.009$; LDL $112 \pm 30$ vs $147 \pm 30 p=.03 ;$ apo B $86 \pm 6$ vs $128 \pm 29 p<.004$ - LDL mg/dl Cho $112 \pm 30$ vs $147 \pm 30 \mathrm{p}=.03 ; \mathrm{Tg} 30 \pm 18$ vs $57 \pm 18 \mathrm{p}=.009$; apo B $65 \pm 18$ vs $105 \pm 23 \mathrm{p}=001$ ). No statistical differences on lipid profile were shown between the 2 transplanted groups 2 and 3 yrs after TX. Conclusion: an earlier improvement of lipid profile is observed after KPW tx ( 1 yr) than after KPS tx ( 3 yrs). This could be due to the higher peripheral IRI levels and to the better metabolic control achieved in the former group.

\section{9}

ABNORMAL GROWTH HORMONE (GH) RELEASE IN IDDM AFTER PANCREAS OR ISLET TRANSPLANTATION

M.E. Malighetti, L. Piceni-Sereni, R. Lanzi, M. Manzoni, A. Battezzati, V. Di Carlo, G. Pozza and L. Luzi. Departments of Medicine, H San Raphael, Milan, Italy and Brigham and Women's Hospital, Boston, U.S.A.

increased circulating $\mathrm{GH}$ and aberrant response to different stimuli (GH-RH, hypoglycemia) characterize both IDDM and chronic uremia and correlate to severe retinal, kidney and heart diabetic complications. To evaluate if pancreas (Ptx) or islet transplantation (Itx) were able to restore a normal secretion of $\mathrm{GH}$ in IDDM and uremia, we studied 5 groups of subjects: (1) 9 IDDM-Uremic patients (IDDUP)(age $35 \pm 4 y$; duration of diabetes $20 \pm 4 \mathrm{yr}$ ); (2) $23 \mathrm{Ptx}$ (age $38 \pm 2$; transplant age $24 \pm 5 \mathrm{mo}$.: pred. $9 \pm 2$ and CyA $327 \pm 30 \mathrm{mg} /$ day); (3) 8 Itx (age 42 \pm 3 ; transplant age $22 \pm 4$ mo; ; pred. $9 \pm 2$ and CyA $280 \pm 13 \mathrm{mg} /$ day); (4) 9 patients with chronic uveitis (UVE) (age 41 \pm 5 ; duration of therapy $50 \pm 15$ mo.: pred. $10 \pm 3$ and CyA $145 \pm 20 \mathrm{mg} / \mathrm{day}$ ); (5) 14 healthy volunteers (CON)(age $31 \pm 6$ yr). Subjects underwent three different tests: a) euglycemic hyperinsulinemic ( 40 $\mathrm{mU} / \mathrm{kg} / \mathrm{min}$ ) clamp; b) insulin ( $0.3 \mathrm{mU} / \mathrm{kg} / \mathrm{min}$ ) hypoglycemic clamp; $\mathrm{c}) \mathrm{GH}-\mathrm{RH}$ (50 $\mu g$ e.v.). Serum GH and FFA were measured in the basal state, during the 150 min of the clamps and after GH-RH (120 min). Basal GH and maximal GH $\Delta$ peak are reported for studies a) and c), and $\Delta G H$ at $60 \mathrm{~min}$ for study b). Study - IDDUP basal $G H=9.0 \pm 3.1 \mathrm{ng} / \mathrm{ml} ; \Delta$ peak $=7.2 \pm 2.8$; Ptx: bas. $G H=2.4 \pm 0.6 ; \Delta$ $=15.3 \pm 3.7(p=<0.01$ vs UVE and CON); Itx: bas. $G H=1.8 \pm 0.3 ; \Delta=18.5 \pm 7.9$ $(p=<0.01)$; UVE: bas $G H=0.7 \pm 0.3 ; \Delta=4.1 \pm 1.5 ; \mathrm{CON}$ : bas. $G H=1.0 \pm 0.2 ; \Delta$ $=1.3 \pm 0.8$. Study $b$-IDDUP: bas. GH $=2.0 \pm 0.4 ; \Delta=14.5 \pm 3.2 ;$ Ptx: bas. $G H$ $=2.4 \pm 0.6 . \Delta=4.0 \pm 0.4 ;$ UVE: bas. $\mathrm{GH}=0.5 \pm 0.2 ; \Delta=0.1 \pm 0.2 ; \mathrm{CON}$ : bas. $\mathrm{GH}=1.9 \pm 0.9 ; \Delta=0.1 \pm 0.2$. Study c) $-\mathrm{Ptx} \Delta=8.9 \pm 5.1 ;$ UVE $\Delta=2.8 \pm 0.5$; CON $\Delta=17.7 \pm 3.7$. Basal FFA were similar in all study groups (IDDUP $=0.6$ $\pm 0.32 \mathrm{Ptx}=0.60 \pm 0.05, \mathrm{Itx}=0.80 \pm 0.11, \mathrm{UVE}=0.80 \pm 0.12, \mathrm{CON}=0.55 \pm$ $0.16 \mathrm{mmol} / \mathrm{L}$ ). The decrement during hyperinsulinemia (study a) was also similar in groups $2-5(72 \pm 4 \% ; 30 \pm 7 \% ; 76 \pm 6 \% ; 77 \pm 5 \%)$, and much lower in group 1 (IDDUP $=57+5 \%$ ) Conclusion: 1) IDDUP show an abnormal $\mathrm{GH}$ response to both euglycemic hyperinsulinemia and hypoglycemia; 2) Ptx does not restore the $\mathrm{GH}$ response to various stimuli; 3 ) $\mathrm{GH}-\mathrm{RH}$ test results suggest an abnormal regulation of the $\mathrm{GH}-\mathrm{RH} / \mathrm{GH}$ axis at the hypothalamic level.

\section{0}

COMBINED KIDNEY-PANCREAS TRANSPLANTATION IN PATIENTS WITH UNRECOGNIZED NIDDM. R. E. Ratner, R.S. Gray, T. Sasaki, and J.A. Light. Washington Hospital Center, Washington, DC. 74 combined kidney-pancreas transplants have been done at our center over the last 6 years. Analysis of pre-transplant $\mathrm{C}$-peptide values identified 17 patients with measurable levels consistent with a diagnosis of NIDDM. 12/17 patients are Black, and $11 / 17$ are male. Mean pre-transplant C-peptide $=3.75 \mathrm{ng} / \mathrm{ml}$ $(0.6-12.5)$ for the 17 patients identified as NIDDMs compared to a mean of $0.11 \mathrm{ng} / \mathrm{ml}(<0.05-0.41)$ for the remaining 57 patients. Mean age at time of transplantation for the NIDDM group was 39.4 years with a mean duration of diabetes of 19.4 years and all 17 patients were insulin treated. Family history of diabetes was positive in first degree relatives in $47 \%$ of the NIDDM patients. HLA phenotyping revealed DR 2 in two patients, DR 3 in five, and DR 4 in six patients. Patient survival in the NIDDM group is currently $88 \%$, with a mean functioning graft survival of 40.8 months. Two patients died and one lost pancreas graft function at a mean of 19.6 months (11-29 months). One year graft survival of $94 \%$ in the NIDDM group is comparable to the $85 \%$ one year graft survival in the 74 patients as a whole. The 14 patients with functioning grafts are normoglycemic on no insulin therapy. The mean creatinine of the surviving kidney grafts in the NDDM group is $1.91 \mathrm{mg} / \mathrm{dl}(1.1-3.1)$, identical to those with IDDM (1.1-4.1). We conclude that our population of diabetic ESRD patients has a significant number of insulin-treated NIDDMs distinguishable from IDDMs only on the basis of C-peptide levels. These patients respond well to pancreatic replacement therapy in a manner no different than IDDMs relative to function, rejection, or survival. We further conclude that the transplantation of a normal pancreas into the insulin resistant milieu of NIDDM is sufficient to maintain normal glycemic control and thus emphasizes the role of the beta cell defect in the pathogenesis of NIDDM. 
501

BENEFTCIAL EFHECT OF A LONG-TERM INCTSEATION WTTH GROWTH HORMONE ON INSULDN BECRETION OF HUMAN FETAL INLETB: AN INELUENCE OF GESTATIONAI AGE

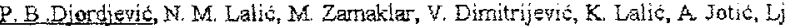
Lukic and N. Rajković, Institute for Endocrinology, Belgrade, Yugoslavia

We have previously shown that a long-term in witro incubation with growth hormone (GH) improves and preserves the insulin secretion capacity of isolated human fetal islets, but the influence of zestational age on this phenorrenon has not yet been elucidated. The aim of this study was to analyse the effect of a long-term (15 days) incubation with 1000mg/1 GH (Genotropin, Kabi Pharnacia) on glucoset theophyliti-3timulated insulin reaponse of isolated human fetal istet originating from 16 wk old (protocol A), 20wk old (protocol B) and 24wk old fetuses (protocol C). The islets were isolated from pancreata of the fetuses by collagenase digestion, and cultured in the media with $10 \%$ fetal calf serm at $37 \mathrm{C}$, $5 \% \mathrm{CO} 2$. The insulin secretion wapacity was evaluated by deterrining insulir levels in the culture media after one hour insubation with 1.67 and $16,7 \mathrm{mmol}$ glucose $5 \mathrm{mmol} / \mathrm{h}$ theophyllin sequentially and expressed as the percentage of the increase in insulin levels after stimulation. We found that the glucose+ theophyllin-stirnulated insulin response after GH-incubation mas significantly higher in protocols $\mathrm{A}$ and $\mathrm{B}$ compared to protocol $\mathrm{C}$, when tested both on day 7 (protocol A: GH+ $729.6+1-63.8 \%$, GH-334.6+1-23.7\%; protocol B: GH+ $713.8+-72.2 \% ; \mathrm{GH}-352.5+1-33.3 \% ;$ protocol $\mathrm{C}$ : GH+ $518.4+1-54.8 \% \%$, GH$3273+1-44.6 \%$; GH+: $A, B$ vs $C, p<0,05$ ), and on day 15 of incubation (protocol $A$ $\mathrm{GH}+732.5+1-64.9 \%, \mathrm{GH}-204,5+1-28.2 \%$; protocol B.GH+ $728.6+1-63.4 \% \%$ GH-

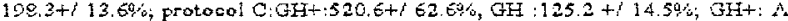
$B$ ws $C, p<0,05$ ). Sirmultaneously, we did not detect the difference in the insulin resporise in the isiet cultures with GH between protocols $A$ and $B$ neither on day 7 nor on day 15. Also, the insulin response remained stable furing 15 days in the cultures with Git in all three protacols, while it prostessively declined in cultures without GH (GH-: A, B and $\mathrm{C}$ i day 7 \% day $15, \mathrm{p}<0,05$ ). Our results have shown that the beneficial effect of incubation with $\mathrm{GH}$ is strongly dependent on the gestational age of the donor fetuses, which might be of elinisal relevanes for the possible use of GH incubation in a pretreatnent before transplantation in diabetio patients.

\section{2}

INSULIN TREATMENT ENHANCES THE GROWTH AND FUNCTION OF ISLET ISOGRAFT

J-H. Juang, C-H. Kuo, S-H. Yeh and H-S. Huang. Division of Endocrinology and Metabolism, Chang Gung Memorial Hospital, Taipei, Taiwan, R.O.C.

Insulin has been shown having beneficial effect on isiet transplantation. To study the mechanism(s), an insufficient number (150) of islets were transplanted syngeneically under left kidney capsule of streptozotocin-diabetic mice. After transplantation, recipients were injected with Ultratard ${ }^{\circledR}$ (UT, $n=30$ ) or normal saline (NS, $n=16$ ) subcutaneously once daily for 2 weeks. From day 3 to 28 after transplantation, the animals in UT had lower blood glucose and higher body weight as compared with those in NS. At 4 weeks, the mean blood glucose level and body weight were $179 \pm 16 \mathrm{mg} / \mathrm{dl}$ and $24.4 \pm 0.2 \mathrm{~g}$ in UT and $377 \pm 17 \mathrm{mg} / \mathrm{dl}$ and $21.8 \pm 0.6 \mathrm{~g}$ in NS $(\mathrm{P}=0.0001)$. The intraperitoneal glucose tolerance and glycosylated hemoglobin in UT were better than those in NS at 2 and 4 weeks after transplantation. In addition, the insulin content of the graft in UT was higher than that in NS $(2.5 \pm 0.3$ vs. $0.5 \pm 0.2 \mu \mathrm{g}, \mathrm{P}<0.01)$. Immunohistochemistry of the graft showed increased B-cell mass in UT. Above beneficial effects were also seen in the other experiments with insulin treatment given 2 weeks before transplantation or 2 weeks before and 2 weeks after transplantation. These data indicate that insulin treatment, before and/or after transplantation, can enhance islet growth and function to improve the outcome of islet transplantation.

\section{3}

TREATING DIABETES WITH A BIO-ARTIFICIAL ENDOCRINE PANCREAS

H. Ohgawara.* **, J. Miyazaki.***, S. Karibe.**, N. Katagiri.* and T. Akaike.*****

* Medical Research Institute, ** Diabetes Center, Tokyo Women's Medical College. ***:Department of Molecular Embryology, Tohoku University. *****Faculty of Bioscience \& Biotechnology, Tokyo Institute of Technology.

Immunoisolation is a potentially important approach to transplanting isler without any immunosuppressive therapy. Immunoisolation systems have been conceived in which the transplanted tissue is separated from the immune system of the host by an artificial barrier.

Pieviously, we described a bioartificial endocrine pancreas (Bio-AEP) which was constructed by placing pancreatic B-cell clusters (ICCs) trapped in a mixed matrix ( mixture of extracellular matrix and agarose, containing maltose-carrying polystyrene and nicotinamide) in the center of a ring holder sandwiched between semipermeable membranes, which were shielded by silicone. In the experiment, a nucleopore membrane, of pore size 0.1 um, was employed as the semipermeable membrane which provided a mechanical barrier between the endocrine pancreatic graft and the host's immune system.

Six streptozotocin ( STZ)-induced diabetic rats were implanted with Bio-AEPs containing mouse insulinoma B-cell clusters ( MIN6-cells) which respond to glucose-stimulation.

In the rats with Bio-AEPs, a return to normoglycemia was observed up to 50 weeks after the Bio-AEP implantation, without the use of any immunosuppressant. During the observation, when the chambers were removed from the STZ-diabetic rats which received Bio-AEPs, the blood glucose immediately returned to the preimplantation levels.

The results indicate that the Bio-AEP with a 0.1 um pore size membrane should be useful for the implantation of xenograft pancreatic endocrine cells in diabetic animals and may open a new field in the therapy of human IDDM.

\section{4}

DEFECTIVE GLUCAGON RESPONSES FROM INTRAHEPATIC ISLETS DURING HYPOGLYCEMIA IS TRANSPLANTATION-SITE DETERMINED V Gupta, DM Kendai\}, DP Rooncy, DG Wahoff, DER Sutherland and RP Roberison. University of Minnesota, Department of Medicine, Minneapolis, MN. The liver is the most commonly employed site for pancreatic islet transplantation. However, glucagon secretion from islets transplanted in liver in humans has been reported to be unresponsive to hypoglycemia yet responsive to arginine. To determine whether this selective glucagon secretory defect is caused by the intrahepatic site of islet implantation or is related to the islet transplantation procedure itself, we studied hormone responses to hypoglycemia in dogs with islet autotransplantation in the hepatic parenchyma $(\mathrm{IH}, \mathrm{n}=9)$ or the peritoneal cavity $(\mathrm{IP}, \mathrm{n}=9)$ following total pancreatectomy, and compared them with responses in normal controls $(n=10)$. Dogs were subjected to a hypoglycemic, hyperinsulinemic clamp for 90 minutes. Immediately following the clamp an IV arginine stimulation test was performed. During the clamp glucagon responses in the IH group (glucagon area under curve $($ AUC) $=713 \pm 1022 \mathrm{ng} / \mathrm{L}-\mathrm{min}$ ) were significantly lower compared to controls ( $A \cup C=6943 \pm 2842 \mathrm{ng} / \mathrm{L}$-min; $\mathrm{p}<0.001$.) Glucagon responses in the IP group ( $\mathrm{AUC}=4090 \pm 1600 \mathrm{ng} / \mathrm{Lmin}$ ) did not differ significantly from controls. Epinephrine responses to hypoglycemia were similar in all groups, whereas, neither of the transplanted groups had pancreatic polypeptide responses. There was a prompt rise in plasma glucagon after intravenous arginine in all groups. These data indicate that selective ghucagon unresponsiveness to hypoglycemia hy intrahepatically transplanted islets is a transplantation site-determined phenomenon, rendering the liver a disadvantageous site for optimal alpha cell function. 
AN OPTIMAL CULTURE MEDIUM TO MAINTAIN PORCINE ISLET FUNCTION FOR THE DEVELOPMENT OF A BIOARTIFICIAL PANCREAS. S. Darquy, C. Rouault, G. Reach. INSERM U 341, Department of Diabetes, Hôtei-Dieu Hospital, Paris, FRANCE.

Various media have been used to maintain porcine islet preparations in culture, and in all cases porcine islets show decreased insulin secretion ove time, which become negligible after 3 weeks. The aim of this work was to find culture conditions optimal for porcine islet function. The following culture media were tested: RPMI+fetal calf serum (FCS) $10 \%$ RPMI+porcine serum 10\% (PS), RPMI+Mediaser $10 \%$ or SFX or Prolofix $10 \%$ (synthetic sera), Uttraculiure a complete synthetic medium and, Hams Flot $2 \%$ Ultroser (synthefic serum). Glucose concentration was $1 \mathrm{~g} / \mathrm{l}$ in all media except UItraculture $(3 \mathrm{~g} / \mathrm{h})$. Porcine islets recovered from one pancreas were split into 20 flasks and fed every two days. Insulin secretion was determined at 6,14 and 20 days by incubating islets for $45 \mathrm{~min}$. in the presence of $2.8 \mathrm{mM}$ or $10 \mathrm{mM}$ glucose using a validated transwell filtermicroplates method. In the first set of screening experiments, RPMI+FCS PS, Prolifix and mediaser were eliminated because insulin secretion was low after 15 days of culture. In a second set of experiments, Uliraculture and Hams F10+Ultroser were compared and the results demonstrate a more appropriate response to glucose when islets were cultured in Hams Fro+UItroser. A long term study was investigated according to the same method described below.1) secreted insulin was significant at 1 and 3 weeks and 2) glucose-indiced insulin response was restored : 1 week:basal $1513 \pm$ $481 \mathrm{mU} /$ pancreas, stimulated $1598 \pm 677 \mathrm{mU} /$ pancreas; 3 weeks :basal $829 \pm$ 350 , stimulated $1163 \pm 449 \mathrm{mU} /$ pancreas $(\mathrm{n}=8)$. Stimulatory indexes (S/B) were $1,91 \pm 0,26,1,99 \pm 0,39$ and $2,38 \pm 0,47$ at 1,2 and 3 weeks respectively. A response to glucose at 3 weeks was demonstrated by a glucose-secretion response curve $(0,0.5,1,2,3 \mathrm{~g} / 1): 517 \pm 216 ; 372 \pm 128$; $612 \pm 257 ; 860 \pm 343 ; 1078 \pm 419 \mathrm{mU} /$ pancreas respectively. In conclusion, Hams F10 medium supplemented with $2 \%$ Ultroser is compatible with maintenance of islet function in culture at least 3 weeks. Under these conditions, the paradoxical inhibitory effect of glucose for insulin secretion is reversible into a normal stimulatory effect.

\section{7}

BOVINE TO PORCINE INTRATHYMIC ISLET XENOTRANSPLANTATION.

S. Del Guerra, R. Giannarelli, A. Coppelli, C. Tellini, P. Marchetti, $R$. Lupi, M. Carmellini, P. Viacava, F. Mosca, R. Navalesi. Cattedra Malattie Metabolismo, Istituto Clinica Medica II, Università di Pisa, Italy.

Intrathymic islet transplantation allows survival of auto- and allo-islet grafts in rodent, dog and pig experimental models. In addition, the thymus is a suitable site for islet survival in concordant xenotransplantation rodent models (rat-to-mouse). In this study we prepared pure bovine islets, of proven in-vitro and in-vivo viability, and cultured them at $37^{\circ} \mathrm{C}$ in medium M199, with $10 \%$ bovine serum and antibiotics for 15 to 21 days. Then, approximately 10,000 islets were transplantanted into the thymus of eight 3-mo old, nonimmunosuppressed pigs, under general anesthesia. The surgical procedure was simple, safe and effective. The glands were retricyed after 3 ( 2 animals), 7 ( 3 animals) and 14 ( 3 animals) days from implantation, and processed for light microscopy (hematoxylin-eosin staining, immunocytochemistry). In 2 pigs of the 14 day study, intravenous glucose was administered at 3,7 and 14 days after islet transplantation, and bovine insulin measured by RIA following HPLC separation from porcine insulin. Well preserved islets were shown both at 3 and 7 days from transplantation, and insulin containing cells were evidentiated by immunocytochemical staining. No apparent modification of the islet surrounding thymus was observed. Bovine insulin was found in the plasma of transplanted pigs, which increased upon glucose stimulation, up to 14 days from implantation. These results demonstrate that bovine islets can survive for a few days in the thymus of non-immunosuppressed pigs, with avoidance of hyperacute rejection. Further studies are ongoing to evaluate how long the islets can function in this discordant xenograft model.
Insulin independence in an IDDM patient one year after allogeneic intraportal islet transplantation.

K.B. Yderstraede, S.A. Birkeland and H. Beck-Nielsen. Odense University Hospital, Dept.'s of Endocrinology and Nephrology.

Human allogeneic islet transplantation is performed in 15 institutions worldwide. Long-term insulin independence is rarely reported. From january 1995 an islet transplantation programme was started in Denmark using a distant islet isolation laboratory (Milan, Italy) and a local transplantation facility. The pancreas is removed in Denmark, canulated and injected with collagenase before being flown by jet plane to Milan reaching the laboratory within a time span of 4-5 hours. After isolation the islets were flown back to Denmark. The case reported here is a male C-peptide negative IDDM patient with severe diabetic nephropathy. During the processing of the pancreas he received a renal transplant from the same donor and within 3 hours he had an islet transplant intraportally. A second islet transplant was performed after 10 days bringing the total islet equivalent number to about 700.000 . The immunosuppressive regimen included induction therapy with anti-lymphocyte globulin and follow-up treatment with Cyclosporine A, recently supplemented with Mycophenolate Mophetil. Insulin was gradually reduced to $1 / 6$ of the initial dosage, and finally discontinued after 3 months. The patient has remained off insulin for one year so far. IVGTT's have shown almost normal values while OGTT's were clearly abnormal indicating glucose intolerance. The fasting values for C-peptide are reported to be somewhat higher in successful transplants from other centres, probably reflecting the effect of adding steroids to the immunosuppressive protocol. $\mathrm{HbAl}_{c}$ values have increased, but are now stabilized at about 0.07 inormal values 0.043 $0.063)$. This successful islet transplantation demonstrates, for the first time, the potential feasability of using an immunosuppressive protocol without steroids.

\section{8}

PRI STRVATION OF FROZFN-THLWFI ISIFTS PRIOR TO TRANSPI.ANTATION ST PPERIORITY OF RPMI OVTR LINIVTRSSITY OI WISCONSIN SOLITTION

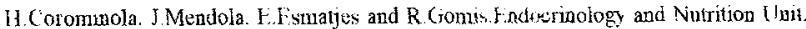
Hospital Clinic of Barcelona. Barcelona. Spain

Although storage by cryogento preservation would allow silet banhing and would simplify transpon betreen centers it rasces the need to find a solution or a nethod to ensure $\beta$-cevil function in the post-than phase and in the transportation of the islets prior to transplantation. Different solutions bave been described for the storage of the whole pancreas. one of these was the University of Wisconsin solution which is also used for torage of the pancreatic digest before islet purification. The aim of this stidy was to determine the optimal conditions and solutions for frozen-flamed islets storage and transportation to av oid loss of vell viablity and function before transplantation. Wistar rat islets nert wo opreserved with DMSO $2 \mathrm{M}$ and DMSO $3 \mathrm{M}$ after an overnight culture in RPMI 1640 (11 I mM glucose). thawed with $0.75 \mathrm{M}$ suetose and maintained under different conditions: group l.not treated -controls- $(\mathrm{n}=6)$ ) group 2:cultured at $37^{\circ} \mathrm{C}$ for $18-24$ hours in RPMI 1640 ( $\mathbf{n}=7$ ) group 3 mantained at $4{ }^{\circ} \mathrm{C}$ for $18-24$ hours in RPMI !640) (1)=7): group 4 maintaned at $4^{\circ} \mathrm{C}$ for $18-24$ hours in University of Wisconsin solution (11-7). In each group. vell viability was performed by ethidium bromide/orange auridiue stamng and mage anals sis (Cue 2) and in vitro functional assessment was carried out by meacurencen of insulin secretion at $2.8 \mathrm{mM}$ and $167 \mathrm{mM}$ glucose in a static incubation. In vir 0 functional assessment was studied in groups 2.3 and 4 by the rate of reversal of drabeter un streptorotocin-treated lewis rats grafted intraportally. Group 2 showed the highesi will viability $(x=87 \% \%)$ and the best insulin secretory response ( $45.6 \pm 13.2$ at 2.8 mM glucose is $112.6 \pm 23.6$ at $16.7 \mathrm{mM}$ glucose) and goup 4 showed the lowest vell riabtlity $(x=52.9 \%)$ and the worst insulin secretory response $(81.0 \pm 19.9$ at $2.8 \mathrm{mM}$ glucose ys $97.1 \pm 13.0$ at $16.7 \mathrm{mM}$ glucose). The in vitro and the in vivo results suggest that cill wability and insulin secretion of frozen-thawed islets is improved after a period of culture and that the University of Wisconsin is not the best solution for storage of frozcnthancd islets Preservation of islets at $4^{\circ} \mathrm{C}$ in RPMI is a good method that allow the sllecestul transplantation of cryoprescrved islets. 
509

INSULIN RELEASE IN VITRO FROM LONG-TERM MOUSE ISLET TRANSPLANTS

C.-L. Shi, S. Persson-Sjögren and I.-B. Täljedal

Department of Histology and Cell Biology, Umeå University, S-901 87 Umeå, Swcden

For unclear reasons, late ( $>1$ year) functional failure may occur in syngeneic or autogeneic islet transplants. Aiming at a better understanding of this phenomenon, we tested the secretory capacity of long-term islet grafts by perifusion in vitro. Normal BALB/c mice $(6$ months old) reccived 150 islets from donors of the same age. After 45 or 90 weeks the grafts were isolated, as were fresh islets from the pancreas of the graft-bearing mice. The insulin content of 90 -week grafts $(9.6 \pm 1.3 \mu \mathrm{g} / \mathrm{graft})$ did not differ from that of 45 -week grafts $(11.0 \pm 0.8 \mu \mathrm{g} / \mathrm{graft}$ ), but the insulin response to glucose (11 and 16.7 mmol/l) of 90 -week grafts $(0.65 \pm 0.2$ and $0.78 \pm 0.1 \mathrm{ng} / \mathrm{graft}$ per min respectively)were generally less pronounced than those of 45 -week grafts $(1.41 \pm(0.3$ and $2.66 \pm 0.7 \mathrm{ng} /$ graft per min). Insulin content $(5.2 \pm 0.9$ and $5.9 \pm 0.7 \mu \mathrm{g} / 50$ islets respectively) and insulin secretion of fresh islets did not differ between 45-week and 90-week graftbearing mice. When $15 \mathrm{mmol} / \mathrm{l}$ arginine was added to the perifusion medium, glucose-induced insulin secretion of fresh islets from $45-$ week graft-hearing mice was increased by $6.8 \pm 0.6$ times, which was stronger than that from 45 -week grafts $(3.8 \pm 1.4)$. 90-week grafts had no response to glucose plus arginine, while the fresh islets increased by $5.9 \pm 0.9$ times. The addition of $1 \mathrm{mmol} / \mathrm{l}$ caffeine increased the glucoscinduced insulin secretion by 4 times in 90 -week grafts. We conclude that long term transplanted islets under the kidney capsule kept their insulin content, although the capacity of insulin secretion was decreased.

\section{0}

THE REVASCULARIZATION PROCESS OF PURIFIED RAT ISLET BETA CELL GRAFTS

J.Mendola, I.Conget, J.M.Manzanares, H.Corominola and R.Gomis. Endocrinology and Nutrition Unit, Hospital Clinic of Barcelona, Barcelona, Spain.

Pancreatic islet transplantation has become recently a therapeutical approach for the cure of insulin-dependent diabetes mellitus. However, it has been suggested that aggregates of purified islet $\beta$-cell allografts can survive in histoincompatible hosts without pharmacological immunosuppression mainly because they are less immunogenic than isolated islets. Since little is known about the revascularization process of purified islet $\beta$-cells after transplantation, the aim of the study was to investigate the time course of the process of angiogenesis of rat islet $\beta$ cell grafts after syngeneic transplantation into the renal subcapsular space by immunocytochemical analysis. Wistar rats were grafted under the renal capsule with either Wistar islets $(n=6)$ or with purified islet $\beta$ cells $(n=9)$. Purified $\beta$-cells were sorted using a flowcytometer after Ioading with Fluo-3, viability being always higher than $90 \%$ (neutral red staining). Purity of $\beta$-cells was assessed by hormonal content, insulin/glucagon ratio being 2000 times higher than in non- $\beta$ cells. A preparation of purified $\beta$-cells was immunohistochemically stained before transplantation with an antiserum to factor VIII related antigen to verify the non-contamination with donor endothelial cells. A nephrectomy of islet and pure $\beta$-cell grafts was performed at days 3,5 and 7 after implantation. Kidneys bearing grafts were snap frozen and immunohistochemically stained with a double immunofluorescence technique using a Rabbit anti factor VIII-related antigen antiserun (which identifies endothelial cells) and a Guinea pig anti-insulin antibody. The immunohistochemical analysis revealed that islet grafts revascularization is completed between days $3-5$ after implantation by the detection of intra and peri-islet endothelial cells. On the other hand, in purified $\beta$-cell grafts an anti factor VIII related antigen staining was firstly observed at day 5 and was much more prominent at day 7 . indicating that revascularization with host vessels is an early phenomenon in pure $\beta$-cell grafts implanted under the renal capsule of syngeneic rats.

\section{1}

AMINOGUANDINE UPHOLDS B-CELL FUNCTION IN RAT PANCREATIC ISLETS TRANSPLANTED TO NORMAL RECIPIENTS

Y. Tajiri and V. Grill. Department of Molecular Medicine, The Endocrine and Diabetes Unit, Karolinska Hospital, Stockholm, Sweden

We previously reported that aminoguanidine (AG), known to inhibit the formation of advanced glycosylation end products (AGEs), restored insulin release and insulin mRNA content of tat islet grafts transplanted to diabetic recipients (15th IDF Congress, Kobe, 1994). Since AGE formation takes place also under non-diabetic conditions and may accelerate aging processes, we presently tested long-term effects of $A G$ on islet grafts transplanted to normal recipients. Pancreatic islets were isolated by collagenase digestion from inbred female Wistar-Furth rats (10-15 weeks old), and 200 islets were transplanted under left kicney capsules of male recipients $110-15$ weeks old). Recipients were randomly divided into two groups and one group was treated by $\mathrm{AG}$ in the drinking water (1g/L). Two and eight weeks after transplantation, graft-bearing kidneys of each grousp were isolated and perfused to measure insulin release from islet grafts. After pertusion, islet grafts were retrieved for the measurement of insulin mRNA content. After eight weeks, in non-treated groun (N) $27.8 \mathrm{mM}$ glucose-stimulated insulin release $(\Delta \mathrm{G} ; 132 \pm 45 \mathrm{fmol} / \mathrm{min})$ and $10 \mathrm{mM}$ arginine-stimulated insulin release (Arg; $154 \pm 37 \mathrm{fmol} / \mathrm{min}$ ) were markedly lower than the release after two weeks of transpiantation $(\Delta G ; 320 \pm 123$, Arg; 374 \pm 88 fmol/min). These declines of insulin release were almost completely restored in the AG-treated group (N-AG; $\Delta \mathrm{G} ; 277 \pm 39, \mathrm{Arg} ; 430 \pm 67 \mathrm{fmol} / \mathrm{min}$ ) after eight weeks. Moreover, insulin mRNA content in N-AG (32.2 $\pm 4.5 \mathrm{fmol} / \mathrm{graft})$ was significantly $(P<0.05)$ higher than $\mathrm{N}(20.1 \pm 2.9$ fmol/graft $)$ after eight weeks, It is suggested that $\mathrm{AG}$ upholds $\mathrm{B}$-cell function in normal recipients through the inhibition of $\mathrm{AGE}$ formation in islet grafts and that AGE formation may, in part at least, be responsible for the time-dependent deterioration of islet transplant function.

\section{2}

HUMAN ISLETS PROTECT MOUSE B-CELLS FROM ALLOXAN INDUCED TOXICITY

B. Tyrberg* , D.L. Eizirik* ${ }^{*}$, N. Blume , O. D. Madsen ${ }^{\dagger}$, D.G. Pipeleers ${ }^{\ddagger}$.

C. Hellerström ${ }^{*}$ and A. Andersson

"Department of Medical Cell Biology, Uppsala University, Uppsala, Sweden tHagedorn Research Institute, Gentofte, Denmark. "Department of Metabolism and Endocrinology, Vrije Universiteit Brussel and $\beta$-Cell Transplant, Brussels, Belgium.

Alloxan is known as a diabetogenic substance in most species. However, wo recently showed that human islets in culture and transplanted to nude mice are resistant to alloxan. In the present study we tested whether human islets could transfer this resistance to mouse islets in a mixed islet graft situation.

For this purpose human and mouse islets were transplanied under the kidney capsule of nude mice, either as separate or as mixed grafts. Two weeks later alloxan $(85 \mathrm{mg} / \mathrm{kg}$ ) or saline (controls) was injected i.v. One week thereafter the mice were killed and the grafts and endogenous pancreata were stained with insulin- or species specific $C$-peptide antibodies, the latter to distinguish between $\beta$-cells from the two species in the mixed grafts. The number of $\beta$-cells was then evaluated with a semi quantitative method.

As expected transplanted human islets were resistant to alloxan, whereas the endogenous pancreata and mouse islet grafts transplanted alone showed total $\beta$-cell destruction. However, mouse $\beta$-cells transplanted together with human islets were partially protected against alloxan-lesions $(p<0.01$ vs mouse islets transplanted alone). Mouse islets transplanted a few millimeter from the human islet graft were also protected against the cytotoxicity $(p<0.001)$. These data evidence a new phenomenon, namely that human islets protect mouse $\beta$-cells against alloxan induced lesions. The mechanisms are unknown, but might involve human islet production of extracelluiar radical scavenging enzymes that are locally distributed to the transplanted mouse $\beta$-cells but not to the endogenous pancreas. 


\section{3}

GRADUAL ABSORPTION OF INTRAPERITONEAL INSULIN RESULTS IN IMPAIRED GIUCOSI: TOLLRANCE IN RECIPIENTS OF MICROENCAPSULATED ISLET GRAFTS

P. De Vos, B.J. De Haan, D. Vegter, J. Strubbe. R. Van Schilfgaarde. Surgical Research Lab. University Hospital Groningen, The Netherlands.

As a consequence of its volume, a microencapsulated islet graft can only be implanted into the peritoneal cavity. We studied the function of intraperitoneally implanted islet allografts microencapsulated in alginate-polylysine capsules. Recipients were subjected to intravenous (IVGTT) and oral glucose tolerance tests (OGTT) 4 to 6 weeks after implantation. All became normoglycemic within 5 days after implantation but were found to be glucose intolerant during IVGTT and OGTT, with maximal bloodglucoses of $11.7 \pm 0.4 \mathrm{mM}$ and $8.8 \pm 0.4 \mathrm{mM}$, respectively, and there was no increase of plasma insulin levels. This impaired glucose tolerance may very well be the consequence of the fact that microencapsulated islets remain freely floating in the peritoneal cavity without vascular access which implies that insulin is not directly absorbed into the circulation, like with vascularized islets, but has to he transferred from the peritoneal fluid to the bloodstream. To investigate whether the transplantation site as such contributes to the interference with optimal transport kinetics between the islets and blood we infused insulin into the peritoneal cavity of conscious and freely moving rats to minic the gradual release of insulin from an encapsulated islet graft. We observed virtually no rise of iusulin levels and it took 30 minutes until glucose levels had dropped significantly, with infusion of $20 \mathrm{pM} / \mathrm{min}$ insulin during $15 \mathrm{~min}$ (the amount of insulin produced by a thative rat pancreas in response to OGTT). With higher insulin doses, there was a dose dependent rise of insulin and decrease of glucose levels. When compared to intraportal infusions with the same insulin dosages, however, they were strongly delayed and reduced as well as prolonged. This strongly delayed effect of peritoneally infused insulin on both plasma insulin levels and glucose levels explains, the lack of elevation of insulin levels and the disturbed glucose tolerance in recipients of an encapsulated islet graft. With view on the clinical efficacy of the bioartificial pancreas, our findings indicate that we should focus on finding or creating a transplantation site which, more than the uumodified peritoneal cavity, permits for close contact between the bloodstream and the encapsulated islet tissue.

\section{4}

NOBMOGLYCEMIA RESTORES BETA CELL REPLICATIVE CAPACITY IN TRANSPLANTED ISLETS EXPOSED TO CHRONIC HYPERGLYCEMIA. V.Nacher, J.F.Merino, M.Raurell, O.Aranda, J.Soler and E.Montanya. Laboratory of Diabetes and Experimental Endocrinology, Endocrine Unit CSUB-Hospital de Bellvitge. Barcelona. Spain.

To study the effects of chronic hyperglycemia on beta cell replication in transplanted ( $T x$ ) islets, four groups of streptozocin diabetic C57BL/6 mice were $T x$ with $100(T x-100)$ syngeneic islets, an insuficient beta cell mass to restore normoglycemia. One month later (day 30 ), groups 3 and 4 received a second $T x$ of 250 islets $(T x-250)$, sufficient to restore normoglycemia which was harvested 30 days later (day 60 ). Tx-100 was harvested 14 (group 1), 60 (groups 2 and 3 ) and 74 (group 4) days after Tx. Beta cell replication was determined by bromodeoxiuridine incorporation and expressed as percentage of positive beta cells. Groups 1 and 2 remained hyperglycemic through the study; groups 3 and 4 were hyperglycemic from day 0 to day 30 and normoglycemic from day 30 to day 60 . Group 4 showed mild hyperglycemia between day 60 and 74 (harvesting of Tx-250 and Tx-100 respectively). Hyperglycemia increased beta cell replication after $14(1.02 \pm 0.18 \%, p<0.05)$, but not after 60 days $(0.60 \pm 0.23 \%)$, compared to islets exposed to normoglycemia (group 3: $0.42+0.07 \%$ ). In group 4 beta cell replication was increased $(1.20 \pm 0.29 \%, p<0.05)$ in response to increased metabolic demand after Tx-250 extraction. In summary, when $\mathrm{Tx}$ beta cells are chronically exposed to hyperglycemia beta cell replication became limited. Normoglycemia restored the beta cell replicative response to glucose even after one month of exposure to severe hyperglycemia, suggesting that prolonged hyperglycemia did not cause permanent damage.

\section{5}

CORRECTION OF GLUCOSE TOLERANCE TEST IN DIABET: MICE BY PORCINE ISLETS ENCAPSULATED IN AN69 MICROTUBES. C. Delaunay, S. Darquy, J. Honiger, F. Capron and G. Reach. INSERM U 341. Department of Diabetes, Hôtel-Dieu Hospital, Paris, FRANCE.

Purified porcine islets were encapsulated within a $80 \mathrm{~cm}$ long, $400 \mu \mathrm{m}$ (i.d.), $600 \mu \mathrm{m} \mathrm{(o.d.)} \mathrm{microtube} \mathrm{made} \mathrm{of} \mathrm{a} \mathrm{hydrogel} \mathrm{of}$ polyacrylonitrile-sodium methallyl sulphonate (AN69, HOSPAL R\&D). Two microtubes in the form of a spire were implanted in the peritoneal cavity of mice made diabetic by 5 consecutive injections of subdiabetogenic doses of streptozotocin. The aim of this work was to study correction of diabetes and the effect of a glucose load. Islets of Iangerhans were isolated and purified from pig pancreas and maintained in culture for 3 days before transplantation. AN69 hollow fibers were seeded with islets suspended in $600 \mu \mathrm{l}$ culture medium, corresponding to approx. 1/100 of the islets recovered from one porcine pancreas. 17 mice were transplanted. 1) In 11 mice plasma glucose decreased from $354 \pm 16$ to $197 \pm 14 \mathrm{mg} / \mathrm{dl}$ within 3 days, and subsequently to $162 \pm 17 \mathrm{mg} / \mathrm{dl}$ at day 17 . Plasma glucose from the other 6 mice was higher at the time of implantation $(444 \pm 41 \mathrm{mg} / \mathrm{dl}$, $\mathrm{p}<0,05)$, decreased to $322 \pm 48 \mathrm{mg} / \mathrm{dl}$ at day 3 , but then gradually increased to reach $410 \pm 34 \mathrm{mg} / \mathrm{dl}$ at day 17 . Implantation of empty tubes had no effect on glycaemia of diabetic mice. 2) Glucose tolerance tests $(1 \mathrm{~g} / \mathrm{kg}$ body weight, IP) were performed in 7 corrected mice 14 days after implantation. Initial plasma glucose concentrations were 368 $\pm 21,181 \pm 5,165 \pm 17 \mathrm{mg} / \mathrm{dl}$ in non treated diabetic mice, normal control mice, and in corrected-transplanted mice, respectively. Peak values, observed at $20 \mathrm{~min}$, were $635 \pm 85,346 \pm 11$, and $308 \pm 19$ $\mathrm{mo} / \mathrm{dl}$, respectively, decreasing at 2 hours to $654 \pm 118,199 \pm 16$, and $192 \pm 6 \mathrm{mg} / \mathrm{dl}$. Fibers were explanted from 2 normalized mice. Thirteen days later, blood glucose concentration was in the diabetic range ( 345 and $407 \mathrm{mg} / \mathrm{dl}$ ). In conclusion, diabetes was corrected in two thirds of the animals, and a complete correction of glucose tolerance test can be achieved in diabetic mice by xenografting porcine islets in AN69 microtubes.

\section{6}

CHARACTERISATION OF XENOGRAFT REJECTION OF MICROENCAPSULATED ISLETS BY FACS-ANALYSIS

U.Siebers, A.Horcher, K.Federlin, R.G.Bretzel and T. Zekorn

Med.Klinik III, Justus-Liebig-Universität Giessen, Germany

Xenotransplantation of islets of Langerhans would help to overcome the limitations of human organ donor shortage. In this study, we present a method for the characterisation of xenograft rejection by Facs analysis of peritoneal celis. Material and Methods: Porcine islets were microencapsulated in barium alginate microbeads ( $\mathrm{mc}-\mathrm{Tx}$ ). Non-encapsulated islets and empty microcapsules (empty mc) served as controls. $12,000-15,000$ islet equivalents were transplanted intraperitoneally into non-diabetic Lewis rats ( $n=3$ in each group). After 7 days cells were harvested by peritoneal lavage and characterised by Facs analysis. The following antibodies were used: 1F4 (CD3 T-lymphocytes), OX33 (Blymphocytes); OX6 (MHC class II); OXA2 (CD11b, iC3b); ED1 and ED2 (subgroups of monocytes and macrophages). Results: Total cell number was highest after mc-Tx $\left(149,4 \pm 30,1 \times 10^{6}\right.$ cells $)$ compared to empty mc $(41,4 \pm 19,7$ $\left.\times 10^{6}\right)$ and non-encapsulated Tx $\left(18,1 \pm 3,3 \times 10^{6}\right)$. After mc-Tx the percentage of CD3 positive T-lymphocytes rose to $44,5 \pm 11,5 \%$ compared with $4,9 \pm 2,4 \%$ for empty controls. In case of non-encapsulated islets CD3 expression was 19,2 \pm $8,2 \%$. Moreover OX6 positivity was dramatically increased in the mc-Tx group $(60,2+8,9 \%$ vs. $15,2 \pm 7,0 \%$ free islets vs. $4,9 \pm 1,2 \%$ empty mo $)$. Only a small amount of OX33 positive cells were detected with a slightly increased number after free Tx. EDl expression was markedly higher after me-Tx $(37,6 \pm 18,9 \%)$, but did not differ between the two other groups $(1,0 \pm 0,6 \%$ vs $1,0 \pm 0,3 \%)$. OX 42 as a constitutive marker of mononuclear cells was strongly positive in all three groups. Conclusion: Facs analysis of peritoneal cells after xenotransplantation shows a considerable T-cell activation and a hyperexpression of MHC class II molecules on mononuclear cells. Further studies have to evaluate the time course of the reaction, the metabolic aspect and the relevance of cut-off modifications. 
EXTRINSIC RE-INNERVATION OF MOUSE ISLETS TRANSPLANTED UNDER THE KIDNEY CAPSULE S. Persson-Sjögren ${ }^{1}$, S. Forsgren ${ }^{2}$, C.-L. Shi ${ }^{1}$ and I.-B. Täljedal ${ }^{1}$ ${ }^{1}$ Department of Histology and Cell Biology, and 2 Department of Anatomy, Umeå University、 S-901 87 Umcå, Sweden

In syngeneic mouse islets transplanted to kidney, the $\beta$-cells develop defective insulin secretory responses to neurotransmitters, perhaps because the normal extrinsic nerves are cut during islet isolation. With the aim of elucidating the re-innervation of islet grafts, we cultured islets overnight and injected them under the renal capsule of syngeneic fernale $\mathrm{C} 57 \mathrm{BL} / 6$ recipients. At several time points from 4 days up to 52 weeks after transplantation, the grafts were removed, sectioned, and immunohisto-chemically stained for tyrosine hydroxylase (TH), calcitonin gene-related peptide (CGRP), and substance P (SP). At 45 and 90 weeks grafts were also perifused in vitro to study insulin secretory dynamics. The sympathetic (TH) and sensory (CGRP, SP) innervation was very sparse during the first weck after transplantation. The number of sympathetic fibers expressing TH-like immunoreactivity (THLI) increased progressively after 6 weeks, and at 52 weeks was even larger in the grafts than in normal islets in situ in the pancreas. CGRP-LI nerve fibers also increased but did not become as frequent as those expressing TH-LI. SP-LI nerve fibers remained rare throughout. The islet grafts released insulin when stimulated by glucose, but showed a decreased inhibitory response to noradrenaline. We conclude that the sympathetic innervation of the islet grafts is extrinsic, as no TH-LI nerve cell bodies were demonstrated. Re-innervation results in a dense sympathetic and a moderate sensory nerve supply of the grafts without restoring a normal beta-cell response to noradrenaline.
GLUCOSE-SENSITIVE INSULIN-SECRETING AtT20 CELLS: A MODEL FOR INSULIN DELIVERY BY SOMATIC CELL GENE THERAPY.

E.L. Davies a. K.IJ. Shennan ", K. Docherty ${ }^{\text {b }}$ and C.J. Bailey ". "Department of Phamaceutical and Biological Sciences, Aston University, Birningham: ${ }^{b}$ Department of Molecular and Cell Biology, University of Aberdeen, Aberdeen.

Mouse pituitary AtT20 cells were used as a model to develop glucose-sensitive insulin-secreting surrogate $\beta$-cells for insulin delivery by somatic cell gene therapy. The cells were transfected with plasmids containing human preproinsulin cDNA directed by a cytomegalovirus promoter, and cDNA for the GLUT-2 glucose transporter isoform directed by the $\beta$-actin promoter. A clone was selected with strong expression of both genes. Analysis of northem blots showed a glucose dependent increase in the expression of GLUT2 mRNA, increasing 10 -fold between $50 \mu \mathrm{M}$ and $5 \mathrm{mM}$ glucose $(\mathrm{p}<0.02)$, but not increasing between 5 and $10 \mathrm{mM}$ glucose. Insulin release during static incubations increased in a glucose dependent manner up to $50 \mu \mathrm{M}$ glucose, but did not increase further when glucose concentrations were raised into the $\mathrm{mM}$ range. Maximum release of immunoreactive insulin was $7.5 \mathrm{ng} / 10^{6}$ cells/24 hours. By differential radioimmunoassay, about $75 \%$ was processed to true insulin. When $2 \times 10^{7}$ of these insulin secreting cells were implanted intraperitoneally into streptozotocin diabetic nude (nu/nu) mice, the mice showed lower plasma glucose concentrations ( $\mathrm{p}<0.02$ ) and resisted lethal hyperglycaemia for longer than control nude mice receiving noninsulin secreting wild type AtT20 cells. The implanted cells formed vascularised tumours with insulin-secreting cells at the periphery and a necrotic core. Thus, genetically modified pituitary AtT20 cells which express both preproinsulin and GLUT2 genes provide a useful model of glucose-sensitive insulin-secreting surrogate $\beta$-cells for somatic cell gene therapy

\section{PS 15 \\ Glycation}

\section{9}

THE AGE PROdUCT PENTOSIDINE IS INCREASED IN URINE FROM SUBJECTS WITH INSULIN-DEPENDENT DIABETIC SUBJECTS

R.A. Smulders ${ }^{3}$, C. Schalkwijk ${ }^{2}$, J. te Koppele ${ }^{3}$, V.W.M. van Hinsbergh ${ }^{3}$ and C.D.A. Stehouwer ${ }^{1}$. Dept. of ${ }^{1}$ Internal Medicine and ${ }^{2}$ Clinical Chemistry, ICaR-VU, Research School Vrije Universiteit, Amsterdam, ${ }^{3}$ Gaubius Laboratory TNO, Leiden, The Netherlands.

The increased formation of advanced glycosylation end products (AGEs) in insulindependent diabetes mellitus (IDDM) is believed to be involved in the structural and functional alterations observed in: microangiopathy in IDDM. However, assays for AGE-products are not available for routine use. We adapsed an HPLC-based method for measurement of pentosidine in unprocessed urine. To study the relevance of this AGE-product, we investigated three groups: 1) a control group [ $\mathrm{n}=60 ; \mathrm{M} / \mathrm{F} 27 / 33$ mean (sd) age $=41.5$ (13.2) yrs], 2) an IDDM group without complications [n=48; M/F 30/18; age 39.7 (16.4) yrs; diabetes duration 17.5 (13.8) yrs; $\mathrm{HbA}_{1 \mathrm{c}} 7.6(1.2) \%$ and 3) a group IDDM subjects with microalbuminuria $[\mathrm{n}=10 ; \mathrm{M} / \mathrm{F} 5 / 5$; age 33.2 (14.0) yrs; diabetes duration $18.6(8.8)$ yrs; $\mathrm{HbA}_{1 \mathrm{c}} 9.0(1.6) \%$ l. Urine samples were collected in the morning between 8.00 and 12.00 hours. Urinary pentosidine was expressed as $\mu \mathrm{mol} / \mathrm{mol}$ creatinine. Mean age was not different between the three groups $(\mathrm{P}=0.25)$. Urinary pentosidine concentration was higher in the (total) IDDM group than in the control group $[4.54$ (3.2) vs $2.78(0.73) \mu \mathrm{mol} / \mathrm{mol}$ creat. $P<0.0001]$. Multiple linear regression analysis using urinary pentosidine as the dependent variable showed that age, and the presence or absence of diabetes significantly influenced urinary pentosidine concentration [both $\mathrm{P}<0.001$; multiple $\left.\mathrm{R}=0.52, \mathrm{R}^{2}=27.4\right]$. Diabetes duration, sex, $\mathrm{HbA}_{10}$, and the presence or absence of microalbuminuria were not statistically significant predictors of elevated pentosidine levels in the IDDM group. These results demonstrate that urinary pentosidine concentration is increased in IDDM subjects compared to age-matched control subjects. Age and the presence of diabetes are the main determinants for predicting the pentosidine concentration. Further studies are needed to investigate the relationship with microangiopathy.

\section{0}

DIRECT EVALUATION OF THE OXIDATION ROLE IN GLOBIN GLYCATION: AN IN VIVO STUDY

A. Lapolla, D. Fedele, R. Aronica, M. Garbeglio, M. D'Alpaos, R. Seraglia and P. Traldi, Istituto di Medicina Interna, Malattie del Metabolismo, Università di Padova, Via Giustinjani 2. 1-35100 Padova, Italy.

Glycation processes are responsible for the long term diabetic complications. Thus the monitoring of glycated proteins is of high interest to determine the glucose exposure during the protein half life. In this contest the $\mathrm{HbA}_{10}$ determination is the most employed method. However, together with simply glycated globins, glyco-oxidation products can be present. Their evaluation is diagnostically relevant, indicating the exposure to both glucose and oxidative stress. The aim of the present study was the development of an analytical method able to distinguish between glycation and glycooxidation products of globins. This was achieved by matrix assisted laser desorption/ionization (MALDI) mass spectrometry, which allows to determine the molecular wcight of large biomolecules with an accuracy in the range $0.1-0.01 \%$. Globine samples from 6 healthy subjects (mean age \pm SD) $54 \pm 12$ years and 14 NIDDM subjects (mean age \pm SD $64 \pm 6$ years, mean disease duration $14 \pm 5$ years) were analyzed. Globine samples were obtained by treating whole blood with acetone with $0.2 \% \mathrm{HCl}$ and extracted by diethylether. For NIDDM, mean fasting plasma glucose levels were significantly higher than those of healthy subjects (172 \pm 46 is 91 $\pm 8 \mathrm{mg} / \mathrm{mll} ; \mathrm{p}<0.001$ ); furthermore the $\mathrm{Hb} A_{1 \mathrm{c}}$ values were for NIDDM $8.9 \pm 1.4 \%$ vs $5.6 \pm 0.5 \%$ for healthy subjects $(\mathrm{p}<0.001$ ). MALDI spectra of healthy subjects show the peaks corresponding to $\alpha$ - and $\beta$-globins; glycated $\beta$-globin is detected, with an abundance in the range $1-5 \%$. In the case of samples coming from NIDDM the abundance of such peak grows up in the range 5-15\%. Interestingly, for some of the samples under investigation, further peaks become detectable, corresponding to $\beta$. globins linked with dehydrationt-oxidation products of glucose. Then MALDI/MS allows to determine directly the level of glycation of globins, as well as to evaluate the relevance of oxidative processes sequential to the simple glycation. 
521

ADVANCED GLYCATION END-PRODUCTS IN DIABETIC PERIPHERAL NERVE AFTER ISLET TRANSPLANTATION. $M$ Sensi, S Morelli, E Sagratella, P Castaldo, S Morano. G Pugliese. V Caltabiano, $M$ Vetri, F Purrello, U Di Mario. Endocrinology Departments of Rome "La Sapienza", Catania and Catanzaro Universities. Italy.

To establish whether return to normoglycemia reduces peripheral nerve advanced glycation end-product (AGE) levels, potentially contributing to the pathogenesis of diabetic neuropathy, 1200-1500 islets were transplanted into the liver of diabetic inbred Lewis rats after 4 or 8 months of disease. This study was then continued for further 4 months and concluded with sciatic nerve removal for protein AGE level determination by ELISA. Age-matched diabetic and control animals were also followed in parallel. The metabolic parameters in both groups of transplanted animals returned to values not significantly different from the respective control groups (glycemia, $\mathrm{mg} / \mathrm{dl}: 50.3 \pm 11.0 \mathrm{SD}$ and $73.7 \pm 22.2$ vs $55.5 \pm 9.4$ and $80.6 \pm 35.7 ; \mathrm{HbAl}, \%: 8.1 \pm 2.2$ and $10.1 \pm 1.9$ vs $6.7 \pm 1.2$ and $8.5 \pm 1.5$; body weight, g: $356 \pm 53$ and $340 \pm 25$ vs $403 \pm$ 34 and $406 \pm 81$ ). The AGE level in the earlier tsansplant group ( $4 \mathrm{mo}$.) was reduced to a value $(1.16 \pm 0.51 \mathrm{SD}$ AGE Units/mmol Nor-Leucine equivalents) which was lower than that of the corresponding diabetic group (2.31 $\pm 1.34 \mathrm{AGE}$ Units), higher than that of the control group $(0.95 \pm 0.53$ AGE Units) but not significantly different from any of the two. In the later transplant group $(8 \mathrm{mo}$.) the AGE level $(1.35 \pm 0.65$ AGE Units) was still lower than that of the diabetic group $(2.48 \pm 0.76$ AGE Units) but it remained significantly $(p<0.05)$ more elevated than that of the control group $(0.58 \pm 0.26 \mathrm{AGE}$ Units). This study has shown that islet transplantation, by normalizing the metabolic control, can reduce peripheral nervous protein AGE levels provided that is performed early in the course of the disease.

\section{2}

THE ROLE OF TYROSINE PHOSPHATASE IN THE SMOOTH MUSCLE CELLS ON ADVANCED GLYCATION END PRODUCTS STIMULATION

N. Seki, N. Hashimoto, B. J. Goldstein', N. Araki? , S. Horiuchi? ${ }^{2}$ H. Makino and Y. Saito Department of Internal Medicine Il, Chiba University School of Medicine, Chiba 260, Japan, and 'Division of Endocrinology and Metabolic Diseases, Thomas Jefferson University Hospital, Philadeiphia, PA, ${ }^{2}$ Department of Biochemistry II, Kumamoto 860, Japan

Advanced glycation end products (AGE), resulting from nonenzymatic glycation and oxidation of proteins, has been proposed as a cause in diabetic complication. We examined the effect of AGE on thymidine uptake in cultured rats aortic smooth muscle cells (SMC). Incubation with 5 . $10 \mu \mathrm{g} / \mathrm{ml}$ AGE for $48 \mathrm{hrs}$ exhibited the 1.2-1.3-fold increased thymidine uptake in SMC. $(p<0.05)$. The phosphorylation of tyrosine residues is one of the essential roles in intracellular signal transduction on hormone and growth factor stimulation. On the other hand, protein tyrosine phosphatases (PTPases), which dephosphorylate tyrosyl residues, take part in signal transduction, suggesting that PTPases may play an important role in overgrowth state, such as in diabetic complications. To investigate the role of PTPase on the proliferation of rat aortic SMC stimulated by AGE, we evaluated the activity of PTPase on AGE stimulation using the phosphorylated peptide derived from insulin receptor kinase domain as a substrate. In the membrane fraction, PTPase activity 1.8 -fold increased by $10 \mu \mathrm{g} / \mathrm{ml}$ AGE stimulation compared with control BSA. $(p<0.05)$. Using specific antibody, we assayed the activity of SHPTP2 ( $\mathrm{SH} 2$ domain containing PTPase) which has been thought to have a positive role on signal transductions. Activities of SHPTP2 increased in the membrane fraction from rat aortic SMC. In the cytosol fraction, PTPases activity did not change. These data demonstrate that AGE has a proliferative effect on the rat aortic SMC through the increase of PTPase activity in the membrane fraction.

\section{3}

MONOCYTE RECEPTORS FOR ADVANCED GLYCOSYLATION ENDPRODUCTS IN PATIENTS WITH TYPE 1 DIABETES MELLITUS A. Festa, *B. Schmölzer, *E. J. Menzel and G. Schernthaner; I. Medical Dept., Rudolfstiftung Hospital, Vienna, Austria and *Dept. of Immunology, University of Vienna, Austria

One of the main pathogenetic mechanisms of diabetes-associated complications is the accelerated formation of advanced glycosylation endproducts (AGEs) as a result of chronic hyperglycemia. AGEs not only cause qualitative and quantitative changes in extraceliular matrix, but also alter cellular function by interacting with specific receptors.

The aim of our study was to determine the expression of AGE- specific receptors on monocytes in 30 patients with type 1 diabetes mellitus (mean age: $33 \pm 12$ years, mean diabetes duration: $13,4 \pm 11$ years) in comparison with 30 age-matched, non-diabetic controls. Monocytes were isolated from heparinized blood by Ficoll-Paque®, washed with RPMI $\mathbb{B}^{\circledR}$, enriched by adhesion on plastic, then washed again, detached and suspended in RPMI 1640 containing $10 \%$ fetal calf serum to a concentration of $1,5 \times 10^{6} \mathrm{cell} / \mathrm{ml}$. Then AGE-BSA, labeled with fluoresceinisothiocyanate (FITC) was added in increasing concentrations $(1,5$ to $48 \mu \mathrm{mol} / \mathrm{l})$. Incubation was performed at $4^{\circ} \mathrm{C}$ for $16 \mathrm{~h}$. After repeated washing, cells were suspended in sheath-solution and analysed by FACS. For data analysis the percentage of positive cells (\%pos.cells) and the mean fluorescence intensity (MFI) were determined. In patients with type 1 diabetes the number of monocytes expressing AGE-receptors was increased compared to controls (\%pos.cells at $6 \mu \mathrm{mol} / \mathrm{l}$ AGE-BSA: $96 \pm 5$ vs. $93 \pm 7 ; p=0,05$ ), as well as the relative number of receptors (MFI at 6 $\mu \mathrm{mol} / 1 \mathrm{AGE}$ ASA (MFI 6): $78 \pm 32$ vs. $59 \pm 23 ; p<0,01$; MFI $12: 115 \pm 50$ vs. $86 \pm 32 ; p<0,01$ ). Patients with good metabolic long-term control (mean HbA 1 -value $<7.5 \% ; n=5$ ) showed a lower relative number of receptors than patients with a mean $\mathrm{HbA} 1 \mathrm{c}^{-v a l u e}>7,5 \%(\mathrm{n}=17):$ MFI $12: 79 \pm 26$ vs. $123 \pm 58 ; \mathrm{p}<0,03$. Our data show, that AGE specific monocyte receptors are overexpressed in patients with type 1 diabetes mellitus compared to non-diabetic controls. The finding, that this overexpression is blunted in patients with good Iong-term metabolic control may suggest a relationship of AGE receptor expression with metabolic control and thus with the occurence of diabetic micro- and macrovascular complications.
524

EFFECTS OF NOVEL GLYCATION NHIBITORS ON DIABETIC RATS AND MECHANISM OF ACTIONS

K. Katsuno, M. Kobayashi, Y. Baba, T. Koizumi. and F. Sato, Kissei Pharmaceutical

Co. Lid., 19-48 Yoshino, Matsuno, Nagano-Pref., 399, Japan.

Non-enzymatic irreversible formation of advanced glycation end products (AGE) is postulated as a causal factor for developing of diabetic complications. We investigated inhibitory effects of KGR-1380 and KGR-1421, novel non-hydrazine compounds, on AGE formation. Both inhibitors were 10 times as potent as aminoguanidine in inhibiting lysozyme polymerization and AGE formation from $B S A$ in vitro. AGE levels were determined using rabbit polyclonal antibody against Keyhole limpet hernocyanin (KLH)AGE. Although this antibody was specifically reactive with AGE formed on various kinds of proteins, it did not recognize Amadori compound. In human monocyte cell line (THP-1), secretion of IL-1 $B$ was stimulated by addition of AGE-BSA and the stimulation was attenuated by anti-AGE antibody. These data suggest that the antibody used in the present study recognizes the AGE epitops which bind to AGE receptor(s) causing biological functions linked to diabetic complications. AGE level in sera increased depending on blood glucose level in streptozotocin-induced diabetic rals. Oral administration of $K G R$ compounds $(25-100 \mathrm{mg} / \mathrm{kg})$ for 10 weeks to diabetic rats significantly lowered AGE content in plasma, whereas aminoguanidine showed no obvious effects. None of these three compounds affected plasma glucose or HEATC levels. To elucidate the possible mechanism of KGR compounds, BSA was incubated with glucose or other Maillard reaction intermediates KGR-1380 and KGR-1421 inhibited formation of AGE from either glucose or Amadori compounds more potently than aminoguanidine. In contrast, inhibitory activity of KGRs were not as potent as that of aminoguanidine against AGE formation from 3-deoxyglucosone or other dicarbonyl compounds. These results suggest that KGRs inhibit Maillard reaction at step(s) different from that of aminoguanidine and they can be useful for preventing diabetic complications. 


\section{Oxidative Stress}

\section{5}

THE POSSIBLE ROLE OF NITRIC OXIDE IN THE EFFECT OF INSULIN ON THE AGGREGATION AND ADENOSINE TRIPHOSPHATE RELEASE OF HUMAN PLATELETS.

1. Wittmann, J. Kátai, M. Molnár and J. Nagy, Second Department of Medicine, University Medical School of Pécs, Hungary.

Recent observations support the possible connection of the nitric oxide (NO)-guanylate cyclase-cyclic GMP messenger system and the instlin effect on cells. The aim of this study was evaluation of the insulin effect on platelet functions in the presence of high concentrations of the substrate $L$-arginine or an inhibitor, $N^{G_{-}}$-nitro-L-arginine (L-NA) of the nitric oxide synthase (NOS) enzyme, in an in vitro system. The aggregation of thrombocytes and ATP release from platelets induced by adenosine diphosphate in piatelet-rich plasma obtained from healthy persones were investigated. The results are summarized in Table I.

Table 1. Summary of the effect of insulin, L-arginine and L-NA* on the aggregation of platelets and ATP release from thrombocytes.

\begin{tabular}{|lll|}
\hline Agents added to PRP** & Aggregation & ATP release \\
\hline Insulin & $\downarrow^{* * *}$ & $\downarrow$ \\
L-arginine & $\downarrow$ & $\downarrow$ \\
L-NA & no effect & $\downarrow$ \\
Insulin+L-arginine & $\downarrow \downarrow \downarrow$ & $\downarrow \downarrow \downarrow$ \\
Insulin+L-arginine+L-NA & $\downarrow$ & $\downarrow \downarrow \downarrow \downarrow$ \\
$* N^{G}$-nitro-L-arginine (L-NA) & \\
$* \star$, platelet-rich plasma (PRP) & \\
$* * *$, platelet functions decreased to different degrees $(\downarrow-\downarrow \downarrow \downarrow \downarrow)$
\end{tabular}

These results support the hypothesis that the increase in cyclic GMP in cells treated by insulin can be the consequence of an increased concentration of NO. Furthermore, the aggregation of thrombocytes and ATP secretion from platelets are differently affected by NO. Inhibition by insulin of platelet aggregation and ATP release can prevent the secretion of growth factors from thrombocytes, which may play a role in the prevention of atheroscierosis in diabetes mellitus.

\section{6}

HIGH-D-GLUCOSE-INDUCED CHANGES IN ENDOTHELIAL Ca ${ }^{2+} /$ EDRF SIGNALING IS DUE TO GENERATION OF SUPEROXIDE ANIONS

W.F. Graier, S. Simecek, H.G. Hoebel, T.C. Wascher ${ }^{*}$ and Gerhard M. Kostner. Dept. Med. Biochem. \& * Int. Med., University Graz, Harrachgasse 21/III, A$8010 \mathrm{Graz}$, Austria

This study was designed to investigate the intracellular mechanisms by which high D-glucose affects endothelial $\mathrm{Ca}^{2}+/ \mathrm{EDRF}$ response. Pretreatment of porcine aortic endothelial cells with high D-glucose results in enhanced EDRF formation (39\%; measured by the conversion from $\mathrm{L}^{-}{ }^{3} \mathrm{H}$-arginine to $\mathrm{L} \cdot{ }^{3} \mathrm{H}$ citrulline) due to increased endothelial $\mathrm{Ca}^{2+}$ signaling to bradykinin $(97 \%$; measured using the fura-2 technique). The aldose-reductase inhibitors, sorbinil and zopolrestat failed to diminish high-D-glucose-mediated alterations in the $\mathrm{Ca}^{2+} /$ EDRF response, suggesting that enhanced aldose-reductase activity does not contribute to high-D-glucose-initiated changes in $\mathrm{Ca}^{2}+/ E D R F$ signaling. Pretreatment of cells with the non-metabolizing D-glucose analog, 3O-methylglucopyranose mimicked the effect of high D-glucose on endothelial $\mathrm{Ca}^{2}+/$ EDRF signaling to bradykinin (114 and $26 \%$ respecitively). High Dglucose and 3-0-methylglucopyranose yielded increases in superoxide anion $\left(\mathrm{O}_{2}{ }^{-}\right)$formation (133 and $293 \%$, respectively; measured by the reduction of ferrocytochrome c). High D-glucose-enhanced $\mathrm{O}_{2}{ }^{-}-$release was insensitive to inhibitors of cyclooxygenase, lipoxygenase and cytochrome $P 450$, while it was diminished by desteral, a heavy metal chelator. The effect of high D-glucose and 3-0-methylglucopyranose were abolished by co-incubation with superoxide dismutase amd the antioxidants, GSH, vitamine $E$ and probucol. Pretreatment of endothelial cells with the $\mathrm{O}_{2}{ }^{-}$-generating system, xanthine oxidase, mimicked the effect of high $\mathrm{D}$-glucose on $\mathrm{Ca}^{2+} / \mathrm{EDRF}$ signaling. We suggest that prolonged exposure to high $\mathrm{D}$-glucose concentration results in enhanced formation of $\mathrm{O}_{2}-$, due to metal-mediated oxidation of D-glucose within the cells. This overshoot of $\mathrm{O}_{2}{ }^{*}$ enhances agonist-stimulated $\mathrm{Ca}^{2+}+$ EDRF signaling via a yet unknown mechanism.

\section{7}

OXIDATIVE STRESS ACTIVATES NUCLEAR FACTOR $\kappa ß$ AND INDUCES NITRIC OXIDE SYNTHASE IN B-CELLS. J.G. Mabley, R. Barry and I.C. Green. School of Biological Sciences, University of Sussex, Brighton BN1 9QG, U.K.

The induction of nitric oxide synthase (NOS) in rat islets and RINm5F cells by interleukin- $1 \beta$ is mediated by activation of the nuclear factor $N F \kappa \beta$. Our aim was to examine if superoxide could activate $N F K B$ and induce NOS activity and if transforming growth factor $\beta 1$ could prevent induction in RINm5F cells. RINm5F cells were pretreated for $2 \mathrm{~h}+100 \mu \mathrm{M}$ diethyldithiocarbamic acid (DETC), an NFאß activation inhibitor, prior to addition of the superoxide generator pyrogallol for 24h. The activity of NOS was determined by cytosolic conversion of ${ }^{14} \mathrm{C}$-arginine to ${ }^{14} \mathrm{C}$-citrulline and nitric oxide. RINm5F cell arginine conversion was increased by pyragallol $[50,100$ and $200 \mu \mathrm{M}]$ from $5.7 \pm 1 \mathrm{fmol}$ arginine $/ \mu \mathrm{g}$ protein $/ 45 \mathrm{~min}$ to $14.7 \pm 1.6$ ( $\mathrm{p}<0.005 \mathrm{vs}$. control), $18.2 \pm 3.1(\mathrm{p}<0.01), 23.5 \pm 3.8(\mathrm{p}<0.05)$ respectively. The rise in NOS activity was prevented by DETC; $5.7 \pm 0.3$ ( $p<0.001$ vs. pyrogallol $50 \mu \mathrm{M}), 6.9 \pm 2.2(\mathrm{p}<0.05$ vs. $100 \mu \mathrm{M}), 3.8 \pm 0.5$ ( $\mathrm{p}<0.05$ vs. $200 \mu \mathrm{M})$. Increased NOS activity was also observed in rat islets treated with pyrogallol or xanthine oxidase/hypoxanthine. TGFB1 pretreatment inhibited $\Pi-1 \beta$ induction of NOS in RINm5F cells as shown by Western blotting and cytosolic enzyme activity. The increased arginine

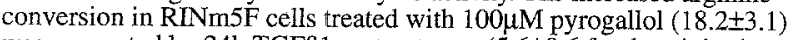
was prevented by $24 \mathrm{~h}$ TGFB1 pretreatment $(5.6 \pm 0.6 \mathrm{fmol}$ arginine $/ \mu \mathrm{g}$ protein $/ 45 \mathrm{~min}(\mathrm{p}<0.05$ vs. $100 \mu \mathrm{M})$ ). Oxidative stress can induce nitric oxide synthase in RINm5F cells and rat islets of Langerhans by NFKB activation. TGF 11 prevents induction of nitric oxide synthase by $I L-1 \beta$ or oxidative stress in RINm5F cells and may inhibit NFk $\beta$ activation.

\section{8}

HYPOGLYCEMIC EFFECT OF LIPOIC ACID: MODE OF ACTION IN NORMAL AND STREPTOZOTOCIN DLABETIC RATS

M. Khamaisi, A. Tirosh, E. Damschek, A. Rudich, H. Tritschler, R. Potashnik and N. Bashan. Dept. of Clinical Biochemistry, Faculty of Health Sciences, BenGurion University of the Negev, Beer-Sheva, Israel 84103.

Lipoic acid (LA), a cofactor of ketoacid dehydrogenases, is a unique antioxidant which was shown to increase peripheral glucose utilization in diabetic patients. We have found that ten days LA injections to streptozotocin (STZ) diabetic rats caused a reduction in both fasting and non-fasting blood glucose, with no changes in insulin levels. This reduction was accompanied by normalization of skeletal muscle GLUT4 protein content and glucose transport activity, as compared to non-diabetic animals. We further investigated whether hepatic glucose metabolism could be an additional site of LA's action. $100 \mathrm{mg} / \mathrm{Kg}$ i.v LA injection to fasting normal rats caused hypoglycemia $1-2 \mathrm{~h}$ after injection $(35 \pm 9$ Vs $68 \pm 13 \mathrm{mg} \%, \mathrm{P}<0.05$ ). In $\mathrm{STZ}$-diabetic rats glucose reduction appeared later, and was progressive up to 8 hours $(185 \pm 64$ Vs $373 \pm 64 \mathrm{mg} \%, P<0.01$ at $8 \mathrm{~b}$ ). This effect of LA was observed only when the experiment was performed under fasting conditions. The hepatic response to exogenous glucagon $(20 \mu \mathrm{g} / \mathrm{rat}, \mathrm{i} . \mathrm{\gamma})$ was completely abolished when administered following LA, suggesting the interference of LA in gluconeogenesis and/or glycogenolysis. Since neither liver glycogen content nor glycogen phosphorylase activity were influenced by $\mathrm{LA}$, we suggest that LA affects gluconeogenesis by either substrate depletion or a direct effect on specific gluconeogenetic enzymes. Insulin stimulated glucose uptake to soleus was decreased by $34 \%$ when muscles were excised during hypoglycemia, representing the effect of counter regulatory hormones activation. The possibility of inhibiting gluconeogenesis by LA, in addition to increasing muscle GLUT4 content, could be beneficial to diabetic patients in whom hepatic glucose production and skeletal muscle insulin resistance are major contributors to fasting hyperglycemia. 


\section{9}

OXYGEN RADICALS INCREASE BASAL GLUCOSE TRANSPORT, BUT REDUCE INSULIN SENSTTIVITY IN MUSCLE CELLS AND ADIPOCYTES A Rudich, N Kozlovsky, R Potashnik and N Bashan. Dept. of Clinical Biochemistry, Faculty of Health Sciences, Ben-Gurion University of the Negev, Beer-Sheva, Israel.

Increased oxidant stress has been suggested to contribute to the development of late diabetic complications. In this study we hypothesized that exposing L6 myotubes and 3T3-L1 adipocytes to Reactive Oxygen Species and advanced glycosylation end products (AGEs) will alter their glucose transport activity and insulin responsiveness. $\mathrm{L} 6$ myotubes exposed to $\mathrm{H}_{2} \mathrm{O}_{2}$ and $\mathrm{O}_{2}-$ generating systems (glucose/glucose oxidase or to xanthine/xanthine oxidase, respectively), exhibited a time and dose-dependent increase in 2-deoxyglucose uptake activity. This effect was first observed after 6 hours incubation, and reached $2.6+0.4$ and $2.2 \pm 0.7$-fold increase at 24 hours exposure to $5 \mathrm{mM}$ glucose and $50 \mathrm{mU} / \mathrm{ml}$ glucose oxidase, or to $50 \mathrm{mM}$ xanthine and $20 \mathrm{mU} / \mathrm{ml}$ xanthine oxidase, respectively. This effect could be attributed to an approximately 2 -fold increase in both GLUT 1 protein and mRNA content. In 3T3-L1 adipocytes incubated with glucose oxidase basal 2DG uptake was increased by $4.2 \pm 0.6$. However, maximal insulin stimulation $(100 \mathrm{nM})$ increased basal glucose transport activity by $750 \%$ in control Vs $185 \%$ in glucose oxidase treated cells. Insulin sensitivity was also reduced, as evident by insulin concentration required for $50 \%$ of maximal transport activation $(0.25$ Vs $2.5 \mathrm{nM}$ ). This correlated with increased GLUT1 mRNA and decreased GLUT4 mRNA content. 3T3-L1 cells incubated for 48 hours with glycated albumin $(0.25 \mathrm{mg} / \mathrm{ml})$ exhibited a significant $60 \%$ increase in basal 2DG uptake activity, resulting in 50\% reduction in the degree of maximal uptake stimulation by insulin. These results suggest that prolonged, low grade exposure of skeletal muscle cells and adipocytes to ROS results in a significant loss in their ability to respond to insulin stimulation. AGEs as inducers of oxidant stress in vivo may therefore contribute to the insulin resistant state in diabetes mellitus.

531

\section{IN VITRO DETERMINATION OF SERUM TOTAL ANTIOXIDANT CAPACITY (TAC) IN TYPE 1 (IDDM) DIABETES MELLITUS \\ E Petruzzi, P Pinzani, AM Stefanescu, E Mannucci, CM Rotella, and M Pazzagli}

Clinical Biochemistry and Endocrinology Unit, Dept of Clin Pathophysiology Instit of Gerontology and Geriatrics; University of Florence - ITALY

In diabetes mellitus, a sharp reduction of antioxidant defences and an increased production of serum free radical seem to coexist (Cross CE et al, Ann Intern Med 107: 526-45, 1987). For this reason there is increasing interest in the study of mechanisms of antioxidant protection against free radical-induced injury and in the identification of suitable biochemical parameters for the measurement of the Total Antioxidant Capacity (TAC) in body fluids. Several methods have been proposed for the assessment of TAC, including that based on enhanced chemiluminescence (Whitehead TP et al, Anal Chim Acta 266:265-77, 1992). This technique is calibrated with TROLOX and assay results are expressed as $\mu \mathrm{mol} \mathrm{L} \mathrm{L}^{-1}$ of Trolox. This procedure implies the determination of TAC both on whole serum (WTAC) and on its deproteinated fraction (DPTAC), obtained by uffrafiltration, using a Centrifree MS-1 micropartition system (Amicon Massachusetts). We have measured WTAC and DPTAC in two subject groups: i) 15 young Type 1 (IDDM) diabetic subjects (Mean $\pm S D=30 \pm 3.5 \mathrm{yr}$ ), affected by medium-term Diabetes Mellitus, in a good metabolic control, evaluated on the basis of $\mathrm{HbA} 1 \mathrm{\%}$ (Mean+SD=5.9+1.3\%) and of fasting glycemia (MeantSD=146 \pm 34 $\mathrm{mg} / \mathrm{dL}$ ); ii) 16 age- and sex-matched healthy control subjects (HS). Results (Mean $\pm S D$ ) in IDDM were: $W T A C=204 \pm 137 \mu \mathrm{mol} / L^{-1}$ and DPTAC $=82 \pm 30$ $\mu \mathrm{mol} / \mathrm{L}^{-1}$; in HS were: $W T A C=366 \pm 145 \mu \mathrm{mol} / \mathrm{L}^{-1}$ and $\mathrm{DPTAC}=204 \pm 116$ $\mu \mathrm{mol} / \mathrm{L}^{-1}$. Statistical analysis showed a significant difference $(p<0.01)$ between IDDM and control subjects both in WTAC and DPTAC values. The significanly reduced WTAC and DPTAC in IDDM subjects may be due to an increased free-radicals production. It may be due, at least in part, to the reduction of circulating vitamin $E$ induced by insulin (Quinones-Galvan $A$ et al, Metabolism 1996, in press). As the good metabolic control of our IDDM patients resulted unable to prevent the serum TAC reduction, it remains to verify the role that a supplementary antioxidant therapy may have in the treatment of Type 1 (IDDM) Diabetes Mellitus.
530

ADMINISTRATION OF GLUTATHIONE IN PATIENTS WTTI NIDDM IS AB!F TO IMPROVE THE TNTRAPLATELET $~ N O S O$ AND TO DECREASE PAI

V. Martina, G.A Bruno, M. Tagliabue, V. Brancaleoni, E. Zumpano, F' Trucco* a G. P. Pescarmona* . Istituto di Medicna Intema Divisione di Endocrinologia; Dipartimento di Genetica Biologia e (himica Medica*. Universita degli Studi di Torino

In NDDM the plasminogen activator inhibitor-1 (PAI-1) is elevated and recent studies document that, related to hyperglicemia, there is an oxidative stress, which is accompanied by coagulative alterations. On the other hand it has been demonstrated that the endothelial cells of diabetic rats present a reduction of nitric oxide (NO) production. Firthermore our data, in press, document a reduced $\mathrm{cNOS}$ intraplatelet activity in NIDDM. These data could explain, at least in part the development of the vascular hypertension and of the cardiovascular complications. Glutathione (GSH), antioxidant substance and activator of costitutive nitric oxide synthase (cNOS), is decreased in NIDDM. GSH administration in patients with NIDDM could improve the lowered antioxidant status and the NO synthesis. Aim of the study was to evaluate the effect of GSH administration on PAI-1 and tissue plasminogen activator inhibitor (t-PA) plasma levels such as intraplatelet activity of eNOS in NIDDM. Subjects and methods: 12 patient with NIDDM, $5 \mathrm{M}$ and $7 \mathrm{~F}$, age (X+SEM) 60.8ะ0.8 yrs, BMI $27 \pm 1.5 \mathrm{Kg} / \mathrm{m}^{2}$, treated with hypoglycaemic oral agents, underwent a ireatment with GSH im ( $600 \mathrm{mg} / \mathrm{die})$ for ten days. Before and after treatment were evaluated intraerythrocytary GSH, plasma levels of PAI- 1 and t-PA. Results: after treatment GSH increased $\left(2.17 \pm 0.3\right.$ vs $1.12 \pm 0.4 \mu \mathrm{mol} / 10^{10}$ erythrocytes; $\left.p<0.01\right)$, the PAI-1 decreased $(69.8 \pm 5.0$ vs $81.5 \pm 4.4 \mathrm{ng} / \mathrm{ml} ; \mathrm{p}<0.005)$, the t-PA didn't modify $(0.5 \pm 01$ vs $0.9 \pm 0.2 \mathrm{U} \mathrm{U} . \mathrm{m} / \mathrm{ml})$ and the $\mathrm{NOS}$ activity increased $(4.3+0.9$ vs $0.8 \pm 0.2$ frnol/m/10 PLTs; $\mathrm{p}<0.001$ ). Comelusions: our data demonstrated that GSH administration is able to increase the intraerythrocytary thiolic group and intraplatelet cNOS and at the same time reduce PAI-1 plasma levels probably because the GSH activates the cNOS and protects against the oxidative action of free radicals. These preliminary data suggest that GSH can be useful in NIDDM to decrease the major cardiovascular events, which are the first cause of death in NIDDM, by improving the fibrinolytic state and by increasing the cNOS activity.
532

REDOX SYSTEMS OF ERYTHROCYTES IN IDDM

R.M.Parhimovich, Moscow Regional Research Clinical Institute, 61/2, Shchepkina str., Moscow, 129110, Russia.

The aim was the evaluation of antioxidative defence in IDDM. Redox systems of erythrocytes (E) and reducing equivalents (RE) transport was evaluated by potentiometric registration of reduction rate of ferrycianide (RRF) added to blood and $E$ suspension in vitro. Ferrycianide is non-penetrating the $E$ acceptor of electrones (e) transported from $E$ partly by shuttle ascorbic acid $=$ dehydroascorbic acid $(A A=D H A A)$. The main source of RE in oxidative load was shown to be glucosomonophosphate shunt (GMPS)-glutathione system. The shuttle $A A=\triangle D H A A$ was evaluated by estimating of RRF after adding of $A A$ in vitro. Increased ( $P<0.01$ ) RRF ( $R E$ flow) was revealed in blood of diabetics: to 1.9 -fold in $\mathrm{pH} 7.4$ and even 2.8 -fold in pH 7.0 (instead of decrease in control in pH 7.0). In the situation of such RE loss in IDDM the lowered (in pH 7.4 and absent in $\mathrm{pH} 7.0$ ) participation of the shuttle $A A==D H A A$ in $\mathrm{H}+$ and (e) transport from $\mathrm{E}$ was established (i.e.decreased intracellular DHAA reduction). Increased RE consumption may deplete antioxidative defence. Evidently the exhaustion of antioxidative cell defence is the main mechanism of diabetogenic effect of alloxan by means of free radicals generating in alloxan $=$ dialuric acid cycle. We observed: 1)rapid depletion of $R E$ output in rat $E$ after injection of diabetogenic dose of alloxan, 2)increase of RRF (RE output) and exhaustion of GMPS in human $E$ (and hemolysis) after adding alloxan in vitro. The antioxidative defence of B-cells is known to be much lower compared to $E$. 
533

RELATION BETWEEN PLASMA AND PLATELET CONCENTRATION OF MALONYLDIALDEHYDE AND ASCORBIC/DEHYDROASCORBIC ACM IN PATIENTS INSULIN-DEPENDENT DIABETES MELLITUS

F Franconi. R Anichini. M.Ciuti. M.De Felice. L.Martinoli. and G Seghicri. Dpt. of Biochemistry. Univ of Sassari. Diabetes Unit. Spedali Rituniti. Pistoia. National Nutrition Institute. Rome. Faculty of Pharmacy. Univ of Rome. Italy

The couple ascorbic acid/deliydroascorbic acid AA/DHAA svstem exerts a scavenging function against peroxides in plasma as well as in the intracellular milieu. Since diabetes mellitus represents a situation where production of peroxides is increased at the intracellular and extracellular milieu. the aim of this study was to investigate the relation of plasma and platelet levels of AADHAA with those of malonyldialdebyde (MDA). an indirect marker of lipoperoxides. both assayed by a HPLC method. in 34 patients insulin-dependent diabetes mellitus (IDDM). compared with 23 age- and sex-matched control subjects. Plasma and platelet MDA were significantly higher in the patients affected with IDDM than in controls. Diabetic group was moreover characterized by a huge decrease in plasna AA $(8.5 \pm 5.5 \mu \mathrm{mol} / \mathrm{l}(\mathrm{SD})$ vs $33.4 \pm 7.6 \mu \mathrm{mol} / \mathrm{l} . \mathrm{p}=0.0(00)$ ). mirrored by a significant increase in plasma DHAA (11.9 $\pm 3.9 \mu \mathrm{mol} / \mathrm{l}$ us $3.9 \pm 2.5 \mu \mathrm{mol} / \mathrm{l} \mathrm{p}=0.01)$. No detectable DHAA was observed in platelets of both diabetic and control subjects. while AA was significantly increased in platelets of the diaberic patients as comparcd with the controls $\left(+2.6 \pm 7.41634 .8 \pm 5.1 \mathrm{nmol} 10^{-4}\right.$ platelets. $\left.\mathrm{p}=0\right)(0001)$ ) Platelet AA in diabetic group was significantly inversely correlated with glycalcd hacmoglobin $(r=-0) 34: p=1), 04)$ and plasma MDA $(r=-1) .36: p=0.04)$ and directs with the sum of plasma AA+DHAA $(r=0.44: p=0.009)$ and with platclet MDA $(r=0) 38: p=0.02)$. In conclusion: 1) plasma AADHAA is significantly lowerce in IDDM. in association with an increase in MDA lerels. 2) only AA is detected In platelets. suggesting a rapid tum-ever of intracellular DHAA. 3) platelet AA is modulated by metabolic control. as well as by the production of peroxides in IDDM.

\section{5}

OXHDTIE STRESS AS A RISK FOK DEVELOPMENT OF DIABETES DUR!NGIOBESTY

G. Koeic, D Pavlovic, R Kocic, 'T. Cvetkovié, M. Milenovic, S. Radenković, 1). Mikid, S.Zivic. Institute of Binchemistry and Clinic for Endocrinology Findly of Medicina Niš, Yugoslavia

Obesity is traquenty associated with insulin resistance and/or impaired stenstivity of $\beta$ cells. Elevated lipid peroxidation and disordered thiol compound metabolism are among the crucial aspects in the pathogenesis of diabetes. The present work was initiated to estimate erythrocyte redued ghtathione content (eGSH), lipid peroxidation intensity and response of erythroytes to oxidative stress in obese - NIDDM patients and obest non-diabetis subjects. $A$ first group of 10 obese diabetics $(\mathrm{BMI}=$ $32,88+4,65$ ) and second of 12 obese subjects (BMI $\pm 34,18+5,98$ ), together with control subjeds ( $B M+20,03 \pm 3,12$ ), matchod in age, were included in study. A marked decrease in eGSH level was present in obese diabetics $(0,76+0,11 \quad \mathrm{p}<0,001$ v.s. control value $1,20 \pm 0,04$ !mol/miEr $)$ together with a rise in lipid peroxide levels in both plasma $(5,72+0,21$ p $<0,05$ v.s control value $3,58+1,22 \mu \mathrm{mol} \mathrm{MDA} /$ ) and erythrocytes $(3,58+0,19 p<0,05$ $\mathrm{v}, \mathrm{s}$ control value $2,63+0.36 \mathrm{nmol} \mathrm{MDA} / \mathrm{ml}$ Eri. The increase in plasma and arythrocyte lipid peroxidation products in basal state was shown to occur in obesity $(3,98 \pm 0,27 \mu \mathrm{mol} \mathrm{MDA} / \mathrm{in}$ plasma and $3,44 \pm 1,08 \mathrm{nmol}$ MI)A/m Er), while eGSH tended to decrease $(1,02+0,14$ p<0,05, v.s. control value. The correlation between eGSH and lipid peroxidation was negative $(r=-0,42 ; y=-1,89 x+6,06)$. There was not difference between lipid peroxidation products in erythrocytes after exposing to oxidative stress with $5 \mathrm{mM} 1202(7,88 \pm 0,4$ in obese diabetics, v.s. $7,38+1,02$ in obese subjects, v.s. $7,06+1,2$ nmolMDAiml $\mathrm{Er}$ in controls. The progressive deterioration of eGSH content from group of obese to group of obese diabeties may reflect an evolution from obesity toward diabetes. This hypothesis is based on evidence of the insulinotropic effect of GSH, since GSHI can affect the sensitivity of $\beta$ cells to respond to glucose. These data indicate that the disfunction of prooxidant/antioxidant status of obese patients can be affected before the development of diabetes. Presented findings permited sound recommendation of drugs with antioxidative properties

\section{4}

NITRIC OXIDE RELEASE AND GLUCOSE METABOLISM ARE IMPAIRED IN SUBJECTS WITH MICROVASCLLAR ANGINA.

P.M. Piatti, G. Fragasso. L.D. Monti, B. Guazzini, V.C. Phan. G. Valsecchi, S. Chierchia. A.E. Pontiroli and G. Pozza. IRCCS H. San Raffaele. Milano. Italy.

The aim of the study was to evaluate whether the insulin stimulated-arterial endothelin-1 (ET-1) and nitric oxide (NO) release, and forearm glucose and lipid metabolism are impaired in subjects with microvascular angina (MA). Nine subjects with MA (mean age $52 \pm 3$ years, mean BMI $24.4 \pm 0.7 \mathrm{~kg} / \mathrm{m}^{2}$ ) and eleven normal subjects with similar mean age and BMI were submitted to an acute intravenous administration of $0.1 \mathrm{U} / \mathrm{kg}$ of insulin combined to a euglycemic clamp technique and forearm indirect calorimetry lasting $60 \mathrm{~min}$. The incremental areas ( $\triangle A$ AUC, $0-60 \mathrm{~min}$ ) of ET-1. NO. glucose and lipid oxidation. glucose storage and non-oxidative glycolysis were measured during this period. Basal ET-1 levels were higher in MA than in normal subjects $(8.23 \pm 0.65$ vs $3.90 \pm 1.16 \mathrm{pg} / \mathrm{mI} ; \mathrm{p}<0.007)$ while basal NO. insulin and glucose levels were similar in both groups. After insulin bolus. the $\triangle A U C S$ of ET-I were similar while the $\triangle A U C s$ of NO were significantly lower in MA than in normal subjects $(-49.40+79.70$ vs $306.82 \pm 131.01 \mu \mathrm{mol} / \mathrm{L}$. $\mathrm{p}<0.01)$. The $\triangle A U C s$ of forearm glucose uptake (FGU) and glucose oxidation were significantly decreased by $40 \%$ and 110 by $\%$ in subjects with MA than in normal subjects. No differences were found measuring the $\triangle A U C S$ of non-oxidative glycolysis. glucosc storage and lipid oxidation. Basal ET-I levels negatively correlated with the $\triangle A$ ULCS of FGU $(r=-0.59, p<0.01)$ while basal NO levels positively correlated with the $\triangle A U C s$ of glucose oxidation $(\mathrm{r}=0.51, \mathrm{p}<0.05)$ and negatively with the $\triangle \mathrm{AUCs}$ of lipid oxidation ( $\mathrm{r}=-0.57, \mathrm{p}<0.02$ ). In conclusion, 1) subjects with MA exhibit high basal ET-1 levels associated to a paradoxical decrease of NO release after insulin stimulation; 2) they show decreased insulin mediated FGU and forearm vlucose oxidation; 3) ET-I and NO levels seem to influence insulin-stimulated forearm glucose metabolism in a opposite manner; 4) with the intravenous insulin bolus associated with forearm indirect calorimetry we were able to detect alterations in ET.1 and NO release which could be responsible for the impairment of glucose metabolisin in subjects with MA.

\section{6}

ELIMINATION OF CHRONIC TONSILUTIS EFFECTS HYDROGEN PEROXIDE LEVEL IN TYPE I DIABETIC PATIENTS

B. Wierusz-Wysocka, D. Zozulińska, A. Majchrzak and H. Wysocki. Insiture of Internal Medicine, Academy of Medicine in Poznań, Poland

Presence of chronic inflammatory focuses in diabetic patients usually results in poor metabolic control of the disease and therefore might influence development of late complications of diabetes. Previously we have noticed very high plasma hydrogen peroxide $\left(\mathrm{H}_{2} \mathrm{O}_{2}\right)$ levels in diabetic patients with chronic inflammatory process. The aim of the study was to estimate plasma $\mathrm{H}_{2} \mathrm{O}_{2}$ concentration before and 14 days after tonsillectomy. The study was performed in 25 patients with type I diabetes ( 15 male, 10 female, aged $28.8 \pm 9.6$ years, diabetes duration $9.8 \pm 5.4$ years, $\mathrm{HbA}_{\mathrm{lc}} 10.4 \pm 2.8 \%$ ) who had chronic purulent tonsillitis (group A) $\mathrm{H}_{2} \mathrm{O}_{2}$ was also estimated in non-diabetic patients with chronic tonsillitis (group B) and in poorly controlled type I diabetic patients without chronic inflammatory process (group C) before and after 14 days of intensified treatment. The plasma $\mathrm{H}_{2} \mathrm{O}_{2}$ concentration was measured acc. to Frew et al.

\begin{tabular}{|c|c|c|c|}
\hline & $\begin{array}{c}\mathrm{H}_{2} \mathrm{O}_{2} \text { level before } \\
(\mu \mathrm{mol} / \mathrm{l})\end{array}$ & $\begin{array}{c}\mathrm{H}_{2} \mathrm{O}_{2} \text { level after } 14 \text { days } \\
(\mu \mathrm{mol} / \mathrm{l})\end{array}$ & $\mathrm{p}$ \\
\hline group $\mathrm{A}$ & $12.82 \pm 1.16$ & $2.81+0.55$ & $<0.001$ \\
\hline group B & $3.45+0.43$ & $2.46 \pm 0.35$ & $<0.001$ \\
\hline group C & $7.81+0.55$ & $4.85 \pm 0.40$ & $<0.001$ \\
\hline
\end{tabular}

Results expressed as mean $\pm \mathrm{SEM}$, paired $\mathrm{t}$ test

The results obtained might indicate the necessity of chronic inflammatory focuses elimination in poorly controlled diabetic patients. 
DOWN-REGULATTON OF THE HUMAN TAURINE TRANSPORTER BY GLUCOSE INVOLVES AN ALDOSE REDUCTASE UNRELATED MECHANISM M.J. Stevens, J.A. Masterson, Y. Hosaka and D. Larkin. University of Michigan, Ann Arbor, MI, USA.

Aldose reductase $(A R)$ related and unrelated mechanisms exacerbate oxidative stress $(\mathrm{OS})$ and contribute to diabetic complications. AR pathway activation (metabolizes glucose (G) to sorbitol (S) and fructose) may exacerbate OS directly or by osmotic depletion (due to $S$ accumulation) of the osmolyte taurine, a B-amino acid and intracellular antioxidant. Taurine depletion may reflect decreased $\mathrm{Na}$-dependent taurine transport (TT) which in high AR-expressing human retinal pigment epithelial (RPE) cells parallels $S$ accumulation, is prevented by AR inhibitors (ARI's), and is associated with decreased TT mRNA. As G-induced OS occurs in low AR-expressing cells (without $S$ accumulation) which activate protein kinase $C$ (PKC), a non-osmotic PKC-mediated mechanism may mediate $G$-induced taurine depletion. The effects of 5$30 \mathrm{mM} \mathrm{G} \pm \mathrm{PKC}$ activation/inhibition on TT activity (measured as linear uptake of 1 $\mu \mathrm{M}^{3} \mathrm{H}$-taurine) and TT mRNA levels (assessed by filter hybridization using a 900 b.p. TT cDNA), were explored in low AR-expressing RPE cells. TT mRNA levels declined in a time and dose-dependent fashion within $2 \mathrm{~h}$ of 10-30 mM G exposure with maximum affect at $12 \mathrm{~h}(65 \%$ of basal, $\mathrm{p}<0.05$ in $30 \mathrm{mM} \mathrm{G})$ and was unaffected by ARI. TT activity paralleled TT mRNA levels, decreasing within $2 \mathrm{~h}(81 \% \mathrm{p}<0.05$ of basal) with maximum reduction of $71 \%$ of basal $(\mathrm{p}<0.05)$ after $8 \mathrm{~h}$ of $30 \mathrm{mM} \mathrm{G}$. PKC activation (100 nM 12-O-tetradecanoyl-phorbol-13 acetate [TPA]) reproduced the inhibitory effects of glucose on TT activity $(40 \% \mathrm{p}<0.05$ of basal after $15 \mathrm{~min}$ exposure), whereas PKC downregulation ( $1 \mu \mathrm{M}$ TPA for $24 \mathrm{~h}$ ) blocked the effect of $\mathrm{G}$ on TT activity (109\% of basal n.s. after $8 \mathrm{~h}$ of $30 \mathrm{G}$ exposure). Thus $\mathrm{G}$ rapidly and specifically decreases TT mRNA and TT activity by non-AR-related (potentially PKC-mediated) as well as AR-related mechanisms. In diabetes, G-induced downregulation of TT may occur independently of AR pathway activity nevertheless predisposing to taurine depletion and OS.

\section{PS 17 \\ Leptin}

\section{8}

A TWO-SITE, MONOCLONAL, IMMUNORADIOMETRIC ASSAY FOR HUMAN LEPTIN

Steve Goodrick', Vidya Mohamed-Ali ${ }^{1}$, Terry Jowett ${ }^{2}$, Alan $\mathrm{O}^{\prime} \mathrm{Shea}^{2}$, John S Yudkin', and Simon Coppack'. 1 Dept of Medicine 2 Dept of Immunology, University College, London.

The recent discovery of leptin, the $o b$ gene product, is a major step in the understanding of the pathophysiology of obesity. Reproducible and accurate measures of circulating levels of leptin production under different conditions will allow a better understanding of the role of this polypeptide in the regulation of adipose tissue. All assays for leptin that have been reported to date rely on polyclonal antibody-based radioimmunoassays. We have raised a panel of monoclonal antibodies to synthetic peptides that span the human leptin sequence. Two of the antibodies, one binding to amino acid sequences 60 to 80 and the second binding in the region of amino acids 117 to 133 of human leptin, have been employed in developing a two-site immunoradiometric assay for human leptin. The assay shows less than $5 \%$ cross-reactivity with mouse leptin, is sensitive to $1.0 \mathrm{ng} \cdot \mathrm{ml}^{-1}$ (nean $+3 \mathrm{SD}$ of zero signal), with inter and intraassay coefficients of variations of $8.4 \pm 2.1$ $(n=15)$ and $5.4 \pm 2.9 \%(n=15)$ respectively. In 12 fasting samples, 6 arterial and 6 venous, levels of leptin were $9.3+8.9$ and $12.6+$ $12.0 \mathrm{ng} . \mathrm{ml}^{-1}$, respectively, agreeing with those previously determined using a polyclonal assay $(r=0.91)$. The 2-site monoclonal IRMA might provide more accurate and specific measures of human leptin, with an assay which would remain consistent over time. This will be an important advantage in longitudinal studies of individuals with changing body weight.

\section{9}

REGULATION OF $O b$ GENE EXPRESSION IN 3T3-L1 AND 3T3F442A ADIPOSE-DERIVED CELL LINES

M Tadayyon', S Holmes', P Trayhurn ${ }^{2}$ and J Arch'. 'Deparment of Vascular Biology, SmithKline Beecham Pharmaceuticals, Welwyn, AL6 9AR, U.K. and ${ }^{2}$ Rowett Research Institute, Aberdeen, AB2 9SB, U.K.

To fully understand the regulation of $o b$ gene expression it is imperative to identify a suitable cell-line(s) in which $o b$ mRNA expression is regulated in a manner similar to that which occurs in vivo. The present study examined two widely used adipose-derived cell lines for their ability to fulfil this criteria. Pre-adipocytes of lineages 3T3-L1 and 3T3-F442A were differentiated using well established conditions for each cell type. Cells were harvested at various time points (2-21 days) following induction of differentiation, and total RNA extracted for analysis of $o b$ mRNA expression using reverse transcriptase-PCR (RT-PCR) or northern blotting coupled with the digoxigenin chemiluminescent detection system. Northern hybridization demonstrated the presence of $o b \mathrm{mRNA}$ in rodent adipose tissue and in differentiated 3T3-F442A cells but not in 3T3-L1 cells, even at day 21 postdifferentiation. RT-PCR of total RNA from differentiated 3T3-L1 cells, using specific $o b$ primers, produced amplicons of the correct size, indicating that 3T3-L1 cells do express ob mRNA, but at a significantly lower level compared to 3T3-F442A cells or rodent adipose tissue. Treatment of each cell-line with insulin ( $800 \mathrm{nM}$ for 48 hours) produced a 2.5 fold stimulation of $o b$ mRNA production. Stimulation of $o b$ gene transcription was accompanied by a time-dependent increase in the appearance of the ob gene product, Ieptin, in the cell culture medium as determined by a specific immunoassay (leptin secretion (ng/ml) mean \pm SEM; control cells: $1.66 \pm 0.29$ $v_{S}$ insulin treated cells: $13.2 \pm 3.4 ; 0<0.005$ by t-test]. It is concluded that $o b$ mRNA expression varies depending on the cell-line with the following rank order: rodent adipose tissue $>3$ T3-F442A cells $>>3$ T3-L1 cells. $O b$ gene expression is positively regulated by insulin at the transcriptional level with a resultant increase in leptin secretion. 
540

A Lrquid Mixed Meal or Exogenous GLP-1 Do not Alter Plasma Leptin CONCENTRATIONS IN HEALTHY VOLUNTEERS

C. Drewes, M. A. Nauck, R. Horn, U. Niedereichholz, R. Ettler, J. Holst, W. Schmiegel and G. Brabant. Dep. of Med., Univ. Hospital, Knappsch.-Krankenh., FRG, Dep, of Med. Physiol, Panum Inst., Uniy, of Copenhagen, DK, Bochum and Div. of Endocrinology, Medizin. Hochschule Hannover, FRG

Glucagon-like peptide 1 [7-36 amide] (GLP-1) and the obese gene product (leptin) are thought to be involved in the central regulation of feeding. Both may act from the peripheral circulation to influence brain function. To study potential interactions, GLP-1 ([7-36 amide): $0.4,0.8 \mathrm{pmol} \cdot \mathrm{kg}^{-1} \cdot \mathrm{min}^{-1}$ or placebo on separate occasions) was infused intravenously (from-30 to $240 \mathrm{~min}$ ) into 9 healthy volunteers (age $26 \pm 3$ y., Body-Mass-Index: $22.9 \pm 1.6 \mathrm{~kg} / \mathrm{m}^{2}, \mathrm{HbA}_{1 \mathrm{c}}: 5.0 \pm 0.2 \%$ [normal: $4.3-6.2 \%$ ], creatinine: $1.1 \pm 0.1 \mathrm{mg} / \mathrm{dl}$ ), and (at $0 \mathrm{~min}$ ) a liquid test meal $(50 \mathrm{~g}$ sucrose in $400 \mathrm{ml}$ $8 \%$ amino acid - Aminosteril Hepa) was administered via a nasogastric tube. Measurements: Plasma leptin (RIA, raising specific antibodies against leptin (125-140) $_{\text {, }}$ coupled to hemocyanine in rabbits), glucose (Beckman glucose-analyser), insulin (IMX insulin, Abbott), C-peptide (ELISA, DRG Marburg), GLP-1 (RIA, Ab 89390). Statistics: repeated-measures-ANOVA, Student's t-lest. Plasma leptin concentrations were $31 \pm 6 \mathrm{pmol} / \mathrm{l}$ in the basal state. They did not change within 240 min after meal ingestion nor in response to the infusion of exogenous GLP-1 [7-36 amide] $(\mathrm{p}=0.99$ for the interaction of experiment and time) reaching steady-state plasma levels of 25 \pm 3 and $36 \pm 3$ (basal $6 \pm 1$ ) pmol/l. On the other hand, there were clear meal-related responses of glucose (from basal $4.7 \pm 0.1$ to $5.9 \pm 0.2 \mathrm{mmol} / \mathrm{h}$ at $15 \mathrm{~min}, \mathrm{p}<0.050$ ) and insulin (from basal $24 \pm 2$ to $325 \pm 78 \mathrm{pmol} / \mathrm{l}$ at $45 \mathrm{~min}, \mathrm{p}<0.0001$ ) with placebo. In conclusion, (1) plasma leptin levels in normal human subjects show no short-term changes after feeding a liquid mixed meal and (2) do not appear to be acutely influenced by physiological and pharmacological elevations in plasma GLP-1 [7-36 amide] concentrations. This does not exclude interactions at the cerebral (hypothalamic) level or on more long-term temporal scales.

\section{1}

CIRCADIAN LEPTIN SECRETION: DIFFERENCES BETWEEN THE LEAN AND OBESE

M. F. Saad, M. R. Gabriel, A. Khan, S. Damani, K. El-Tawil, A. Sharma, R. Boyadjian, S. Jinagouda, and G. M. Steil, University of Southern California Medical School, Los Angeles, CA, USA.

Little is known about the secretory pattern of leptin, the putative weight regulating hormone. The 24 -hour leptin profile was evaluated in 5 lean (age $34 \pm 3 \mathrm{yr}$ [mean \pm SEM], body mass index [BMI] $23.1 \pm 07$ $\mathrm{kg} / \mathrm{m}^{2}$ ) and 5 obese (age $36 \pm 2$, BMI 32.4 \pm 2.2 ) men. Plasma glucose, insulin, and leptin levels were measured every 20 minutes starting at 06:00 a.m.; meals were served at 08:00,13:00, and 18:00. Fasting $(2.3 \pm 0.6$ vs. $8.3 \pm 2.3 \mathrm{ng} / \mathrm{ml}, p<0.01)$ and the average 24 -hour $(2.5 \pm 0.6$ vs. $7.9 \pm 2.6 \mathrm{ng} / \mathrm{ml}, p<0.01$ ) leptin levels were higher in the obese. Fasting leptin correlated with $\mathrm{BMI}$ and fasting insulin $(r=0.87,0.71$, $p<0.001$ for each), but not with fasting glucose. In all subjects, nighttime (23:00-05:40) mean leptin level was higher than daytime (06:00-22:40) mean $(7.4 \pm 2.6$ vs. $5.8 \pm 2.0 \mathrm{ng} / \mathrm{ml}, \mathrm{p}=0.01)$. Cosinor analysis confirmed the presence of a strong circadian rhythm for leptin in all subjects $\left(r^{2}=\sim 0.85\right)$. The acrophase (time of the peak) occurred earlier in the lean $(00: 15 \pm 00: 24$ vs. 01:12 $\pm 00: 18$ hour, $p=0.02)$ and the amplitude (difference between nadir and peak) was higher $(53 \pm 5$ vs. $38 \pm 4 \%, p=0.04$ ). Autocorrelation analysis showed a second smaller oscillatory component with a period of approximately 11 hours. The amplitude of this component was similar in the lean and obese $(5.9 \pm 1.0$ vs. $7.6 \pm 2.3 \%)$, but the acrophase occurred earlier in the lean $(14: 06 \pm 00.26$ vs. $16.36 \pm 00: 12$ hour, $p=0.001)$. Conclusions: 1) human obesity is associated with increased leptin levels, 2) the nocturnal rise in leptin levels may help suppress appetite during sleep, 3) leptin rhythmicity is blunted in obesity.

\section{2}

\section{ADIPOSE TISSUE LEPTIN PRODUCTION AND PLASMA LEPTIN KINETICS IN HUMANS:}

$\checkmark$ Mohamed-Ali!, S Klein ${ }^{2}, M$ Landt$^{2}$, and SW Coppack'. 1 University College, London, 2 University of Washington, USA. Abdominal adipose tissue leptin production was determined in vivo by arteriovenous balance in 14 lean and obese men (BMI mean= $27.0 \pm 1.9$, range $\left.=21.4-45.2 \mathrm{Kg} \cdot \mathrm{m}^{-2}\right)$. Blood samples were taken simultaneously from an abdominal vein, draining subcutaneous adipose tissue, and a radial artery. Adipose tissue blood flow was measured by xenon washout. Abdominal vein leptin concentrations (mean $=8.9 \pm 2.4 \mathrm{ng} \cdot \mathrm{ml}^{-1}$, range $=2.1-36.5 \mathrm{ng} \cdot \mathrm{ml}^{-1}$ ) were consistently greater than arterial values (mean $=6.6 \pm 1.9 \mathrm{ng} \cdot \mathrm{ml}^{-1}$, range $=1.7-$ $28.2 \mathrm{ng} . \mathrm{ml}^{-1} ; \mathrm{p}<0.001$ ). The net rate of abdominal adipose tissue leptin production (mean $=3.2 \pm 0.5 \mathrm{ng} .100 \mathrm{~g}^{-1} \mathrm{~min}^{-1}$ ) correlated directly with percent body fat $\left(r_{s}=0.59 ; p=0.016\right)$. Estimated whole body leptin production rate $\left(797 \pm 283\right.$ ng.person. $\left.\mathrm{min}^{-1}\right)$ correlated directly with percent body fat $\left(r_{s}=0.93 ; p<0.0001\right)$ and with regional leptin production $\left(r_{s}=0.81 ; p<0.001\right)$. In contrast, the rate of leptin clearance from plasma (mean $=1.50 \pm 0.23 \mathrm{ml} . \mathrm{kg}$ $\left.{ }^{\prime} \mathrm{min}^{-1}\right)$ and plasma leptin half-life (mean $=24.9 \pm 4.4 \mathrm{~min}$ ) was unrelated to adiposity $\left(r_{s}=0.06 ; p=0.03\right.$ and $r_{s}=0.16 ; p=0.30$, respectively). These results provide direct evidence that leptin is produced by adipose tissue in humans and the rate of production is directly related to adiposity. A combination of greater leptin production per unit of body fat and increased production from expanded total body fat mass, rather than alterations in leptin clearance, account for the increase in plasma leptin concentrations observed in obese humans.

\section{3}

ELEVATED SERUM LEPTIN IN GIRLS WITH DIABETES DEVELOPING OBESITY DURING PUBERTY

T. Danne, A. Wadimirova, O. Kordonouri, A. Gruters, R. Horn ${ }^{\star}$, B. Mayr , G. Brabant*. Zentrum für Kinder und Jugendmedizin, Virchow-Klinikum, Augustenburger Platz 1, 13353 Berlin, and "Department Klinische Endokrinologie, Medizinische Hochschule Hannover, Konstanty-Gutschow Str.8, 30625 Hannover, Germany

Resistance to leptin, a satiety hormone, has been proposed as a mechanism contributing to obesity. Unwanted weight gain is also a frequent clinical problem in the treatment of type I diabetes. In the present study we investigated whether serum leptin levels are associated with the development of obesity during diabetes therapy in adolescents. A "matched pairs" siudy was conducted. Retrospective analysis of serum leptin-like immunoreactivity by a specific radioimmunoassay was performed in 32 (14 boys, 18 girls) adolescents with type I diabetes. All patients had prepubertally normal BMI values $(<+1 S D S)$. Sixteen patients developing obesity during puberty and remaining obese after sexual maturation (ob;BMl $>+2 S D S$ ) were matched for age, diabetes duration and sex with patients maintaining normal body weight (nw). No significant differences in glycemic control (HbA1c, HPLC, Diamat), insulin dose or therapeutic regimen were present between these groups at any time point of this investigation. Prepubertal serum samples were available in seven pairs, showing no differences in serum leptin. Significant increases in BMI were observed during puberty while serum leptin remained comparable (boys: $43 \pm 17$ vs. $36 \pm 29$; girls: $76 \pm 40$ vs. $80 \pm 45$ pmol/l; ob vs. $n w, x \pm 1 S D$ ). However, further weight gain in the obese group was associated with a significant elevation of leptin in obese girls (BMl: $26 \pm 1$ vs. $21 \pm 1 \mathrm{~kg} / \mathrm{m}^{2}$; leptin: $171 \pm 99$ vs. $67 \pm 43$ pmol/l; $p<0.05$; insulin: $1.0 \pm 0.1 \mathrm{vs}$. $1.0 \pm 0.1 \mathrm{U} / \mathrm{kg}$ ) but not in boys (BM!: $26 \pm 1 \mathrm{vs}, 21 \pm 2 \mathrm{~kg} / \mathrm{m}^{2}$; leptin: $49 \pm 19 \mathrm{vs}$. $40 \pm 30 \mathrm{pmol} / \mathrm{l}$; insulin: $1.0 \pm 0.3 \mathrm{vs}$. $0.9 \pm 0.1 \mathrm{U} / \mathrm{kg}$ ). In summary, girls with diabetes had two times higher serum leptin values at all times giving evidence for gender specific differences in the regulation of leptin secretion. This was independent from exogenous insulin substitution. Thus, insulin-dependent effects appear to be of lesser importance for the regulation of leptin secretion in obese adolescents with type I diabetes. 


\section{4}

Cloning and Mutational Analysis of the $o b$ Gene in Juvenile Onset Obesity and Identification of a Phe->Leu Amino Acid Substitution S.M. Echwald ', S.B. Rasmussen' ', L. Hansen ${ }^{1}$, A. Tybjerg-Hansen ${ }^{2}$, T. Andersen ${ }^{2,4}$, T. I. A. Sørensen ${ }^{2,3}$ and O. Pedersen ${ }^{1}$. ${ }^{1}$ Steno Diabeles Center and Hagedorm Research Insritute, Gentofte, Denmark. ${ }^{2}$ Copenhagen City Heart Study, National University Hospital, Copenhagen, Denmark. ${ }^{3}$ Danish Epidemiological Science Center at the Institute of Preventive Medicine, University of Copenhagen, Denrnark. Obesily represents one of the major factors predisposing to NIDDM. The recently cloned ob gene, mutations in which are responsible for an early onset autosomally inherited recessive obesity in rodents, represents an interesting candidate for the genetic basis of obesity in humans. To analyse for the presence of mutations in the gene in human subjects we have cloned the cDNA sequence and gene of the human $o b$. The entire coding region of the ob gene including flanking intronic sequences was scanned by single strand conformational polymorphism (SSCP) analysis in 156 male subjects with juvenile onset obesity. All subjects were originally selected at the draft board examination and had a $B M I \geq 31 \mathrm{~kg} / \mathrm{m}^{2}$ and all had a history of juvenile obesity. They were examined at the Copenthagen City Heart Study in 1992-94, and had at this time a mean BMI of $35.4 \mathrm{~kg} / \mathrm{m}^{2}$ (SD $\left.5.5 \mathrm{~kg} / \mathrm{m}^{2}\right)$. The mutational analysis identified one obese subject being a carrier of an amino acid substitution at codon

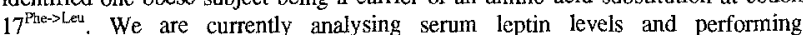
transfection studies to asses the functional implications of this mutation. A splice form exists of ob, missing codon 49 , and PCR primers flanking codon 49 in the human cDNA sequence were used to amplify a 120 bp DNA segment from 64 muscle CDNA samples from 56 NIDDM patients and 8 control subjects. Analysis of the product by gel electrophoresis revealed 5 subjects, all NIDDM patients, expressing splice variants in varying degree. In conclusion: Mutations in the coding region of the ob gene are unlikely to represent a major factor in common familial obesity. However, we cannot exclude that mutations in the coding region affecting expression, or splice variations may be involved in regulating body weight.

\section{5}

LEPTIN ARE INCREASED IN NON-DIABETIC SUBJECTS WITH INCREASED INSULIN AND PRO-INSULIN CONCENTRATIONS. S.M. Haffner, H. Miettinen and M.P. Stern. The Universily of Texas Health Science Center at San Antonio, Texas. USA.

Leptin, the product of the human $O B$ gene is increased in obese individuals suggesting resistance to its effect. However, there is considerable variability in leptin levels at each level of body mass index suggesting that genetic and environmental factors may regulate leptin concentrations. We examined whether non-diabetic subjects with elevated specific insulin and proinsulin levels had increased leptin levels. We used a radioimmunoassay to measure serum leptin levels in 197 non-diabetic Mexican Americans and non-Hispanic whites from the San Antonio Heart Study. Leptin levels were significantly correlated with fasting specific insulin $(r=0.608, p<0.00 \mathrm{I})$ and proinsulin $(r=0.608, p<0.001)$ but not with the proinsulin/insulin ratio $(r=0.012, p=0.832)$. These associations were similar in men and women and in Mexican Americans and in non-Hispanic whites. After adjustment for body mass index and waistto-hip ratio, leptin levels remained significantly correlated with specific insulin $(r=0.260, p<0.001)$ and proinsulin $(r=0.224, p=0.002)$ although the magnitude of the associations were considerably attenuated. We conclude that specific insulin and proinsulin are positively related to leptin levels and that these associations are to some degree independent of obesity and body fat distribution. Thus, subjects with increased insulin levels may be relatively resistant to the effects of leptin. It is possible that these effects could explain weight gain in insulin resistant subjects predisposed to diabetes and also in diabetic subjects given exogenous insulin and sulfonylurea.

\section{6}

DETERMINANTS OF SERUM LEPTIN CONCENTRATION IN OBESE SUBJECTS WITH IMPAIRED GLUCOSE TOLERANCE AK Turpeinen', SM Haffner', AM Louheranta', LK Niskanen ${ }^{3}$, H Miettinen', MIJ Uusitupa' 'Dept. of Clinical Nutrition, University of Kuopio. Finland, ${ }^{2}$ University of Texas, San Antonio, Texas, USA and ${ }^{3}$ Dept. of Internal Medicine, Kuopio University Hospital, Finland Leptin, the product of the ob gene, regulates body weight in mice, but its role in humans is largely unknown. It has been suggested that its secretion could be regulated by insulin. The aim of this study was to evaluate the association between insulin metabolism and leptin in subjects with impaired glucose tolerance (IGT). We studied 39 obese subjects (17 men, 22 women, body mass index (BMI) $30.6 \pm 0.6$ $\mathrm{kg} / \mathrm{m}^{2}$, age $54 \pm 1$ years, mean $\pm \mathrm{SEM}$ ) with IGT according to the WHO criteria. Insulin metabolism was assessed with frequently sampled intravenous glucose tolerance test (FSIVGTT) and body composition with infrared light $\left(\right.$ Futrex $\left.{ }^{R}\right)$. Mean serum leptin concentration was $20.7 \pm 1.8 \mathrm{ng} / \mathrm{ml}$ and it was higher in women than in men even after controlling for fat mass $(28.4 \pm 1.6$ vs. $10.8 \pm 1.3$ $\mathrm{ng} / \mathrm{ml}, \mathrm{p}<0.001)$. Leptin correlated with BMI $(r=0.36, p=0.022)$, fat percent $(r=0.74, p<0.001)$ and fat mass $(r=0.53, p<0.001)$. After adjustment for sex and fat mass, leptin had a positive correlation with hip $(r=0.58, p<0.001)$ and waist $(r=0.55, p<0.001)$ circumferences, but not with BMI or body weight. Fasting plasma insulin was $12.9 \pm 0.8 \mathrm{mU}$, insulin sensitivity index $\left(S_{1}\right) 1.83 \pm 0.17$ $.10^{-4} \mathrm{~min}^{-1} / \mu \mathrm{U} / \mathrm{ml}$, glucose effectiveness $\left(S_{G}\right) 0.015 \pm 0.001 \mathrm{~min}^{-1}$ and first-phase insulin response (FPIR) $274 \pm 43 \mathrm{mU} / \cdot$ - min. Leptin showed no association with $S_{1}, S_{G}$ or FPIR, nor did it correlate with plasma glucose. In conclusion, in obese IGT subjects fat mass is the main determinant of serum leptin concentration. Insulin sensitivity or insulin secretion capacity are not associated with leptin in IGT.

\section{7}

UKPDS: PLASMA LEPTIN, OBESITY AND PLASMA INSULIN IN TYPE 2 DIABETIC SUBJECTS

A. Widjaja' I.M. Stratton', R. Horn' ${ }^{2}$, R.R. Holman', R.C. Turner' ${ }^{1}$ and G.Brabant ${ }^{2}$ 'Diabetes Research Laboratories, Radcliffe Infirmary, Oxford, UK, ${ }^{2}$ Abteilung Klinische Endokrinologie, Medizinische Hochschule Hannover, Hannover, Germany

Adipocytes specifically express the ob gene product, a hormone called leptin, which decreases food intake. In animal models, obesity with diabetes can develop from leptin deficiency or from an impaired satiety response to leptin. Insulin can increase expression of the leptin gene both in vitro and in vivo. To assess whether obesity with diabetes in man is associated with abrormal leptin concentrations and whether leptin concentrations are associated with insulin concentrations. We measured plasma leptin concentrations across a range of obesity in 829 White Caucasian, 154 Afro-Caribbean and 204 Asian type 2 diabetic patients. Obesity was expressed as body mass index and insulin sensitivity assessed by fasting plasma insulin levels. In all three ethnic groups, leptin concentrations correlated with body mass index and were higher in females than males, with no difference between ethnic groups. In a multivariate regression analysis, plasma leptin was associated with gender and body mass index, (both $p=1 \times 10^{-17}$ ), and fasting plasma insulin concentrations $\left(\rho=5 \times 10^{-9}\right)$. Patients treated with insulin had both raised insulin and leptin concentrations. Patients with unusually high leptin levels, $>90$ th percentile in relation to body mass index, had significantly higher fasting insulin concentrations than in a gender, ethnic group, age, therapy and body mass index matched diabetic group, mean (SD range) 19 (1032) $\mathrm{mU}$ vs $14(8-25) \mathrm{mU}(\mathrm{p}<0.0009)$. Plasma leptin levels were increased in parallel to obesity in the White Caucasian, Afro-Caribbean and Asian diabetic patients. In patients with type 2 diabetes high leptin concentrations were associated with high fasting plasma insulin concentrations. suggesting that high leptin concentrations may in part be influenced by hyperinsulinaemia or impaired insulin sensitivity. 
548

PLASMA LEPTIN CONCENTRATION IS AFFECTED BY NIDDM, GENDER, AND BODY MASS INDEX

Aa. Handberg, J. Vinten, P. Thye-Rønn, P. Stæhr, C. Levin, D. Worm and H. Beck-Niclsen, Dept. of Medical Physiology, Panum Inst., University of Copenhagen and Dept. of Endocrinology, University Hospital of Odense, Denmark

Leptin, a recently described hormone secreted by adipocytes, has been proposed to convey the feeling of satiety. To characterize the effect of NIDDM, obesity, and gender on lepin, fasting plasma leptin concentrations were measured by RLA. Male NIDDM patients ( $\mathrm{N}=12$ ), who were brought to, and kept at nomoglycemia for 2 hours, showed elevated leptin concentrations when compared to control subjects $(\mathrm{N}=21)(8.0$ vs. $5.1 \mathrm{ng} / \mathrm{ml}$, $\mathrm{p}<0.005$ ). However, mean body mass index (BMI) was higher in the diabetic group (29.5 vs. 26.6. p<0.01). When leptin concentrations were corrected for BMI individually, the difference between NIDDM patients and controls was maintained $\left(0.27 \mathrm{vs} .0 .18 \mathrm{ng} \mathrm{m}^{2} \mathrm{ml}^{-1} \mathrm{~kg}^{-1}, p<0.0005\right)$. Female control subjects showed elevated leptin levels compared to male controls $(14.5 \mathrm{vs} .5 .1 \mathrm{ng} / \mathrm{ml}$, $p<0.005)$, even after corrections for differencies in BMI $\left(0.51 \mathrm{vs} .0 .18 \mathrm{ng} \mathrm{m}^{2}\right.$ $\left.\mathrm{ml}^{-1} \mathrm{~kg}^{-1} \cdot \mathrm{p}<0.0005\right)$. In both females and males leptin concentrations were tightly correlated with BMI $(r=0.89$ and $r=0.76)$ and percentage body fat $(r=0.72$ and $r=0.76)$. We were unable to detect any effect of 3 hours of insulin infusion (20 mU/m $\mathrm{min}^{-1}$ ) at cuglycemia on leptin concentrations in control persons. In conchusion, plasma leptin levels are significantly higher in females compared to males and lightly correlated to BMI in both groups. Male NIDDM patients (kept at englycemia for 2 hours) seems to have clevated leptin concentrations that can not be accounted for solely by elevated BMI.

\section{9}

LEPTIN RESPONSE TO A FAT-RICH MEAL IN POLYNESIANS WITH A HIGH PREVALENCE OF NIDDM AND OBESITY

M. de Courten ${ }^{1}$, V. Collins ${ }^{1}, A$. Dart ${ }^{2}, G$. Jennings ${ }^{2}, G$. Collier ${ }^{1}$, and $P$. Zimmet $^{1}$, International Diabetes Institute, Caulfield, Victoria ${ }^{1}$, and Baker Medical Research Institute, Prahran, Victoria ${ }^{2}$, Australia.

Leptin, the encoded product of the ob (obese) gene, reflects the size of the adipose tissue mass and may have a role in regulating appetite, energy expenditure and insulin sensitivity. As obesity in humans correlates with high leptin levels, resistance to the putative weight reducing role of leptin is suspected. Currently there is not much known about factors regulating serum leptin levels other than observations of similar levels before and after a glucose load. Studies in normal and brown fat deficient mice indicated a stimulation of leptin after ingestion of a high-fat diet. To obtain information on the regulation of leptin in humans, we measured leptin concentration (RIA assay) in fasting serum and 5 hours after ingestion of a fat rich meal $(45 \%$ fat, $35 \%$ carbohydrates, $20 \%$ protein) providing $66 \%$ of the daily energy requirements in 96 Western Samoans. The subjects were part of a study investigating risk factors for coronary heart disease. $13 \%$ of the 46 men and $20 \%$ of the 50 women had diabetes, Mean BMI was 29.5 in men and 31.2 in women. Serum triglycerides increased significantly in both sexes after the fat challenge. Women showed on average higher leptin levels than men ( $6.2 \mathrm{vs} .3 .1 \mathrm{ng} / \mathrm{ml}$ fasting; 6.1 vs $3.15 \mathrm{hr}$ levels respectively). Paired t-test showed no significant difference between measurements fasting or after $5 \mathrm{hr}$ in both sexes. Assessing agreement of the two measurements, $94 \%$ of the differences were within 2SD of the mean difference. In conclusion there was no indication that serum leptin levels in humans are regulated over a $5 \mathrm{hr}$ time span by a fat-rich meal. Despite evidence for the appetite-suppressing function of leptin in mice, a highenergy/high-fat meal appears not to be part of a proposed feed-back mechanism in humans.

\section{0}

Diurnal variation of the plasma leptin concentration in anorexia nervosa patients and control subjects.

J. Vinten, R. Stoving, Aa. Handberg and C. Hagen, Dept. of Medical Physiology, Panum Inst., University of Copenhagen and Dept. of Endocrinology, University Hospital of Odense, Denmark.

Insulin is the main mediator of storage of chemical energy in adipose tissue, and insulin sensitivity is inversely related to body fat mass and also shows diurnal variations, likely mediated by antagonistic hormones. It has recently been demonstrated that adipocytes secrete the satiety hormone leptin. To characterize the secretion of leptin in groups of subjects with large differences in body fat mass, its plasma concentration was measured in blood samples taken at 9 a.m. after an overnight fast by RIA in 10 female anorexia nervosa patients (age 15-32 years, BMI 14.0-17.5, range) and 8 female control subjects (age 23-33 years, BMI 18.8-23.8). Twelve subjects were hospitalized for the following 24 hours and offered a standardized diet rich in vegetables at regular meal hours, and 6 blood samples were obtained with $4 \mathrm{~h}$ interval from each subject. The measurements showed that the mean fasting leptin concentration was lower in anorexia nervosa patients than in controls $(2.9 \mathrm{vs} 6.8 \mathrm{ng} / \mathrm{ml}$, $\mathrm{p}=0.03$ ) and that there was a highly significant positive correlation between leptin concentration and BMI $(r=0.73, p=0.001)$. The relative changes throughout $24 \mathrm{~h}$ were moderate $(\mathrm{CV}=0.23)$ and no difference in pattern could be detected between patients and control subjects. The time course of the mean of the individual relative leptin concentrations showed a monophasic variation with minimum at 9 a.m., a slow increase throughout day and evening, maximum at $1 \mathrm{a} . \mathrm{m}$. and a faster decline from 5 to 9 a.m. In conclusion, the pattern of leptin secretion seemed to mirror energy balance with a delay of about $4 \mathrm{~h}$, and the mean leptin level was related to body fat mass, also in anorexia nervosa patients.

\section{1}

SERUM LEPTIN CONCENTRATION IS NORMAL IN CUSHING SYNDROME AND IN ACROMEGALY

H.A. Koistinen', M. Välimäki', K. Sund ${ }^{1}$, L. Tervo', S-L. Karonen', M.L. Heiman $^{2}$, T.W. Stephens ${ }^{2}$ and V.A. Koivisto. 'Helsinki University Central Hospital, Finland; ${ }^{2}$ Lilly Research Laboratories, Indianapolis, IN, USA. The recently discovered leptin is a novel hormone, which regulates energy intake and expenditure. Insulin and corticosteroids increase leptin mRNA synthesis in rodents, and data in man suggests an inverse relationship between serum growth hormone (HGH) and leptin concentrations. To investigate whether increased serum cortisol and $\mathrm{HGH}$ influence leptin concentrations in humans, we analysed fasting serum leptin (RIA) in 11 females with Cushing syndrome ( $40 \pm 3$ yrs, BMI $\left.31 \pm 3 \mathrm{~kg} / \mathrm{m}^{2}\right)$, in 15 females with acromegaly $(52 \pm 3$ yrs, BMI $\left.30 \pm 2 \mathrm{~kg} / \mathrm{m}^{2}\right)$ and in 12 healthy females $\left(50 \pm 1 \mathrm{yrs}, \mathrm{BMI} 30 \pm 1 \mathrm{~kg} / \mathrm{m}^{2}\right)$. Serum leptin concentration was normal in Cushing patients $(36.3 \pm 7.8 \mathrm{ng} / \mathrm{ml}$ vs $23.2 \pm 3.4 \mathrm{ng} / \mathrm{ml}$ in controls, $\mathrm{p}=0.18)$ and in acromegalic females $(18.4 \pm 3.0$ $\mathrm{ng} / \mathrm{ml}$ ). Leptin was similar in 11 males with acromegaly (leptin $4.1 \pm 0.4 \mathrm{ng} / \mathrm{ml}$, $49 \pm 4$ yrs, BMI $\left.29 \pm 1 \mathrm{~kg} / \mathrm{m}^{2}\right)$ and 9 healthy male controls $(6.5 \pm 1.3 \mathrm{ng} / \mathrm{ml}, 35 \pm 3$ yrs, BMI $\left.27 \pm 2 \mathrm{~kg} / \mathrm{m}^{2}\right)$. Leptin level was higher both in healthy and acromegalic females than in males $(\mathrm{p}<0.001)$. Leptin correlated positively with BMI in healthy $(r=0.90, p<0.001)$ and acromegalic males $(r=0.80, p<0.01)$, and in healthy $(r=0.57, p<0.05)$ and Cushing syndrome females $(r=0.68, p<0.05)$. Leptin correlated positively with serum insulin both in acromegalic females $(r=0.56, p<0.05)$ and females with Cushing syndrome $(r=0.67, p<0.05)$. No correlation was observed between serum leptin and cortisol or HGH concentrations. In stepwise multiple regression analysis in Cushing patients $70 \%$ of variation in leptin was explained by $\mathrm{BMI}$ alone, $77 \%$ when insulin and $85 \%$ when insulin and cortisol were added to the model ( $p<0.001$ in all). In conclusion: 1) chronic hypercortisolinemia or $\mathrm{HGH}$ excess do not affect serum leptin concentration, 2) adiposity and insulin are the major regulators of serum leptin levels also in patients with acromegaly and Cushing syndrome. 
552

\section{PHENOTYPE-LINKED AMINO ACID ALTERATION IN LEPTIN RECEPTOR CDNA FROM ZUCKER FATTY RAT}

T. Murakami, M. lida, K. Ishida and K. Shima

Department of Laboratory Medicine, School of Medicine,

University of Tokushima, Tokushima 770, Japan

The mouse obese (ob) gene product (leptin), expressed specifically in adipose cells, regulates energy balance in mice. Both mouse diabetes ( $d b$ ) and rat fatty (fa) gene products are thought to play major roles in leptin signaling pathways in the hypothalamic area. Mutations of these genes in murines result in marked obesity and type II diabetes as part of a syndrome that resembles morbid obesity in humans. The main aim of this report is the clarification of mutaitons in the leptin receptor (OBR) gene from the Zucker (fa/fa) rat. By the reverse transcriptionpolymerase chain reaction (RT-PCR) with primers which were specific for the sequence corresponding to the mouse OB-R CDNA, we cloned and sequenced one of spliced variant forms of rat leptin receptor (OB-R) CDNA with short intracellular domain. In the Zucker ( $\mathrm{fa} / \mathrm{fa}$ ) rat, no changes in either the gene structure or the expression levels were observed. However, obese phenotype-linked nucleotide alteration exists in the CDNA from Zucker ( $f a / f a)$ rat, which results in an amino acid substitution, glutamine to proline.

\section{4}

\section{LEPTIN TREATMENT IN PSAMMOMYS ORESUS.}

K. Walder ${ }^{1}$, P. Lewandowski ${ }^{1}$, A. Sanigorski ${ }^{1}$, S. Lee ${ }^{1}, P$. Zimmet $^{2}$ and G.R. Collier'. School of Nutrition And Public Health. Deakin University, Geelong, Victoria, Australia ${ }^{1}$ and International Diabeles Insitutue. Caulfield, Victoria, Australia 2 .

Administration of leptin, the circulating product of the of gene dramatically reduces food intake and bodyweight in ob/ob mice (whic) have no endogenous circulating leptin). However, exogenous leptin is ineffective in $d b / d b$ mice, which have a defective hypothalamic leptin receptor. Our aim was to test the effect(s) of exogenous leptin in Psammomys obesus, a polygenic animal model of obesity and NIDDM When fed ad libitum laboratory chow, a significant proportion of Psammomys obesus develop obesity, insulin resistance, glucoso intolerance and at the extreme end of the spectrum, frank diabetes. We have previously shown that obese, diabetic $P$ sammomys obestis have hyperleptinemia compared with their lean, non-diabetic litermates. In this study mature, obese Psammomys obesus with hyperglycemia and hyperinsulinemia were given daily injections of murine leptin $(5 \mathrm{mg} / \mathrm{kg}$ bodyweight, kindly supplied by Amgen, USA; $n=8$ ) or placebo $(n=8)$ for a period of 14 days. There was no significant change in mean food intake during this period in the leptin-treated group $(+1.9 \pm 0.1 \mathrm{~g} / \mathrm{d})$ or the control group $(-1.8 \pm 0.1 \mathrm{~g} / \mathrm{d})$. Similarly, bodyweight was unchanged in the leptin-treated group $(+0.7 \pm 0.1 \%)$ and the control group $(-1.1 \pm 0.1 \%)$ during the study. Mean blood glucose concentration was also unaffected in both groups; leptin-treated - $0.15 \pm$ $0.10 \mathrm{mmol} / \mathrm{l}$, control $-0.44 \pm 0.2 \mathrm{mmol} / \mathrm{l}$, over the 14 day period. Leptin administration also had no significant effect on plasma insulin concentration in either group. In conclusion, daily leptin administration of $5 \mathrm{mg} / \mathrm{kg}$ for 14 days had no significant effect on food intake, bodyweight, blood glucose or plasma insulin concentrations in Psammomys obesus.

\section{3}

PLASMA LEPTIN AND PLASMA INSULIN: EFFECTS OF A HIGH FAT DIET AND FASTING IN TWO MOUSE STRAINS

P.J. Havel, R.L.Gingerich, J.S. Stern, and B. Ahren. Dept. of Nutrition, Univ. of CA, Davis, Linco Res., St. Charles, MO, and Dept. of Medicine, Malmo, Sweden.

Leptin, the product of the mouse gene $(\mathrm{Ob})$ is defective in the obese, insulin resistant (ob/ob) mouse. We examined the relationship of plasma leptin with plasma insulin in mice on normal (LF; $11 \%$ fat) and high fat (HF; $58 \%$ ) diets, before and after fasting. Plasma leptin was measured with sensitive radioimmunoassay for murine leptin. In C57/B1/6J (C57) mice (the background strain for the ob/ob mouse), plasma leptin was increased after 2 months in HF $(7.4 \pm 0.8 \mathrm{ng} / \mathrm{ml} ; \mathrm{n}=26$ vs $5.4 \pm 0.5 \mathrm{ng} / \mathrm{ml} ; \mathrm{n}=23$, $\mathrm{p}<0.05)$, coincident with increased plasma insulin $(238 \pm 26 \mathrm{vs} 148 \pm 19 \mathrm{pM}, \mathrm{p}<0.0025)$ and plasma glucose $(6.7 \pm 0.2 \mathrm{vs} 5.7 \pm 0.2 \mathrm{mM})$, without an increase of body weight (BW). After 10 months, BW and plasma leptin were higher than at 2 months in both HF and LF. BW $(31 \pm 1.2$ vs $25.4 \pm 0.5 \mathrm{~g}, p<0.001)$, plasma insulin $(303 \pm 43$ vs $169 \pm 14 \mathrm{pM}, \mathrm{p}<0.01)$, and plasma leptin $(18.5 \pm 1.5$ vs $14.2 \pm 1.5 \mathrm{ng} / \mathrm{ml}, \mathrm{p}<0.05)$ were all elevated in HF vs LF. Plasma leptin was highly correlated with $\mathrm{BW}$ ( $r=0.84$, $\mathrm{p}<0.0001, \mathrm{n}=110)$ and correlated with plasma insulin independently of $\mathrm{BW}(\mathrm{r}=0.63$, $p<0.005)$. Fasting for 48 tours docreased BW, plasma glucose, plasma insulin $(-67 \pm 9$ and $-44 \pm 14 \%$ ) in both 2 month LF and HF, but plasma leptin decreasedmore in LF $(-44 \pm 3 \%, p<0.0001)$ than in HF $(-16 \pm 7 \% p<0.01 ; p<0.005$ vs LF). After 10 months, plasma leptin decreased during fasting in LF $(-24 \pm 9 \%, \mathrm{p}<0.01)$, but not $\mathrm{HF}(+1 \pm 5 \%$, $p<0.02$ vs LF). Plasma leptin in NMRI mice was elevated after 1 month HF $(8.8 \pm 0.8$ vs $5.9 \pm 0.4 \mathrm{ng} / \mathrm{ml}, \mathrm{p}<0.002)$, but not after 3 months $(6.1 \pm 0.9$ vs $4.9 \pm 0.5 \mathrm{ng} / \mathrm{ml}, \mathrm{NS})$, was less well correlated with $B W(r=0.50, p<0.001, n=40)$ and did not decrease with fasting in either LF $(+11 \pm 12 \%)$ or HF $(+46 \pm 47 \%)$ mice. We conclude: 1$) \mathrm{HF}$ and age increase plasma leptin in C57 mice. 2) Plasma leptin correlates with BW andplasma insulin in mice. 3) Plasma leptin decreases after 48 hours fasting in young LF and HF C57 mice, but not older HF mice. 4) There are marked differences in the regulation of plasma leptin between mouse strains. Thus, plasma leptin concentrations are altered with age and nutritional status and associated with parallel changes of plasma insulin.
555

\section{DEVELOPMENT OF OBESITY AND HYPERLEPTINEMIA IN PSAMMOMYS OBESUS.}

G.R. Collier ${ }^{1}$, K. Walder ${ }^{1}$, P. Lewandowski ${ }^{1}$, A. Sanigorski ${ }^{1}$, S. Lee ${ }^{1}$ and P. Zimmet ${ }^{2}$. School of Nutrition And Public Health, Deakin University, Geelong, Victoria, Australia ${ }^{1}$, and International Diabetes Institute, Caulfield, Victoria, Australia ${ }^{2}$.

The recently discovered $o b$ gene and its circulating product, leptin, may be critical factors in control of energy balance. Recent studies in $o b / a b$ mice, which lack circulating leptin, have shown dramatic reductions in food intake and body weight following leptin treatment. In addition, studies in both obese humans and animal models of obesity have demonstrated hyperleptinemia. We report a longitudinal study examining changes in circulating leptin during the development of obesity and diabetes in Psammomys obesus over an 8 week period.

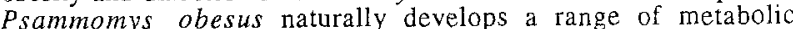
disturbances including hyperphagia, hyperglycemia, hyperinsulinernia, insulin resistance and dyslipidemia when fed standard laboratory chow. During the study, lean animals $(n=10)$ increased their body weight by $154 \%$, from $67 \pm 4$ to $170 \pm 9 \mathrm{~g}$. During this period blood glucose and plasma insulin levels remained unchanged (glucose: $4.2 \pm 0.3$ to $4.1 \pm$ $0.3 \mathrm{mmol} / \mathrm{l}$; insulin: $5 \mathrm{l} \pm 11$ to $55 \pm 10 \mathrm{mU} / \mathrm{l}$ ). Despite the increasc in bodyweight during this period, leptin levels atso remained unchanged $(61 \pm 8$ to $54 \pm 12 \mathrm{ng} / \mathrm{ml})$. In contrast, animals that developed obesity $(n=10)$ (223\% increase in body weight, from $64 \pm 3$ to $207 \pm 5 \mathrm{~g}$ ), also developed hyperglycemia $(6.7 \pm 0.9$ to $12.0 \pm 1.0 \mathrm{mmol} / \mathrm{l})$ and hyperinsulinemia $(98 \pm 17$ to $460 \pm 69 \mathrm{mU} / 1)$. Interestingly, these animals also developed hyperleptinemia during this period $(65 \pm 6 \mathrm{to}$ $151 \pm 14 \mathrm{ng} / \mathrm{ml})$. These results suggest that leptin resistance and the resulting hyperleptinemia may be neccessary factors in the development of obesity and subsequent metabolic abnormalities. 


\section{Relatives of NIDDM}

556

FIRST PHASE INSULIN RELEASE IS THE MAIN DETERMINANT OF GLUCOSE TOLERANCE.

TW van Haeften, ML Zonderland, S Dubbeldam and DW Erkelens Utrecht University Hospital, Utrecht, The Netherlands.

Since NIDDM is under strong genetic influence, we aimed to assess the relationship of NIDDM family history, insulin secretion, insulin sensitivity, body mass index (BMI), and aerobic capacity with oral glucose tolerance (OGTT 75 gram glucose). This was studied in 18 normo-glucose-tolerant (NGT) first-degree family-members of NIDDM subjects and in 18 NGT controls, matched for age, gender, BMI, and $\mathrm{VO}_{2} \max$ (aerobic capacity). We assessed $\mathrm{VO}_{2} \max$ and $\mathrm{RQ}$ at $60 \%$ of $\mathrm{VO}_{2}$ max (measure of athletic performance) with a step-wise endurance exercise-test on a bicycle ergometer, and insulin secretion (Area Under Curve 0-10 min plasma insulin (AUCINS1) and AUC 60-120 min plasma insulin (AUC-INS2) for first and second phase) with a hyperglycemic clamp $(10 \mathrm{mmol} / \mathrm{L})$ in the 36 volunteers (mean \pm SD age $46.3 \pm 6.7 \mathrm{yr}$, BMI $25.8 \pm 2.9 \mathrm{~kg} / \mathrm{M}^{2}$, $28 \mathrm{~F} / 8 \mathrm{M}$ ). Insulin sensitivity was assessed by the ratio of Glucose Infusion Rate (GIR) and mean plasma insulin (I) during the second hour of the clamp (GIR/I). Step-wise multiple linear regression showed that AUC-INS1 determined $20 \%$ of the variance of the OGTT, while family history and BMI added 3 and $2 \%$ respectively (Multiple $\mathrm{R}=0.569, \mathrm{~F}=4.95, \mathrm{p}=0.0064$ ); Age, $\mathrm{VO}_{2} \max$, RQ 60\%, AUC-INS2, and GIR/I did not add to the multiple linear regression. Conclusion: Normal glucose tolerance is determined by first phase insulin release with only a small additional effect of family history of NIDDM and body mass; insulin sensitivity does not appear to play an additional role, presumably due to compensation by insulin release.

\section{7}

INSULIN SECRETION COMPENSATES INSULIN RESISTANCE IN RELATIVES OF PATIENTS WITH NIDDM. INSULIN SECRETION AND CLEARENCE ESTIMATED FROM INDIVIDUAL C.PEPTIDE KINETICS.

P. Thye-Rønn, C. Knudsen", K. Levin, S. Madsbad". H.J. Gjessing and H. BeckNielsen. Mathematical dept., University of Leeds", Hvidovre Hospita ${ }^{* *}$ and Dept. of Endocrinology M, Diabetes Research Centre. Odense University Hospital. DK. We have studied the $\beta$-cell function in first-degree relatives in order to evaluate the prediabetic NIDDM condition. Relatives of patients with NIDDM have previously been shown to he insulin resistant several decades before the possible development of diabetes. Insulin secretion was derived from peripheral C-peptide using individual C-peptide kinetics in a deconvolution model. Insulin sensitivity was determined by the euglycemic hyperinsulinaemic clamp $\left(40 \mathrm{mU} / \mathrm{m}^{2} / \mathrm{min}\right)$. Insulin secretion was measured in an oral glucose tolerance test (OGTT) and in a hyperglycemic clamp (HA, $25 \mathrm{mmol} / \mathrm{l}$ ). Individual C-peptide kinetics was derived from a $C$-peptide bolus. We have investigated 25 (30.4 \pm 1.3 year, 25.2 $\pm 0.8 \mathrm{BMI}$ (body mass index: $\mathrm{kg} / \mathrm{m} 2)$. $0.83 \pm 0.02$ WHR. $76.9 \pm 2.8 \mathrm{~kg}$ ) first-degree relatives of patients with NIDDM and $25(31.4 \pm 2.2$ year, $25.5 \pm 0.9 \mathrm{BMI}, 0.84 \pm 0.02$ WHR, $78.5 \pm 3.4 \mathrm{~kg}$ ) matched control persons without any history of diabetes. Insulin sensitivity was reduced in relatives compared to controls: $6.6 \pm 0.4$ vs. $8.3 \pm 0.5 \mathrm{mg} / \mathrm{min} / \mathrm{kg}$ FFM: $p<0.01$. Fasting insulin secretion in relatives and controls were $165 \pm 16$ vs $120 \pm 11 \mathrm{pmol} / \mathrm{min}(p<0.03)$ and $2.12 \pm 0.15$ vs $1.48 \pm 0.10 \mathrm{pmol} / \mathrm{min} / \mathrm{kg}$ ( $\mathrm{p}<0.001)$. Fassing C-peptide $(689 \pm 56$ vs $492 \pm 36 \mathrm{pmol} / \mathrm{l}, \mathrm{p}<0.005$ ) and fasting insulin (62 27 vs $44 \pm 5 \mathrm{pmol} / \mathrm{l}, \mathrm{p}<0.05)$ were increased. Fasting glucose $(5.2 \pm 0.1$ vs $5.1 \pm 0.1 \mathrm{mmol} / \mathrm{l})$ and 2 -hour glucose $(5.4 \pm 0.2$ vs $5.0 \pm 0.3)$ were identical. Insulin secretion in OGTT $978 \pm 60 \mathrm{pmol} / \mathrm{min} / \mathrm{kg}(\mathrm{p}<0.03)$ and $\mathrm{AUC}_{\text {(it-188. incerementat) }} 792 \pm 59$ vs $710 \pm 57(\mathrm{p}<0.4)$.

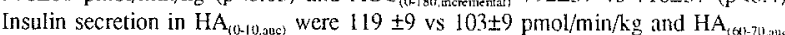
$372 \pm 2.3$ vs $335 \pm 22 \mathrm{pmol} / \mathrm{min} / \mathrm{kg}(\mathrm{p}=0.3)$. However, when taking individual insulin sensitivity into account the OGTT

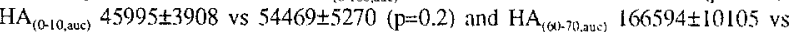
$197016 \pm 12208(\mathrm{p}=0.06) \mathrm{pmol} / \mathrm{min} / \mathrm{kg}$. Similar results were oblained with population based $\mathrm{C}$-peptide kinetics. The meta-bolic clearance rate of $\mathrm{C}$-peptide were identical. $257 \pm 10$ vs $259 \pm 10 \mathrm{ml} / \mathrm{min}(\mathrm{p}=0.9)$. We conclude that relatives of NIDDM patients have a genetically determined insulin resistance and a normal beta cell function. Furthermore, that relatives have a normal clearance (MCR) of C-peptide.

\section{8}

INSULIN RESISTANCE AND INSULIN SECRETION IN Y YOUNG, HEALTHY FIRST DEGREE RELATIVES OF NIDDM PATIENTS

G.A. Brunner, Z. Trajanoski, W. Radkohl, T.R. Pieber. Department of Internal Medicine, Diabetes and Metabolism, Karl-Franzens-University Graz, Auenbruggerplatz 15, A-8036 Graz, Austria.

AlM: insulin resistance is a well known feature in first degree relatives of NIDDM patients. Aim of this study was to measure insulin resistance and insulin secretion in very young, healthy first degree relatives of NIDDM families.

METHODS: In a population based screening 21 healthy, male subjects with NIDDM in both parental lines were identified. 8 subjects with positive family history $(\mathrm{FH}+$ ) and in 8 age and BMI matched controls without any family history of NIDDM (FH-) were investigated. An OGTT over 4 hours, a mixed meal test over 4 hours, and a hyperinsulinemic, euglycemic clamp $(1 \mathrm{mU} / \mathrm{kg} / \mathrm{min})$ were performed

RESULTS: Mean age $( \pm S D)$ in all subjects was 19 years $( \pm 1)$, BMl was $22,5 \mathrm{~kg} / \mathrm{m}^{2}( \pm 1,8)$. Physical activity, smoking habits and blood pressure were comparable in the $\mathrm{FH}_{+}$and the control group $\left(\mathrm{FH}_{-}\right)$. After an oral glucose load $(75 \mathrm{~g})$, plasma glucose levels over 4 hours were identical in both groups investigated, and none of the subjects had impaired glucose tolerance. Insulin levels in the $\mathrm{FH}+$ group were significantly higher as compared to $\mathrm{FH}$ - group (AUC $8420 \pm 1221$ versus $6552 \pm 912, \mathrm{p}<0.05$ ). In the mixed meal test plasma glucose was in the same range in both groups, and insulin levels were slightly higher in the $\mathrm{FH}+$ group (AUC 15235 $\$ 2467$ versus $11988 \pm 1970, \mathrm{n.S}$.). In the hyperinsulinemic, euglycemic clamp glucose infusion rate (GIR) was $6,5 \pm 1,7 \mathrm{mg} / \mathrm{kg} / \mathrm{min}$ in the group with positive family history compared to $8,3 \pm 1,0 \mathrm{mg} / \mathrm{kg} / \mathrm{min}$ in the group with negative family history $(p<0.05)$.

CONCLUSION: Insulin resistance and hyperinsulinemia are already present in very young (19 years old), healthy first degree relatives of NIDDM patients. This results indicate the strong genetic link of insulin resistance and hyperinsulinemia in the development of the disease.

\section{9}

INSULIN SENSITIVITY, SECRETION AND EARLY METABOLIC DEFECTS N LEAN NORMOGLYCEMIC CAUCASIAN OFFSPRING OF NIDDM PARENTS. G. Perseghin and G.I. Shulman. Yale University, Now Haven, USA.

First degree relatives of NIDDM patients have $40 \%$ life-time risk of developing diabetes and insulin resistance is the best predictor of it occurring. However insulin resistance is altered by many other factors including age, diet, exercise and medications. To investigate the metabolic and endocrine alterations associated with insulin resistance when these confounding factors are excluded, we examined 49 caucasian, normoglycemic $(4.99 \pm 0.05$ vs $4.94 \pm 0.05 \mathrm{mM}$ ), non-exercising, lean (BMI: $24=1$ vs $23 \pm 1 \mathrm{~m}^{2} / \mathrm{kg}$; IBW: $106 \pm 1$ vs $105 \pm 1 \%$ ) offspring of NDDM patients (OFF), compared to 29 matched healthy subjects (CON) by means of the study of the first phase of insulin secretion (FP) induced by an i.v. glucose bolus $(0.3 \mathrm{~g} / \mathrm{kg} \mathrm{BW}$ ) immediately followed by a euglycemic hyperinsulinemic ( $420 \mathrm{pM}$ ) clamp, along with lipid and amino acid (AA) profiles. OFF showed fasting hyperinsulinemia $(40.8 \pm 2.4$ vs $30.6 \pm 2.4 p M ; p<0.005)$ and higher FFA $(582 \pm 27$ vs $470 \pm 26 \mu \mathrm{M} ; p<0.007)$ while triglycendes, tolal cholesterol, HDL-chol and LDL-chol were comparable to CON. Alanine ( $320 \pm 10$ vs $361 \pm 14 \mu \mathrm{M} ; p<0.02)$, serine $(p<0.05)$, glutamine and glycine $(p<0.01)$ were lower in OFF vs CON, while branched chain AA ( $343 \pm 8$ vs $357 \pm 10 \mu \mathrm{M} ; p=N S$ ) were not different. Insulin sensitivity (IS) $(M=4.86 \pm 0.24$ vs $6.17 \pm 0.29 \mathrm{mg} /[\mathrm{kg} \cdot \mathrm{min}] ; \quad p<0.0009)$ was lower and correlated to fasting FFA in OFF $(r=-0.48, p<0.0005)$ but not in CON $(r=-$ $0.17, p=0.4$ ). Since is in OFF did not fit a single normal distribution but appeared to be a mixture of three distributions, OFF were subdivided into three subgroups with Very Low (OFF-VL: M<4; $24 \%$ ), Low (OFF-L: $4<M<5.5$; $45 \%$ ) and Normal (OFF-N: $M>5.5 ; 31 \%$ ) IS. The same alterations in FFA and $A A$ metabolism were observed in OFF. $V L$ and OFF.L but not in OFF-N. FP of insulin secretion appears to compensate significantly for insulin resistance in $O F F-L$ vs OFF-N and CON, but it was inappropriate for the degree of insulin resistance in OFF-VL. In summary insulin resistant OFF have: 1) tri-modal distribution of insulin sensitivity, 2) defective FP of insulin secretion, 3) high fasting FFA and low gluconeogenic AA concentrations, 4) inverse correlation between IS and FFA levels. In conclusion these results suggest that, in this carefully screened population, insulin sensitivity is under the genetic control of a single gene and that FFA metabolism may play a role in the pathogenesis of a single
NIDDM. 
560

EVIDENCE OF DISORDERLY AND NON-STATIONARY INSULIN SECRETION IN GLUCOSE TOLERANT RELATIVES OF NIDDM PATIENTS O. Schmitz', N. Pørksen', B. Nyholm', C. Skjærbæk', P. C. Butler ${ }^{2}$, J. D.

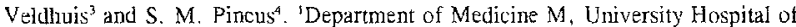

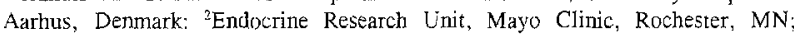
${ }^{3}$ Endocrinology Division, University of Virginia, Charlottesville, VA and ${ }^{4} 990$ Moose Hill Road, Guildford, CT, USA.

To further explore the role of the $\beta$-cell in the pathogenesis of NIDDM, insulin secretion at modest hyperglycaemia was examined in 15 healthy first-degree relatives of NIDDM patients and 13 antluropometrically and age matched controls. Oral glucose tolerance test was normal in all, but the relatives had impaired insulinstimulated glucose uptake $(\mathrm{P}<0.05)$ as assessed by the euglycaemic clamp technique. During a constant intravenous glucose infusion $(4-5 \mathrm{mg} / \mathrm{kg} / \mathrm{min})$ for 135 minutes, we performed a time-series analysis of serum insulin in samples obtained at one-minute intervals for 75 mimutes (time, 60 to $135 \mathrm{~min}$ ). Plasma glucose was comparable in the two groups $(8.2 \pm 0.3$ and $7.7 \pm 0.3 \mathrm{mmol} / 1, \mathrm{P}=0.17$; relatives vs controls), while AUC of serum insulin during the study period was higher in the relatives $(P<0.05)$. The recently introduced scale- and model-independent statistic, Approximate Entropy ( $\mathrm{ApEn}$ ), as well as the coefficient of variation for a 6 point moving average (MA) were applied to test regularity and stationarity of insulin secretion, respectively. Both ApEn and 6 point MA were able to significantly discern the insulin time-series of the two groups $(\mathrm{P}<0.05)$, demonstrating a higher degree of irregularity and non-stationarity among the offspring. Moreover, when combining the two complementary sets of statistics into a single "index of nonpulsatility", an even more notable distinction was available $(P<0.01)$. In conclusion, this novel approach demonstrates that the stimulated insulin secretion of glucose tolerant relatives of NIDDM patients is abnormal and characterized by disorderliness. Whether this experimental and statistic model can predict the risk in nom-diabetic people for subsequent development of clinically important $\beta$-cell dysfunction and NIDDM remains to be clarified.

\section{1}

LOWERED INSULIN SENSITIVITY (Sr-MINMOD) IN SONS WITH HIGH TRIGLYCERIDE RESPONSE DURING AN ORAL METABOLIC TOLERANCE TEST AND FAMLY Y HISTORY OF THE METABOLIC SYNDROME

E. Wagner, W. Stürmer, H. Kasper and J. Schrezenmeir

Departement of Internal Medicine, University of Würzburg, Germany

Introduction: Previous studies have established the phenomenon of high triglyceride response after an standardized oral metabolic tolerance test (OMTT). We have found that 19 out of $28(69 \%)$ young sons with family history of the metabolic syndrome have pathological triglyceride levels (HR-sons) during OMTT. Because these subjects were normotensive and glucosetolerant during a conventional $75 \mathrm{~g}$ OGTT, we asked whether the triglyceride high response could be an early indicator of further metabolic disturbances.

Question: Are sons with positive family history for the metabolic syndrome and a pathological result during OMTT less insulin sensitive than age- and BMI-matched controls without such a history?

Methods: Insulin sensitivity index ( $\mathrm{S}_{1}$ ) was assessed dynamically by the minimal model approach with frequent sampling intravenous glucose tolerance test like described by R.N.Bergman ( $300 \mathrm{mg}$ glucose/kg body wt; $3 \mathrm{mg}$ tolbutamide $/ \mathrm{kg}$ body wt).

\begin{tabular}{|c|c|c|c|c|c|c|c|c|}
\hline Results: & $\mathbf{n}$ & age & BMI & WHR & $\mathrm{TG}_{\text {tast }}$ & $B \mathrm{G}_{\text {fast }}$ & $\mathrm{IRI}_{\text {irst }}$ & ${ }_{-4} \mathrm{~S}_{\mathrm{It}}$ \\
\hline HR-sons & 10 & $\begin{array}{l}\text { (years) } \\
28\end{array}$ & $\begin{array}{l}(\mathrm{kg} / \mathrm{m} 2) \\
27,5\end{array}$ & 0,966 & $\begin{array}{c}(\mathrm{mmol} / \mathrm{)}) \\
2,20\end{array}$ & $\begin{array}{c}\text { (mnoli) } \\
4,7\end{array}$ & $\begin{array}{l}(\mathrm{u} \pi / \mathrm{m} t) \\
15,5\end{array}$ & $\begin{array}{c}10^{-4} \mathrm{~L} \mathrm{mU}^{-1} \min ^{-1} \\
3,76 \pm 2,3\end{array}$ \\
\hline NR-controls & 10 & 30 & 27,5 & 0,892 & 0,95 & 4,5 & 13,6 & $8,60 \pm 5,3$ \\
\hline value & . & $n . \mathrm{s}$ & n.s & 0,031 & 0,0076 & n.s & n.s & 0,026 \\
\hline
\end{tabular}

Conclusion: HR-sons showed a significantly lower $S_{I}$ index compared to matched controls without family history. That finding support our assumption, that in male offsprings of patients with NIDDM the OMTT might be simple approach to detect early metabolic defects which predispose to a later metabolic syndrome.

\section{2}

FIRST DEGREE RELATIVES OF NIDDM FAMILIES HAVE HYPERINSULINAEMIA BUT NOT HYPER PROINSULINAEMIA.
DB Humphriss ${ }^{\mathrm{a}}$, TS Berrish ${ }^{\mathrm{a}}$, MW Stewart ${ }^{\mathrm{a}}$, C Dark ${ }^{\mathrm{b}}$, L Ashworth ${ }^{\mathrm{a}}$, L Barriocanal $\mathrm{I}^{\mathrm{a}}$, L Trajano ${ }^{\mathrm{a}}$ and $\mathrm{M}$ Walker ${ }^{\mathrm{a}}$. Department of Medicine, University of Newcastle upon Tyne and DAKO Diagnostics Ltd, Ely, UK.

First degree relatives of NIDDM patients are at increased risk of developing NIDDM. NIDDM patients have an increased proportion of proinsulins to insulin which is thought to reflect abnormal $\beta$-cell function. We examined whether this is a feature in at risk relatives. We measured insulin, intact (IP) and total (TP) proinsulin levels using specific 2 site monoclonal assays, fasting and after a $75 \mathrm{~g}$ OGTT, in 126 normal glucose tolerant first degree relatives of NIDDM patients and 147 control subjects with no family history of NIDDM. Post glucose areas under the curve (AUC) were calculated. Insulin and proinsulin results were log transformed. The relatives were younger and had a greater BMI but there were no significant differences in sex distribution, waist hip ratio or blood glucose levels. The relatives had significantly higher fasting $(10.1(3.7,27.4)$ v $7.8(2.7,22.4) \mathrm{mU} / 1$, geometric mean $(95 \% \mathrm{CI}), \mathrm{p}=0.0004$, Mann Whitney) and AUC $(6758 \quad(2195,20800)$ v $5568 \quad(1949,15906)$ $\mathrm{mul}^{-1}$. min, $\mathrm{p}=0.0049$ ) insulins. There were no differences in the measured levels of fasting $(10.6(3.5,31.9)$ vs $9.7(3.3,28.0)$ pmol $\left.1^{-1}\right)$ or AUC $(5180(1704,15641)$ vs $(5145(1646,16086)$ pmol $\left.1^{-1} \cdot \min \right)$ TP, nor were there any differences in IP levels. The insulin levels remained significantly higher after analysis of covariance including age, sex, BMI, WHR and 2 hour glucose as covariates. Normal glucose tolerant first degree relatives have true hyperinsulinaemia compared to control subjects but there are no differences in total or intact proinsulin levels.

\section{3}

PROINSULIN SECRETION IN NON DIABETIC FIRST DEGREE RELATIVES OE NIDDM PATIENTS

J. Biarnés, M. Eernández-Castañer, I. Camps, J. Ripollés and $J$. Soler. Endocrine Unit, csU Bellvitge. Barcelona (Spain).

The aim of our study was to analyze proinsulin responses to an intravenous infusion of glucose in non diabetic first-degree relatives of NIDDM with normal and impaired glucose tolerance. Thirtythree first-degree relatives and twenty-four age, sex and BMI matched controls were studied. A sixty-minute intravenous continous infusion of 5 mg of glucose/kg of ideal body weight (CIGMA) was performed. Fasting and achieved serum glucose, insulin, c-peptide and proinsulin levels were measured. Beta celi function and insuiin sensitivity were obtained by CIGMA. Relatives had higher fasting glucose levels $(5.4 \pm 0.7$ vs $5.1 \pm$ $0.6 \mathrm{mmol} / \mathrm{l}, p=0.05)$ and lower insulin sensitivity $(54.7 \pm 26.6$ vs $75.7 \pm 41.8$ g $p=0.04\rangle$ than controls. Fasting $(8.5 \pm 7.6$ vs $9.5 \pm 10.5 \mathrm{pmol} / 1$, $\mathrm{p}=0.6)$ and achieved proinsulin $(20.0 \pm 14.4$ vs $22.4 \pm 16.7 \mathrm{pmol} / \mathrm{l}, \mathrm{p}=0.5)$ were not different. Eight relatives had impaired glucose tolerance (IGT). IGT subjects had lower beta cell function than glucose tolerant relatives ( $106.6 \pm 20.9 \mathrm{vs}$ $149.9 \pm 53.8 \pm, p=0.002)$ but there were no differences in fasting $(7.4 \pm 5.1$ vs $8.8 \pm 8.4$ pmol/l, $p=0.8)$ and achieved proinsulin $(16.5 \pm$ 6.2 vs $21.1 \pm 16.2$ pmol/1, $p=0.7$ ) levels and proinsulin/insulin ratios. Therefore, we concluded that, in oux population, hyperproinsulinemia is not present in first-degree NIDnM relatives even in the IGT ones. 


\section{4}

EVIDENCE OF AN IMPAIRED ANTILIPOLYTIC EFFECT OF INSULIN IN HEALTHY FIRST DEGREE RELATIVES OF NIDDM P-A Jansson and J.W. Eriksson, Lundberg Laboratory for Diabetes Reasearch, Sahlgrenska University Hospital, S-413 45 Göteborg, Sweden

It is established that resistance as to insulin's glucoregulatory function is an early event in the development of NIDDM, but it is less clear if the insulin resistance also involves the antilipolytic effect of insulin. In the present study, we combined abdominal subcutaneous (sc) microdialysis and ${ }^{133} \mathrm{Xe-clearance}$ during a two-step euglycemic $(5.0 \mathrm{mM})$ insulin clamp to assess sc adipose tissue lipolysis and glycerol release. Eight healthy subjects with two first degree relatives with NDDM (R-NIDDM) (Age: $35 \pm 1$ yrs (Mean \pm SE), $4 \mathrm{~F} / 4 \mathrm{M}$, BMI: $23.2 \pm 0.7 \mathrm{~kg} / \mathrm{m}^{2}$ ) and 8 pairwise matched control subjects (C) without any family history of NIDDM (Age: $35 \pm 1,4 \mathrm{~F} / 4 \mathrm{M}, \mathrm{BMI}: 23.0 \pm 0.4$ ) were studied after an overnight's fast.

Baseline data before the insulin infusion were obtained: Plasma insulin: $7 \pm 1$ vs $6 \pm 1$ $\mathrm{mU} / \mathrm{l}$, Glucose: $4.9 \pm 0.1$ vs $4.9 \pm 0.1 \mathrm{mM}$, FFA: $505 \pm 32$ vs $482 \pm 51 \mu \mathrm{M}$, Interstitialarterial (LA) Glycerol: $150 \pm 12$ vs $117 \pm 13 \mu \mathrm{M}(\mathrm{p}=0.05)$, Adipose tissue blood flow (ATBF): $3.7 \pm 0.6$ vs $2.7 \mathrm{ml} / 100 \mathrm{~g} / \mathrm{min}$ ( $p=0.1$ ). Net sc glycerol release: $2.14 \pm 0.26 \mathrm{vs}$ $2.14 \pm 0.19 \mu \mathrm{mol} / \mathrm{kg} / \mathrm{min}$ in the R-NIDDM and C-group, respectively. During a low insulin infusion rate $\left(10 \mathrm{mU} / \mathrm{m}^{2} / \mathrm{min}\right)$ : Plasma insulin: $23 \pm 2$ vs $18 \pm 1 \quad(\mathrm{p}=0.09)$, FFA: $166 \pm 43$ vs $71 \pm 14$ ( $\mathrm{p}=0.07$ ). Glucose disposal (GIR): $2.58 \pm 0.33$ vs $3.33 \pm 0.29$ $\mathrm{mg} / \mathrm{kg} / \mathrm{min}(\mathrm{p}=0.06$ ), IA Glycerol: $81 \pm 12$ vs $45 \pm 9$ ( $\mathrm{p}<0.05$ ), ATBF: $2.5 \pm 0.7$ vs $3.5 \pm 0.7$ (ns), Net sc glycerol release: $0.89 \pm 0.11$ v $0.75 \pm 0.19$, respectively. During a high insulin infusion rate $\left(60 \mathrm{mU} / \mathrm{m}^{2} / \mathrm{min}\right)$ : Plasma insulin: $98 \pm 7$ vs $83 \pm 4$ $(\mathrm{p}=0.06)$, FFA: $89 \pm 29$ vs $30 \pm 6(\mathrm{p}=0.05)$. GIR: $9.03 \pm 0.90$ vs $10.70 \pm 0.58$ (ns), IA Glycerol: $64 \pm 9$ vs $33 \pm 8$ ( $p<0.01$ ), ATBF: $2.6 \pm 0.6$ vs $2.1 \pm 0.4$, Net sc glycerol release: $0.78 \pm 0.11$ vs $0.37 \pm 0.12(\mathrm{p}<0.05)$, respectively. Furthermore, ATBF was decreased during the high insulin infusion rate compared to baseline in $\mathrm{C}$ but this effect was absent in R-NIDDM ( $p<0.05)$.

In conclusion, the data indicate that the effect of insulin to suppress FFA levels and net sc glycerol release is impaired in healthy first degree relatives of NIDDM patients. Moreover, the clamp insulin levels tended to be higher in R-NIDDM which can be due to a decreased rate of insulin clearance. In addition, there also seems to be a dysregulation of ATBF in this group.

\section{PS 19}

\section{Insulin Resistance and NIDDM}

\section{5}

POST-PRANDIALAMYLN CONCENTRATIONS ARE INCREASED BY SULPHONYLUREA, BUT UNCHANGED BY BASAL INSULIN, IN NIDOM J. Rachman, M.J. Payne, J.C. Levy, B.A. Barrow, S.E. Manley, R.R. Holman and R.C. Turner. Diabetes Research Labs, Radcliffe Infirmary, Oxford, U.K.

Amylin (islet amyloid polypeptide) is the constitutive peptide of the islet amyloid characteristic of NIDDM. Changes in amylin secretion by the $\beta-$ cell, in response to therapies, may influence rate of formation of islet amyloid. Amylin concentrations were assessed in a randomised, crossover design, after eight-week treatment periods with diet alone, exogenous insulin (ultralente) or sulphonylurea (gliclazide) in 8 NIDDM subjects: (mean $\pm \mathrm{SD}$ ), age $63 \pm 8 \mathrm{yr}, \mathrm{BMl} 31 \pm 4 \mathrm{~kg} \cdot \mathrm{m}^{-2}$, time since diagnosis $10 \pm 3 \mathrm{yrs}$. Subjects had a standard breakfast after each period. Fasting plasma glucose was reduced from $11.8 \pm 5.2 \mathrm{mmol} .1^{-1}$ on diet, by both sulphonylurea: $8.7 \pm 3.5 \mathrm{mmol} . \mathrm{f}^{-1}(\mathrm{p}=0.009)$ and insulin: $6.0 \pm 2.3 \mathrm{mmol}^{-1} \mathrm{f}^{-1}$ $(p=0.002)$. Amylin and amylin-like peptide (ALP) were measured in samples taken into trasylol, using sandwich ELISAs with capture antibodies F024 and F002 respectively. Interassay CVs were $<15 \%$ across the assay range. Minimum detectable amylin and ALP concentrations were 1.9 and 2.7 pmol. $\mathrm{I}^{-1}$. Post-prandial peptide areas under the curve, geometric mean (1SD range) were:

\begin{tabular}{|c|c|c|c|c|}
\hline & Diet & Insulin & Sulphonylurea \\
\hline c-pept & $\left(\mathrm{nmol.1}^{-1}\right)$ & $1.7(1.1-2.7)$ & $1.6(1.1-2.4)$ & $2.3(1.6-3.3)^{*}$ \\
\hline amylin & $\left(\mathrm{pmol.I^{-1 } )}\right.$ & $3.3(1.9-5.9)$ & $3.3(1.8-6.3)$ & $5.2(2.4-11.2)^{\circ}$ \\
\hline ALP & $\left(\mathrm{pmol} .1^{-1}\right)$ & $10.1(5.0-20.7)$ & $10.0(5.5-18.2)$ & $16.4(8.5-31.6)$ \\
\hline
\end{tabular}

$p<0.01$ compared with diet or insulin

Amylin and ALP concentrations were increased in parallel with c-peptide by sulphonylurea. Basal insulin supplement alone was not sufficient to suppress post-prandial amylin concentrations. Sulphonylurea therapy, by increasing amylin secretion, might accelerate islet amyloid formation and cause faster decline in $\beta$-cell function than therapy with diet or insulin.

\section{6}

AMYLIN RESPONSE FOLLOWING SUSTACAL ${ }^{\text {is }}$ INGESTION IS DIMINISHED IN TYPE II DLABETIC PATIENTS TREATED WITH INSULIN.

M.S. Fineman, M.P. Giotta, R.G. Thompson, O.K. Kolterman and J.E. Koda. Amylin Pharnaceuticals, Inc, San Diego, CA, USA.

Amylin is cosecreted with insulin from pancreatic beta cells. Assays to measure both unmodified and glycosylated amylin have been previously reported. We measured unmodified amylin (UA) and unmodified amylin plus glycosylated amylin (AG) in 12 insulin treated type II diabetic patients (DI), 12 diet or oral hypoglycenic agent treated diabetic patients (DD) and 25 age matched lean non-diabetic controls (C) during a 3 hour Sustacal ${ }^{\mathbb{E}}$ challenge. The duration of disease for both diabetic groups was 2-10 years and both had elevated Body Mass Index compared to $C(D I=28.3 \pm 1.0, D D=29.4 \pm 0.8$, $\mathrm{C}=24.1 \pm 0.5, \mathrm{p}<0.01$ ). Glucose area under the curve during the Sustacal ${ }^{*}$ challenge was elevated for both diabetic groups compared to $\mathrm{C}(\mathrm{DI}=757 \pm 53, \mathrm{DD}=570 \pm 59, \mathrm{C}=298 \pm$ $9 \mathrm{mg} \bullet / \mathrm{dL}, \mathrm{p}<0.0001$ ). In results from the AG assay. Cmax and Cmax adjusted by the fasting value (adj Cmax) were significantily lower in $\mathrm{DI}$ compared to $\mathrm{C}$ but no differences were seer between DD and $\mathrm{C}$. Using the UA assay, there was no difference between Cmax for either DI or DD compared to $\mathrm{C}$, but the adjusted Cmax was significantly lower in DI compared to $C$. Additionally, Tmax was delayed for DD compared to $C$ in both assays.

Results = Control Insulin Diet/oral P value Pvalue

Mean \pm SEM Group (C) Group (DI) Group (DD) DIvs C DD vsC $\begin{array}{llllll}\text { UA Cmax (pM) } & 13.6 \pm 1.9 & 12.2 \pm 2.6 & 11.8 \pm 2.3 & \text { NS } & \text { NS }\end{array}$ $\begin{array}{llllll}\text { UA Adj Cmax (pM) } & 8.7 \pm 1.2 & 4.6 \pm 1.2 & 6.2 \pm 1.0 & 0.032 & \text { NS }\end{array}$ $\begin{array}{llllll}\text { UA Tmax (min) } & 62.4 \pm 6.0 & 82.5 \pm 10.5 & 87.5 \pm 11.9 & 0.083 & 0.042\end{array}$ $\begin{array}{llllll}\text { AG Cmax (pM) } & 26.2 \pm 2.7 & 15.9 \pm 3.7 & 25.7 \pm 3.5 & 0.034 & \text { NS }\end{array}$ $\begin{array}{llllll}\text { AG Adj Cmax (pM) } & 18.8 \pm 2.0 & 7.5 \pm 2.0 & 16.3 \pm 2.2 & 0.001 & \text { NS }\end{array}$

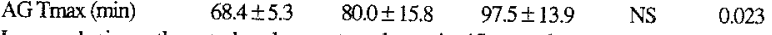
In conclusion, the study demonstrated a significant decrease in the maximum unmodified amylin response in insulin treated Type II diabetic subjects compared to lean non-diabetic controls. A similar decrease was seen in these two groups for both the maximum absolute level and response using the anylin plus glycosylated amylin assay. 


\section{7}

\section{SALT DEPLETION DECREASES INSULIN SENSITIVITY IN NIDDM}

JR Petrie, AD Morris, ${ }^{1} \mathrm{~K}$ Minamisawa, HL Elliott, M Small, ${ }^{2}$ and JMC Connell. Department of Medicine and Therapeutics, University of Glasgow; ${ }^{1}$ Department of Clinical Pharmacology, University of Dundee; ${ }^{2}$ Diabetes Centre, Gartnavel General Hospital, Glasgow, UK.

It has been suggested that angiotensin converting enzyme inhibitors increase insulin sensitivity in diabetic patients, possibly via angiotensin II (ANG II) withdrawal. In contrast, we have previously shown that acute administration of ANG II increases insulin sensitivity in patients with non-insulin-dependent diabetes mellitus (NIDDM). We therefore aimed to determine the effect of more chronic activation of the renin-angiotensin system on insulin-mediated glucose uptake in these patients. 9 normotensive patients with diet-controlled NIDDM [age (mean \pm SD) $57 \pm 9.6$ years; HbAlc $5.7 \pm 0.8 \%$; cholesterol $\left.5.76 \pm 1.25 \mathrm{mmoll}^{-1}\right]$ underwent hyperinsulinaemic euglycaemic clamp studies (Actrapid $1.5 \mathrm{mU} / \mathrm{kg} / \mathrm{min}$ ) in a sodium-deplete (D) and replete (R) state in a double-blind placebo-controlled crossover design. Patients were studied after two four-day periods of low sodium diet $(40 \mathrm{mmol} / 24$ hours $)$ during which they took either $120 \mathrm{mmol} / 24$ hours of sodium tablets (slow sodium. CIBA GEIGY) or placebo. Mean \pm SD 24 hour urinary sodium was $197 \pm 76.0 \mathrm{mmol}$ (R) and $67 \pm 19.5 \mathrm{mmol}(\mathrm{D}), \mathrm{p}<0.01$. Baseline ANG II levels were $4.9 \pm 5.8$ (R) and $16.0 \pm 15.6(\mathrm{D}) \mathrm{pg} / \mathrm{ml}, \mathrm{p}<0.05$. A reproducible insulin stimulus was achieved. Insulin sensitivity (M-value) was $7.8 \pm 2.0 \mathrm{mg} \mathrm{kg}^{-1} \mathrm{~min}^{-1}(\mathrm{R})$ and $6.5 \pm 2.2 \mathrm{mg} \mathrm{kg}^{-1} \mathrm{~min}^{-1}$ (D), $\mathrm{p}<0.05$. There were no significant differences in hepatic glucose production between the two conditions. Blood pressure was $130 \pm 20.6 / 78 \pm 11 \mathrm{mmHg}(\mathrm{R})$ and $128 \pm 12 / 73 \pm 10 \mathrm{mmHg}(\mathrm{D})$. These data suggest that chronic activation of the renin-angiotensin system by sodium depletion leads to a decrease in insulin sensitivity in normotensive patients with NIDDM. As insulin sensitivity is an important pathophysiological feature of NIDDM, larger studies should be performed examining the effect of following current dietary sodium recommendations (restriction to $40.50 \mathrm{mmol}$ daily) on glycaemic control in hypertensive patients with NIDDM.

\section{8}

INSULIN SENSITIVITY AND IRON STORES IN CONTROL AND NIDDM SUBJECTS

J.M. Fernández-Real, W. Ricart,"*R. Casamijana, ${ }^{* *}$ M. Fernández-Castañer and **:J. Soler.

Department of Endocrinology, Hospital "Dr Josep Trueta". Girona. "Hormonal Laboratory, Hospital Clínic, Barcelona. **Department of Endocrinology, Hospital de Bellvitge, Barcelona. SPAIN.

Objective: Insulin resistance and hyperinsulinemia have been found in patients with chronic iron overload and normal glucose tolerance, as idiopathic hemochromatosis and patients with thalassemia major treated with hypertransfusion therapy. It appears that the initial and the most common defect in these patients with an earlier stage of damage induced by iron overload is one of liver-mediated insulin resistance. We investigated the influence of iron stores, measured as serum ferritin, on insulin sensitivity in lean and obese subjects without evidence of iron overload. Subjects and Methods: Oral glucose tolerance and insulin sensitivity were evaluated in 23 subjects [9 lean, 14 obese, defined as a BMI between 30 and $40 \mathrm{Kg} / \mathrm{m}^{2}$ ] through standard OGTT and the Bergman's Minimal Model. Conditions that determine altered levels of serum ferritin were excluded in all subjects. Results: Oral glucose tolerance was normal in all lean subjects and in seven of the obese subjects. Glucose intolerance was present in 2 obese subjects, and diabetes mellitus was diagnosed by OGTT criteria in 5 subjects. Significant differences in $S_{I}$ were observed between lean, obese and NIDDM obese subjects $\left(4 \pm 1.5,2.5 \pm 1\right.$ and $1 \pm 1.0510^{-4} \mathrm{~min}^{-1} / \mu \mathrm{U} / \mathrm{mL}$, respectively, $\left.\mathrm{p}=0.0004\right)$. A negative correlation between $S_{I}$ and serum ferritin $(r=-0.43, p=0.03)$ was found, which persisted after correction for BMI and age. The relationship between $S_{I}$ and $\log$ transformed serum ferritin was even stronger among males $(r=-0.76, p=0.007, n=11)$. Conclusion: Iron stores, measured as serum ferritin, may influence insulin sensitivity even in the absence of iron overload.

\section{9}

ABDOMINAL FAT DISTRIBUTION AND INSULIN SENSITIVITY IN NONINSULIN-DEPENDENT DIABETES MELLITUS : ETHNIC DIFFERENCES. J.F. Gautier, A. Mourier, E. De Kerviler, C. Alvarez, P. Vexiau and G. Cathelineau. Departments of Endocrinology and Radiology, SaintLouis Hospital , 75010, Paris, France.

Visceral adipose tissue accumulation is an important factor in the development of NIDDM in white people. The aim of the study was to investigate abdominal fat distribution and insulin sensitivity in black NIDDM patients in comparison to white NIDDM patients with similar age and duration of diabetes. Eighteen black patients (age $46 \pm 2$ (SEM) yr, duration of diabetes $58 \pm 9$ months, BMI $24.9 \pm 1.1 \mathrm{~kg} \cdot \mathrm{m}^{-2}$, total body fat using skin fold thickness $25.8 \pm 1.4 \%$, waist-to-hip ratio $0.92 \pm 0.03, \mathrm{HbA}_{1} \mathrm{c} 9.1 \pm 0.6 \%$ ) and 14 caucasian patients (age $47 \pm 2 \mathrm{yr}$, duration of diabetes $65 \pm 13$ months, BMI $29.6 \pm 1.2$ $\mathrm{kg} \cdot \mathrm{m}^{-2}$, total body fat $25.2 \pm 1.1 \%$, waist-to-hip ratio $0.99 \pm 0.01$, $\mathrm{HbA} 1 \mathrm{c} 8.0 \pm 0.4 \%$ ) were studied. Areas of visceral and subcutaneous abdominal fat were calculated from axial magnetic resonance images obtained at the level of the umbilicus. An insulin tolerance test $(0.1$ U. $\mathrm{kg}^{-1}$ ) was performed after an overnight fast, at least 48 hours after the withdrawal of metformin. Areas of subcutaneous abdominal fat did not differ statistically between black and white patients $(2.0 \pm 0.17$ and $2.39 \pm 0.21 \mathrm{~cm}^{2}$ per $\mathrm{kg}$ of body weight, respectively). In contrast, areas of visceral adipose tissue were significantly smaller in black patients: $1.24 \pm 0.12$ vs $1.73 \pm 0.19 \mathrm{~cm}^{2} \cdot \mathrm{kg}^{-1}, \mathrm{p}<0.05$ (ANOVA). The blood glucose disappearance rate KITT was similar in both groups $\left(2.1 \pm 0.3\right.$ and $2.0 \pm 0.2 \% \cdot \mathrm{min}^{-1}$, respectively). Subcutaneous abdominal fat correlated positively to BMI in both groups (black $\mathrm{r}=0.52$, white $\mathrm{r}=0.88$ ) while visceral fat did not (black $\mathrm{r}=0.30$, white $\mathrm{r}=0.23$ ). In conclusion black NIDDM patients had less visceral adipose tissue accumulation which is of interest to better understand the pathogenesis of NIDDM in this population.

\section{0}

VISCERAL FAT ACCUMULATION INCREASES IRI AREA ON OGTT IN NORMAL, IGT AND EARLY NIDDM SUBJECTS C. Nakagawa, I. Mineo, M. Gomi and S. Tarui. Otemae Hospital, Osaka, Japan

Visceral fat accumulation has been shown to give a marked affection on glucose and lipid metabolism. In the absence of subcutaneous adiposity, visceral fat accumulation does not necessarily result in significant overweight. In fact, the major parts of newly diagnosed NIDDM cases in Japan have been demonstrated to have excess visceral adiposity and normal range BM! $(<25)$. In order to analyze the effect of visceral adiposity distinctly, we investigated correlation between visceral fat accumulation and $|R|$ area on $75 \mathrm{gOGTT}$ in male adults with normal range BMI. Visceral and subcutaneous fat area was measured at the level of umbilicus on CT scan. Eighty subjects were divided into the following groups; N: normal glucose tolerance $(n=13)$, l: IGT $(n=24)$ and D: early NIDDM $(n=43)$. There was significant positive correlation between $\mid \mathrm{RI}$ area and visceral fat area in the groups of $N(r=0.634$, $p<0.05), I(r=0.426, p<0.05)$ and $D(r=0.442, p<0.005)$. Furthermore, $|R|$ area was significantly greater in subjects with excess visceral adiposity than in those without visceral adiposity in each of these three groups $(p<0.01, p<0.05$ and $p<0.005$, respectively). There was no correlation between blood glucose area and visceral fat area in any groups. These results indicate that excess visceral adiposity increases IRI area possibly through reducing insulin action in those groups with varying glucose tolerance. We conclude that excess visceral adiposity could cause insulin resistance and is a potent risk factor of diabetes even if BMl is within normal range. 
571

\section{COMPARISON OF DTFEERENT METABOLIC AND ANTROPOMTETRIC PARAYETERS IN REFLECTION OF INSULIN SENSTITVITY IN PRENLENAPAUSAL WOMEN}

Hekim N, Korugan Ü, Altuntaş Y.

Cerrahpaşa Medical Faculty of Istanbul University, Departmant of Endocrinology and Metabolism, TURKEY.

The aim of this study is to find safe and simple metabolic and antropometric parameters in reflection of insulin sensitivity. This study is conducted in 83 and 42 non obese control subjects [BMI $31.6 \pm 4.1$ vs $23.8 \pm 2 \mathrm{~kg} / \mathrm{m} 2, \mathrm{p}=0.005$; waist-hip ratio (WHR) $0.83 \pm 0.18$ vs $0.73 \pm 0.13, \mathrm{p}=0.027$; body fat distribution (BFD) $4 \pm 4.9$ vs $35.8 \pm 5.1 \%, p>0.05$ ]. Fasting(F) levels for insulin were $103.6 \pm 77.8$ vs $70 \pm 46 \mathrm{pM}(\mathrm{p}=0.037)$, C-peptid (Cp) were $1 \pm 0.6$ vs $0.8 \pm 0.35 \mathrm{nM}$ (NS), insulin glucose ratio (IGR) were $20.3 \pm 15.3$ vs $15,6 \pm 13.7 \mathrm{pM} / \mathrm{mM}$ (NS), insulin C-peptid ratio (ICR) were $0.17 \pm 0.13$ vs $0.1 \pm 0.07 \mathrm{M}(\mathrm{p}=0.007) ; 1$-h postglucose $(\mathbf{P})$ levels for insulin were $839.4 \pm 634.8$ vs $512 \pm 420$ pM ( $=0.006)$, C-peptid were $4.04 \pm 1.88$ vs $3.6 \pm 2.7 \mathrm{nM}$ (NS), IGR were $130 \pm 96$ vs $82 \pm 52.3 \mathrm{pM} / \mathrm{mM}$, ICR were $0.21 \pm 0.14$ vs $0.17 \pm 0.05 \mathrm{M}(\mathrm{NS})$ found in obese and nonobese subjects. FFA and lipid values were not different obese and nonobese groups (NS). BMI and BFD are not correlated with insulin, $C p$ level, IGR ang ICR. WHR correlated with fasting and $1-h$ insulinemia $(r=0.45, p=0.016 ; \quad r=0.54$ $p=0.004)$, Fop level $(=0.69, p=0.012)$, fasting IGR and $1-h$ IGR $(r=0.4$, $\mathrm{p}=0.012 ; \mathrm{p}=0.64, \mathrm{p}<0.0001)$. Triglyceride correlated with FFA $(r=0.5$, $\mathrm{p}=0.007$ ), $1-\mathrm{h}$ insulinemia ( $=0.35, \mathrm{p}=0.012$ ), fasting and $\mathrm{l}$-h $\mathrm{Cp}$ levels $(r=0.4, p=0.042 ; r=0.49, p=0.012)$. As a conclusion WHR and elevated triglyceride leveis are simple antropometric and metabolic parameters in reflecting the insulin sensitivity in obesity.

\section{2}

LACK OF INFLUENCE OF BODY MASS INDEX ON THE DELAYED ACTIVATION OF INSULIN-MEDIATED GLUCOSE DISPOSAL IN OBESITY G. Biolo, G. Toigo, B. Ciocchi, R. Situlin, and G. Guarnieri, Istituto di Clinica Medica, University of Trieste, Italy.

Obese subjects are characterized by insulin resistance and slow activation of insulin action. The aim of the present study was to evaluate the influence of body mass index (BMI) on the timing of activation of the insulin effect on glucose disposal in obesity. We performed prolonged ( 6 hours) euglicemic-hyperinsulinemic glucose clamps $\left(40 \mathrm{mU} / \mathrm{m}^{2} / \mathrm{min}\right)$ in 12 abese subjects with impaired glucose tolerance. During the first 2 hours of clamp, insulin-mediated glucose disposal $(M)$ was greater than $3 \mathrm{mg} / \mathrm{kg} / \mathrm{min}$ in 5 subjects $(M>3)$ and lower than $3 \mathrm{mg} / \mathrm{kg} / \mathrm{min} \quad(M<3)$ in 7 subjects. The two groups (i.e., $M>3$ and $M<3$ ) were matched for $B M l(40 \pm 4$ and $41 \pm 3$

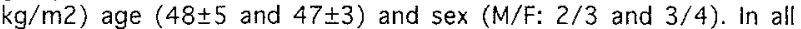
subjects, the rate of insulin-mediated glucose disposal progressively increased during the first 4 hours of clamp to reach constant values during the $5^{\text {th }}$ and $6^{\text {th }}$ hour of clamp. In the $M>3$ group, $M$ at the $2^{\text {nd }}$ hour $(5.05 \pm 0.80 \mathrm{mg} / \mathrm{kg} / \mathrm{min})$ was $91 \pm 3 \%$ of $M$ at the $6^{\text {th }}$ hour $(5.60 \pm 0.87 \mathrm{mg} / \mathrm{kg} / \mathrm{min})$. In the $M<3$ group, $M$ at the $2^{\text {nd }}$ hour (2.26 $\pm 0.13 \mathrm{mg} / \mathrm{kg} / \mathrm{min})$ was only $59 \pm 4 \%(P<0.01$ vs. $M>3$ group) of $M$ at the $6^{\text {th }}$ hour $(3.99 \pm 0.49 \mathrm{mg} / \mathrm{kg} / \mathrm{min})$. In all subjects $(n=12)$, BMI poorly correlated with $M$ at the $2^{\text {nd }}$ hour $(R=-0.42$; NS), whereas BMI significantly correlated with $M$ at the $6^{\text {th }}$ hour $(R=-0.60 ; P<0.05)$. In conclusion, insulin-resistance of obesity is characterized by at least two components: 1) a delayed activation of the insulin effect on glucose metabolism, which is not influenced by the degree of obesity and is greater in obeses with marked insulinresistance; 2) a defect of insulin action which is not dependent on the duration of hyperinsulinemia and correlates with BMI.

\section{3}

LOW FAT DIET-INDUCED WEIGHT LOSS IMPROVES OXIDATIVE AND NONOXIDATIVE GLUCOSE DISPOSAL JM Bryson, SE King, CM Burns, S Swaraj and ID Caterson. Dept of Endocrinology, Royal Prince Alfred Hospital, Sydney, Australia, 2050.

Reductions in both oxidative (GOX) and non-oxidative (NOX) pathways of glucose disposal are associated with decreased glucose utilisation in insulin resistance, with these changes being attributed to increased availability of fatty acids as an alternative energy supply. The aim of this study was to examine the effects in obese subjects of a low fat diet (LFD) of $30 \mathrm{~g} \mathrm{fat} /$ day on insulin-stimulated glucose disposal (ISGD), GOX, and NOX, and on the activities in skeletal muscle of the key enzymes of glucose disposal, pyruvate dehydrogenase complex (PDH) and glycogen synthase (GS). RQ was measured in eight obese non-diabetic subjects $(6 \mathrm{~F}, 2 \mathrm{M}$; $\mathrm{BMI}: 38.1 \pm 1.5)$ before and during a euglycemic hyperinsulinemic clamp $(40 \mathrm{mU} / \mathrm{m} 2 / \mathrm{min})$ and muscle biopsies were taken after the clamp for PDH and GS determination in 7 subjects. These tests were repeated after 12 weeks of LFD. LFD treatment resulted in significant weight loss (pre:105.9 \pm 4.0 , post: $100.2 \pm 3.7 \mathrm{~kg} ; \mathrm{p}=0.006$ ) due to reduced body fat. Fasting serum insulin levels were decreased (pre: $101.5 \pm 15.3$, post: $79.6 \pm 11.9 \mathrm{pM} ; \mathrm{p}=0.023$ ). Increases were seen in insulin-stimulated RQ (pre: $0.87 \pm 0.01$, post: $0.90 \pm 0.01 ; \mathrm{p}=0.002$ ), ISGD (pre: $4.94 \pm 0.84$, post: $7.20 \pm 1.03 \mathrm{mg}$ gluc/min/g body wt/pM I; $p=0.017$ ), GOX (pre: $2.57 \pm 0.33$, post: $3.06 \pm 0.32, p=0.084$ ) and NOX (pre: $2.38 \pm 0.65$, post: $4.13 \pm 0.77$; $\mathrm{p}=0.022$ ). There was a significant increase in muscle PDH (pre: 14.26 \pm 1.82 ; post:22.09 $2.79 \mathrm{mU} / \mathrm{U} \mathrm{CS} ; \mathrm{p}=0.027$ ) with $\mathrm{PDH}$ levels post LFD being strongly correlated with GOX $(r=0.755, p=0.049)$. There was no change in the \% active or in the total GS activity suggesting that there was no change in the rate of glycogen synthesis. These results show that the decrease in resistance to insulin following LFD-induced fat loss is due to improvements in both the oxidative and the nonoxidative glycolytic pathway of glucose disposal.

\section{4}

INCREASE IN PERIPHERAL BLOOD FLOW IMPROVES METABOLIC DERANGEMENT AND INHIBITS APPEARANCE OF HYPERGLYCEMIA IN NIDDM MODEL RAT

I. Nakahara ${ }^{1}$, Y.shiba ${ }^{1}, \quad$ M. Kubota ${ }^{1}$, T.Tbmita ${ }^{1}$, M.Matsuhisa ${ }^{1}$, R.Kawamori ${ }^{2}$, Y.Yasuda ${ }^{3}$, Y.Kimura ${ }^{3}$, and Y.Yamasaki ${ }^{1}$

'Osaka University, Osaka, 2Juntendo University, Tokyo, and ${ }^{3} \mathrm{Otsuka}$ Research Institute, Tokushima, Japan

Impaired increase in muscle blood flow against insulin might play one of crucial roles in insulin resistance. The aim of this study was to investigate the increase in blood flow improves the abnormality of glucose metabolism or not. Using dual tracer method $\left(6,6-{ }^{2} \mathrm{H}_{2}\right.$-glucose, U- ${ }^{12} \mathrm{C}-$ glucose), we measured hepatic glucose production (HGP), initial hepatic ghcose uptake (IHGU) and glucose metabolic clearance rate (MCR) during portal glucose infusion in obese NIDDM model, OLETF (Otsuka Long Evans Tbkushima Fatty) rats fed with anti-platelet drug, cilostazol (33 $\mathrm{mg} / \mathrm{kg} / \mathrm{day})$ containig chow $(\mathrm{CI} ; \mathrm{n}=6)$. Controls are OLETF rats (DM; $\mathrm{n}=5)$ and normal LETO rats $(\mathrm{NC} ; \mathrm{n}=6)$ fed with normal chow. At 28 weeks body weight in DM $(604 \pm 45 \mathrm{~g})$ and $\mathrm{CI}(583 \pm 11 \mathrm{~g})$ were similar and significantly higher than $\mathrm{NC}(429 \pm 25 \mathrm{~g})$. Fasting plasma glucose in CI $(5.5 \pm 0.9 \mathrm{mM})$ was significantly lower than DM $(10.1 \pm 1.9 \mathrm{mM}, \mathrm{P}<0.01)$ and similar to NC $(6.3 \pm 0.9 \mathrm{mM})$. Fasting plasma insulin concentration in CI $(1013 \pm 641 \mathrm{pM})$ was significantly lower than DM $(2197 \pm 430 \mathrm{pM}$, $\mathrm{P}<0.05)$ but significantly higher than NC $(405 \pm 76 \mathrm{pM})$. MCR in CI $(9.9 \pm 2.2 \mathrm{ml} / \mathrm{kg} / \mathrm{min})$ was significantily higher than DM $(5.7 \pm 0.4 \mathrm{~m} / \mathrm{kg} / \mathrm{min}$, $\mathrm{P}<0.05)$ and similar to $\mathrm{NC}(8.7 \pm 2.9 \mathrm{ml} / \mathrm{kg} / \mathrm{min})$, while there were not significant differences in HGP $(0.054 \pm 0.014,0.061 \pm 0.009 \mathrm{mmol} / \mathrm{kg} / \mathrm{min})$ and IHGU $(12.4 \pm 4.3,7.5+2.8 \%)$ between $\mathrm{CI}$ and DM. Acute intra-venous administration of cilostazol significantly increased muscle blood flow from $11 \pm 4$ to $15 \pm 4 \mathrm{ml} / \mathrm{min} / 100 \mathrm{~g}$ tissue in OLETF rats. In summary, antiplatelet drug, cilostazol, could improve derangement of peripheral glucose metabolism in NIDDM model rat by increasing peripheral blood flow and successfully inhibit appearance of hyperglycemia. 
575

Effects of L-arginine on insulin-mediated vasodilation and insulin sensitivity in NIDDM and obesity.

The Diabetic Angiopathy Research Group: T.C. Wascher, W.F. Graier* B. Bahadori, H. Toplak. Dept. Internal Medicine and "Inst. of Medical Biochemistry, Univ. Graz, Austria

The direct vascular effect of insulin, as well established, is vasodilatation for adequate substrate supply to target organs (e.g. sceletal muscle). Very recently, this vasodilatation has been shown to depend on the formation of endothelium-derived relaxing factor (EDRF) in vascular endothelial cells. In obese patients and NIDDM endothelial dysfunction by means of a reduced endothelium-dependent vasodilatation upon stimulation, as well as a lack of insulin to induce vasodilatation are described. Intravenous L-arginine has been shown to improve insulin sensitivity in NIDDM patients as well as to restore endothelial dysfunction. The aim of the present study was therefore to investigate the effect of intravenous L-arginine on insulinmediated vasodilation and insulin sensitivity in NIDDM and obesity compared to healthy volunteers. The study was done in 6 NIDDM patients. 6 obese nondiabetics and 6 healthy volunteers. Insulin-induced vasodilation (by venous occlusion plethysmography) and insulin sensitivity were assesed twice in each patient during a 3-hour insulin-suppression test (as described by G.M. Reaven) in absence and presence of concomitant L-arginine infusion ( $0.52 \mathrm{mg} / \mathrm{kg}$ bodyweight $/ \mathrm{min}$ ). In the absence of L-arginine forearm blood-flow was unchanged in obese and NIDDM subjects but increased by $16.3 \pm 9.3 \%$ ( $p<0.01$ vs. baseline) in healthy subjects. In the presence of L-arginine however, Insulin-mediated vasodilation was restored in obesity $(118.8 \pm 11.2 \%, \mathrm{p}<0.01)$ and NIDDM $(117 \pm 10.3 \%, \mathrm{p}<0.01)$. In healthy subjects $\mathrm{L}$-arginine resulted in a nonsignificant increase to $121.3 \pm 8.8 \%$. Interestingly, insulin sensitivity, by means of steady state blood glucose improved significantly $(p<0.001)$ in all groups investigated.

In conclusion, our data clearly demonstrate that intravenous L-arginine restores insulin-mediated vasodilation in obesity and NIDDM while improving insulin-sensitivity. These results support the hypothesis of a contribution of impaired insulin-mediated vasodilation to insulin resistance in obesity and NIDDM.

\section{6}

ENHANCED SERUM- AND INSULIN-STIMULATED GROWTH OF SMOOTH MUSCLE CELLS FROM TYPE II DIABETIC GK RATS

N. Parés-Herbuté, A. Avignon, E. Fliche and L. Monnier. Depart. Metabolic Diseases, CHU Lapeyronie, 34295 Montpellier, France.

Hyperinsulinaemia could contribute to the development of atherosclerosis in the diabetic population. This vascular desease is associated with abnormal proliferation of intimal smooth muscle cells (SMC). However, the role of insulin in SMC growth remains unclear. In this work, we studied the growth curves and the effect of insulin on primary cultures of aortic SMC from hyperinsulinaemic type II diabetic GK rats (source obtained from Dr. B. Portha, CNRS URA 307, Paris VI, France) and from age-matched Wistar control rats. Proliferation was evaluated as the measurement of DNA synthesis $([3 \mathrm{H}]$ thymidine incorporation) and cell number. Growth curves in $2.5 \%$ serum showed that SMC from diabetic GK rats presented an enhanced proliferation (2.7 fold) compared with those in SMC from control rats, on days 5 to 9 of culture. However, in 10\% serum, significant enhancement $(\mathrm{p}<0.01)$ was only observed at day 9 of culture. Insulin $(1 \mu \mathrm{mol} / \mathrm{l})$ significantly stimulated the proliferation of SMC from diabetic GK rats on day 5 to $9(30 \%, p<0.01)$, but no significant enhancement was observed in SMC from control rats. Insulin $(48 \mathrm{~h})$ stimulated dosedependently (1 to $1000 \mathrm{nmol} / 1$ ) [3H]thymidine incorporation into DNA from diabetic GK SMC. In contrast, no change was observed in [3H]thymidine incorporation in control SMC. Worthmannin (1 $\mu \mathrm{mol} / \mathrm{l}$ ), an inhibitor of phosphatidyl inositol (PI) 3-kinase, partially inhibited insulin-stimulated DNA synthesis, suggesting that PI 3-kinase may be involved in the insulin mitogenic effet. These results suggest that serum- and insulin-stimulated proliferation was enhanced in the SMC from GK rats. This increased responsiveness could contribute to alterations in vascular smooth muscle growth that may be associated with the atherogenic process observed in type II diabetes mellitus.
577

PYRUVATE DEHYDROGENASE ACTVITY IN MONONUCLEAR LEUKOCYTES: AN INDEX OF RESTORED PERIPHERAL INSULIN SENSITIVITY AFTER DEXFENFLURAMINE TREATMENT IN NIDDM PATIENTS WITH OBESITY

A. Pellacani, I. Rabbone, *S. Gamba M. Curto, S. Mioletti, C. Ceruti, M. Piccinini, \#R. Lavielle, R. Bruno, and M.T. Rinaudo. Dept. of Medicine and Experimental Oncology, Lavielle, R. Bruno, and M.T. Rinaudo. Dept. of Medicine and Experimental Oncolog
University of Turin;* Maria Vittoria Hospital, \# Servier Research Inst., Roma, Italy. Pyruvate dehydrogenase (PDH), the key enzyme in glucose oxidative
Pyose breakdown, appears to be deranged in skeletal muscle of obese, NIDDM patients. We have previously shown that in different models of altered glucose tolerance the activity of PDH in the active form (FDHa) is reduced in circulating mononuclear leukocytes (CML) and significantly related to the glycemic area under the curve (GAUC) during an OGTT The aim of the present study was to determine basal (bPDHa) and in vitro insulin-stimulated (iPDHa insulin at 33 PM) $\mathrm{PDH}$ aclivity (nmol (PDHa, insulin at $33 \mathrm{pM}$ ) PDHa activity (nmol acetylCoA/10 cells/h) in CML from 15 obese, NIDDM patients (age $48.7 \pm 7.7 \mathrm{y}$, BM $31.0 \pm 2.3 \mathrm{Kg} / \mathrm{m}^{2}$ ), before and after 21 days of dexfenfluramine treatment (DEX, $15 \mathrm{mg}$ b.i.d), and to relate both activities to indexes of glycemic control, represented by fasting glycemia ( $\mathrm{FG}$, mmol/l), fasting insulinemia ( $\mathrm{Fl}, \mathrm{mU} / \mathrm{l}), \mathrm{GAUC}(\mathrm{mmol} / \mathrm{l} / 180 \mathrm{~min})$ and insulinemic AUC (IAUC, mU//180 min) during an OGTT (75 g glucose). CML were isolated from peripheral blood by density gradient centrifugation. Comparisons were performed by Wilcoxon sioned rank test; correlation coefficients were calculated by

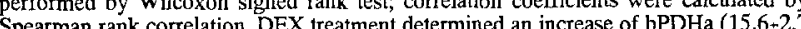
ys $25.9-2.90<00001$ ). In addition, while before treatment iPDHa did not differ from bPDHa $(15.8+2.5$ vs $15.6 \pm 2.7, \mathrm{~ns})$, DEX restored PDHa responsiveness to insulin, bPDHa (15.8 \pm 2.5 vs $15.6 \pm 2.7$, ns), DEX restored PDHa responsiveness to insulin,
resulting in a significant increase of $\mathrm{PDH}$ over bPDHa (30.2 4.7 vs $25.5 \pm 2.6$, resulting in a significant increase of iPDHa over bPDHa $(30.2 \pm 4.7$ vs $25.5 \pm 2.6$,
$p=0.0004)$. DEX reduced GAUC (2462.6 376.5 vs $2014.9 \pm 327.1, p=0.0004)$, FG $(8.3 \pm 0.6$ vs $7.2 \pm 0.9, \mathrm{p}=0.0002)$ and $\mathrm{FI}(24.8 \pm 7.5$ vs $17.6 \pm 6.0, \mathrm{p}=0.0001)$, while IAUC was unchanged $(10250 \pm 4199.4$ vs $10653=4533.8, \mathrm{~ns}$ ). bPDHa was related to GAUC $(\mathrm{r}=-0.49, \mathrm{p}=0.006), \mathrm{FG}(\mathrm{r}=-0.52, \mathrm{p}=0.003)$, and $\mathrm{FI}(\mathrm{r}=-0.56, \mathrm{p}=0.001)$, but not to IAUC. iPDHa was also related to GAUC ( $r=-0.47, p=0.009)$, FG $(r=-0.42$, $\mathrm{p}=0.023)$ and $\mathrm{FI}(\mathrm{r}=-0.49 \mathrm{p}=0.007)$, but again not to IAUC. In conclusion, both $\mathrm{p}=0.023)$ and $\mathrm{FDHa}(\mathrm{r}-\mathrm{CML}$ from obese, NIDDM patients increased after 3 weeks of DEX treatment. Both enzyme activities were related to the GAUC, FG and FI, which in DEX treatment. Both enzyme activities were related to the GAUC, FG and FI, which in
turn indicated a better glycemic control after DEX. The improved in vivo insulin sensitivity suggested by the contemporary decrease in $F G$ and $F I$ and by decreased GAUC during OGTT, along with unchanged IAUC, was paralleled in CML by increased bPDHa and restored insulin-sensitivity of PDH in vitro. Thus, evaluation in CML of PDHa basal activity and of the enzyme response to insulin in vitro might be useful model to investigate the pharmacodynamics of drugs affecting glucose disposal in NIDDM patients.

\section{8}

RELATIONSHIP OF PLASMA PAI-1 ANTIGEN/ACTIVITY TO CLINICAL AND METABOLIC ASPECTS OF INSULIN RESISTANCE SYNDROME IN NON-INSULIN DEPENDENT DIABETES MELLITUS.

L.A De Giorgio, A. Bastreri. R. Anichini, S. Vichi, A.M Sironi. E. Romano, A. Zappa, S. Lenzi, E. Ferrannini, and G. Seghieri. Dpt of Medicine and Diabetes Unit, Ospedale S.Andrea, La Spezia, Diabetes Unit, Spedali Riuniti, Pistoia, CNR Inst. of Clinical Plyysiology, Univ, of Pisa, Pisa, Italy To measure plasma concentrations of PAI-1 Antigen (PAI-1 Ag) or Activity (PAI-1 Act) in non-insulin dependent diabetes mellitus (NIDDM) and their relationship to the clinical correlates of the insulin resistance syndrome (syndrome $\mathrm{X} ; \mathrm{S}-\mathrm{X}$ ), we assayed plasma from 97 NIDDM patients and 33 control subjects matched for sex, age and smoking habits. In a subset of 40 patients insulin sensitivity was moreover measured by the insulin suppression test (IST). Mean PAI-1 Ag and PAI-1 Act $( \pm$ SD) were significantly higher in NIDDM, as compared to controls $(39 \pm 2 \mathrm{ng} / \mathrm{ml}$ and $14 \pm 2 \mathrm{U} / \mathrm{ml}$ in diabetic group and $28 \pm 2 \mathrm{ng} / \mathrm{ml}$ and $10 \pm 2 \mathrm{U} / \mathrm{ml}$ in the control subjects; $\mathrm{p}=0.02$ for both). Significant correlations were found between PAI-1 Ag or PAI-1 Act and plasma triglycerides $(r=0.25, p=0.01)$, basal plasma insulin $(r=0.35$ $p=0.03)$, total cholesterol $(r=0.21 ; p=0.04)$, body mass index (BMI) ( $r=0.24$; $\mathrm{p}=0.01)$ or steady-state glucose during the IST $(\mathrm{r}=0.43 ; \mathrm{p}=0.005)$, but not glycated haemoglobin, or mean blood pressure. When diabetic patients were stratified for absence of $\mathrm{S}-\mathrm{X}$ ie $\mathrm{TG}<2.3 \mathrm{mmol} / \mathrm{l}$, arterial blood pressure $<$ $160 / 90 \mathrm{mmHg}$ or no antihypertensive therapy, and BMI $<27 \mathrm{Kg} / \mathrm{m}^{2}$ (group A), or for presence of only one (group B), two (group C) or three of these correlates (group D), a parallel increase in both PAI-l Ag/Act was observed : $29 \pm 2 / 9 \pm 3$ in group A, $33 \pm 2 / 13 \pm 3$ in group B, $43 \pm 2 / 17 \pm 2$ in group $C$ and $57 \pm 2 / 18 \pm 2$ in group D ( $p<0.05$ for trend). In summary, PAI-I antigen and activity are significantly higher in NIDDM, and are associated with insulin 


\section{9}

NO EFFECT OF NEUTRALIZATION OF TNF- $\alpha$ ON INSULIN-MEDIATED GLUCOSE DISPOSAL IN OBESE INSULIN-RESISTANT SUBJECTS.

A.J. Scheen, M.J. Castillo, N. Paquot and P.J. Lefêbvre. Division of Diabetes, Nutrition \& Metabolic Disorders, CHU Sart Tilman, B-4000 Liège, Belgium.

Overexpression of tumor necrosis factor alpha (TNF- $\alpha$ ) gene occurs in animal models of obesity/insulin resistance and in human obesity. In the former, neutralization of peripheral TNF- $\alpha$ results in marked increase in insulin sensitivity. In patients presenting android obesity, we have investigated the effect of Ro 45-2081 (a soluble TNF p55-receptor-IgG fusion protein neutralizing endogenous TNF- $\alpha$ ) on insulin sensitivity. Seven patients $(5 / 2$ women/men, mean \pm SD age: $41.1 \pm 4.0$ years, BMI: $36.1 \pm 4.7$ $\mathrm{kg} / \mathrm{m}^{2}$, waist/hip ratio: $0.99 \pm 0.11$ ) were studied. Three patients had normal glucose tolerance and four impaired glucose tolerance or mild diabetes. Hyperinsulinism was present in all cases. Each patient underwent two consecutive euglycaemic glucose-clamp tests $(1 \mathrm{mU} / \mathrm{kg}$ body weight of insulin for 120 minutes): $48 \mathrm{~h}$ after injection of placebo, and $48 \mathrm{~h}$ after a single i.v. injection of $50 \mathrm{mg}$ of Ro $45-2081$. In both tests, steady-state plasma glucose $\left(5.51 \pm 0.61\right.$ vs $\left.5.42 \pm 0.73 \mathrm{mmol} . \mathrm{l}^{-1}\right)$ and insulin levels $(649 \pm 130$ vs 688 \pm 160 pmol. $\left.1^{-1}\right)$ were similar. Insulin-mediated glucose disposal, i.e. the glucose infusion rate (GIR) at steady-state, was similar after placebo $(12.4 \pm$ 4.1, range: $\left.8.4-18.8 \mu \mathrm{mol} \cdot \mathrm{kg}^{-1} \cdot \mathrm{min}^{-1}\right)$ and after the drug $(13.2 \pm 5.5$, range: $\left.8.4-23.6 \mu \mathrm{mol} \cdot \mathrm{kg}^{-1} \cdot \mathrm{min}^{-1}\right)$. Similarly, no statistically significant changes occurred in glucose metabolic clearance rate $(2.28 \pm 0.85$ vs $2.48 \pm 1.03$ $\mathrm{ml} \cdot \mathrm{kg}^{-1} \cdot \mathrm{min}^{-1}$ ) or GIR/Insulin ratio. Indirect calorimetry showed no differences in lipid and carbohydrate oxidation rates between the two experimental conditions. Therefore, under the conditions of this study, no improvement in peripheral insulin sensitivity was observed in obese insulin-resistant patients following peripheral neutralization of endogenous TNF- $\alpha$ with a single i.v. dose of $50 \mathrm{mg}$ Ro $45-2081$.

\section{1}

DISPROPORTIONATE HYPERPROINSULINAEMIA REFLECTS THE DEGREE OF IMPAIRED B-CELL SECRETORY CAPACITY IN NIDDM M.E. Røder, D. Porte Jr., R.S. Schwartz ${ }^{*}$ and S.E. Kahn. Division of Melabolism, Endocrinology and Nutrition, VA Medical Center; *Gerontology and Geriatric Medicine, "Harborview Medical Center, University of Washington, Sedtle, WA, USA.

An increased proportion of fasting proinsulin (PI) relative to immunoreactive insulin (IRI; increased PI/IRI) occurs in NIDDM. To deternine whether the magnitude of the increase in PI/IRI is an indicator of the degree of B-cell dysfunction, we measured fasting plasma glucose, PI, IRI and PI/IRI and related them to maximal B-cell secretory capacity (AIRmax) in 10 subjects with NIDDM (age: $61 \pm 3 \mathrm{yr}$, BMI: $28.2 \pm 1.3 \mathrm{~kg} / \mathrm{m}^{2}$, duration of NIDDM: $10.1 \pm 1.8 \mathrm{yr}$; mean $\pm \mathrm{sem}$ ) and in 10 healthy subjects matched for age and BMI (age: $61 \pm 6 \mathrm{yr}$, BMI: $27.9 \pm 1.5$ $\mathrm{kg} / \mathrm{m}^{2}$ ). AIRmax was quantified as the incremental insulin response to i.v. arginine at maximal glycemic potentiation (> $25 \mathrm{mmol} / \mathrm{l}$ ).

Mean fasting plasma glucose was $13.0 \pm 1.4 \mathrm{mmol} / 1$ (range: $6.9-18.3 \mathrm{mmol} / \mathrm{l}$ ) in NIDDM subjects and $5.0 \pm 0.1 \mathrm{mmol} / 1$ in the controls. Fasting PI was higher in $\operatorname{NIDDM}(31.1 \pm 5.0)$ than in controls $(9.4 \pm 2.5 \mathrm{pmol} / 1 ; \mathrm{p}<0.01)$, hul IRl levels were similar $90.7 \pm 10.1$ vs. $82.8 \pm 23.4 \mathrm{pmol} / \mathrm{l}$, ( $\mathrm{p}=\mathrm{NS}$ ). The $\mathrm{PI} / \mathrm{IRI}$ ratio was significantly elevated in NIDDM compared to control subjects $(34.2 \pm 4.0 \%$ vs. $12.8 \pm 0.8 \%$; $\mathrm{p}<0.01$ ). Following elevation of the glucose level to $30.2 \pm 0.4 \mathrm{mmol} / 1$ (NIDDM) and $30.3 \pm 0.5 \mathrm{mmol} / 1$ (controls), AIRmax was quantified as $837 \pm 224 \mathrm{pmol} / 1$ in NIDDM and $1997 \pm 315 \mathrm{pmol} / 1$ in controls, $(p<0.01)$. Fasting plasma glucose was inversely correlated with AIRmax $(\mathrm{r}=-0.76, \mathrm{p}<0.001)$ and positively correlated with fasting PI/IRI $(r=0.80, p<0.001)$. The PI/ IRI ratio correlated inversely with AIRmax $(\mathrm{r}=-0.80, \mathrm{p}<0.001)$.

We conclude that the magnitude of the elevation in fasting PI/ IRI is related to the reduction in B-cell secretory capacity (AIRmax) in NIDDM. Thus, the fasting $\mathrm{PI} / \mathrm{IRI}$ ratio appears to be a marker of the degree of B-cell dysfunction in NIDDM.
580

PREDIABETIC STATE DUE TO PHOSPHOFRUCTO-1KINASE-DEFICIENCY IN HUMANS

M.Ristow, M.Mōhlig, M.Vorgerd, H.Schatz, A.Pfeiffer University Hospital Bergmannsheil Bochum, Germany Background: Phosphotructo-1-kinase (PFK1) is a rate limiting enzyme in glycolysis, and it's muscle subtype (PFK1-M) deficiency leads to an disorder known as glycogenosis type VII or Tarui's disease. It was evaluated whether PFK1-M deficiency leads to NIDDM in humans. Methods: A core family of four was biochemically and by DNA-analysis evaluated for PFK1-M deficiency. All members underwent oral and intravenous glucose tolerance test (oGTT/ivGTT), as well as an insulin resistance test (IRT) using octreotide. Results: Father (46 yrs., BMI 22.4) and older son (19 yrs.. BMI 17.8) showed homozygous PFK1-M deficiency. while mother ( 47 yrs., BMI 28.4$)$ and younger son $(13$ yrs.. BMI 16.5) were shown to be heterozygously PFK1-Mdeficient biochemically. DNA anaylsis revealed an exon 5missense-mutation at one allele of all four members, and an exon 22-frameshift-mutation at the other allele of the two homozygously affected. By oGTT the father showed impaired glucose tolerance, and the mother clinical diabetes. By ivGTT both parents and the older son had a decreased first phase insulin secretion. The IRT showed severe insulin resistance in both parents, and moderate in the older son. Conclusions: PFK1-M-deficiency leads to a metabolic state typical for NIDDM in homozygously affected humans, especially insulin resistance and loss of first phase betacell function, and may additionally contribute to the clinical manifestation of NIDDM in heterozygously affected humans if combined with risk factors like obesity or age.

\section{2}

THE METABOLIC X SYNDROME IN DERIVATIVES OF THE PROGENITOR STRAIN OF DIABETES-PRONE BB RATS Voigt, B., Berg, S., Kovacs, P., L. Vogt and I. Klöting „Gerhardt Katsch“ Institute of Diabetes, University of Greifswald, Karlsburg

It is well-known that all BB rats descende from an outbred Wistar rat strain characterized by a high degree of genetic heterogeneity including $2 \mathrm{MHC}$ haplotypes RTla and RT1u. In 1981 animals of this outbred stock were transfered to our animal facility. Homozygous RT1a and RT1u (diabetes susceptible haplotype) animals were selected, inbred separately and only selected for a ,healthy fertility" named WOK. $1 \mathrm{~A}$ (RT1a) and WOK.1W (RT1u), After more than 35 inbred generation the fertility and survival of pups droped drastically in WOK. IW rats which prompted us to search for causes of this observation. Therefore, 21 WOK. $1 \mathrm{~W}(9 \mathrm{M}: 12 \mathrm{~F})$ and 18 nondiabetic BB $(8 \mathrm{M}: 10 \mathrm{~F})$ rats were longitudinally studied for body weight gain, glucose tolerance (i.p.), serum and urine constituents and blood pressure (tail cuff method) from the 3rd until the 32nd weeks of life at defined intervals. The body weight gain was comparable between male as well as female WOK. $1 \mathrm{~W}$ and $\mathrm{BB}$ rats. However, the nonfasting blood glucose was significantly lower $(5.2 \pm 0.7$ vs. $6.2 \pm 0.8 \mathrm{mmol} / \mathrm{l} ; \mathrm{p}<0.001)$ and insulin was significantly higher in WOK. 1 W than $B B$ rats $(5.9 \pm 0.7$ vs. $2.1 \pm 0.6 \mathrm{ng} / \mathrm{ml}$ $\mathrm{p}<0.01)$. Compared with nondiabetic BB rats the glucose tolerance was disturbed in WOK.I W rats indicated by significantly lower blood glucose $10 \min (11 \pm 3$ vs. $16 \pm 95$ $\mathrm{mmol} / \mathrm{h})$ and significantly higher values $30 \mathrm{~min}(12 \pm 2.5 \mathrm{vs} .9 .3 \pm 1.7 \mathrm{mmol} / \mathrm{l})$ after glucose load. Furthermore, serum triglycerides ( $3.4 \pm 1$ vs. $1.8 \pm 0.8 \mathrm{mmol} / \mathrm{l} ; \mathrm{p}<0.0001)$ and the blood pressure $(147 \pm 13$ vs. $126 \pm 10 \mathrm{mmHg} ; \mathrm{p}<0.001)$ were significantly higher in WOK.1W than BB rats.Comparing male and female WOK. $1 \mathrm{~W}$ rats significantly higher values were found in males for glucose, insulin and in females for triglycerides and blood pressure. Interestingly, the blood pressure increased (136 to $151 \mathrm{mmHg}$ ) and the triglycerides ( 3.8 to $2.6 \mathrm{mmol} / \mathrm{l}$ ) decreased continuously in male, but not in female WOK. $1 \mathrm{~W}$ rats from 8 th to 32 nd weeks. This findings indicate that WOK.IW rats are characterized by nonfasting hypertriglyceridaemia, hyperinsulinaemia, impaired glucose tolerance and moderate hypertension which most probably cause the low reproductive performance in this rat strain. Despite this fact, WOK.1W rats may become an interesting animal model not only for metabolic but also for genetic studies of the known human metabolic $\mathrm{X}$ syndrome especially as the genetic profile of WOK.1W comprising 97 marker loci is already known. 
583

GLUCAGON AND GLP-1 RECEPTORS ON B-CELLS AND HEPATOCYTES

K. Moens, C. Van Schravendijk, A. Foriers*, D. Pipeleers, V. Rogiers*, D Flamez and F. Schuit. Diabetes Research Centre \& *Laboratory for Pharmacognosy and Toxicology, Vrije Universiteit Brussel, Laarbeeklaan 103 , B-1090 Brussels, Belgium.

Rat B-cells express functional receptors for glucagon and glucagon-like peptide$\mathrm{I}(7-36) \mathrm{NH}_{2}$ (GLP-I), two homologous insulinotropic peptides produced by alternative processing of the preproglucagon gene. The present study compares both peptides in terms of their receptor-binding and stimulation of cAMP production in 3 -cells. Exendin $(9-39) \mathrm{NH}_{2}$ and des-His ${ }^{1}\left[\mathrm{Glu}^{9}\right]$ glucagon- $\mathrm{NH}_{2}$ were used as receptor-specific antagonists, and isolated rat hepatocytes as reference system. No specific binding of [125I]-GLP-I was detected on rat hepatocytes, while its binding to B-cells was characterised by linear Scatchard plots with $3.6 \pm 0.3 \times 10^{4}$ sites per cell and $K_{D}$ of $0.43 \pm 0.04 \mathrm{nM}$. GLP- 1 binding was completely displaced by $1 \mu \mathrm{M}$ exendin, slightly altered by $1 \mu \mathrm{M}$ des-His-glucagon and half-maximally displaced by $0.3 \pm 0.1 \mu \mathrm{M}$ glucagon. Low affinity glucagon binding to the GLP-1 receptor is in agreement with the curvi-linear Scatchard plot of [125] -glucagon binding to purified B-cells. Hepatocytes exhibited high affinity glucagon binding $\left(\mathrm{K}_{\mathrm{D}}=3.9 \pm 0.1 \mathrm{nM}\right)$, which was completely displaced by $1 \mu \mathrm{M}$ des-His-glucagon, but unaffected by $1 \mu \mathrm{M}$ GLP-1 or exendin. On the contrary, 1 $\mu \mathrm{M}$ des-His-glucagon competed for $82 \pm 12 \%$ with high-affinity glucagon binding on B-cells, while displacement was significant with $1 \mu \mathrm{M}$ exendin $(33 \pm 26 \%)$ and 1 MM GLP-1 (43 $\pm 23 \%)$. Receptor-mediated cAMP production in hepatocytes and $B$-cells was consistent with these binding data. In hepatocytes, no GLP-1 induced cAMP formation was detected, while glucagon-induced CAMP production was suppressed effectively by $1 \mu \mathrm{M}$ des-His-glucagon but only marginally by $1 \mu \mathrm{M}$ exendin. In B-cells, $1 \mu \mathrm{M}$ exendin completely suppressed glucagon-induced cAMP production, while $1 \mu \mathrm{M}$ des-His-glucagon only counteracted the effects of up to $1 \mathrm{nM}$ glucagon. In conclusion, glucagon interacts with a des-His-glucagonsensitive receptor on hepatocytes, but with both a des-His-glucagon-sensitive and an exendin-sensitive receptor on $B$-cells.

\section{4}

AMYLIN INHIBITION OF ARGININE-INDUCED GLUCAGON SECRETION: COMPARISON WITH GLUCAGON-LIKE-PEPTIDE-1 (7-36)-AMIDE (GLP-1) B. Gedulin, C. Jodka, D. Green, R. Lawler and A. Young. Amylin Pharmaceuticals Inc., San Diego, CA 92121, USA

Pancreatic glucagon secretion is inhibited by insulin from pancreatic B-cells and, as reported recently, also by GLP-1. We have shown that amylin, a 37 amino acid peptide co-secreted with insulin can also inhibit glucagon secretion. To compare the potency and efficacy of the glucagonostatic effects of amylin and GLP-1, changes in arginine-stimulated glucagon secretion were measured in anesthetized male Harlan Sprague Dawley rats infused intravenously with either rat amylin $(0,0.1,0.3,1,3$ or $10 \mu \mathrm{g} / \mathrm{h} ; \mathrm{n}=23,7,8,6$, 4,7 , respectively) or with GLP-1 $(0,0.3,1,3,10,100 \mu \mathrm{g} / \mathrm{h} ; \mathrm{n}=19,4,4,6,5,7$, respectively) from $30 \mathrm{~min}$ before until $120 \mathrm{~min}$ after arginine ( $2 \mathrm{mmol}$ infused over $7 \mathrm{~min}$ ). To equalize the effects of glucose and insulin upon glucagon secretion, plasma glucose was clamped at $5.8 \mathrm{mM}$ following a primed/ continuous insulin infusion ( $12 \mathrm{mU}+5 \mathrm{mU} / \mathrm{kg} / \mathrm{min})$, beginning 60 min before the arginine infusion. Amylin infusion could inhibit the plasma glucagon response to arginine (measured as the $60 \mathrm{~min}$ increment in area-under-thecurve above the pre-arginine baseline $)$ by a maximum of $54.9 \pm 5.3 \%(P<0.01$ cf saline controls). GLP-1 infusions inhibited the glucagon response by a maximum of $38.5 \pm 2.6 \%$ ( $P<0.05$ cf saline controls). Concentration-responses were obtained for amylin and GLP-1 by relating glucagon responses to steady-state plasma concentrations obtained at the same infusion rate in separate studies. The $\mathrm{EC}_{50}$ thus derived for amylin-inhibition of glucagon secretion was $24 \mathrm{pM} \pm 0.17 \mathrm{log}$ units, and the $\mathrm{EC}_{50}$ for GLP. 1 in the same preparation was $162 \mathrm{pM} \pm 0.19 \mathrm{log}$ units. These data indicate that, in this rat preparation, amylin is $\sim 1.4$-fold more effective $(P<0.05)$ and $\sim 6$-fold more potent $(P<0.05)$ than GLP-1 in suppressing arginine-stimulated glucagon secretion.

\section{5}

GLUT-4 IN DIABETIC ADIPOSE TISSUE. EFFECT OF GLP-1. J. Puente, F. Clemente, L. Márquez, I. Valverde and M.L. Villanueva-Peñacarrillo. Fundación Jiménez Díaz, Madrid, Spain.

Glucose transport in adipose tissue is altered in diabetic states, and it has been shown that in diabetic rats, induced by streptozotocin (STZ) treatment in the adult age, the amount of GLUT-4 is decreased. In this work we have explored whether GLP-1(7-36)amide (GLP-1) treatment, peptide with insulin-like effects upon the glucose metabolism in some extrapancreatic tissues, affects the adipose GLUT-4 levels in normal and in two models of diabetic rats -treated with STZ in the adult age (IDDM) or at day 1 of birth (NIDDM)- The GLUT-4 protein was detected by Dot-Blot analysis in the epididymal fat of normal, IDDM and NIDDM rats, receiving no treatment or either GLP-1 $10.4 \mathrm{nmol} / \mathrm{kg} / \mathrm{h})$ or insulin $(450 \mathrm{mU} / \mathrm{kg} / \mathrm{h}$, only diabetic rats through an osmotic pump during three days. GLP-1 or insulin treatment induced in all groups a decrease in plasma glucose of about $30 \%$. In normal rats, GLP-1 treatment did not change GLUT-4 levels. Both groups of riats 4 levels $169 \pm 3 \%$ of normal rats, $p<0.01, n=3$, in NIDDM, and $56 \pm 3 \%, p<0.001, n=3$, in IDDM). In NIDDM rats, the treatment with GLP-1 induced a significant increment $(p<0.01$ vs untreated NIDDM $n=3$ ) of GLUT-4 levels up to normal values $(102 \pm 7 \%$ of normal ratsl; insulin treatment also induced an increment $(p<0.01 \mathrm{vs}$ untreated NIDDM, $n=3)$ of the glucose transporter value (124 $\pm 14 \%$ of normal rats). In IDDM rats, the treatment with GLP-1 induced a recovery of the GLUT-4 levels $1107 \pm 8 \%$ of normal rats, $p<0.001$ vs untreated IDDM, $n=3$ ); the same was observed after insulin treatment (116 $\pm 8 \%$ of normal rats, $p<0.001$ vs untreated IDDM, $n=3$ ). In conclusion, these data document a decreased GLUT-4 levels in the adipose tissue of IDOM and NIDDM rats and their normalization after treatment with GLP-1, and add support to the role GLP-1 in the adipose tissue glucose disposal.

\section{6}

GLP-1(7-36)AMIDE STIMULATES AN INOSITOL-PHOSPHOGLYCAN GENERATION IN RAT LIVER AND FAT.

L. Márquez, M.A. Trapote, M.A. Luque, A.I. Alcántara, I. Valverde and M.L. Villanueva-Peñacarrillo. Fundación Jiménez Díaz, Madrid, Spain.

GLP-1(7-36)amide (GLP-1) exerts insulin-1ike effects upon the glucose metabolism in liver and fat; the GLP-1 effect in liver is not mediated by an activation of adenyl cyclase. An inositolphosphoglycan (IPG) has been proposed as a second messenger in the insulin action. We have documented the possible mediation of an IPG on the glycogenic effect of GLP-1 in myocytes $\mathrm{BC}_{3} \mathrm{H}-1$ and $\mathrm{G} 2$-human hepatoma cells. In this work, we studied the kinetic of the glycosylphosphatidylinositols (GPIs), precursors of IPGs in rat hepatocytes and adipocytes, prelabelled with myo- $\left[{ }^{3} \mathrm{H}\right]$ inositol and further incubated in the absence (control) or presence of insulin or GLP-1. GPIs were analyzed in the cell organic extracts by sequential TLC autoradiography and B-counting. In hepatocytes, as well as in adipocytes, both GLP-1 and insulin induced a rapid decrease of the GPI levels, which were recovered to control value at $2 \mathrm{~min}$, followed by an increment at 5 and $10 \mathrm{~min}$ : HEPATOCYTES $10^{-9} \mathrm{M}$ insulin, $0.5 \mathrm{~min}:-23 \pm 5 \%$ of control, $\mathrm{p}<0.001$, $\mathrm{n}=17 ; 1 \mathrm{~min}:-25 \pm 7 \%, \mathrm{p}<0.01, \mathrm{n}=12 ; 2 \mathrm{~min}:-1 \pm 7 \%, \mathrm{n}=15 ; 5 \mathrm{~min}:$ $+13 \pm 5 \%, \quad p<0.05, \quad n=16 ; 10$ min: $+15 \pm 4 \%, p<0.01, n=15 ; 1090$ GLP $-1,0.5$ min: $-22 \pm 3 \%$ of control, $p<0.001, n=15 ; 1$ min: $-13 \pm 5 \%$, $\mathrm{p}<0.05, \mathrm{n}=14 ; 2$ min: $-8 \pm 5 \%, \mathrm{n}=15 ; 5 \mathrm{~min}:+19 \pm 6 \%, \mathrm{p}<0.01, \mathrm{n}=17$; $10 \mathrm{~min}:+12+5 \%, p<0.05, \mathrm{n}=16$. ADIPOCYTES $10^{\circ} \mathrm{M}$ insulin, $0.5 \mathrm{~min}$ : $-26 \pm 4 \%$ of control, $\mathrm{p}<0.001, \mathrm{n}=7 ; 1 \mathrm{~min}:-19 \pm 5 \%, \mathrm{p}<0.02, \mathrm{n}=6$; 2 min: $-10 \pm 5 \%, n=7 ; 5$ min: $+31 \pm 13 \%, n=6 ; 10 \mathrm{~min},+30 \pm 11 \%$, $\mathrm{p}<0.02, \mathrm{n}=7 ; \quad 10^{-9} \mathrm{M}$ GLP-1, $0.5 \mathrm{~min} ;-24 \pm 7 \%$ of control, $\mathrm{p}<0.02$, $\mathrm{n}=7 ; 1$ min: $-24 \pm 5 \%, \mathrm{p}<0.01, \mathrm{n}=8 ; 2 \mathrm{~min}:+11 \pm 7 \%, \mathrm{n}=8 ; 5 \mathrm{~min}:$ $+38+7 \%, \mathrm{p}<0.01, \mathrm{n}=5 ; 10 \mathrm{~min}:+28 \pm 7 \%, \mathrm{p}<0.02, \mathrm{n}=6$. An IPG seems to be involved in the GLP-1 action in normal rat liver and adipose tissue. 


\section{7}

POTENTIATION OF GLP-1 INSULINOTROPIC ACTION BY A NON-GLUCIDIC NUTRIENT IN THE PANCREAS OF NORMAL AND DIABETIC RATS

V. Leclercq-Meyer and W.J. Malaisse. Laboratory of Experimental Medicine, Brussels Free University, Brussels, Belgium.

The glucagon-like peptide 1 (GLP-1), often referred to as a glucosedependent insulinotropic agent, is currently under investigation as a tool in the treatment of non-insulin-dependent diabetes. Since pancreatic islet B-cells often display an impaired sensitivity to glucose in NIDDM, we have investigated whether a non-glucidic nutrient, susceptible to bypass site-specific defects in glucose transport and metabolism, could be used to support the insulinotropic potential of GLP-1 and restore its full efficiency in an animal model of type- 2 diabetes. For this purpose, the pancreas of either control rats or diabetic GK rats was first perfused with a nutrient-free medium and then exposed to the dimethyl ester of succinic acid (SAD, $10 \mathrm{mmol} / \mathrm{l})$. Human GLP-1 (7-36) amide (1.0 nmol/l) was administered for $30 \mathrm{~min}$ under each of these two experimental conditions. In the absence of any exogenous nutrient, GLP-1 failed to affect insulin and glucagon release in control animals, whilst causing a modest stimulation of insulin secretion and inhibition of glucagon output in $\mathrm{GK}$ rats. In the presence of $\mathrm{SAD}$, however, the secretory behaviour of the pancreas was virtually identical in control and GK rats. Thus, in both control and GK rats, GLP-1 dramatically increased insulin output evoked by SAD, whilst failing to affect glucagon output, that was suppressed by the succinic acid ester. It is proposed, therefore, that non-glucidic nutrients, such as SAD, may indeed optimalize the B-cell secretory response to GLP-1 in noninsulin-dependent diabetes.

\section{8}

GLUT-2 IN RAT LIVER. EFFECT OF GLP-1 N DIABETES.

F. Clemente, J. Puente, M.I. López-Delgado, I. Valverde and M.L. Villanueva-Peñacarrillo. Fundación Jiménez Díaz. Madrid. Spain.

It has been reported that, in diabetic states, the glucose transport is altered in several tissues. In the liver of diabetic rats induced by streptozotocin (STZ) treatment in the adult age, it has been documented an increase in the total amount of glucose transporter GLUT-2, which is reverted to normal levels by insulin treatment. In this work, we have explored the effect of GLP-1(736)amide (GLP-1), peptide with insulin-like actions in some extrapancreatic tissues, upon GLUT-2 levels in the liver of two models of diabetic rats, treated with STZ in the adult age (IDDM) or at day 1 of birth (NIDDM). Both groups of diabetic rats received no treatment or either GLP-1 $(0.4$ $\mathrm{nmol} / \mathrm{kg} / \mathrm{h})$ or insulin $(450 \mathrm{mU} / \mathrm{kg} / \mathrm{h})$ through an osmotic pump, during three days; then, rats were killed, and the livers were dissected and stored at -70 ${ }^{\circ} \mathrm{C}$. Liver plasma membranes were isolated and solubilized, and GLUT-2 content was quantified by slot-blot. It was observed that, in both diabetic rat models, GLP-1 or insulin treatment induced a reduction of the glucose plasma levels of $\approx 30 \%$. In IDDM rats, GLUT -2 content was significantly higher than in normal control rats $(126 \pm 11 \%, \mathrm{p}<0.05, \mathrm{n}=3)$; GLP-1 treatment decreased ( $p<0.05$ vs untreated IDDM, $n=3$ ) the GLUT-2 levels down to $92 \pm 9 \%$ of normal control; similar results were observed with insulin ( $97 \pm 11 \%$ of normal control, $n=3$ ). In NIDDM rats, GLUT-2 levels were significantly lower ( $39 \pm 5 \%$ of normal control, $p<0.05, n=3$ ), and the treatment with GLP-1 or insulin induced an increase of GLUT-2 values to $67 \pm 10 \%$ and $66 \pm 9 \%$ of normal control, respectively. In conclusion, the restoration to normal values after GLP-1 treatment in diabetic states of their aitered liver GLUT-2 levels, aclds support to the role of GLP-1 in the hepatic glucose metabolism.

\section{9}

INSULN SAVING BY GLP-I IN FASTING NORMOGLYCAEMIC IDDM

E.J. Freyse, S. Knospe, T. Becher, O. El Hag, B. Göke*and U. Fischer; Institute of Diabetes "Gerhardt Katsch" of the Ernst-Moritz-Arndt-University Greifswald and Clinical Research Unit for Gastrointestinal Endocrinology of the Philipps-University Marburg*

GLP-1 is known to reduce glucagon secretion and meal-related requirement for insulin. To verify the effect of GLP-1 on basal insulin supply in IDDM chronic diabetic dogs $(\mathrm{n}=9)$ were overnight iv treated with glucose-controiled insulin infusion (GCII). Additionally, GLP-1 $\left(10 \mathrm{pmol} \cdot \mathrm{kg}^{-1} \cdot \mathrm{min}^{-1}\right)$ or in control experiments (C) $\mathrm{NaCl}(0.154 \mathrm{~mol} / \mathrm{l})$ were infused for $480 \mathrm{~min}$. Insulin infusion rates were estimated hourly. Hormones, alanine and turnover rates of glucose $\left(6-{ }^{3} \mathrm{H}\right.$-glucose) and alanine ( $\mathrm{U}-{ }^{14} \mathrm{C}$-alanine) were measured in normoglycemic steady state $(390-480 \mathrm{~min})$. In both series of experiments insulin was infused in comparable amounts overnight ( $31.2 \pm 3.6$ vs. C $34.2 \pm$ $4.8 \mathrm{mU} \cdot \mathrm{kg}^{-1} \cdot \mathrm{h}^{-1}$, NS). Plasma GLP-1 increased by the infusion from $3 \pm 1$ to $41 \pm 10$ pmol/l. Plasma insulin (0 min: $108 \pm 6,480 \mathrm{~min}: 121 \pm 14$; C $91 \pm 11,99 \pm$ $13 \mathrm{pmol} / \mathrm{l})$ and pancreatic glucagon $(24 \pm 8,31 \pm 1 \mathrm{i} ; \mathrm{C} 27 \pm 9,21 \pm 5 \mathrm{pmol} / \mathrm{l})$ were not altered and normoglycemia was maintained throughout $(0 \mathrm{~min}$ : $5.1 \pm 0.3$, $480 \mathrm{~min}: 4.8 \pm 0.2 ; \mathrm{C} 4.6 \pm 0.2,4.5 \pm 0.1 \mathrm{mmol} / 1$ ). A significant decline in insulin infusion rate was observed $60 \mathrm{~min}$ after initiation of GLP-1 infusion up to 360 min (minimum rate: $22.2+3.5 \mathrm{mU} \cdot \mathrm{kg}^{-1} \cdot \mathrm{min}^{-1}, \mathrm{p}<0.05$ ). C, however, showed a later and lower decline between 240 and $480 \mathrm{~min}$ (minimum rate: $24.1+2.8$ $\left.\mathrm{mU} \cdot \mathrm{kg}^{-1} \cdot \mathrm{min}^{-1}, \mathrm{p}<0.05\right)$. There was no effect on glucose tumover $(22.4 \pm 4.3$, $\left.\mathrm{C} 16.3 \pm 1.4 \mu \mathrm{mol} \cdot \mathrm{kg}^{-1} \cdot \mathrm{min}^{-1}, \mathrm{NS}\right)$, glucose clearance $(4.56 \pm 0.7 ; \mathrm{C} 3.8 \pm 0.4 \mathrm{ml}$ $\left.\cdot \mathrm{kg}^{-1} \cdot \mathrm{min}^{-1}, \mathrm{NS}\right)$, plasma alanine $(0.34 \pm 0.06 ; \mathrm{C} \quad 0.38 \pm 0.08 \mathrm{mmol} / \mathrm{l}, \mathrm{NS})$ alanine turnover $\left(9.8 \pm 2.6 ; \mathrm{C} 10.1 \pm 1.9 \mu \mathrm{mol} \cdot \mathrm{kg}^{-1} \cdot \mathrm{min}^{-1}\right.$, NS) or glucose production from alamine $\left(2.4 \pm 0.5 ; \mathrm{C} 1.5 \pm 0.4 \mu \mathrm{mol} \cdot \mathrm{kg}^{-1} \cdot \mathrm{min}^{-1}, \mathrm{NS}\right)$. Conclusion: There is no real saving of insulin during GCII with concomitant GLP-I infusion. It is argued that GLP-1 gains therapeutic significance in IDDM.

\section{0}

FAILURe to LoWer 24 H-Glucose Profiles through Normalizing FASTING VALUES BY OVERNIGHT INFUSIONS OF GLP-1 IN NIDDM B. Willms, K. Idowu, J. J. Holst, M. A. Nauck, Fachklinik für Diabetes und Stoffwechselkrankheiten, Bad Lauterberg, FRG, Dep. of Medical Physiology, Panum-Inst., Univ. Copenhagen, DK and University Hospital, Knappschafts-Krankenhaus Bochum. FRG

GLP-1 [7-36 amide] normalizes fasting plasma glucose in NIDDM patients It was the aim to study the effect of overnight intravenous GLP-1 [7-36 amide] on $24 \mathrm{~h}$-glucose profiles after stopping GLP-1. Ten NIDDM patients ( 7 female, 3 male; age $62 \pm 4$ y., BMI $29.6 \pm 3.9 \mathrm{~kg} / \mathrm{m}^{2}$, duration $10 \pm 7 \mathrm{y}$., $\mathrm{HbA}_{16} 10.9 \pm 1.3 \%$ [normal $4.0-6.1 \%$ ]) were studied on two occasions in random order: Either GLP-1 [7.36 amide] (Saxon Biochemicals, Hannover, FRG, 1 pmol $\left.\mathrm{kg}^{-1} \cdot \mathrm{min}^{-1}\right)$ or placebo $(0.9 \% \mathrm{NaCl}$ with $1 \%$ human serum albumin, Behringwerke, Marburg. FRG) were infused intravenously from $22: 00$ to 7:00 o'clock $(9 \mathrm{~h})$. 16-point plasma glucose profiles were then obtained over $24 \mathrm{~h}$ overnight (patients continued their usual oral treatment with glibenclamide [ $n=5]$, metformin [ $n=1]$, or both $[n=4]$ ). GLP-1 [7-36 amide] (plasma concentration $110 \pm 12 \mathrm{pmol} / \mathrm{l}$ ) raised C-peptide $(\mathrm{p}=0.0005)$, suppressed glucagon $(p=0.01)$ and lowered plasma glucose to $5.5 \pm 0.6$ and $6.3 \pm 0.4 \mathrm{mmol} / \mathrm{h}$ at $3: 00$ and $7: 00 \mathrm{a} . \mathrm{m}$. (vs. $10.3 \pm 0.9$ and $11.3 \pm 0.6 \mathrm{mmol} / \mathrm{h}$, $p=0.0003$ and $p<0.0001$, respectively, with placebo). However, starting $1 \mathrm{~h}$ after breakfast $(14.0 \pm 0.8$ vs. $16.1 \pm 0.9 \mathrm{mmol} / \mathrm{l}, \mathrm{p}=0.075)$, there were no major differences in plasma glucose, insulin, C-peptide or glucagon profiles between experiments with GLP-1 [7-36 amide] and placebo. In conclusion, (1) overnight GLP-1 [7-36 amide] normalizes fasting plasma glucose, but (2) has no sustained effect on meal-induced glucose, insulin or glucagon concentrations once its administration has been stopped. (3) Normalization of fasting plasma glucose alone does not improve daytime metabolic control in NIDDM patients on oral agents. 


\section{Gastric Emptying}

591

ERYTHROMYCIN ANTAGONIZES THE INHIBITION OF GASTRIC EMPTYING BY GLP-1 AND UNMASKS ITS POSTPRANDIAL INSULINOTROPIC ACTIONS.

M. A. Nauck, S. Grosser, G. Kemmeries, C. Orskov, J. J. Holst and W. Schmiegel, University Hospital, Knappschafts-Krankenhaus Bochum and Dep. of Medical Physiology, Panum-Inst., Univ. Copenhagen, DK

Glucagon-like peptide 1 [7-36 amide] (GLP-1) is an insulinotropic enteric hormone synthesized in enteroglucagon-producing L-cells in the ileum, colon and rectum. Its function to augment postprandial insulin secretion is difficult to evaluate because GLP-1 also slows gastric emptying. Therefore, in 10 healthy volunteers (age $25 \pm 4$ $\mathrm{y}$, Body-Mass-Index: $\left.24.6 \pm 4.7 \mathrm{~kg} / \mathrm{m}^{2}\right)$, GLP-1 ([7-36 amide]: $0.8 \mathrm{pmol} \cdot \mathrm{kg}^{-1} \cdot \mathrm{min}^{-1}$ or placebo) was infused intravenously (from -30 to $240 \mathrm{~min}$ ) and (at $0 \mathrm{~min}$ ) a liquid test meal (50 g sucrose in $400 \mathrm{ml} 8 \%$ amino acids - Aminosteril Hepa) was adrninistered. On seperate occasions, the prokinetic drugs metoclopramide (10 mg), domperidone ( $10 \mathrm{mg}$ ), cisapride $(10 \mathrm{mg}$, all at $-30 \mathrm{~min}$ by mouth) or erythromycin (200 mg intravenously at 0-15 min) were given in addition to GLP-1. Measurements: Plasma glucose (Beckman glucose-analyser), insulin (IMX insulin, Abbott), C. peptide (ELISA, DRG Marburg) GLP-1, glucagon (RLAs, Abs 89390, 4305). gastric emptying (phenol red - dye-dilution, "double-sampling"). Statistics: repeatedmeasures-ANOVA, Student" t-test. GLP-1 significantly decelerated gastric emptying $(p<0.0001)$. Metoclopramide slightly $(p<0.05$ at $120 \mathrm{~min})$ and erythromycin profoundly ( $p<0.05$ from 60 to $240 \mathrm{~min}$ ) accelerated gastric emptying vs. GLP-1 alone. With GLP-1 and erythromycin, gastric empyting followed the same time course as with placebo. Glucose increments and the stimulation of insulin secretion (insulin and C-peptide: $\mathrm{p}<0.05$ at 30 and $60 \mathrm{~min}$ ) were more pronounced than with GLP-1 alone, but glucose increments were smaller with GLP-1/erythromycin than with placebo, while insulin secretory responses were similar. Conclusions: (1) Erythromycin is the only prokinetic drug that potently counteracts the deceleration of gastric emptying caused by GLP-1 [7-36 amide]. (2) Erythromycin unmasks the insulinotropic action of a pharmacological dose of exogenous GLP-1, when administered together with a liquid mixed meal in healthy volunteers, that otherwise remains hidden by the net inhibitory influence of decelerated gastric emptying on postprandial glucose and insulin responses.

\section{3}

EUGLYCAEMIC HYPERINSULINAEMIA DOES NOT DELAY GASTRIC EMPTYING IN NORMAL SUBJECTS

M.-F. Kong, P.E. Blackshaw, P. King, I.A. Macdonald, A.C. Perkins and R.B.Tattersall, Queen's Medical Centre, Nottingham, U.K.

The rate of gastric emptying is affected by different factors, such as meal composition, blood glucose and levels of various gut hormones. The effect of insulin is uncertain. The present study assessed the effect of insulin on emptying of a solid and liquid meal in normal subjects. Eight non-obese men (age 20-32 years, mean 23.6) were studied on three occasions in a random manner. After an overnight fast the subjects were infused with $0.9 \%$ saline on two occasions (to assess reproducibility of the gastric emptying studies) and with insulin, at $40 \mathrm{mU} / \mathrm{m}^{2} / \mathrm{min}$, on the third occasion with $20 \%$ glucose being infused intravenously to maintain euglycaemia. Steady-state glucose infusion rate was ensured before the subjects ate a meal consisting of a pancake (labelled with $3 \mathrm{MBq}$ non-absorbable ${ }^{99 \mathrm{~m}} \mathrm{Tc}$ ) and milkshake (labelled with $0.33 \mathrm{MBq}$ non-absorbable ${ }^{111}$ In-DTPA), providing $400 \mathrm{kcal}$ (57\% carbohydrate). Gamma-scintigraphic images were then obtained at $20 \mathrm{~min}$ intervals for the next 3-4 hours to quantify gastric emptying. The median lag time for emptying of the solid was 36.5 min during $0.9 \%$ saline infusion and 40.4 min during insulin infusion (difference $3.97 \pm 2.65$, NS). The $t_{50}$ for emptying of the solid was 137.4 min during $0.9 \%$ saline and 152.2 min during insulin infusion (difference $14.7 \pm 6.5, \mathrm{NS}$ ) and for the liquid the $\mathrm{t}_{50}$ was $41.7 \mathrm{~min}$ and $40.8 \mathrm{~min}$ respectively (difference $-0.86 \pm 2.48, \mathrm{NS}$ ). Our data show no effect of insulin on gastric emprying of a solid and liquid meal in normal subjects.
592

INGHBITORY LFHECTS OF HYPERGLYCEMA AND HYPERINSULINEMLA ON

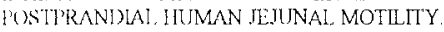

I1 M Bumc K Pluntke R. Amold. B. Göke, J Schirra, and M Katschinski Dept of Gastrointestinal Endocrinolog. University of Marburg. Germany.

This study was undertaken to establish the effects of hyperglycemia on posiprandial jejunal notility and small intestinal transit time, and to establish if the effects of hyperglycemia are mediated in part by coexisting hyperiusulinemia. Methods: 9 healthy male volunteers were studied in random order using three experimental conditions:a) euglycemic clamp (glucose $5 \mathrm{mM}$ ) b) hyperglycemic clamp (glucose $15 \mathrm{mM}$ ) c) euglycemic hyperinsulinemic clamp (insulin infused in stepped doses from $50-150 \mathrm{mU} /\left(\mathrm{m}^{2} \mathrm{~min}\right)$. Postprandial jejunal motility was induced by an intrajejunal perfusion of a lipid solution (Lipofundin MCT $10 \%$ ) $1.5 \mathrm{ml} / \mathrm{min}(1.5 \mathrm{kcal} / \mathrm{min}$ ) infused for $180 \mathrm{~min}$ through the nost proximal port of a manometry catheter $(8$ ports spaced at $2 \mathrm{~cm}-$ intervals) located just distal to the ligament of Treitz. One minute prior to the lipid perfusion $15 \mathrm{~g}$ of lactulose dissolved in $20 \mathrm{ml}$ of tap water was infused. Small intestinal transit time was measured by the hydrogen breath test.

\begin{tabular}{|c|c|c|c|}
\hline Parameter & Euglycemia & Hyperglycemia & Hyperinsulinemia \\
\hline ntestinal transit time (min) & $36.3 \pm 6.5$ & $95.0 \pm 20.5^{*}$ & $70.0 \pm 12.8^{*}$ \\
\hline contractions/180 $\mathrm{min}$ & $292 \mathrm{l} \pm 365$ & $321 \pm 256^{*}$ & $2358 \pm 201 *$ \\
\hline lean amplitude (nunHg) & $23.7 \pm 1.4$ & $21.1 \pm 2.9^{*}$ & $22.7 \pm 1.1$ \\
\hline Iotility index (mmings $180 \mathrm{~mm}$ & 117) $162676 \pm 29303$ & $3105913 \pm 17814^{*}$ & $120578 \pm 13889^{*}$ \\
\hline propagated peaks $180 \mathrm{~min}$ & $11+46 \pm 250$ & $1092 \pm 173^{*}$ & $1187 \pm 133$ \\
\hline propagations over $2 \mathrm{~m}$ & $3+2 \pm 45$ & $281 \pm 37^{*}$ & $297 \pm 27$ \\
\hline opagations over $4-6 \mathrm{~cm}$ & $159 \pm 29$ & $117 \pm 22^{*}$ & $132 \pm 17$ \\
\hline opagations over $8-12$ & $42 \pm 13$ & $26 \pm 7$ & $30 \pm 6$ \\
\hline
\end{tabular}
$\sum$ propagations over $8-12 \mathrm{~cm} \quad 42 \pm 13$ leant SEYY *P<0.05 visuglycamia

Summary: Acute hyperglycemia reduced the total number of contractions, progradely propagated contractions, the motility index, the mean amplitude of contractions, and the contractions propagated for $2 \mathrm{~cm}$ and $4-6 \mathrm{~cm}$. Hyperinsulinemia reduced the total number of contractions and the motility index. Small intestinal transit time was delayed under both conditions. Conclusion: The inhibitory effects of hyperglycemia on jejunal motility and small intestinal transit time may be partially mediated by coexisting hyperinsulinemia.

\section{4}

EXAGGERATED SECRETION OF GLUCAGON-LIKE PEPTIDE-1 (GLP-1) CAN EXPLAIN REACTIVE HYPOGLYCAEMIA.

M. Toft-Nielsen ${ }^{1,2}$, S. Madsbad ${ }^{1}$ and J.J. Holst ${ }^{2} .{ }^{1}$ Dept. of Endocrinology, Hvidovre Hospital and ${ }^{2}$ Dept. of Medical Physiology, the Panum Institute, Denmark.

We previously measured peripheral venous concentrations of GLP- 1 in partially gastrectomised subjects with reactive hypoglycaemia after oral glucose and found 10-fold increased GLP-1 responses compared to matched controls. However, because GLP-1's insulinotropic effect is glucose dependent, it is impossible to bring about hypoglycaemia in normal subjects in the basal state by exogenous GLP-1 regardless of dose, and exaggerated GLP-1 secretion, therefore, was not considered responsible for reactive hypoglycaemia in these patients. To further asses the role of GLP-1 in reactive hypoglycaemia we now infused GLP-1 i.v. (1.5 $\mathrm{pmol} / \mathrm{kgxmin}$ for $60 \mathrm{~min}, 0.75 \mathrm{pmol} / \mathrm{kgxmin}$ for $60 \mathrm{~min}$ and $0.37 \mathrm{pmol} / \mathrm{kgxmin}$ for $60 \mathrm{~min}$ ) into 8 healthy volunteers together with intravenous glucose, attempting to copy the peripheral concentrations of both as measured in the patients. In two series of control experiments glucose ( $25 \mathrm{~g}$ over $30 \mathrm{~min}$ ) was infused alone or together with a similar infusion of human glucose-dependent insulinotropic polypeptide (GIP), the other important incretin hormone. Radioimmunoassays of peripheral hormone concentrations confirmed that the GLP-1 and GIP concentrations were comparable to those of the dumping patients. With glucose alone blood glucose increased from $5.3 \pm 0.1$ to $12.5 \pm 0.6 \mathrm{mmol} / 1$ after $30 \mathrm{~min}$; with GIP from $5.4 \pm 0.1$ to $12.6 \pm 0.7 \mathrm{mmol} / 1$ requiring an additional infusion of 28.0 $\pm 1.2 \mathrm{~g}$ of glucose; and with GLP-1 from $5.4 \pm 0.1$ to $11.6 \pm 0.8 \mathrm{mmol} / \mathrm{l}$ requiring additionally $33.4 \pm 1.3 \mathrm{~g}$ glucose. At $30 \mathrm{~min}$ plasma insulin concentrations were $101 \pm 13$ (glucose alone); $429 \pm 64$ with GIP; and $866 \pm 57 \mathrm{pmol} / 1$ with GLP- 1 . During the subsequent $150 \mathrm{~min}$ period lowest glucose concentrations were 4.5 ( 3.7 - 4.9) (median, range) glucose alone; $3.7(2.6$ - 4.0) with GIP; and $2.4(1.9-2.8)$ mmol/1 with GLP-1. We considered that the exaggerated GLP-1 responses to nutrients (but not GIP-responses alone) in patients with the dumping syndrome can fully account for their high incidence of reactive hypoglycaemia. 


\section{5}

PHYSIOLOGICAL HYPERGL YCAEMIA SLOWS GASTRIC EPAPTYING IN TYPE 1 DIABETES PAELLITUS

E Schvarcz1, M Palmér1, J Ånanze, B Horowitz3, and C Berne4, Dept of Incernal Medicinel and Paediatrics2, Örebro Medical Centre Hospital, Örebro, Sweden, Dept of Fledicine3, University of Adelaide, Royal Adelaide Hospital, Australia, and Dept of Internal Medicine4, University Hospital, Uppsala, Sweden

Marked hyperglycaemia (B-glucose $>15 \mathrm{mmol} / \mathrm{L}$ ) slows gastric emptying in both nornal subjects and patients with type 1 diabetes mell itus. Whether small changes of the blood glucose concentration within the physiological range also affects gastric emptying has not been reported.

The aim of this work was to study if changes in the blood glucose concentration within the normal postprandial range affect gastric emptying in nine C-peptide negative males with type 1 diabetes mellitus. The rate of gastric emptying of a mixed liquid and solid meai was measured by a scintigraphic technique on two occasions at a blood glucose concentration of $4 \mathrm{mmol} / \mathrm{L}$ and $8 \mathrm{mmol} / \mathrm{L}$ respectively. Blood glucose concentrations were stabilized by an insulin glucose clamp.

The intragastric retention of the solid meal component at 100 minutes was $44.2 \pm 4.2 \%$ at $8 \mathrm{mmol} / \mathrm{L}$ vs $35.7 \pm$ $4.2 \%$ at 4 mol $/ L(p=0.002)$ and the $50 \%$ gastric emptying time of the liquid meal was $41.3 \pm 3.4$ ninutes vs 29 . +3.5 minutes $(p=0.002)$ respectively. In conclusion, changes in blood glucose within the physiological range have a major impact on gastric enptying in patients with tyoe 1 diabetes mellitus.

\section{PS 22}

\section{Kinetic Measurements in Vivo}

\section{6}

KINETIC MODELLING OF ALANINE CARBON FLUXES IN EXPERIMENTAL IDDM: EFFECT OF PORTAL VERSUS SYSTEMIC INSULIN SUBSTITUTION U. Fischer, P. Junghans, P. Heinke, E. .J. Freyse, Gerhardt Katsch Institute of Diabetes Karlsburg, Germany

The gluconeogenic efficiency of alanine is increased in IDDM on posthepatic but not on prehepatic insulin administration. To elucidate physiological consequences, 5 chronic experimental IDDM dogs were kept over 1 week on wearable insulin pumps, either via portal or via systemic venous route at random oxder. $\mathrm{U}_{-}{ }^{14} \mathrm{C}$-alanine boluses were injected when the animals were in normoglycaemic steady state, and specific ${ }^{14} \mathrm{C}$ activities of circulating alanine, lactate, and glucose were followed over $4 \mathrm{hrs}$. Also, in liver biopsy samples the concentrations and ${ }^{14} \mathrm{C}$ activities of glycogen and alanine were analysed at the end. Data were subjected to three-step kinetic modelling according to a compartment approach comprising five subsystems: (1) free alanine (+pyruvate), (2) lactate, (3) free glucose, (4) liver glycogen, and (5) amino acids other than alanine + fast-turnover proteins. - Results: In comparison to non-diabetic controls, hepatic insulin:glucagon ratios were reduced, and the sizes of all pools mentioned above were enlarged $(\mathrm{p}<0.05)$ in the diabetic animals on systernic, but not on portal insulin. The fractional rate constants $\mathrm{L}_{\mathrm{ij}}$ of carbon fluxes from alanine to the other compartments and amongst them were $\left(\mathrm{min}^{-1}\right.$, means $+\mathrm{SD},{ }^{*} \mathrm{p}^{<0.005) \text { : }}$

\begin{tabular}{|c|c|c|c|}
\hline & controls & diab./systemic ins. & $a b /$ portal ins. \\
\hline L21alanine > lactate & $0.050 \pm 0.002$ & $0.175 \pm 0.010^{*}$ & $0.081 \pm 0.004^{*}$ \\
\hline L3lalanine $>$ glucose & $0.031 \pm 0.002$ & $0.097 \pm 0.005^{*}$ & $0.058+0.005$ \\
\hline L4lalanine $>$ glycogen & $0.030 \pm 0.003$ & $0.052 \pm 0.031$ & $0.046 \pm 0.055$ \\
\hline L51alanine > amino acids & $0.227 \pm 0.010$ & $0.158 \pm 0.027^{*}$ & $0.234 \pm 0.018$ \\
\hline L43glucose $>$ gjycogen & $0.093 \pm 0.022$ & $0.017 \pm 0.052^{*}$ & $0.724 \pm 0.775$ \\
\hline L34 glycogen > glucose & $0.024+0.002$ & $0.013+0.002^{*}$ & $0.128+0.023^{*}$ \\
\hline
\end{tabular}

Resulting glucose flux rates were validated by comparing them to parallel estimates from $6{ }^{3} \mathrm{H}$-glucose kinetics. - Conclusion: In IDDM on normoglycaemic posthepatic insulin substitution which included normal overall glucose turnover, alanine carbon fluxes are redistributed into gluconeogenesis and indirect glycogen synthesis, but direct glycogen synthesis is reduced, and less glucose is provided from liver glycogen. The effects are restored to near-normal by means of chronic prehepatic insulin administration which may be potentially important in hypoglycaemia prevention.

\section{7}

MEASUREMENT OF $\left[\mathrm{U}^{-13} \mathrm{C}\right]$-LACTATE FORMATION FROM $\left[\mathrm{U}^{13} \mathrm{C}\right]$-GLUCOSE IN NIDDM PATIENTS AND CONTROLS

H.G. Wahl, D. Overkamp, F. Baier, H.-U. Häring and R.-M. Schmülling, Medizinische Universitätsklinik Abt. IV, 72076 Tübingen, Germany

$\left[\mathrm{U}^{13} \mathrm{C}\right]$-glucose can be used in tracer bolus experiments for determination of glucose kinetics. Further refinement of existing models can be achieved by measuring simultaneously ${ }^{13} \mathrm{C}$-lactate kinetics and mass isotopomer analysis of $\left[{ }^{13} \mathrm{C}_{3}\right]$-glucose in addition to $\left[\mathrm{U}-{ }^{13} \mathrm{C}\right]$-glucose and ${ }^{13} \mathrm{CO}_{2}$. We developed an experimental setup in which we are able to measure ${ }^{13} \mathrm{C}$ enrichment in glucose, lactate and carbon dioxide and perform glucose mass isotopomer analysis with high precision. Plasma $\left[\mathrm{U}-{ }^{13} \mathrm{C}\right]$-glucose was determined as bis(butylboronate)-acetate derivative by GC-MS and breath ${ }^{13} \mathrm{CO}_{2}$ by a special modified quadrupole mass spectrometer as reported earlier. Quantification of ${ }^{13} \mathrm{C}$-lactate enrichment was achieved by GC-MS analysis of $100 \mu \mathrm{l}$ plasma in form of the n-propylamide-heptafluorobutyrate derivative, which yields prominent ions at $\mathrm{m} / \mathrm{z} 327(\mathrm{Cl}, \mathrm{C} 2$ and $\mathrm{C} 3$ of lactate) and $\mathrm{m} / \mathrm{z} 241$ (C2 and $\mathrm{C} 3$ of lactate) for the unlabelled lactate. Due to a high signal to noise ratio of 92.6 and low isotopic background for the natural abundance of $U$ ${ }^{13} \mathrm{C}$-lactate at ion $\mathrm{m} / \mathrm{z} 330$ the method shows high precision: for total lactate concentrations between 0.5 and $3.0 \mathrm{mmol} / \mathrm{L}$ and ${ }^{13} \mathrm{C}$-enrichments from 0.04 to $1.19 \mathrm{~mol} \%$ excess the intra-assay coefficient of variation was 0.7 to $2.6 \%$, and 1.3 to $3.6 \%$ for inter-assay. Calibration curves in the same range of ${ }^{13} \mathrm{C}$-enrichment showed correlation coefficients between 0.9961 and 0.9990 . For the clinical study 6 obese NIDDM patients and 6 weight and age matched controls were investigated. After an i.v. bolus of [U $\left.{ }^{15}{ }^{3} \mathrm{C}\right]$-glucose $(7 \mathrm{mg} / \mathrm{kg})$ blood and breath samples were taken at frequent intervals over 180 min. There was a fast increase in lactate enrichment in the first $20 \mathrm{~min}$ in both groups with a maximum ${ }^{13} \mathrm{C}$-enrichment between 0.4 and $0.8 \mathrm{~mol} \%$ excess over basal. The method described here with its high sensitivity and precision is suitable for lactate kinetic measurements in $\left[\mathrm{U}-{ }^{13} \mathrm{C}\right]$-glucose bolus experiments. 


\section{8}

UNDERESTIMATION OF NON-OXIDATIVE GLUCOSE TURNOVER WITH THE CONVENTIONAL TRACER DILUTION TECHNIQUE. O. Hother-Nielsen, J.E. Henriksen, P. Staehr, and H. Beck-Nielsen. Medical Department M, Odense University Hospital, Denmark.

It is now recognized that the conventional tracer dilution technique may underestimate glucose turnover rates during clamp studies, whereas more appropriate rates can be obtained when plasma specific activity is maintained constant. During clamp studies non-oxidative glucose metabolism is calculated as tracer determined total glucose disappearance (Rd) minus glucose oxidation rates. Therefore, when tracer determined $\mathrm{Rd}$ is underestimated, non-oxidative glucose metabolism may also be underestimated. To evaluate the magnitude of such errors we performed paired euglycaemic clamp studies ( $2 \mathrm{~h}$ insulin infusion: $5,10,20,40,80$, and $160 \mathrm{mU} \mathrm{m}^{-2} \mathrm{~min}^{-1}$ ) in 30 normal lean subjects using $3{ }^{-3} \mathrm{H}$-glucose infusion and conventional unlabeled glucose infusates (Cold-GINF) or labeled glucose infusates (Hot-GINF) to maintain constant plasma specific activity. Glucose oxidation rates were determined with indirect calorimetry. Glucose oxidation rates averaged $69 \pm 4,83 \pm 5,107 \pm 9,109 \pm 8,124 \pm 11$, and $122 \pm 6 \mathrm{mg} \mathrm{m}^{-2} \mathrm{~min}^{-1}$ in $5,10,20,40,80$, and $160 \mathrm{mU}$ studies, and non-oxidative glucose metabolism in Cold-GINF vs Hot-GINF studies were $23 \pm 3$ vs $41 \pm 7,32 \pm 3$ vs $50 \pm 12,63 \pm 17$ vs $106 \pm 19,170 \pm 12$ vs $244 \pm 41$ $213 \pm 23$ vs $266 \pm 20$, and $236 \pm 15$ vs $293 \pm 20 \mathrm{mg} \mathrm{m}^{-2} \mathrm{~min}^{-1}, \mathrm{p}<0.05$. Thus, with conventional tracer dilution technique (Cold-GINF) non-oxidative glucose metabolic rates were only $55,63,59,70,80$, and $80 \%$ of the rates calculated in constant specific activity studies (Hot-GINF). In conclusion, due to underestimation of glucose turnover with the conventional tracer dilution technique non-oxidative glucose metabolic rates were markedly underestimated. This error varied from $45 \%$ in low-dose to $20 \%$ in highdose insulin infusion studies.

\section{9}

GLUCOSE RECIRCULATION DETERMINED FROM KINETIC ANALYSIS OF $\mathrm{M}_{+6} \mathrm{AND}_{\mathrm{M}} \mathrm{M}_{+3}$ TRACER ISOTOPOMER ENRICHMENT FOLLOWING A SINGLE BOLUS INJECTION OF U $\left.{ }^{13} \mathrm{C}\right]$ GLUCOSE

D.Overkamp, H.G. Wahl, W. Renn, M. Haap, H.U. Hăring and A.Pickert.

Medizinische Universitätsklinik, Abt. IV, D-72076 Tübingen, Germany

Glucose recirculation via the Cori cycle is thought to contribute to the increase in endogenous glucose production observed in Type II diabetic patients and may thus be part of the pathophysiology leading to a raised glucose concentration. Various methods for determination of glucose recirculation have been presented, based either on the difference in glucose turnover measured with recirculating vs. nonrecirculating tracers or the redistribution of labelled glucose carbon atoms to specific intramolecular localisation. We here present a new method based upon the simultaneous measurement of the $\mathrm{m}_{+6}$ and $\mathrm{m}_{+3}$ isotopomer enrichment of plasma glucose following a single $\mathrm{U}\left[{ }^{13} \mathrm{C}\right]$ glucose bolus injection. 6 obese Type II diabetic patients (mean BMI $28 \mathrm{~kg} / \mathrm{m}^{2}$ ) in poor metabolic control (mean $\mathrm{HbAl}_{\mathrm{c}} 10.5 \%$ ) were studied at their ambient glucose concentration $\left(206 \pm 18 \mathrm{mg} / \mathrm{dl}\right.$ ). 6 obese subjects (mean $\mathrm{HbAl}_{c} 5.6 \%$, BMI $33 \mathrm{~kg} / \mathrm{m}^{2}$ ) served as controls. The timecourse of the $m_{+6}$ and $m_{+3}$ glucose isotopomer enrichment was simultaneously fitted to a six compartmental model, describing the kinetics of glucose and the pathways of glucose recirculation. The proportion of glucose recirculated relative to total endogenous glucose production was $11 \pm 1,6 \%$ (meantSEM) in the obese control subjects, thus comparing favourably with published figures. In the Type II diabetic patients, this proportion was $18 \pm 4,3 \%$, not significantly different from the figure in control subjects. The rate constants describing the efficiency of the metabolic steps from $\mathrm{m}_{+6}$ to $\mathrm{m}_{+3}$ glucose appeared unaltered in the diabetic patients, whereas alternative paths of glucose metabolism (oxidative and / or nonrecirculation nonoxidative paths) were significantly diminished. Thus, a putative increase in glucose recirculation appears to be secondary to a defect in alternative pathways of glucose elimination and not to an increase in the efficiency of glucose recirculation per se.

\section{0}

RQLIVALENT TOLERANCE INDEXES FROM REGULAR AND INSULIN.MODIFIED IVGTT IN NORMAL MEN: USE IN NIDDM G. Pacini G Tonolo. M. Cíccarese, M. Sambataro, E. Brocco, M. Maioli, $\mathrm{R}$ Nosadini $L A D S E B-C N R$ and Institute of Internal Medicine, Padua; Metabolic Disease Unit, Sassari, haly.

Reduced insulin sensitivity (SI), glucose effectiveness (SG), B-cell function (IR) are factors of impaired tolerance (IT) of NIDDM. Minimal model analysis (MM1) of i.x. glucose test (FSIGT) is commonly used to calculate relative contributions to IT. As NIDDM have reduced $\mathrm{B}$-cell response, insulin infusion $(0.3 \mathrm{nmol} / \mathrm{kg}$ from $201025 \mathrm{~min})$ increases circulating insulin, augments glucose dynamics. improves SI precision. Aims of this study: (i) to compare the MM parameters in 10 healthy controls ( $\mathrm{C}, 70 \pm 5 \mathrm{~kg}$ ) obtained with regular (RT) and insulin-modified (IM) FSIGT, since, as far as we know, the two protocols have never been evaluated in the same subjects; (ii) to quantify some factors responsible for IT in 15 NIDDM (D, 86 $\pm 5 \mathrm{~kg})$. In C SI was $1.2 \pm 0.110^{4}$ $\mathrm{min}^{-1} / \mathrm{pM}$ with RT and $1.2 \pm 0.2$ with $\mathrm{MM}$ (range $0.5-6.0, \mathrm{p}>0.7$ ) with strong correlation (slope $=0.96$, intercept $=0.4$ not different from 1 and $0 ; \mathrm{r}=0.87, \mathrm{p}=$ $0.012)$. SG was $25 \pm 410^{3} \mathrm{~min}^{-1}$ and $32 \pm 4, \mathrm{p}=0.06$, well correlated $(\mathrm{r}=0.73$, $\mathrm{p}=0.02$ ). In $\mathrm{D} \mathrm{SG}$ was $19 \pm 3, \mathrm{p}<0.02 ; \mathrm{SI} 0.2 \pm 0.1, \mathrm{p}<0.0001$ vs. IM of $\mathrm{C}$. $30 \%$ of $\mathrm{D}$ had SI $(0.5 \pm 0.1)$ within lower part of normal range and markedly reduced IR (insulin area prior to exogenous input (AUC) $0.78 \pm 0.18 \mathrm{nM} \cdot 15 \mathrm{~min}$ vs. $1.68 \pm 0.18$ of other $D$ and $3.78 \pm 0.54$ of $C, p<0.0004)$. SI and AUC inversely correlated $(r=-0.6, p=0.03$ ) in all $D$. Conclusions: in subjects with normal or elevated endogenous insulin response $\mathrm{RT}$ and IM give equivalent SI SG are similar though slightly underestimated with RT. IM provides a precise approach to identify NIDDM with near-normal SI and severely reduced IR.

\section{1}

THE HOT IVGTT TWO COMPARTMENT MINIMAL MODEL (2CMM): INDICES OF GLUCOSE EFFECTIVENESS (GE) AND INSULIN SENSITIVITY (IS). P. Vicini ${ }^{1}$, A. Caumo ${ }^{2}$ and C. Cobelli ${ }^{1}$. 1Department of Electronics and Informatics, University of Padova, Italy. 2Scientific Institute San Raffaele, Milano, Italy.

A new 2CMM has been proposed (Am. J. Physiol. 264: E829-E841, 1993) which allows the estimation of a physiologically plausible profile of hepatic glucose release (HGR). We show that this model can also measure GE $\left(\mathrm{S}_{\mathrm{G}^{2 *}}, \mathrm{ml}^{-1} \mathrm{~kg}^{-1} \mathrm{~min}^{-1}\right)$ and IS $\left(\mathrm{S}_{\mathrm{I}^{2}}, \mathrm{ml} \mathrm{kg}^{-1} \mathrm{~min}^{-1} / \mu \mathrm{U} \mathrm{ml}^{-1}\right)$, in addition to plasma clearance rate PCR $\left(\mathrm{ml} \mathrm{kg}^{-1} \mathrm{~min}^{-1}\right) . \mathrm{S}_{\mathrm{G}^{2}}{ }^{2 *}$ and $\mathrm{SI}^{2^{*}}$ are different from the one compartment MM (1CMM) indices $\mathrm{S}_{\mathrm{G}^{*}}\left(\mathrm{~min}^{-1}\right)$ and $\mathrm{S}_{\mathrm{I}}^{*}\left(\mathrm{~min}^{-1} / \mu \mathrm{U} \mathrm{ml}^{-1}\right) \cdot\left[6,6^{-2} \mathrm{H}_{2}\right]$ glucose labeled IVGTTwere performed in 14 normals. Results (mean $\pm \mathrm{SEM}$ ): $\mathrm{S}_{\mathrm{G}^{2 *}}=0.85 \pm 0.14 \mathrm{ml} \mathrm{kg}^{-1} \mathrm{~min}^{-1}$, PCR $=2.02 \pm 0.14 \mathrm{ml} \mathrm{kg}^{-1} \mathrm{~min}^{-1}$ and $\mathrm{S}_{\mathrm{I}}{ }^{2 *}=13.83 \pm 2.5410^{-2} \mathrm{ml} \mathrm{kg}^{-1} \mathrm{~min}^{-1 /}$ $\mu \mathrm{U} \mathrm{ml}-1$. Precision was acceptable (mean $21 \%$, range 5-65\%) for PCR and $\mathrm{S}_{\mathrm{G}^{2 *}}$, while for $\mathrm{S}_{\mathrm{I}^{2}}$ it was satisfactory in $11(26 \%, 5-71 \%)$ but not $(>100 \%)$ in 3 subjects. To compare the $1 \mathrm{CMM}$ with the $2 \mathrm{CMM}$ indices, $\mathrm{S}_{\mathrm{G}}{ }^{*}$ and $\mathrm{S}_{\mathrm{I}}{ }^{*}$ have been multiplied by the distribution volume $\mathrm{V}$ of the 1CMM: $\mathrm{S}_{\mathrm{G}}^{*} \mathrm{~V}$ was $1.36 \pm 0.08 \mathrm{ml} \mathrm{min}{ }^{-1} \mathrm{~kg}^{-1}$ and $\mathrm{S}_{\mathrm{I}}^{*} \mathrm{~V}$ was $12.98 \pm 2.21$ $10^{-2} \mathrm{ml} \mathrm{min}{ }^{-1} \mathrm{~kg}^{-1} / \mu \mathrm{U} \mathrm{ml}{ }^{-1} . \mathrm{S}_{\mathrm{G}}{ }^{*} \mathrm{~V}$ was significantly lower $(\mathrm{p}<0.001)$ than PCR and higher $(p<0.001)$ than $\mathrm{S}_{\mathrm{G}}{ }^{2 *}$, and did not correlate with either ( $r=0.45, N S$, and $r=0.50, N S$, respectively), while $S_{I}^{*} V$ was not different and correlated with $\mathrm{SI}^{2 *}(\mathrm{r}=0.95 ; \mathrm{p}<0.001)$. PCR underestimation by $\mathrm{S}_{\mathrm{G}}{ }^{*} \mathrm{~V}$ is due to the single compartment approximation. When $\mathrm{S}_{\mathrm{G}}{ }^{*}$ is multiplied by the total distribution volume, $\mathrm{V}_{\mathrm{D}}$ (from $2 \mathrm{CMM}), \mathrm{S}_{\mathrm{G}}{ }^{*} \mathrm{~V}_{\mathrm{D}}$ is not different and well correlated with PCR ( $\mathrm{r}=0.88$; $\mathrm{p}<0.001)$. However, $\mathrm{S}_{\mathrm{G}}{ }^{*} \mathrm{~V}_{\mathrm{D}}$ is different from $\mathrm{S}_{\mathrm{G}^{2}}{ }^{*}(\mathrm{p}<0.001)$, even if correlation is good $(r=0.83 ; p<0.001)$.In conclusion, the $2 \mathrm{CMM}$, by allowing the estimation from a hot IVGTT of GE, IS and PCR, in addition to HGR, is a powerful tool for the study of glucose metabolism in vivo. 
602

COMPARISON OF GLUCOSE LOADS IN THE FREQUENT SAMPLING INTRA-VENOUS GLUCOSE TOLERANCE TEST IN NORMAL SUBJECTS S. Batty, V.N. Anyaoku, P. Chong, S. Franks, D.G. Johnston and S. Robinson. Unit of Metabolic Medicine, St Mary's Hospital Medical School, Paddington, London W2 IPG.

The glucose load given for the frequent sampling intra-venous glucose tolerance test (FSIVGTT) has been the subject of previous investigation. If the glucose load is too low then the insulin increment is not high enough for satisfactory modelling. However, an excessive glucose load causes unphysiological hyperglycaemia. The intravenous insulin added to the IVGTT increases the sensitivity of the modelling and has reduced the need for a high glucose load Previously assays for insulin have measured all circulating insulins where only a proportion are biologically active. We therefore aimed to compare low $\left(0.15 \mathrm{gm} \cdot \mathrm{kg}^{-1}\right)$ and standard $\left(0.3 \mathrm{gm} \cdot \mathrm{kg}^{-1}\right)$ glucose loads in the IVGTT and model using an assay for specific insulin. A low and normal dose IVGTT with minimal modelling was compared in ten normal subjects. Median and [interquartile range] are stated, Wilcoxon paired rank sum test was used. Glucose at 4 minutes was higher in the standard group (low 13.7[0.7) v standard $9.9[0.5] \mathrm{mmol.} \mathrm{I}^{-1}$, $p<0.05$ ). First phase insulin area was similar in the two groups (low 404[1312] v standard $460[625]$ pmol. $\left.I^{-1} .24 \mathrm{mins}^{-1}\right)$ suggesting maximal insulin stimulation with either Ioad. Glucose tolerance was similar with the two tests (low $1.88[0.58]$ v standard $1.66[1.10] \%$ ). Insulin sensitivity was similar in the two tests (low

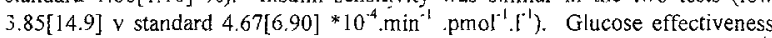
was greater in the low dose test (low $0.029[0.015]$ v standard 0.017 [0.012] $\mathrm{min}^{-1}$, $p<0.02$ ). In conclusion the two glucose loads give similar results. However, glucose effectiveness appears reduced with the higher glucose load possibly because the mechanisms for glucose uptake are saturated by the unphysiological glucose concentrations achieved with $0.3 \mathrm{gm} / \mathrm{kg}$ glucose. This may have consequences for the interpretation of reduced glucose effectiveness in NIDDM.

\section{4}

THE HOMEOSTASIS MODEL (HOMA) IN MEXICAN AMERICANS AND NON-HISPANIC WHITES IN THE SAN ANTONIO HEART STUDY H. Miettinen and S.M. Haffner. The University of Texas Health Science Center at San Antonio, TX. USA.

The homeostasis model (HOMA) has been widely used to assess insulin sensitivity and resistance using only the fasting insulin and glucose but there are few data regarding its use in multiple ethnic groups. We examined the HOMA model including B-cell function (Bcell) and insulin resistance (IR) in relation to fasting insulin (F!) and change in insulin to change in glucose over the first 30 minutes of an oral glucose tolerance test $\left(\Delta \mathrm{l}_{3} / \Delta \mathrm{G}_{30}\right.$ ) in 2735 Mexican Americans (MAs) and non-Hispanic whites (NHWs) from the 1984-88 cross-sectional examination of the San Antonio Heart Study. Spearman correlation between these measures are shown below (all $p<0.001$ )

\begin{tabular}{lcccccc}
\hline & \multicolumn{2}{c}{ MA } & \multicolumn{3}{c}{$\mathrm{NHW}$} \\
\hline & Bcell & $\mathrm{FI}$ & $\Delta \mathrm{Al}_{30} / \Delta \mathrm{G}_{30}$ & Bcell & $\mathrm{FI}$ & $\Delta \mathrm{I}_{30} / \Delta \mathrm{G}_{30}$ \\
\hline HOMA Bcell &.- & .68 & .41 & $\ldots$. & .74 & .33 \\
HOMA IR & .51 & .97 & .16 & .64 & .99 & .21 \\
\hline
\end{tabular}

HOMA IR and fasting insulin are virtually identical while HOMA Bcell and $\Delta l_{30} / \Delta G_{30}$ are significantly correlated. HOMA IR increases with worsening glucose tolerance in both MAs (normal glucose tolerance (NGT): 2.0; impaired glucose tolerance (IGT): 3.6 ; and NIDDM: 7.2 ) and in non-Hispanic whites (NGT: 1.5; IGT: 3.2; and NIDOM: 5.0). HOMA Bcell declines in NIDDM in both Mexican Americans (NGT: 5.2 ; IGT: 5.3 and NIDDM: 4.4) and in non-Hispanic whites (NGT: 4.9; IGT: 5.2 and NIDDM: 4.6). After adjustment for HOMA IR, HOMA Bcell also declined from NGT to IGT. We conclude that the HOMA model may provide a simple measure of insulin sensitivity and resistance that may apply in both low risk populations for NIDDM (NHWs) and high risk populations (MAs).

\section{3}

RELATIONSHIP BETWEEN FASTING PLASMA INSULIN LEVEL AND MINIMAL MODEL-DERIVED $S_{1}$ INDEX IN NON-DIABETIC SUBJECTS, M.R. Letiexhe, A.J. Scheen and P.J. Lefebvre. Division of Diabetes, Nutrition \& Metabolic Disorders, CHU Sart Tilman, B-4000 Liège, Belgium.

In epidemiological studies, fasting hyperinsulinaemia is frequently used as a simple index of insulin resistance. In order to further assess the validity of such approach, fasting plasma insulin level $\left(F_{1}\right)$ was compared to the insulin sensitivity index $S_{1}$ derived from Bergman's minimal model method applied to a frequently-sampled intravenous glucose tolerance test. A total of 108 nondiabetic individuals participated in the study : $29 \mathrm{M}$ and $79 \mathrm{~F}$; age : $33.9 \pm$ 1.2 years (range : $16-65$ ); body mass index - BMI - : $29.0 \pm 0.7 \mathrm{~kg} / \mathrm{m}^{2}$ (range : 17.5-47.8). In this heterogeneous population, $F_{1}$ varied between 1.3 and $67.3 \mathrm{mU} \cdot \mathrm{L}^{-1}$ (mean \pm SEM : $11.1 \pm 0.9$ ) and $S$, varied between 0.3 and $39.310^{-4} \mathrm{~min}^{-1} / \mathrm{mU}^{-L^{-1}}$ (mean \pm SEM : $6.5 \pm 0.6$ ). The population was divided in quartiles $(n=27)$ according to $S$ values $(2.0 \pm 0.1,4.0 \pm 0.1$, $6.5 \pm 0.1$ and $13.6 \pm 1.510^{4} \mathrm{~min}^{-1} / \mathrm{mU} \cdot \mathrm{L}^{-1}$ respectively) and the corresponding $\mathrm{F}_{1}$ levels were calculated $(18.5 \pm 2.0,12.1 \pm 2.3,7.5 \pm 0.8$ and $6.4 \pm 0.8 \mathrm{mU} \cdot \mathrm{L}^{-1}$ respectively). Analysis of the data demonstrated that the inverse relationship between $S_{t}$ and $F_{t}$ is nonlinear and can best be explained by a hyperbolic function. In this population, both $F_{1}$ and $S_{1}$ were highly correlated with BMI $(p<0.001)$, but not with age. When considering a $S_{1}$ value $<410^{-4} \mathrm{~min}^{-1} / \mathrm{mU}^{-\mathrm{L}^{-1}}$ as an index of decreased insulin sensitivity, we found that a $F_{1}$ level $>18 \mathrm{mU} . \mathrm{L}^{-1}$ has a good specificity $(98.5 \%)$, but a poor sensitivity $(29.3 \%)$ as criteria of insulin resistance. $F_{1}$ was $\leq 18$ mU. $\mathrm{L}_{-1}$ in all 58 non-obese (BMI $\leq 28: 23.0 \pm 0.3 \mathrm{~kg} / \mathrm{m}^{2}$ ) subjects (nevertheless 7 had $\mathrm{S}_{1}<4$ ) and in 37 out of the 50 obese (BMI $>28: 35.9 \pm 0.7 \mathrm{~kg} / \mathrm{m}^{2}$ ) subjects (nevertheless 22 had $S_{1}<4$ ). In conclusion, $F_{1}$ is inversely related to $S$, according to an hyperbolic relationship. In absence of diabetes, elevated fasting insulin level ( $\left.>18 \mathrm{mU} \cdot \mathrm{L}^{-1}\right)$ may be considered as a highly specific, but only poorly sensitive, marker of insulin resistance.

\section{5}

COMPARISON OF IN VIVO TESTS FOR GLUCOSE TOLERANCE IN SUBJECTS WITH NORMAL, IMPAIRED GLUCOSE TOLERANCE AND TYPE 2 DIABETES

M.P. Hermans, J. Levy, S. Coppack and R.C. Turner. Diabetes Research Laboratories, Radchiffe Infirmary, Woodstock Rd, Oxford, UK

Standard $120 \mathrm{~min}$ OGTT glucose used for diagnosing givcose tolerance (GT) abnormalities is cumbersome and lacks reproducibility. Fasting plasma glucose (FPG) from arterialised venous blood was recently shown to be as valid in discriminating individuals for GT. We compared the efficiency of FPG against non-OGTT tests to estimate GT in vivo using discriminant ratio (DR, underlying between-subiect $S D$ to withinsubject SD) and rank correlation analysis for duplicate tests to compare their performance in 24 subjects across the range of GT (7 non-diabetic. 8 with impaired GT and 9 NIDDM $)$. The duplicate tests in random order were assessed by: 1) FPG. from single [o mm or 5 min-interval repeat samples ( $\left.F P G_{\text {io }} 5.10 \mathrm{~min}\right)$ taken on arrival $(8.00 \mathrm{am})$ or after $2 \mathrm{~h}$ rest $(10$ samples ${ }_{1120}$ to $\left.\left.185 \mathrm{~mm}\right) ; 2\right) \mathrm{HbA}_{1 c}$; 3) achieved plasma glucose (mean of 3 samples, 5 min interval) after 60 or 120 min glucose infusion (ClGMA) 4) Frequently Sampled intravenous Glucose Tolerance Test (FSIVGTT) with $\operatorname{MinMod}^{\prime \prime}\left(\mathrm{S}_{g}\right)$ and glucose disappearance slope na-ro $\operatorname{rin} ;\left(\mathrm{K}_{g}\right)$. Respective DRs for each test were: $0.60\left[S_{g}\right.$ FSIVGTT]: $0.94\left[\mathrm{~K}_{g}\right.$ IVGTT]; 4.02 [CIGMA $60 \mathrm{~min}$ ]; 4.15 [FPG [0 man]] 4.46 [CIGMA $120 \mathrm{mu}$ ]; 5.28 $\left[\mathrm{HbA}_{10}\right] ; 6.07$ [FPG $[120$ ic $165 \mathrm{~mm}$ ] and 8.02 [mean FPG $10.5,10 \mathrm{~min}$ ]. Spearman Rank correlations between tests were $>0.90$ between FPG and CIGMA measurements. which correlated $0.85-0.90$ with $\mathrm{HBA}_{1 \mathrm{C}}$ and $0.75-0.85$ with $K_{g}$ and $<0.20$ with $S_{g}$. We conciude that the mean from 3 FPG determinations provides a larger DR and is likely to distinguish differences in GT better than (FS)IVGT. CIGMA and $\mathrm{HBA}_{11}$. In conclusion, the highest DR was achieved by the mean of 3 FPG from venous blood taken at $5 \mathrm{~min}$ intervals immediately on admission. This provides a simple routine test for assessing glucose tolerance. 
606

VALID SINGLE-DAY METHOD FOR CALCULATING INSULIN SECRETION IN NORMAL AND NIDDM SUBJECTS.

L.L. Kjems, ${ }^{1}$ E. Christiansen ${ }^{2}$, Aat Volund ${ }^{3}$, and S. Madsbad ${ }^{1}$. Dept. of Endocrinology, Hvidovre Hospital ${ }^{1}$, Rigshospital ${ }^{2}$, and Novo-Nordisk $\mathrm{A} / \mathrm{s}^{3}$, Copenhagen, Denmark

In order to detect and understand the changes in $\beta$-cell function in the pathogenesis of NIDDM, an accurate and precise estimation of pre-hepatic insulin secretion rate (ISR) is essential. There are two common methods to assess ISR: (1) deconvolution method (by Eaton/Polonsky ("E/P"), often considered the "gold standard", is a twoday method which generally requires separate assessment of C-peptide (CPEP) kinetics; (2) combined model (by Volund et al.; "COMB"), a single-day method, that uses insulin and CPEP data from a single test of interest. Given the relative simplicity of the COMB model to assess ISR, we examined its ability to quantify ISR by comparing ISR from COMB test with that obtained by E/P. We also tested the ability of both methods to recover the known ISR from the oral glucose tolerance test (OGTT). Three protocols were performed in NIDDM and normal subjects (N $=8$ for each). (1) assessment of CPEP kinetics, (2) OGTT, and (3) stepwise infusion of CPEP and insulin (molar ratio 2:1) for 210 min, during somatostatin, to simulate secretion profile from the OGTT; this test yields the "known ISR". Both maximal and total ISR from the COMB model was strongly correlated to that obtained by E/P:

\begin{tabular}{l|c|c|c}
\hline \multicolumn{1}{c|}{$n=16$} & Known ISR & Deconvolution & Combined \\
\hline $\begin{array}{l}\text { Maximal ISR } \\
\text { (pmol } / \text { min } / \mathrm{kg})\end{array}$ & $10.6 \pm 1.6$ & $8.9 \pm 1.0$ & $10.0 \pm 1.1$ \\
\hline $\begin{array}{l}\text { Total ISR } \\
\text { (pmol } / \mathrm{kg})\end{array}$ & $1120 \pm 156$ & $84 \% \mathrm{known}$ ISR) & $(94 \%)$ \\
\hline
\end{tabular}

$(r=0.89$ and 0.82 , respectively). Furthermore, ISR from both COMB model and E/P were accurate, i.e. recovery of true ISR was not different from $100 \%$ ( $p>0.05$ for both). These results indicate that the both approaches provide accurate assessment of pre-hepatic insulin secretion rates in NIDDM subjects. Given the simplicity and cost effectiveness of a single-day procedure, combined model method is well suited to assess $\beta$-cell function in pathogenesis studies of diabetes mellitus.

\section{7}

\section{CONTRIBUTION OF GLUCONEOGENESIS TO GLUCOSE PRODUCTION IN FASTING HUMANS}

K. Ekberg', B.R. Landau ${ }^{2}$, V. Chandramouli ${ }^{2}$, C. Schumann ${ }^{2}$, S.C. Kalhan ${ }^{2}$ and J. Wahren ${ }^{1}$. Karolinska Institute ${ }^{x}$, Stockholm and Case Western Reserve University ${ }^{2}$, Cleveland, Ohio.

Methods for the evaluation of gluconeogenesis have been presented, but all involve indirect measurements or several assumptions. We have tried a new approach which allows direct measurement of the proportion of gluconeogenesis in total glucose production by use of ${ }^{2} \mathrm{H}_{2} \mathrm{O}$, at a low dose. The fraction of gluconeogenesis equals the ratio of deuterium $\left({ }^{2} \mathrm{H}\right)$ enrichment in hydrogens at carbon 5 to that at carbon 2 of blood glucose. Eight healthy subjects (mean age 32 yrs and BMI $23.4 \mathrm{~kg} / \mathrm{m}^{2}$ ) ingested ${ }^{2} \mathrm{H}_{2} \mathrm{O}$ to achieve $0.5 \%{ }^{2} \mathrm{H}$ enrichment in body water. After $14 \mathrm{~h}, 22 \mathrm{~h}$ and $42 \mathrm{~h}$ of fasting blood samples were drawn. Urine was collected between $14-18 \mathrm{~h}$ and $38-42 \mathrm{~h}$. Enrichment of ${ }^{2} \mathrm{H}$ in the hydrogens bound to carbon 2 and 5 of blood glucose were determined in hexamethylenetetramine (HMT) using GC/MS. To facilitate transfer of hydrogens bound to carbon 5 and 2 into HMT, glucose was chemically converted to xylose and ribulose-5-P, respectively, and treated with periodine and ammonia. Less than $2 \mathrm{mg}$ of glucose was required for the analysis. To determine deuterium enrichment in body water, enrichment in urine was measured by isotope ratio MS. After $14 \mathrm{~h}$ of fasting gluconeogenesis contributed $47 \pm 4 \%$ and after $22 \mathrm{~h} 67 \pm 4 \%$ of total glucose production. After $42 \mathrm{~h}$ the corresponding value was $93 \pm 2 \%$, in accordance with almost total depletion of hepatic glycogen. Thus, the method allows direct determination of gluconeogenesis in humans at a safe dose of ${ }^{2} \mathrm{H}_{2} \mathrm{O}$.

\section{PS 23}

\section{Hepatic Metabolism}

\section{8}

PROTEIN PHOSPHATASE 2C IS RELATED TO GLYCOGEN SYNTHASE IN LIVER OF NORMAL AND DIABETIC MONKEYS. H.K. Ormeyer and B.C. Hansen. Deparment of Physiology, University of Maryland at Baltimore, Baltimore, MD 21201 USA

The activation of skeletal muscle and adipose tissue glycogen synthase (GS) by in vivo insulin has been shown to be defective in insulin-resistant primates. The defect in insulin activation of GS may be due to a defect(s) in the ability of insulin to dephosphorylate/phosphorylate one or more protein (serine/threonine) phosphatases and kinases, including protein phosphatase (PP) 1, PP2A, PP2C, PP1-G, cAMP-dependent protein kinase and GS kinase3. In order to determine whether PP1, PP1/PP2A or PP2C activities were related to GS activity, these enzymes were measured in liver (freeze-clamped ex situ) of 16 monkeys under basal fasting conditions. The PP activities were measured in "thick" homogenates $(18 \mu \mathrm{L} / \mathrm{mg}$ tissue) and corrected for protein content. Four of the monkeys were normoglycemic and normoinsulinemic, 5 were hyperinsulinemic (fasting plasma insulin $150 \pm 18 \mu \mathrm{U} / \mathrm{ml}$ ) and 7 had type 2 diabetes (fasting plasma glucose $230 \pm 28 \mathrm{mg} / \mathrm{dl}$ ). PP2C (measured in the presence of $1 \mu \mathrm{mol} / 1$ okadaic acid) was positively correlated to the independent activity of $G S(r=0.61, p<0.01)$ and to GS fractional activity $(\mathrm{r}=0.63, \mathrm{p}<0.01$ ). PP2C was inversely related to glucose $6-$ phosphate content $(r=-0.79, \mathrm{p}<0.001)$. PP1/2A activity (measured in the absence of okadaic acid) and PP1 activity (measured in the presence of $1 \mathrm{nmol} / 1$ okadaic acid) were not significantly related to GS activity. The activities of PP2C (range 1.09 to $6.71 \mathrm{nmol} / \mathrm{min} \cdot \mathrm{mg}$ protein) and PP1/2A (range 0.17 to $0.58 \mathrm{mmol} / \mathrm{min} \cdot \mathrm{mg}$ protein) were positively correlated $(\mathrm{r}=0.79, \mathrm{p}<0.001)$. We conclude that protein phosphatase $2 \mathrm{C}$ may be an important regulator of glycogen synthase in liver of rhesus monkeys ranging from normoglycemic to hyperglycemic.
609

MODIFICATIONS OF KREBS CYCLE ACTTVITY AND GLUCONEOGENESIS IN STREPTOZOTOCIN INDUCED DIABETES AND EFFECTS OF METFORMIN ADMINISTRATTON. M. Beylot, M. Odéon and V. Large. INSERM U. 197, Faculté de Médecine R.Laënnec, Lyon, France.

To better define the abnormalities of liver metabolism responsible for the increased gluconeogenesis and glucose production of diabetes and the effects of metformine on these abnormalities we infused (open circuit) livers isolated from starved normal (control, $n=5$ ) and diabetics (streptozotocin, $n=6$ ) rats with lactate $(\mathrm{L}, 1.5 \mathrm{mmol} / \mathrm{l}, 10 \%$ enriched with $\left[3-{ }^{13} \mathrm{C}\right]$ lactate) and pyruvate $(P, 0.2 \mathrm{mmol} / \mathrm{l})$ without or with a simultaneous infusion of metformine. $L+P$ uptake and glucose output were measured. Glutamate was purified from livers freeze-clamped at the end of the experiments; its ${ }^{13} \mathrm{C}$ labelling pattern was determined by mess spectrometry in order to calculate according to Magnusson's model the activities of the main enzymes of Krebs' cycle and of the gluconeogenic pathway expressed relative to citrate synthesis. These relative fluxes were then converted into absolute values using glucose output as a measure of gluconeogenesis. Compared to control livers, livers from diabetic rats had increased $L+P$ uptake $(1373 \pm 71$ vs $972 \pm 119 \mathrm{nmoles} / \mathrm{min} / \mathrm{g}$ of liver, mean $\pm \mathrm{sem}, \mathrm{p}<0.05)$ and glucose production $(704 \pm 49$ vs $368 \pm 24$ nmoles $/ \mathrm{min} / \mathrm{g}$ of liver, $\mathrm{p}<0.01)$; calculated flux through phosphoenolpyruvate carboxykinase (PEPCK) $(2196 \pm 189$ vs $949 \pm 150 \mathrm{nmoles} / \mathrm{min} / \mathrm{g}$ of liver, $\mathrm{p}<0.01)$ was also increased as well as citrate synthesis $(197 \pm 12$ vs $70 \pm 13, p<0.01)$ and fatty acids oxidation $(193 \pm 10$ vs $57 \pm 13, p<0.01)$. Metformine had no significant effects in control livers but decreased in diabetic livers glucose production $(550+97$ vs $704 \pm 49, \mathrm{p}<0.05)$ and the flux through PEPCK $(1091 \pm 200$ vs $2196 \pm 189, p<0.05)$ without modifying $L+P$ uptake (1302 \pm 124 vs $1373 \pm 71$ ). We conclude: 1) in this model of diabetes the increased gluconeogenesis is explained by increased uptake of gluconeogenic substrates and PEPCK activity, 2) metformine decreased glucose production by decreasing PEPCK activity. 
610

OLEIC ACID KINETICS AND KETONE PRODUCTION IN ISOLATED RAT HEPATOCYTES USING A MATHEMATICAL MODEL

A.Valerio, B.Boniollo, K. Thomaseth, A.Avogaro, G.Pacini. Dipartimento di Medicina Clinica e Sperimentale; LADSEB CNR.Padova.

Several methods have been employed to assess ketone body production ( $K B \mathrm{Ra}$ ) either in perfused liver or in isolated hepatocytes. Here we propose a new method for quantifying $K B$ Ra in isolated rat liver cells by using an appropriate mathematical model of their individual concentration inside the hepatocytes after adding increasing amounts of oleic acid in the medium. Hepatocytes from male Wistar rats $(130-250 \mathrm{~g})$ were incubated in the presence of: A) oleate $(0.25 \mathrm{mM})$; B) $(0.5 \mathrm{mM})$; C) (1mM). Aliquots of suspension were obtained at $0,5,7,10,15,20,30,45,60,75$ 90,120 min.Model consists of two compartments :oleate pool, and acetoacetate $(\mathrm{ACAC})+3$-hydroxybutyrate $(3-\mathrm{BOH})$ pooled in one variable. Two processes are accounted for the disappearance of the oleic acid : the conversion to $K B\left(K_{i n}\right)$ and to other pathways (Kout). The ratio AcAc/3-BOH was found to be appropriately described by $\lambda_{1} \exp \left(\lambda_{2}\right.$ t) $+\lambda 3$. Kin was significantly lower $(p<0.05)$ in $C\left(0.0035 \pm 0.0006 \mathrm{~min}^{-1}\right)$ than in $B(0.0089+0.0017)$ and $A(0.0105 \pm 0.0021)$. No differences were observed in Kout among the three studies $\left(0.0121+0.0039 \mathrm{~min}^{-1}\right.$ in $\mathrm{A}$, $0.0224+0.0044$ in $B, 0.0136+0.0017$ in C). The fraction of $K_{\text {in }}$ of the total FFA disappearance was not different in the three groups $(48+15 \%$ in $A, 30+6 \%$ in $B, 20+4$ in $C$, respectively). Similarly $\lambda_{2}$ was similar in the three groups while the sum of $\lambda_{1}+\lambda_{3}$ was significantly higher in $C$ $(0.5352 \pm 0.1844)$ than in $A$ and $B(0.0425 \pm 0.0387,0.1384 \pm 0.0579)$. In conclusion, this mathematical model can reliably assess ketone body metabolism in isolated rat hepatocytes; the fractional conversion of FFA to ketones is reduced in the presence of elevated FFA concentration; the initial ACAC/3-BOH is affected by the initial FFA concentration, while its time course is not.

\section{1}

ENDOTHELIN-1 REDUCES GLUCOKINASE ACTIVITY THROUGH NITRIC OXIDE PATHWAY IN ISOLATED RAT HEPATOCYTES L.D. Monti, G. Valsecchi, P.M. Piatti, S. Costa, C. Stangalini. C. Socci and G. Pozza. IRCCS H. San Raffaele. Milano, Italy.

Glucokinase (GK) plays a key role in hepatocytes as a glucose sensor by controlling the rate of giucose metabolism. Since elevated endothelin-1 (ET-1) leveis have bectn found in diabetes mellitus, we postulated that increased ET-1 levels might cause an impairment of GK activity and evaluated whether ET-1 action on GK activity was dependent through NO reduction, or due to ET-1 activity per se. Hepatocytes were isolated from Sprangue-Dowley rats (approximately $200 \mathrm{gr}$ ) according with Seglen's method. Routinely more than $90 \%$ of the cells excluded Trypan blue. Culture medium was RPMI 1640 supplemented with penicillin and streptomycin. Hepatocytes $\left(10^{6}\right.$ cells/dish $)$ were stimulated for $I$ hour under the following conditions: insulin (1200 pmol//) and glucose (12 mmol/h) (A): insulin, glucose aild ET-1 (2.0 nmol/1) (B); insulin, glucose and L-arginine (1720 $\mu \mathrm{mol} / \mathrm{l})$ to increase NO levels (C): and insulin, glucose. L-arginine and ET-1 (D), At the end of 1 hour period. GK activity was $0.56 \pm 0.07 \mathrm{nmol} / \mu \mathrm{g}$ protein/h during A. The addition of ET1 in B, significantly decreased GK activity by $50 \%(0.29 \pm 0.03 \mathrm{nmol} / \mathrm{Mg}$ protein/h: $p<0.05$ vs A) accompanied by a concomitant $32 \%$ reduction of nitric oxide (NO) levels (106.11 \pm 11.8 vs $156.8 \pm 17.1 \mu \mathrm{mol} / \mathrm{l}: \mathrm{p}<0.005 \mathrm{~B}$ vs A). A highly significan correlation was found between the decrease in $\mathrm{GK}$ activity and NO levels $(\mathrm{R}=0.95$. $p<0.0001)$. L-arginine slightly increased GK activity $(0.67+0.11 \mathrm{nmol} / \mu \mathrm{g}$ protein $/ \mathrm{h}$ $p<0.05$ vs $B$ ) and NO levels $(247.9 \pm 3.4 \mu$ molil; $p<0.01$ vs $A$ and $B)$. During D. Larginine was able to counteract the decrease of NO levels in the presence of ET-I $(257.2 \pm 10.0 \mu \mathrm{mol} / \mathrm{l} ; \mathrm{p}<0.01$ vs $\mathrm{A}$ and $\mathrm{B}$ : NS vs $\mathrm{C})$. Under this condition, no decrement of GK activity was observed $(0.68 \pm 0.08 \mathrm{nmol} / \mu \mathrm{g}$ protein $/ \mathrm{h}$ : $\mathrm{p}<0.05 \mathrm{vs} \mathrm{B}$; NS vs C). In conclusion. ET-I reduction of hepatic GK activity is mediated hy concomitant decrease of NO levels. L-arginine, mainlaining stable NO levels. is able 10 counteract ET-1 effects on hepatic GK.

\section{2}

TRANSLOCATION OF GLUCOKINASE BETWEEN NUCLEUS AND CYTOPLASM IN PRIMARY CULTURES OF RAT HEPATOCYTES

Y.Toyoda, Y.Ito, and I. Miwa, Department of Pathobiochemistry, Faculty of Pharmacy, Meijo University, Nagoya, Japan

Glucokinase (GK) is considered to play a key role in hepatic glucose metabolism. We have previously shown that GK is present predominantly in the nucleus of hepatocytes of adult rats and is translocated from the nucleus to the cytoplasm when liver is perfused with a high concentration of glucose. In the present immunohistochemical study, we have investigated the effects of various concentrations of glucose and fructose on the subcelluar distribution of GK in cultured hepatocytes. Rat hepatocytes wcre cultured in MEM medium containing $5 \%$ neonatal calf serum. After incubation of the hepatocytes with various sugars, the cells were fixed with $4 \%$ paraformaldehyde and stained by the $A B C$ peroxidase procedure. In hepalocytes cultured in $5 \mathrm{mM}$ glucose, GK was detected almost exclusively in the nucleus. Incubation with glucose $(10-40 \mathrm{mM})$ for 30 min caused a marked decrease in nuclear GK and an increase in cytoplasmic GK. Within 30 min after the return of the hepatocyles to 5 $\mathrm{mM}$ glucose, GK was again observed in the nucleus. Addition of fructose $(0.1-1 \mathrm{mM})$, which stimulates the phosphorylation of glucose, to a medium containing $5 \mathrm{mM}$ glucose also caused the translocation of GK from the nucleus to the cytoplasm. In contrast, no change took place when the cells werc incubated with $20 \mathrm{mM}$ L-glucose, 2-deoxyglucose, or 3-O-methylglucose. These results clearly indicate that glucokinase is translocated between the nucleus and the cytoplasm in response to external stimuli, and support the ideas that GK is localized in the nucleus under metabolically static conditions and metabolic conditions involving an increase in glucose metabolism lead to transiocation of GK from the nucleus to the cytoplasm. 


\section{Muscle Metabolism}

\section{3}

FUEL SUPPLY AND ENERGY EXPENDITURE ALTER MALONYL-COA LEVELS IN SKELETAL MUSCLE BY DIFFERENT MECHANISMS.

D. Vavvas, A.K. Saha, A. Apazidis, T.G. Kurowski, L.A. Witters, E. Shafrir and N.B. Ruderman. Boston University Medical School, Boston, MA., 02118, U.S.A.

The concentration of malonyl-CoA, a regulator of cannitine palmitoyl transferase I and thus of fatty acid oxidation, changes acutely in skeletal muscle in response to alterations in its fuel supply or energy expenditure. This study was designed to elucidate the responsible mechanisms. As previously reported, incubation of rat soleus muscles with $5.5 \mathrm{mM}$ glucose and $10 \mathrm{mU} / \mathrm{ml}$ insulin for $20^{\prime}$ increases the concentration of malonyl-COA by 4 fold $(0<0.05)$. This increase in malonyl-CoA was paralleled by increases in the levels of citrate $(150 \%, \mathrm{p}<0.05)$ and of malate $(200 \%, p<0.05)$, an antiporter for citrate efflux from the mitochondria. In contrast the stable activity of acetyl-CoA carboxylase, the ultimate enzyme in the malonylCoA synthetic pathway, was unchanged ( $644 \pm 61$ vs $641 \pm 90 \mathrm{pmole} / \mathrm{min} / \mathrm{mg}$ ) Incubion with $10 \mathrm{mM}$ OH-Citrate, an inhibitor of ATP citrate lyase, blocked the increase in malonyl-CoA by $80 \%$, suggesting that citrate is a major precursor of the cytosolic acetyl-COA from which malonyl-CoA is synthesized. Incubations with the ketone body, acetoacetate, caused similar increases of malonyl-CoA. Thus the increase in malonyl-CoA produced by glucose and acetoacetate can explain, at leas in part, how these fuels diminish the oxidation of fatty acids. In contrast to the changes produced by glucose and acetoacetate, muscle contractions induced by electrical stimulation (tetanic) of the sciatic nerve for 5 minutes, diminished malonyl-CoA levels by $50 \%(\mathrm{p}<0.05)$ and were associated with a $90 \%(\mathrm{p}<0.05)$ decrease in acetyl-CoA carboxylase activity. This could be attributed to observed changes in enzyme phosphorylation state. Conversely, denervation (inactivity) of skeletal muscle, caused by sectioning of the sciatic nerve, increased malonyl-COA levels by two-fold $(\mathrm{p}<0.05)$ with a concomitant increase in the activity of acetylCo A carboxylase $(175 \%, 0<0.05)$. The results suggest that changes in fuel supply ilter manyl-CoA levels in skeletal muscle by affecting the concentration of citrate and malate. In contrast, contractions and inactivity act by altering the intrinsic activity of acetyl-COA carboxylase.

\section{5}

INHIBITION OF GUANYLATE CYCLASE BY LY 83583 DECREASES INSULIN-MEDIATED GLUCOSE UPTAKE IN SOLEUS MUSCLES IN VITRO

J. Jensen*, M.E. Young, G.K. Radda and B. Leighton

Department of Biochemistry, University of Oxford, Oxford, OX1 3QU, U.K. Department of Anatomy, University of Oslo, Norway

Glucose transport and metabolism can be stimulated in skeletal muscle by two different mechanisms, namely by insulin and contraction. Less is known about how the contraction-mediated pathway stimulates glucose utilisation. Contraction of skeletal muscle leads to the formation of nitric oxide which activates guanylate cyclase and increases the formation of cyclicGMP. It is not known whether insulin affects cyclicGMP levels. If cyclicGMP is involved in stimulation of glucose utilisation in muscle then inhibition of guanylate cyclase should affect rates of glucose metabolism. We incubated rat soleus muscle preparations with insulin (at a sub-maximalstimulating concentration, $100 \mu \mathrm{Units} / \mathrm{mI}$ ) and the guanylate cyclase inhibitor $L Y 83583(10-\mu \mathrm{M})$ and measured the effects this had on the rate of glucose utilisation.

net lactate release ${ }^{14} \mathrm{C}$ lactate release glycogen synthesis

$$
\begin{array}{ll}
\frac{\text { Insulin }}{9.78 \pm 0.68(6)} & \frac{\text { Insulin }+ \text { LY83583 }}{5.38 \pm 0.51(6)^{* .4}} \\
9.37 \pm 1.34(6) & 3.60 \pm 0.41(6)^{m *} \\
5.99 \pm 0.66(6) & 1.54 \pm 0.33(6)^{\cdots *}
\end{array}
$$

Thus, LY 83583 markedly inhibited the insulin-mediated rates of net lactate and ${ }^{14} \mathrm{C}$-lactate release (which indicates glucose transport) and glycogen synthesis which suggests that inhibition of guanylate cyclase, and therefore a likely decrease in cyclicGMP content, has dramatically interfered with a specific mechanism for increasing glucose utilisation in skeletal muscle.
614

THE cyclicGMP PHOSPHODIESTERASE INHIBITOR ZAPRINAST STIMULATES GLUCOSE UTILISATTON IN SKELETAL MUSCLE IN VITRO

M.E. Young, G.K. Radda and B. Leighton

Department of Biochemistry, University of Oxford, Oxford, OXI3QU, U.K.

We hypothesise that in skeletal muscle nitric oxide increases cyclicGMP content by stimulating guanylate cyclase activity and this could be a new mechanism for increasing glucose utilisation. CyclicGMP is normally rapidly hydrolysed by cyclicGMP phosphodiesterases. We tested our hypothesis by treatment of rat soleus muscle preparations in vitro with the cyclicGMP phosphodiesterase inhibitor zaprinast $(27-\mu \mathrm{M})$ and we determined the effects zaprinast had on cyclicGMP content and rates of glucose utilisation. CyclicGMP content was significantly $(\mathrm{P}<0.001)$ increased by $27-\mu \mathrm{M}$ (2-fold) and $100-\mu \mathrm{M}(2.5$-fold) zaprinast. In the presence of a basal concentration of insulin (i.e. $10 \mu \mathrm{Units} / \mathrm{ml}$ ) zaprinast significantly stimulated all aspects of glucose utilisation: ${ }^{14} \mathrm{C}$-lâctate release, $(4.34 \pm 0.49$ [control] versus $6.73 \pm 0.14$ [zaprinast] $\mu \mathrm{mol} / \mathrm{h} / \mathrm{g}$ wet wt; $\mathrm{P}<0.01)$; net lactate release, $(6.25$ \pm 0.54 versus $8.23 \pm 0.52 \mu \mathrm{mol} / \mathrm{h} / \mathrm{g} ; \mathrm{P}<0.05$ ); glucose oxidation, ( $0.30 \pm 0.05$ versus $0.60 \pm 0.14 \mu \mathrm{mol} / \mathrm{h} / \mathrm{g} ; \mathrm{P}<0.05$ ); glycogen synthesis, $(2.00 \pm 0.36$ versus $3.05 \pm 0.38 \mu \mathrm{mol} / \mathrm{h} / \mathrm{g} ; \mathrm{P}<0.05)$. These results clearly show that zaprinast, at $27-\mu \mathrm{M}$, significantly increased cyclicGMP content and increased the rates of glucose conversion to lactate and glycogen and glucose oxidation. Our results suggest that a cyclicGMP mechanism, activated by nitric oxide, does exists in skeletal muscle as a novel mechanism for increasing glucose utilisation as might occur during muscle contraction.

\section{6}

ANTIHYPERTENSIVE AGENT MOXONIDINE ENHANCES GLUCOSE TRANSPORT IN INSULIN-RESISTANT MUSCLE. D.L. Fogt, E.J. Henriksen, S. Jacob, D. Dal Ponte and J. Gödicke. University of Arizona, Tucson, AZ U.S.A.; Max-Grundig-Klinik, Bühlerhöhe, Germany; and Beiersdorf-Lilly, Hamburg, Germany

The antihypertensive agent moxonidine (MOX), a centrally-acting selective $\mathrm{I}_{1}$-imidazoline receptor modulator (putative agonist) that lowers sympathetic outflow, may be beneficial in hypertensive patients with insulin resistance. In the present study, the effects of chronic in vivo MOX treatment of obese Zucker (falfa) rats -- a model of severe glucose intolerance, hyperinsulinemia and insulin resistance, and dyslipidemia -- on whole body glucose tolerance, plasma lipids, and insulin-stimulated skeletal muscle glucose transport activity (2deoxyglucose uptake) were investigated. MOX was administered orally $(10 \mathrm{mg} / \mathrm{kg}$ body weight) for 21 consecutive days. Final body weight was $17 \%$ lower in the MOX-treated animals relative to the controls. The MOX-treated obese animals had significantly lower fasting plasma levels of insulin $(-19 \%)$ and free fatty acids $(-28 \%)$, whereas plasma glucose was not significantly altered. During a 1 $\mathrm{g} / \mathrm{kg}$ body weight oral glucose tolerance test, the glucose response (area under the curve) was 67\% lower in the MOX-treated obese group, while the insulin response was not significantly altered. Moreover, glucose transport activity in the isolated epitrochlearis muscle stimulated by a maximally-effective insulin dose $(13.3 \mathrm{nM})$ was $70 \%$ greater in the MOX-treated group (all effects $\mathrm{P}<0.05$ ). In conclusion, these findings indicate that in the severely insulinresistant and dyslipidemic obese Zucker rat, chronic in vivo treatment with MOX can significantly improve whole body glucose tolerance, possibly as a result of the enhanced insulin-stimulated skeletal muscle glucose transport activity and reduced circulating free fatty acids. 


\section{Adipose Tissue and Brain Metabolism}

617

INSULIN-LIKE EFFECTS OF PEROXOVANADATE ON LIPOLYSIS AND GLUCOSE UPTAKE IN ADIPOCYTES FROM NIDDM PATIENTS

Z.W. Yu. P.-A. Jansson and J. W. Eriksson. The Lundberg Laboratory for Diabetes Research, Department of Medicine, University of Göteborg, Sahlgrenska University Hospital, S-413 45 Göteborg, Sweden

Vanadate and peroxovanadate are powerful phosphotyrosine phosphatase inhibitors and may be promising agents for the treatment of diabetes. The aim of this study was to explore the effects of a stable peroxovanadate compound (bpV(pic)) as well as that of insulin on lipolysis and glucose uptake in adipocytes from well-controlled NIDDM patients. Needle biopsies of abdominal subcutaneous fat tissue were performed in 10 male NIDDM patients (HbAlc 6.9 $\pm 0.4 \%$ ) and 10 tnale non-diabetic control subjects matched for age ( $52 \pm 2$ vs $50 \pm 2 \mathrm{yrs}$ ) and BMI $\left(27.5 \pm 1.2\right.$ vs $\left.27.5+1.1 \mathrm{~kg} / \mathrm{m}^{2}\right)$. Incubation of cells with either a beta-adrenergic receptor agonist isoprenaline $(0.01-1 \mu \mathrm{M})$ or the cAMP analogue 8-bromo-cAMP $(0.5-10 \mathrm{mM})$ at $37^{\circ} \mathrm{C}$ for $1 \mathrm{~h}$ dose-dependently enhanced lipolysis assessed as glycerol release from the adipocytes, but the maximal stimulation was reduced by $-40 \%$ in NIDDM compared to control $(p<0.05)$. Similar to insulin, bpV(pic) exerted a clear antilipolytic effect in the presence of 8-bromo-cAMP in both groups $\left(-50 \%\right.$ inhibition). Basal ${ }^{1+C}$-U-glucose clearance was not differen between the two groups ( $12.2 \pm 2.1$ vs $12.3 \pm 2.5 \mathrm{fl} / \mathrm{cell} / \mathrm{s}$ for NIDDM vs control) whereas maximal insulin-stimulated glucose uptake was decreased in NIDDM (2.2 told vs 3.4 -fold, $p<0.05$ ). bpV(pic) increased giucose uptake both in control and NIDDM subjects, but this effect was also impaired in NIDDM subjects (1.6-fold vs 2.4-fold, $\mathrm{p}<0.05)$. In addition, low concentrations of $\mathrm{bpV}(\mathrm{pic})(0.005-0.05$ $\mathrm{mM}$ ) did not influence insulin-stimulated glucose uptake, although tyrosine phosphorylation of the insulin receptor beta-subunit was clearly increased by opV(pic). Conclusions: 1) Beta-adrenergic stimulation of lipolysis is biunted in subcutaneous adipocytes in NIDDM due to post-receptor mechanisms. 2) The stimulating effect of insulin on glucose uptake but not the antilipolytic effect is impaired in NIDDM. 3) Tyrosine phosphatase inhibition with peroxovanadate leads to insulin-like effects both in NIDDM and control adipocytes and the effect on glucose uptake is impaired in NIDDM. Peroxovanadate does not improve insulin sensitivity, suggesting that insulin-resistant glucose uptake in NIDDM is not merely caused by phosphotyrosine-related mechanisms.

\section{8}

HYPERINSULINEMIA ENHANCES LIPOPROTEIN LIPASE ACTIVITY AND MRNA EXPRESSION IN BOTH ADIPOSE TISSUE AND MAMMARY GLAND OF PREGNANT RATS.

A. Martin-Hidalgo, P. Ramos and E. Herrera.

Dpto. de Investigación, Hospital Ramón y Cajal, and Universidad San Pahlo-CEU. Madrid (Spain).

Lipoprotein lipase (LPL) catalyses the hydrolysis and tissue uptake of circulating triglycerides, and during late gestation its activity is decreased in white adipose tissue (WAT) and enhanced in mammary gland (MG). Sustained hyperinsulinemia caused by prolonged glucose infusion is known to revert the insulin resistance normally present during late pregnancy (Ramos \& Herrera, Am. J. Physiol. 269, E858E863, 1995). To determine whether this experimental condition modify LPL activity and mRNA in those tissues, 17-day pregnant (P) and virgin (V) rats were subjected to a continuous i.v. infusion with $50 \%$ glucose ( $35 \mathrm{ml} /$ day) or medium for $72 \mathrm{~h}$. The glucose infusion did not modify glucose levels but enhanced insulin levels more in P than in V rats. LPL activity and mRNA in WAT were lower in $P$ than in $V$ receiving the medium but increased in both groups after the glucose infusion, the effect being greater in $P$ than in V. In MG, LPL activity and mRNA were higher in $P$ than in $V$ receiving the medium, and they increased similarly in both groups after the glucose infusion, values remaining higher in P than in V. Conclussions: 1/ During late gestation, whereas LPL activity and mRNA in WAT are decreased as consequence of the insulin resistant condition, MG LPL activity and mRNA remain sensitive to insulin, and 2/ Parallel changes in LPL activity and mRNA indicate that long-term (e.g.-transcriptional) regulation of the LPL gene expression is an important mechanism for the control of triglycerides uptake in WAT and MG during late gestation.

\section{9}

\section{ROLE OF CALCIUM IN BRAIN NEUROTRANSMISSION IN STREPTOZOTOCIN - INDUCED DIABETES}

T. Kuchmerovskaya, G. Donchenko, I. Obrosova, A. Klimenko N. Kuchmerovsky and A. Yefimov. Institute of Biochemistry, Kiev, Ukraine

The aim of this study was to assess the role of $\mathrm{Ca}^{2+}$ in brain neurotransmission in diabetes. We have studied the ${ }^{45} \mathrm{Ca}$ uptake into brain cortex synaptosomes of 3 rat groups: control and two groups of rats 30 days after induction of diabetes by streptozotocin $(70 \mathrm{mg} / \mathrm{kg}$ of body weight), one treated by nicotnamide ( $N A m, 200 \mathrm{mg} / \mathrm{kg}$ daily, 14 days) the other untreated. The rats with blood glucose level over 300 $\mathrm{mg} / \mathrm{dl}$ were taken into experiment. The finding showed that $45 \mathrm{Ca}$ uptake by brain cortex synaptosomes was elevated by $36 \%$ in diabetic rats as compared to control. Synaptosomes treatment by $10^{-}$ $3 \mathrm{M}$ monoiodacetate ( MIA, inhibitor of glycolysis) has been found to decrease the calcium uptake by $52 \%$ in control. Under the same conditions the ability of MIA to decrease of ${ }^{45} \mathrm{Ca}$ uptake was negligible in diabetes. It was concluded that glycolysis energy had no marked significance for the calcium permeability into synaptosomes. The NAm administration partial restored synaptosomal $45 \mathrm{Ca}$ uptake in diabetic rats. Recently we reported that serotonin and GABA release from the neuro-transmitters pre-loaded synaptosomes showed a significant elevation. Evidently the elevated uptake of $\mathrm{Ca}^{2+}$ was connected with these processes. These data suggested that NAm was an importent factor in the control of $\mathrm{Ca}^{2+}$ homeostasis. Thus pool of $\mathrm{Ca}^{2+}$ contained within the synaptosomes played a significant role in the mechanism of neurotransmitters release and was controlled by the level of synaptosomal metabolism in diabetes. 


\section{Regulation of Insulin Secretion}

620

THE PRO-REGION OF PC2 RESTRICTS MATURATION TO ACIDIC $\mathrm{PH}$

K.T. Scougall, N.A. Taylor, K. Docherty and K.I.J. Shennan. Department of Molecular and Cell Biology, University of Aberdeen, Aberdeen, U.K

The conversion of proinsulin to insulin is performed by the prohormone convertases PC2 and PC3. Proinsulin is initially cleaved by PC3 at the Bchain/C-peptide junction and subsequently by $\mathrm{PC} 2$ at the $\mathrm{C}$-peptide/A-chain junction to leave mature insulin and C-peptide. Defects in this processing pathway can lead to elevated levels of proinsulin secretion and impaired glucose tolerance. PC2 and PC3 are synthesised as inactive zymogens and their maturation is autocatalytic, occurring during transit through the secretory pathway. Pro-PC2 maturation is thought to occur in a later, more acidic part of the secretory pathway than pro-PC 3 and may be regulated by the neuroendocrine protein $7 \mathrm{~B} 2$. In this study the molecular basis for the difference in maturation profiles was examined by performing domain swap experiments. The pro-region of $\mathrm{PC} 3$ was replaced with that of $\mathrm{PC} 2$, retaining the normal pro-PC3 cleavage sites. When this mutant (PC3M9) was expressed in an in vitro translation/translocation system maturation was found to be calcium dependent (optimal at $10 \mathrm{mM}$ ) and occurred optimally at $\mathrm{pH} 5.5$. These conditions are similar to the conditions required for pro-PC2 maturation and different to the conditions required for wild-type pro-PC3 maturation (calcium independent, optimal at $\mathrm{pH} 7$ ). Maturation of pro- $\mathrm{PC} 2$ is inhibited by co-expression with $7 \mathrm{~B} 2$ in the in vitro translation system while pro-PC3 maturation is unaffected. This study revealed that maturation of the domain swap mutant, PC3M9, was also unaffected by co-expression with 7B2. Results suggest that the pro-domain of $\mathrm{PC} 2$ is important for restricting maturation of pro-PC2 to a late compartment of the secretory pathway and that the interaction of pro- $\mathrm{PC} 2$ with the molecular chaperone $7 \mathrm{~B} 2$ is not dependent on the pro domain alone.

\section{1}

INVESTIGATION OF THE EFFECTS OF A PERIPHERAL-TYPE BENZODIAZEPINE RECEPTOR LIGAND ON INSULIN SECRETION

P. Petit, D. Pujalte, S. Claevsen, J. Chapal, D. Hillaire-Buys and MM. Loubatières-Mariani. Laboratoire de Pharmacologie (Unité de Recherche EA 1677), Faculté de Médecine, Boulevard Henri IV, 34060 Montpellier Cedex. France.

We have recently shown that benzodiazepines with high affinity for peripheral-type receptors, such as 4'-chlordiazepam, inhibit insulin secretion in vitro. PK 11195 [1-(2-chlorophenyl)-N-methyl-N-(1methylpropyl)-3-isoquinoline-carboxamide], a potent and selective ligand for peripheral benzodiazepine binding sites, was also shown to inhibit insulin release from rat pancreatic islets. Both substances have been reported to interact with mitochondrial binding sites. Hence, the present study was performed to investigate the effects of PK 11195 on insulin secretion induced by either a metabolic or a non metabolic stimulus. In the rat isolated pancreas perfused at a constant pressure with a Krebsbicarbonate buffer containing $8.3 \mathrm{mmol} / \mathrm{l}$ glucose, PK $11195\left(10^{-6}\right.$ and $10^{-5}$ $\mathrm{mol} / \mathrm{l})$ induced a progressive and concentration-dependent decrease in insulin secretion $(p<0.01)$. Simultaneously, we recorded the effects on the pancreatic flow rate; in contrast to 4'-chlordiazepam, previously shown to induce vasodilation in the same preparation, PK 11195 was ineffective. The differential effects of these two substances on vascular resistance and insulin secretion may suggest the existence of different subtypes of peripheral benzodiazepine receptors on pancreatic B cells and vessels. A metabolic stimulation of insulin secretion induced by a glucose increment from 4.2 to $8.3 \mathrm{mmol} / \mathrm{l}$ could be reduced by $10^{-5} \mathrm{~mol} / 1$ PK $11195(\mathrm{p}<0.02)$. In contrast, the drug had no significant effect on the insulin secretion induced by $5 \mathrm{mmol} / \mathrm{KCl}$ in the presence of a non stimulating glucose concentration $(4.2 \mathrm{mmol} / \mathrm{l})$. These results suggest that PK 11195 inhibits insulin secretion by interfering with glucose metabolism.

\section{3}

DISSOCIATED INSULINOTROPIC SENSITIVITY TO GLUCOSE AND CARBACHOL INDUCED BY HIGH-FAT DIET IN C57/BL/6J MICE E. Simonsson, H. Mulder, U. Myrsén, F. Sundler and B. Ahrén. Departments of Medicine, Malmö and Physiology and Neuroscience, Lund, Lund Univ, Sweden.

To study mechanisms underlying islet adaptation to reduced insulin sensitivity, we examined mice of the $\mathrm{C} 57 / \mathrm{BL} / 6 \mathrm{~J}$ strain, the genotype of which carries an increased propensity to develop glucose intolerance when metabolically challenged. The mice were given either a high-fat diet ( $58 \%$ fat on an energy basis) or a controi diet ( $11 \%$ fat). After 12 weeks of treatment, body weight had increased more in mice given high-fat diet (to $25.8 \pm 0.4 \mathrm{~g}$ ) than in controls (to $21.3 \pm 0.2 \mathrm{~g}$; $\mathrm{p}<0.001$ ). Plasma glucose levels in high-fat diet-treated mice were $7.5 \pm 0.1 \mathrm{mM}$ versus $6.5 \pm 0.1$ in controls $(p<0.001$ ), while plasma insulin levels were $248 \pm 17 \mathrm{pM}$ versus $104 \pm 69 \mathrm{pM}$ $(p<0.001)$, indicative of insulin resistance. After 4,8 and 12 weeks of treatment glucose $(2.8,8.3$ or $16.7 \mathrm{mmol} / \mathrm{kg}$ ) or the cholinergic agonist carbachol (30 or 100 $\mu \mathrm{mol} / \mathrm{kg}$ ) was injected intraperitoneally. The insulinotropic response to glucose was not different between the two groups after 4 or 8 weeks, whereas after 12 weeks, glucose-induced insulin secretion was markedly impaired in high-fat diet-treated mice $(p<0.001)$. Also insulin secretion from incubated islets was impaired at glucose levels at $8.3,11.1$ and $16.7 \mathrm{mM}(\mathrm{p}<0.05)$. In contrast, after 8 and 12 weeks of treatment with high-fat diet, carbachol-stimulated insulin secretion was potentiated $(p<0.01)$, whereas carbachol-stimulated glucagon secretion was not significantly altered. Islet morphology and islet innervation, as examined by immunocytochemistry, were unaffected by treatment with high-fat diet. However, insulin gene expression, as determined by quantitative in situ hybridisation, was increased 3.5 fold by high-fat diet treatment $(\mathrm{p}<0.001)$. We conclude that, concomitantly with increased gene expression, the insulin response to glucose is impaired but that to cholinergic activation is augmented, when insulin resistance occurs in $\mathrm{C} 57 / \mathrm{BL} / 6 \mathrm{~J}$ mice. This suggests that the increased insulin demand in insulin resistance is compensated by enhanced islet sensitivity to cholinergic activation and exaggerated insulin gene expression. 
624

B-CELL HIPERSENSITIVITY TO GLUCOSE IN PARTIALLY PANCREATECTOMIZED BUT NORMOGLYCAEMIC MICE.

B. Soria", E. Anóreu", M. Raurell', J.V. Sanchez-Andrés", F. Martin", and E. Montanya'. 'Dept. of Physiology, University of Alicante, Aptdo. 374, 03080 Alicante, Spain., CSUB-Hospital of Bellvitge, University of Barcelona, 08907 L'Hospitalet de Llobregat, Barcelona, Spain.

To study the adaptation of insulin secretion mechanisms in order to maintain normogiycaemia when pancreatic $\beta$-cell mass is reduced. we studied the effects of glucose on the $\beta$-cell membrane potential and crtosolic calcium $\left(\left[\mathrm{Ca}^{2}+\right]\right)$ of isiets isolated from $60 \%$ pancreatectomized (Px) mice, 2 and 8 weeks after pancreatectomy. The electrical activity was measured by intracellular recording of pancreatic $\beta-c e l l .\left[\mathrm{Ca}^{2}\right]$; was monitored using duai-emission microfluorimetry techniques with indo- 1 loaded islets. $\beta$-cell mass was measured by point counting morphometry on immunoperoxidase-stained sections of the pancreas. All mice were normoglycemic throughout the study ( 2 weeks after $\mathrm{Px}(\mathrm{n}=15): 4.2 \pm 0.1$ mmol/l; 8 weeks after $P x(n=10): 5.0 \pm 0.2$ mmol/l). $\beta$-cell mass (control $(n=4)$ : $1.52 \pm 0.38 \mathrm{mg}$ ) in $60 \%$ pancreatectomized mice increased from $40 \%$ to $65 \%$ after 2 weeks ( $\mathrm{n}=4)(0.99 \pm 0.19 \mathrm{mg})$ and to $80 \%$ after 8 weeks $(\mathrm{n}=4)(1.23 \pm 0.16$ $\mathrm{mg})$. In 2-week $\mathrm{Px}$ islets $(\mathrm{n}=12)$ there were a lef-shift displacement on the doseresponse of the glucose-induced bursts of electrical activity and $\left[\mathrm{Ca}^{2}\right]$ : oscillations. The activity observed at $5.6 \mathrm{mmol} / \mathrm{l}$ in 2-weeks Px islets $(\mathrm{n}=11)$ were similar to that observed at $11 \mathrm{mmol} / \mathrm{l}$ in control islets $(\mathbf{n}=9)$. Moreover, in 2-weeks Px islets $(\mathrm{n}=8)$ maximal electrical activity and $\left[\mathrm{Ca}^{2}\right]$, responses were achieved at lower glucose concentration $(16.7 \mathrm{mmol} / \mathrm{h})$ than in control islets $(22 \mathrm{mmol} / \mathrm{l})(\mathrm{n}=8)$. This left-shift displacement dissappeared when islets from 2-week $\mathrm{Px}$ mice were cultured during $48 \mathrm{~h}$ in $5.6 \mathrm{mmol} / \mathrm{l}$ glucose $(n=6)$. No differences were found in the electrical activity and in $\left[\mathrm{Ca}^{2+}\right]_{i}$ between 8 -week Px islets $(n=9)$ and controls $(n=$ 14). In summary, pancreatectornized but normoglycaemic mice showed an increased sensitivity to glucose when the total $\beta$-cell mass was reduced about $40-35$ $\%$, but not when the $\beta$-cell mass recovery reached about $80 \%$ of the control values. We suggest that an increased sersitivity to glucose might be an adaptative response that maintain the normoglycaemia with a limited $\beta$-cell mass.

\section{PS 27}

\section{Insulin Receptors}

\section{5}

PROPERTIES OF A CROSS-LINKING MODEL OF INSULIN RECEPTOR BINDING

R. M. Shymko and P. De Meyts. Hagedorn Research Institute, Niels Steensens Vej 6, DK-2820 Gentofte, Denmark

Experiments have suggested that each $\alpha$-subunit of the insulin receptor contains at least two or three separate low affinity binding sites for insulin, but that the $\alpha_{2} \beta_{2}$ holoreceptor dimer binds only one insulin molecule with high affinity. Kinetic binding studies of insulin analogues mutated at either their dimer-forming or hexamer-forming surfaces have led to models whereby a single insulin molecule forms a bridge between the two $\alpha$-subunits, similar to the ligand-induced dimerization phenomenon prevalent in the cytokine receptor family. The acceleration of the dissociation of tracer bound insulin in the presence of high concentrations of unlabelled insulin suggests that some cooperation between the two receptor halves is required to generate high affinity binding, in a way that is disrupted by further saturation of the binding sites. Furthermore, the degree of acceleration of dissociation does not increase uniformly with unlabelled insulin concentration; rather, the acceleration increases to a maximum and then decreases at higher concentrations ("bell-shaped curve"). We have proposed that these phenomena may be explained by the possibility of a second bridge occurring between the two $\alpha$-subunits upon partial dissociation of the first bridge. We present here a quantitative analysis of the properties of such a binding model for the insulin receptor in the simple case in which two halves of the receptor can be crosslinked at a single bridge through a single insulin molecule, thereby creating a high-affinity state of the receptor. We show that the crosslinking reaction indeed provides for the non-first order dissociation and association in agreement with experimental observations. The model further demonstrates that high concentrations of hormone can compete for the crosslinking reaction, predicting a bell shape for the plot of number of crosslinked receptors versus hormone concentration. Therefore, if crosslinking of receptors is required for insulin receptor signalling, the prediction of the model is that the biological response should be bell-shaped as well. This is indeed found for an increasing number (but not all) of biological effects induced by insulin and $|G F$.

\section{6}

973 VALINE TO METHIONINE MUTATION OF HUMAN INSULIN RECEPTOR (HIR): FUNCTIONAL CHARACTERISATION IN 293 CELLS. V. Strack, B. Bossenmaier, J. Mushack and H.-U. Häring. Medizinische Klinik und Poliklinik (IV. Abteilung), Eberhard-Karls-Universität, Otfried-Müller-Str. 10, 72076 Tübingen, Germany.

Possible reasons for the insulin resistance in NIDDM patients include structural changes in the human insulin receptor (HIR). It was recently reported that the 973 Val to Met mutation of HIR occurs in NIDDM patients with a prevalence of $5 \%$ compared to $0.5 \%$ in a control population. The aim of the present study was to asses whether this mutation is associated with altered autophosphorylation of the human insulin receptor $\beta$-subunit or altered susceptibility to hyperglycemia induced inhibition. Furthermore we studied the HIR/RS-1 interaction of the mutated receptor (HIR-973). HIR-973 (valine to methionine) was prepared by in vitro mutagenesis and was overexpressed in 293-cells. Wild type HIR and HIR-AJM (deletion of the 12 amino acids $954-965$ in the juxtamembranc region of the insulin receptor) were used as controls. To study HIR/IRS-1 interaction cells were cotransfected with IRS-1 and HIR, HIR-973 and as an IRS-1 negative control HIR$\Delta \mathrm{JM}$. Using specific antibodies (anti-pY and anti- $\beta$-subunit) it was shown that insulin stimulated autophosphorylation of HIR-973 was identical to wild type, furthermore hyperglycemia ( $25 \mathrm{mM}$ 2-deoxyglucose) inhibited insulin-induced autophosphorylation in all constructs equally by more than $90 \%(n=5)$. Cotransfection of HIIR and HIR-973 with IRS-1 increased tyrosine phosphorylation of IRS1 and other cellular substrates more than 10 -fold. This effect was even further pronounced (1.3-fold, $n=7$ ) by cotransfection of IRS-1 with HIR-973. In summary the data show that HIR-973 1) exhibit unaltered autophosphorylation 2) exhibit unaltered susceptibility to hyperglycemia inbibition 3) shows slightly enhanced phosphorylation of IRS-1 and other cellular subsirates. The latter effect might be explaned by the close vicinity of this mutation to the known IRS-I binding domain of HIR (Tyr960). This might be an effect which compensates other defects in the cells of the NIDDM patients. 
THE ROLE OF THE INSULIN RECEPTOR IN CELLULAR GROWTH AND TUMORIGENESIS.

E Wertheimer ${ }^{1}$, T Tennenbaum ${ }^{2}$, DE Graham ${ }^{3}, K_{\text {Barth }}^{3}$, SH Yuspa ${ }^{4}$, and SI Taylor'. 'Dept. of Pathology, Sackler Medical School, Tel Aviv Univ., Israel ${ }^{2}$ Dept of Life sciences, Bar ilan Univ., Israel. ${ }^{3}$ Diabetes Branch, NIDDK, NIH ${ }^{4}$ Laboratory of Cellular Carcinogenesis and Tumor Promotion, NCI, NIH, Bethesda, MD.

The insulin receptor (IR) is a ubiquitously expressed tyrosine kinase which mediates insulin signaling. While most research efforts focus on its role in regulation of glucose metabolism in the insulin-target tissues, i.e. muscle fat and liver, its role in other tissues is neglected. Recently we and others have demonstrated that insulin signaling is involved in regulation of various basement membrane proteins, that are involved in cellular growth and differentiation. In addition, there is increasing evidence that IR and the closely related IGF-I receptor are involved in malignant transformation. However, the role of IR in cellular growth and its transforming potential were never investigated properly. Using a sensitive transformation assay we have studied the IR transforming activity. In this model system we co-express in NIH-3T3 cells the IR with the bovine papilloma virus (BPV), which also has weak transformation activity, thus increasing the sensitivity of the cell to a given level of IR transforming activity. Overexpressing IR-BPV induces a transformed phenotype, characterized by loss of contact inhibition and increased cellular growth density. When injected subcutaneously into nude mice, cells overexpressing IR-BPV form significantly more tumors then parental cells or cells expressing BPV vector alone. In contrast, when the mutant Ser323Leu IR that lacks the ability to bind insulin was co-expressed with BPV, the mutant receptor lacked transforming activity as evidenced by the inability of the transfected cells to form tumors. Thus, we conclude that insulin binding is required for the co-transforming activity of IR in this experimental model

Determining the role of IR in tumor promotion as well as understanding its involvement in cellular growth will provide insights into its function in the non-classical as well as classical target tissues for insulin. Futhermore it has the potential to elucidate the role of the IR family of proteins in cancer diabetes and the associated complications of both diseases.

\section{PS 28}

\section{Signal Transduction}

628

SERINE PHOSPHORYLATION SITES 1293/94 AND 1308/09 IN THE C-TERMINAL END OF THE INSULIN RECEPTOR $\beta$-SUBUNIT MODULATE INSULIN DEPENDENT TYROSINE PHOSPHO RYLATION OF AN UNIDENTIFIED 66kDa PROTEIN.

B.Stoyanov, B.Bossenmaier, J.Mushack and H-U.Häring, Medizinische Klinik und Poliklinik (IV Abteilung), Eberhard-KarlsUniversität Tübingen, Otfried-Müller-Str. 10, 72076 Tübingen, Germany.

The aim of the present study was to assess the role of serine phosphorylation sites in the human insulin receptor (HIR) $\beta$-subunit for the insulin induced tyrosine phosphorylation of HIR substrate proteins. Serine to alanine mutations were introduced in HIR at positions 1023/25, 1293/94 and 1308/09. 293 cells were transfected with wild type HIR, the HIR mutants and and a HIR with a C-terminal deletion of 43 amino acids ( $\triangle C T$-HIR). After insulin stimulation total cell lysates were analyzed by anti-pY antibodies. Major differences were seen with respect to the phosphorylation of a $66 \mathrm{kDa}$ protein. The $\triangle C T$-HIR as well as the HIR $1308 / 09$ and 1293/94 point mutants induce enhanced phosphorylation of pp66 (2.1 fold $\triangle \mathrm{CT}$-HIR, 1.9 fold 1293/94, 2.1 fold 1308/09, $\mathrm{n}=10$ ). Antibodies against PTP IC, PTP1D, p55pik do not crossreact with pp66, pp66 does not co-precipitate with HIR or IRS-1. Our data suggest that 1) pp66 is an unidentified substrate of HIR; 2) serine phosphorylation sites $1293 / 94,1308 / 09$ in the C-terminal tail of the insulin receptor appear to suppress phosphorylation of $\mathrm{p} 66$. As it is known that the $\Delta \mathrm{CT}$ receptor contains an increased mitogenic activity we speculate that pp66 might be involved in mitogenic signalling.
629

PC- 1, TNF- $\alpha$ AND INSULIN RECEPTOR TYROSINE-KINASE IN HUMAN ADIPOSE TISSUE- RELATIONSHIP WITH INSULIN SENSITIVITY

V. Trischitta, J. Youngren*, P. Sbracciat, M. D'Adamo+, A. Buongiomo+t D. Spampinato, L. Frittitta, R. Vigneri, and I.D. Goldline*. Internal Medicine, Endocr and Mctab, Univ of Catania, +Division of Endocrinology 1, Univ "La Sapienza" and ++Istituto Supcriore Sanità, Rome, Italy, and *Division of Diabetes and Endocr Res, Univ of San Francisco, USA.

PC-1 and TNF- $\alpha$ have been reported to reduce insulin action in cultured cells by inhibiting insulin receptor tyrosine-kinase aclivity (IR-TK) and, therefore, insulin signaling. Morevorer, PC-1 and TNF- $\alpha$ are overexpressed in adipose tissue from obese and/or NIDDM paticnts. It is not known, however, whether PC-1 and TNF$\alpha$ overexpression in adipose tissue is an early event in the development of human $\alpha$ overexpression in adipose tissue is an early event in the development of human
insulin resistance or is a consciuence of obcsity and/or NIDDM. Aim: to answer this question we studied PC-1 protcin and mRNA TNF- $\alpha$ tanscript content, and insulin receptor tyrosine-kinase activity in adipose tissue of non-obese (BMI < 28), non-diabetic (by OGTT) subjects with a wide range of insulin sensitivity as assessed by an intravenous insulin tolerance test (Kitt values: 2.9-8.1). Methods: assessed by an intravenous insulin tolerance test (Kitt values: 2.9-8.1). Methods:
subcutancous abdominal adipose tissue was obtained at elective surgery. PC-1 content was assessed in solubilized membrane extracts by RIA $(n=19)$. TNS $-\alpha$ mRNA content was measured by quantitative compecitive RT-FCR and $20 \%$ PAGE $(\mathrm{n}=12)$. Insulin (0.1-100 $\mathrm{nM})$ stimulared IR-TK was studied in solubilized adipose tissue on immunopurificd $\mathbb{R}$ by poly-glu-tyr $4 / 1$ phosphorylation $(n=8)$. Results: 1) adipose PC-1 content significantly correlated with: Kitt values ( $r=$ $0.5, p=0.04)$ and both plasma glucose $(r=0.5, p=0.032)$ and insulin $(r=0.7$, $p=0,001)$ levels 120 min during OGTT. Morcover, the less insulin sensitive subjecs (Kitt value $<6$, median value, $n=10$ ) have a significanlly higher $\mathrm{PC}-1$ content than subejcts with $\mathrm{K}$ values $>6(\mathrm{n}=9)(525 \pm 155$ vs $336 \pm 134 \mathrm{ng} / \mathrm{mg}$ content than subejcts with $K$ values $>6(\mathrm{n}=9)(525 \pm 155$ vs $336 \pm 134 \mathrm{ng} / \mathrm{mg}$
protcin, mean \pm S.E., $\mathrm{p}=0012)$. 2) No correlation was found beiween TNF-a mRNA content and any of the above mentioned indicators related to insulin sensitivily 3) Insulin stimulation of IR-TK was significantly reduecd ( $p=0,02$, by 2-way ANOVA test) in adipose tissue from subjects $(n=4)$ with Kit $<6(n=4)$ as compared to subjects with Kitt $>6$. Conclusions: healthy, non-obese, noncompared to subjects with Kitt $>6$. Conclusions: healthy, non-obese, non-
diabetic subjects with reduced insulin sensitivity have an increased adipose tissue diabetic subjects with reduced insulin sensitivity have an increascd adipose tissue
content of PC-1, but not TNF- $\alpha$ mRNA. The increased PC- 1 content in adipose tissue is associated to a reduccd IR-TK activity in response to insulin. PC-1 but not TNF- $\alpha$, thercfore, may play a role in the early stage of human insulin resistance. 
630

SERINE RESIDUES IN HIR CYTOPLASMIC PART CONTRIBUTE TO A SECOND DOMAIN REQUIRED FOR IRS-1 PHOSPHORYLATION. B. Bossenmaie* ${ }^{* *}$ B. Stoyanov ${ }^{*}, V$. Strack ${ }^{*}$, and H. Häring ${ }^{*}$ Medizinische Klinik a. Poliklinik (Abt. 4) Eberhard Karls Universität Tübingen+ Boehringer Mannheim GmbH Abt. Molekulorbiologie

We studied potential functions of serine residues in human insulin receptor (HIR) for activation of the HIR/IRS-1 signaling chain. HIR with aminoacid exchanges (serine to alanine) at position 1023/1024, 1055, 1074/78, $1177 / 78 / 82,1294 / 95$ and 1308/09 were transiently overexpressed in 293 cells together with IRS-1. As a conirol IRS-1 was cotransfected with JMAHIR which does not contain the domain required for IRS-1 phosphorylation. Tyrosine phosphorylation was detected with anti-pY antibodies. Expression levels of HIR and mutants as well as those of IRS-1 were monitored by immunoblotting with the respective antibodies. Coexpression of HIR with IRS-1 enhanced tyrosine phosphorylation of IRS-1 ( $>10$ fold). The same effect was observed in cells overexpressing IRS-1 together with HIR1023/25. HIR1055, HIR129394 and HIR1308/09. As expected cells expressing HIRAJM show no tyrosine phosphorylation of IRS-1. Surprisingly IRS-1 phosphorylation was also impaired for HiR1177/78/82 despite its normal extent of aufophosphorylation. Immunodetection of total cell lysates with anti-pY antibodies showed that besides IRS-1 a number of other cellular proteins displayed reduced levels of tyrosine phosphorylation in cells overexpressing HIR $\triangle \mathrm{JM}$ and HIR1177/78/82 as compared to the overexpression of HIR or other mutated forms. The results obtained with HIRAJM are in agreement with the previously described role of the JM domain for HIR/IRS-1 coupling. Our finding that serines 1177/78/82 in HIR are required for efficient tyrosine phosphorylation of IRS-1 defines a second region in the receptor that is involved in coupling the IRS-1 and other cellular proteins. As mutations in the $\mathrm{JM}$ region as well as in the 1177/87/82 region around the serine residues $1177 / 78 / 82$ are sufficient to several impair HIR/IRS-1 interaction, it seems that these regions are functionally independent. To analyse this phenomenon more carefully we established stable NIH3T3 fibroblast cell lines overexpressing wildtype HIR and HIR1177/78/82. In these cell lines we analysed tyrosine phosphorvlation of endogenous substrates, mitogenic response, glucose transport, Fl3-kinase and MAP-kinase activity.

\section{1}

REDUCED IRS-1, GLUT-4 PROTEIN CONTENT AND PI3-KINASE ACTIVITY IN ADIPOCYTES FROM SUBJECTS WITH TYPE 2, BUT NOT TYPE 1, DIABETES.

U.Smith, C.M.Rondinone, P.Lönnroth and C. Wesslau. Lundberg Laboratory for Diabetes Research, Department of Internal Medicine, Sahlgrenska University Hospital, S-413 45 Göteborg, SWEDEN.

Insulin stimulates tyrosine phosphorylation of IRS-1 which in turn binds and activates PI3-kinase. PI3-kinase lipid kinase seems to be critical for GLUT4 translocation. The aim of this study was to examine potential defect(s) in insulin action and insulin's signaling pathway in human adipocytes from normal subjects and insulinresistant states. Insulin-stimulated glucose transport, protein content and tyrosine phosphorylation of key proteins inolved in insulin action as well as PI3-kinase activity in antiphosphotyrosine immunoprecipitates were measured in adipocytes from healthy subjects, type 1 and type 2 diabetic patients. Insulin-stimulated glucose transport was reduced by $-50 \%$ in type 2 diabetic patients while IRS -1 and GLUT 4 content were reduced by $\sim 75 \%$. In contrast, other key proteins such as the insulin receptor, PI3-kinase (p85 and p110) and MAP kinases were unaltered. There was also a decrease in IRS-1 tyrosine phosphorylation due to the lower amount of protein in these patients and a 90\% reduction in PI3-kinase activity. Interestingly, these defects were not detected in cells from subjects with type 1 diabetes.

Conclusions: A) IRS-1 and CLUT4 content as well as PI3-kinase activity are reduced in fat cells from subjects with type 2 but not type 1 diabetes. B) PI3-kinase activity is reduced in type 2 diabetes probably due to the lower content of IRS -1 in these patients. C) Low IRS-1 and GLUT4 seem to be markers of insulin resistance.

\section{2}

IN VTVO REGULATION OF IRS-1 ASSOCLATED PI 3-KINASE BY INSLLIN IN HEART AND LIVER OF hHTg INSULIN-RESISTANT RAT

D. Gašperíková, E. Šeböková, T. 'Kolter, J. 'Eckel, I. Klimes. Institute of Experimental Endocrinology, Slovak Academy of Sciences, Bratislava, Slovak Republic and Diabetes Research Institute, Düsseldorf, Germany

We have recently shown a marked increase of the total phosphatidylinositol (PI) 3-kinase activity in heart of insulin resistant rats which failed to increase further in response to hyperinsulinemia. Therefore, this time the activity (incorporation of [ $\left.{ }^{32} \mathrm{P}\right]$ dATP into PI) and immunoproteinlevels (Western blot) of the insulin-receptor-substrate-1 (IRS-1) associated PI-3-kinase were measured in hearts and liver of hereditary hypertriglyceridemic and insulin resistant $(\mathrm{hHTg})$ rats subjected to an euglycemic hyperinsulinemic $6.4 \mathrm{mU}$. $\mathrm{kg}^{-1} \cdot \mathrm{min}^{-1}$ ) clamp. The data obtained were compared to results of control (normotriglyceridemic) rats which underwent an identical protocol. The IRS-1 associated PI-3 kinase activity was two times lower in hearts of $\mathrm{hHTg}$ rats (hHTg: $0.6 \pm 0.08$ vs. controls: $1.2 \pm 0.05$ arbitrary units (AU); $p<0.01$ ). This decrease was accompanied by a similar change of the IRS- 1 associated PI-3 kinase protein levels (hHTg: $1.9 \pm 0.3$ vs. controls: $3.3 \pm 0.3$ AU; $p<0.05$ ) Hyperinsulinemia (approx. 10-fold increase above fasting insulinemia) changed neither the activity $(0.5 \pm 0.02 \mathrm{AU}$; NS), nor the immunoprotein amount (2.8 $0.43 \mathrm{AU} ; \mathrm{NS})$ of the IRS-1 associated PI-3 kinase in heart of hHTg rat. However, in hearts of control rats hyperinsulinemia led to a reduction of the IRS-1 associated PI-3 kinase activity $(0.3 \pm 0.02 \mathrm{AU} ; \mathrm{p}<0.01)$ and immunoprotein $(1.4 \pm 0.2 \mathrm{AU} ; \mathrm{p}<0.01)$. There were no changes either in the activity or in the immunoprotein levels in liver of $\mathrm{hHTg}$ rats. Hyperinsulinemia did not further change the aforementioned parameters. In summary, insulin signalling via the IRS-1 pathway seems to be decreased in heart but not in the liver of the insulin resistant hHTg rat. (Supported in part by $D F G, S F B 35$ I C2, SDS).

\section{3}

Role of Map Kinase in Insulin Signalling Pathways Regulating Glucose Metabolism in Human Skeletal Muscle.

*B.T. Navé, §P.R. Shepherd, +E. Wahlström-Ødegaard, ${ }^{\star} R$.J.Haigh, + L.A. Nolte, ${ }^{+} D$. Galuska, ${ }^{+} J . R$. Zierath, ${ }^{*}$ K. Siddle \& ${ }^{+} H$. WallbergHenriksson.*Dept. of Clinical Biochemistry, University of Cambridge, \$Dept. of Biochemistr, University College London, U.K. and + Dept. of Clinical Physiology, Karolinska Hospital, Sweden.

Muscle is the major site for insulin stimulated glucose disposal in vivo. However, little is known about the signalling pathways involved in regulating glucose metabolism in muscle. We have investigated the role of Map Kinase in insulin stimulation of glucose transport and activation of glycogen synthase in human skeletal muscle. Biopsies of the vastus lateralis muscle were obtained from overnight fasted, healthy young male volunteers and muscle strips were prepared from biopsies. Strips were preincubated for 5 minutes with the PI 3-kinase inhibitor LY294002 (30 $\mu \mathrm{M})$ or the Map Kinuse Kinase inhibitor PD98059 (up to $20 \mu \mathrm{M}$ ). Strips were then incubated in the presence of inhibitor and insulin $(1000 \mu \mathrm{U} / \mathrm{ml})$ for a total of 30 minutes. Insulin acutely stimulated phosphorylation of Map Kinase with the amount of the active form rising from $57 \% \pm 4.4$ to $80 \% \pm 1.8$ $(p<0.05)$. This stimulation was blocked by $15 \mu \mathrm{M}$ PD 98059 but not by $30 \mu \mathrm{M}$ LY294002. Conversly, insulin stimulated 3-Omethylglucose uptake approximately 2 fold and this was blocked by $30 \mu \mathrm{M}$ LY294002 but not by $20 \mu \mathrm{M}$ PD 98059 . Further, PD 98059 did not block the insulin induced increase in the glycogen synthase activity ratio. Taken together these results demonstrate that while both Map Kinase and PI 3-kinase are activated by insulin in human skeletal muscle, Map Kinase is not involved in insulin's regulation of glucose transport or glycogen synthase. 
INCREASED GLUCOSE UTILISATION IN SKELETAL MUSCLE VIA A MECHANISM INVOLVING NITRIC OXIDE AND cyclicGMP

B. Leighton, M. E. Young, J. Jensen" and G.K. Radda

Department of Biochemistry, University of Oxford, Oxford, OX1 3QU, U.K. Department of Anatomy, University of Oslo, Norway

Nitric oxide synthase enzyme is located inside skeletal muscle cells to produce nitric oxide (NO), for example, during muscular contraction. We hypothesise that $\mathrm{NO}$ is a novel means for mediating increased glucose transport and metabolism in muscle via a cyclicGMP dependent mechanism. Soleus muscle preparations were incubated with sodium nitroprusside (SNP; $15 \mathrm{mM}$ ), a NO donor, and this significantly increased the basal rate of 2deoxyglucose transport $(57 \%)$. SNP significantly $(\mathrm{P}<0.001)$ increased the insulin-mediated $(100 \mu \mathrm{U} / \mathrm{ml})$ rates of ${ }^{14} \mathrm{C}$-lactate release $(38 \%)$, net lactate release $(45 \%)$ and glucose oxidation $(172$ $\%$ ). SNP, at $15 \mathrm{mM}$ for $15 \mathrm{~min}$, stimulated a 10 -fold rise in soleus muscle cyclicGMP content. A cell permeable cyclicGMP analogue, 8-bromocyclicGMP, was incubated with muscle preparations and the effect on basal rates of lactate release was determined. 8 BromocyclicGMP $(100 \mu \mathrm{M})$ significantly increased the rate of lactate release $(7.55 \pm 0.37$ versus $6.10 \pm 0.49 ; P<0.05)$. Therefore, the NO donor SNP stimulates glucose transport and utilisation and cyclicGMP content in an isolated incubated rat skeletal muscle preparation. A cyclicGMP analogue stimulated the rate of conversion of glucose to lactate. All this points to a novel mechanism for increasing glucose utilisation in skeletal muscle involving cyclicGMP via activation of nitric oxide synthase to produce NO which will activate guanylate cyclase.

\section{6}

POSITIVE AUTOREGULATION OF PROTEIN KINASE C $B_{1}$ - and $B_{2}$-mRNA BY HYPERGLYCEMIA IN HUMAN LYMPHOCYTES IN VIVO

R. Assert, K. Haupt, V. Pirags, H. Schatz and A. Pfeiffer; Medizinische Universitätsklinik Bergmannsheil, D-44789 Bochum, Germany

Protein Kinase $C$ B (PKCB) was shown to be activated by hyperglycemia in human thrombocytes and there is strong experimental evidence implicating PKC $B$ in the pathogenesis of diabetic complications. The transcription of PKCB-mRNA is positively autoregulated by its promoter resulting in upregulation upon activation of PKC. We therefore investigated the autoregulation of $P K C \beta_{1}$ - and $B_{2}-m R N A$ during 150 min of hyperglycemia in human lymphocytes in vivo. Methods: Twenty-one subjects were infused with octreotide and glucose plus insulin. At 0,60 and 150 min lymphocytes were isolated by densitygradient centrifugation. PKCB protein was determined by quantatitive immune blots. For PKCB1 - and $B_{2}$-cRNA probes labeled to high specific activity were hybridized in solution for ribonuclease protection assays. The housekeeping gene GAPDH was cohybridized as internal standard and laser densitometry was used for quantification of autoradiographs. Results: Under conditions of hyperglycemia in the presence of $40 \mu \mathrm{U} / \mathrm{ml}$ insulin $\mathrm{PKCB}_{1}$ and $\mathrm{B}_{2}$ protein was elevated in both cytosol and membrane compartments. The mRNA of $\mathrm{PKCB}_{1}$ showed a highly reproducible increase by $28 \pm 6 \%$ (n $=2 \hat{i}$, $p$ $<0.001)$ and $P K C B$ by $19 \pm 7 \%(n=15, p=0.011)$. The increases in mRNA were related to the increase in blood sugar. Conclusions: Hyperglycemia is shown to increase $P K C B_{1}$ - and $B_{2}-m R N A$ in vivo in man. This may be explained by an activation of $P K C B$ by hyperglycemia and subsequent positive transcriptional autoregulation. The autoregulation may prevent downregulation of $\mathrm{PKCB}$ despite chronic activation due to an elevated rate of resynthesis. This may explain why PKCB plays a particular role in the pathogenesis of diabetic complications.
HIGH GLUCOSE INDUCED MODULATION OF PROTEIN KINASEC ACTIVATION IN CULTURED ENDOTHELIAL CELLS

G. Reining, S. Baumgartner-Parzer and W. Waldhäusl; Medicine III Endocrinol. \& Metabol.; Währinger Gürtel 18-20, 1090 Vienna, Austria

It has been shown in cells derived from vascular tissue that elevated concentrations of glucose activate Protein Kinase $C$ (PKC). This study was designed to evaluate the effect of $30 \mathrm{mM}$ giucose long-term incubation on ligand induced activation of PKC (i. e. translocation from the cytosol to the membran fraction) in cultured human umbilical vein endothelial celis (HUVEC). Paired cultures of 12 individual isolates were incubated in $5 \mathrm{mM}$ and $30 \mathrm{mM}$ glucose, respectively, for $16 \pm 2$ days. Confluent cultures were stimulated with increasing concentrations of thrombin for 30 seconds. Equal amounts of protein from the membrane fraction, gained by high speed centrifugation, were analysed by immunoblotting using antibodies against PKC isoforms alpha (PKC $\alpha$ ) and epsilon (PKC $\varepsilon$ ). As long-term incubation with $30 \mathrm{mM}$ glucose did not affect the total cellular content of PKC $\alpha$ and $\varepsilon$, an increase in intensity of the isoformspecific band in the membrane fraction was set equivalent to PKC activation. Cultures grown in $30 \mathrm{mM}$ glucose showed an increase in membrane bound $\mathrm{PKC \alpha}$ $(169 \pm 77 \%$ of control, Student's paired t-test; $p<0.05 ; n=12)$ and $P K C \varepsilon$ $(135 \pm 47 \%$ of control, $p<0.05 ; n=12)$ compared to cultures kept in $5 \mathrm{mM}$ glucose. Stimulation of confluent cultures with thrombin $(0.001,0.01,0.1$ 1 and $10 \mathrm{nM}$ ) displayed a concentration dependent increase in membrane bound $\mathrm{PKC} \alpha$ and $\varepsilon$. The resulting thrombin-stimulationcurves derived from cells grown in $5 \mathrm{mM}$ and $30 \mathrm{mM}$ glucose did not differ for $P K C \alpha$, but activation of $\mathrm{PKC} \varepsilon$ in cells grown in $30 \mathrm{mM}$ glucose was reduced at concentrations of 1 and $10 \mathrm{nM}$ thrombin (AUC, $p<0.05$ ) versus control cultures kept in $5 \mathrm{mM}$ glucose. These data suggest that elevated glucose might influence a ligand induced PKC activation in an isoformspecific manner.

\section{7}

HIGH LEVELS OF GLUCOSE ACTIVATE GLUCOSE TRANSPORT BY A MECHANISM WHICH INVOLVES PKC BUT NOT PLC.

Y. Kawano, A. Soler, L. Nolte, J. Rincon, J.R. Zierath and H. WallbergHenriksson. Department of Clinical Physiology Karolinska Hospital, Stockholm, Sweden.

Insulin and contraction have been accepted as two separate stimulators of glucose transport in skeletal mucle. Recently, we demonstrated that increased glucose uptake to muscle by high levels of extracellular glucose is blocked by Dantrolene, an inhibitor of $\mathrm{Ca}^{2+}$ release from SR, but not by Wortmannin, a PI3 kinase inhibitor. This observation cannot be explained by a mass action effect of glucose, and suggests the existence of a $\mathrm{Ca}^{2+}$ dependent signaling pathway for activation of glucose transport that is distinct from the insulin signaling pathway. The airn of this study was to further elucidate the signaling mechanism by which hyperglycemia can autoregulate it's own transport. Isolated rat soleus muscle were incubated with [ $\left.{ }^{3} \mathrm{H}\right] 3-0$-methylglucose for measurement of glucose transport. Glucose transport increased in a dose dependent manner by up to 3 -fold $(<0.01)$, followed by a $35 \%$ decrease in glucose transport at $35 \mathrm{mM}(p<0.05)$. This concentration dependent curve can be separated from a curve obtained by adding Dantrolene $(25 \mu \mathrm{M})$, and reflects the mass effect. Based on these results, the high glucose effect occurs over a range of physiological concentrations of glucose $(p<0.05)$. $\mathrm{H}-7$, a protein kinase $\mathrm{C}$ inhibitor $(\mathrm{PKC})$ abolished the high glucose effect on glucose transport $(p<0.01)$, whereas insulin-stimulated glucose transport was unaltered. U-73122 (PLC inhibitor) did not alter glucose- or insulin-stimulated glucose transport. Thus, PKC, but not PLC is involved in this glucose sensitive pathway. Furthermore, the pathway by which hyperglycemia increases glucose transport is clearly separate from the insulin signaling pathway. 


\section{NUCLEAR LOCALIZATION OF PROTEIN KINASE C $\delta$ IN THE BETA CELL}

M. Hoenig and K.L. Knutson. Dept. of Physiology and Pharmacology, University of Georgia College of Veterinary Medicine, Athens, GA, USA

Beta-cells express multiple isoforms of protein kinase $\mathrm{C}$ (PKC) and some isoforms are located to multiple pools within the cell, including the cytoskeletal elements. In this study we analyzed the localization of the $\delta, \varepsilon, \xi, B$ and $\alpha$ isoforms of PKC to the nucleus. Nuclei were isolated from insulinoma beta-cells and fractionated by centrifugation to give the nuclear soluble fraction, nuclear membrane fraction, and the insoluble matrix. The nuclear pellet was enriched in DNA and contained less than $5 \%$ of the total cellular nucleotidase activity. The nuclear membrane contained less than $2 \%$ of the total cellular nucleotidase activity, suggesting negligible plasma membrane contamination. Analysis of nuclear fractions by immunoblotting with isoform-specific anti-PKC antibodies showed that PKC $\alpha, B, \xi$, and $\varepsilon$ could be detected in the soluble fraction of the cell but not in the nucleus. Only PKC $\delta$ could be detected in the nucleus and was mostly present in the nuclear membrane fraction. There was light staining in the nucleosol and the nuclear matrix but the enzyme in the nuclear membrane represented $\sim 76 \%$ of the total nuclear enzyme. Nuclear PKC $\delta$ constituted $\sim 9 \%$ of the total cellular enzyme. Phorbol ester $(1 \mu \mathrm{M})$ increased the levels associated with the nuclear membrane -3 fold but not to the nuclear matrix or nucleosol. Inhibition of PKC with MDL 29152 increased levels of preproinsulin mRNA relative to B-actin mRNA levels while prolonged (24 hour) phorbol ester treatment led to a slight downregulation. Taken together, these data suggest that PKC $\delta$ is constitutively active in the nucleus and may be important in modulating preproinsulin mRNA levels.

\section{0}

LOCATION AND TISSUE SPECIFICITY OF PHOSPHATIDYLINOSITOI. 3-KINASE PROTEIN SUBSTRATES M.J. Marcucci. N.B. Ruderman, and S.J. Heydrick. BOSTON UNIVERSITY MEDICAL SCHOOL, BOSTON, MA., 02118, U.S.A.

Phosphatidylinositol 3-kinase (PI3K) has recently been shown to act as a protein serine kinase on its own $85 \mathrm{kDa}$ regulatory subunit (p85) and insulin receptor substrate 1 (IRS-1) in whole cell extracts of rat adipocytes. To further understand the role of this serine kinase activity in insulin signal transduction, we chose to examine two questions: 1) whether additional endogenous substrates interact with PI3K, and 2) whether such substrates differ in adipocytes and hepatocytes. Isolated adipocytes and in situ livers were treated with insulin, fractionated, and $\mathrm{Mn}^{2+}$ - dependent kinase activity was assessed in anti-p85 immunoprecipitates in the presence and absence of Wortmannin (100 nM), a specific inhibitor of PI3K. In adipocytes, Wortmanninsensitive kinase substrates corresponding to $\mathrm{p} 85$ and the $110 \mathrm{kDa}$ PI3K catalytic subunit (p110) were present in all fractions, and IRS-1 and two additional protein substrates (160 and $63 \mathrm{kDa}$ ) were present in the low density membrane (LDM) and cytosolic (CYT) fractions. Interestingly, six additional substrates were found in the CYT. This trend was also evident in the liver in which the Wortmannin-sensitive substrates p85 and p110 were present in all fractions, IRS-1 in the LDM and CYT only, and seven additional substrates in the cytosol. In comparing liver and fat cytosol, four of the nine substrates identified were found in both tissues and the others were tissue specific. Total Wortmannin-sensitive kinase activity was greatest in the CYT in both fat and liver; however, the activity corrected for the amount of p85 was greatest in the LDM fraction. Interestingly, all of the anti-p85 immunoprecipitates (both tissues) contained Wortmannin-insensitive kinase activity that was greatest in the LDM fraction. In summary, the data demonstrate that a greater number of PI3K protein substrates exist than previously thought, including the p110 catalytic subunit. The presence of Wortmannin-insensitive kinase activity in p85 immunoprecipitates indicates that PI3K exists in a complex with at least one other protein kinase. Finally, the finding of unique substrates in each tissue supports the notion that liver and fat each have unique insulin signaling pathways.

\section{9}

REGULATION OF MYO-INOSITOL TRANSPORT BY PROTEIN KINASES AND GLUCOSE IN RETINAL PIGMENT EPITHELIAL CELLS

T.P. Thomas, A. K. Karihaloo, K. Kato and D. A. Greene, University of Michigan, Ann Arbor, MI, USA

In cells prone to diabetic complication, glucose (G) induces Ml-depletion. The regulation of Ml-uptake and -efflux, by Na-dependent MI transport (SMIT) and the volume sensitive organic osmolyte anion channel (VSOAC), is poorly understood. Human retinal pigment epithelial (RPE) celis are known to model $\mathrm{G}$-induced $\mathrm{Ml-depletion}$ in vitro. The aim of the study is to examine the role of protein kinases and G on the regulation of SMIT and VSOAC. In RPE 47 cells, protein kinase $\mathrm{C}$ (PKC) activation by TPA and DiC8 decreased SMIT to $78.7 \pm$ $4.3^{*}$ and $84.8 \pm 6.8 * \%$ of control (C) (measured as linear uptake of $1 \mu \mathrm{M}{ }^{3} \mathrm{H}-\mathrm{MI}$. ${ }^{*} \mathrm{p}<0.05$ by t-test). DiC8 increased Ml-efflux from $28.8 \pm 0.3$ to $70.8+3.0^{*}$ (measured as linear release of ${ }^{3} \mathrm{H}-\mathrm{Ml}$ in pre-loaded cells and expressed as $-/ \mathrm{h}$ of fraction remaining intracellularly following a 60 min washout). Protein kinase A(PKA)-agonists (forskolin, dibutyryl cAMP) increased SMIT respectively to $200.2 \pm 13.3 \%{ }^{*}$ and $180.1 \pm 6.7^{*} \% \mathrm{C}$. Forskolin increased MI-efflux from 32.4 \pm 9.5 to $67.2 \pm 8.0^{*}$. $30 \mathrm{mM} \mathrm{G}$ induced Ml-depletion and inhibited SMIT activity in a competitive fashion. In addition, pre-incubation with $\mathrm{G}$ decreased SMIT activity, in a time- and dose-dependent fashion with half-maximal inhibition at $\sim 10 \mathrm{mM} \mathrm{G}$ (uptake measured at $5 \mathrm{mM} \mathrm{G}$ ). $30 \mathrm{mM} \mathrm{G}$ maximally inhibited SMIT activity to $69.0 \pm 3.7 \% \mathrm{C}$ at $12 \mathrm{~h}$. $30 \mathrm{mM} \mathrm{G}$ failed to show any effect on Mlefflux. PKC-down regulation (by $24 \mathrm{~h}$ exposure to $14 \mathrm{M}$ TPA) increased SMIT to $109.3 \pm 2.6^{*} \% \mathrm{C}$ at $5 \mathrm{mM} \mathrm{G}$, and reversed the inhibition by $30 \mathrm{mM} \mathrm{G}$ to 91.8 $\pm 4.5 \% \mathrm{C}$. In RPE cells, (1) PKC activation reduces Ml content by inhibiting SMIT and activating efflux, perhaps functioning as a feed-back loop to stabilize: intracellular MI content, (2) PKA by activating both SMIT and VSOAC, may modulate trans-epithelial MI transport, (3) G inhibits SMIT through a PKC.mediated pathway. Differential regulation of SMIT and VSOAC by protein kinases may modulate cellular MI content under hyperglycemia.

\section{1}

DEFECT IN ACTNATION OF PI 3-KINASE BY INSULIN IN VTVO IN hHTG INSULIN RESISTANT RAT IS TISSUE SPECIFIC

E. Šeböková, D. Gašperíková, J.A. Maassen ', J. Eckel'², I. Klimeš. Institute of Experimental Endocrinology, Bratislava, Slovakia, 'University of Leiden, Netherlands and 'Diabetes Research Institute, Düsseldorf, Germany To evaluate the possible involvement of phosphatidylinositol(PI) 3-kinase in insulin resistance, the regulation of this enzyme in conditions of an intensive insulin-induced glucose utilization was studied in hereditary hypertriglyceridemic (hHTG) and insulin resistant rat. The animals were subjected to 90 min euglycemic hyperinsulinemicclamp (EHC, $6.4 \mathrm{mU}_{\mathrm{kg}} \mathrm{kg}^{-1} \cdot \mathrm{min}^{-1}$ ) to achieve about 10 -fold of basal fasting insulin levels. Total and insulin receptor substrate-1 (IRS-1) associated PI 3-kinase activities were measured in muscle and adipose tissue. The results were compared with the data obtained in control (C) rats. In quadriceps femoris (red and white fibres) muscle, no stimulation of total (Basal: $686.5 \pm 45.9$ vs. EHC: $710.7 \pm 85.1$ arbitrary units [AU], n.s.) or IRS-1 associated (Basal: $5.1 \pm$ 0.3 vs. EHC: $5.2 \pm 0.3 \mathrm{AU}$, n.s.) PI 3 -kinase activities were found after hyperinsulinemia in both $\mathrm{hHTG}$ and $\mathrm{C}$ rats. In soleus muscle (red fibre) insulin did stimulate total PI 3-kinase activity in controls (Basal C: $19.5 \pm 2.8$ vs. EHC C: $77.3 \pm 9.4$ $\mathrm{AU}, \mathrm{p}<0.001$ ) without having any effect in hHTG rats (Basal hHTG: $21.0 \pm .3 .5$ vy. EHC hHTG: $27.5 \pm 2.6$ AU, n.s.). Insulin did not show any stimulatory effect in soleus muscle on IRS-1 associated PI 3-kinase activity (Basal C: $13.3 \pm 1.2 \mathrm{vs}$. EHC C: $12.9 \pm 0.8$ AU, n.s.; Basal hHTG: $7.0 \pm 0.3$ vs. EHC hHTG: $5.2 \pm 0.5$ AU, n.s.) which was significantly decreased in hHTG rats in comparison to controls $(\mathrm{p}<0.001)$. In white adipose tissue (WAT), both the total (Basal: $21.4 \pm 2.8$ vs. EHC: $93.8 \pm 28.3 \mathrm{AU}, \mathrm{p}<0.05$ ) and the IRS-1 associated (Basal: $9.8 \pm 1.4$ vs. EHC: $39.5 \pm 12.6 \mathrm{AU}, \mathrm{p}<0.02)$ PI 3-kinase activities were increased after 90 min hyperinsulinemia in controls but not in the hHTG animals. In summary, a) activation of PI 3-kinase activity by insulin in vivo may involve the IRS-1 in white adipose tissue but not in skeletal muscles which implies tissue specificity; b) defect in the activation of PI 3-kinase activity by insulin in hHTG rats may participate in insulin resistance of these animals. This is the first report showing the involvement of PI 3-kinase in insulin induced glucose utilizationduring euglycemic hyperinsulinemia. (Supported partly by GAV and GAT COST B5). 
ADENOVIRUS-MEDIATED GENE TRANSFER OF DOMINANT NEGATIVE RAS: EFFECTS ON THE ACTIVATION OF PI3 KINASE AND GLUCOSE TRANSPORT IN 3T3L1 ADIPOCYTES.

L.Gnudi, E.U.Frevert, K.L.Houseknecht and B.B.Kahn. Diabetes Unit, Beth Israel Hospital-Harvard Medical School, Boston, MA, 02214, USA.

We sought to elucidate the relationship between the ras and PI3 kinase signalling pathways in whole cell physiology as well as to study the role of p21-H-ras in the activation of glucose transport by insulin. We used a gene transfer system which could achieve high efficiency in terminally differentiated cells, rapid enough to prevent chronic compensatory changes in the expression of other genes in the cell. We generated a recombinant adenovirus encoding dominant negative ras (ras-asn17). Efficiency of gene transfer was assessed by infecting 3T3L 1 adipocytes with a recombinant adenovirus expressing $\beta$-galactosidase ( $\beta$-gal). We achieved $60-70 \%$ infection efficiency and high level ras-asn 17 expression ( 10 -fold over endogenous ras). The dominant negative ras effect was assessed by map kinase gel mobility shift assay. In cells which were uninfected or infected with $\beta$-gal virus, insulin $(100 \mathrm{nM})$ or EGF (100 $\mathrm{ng} / \mathrm{ml}$ ) stimulation resulted in the appearance of a hyperphosphorylated species of 442 map kinase which was not seen in the cells infected with rasasn17. Hence adenovirus-mediated transfer of the ras-asn 17 clearly had a dominant negative effect on the activation of map kinase. In uninfected cells and $\beta$-gal-infected cells, insulin stimulated IRS-1-associated PI3 kinase activity $\sim 10$-fold and high level overexpression of the ras-asn17 did not impair this effect. Similarly, insulin and EGF activation of total PI3 kinase activity, measured in both the cytosol and in total membranes was normal in ras-asn 17 -expressing cells compared to $\beta$-gal-infected cells. Insulin stimulated glucose transport was also normal in cells expressing dominant negative ras. These data indicate that relatively acute inhibition of ras activation: a) inhibits the phosphorylation of map kinase; b) does not affect insulin or EGF stimulation of PI3 kinase; c) is not essential for insulin's effect on glucose transport. Thus in normal cell physiology, PI3 kinase does not appear to be downstream of ras in mediating the acute actions of insulin and EGF.

\section{4}

\section{RAC AND RHO IN NSSLIN-STIMULATED GLUCOSE TRANSPORT}

\section{G.C.M. van der Zon, J. Dorrestijn and J.A. Maassen}

Department of Medical Biochemistry, university of Leiden; The Netherlands.

Insulin-stimulated hexose uptake requires both the presence of small guanine nucleotide binding proreins (G-proteins) and an intact network of actin filaments. In most celis the structure of the cycoskeleton is regulated by smal! G-proteins such as Rac and Rho. In the present study we examined the contribution of Racand Rho-mediated signals to insulin-stimulated hexose uptake.

Signalling by RacGTP was inhibited by expression of the dominant-negative mutant $\mathrm{Rac}^{\mathrm{N} 17}$ in 3 T3-L1 adipocytes. A vaccinia virus-mediated gene transfer system was used. Western blor analysis showed an approximately five-fold overexpression of the mutant Rac in adipocytes with respect to endogenous Rac after infection. Subsequently the effect of $\mathrm{Rac}^{\mathrm{N1} 7}$ expression on insulin stimulated hexose transport was examined. No effecr on basal uptake and the magnitude of insulin-stimulated hexose uprake was detecred.

The contribution of RhoGTP-induced signals to hexose uptake was examined by incubation of 3T3-L1 adipocytes with lysophosphatidic acid (LPA). This compound is an activator of Rho in most cells. To examine whether 3T3-L1 adipocytes are sensitive to the action of LPA we have monitored the effect of LPA on the degree of tyrosine-phosphorylation of Focal adhesion kinase (FAK). This procein undergoes increased tyr-phosphorylation in response to LPA. FAKphosphorylation, analyzed by western blot using anti-phosphotyrosine antibody, was increased in response to LPA indicating that $3 T 3-\mathrm{Ll}$ adipocytes express functional LPA receptors. Incubation of adipocytes with LPA had no effect on the dose-response relation of insulin-stimulated hexose uptake. We conclude that RacGTP mediated signals are not required for insulin-stimulated hexose uptake and that activation of Rho is unlikely to contribute significantly to this process. Together these data suggest that the Rac/Rho cascade of small G-proteins is not involved in the stimulation of glucose uptake by insulin.
PHOSPHATIDYLINOSITOL 3-KINASE AS A POTENTIAL MEDIATOR OF INSULIN RECEPTOR AND FOCAL ADHESION KINASE INTERACTIONS S. Clark* G. Eckardt and N. Konstantopoulos. *Institute of Human Nutrition, Deakin University \& University of Melbourne Department of Medicine, Royal Melboume Hospital, Victoria AUSTRALIA

An insulin receptor (IR) mutant (K164 \& K582 $\rightarrow \mathrm{N})$, developed by us, provides evidence for IR involvement in cytoskeletal interactions. The resultant cell-line (CHO.2N) has altered morphology associated with decreases in cell adhesiveness, paxillin protein levels and basal focal adhesion kinase (FAK) phosphorylation. We have investigated potential mediators of this effect and report here on phosphatidylinositol 3-kinase (PI 3-K), an insulin signalling intermediate (via PI 3$\mathrm{K}$ interaction with IRS-1) which has been implicated in insulin-stimulated actin fibre reorganisation and membrane ruffling. PI 3-K may also associate with FAK. Using CHOT cells (overexpress wild type IR) and CHO.2N cells we examined the effect of the PI 3-K inhibitor wortmannin (WTMN) on FAK phosphorylation in control and insulin-stimulated cells. After insulin stimulation, FAK was immunoprecipitated, separated by SDS-PAGE and then immunoblotted with an antiphosphotyrosine antibody. We show that, in CHO.2N cells, but not in CHOT cells, inhibition of PI $3-\mathrm{K}$ with $100 \mathrm{nM}$ WTMN for $10 \mathrm{~min}$ at $37^{\circ} \mathrm{C}$ leads to a 367 $\pm 17 \%(p<0.01, n=3)$ increase in basal FAK phosphorylation. Insulin, unlike other growth factors, stimulates dephosphorylation of FAK. In both CHOT \& CHO.2N cells insulin stimulation results in - 50\% decrease in FAK phosphorylation. However, $100 \mathrm{nM}$ WTMN prevented the insulin-stimulated dephosphorylation of FAK in CHO.2N cells ( $114 \pm 23 \%$ of no insulin) but was without effect on FAK dephosphorylation in CHOT cells $(45 \pm 13 \%$ of no insulin $p<0.05$ ). Finally, CHO.2N cells contain - twice the level of PI $3-\mathrm{K}$ p85 subunit and, upon insulin stimulation, there was a 5 fold greater association of p85 with IRS- 1 in these cells $(n=3)$. Thus wo have demonstrated changes in parameters associated with PI 3-K in CHO.2N colls which express a mutant IR and have cytoskeletal alterations. Thosc results imply a rolc for PI 3-K in the altered phenotype of $\mathrm{CHO} .2 \mathrm{~N}$ cells and further strengthens a role for this enzyme in cytoskeletal organisation.

\section{5}

RT-PCR ANALYSIS OF TISSUE SPECIFIC GENE EXPRESSION ON MICROSPECIMEN OF SUBCUTANEOUS DEPOTS IN OBESE SUBJECTS A. Napolitano, P. Maffei, R. Perin, C. Martini, E. De Carlo, C. Scandellari and N. Sicolo - Ist Semeiotica Medica, Patologia Medica III^ University of Padua, Italy. Molecular studies on adipocyte gene expression and regulation of adipsin (ADN), leptin and TNF- $\alpha$ have recently improved our understanding on adipose tissue. In human, extensive in situ studies have been prevented for the difficulties in obtaining enough tissue for most conventional analytical tecniques. We developed a method in which through needle biopsies we obtained enough adipose tissue to perform a semiquantitative RT-PCR analysis. Needle biopsies (Vasofix 14G) have been performed on abdominal and gluteofemoral depots in 6 lean (M/F=5/1; age $32 \pm 3 \mathrm{yrs}$; $\mathrm{BMI}=23 \pm 3$ ) and 7 obese subjects ( $\mathrm{M} / \mathrm{F}=1 / 6$; age $47 \pm 10 \mathrm{yrs} ; \mathrm{BMI}=37 \pm 7$ ). After extraction of total RNA, a semiquantitative RT-PCR has been carried out Since the impossibility of detection of RNA concentrations through spectrophotometry, an unknown amount of RNA has been reverse transcribed and cDNA has been coamplified for ADN and glyceraldeide-3-phosphate-dehydrogenase (GAPDH) gene, the latter used as control. The PCR has been done in the presence of digoxigenin and labelled nucleotides; we utilized an ELISA PCR method with biotin-labelled oligos as specific capture probes. The cDNA levels have been quantitated by an antidigoxigenin peroxidase conjugate followed by a colorimetric reaction $(405 \mathrm{~nm})$. The data have been expressed in log scale and as a ratio of cDNA: ADN/GAPDH. Preliminary results seem to indicate that ADN mRNA levels in obese were significantly reduced in comparison with lean controls $(0.01 \pm 0.9$ vs $0.66 \pm 0.5 ; \mathrm{p}$ $<0.05$ ) while no significant differences of $A D N$ gene expression have been found in relation to gender or depots. Furthermore, we demonstrated a negative correlation between ADN mRNA levels and BMT $((p<0.02, r=-0.6)$ and weight $(p<0.0001, r=-$ $0.7)$. In conclusion, we observed a significative reduction of ADN mRNA levels in subcutaneous depots of obese subjects. This method could be utilized to carry out in humans further studies on the expression of various metabolic important genes involved in the regulation of energy balance. 
646

The Contribution of Cytosolic and Secretory Phospholipase $A_{2}$ to Phospholipase A2 Activity in Control and Diabetic Glomeruli R. J. Keogh, M. E. Dunlop, and R. G. Larkins. Department of Medicine, Uni. of Melbourne, PO Royal Melbourne Hospital, Parkville, Victoria, 3050, AUSTRALIA,

An increase in the production of vasoactive prostaglandins is one mechanism thought to contribute to glomerular hyperfiltration at the onset of diabetes. The enzyme phospholipase $\mathrm{A}_{2}\left(\mathrm{PLA}_{2}\right)$ catalyses the release of arachidonic acid from membrane phospholipids for prostaglandin production and PLA2 activity has been shown to be increased in diabetic glomeruli. PLA 2 exists in several forms including a cytosolic form (cPLA 2 ) and a secretory form (SPLA $)$. To date, no study has identified the forms of PLA2 in the diabetic glomerulus, hence the aim of this work was to determine which forms of PLA2 are present and contribute to PLA 2 activity in control and diabetic glomeruli. Male Sprague-Dawley rats weighing approximately $180 \mathrm{~g}$ were made diabetic by a single intra peritoneal injection of Streptozotocin (STZ, $70 \mathrm{mg} / \mathrm{kg}$ body weight). Animals were killed following one or three weeks of STZdiabetes and glomeruli prepared by differential sieving of kidney cortices. Total PLA 2 activity of glomenular cytosolic preparations was measured in vitro by the hydrolysis of an exogenous ${ }^{14} \mathrm{C}$-labelled arachidonyl phosphatidylcholine substrate. Dithiothreitol (DTT) pre treatment of samples $\left(10 \mathrm{mM}, 30\right.$ minutes, $\left.37^{\circ} \mathrm{C}\right)$ was used to inactivate cell associated SPL. $A_{2}$. Results are expressed as \% of the timed control value in the absence of DTT and represent the mean \pm SEM of $n=4$ separate experiments with $n \geq 3$ independent determinations in each. ${ }^{*} p<0.05$ with respect to relevant control (Student's $t$ test).

relevant control (Student's $t$ test).
\begin{tabular}{|l|c|c|c|c|}
\hline Duration of STZ-diabetes & \multicolumn{2}{c|}{ One Week } & \multicolumn{2}{c|}{ Three Weeks } \\
\hline \hline DTT treatment & - & + & - & + \\
\hline \hline Control & $100 \pm 16$ & $80 \pm 10$ & $100 \pm 17$ & $106 \pm 24$ \\
STZ-Diabetic & $133 \pm 29$ & $90 \pm 14$ & $185 \pm 42 *$ & $209 \pm 74^{*}$ \\
\hline
\end{tabular}

Total PLA 2 activity is not altered following one week of STZ-diabetes but is significantly increased after three weeks. DTT pre treatment is without effect on $\mathrm{PLA}_{2}$ activity in control or STZ-diabetic glomeruli after STZ-diabetes of one or three weeks duration. From this we conclude that no sPLA 2 activity is detectable in glomeruli immediately ex vivo and that $\mathrm{CPLA}_{2}$ is the form contributing to measurable PLA 2 activity in control and STZ-diabetic glomeruli.

\section{PS 29}

\section{Glucose Transporters}

648

\section{EVIDENCE FOR GLUCOSE TRANSPORT INHIBITORS IN} MOMORDICA CHARANTIA L

C. Lau ${ }^{1}$ A. Raman ${ }^{1}$, M. Noel ${ }^{2}$, M. Kergoat ${ }^{2}$ M.J. I awrence ${ }^{1}$ and A.N.O. Dodoo'. 'Department of Pharmacy, King's College London, Manresa Road, l.ondon SW3 6LX. United Kingdom; ${ }^{2}$ Pharmacology Research Laboratories, Lipha, Chilly-Mazarin, France F-91380.

The unripe fruit of Momordica charantia L. (Cucurbitaceae) has been used traditionally in the treatment of diabetes mellitus. Its hypoglycaemic activity in both human and animal models has been widely reported. The aim of our study is to investigate whether $M$. charantia inhibits glucose absorption from the gut using in vivo and in vitro models. Following confirmation of improvement in oral glucose tolerance by the fruit juice $(10 \mathrm{ml} / \mathrm{kg}$ body weight) in streptozotocin-induced NIDDM rats $(\mathrm{n}=6)$, we found that in vivo anti-hyperglycaemic activity resided in a hexane extract of the juice. However, this extract did not improve intravenous glucose tolerance, suggesting that it inhibited glucose uptake from the gut. This was further investigated using brush border membrane vesicles (BBMV), prepared from frozen small intestine of rabbit. Uptake of radiolabelled Dglucose was measured at $25^{\circ} \mathrm{C}$. The reaction was initiated by adding $20 \mu \mathrm{l}$ BBMV to a mixture of $20 \mu$ l glucose $(0.2 \mathrm{mM})$ and $20 \mu 1$ of $0.3 \%$ Tween 80 or hexane extract dissolved in $0.3 \%$ Tween 80 . At 20 seconds, glucose uptake with Tween 80 (control) was $428.49 \pm 20.31$ pmoles $/ \mathrm{mg}$ protein (Mean \pm SEM, $n=6$ ). The hexane extract at concentrations of $0.073 \mu \mathrm{g} / \mu \mathrm{l}$ and $0.73 \mathrm{Hg} / \mu \mathrm{l}$ resulted in percentage inbibitions of $10.76 \pm 1.58 \%(\mathrm{p}<0.1$, compared to control by unpaired t-test) and $33.50 \pm 2.13 \%(p<0.0001)$ respectively $(\mathrm{n}=6)$. There was no significant change in equilibrium glucose concentration at 3600 seconds, indicating that the BBMV remained intact. These results suggest that $M$. charantia fruit contains non-polar glucose uptake inhibitor(s), the chemical structure of which remains to be found. The BBMV model will be used as a guide for isolation of the inhibitor(s).
647

THIS ABSTRACT HAS BEEN .WITHDRAWN BY THE AUTHOR.

\section{9}

VANADATE REVERSES CYCLIC AMP-INDUCED SUPPRESSION OF GLUT 4 GENE EXPRESSION IN 3T3-F442A CELLS.

J. W. Eriksson and Z.W. Yu. The Lundberg Laboratory for Diabetes Research, Department of Medicine. University of Göteborg. Sahlgrenska University Hospital. S- $+13+5$ Göteborg. Sweden.

To further elucidate the mechanisms of cAMP-induced insulin resistance we exumined effects of CAMP. insulin and the tyrosine phosphatase inhibitor vanadate on GLUT + and insulin receptor (IR) gene expression in cultured 3T3-F442A mreadipocytes. 3T3-F442A preadipocytes were cultured in low-glucose $(5.6 \mathrm{mM})$ medium (DMEM) until day 8 after confluence, and then treated with the indicated agents. Northem blots showed that both insulin $\left(10^{4}, \mathrm{U} / \mathrm{ml}\right)$ and vanadate $(5-10$ 1) clearly increased GLUT + nRNA levels. whereas 8-bromo-cAMP or $\mathrm{N}^{6}$. mbcAMP $(1-4 \mathrm{mM})$ produced a marked, dose-dependent reduction in basal and insulin-stimulated GLUT 4 mRNA. Accordingly. GLUT 4 protein content in whole cell assessed by Western blots was decreased by the cAMP analogs. In addition. ${ }^{1+} \mathrm{C}$-deoxyglucose uptake was increased by $\sim 6$-fold $(\mathrm{p}<0.01$ ) after a shorttem (30 min) insulin treatment, and this effect was reduced, whereas basal glucose uptake was increased by cAMP pretreatment for $16 \mathrm{~h}$. To study the involvement of IR on the requlation of GLUT 4 gene expression. IR $\mathrm{mRNA}$ and protein as

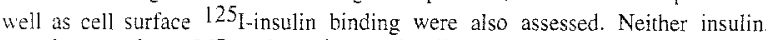
vanadate nor the cAMP analogs changed insulin receptor mRNA content during 16-48 h treatments. Insulin treatment for $16 \mathrm{~h}$ reduced 125 [-insulin binding at the cell surface by $\sim 40 \%$ ( $<<0.05$ ), and this was opposed by the cAMP analogs which by themselves slightly increased binding. Essentially parallel changes were seen in IR protein levels. Conclusions: GLUT 4 gene expression in 3T3-F442A preadipocytes cultured in low-glicose medium is enhanced by insulin, which is blunted by cAMP. Vanadare alone stimulates GLUT 4 gene expression, and also is capable to reverse the cAMP-induced reduction in insulin action suggesting the importance of tyrosine kinase-related mechanisms on the reglation of GLUT 4 gene expression. The impairment of insulin-stimulated glucose uptake in adipocytes following long-term cellular cAMP elevation can be partly due to the reduction of GLUT 4 gene and protein expression. In contrast, IR gene expression seems unaffected by insulin, vanadate or cAMP treatment alone or in combination. 
650

EXPRESSION AND LOCALIZATION OF GLUT1 AND GLUT3 IN PERIPHERAL NERVE OF DIABETIC BB/W RAT.

P. V. Cherian ${ }^{1}$, F. C. Brosius, III ${ }^{1}$, D. A. Greene ${ }^{1}$, and A. A. F. Sima ${ }^{1,2},{ }^{1}$ University of Michigan, Ann Arbor, MI, U. S. A., ${ }^{2}$ Wayne State University, Detroit, MI, U. S. A.

We have previously demonstrated immunocytochemical localization of GLUT1 to endoneurial endothelium, the perineurium, the node of Ranvier and Schmidt-Lanterman incisures of peripheral nerve in non-diabetic 6 and $13-15$ month old $\mathrm{BB} / \mathrm{W}$ rats. In the same animals GLUT 3 was localized to axonal membranes, endothelial and perineurial cells in 13-15 mo but not in 6 mo old rats. In the present study we examined by Western blot and immunocytochemistry the expression and localization of GLUT1 and 3 in age-matched diabetic $\mathrm{BB} / \mathrm{W}$ rats. Surprisingly, we were not able to demonstrate decreased expression of either GLUT1 or 3 in hyperglycemic diabetic rats. No differences were found with respect to GLUT1 or 3 localization when compared to age-matched control rats. On the other hand the localization of both transporters at the node of Ranvier was not confined to the paranode as previously reported in control rats, but was abnormally distributed along the internodal Schwann cell and axon. These findings may be consequent to the break down in the paranodal barrier in the diabetic rats. In conclusion the present study demonstrated a failure by peripheral nerve to down regulate glucose transporters as a protection against glucotoxicity, which was coupled with redistribution of GLUT1 and 3 in peripheral nerve fibers.

\section{1}

OVEREXPRESSION OF GLUT4 GLUCOSE TRANSPORTER OR HEXOKINASE II IMPROVES IN VIVO MUSCLE INSULIN SENSITIVITY IN TRANSGENIC MICE

AM. Lombardi, PY Chang*, M. Loizeau, J. Girard, D. Moller* and A. Leturque, Meudon, France \&*Boston, MA

Glucose clearance is a two step process involving transport across the plasma membrane and phosphorylation of the internalized hexose. The GLUT4 glucose transporter and type II hexokinase (HKII) are predominately expressed in skeletal muscle and adipose tissue. The aim of our study was to investigate glucose metabolism in transgenic (tg) mice overexpressing the glucose transporter GLUT4. HKII and GLUT4/HKII in striated muscle. An improvement of oral glucose tolerance test $(1 \mathrm{mg} / \mathrm{g}$ bw) was observed in GLUT4 and GLUT4/HKII tg mice versus control mice. The GLUT4 and GLUT4/HKII mice underwent intraperitoneal insulin tolerance test (1.6 $\mathrm{U} / \mathrm{kg}$ ). 4 days after streptozotocin induction of diabetes. The insulin tolerance tests werc improved in GLUT4 tg mice when compared to control but not further improvement was ohserved in GLUT4/HKI compared to GLUT4 transgenic mice. To study glucose uptake in different muscles, a bolus $(30 \mu \mathrm{Ci})$ of 2 -deoxy- $\mathrm{H}$-glucose was injected into the tail vein under basal conditions and in mice subjected to hyperglycaemic clamp. A variable glucose infusion rate maintained the blood glucose at $240 \mathrm{mg} / \mathrm{dl}$. The plasma insulin levels were increased 5 fold in control and 2 fold in tg mice over basal levels. Our results show increased glucose uptake in basal conditions in heart of the GLUT4 and GLUT4/HKII transgenic mice $(p<0.05)$. During hyperglycaemic clamp, increased glucose uptake was also evident in soleus from GLUT4 tg mice $(p<0.005)$, in gastrocnemius and heart from HKII and GLUK4 tg mice $(\mathrm{p}<0.05)$ compared to control mice. In conclusion, these results indicate that overexpression of either GLUT4 or HKII in muscle improves glucose utilization and insulin action whereas the overexpression of both gencs does not seem to further increase these parameters.
652

EFFECT OF TRIYODOTIRONINE ON GLUT4 MRNA EXPRESSION IN 3T3-LI ADIPOCYTES

R. Romero, B. Casanova, N. Pulido, A. Suárez, E. Rodríguez and A. Rovira. Fundación Jiménez Díaz. Madrid. Spain.

The excess of triyodotironine (T3) increases the GLUT4 content in skeletal muscle. The aim of this work was to know the effect of $T 3$ on the GLUT4 protein levels and its mRNA in 3T3L1 adipocytes. Differenciated cells were incubated in the absence and presence of $\mathrm{T} 3$ (50 nM) for three days. Protein GLUT4 was measured by Western-blot and its mRNA levels by Northern-blot. Cells treated with T3 had higher GLUT4 protein levels (1194 \pm 165 vs $626 \pm 140 \mathrm{AU} / 15 \mu \mathrm{g}$ of protein, mean $\pm \mathrm{SEM}, \mathrm{p}<0.05)$ and higher mRNA levels $(8.5 \pm 0.9$ vs $2.3 \pm 0.6$ $\mathrm{AUJ} / 15 \mu \mathrm{g}$ total RNA). In order to know whether the increase in GLUT4 mRNA induced by $T 3$ was due to a higher stability of transcripts, adipocytes incubated in the absence and presence of $\mathrm{T} 3$ were exposed to actinomycin-D $(5 \mu \mathrm{g} / \mathrm{mI})$ for $2,4,8$ and 24 hours. The halflife of GLUT4 mRNA was 8 hours in the absence of $\mathrm{T} 3$ and 13 hours in the presence of the hormone. The high GLUT4 gene expression produced by $\mathrm{T} 3$ was not changed by inhibiting protein synthesis with cicloheximide 15 $\mu \mathrm{g} / \mathrm{ml})$. In conclusion, thyroid hormone increases the GLUT4 protein content and its mRNA levels due, at least in part, to a higher MRNA stabilization.

\section{3}

TISSUE-SPECIFIC REGULATION OF GLUT4 EXPRESSION IN FRUCTOSEINDUCED INSULIN RESISTANCE IN THE RAT

JP Bastard 1, E Rossini 2, C Jardel 1, S Halimi 2 and B Hainque 1 1Biochimie: Hôpital Salpétriêre, Paris. 2 Laboratoire de recherches métaboliques, UFR Médecine Pharmacie, Grenoble, France.

It is known that fructose rich diet induces insulin resistance in rats and we have previously shown that metformin improved it. The present study was performed to investigate whether an alteration of the expression of the insulin regulatable glucose transporter GLUT4 occurs in this model. We studied 12 males Wistar rats after weaning at $50 \mathrm{~g}$ body weight. The animals were divided into 3 groups and fed ad libitum one of the following diets for 6 weeks: 1) normal chow diet, 2) high-fructose diet (HFD), 3) HFD plus metformin. GLUT4 protein content was assessed by immunoblotting in membranes from epididymal and abdominal fat pads, interscapular brown adipose tissue, heart, diaphragin and hindlimb muscles. GLUT4 protein levels are strongly increased in both epididymal and abdominal fat pads in HFD rats by 3.8 fold, $p<0.05$ and 1.8 fold, $p<0.05$ respectively, as compared to control rats, but remains unchanged in HFD rats treated by metformin. No change in GLUT4 expression was observed within the three groups studied in brown adipose tissue. As compared to controls, the following were decreased in both heart and diaphragm respectively: GLUT4 protein contents by $40 \%, \mathrm{p}<0.05$ and $38 \%, p=0.08$ in HFD rats and by $66 \%, p<0.05$ and $49 \%, p<0.05$ in HFD rats treated by metformin. In contrary, a slight increase in GLUT4 expression was observed in leg skeletal muscle in HFD rats by $27 \%$, p<0.05 without any change in HFD rats treated with metformin. This sudy clearly shows that HFD may regulate GLUT4 expression in insulin sensitive tissues. Moreover, among the different tissues studied, HFD seems differentially alterate GLUT4 expression, suggesting a tissue-specific regulation of GLUT4 in this model. The impact of metformin treatment on GLUT4 expression in HFD rats remains unclear and needs further investigations. 


\section{4}

AEGULATION OF GLUCOSE TRANSPORTERS IN MUSCLE AND ADIPOCYTES OF INSULIN RESISTANT RATS: EFFECTS OFN-3 POLY- AND MONO- UNSATURATED FATTY ACIDS

S.Fluteau-Nadler, S. W. Rizkalla, M. Kabir, M. Guerre-Millo, J. Luo, F. Bruzzo and G. Slama Depatment of Diabetes, iNSERM U 341, Hôtel-Dieu Hospital, INSERM U 177 , Paris, FRANCE

We aimed to determine the effects of dietary n-3 poly- or mono-unsatured fatty acids in insulin-resistant rets, and to evaluate whether there is a difference in the underlying celluiar and molecular mechanisms. Fourty eight Sprague-Dawley rats, aged five weeks, were randomly divided into four groups : three groups were fed a diet containing (w/W): $57.5 \%$ sucrose and $14 \%$ lipio's as either fish oil (Maxepa ${ }^{\circledR}(\mathrm{SF}$ ), olive oil (SO) or a mixture of standard oils (SMS). The control group was fed a diet containing $57.5 \%$ starch and $14 \%$ standard oils (C). After 3 week diet, body weight was comparable in the four groups. The sucrose-fed rats (SMS, SO) showed high glycaemic and insulinaemic responses to a glucose load. Fish oll corrected the sucrose-induced high insulin responses, but had no effect on plasma glucose resoonses. Nonfasted plasma lipids were lower in the SF fed rats than those fed SMS or SO diet (triacylglycerols: $p<0.001$, cholesterol: $p<0.001$, free fatty acids: $p<0.001$, ANOVA). Maximal insulin-stimulated glucose transport increased under fish oil feeding in adipocytes (C: $2.5 \pm 0.3$, SMS: $1.7 \pm 0.3$, SF: 4.0 00.8 , SO: $1.7 \pm 0.3 \mathrm{pmol}$ glucose/ $10 \mathrm{mg}$ lipids/ $30 \mathrm{sec}$., $\mathrm{p}<0.005$ ). This was due mainly to an increase in Glut- 4 proteins $(p<0.05)$ and GLUT4 gene expression. in muscle, no detected effect was found on GLUT4 proteins, however, GLUT4 gene expression was markedly increased. Olive oil, however, could not ameliorate either glucose or lipid metabolism. In conclusion, 3 weeks of n-3 poly-unsatured fatty acid rich diet to sucrose-fed rats decreased plasma triacyiglycerols, corrected hyperinsulinaemia, restored the glucose transport in adipocytes by a transcriptional up requiation of the glucose transporters GLUT4. Thus, fish oil and not olive oil could have beneficial effects in insulin-resistant rats, that is essentially due to gene expression regulation at the adipocyte level before the appearance of any net effect at the muscle level.

\section{5}

INSULIN RESISTANCE IN SKELETAL MUSCLE FROM SMOKERS DOES NOT INVOLVE ALTERATIONS IN GLUT4 EXPRESSION. J. Rincon, J.R. Zierath, E. Ödegaard and H. Wallberg-Henriksson. Department of Clinical Physiology, Karolinska Hospital, Stockholm, Sweden.

Chronic cigarette smoking is associated with reduced whole body insulin mediated glucose uptake. The aim of the present study was to investigate the cellular mechanism(s) behind this whole body insulin resistance. We obtained vastus lateralis skeletal muscle specimens $(600 \mathrm{mg})$ frot 10 male healthy cigarette smokers (smoking $+12 \mathrm{yrs})$ and 10 controls $(28 \pm 2$ yrs) using an open muscle biopsy procedure. The smokers were instructed to abstain from smoking 12 hrs prior to investigation. Five smokers were randomly asked to smoke two cigarettes within 20 min before undergoing a second muscle biopsy. Basal in vitro glucose transport was 2.2-fold $(\mathrm{p}<0.01)$ and 2.1-fold $\mathrm{p}<0.01$ ) higher in muscle from the chronic and acute smokers, respectively when compared to controls. Insulin $(100 \mu \mathrm{U} / \mathrm{ml})$ increased glucose transport 2.7-fold $(p<0.05)$ in muscle from the controls, whereas no significant increase was detected in muscle from acute or chronic smokers. Total GLUT4 expression in muscle was unaltered by chronic cigarette smoking (400 \pm 102 vs $457 \pm 21$ arbitrary units, for smokers and controls, respectively). Similarly, muscle plasma membrane GLUT4 content did not differ significantly with chronic or acute smoking $(820 \pm 91,609 \pm 141$ and $742 \pm 150$, arbitrary units for controls, acute smokers and chronic smokers, respectively). Plasma FFA levels were markedly elevated in the smokers ( $748 \pm 138$ vs $264 \pm 49 \mu \mathrm{mol} / \mathrm{f}$ for smokers vs controls respectively, $\mathrm{p}<0.001$ ). In conclusion, skeletal muscle insulin resistance in cigarette smokers cannot be explained by altertions in total GLUT4 expression. Thus, the increased circulating FFA levels in chronic cigarette smokers may be the mechanism by which smoking alters basal glucose metabolism and impairs insulin action muscle glucose transport.
656

EFFECT OF TRIYODOTIRONINE ON GLUCOSE TRANSPORT AND GLUT4 DISTRIBUTION IN 3T3-L1 ADIPOCYTES E. Rodríguez, R. Romero, N. Dulido, A. Suárez, B. Casanova and A. Rovira. Fundación Jimënez Diaz (UAM). Madrid. Spain

Triyododotironine (T3) has an important role in the regulation of glucose metabolism. The aim of this study was to know the effect of $T 3$ on glucose transport and on the GLUT4 subcellular distribution in 3T3-LI adipocytes. Cells were incubated in the absence and presence of $T 3$ (50 nM) during three days. $\left[{ }^{3} \mathrm{H}\right]-2$ deoxy-glucose uptake was measured in the absence and presence of insulin $\left(10^{-11}-10^{-7} \mathrm{M}\right)$. Subcellular fractions, plasma membranes (PM), low density microsomes (LDM) and high density microsomes (HDM), were isolated under basal and insulin-stimulated $\left(10^{-3}\right.$ M) conditions. GLUT4 was measured by Western-blot using a polyclonal rabbit antisera. T3 produced a 5 -fold increase $(p<0.05)$ in the basal glucose uptake and a 2 -fold increase $(p<0.05)$ in the maximal insulin-stimulated glucose uptake. Total GLUT4 was higher in celis treated with T3 $(1194 \pm 165$ vs $626+140$ U.A/15 $\mu g$ of protein. $p<0.05)$ ). In the absence of insulin, T3 modified the GLUT4 subcellular distribution producing a 2 fold increase $(\mathrm{p}<0.05)$ in the amount present in PM relative to the total. The subcellular distribution of GLUT4 after insulin exposure was not modified by T3. In conclusion: thyroid hormone stimulates glucose uptake by increasing total GLUT4 content and by favouring its recruitment in the plasma membrane.

\section{7}

EFFECTS OF HYPERGLYCEMIA IN VITRO ON THE GLUT 1 GLLCOSE TRANSPORTER SYSTEM OF ISOLATED HUMAN TERM TROPHOBLASTS. G. Desoye, T. Hahn, S. Barth, M. Hartmann and A. Blaschitz. Department of Obstetrics and Gynecology and Institute of Histology and Embryology, University of Graz, A-8036 Graz, Austria

The trophoblast forms the main barrier for maternal-fetal glucose transport in human placentas. The present study investigated the location of the glucose transporter isoform GLUT1 and the effect of hyperglycemia in vitro on the GLUT1 system of trophoblast cells in term human placentae. Trophoblast cells were isolated by a standard procedure and subsequently immunopurified using an HLA class-l antibody. Cells were cultured for $48 \mathrm{hrs}$ in DMEM containing either 5.5 (control) or $25 \mathrm{mmol} / 1 \mathrm{D}$-glucose (hyperglycemia) or $25 \mathrm{mmol} / \mathrm{l} \mathrm{L}$-glucose (osmotic control), respectively. Initial uptake of glucose was measured after $5 \mathrm{~s}$ using the non-metabolising glucose analogue 3-O-[ $\left.{ }^{14} \mathrm{C}\right]$ methyl-D-glucose at a total concentration of either 5.5 or $15 \mathrm{mmol} / \mathrm{l}$ exogenous glucose. GLUT1 immunoreactivity was abundant in the microvillous membrane of the syncytiotrophoblast, in cytotropboblast and endothelial cells of chorionic vilii. Uptake rates of cells cultured under hyperglycemic conditions did not differ at 5.5 mmol/l glucose, but were decreased by $25 \%(p<0.05)$ at $15 \mathrm{mmol} / \mathrm{l}$ substrate concentration as compared to control conditions. The levels of GLUT1 transcripts (Northern blotting) were lower by $20 \%(\mathrm{p}<0.05)$ and of GLUT1 protein (Western blotting) were $40 \%$ greater $(\mathrm{p}<0.05)$ in cells cultured under hyperglycemic as compared to control conditions. We conclude that 1) GLUT1 is expressed in all major cells of the human term placenta, 2) prolonged hyperglycemia in vitro stimulates glucose uptake only at diabetes-like substrate concentrations, and 3) transcription of GLUT1 in the term human trophoblast is not inducible by glucose. (Grant P9140 Austrian Science Foundation) 
658

ROLE OF EICOSANOIDS IN THE REGULATION OF GLUCOSE TRANSPORT IN AORTIC VASCULAR CELLS

S. Sasson, R. Reich, $\mathbb{I}$ N. Kaiser, A. Davarashvili, and $\mathbb{T E}$. Cerasi. Departments of Pharmacology and "Endocrinology \& Metabolism, Hebrew University-Hadassah Medical Center, Jerusalem, Israel

Arachidonic acid metabolites are involved in many regulatory mechanisms in vascular cells. We studied the effect of esculetin, a non-specific lipoxygenase inhibitor, on the glucose transport system of bovine aortic smooth muscle (SMC) and endothelial cells (EC). Both types of cells increased the rate of hexose transport under esculetin treatment in a dose- and time-dependent manner: maximal effect (2-3-fold increase) being observed with 50-100 $\mu \mathrm{M}$ within $8 \mathrm{hr}$. This effect of esculetin was accompanied by increased GLUT-1 level in the plasma membrane of the cells, as determined by the cell surface biotinylation technique. Specific cyclooxygenase and 5-lipoxygenase inhibitors had no effect on hexose transport, indicating that neither prostaglandins nor leukotrienes are involved. The identification of the eicosanoids involved is currently under investigation. The similar effect of esculetin in both types of cells is in contrast with the autoregulatory effect of glucose on hexose transport which is restricted to SMC. These results show, for the first time, that eicosanoids participate in the regulation of hexose transport in vascular cells and may affect overall glucose disposal while protecting the vascular cells from toxic effects of glucose under hyperglycemic conditions.

\section{9}

SULFONYLUREAS STIMULATE GLUT4 TRANSLOCATION IN RAT SKELETAL MUSCLE

N. Pulido, E. Romero, A. Suárez, E. Rodríguez, B. Casanova, F.J. Arrieta and A. Rovira. Fundación Jiménez Díaz (UAM). Madrid. Spain.

We have previously reported that gliclazide stimulates the glucose uptake by skeletal muscle through an ATP-sensitive $\mathrm{K}^{+}$channel dependent mechanism. In this study we investigated whether gliclazide can promote the GLUT4 translocation in skeletal muscle. Rat hindquarters were perfused $20 \mathrm{~min}$ in the absence of drugs and $30 \mathrm{~min}$ with either $300 \mu \mathrm{g} / \mathrm{ml}$ of gliclazide or insulin $\left(10^{-9} \mathrm{M}\right.$ or $\left.10^{-7} \mathrm{M}\right)$, or with the combination of gliclazide $(300 \mu \mathrm{g} / \mathrm{ml})$ and insulin $\left(10^{9} \mathrm{M}\right)$. Plasma membranes (PM) and low density membranes (LDM) were isolated from gastrocnemius muscles taken at the end of the perfusion. GLUT4 was measured by Western-blot using a polyclonal rabbit antisera. The basal glucose uptake was increased 2.7 -fold by gliclazide $(p<0.05$ ). Gliclazide produced a 2.5 -fold enrichment in the GLUT4 content in PM $(\mathrm{p}<0.05)$. Insulin at the concentration of $10^{-9} \mathrm{M}$ produced a 2.8-fold increase in the glucose uptake and a 2.5 -fold increase in the GLUT4 level in PM, which were similar to those produced by gliclazide. The effect of the combination of gliclazide $(300 \mu \mathrm{g} / \mathrm{ml})$ and insulin $\left(10^{-9} \mathrm{M}\right)$ on the glucose uptake $(3.4$-fold respect to the basal value) and on the PM enrichment in GLUT4 (3.7fold respect to the basal value) were higher to those produced by either gliclazide or insulin alone, but similar to those obtained with insulin $\left(10^{-7} \mathrm{M}\right)$. In conclusion, the direct stimulatory effect and the enhancing insulin stimulatory action of gliclazide on glucose uptake by skeletal muscle are produced by promoting the movement of GLUT4 to the plasma membrane.

\section{0}

HIGH FAT FEEDING IMPAIRS INSULIN-STIMULATED GLUT4 RECRUITMENT IN MUSCLE VIA AN EARLY SIGNALING DEFECT. J.R. Zierath, K.L. Houseknecht, L. Gnudi, and B.B. Kahn. Department of Medicine at Harvard Medical School and Beth Israel Hospital, Boston MA, USA.

Glucose transport in skeletal muscle can be mediated by two separate pathways, one stimulated by insulin and the other by contraction. High fat feeding impairs glucose transport in muscle, however the mechanism remains unclear. FVB mice ( 3 wks old) were fed either a high fat diet (55\% fat, $24 \%$ carbohydrate, $21 \%$ protein) or standard chow for $3-4$ wks or 8 wks. Insulin-stimulated glucose transport assessed with either 2-deoxyglucose or 3-O-methylglucose was decreased $35-40 \%(p<0.001)$ in isolated soleus muscle, regardless of diet duration. Similarly, glucose transport stimulated by okadaic acid, a serine/threonine phosphatase inhibitor, was also $35 \%$ lower with high fat feeding $(p<0.001)$, but the glucose transport response to hypoxia or W-7 (stimulators of the "contraction pathway") was intact. Hexokinase I, II and total activity was normal in soleus muscle from high fat fed mice. GLUT4 expression in soleus from high fat fed mice was normal, but insulin-stimulated cell surface recruitment of GLUT4 assessed by exofacial photolabeling with ${ }^{3}[\mathrm{H}$-ATB bis-mannose, was reduced by $47 \%$ $(\mathrm{p}<0.001)$. IRS-1 associated PI3 kinase activity stimulated by insulin was also reduced $36 \%(p<0.001)$ and expression of $p 85$ and $\mathrm{p} 110_{\beta}$ subunits of PI3 kinase was normal. In conclusion, high fat feeding selectively impairs insulin-stimulated, but not contraction-pathway-mediated glucose transport by reducing GLUT4 translocation and/or fusion with the plasma membrane. This appears to result from an acquired defect in insulin activation of PI3 kinase. Since the effects of okadaic acid on glucose transport are independent of PI3 kinase, a second signaling defect may also by induced. 
PS 30

\section{Insulin Action in Vivo}

661

THE RELATIONSHIP BETWEEN INSULIN-MEDIATED INCREASES IN LEG BLOOD FLOW AND GLUCOSE UPTAKE IS TIME-DEPENDENT.

J.C. ter Maaten, A. Voorburg, R.J. Heine, A.J.M. Donker and R.O.B. Gans. Department of Internal Medicine, ICaR-VU, Research School V.U.-Hospital, Amsterdam, The Netherlands. Insul in has several hemodynamic effects, apart from its metabolic actions. The aim of this study was to assess the relationship between the hemodynamic and metabolic effects of insulin. We investigated the effects of exogenous insulin $(50 \mathrm{mU} / \mathrm{kg} / \mathrm{hr})$ on blood pressure, heart rate, stroke volume and leg blood flow, and their relation to the insulin-mediated glucose uptake during a four hour euglycemic clamp in 12 heal thy subjects. Systolic blood pressure was related to basal plasma insul in levels $(r=0.63, p=0.03)$. Whole body glucose uptake, expressed as M/I value, increased from $4.04 \pm 1.83$ during the second hour to $4.40+1.16$ $\mathrm{mg} / \mathrm{kg} / \mathrm{min}$ per pmol insulin/1x100 during the fourth hour of the clamp. Systolic and diastolic blood pressure increased during insulin infusion $(120.5 \pm 6.8$ to $129.1+8.6 \mathrm{mmHg}$, and $62.7+6.0$ to $68.9+7.4 \mathrm{mmHg}, p<0.001$ ) whereas heart rate was unaltered. Stroke volume index increased from $56.5+13.3$ to $63.7 \pm 16.3 \mathrm{ml} / \mathrm{m}^{2} \quad(p<0.01)$. Leg blood flow gradual $1 \mathrm{y}$ increased from $0.94+0.30$ to $1.36+0.57 \mathrm{ml} / \mathrm{min} / \mathrm{dl}$ during the second hour and to $1.57+0.65 \mathrm{ml} / \mathrm{min} / \mathrm{dl}$ during the fourth hour of the clamp $(p=0.01)$. Increments in leg blood flow correlated with insul in-mediated alucose disoosal during the fourth hour of the clamp $(r=0.86, p<0.01)$, but not the second hour $(r=0.21, p=0.51)$. A control study in 7 of them showed no significant changes in systemic hemodynamics.

Thus, a time-dependent effect of insul in on blood flow and glucose uptake was shown. The presence of a close but not complete relationship between both actions of insulin suggests that insulin-induced increases in blood flow can only partially explain increases in glucose uptake.

\section{3}

IS INSULIN-INDUCED VASODILATION SECONDARY TO CHANGES IN GLUCOSE METABOLISM?

C. de Haan, A. Houben, F. Huvers. P. de Leeuw, B. Wolffenbuttel and N. Schaper. Dept. of Medicine, University Hospital Maastricht, The Netherlands.

Physiological hyperinsulinemia (INS) induces vasodilation in systemic, englycemic clampstudies, possibly via increased production of endothelial derived nitric oxide (EDNO). To study regional vasoactive effects of insulin, forearm blood flow (plethysmography, FBF) was determined during 4 hrs regional ins. To determine the role of glucose in insulin-induced vasodilation, on a second occasion, the offect of combined INS and hyperglycemia (GLYC) was studied. To determine the role of EDNO, the vasoreactivity to L-NMMA was studied. Suhjects and methods: 15 healthy men, age 22 (19-23) yrs (median + intery. ranges), BMI 22.8 (20.5$23.7) \mathrm{kg} / \mathrm{m}^{2}$, were studied. Insulin $(7 \mathrm{mU} / \mathrm{min})$, with or without concomitant glucose $5 \%$ (regional GLYC $15 \mathrm{mmol} / \mathrm{h}$ ), was infused into the brachial artery (i.a.). FBF was measured st baseline, and 45,90, 135, 180 and 225 minutes after start of insulin infusion. L-NMMA (1-2-8 $\mu \mathrm{mol} / \mathrm{mmin}$, each dose for $5 \mathrm{~min})$ was infused i.a. at baseline and after $225 \mathrm{~min}$ INS or INS/GLYC. Flow data are expressed as index (infused/contralateral arm), to correct for systemic changes. Vasoreactivity to L-NMMA was calculated as \% change index. Results: Mean regional insulin levels were approximately $90 \mathrm{mU} / 1$ on both days, systemic glucose levels did not change. INS: FBF index showed a minor increate of $18 \%(8-45)$ at $t=45 p<0.01$ (Wilcoxon), and rose to $45 \%(14-93)$ at $t=225(\mathrm{p}<0.04$ vs $t=0$ and $45 \mathrm{~min})$. INS/GLYC: FBF index increased $59 \%$ (39-72) at $\mathrm{t}=45$ (also higher than $\mathrm{t}=45 \mathrm{INS}$ ), and rose up to $77 \%(56-134)$ at $t=225$ (all $n<0.01)$. Maximal achieved vasodilation during INS/GLYC was higher than during INS $\mathrm{p}<0.04$. During INS $/$ GLYC LNMMA-induced constriction $(8 \mu$ mol) increased relatively to baseline: FBFindex $52 \%(33-64)$ vs $35 \%(14-49), p<0.03$. The same trend was seen during ins. Conclusions: Insulin-induced vasodilation has a relative slow onset, and concomitant hyperglycemia induces a more rapid onset and a higher maximal effect. This vasodiation is (partially) mediated by EDNO. Stimulation of EDNO by insulin is possibly secondary to insulin-induced changes in glucose metaholism.

\section{2}

VASOCONSTRICTOR-MEDIATED BLOOD FLOW REDISTRIBUTION AND ACUTE INSULIN RESISTANCE IN RAT SKELETAL MUSCLE , ITI\%

M.G. Clark* S. Rattigan and E.J. Barrett. *Department of Biochemistry. University of Tasmania. Australia and University of Virginia Diabeles Cenier. Charlottesville. USA. Dysregulation of muscle blood fow may contribute to insulin resistance (IR) in NIDDM and hypcrension. Scrotonin (5HT)-mediated vasoconstriction mimics the effect of sympathetic nerve stimulation to cause IR in perfused skeletal muscle. 5HT has no dircel metabolic action on muscle cells but in the constant-flow perfused hindlimb alters the perfusate flow distribution. Metabolism of 1-methylxanthine (1MX), a substrate for capillary-endotheial xanthine oxidase is decreased by $5 \mathrm{HT}$ in the perfused hindlimb confirning its usefunness as a marker of now distribution. Thus our aim in the present study was to determine the effects. in wivo, of the peripheral acting SHT agonist. ( $x$-methylscrotonin $(x-$-mel5HT). on muscle blood flow distribution and insulin-mediated glucose uptake. Anesthelized rats were treated with either saline (CON) or $\left(x-\mathrm{met} 5 \mathrm{HT}(20) \mathrm{gg} \cdot \mathrm{min}^{-1} \cdot \mathrm{kg}^{-1}\right.$ ) alone or in combination with an euglycaemic insulin clamp ( $\left(10 \mathrm{mU}\right.$. minn $\left.{ }^{-1} \mathrm{~kg}^{-1}\right)$. Mcan arterial blood pressurc (BP). heart rate (HR). and femoral blood flox (FBF) were continuously monitored. Hindlimb blood flow distribution was assessed by arteriat-renous femoral blood sampling to determine the mctabolism of 1-MX. Whole body and leg glucose metabolism was cstimatcd from the glucose infusion rate (GIR) necded to maintain cuglycemia during the clamp and by the artcrial femoral venous glucose measurement. Compared to CON. $\alpha$-met5HT alone increased BP (113 \pm 5 is $135 \pm 3 \mathrm{mmHg}$. p $<1.01)$ and HR (332 \pm 19 vs $459 \pm$ 21 bpm. $p-0.002)$ but did not change FBF $\left(0.81 \pm 0.07\right.$ is $\left.0.69 \pm 0.10 \mathrm{ml} \mathrm{min}^{-1}\right)$. Despitc unchanged FBF. hindlimb 1-MX disappearance decreased (9.52 \pm 1.13 is 5.14 $+1.55 \mathrm{nmol} \mathrm{min}^{-1}, \mathrm{p}-(0.05)$ indicating less capillan flow: During the clamp $\alpha$-meisHT also decreased GIR (25.1 \pm 0.7 is $14.4 \pm 1.5$ mg glucose. $\mathrm{min}^{-1} \cdot \mathrm{kg}^{-1} \cdot \mathrm{p}-0.0(0) 1$ ) and hindlimb muscle glucose uptake $\left(324 \pm 5+\right.$ is $162 \pm 26 \mu \mathrm{g} \cdot \mathrm{min}^{-1}$. $\left.\mathrm{p} 00.05\right)$. It is concluded that it - met5HT. acts in skelctal muscle in vimo to increase BP. decrease muscle capillary blood flow and cause acure IR. Changes in blood flow distribution within skeletil muscle in hypertension may contribute to insutin resistance

\section{4}

DOES INSULIN PLAY A ROLE IN POSTPRANDIAL HYPOTENSION IN THE ELDERLY?

MT Keamey, TA Stubbs, A Evans, AJ Cowley and IA Macdonald. Queens? Medical Centre Nottingham.

Posiprandial (PP) hypotension is common in the elderly but the mechanisms underlying this are unclear. After a high fat meal (HF) when blood pressurc (BP) is well mainlained there is an increase in calf vascular resistance (CVR) not seen after a high carbohydrate meal (HC). Insulin (1), a skcletal muscle vasodilator, may account for this failure of vasoconstriction after $\mathrm{HC}$, leading to hypotcnsion. This study aimed to assess the role of 1 in PP cardiovascular (CV) homeostasis in the clderly. Wo measurcd the $\mathrm{CV}$ responses to a $\mathrm{HF}$ meal accompanied by a cuglycaemic I infusion reproducing the plasma profile seen after $\mathrm{HC}$, and compared the responses to those occurring alter HC and HF meats alone in 10 healthy clderly subjects (mean age 71.4 22.2). Fasted medsurements of heart ralc (HR), BP, cardiac.output (CO) using Doppler ultrasound and calf blood flow (CBF) using venous occlusion plethysmography were taken. Thesc were repcated on 3 occasions after eating $\mathrm{HC}, \mathrm{HF}$ or $\mathrm{HF}$ accompanied by the I infusion (HFI). Table. CV variables before and after the 3 meals. Data expressed as mean \pm SEM * denotes $\mathrm{p}<0.05$

\begin{tabular}{|l|l|l|l|}
\hline Meal & HF & HC & HFI \\
\hline & Fasted & Fasted & Fastcd \\
\hline HR $(\mathrm{bpm})$ & $35 \mathrm{~min}$ & $35 \mathrm{~min}$ & $35 \mathrm{~min}$ \\
\hline & $62 \pm 3$ & $63 \pm 2$ & $62 \pm 2$ \\
\hline $\mathrm{CO}(1 / \mathrm{min})$ & $68 \pm 2^{*}$ & $69 \pm 2^{*}$ & $71 \pm 2^{*}$ \\
\hline $\mathrm{BP}(\mathrm{mmHg})$ & $3.7 \pm 0.2$ & $4.4 \pm 0.3$ & $4.4 \pm 0.2$ \\
& $5.5 \pm 0.3^{*}$ & $5.4 \pm 0.3^{*}$ & $6.1 \pm 0.4^{*}$ \\
\hline $\mathrm{CBF}$ & $96 \pm 4$ & $98 \pm 4$ & $100 \pm 3$ \\
$\mathrm{(mL} / 100 \mathrm{~m} / \mathrm{min})$ & $39 \pm 3$ & $90 \pm 3^{*}$ & $89 \pm 4^{*}$ \\
\hline $\mathrm{CVR}(\mathrm{U})$ & $2.0 \pm 0.2$ & $2.9 \pm 0.3$ & $3.1 \pm 0.3$ \\
& $33 \pm 3$ & $3.2 \pm 0.4$ & $3.2 \pm 0.4$ \\
\hline
\end{tabular}

Despite an increase in cardiac output there was a fall in BP aficr HC and HFI. The failure to increase CVR duc insulins' action orl the skelctal muscle vascular bed may account for the fall in BP scen in the clderly after $\mathrm{HC}$. 


\section{5}

CHLANGES IN INTRACELLULAR GLUCOSE METABOLISM IN PATIENTS WITH DNSULIN-DEPENDENT DIABETES: A FOLLOW-UP STUDY

N.M Lalic, P.B.Djordjevic, M.Zamakdar, K.Lalic, V.Dimitrijević, A.Jotic, M.Ilic, Diabetes Center, Institute for Endocrinology, Belgrade, Yugoslavia

Previous studies have revealed the impairements in insulin sensitivity in patients with recent-onset insulin-dependent diabetes mellitus (IDDM), but the intracellular oxidative and nonoxidative pathways of glucose metabolism in these patients have not yet been clarified. Therefore, the aim of this follow-up study uras to analyse changes in oxidative and nonoxidative glucose disposal in 25 recentonset IDDM patients (a) in insulin-requiring state immediately atter initiation of insulin tretment (RRS I); (b) in IRS after long-term (3 months) insulin treatment (IRS II) (c) in clinical remission (non-insul in-requiring state (NIRS) lasting more than 30 days) and (d) in relapse of IRS after rernission (IRS III) and to compare the results with those in 15 age-matched healthy controls. The analysis has been done by using a $3 \mathrm{hr}$ euglycenic hyperinsulinemic clamp (inaulin infusion $1 \mathrm{mU} / \mathrm{kg} / \mathrm{min}$, glycernia targeted to $90 \mathrm{mg} / \mathrm{d})$ conbined with indirect calorimetry. All patierts were analysed in euglycemic state obtained in insulin-treated subjects by insulin purnp therapy. We found that total, oxidative and nonoxidative glucose untake were decreased in IRS I $(4.3+1-0.3 ; 1.0+1-0.2 ; 3.3+1-0.4 \mathrm{mg} / \mathrm{kg} / \mathrm{min}$ respectively) compared to controls $(8.4+i-1.1,3,4+1-0.3 ; 5.0+1-0.5 \mathrm{mg} / \mathrm{kg} / \mathrm{min}$ respectively; $p<0.05$ ) The total and oxidative glucose disposal increased significantly in IRS II and NIRS (IRS II: $6.9+1-0.9$ and $3.0+1-0.4 ;$ NIR3: $7.3+1-0.9$ and $3.3+1-0.4 \mathrm{mg} / \mathrm{kg} / \mathrm{min}$, respectively; $0<0.05$ ) to the levels comparable and not different from those in the controls, and then declined again in the RSS III (4.7+\% 0.5 and $1.2+(0.2$; mglkg/min, rospootivoly; $p=0.05$ ). Howeyor, the nonoxidative glucose disposal did not increase in IRS II and NIR.S and remained significantly lower than in the controls (IRS II: $3.9+1-0.5$ and NIR.S: $3.9+1-0.4 \mathrm{~m}$ g/kg/min $\mathrm{p}<0.05)$, as well as it did not change signiticantly in IRS III $(3.5+1-0.4 \mathrm{mg} / \mathrm{kg} / \mathrm{min})$ Our results have demonstrated a defect in nonoxidative pathway of glucose metabolism in recent-onset. IDDM patients which persisted both in IRS and in clinical remission. The results imply that, in contrast to the oxidative glucose uptake, the defect in nonoxidative glucose disposal was not reversible, neither by an adequate exagenous insulinization in IRS nor by the recovery of endogenous insulin secretion in clinical remission.

\section{6}

ALTERED SENSITIVITY OF MUSCLE TO INSULIN IN THE MALE OFFSPRING OF PROTEIN-MALNOURISHED RATS

S.E. OZANNE, C.L. WANG and G.D. SMITH

Dept. of Clinical Biochemistry, University of Cambridge, U.K.

Epidemiological studies have suggested that there is a relationship between fetal growth and the subsequent development of non-insulin dependent diabetes mellitus (NIDDM). Animal studies have shown that the offspring of rats fed a low ( $8 \%$ ) protein diet are significantly less glucose tolerant than those of dams fed a $20 \%$ protein diet during pregnancy and lactation. The livers of these rats are insulin resistant, but peripheral insulin sensitivity has not been addressed. The aim of the present study was to determine the ability of insulin to stimulate glucose uptake in skeletal muscle in the offspring of control and low protein rats. Isolated muscle strips from low protein animals took up significantly (p $<0.001)$ more ${ }^{3} \mathrm{H}$-methyl glucose than controls $(4.26 \pm 0.45$ and 19.2 $+2.5 \mathrm{nmol} / \mathrm{min} / \mathrm{mg}$ muscle for control and low protein respectively). Insulin $(10 \mathrm{nM})$ stimulated glucose uptake in both experimental groups but had a significantly $(p<0.001)$ greater effect on control muscle $(6.2$ \pm 1.0 fold stimulation compared to low protein muscle $(1.4 \pm 0.1$ fold stimulation). Western blot analysis revealed no difference in total Glut 4 protein content in control and low protein muscles. However, subcellular fractionation showed that there was significantly $(p<0.001)$ more Glut 4 in plasma membranes of low protein animal muscles compared to control animals with similar plasma insulin concentrations. Insulin binding studies indicated that this may be related to a two-fold increase in the number of insulin receptors in low protein muscle membranes $\left(1.28 \pm 0.10 \times 10^{11}\right.$ and $2.35 \pm 0.17 \times 10^{11}$ insulin receptors $/ \mathrm{mg}$ muscic membrane protein for control and low protein, respectively, $\mathrm{p}<0.01$ ). These results suggest that programming of muscle insulin sensitivity can occur during fetal life.

\section{8}

IMPROVED GLUCOSE UPTAKE IN EUGLYCEMIC HYPONSULINEMIC $60 \%$ PANCREATECTOMIZED MICE

J.M. Ruiz, F. Martín and B. Soria. Department of Physiology, Facultad de Medicina, Universidad de Alicante, Apdo. 374, 03080 Alicante, Spain.

To study the effects of a reduction in plasma insulin levels on peripheral tissue sensitivity to insulin, we performed $60 \%$ pancreatectomies in $8-10$ weeks old male Swiss mice. 3 groups were studied: Control $(\mathrm{Ctl}, \mathbf{n}=19)$, Sham Pancreatectomy $(\mathrm{Sx}, \mathrm{n}=8)$, and $60 \%$ Pancreatectomy $(\mathrm{Px}, \mathrm{n}=15)$. Blood was obtained by intracardiac puncture on day $\mathrm{O}$ in $\mathrm{Ctl}$, and on day 14 in $\mathrm{Sx}$ and $\mathrm{Px}$ for measurements of glycemia (glucose oxidase), insulinemia (RLA), C-peptide (RIA), and FFA (enzymatic method). Adipocytes were isolated from the epididymal fat pad on the same dates for measurements of glucose uptake at 0 and $1.25 \mathrm{nmol}^{-l^{-1}}$ insulin concentrations ( ${ }^{3} \mathrm{H}-2-\mathrm{DOG}$ method, average of 5 measurements per animal). RESULTS: Plasma glucose was similar in the three groups $(\mathrm{CL}=10.22 \pm 0.56, \mathrm{n}=14$; $\mathrm{S} x=10.89 \pm 0.72, \mathrm{n}=8$; and $\mathrm{Px}=10.94 \pm 0.5 \mathrm{mmol} / 1 ; \mathrm{n}=13)(\mathrm{p}=\mathrm{NS})$. Plasma FFA were also similar in the 3 groups $(\mathrm{Px}=0.694 \pm 0.045, \mathrm{n}=6 ; \mathrm{Ct}=0.62 \pm 0.06, \mathrm{n}=7$; $\mathrm{S} x=0.501 \pm 0.051 \mathrm{mmol} / \mathrm{l}, \mathrm{n}=3)(\mathrm{p}=\mathrm{NS})$. Senum insulin was about $50 \%$ lower in $\mathrm{Px}$ $(156 \pm 39, \mathrm{n}=7)$ than in Ctl $(312 \pm 32, \mathrm{n}=7)$ and $\mathrm{Sx}(320 \pm 67 \mathrm{pmol} / 1, \mathrm{n}=3)(\mathrm{p}<0.03 \mathrm{Px}$ vs $\mathrm{Cu}$ and $\mathrm{Sx}$.). C-Peptide was about $50 \%$ lower in $\mathrm{Px}(0.331 \pm 0.017, \mathrm{n}=9)$ than in $\mathrm{Ctt}(0.762 \pm 0.027, \mathrm{n}=9)$ and $\mathrm{Sx}(0.708 \pm 0.059, \mathrm{n}=3)(\mathrm{p}<0.0001 \mathrm{Px}$ vs $\mathrm{Ctl}$ and $\mathrm{Sx})$. Basal glucose uptake was similar in $\mathrm{Ctl}=18.18 \pm 4.14, \mathrm{n}=4$; $\mathrm{Sx}=24.52 \pm 10, \mathrm{n}=4$; and $\mathrm{Px}_{\mathrm{x}}=12.20 \pm 2.49 \mathrm{fmol}^{-a_{i}}$ uptake was about double in $\mathrm{Px}(214.32 \pm 33.95, \mathrm{n}=3)$ compared to both $\mathrm{Ct}$ $(113.08 \pm 22.3, \mathrm{n}=4)$ and $\mathrm{Sx}\left(98.39 \pm 23.3\right.$ fmoladipocyte $\left.{ }^{-1} \cdot \mathrm{min}^{-1}, \mathrm{n}=4\right)(\mathrm{p}<0.3 \mathrm{Px}$ vs $\mathrm{Ctl}$ and $\mathrm{Sx}$ ). CONCLUSIONS: In non-diabetic animals a reduction insulinemia of about 50\%: 1) did not induced a significant change in glycemia and FFA levels; 2) caused a compensatory increase of insulin induced glucose uptake in adipocytes which we suggest is largely responsible for the maintenance of euglycemia. We also propose the $60 \%$ pancreatectomized mice as a suitable animal model to study the effects of hypoinsulinemia on insulin sensitivity in normoglycemic animals. We conclude that 1 ) muscle insulin resistance induced by HFF is readily ameliorated by three independent, short term physiological manipulations, likely to lower muscle ipid availability. 2) a single-meal reduction in lipid intake in the chronic HFF ta esuls in sthes focal lipid availability is important to the nanifestation of muscle insulin resistance in HFF rats, but mechanisms additional is nanifestation of muscle insulin resistance
ia glucose-fatty acid cycle are involved. 
669

COMPARISON OF VANADIUM BASED PHOSPHATASE INHIBITORS: BIOCHEMICAL, CELLULAR AND IN VIVO STUDIES

C.L. Brand, A.R. Sørensen, V.J. Jensen, S. Branner, H.S. Andersen, B. Hansen, S. Rasmussen, L.L.N. Schmidt and N.P.H. Møller. Novo Nordiz'n, Bagsværd, DK

Vanadate and vanadium complexes are phosphatase inhibitors with insulin-like properties. We compared the biochemical (i.e. protein tyrosine phosphatase (PTPase) inhibition), cellular (i.e. lipogenesis) and in vivo (i.e. glucose lowering) activities of two peroxovanadium compounds (bpV(phen); bpV(pic)), bis(maltolato) oxovanadium (BMLOV) and orthovanadate (OV). Biochemical PTPase inhibition was analyzed with an intracellular (PTP1B) and a receptortype enzyme (PTP $\alpha$ ) using p-nitrophenyl phosphate as substrate. The observed $K i$ values were similar for the all three types of vanadium complexes (PTP†B: 17-20 $\mu \mathrm{M} ;$ PTP $\alpha ; 45 \mu \mathrm{M}$ ) and 1.5-2 fold higher for OV against both PTPases. In vitro: Lipogenesis was measured as conversion of $3 \mathrm{H}$-glucose into extractable lipiós in primary mouse (B6D2F1) adipocytes. The relative potency of the compounds can be summarized as: $\operatorname{bpV}($ phen $)>\operatorname{bpV}($ pic $)>>$ BMLOV > OV. In vivo: Compounds were administered (10 $\mu \mathrm{mol} / \mathrm{kg}, \mathrm{s.c}$.) to groups $(n=5)$ of male B6D2F1 mice and blood samples $(10 \mu)$ were obtained from the tail tip pre-dose and at 30 to $60 \mathrm{~min}$ intervals for 2 hours post-dose enabling calculation of the decrimental or incremental areas under the giucose curves ( $\triangle A U C$; t-test vs vehicle; mean $\pm S E$ ). In 18 hour fasted mice the effects of $\mathrm{bpV}(\mathrm{phen}), \mathrm{bpV}(\mathrm{pic}), \mathrm{BML} . \mathrm{OV}, \mathrm{OV}$ and vehicle on $\triangle \mathrm{AUC}$ were $-81 \pm 12$ $(p<0.002),-123 \pm 6 \quad(p<0.006), 2 \pm 17,-26 \pm 21$ and $16 \pm 18 \mathrm{~min} \cdot \mathrm{mmol} / \mathrm{l}$ respectively. In glucose challenged (2 $\mathrm{g} / \mathrm{kg}$, s.c.) 3 hour postprandial mice, the effects of the compounds (same order as above) were - $131+24(p<0.001)$, $-29 \pm 39,59 \pm 24,16 \pm 12$ and $-8 \pm 9 \mathrm{~min} \cdot \mathrm{mmol} / \mathrm{l}$ respectively, In conclusion, the observed differences in acute effects of vanadate and vanadium compley.es in vitro ard in vivo are due to the different properties of the ancillary ligands ather than different PTPase inhibitory potencies.

\section{0}

ENDOGENOUS DIGITALIS-IIKE SUBSTANCES IN DIABETIC SUBJECTS, E.Martinka, P.Galajda, M. Mokan.

Jepartment of Internal Medicine I, Jesenius Medical School, Martin, SIOVAKIA.

NTRODUCIION: Bndogenous digitalis-like substances (EDLS) represent recently identified substances which are close to cardiac glycosides. These substances via inhibition of $\mathrm{Na}^{+}-\mathrm{K}^{+}-\mathrm{ATP}$ ase increase $\mathrm{Ca}^{2+}$ concentrations and may moduiate various $\mathrm{Ca}^{2+}$, - dependent processess. In regards to our topic: $1 . /$ both insulin sensitivity and insulin secretion are Ca-dependent mechanisms and 2./ EDIS has been found increased in both types of diabetes and to correlate with insulin levels and degree of insulin resistance. AIM OE STODY: In previous studies we have docutiented higher levels of IDLS in pregnant women with gestationai diabetes nellitus as well as correlation of BDLS with glucose induced insulin secretion during 75g-OGMY in these women. In this study we were interested if there are relationships of EDIS to sone basic diabetes paraneters (plasma glucose, insulin and C-peptide leveis) also in non-pregnant patients with both (type-1 and type-2) diabetes mellitus parameters. SUBJECTS AND MEIHODS: 27 type-2 diabetic subjects (NIDDM), 12 type-1 diabetics (IDDM) and 11 healthy controls (HC). FDIS has been measured as digoxin-like innunoactivity. Anti-digoxin antibodies used in this method did not correlate with other steroid substances such as progesteron, estradiol, cortisol and aldosteron, RESULTS: EDLS plasma leveis in NTDDM $(0.19+0.15 \mathrm{nmol} / \mathrm{l})$ were significantly $(\mathrm{p}=0.0006)$ higher than in IDDM (0.04t0.04 nirol $/ 1)$ and non-significantly $(p=0.067)$ higher than in $B D$ (0.10 $0.07 \mathrm{nmol} / 1)$. Moreover, in all subjects EDIS significantly correlated with $C$-peptide levels $(r=0.492 ; \quad p=0.00028)$. Beside this EDLS correlated with age $(I=0.32 ; \mathrm{p}=0.021)$, but did not correlate with other measured parameters such as body mass index, plasma glucose, imunoreactive insulin (IRI), blood pressure, cholesterol levels, triglycerides levels and cortisol levels. CONCLUSTONS: These findings suggest a possible (contributive/modulating) role od BDLS in mechanisms involved in insulin sensitivity and insulin secretion.

\section{PS 31}

\section{Regulation of Metabolism}

\section{1}

THE ANTILIPOLYTIC EFFECT OF INSULIN IN HUMAN ADIPOSE VERSUS SKELETAL MUSCLE TISSUE; ROLE OF PHOSPHODIESTERASE III.

E. Hagström-Toft, S. Enoksson and P. Arner. Departments of Medicine, Surgery and the Medical Research Center, Huddinge Hospital, Karolinska Institute, Sweden. Lipolysis in adipose tissue is inhibited by insulin through different phosphodiesterases (PDE), mainly PDE III. In vivo lipolysis is recently shown to occure also in human skeletal muscle and the aim of this study was to investigate the regulation of insulin-antilipolysis in muscle. Ten healthy, normal-weight (age 31 $(26-49)$ yrs, BMI $\left.23 \pm 1.4 \mathrm{~kg} / \mathrm{m}^{2}\right)$ were investigated with microdialysis of abdominal subcutaneous adipose and skeletal muscle (M. gastrocnernius) before and during a $135 \mathrm{~min}$ euglycemic, hyperinsulinemic $\left(40 \mathrm{U} / \mathrm{m}^{2}\right)$ clamp. Three microdialysis catheters in each tissue $30 \mathrm{~mm}$ polyamide membrane, $20,000 \mathrm{MW}$ cur-off) were perfused at $2 \mu \mathrm{l} / \mathrm{min}$ with Ringer' solution and the general and selective PDEinhibitors theophylline $10^{-2} \mathrm{M}$ and amrinone $10^{-3} \mathrm{M}$ respectively and glycerol (lipolytic index) was analyzed in $15 \mathrm{~min}$ fraction of the dialysate. Ethanol $50 \mathrm{mM}$ was added to the perfusate to estimate changes in the local blood-flow. The insulininduced antilipolysis was attenuated in both tissues by the unselective PDE-inhibitor theophylline (ANOVA adipose tissue $F=12.1$, muscle $F=5.7$ vs. Ringer's pertusion) whereas the PDE-III inhibitor anrinone only counteracted antilipolysis in adipose tissue (ANOVA F=29.4 vs. Ringer's). No major changes in the local blood flow in fat or muscle were observed during the insulin clamp. It is concluded that insulininduced antilipolysis in adipose tissue and skeletal muscle is tegulated through different phosphodiesterases. In subcutaneous fat, PDE III is the main regulating enzyme, but in skeletal muscle other PDE isoenzyme(s) are involved.

\section{2}

EFFECTS OF ANTILIPOLYSIS ON GLUCOSE PRODUCTION AND UPTAKE IN OVERNIGHT FASTED HUMANS.

F. Féry, L. Plat, M. Baleriaux and E.O. Balasse. Laboratory of Experimental Medicine, University of Brussels, Brussels, Belgium.

The role played by circulating FFA levels in the regulation of whole body glucose uptake at low basal insulin levels remains a controversial issue. In the present study we reexamine this question in 8 normal overnight fasted volunteers in whom glucose kinetics ( $\mathrm{Ra}, \mathrm{Rd}$ and MCR) ( $3 \cdot^{3} \mathrm{H}$-glucose infusion) and substrate oxidation rates (indirect calorimetry) were measured during $10.5 \mathrm{~h}$ both under placebo $(\mathrm{P})$ conditions and during experimental antilipolysis induced by Acipimox (APX) (1.5 g given in divided doses during the last $8 \mathrm{~h}$ of the study). At the end of the $8 \mathrm{~h}$, the following changes from baseline were recorded in APX $v s \mathrm{P}: \triangle \mathrm{FFA}:-0.31 \pm 0.05$ vs $+0.35 \pm 0.07$ $\mathrm{mmol} / \mathrm{l}(\mathrm{P}<0.001) ; \Delta$ glucose : $-12 \pm 1$ vs $-13 \pm 1 \mathrm{mg} / \mathrm{dl}(\mathrm{NS}) ;$ insulin : $-9 \pm$ $1 v s-6 \pm 1 \mu \mathrm{U} / \mathrm{ml}(\mathrm{P}=0.02) ; \Delta$ glucagon : $+25 \pm 16$ vs $-14 \pm 12 \mathrm{pg} / \mathrm{ml}(\mathrm{P}<$ $0.05) ; \Delta \mathrm{GH}:+46 \pm 10 v s+2 \pm 2 \mathrm{ng} / \mathrm{ml}(\mathrm{P}<0.001) ; \Delta$ cortisol : $+30 \pm 12 v s$ $-45 \pm 29 \mathrm{ng} / \mathrm{ml}(\mathrm{P}<0.05) ; \Delta R \mathrm{R}:+15 \pm 6 v s-19 \pm 4 \mathrm{mg} / \mathrm{min}(\mathrm{P}<0.005) ;$ $\Delta R . d:+13 \pm 6 v s-15 \pm 4 \mathrm{mg} / \mathrm{min}(\mathrm{P}<0.02) ; \Delta \mathrm{MCR}:+34 \pm 3 v s+4 \pm 4$ $\mathrm{ml} / \mathrm{min}(\mathrm{P}<0.005)$. APX inhibited fat oxidation $(23 \pm 1$ vs $36 \pm 3 \mathrm{~g} / 8 \mathrm{~h} ; \mathrm{P}<$ $0.005)$ and enhanced carbohydrate oxidation $(57 \pm 4 v s 3 \pm 6 \mathrm{~g} / 8 \mathrm{~h} ; \mathrm{P}<0.02)$. A stimulation of protein catabolism was observed $(29 \pm 3$ vs $21 \pm 7 \mathrm{~g} / 8 \mathrm{~h} ; \mathrm{P}<$ 0.005 ) that could account for $\sim 40 \%$ of the rise in Ra. Thus (1) the prolonged lowering of FFA levels stimulates glucose production through changes in the hormonal milieu consisting in a fall in insulin and a rise in glucagon, cortisol and GH levels. (2) Despite these hormonal changes, antilipolysis stimulates glucose MCR and Rd so that glucose concentration remained unaffected. Our data suggest that contrary to current opinion, the glucose-fatty cycle operates even at low basal insulin levels. 
673

GLUCOSE-FATTY ACID INTERACTIONS ARE DIFFERENT BETWEEN PREPUBERTAL AND PUBERTAL CHILDREN: EFFECTS OF LIPID INFUSION ON BASAL AND INSULIN STIMULATED GLUCOSE METABOLISM.

S.Arslanian and C.Suprasongsin, Children's Hospital, Univ of Pittsburgh, Pittsburgh, PA, USA.

It has been suggested that the insulin resistance of puberty may be caused by substrate competition between glucose and free-fatty acids (FFA) (Randle Cycle). This investigation examined whether or not puberty differs from prepuberty in regards to the effects of increased FFA on in vivo glucose metabolism. Nine prepubertal (age 10.6 $\pm 0.3 \mathrm{yrs}$ ) and 13 pubertal (age 13.4 \pm 0.2 yrs) healthy children were studied. Each subject was studied twice, at random order, within 1-2 weeks, once with normal saline (control study) and once with $20 \%$ intralipic infusion. Glucose turnover, oxidation, nonoxidative disposal and fat oxidation were measured in the basal state and during a 3-hour hyperinsulinemic $\left(40 \mathrm{mu} / \mathrm{m}^{2} / \mathrm{min}\right)$-euglycemic clamp with the use of $\left[6,6-{ }^{2} \mathrm{H}\right]$ glucose and indirect calorimetry. Basal fat oxidation was higher in pubertal vs prepubertal subjects (3.2 \pm 0.3 vs $1.8 \pm 0.4 \mu \mathrm{mol} / \mathrm{kg} / \mathrm{min}, p=0.006$ ). Basal glucose turnover was lower in pubertal than prepubertal children $(19.7 \pm 1.2$ vs $23.5 \pm 2.2 \mu \mathrm{mol} / \mathrm{kg} / \mathrm{min}, p=$ 0.05 ). During hyperinsulinemia, insulin mediated total, oxidative and nonoxidative glucose disposal were significantly lower in pubertal vs prepubertal subjects. The response to intralipid ${ }^{\otimes}$ infusion was different between prepubertal children and pubertal adolescents. In the basal period, Intralipid ${ }^{\circ}$ infusion resulted in increased whole body glucose uptake and insulin concentration in prepubertal but not pubertal subjects. During hyperinsulinemia and Intralipid infusion, insulinstimulated whole body glucose disposal was impaired in pubertal adolescents (saline study: $55.0 \pm 3.6$, Intralipid study: $46.7 \pm 3.4 \mu \mathrm{mol} / \mathrm{kg} / \mathrm{min} p=0.01$ ), but not in prepubertal children (saline: $77.6 \pm 8.9$, Intralipid $: 84.5 \pm 13.3 \mu \mathrm{mol} / \mathrm{kg} / \mathrm{min}$ ) despite comparable decrements in glucose oxidation. We conclude that in prepubertal children, lipids exert effects in the basal state by stimulating hepatic glucose production and increasing glucose disposal, while in pubertal adolescents they induce peripheral tissue insulin resistance by decreasing insulin-stimulated glucose uptake. This differential response could be due to developmental/maturational changes in tissue sensitivity and/or specificity to the glucose-FFA interaction

\section{5}

EFFECT OF FREE FATTY ACIDS ON THE FATE OF GLUCONEOGENIC CARBONS FROM LACTATE IN HUMAN Y. Khalfaliah, S. Vinoy and J. P. Riou. INSERM! U449, Faculté de Médecine R. Laënnec, 69372 Lyon, France.

To determine whether free fatty acids (FFA) stimulate gluconeogenesis and/or glycogen synthesis from lactate, we randomly performed in 5 healthy men a 4 hs euglycaemic mildhyperinsulinemic clamp with FFA maintained at fasting basal (BFFA) or low (L-FFA) levels. The apparent flux of carbon 13 from [3-13C] lactate to glucose was measured. To quantify the amount of gluconeogenic carbons stored as glycogen, glycogenolysis was induced by a perfusion of glucagon for $1 \mathrm{~h}$ at the end of insulin infusion. $[6,6-2 \mathrm{H} 2] g$ lucose and [2$2 \mathrm{H} 1 \mathrm{jglucose}$ were used to determine the endogenous glucose production during ciamp (EGPC) and glucagon infusion (EGPg), respectively. A two-compartment model was performed for the calculation of glucose dynamic parameters under glucagon. The results showed that neither EGPC nor EGPg were modified when basal level of FFA was maintained. However, the $13 \mathrm{C}$ glucose appearance during clamp was significantly increased by FFA : $1.0 \pm 0.5$ (mean $\pm S E M)$ vs. $0.5 \pm 0.1 \mu \mathrm{mol} / \mathrm{kg} / \mathrm{min}$ $(p<0.05)$ for B-FFA and L-FFA, respectively. Moreover, the glucagon stimulated release of $13 \mathrm{C}$-glucose was significantly higher following B-FFA study : $63 \pm 7$ vs. $39 \pm 14 \mu \mathrm{mol} / \mathrm{kg} / 60 \mathrm{~min}$ for L-FFA $(p<0.05)$. We conclude that FFA at basal level induce an increase of gluconeogenesis from lactate which lead to an increase of carbon appearance in plasma glucose and in glycogen store.
674

EFFECT OF GLUCOSE ON UPTAKE AND RELEASE OF NEFA AND GLYCEROL BY MUSCLE AND ADIPOSE TISSUE S.W.Coppack and J.M.Miles*. UCL Medical School, London and Mayo Clinic, Rochester, MN U.S.A.

Lipolysis is relevant to the glucose-fatty acid cycle, to dyslipidaemia and to ketoacidosis. Previous studies of the regulation of lipolysis in wivo have used systemic tracers of fatty acids and glycerol, but interpretation of the results has been controversial. We studied 13 healthy men (age $26.8 \pm 1.3 \mathrm{y}$. BMI $23.4 \pm 0.5 \mathrm{~kg} \cdot \mathrm{m}^{-2}$ ) after an overnight fast (F) and $90.120 \mathrm{~min}$ after commencing a glucose infusion at $6-8 \mathrm{mg} \bullet \mathrm{kg}^{-1} \cdot \mathrm{min}^{-1}(\mathrm{G})$. Deep forearm and abdominal adipose tissue metabolism was studied by arteriovenous difference techniques. Constant infusion of ${ }^{3} \mathrm{H}$-glycerol and ${ }^{3} \mathrm{H}$ palmitate were used to partition uptake and release in the tissue bed. Concentrations and specific activities were ineasured by HPLC. The glucose infusion provoked an insulin response and inhibited lipolysis in adipose tissue. The results slowed (1) muscle uptake of glycerol, with fractional exiraction (FE) of $35 \pm 1[\%(\mathrm{~F})$ and $56 \pm 5 \%(G)$, boch $p<0.01:(2)$ muscle uptake of palmitate ( $\mathrm{FE}=36 \pm 5 \% \mathrm{~F}$ and $43 \pm 6 \% \mathrm{G}$, both $\mathrm{p}<0.001$; (3) unmeasurably low adipose tissue uptake of glycerol $(F E=-6 \pm 6 \% \mathrm{~F}$ and $7 \pm 13 \%$ $\mathrm{G}$, both $\mathrm{p}=\mathrm{NS}$; (4) unmeasurable adipose tissue uptake of palmitate during $F(F E=-4 \pm 7 \%, p=N S$ ) but significant uptake during $G$ $(\mathrm{FE}=66 \pm 6 \%, p<0.001)$; and (5) a decrease in adipose tissue release of palmitate (by $95.9 \pm 0.6 \%$ ) which was significantly greater $(p<0.01)$ than the decrease in glycerol release (by $89.9 \pm 1.6 \%$ ) during $G$ infusion. Thus, under postabsorptive conditions glycerol and NEFA are both cleared from the circulation by muscle, but not adipose tissue. Significant NEFA uptake in adipose tissue was observed during glucose infusion.

\section{6}

\section{DIVERGENT EFFECTS OF LACTATE ON INSULIN SENSITIVITY UNDER NORMO- OR HYPERGLYCAEMIA.}

C. Pagano, R. Fabris, AM. Lombardi, M. Granzotto, R. Serra, G. Federspil and R. Vettor - Endocrine-Metabolic Lab, Inst. of Semeiotica Medica, University of Padova, via Ospedale 105, 35100 Padova, Italy

I.actate metabolism is altered in several insulin-resistant states. In particular obesity and diabetes show increased fasting plasma lactate while the lactate rise after oral or intravenous glucose load is impaired. Recently jt has been proposed that lactate itself may influence insulio seusitivity thus playing a role in the devejopment of insulin resistance. The aim of our studies was to evaluate if the induction of increased lactate concentration to normal rats may change insulin sensitivity in vivo under different glucose levels. Anesthetized Sprague-Dawley rats were infused with sodium lactate or corresponding equivalents of sodium bicarbonate as controls. The following studies were carried oul: Study 1: euglycaemic hyperinsuline mic clamp combined with $3 \cdot{ }^{3} \mathrm{H}$ glucose technique to evajuate glucose turnover and endogenous glucose production Study 2: euglycaemic byperinsulinemic clamp combined with 2 -deoxy- ${ }^{7} \mathrm{H}$-glucose technique to evaluate the effect of lactate on glucose uptake in different tissues under normoglycaemia. Study 3: hyperglycaemic clamp combined with 2-deoxy- ${ }^{3} \mathrm{H}$-glucose techuique to evaluate the effect of lactate on glucose uptake in different tissues under byperglycaemia. Study 4: hyperglycaemic clamp to evaluate the effect of lactate on insulin secretion in vivo. Our results show that lactate does not increase endogenous gjucose production in basal condition or duriog hyperinsulinemia while glucose turnover at the end of clamp was reduced (Rd: $26.6 \pm 1.1$ vs $19.5 \pm 1.1 \mathrm{mg} \cdot \mathrm{min}^{-1} \cdot \mathrm{kg}^{-1}$; $p<0.01)$. This was associated to a reduced ghucose utilization in soleus $(\mathrm{p}<0.01)$, diaphragm $(p<0.05)$ and heart $(p<0.01)$. On the contrary, during byperglycaemic clamp, overall glucose utilization during lactate infusion was increased (M: $23.9 \pm 1.9$ vs $\left.30.6 \pm 0.7 \mathrm{mg} \cdot \mathrm{min}^{-1} \cdot \mathrm{kg}^{-1} ; \mathrm{p}<0.01\right)$ and an increased glucose uptake was observed in diaphragm ( $p<0.05$ ), white and red quadriceps $(p<0.01)$, tibialis and EDL $(p<0.05)$. Moreover lactate infusion did not induce any change in both first and second phase of insulin secretion. In conclusion our data show that, at different glucose levels, an increased lactate availability induces opposite effects on muscle insulin sensitivity in rat. They suggest that an aitered lactate metabolism may influence insulin sensitivity in obesity and diabetes. 
677

ROLE OF AMINO ACID AND INSULIN CONCENTRATION ON SPLANCHNIC UPTAKE OF DIETARY LEUCINE IN TYPE 1 DIABETES

M.Zanetti, R.Barazzoni, D.Bruttomesso, G.Biolo, M.Vettore, P.Tessari. Dept. of Metabolic Diseases, University of Padua, Italy.

To study the role of insulin availability vs amino acid concentrations on splanchnic and whole-body metabolism of dietary amino acids, leucine kinetic was determined in 5 normal subjects (group 1), in 5 insulin-deprived IDDMs (group 2) and in 6 IDDMs undergoing a peripheral insulin infusion (group 3). The subjects were infused for 8 hours with $\mathrm{L}-\left[5,5,5-{ }^{2} \mathrm{H}\right]$-leucine intravenously, A mixed meal containing L- $\left[1-{ }^{13} \mathrm{C}\right]$-leucine was administered continuously in the last 4 hours. In the fasting state, glucose and leucine concentrations and intracellular leucine rate of appearance $\left(\mathrm{Ra}_{\mathrm{i}}\right)$, were significantly higher $(\mathrm{p}<0.001, \mathrm{p}<0.001, \mathrm{p}<0.02$ respectively) in group $2\left(17.6 \pm 1.2 \mathrm{mmol} / \mathrm{L}, 218 \pm 8 \mu \mathrm{mol} / \mathrm{L}\right.$ and $\left.2.64 \pm 0.21 \mu \mathrm{mol} \cdot \mathrm{kg}^{-1} \cdot \mathrm{min}^{-1}\right)$ than in group $1(4.8 \pm 0.02,125 \pm 10$ and $2.02 \pm 0.09)$ and $3(7.2 \pm 0.5,127 \pm 12$ and $2.14 \pm 0.07)$. During the meal, glucose and leucine concentrations rose more $(\mathrm{p}<0.001)$ in group 2 $(25.5 \pm 1.3 \mathrm{mmol} / \mathrm{L}$ and $394 \pm 15 \mu \mathrm{mol} / \mathrm{L})$ than in group $1(7.2 \pm 0.3$ and $204 \pm 12)$ and 3 $(9.6 \pm 1.0$ and $173 \pm 12)$. Total leucine $\mathrm{Ra}$ was $2.93 \pm 0.19 \mu \mathrm{mol} \cdot \mathrm{kg}^{-1} \cdot \mathrm{min}^{-1}$ in group 2, $2.51 \pm 0.15$ in group 1, and $2.73 \pm 0.08$ in group 3 (NS among groups). First-pass splanchnic uptake (SU) of dietary leucine was significantly lower $(\mathrm{p}<0.02)$ in group $3\left(0.25 \pm 0.03 \mu \mathrm{mol} \cdot \mathrm{kg}^{-1} \cdot \mathrm{min}^{-1} ; 20.4 \pm 2.3 \%\right)$ than in group $1(0.36 \pm 0.03 ; 29.6 \pm 2.8 \%)$ and $2(0.41 \pm 0.05 ; 33.8 \pm 4.2 \%)$. Endogenous leucine $\mathrm{Ra}_{\mathrm{ic}}$ was significantly more suppressed ( $\mathrm{p}<0.005)$ in group $1\left(1.46 \pm 0.14 \mu \mathrm{mol} \cdot \mathrm{kg}^{-1} \cdot \mathrm{min}^{-1} ;-27.3 \pm 6.6 \%\right)$ than in group $2(2.07 \pm 0.17 ;-21.1 \pm 3.8)$ and $3(1.42 \pm 0.06 ;-33.1 \pm 3.7 \%)$. In summary, in IDDM: 1) A peripheral insulin infusion can normalize the high post-prandial endogenous leucine $\mathrm{Ra}_{i c} ; 2$ ) SU of dietary leucine is normal in the insulin-deficient state, probably because of hyperleucinemia; however: 3) SU uptake of dietary leucine is decreased following insulin-infusion in the face of euleucinemia. In conclusion, a peripheral insulin infusion can normalize leucine concentrations and $\mathrm{Ra}$ in IDDM, but it impairs dietary leucine uptake by splanchnic tissues, probably because of portal hypoinsulinemia.

\section{8}

LOW-PROTEIN DIET IMPROVES INSULIN SENSITIVITY OF ENDOGENOUS GLUCOSE PRODUCTION IN PREDIALYTIC UREMIC PATIENTS.

Gin H, Rigalleau V, Blanchetier V, Combe C, Deleris G. Guillot $C$, Aubertin J and Aparicio M. Hopital Pellegrin-Tripode, Bordeaux, France.

Low-protein, low phosphorus diet (LPD) has been shown to improve insulin sensitivity in uremic patients, but this improvement has not been studied at low, physiologica! levels of insulinemia. In the present study we used the glucose clamp technique at low (0.25 mu. $\left.\mathrm{kg}^{-1} \cdot \mathrm{min}^{-1}\right)$ level of hyperinsulinemia, associated with the infusion of $D\left[6,6^{2} \mathrm{H}_{2}\right]$ glucose to assess endogenous glucose production. Eight non-dialyzed uremic patients were studied before and after 3 months on a LPD providing $0.3 \mathrm{~g} \cdot \mathrm{kg}^{-1}$ protein, $5-7 \mathrm{mg}$ phosphorus. $\mathrm{kg}^{-1}$, and $146 \mathrm{KJ} \cdot \mathrm{kg}^{-1}$ energy supply $(67 \%$ carbohydrates, $30 \%$ lipids) per day, supplemented with ketoanalogue aminoacids. At the postabsorptive state, plasma glucose and insulin declined after 3 months of diet (Plasma glucose: $5.0 \pm 0.1$ before vs $4.7 \pm 0.1 \mathrm{mmol}^{-1}$ after $L P D$; $p<0.05$; plasma insulin: $12.3 \pm 3.1 \mu \cup \mathrm{ml}^{-1}$ before vs $7.3 \pm 0.9$ after; $p<0.05$ ), and glucose turnover rates did not change $\left(2.06 \pm 0.14\right.$ before vs $2.17 \pm 0.17 \mathrm{mg} \cdot \mathrm{kg}^{-1} \cdot \mathrm{min}^{-1}$ after LPD; NS). Insulin metabolic clearance rate was enhanced after the diet, so a lower level of hyperinsulinemia was obtained during the clamp $\left(25.2 \pm 4.2 \mu \mathrm{U} . \mathrm{ml}^{-1}\right.$ before vs $17.2 \pm 2.2$ after: $p<0.05)$. However, endogenous glucose production was more inhibited after the diet $(0.90 \pm 0.31$ before vs $0.30 \pm 0.17$ $\mathrm{mg} \cdot \mathrm{kg}^{-1} \cdot \mathrm{min}^{-1}$ after LPD, during clamp; $\mathrm{p}<0.05$ ), evidencing an improved insulin sensitivity.

\section{9}

SKELETAL MUSCLE PROTEIN METABOLISM IN IDDM WITH PANCREAS TRANSPLANTATION.

L. Piceni-Sereni, 1. Terruzzi, M. Spessot, M. R. Pastore, V. Di Carlo, G. Pozza, L. Luzi. Departments of Medicine San Raphael Hospital, Milan, Italy and Brigham and Women's Hospital, Boston, U.S.A.

To evaluate whether pancreas-kidney transplantation (Ptx) can correct the altered muscle protein metabolism of IDDM, 4 patients after Ptx (FPG $=76 \pm 1$ $\mathrm{mg} / \mathrm{dl} ; \mathrm{HbA}_{\mathrm{tc}}=6.1 \pm 0.1 \%$; Pred. $=10 \pm 2 \mathrm{mg} /$ day; $\mathrm{CyA}=5 \mathrm{mg} / \mathrm{kg} / \mathrm{day}$ Azathioprine $=1 \mathrm{mg} / \mathrm{kg} / \mathrm{day}$ ), 7 IDDM (FPG $=145 \pm 9 ; \mathrm{HbA}_{1 \mathrm{c}}=7.5 \pm 0.5$; daily insulin $=42 \pm 4 \mathrm{U}$ ) and 6 healthy volunteers (CON) received $\left[1-{ }^{14} \mathrm{C}\right]$-leucine and $\left[2,6-{ }^{3} \mathrm{H}\right]$-phenylalanine by i.v. with brachial artery and vein catheterization and underwent: Study 1, euglycemic hyperinsulinemic $\left(40 \mathrm{mU} / \mathrm{m}^{2} / \mathrm{min}\right)$ clamp; Study 2 , same as in 1 with a balanced amino acid infusion ( $A A \times 2$ ) (IDDM, CON). Fasting free- $|R|$ was similar in Ptx and IDDM $(17 \pm 5$ and $17 \pm 3 \mu \mathrm{U} / \mathrm{ml})$, higher than in $\operatorname{CON}(p<0.01)$. Basal plasma Leu and Phe were lower in Ptx than in IDDM $(p<0.05)$. Whole body AA kinetics were comparable in PTx IDDM and CON, respectively: Endogenots Leucine Flux $(E L F)=1.0 \pm 0.14 ; 1.04 \pm 0.15$; $1.26 \pm 0.14 \mu \mathrm{mol} / \mathrm{kg} / \mathrm{min}$; Non-Oxidative Leu Disposal (NOLD) $=0.93 \pm 0.14 ;$ $0.96 \pm 0.14 ; 1.02 \pm 0.12$; Phe Flux $=0.52 \pm 0.12 ; 0.52 \pm 0.09 ; 0.5 \pm 0.06$. Forearm Phe Balance (Bal) was negative in the basal state: $\mathrm{Ptx}=-1.6 \pm 6.9$, $I D D M=-8.7 \pm 9.8, C O N=-16.4 \pm 3.4 \mathrm{nmol} / 100 \mathrm{ml} / \mathrm{min}(\mathrm{p}<0.05, \mathrm{Ptx}$ and IDDM vs CON; $p=0.05$ Ptx vs IDDM). Study $1: E L F=0.79 \pm 0.02 ; 0.58 \pm 0.1 ; 0.48$ \pm 0.18 ; (NOLD-ELF) $=-0.07 \pm 0.06 ;-0.08 \pm 0.01 ;-0.24 \pm 0.04$; Phe Flux $=0.34$ $\pm 0.05 ; 0.37 \pm 0.17 ; 0.21 \pm 0.09$ in PTX, IDDM and CON, respectively ( $P=N S$ ). Insulin caused a higher reduction of Phe Ra $(25 \rightarrow 12 \pm 9 ; 24 \rightarrow 9 \pm 9$ $\mathrm{nmol} / 100 \mathrm{ml} / \mathrm{min})$ respect to Phe $\mathrm{Rd}(12 \rightarrow 14 \pm 4 ; 9 \rightarrow 11 \pm 1)$ and Phe Bal became neutral $(2.1 \pm 5.2 ; 2.5 \pm 9.9)$. During the last hour of study 2 , ELF $(0.59 \pm 0.12)$ and Phe Flux $(0.35 \pm 0.01)$ decreased similarly in IDDM and CON while NOLD nearly doubled; Phe Bal became positive in IDDM $(4 \pm 13)$ and in CON $(37 \pm 6)$ In summary: 1) in the basal state: Ptx show reduced plasma Leu and Phe concentrations with normal Leu and Phe fluxes; $P$ he Bal is less negative in Ptx and IDDM than in CON; 2) the forearm Phe Bal of Ptx and IDDM becomes neutral during hyperinsulinemia; 3 ) combined insulin and $A A$ infusion is required for a positive Phe balance.

\section{0}

AMINO ACID AND PROTEIN METABOLISM IN GLUCAGONOMA. R. Barazzoni, M. Zanetti, A. Tiengo, and P. Tessari. Dept. of Metabolic Diseases, University of Padova, Italy.

Glucagonoma (G) patients often exhibit weight loss, protein wasting and hypoaminoacidemia. In order to study the mechanism(s) of protein catabolism in this disease, two $G$ and twelve normal $(\mathbb{N})$ subjects were infused with $L$ $\left[{ }^{2} \mathrm{H}_{3}\right]$-leucine (Leu), $\mathrm{L}-\left[{ }^{2} \mathrm{H}_{5}\right]$-phenylalanine (Phe) and $\mathrm{L}-\left[{ }^{2} \mathrm{H}_{2}\right]-$ tyrosine (Tyr) for 3 hrs. $G$ subjects were studied before anti-tumor therapy. Leu, Phe and Tyr plasma rates of appearance $\left(R a_{0,}\right)$, Leu intracellular $R a\left(R a_{i s}\right)$, an index of protein degradation, and Phe hydroxylation to Tyr [Phe $\rightarrow$ Hy] (the first step of Phe catabolism), were measured at steady-state. In $G$, plasma glucagon $(2677 \pm 2086$ vs $86 \pm 13 \mathrm{pg} / \mathrm{ml})$ and insulin $(20 \pm 3$ vs $8 \pm 2 \mu \mathrm{U} / \mathrm{ml})$ were $>2$ standard deviations (SD) greater than in N. Also plasma glucose $18.7 \pm 1.8$ vs $4.6 \pm 0.4 \mathrm{mmol} / 1)$ was higher in $\mathrm{G}$. Plasma amino acid concentrations were dramatically lower in $G$ than in $N$ [iLeu]: $50 \pm 2$ vs $143 \pm 11$; [Phe]: $34 \pm 2$ vs $54 \pm 3$; [Tyr]: $16 \pm 1$ vs $55 \pm 4 \mu \mathrm{mol} / /$ ). Leu, Phe and $T y r R a_{p 1}$ were similar in the two groups (Leu: $1.62 \pm 0.17$ vs $1.42 \pm 0.05$; Phe: $0.70 \pm 0.03$ vs $0.65 \pm 0.04 ;$ Tyr: $0.40 \pm 0.01$ vs $0.53 \pm 0.03 \mu \mathrm{mol} / \mathrm{kg}$. min). in contrast Leu $\mathrm{Ra}_{\mathrm{ic}}$ was $>2$ SD greater in $\mathrm{G}(3.17 \pm 0.11 \mu \mathrm{mol} / \mathrm{kg} \cdot \mathrm{min})$ than in $\mathrm{N}$ $(1.88 \pm 0.08)$. [Phe $\rightarrow$ Hy] was positively correlated with Phe plasma concentration ([Phe]) in $\mathrm{N}(\mathrm{r}=0.78, \mathrm{p}=0.002)$. [Phe $\rightarrow \mathrm{Hy}$ ] was comparable in the two groups (G: $0.106 \pm 0.012 ; \mathrm{N}: 0.091 \pm 0.005 \mu \mathrm{mol} / \mathrm{kg} \cdot \mathrm{min}$ ). However, Phe hydroxylative clearance (i.e. [Phe $\rightarrow$ Hy] expressed as a function of [Phe]), was sharply increased (more than $2 \mathrm{SD}$ ) in $\mathrm{G}(3.15 \pm 0.30$ vs $1.69 \pm 0.06$ $\mathrm{ml} / \mathrm{kg} \cdot \mathrm{min}$ in $\mathrm{N} /$. In summary, in $\mathrm{G}$ patients plasma amino acid concentrations are sharply decreased, Leu, Phe and Tyr plasma Ra are normal, while protein degradation and Phe fractional catabolism are increased. These data suggest in $G$ patients both increased proteolysis and phenylalanine degradation, as well as an altered transmembrane Leu transport, possibly due to pathologic hyperglucagonemia. 
681

BASAL INSULIN MODULATES GLUCOSE-MEDIATED GLUCOSE METABOLISM IN TYPE 2 DIABETIC PATIENTS (NIDDM).

S. Del Prato, S. Marchetto, S. Vigili de Kreutzenberg, M. Zanon, M.C. Marescotti, and A. Tiengo. Cattedra di Malattie del Metabolismo, University of Padova, Italy.

In NIDDM hyperglycemia is claimed to compensate for insulin resistance. Whether basal plasma insulin modulates glucose-mediated glucose utilization is unknown. Six NIDDM (4M/2F; $57 \pm 5$ yrs; $28 \pm 1$ $\mathrm{kg} / \mathrm{m}^{2} ; \mathrm{FPG}=7.5 \pm 0.5 \mathrm{mmo} / \mathrm{L}$ ) underwent two $240 \mathrm{~min}$ hyperglycemic $(+9.7 \mathrm{mM})$ clamps with somatostatin $(\mathrm{SRIF}=750 \mu \mathrm{g} / \mathrm{h})$, glucagon $(0.5$ $\mathrm{ng} / \mathrm{kg} / \mathrm{min})$ and with or without replacing $(0.9 \mathrm{mmol} / \mathrm{kg} / \mathrm{min})$ insulin infusion. Once a new hyperglycemic plateau $(120 \mathrm{~min}=17.4 ; \mathrm{CV}=2 \%)$ was attained, a primed-continuous infusion of $3-{ }^{3} \mathrm{H}$ - and U-14 $\mathrm{C}$-glucose was started. Metabolic determinations and indirect calorimetry readings were obtained during the last hr of clamp, under steady state condition. In both studies, C-peptide levels were suppressed $(0.2$ and $0.2 \mathrm{nmol} / \mathrm{L})$ by SRIF. Basal plasma insulin ( $95 \mathrm{pmol} / \mathrm{L}$ ) dropped $(36 \pm 4)$ with SRIF, but was constant with replacing infusion $(97 \pm 6 ; p<0.01$ vs SRIF). Glucose utilization $\left({ }^{3} \mathrm{H}-\mathrm{G}\right.$ turnover $=27.2 \pm 1.7$ vs $27.2 \pm 2.2$ $\mu \mathrm{mol} / \mathrm{kg} / \mathrm{min}$ ) was equal in both studies. Hypoinsulinemia was associated with lower total glucose oxidation (calorimetry $=8.9 \pm 1.1$ vs $6.6 \pm 1.1 \mu \mathrm{mol} / \mathrm{kg} / \mathrm{min}$ ), but higher oxidation proportion of intracellularly derived glucose (calorimetry $-{ }^{14} \mathrm{C}-\mathrm{G}$ ox $=38$ vs $20 \%$; $\mathrm{p}<0.05$ ), higher non-ox glucose metabolism ( $20.6 \pm 1.1$ vs $18.3 \pm 1.3$ ). lower exogenous glucose infusion $(11.1 \pm 1.7$ vs $16.7 \pm 2.7 ; \mathrm{p}<0.01)$, higher hepatic glucose output ( $\mathrm{HGP}=16.1 \pm 1.7$ vs $10.5 \pm 2.7)$. Moreover, the Cori cycle was more active and related to $\mathrm{HGP}(\mathrm{r}=0.52 ; \mathrm{p}<0.05)$. Plasma FFA $(1.0 \pm 0.1$ vs $0.3 \pm 0.1 \mathrm{mM})$ and lipid oxidation $(10.9 \pm 0.4$ vs $8.8 \pm 0.4 \mu \mathrm{mol} / \mathrm{kg} / \mathrm{min}$ ) were increased $(\mathrm{p}<0.05)$ and inversely related to glucose oxidation $(r=0.88 ; p<0.01)$. In conclusion, basal insulin modulates glucose-mediated glucose metabolism without affecting glucose utilization demonstrating that a qualitative compensatory effect of hyperglycemia requires adequate basal insulinization.

\section{3}

DECREASED GLUCOSE CLEARANCE IN DIABETIC RATS IS DUE TO REDUCED HEXOKINASE ACTIVITY IN SKELETAL MUSCLE. J. K. Wi and J. H. Youn, USC, Los Angeles, Califormia, USA

We have previously suggested that postabsorptive hyperglycemia in streptozotocin diabetic (STZD) rats is due to decreased peripheral glucose clearance rather than to elevated hepatic glucose output. To examine whether the decreased glucose clearance in STZD rats is caused by an impairment in glucose transport $v s$. an impairment in glucose phosphorylation or metabolism, we measured in vivo glucose transport activity and muscle glucose 6-phosphate (G-6-P) levels in normal and STZD rats. In vivo glucose transport activity was assessed by analyzing plasma kinetics of $3-\mathrm{O}-\left[{ }^{3} \mathrm{H}\right]$ methylglucose and $\mathrm{L}-\left[1-{ }^{1+} \mathrm{C}\right]$ glucose following an i.v. injection. Normal rats were studied either at euglycemia $(\sim 7 \mathrm{mM} ; \mathrm{n}=7)$ or at hyperglycemia $(\sim 12 \mathrm{mM} ; \mathrm{n}=8)$ matched with the postabsorptive levels in STZD rats $(\sim 12 \mathrm{mM}$; $n=7$; STZ [50 mg/kg] injected the day before the experiments). When glucose was clamped at hyperglycemia in normal rats, somatostatin $(5 \mu \mathrm{g} / \mathrm{kg} / \mathrm{min})$ was infused to suppress insulin response. The time profiles of plasma 3-O-methylglucose and L-glucose were superimposable between normal (at euglycemia or hyperglycemia) and STZD rats. Compartmental analysis showed that the rate constants for extracelfular glucose distribution and cellular glucose transport were not altered by hyperglycemia in normal rats and that the rate constants were not different between normal and STZD rats $(\mathbf{P}>0.05)$. Conversely, G-6-P levels in soleus muscles were increased by hyperglycemia in normal rats $(0.18 \pm 0.01$ vs. $0.11 \pm 0.02 \mu \mathrm{mol} / \mathrm{g}, \mathrm{p}<0.01)$. Muscle G-6-P levels were significantly lower in STZD $(0.07 \pm 0.01 \mu \mathrm{mol} / \mathrm{g})$ rats, compared to normal rats at hyperglycemia or euglycemia ( $p<0.01$ for both), suggesting that the supply of G-6-P (via glucose transport and phosphorylation) is rate limiting for glucose utilization. Taken together, these data suggest that reduced hexokinase activity is responsible for decreased glucose clearance and thus hyperglycemia in STZD rats.
682

PARACRINE ACE EFFECTS ON INTERSTITIAL GLUCOSE CONCENTRATION IN HUMAN SKELETAL MUSCLE: AN IN VIVO MICRODIALYSIS STUDY

M.Müller, P.Fasching**, T.Burgdorft, F.Schmid ${ }^{* * *}$, W.Waldhäust ${ }^{* *}$ and HG. Eichler*

From the Departments of "Cinical Pharmacolagy, ${ }^{* *}$ Internal Medicine III, Division of Endocrinology and Metabolism and ${ }^{* * *}$ Clinical and Laboratory Investigation; University Hospital Vienna, Austria

Introduction: From previous studies it has been concluded that ACEInhibitors may enhance insulin responsiveness. Due to methodological limitations, however, it was not possible to assess the relative contribution of the local-paracrine and the systemic-endocrine renin angiotensin system to this overall effect. Furthermore, the mechanism by which ACE Inhibitors are to affect carbohydrate metabolism remained unclear. Methods: In the present study, time versus interstitial concentration protiles of glucose and lactate were assessed by means of in vivo microdialysis in skeletal muscle of healthy volunteers $(n=8$ ) during local inhibition of ACE by enalaprilate. Each volunteer was studied twice, once during basal conditions and once during a $1 \mathrm{mU} / \mathrm{kg}$ min euglycemic insulin clamp. Besults: There was no significant change in blood glucose or lactate concentrations from baseline, neither during the basal, nor during the clamp study. Continuous local administration of cumulative doses of enataprilate $(0.2,2,20 \mu \mathrm{g} / \mathrm{ml})$ led to a dose dependent increase in interstitial glucose $(+25.5 \pm 10.1 \%)$ and lactate $(+39.9 \pm 5.1 \%$ ) concentrations ( $p<0.05$ versus baseline). This effect was more pronounced during clamp experiments (glucose, + $38.8 \pm 16.1 \%$; lactate, $+94.9 \pm 47.8 \% . p<0.05$ versus baseline). Conclusion: Selective inhibition of paracrine muscle ACE led to a significant change of interstitial glucose and lactate concentrations, which may be explained by increased glucose transport from the blood into the interstitial space but does not suggest any exlusive increase in cell glucose uptake. From this it appears that paracrine ACE modulates glucose transport via a hemodynamic mechanism which is more pronounced during hyperinsulinemic conditions.

\section{4}

SENSITIVITY TO EXOGENOUS INSULIN AND ITS CLEARANCE DECREASE AFTER SYSTEMIC DIVERSION OF PANCREATIC DRAINAGE S. Pye, P. Barron, A. Humar, J. Davies, L. Welsh and J. Radziuk, Ottawa Civic Hospital, Ottawa, Canada

Previous data indicate that sensitivity to low levels of insulin is decreased following the transposition of the veins draining the pancreas from the portal vein to the inferior vena cava. To characterize this change further, parallel determinations were máde of the relationships between the metabolic clearance rate (MCR) of glucose (MCR $)$ and the insulin level (IRI) and between the MCR of insulin (MCR $)$ and insulin dose when this was infused for $3 \mathrm{~h}$ at rates of 5.6 , 11 and $56 \mathrm{pmol} / \mathrm{kg}$-min. 7 diverted and 7 sham-operated dogs were studied after an $18 \mathrm{~h}$ fast. Somatostatin was infused concurrently and glucose was clamped at basal levels. The metabolic clearance rate of glucose and its total production were calculated using an infusion of $\left[6_{-}{ }^{3} \mathrm{H}\right] \mathrm{glucose}$ and a 2 -compartment model of glucose kinetics. Endogenous production was estimated from the difference in total production and the rate of glucose infusion. Basal IRI were $35 \pm 7$ and $84 \pm 21 \mathrm{pM}$ $(p<0.02)$ and $M_{C R}$ was $3.0 \pm 0.3$ and $2.7 \pm 0.4 \mathrm{ml} / \mathrm{kg}$-min (n.s.) for diversions and shams respectively. The 3 dose rates used, yielded IRI which were $0.27+0.03,(n=9), 0.64+0.06(n=5)$ and $6.3 \pm 0.5(n=5) n M$ for the diverted animals and $0.22 \pm 0.03(n=5), 0.43 \pm 0.04(n=3)$ and $5.3 \pm 0.4(n=4) n M$ for the shams. This corresponded to $\mathrm{MCR}_{\mathrm{i}}$ of $20 \pm 2,17 \pm 4$ and $8.9 \pm 0.7 \mathrm{ml} / \mathrm{kg}$-min in the diverted animals and $25 \pm 2,26 \pm 3$ and $10.7 \pm 0.8 \mathrm{ml} / \mathrm{kg}$-min in the shams. $\mathrm{MCR}_{\mathrm{g}}$ was $6 \pm 1,18 \pm 3$ and $19 \pm 4 \mathrm{ml} / \mathrm{kg}$-nin in the diverted dogs and $11 \pm 2$, $25 \pm 5$ and $24 \pm 3 \mathrm{ml} / \mathrm{kg}$-min for shams $(p<0,05)$. There is a decrease in the MCR of exogenous insulin following diversion which is paralleled by a fall in $\mathrm{MCR}_{\mathrm{g}}$. $\mathrm{MCR}_{g}$ is characterized by a decrease in its maximal response to insulin and a shift to the right in the dose response. Chronic peripheral venous drainage of the pancreas therefore induces a concurrent decrease in the MCR of insulin and in sensitivity to it, suggesting an association between the two parameters. 
PS 32

\section{Regulatory Peptides}

685

\section{EFFECTS OF C-PEPTIDE ON BLOOD FLOW IN IDDM PATIENTS:} AN ENDOTHELIUM-MEDIATED FUNCTION?

E. Fernquist-Forbes, B-L. Johansson, M. Eriksson, B.Lind and J.Wahren. Dept of Clinical Physiology, Karolinska Hospital, Stockholm, Sweden

Recent studies suggests that C-peptide increases blood flow in both exercising and resting forearm skeletal muscle and augments capillary diffusion capacity, glucose and oxygen uptake in patients with type 1 diabetes. In order to find out whether the effects of C-peptide on forearm vascular bed is dependent on endothelial cell function we have performed non-invasive measurements of endothelium-mediated anterial response by using an high-resolution ultrasound technique. Six type 1 C-peptide negative diabetic patients, age $29 \pm 2$ yrs with a diabetes duration of $17 \pm 3$ yrs, were studied They were treated with insulin s.c. four times per day, short acting before meals and intermediate acting at bedtime. Their mean insulin dosage and $\mathrm{HbAlc}$ were $0.68 \pm 0.08 \mathrm{U} / \mathrm{kg} / 24 \mathrm{hrs}$ and $7.8 \pm 0.5 \%$, respectively, prior to the study. No clinical sign of neuropathy, nephropathy and retinopathy were found. All were studied twice within one week. They recived i.v. insulin the night before and during the study so regulated that euglycemia $(5-6 \mathrm{mmol} / 1)$ was achieved. Blood flow and the diameter of the brachial artery were measured in basal state, 1 min after reactive hyperemia ( $4 \mathrm{~min}$ distal arterial occlusion) and $10 \mathrm{~min}$ later. Subsequently, C-peptide $(6 \mathrm{pmol} / \mathrm{kg} / \mathrm{min})$ or saline was given $\mathrm{i} . v$. in a randomized double blind order. Repeated measurements were performed after 60 min infusion as well as after a doasge of sublingual glyceryl trinitrate. C-peptide infusion resulted in a $36 \pm 8 \%(\mathrm{p}<0.01)$ increase of basal blood flow and a dilatation of the brachial-artery by $5 \pm 1 \%$ $(p<0.05)$ as compared to the saline infusion period. However, the vascular response to reactive hyperemia (endothelium-mediated) and glyceryl trinitrate did not differ between the periods. It is concluded that $\mathrm{C}$-peptide at physiological levels $(1.5 \mathrm{nmol} / 1)$ increases resting forearm blood flow and brachial diameter in type 1 diabetic patients, but the effect does not seem to be endotelium-mediated.

\section{6}

C-PEPTIDE AUGMENTS THE VASOCONSTRICTOR EFFECT OF NPY AND INCREASES FOREARM BLOOD FLOW IN IDDM PATIENTS

Bo-Lennart Johansson, John Pernow and John Wahren, Depts of Clinical Physiology and Cardiology, Karolinska Hospital, Stockholm, Sweden.

Recent findings suggest that C-peptide may have beneficial effects on renal and nerve function as well as microcirculation in patients with IDDM, possibly by stmulating $\mathrm{Na}^{+}, \mathrm{K}^{+}-\mathrm{ATPase}$ activity. In vitro studies on rat renal tubuli cells show that the effect of $\mathrm{C}$-peptide on $\mathrm{Na}^{+}, \mathrm{K}^{+}$-ATPase activity is potentiated in the presence of neuropeptide Y (NPY). The aim of the present study was to examine whether the effects of NPY on resting forearm blood flow in IDDM patients is altered in the presence of C-peptide. Eight patients, aged $29 \pm 2$ yxs with a diabetes duration of $16 \pm 2$ yrs were studied. Their mean $\mathrm{HbAlc}$ was $6.2 \pm 0.3 \%$. All were treated with insulin s.c. four times per day - short acting insulin before meals and intermediate acting at bedtime. Their mean insulin dosage was $0.71 \pm 0.06 \mathrm{U} / \mathrm{kg} / \mathrm{bwt}$. They were studied in the morning after an overnight fast without morning insulin dosage. Forearm blood flow was measured by a pletysmographic method before, during and 10 and 20 min after NPY infusion. The NPY infusion was given i.a. as a bolus in three different dosages, 40,400 and $4000 \mathrm{pmol}$. C-peptide was then administered i.v. in a dosage of $5 \mathrm{pmol} / \mathrm{kg} / \mathrm{min}$ for $40 \mathrm{~min}$ before the forearm blood flow measurements were repeated as before, i.e. before, during and after three different dosages of i.a. NPY. Basal blood flow was $36.7 \pm 2.2 \mathrm{ml} / \mathrm{min} / 1000 \mathrm{ml}$. It decreased in a dose dependent manner during NYP infusion by 10,20 and $30 \%(p<0.001)$ during low, middle and high dose of NPY, respectively. Basal blood flow increased by $30 \%$, to $46.3 \pm 3.5$ $\mathrm{ml} / \mathrm{min} / 1000 \mathrm{ml},(p<0.01)$ during C-peptide infusion and the reduction in blood flow became even more apparent during the different doses of NPY infusion (20,30 and 45\%, during low, middle and high dose, respectively. In conclusion, the present data demonstrate that C-peptide increases resting forearm blood flow and augments the vasoconstrictor effects of NPY in patients with type 1 diabetes.

\section{7}

EFFECT OF RAT C-PEPTIDE ON IN VIVO INSULIN ACTION IN DIABETIC RATS

Y. Sato, Y. Oshida, L. Li, J.Sato, B.L Johansson* and J. Wahren* Nagoya University. Nagoya, Japan, *karol inska Hospital, Stockholm, Sweden

Recent studies from our laboratories suggest that supraphysiological levels of human C-peptide (20-40nmol/1) augments glucose utilization in diabetic rats. The present study was undertaken to determine the influence of physiological plasma concentrations of rat C-peptide on in vivo glucose utilization using the sequential euglycemic clamp technique. Two different insulin in fusion rates, 3.0 (low-dose) and $30.0(h i g h$-dose) $\mathrm{ml} / \mathrm{kg} \cdot \mathrm{min}$ were employed in the awake condition. Diabetes was induced by streptozotocin at a dose of $60 \mathrm{mg} / \mathrm{kg}$. Rat C-peptide $\mathrm{I}$ or $\Pi$ was infused at a rate calculated to result in physiological C-peptide levels $(0.05 \mathrm{nmol} / \mathrm{kg} \cdot \mathrm{min})$ in diabetic rats. Plasma insulin le vels during the low-dose and high-dose insulin infusion were 30 $\mu \mathrm{J} / \mathrm{ml}$ and $400 \mu \mathrm{U} / \mathrm{ml}$. respectively and blood glucose was clamped at $7.5 \mathrm{mmol} / 1$ by periodic adjustment of the $i, \mathrm{~W}$ glucose infusion rate. Glucose metabolic clearance rate (MCR) in diabetic rats given $C$-peptide I during the low-dose insulin clamp was 17. $9 \pm 4.0 \mathrm{ml} / \mathrm{kg} \cdot \mathrm{min}(\mathrm{n}=6)$ as compared to $7.2 \pm 0.8 \mathrm{~m} 1 / \mathrm{kg} \cdot \mathrm{min}(\mathrm{n}=6)$ in diabetic control rats $(P<0.05)$. Likewise, MCR in diabetic rats infused with $C$-peptide $I(13.6 \pm 3.3 \mathrm{ml} / \mathrm{kg} \cdot \mathrm{min}, \mathrm{n}=6)$ tended to be greater than in control diabetic rats $(0.05<p<0.1)$. MCR of diabetic rats infused with either C-peptide I or II was similar to the HCR for non-diabetic control rats $(15.1 \pm 2.0 \mathrm{mI} / \mathrm{kg} \cdot \mathrm{min})$. Cpeptide $I$ and II did not significantly influence $M C R$ at the high -dose insulin clamps. It is concluded that rat C-peptide $I$ and $I$ stimulate whole body glucose utilization in awake diabetic rats.

\section{8}

MICE LACKING IAPP DEVELOP AND REPRODUCE NORMALLY AND APPEAR METABOLICALLY UNAFFECTED S Gebre-Medhin 1 , H Mulder ${ }^{2}$ B Abrén ${ }^{3}, M$ Pekny ${ }^{1}$. J Törnell ${ }^{4}, F$ Sundler ${ }^{2}$, P Westermark $\mathrm{k}^{5}$ and $\mathrm{C}$ Betsholtz ${ }^{1}$. Dept of Medical Biochemistry, Göteborg Universityi : Dept of Physiology and Neuroscience, Lund University ${ }^{2}$; Dept of Medicin, Malmö University Hospital ${ }^{3}$; Dept of Physiology, Göteborg University ${ }^{4}$; Dept of Pathology, University of Linköping ${ }^{5}$; Sweden. Islet amyloid polypeptide (IAPP), also named amylin, is a pancreatic $\beta$-cell peptide with structural similarities to calcitonin-gene related peptide. IAPP is the major constituent of amyloid occurring in pancreatic islets in NIDDM. Additionally, IAPP is expressed in gastrointestinal endocrine cells and in sensory neurons. The co-localization of IAPP with insulin and their co-release in response to $\beta$-cell secretagogues has led to several hypotheses concerning the physiological role of IAPP; these include regulation of glucose metabolism insulin secretion and satiation. Yet, the physiological function of IAPP has remained unclear. In an attempt to elucidate the functional role of IAPP, we used gene targeting techniques to generate IAPP-deficient (ko) mice lacking the entire sequerce coding for mattre IAPP. These mice were found to develop and reproduce normally. In ko mice, IAPP-like immunoreactivity was not detectable, while expression of other neurohormonal peptides in the islets, gastrointestinal tract and sensory neurons seemed unaffected. Weight gain, food intake or -preference did not differ between ko and wild type (wt) animals. There was no difference between $k o$ and $w t$ in basal plasma levels of glucose $(8.2+0.6$ vs $7.5+0.3 \mathrm{mM}$ ) or insulin $(283 \pm 51$ vs $294 \pm 35 \mathrm{pM} ; \mathrm{n}=6$ in each group). Similarly; following an iv giucose challenge $(1 \mathrm{~g} / \mathrm{kg})$, the peak plasma insulin level did not differ between ko and wt ( $1783 \pm 437$ vs $1558 \pm 329 \mathrm{pM})$. These results indicate that the lack of IAPP in mice is not associated with any gross abnormalities in eating behaviour, insulin sensitivity or glucose-stimulated insulin secretion. Adaptive mechanisms or functionally overlapping peptides may have come into play. Further studies are are required to resolve these questions. 
689

KINETICS AND DISTRIBUTION OF INTRAVENOUSLY ADMINISTERED IAPP IN HUMANS

M. Clodi', K. Thomaseth ${ }^{2}$, K. Hermann', G. Pacini ${ }^{2}$, A. Kautzky-Willer', K. Borchert', R. Prager and B. Ludvik'. ' Department of Medicine III, University of Vienna, Vienna Austria; ${ }^{2}$ LADSEB-CNR, Padova, Italy

Islet amyloid polypeptide (IAPP) has been shown to modulate insulin secretion and to have a potential therapeutic effect in restoring accelerated gastric emptying in subjects with IDDM. However, the dynamics, distribution space, and clearance rate of IA.PP in humans have not been directly evaluated in vivo so far. The aim of the study was to evaluate the above mentioned parameters. We injected $50 \mu \mathrm{g}$ of human IAPP in 10 healthy, lean subjects $(73.4 \pm 2.4 \mathrm{~kg}$ ). Endogenous IAPP release was suppressed by a primed continous infusion of somatostatin (SRIF; $1.8 \mu \mathrm{g} / \mathrm{kg}$. $0.06 \mu \mathrm{g} / \mathrm{kg} / \mathrm{min}$ ). The disappearance curve of plasma IAPP concentration was analysed by a two exponential model which yielded the best data description. Combination of the estimated parameters provides the clearance rate (CL) and the distribution volume (VD). Glomerular fittration rate (GFR) and renal plasma flow (RPF) were assesed by infusion of iothalamate and PAH, respectively. VD was $132 \pm 13 \mathrm{~m} / \mathrm{kg}$, not different from that of insulin (157, Sherwin JCI 1974). CL and fractional CL were $6.7 \pm 0.4 \mathrm{ml} / \mathrm{kg} / \mathrm{min}$ and $0.054 \pm 0.004 \mathrm{~min}^{-1}$, respectively. GFR and RPF decreased following SRIF from $109 \pm 15 \mathrm{ml} / \mathrm{min} / 1,73 \mathrm{~m}^{2}$, and $640 \pm 105 \mathrm{ml}$ (min to $78 \pm 10$ and $448 \pm 67 \quad(p<0.05)$ and were restored following IAPP infusion to $106 \pm 10$ and $642 \pm 66$, respectively. In conclusion, clearance of IAPP is similar to that reported for C-peptide (0.06, Polonsky JCI 1986) and much lower than that of insulin (0.12-0.2, Ferrannini Diab. Met. Rev. 1987) indicating that the commonly used insulin/APP molar ratio does not reflect the correct relationship of the two peptides. High doses of IAPP restore the decrease in GFR and RPF caused by SRIF presumbly by excerting its known vasodilating effects.

\section{0}

\section{i.v. NEUROPEPTIDE Y INFUSION INCREASES MUSCLE INSULIN SENSITIVITY WITHOUT AFFECTING INSULIN SECRETION IN RATS.}

R. Vettor, C. Pagano, R. Fabris, AM. Jonthardi, *F. Rohner-Jcanrenand, C. Federspil and *B. Jeanrenaud - Inst. of Semeionca Medica, Endocrine-Merabolic Lah. University of Padowa, via Ospedale 105. /-351/0) Padeva, Italy and *aboratoire de Recherches Metaboliques, University of (ienewa, Switzerland

An inereased NPY genc expression bas hec found in the hypothalamus of genetically ohese rats and $i, c . V$ administration of NPY 10 normal rats induces a syndeome characterizd by obesity. hyperiusulinemia and insulin resistance. I ittle is kuown abeut any posithle effect of circulaling NPY on yitcose netabolisn and on insulin secretion. We aimed to evaluate the efiec of i.v. infusion of NPY on glueose metabolism and iusulin sensitivity as ascessed under euglycateme hyperinsulinemi clamp combined with the 3-"IJ-glucose and 2-deoxy-[1-"If]-glucose lechniques. Moreover its influevec on insulin secretion was evaluated by hyperglyecmic clamp. Glucose turnover (Rd), endogenous glucose production (li(3)), whole body glycolytic flux (GF), glucose storage (GS), insulin-stimulated glucose utilization index (CUM) in tissues, plasma lactate and HWA were evaluated in hasal condition, after NPY infusion and at the end of euclyeatmic clamp. NPY infusion did not induce any change in FEP either in hasal condition or at the end of clamp. Rd at the end of clamp was signilicantly incrascd in NPY-intused group $\left(27.8 \pm 1.3\right.$ vs $24.0 \pm 1.6 \mathrm{mg}^{*}{ }^{*}{ }^{2}{ }^{1}{ }^{1}{ }^{*} \mathrm{~kg}^{1}$; $\mathrm{p}<0.05)$ as was the GF $\left(18.9 \pm 1.6 \mathrm{vs} 14.4 \pm 0.8 \mathrm{mg}^{*} \mathrm{~min}{ }^{*} \mathrm{~kg}^{\mathrm{j}} ; \mathrm{p}<0.05\right)$, while GS was comparable in the two groups. Plasma FIA were unaltered by NPY infusion and were similarly suppressed in both groups during byperinsulinemia, while the lactate rise at the end of clann was markedly decreased in the NPY-infused rats (p<t).t75). i.v. NPY administration to nomal rats increascd (GUI in diaphragm $(p<0.05)$, red quadriceps $(p<0.05)$, red and white gastrocnemius $(p<0.05)$. No significant differences were present in both subcutaneous and epididimal adipose tissue between the two groups. The first and second phase of insulin response to hyperglycemia resulted unaffected by NPY-infusion. In conclusion our data show that acute i.v. NPY infusion may increasc insulin-induced glucose disposal in normal rats accelerating its utilization through the glycolytic pathway without affecting insulin secretion.

\section{1}

IDENTIFICATION OF TWO MISSENSE MUTATIONS IN THE HUMAN GIP RECEPTOR GENE, A FUNCTIONAL STUDY AND ASSOCIATION ANALYSIS WITH NIDDM

A. Kubota, Y. Yamada, S. Kagimoto, H. Ishica, and Y. Seino Department of Metabolism and Clinical Nutrition, Kyots University Faculy of Medicine, 54 Shogoin-Kawahara-cho, Sakyo-ku, Kyoto 606, Japan,

[AIM] Since the insulinotropic effect of gastric inhibitory polypeptide (GIP) is reduced in non-insulin-dependent diabetcs mellitus (NIDDM), it should be clarifiec whether defects in the GIP receptor gene contribute to the impaired insulin secretion in NIDDM. [METHODS] We have investigated the entire coding region of the GIP receptor gene by PCR-SSCP analysis using genormic DNA samples from Japanese NIDDM and control subjects. We subsequently examined the function of the receptors with the mutations detected and performed association analysis with NIDDM. [RESULTS] Wo have identified two missense mutations, one from GGC198 to TGC $^{198}$ in exon 7 resulting in an amino acid substitution from lycine to cysteine (Gly198Cys) and the other from GAG354 to CAG354 in exon 12 resulting in an amino acid substitution from glutamic acid to glutamine (Glu354GIn). Inyestigation of the function of the GIP receptor with either of these mutations reveals an $\mathrm{ED}_{50}$ value of GIP-induced cAMP response in $\mathrm{CHO}$ cells expressing GIP receptor with Gly 198 Cys of $6.3 \pm 1.2 \times 10^{-10}(n=3)$, which was considerably lower than that of normal GIP receptor, $9.4 \pm 3.8 \times 10^{-12} \mathrm{M}$ GIP $(n=3)$, while that of GIP receptor with Glu354Glin was not significantly different from that of normal GIP receptor. Association studies, however, showed no relationship between NIDDM and either of the two mutations. [CONCLUSION] We have identified two missense mutations in the GIP receptor gene, one of which impairs the function of the GIP receptor. Association studies, however, find no significant association between these mutations and NIDDM.

\section{2}

SOMATOSTATIN EFFECT ON GLUCOSE METABOLISM IN MUSCLE M.A. Luque, A. Alcántara, M. Morales, M.I. López-Delgado, F. Clemente, M.L. Villanueva-Peñacarrillo and I. Valverde. Fundación Jiménez Díaz, Madrid, Spain.

It has been obtained controversial results, in vivo, on the possible potentiating effect of somatostatin (SS) upon the insulin-induced glucose disposal. On the other hand, an insulin independent effect of GLP-1(7-36)amide (GLP-1), a peptide with insulin-like effect on glucose disposal in several extrapancreatic tissues, could not be demostrated during a SS infusion. In order to explore whether SS has a direct effect on muscle glucose metabolism, we have measured the glucose incorporation into glycogen (Gly), glucose oxidation (GO) and utilization (GU), and glycogen synthase $a(\mathrm{GS} a$ ) and phosphorylase $a(\mathrm{GP} a)$ activities in rat isolated paired soleus muscles incubated in $\mathrm{KRB}$, in the absence or presence of SS (20 or $200 \mathrm{ng} / \mathrm{ml})$, GLP-1 $\left(10^{-10} \mathrm{M}\right)$ and insulin $\left(10^{-\circ} \mathrm{M}\right)$, alone or in combination. At $20 \mathrm{ng} / \mathrm{ml}$, SS did not modify the control vilue (one of the paired muscles ineubated in the absence of peptide, or otherwise stated) of any of the parameters studied; at $200 \mathrm{ng} / \mathrm{ml}$, SS induced an increase in Gly $(122 \pm 9 \%$ of control, $\mathrm{p}<0.05), \mathrm{GO}(143 \pm 19 \%$, $\mathrm{p}<0.05), \mathrm{GSa}(133 \pm 11 \%, \mathrm{p}<0.01)$ and in GU $(170 \pm 46 \%)$. When SS was tested in combination with GLP-1 or insulin, further increases were observed in some parameters: $10^{-10}$ M GLP- 1 , Gly: $117 \pm 8 \%$ of GLP-1 alone, $p<0.05$; GO: $125 \pm 9 \%, p<0.05$; GSa: $153 \pm 14 \%, p<0.01 ; 10^{-9} \mathrm{M}$ insulin, Gly: $124 \pm 11 \%$ of insulin alone, $\mathrm{p}<0.05$; GS $a: 143 \pm 8 \%, \mathrm{p}<0.001$. These data demonstratte that somatostatin directly affects glucose metabolism, and has a glycogenic effect which seems to be synergic to that of insulin or GLP-1. 
693

SOMATOSTATIN EFFECT ON GLUCOSE METABOLISM IN THE LIVER M.I. López-Delgado, M. Morales, M.A. Luque, J. Puente, M.L. Villanueva-Peñacarrillo and i. Valverde. Fundación Jiménez Díaz, Madrid, Spain.

Somatostatin has been used in vivo to inhibit the release of several hormones, and to study the actions of these hormones on glucose metabolism when administered exogenously. In order to explore whether somatostatin has a direct effect on liver glucose metabolism, we have measured the giucose incorporation into givcogen (Gly), glucose oxidation (GO) and utilization (GU), lactate formation (LA) and glycogen synthase a (GSa) and phosphorylase a (GPa) activities, in isolated rat hepatocytes $\left(2\right.$ or $4 \times 10^{6}$ cells) incubated in KRB, in the absence or presence of somatostatin, GLP-1(7-36)amide (GLP-1), insulin and glucagor, alone or in combination. Somatostatin $(20 \mathrm{ng} / \mathrm{ml})$ induced a significant increase in Gly $(115 \pm 7 \%$ of control in the absence of peptides, $n=29, p<0.05), G 0(188 \pm 30 \%, n=20, p<0.01)$, GU $(147 \pm 14 \%, n=20, p<0.01)$, LA $(112 \pm 4 \%, n=20, p<0.01)$ and $G S a$ $(111 \pm 3 \%, n=21, p<0.01)$. At $200 \mathrm{ng} / \mathrm{ml}$ somatostatin, a higher increase was observed in all parameters except in GU and LA, and a decrease of the GPa activity $(83 \pm 5 \%, n=12, p<0.05)$, similar to that of $10^{.9} \mathrm{M} \mathrm{GLP}-1(72 \pm 4 \%, n=9, p<0.001)$ or $10^{-9} \mathrm{M}$ insulin $(72 \pm 5 \%$, $n=9, p<0.0011$. When somatostatin was tested in combination with GLP- 1 or insulin, elicited a higher increase of Gly and GSa, which were not significantly different to the sum of the effects of each peptide. Somatostatin also abolished the inhibition of GSa produced by $10^{-\mathrm{A}} \mathrm{M}$ glucagon, as well as the stimulation of GPa. These data demonstrate that somatostatin, in the liver, directly provokes an increase of glucose incorporation into glycogen and of GSa activity, and that these effects may be additive to those induced by GLP-1 or insulin, and could counteract those of glucagon.

\section{5}

GLUCAGON DEPLETES CIRCULATING GLUTAMINE BY INCREASING GLUTAMINE UPTAKE AND NOT AFFECTING ITS RELEASE.

A. Battezzati, D.C. Simonson, L. Luzi and D.E. Matthews. Boston, MA, USA; Mitan, Italy; New York, NY, USA.

The effect of glucagon on glucose production is transient but the mechanism of this action is unclear. To examine whether it is due to an effect of glucagon on circulating glutamine, a major gluconeogenic precursor, 7 young healthy subjects each underwent 3 pancreatic clamp studies (octreotide $30 \mathrm{ng} / \mathrm{kg} / \mathrm{min}$, insulin $0.15 \mathrm{mU} / \mathrm{kg} / \mathrm{min}$, glucagon $1.4 \mathrm{ng} / \mathrm{kg} / \mathrm{min}$ ) for $7 \mathrm{hrs}$. During the last $3.5 \mathrm{hrs}$, glucagon infusion was either unchanged (Study 0) or increased to 4 and $7 \mathrm{ng} / \mathrm{kg} / \mathrm{min}$ (Studies 1 and 2). ${ }^{2} \mathrm{H}_{2}$-glucose (gle), ${ }^{15} \mathrm{~N}$ glutamine (g|n), d5-phenylalanine (phe) and ${ }^{2} \mathrm{H}_{3}$-leucine (leu) were infused to quantitate the respective fluxes. Glucagon infusions increased piasma givcagon by $38 \pm 8 \%$ (Study 1) and $90 \pm 11 \%$ (Study 2) over Study 0 . The effects of glucagon in the first and the second 1.75 hrs of the study periods were anahzed separately.

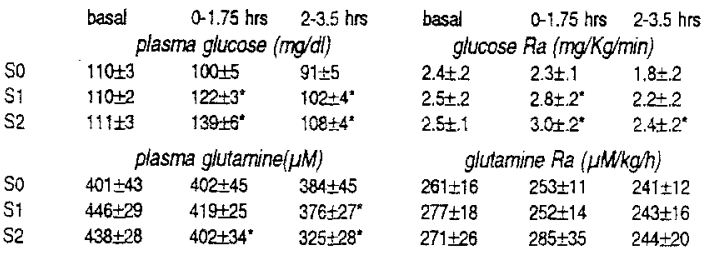

Compared to the control study, hyperglucagonemia increased glc concentration (ANOVA $0<0.001)$ and $\mathrm{Ra}(\mathrm{p}<0.001)$ in a dose-dependent manner. This effect peaked within the first $1.75 \mathrm{hrs}$, then it declined. Gin concentration decreased in a dose- and timedependent manner $(p<0.001)$. The drop in gin concentration was due uniquely to the stimulation of gin clearance $(p<0.001)$, in the absence of any effect of glucagon on gln release. Likewise, the release of leu and phe from proteolysis was not affected. These data show that hyperglucagonemia stimulates glutamine uptake but it does not stimulate the glutamine release from proteolysis or de novo synthesis. A reduction in glutamine concentration results which may limit the ability of glucagon to sustain glucose production.
694

EFFECT OF GLUCAGON ON FIBRINOGEN SYNTHESIS IN HUMANS. P. Tessari, E. lori, M. Vettore, M. Zanetti, R. Barazzoni, E. Kiwanuka. Dept. of Metabolic Diseases, University of Padova, Italy.

Both fibrinogen and glucagon concentrations, and fibrinogen fractional synthetic rate (FSR), are elevated in uncontrolled diabetes mellitus. However, the mechanism(s) of these alterations are poorly known. Therefore, we have studied the effects of selective hyperglucagonemia on fibrinogen FSR in 9 normal subjects, during suppression of endogenous pancreatic hormone secretion by means of somatostatin infusion and replacement doses of insulin, glucagon and growth hormone. The subjects were studied both before ( $\approx 3 \mathrm{hrs}$ ) and following selected hyperglucagonemia (from $\approx 200$ to $\approx 600$ pgrams $/ \mathrm{mll}$ for $3 \mathrm{hrs}$. Fibrinogen FSR was evaluated with precursorproduct relationships using $\mathrm{L}-\left[1{ }^{19} \mathrm{C} C\right]$ hentenlalanine $(\mathrm{n}=7), \mathrm{L}-[2,6$ $\left.{ }^{3} \mathrm{H}\right]$ phenylalanine $(n=2)$, and $\mathrm{L}-\left[1-{ }^{14} \mathrm{C}\right]$ leucine $(n=2)$ primer-constant tracer infusions. $L=\left[5,5,5-{ }^{2} \mathrm{H}_{3}\right.$-leucine was also infused along with radioactive phenylalanine. Hyperglucagonemia did not change either plasma phenylalanine (Phe) specific activity (SA) (from $4.93 \pm 0.52$ to $5.21 \pm 0.53$ DPM/nmol, $p=0.061$ ) or plasma tyrosine (Tyr) SA (from $0.85 \pm 0.11$ to $0.91 \pm 0.07 \mathrm{DPM} / \mathrm{nmol}, \mathrm{p}=0.307$ ). Using plasma Phe SA as precursor pool, fibrinogen FSR increased by $\approx 56 \%$ (from $12.1 \pm 2.5$ to $18.9 \pm 3.9$ percent per day, $p<0.001)$. FSR increased by $\approx 51 \%$ also using plasma Tyr SA as precursor pool $(p<0.01)$. When intracellular Phe SA was calculated after correction for the KIC/Leu enrichment (or SA) ratio, fibrinogen FSR increased by $\approx 63 \%$ (from $15.0 \pm 3.3$ to $24.4 \pm 5.3$ percent per day, $p<0.001$ ). In the two subjects infused with ${ }^{14} \mathrm{C}$-leucine, fibrinogen $\mathrm{FSR}$ increased by $\approx 6 \%$ when plasma ${ }^{14} \mathrm{C}-\mathrm{KIC}$ SA was used as precursor pool. In summary, isolated hyperglucagonemia increases fibrinogen FSR using a phenylalanine tracer technique. However, such an increase appears to be greater than that restimated using the classic leucine tracer method. Additional studies are in progress to confirm these findings.

\section{6}

EFFECT OF GROWTH HORMONE REPLACEMENT THERAPY ON GLUCOSE METABOLISM IN GROWTH HORMONE DEFICIENT ADULTS

M. Ried!', H. Kotzmann', M. Clodi', G. Pacini ${ }^{3}$ O. Wagner² D Rabensteiner! R. Prager', and A. Luger'. 'Department of Medicine III, Division of Endocrinology and Metabolism, "Department. of Laboratory Medicine, University of Viema, Vienna, Austria, ${ }^{3}$ LADSEB-CNR, Padoya italy

Growth hormone deficiency (GHD) is associated with decreased lean body mass, increased fat mass, increased lipid levels and insulin resistance On the other hand growth hormone (GH excess leads to hyperinsulinemia and insulin resistance. To assess the influence of growth hormone replacement therapy (GHRT) on carbohydrate metabolism in adults with GHD we examined 12 patients $(2 \mathrm{~m} / 10$ f) mean age $47 \pm 2.5$ yrs) before and after 18 months of GHRT in a dose of $0.25 \mathrm{IU} / \mathrm{kg} /$ week. Patients with additional anterior pituitary deficiencies were on stable replacement therapy throughout the study. A frequertly sampled i.v. glucose tolerance test was performed and glucose, insulin and $\mathrm{C}$-peptide concentrations were measured over a 3 hours period. Insulin sensitivity, basal and total insulin secretion rate, and hepatic extraction rate of insulin were analysed using the minimal model method of Bergman. IGF-l levels increased significantly after 6 months GHRT $(80.1 \pm 12.3 \mu \mathrm{g} / \mathrm{L}$ vs. $203.4 \pm 22.8 \mu \mathrm{g} / \mathrm{L} ; \mathrm{p}<0.0005$ ). Fasting glucose and $\mathrm{HbA} 1 \mathrm{c}$ levels remained unchanged after 18 months GHRT(FG: $85.7 \pm 2.5 \mathrm{mg} / \mathrm{dL}$ vs. $86.3 \pm 2.2 \mathrm{mg} / \mathrm{dL}$; HbAlc: $5.6 \pm 0.09 \%$ vs. $5.88 \pm 0.14 \%$ ). Insulin sensitivity $\left(\mathrm{S}_{\mathrm{i}}\right)$ was significantly reduced after 18 months GHRT (Si: $8.9 \pm 2.8 \mathrm{~min}_{-1} / \mathrm{mU} / \mathrm{L}$ vs. $5.3 \pm 2.3$ $\mathrm{min}_{.4} / \mathrm{mU} / \mathrm{L} ; \mathrm{p}<0.004$ ), while basal (BIS) and total insulin secretion rate (TIS) increased significantly (BIS: $31.03 \pm 5.8 \mathrm{pMol} / \mathrm{L} / \mathrm{min}$ vs. $49.9 \pm 12.2 \mathrm{pMol} / \mathrm{L} / \mathrm{min} ; \mathrm{p}<$ 0.03 ; TS: $11.4+2.3 \mathrm{nMot} / \mathrm{L}$ in $180 \mathrm{~min}$ vs. $49.9 \pm 12.2 \mathrm{nMol} / \mathrm{L}$ in $: 80 \mathrm{~min} ; \mathrm{p}<0.05$ ). Hepatic insulin extraction rate (HE) remained unchanged $(84.2 \pm 2.7 \%$ vs. $80.3 \pm 3.9 \%$ ). In conclusion 18 -months growth hormone replacement therapy in adults with GHD lead to a significant reduction of insulin sensitivity and significant increase in basal and total insulin secretion rate, while fasting glucose levels were unaffected. 


\section{7}

GROWTH HORMONE RESPONSE TO GHRH, GHRP-6 AND GHRH + GHRP-6 IN NON-INSULIN-DEPENDENT DIABETES MELLITUS

D. Micić, A. Kendereški, V. Popović, M. Šumarac, Dj. Macut, S. Zorić, M Colic, D. Zivkovic, C. Dieguez* and F. Casanueva*. Institute of Endocrinology, Dr Subotića13, 11000 Beograd, Yugoslavia and *Department of Medicine, Santiago de Compostela Universily, PO Box 563, Santiago de Compostela, Spain

A suppressed growth hormone $(\mathrm{GH})$ response to Growth Hormone Releasing Hormone (GHRH) in NIDDM has been reported. A novel GH secretagogue: Growth Hormone Relcasing Peptide-6 (GHRP-6) release GH specifically, in a dose related manner, acting at the pituitary and hypothalamic level. The aim of our study was to compare the GH response to GHRH (GRF 1-29 $\mathrm{NH}_{2}, 100 \mu \mathrm{g}$ iv), GHRP-6 (His-D Trp-Ala-Trp-D Phe-Lys- $\mathrm{NH}_{2}, 90 \mu \mathrm{g}$ iv) and combined GHRH+GHRP-G in 10 well regulated patients with NIDDM (age: $45.89 \pm 1.84$ years, BMI: $25.53 \pm 0.72 \mathrm{~kg} / \mathrm{m}^{2}$ ) and $10 \mathrm{age}$ and BMI matched healthy controls. Serum GH was measured with fluoroimmunoassay (Delphia, Pharmacia, $\mathrm{mU} / \mathrm{l}$ ) at $-30,-15,0,15,30,45,60,90$ and $120 \mathrm{~min}$ in all three tests. GH was analysed as area under the curve (AUC)(mU per $1 / 120 \mathrm{~min}$ ) using Student's $\mathbf{t}$ test. Comparing GH response in patients vs. controls following results were obtained: a) GHRH-AUC $(444,23 \pm 115.28$ vs. $1342.70 \pm 396.73, p<0.05)$, b) GHRP-6-AUC $(2058.81 \pm 506.68$ vs. $5010.51 \pm 1879.68, \mathrm{p}>0.05)$ and $\mathrm{c})$ GHRH+GHRP-6-AUC $(3837.25 \pm 450.02$ vs $10613.90 \pm 2482.07, \mathrm{p}<0.05)$. In conclusion, patients with NIDDM have significantly blunted GH response to GHRH and GHRH+GIRP-6 in comparison with healthy controls, while there were no difference in $\mathrm{GH}$ response to GHRP-6, indicating that deranged $\mathrm{GH}$ response to GHRH in NIDDM is a functional defect.

\section{PS 33}

\section{IGF and IGFBP}

\section{8}

IGF-|| EXPRESSION IN TRANSFECTED KIDNEY FIBROBLASTS INDUCES IGFBP-5 EXPRESSION BUT DOES NOT SERVE AS A GROWTH STIMULUS. W. Kiess, G. Köpf, S. Mohan\#, W.F. Blum, L. Bach§, and E. Wolf*. Children's Hospital Giessen, Germany, *Institute of Molecular Animal Production, Munich, Germany, \#Loma Linda University, Ca, USA, § Austin Hospital, VIC, Australia. Insulin-like growth factor-II (IGF-Ii) is a mitogen for cells in culture. IGF binding proteins (IGFBPs) modulate IGF action. We have asked whether overexpression of prolGF-1l leads to increased cellular proliferation and to changes in IGFBP expression. We have reported that human embryonal kidney fibroblasts (293 cells) which were stably transfected with the human IGF-II cDNA express large quantities of IGF-II mRNA and secrete 1-5 ug/ml and 150-230 ng/ml IGF-II within 24 hours of serum-free culture (transfectant 293-9 and -11 respectively) (Eur. J. Biochem. 232:172-178 (1995)). Interestingly, media from overexpressing 2939 and to a lesser degree from 293-11 cells contained large amounts of a $22 \mathrm{kDa}$ IGFBP as shown by ligand blot analysis using ${ }^{125}$ |-IGF-II while media from parental 293 and control transfected 413 cells do not. Specific 32P-labeled riboprobes for IGFBP-1, $-2,-3,-4,-5$ and -6 were used in solution hybridisation RNase protection assays to measure IGFBP mRNA expression in the different cell lines. While no significant difference in IGFBP-1, -2, -3, -4 and -6 mRNA expression was measured, IGFBP-5 mRNA expression was twofold higher in cells overexpressing prolGF-II. In addition, $292-0$ cells secreted $19.0+1.6$, 29311 cells $16.2 \pm 1.5$, and 413 cells $19.7 \pm 2.3 \mathrm{ng} / \mathrm{ml}$ IGFBP- 5 as measured by a specific radioimmunoassay while 293-9 cells secreted more IGFBP-5 $(41.0 \pm 5$ $\mathrm{ng} / \mathrm{ml}$ per $24 \mathrm{hrs}, \mathrm{n}=6, p<0.005)$. Surprisingly, parental cells or control transfected cells grow as well as cells overexpressing prolGF-II as measured by transfected cells grow as well as cells overexpressing prolGF-ll as measured by diphenyltetrazolium bromide) assay and BrdU (5-bromo-2'-deoxy-uridine) assay. In order to assess the mitogenic effect of prolGF-II further, prolGF-II secreted into media conditioned by transfected 293-9 cells was purified over an anti-|GF-II affinity column. Addition of IGF-I, IGF-II or prolGF-II (1 to $1000 \mathrm{ng} / \mathrm{ml}$ ) to 293-0 parental cells showed that IGF-I stimulated MTT activity at low concentrations (1 $10 \mathrm{ng} / \mathrm{ml}$ ), while IGF-|| was less potent and prolGF-II had no effect. In conclusion, (1) overexpression of prolGF-II in human kidney embryonal fibroblasts leads to an increased expression of IGFBP-5 mRNA and also to an increased secretion of IGFBP-5. (2) prolGF-11 does not seem to be a potent mitogen for human kidney embryonal fibroblasts in culture. (supported by DFG, Bonn, Germany).

\section{9}

INSULIN-LIKE GROWTH FACTOR BINDING PROTEIN1 REGULATES SKELETAL MUSCLE GLUCOSE TRANSPORT. H. Wallberg-Henriksson, B.W. Craig, M. Tally, L.A. Nolte, K. Hall and J.R. Zierath. Department of Clinical Physiology and Department of Endocrinology, Karolinska Hospital, Stockholm, Sweden.

Insulin-like growth factors (IGF's) are known to activate muscular glucose transport by the same mechanism as insulin. One striking difference between insulin and IGF's is that the growth factors have binding proteins. The regulatory effect of insulin-like growth factor binding protein 1 (IGFBP1) on insulin-, IGF1-, exercise and hypoxia induced glucose transport was studied in isolated rat epitrochlearis- and split soleus muscle. Increasing concentrations of IGF1 resulted in a dose-dependent increase in 3-0-methylglucose transport in split soleus muscle. IGFBP1 ( $375 \mathrm{ng} / \mathrm{ml}$ ), inhibited the IGF1 induced increase in glucose transport by $80 \%$ $(\mathrm{p}<0.001)$ in the split soleus muscle and by $70 \%(\mathrm{p}<0.01)$ in the epitrochlearis muscle. Furthermore, exposure to IGFBP1 $(375 \mathrm{ng} / \mathrm{mI})$, abolished the exercise induced (swimming) increase in glucose transport by $68 \%(\mathrm{p}<0.05)$ and the hypoxia induced increase by $52 \%(\mathrm{p}<0.05)$. Dantrolene $(25 \mu \mathrm{M})$, a blocker of $\mathrm{Ca}^{2+}$ release from the sarcoplasmic reticulum, decreased the IGF induced increase in glucose transport from $1.02 \pm 0.05 \mu \mathrm{mol} \mathrm{x} \mathrm{ml}{ }^{-1} \times \mathrm{x} \mathrm{hr}^{-1}$ to $0.28 \pm 0.04 \mu \mathrm{mol} \mathrm{x} \mathrm{ml}{ }^{-1} \times \mathrm{hr}^{-1}(\mathrm{p}<0.05)$ and totally abolished the exercise effect on glucose transport $(p<0.01)$. Serum levels of immunoreactive IGF1 were decreased by $50 \%(\mathrm{p}<0.001)$ in rats immediately following acute swim exercise compared to sedentary controls. No change was observed in serum levels of IGF2 following exercise. In conclusion, IGFBP1 alone or in conjunction with the IGF's, constitute an important regulator of the exercise induced increase in muscular glucose transport. This novel regulatory mechanism appears to be $\mathrm{Ca}^{2+} \mathrm{de}$ pendent and might operate via increased binding and degradation of IGFI by skeletal muscle. 
700

HYPERINSULINEMIA ASSOCIATED TO LOW INSULIN-LIKE GROWTH FACTOR BINDING PROTEIN 1 \& 2 IN OBESITY M.H. Rasmussen, A. Juul, N.E. Skakkebre and J. Hilsted. Department of Endocrinology, Hvidovre University Hospital and Department of Growth and Reproduction, Rigshospitalet, Denmark.

This is the first study investigating Insulin-like growth factor binding protein-1 (IGFBP-1) and IGFBP-2 before and after weight loss in obese subjects. In obesity insulin mediated suppression of IGFBP-1 has been suggested, but it is not known whether the decrease in IGFBP-1 is reversible with weight loss or a resistant finding in obesity. To address this question we have measured IGFBP $-1,-2$, and insulin levels in 18 obese subjects (BMI; $40.9 \pm 1.1 \mathrm{~kg} / \mathrm{m}^{2}$, mean \pm SEM) before and after an average weight loss of $30.3 \pm 4.6 \mathrm{~kg}$ and in 18 age-and sex matched normal subjects (BMI; $23.0 \pm 0.4 \mathrm{~kg} / \mathrm{m}^{2}$ ). Body composition was determined using anthropometric measurements and dual-energy $x$-ray absorptiometry scanning. IGFBP-1 as well as IGFBP-2 were decreased in the obese subjects compared to age-and sex matched normal controls $(2.1 \pm 0.9$ ys. $4.4 \pm 1.6 \mu \mathrm{g} / \mathrm{L}$; $P<0.01)$ and $(88 \pm 17 v s .185 \pm 19 \mathrm{ng} / \mathrm{ml} ; P<0.01)$, respectively. The insulin level was elevated in obese subjects compared to normal subjects $(43 \pm 3$ vs. $82 \pm 8 \mathrm{pmol} / \mathrm{L} ; P<0.001)$. After diet-induced massive weight loss IGFBP-1, IGFBP-2 and insulin levels were restored to nomal in obese subjects (BMI; $27.8 \pm 1.4 \mathrm{~kg} / \mathrm{m}^{2}$ ). Multiple step-wise regression analysis revealed that changes in insulin levels during weight loss was the major determinant of, and accounted for $46 \%$ of the increase in IGFBP-1, whereas changes in body weight had no independent effect. We conclude that the decreased IGFBP-1 and IGFBP-2 levels in obesity is normalized after weight loss and is mainly a consequence of the diminished hyperinsulinemia.

\section{2}

METABOLIC EFFECTS OF RECOMBINANT HUMAN INSULIN-LIKE GROWTH FACTOR-I IN PATIENTS WITH PRIMARY SEVERE INSULIN RESISTANCE AND DIABETES MELLITUS

H. Vestergaard, M. Rossen, S. Urhammer, J. Müller, P.C. Eskildsen and O. Pedersen. Steno Diabetes Center, Koege Hospital and National University Hospital, Denmark.

A series of different mutations in the insulin receptor gene is associated with some of the syndromes of severe insulin resistance, whereas postreceptor molecular defects are likely to be dominant in other cases of severe insulin resistance. In patients suffering from the genetic syndromes of severe insulin resistance it appears the diabetes develops when the adoptive hypersecretion of insulin fails and often these forms of diabetes will be insensitive to insulin treatment. The present study was undertaken to examine the potential extent of metabolic and hormonal responses to subcutaneously administered recombinant human IGF-I (rhIGF-l: $80 \mu \mathrm{g} / \mathrm{kg}$ body weight twice a day) for two weeks in 4 patients with extreme insulin resistant diabetes mellitus. Two siblings had known mutations in the tyrosine kinase domain of the insulin receptor and a deletion of exon 17 in part of their insulin receptor mRNA whereas the remaining 2 patients were suspected to have defects at receptor and/or postreceptor sites. In all but one patient plasma glucose levels decreased more than $35 \%$ in response to rhiGF-I which was parallelled by reduced levels of serum insulin (25-50\%), proinsulin (40-50\%) and C-peptide $(10-65 \%)$ and an improvement in glycemic control as evaluated by decreased $\mathrm{HbA}_{1 \mathrm{C}}$ and serum fructosamine. In one patient, characterized by having very low levels of serum IGF-I and plasma IGFBP-3 and very high levels of plasma IGFBP-1, plasma glucose was hardly reduced $(18 \%)$ and an increase in serum insulin, proinsulin and $C$-petide was seen at the end of the study period. No severe adverse effects were reported or observed during the study period

Taken together, the results of the present study indicate that short-term rhIGF. I treatment is clinically useful in some patients with extreme insulin resistance syndromes and diabetes mellitus in whom insulin treatment fails to maintain acceptable glycaemic control.

\section{1}

IGF-I STIMULATES THE TYROSINE PHOSPHORYLATION OF PAXILLIN AND FOCAL ADHESION KINASE

P. S. Leventhal and E. L. Feldman, Department of Neurology, University of Michigan, Ann Arbor, MI

Because insulin-like growth factor-I (IGF-I) promotes neurite outgrowth and neuronal survival, IGF-I may be useful for treating diabetic neuropathy. We were therefore interested in the biochemical mechanisms by which IGF-I stimulates neurite outgrowth. We found that the initial effect of IGF-I was to stimulate membrane ruffling and the extension of highly motile veil-like membranes (lamellipodia). Because the tyrosine phosphorylation of focal adhesion kinase (FAK) and paxillin are thought to help direct cell membrane motility, we examined the localization of these proteins and whether IGF-I would promote their phosphorylation. Paxillin, FAK, and phosphotyrosine were localized in the lamellipodia. Furthermore, we found that IGF-I enhanced the tyrosine phosphorylation of both $F A K$ and paxillin with a similar concentration profile as that of the IGF-I receptor. However, maximal lyrosine phosphorylation of FAK and paxillin was delayed by at least 10 min compared to that of the IGF-I receptor. IGF-I stimulated phosphorylation of paxillin, FAK, and the IGF-I receptor was reduced by the receptor blocking antibody aIR-3. Finally, the phosphorylation of FAK and paxillin, but not of the IGF-I receptor was inhibited by cytochalasin $\mathrm{D}$. These results indicate that the yrosine phosphorylation of FAK and paxillin occurs downstream of the IGF-I receptor. These phosphorylations may help direct IGF-I stimulated lamellipodial extension, which in turn determine cell, neurite, and growth cone shape.

Supported by NIH grants R29 NS32843 (E.L.F.) and NRSA IF32NS09912-01 (P.S.L.)

\section{3}

POTENT INSULIN-LIKE ACTION OF IGF-I IN RAT LIVER R. Englisch, C. Fürnsinn, C. Ludwig, W. Waldhäusl, and M. Roden Dept. Internal Med. III, Div. Endocrinol. Metab., Univ. Vienna, Austria Administration of insulin-like growth factor-I (IGF-I) has been shown to suppress hepatic glucose production in vivo, but the direct action of IGF-I on the liver is still unclear. In order to study the effects of IGF-I on glucose metabolism, isolated livers of fed rats were perfused (flow rate: $-3 \mathrm{ml} / \mathrm{min} \cdot \mathrm{g}$ liver) in a non-recirculating system (oxygenated $\mathrm{K}-\mathrm{H}$ buffer, $5 \mathrm{mM}$ Dglucose). Basal glucose production ( $\mu \mathrm{mol} / \mathrm{min} \cdot \mathrm{g}$ liver) remained unchanged during control experiments $(t=30 \mathrm{~min}: 0.72 \pm 0.19$ vs $t=0 \mathrm{~min}: 0.88 \pm 0.29$; means $\pm S E ; n=4$ per group), while it was dose-dependently inhibited by insulin (INS, $0.1 \mathrm{nM}: 0.51 \pm 0.21, \mathrm{p}<0.05 ; 1 \mathrm{nM}:-0.13 \pm 0.17, \mathrm{p}<0.001$ vs control) and by IGF-I (0.1 nM: $0.59 \pm 0.13 ; 1 \mathrm{nM}: 0.13 \pm 0.06, \mathrm{p}<0.001$ vs control). IGF-I was equally potent at both concentrations (ns vs. INS). Stimulation by epinephrine (EPI, $0.05 \mu \mathrm{M}$ ) caused a 4 fold increase of hepatic glucose production ( $2.68 \pm 0.22,0<0.001$ vs baseline), which was reduced by $44 \%$ and $71 \%$ in the presence of 0.1 and $1 \mathrm{nM} \mathrm{NNS}$, respectively $(1.58 \pm 0.14, p<0.01 ; 0.79 \pm 0.18 p<0.001$ vs control). IGF-I (1 $\mathrm{nM})$ inhibited EPI-induced glucose release by $54 \%(1.25 \pm 0.11 \mathrm{p}<0.001$ vs control, ns vs $1 \mathrm{nM}$ INS). Net rates of lactate production (mmol/min $\mathrm{g}$ liver) increased $\sim 5$ fold by EPI, but were neither affected by INS nor by IGF-I at InM $(0.49 \pm 0.03 ; 0.52 \pm 0.07 ;$ control: $0.46 \pm 0.031$. Mean portal pressure was similar under basal $\left(-4.5 \mathrm{~cm} \mathrm{H}_{2} \mathrm{O}\right)$ and EPI-stimulated conditions $(-12.0 \mathrm{~cm}$ $\mathrm{H}_{2} \mathrm{O}$ ) during INS, IGF-I, and control studies. Conclusions: the results indicate that at physiological concentrations IGF-I inhibits (a) basal and (b) epinephrine-stimulated hepatic glucose release, and (c) does not affect portal perfusion in vitro. These findings suggest that IGF-I might play an important role for the regulation of liver metabolism, which could be mediated by specific IGF-I receptors or hybrid insulin/IGF-I receptors. 


\section{Stress Hormones in Diabetes- Catecholamines}

704

\section{ACUTE INDUCTION OF MUSCULAR INSULIN RESISTANCE BY EPINEPHRINE IS ACCOMPANIED BY AN INCREASE IN INTERSTITIAL GLUCOSE CONCENTRATION}

M Niklasson, A Holmäng and P Lönnroth', The Wallenberg Laboratory and ${ }^{1}$ The Lundberg Laboratory for Diabetes Research, Sahlgrenska Hospital, S-413 45 Gothenburg, Sweden The effects of epinephrine (E) and propranolol (P) on muscle glucose uptake and interstitial insulin concentrations were investigated by the microdialysis method during euglycemic hyperinsulinemic $(130 \mathrm{mU} / \mathrm{L})$ clamp in female rats $(277 \pm 6 \mathrm{~g})$. To assess the interstitial concentrations of insulin and glucose simultaneously microdialysis was performed in medial femoral muscles in both legs. Infusion with $\mathrm{E}(0.15$ $\mu \mathrm{g} / \mathrm{kg} \cdot \mathrm{min}$ ) started $120 \mathrm{~min}$ after initiation of the clamp, followed by a $B$-adrenergic blockade with $P(2 \mu \mathrm{g} / \mathrm{kg} \cdot \mathrm{min})$ after $200 \mathrm{~min}$. In additional experiments somatostatin (S) was infused $(0.1 \mu \mathrm{g} / \mathrm{min})$ starting at 110 $\mathrm{min}$. Glucose infusion rate was $8.6 \pm 0.7 \mathrm{mg} / \mathrm{kg} \cdot \mathrm{min}$ during clamping with insulin alone, $\mathrm{E}$ reduced this by $78 \pm 12 \%$ and this effect of $\mathrm{E}$ was fully reversed by $P$. Mean interstitial glucose rose from $6.7 \pm 0.6$ $\mathrm{mmol} / \mathrm{L}$ to $7.8 \pm 0.7 \mathrm{mmol} / \mathrm{L}$ with $\mathrm{E}$, then down to $4.3 \pm 0.5 \mathrm{mmol} / \mathrm{L}$ with $P$. The arterial interstitial glucose difference was unchanged by $\mathbf{E}$ but rose by $50 \pm 10 \%$ with $P$. E induced a small S-inhibited increase in plasma insulin concentration but interstitial insulin concentration were low and unchanged. The results demonst-rate that interstitial glucose but not insulin concentration increases during the severe insulin resistance induced by $E$. The effect is reversed by blocking $B$ adrenergic receptors with $P$. It may be suggested that induction of insulin resistance in resting muscle increases interstitial glucose levels by a shift in rate-limiting step for glucose uptake in the muscle cells.

\section{6}

TISSUE-SPECIFIC MODULATION OF INSULIN RECEPTOR MRNA LEVELS IN ADRENALINE-TREATED RATS.

C. Calle, J. Campión, N. Dávila', M.C. Carranza and P. Aller ${ }^{2}$. Dept. Bioquímica III, U.C.M., Hospital C.P.H. ${ }^{1}$ and C.I.B. ${ }^{2}$ Madrid.Spain.

We have previously reported that human insulin receptor $(\mathrm{IR})$ mRNA levels may be modulated in vivo and in vitro by high levels of catecholamines. In the present work we comparatively analyze $\mathbb{R}$ gene expression at the mRNA level in liver, himdlimb skeletal muscle, and epididymal adipose tissue of rats with increased levels of catecholamines, induced by a prolonged administration in vivo of adrenaline $(2 \times 0.05 \mathrm{mg} / \mathrm{rat} / \mathrm{day}$, for 7 days) $(n=6)$, in relation with control rats that received a sham-treatment $(n=6)$. All the animals were killed without anesthesia. Trunk blood samples were collected and the three tissues in study were immediately removed and frozen in liquid nitrogen. Rat body weights were markedly reduced by adrenaline treatment, and plasma insulin and glucose levels were increased and unaltered respectively, as compared to control rats. In the liver of treated rats, there were not differences in relation to controls when total DNA and pratein content were measured. In the skeletal muscle of treated animals, only a light decrease in protein concentration was detected. However, a clear increase in both protein and DNA content was observed in the adipose tissue of treated animals, as compared to controls. Northern blot assays revealed two IR mRNA species of approximately 9.5 and $7.5 \mathrm{~Kb}$, in the three tissues. Adrenaline treatment induced an approximately $60 \%$ increase in the levels of both RNAs in adipose tissue but not in liver or skeletal muscle. These results provide evidence for an in vivo tissue-specific regulation of IR gene expression at the mRNA level, in rats under an experimental condition of excess of catecholamines.

\section{5}

THIS ABSTRACT HAS BEEN .WTTHDRAWN BY THE AUTHOR.
707

THE IMIDAZOLINE ANTAZOLINE STIMULATES INSULIN SECRETION AND IMPROVES GLUCOSE TOLERANCE IN RATS. G. Bertrand, D. Berdeu, R. Puech and M.M. Loubatières-Mariani. UPR 9023 CNRS; Lab. Pharmacol, Fac. Méd., Montpellier, FRANCE.

It is known that some imidazoline $\alpha_{2}$-adrenergic blockers stimulate insulin secretion in vivo. On the other hand, in vitro studies have shown that imidazolines with or without $\alpha_{2}$-adrenergic antagonist activity increase insulin release by acting on specific imidazoline receptors. In this study, we investigated in vivo the effects of antazoline, an imidazoline devoid of $\alpha_{2}$-adrenergic antagonist activity, on insulin secretion and glycemia. The experiments were performed in anaesthetized or conscious rats, fasted overnight. In anaesthetized rats, the intravenous administration of antazoline $(1.5 \mathrm{mg} / \mathrm{kg})$ induced an immediate $(+49.9 \pm 7.9 \mathrm{mU} / \mathrm{l}$ at $2 \mathrm{~min})$ and transient $(8-10 \mathrm{~min})$ increase in plasma insulin levels without affecting basal plasma glucose levels. During an intravenous glucose tolerance test $(0.5 \mathrm{~g} / \mathrm{kg})$, the imidazoline induced an 3-fold increase in insulin secretion during the first $20 \mathrm{~min} \quad(p<0.001)$ and increased the glucose disappearence rate $(p<0.01)$. Moreover, in conscious rats, during an oral glucose tolerance test $(1 \mathrm{~g} / \mathrm{kg})$, the intragastric administration of antazoline $(1.5 \mathrm{mg} / \mathrm{kg})$ clearly enhanced the insulin response during the first $30 \mathrm{~min}$ (the integrated insulin response was $+1558.7 \pm 204.6$ vs $+929.4 \pm 131.6 \mathrm{mU} / \mathrm{l}$ with glucose alone; $p<0.05$ ) and reduced the hyperglycemia during the $120 \mathrm{~min}$ (the integrated glucose increment was $+312.81 \pm 21.99$ vs $+422.61 \pm 31.29 \mathrm{mmol} / \mathrm{l}$ with glucose alone; $p<0.05$ ). In conclusion, the imidazoline antazoline is able to stimulate insulin secretion and improve glucose tolerance. Thus, the imidazoline sites of $B$ cells may be a new target for antidiabetic drugs. 
DISCREPANCY OF CARDIODYNAMIC AND METABOLIC COMPONENTS OF ADRENERGIC REACTIONS IN DIABETIC DOGS A.I. Khomazjuk, A.P. Nescheret and I.V. Gonchar. Institute of Endocrinology \& Metabolism. Vyshgorodska 69, Kiev, Ukraine, 254114. With the aim to study conformity between cardiodynamic and metabolic components of adrenergic heart reactions (AR) experiments in 12 dogs only with overt diabetes (D; alloxan $0.75 \mathrm{mkg} / \mathrm{kg}$ iv, fasting glycemia $18,3 \pm 1.2 \mathrm{mmol} / \mathrm{l}$ ) and12 control dogs (C) were performed under chloralose anaesthezia. Catheterisation and programmed extracorporal autoperfusion of left coronary artery (CA), catetherization of heart and drainage of coronary sinus (CS) were used. The AR was provoked by injection of epinephrine $(0.05-5.0 \mathrm{mkg})$ into coronary blood stream. In $\mathrm{C} A \mathrm{AR}$ included short term dose dependent increase of left ventricular pressure, $\mathrm{dP}^{2} / \mathrm{dt}$ and heart rate. On 15-30s the AR was interrupted by cholinergic counterregulatory heart rate slowing. decrease of contractility and myocardial $\mathrm{O}_{2}$ consumption. During the AR myocardial consumtion of glucose and FFA was diminished and glycerol was released into CS due to activation of glycolisis and lypolisis. Dynamic changes of ECG included augmentation and conversion of T wave, elevation or depression of ST segments and extrasystolia.Counterregulatory components of the AR were blocked by atropine $(0.5 \mathrm{mg} / \mathrm{kg})$ and most of all beta-adrenergic components but not lypolisis were blocked by propranolol ( $2 \mathrm{mg} / \mathrm{kg}$ ). In D counterregulatory components of the AR were absent or become weakened and beta-adrenergic components were less attenuated. On the contrary metabolic components of AR were enhanced and followed by release of glucose, lactate and glycerol (AVD: $-4.1 \pm 0.02 ;-0.2 \pm 0.09 ;-0.3$ $\pm 0.08 \mathrm{mmol} / 1$, resp.) into CS blood. Changes of ECG were enhanced as well too. This data suggest that disturbances of cholinergic heart control in D increase possibility of adrenergic deterioration of myocardial metabolism and function.

\section{PS 35}

\section{Stress Hormones in Diabetes- Steroids}

\section{9}

THE EFFECT OF ELEVATED STRESS HORMONES ON INSULIN LEVELS AND TISSUE LIPOGENESIS IN RATS.

GJ Cooney, JL Phuyal and SC Blair. Department of Endocrinology, Royal Prince Alfred Hospital, Camperdown, NSW 2050, Australia.

Counter-regulatory stress hormones such as corticosterone (CORT) are known to cause insulin resistance in skeletal muscle by increasing lipolysis, elevating NEFA levels and thereby reducing glucose utilisation. However increased stress responses and increased levels of CORT are also associated with increased adiposity in obese animal models. To determine what effect increases in CORT might have on lipid synthesis during normal feeding patterns we supplied different concentrations of CORT in the drinking water to different groups of rats. Lipogenesis was assessed in liver and adipose tissues by measuring the incorporation of tritiated water into saponifiable lipid over a 1 hour period 4 hours after the commencement feeding (lights off).

\begin{tabular}{|c|c|c|c|c|}
\hline \multirow{3}{*}{$\begin{array}{l}\text { CORT } \\
\text { (in water) }\end{array}$} & \multirow{3}{*}{$\begin{array}{l}\text { insulin } \\
(\mu U / \mathbf{m I})\end{array}$} & \multicolumn{3}{|c|}{ lipogenesis ( $\mu$ gatoms/h/tissue) } \\
\hline & & & epididym: & mesenteric \\
\hline & & liver & adipose & adipos \\
\hline $0 \mu \mathrm{g} / \mathrm{ml}$ & $126 \pm 15$ & $51 \pm 5$ & $12 \pm 2$ & $16 \pm 2$ \\
\hline $10 \mu \mathrm{g} / \mathrm{ml}$ & $151 \pm 11$ & $33 \pm 3^{*}$ & $32 \pm 7^{*}$ & $22 \pm 4$ \\
\hline $25 \mu \mathrm{g} / \mathrm{ml}$ & $182 \pm 18^{*}$ & $28 \pm 6^{*}$ & $38 \pm 2^{*}$ & $26 \pm 5^{*}$ \\
\hline $50 \mu \mathrm{g} / \mathrm{mi}$ & $192 \pm 19^{*}$ & $26+2^{*}$ & $28 \pm 5$ & $24 \pm 2$ \\
\hline
\end{tabular}
${ }^{*} \mathrm{p}<0.05$ for difference from $0 \mu \mathrm{g} / \mathrm{ml}$

Increasing the CORT dose significantly increased the prandial insulin levels and the rates of lipid synthesis in epididymal, and to a lesser extent mesenteric adipose tissue. However the lipogenic rate in liver was depressed by CORT supplementation even though the insulin levels were increased. These results suggest that slight increases in the levels of the stress hormone CORT during feeding may cause insulin resistance in liver but increase lipid synthesis in abdominal adipose tissue depots.

\section{0}

Glucose intolerance and increased glucose-6-phosphatase activity in transgenic mice with overexpressed $\beta$-cell glucocorticoid receptor.

A.Khan, F.Delaunay, ZC.Ling, B.Davini, A. Cintra, C-G Östenson, A.Andersson, J.A.Gustafsson, S.Okret, S.Efendic. Dept. of Molecular Medicine, Karolinska Hospital, Dept, of Medical Nutrition, Huddinge Hospital, Karolinska Institute, Stockholm, and Dept. of Medical Cell Biology, Uppsala University, Uppsala, Sweden.

Glucocorticoid treatment in vivo increases glucose cycling (glucose-glucose-6-P--glucose) (GC) and glucose-6 phosphatase (G6Pase) activity and decreases glucose stimulated insulin release in ob/ob mice islets. In the present study we have used transgenic mice (TG) with overexpressed glucocorticoid receptors in the B-cells to further investigate the impact of glucocorticoids on islet glucose metabolism and insulin release. TG mice exhibited significantly higher blood glucose concentrations compared to control mice $120 \mathrm{~min}$ after administration of $2 \mathrm{~g} / \mathrm{Kg}$ glucose load I. V. $(8.57 \pm 0.51$ vs $6.18 \pm 0.34 ; \mathrm{p}<0.001)$. The rate of $\mathrm{GC}(\%)$ and G6Pase activity (pmol/islet/h) were higher in TG mice islets while insulin release $(\mu \mathrm{U} /$ islet/h) was lower. Dexamethasone (dex) treatment in vivo for 3 days $(25 \mu \mathrm{g} /$ day) increased G6Pase activity and decreased insulin response in $T G$ and normal animals.

$\begin{array}{llll}\text { mice } & \text { TG }(\mathrm{n}=6) & \text { control }(\mathrm{n}=6) & \text { significance } \\ \text { GC } & 16.4 \pm 0.4 & 6.0 \pm 1.1 & \mathrm{p}<0.05 \\ \text { G6Pase } & 3.65 \pm 0.4 & 2.32 \pm 0.4 & \mathrm{p}<0.05 \\ \text { G6Pase(dex) } & 6.21 \pm 0.4 & 4.01 \pm 0.5 & \mathrm{p}<0.01 \\ \text { Insulin (5.5) } & 21.9 \pm 0.5 & 33.8 \pm 2.4 & \mathrm{p}<0.001 \\ \text { Insulin(16.7) } & 96.5 \pm 7.1 & 149.9 \pm 13.1 & \mathrm{p}<0.01 \\ \text { Insulin(dex) }(5.5) & 15.7 \pm 0.4 & 25.1 \pm 1.2 & \mathrm{p}<0.001 \\ \text { Insulin(dex)(16.7) } & 70.7 \pm 3.1 & 118.7 \pm 7.2 & \mathrm{p}<0.001\end{array}$

In conclusion, glucocorticoids enhance islet G6Pase activity which results in increased GC. This in turn leads to decreased insulin response to glucose with impaired glucose tolerance. 
711

EFFECTS OF ACUTE AND CHRONIC DEXAMETHASONE ADMINISTRATION ON GLUCOSE TOLERANCE AND HEPATIC GLUCOSE METABOLISM IN HEALTHY HUMANS. $\mathrm{Ph}$. Schneiter and L. Tappy. Institut de Physiologie, Lausanne, Switzerland.

To determine wether early or late alterations of glucose clearance or hepatic glucose metabolism contribute to glucocorticoid-induced impaired glucose tolerance, 6 healthy humans were studied during 4 hours after ingestion of a ${ }^{13} \mathrm{C}$ labeled oral glucose load. Total glucose turnover was measured with $6,6^{2} \mathrm{H}$-glucose, and exogenous glucose appearance was monitored from plasma ${ }^{13} \mathrm{C}$ glucose enrichment. These measurements were performed after a 2-day chronic treatment with $2 \mathrm{mg}$ /day dexamethasone (CD) or placebo (P) or after an acute administration of $0.5 \mathrm{mg}$ dexamethasone 2 hours before the test (AD). $\mathrm{AD}$ and $\mathrm{CD}$ increased post prandial plasma glucose concentration by $27 \%(\mathrm{p}<0.04)$ and $32 \%(\mathrm{p}<0.02)$ and decreased glucose clearance by $22 \%(\mathrm{p}<0.05)$ and $28 \%(\mathrm{p}<0.02)$ respectively, but only CD increased plasma insulin concentration $(+160 \%, \mathrm{p}<0.0003)$. Total glucose turnover, suppression of bepatic glucose production by oral glucose, and splanchnic uptake of oral glucose were not affected by $A D$ or $C D$. It is concluded that 1) an impaired glucose tolerance, secondary to a decrease of glucose utilization by peripheral tissues, develops within hours of dexamethasone administration, 2) an increase in insulin concentration occurs after 2 days treatment with dexamethasone, without further deterioration of glucose tolerance, and 3) post glucose inhibition of hepatic glucose production and splanchnic uptake of exogenous glucose are not altered by acute or chronic dexamethasone administration.

\section{2}

INSULIN SECRETION, INSULIN SENSITIVTTY AND GLUCOSE EFFECTIVENESS IN CUSHING'S SYNDROME: MINIMAI MODEL ANALYSIS A.Kendereški, D.Micić, M.Šumarac, S.Zorić, Dj.Macut, M.Čolić and D.Manojlović Institute for Endocrinology, Clinical Center of Serbia, Dr Subotića 13, 11000 Belgrade, Yugoslavia

Mechanisms by which glucocorticoids infuence plasma glucose and insulin levels in Cushing's syndrome (CS) are not fully understood. The aim of the study was to investigate insulin secretory response during oral glucose tolerance test (OGTT), insulin sensitivity $(\mathrm{Si})$ and glucose effectiveness $(\mathrm{Sg})$ in patients with Cushing's syndrome. Nine patients $\left(5 \mathrm{f}, 4 \mathrm{~m}\right.$; age: $30.43+/-5.04$ yrs; BMI: $26.63+/ 1.05 \mathrm{~kg} / \mathrm{m}^{2}$ ) with clinical and biochemically proven CS and 9 age, sex and weight matched controls were studied. Two tests were performed at random order: OGTT and frequently sampled i.v. glucose tolerance test (FSIGT) in each investigated subject. Area under the curve for glucose (AUCG) and insulin (AUCD) was calculated during OGTT using trapezium rule while Si and Sg were calculated from FSIGT using MINMOD program (copyright R.N.Bergman). Basal glucose value $(5.78+/ /-0.30$ vs $4.43+/-0.35 \mathrm{mmol} / \mathrm{p}, \mathrm{p}=$ $0.01)$ and AUCG $(1303.25+/-93.13$ vs $717.5+1.80 .89 \mathrm{mmol} / / \mathrm{min}, \mathrm{p}<0.01)$ were significantly higher in CS than in controls. One patient had nonnal glucose tolerance, one patient had mild diaberes mellitus, and rest fullfiled WHO criteria for diabetes mellitus during OGTT. Patients with CS had significantly higher basal insulin levels $(13.61+/ 2.04$ vs $6.24+1 / 0.89 \mathrm{mU} / \mathrm{l}, \mathrm{p}<0.0 \mathrm{I}$, RIA INEP, normal values $0-20 \mathrm{mU} / \mathrm{l})$ while insulin response during OGTT was not significantly higher in comparison to controls (AUCI: $5295+/-1339,03$ vs $3776+/-1225.23 \mathrm{mU} / / \mathrm{min}, \mathrm{p}>0.05$ ). Insulin sensitivity was decreased in patients with $C S$ in comparison to controls $(1.63+1-0.42$ vs $\left.5.05+/-0.36 \times 10^{4} \mathrm{~min}^{-1} / \mathrm{mU} / /, \mathrm{p}<0.01\right)$ while there was no difference in $\mathrm{Sg}(0.029+1-$ 0.012 vs $0.019++-0,002 \mathrm{~min}^{-1}, \mathrm{p}>0.05$ ). In conclusion, our results demonstrate impaired glucose tolerance and insulin sensitivity, higher basal insulinemia without exagerated insuljn response to oral glucose challenge in patients with Cushing's syndrome.

\section{3}

REḠULATION BY ALDOSTERONE OF INSULIN RECEPTOR MRNA LEVELS IN HUMAN PROMONOCYTIC CELLS.

J. Campión, P. Aller', F. Mata, N. Dávilaª, B. Maestro and C. Calle. Depts. Bioquímica I and III. U.C.M, C.I.B. ${ }^{\prime}$ and Hospital C.P.H. ${ }^{2}$, Madrid. Spain.

Earlier studies of our laboratory and others have demostrated regulation of the insulin receptor (IR) gene expression in established cell lines by different steroid hormones, such as glucocorticoids, 1,25-dihydroxivitamin $D_{3}$, progestins and androgens. Nonetheless, there are no studies on the possible effects of mineralocorticoids on IR gene expression. For these reasons, in the present work we analyze the effect of aldosterone, the major biologically active mineralocorticoid in humans, on IR mRNA levels in U-937 human promonocytic cells. This cell line possesses steroids receptors and is considered to be an useful in vitro monocyte-like model to study IRs and their modulation by diverse agents. Aldosterone (Sigma) was applied to the cells at the final concentrations of $10^{-10}, 10^{-9}, 10^{-8}$ and $10^{-7} \mathrm{M}$ for $24 \mathrm{~h}$. For Northern blot assays, RNA samples were electrophoresed in agarose-formaldehyde gels, blotted to nylon membranes, hybridized with a ${ }^{32} \mathrm{P}$-labeled human IR cDNA probe and autoradiographed. The autoradiographs were scanned with a laser densitometer and the reading normalized with the amount of 285 rRNA, as revealed by ethidium bromide staining. Northern assays showed the presence of two major IR-related mRNA species of approximately $11 \mathrm{~Kb}$ and $8.5 \mathrm{~Kb}$ in size. The levels of both RNAs were decreased upon treatment with $10^{-10} \mathrm{M}$ aldosterone, reaching minimum values $150 \%$ decrease) with $10^{-9} \mathrm{M}$ aldosterone, and increasing thereafter at higher aldosterone concentrations. At present, the exact mechanism by which aldosterone regulates IR gen expression in U-937 is not known. However, this effect may be mediated by receptor proteins that are closely related with those of glucocorticoid receptors. The hormone-receptor complex after being translocated into the nucleus could bind to common consensus DNA sequences, designated as hormone response elements, confering in this manner mineralocorticoid regulation on the human IR gene.

\section{4}

INSULIN ACTION AND NON-INSULIN DEPENDENT GLUCOSE UPTAKE IN NORMOGLYCAEMIC WOMEN DURING ORAL CONTRACEPTION . K.R. Petersen, E. Christiansen, C. Haakonson, S. Madsbad, S. Skouby. Diabetes Centre, Rigshospitalet, Copenhagen and Dept. of Endocrinology, Hvidovre Hospital, Hvidovre, Denmark.

The decreased insulin sensitivity described during intake of older oral contraceptives (OCs) containing 1 , and 2 . generation progestogens is part of the cluster of metabolic alterations associated with vascular complications in users of these compounds. The aim of the present study was to evaluate the effect on insulin sensitivity and glucose effectiveness (non-insulin dependent glucose uptake) in non-diabetic women treated with an OC containing a newly developed 3. generation progestogen, norgestimate. Intravenous glucose tolerance test with frequent blood sampling and tolbutamide injection to elicitate a second insulin response was used to estimate insulin sensitivity index $\left(S_{1}\right)$ and glucose effectiveness $\left(S_{0}\right)$ in 16 healthy women (aged 21 to 27 years) before and after 6 months of treatment with a triphasic combination of ethinyl estradiol and norgestimate. Statistical evaluation was performed with paired non-parametrical methods. Fasting plasma insulin increased after 6 months of treatment 125.9

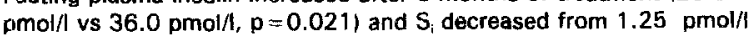
$x: \min ^{-1}$ before to $0.95 \mathrm{pmol} / \mathrm{x} \times$ min $^{-1}$ after 6 months $(\mathrm{p}=0.004)$. Fasting plasma glucose and $S_{8}$ were unchanged during treatment. Our results indicate that newer oral contraceptives have no effect on glucose effectiveness; they do, however, decrease insulin sensivity. These findings may have consequences for the contraceptive counselling of women with previous gestational diabetes or women with a family history of vascular disease. 


\section{5}

PROGESTERONE-INDLICED INSULIN-RESISTANCE IN FEMALE RAT ADIPOCYTES: LIPOLYTIC ACTION AND PHOSPHODIESTERASES ACTIVITIES.

M.-Th. Sutter-Dub, P. Cordoba and B.Ch.J. Sutter, Lab. Endocrinologie, Univ. Bordeaux I, Avenue des Facultés, F-33405 Talence Cedex, France.

Progesterone is one of the hormones responsible for insulinresistance in rats. Its effects occur within $20 \mathrm{~min}$ in vivo and in vitro. Recent evidence has indicated that cAMP and lipolysis are inhibited by progesterone in the female rat adipocytes. The purpose of the present study was to determine the role of the phosphodiesterases (PDE) in this action. - In the presence of Ro $20.1724 \%$, a specific inhibitor of PDE N (cAMP-dependent PDE), the inhibitory effect of progesterone $\left(10^{-9} \cdot 10^{-4} \mathrm{M}\right)$, on cAMP accumulation, disappeared. When the PDE I (calcium-calmodulin dependent PDE) was specifically inhibited by $\mathrm{CP} 41,757^{*}$, this effect remained. We then measured directly the enzyme activities in the presence of the steroid $\left(10^{-10_{-}}-0^{-3} \mathrm{M}\right)$. Progesterone had no effect on PDE activities, when the whole homogenate of adipocytes was considered. However, when the cytosolic and the particulate fractions of the homogenate were analyzed separately, the steroid increased the activity of the cytosolic PDE $\left(70 \%\right.$ at $\left.10^{-5} \mathrm{M}\right)$ but decreased that of the particulate fraction $\left(55 \%\right.$ at $\left.10^{-7} \mathrm{M}\right)$. These opposite effects of progesterone on the two subcellular fractions hide the real action of the steroid on PDE activities, when intact cells were considered. Thus, these results seem to indicate that one of the mechanisms through which progesterone decreased cAMP levels and, further lipolysis, is based on the increase of CAMP-PDE activity.

*Generous gifts of Hoffmann La Roche (Switzerland) and Pfizer (USA).

\section{PS 36 \\ Exercise}

716

'DIABETES WATCH': A COMMUNITY BASED PHYSICAL ACTIVITY PROGRAMME FOR NON-INSULIN-DEPENDENT DIABETIC PATIENTS N Latham, H J Bodansky² and M Dudfield ${ }^{3}$. Leeds Metropolitan University $y^{1}$, The General Infirmary at Leeds ${ }^{2}$, Leeds Sports Development Unit ${ }^{3}$.

The aim of this inter-sectorial collaborative study, by Leeds General Infirmary, Leeds Sports Development and Leeds Metropolitan University, was to develop an effective programme to improve and maintain the physical and mental well-being of people with NIDDM. 'Diabetes Watch' is a physical activity programme which consists of: (i) exercise testing and lifestyle counselling and (ii) supervised physical activity sessions. The programme was initiated to provide recommendations for the health care team due to the current poor success rate of promoting exercise. Over a period of 6 weeks, 234 patients (107 female, 127 male, mean age $57 \pm 10$ duration of NIDDM $17 \pm 3$ years) attending for their routine check up at the outpatients, were approached to determine their interest in 'Diabetes Watch'. The responses were: interested and registered for exercise testing $26 \%$; not interested $28 \%$; interested but constraints preventing involvement $40 \%$; not interested as autonomously active $6 \%$. Following this initial contact phase, exercise testing appointments were arranged with 35 of those registering an interest. From these arranged appointments, confirmed by telephone and in writing, 27 were tested $(11 \%$ of the overall study group), from which 14 attended the supervised physical activity classes (6\% of the study group). Positively, from a questionnaire appraisal, $98 \%$ had enhanced physical and mental well-being since joining the programme. This study demonstrates that although $66 \%$ of the study group valued, and were interested in exercise, only a small percentage finally participated in the physical activity sessions. In conclusion, few patients are self-motivated towards exercise in the control and management of NIDDM. To counter this, there is a need to promote exercise at the initial diagnosis of NIDDM. Further, to overcome perceived barriers to exercise, identified by patients, and enhance their motivation, there is a call for the provision of lifestyle counselling and a structured exercise programme stressing not only the physical, but also the mental and social benefits of exercise.

\section{7}

EFFECTS OF PHYSICAL TRAINING ON HEART RATE VARIABILITY IN PATIENTS WITH CARDIOVASCULAR AUTONOMIC NEUROPATHY K. Howorka, J. Pumprla, P. Haber ${ }^{*}$, J. Koller-Strametz ${ }^{*}, J$. Mondrzyk ${ }^{*}$ and $H$. Thoma, Department of Biomedical Engineering and Physics, ${ }^{*}$ Department of Internal Medicine IV, University of Vienna, Austria

We investigated effects of a regularly performed physical training (12 weeks, $2 \times 30$ min with $60 \%$ of maximal performance) on heart rate variability (HRV) in 22 diabetic patients ( $n=20$ with IDDM) with different degrees of cardiovascular autonomic neuropathy (CAN): 8 subjects with no CAN (BMI $=24,4 \pm 2,8 \mathrm{~kg} / \mathrm{m}^{2}$, age $=44,1+8,5$, diabetes duration $=18,9+12,7$ ), 8 with early CAN ( $\mathrm{BMl}=25,3 \pm 3,1 \mathrm{~kg} / \mathrm{m}^{2}$, age $=51,8 \pm 7,2$, diabetes duration $=15,5 \pm 8,5$ ), and 6 patients with definite/severe CAN $(B M)=25,8 \pm 4,8 \mathrm{~kg} / \mathrm{m}^{2}, \quad$ age $=50,3 \pm 11,5$, diabetes duration $=21,7 \pm 8,1$ years). A standard battery of cardiovascular reflex tests was used for grading of CAN, a short-term spectral analysis of HRV (in supined and standing positions, 3×256 heart beats, system VariaPulse $T F 3^{3}$ ) for follow-up monitoring of its changes before and after training-free as well as before and after training intervals. Every patient represented in this way his own control. While the training-free intervals showed no changes in spectral indices, the 12-weeks training period increased in patients with no CAN the spectral power in high-frequency (HF) band $/ 0,15-0,50 \mathrm{~Hz} /$ from $\ln \left[\mathrm{ms}^{2}\right]_{\text {betore }} 6,1 \pm 0,7$ to $\left.\ln \left[\mathrm{ms}^{2}\right]_{\text {ater }} 6,9 \pm 0,8 ; \mathrm{p}<0,01\right)$, as well as in low-frequency (LF) band $10,06-0,15 \mathrm{~Hz}$ / (from in [ $\mathrm{ms}^{2}$ ] $7,1 \pm 0,5$ to In $\left[\mathrm{ms}^{2}\right]_{\text {toro }}=7,8 \pm 0,7 ; \quad$ N.S.), which resulted in increase of total spectral power $10,06-0,50 \mathrm{~Hz}$ (from in $\left[\mathrm{ms}^{2}\right]_{\text {betore }}=7,4 \pm 0,5$ to in $\left.\left[\mathrm{ms}^{2}\right]_{\text {ata }}=8,2 \pm 0,6 ; \quad p<0,03\right)$. Patients with early form of CAN showed an increase of total spectral power (from in $\left[\mathrm{ms}^{2}\right]_{\text {e }}=6,2+0,3$ to In $\left.\left[\mathrm{ms}^{2}\right]_{\text {atto }}=6,7 \pm 0,4 ; \mathrm{p}=0,04\right)$ whereas those with definite/severe CAN showed after the training period no changes, in three subjects we recorded even an on-going loss of total spectral power. In all groups there were not significant trends towards improvement of fitness. In patients with no or early CAN the regularly performed physical training improved the sympathetic and parasympathetic activity indices, whereas in subjects with definite/severe CAN no beneficial influences on spectral parameters could be demonstrated after this kind of training. 


\section{8}

THE EFFECT OF RESISTANCE TRAINING ON LONG-TERM GLYCEMIC CONTROL IN NIDDM SUBJECTS.

S. Taimela, J. Eriksson, K. Eriksson, S. Parviainen, J. Peltonen and U. Kujala. Unit for Sports and Exercise Medicine, University of Helsinki and National Public Health Institute, Helsinki, Finland

OBJECTIVES: To determine 1) the effect of resistance training on long-term glycemic control in NIDDM subjects 2) whether cardiorespiratory fitness and glycemic control are associated in NIDDM subjects 3) whether muscle size and glycemic control are associated in NIDDM subjects 4) whether a resistance training program is feasible for sedentary NIDDM subjects.

METHODS: Eight NIDDM patients $(55 \pm 7$ years; NIDDM duration $7 \pm 3$ years; BMI $30.4 \pm 3.4 \mathrm{~kg} / \mathrm{m}^{2}$ ) participated in a 3 month individualized progressive resistance training program twice a week with measurements of $\mathrm{HbA} 1 \mathrm{c}$, lipids, blood pressure, $\mathrm{VO}_{2}$ max and thigh muscle cross-sectional area (Magnetic Resonance Imaging) at baseline and after the exercise period.

RESULTS: There was a significant improvement in $\mathrm{HbA} 1 \mathrm{c}$ (from $8.8 \%$ to $8.2 \% ; p=0.037$ ). The HbA1c concentration post-study correlated inversely with the cross-sectional area of the knee extensor muscles $(r=-0.7288 ; p=0.040)$. Muscle strength increased by $32 \pm 23 \%(p=0.016)$, while the cross-sectional area of $m$.vastus lateralis increased by $20.9 \% \quad(p=0.0009)$. No changes were observed in VO2max although overall submaximal physical performance capacity tended to increase. The program was safely fulfilled.

CONCLUSIONS: These data suggest that 1) resistance training is effective in improving glycemic control in NIDDM subjects 2) there was no significant association between $\mathrm{VO}_{2}$ max and $\mathrm{HbA1c} 3$ ) the size of muscle tissue is of importance for glycemic control 4) resistance training is feasible in sedentary elderly NIDDM subjects.

\section{PS 37}

\section{Epidemiology}

\section{9}

\section{TYPE 1 DIABETES IN CHILDREN UNDER 5 YEARS IN GERMANY: INCIDENCE AND GEOGRAPHICAL DISTRIBUTION}

J. Rosenbauer, T. Fabian-Marx, P. Herzig and G. Giani. Department of Biometrics and Epidemiology, Diabetes Research Institute at Heinrich-Heine-University Düsseldorf, Germany.

Objective: This prospective study was designed to estimate the incidence of Type 1 diabetes in children under 5 years of age in Germany during 1993-94 and to analyse spatial variation of incidence. Methods: During 1993-94 new cases of Type 1 diabetes diagnosed in children under the age of 5 years were registered through the 'Surveillance Unit for Rare Paediatric Diseases in Germany (ESPED)'. ESPED is an active nationwide surveillance system questioning all paediatric departments in Germany through monthly postcards on the number of cases of certain paediatric diseases. Based on the number of newly diagnosed cases of Type 1 diabetes in children under 5 years of age incidence rates were estimated using the Poisson approximation. Poisson regression models were used to assess the effect of age at diagnosis. sex. year of diagnosis, month of diagnosis and geographical region (Federal States of Germany) Crude and directly standardized (for age and sex) incidence rates were calculated for each geographical region. Geographical incidence mapping was based on comparative incidence figures (CIF) using a quintile scale as well as a continuous scale. A colour scale (red-yellow-green) was used for representation of CIFs with red and green corresponding to high and and low incidence rates, respectively. Results: The national incidence rate $\left[10^{-5}\right.$ * year $\left.^{-1}\right]$ was 6.55 (95\%-CI: $\left.6.02-7.11\right)$, thus more than twice as high as estimated on the basis of the diabetes register of the former East-Germany. Poisson regression modeling showed a significant effect of ge $(\mathrm{p}<0.001)$, geographical region $(\mathrm{p}=0.002)$ and month of diagnosis $(\mathrm{p}=$ $0.013)$, but no effect of sex and year of diagnosis $(p>0.1)$. Contrary to other studies. the highest incidence rate was seen in midsummer. The geographical distribution of diabees incidence showed a north-south but no west-east gradient. Conclusions: The incidence of Type 1 diabetes in children under 5 years in Germany seems to have considerably increased in the last years. Apparently there is a marked northsouth gradient of the diabetes incidence. Both the observed time trend and the veouraphical distribution of the incidence of Type 1 diabetes has to be validated by a further continuous surveillance over a longer time period. A substantial increase of the incidence of Type 1 diabetes would be of considerable public health concern.

\section{0}

Comparison of incidence and seasonality in clinical onset of TDOM in children aged 14 years or under in Poland and Finland (1987-1994).

Z. Szybiński', M. Karvonen². H. Dziatkowiak', M. Szurkowska', D. Pach and J.Tuomilehto ${ }^{2}:$ Chair and Department of Endocrinology. Collegium Medicum. Jagiellonian University. Krakow. Poland: ' National Public Health Institute, Helsinki. Finland

The incidence of IDDM among children aged 14 years or under in Finland is the highest in the world, whereas in Poland it is one of the lowest in Europe and also low in global scale. The average annual IDDM incidence during 1987 to 1994 was 35.6 per $100.000 /$ year in Finland and 4.3 per $100.000 /$ year in Poland. In spite of the striking over 8 -fold difference between countries in the incidence rates. there were certain similarities in the age distribution and seasonal variation of clinical onset of IDDM. In both countries the highes incidence was found in the age group 10-14 years old. The yearly seasonal pattern in incidence was estimated presenting the data as short Fourier series up to three harmonics together with possible linear trend. Likelihood ratio test and Akaike's information criterion were used to determine the number of harmonics necessary to model the seasonal pattern. The most visible seasonal pattern during the calendar year in both countries was one cycle with a lower incidence of IDDM among children aged 14 years or under during the summer months than during the rest of the year. No "epidemic peaks in incidence were found in either population. In Poland a significant seasonal pattern was seen in both sexes. whereas in Finland only in boys. Among youngest children ( $0-4$ years) no seasonality was seen. 
721

MAPPING OVERT IDDM IN SARDINIA (1989-94): FURTHER EVIDENCE FOR AN ENVIRONMENTAL COMPONENT IN ITS AETIOLOGY

M Songini*, L Bernardinelli**, D Clayton ***, C Montomoli**, C Pascutto**, M Ghislandi**, G F Bottazzo and the Sardinian Collaborative Group for Epidemiology of IDDM. *Centre for Metabolic Diseases, Ospedale San Michele, Cagliari; **Istituto di Scienze Sanitarie Applicate, Università di Pavia; ***Medical Research Council, Biostatistics Unit, Cambridge, ${ }^{+}$Department of Immunology, St Bartholomew's and the Royal London School of Medicine \& Dentistry, London.

Sardinia, together with Finland, has the highest incidence of IDDM in the world, despite it is placed in the middle of the 'cold' Mediterranean area for IDDM. The analysis of variation of risk for a given disease in space and time may give important insights for the identification of the environmental factor(s), possibly involved in its aetiology. In the case of IDDM, time-trend of prevalence/ incidence of IDDM within a stable population cannot be explained only in terms of variations in IDDM genetic susceptibility. We have organised 'The hot and cold spots-IDDM' project in Sardinia, whose aim is to map the Island for over IDDM and pre-IDDM. We concentrate here in defining the geographical distribution and the time-trend of overt IDDM risk. We used the EURODIAB ACE Sardinian IDDM Register (1989-1994) and analysed for the above parameters across the Sardinian communes and over the whole region. We adopted a Bayesian approach to filter out the random variation of the estimates due to the small number of events in each areal/temporal unit. We also investigated the possible relationship between IDDM and malaria, which was endemic for centuries in the Island. The data have confirmed that the SR is quite high (35.6/100.000). All the communes at high risk are situated in the CentralWestern part of Sardinia, while most of the low risk areas lie in the NorthernWestern part. The RR of the low-high lying communes (mean $500 \mathrm{~m}$ a.s.l.) is not very high, although its corresponding $95 \%$ CI shows a significantly higher risk for IDDM in mountainous/hilly areas compared to low-lying areas, where malaria was more prominent. By continuing this work, one could identify areas within the Island, where individuals are at higher or lower risk of developing overt IDDM and subsequently compare them for genetic susceptibility, immunologic predisposition and a series of ecological events.

\section{2}

THE I NCREABE OF TYPE I DIABETES INCIDENCE AMONG CHILIREN IN NOVOSIBLKSK CITY J.Choubnikova, E.Shubnikot and L.Kalashnikova. center of Endocrinojugy bidudid, Institute of Enternal Medicine, Novosibirsk, Russia

We bave esilimated line inciderce of trype 1 diabetes among children trom 0 to 14 years in Novositixsik city according to standards of who program "Diamond". The study period coverer

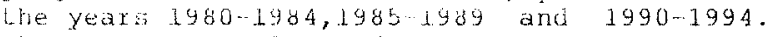
The mean annual incidence of type 1 diabetes was significantly highex in 1990-1994 study period-7,9 per $100000(95 \%$ confidence interval (CI $): 6,09,8)$ than in $1980 \cdots 1984$ study period where it was 4,4 per $100000(95 \%$ CI:3,2-5,6). The conval incidence of 'pype 1 diabetes in 1985-1989 stulay period wa: 6.4 per $100000(95 \%$ $(\mathrm{C}: 5,0 \%, 8)$. We have not tound significant difference in incidence of Type 1 diabetes dimong males in these study periods, but we have found such difference among females, also for 1990-1994 and 1980-1984 periods. We have not also found the signiticant time trends of incidence of I'ype 1 diabetes amorig childxen of small towns and villages (with, the same genetic background around the industrial Novosibirsk Citylabold 1,5 million of inhabitants). The most likely reason for these phenomena is increasing power of environmental casual agents at the big cities in Siberia.

\section{3}

PREVALENCE OF ANTIBODIES TO GLUTAMIC ACID DECARBOXYLASE IN DIFFERENT GLUCOSE TOLERANCE CATEGORIES AMONG ELDERLY CAUCASIANS

J.B. Ruige, J.N.D. de Neeling, M.R. Batstra, H.J. Aanstoot, G.J. Bruining, L.M. Bouter and R.J. Heine. Institute for Research in Extramural Medicine, Vrije Universiteit, Van der Boechorststraat 7, 1081 BT, Amsterdam \& the Department of Clinical Genetics, Erasmus Universiteit, Rotterdam, The Netherlands

In order to test the hypothesis that latent autoimmunity is of substantia importance in the pathogenesis of diabetes in adults, we assessed the prevalence of antibodies to the $65 \mathrm{kD}$ isoform of Glutamic Acid Decarboxylase $\left(G A D_{65}-A\right)$ in different glucose tolerance categories among elderly Caucasians. A general population sample of 2,484 50-74 year-old subjects underwent an oral glucose tolerance test. They were classified according to WHO-criteria as having normal glucose tolerance (NGT), impaired glucose tolerance (IGT), newly detected diabetes (NDM) and known diabetes (KDM). $\mathrm{GAD}_{65}-\mathrm{A}$ was measured in serum, stored at $-70^{\circ} \mathrm{C}$, with the help of a radioligand assay. $G_{A D_{65}-A}$ titers were expressed as GAD-index: (sample value - negative control) / (positive control - negative control). The Table shows the prevalence (\%) of $\mathrm{GAD}_{65}-\mathrm{A}$ positivity by glucose tolerance categories at different cut-off values of the GAD-index (mean titer $+1 \mathrm{SD}, 2 \mathrm{SD}$ and $3 \mathrm{SD}$ ).

\begin{tabular}{cccccc}
\hline missing $=16$ & NGT & IGT & NDM & KDM & Total \\
& $n=2037$ & $n=231$ & $n=110$ & $n=90$ & $n=2468$ \\
\hline GAD-ind. $>0.20(1 \mathrm{SD})$ & 1.0 & 1.3 & 0.9 & 3.3 & 1.1 \\
GAD-ind. $>0.39(2 \mathrm{SD})$ & 0.6 & 0.9 & 0.9 & 3.3 & 0.7 \\
GAD-ind. $>0.61(3 \mathrm{SD})$ & 0.6 & 0.9 & 0.9 & 3.3 & 0.7 \\
\hline
\end{tabular}

Of the $3 \mathrm{GAD}_{65}-\mathrm{A}$ positive subjects with $\mathrm{KDM}$, two subjects, aged 50 an 54 years, were on insulin therapy (age at onset of diabetes 35 an 49 years, respectively), the third was 68 year old and used a diet only (age at onset 44). Of the $G D_{65}-A$ negative subjects with $K D M, 19 \%$ was treated with insulin, $66 \%$ with blood glucose lowering agents, and $15 \%$ with diet only. We conclude that the prevalence of $\mathrm{GAD}_{65}-\mathrm{A}$ is higher in $\mathrm{KDM}$ patients compared to the control population, but also that latent autoimmunity (based on $G A D_{65}-A$ ) plays a lesser role than previously suggested. Additional studies may clarify if such patients indeed have autoimmune diabetes.

\section{4}

MICROALBUMTNURIA IN THE DIABETTC AND NON-DIAEETIC POPULATION

T.G.Jenssen, K.H.Bonaa, I.Toft and M.BerdaI. University of Tromsø, Norway

Microalbuminuria is a predictor of diabetic nephropathy in type I diabetes, and is frequently associated with cardiovascular disease in type II diabetes and in norai abetics. In a community health gurver all persons older than 25 years a corer than 25 years were screened Eor cardiovascular cisk iactors. A representative sample of 7.000 persons delivered 3 first morning vold urine samples produced on separate days. Microalbuminuria was defined as albumin/creatinine (a/c) ratio of $1.7-25 \mathrm{mg} / \mathrm{mmol}$ without bacterial growth in 2 out of 3 firgt moming void samples. Here. we present the results of the first 4.405 persons without bacteruria in the database. AlI results are adjusted for age and gender. normalized by logarithmic transformation when appropriate, and
presented as meantstandard deviation. Diabetics ( $n=117)$ compared with non-diabetics ( $1=4.288$ ) had significantly higher triglycerides $(2.26 \pm 1.75$ vs. $1.73 \pm 1.03 \mathrm{~m} w)$

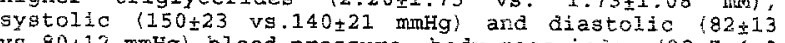
vs. $80 \pm 12 \mathrm{mmfg})$ blood pressure, body mass index $(28.7 \pm 4.9$ vs. $26.0 \pm 3.9)$, waist-hip $(0.9 \pm \pm 0.08 \mathrm{vs} .0 .87 \pm 0.08)$ and a/c ratio ( $1.4 \pm 33.8 \mathrm{vs} .0 .67 \pm 7.2 \mathrm{mg} / \mathrm{mmol})$. The prevalence of microalbuminuria in the diabetic population was 30.9 . compared to $10.0 \%$ in the non-aibetic population $30.9 \%$ was a gignificant association between population. There was a excretion rate even in the non-diabetic population: HbAlC was $5.60 \pm 0.01 \%$ in persons with normoalbuminuria, and $5.80 \pm 0.05 \%$ in persons with microalbuminuria $(\mathrm{p}=0.003)$. Independent correlates of microalbuminuria, calculated as odds ratio (95\% confidence intervals), were hypertension $3.1(2.5-3.8)$, diabetes $3.1(2.0-4.8)$, smoking $2.5(2.0-$ $3.1)$, male gender $1.8(1.4-2.2)$, age $1.6(1,4-1.8)$ and cardiovascular disease $1.3(1.0-1.7)$ conclusion likelihood of developing microlion: The fold in diabetes melitus fold in diabetes mellitus. Diabetes, hypertension and smoking are the three factors that most strongly
associate with micxoalbuminuria. 
CLUSTER OF GLUCOSE INTOLERANCE AND OF MICROALBUMINURIA IN FAMILIES OF PATIENTS WITH NON-INSULIN-DEPENTENT DIABETES A.E. Pontiroli, P.M. Piatti, L. Baruffaldi, T. Pezzoni, B. Solerte and P. Pugni, Istituto Scientifico San Raffaele, Università di Milano, Ospedale di Tradate Polićlinico San Matteo. Università di Pavia, Ospedale di Niguarda, Milan, Italy SPIDER (an acronyme meaning Studio di Prevenzione di Insorgenza del Diabete ad Estensione Regionale) is an ongoing family study aimed at recruiting brothers/sisters (relatives) and spouses of patients (probands) with Type 2 (non-insulin-dependent) diabetes mellitus in the Lombardia region. All subjects (probands, relatives and spouses) fill a questionnaire about dietary and physical activity, and undergo a fasting blood sampling for DNA studjes and for endocrine-metabolic evaluations (glucose. lipids, insulin. proinsulin, C-peptide, glucagon); relatives and spouses undergo an OGTT (75 g glucose p.o.) during which endocrine-metabolic evaluations are repeated So far, 45 families have been recruited and studied; OGTT has yielded impaired glucose tolerance (IGT) or unknown Type 2 diabetes in a total of $37 / 88$ relatives, and in $1 / 35$ spouses $\left(X^{2}=16.2, p<0.01\right)$. Microalbuminuria $(\mu \mathrm{A})$ has been evaluated in 28 families, $\mathrm{t} 1 \mathrm{in}$ which the proband had $\mu \mathrm{A}$, and 17 in which the proband had no $\mu \mathrm{A}$, relatives belonging to $4 / 11$ families had $\mu \mathrm{A}$, as opposed to $1 / 17$ families $\left(\mathrm{X}^{2}=8.3, \mathrm{p}<0.01\right)$; the total number of relatives with $\mu A$ was $12 / 29$ in the first group, as opposed to $1 / 50\left(X^{2}=30.5\right.$, $p<0.01$ ) in the second group, independently of results of OGTT, and to 1/28 spouses. Probands, relatives and spouses were not different as to body weight, diet and physical activity, arterial blood pressure and kidney function These data suggest that both glucose tolerance and microalbuminuria show a familiar clustering.

\section{6}

PREVALENCE OF ALBUMINURIA IN TYPE2 DIABETIC PATIENTS IN NORTH SPAIN

Goicolea I, Villar G., Garcia Y., Vazquez J.A. Servicio Endocrinologia. Hospital de Cruces Baracaldo.Spain.

Diabetic nephropathy (DN)is fast becoming the leading cause of end stage renal disease in developped countries. The aim of study was twofold.First, to know the prevalence of $\mathrm{DN}$ in type 2 diabetics in our country. Second, to anlyze the association of DN with cardiovascular and other diabetic chronic complications. Material and methods: 801 consecutive type2 diabetics (mean age $61+/-7$ years, diabetes duration $10,5+1-6,5$ years) attending 1 hospital , 7 specialized and 1.2 family doctors consultations from an area of 400.000 persons were chequed for diabetic complications ,cardiovascular risk factors (lipids,blood pressure,smoking) ,body mass index (BMI)and waist and hip circumferences and its ratioW-H.A.n overnight urine sample was collected to exam urinary albumin excretion (UAE) and if was $>20 \mathrm{ug} / \mathrm{min}$,an 24 hours urine sample was taken again to confirm DN. Reiative risk ratios were calculated and adjusted for stratificacion variables as Mantel-Haenszel risks.Results:The prevalence of DN was $33 \%, 6 \%$ macro and $27 \%$ of microalbuminuria. DN patients were older,increase with diabetes duration $(p<0,01)$.DN patients have higher risk of retinopathy $R R=2,09(1,74-2,52)$,symptomatic neuropathy $R R=1,56(1,28-1,92)$ and peripheral vasculopathy $\mathrm{RR} \approx 2,27(1,54-3,35)(\mathrm{p}<0,001)$ than normoalbuminurics as were known hypertension and hypertrygliceridemia $(p<0,01)$.Systolic $(p<0,01)$ and diastolic $(\mathrm{p}<0,05)$ blood pressure were significantly raised in ND. Hip and waist circumferences were lower in normoalburninuric $(p<0,01)$ but $B M I$ and the ratio $\mathrm{W}-\mathrm{H}$ were not different in spite of being stratified by age,sex and diabetic duration. Conclusion ND prevalence in type 2 diabetic in our area is more elevated than observed in others countries.ND is associated. with several microangiopatic diabetic complications and cardiovascular risk factors.

\section{7}

\section{EPIDEMIOLOGICAL CHARACTERIZATION OF THE ASSOCIATION BETWEEN HYPERTENSION AND HYPERINSULINEMIA IN THE GENERAL POPULATION}

O.Vaccaro, G.Ruffa, G.Imperatore,C.Iovine and G.Riccardi. Department of Clinical and Experimental Medicine - Federico II University. Naples, Italy.

The inconsistent association between hypertension and hyperinsulinemia could be partly explained by the etiological heterogeneity of hypertension. We evaluate the extent to which this association is explained by the concomitant presence of obesity, central adiposity or Sindrome X (SX). In a working population of 2600 non diabetic men (35-65 yrs), supine blood pressure, anthropometry and biochemical variables were measured in a standardized way. Hypertension was blood pressure $\geq 160 / 95$ $\mathrm{mmHg}$ or treatment. In the whole population hyperinsulinemia (fasting insulin $\geq 8.9$ $\mathrm{mU} / \mathrm{l}$, upper tertile of distribution), obesity and central adiposity (WHR $>0.98$ ) were associated with hypertension independently of each other $(p<.01, p<.0001, p<.0001$ respectively). However, of 453 hypertensives, $267(59 \%)$ were not hyperinsulinemic and hyperinsulinemia in the absence of obesity or central adiposity was present only in $11 \%$ of hypertensives. To further clarify this issue, hypertensives with $\mathrm{SX}$ ( $=$ one or more factors among glucose, uric acid or triglycerides in the upper quintile, HDL cholesterol in the lower) were compared to both hypertensives without SX and normotensives; insulinemia was significantly higher in hypertensives with $\mathrm{SX}$ than in the other two groups $(10.0 \pm 6.7$ vs $7.9 \pm 3.7$ and $7.6 \pm 3.2 \mathrm{mU} / 1, \mathrm{p}<0.001)$.

Concluding, hyperinsulinemia, obesity and central adiposity are associated with hypertension independently of one another, yet hyperinsulinemia is not an ineherent feature of hypertension and is observed only when hypertension is associated with SX.

\section{8}

Standardised study of glucose tolerance and diabetes prevalence in 4 African (origin) populations in Cameroon, Jamaica and migrants to Btitain. IC Mbanya ${ }^{1}, \mathbf{R}$ Wilks ${ }^{2}$, F Bennett ${ }^{2}, M$ Jackson ${ }^{2}$, T Forrester $^{2}$, L Riste $^{3}, A$ Forhan ${ }^{4}$, B Balkau ${ }^{4}$ and J.K Cruickshank ${ }^{3}$. Universities of: ${ }^{1}$ Yaounde 1 Cameroon; West Indies, ${ }^{2}$ Jamaica; ${ }^{3}$ Manchester, UK and ${ }^{4}$ InsermU21 Paris. To assess the emergence of diabeter 112 African (-origin) populations usm: the same protocol we carried sut 75 g glucose tolerance tests in representatry"s community ramples ared 25-74 years. In Jamaica and the LK, AfricanCaribbean was defined by grandparental etlmicty. Diabete's was defined by takingr hy poglycemic tratement or $2 \mathrm{hr}$ plasma gilusose (WHO criterial).

\begin{tabular}{|c|c|c|c|c|}
\hline & $\begin{array}{l}\text { Cameroon } \\
\text { Rural }\end{array}$ & $\begin{array}{l}\text { Cameroon } \\
\text { Urban }\end{array}$ & Jamaica & $\begin{array}{r}\text { Manchester } \\
\text { LKK }\end{array}$ \\
\hline $\operatorname{MEN}(n)$ & 188 & 138 & 199 & 1.1 \\
\hline Age (years) & 49 & 42 & 50 & 5 \\
\hline $\mathrm{BMI}\left(\mathrm{kg} / \mathrm{m}^{2}\right)$ & 21.3 & 25.2 & 22.5 & $26 x$ \\
\hline Ohr glucose $(\mathrm{mM})^{\star}$ & 3.9 & 4,2 & 4.9 & 5.1 \\
\hline $2 \mathrm{hr}$ glucose $(\mathrm{mM})^{*}$ & 5.6 & 4.4 & 5.7 & 6.1 \\
\hline Diabetes prev. ${ }^{*} 1$ & $1.0(0.1-3.5)$ & $1.2(0.1-4,2)$ & $5.7(2.8-10.1)$ & $12.8(7.9-19.0)$ \\
\hline WOMEN $(n)$ & 196 & 157 & 198 & 21.3 \\
\hline Age (years) & 50 & 44 & 49 & 51 \\
\hline $\mathrm{BMI}\left(\mathrm{kg} / \mathrm{m}^{2}\right)$ & 21.9 & 26.8 & 26.4 & 28.6 \\
\hline Ohr glucose $(\mathrm{mM})^{\star}$ & 4.1 & 4.2 & 5.0 & 5.11 \\
\hline $2 \mathrm{hr}$ glucose $(\mathrm{mM})^{*}$ & 5.0 & 4.9 & 6.5 & 5.5 \\
\hline
\end{tabular}
2hr glucose $(\mathrm{mM})^{*} \quad 5.0 \quad 4.4 \quad 50.5$ Diabetes prev. ${ }^{* *} \quad 0.6(0.0-3.1) \quad 2.8(0.9-6.5) \quad 9.0(5.5-13.8) \quad 9.9(6.2-14.8)$ * in subjects not treated for diabetes. ${ }^{* *}$ prevalence standardised according to the 'SEGl' world population ( $95 \%$ confidence interval)

In men and women, diabetes prevalence (arge-adjusted) increatied frum Africis to the Caribbean to Europe - with particularly high prevalence in Manchenter men. Body mass index showed a similar trend, with a itriking incereane between rural \& urban Cameroon, despite younger age. Incriasing prevalence of diabetes (NIDDM) is paraljeled within and acrom site by changes in nutritional \& life style factors, also measured usingr standurdincd meihods. Even in Cameroon, prevalence exceeds rate's in Eurupe. 
729

DIABETES, FASTING GLYCAEMIA AND INSULINAEMIA IN EUROPEAN POPULATIONS AGE 70-75 YEARS

A.U. Teuscher and A. Teuscher, on behalf of the EC/EURONUT

SENECA Investigators, Department of Medicine, University of Bem, Switzerland

Diabetes mellitus and impaired glucose tolerance increase with age and hyperinsulinaemia as a potential vascular risk factor may be present many years before the onset of NIDDM. However, data in the elderly concerning prevalence of diabetes mellitus, abnormal fasting glycaemia and insulinaemia are scarce. Data comparing their prevalence in geographically different countries in Europe are unknown. We analyzed fasting glucose, fructosamine and fasting insulin levels in 1976 subjects (age $70-75$ years) from 11 European countries which took part in the coordinated study EC/EURONUT 'Nutrition and the Elderly', a concerted action on nutrition and health in the European Community. In total $251(12.7 \%)$ subjects had diabetes: $128(6.5 \%)$ had self reported diabetes, $123(6.2 \%)$ had newly diagnosed diabetes based on fasting plasma glucose levels $\geq 7.8$ $\mathrm{mmol} / \mathrm{l}$. Prevalence of diabetes varied from $6.9 \%$ (Denmark) to 25.6 $\%$ (Hungary). 806 subjects $(41 \%)$ had abnormal fasting plasma glucose between $5.6-7.7 \mathrm{mmol} / \mathrm{l}$. Fructosamine was elevated in 158 (8\%). Hyperinsulinaemia was present in $344(17.4 \%)$. In conclusion, prevalence of undiagnosed diabetes in this age group is about equal to that of known diabetes. Diabetes prevalence varies considerably among different European populations. Abnormal fasting glycaemia, diabetes and hyperinsulinaemia as potential vascular risk factors are present in a large group of elderly European subjects.

\section{1}

AN OVERVIEW OF RANDOMIZED CONTROLLED TRIALS IN NON-INSULIN DEPENDENT DIABETES MELLITUS B. McIver and S.F. Dinneen. Mayo Clinic, Rochester, MN The randomized controlled trial (RCT) represents the highest level of evidence used to assess an intervention. An RCT's ability to influence clinical practice depends on: 1) its size, 2) the outcomes measured, and 3) the methodologic quality of the trial. To assess these factors in RCTs involving NIDDM patients we performed two independent searches of the MEDLINE database looking for RCTs published between January 1994 and November 1995. Information relating to trial design was independently extracted by two investigators. We retrieved 195 unique citations of which 103 were excluded as not being RCTs. Of the remaining 92 RCTs 79 were available for analysis. Forty-two percent of RCTs were published in either Diabetic Medicine or Diabetes Care. The median number of patients randomized was 40 (range 5 to 2769). Median duration of study was 22 weeks (.2-260). Glycemic control was reported as an outcome measure in all but 4 RCTs $(95 \%)$. The next most frequently reported outcomes were lipid levels (63\% of RCTs), body weight (56\% of RCTs), and a measure of insulin secretion and/or insulin action ( $44 \%$ of RCTs). Only two studies $(2.5 \%)$ reported major clinical endpoints and five studies $(6.3 \%)$ reported a formal measure of quality of life. Ninety-one percent of patients randomized had complete follow-Lp. The method of randomization was described in only $15 \%$ of RCTs. In the 46 RCTs which were described as doubleblind, an appropriate method of blinding was reported in only 21 RCTs (46\%). Our data suggest a need for more large RCTs with major clinical endpoints and a greater awareness of patient related outcomes (quality of life). The development of a valid instrument to assess the quality of NIDDM RCTs is needed.
730

WABSELS. CHOLESEROL AWDLONG-TERM PROGNOSIS IN MDDLE AGED SWEDSH MES

A. Adertertin. A. Rosengren. L.C. Ohisson. L.E. Bresäter and L. Whochen. Secion of Preventive Cardiology and Diabetes Section. Deparmen of Mediche. Ostra Haspital. Goteborg. Sweden.

Ins study presents a 16 year follow-tip of the Primary Prevention Stuty in Goteborg. Sweden. We investigated the importance of elevated serum cholesterol levels for long-tern prognosis in 249 men wht diabetes out of a popuation sample of 7122 men aged $5 i$ to 59 at baseline in 1974 to 1977 . After a mean of 16 years follow-up $31 \%$ of the men with diabeies had died from coronary disease (CHD) and $52 \%$ had died trom any calise, compared to $9 \%$ and $29 \%$ of the nen without diabetes. Diabetic men with serum choiesterol above $7.2 \mathrm{mmol} / \mathrm{l}$ had a montality from $\mathrm{CHD}$ of $47 \%$. compared to $24 \%$ among diaberic nen with seram cholesterol below $5.2 \mathrm{mmolf}$ (adjusted relative risk 3.6 (1.8-7.5\%. Mortality from all causes was $78 \%$ in diabetic men with high cholesterol and $58 \%$ in men with low cholesterol (adjusted relative risk $2.4(1.5-4.0)$. In conclusion, mortality from $\mathrm{CHD}$ and from all causes was higher ir men with diabetes than in non-diabetic men, and high cholesterol increased the mortality rates from CHD and from all causes among diabetic men in the present study.
732

Risk stratification in diabetes by the RECPAM approach; results of an Italian study.

F.Carinci $\mathrm{MS}^{1}$. A.Nicolucci $M D^{1}$, R. Arboreui $\mathrm{MS}^{\mathrm{l}}$. A. Ciampi $\mathrm{PhD}^{2}$ on behalf of The flalian Study Group $f:$. the Implementation of the St. Vincent Declaration - Società Klaliana di Diabetolugia - Associazione Medici Diabelolog 3

1.Deparment of Clinical Pharmacology and Epidemiology. Consorzio Mario Negri Sud. S. Maria Imbaro, Chieti, Italy.2.Mc Gill University and Montreal Heart Institute. Montreal, Quebec, Canada 3.Scientific commente: M. Massi Benedeti (chairman), F. Capani, Q. Carta, E. De Feo, C. Taboga. G. Tognoni.

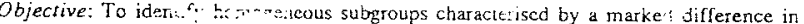
risk of developing major long-term diabclic complications (i.e. critical limb ischemia, amputation, chronic renal failure (creatinine $>3 \mathrm{mg} / \mathrm{dl}$ ), dialytic treatment, proliferative retinopathy and biindness). The independent effect of, and the mutual interaction among retinopathy and biindness). The independent effect of, and the mutual interactic
clinical, socio-economical and health carc-related risk factors were explored. Design: Case-control study.

Patients and setting: 886 patients with long-tern diabelic complications and 1888 controls without complications were enrolled from 35 diabetic outpatient clinics and 49 general practitioners' offices during a period of 6 months.

Staristical methods: The main results were obiained using RECPAM (RECursive Partitioning and AMalgamation), a technique which antempts to integrate the advantages of main effect logistic regression and tree growing

Results: The application of RECPAM led to the detection of important interactions involving clinical, socio-economic and heath care-related characteristics. Among IDDM patients ( $\mathrm{n}=376$ ), the interaction between hypertension and smoking habiss led to a dramatic increase in complication risk; for those without hypertension, the interaction between blood glucose self monitoring practice and low socio-economic status further differentiate the complication risk. Among NIDDM subjecis not reated with insulin $(\mathbf{n}=1371)$, a poor compliance with visit scheduling and a present or past history of smoking defined the subgroup with the highest complicati-n risk. For NIDDM patients ueated with insulin $(n=997)$, not attending reguiarly the health care-facility was associated with the highest risk; among the others, those with hypertension and males without hypertension with low level of education aiso showed an increased risk. Conclusions: Our data suggest that individual complication risk heavily depends upon interactions among several factors. Of particular importance, socio-economic status and level of education can determine a complication risk not dissimilar to that related to hard clinical variables, such as hypertension and diabetes duration. New statistical lechriques. such as RECPAM, can help in identifying complex interactions when the effect of many variables on outcome are simultaneously investigated. 
733

LONG-TERM MORTALITY RISK IN DIABETES BASED ON THE TIME- AND PLACE-MATCHED POPULATION COHORTS D. Janeczko, J.Kopczyński, A.Czyżyk, Z.Lewandowski, E.Janeczko-Sosnowska, A.Tuszyńska From the Departments of Gastroenterology \& Metabolic Diseases, and of Epidemiology, Warsaw Medical A.cademy.

A renewed estimate of mortality risk was attempted with the data from 22-year cohort of 4591 diabetic patients of originally short-term diabetes, compared with three population cohorts of 8121 adult Warsaw dwellers followed at various instances during the same time period. 3530 and 1380 deaths in diabetics and population cohorts, respectively, were uniformly scrutinized for the causes of deaths, with the same correcting rules applied. For 4420 NIDDM patients standardized rate ratio (SRR) was 2.17 (95\% CI: $2.0-2.4$ ), with little sex difference, whereas in 171 IDDM persons SRR amounted to 5.02 (CI: 3.5-7.1), much larger for women ( $S R R=7.55$ ) than men (3.47), SRR for cardiovascular deaths for NIDDM equalled over 3.00 (CI: 2.7-3.3); for ischaemic heart disease - 5.4, being higher for females (6.3) than for males (4.8). The risk estimates were generally higher than in most other investigations, with might result from the long follow-up and/or closely matched control groups.
734

BODY WEIGHT VARIABILITY AND GLUCOSE CONTROL AS PREDICTORS OF MORTALITY IN ELDERLY NIDDM PATIENTS

G. Zoppini, G. Verlato, R. de Marco and M. Muggeo - Division of Metabolic Diseases and Institute of Medical Statistics, University of Verona, Italy.

In the non-diabetic population fluctuation of body weight is a predictor of mortality. The aim of the present study was to verify whether body weight variability retained the same negative prognostic value also in elderly NIDDM patients. The investigation was performed in the framework of the Verona Diabetes Study on 542 NIDDM patients, aged 75 years and over, who were followed-up from 1.1.1987 to 31.12.1991 to assess mortality. From their clinical records all available body weight and height determinations for the years 1977 to 1986 were collected and the corresponding values of body mass index (BMI) were computed. Mean and coefficient of variation (CV) of BMI were used to group the patients in tertiles and the effect of these variables on all-cause and cardiovascular mortality was assessed through a Poisson regression model. When controlling for sex, age, duration of disease, insulin treatment and mean BMI, CV of BMI emerged as a significant predictor of both all-cause mortality $(p=0.011)$ and cardiovascular mortality $(p=0.020)$ : with respect to patients in the lowest tertile, the relative risk of death from all-cause in patients in the highest tertile was 1.75 (95\% CI 1.19-2.58) and the the relative risk of cardiovascular death was 2.34 (1.27-4.33). After adding to the model the average value and the coefficient of variation of fasting plasma glucose during the period 198486 , the effect of BMI variability on mortality tended to decrease and was no longer significant $(p=0.081$ for all-cause mortality and $p=0.087$ for cardiovascular mortality). In conclusion, long-term instability of body weight seems to be a predictor of mortality also in elderly NIDDM patients, although in the present investigation the effect of fasting plasma glucose variability was much more pronounced.

\section{5}

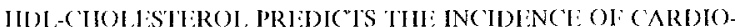
VASCUIAR DISIASI IN PIERSONS WITI IMPAIRIID GI.UCOSI TOIJIRANCL:

(iicl Nijpels', Iiric T.P. Keulen', Iex M. Bouter ${ }^{1.2}$, Robert J. Iteine ${ }^{1-3}$ Institute for Research in lixtramural Medicine, -Isepartment of Epidemiology and Biostatistics, ${ }^{3}$ Research Institute for Endocrinology, Reproduct on and Metabolism, Vrije Universiteit Amsterdam, Van der Bocchorststraat 7, LOSI B'l Amsterifam, The Netherlands

The aim of this prospective study in persons with impaired glucose tolefance was to analyse the cardiovascular risk factors (CVRI) as predictors for developing cardiovaseular disease (CVD), defined by I: (' $G$ or anklebachial pressure index $(\Lambda \mathrm{BPI})$ ahoormalities. $\Lambda \mathrm{t}$ baseline subjects were classilicd according to the World IIealth Organization criteria hased on the nean values of two oral glucose tolcrance tests. JiCG abnomality was defincd as Minnesota codes $1-1 / 3,4-1 / 3,5-1 / 3$ and $7-1$. Abnornal $A B P I$ was delined as an index $<0.90$. The nean duration of follow-up was 36 months (25-75\% pere: $32-43.5$ months). $A$ complete datasel was obtained from 119 subjects. The prevalence of any $\mathrm{BC} C \mathrm{C}$ or $\mathrm{ABPI}$ abnormality at follow-up was $33 \%(\mathrm{n}=39 ; 95 \%$ (I: $24.5 \%-41.5 \%)$. For analysing the predictive value of developing (VD) for the various parameters, subjects with existing $\mathrm{I}: \mathrm{C}(\mathrm{r}$ and $\mathrm{ABPI}$ abnomalities at baseline $(\mathrm{u}=20)$ were excluded. The cumulative incidence of (VD) was $19 \%(95 \%(\mathrm{I}: 15 \%-23 \%)$. Iogistic regression analyses showed that only IIDI-cholesterol at baseline was negatively associated with the development of (CVD) (odds ratio 0.(6) per 1 mInul// difference, $p<0,04)$. In conclusion: subjects with well-delined IG' hasl a relatively high incidence of cardiovascular disease and IIDI-cholesterol was reversely associated with the development of cardiovascular discasc.
736

\section{GENDER DIFFERENCE IN THE RELATIVE RISK OF ISCHAEMIC HEART DISEASE IN DIABETES}

F.W.F. Hanna' ${ }^{1}$ C.J. Currie ${ }^{2}$, A Rees ${ }^{3}$ and J.R. Peters ${ }^{1}$

1 - Department of Medicine, University Hospital of Wales, Cardiff, UK 2 - Department of Public Health Medicine, SGHA, Cardiff, UK

Introduction: Ischaemic heart disease (IHD) accomts for $70 \%$ of mortality in diabetes. It is belicied that there is loss of the biological protective effect in wonen with ovulatory cycles

Aim: We aimed to re-explore this hypothesis by looking at the sex difference in the incidence of IFHD-related events in diabetics in comparison with a non-diabetic control population

Methods: Using the diabetic dalabase in South Glamorgan (5602 diabetics ont of a total population of 402,798 ), the number of in-patient and out-patient IHD-related events were assessed, including angina, acute myocardial infarction (MI) and hear failure (HF)

Results: Diabetics had an increased rclative risk (RR) of IHD-related events of more than 20 fold in both sexes below 50 rears and at least 3.5 fold in those above 50 comparcd to the general population. This pattern was consistent in angina. MI and IFF. As expected. non-diabetic women below 50 years had a significantly lower incidence rate of IHD events. In DM. below 50. men had a significantly higher RR of developing IHD compared to women. Above 50. both sexes shared the same relative risk. The table summarises the male to female RR in DM.

\begin{tabular}{ccccccccc}
\hline & Ml & \multicolumn{3}{c}{ Ang } & HF & & All & \\
\hline$<50$ s & 5.5 & $\mathrm{p}=0.002$ & 3.0 & $\mathrm{p}=0.004$ & 1.17 & NS & 2.3 & $\mathrm{p}=0.0018$ \\
$\mathbf{5 0 + \mathrm { s }}$ & 1.2 & $\mathrm{p}=0.04$ & 1.1 & NS & 0.8 & NS & 0.93 & NS \\
\hline
\end{tabular}

Conclusion: DM significantly increases the relative risk of IHD to a greater extent than previously reported. Although diabetic women below 50 years are at increased risk when compared to non-diabetic women. their risk of 1HID-rclated exconts is significantly less than in diabetic men. This suggests that, contrary to previous studies, diabetic women retain their relative cardioprotective effect. 


\section{7}

PRESCRIPTION DRUG USE AMONG DIABETTC PATIENTS

W. Rathmann ${ }^{1}$, B. Haastert ${ }^{1}$, B. Delling ${ }^{2}$, J.M. Roseman ${ }^{3}$, G. Giani $^{1}$ and F.A Gries $^{1}$ Diabetes Research Institute, Düsseldorf ${ }^{1}$, Germany; IMS ${ }^{2}$, Frankfurt, Germany; $\mathrm{UAB}^{3}$, Dept. of Epidemiology, Birmingham, AL, USA.

Objective: To evaluate prescription drug use among diabetic patients treated by general practitioners and internists. Design: Computerized data (MediPlus, IMS) on drug prescriptions in 1994 were assembled among a large sample of general practitioners and intemists throughout Germany. Diabetic patients were identified based on diabetes-related diagnoses and/or prescription of diabetes care products. Setting: 386.520 patients in 286 practices. Outcome: OR (diabetics/non-diabetics: patients with/without $\geq 1$ prescription/year) obtained from logistic regression models controlling for age, sex, type of health insurance, region and speciality of practice. Results: $30.558(7.9 \%)$ diabetic patients were identified. Twenty per cent were treated with insulin and $45 \%$ received orai antidiabetic drugs. There was an increased prescription use among diabetics in most drug groups. However, after controlling for age and the other covariates, substantial increases were found mainly in cardiovascular drugs (loop diuretics: $O R=2.7 ; 95 \%$ CI: $2.4-2.9$; digitalis: $\mathrm{OR}=2.5 ; 2.3-2.7$; ACE-inhibitors: $\mathrm{OR}=2.5 ; 2.3-2.7$; nitrates: $\mathrm{OR}=1.9 ; 1.7-2.0$; thiazides: $\mathrm{OR}=1.8 ; 1.7-2.0 ;$ Ca-antagonists: $\mathrm{OR}=1.8 ; 1.7-1.9)$, and for fibrates $(\mathrm{OR}=2.5 ; 2.2-2.9)$, gout medication $(\mathrm{OR}=1.9 ; 1.7-2.1)$, and wound care products $(\mathrm{OR}=1.4 ; 1.3-1.6)$. No increase was observed for drug treatment of respiratory system disorders, for many gastrointestinal drugs (antidiarrheal, prokinetics, antiulcer, spasmolytics), and for a variety of other groups including thyroid drugs, steroids, vaccines, and prostate medication. Decongestants, flu medicine, migraine drugs, and muscle relaxants were significantly less frequently prescribed among diabetic patients. Overall, $33 \%$ of the diabetics received more than 10 different drug prescriptions/year compared to only $12 \%$ among non-diabetics. Conclusions: Prescription drug use among diabetic patients was predominately increased for cardiovascular drugs, and for medications to treat diabetes-related metabolic disturbances or complications. Use of multiple drugs due to multimorbidity occured frequently, enhancing the risk for adverse drug effects.

\section{PS 38}

\section{Nutrition and Diet}

\section{8}

VERY LOW CALORIE DIET IN THE TREATMENT OF TYPE 2 DIABETICS

P. Hannc-Parikka and S. A Salo. Finnish Diabcics Association, Diabcles Center,Kirjoniementie 15, 33680 Tampere. Finland

The Finnish Diabetes Association has an education center for diabetics. In $194+$ we started a new programme for severe obese type 2 diabetics, who had not succeeded in weight reduction. A non-controlled assessment of the feasibility; safety and efficacy of a very low calorie diet (VLCD) has been conducted in 34 obese type 2 diabetic subjects, who have so far taken part in this programme. In the first part of the course ( 3 days) the participants start VLCD for 6-12 weeks. Visits at their own health care centre take place every second week. The other part of the course ( 4 days) takes place after three months and concentrates in maintaining the weight loss achieved by normal hypocaloric diet and exercise and creating a realistic treatment plan together with the participants. The third part at 9 to 12 months ( 2 days) deals with the behavioural and psyhosocial aspects of eating. weight control and diabetes. 31 of 34 subjects had VLCD for 6-12 weeks and it was tolerated well. Most of the patients found it easy to comply with the diet. The side-effects were few. All continued their usual work and their overall well-being was categorised as good or better than usual. The mean weight was $108,7(80,3-164,6) \mathrm{kg}$ at the beginning and $98,9(70,1$ $145,5) \mathrm{kg}$ at $9-12$ months. The mean weight loss was $13,7(-4,7--30,9) \mathrm{kg}$ at 3 months $(\mathrm{p}<0.001)$ and $9,8(+5,3--24) \mathrm{kg}$ at $9-12$ months $(\mathrm{p}<0.001)$. The mean $\mathrm{HbAlc}$ value was $8,3 \%$ before the programme and $7,0 \%$ at $9-12$ months $(p<0.01) .23$ out of 34 subjects had oral antihyperglycaemic agents at the beginning. At $9-12$ months, 6 had stopped and 13 reduced their medication. We conclude that VLCD is a treatment of choice for obese type 2 diabetics in order to lose weight and improve metabolic control. The most difficult part seemed to be the change back to a normal hypocaloric diet and the maintenance of the weight loss.
739

THE EFFECT OF ACARBOSE ON DIETARY NUTRIENT INTAKE AND METABOLIC CONTROL IN NIDDM PATIENTS. J. Lindström, J. Tuomilehto and M. Spengler. Diabetes and Genetic Epidemiology Unit, National Public Health Institute, Helsinki, Finland

It has been speculated that some of the effect on metabolic control with acarbose might be due to concomitant changes in diet. Therefore during the 24-week double-blind study the aim was to determine whether poor metabolic control can be improved by acarbose, and secondly to find out whether the acarbose supplementation results in changes in habitual nutrient intakes which may have an effect on metabolic control. Poorly controlled NIDDM patients treated with oral hypoglycaemic agents (OHA) were randomized into either acarbose ( $100 \mathrm{mg}$ t.i.d.) or placebo treatment. Nutrient data were collected using 3-day food diary at $0,4,12$ and 24 weeks. 10 acarbose (A) and 11 placebo $(\mathrm{P})$ treated patients dropped out, and in the final analyses there were 36 acarbose and 39 placebo treated patients. The median duration of NIDDM was 76 and 60 months, mean age 58 and $62 \mathrm{yrs}, \mathrm{BMI} 28.3$ and $29.1 \mathrm{~kg} / \mathrm{m}^{2}$, fasting blood glucose (BG) 10.0 and $10.0 \mathrm{mM} / \mathrm{L}$ and $\mathrm{HbA} 10.1$ and $9.8 \%$, respectively. No significant differences in nutrient intakes were observed. During the randomized treatment the energy intake (appr. $2000 \mathrm{kcal} / \mathrm{day}$ in men and $1500 \mathrm{in}$ women) and energy \% of carbohydrates (appr. 50\%) remained unchanged in both tretment groups. At 24 weeks the baseline adjusted means of fasting, 1-hr and 2-hr postprandial BG were 9.3 vs $10.5(\mathrm{p}=0.02), 11.6$ vs 14.5 $(\mathrm{p}<0.001)$ and 11.0 vs $13.7 \mathrm{mM} / \mathrm{L}(\mathrm{p}<0.001)$ and $\mathrm{HbA} 9.3 \%$ vs $10.2 \%(p=0.002)$ in acarbose and placebo groups, respectively. The difference in $\mathrm{HbA}_{1}$ between groups increased continuously and became statistically significant at 12 weeks. In conclusion, acarbose improves significantly metabolic control in NIDDM patients poorlycontrolled with OHA. This effect seems to be due to acarbose itself since concomitant changes in dietary intakes during the study were non-significant. 
740

FACTS AND RESERVATIONS ABOUT THE DIET RECOMMENDATIONS FOR DIABETIC INDIVIDUALS.

D. Karamitsos, G. Kourtoglou, T. Didangelos and A. Romanidou.

Diabetes Center, 2nd Prop. Medical Department of Aristotelian University. Hippocration Hospital, Thessaloniki, Greece

According to the ADA's 1996 nutrition recommendations and principles for people with diabetes mellitus "the dietary protein $(P)$ may contribute $10-20 \%$ of the total caloric content of the diet. The rest of the calories $(80-90 \%)$ remain to be distributed between dietary fat $(F)$ and carbohydrate $(\mathrm{CH})$. Less than $10 \%$ of these calories should be from saturated F". Aim: To test if the dietary recommendations are realistic and applicable. Method: We developed a computer program for diet prescription and calculations using File Maker Pro software. Various proportions of $\mathrm{P}, \mathrm{CH}$ and $\mathrm{F}$ were tested in low caloric diets (<1500 Cal) and isocaloric diets. Results: 1) A diet of $20 \%$ of calories from $\mathrm{P}, 50 \%$ from $\mathrm{CH}$ and $30 \%$ from $\mathrm{F}$ is unrealistic because of the very little amount of $F$ that remains to be given as vegetable oil. Moreover this diet includes saturated $F$ in very high percentage. 2) A realistic and applicable diet can be prescribed with $15 \%$ of calories from $P, 45 \%$ from $\mathrm{CH}$ and $40 \%$ from $F$, (butter isn't included). 3) Problems arise in slimming diets. In these diets we must give at least $17 \%$ of calories from $\mathrm{P}, 40 \%$ from $\mathrm{CH}$ and $43 \%$ from $\mathrm{F}$ to make the diet applicable. However in these cases the percentage of calories ofrom saturated $F$ rises far from the recommended. Conclusion: The diet recommendations of ADA must be tested in practice and revised to become realistic and applicable. Special recommendations are needed for obese NIDDM patients.

\section{2}

DIETARY PROTEIN RESTRICTION AND MICROALBUMINURIA IN TYPE 2 DIABETES PATIENTS WITHOUTNEPHROPATHY

l.TJ Pijls', H de Vrics', AJM Donker', JTM van Eijk'. 'Institute for Research in Extramural Medicine (EMGO-Institute), 'Department of Internal Medicine, Verie Universitcit Amsterdam

Introduction: Most trials on protein restriction and renal function in patients with diabeles concern macroalbuminuric, insulin-dependent paticnts. Patients and methods: Therefore, we present data on the first 33 patients (out of 150; data available in July), after the first six months of intervention, in a randomized trial on protein restriction and microalbuminuria (ALB) in NIDDM patients, at risk of nephropathy (ALB $>6.5 \mathrm{mg} \mathrm{l}^{-1}$ (=detection Icvel) in two 24hr urines, or $>20 \mathrm{mg} 24 \mathrm{hr}^{-1}$ in at least one sample, or diabetes duration $\geq 5 \mathrm{yrs}$ ). All subjects had a protein intake $>0.80 \mathrm{~g} \mathrm{~kg}^{-1}$ body weight, and no leukocyturia. Clearance was measured while blocking renal-tubular creatinine secretion by cimitedine. All subjects received dictary guidance according to the European consensus, with intake of (saturated) fat as main topic. In addition, guidance in the experimental group (E) was aimed at protein restriction, isocalorically, to $0.65 \mathrm{~g} \mathrm{~kg}^{-1}$. At baseline, in group $\mathrm{E}$ (11 , 5\%) and control group (C) $\left(110^{\delta}, 6 \%\right)$ respectively, onc and three used an ACE-inhibitor, mean ages werc 65 and 64 yrs, diastolic blood pressure (DBP) 79 and $76 \mathrm{mmllg}$, and HbAlc 7.8 and $7.3 \%$. Baseline protein intake in group $\mathrm{F}$ and $\mathrm{C}$ was 1.22 and $1.13 \mathrm{~g}$ protcin $\mathrm{kg}^{-1}$, ALB 19 and $35 \mathrm{mg} \mathrm{24hr}^{-1}$,

creatinine clearance $(\mathrm{CrCl}) 76$ and $82 \mathrm{ml} \mathrm{min}^{-1} 1.73 \mathrm{~m}^{-2}$, and fractional albumin cicarance (FAC) 4.0 and $6.3 \times 10^{-5}$. Results: The following changes in group $\mathrm{E}$ and $\mathrm{C}$ were obscrved: protein intake -0.08 and $+0.08 \mathrm{~g} \mathrm{~kg}^{-1}$, DBP -5 and +1

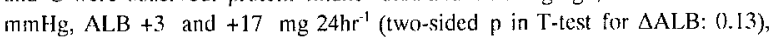
$\mathrm{CrCl}-5$ and $+8 \mathrm{ml} \mathrm{min}^{-1} 1.73 \mathrm{~m}^{-2}$. $\mathrm{FAC}+1.0$ and $+1.8 \times 10^{-6}$. Conclusion: These preliminary results are in line with the hypothesis that dictary protein restriction bencficially influences albuminuria in NIDDM patients at risk of nephropathy.

\section{1}

METABOLIC EFFECTS OF RESISTANT STARCH IN PATIENTS WITH NIDDM R.Giacco, M.Mancini*, G.Clemente, S.Coppola, G.Lasorella, A.D'Avanzo*, A.Rivieccio $0^{\circ}$, G. Ruffa, F.Brighenti^, A.A.Rivellese ${ }^{\circ}$ and G.Riccardi ${ }^{\circ}$. Institute of Food Science and Technology of C.N.R., Avellino, *S.G.Moscati Hospital, Avellino, 'DISTAM,University of Milan, 'Department of Clinical and Experimental Medicine, Federico II University, Naples, Italy.

Resistant Starch (RS) is starch not digested by $\alpha$-amylase in human bowel. Aim of this study was to evaluate in patients with NIDDM:1) the metabolic response to a meal rich in RS; 2) the effects of RS consumption on the metabolic response to the following meal. Ten patients with NIDDM ( 6 male and 4 female, age $50 \pm 2$ yrs $\left(\mathrm{M} \pm \mathrm{SE}\right.$ ), BMI $27 \pm 1 \mathrm{~kg} / \mathrm{m}^{2}$, duration of diabetes $6 \pm 1 \mathrm{yrs}$, fasting plasma glucose $153 \pm 4 \mathrm{mg} / \mathrm{dl}$, treated with diet alone or diet + sulphonylureas) were given, after a 12 hours overnight fast, on different days and in random order, two meals similar in composition (Protein 15\%, Fat 30\%, Carbohydrate 55\%, Fibre 3g, kcal 800 ) but differing in RS content $(16 \mathrm{~g}$ vs $1 \mathrm{~g})$. Four hours after the first meal patients were given a second meal which was identical on both occasions (Protein 15\%, Fat 30\%, Carbohydrate $55 \%$, Fibre $3 \mathrm{~g}, \mathrm{kcal} 600$ ). Blood samples were collected at fasting and every hour for 4 hours after each meal for measurements of plasma glucose, insulin, triglycerides and NEFA. Blood glucose response was lower after the high RS than after the low RS meal $(-38 \% ; \mathrm{p}<0.04)$. Also, the plasma insulin response was significantly lower after the RS rich meal $(-26 \% ; \mathrm{p}<0.05)$. Conversely, plasma concentrations of NEFA and triglycerides were similar after the two meals Consumption of RS had no effect on the subsequent meal; in fact there were no differences in plasma glucose, insulin, NEFA and triglyceride responses between the standard meals consumed 4 hours after either the high RS or the low RS meal. In conclusion, RS consumption improves the plasma glucose and insulin response to the meal. The availability of various starchy foods enriched in RS would enlarge the choice of low glycemic index foods for diabetic patients, thus improving diet compliance.
743

Differential effects of saturated and monounsaturated fat on postprandial lipaemia and GIP/GLP-1 responses in healthy subjects.

O Rasmussen, C Thomsen, T Lousen, Hermansen K, JJ Holst" Aarhus Amtssygehus, Aarhus University Hospital, Panum Institute", University of Copenhagen.

Postprandial lipaemia may influence the development of coronary heart disease through an elevation of postprandial chylomicrons (CM) and very low density lipoproteins (VLDL). Diet-induced alterations in insulin and GIP (gastrointestinal polypeptide) seems to indirectly influence postprandial TG-metabolism, whereas no data concerning GLP-1 (glucagon-like peptide 1) are available. Long-term studies have demonstrated beneficial effects of monounsaturated fatty acids (MUFA) on fasting lipid and lipoprotein levels. In the present study we compared the acute responses of glucose, insulin, free fatty acids (FFA), TG, GIP and GLP-1 in ten young, lean, healthy persons ( 5 men, 5 women) after three meals over 8 hour study periods. Meal A: a non-caloric soup taken with $50 \mathrm{~g}$ carbohydrate as white bread, meal B: meal A plus $100 \mathrm{~g}$ butter, and meal C: meal A plus $80 \mathrm{~g}$ olive oil. The TGresponses were measured in total plasma, in a $\mathrm{s}_{\mathrm{z}} \geq 1000 \mathrm{CM}$-fraction and in a $\mathrm{s}_{\mathrm{f}}$ 1000 non-CM fraction (VLDL-rich fraction).

Results: During the initial 4 hours the glucose and insulin responses were highest after meal A whereas no differences were seen between meal B and C. The FFA showed an initial depression after all meals, with the lowest value seen after meal $\mathrm{A}$. The two fat-rich meals caused the most prominent GIP increment. In addition, the 6 and 8 hour GIP responses after meal $\mathrm{C}$ were higher than after meal $\mathrm{B}$. Interestingly, the GLP-1 responses showed a different pattern with the highest responses seen after meal $\mathrm{C}$ during the initial 3 hours. The TG-responses were highest after meal $\mathrm{B}$, with CM-TG rising 2.5-5 fold as compared with meal $A$ and $C$. The non-CM-TG after meal B seemed to have a slow clearence.

Conclusion: Butter, but not olive oil, induced high postprandial TG levels in healthy subjects, while no difference were seen in insulin responses. However, olive oil induced an intial high response of GLP-1 and a sustained high response of GIP. These observations demonstrates for the first time a possible relationship between postprandial TG metabolism and GLP-1. 
744

SIMILAR ENERGY BALANCE AFTER THREE ISOCALORIC, BUT DIFFERENTLY COMPOSED, ORAL LOADS IN NON -OBESE SUB.IECTS

E. Bobbioni-Harsch. F. Habicht. T. Lehmann. F. Rohner-Jenarenaud. A. Golay, University Hospital Geneva. Switzerland.

This study was aimed to investigate the energy balance and the substrates oxidative pattern in ten normal weight subjects (5 males, 5 females) submitted to three isocaloric (370 $\mathrm{Kcal} / \mathrm{m}^{2}$ body surface) but differently composed oral loads. i.e. glucose load (GLU) (glucose $100 \mathrm{gr} / \mathrm{m}^{2}$ ), mixed load (Mix) (glucose $50 \mathrm{gr}+$ Fat $19.2 \mathrm{gr} / \mathrm{m}^{2}$ ) and lipids load (LIP) (Fat $38.5 \mathrm{gr} / \mathrm{m}^{2}$ ).

Energy Expenditure (EE) was measured before and during 8 hours after the ingestion of each load, by indirect calorimetry. Plasma insulin. glucose and FFA were measured every hour. EE was the same after each load. (GLU: $425 \pm 26$. MIX: $435 \pm 30$. LIP: $427 \pm 28$. Kcal $/ 8 \mathrm{~h}$ )

Glucose oxidation was increased after glucose (from $30 \pm 4$ to $70 \pm 4 \mathrm{gr} / 8 \mathrm{~h}$ ), and mixed load (from $36 \pm 3$ to $62 \pm 3 \mathrm{gr} / 8 \mathrm{~h}$ ) while fat oxidation, already high in basal condition $(32 \pm 3 \mathrm{gr} / 8 \mathrm{~h})$ was slightly but significantly increased after lipids load $(35 \pm 3$ $\mathrm{gr} / 8 \mathrm{~h}) \mathrm{p}<0.05$. Fat oxidation was drasticaly diminished after both glucose load $14 \pm 2 \mathrm{gr} / 8 \mathrm{~h}$ and mixed load $(23 \pm 3 \mathrm{gr} / 8 \mathrm{~h})$ $\mathrm{p}<0.0001$

FFA plasma levels were negatively linked to glucose oxidation $(r=-0.74 p<0.0001)$ and positively to lipids oxidation $(\mathrm{r}=0.54, \mathrm{p}<0.002)$.

We conclude that the energetic cost of lipids utilization is similar to the one of glucose. Futhermore FFA play an important role in regulating lipids oxidation. We also suggest that alimentary fat can contribute to lipids oxidation by maintening elevated FFA plasma levels.

\section{6}

EFFECT OF A HIGH MONOUNSATURATED FAT DIET ENRICHED WITH HAZELNUT IN NIDDM PATIENTS M.E. Alphan(1), M. Pala(2), F. Acckurt( ${ }^{(3)}$ and M.T. Yilmaz(1). (1) Istanbul University, Istanbul Medical Faculty, Div. of Diabetes and DETAE, (2)Yildız Technical University, Faculty of Chemical Engineering, (3)TÜBITAK Marmara Research Centre, TURKEY.

In this study the effects of high carbohydrate diet ( $H C D$ ) versus high monounsaturated fat diet enriched with hazelnut were compared on metabolic regulation in 19 NIDDM patients (14F/5M, mean chr. age 51.6 $\pm 6.1 \mathrm{yrs}$, duration of diabetes $3.4 \pm 2.2 \mathrm{yrs})$. The study was accomplished in three steps. In the first step patients were given $\mathrm{HCD}$ for 30 days $(60 \% \mathrm{CH}, 25 \%$ fat). This was followed by 15 days of washout period with standard diet $(50 \% \mathrm{CH}, 30-35 \%$ fat $)$. The last step included the hazelnut diet $(40 \% \mathrm{CH}, 45 \%$ fat). Blood samples were taken initially and then at $15 \mathrm{th}, 30 \mathrm{rd}, 45 \mathrm{th}, 60 \mathrm{th}, 75 \mathrm{th}$ days and were analyzed. The fatty acid composition of hazelnuts were assessed by gas chromotography. There is no significant change regarding fasting and postprandial glycemia, insulin and C-peptid levels and BMI at the beginning and at the end of each diet. There is no significant change in all parameters during $H C D$ except LDL. LDL levels were significantly increased $(130.0 \pm 39.6$ vs $144.9 \pm 53.5, \mathrm{p}<0.01)$. Compared to the $\mathrm{HCD}$, the hazeinut diet resulted in lower HbAlc $(8.3 \pm 2.1$ vs $7.2 \pm 1.9 \%$, $p<0.05)$, lower levels of total cholesterol $(234.3 \pm 41.3$ vs $207.5 \pm 30.6$ $\mathrm{mg} / \mathrm{dl}, \mathrm{p}<0.01)$. LDL $(178.0 \pm 42.9$ vs $130.2 \pm 38.7 \mathrm{mg} / \mathrm{dl}, \mathrm{p}<0.001)$ and apoprotein $B(202.6 \pm 46.4$ vs $187.3 \pm 49.5 \mathrm{mg} / \mathrm{dl})$ and higher levels of $\mathrm{HDL}(36.6 \pm 12.4$ vs $39.4 \pm 13.6 \mathrm{mg} / \mathrm{dl})$ and apoprotein $\mathrm{Al}(228.7 \pm 82.6$ vs $292.2 \pm 78.5 \mathrm{mg} / \mathrm{dl})$. These results indicate that monounsaturated fat diet enriched with hazeinut does not influence carbohydrate metabolism and has a beneficial effect on lipid parameters. For this reason hazeinut should be recommended to the NIDDM patients.
745

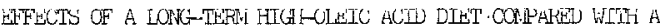

HTGH-CAKBOYMDHAIE DTET IN NDLM PATIETIS.

I. Ioseri, A. Bonalumi, F. Cortinovis, C. Mora, H. Casati, G. Lepore

Diábetes Uiit, 00.Rir., Bergano, Italy

Aim ol' the study was to assess the effect of a hiyh-honounsatured fut inet substituting for part of CHO on glucose and lipid levels, blood pressire

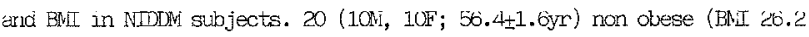
土1.1) INIDDS, ireated with diet alone on plus SU, containing 51-5\%\% of Culories as $\mathrm{C}$ iO, were stuxied. They were randomized to 3 nonttis treatment

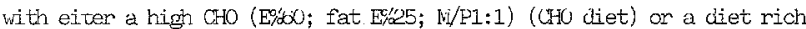
in olive oil (NUFA diet) (4OE\%; M/P2:1; $454 \%$ (UTO). Both diets were isocaloric, containing $22-25 \mathrm{~g}$ of dietary fiber and $0.9 \mathrm{~g} / \mathrm{kg}$ of protein. Hivery patient received the first scherie for a period of 3 months, followeu by a wash-out period of 6 weeks, after which patients were swiched to the opposite diets for an additional 3 months. Body weight, BMI, BP, lipids, Hodic glucose, insulin and C-pepticie were measured initially and at monthly intervals. The two ciets significantly reduced diastolic BP $(92.6 \pm 1.9 \mathrm{~mm}$ before treatuient, $37.8 \pm 2$ after MLFA, p<0.05; 86.3 $\$ 1.4$ after $\mathrm{CHO}, \mathrm{p}<0.01$ ) and enhanced insulin $(16.8 \pm 2 \mathrm{mU} / 1$ before treatment, $19.1 \pm 2$ af uer $\mathrm{MJ} A$, $20.1 \pm 2.1$ after $\mathrm{CHO}$ diet, $\mathrm{p}<0.05$ ) and C-jeptide (3.04 10.27 berore diet, $3.79 \pm 0.47$ after $\mathrm{AUFA}$, $3.7 \pm 0.35$ after Cho diet, $\mathrm{p}<0.05$ ) preprandial concentrations. Moreover fasting both C-peptide (2.42 \pm 0.1 vs $2.05 \pm 0.21$ and 2.07

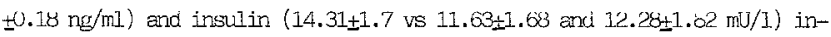
creased after MUFA diet ( $p 40.05$ vs baseline and 010 diet respectively).

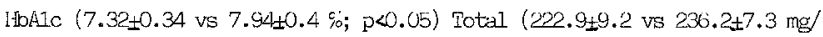

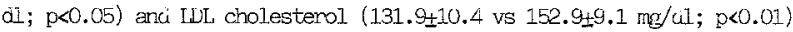
were lower after MIFA scheme combered with CHO diet. A long-tem MFA treatment had beneficial effects on insulin secretion, diastolic BP, chol. and HBAlc. The differeances in chol. and FbAlc were peculiarly related to IUFA compared with CHO diet.

\section{7}

THE IMPACT OF INTENSIFIED DIET EDUCATION ON FAT INTAKE IN NIDDM PATIENTS WITH PERSISTENT MICROALBUMINURIA. M. Beck, P. Vedel, P. Rossing, J. Obel and O. Pedersen, Steno Diabetes Center, Copenhagen, Denmark

The aim was to assess the effects of intensified diet education as a part of a multifactorial intervention on fat intake and fasting serum lipids. BMI and $\mathrm{HbA}_{1 \mathrm{c}}$ in NIDDM patients with persistent microalbuminuria. Protocol: 160 patients, age $45-65 \mathrm{yrs}, \mathrm{HbA}_{\mathrm{co}} 8.6 \%\left( \pm 1.6\right.$ ), (mean $\pm \mathrm{SD}$ ), $\mathrm{BMI} 29.8 \mathrm{~kg} / \mathrm{m}^{2}$ ( \pm 4.4 ), total s-cholesterol $5.6 \mathrm{mmol} / \mathrm{l}( \pm 1.2)$, s-triglycerid $2.6 \mathrm{mmol} / \mathrm{l}( \pm 2.0)$ s$\mathrm{HDL}$-cholesterol $1.0 \mathrm{mmol} / \mathrm{l}$ ( \pm 0.2 ), microalbuminuria $(30-300 \mathrm{mg} / 24 \mathrm{~h}$ ), were randomly assigned either to polypharmacological treatment and intensified diet education $(n=80$ ) focusing on qualitative and quantitative changes in dietary fat (high intake of PUFA and MUFA and low intake of SAFA) or unchanged conventional treatment $(n=80)$. Measurement of diet intake was based on dietary history interviews. In this interim report 2 yrs results are presented. Diet intake in the two groups was comparable at baseline. Results after 2 years: $33 \%$ total fat ( $36 \%$ MUFA, $28 \%$ PUFA, 36\% SAFA), $44 \%$ carbohydrate, $18 \%$ protein and $5 \%$ alcohol in the intervention group vs $42 \%$ total fat $(37 \%$ MUFA, $16 \%$ PUFA, $47 \%$ SAFA), $38 \%$ carbohydrate, $16 \%$ protein and $4 \%$ alcohol in the conventional group. The intake of total fat was significantly lower $(p<0.001)$ and the intake of PUFA was significantly higher $(p<0.001)$ in the intervention group compared to the control group. $\mathrm{HbA}_{1 \mathrm{c}}$ was $8.0 \pm 1.3 \%$ in the intervention group vs $9.3 \pm 1.7 \%$ in the control group $(p<0.001)$. The decline in $\mathrm{HbA}_{1 c}$ was obtained without an increase in BMI. No significant differences between the intervention group and the control group were seen in total s-cholesterol $4.9 \pm 0.7 \mathrm{mmol} / /$ vs $5.5 \pm 1.6 \mathrm{mmol} / /$ and s-triglycerides $1,4 \pm 0,7 \mathrm{mmol} / \mathrm{l}$ vs $3.3 \pm 0.3 \mathrm{mmol} / \mathrm{l}$ or $5-\mathrm{HDL}$-cholesterol which was $1.0 \pm 0.3$ mmol/l in both groups. Conclusion: Intensified diet education resulted in the intended reduction in intake of total fat and a relatively higher intake of PUFA. The decline in $\mathrm{HbA}_{1}$ in the intensively treated group was achieved without the expected increase in BMI. The intensive dief and pharmacological intervention has not yet shown significant benefits on fasting levels of serum lipids. 


\section{8}

PIASMA AND IIRINARY EXCRETION OF MAGNESIUM, COPPER AND ZINC IN NON-INSILIN DEPENDENT DIABETES MELLITUS

MP Saavedı F. F Ameta*. J Fsc|uiroz. L. Hergtteta*. L!niversity Hosp. Principe de Astunas (LAH): * Eniversity Hosp. (jregorio Marañon. Madrid. Spain.

Recent studies have referred alterations of mineral status in diabetic subjects: however these studies yiclded inconsisten results. The objetive of these work was to evaluate magnesium ( $\mathrm{M} g$ ). copper ( $\mathrm{Cu}$ ) and zinc ( $\mathrm{Zn}$ ) metabolism in NIDDM subjects. We measured plasma and 24 h. urinay $M / g$. $C$ and $Z n$ levels by atomic absorption spectophotometry in 92 out-patients $(34 \mathrm{~m} / 58 \mathrm{n})$ with NIDDM (aged: 60) $3 \pm 11.4$. B MII: $28.6+5.1 \mathrm{~kg} / \mathrm{m}^{2}$. HbAle: $\left.8 \pm 1.8 \%, \mathrm{x} \pm 0\right)$ and $+5(14 \mathrm{~m} / 31$ f) non diabetic subjects (aged:49+ 17 vears. BNI: $28.7 \pm 5.3 \mathrm{Kg} / \mathrm{m}^{2}: \mathrm{x} \pm$ ol exposed to de same risk factor except to be diabetic. The plasma metals concentrations were signilicant lower $(p<0.05)$ in diabetics $(\mathrm{Mg}-0.79 \pm 0.29$

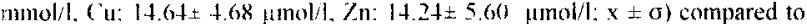
controls ( $\mathrm{Mg}: 1.04 \pm 0.45 \mathrm{mmol} / \mathrm{l}$. ( $\mathrm{u}$ : $20.26 \pm 8.16 \mu \mathrm{mol} / \mathrm{l}$, Zn: $16.46 \pm 6.42$ amol/l; $\mathbf{x} \pm 0)$. The metals fraccional excretion were used as slinical assessment of its renal excretion. These were significant higher $(\mathrm{p}<0.05)$ in diabetics $(\mathrm{Mg}: 4.9 \pm 3.35 \%$ ( (u: $0.51 \pm 0.68 \% \mathrm{k}): \mathrm{Zn}: 1.09 \pm 0.75 \%: \mathrm{x} \pm 0$ ) than in non-

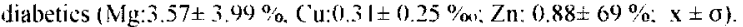

in conclusion: NIDIDM patients present a decreased plasma levels of $\mathrm{Mg}$. $\mathrm{Cu}$ and $\mathrm{Zn}$. These could be explained by the increase of $\mathrm{Mg}$. $\mathrm{Cu}$ an $\mathrm{Zn}$ urinary excretion.

\section{9}

EFFECTS OF DIFFERENT FOOD STRUCTURE AND GLYCEMIC INDEX IN NON-INSULIN-DEPENDENT DIABETES MELLITUS PATIENTS A. Järvi ${ }^{1)}$ B. Kariström ${ }^{1)}$, Y. Granfeldt' ${ }^{2)}$, I. Björck ${ }^{2)}, \mathrm{N}-\mathrm{G} . \mathrm{Asp}^{2)}$ and B Vessby ${ }^{1) .}$

1) Dept of Geriatrics, Unit for Clinical Nutrition Research, Uppsala University, Uppsala 2) Dept of Applied Nutrition and Food Chemistry, Chemical Center, Lund University, Lund.

The aim was to evaluate the metabolic response of carbohydrate-rich foods with different food structure and glycemic index (GI) in NIDDM patients. In a cross-over study 20 patients were given diets with either a low or a high glycemic index in randomised order during two consecutive 3.5 week periods. The diets were individually prepared and composed in accordance with the present dietary recommendations. The nutrient composition, type and amount of of dietary fibre was identical in both diets except for large differences in GI mainly achieved by altering the structure of the starchy foods included. The pheripheral insulin sensitivity increased significantly and the fasting blood glucose decreased during both treatment periods with a reduction of fructosamine, $-2.7 \%,(p<0.05)$ atter the diet with low Gl. The incremental area under the curve for both blood glucose, $30 \%,(p<0.05)$ and plasma insulin, $-27 \%,(p<0.01)$ were significantly lower after the diet period with the low GI. Serum cholesterol and LDL cholesterol were significantly lower after the period with low GI food, $-5.2 \%,(p<0.01)$ and $-8.3 \%,(p<0.01)$, respectively. The PAl-1-activity, which was high on the high GI diet was normalised on the low Gl diet, $-54 \%,(p<0.001)$. A strictly controlled diabetic diet with an intact structure of the starchy foods and a low GI but with all other nutrients including the dietary fiber content kept constant, showed a lower glucose and insulin response throughout the day as well as an improved lipid profile and an improved capacity for fibrinolysis, resulting in a considerably improved metabolic profile.

\section{PS 39}

\section{Health Care, Education, Psychosocial Problems}

\section{0}

Factors influencing quality of life in patients with diabetes.

RJ Young, CA Lewis, JP New, J Lloyd, S Hollis, and D McDowell. Diabetes Centre, Hope Hospital, Manchester. UK.

The aim of this study was to determine which aspects of diabetes are perceived by patients to most influence their quality of life. As part of an annually updated population based diabetes register, in our urban district of 230,000 , a single question (SQ) is routinely asked about diabetes specific interference with life and scored on a five point scale. Using a randomised sample of 100 patients attending hospital clinics, the SQ was compared to the more complex, carefully validated, diabetes specific well being (DSWB) section of the Well Being Questionnaire (Bradley); a significant correlation was observed $(r=0.43, p<0.001)$. Using multiple logistic regression the $S Q$ was compared with variables collected concurrently using DIABCARE diabetes dataset. Information was available for 2625 patients. Neuropathic pain $(\mathrm{r}=-0.20, \mathrm{p}<0.001)$; previous amputation $(r=-0.17, \mathrm{p}<0.001)$; poor glycaemic control $\left(\mathrm{HbA}_{1 \mathrm{c}}\right)(\mathrm{r}=$ $0.11, \mathrm{p}=0.002)$; impotence $(\mathrm{r}=-0.08, \mathrm{p}=0.012)$; and sight threatening retinopathy requiring photocoagulation $(\mathrm{r}=-0.07, \mathrm{p}=0.03)$ were each independently associated with poorer well being assessed by the SQ. Amputation and painful sensory neuropathy had the greatest adverse impact on quality of life. Good glycaemic control was the only identified factor which associated with better quality of life and hypoglycaemia was not found to have an independent adverse effect. This study suggests that by achieving improved glycaemic control diabetic services may improve quality of life not only by reducing amputations but by improving well being directly.

\section{1}

VALIDATION OF A DIABETES QUALITY-OF-LIFE MEASURE U. Bott, I. Mühlhauser, H. Overmann and M. Berger. Clinic of Metabolic Diseases and Nutrition, Heinrich-Heine-University Düsseldorf, Germany There is still a lack of validated diabetes quality-of-life scales with sufficient sensitivity to different treatment and dietary regimen. In a population-based study, a diabetes quality-of-life scale was validated in adult IDDM patients. A total of 530 patients $(41 \%$ women, age $36 \pm 11$ years, diabetes duration $18 \pm 11$ years, $\mathrm{HbA}_{1 \mathrm{c}} 8.0 \pm 1.5 \%$; mean $\pm \mathrm{SD}$ ) completed the measure comprising 71 items on: therapeutic goals (10 items), satisfaction with treatment success (10 items) a validated general well-being scale ( 7 items), and 44 iterns dealing with diabetes-related burdens. Factor analysis of the 44 statements revealed 7 reliabie components (Cronbach's alpha): close social relations (.87), physical complaints $(.84)$, worries about future $(.84)$, leisure time flexibility $(.83)$, social contacts $(.78)$, diet restrictions $(.70)$ and daily hassles $(.70)$. All 7 subscales were negatively correlated with the general well-being scale $(r=-34$ to $.54, p<.001)$. Physical complaints $(r=.28)$ and worries about future $(r=$ 18) showed the highest correlations with $\mathrm{HbA}_{1 \mathrm{c}}(\mathrm{p}<.001)$. Patients who had participated in structured group treatment and teaching programmes perceived less dietary restrictions $(\mathrm{p}<.005)$ and more leisure time flexibility $(\mathrm{p}<.05)$ Patients with a flexible insulin therapy ( $>3$ injections / day or insulin pump), those adjusting insulin dosages according to carbohydrate intake and those who didn't follow a meal plan scored better on the diet scale $(p<.001)$, perceived better close social relations $(p<.01)$, a higher leisure time flexibility and less worries about future $(p<.05)$. Patients with late complications had poorer scores regarding close social relations and social contacts $(\mathrm{p}<.05)$, physical complaints, worries about future and leisure time flexibility $(p<.001)$. A low social status was associated with poorer scores concerning physical complaints $(p<.001)$, leisure time flexibility and diet restrictions $(p<.005)$. In conclusion, the questionnaire is a reliable and valid measure of diabetes quality-of-life. Using this scale, the absence of late complications, a flexible insulin therapy and a liberalized diet were strongly associated with a better quality-of-life. 


\section{2}

\section{SELF ASSESSED WELLBEING AND METABOLIC CONTROL IN TYPE I} DIABETES

J. Sumner and M. Sumner. Diabetes Centre Radcliffe Infirmary NHS Trust. Woodstock Road. Oxford. OX2 6HE LK

The aim of the study was to analyze the association of patient assessed wellbeing and its relationship to glycaemic control measured by HbAlc. The study also examines the optimum $\mathrm{HbAl}$ level to maximise health scores. and compares the health of the study group with that of the general population. 308 consecutive paticnts attending the diabetes clinic completed the Short-Form 36 Hcalth Questionnaire (SF 36) which measures eight aspects of health: physical function (PF), role limitation physical (RLP), role limitation mental (RLM), social function (SC). mental health $(\mathrm{MH})$. energy \& vitality ( $\mathrm{EV}$ ). pain (P). and health perceptions (HP). The routine HoAlc was collected simultaneously. The results showed an inverse relationship between $H P$. PF and HbAlc. Cuirvilinear regressioni indicated that RLP. RLM, SC, MH. EV, P, achieve better scores when HbAlc was between $8 \%$ and $9 \%$. Scores were compared with published normative data for the Oxford region. The sample group scored less than the normative group in all aspects: $\mathrm{PF}^{*}$ RLP**, RLM*, SC*, MH, EV, P*, and HP** (*p<0.05, ${ }^{* *}$ p<0.000001). Other factors shown to affect health scores were gender (male scores were better: mean 77.5 vs $71.9 \mathrm{p}<0.05$ ) and length of time on insulin ( $\mathrm{HbA}$ ic peaks at 25 years on insulin). Only $23 \%$ reported diabetes as a long standing or chronic illness. The main findings showed that the majority of health scores are optimised when $\mathrm{HbAlc}$ levels are between $8 \%$ and $9 \%$. The perceived wellbeing of people with Type 1 diabetes is lower than the general population in Oxfordshire. Metabolic measures alone cannot predict the wellbeing of a person and routine use of an objective measure such as that of the SF36, is a valuable tool to enhance understanding of patients' perceptions, and to quantify their feelings.

\section{3}

\section{WHICH PATIENTS WITH DIABETES CONSULT: A MULTIVARIATE ANALYSIS OF SOCIO-ECONOMIC FACTORS}

NJA Vaughan!, JRH Charlion' and D Rothman ${ }^{3}$ 'Roval Sussex County Hospital Brighton; 'Office of Population Censuses and Surcys and "British Diabetic Association, London

The influence of socio-economic factors on consultation rates in patients with diabetes with an analysis of complication risk was undertaken. The study covered a representative $1 \%$ sample of the population of England and Wales $(502.493$ patients; 5184 with diabetes, prevalence $1.03 \%$ ) from 60 prinary care practices. At least $95 \%$ of all general practitioner contacts were recorded during the vear of the study. Socio-economic information was collected by interviewers for $83 \%$ of patients. Variations in the proportion of people who consulted according to single socio-economic and geographical characteristics were examined and a multivariate analysis was employed to estimate the effect of each variable while controlling for all other variables. These are expressed as odds-ratios. These can be multiplied for risk combinations. Consultation rates increased with age in both sexes when compared with white men aged $16-34,3.5: 1^{* * *} 35-54 y s, 9.9: 1^{* * x} 55-$ $74 \mathrm{ys}, 12.6: 1^{*+*} 75+\mathrm{ys}$, although men were significantly more likely to consult than women. Socio-economic factors associated with increased consultation rates included: rented council property tenants $1.4: 1^{* \times *}$, living alone $(50-64 \mathrm{ys}) 1.5: 1^{* *}$. ethnic minorities - Asian $6.5: 1^{*+*}$, Afro-Caribbean $3.2: 1^{* * * *}$ and Social classes IIIM, IV and V (manual occupations) in women $1.4: 1^{* * *}$. Couples with children were less likely to consult $0.5: 1^{* * *}$. Smoking did not increase the likelihood of consulting. Patients consulting with mactovascular complications including ischaemic heart disease, peripheral vascular disease and stroke were more likely to be; Asian $2.4: 1^{* *}$ or Social class IV \& V 1.5: $1^{*}$. Similarly patients consulting with hypertension were more likely to be Asian 3.2:1** Few differences existed for patients consulting with microvascular complications except retinopathy was more common in Social classes IV \& V 1.6: $1^{*}$. In conclusion, healtheare resource usage and complication rates in diabetes are significantly influenced by socioeconomic factors and must $b e$ accommodated in healthcare planning. $\left(p<0.05^{*}, p<0.01^{* *}, p<0.001^{* * *}\right)$

\section{4}

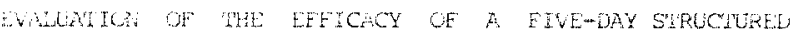

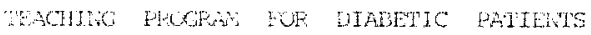

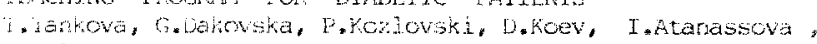

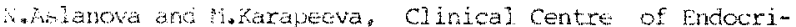
nojogy, Sofja, Butgaria

'tis study wa designed to evaluate the efficacy of a fiveday structured teaching program for diabetio patients. 117? insulin-treated liabetio patients 189 with Iifh and 28 with Ninim?, of hean age $37 * 4+12$. years and mean duration of the disedse $9,6+6.1$ years, were followed up during the five-day projkam as well as 6 months later. The quality of life of dialetic patients was assessed using a standara wel1-being ghestionraire. our results demonstrate a significant increase in overall guality of life (score 56 vs $42, p<0.01$ due to reduction in depression $(3.12 \mathrm{vs} 4.61, \mathrm{p}<0.001)$ and arxisty $(4.13 \mathrm{vs} 7.71,0<0.001)$ and increase in well-being $(14.88$ vs $13.1, p<0.05)$ and energy $(9.03$ vs $8.12 . p>0.1)$. The metabolic control improved significantly, judging by HibAlc level $(7.6 \%$ vs $8.91 \%, 0<0.05)$. There was a significant increase in diabetes-related knowledge at the end of the five-day program ( $81 \%$ vs 59g, p<0.01), being maintained unchanged 6 months later $(80 \%$ vs $81 \%)$. The rate of overall bypoglycaenia decreased from 0.68 to $0.43 \mathrm{cas} / \mathrm{pat} / \mathrm{yr}$ $(p<0.01)$ and the rate of severe hypoglycaemia - from 0.10 to $0.04 \mathrm{cas} / \mathrm{pat} / \mathrm{yr} \quad(\mathrm{p}<0.01)$. The incidence of diabetic ketoacidosis fell from 0.28 to 0.11 cas/pat/yr ( $0<0.001$ ). $9 \%$ of patients reduced smoking. These results demonstrate that structured patient education improves the quality of life of diabetic patients and their metabolic control, significantly reduces the rate of acute complications . thus helping patients to live with the disease.
755

THERAPY. AND EDUCATION RELATED DIFFERENCES BETWEEN PATIENTS WITH IDDM ONE YEAR AFTER A STRUCTURED EDUCATION IN ICT

E.Biermann, W.Heinlein, Th. Kothny and E.Standl

3. Med. Department, Schwabing City-Hospital, Munich, Germany

Intensified Conventional Therapy (ICT) permits patients with IDDM to adapt their dose of normal insulin to preprandial glucose values, intended carbohydrate intake and physical activity. The question raises if differences between groups with poor and good metabolic control can be found, to explain, that the desired therapeutic goal (Hba1c $<7.9$ ) has not been achieved. 60 patients with IDDM (mean age $\mathbf{3 4 , 3} \mathrm{yrs}$., diabetes duration 12,7 yrs.) were reinvestigated one year after a diabetes education. Mean $\mathrm{HbAtc}$ declined from $8.7 \%$ before education to $7.9 \%$ (normal range $4.0-6.5 \%$ ). Three groups were formed : very tight $(\mathrm{HbA1c}<6.5 \%, \mathrm{n}=6)$, good ( $\mathrm{HbA} 1 \mathrm{c} 6.5-8.0 \%$, $n=27)$ and poor ( $\mathrm{HbA1c}>8.0 \%, \mathrm{n}=27$ ) metabolic control. The frequency of severe hypoglycemia was different in these groups (1.2, 0.8 and 0.3 events / patient year, n.s.). Using a standardized log-book evaluation, it was found that in those patients with $\mathrm{Hba1c}>8.0 \%$ BG-values above acceptable range (definded as BG preprandial $70-150 \mathrm{mg} / \mathrm{dl}$, where adding normal insulin to the regular dose was not expected) occured in $59 \%$ of all self control measurements. Patients did not respond in $58 \%$ of all cases to elavated glucose values with adding normal insulin to their regular dose. In contrast, the group with good metabolic control had significantly less values above acceptable range (43\%) and responded more frequently $(55 \%)$. In the very tight controlled group BG-values were even better (13\% too high) and the response rate was better as well $(75 \%)$. We conclude that the problem in patients with poor metabolic control is either insufficient knowledge or non-acceptance of target values for preprandial BG. Furthermore, they do not dare to increase insulin dose or they underestimate their regular insulin dose when BG is in target range. Based on these observation, patients with poor metabolic control and a low response rate to elevated preprandial $B G$-values should be identified and controlled more intensly. 
756

EMPOWERMENT BY FUNCTIONAL INSULIN TREATMENT: LONG- AND SHORT-TERM EFFECTS ON PERCEIVED CONTROL OF DIABETES D. Wagner-Nosiska, K. Howorka, J. Pumpria, Ch. Schiusche and H. Thoma Dept. of Biomedical Engineering \& Physics, University of Vienna, Austria Functional insulin treatment (FIT) requires a clearly defined education program in functional use of insulin (by multiple injections or continuous delivery) either for eating or for fasting or for correction of hyperglycemia. It allows flexible food intake and good metabolic control. Study 1 investigated the effect of such training on health locus of control in 26 Type I (insulindependent) diabetic patients (age: $34+17$, diabetes duration: $15+11$ yrs, $\mathrm{HbA} 1 \mathrm{c}: 6.5 \pm 0.7 / \mathrm{x} \pm \mathrm{SD} /$ ) treated with multiple daily injections after a general diabetes education. Bradley's questionnaires relating to perceived control of diabetes and health beliefs concerning diabetes were administered before and after the FIT-program in the intervention grous $(n=16)$ and after a general diabetes education and 4 weeks later in the control group $(n=10)$. Results of health locus of control ( $\mathrm{HLC}$ ) scales showed no changes in the control group and in the intervention group increase of Internality $(\boldsymbol{p}<0.05)$. Treatment $(p<0.7)$; Externality $(p<0.6)$; Chance $(p<0.1)$; Patient Control $(p<0.1)$; Doctor Control $(0<0.4)$; Foreseeability $(\mathrm{p}<0.4)$ were not influenced. Study 2 investigated the long-term effect of FIT on HLC in 68 Type / diabetic patients (age $38 \pm 16$, duration of diabetes $19 \pm 12$, HbA1c $7,3 \pm 1,4 \%$ ) using HLC scale (maximum score up to 36 ) before and $38+20$ months after the FIT-program. HLC scales demonstrated influence on Treatment (before FIT: $15.7 \pm 6.7 /$ after: $12.8 \pm 5.8, p<0.004)$, on Doctor Controi $(12.3 \pm 7.8 / 8.8 \pm 6.7, p<0.002)$ and on Medical Control $(14+6.6 / 10.8+5.6, p<0.001\}$. Other scales - Internality $(p<0.9)$, Externality $(p<0.4)$, Chance $(p<0.6)$, Foreseeability $(p<0.3)$, Personal Control $(p<0.2)$, Patient Control $(p<0.1)$ and Situational Control $(p<0.4)$ demonstrated no significant changes. The increase of patient's selfresponsibility was demonstrated as a short-term effect (Internality), whereas the feeling of "Empowerment" (decrease of Treatment and Doctor Control and numerical increase of Patient (Contro) were shown as long-term effects. Thus, FIT allows flexible food intake and immediate control of hyperglycemia in a more self-responsibie and self-controlled patient.

758

METABOLIC CONTROL AND HYPOGLYCAEMIC EPISODES IN INSULIN-TREATED PATIENTS IN ITALY: THE SIEMTIC STUDY

M.P. Garancini (1), D. Cucinotta (2), G. Gallus (3), G. Riccardi (4), and A. Rossi (5) (reps. of the S.I.E.M.T.I.C. Group), (1) S. Raffaele Institute, University of (2) Messina, (3) Milan and (4) Naples, (5) Eli Lilly Italia SpA

The Italian Multicenter Survey on Insulin Treatment and Metabolic Control (SIEMTIC) was carried out in 17 randomly selected outpatients diabetes clinics in the whole Country to provide a quantitative picture of the insulin treatment in Italy. 50 insulin treated patients, randomly selected from all the active patients of each clinic (25 IDDM, 25 NIDDM), were interviewed. A glucose meter for home monitoring was given to each patient together with a diary to register the hypoglycaemic episodes occurring during the 15 days after the visit. HbAlc values were standardized using blood standard samples. 770 out of the 850 expected patients $(90.6 \%)$ were available for the final evaluation ( 709 aged $<80 \mathrm{yrs})$; 319 were IDDM patients $(58.5 \%$ males, age $38.0+14.3$ yrs, diabetes duration $16.9+11.3$ yrs) and 390 NIDDM $(45.0 \%$ males, age $63.5 \pm 10.0$, known duration $17.2 \pm 7.9)$. IDDM patients showed an average daily glycaemia of $165.8 \pm 37.1 \mathrm{mg} / \mathrm{dl}$, and the highest values were mainly recorded after lunch ( $23.3 \%$ of patients) and after supper $(26.8 \%)$, the lowest ones were before lunch $(33.0 \%)$. Average $\mathrm{HbAlc}$ was $8.1+1.7$; $68.9 \%$ of patients were on 3 or more injections/day. NIDDM patients showed an average daily glycaemia of $180.6 \pm 38.9 \mathrm{mg} / \mathrm{dl}$, with the highest values after meals. HbAlc was $8.1+14 ; 41.3 \%$ were on 2 injections/day, $43.3 \%$ of NIDDM patients used oral hypoglycaemic agents, too. Intensive insulin therapy, qualified occupation, low BMI and fundus examination were significantly and independently associated to low HbA1c values (average difference approx. 0.5) for both IDDM and NIDDM patients. No serious hypoglycaemia was reported; IDDM and NIDDM patients experienced 1.3 and 0.4 hypos/month, respectively. Cases with at least 1 hypos were associated to IDDM $(\mathrm{p}<0.000 \mathrm{I})$ independently of HbAlc levels, insulin injections, gender insulin $/ \mathrm{kg}$ and disease duration. Presence of hypos was also associated to low HbAlc $(\mathrm{p}=0.05)$ and intensive insulin therapy $(\mathrm{p}=0.012)$, independently of type of diabetes. Among IDDM, hypos were always significantly associated to young age and long disease duration. This study identifies the factors related to a good glycaemic control $\left(\mathbf{n}^{\circ}\right.$ of injections, social level, dietary compliance, clinical management) and suggests that good control can be achieved without a strong impact in terms of hypoglycaemias.

\section{7}

DIABETIC KETOACIDOSIS COSTS RELATIVE TO MEDICAL COSTS OF PATIENTS WITH TYPE I DIABETES. K.A. Javor, J.G. Kotsanos, R.C. McDonald, A.D. Baron, J.G. Kesterson, and W.M. Tierney. Eli Lilly and Company, Lilly Corporate Center Indianapolis, IN 46285

The purpose of this study was to determine the proportion of medical care costs of patients with type I diabetes due to the cost of treating diabetic ketoacidosis (DKA) episodes, and the proportion of costs due to those patients who experience multiple episodes of DKA. Using an electronic medical record system, we conducted a retrospective cohort study of $\mathbf{2 2 8}$ patients with type I diabetes who received medical care between 1/1/93 6/30/94. Patient resource utilization and charge data, including hospitalizations, emergency room and outpatient visits, pharmacy charges, and number of DKA episodes were recorded during this time period. One additional year of data was collected to assess the resources and charges associated with multiple DKA episodes.

We identified 72 patients who experienced $163 \mathrm{DKA}$ episodes. These episodes represented $25 \%$ of the medical charges for all patients with type I diabetes. The average charge per-episode was $\$ 6,416$. The estimated annual medical charges for patients experiencing DKA episodes was $\$ 13,152$ per-patient versus $\$ 5,570$ for patients not experiencing an episode. Twenty-four patients experienced multiple DKA episodes accounting for $70.5 \%$ of DKA episodes and represented $16 \%$ of the medical charges for all patients with type I diabetes. In conclusion, we found that DKA represented 1 out of every 4 dollars spent on medical care for patients with type I diabetes in our cohort. Interventions directed at reducing the number of recurrent DKA episodes in patients with type $I$ diabetes could produce a significant cost savings in a bealth care system.
759

QUALITY OF CARE FOR ADULT PEOPLE WITH IDDM IN GERMANY - A POPULATION BASED STUDY

I. Mühlhauser, H. Overmann, U. Bott, V. Jörgens and M. Berger. University Düsseldorf, Germany.

Until now no data have been made available on the degree of diabetes care and education achieved at the community level in Germany. In a population based study 530 adult IDDM patients (18 years or older, initiation of insulin therapy before age 31) living in the Federal State of Nordrhein-Westfalen (approx. 17 Mio. inhabitants), Germany, were examined at their homes using a mobile van. Mean (SD) age was 36 (11) years, diabetes duration 18 (11) years, women $41.3 \%$, body mass index 24.8 (3.5), $\mathrm{HbAlc}$ (reference range 4.3-6.1\%) $8.0(1.5 \%)$, incidence of severe hypoglycaemia (injection of glucose or glucagon) 0.23 and of ketoacidosis 0.03 cases per patient during the preceding year; $62 \%$ of patients had participated in a structured $5-12$ days group treatment and teaching programme for intensification of insulin therapy; $70 \%$ used 3 or more insulin injections per day, $9 \%$ CSII: $97 \%$ used blood glucose self monitoring, $79 \%$ continuously adapted insulin dosages according to blood glucose values and $61 \%$ according to variable amounts of carbohydrate intake; $63 \%$ reported to have had 3 or more measurements of $\mathrm{HbAlc}$ during the preceding year and $28 \% \mathrm{I}$ to 2 measurements. During the preceding year $82 \%$ had a blood pressure measurement by the family physician, $80 \%$ had an examination of the retina by an ophthalmologist (pupils dilated in $85 \%$ at last examination) and $38 \%$ a neurological and vascular examination of the feet; $82 \%$ had an examination of the feet with the tuning fork at some time and $68 \%$ were aware of an examination of protein in the urine: $27 \%$ had been in contact with a consultant diabetologist during the preceding year. The data show, that recommended standards of diabetes care for IDDM have been widely implemented at the community level in Nordrhein-Westfalen, Germany. 


\section{0}

IMPLEMENTATION OF THE DIABETES EDUCATION SYSTEM IN GEORGIA.

R.Shaginyan, R.Kurashvili, N.Asatiani and K.Vashakidze, Diabetes Center, Georgia. The aim of the study is to develop an effective programme to supply the necessary diabetes education under existing conditions in Georgia. The limited available resources, shift from the public health care to the paid service, the isolation of many regions from the capital, the variety of insulin and other sugarlowering drugs and lack of educational materials in Georgia have led the majority of people, suffering from diabetes to be without permanent control and effective management. After organisation of the TV program on "The Problem of Diabetes and the Role of Patient Education" the project "Outpatient Educational Programmes for 230 IDDM and NIDDM patient from needy groups of society" sponsored by The Royal Netherlands Embassy was started. The parameters measured before the start of a 5 day teaching course, after 3- and 6-month period in both groups (Group1-IDDM, Group2NIDDM) included BMI, mean blood glucose (MBG), HbA1, average insulin dose $(\mathrm{U} / \mathrm{kg} / \mathrm{day})$, number of daily insulin injections (NDII), the quantity of sulfonylureas used, the frequency of appropriate self-monitoring (FSM) and the scores of knowledge (SK). The significant improvement of metabolic control after completing of the course in both groups: MBG $(9.4 \pm 2.3$ vs. $11.8 \pm 4.2$ in Group1, $9.1 \pm 1.9$ vs. $11.5 \pm 3.8$ in Group2, $p<0.01)$ and $\mathrm{HbA} 1(9.0 \pm 1.6$ vs. $11.6 \pm 2.1,9.2 \pm 1.3$ vs. $11.7 \pm 2.5$, respectively, $p<0.01$ ) followed as by the increasing of NDII in Group1 ( $<<0.01$ ), the lesser use of sulfonylureas in Group2 ( $1.3 \pm 0.6$ vs. $2.9 \pm 1.5$ tablet/day, $p<0.01)$ as well as statistical significance in FSM $(70 \%$ vs. $6 \%$ in both Groups, $p<0.001)$ and SK (p<0.001). Whereas the scope of diabetes education was restricted to the capital Tbilisi, at the same time the another project "Training of people, suffering from diabetes by means of publishing of educational materials in Georgian and their distribution free of charge among of 4000 diabetic patients" sponsored by Open Society- Georgian Foundation was realized. As a result the better understanding of necessity of education and increasing of general knowledge of urgent topics of diabetes management were achieved. In conclusion: the present study suggests applicability and effectiveness of this strategy to create the integral system of patient care in Georgia.

\section{2}

HEALTH CARE FOR IDDM AND NIDDM PATIENTS-CENTRALIZED VERSUS NON-CENTRALIZED SYSTEMS (JEVIN)

R. Schiel, U.A. Müller, H. Janz and I.S. Ross. Univ. of Jena Med. School, Dept. of Int. Med. II, Jena, FRG and ${ }^{*}$ Univ. of Aberdeen, Dept. of Clin. Biochemistry, Aberdeen, UK

Since 1990 in Eastern European countries health care systems were decentralized or processes are ongoing. Increasingly, patients were treated not longer by diabetologists and specialized doctors, but by general practitioners (GP). The effect of this shift on quality of care is unclear. In 1989 a population-based trial of insulin-treated diabetic patients was started. Afterwards, the centralized diabetes care system was changed into a private one. Structured education programmes were established and health care is increasingly influenced by the St. Vincent Declaration. In $198983 \%$ $\langle n=190\rangle$ and in $199590 \%(n=244)$ of all insulin-treated diabetic patients aged $16-60$ years, living in the city of Jena $(100,247$ inhabitants) were studied. Results: For IDDM patients under specialized diabetes care $\mathrm{HbA} 1 \mathrm{c}$ was 1995 similar $(1.55 \pm 0.32 \mathrm{HbA} 1 \mathrm{c} /$ mean normal $\mathrm{HbA} 1 \mathrm{c}, \mathrm{n}=51 ; \mathrm{HPLC}$, Diamat $\left.^{\mathrm{R}}\right)$ to $1989(1.52 \pm 0.32, n=131, \mathrm{p}=0.4)$. It was higher for IDDM patients treated by GP's only $(1.71 \pm 0.37$, $n=80, p=0.0004)$. In NIDDM patients there were no differences $(1995: 1.78 \pm 0.43, n=113$ vs 1989:1.75 \pm 0.31 , $n=59, p=0.42$ ). No changes must be observed in respect to incidence of severe hypoglycemias, ketoacidoses, and the prevalence of retino-, nephro- and neuropathy (YOUNNG et al.) $(p<0.05)$. Quality of life (BRADLEY et al., IDDM:51.7 \pm 9.9 . NIDDM:50.2 \pm 10.6 ) was as good as in a control-group of healthy subjects $(47.5 \pm 7.8, n=53, p=0.46)$. Although since 1990 intervention was enforced, no improvement in diabetes care was registered. But, in respect of costly treatment of diabetic complications result from lower quality of care in a few years, the shift of health care systems must be modified.

\section{1}

IMPACT OF TYPE || DIABETES ON EMPLOYMENT STATUS AND WORK PRODUCTIVITY

AC Lloyd, Z Williams, LA Lacey and ML Keech, Glaxo Weilcome, Greenford, UK. Objective: The literature suggests that cardiovascular complications cause the most significant impact on type II diabetics' ability to work. This study assessed the impact of type II diabetes on employment status and work productivity in patients without serious diabetic complications. Methods: 284 type II diabetics previously treated with diet or oral agents with fasting capillary blood glucose stable in the range $7.0-15.0 \mathrm{mmol} / \mathrm{A}$ after wash-out were entered in an international, randomised, double-blind, placebocontrolled study of an investigational oral hypoglycaemic drug. Patients with serious cardiac, renal or hepatic disease were excluded. Data on employment status and productivity impact of diabetes were collected at baseline and after 4, 8, 12 and 16 weeks of therapy. Wage and employment figures for the general population came from the International Labour Office. Results: Patients had a mean age 60 years (range 35-84) and had a mean duration of diabetes of 5 years (range 0.3-32.0). At baseline $76(34 \%)$ were economically active, defined as either employed part or full-time (69) or looking for work (7), compared to $46 \%$ of the general population of the same age, gender and nationality. $101(45 \%)$ patients were retired and $14(6 \%)$ disabled. Of patients not in employment, $5(3 \%)$ gave diabetes as the reason they were not working. Using average UK wages ( $\$ 54.57$ per working day) as an example, productivity lost due to 5 patients unable to work because of diabetes cost $£ 21,828$ over 16 weeks. Had the same proportion of patients been economically active as the total population, expected productivity would have been over $£ 135,000$ higher over 16 weeks. Conclusions. The impact of type II diabetes on employment status and work productivity is substantial, even in patients without serious complications.

\section{3}

DISCREPANCY BETWEEN HBA1C AND GLUCOSE IN NIDDM M Bouma ${ }^{2}$, FEE van der Does ${ }^{2}$, JJJ de Sonnaville, $\mathrm{H}$ de Vries ${ }^{2.2}$, JH Dekker ${ }^{1.2}$, RJ Heine ${ }^{2.3}$ and JThM van Eijk ${ }^{1.2}$. 'Department of General Practice/Institute for Research in Extramural Medicinc/"Department of Interna Medicine, Vrije Universiteit, Amsterdam.

In general practice fasting plasma glucose (FPG) is commonly used to monitor glycaemic control in patients with NIDDM. The aim of this study was to assess the prevalence and the determinants of the occurrence of a diserepancy between HbAlc and FPG in NIDDM patients, not using insulin. Discrepancy between HbAlc and FPG was defined as FPG $\geq 7.8$ mmol// with HbAlc $<6.5 \%$ (ref. value: $<6.1 \%$ ) or FPG $<7.8 \mathrm{mmol} / \mathrm{l}$ with HbAlc $\geq 6.5 \%$. 1020 GP-treatcd NIDDM patients were included in the study: age 70 years (range $25-96) ; 43 \%$ malc; duration of diabctes 4.3 years (0)-45); treatment with diet only $37 \%$, sulphonyl urea drugs $60.7 \%$ and metformin $12.1 \%$. The prevalence of FPG $\geq 7.8 \mathrm{mmol} / \mathrm{f}$ with $\mathrm{HbAIC}$ $<6.5 \%$ was $7.9 \%$. After adjusting for age, scx, duration of diabetes and BMI the usage of sulphonylurea drugs remained associated with this: odds ratio (OR) 2.8 (95\% Confidence Interval (C) 1.6-5.0). The prevalence of $\mathrm{FPG}<7.8 \mathrm{mmol} / \mathrm{l}$ with $\mathrm{HbAlc} \geq 6.5 \%$ was $11.7 \%$. In a model with age, sex, duration of diabetes and BMI the association with sex was statistically significant: OR for female sex 1.6 ( $95 \%$ Cl 1.02- 2.7). After 4 to 13 weeks a second measurement of FPG and HbA1c was available in 6.58 of the 1020 patients. The prevalences of discrepancy then were $9.3 \%$ and $13.2 \%$, respectively. In only 2.9 and $2.7 \%$ of the patients the same kind of discrepancy was found, respectively. In conclusion, a discrepancy between HbAlc and FPG in non-insulin-treated NIDDM paticnts occurs frequently, especiatly in those using sulphonylurea drugs, and is difficult to predict from patient characteristics. Therefore, the assessment of glycacmic control, also in non-insulin-using NIDDM patients, requires measuring of both HbAlc and FPG when strict glycaemic control is desircd. 


\section{4}

NUTRI-EXPERT, A TELEMATIC SYSTEM FOR DIET SELF MONITORING : MULTICENTER EVALUATION IN OBESE SUBJECTS M.C. Turnin ${ }^{1}$, O. Bourgeois ${ }^{1}$, P. Martini ${ }^{1}$, G. Cathelineau ${ }^{2}$, A.M. Leguerrier ${ }^{3}$ S. Halimi ${ }^{4}$ D. Sandre-Banon ${ }^{5}$ V. Coliche ${ }^{6}$ M. Breux ${ }^{7}$, E. Verlet ${ }^{8}$, F. Labrousse 9 D. Bensoussan 10 J.L. Grenier 11 M.F. Poncet ${ }^{12}$ F. Tordjmann 13 J.M. Brun ${ }^{14}$, J.F. Blicklc ${ }^{15}$ C. Malléi ${ }^{16}$ C. Pène ${ }^{\prime}$, M.L. Solera ${ }^{1}$ D. Fabre ${ }^{1}$ and J.P. Tauber ${ }^{1}$ I CHU Rangueil-Toulouse, 2 Paris St Louis, 3 Rennes, 4 Grenoble, 5 Bondy, 6 Boulogne sur Mer, 7 Poitiers, 8 Dunkerque, 9 Lyon, 10 Vallencicnnes, 11 Roubaix, 12 Albi, 13 Corbeil, 14 Dijon, 15 Strasbourg, 16 Marscille (France)

Nutri-Expert, a telematic system for dict self monitoring and nutritional education was evaluard in a population of non diabetic obese subjects, in 16 french diabelology and nutrition centers. 557 patients were included, randomized in two groups : the first group had nutritional education provided during one year by a registered dictelician ( 7 consullations), whereas the second one had NutriExpert in addition. The Nutri-Expert igroup used the system at home, it was free of charge for 12 monlhs. 34 I subjecs $(92.3 \%$ women, $7.7 \% \mathrm{men}$ ) compleled the study. Evaluation criteria at $T_{0}$ and $T_{12}$ months were $B M I$, dieletical knowledge tests, and diet consumption reports. Centralized sampling of tipoprotein and insulinemia was carried out. Nutritional education permitted a significant improvement of $\mathrm{BMI}\left(\mathrm{T}_{0}=33.5 \pm 0.3\right.$ versus $\mathrm{T}_{12}=31.5 \pm 0.4 ; n=241$ $p<0.0001$ ), dietetical knowledge tests, lipoprolein profile and insulinemia. Concerning diet consumption reports : rate of carbohydrate increased significanlly : $41.6 \% \pm 0.5$ versus $43.3 \% \pm 0.5(n=199, p<0,01)$, lipids decreased significanlly: $39.9 \% \pm 0.5$ versus $35.7 \% \pm 0.5(n=199 ; \mathrm{p}<0.0001)$. The group using Nutri-Expert had significantly beter scores for the dictetical knowledge tests $(p<0.05)$. Dict consumption reports were improved but non significantly probably due to few patients in each group. Intensive management with dieletician or telematic tool Nutri-Experl, improve significantly BMI, dietetic knowledges, diclary habils and metabolic balance in obese patients.

\section{5}

EMPLOYMENT AND DIABETES IN AN ITALIAN POPULATION

M.P. Lanti, A. Bertoli* E. Latini, G.M. Pirone and EDIS Group

Istituto Italiano di Medicina Sociale, ${ }^{*}$ Cattedra di Medicina Interna Università di Roma Tor Vergata, Roma Italy.

The aim of the study was to evaluate the relationship between employment status and metabolic control in DM. A randomized sample of diabetics attending to italian "Diabetes Care Centres" was evaluated. Patient's data were recorded on a questionnaire administered by trained and standardized local medical team aimed to obtain demographic, socio-economic, anthropometric and clinical informations; particular attention was given to various aspects of patient's employment history. Anthropometric measures, the details of clinical conditions and the presence of complications were derived from medical records. Diabetes was classified according to WHO criteria. The job related physical activity was graded as sedentary, light, mean and tiring. Data were obtained from 5011 diabetics, 2053 of them were employed (1548 males and 505 females). Data are presented as mean $\pm S D$ and are age adjusted. Mean age of was $49 \pm 10$ in males and $43 \pm 12$ in females. After diagnosis the job had been modified because of DM in $13.8 \%$ of males and $10.6 \%$ of females; changes were undertaken by employer in $77.5 \%$ and $84.2 \%$ respectively in males and females and consisted in decreasing physical acitvity, job related stress, hours and shifts. About $1 / 3$ of diabetics (34.4\% of males and $29.9 \%$ of females) takes some meal on job; more than $50 \%$ can have their lunch at the canteen and $88.7 \%$ of males and $78.6 \%$ of females can choose their suitable foods.

Fasting plasma glucose was higher in unemployed compared to employed subjects in both males and females (ANOVA $F=8.75 ; D F 3,3391 ; p>0.001$ )

\begin{tabular}{|lllll|}
\hline & Employed & Females & Unemployed & Males \\
Fasting plasma & Males & Females \\
glucose & $168 \pm 56$ & $173 \pm 68$ & $181 \pm 64$ & $179 \pm 57$ \\
\hline
\end{tabular}

The blood glucose values are not correlated to the grade of physical activity in type 1 and type 2 DM and are not modified by the possibility to take the lunch in a suitable canteen. Our data indicate that DM induced a job modification only in a few subjects; moreover changes hed been proposed by employers rather than by patients suggesting that diabetic patients are not conditioned in their activities by DM. Furthermore our data suggest that employment status doesn't worsen metabolic control of diabetic data sug

EDIS (Employment and Diabetes Italian Study)

\section{7}

Initiation of insulin therapy in type 2 diabetic patients - Comparison of ambulatory versus in-patient care and education (AMBIT) R. Müller, U.A. Müller, A. Starrach, R. Schiel and V. Jörgens'. Dept for Internal Medicine II, Friedrich-Schiller-University Jena, Germany: Central Research Institute of Ambulatory Health Care for the Federal Republic of Germany, Cologne.

In a prospective controlled trial we compared ambulatory initiation of insulin therapy with the results of an inpatient programme. Methods: The same structured teaching and treatment programme (TTP) was used on an ambulatory basis and with inpatients. 70 Type 2 diabetic patients were recruited in 10 primary care practices (Group A). Starting insulin therapy the patients participated in the TTP $(5$ sessions of 1.5 hours within 4 weeks). The physicians and their assistants participated in a preparatory course and received teaching material (posters, pictures of nutrients, curriculum). The control group (group H) consisted of 70 type 2 diabetic in-patients matched for age, gender and diabetes duration participating in the same TTP on an inpatient basis. Patients were reevaparticipatig in the Preliminary results: Until now 40 patients were luated after one year. Prelliminary results. 9 ; $62 \pm 8$ years, duration reexamined in each group age $62 \pm 9 / 62 \pm 8$ years, duration
$9.5 \pm 4.8 / 9.5+5.3$ years, HbA1c initially $10.1 \pm 2.45 / 10.1 \pm 1.80 \%$ $9.5 \pm 4.8 / 9.5 \pm 5.3$ years, HbA1c initially $10.1 \pm 2.45 / 10.1 \pm 1.80 \%$
(mean normal value $5 \%$ ). BMl $30.0 \pm 8.1 / 27,5 \pm 4,1 \mathrm{~kg} / \mathrm{m}^{2}$ (all diff. $\mathrm{n.s}$.). (mean normal value $5 \%$ ), BMl $30.0 \pm 8.1 / 27,5 \pm 4,1 \mathrm{~kg} / \mathrm{m}^{2}$ (all diff. $\mathrm{n} . \mathrm{S}$.).
At reevaluation $\mathrm{HbA} 1 \mathrm{c}$ decreased significantly in both groups (A:$2.35 \pm 2.2 \%, \mathrm{H}:-1.85 \pm 1.95 \%$, diff.between groups n.s.) with only a little weight gain $(\mathrm{A}:+0.9 \mathrm{~kg} ; \mathrm{H}:+1.8 \mathrm{~kg})$. Compliance to blood glucose little weighting was high in both groups $(A): 95 \% ; H: 83 \%$ after one year, with $A \cdot 13 \pm 9 ; \mathrm{H}: 13+9$ tests/week). Severe hypoglycemia occurred only in 1 . in 1 pats groups. Summary: Ambulatory initiation of insulin therapy in type 2 diabetic patients is as effective as a TTP on an inpatient basis if a structured programme is used. The cost benefit analysis of the study demonstrates tremendeous savings in health care costs using the ambulatory TTP. Following the study, remuneration for the ambulatory programme was introduced by the health authorities (1000 DM for a course of with 4 patients). 
PS 40

\section{Gestational Diabetes}

768

\section{GESTATIONAL DIABETES MELLITUS IS ASSOCIATED WITH SHORT STATURE}

E.Anastasiou, Si.Grigorakis, M.Alevizaki, G.Philippou, M.Kyprianou, A Souvatzoglou. $1^{\text {st }}$ Endocrine Section, Alexandra Hospital, Athens GR.

Decreased body height has been reported to be associated with various degrees of abnormal glucose tolerance, most reports referring to middle aged populations. The aim of the present study was to evaluate body stature in women with gestational diabetes melitus (GDM) which represents a model of temporary impaired glucose tolerance in a young population, prone to develop diabetes later. We

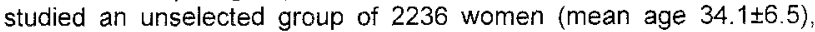
who were referred for screening for GDM from 1990 to 1995. Age height and reported pre-pregnancy weight were recorded. After an $100 \mathrm{~g}$ OGTT, 1533 women were classified as normal $(\mathrm{N}), 195$ women had one abnormal value (IGT), 508 women had GDM, according to the NDDG criteria. A further 38 pregnant women with preexisting type 2 DM (DM2) and 65 with preexisting type 1 DM (DM1) were studied Their heights were as follows (median, mean,SD) $\mathrm{N}: 161,160.9,6.2$, IGT: $160,159.9,5.9$, GDM: $159,158.8,6.5$, DM1:160, 159.7, 5.9, $D M 2: 158,158.1,5.0$. Significant differences from the $N$ group were: IGT $(p<0.05)$, GDM $(p<0.001)$ DM2 ( $p<0.01)$ (Mann-Whitney test); from the IGT group were: GDM $(p<0.05)$, DM2 $(p<0.05)$. The percentage of women whose height was lower than the median value of controls was $56 \%$ in 1 GT, $63 \%$ in GDM and $72 \%$ in DM2 [p $<0.001, x^{2}$ for trend]. Age and weight was significantly higher in GDM than in the $N$ group However, there was no correlation between age or weight and height. We conclude that women with GDM are shorter as a group and this difference becomes more striking as the impairment in glucose tolerance becomes more severe. Height in GDM should be unaffected by osteoporosis. The biological significance of this observation for the development of DM2 remains to be clarified

\section{9}

SCREENING AND TREATMENT OF GESTATIONAL DIABETES (G.D.M.): THE EXPERIENCE OF SEVEN YEARS.

M. Dolci*, G Bianchini^, G. Andreani*, M. Bongiomi*, P. Bay ${ }^{\circ}$, P. Migliorini ${ }^{\circ}$, $M$ Gattini" ${ }^{\circ}$ S. Barberi* and G. Saba*. * U.O. Medicina and ${ }^{\circ}$ U.O. Ostetricia e Ginecologia Ospedale di Massa, ^U.O. Medicina I - Ospedale di Carrara, Italy. We searched for G.D.M. (A.D.A. criteria modified by Carpenter) in 2214 pregnant women $50.03 \%$ of the total deliveries in Massa Hospital), from 1989 to 1995. G.D.M. was present in 64 women (2.89\%) and impaired gravidic glucose tolerance (I.G.G.T.) in $141(6.37 \%)$. The 1-hour after $50 \mathrm{mg}$-oral-glucose challenge test was pathologic in 436 women $(19.69 \%)$. Risk factors for G.D.M. were present in 823 women $(37.17 \%$ ). HbAlc was $5.25 \pm 0.60 \%$ in G.D.M. and $4.73 \pm 0.44 \%$ in all other pregnants ( $\mathrm{P}<0.0001)$. At delivery the drop-outs were $217(9.82 \%), 5$ of whom with G.D.M. (7.94\% of all G.D.M.). Insulin therapy was necessary for $55.93 \%$ of G.D.M. patients and for $11.45 \%$ of women with initial diagnosis of I.G.G.T. at a dosage ranging, at delivery, from 12 to $50 \mathrm{U} / \mathrm{die}$. All pregnants with G.D.M. performed diet-therapy and self-monitored blood-glucose levels (7 points/day/once a week). We started insulin-therapy if fasting glucose or postprandial glucose were $>80 \mathrm{mg} \%$ or $>120 \mathrm{mg} \%$, respectively. For the outcome of delivery we evaluated the percentage of spontaneous abortion, premature delivery, caesarean section, macrosomia, Apgar $\leq 7$, congenital malformations, perinatal mortality and morbidity, hypoglycaemia, hypocal caemia, hyperbilirubinaemia. "G.D.M." and "I.G.G.T. insulin-treated" women concluded pregnancy in every case: gravidic and perinatal complications, with the exception of a higher caesarean section rate, were the same of all other pregnant women; none had perinatal mortality. Macrosomia incidence was $4.36 \%$ in women with normal challenge test and without risk factors for G.D.M. and $5.48 \%$ in women with G.D.M. and I.G.G.T.-insulin-treated. Macrosomia was higher $(\mathrm{P}<0.0001)$ in pregnants with normal challenge test but with risk factors for G.D.M. (7.72\%), with pathologic challenge test (10.63\%) and with I.G.G.T. (13.27\%). The actual diagnostic criteria for G.D.M. probably lacks in prognostic sensibility. Proper screening and treatment for G.D.M. nomalise the incidence of pregnancy complications, included macrosomia.

\section{0}

THE VALUE OF FASTING AND POSLOAD BLOOD GLUCOSE AT OGTT FOR DIAGNOSIS OF GESTATIONAL DIABETES

M.B.Gomes. L.G.K. Aguiar

Discipline of Diabetes- State University of Riode Janeiro - Brazil

The N.D.D.G. criteria has been made according to pregnancy, although questions about its cutoff points (CP) have always been made. The aim of our study was to evaluate in high-risk population at what $\mathrm{CP}, \mathrm{FBG}$ at OGTT could predict the diagnesis of GD. During a one year period, 261 high-risk pregnant women with at least one known risk factor for GD were screened with a 50gr OGTT. None of them had FBG upper $105 \mathrm{mg} / \mathrm{dL}$ before this test. One hundred had a positive screening test, and 77 performed the OGTT according to N.D.D.G. criteria. This group had only higher maternal ages than the one who have not performed the OGTT $(35,2 \pm 5,6$ vs $32,0 \pm 5,4 y$., $p<0,05)$. The incidence of GDM was $9,6 \%$ $95 \% \mathrm{CI}[6,3-13,8]$. In a logistic regression model, we observed that only a positive past history of $\mathrm{GD}(\mathrm{OR}=15.7, \mathrm{p}=0,03)$ and a high body mass index $(O R=1,57, p<0,01)$ independently predicted GD. Considering all OGTT $\left(\mathbf{n}=77\right.$ ), we noticed an excellent correlation between $2^{\text {nd }}$ hour at OGTT and tha area under the curve for glucose $\left(r^{2}=0,89, p<0,001\right)$. The ROC curves for FBG which better predicted the diagnosis of GD was the one associated with altered blood glucoses (BG) on the $1^{\text {st }}$ and $2^{\text {nd }}$ hours at OGTT(area $=0,7743, \mathrm{ep}=0,018$ ), with a cut-off point at $93 \mathrm{mg} / \mathrm{dL}$ (sensitivity $=81,3 \%$, specificity $=74,4 \%$ ). A good reproducibility have been found between FBG at $93 \mathrm{mg} / \mathrm{dL}$ and the $1^{\text {sl }}$ and $2^{\text {nd }}$ hours $B G$ during OGTT $(\kappa=0,48)$. Considering only GD group $(n=25), 68 \%$ had FBG under the NDDG's $\mathrm{CP}$, and $64 \%$ had been diagnosed with altered $\mathrm{BG}$ on $1^{1^{\text {st }}}$ and $2^{\text {nd }}$ hours at OGTT. Our data suggest that the posload BG of NDDG criteria, mainly at $1^{\text {st }}$ and $2^{\text {thd }}$ hours, have been the most important for diagnosing GD and we believe that the CP for FBG at OGTT could be smaller than actually recomended.
771

DOES ONE HOUR PLASMA GLUCOSE PREDICT GESTATIONAI DIABETES AND IMPAIRED GLUCOSE TOLERANCE?

J.M.D.C. Pousada, A.J. Reichelt, E.R. Spichler, T. Yamashita, L. Branchtein, M.M. Teixeira and M.I. Schmidt for the EBDG Study Group. UFRGS - Porto Alegre - Brasil.

The World Health Organization recommends the 2 hour $75 \mathrm{~g}$ oral glucose tolerance test (OGTT) as a screening and as a diagnostic tool for gestational diabetes mellitus (GDM) and impaired glucose tolerance (GIGT). The purpose of this study was to evaluate the validity of using the 1 h glucose as a screen to decide who should proceed to a full $2 \mathrm{~h} 75 \mathrm{~g}$ OGTT. Results on 4031 consecutive oGTTs performed under standard conditions between weeks 24 and 28 of pregnancy were analyzed. Sensitivity (Se) and specificity (Sp) of various cutpoints for the lh glucose in correctly identifying GDM (2h glucose $\geq 11.1 \mathrm{mmol} / \mathrm{L})$ and GIGT (2h glucose $\geq 7.8$ mol/I) were established. Plots of receiver-operator characteristic curves allowed the evaluation of various screening strategies. Maximizing $\mathrm{se}$ without undue loss of $\mathrm{sp}$ led to a cutpoint of 8.4 mmol/L for GDM (I3 of subjects screen positive, $S e=1008, S p=878)$ and of $6.8 \mathrm{mmol} / \mathrm{L}$ for GIGT $(40 \%$ screen positive, Se=96\%, $\mathrm{sp}=648)$. A standard cutpoint (7.8 mmol/L; 218 screen positive) showed se and Sp for GIGT of $86^{\circ}$ and 848 , respectively. In conclusion, the in glucose is a valid and useful screening test during pregnancy, reducing considerably the number of women requiring the full 2 hour test. 
772

GESTATIONAL DIABETES: IMPACT OF AN ADDITIONAL O'SULLIVAN TEST AT 30th-34th GESTATIONAL WEEK. J.Vidal, L.Flores, P.Cristobal*, S.Logwin, F.Carmona*, V.Cararach*, I.Levy and R. Gomis. Endocrinology and Diabetes Unit, *obstetrics and Gynecology Unit. Hospital Clínic Universitari.Barcelona. Spain.

Despite widespread screening programs and optimal metabolic control, GDM is associated with an increased prevalence of adverse outcomes. The aim of our study was to evaluate the impact of the addition of an O'Sullivan test at 30th-34th gestational week to the 3rd International Workshop recommendations for GDM screening, on GDM and gestation adverse outcomes incidence. All women ( $n=704)$ refered to our Unit between june 1994 and june 1995 for GDM screening were eligible for the study. A 100-g OGTT was performed in women presenting with plasma glucose $>7.8 \mathrm{mmol} / \mathrm{l}$ at any of the O'Sullivan tests. NDDG criteria were applied for GDM diagnosis. Gestational age at delivery, mode of delivery, neonatal birtbweight, Apgar score after 1 and 5 minutes, umbilical artery $\mathrm{pH}$ and requirement of neonatal admission to the pediatric unit were recorded. $351(49.9 \%)$ women completed the screening schedule. $196(27.8 \%)$ enterel the program just at the 30th34 th week test, while $157(22.3 \%)$ had the 24th-28th weeks test made but did not complete the schedule. Addition of an O'Sullivan test at 30th-34th gestational week increased GDM prevalence from $4.83 \%$ to $9.23 \%$ of the studied population. The incidence of prematurity, macrossomia, low-birth-weight for gestational age, Apgar score $<7$, foetal sufferance and rate of admission at the pediatric unit were not significantly different when patients with 24 th-28th negative screening who did not complete the screening program were compared to those who completed the schedule with a negative result. Patients diagnosed of GDM due to the additional test (n=31, gestational age at diagrosis $32.1 \pm 2.0$ weeks) required insulin treatment less often $(p<0.05)$ than those diagnosed at $24-28$ weeks. No significant differences in any of the studied parameters were found when those patients were compared either to GDM diagnosed at 24-28 woeks or patients who completed the screnning program with a negative result. Conclusion: GDM is more often diagnosed when an additonal O'Sullivan test at 30th-34th gestational week is performed. In our study, the additional test does not appear to decrease the incidence of adverse outcomes.

\section{3}

LOW PREVALENCE OF GAD65 AND IA2 AUTOANTIBODIES AMONG ICA POSITIVE GESTATIONAL DIABETES M. Puig-Domingo ${ }^{1}$, D. Mauricio, J. Morales ${ }^{1}$. R Corcoy ${ }^{1}$, S. Piquer', V. Lampasona ${ }^{2}$, E Bonifacio and A de Leiva ' Endocrinology Dept. Hospital Sant Pau, Barcelona, Spain. ${ }^{2}$ Laboratory of Medicine and ${ }^{3}$ Dept of Internal Medicine, Istituto Scientifico San Raffaele, Milan, Italy

Gestational diabetes (GD) is a heterogeneous disorder which may include individuals carrying pre-type 1 or pre-type 2 diabetes risk. Different frequencies of islet cell antibody (ICA) positivity have been described in GD. We aimed to enhance the characterisation of islet humoral autoimmunity by studying a group a 40 ICA positive GD women (ICA+ GDW) in comparison with 50 ICA negative GD women (ICA-GDW), and a control group of 30 pregnant women without $\mathrm{GD}$. ICA were detected by indirect immunofluorescence onto human pancreas, while GAD65 and IA2 antibodies were performed by using a combined radiobinding assay. ICA titres in GD were predominantly low, with $80 \%$ being below $20 \mathrm{JDF}$ units. GAD65-Ab frequency did not differ between ICA+ and ICA- GDW ( $5 \%$ vs. $6 \%$ ), while $0 \%$ was found among control pregnancies. IA2-Abs were found in $8.6 \%$ of ICA+ GDW and in none of the ICA- GDW. Non-diabetic pregnancies showed $0 \%$ of IA2-Abs. Among 14 ICA+ GDW in which follow-up was possible, 4 developed glucose intolerance or overt diabetes short term post-partum; none was GAD65 or IA2 Abs positive. We conclude that GAD65 and IA2 do not contribute significantly to ICA reactivity in ICA+ GDW and do not seem to be a valuable tool for type 1 diabetes prediction in this particular group of prediabetic subjects shortly after pregnancy.

\section{4}

CARDIOVASCULAR RISK FACTORS IN WOMEN WITH GESTATIONAL DIABETES AT MID-TERM FOLLOW-UP M. Albareda, A. Caballero, G. Badell, R. Corcoy and $A$. de Leiva. Hospital de Sant Pau, Barcelona.

Women with gestational diabetes (GD) are known to have a high prevalence of diabetes mellitus (DM) and other cardiovascular risk factors (RF) later in 1 ife. We aimed to analyze the prevalence of DM and RF in a population of 156 women of caucasian ethnicity with former GD. The study protocol included the assessment of weight, height, colesterol, triglycerides (TG), HDL-colesterol, blood pressure and smoking habit and the performance of a 75 gr oral glucose tolerance test in women without known DM. Dyslipemia was defined as colesterol $>=6.2 \mathrm{mmol} / 1$, TG $>=2.25$ mol/l, HDL-colesterol $=<0.9 \mathrm{mmol} / 1$ or treatment for known dyslipemia. Hypertension was defined as a blood pressure $>=140 / 90 \mathrm{~mm} \mathrm{Hg}$ or treatment for known hypertension. At a mean follow-up of $6.82 \pm$ 2.03 years $8.33 \%$ women were diabetic and $11.54 \%$ had abnormal glucose tolerance. Body mass index was $>=25 \mathrm{~kg} / \mathrm{m} 2$ in $45.39 \%$, dyslipemia was present in $11.11 \%$, hypertension in $18.94 \%$ and $28.47 \%$ were smokers. There have been 49 additional pregnancies after the index one, and GD had been diagnosed in $31(63.3 \%)$. Overali, $73.7 \%$ of women had at least one RF. In conclusion, in these population of former GD women, the prevalence of disturbed glucose tolerance is lower than in other series but the RF prevalence is very high.

\section{5}

\section{ASYMPTOMATIC BACTERIURIA IN GESTATIONAL DIABETES}

M. Zloczower, I. Zaidise, Y. Kanter, A. Drugan, P. Jakobi and J. Itskovitz-Eldor Dept. Ob/Gyn and Diabetes/Metab. Unit, Rambam Medical Center, Faculty of Medicine - Technion, Haifa, Israel.

Because about $15 \%$ of women with Asymptomatic Bacteriuria (ASB) develop overt urinary tract infections and/or pyelonephrits, our study was design to evaluate the yield of monthly urinary cultures in the detection of ASB in pregnancies complicated by Gestational Diabetes (GDM). Urinary cultures were performed in 221 pregnant affected with GDM, and in 238 non diabetic pregnant women who served as controls. Both groups were devoid of urinary symptoms at time of culture. All women had negative urine cultures at the first trimester. The prevalence of positive cultures was compared by chi square analysis.

Positive urinary cultures were observed in $33(15 \%)$ diabetic patients and in $17(7.1 \%)$ non diabetic pregnancies $(\mathrm{p}<0.01)$. Following appropriate antibiotic treatment, recurrent positive cultures were found in $4(12 \%)$ pregnancies in the study group and in $1(6 \%)$ of the controls.

We conclude that ASB is more common in pregnancies complicated by GDM than in non diabetic gestations. Moreover, recurrent ASB appears to be more common in diabetic pregnancies. Based on these results we suggest that monthly urinary cultures should be pursued in pregnancies affected by GDM. 
776

INCREASED CONCENTRATIONS OF SOLUBLE E-SELECTIN AND V-CAM IN GESTATIONAL DIABETES

O. Wagner, A. Kautzky-Wilier, P. Fasching and W. WaldhäusI, Deparment of Medicine III, Division of Endocrinology \& Metabolism and Department of Medical and Chemical Laboratory Diagnostics, University of Vienna, Austria

Recent research has demonstrated increased concentrations of soluble adhesion molecules (SAM) in NIDDM and a relation to glycemic metabolic control has been postulated. It is, however, unknown, if elevated sAM are only an epiphenomenon of NIDDM or it they might be involved in the sequelae of this disease. To address this question we analyzed serum concentrations of circulating intercellular adhesion molecule-1 (cICAM-1), vascular cell adhesion molecule-1 (cVCAM-1), and endothelial leukocyte adhesion molecule-1(E-selectin) in pregnant women with gestational diabetes (GDM), which can serve as a model for early NIDDM, in comparison to pregnant women with normal glucose tolerance (NGT) and to non-pregnant control women (CS) at fasting and after 180 min following an oral glucose load (OGTT: 100g). The OGTT were repeated 12 weeks after delivery. There were no differences between the fasting and the stimulated levels for SAM in each group arguing against an acute effect of hyperglycemia on SAM. As to basal values ICAM-1 did not differ between groups, while E-selectin and VCAM- 1 were elevated in GDM and NGT vs. CS (E-selectin: $51 \pm 8$ and $56 \pm 4$ vs $26+5 \mathrm{ng} / \mathrm{ml}$; VCAM: $594 \pm 75$ and $700 \pm 58$ vs $379 \pm 10 \mathrm{ng} / \mathrm{ml}$, p<0.01). Following delivery and after restoration of normal glucose tolerance E-selectin and VCAM remained increased vs. CS $(\mathrm{p}<0.01)$ in GDM, while they fell to normal in NGT $(p<0.05)$. Circulating AM were positively related to the total area under the curve (AUC) of glucose within abnormal groups ( $p<0.05, \mathrm{r}=0.5$ ). In GDM E-selectin was additionally related to HbA le values and fasting glucose $(\mathrm{p}<0.1, \mathrm{r}=0.9\}$ as weil as to AUC for insulin $(p<0.05, r=0.7)$. Persistingly raised levels of E-selectin and VCAM characterize GDM even in the early postpartum period i 3 months after delivery). From this it appears that 1. ) raised SAM are related 10 chronic gycentic homeostasis and 2.) increased E-selectin and VCAM mighn not only be an epiphenomenon of NIDDM but carly marker of a prediabetic state.

\section{7}

Maternal Obesity - an underestimated Risk Factor for Newborn Macrosomia

CLAUDIA LUDWIG,PETER FASCHING,ULRIKE DI PIETRO,BARBARA SCHNEIDER;WERNER WALDHÄUSL, Vienna, Austria

The aim of this prospective study was to determine the incidence of neonatal macrosomia and its influencing factors in 171 gravidas screened for gestational diabetes (GDM). According to the results of a 2 $h$ oral glucose tolerance test with $75 \mathrm{~g}$ glucose (normal: fasting $<100$ $\mathrm{mg} / \mathrm{dl}, 1-\mathrm{h}:<180 \mathrm{mg} / \mathrm{dl}, 2-\mathrm{h}:<140 \mathrm{mg} / \mathrm{dl}$ ), hemoglobin $A_{\mathrm{tc}}$ (normal: 3.5 $5.8 \%$ ) and prepregnancy body mass index (pBMl; normal $18-24 \mathrm{~kg} / \mathrm{m}^{2}$ ) gravidas were grouped $A(N=48)$ as healthy, nonobese gravidas (OGTT, $\mathrm{HbA}_{1 \mathrm{c}}$ : normal,pBMl $\left.\leq 24 \mathrm{~kg} / \mathrm{m}^{2}\right), \mathrm{B}(\mathrm{N}=54)$ as healthy obese gravidas ( $O G T T, H b A_{1 c}$ : normal,pBMl $\geq 25 \mathrm{~kg} / \mathrm{m}^{2}$ ) and $\mathrm{C}(\mathrm{N}=69)$ as gravidas with GDM (impaired oGTT and/or elevated $\mathrm{HbA}_{1 \mathrm{c}}$ and $\mathrm{pBMI}$ between 17 and $49 \mathrm{~kg} / \mathrm{m}^{2}$ ). The obtained data demonstrated that women with elevated prepregnancy body mass index but without any clinical evidence of impaired glucose metabolism have a significant higher risk to give birth to macrosomic infants than nonobese healthy gravidas and women with treated GDM. In the obese gravidas $50 \%$ of the newborns were macrosomic (birthweight $>4000 \mathrm{~g}, 90^{\text {th }}$ percentile $/ 40^{\text {th }}$ gestational week) compared to $8 \%$ in nonobese, healthy gravidas and $31 \%$ in gravidas with treated GDM (A vs B,C:p<0.0001, B vs C: $p<0.0003$ ). In evaluation by multiple regression analysis $\mathrm{pBMl}$ appeared as the only independent predictor for newborn macrosomia in obese gravidas $(\mathrm{p}<0.0002)$.

Therefore we conclude that maternal obesity is an important risk factor and independent predictor for newborn macrosomia.

\section{8}

Gestational Diabetes and Neonatal Outcome: Effects of Prepregnancy BMI.

G.Di Cianni, L.Benzi, L.Volpe, P.Orsini, L.Marselli and R.Navalesi Department of Metabolic Disease, University of Pisa (Italy).

This study investigates the clinical characteristics of gestational diabetes mellitus (GDM) and neonatal outcome in relation to prepregnancy body mass index (BMI). Data were obtained using a computerized data system for all deliveries (n.4171) that occurred at the Departments of Obstetrics and Gynecology of the University of Pisa (Italy) from Jan.1, 1987 through to Dec. 31, 1992. 93 women with GDM (prevalence 2.3\%) were found and divided into three groups in relation to their pre-pregnancy BMI: normalweight (Nw; BMI $<25 \mathrm{Kg} / \mathrm{m}^{2}$, n.57, mean age $33.5 \pm 5.8$ years), overweight (Ow; BMI 25-30, n.16, 31.7 \pm 7.2 yrs.) and obese (Ob; BMI >30, n.20, 32.1 \pm 4.5 yrs). 110 control subjects (CG), matched for age, pre-pregnancy BMI and weight gain during pregnancy (WG) were also evaluated. The diagnosis of GDM before $24^{\text {th }}$ week was noted in $66.6 \% \mathrm{Ow}, 82 \% \mathrm{Ob}$ and $49 \% \mathrm{Nw}$ ( $p>0.02$ ). During the last 10 weeks of gestation Nw-GDM had mean fasting glucose of $73.15 \pm 17 \mathrm{mg} \%$, (Ow $85.6 \pm 8 \mathrm{mg} \%$ and $\mathrm{Ob} 83.7 \pm 24.1 \mathrm{mg} \% ; \mathrm{p}<0.02$ vs $\mathrm{Nw}$ ). HbAlc values were superimposable in the three groups. Insulin-therapy was necessary in $77 \%$ of Nw-GDM, $91 \%$ of Ow-GDM and $86 \%$ of Ob-GDM ( $1<0.02)$. WG was similar in all groups (Nw-GDM: $11.3 \pm 3.6$ $\mathrm{Kg}$; Nw-CG: $11.7 \pm 2 \mathrm{Kg}$; Ow-GDM $12.3 \pm 4.2 \mathrm{Kg}$; Ow-CG $13 \pm 4 \mathrm{Kg}$; Ob-GDM $10.1 \pm 6.6 \mathrm{Kg}$; Ob-CG $11.9 \pm 4.1 \mathrm{~kg}$ ). Time of delivery resulted earlier in Ow-GDM $\left(37.3^{\text {th }}\right.$ week) and Ob-GDM $\left(38.7 \pm 1.8^{\text {th }}\right)$ than Nw-GDM $\left(39 \pm 2^{\text {th }}\right)$. This was not confirmed in CG. Preterm deliveries ( $\left(38^{\text {th }}\right.$ week) showed a trend to increase from $\mathrm{Nw}$ to $\mathrm{Ow}$ and $\mathrm{Ob}$ in GDM, but not in CG. The frequency of cesarean sections (CS) was higher in GDM than in CG (44.9 vs 12.9, p>0.01). Ow-GDM and Ob-GDM were more exposed to the CS than Nw-GDM (Nw 35.8, Ow 57.4\% and OB 76.9\%; $\mathrm{p}<0.01)$. In CG the incidence of CS was similar in the three BMI categories. Prevalence of neonatal macrosomia (LGA) was correlated ( $>0.01$ ) with prepregnancy BMI both in GDM (Nw: 41.5\%, Ow: 57.4\%, 0b: 76.92) and CG ((Nw 6.8\%, Ow $10.2 \%, \mathrm{Ob} 25.9 \%$ ); nevertheless in each BMI category prevalence of LGA was significantly higher in GDM.The WG during pregnancy was not associated with LGA neither in GDM nor in CG. This study shows that the degree of overweight is related to an earlier diagnosis of GDM and to higher incidence of insulin treatment. Only in GDM the preterm deliveries and the CS are related to prepregnancy BMI, probably related to patterns of physician decision making. Moreover prepregnancy BMI is more predictive of macrosomia than WG specially in women suffering from GDM.

\section{9}

Impact of Gestational Diabetes on The Mother and her Offspring E. Wender-Ożegowska, E. Biegańska, R. Biczysko

Institute of Obst. and Gymecology, Medical Academy of Science, PoznaniPoland

The aim of our study was to evaluate the effect of Gestational Diabetes (GDM) on the course of pregnancy, the mothers condition and fetal outcome.

Thwo groups of pregrant women with GDM, were analysed: the G1-group, consisting of 136 patients, treated with diet only, and the G2-group, consisting of 85 patients requiring insul in treatment. GDM was recognised_according to WHO criteria using the $75 \mathrm{~g}$ OGTT. GDM in the G1 was recognised on the average in the $30^{\mathrm{h}} \pm 1$ week and in the $G 2$ in the $27 \pm 1$ ( $\left.p<0,005\right)$ week. We established a signifi-cant $(0<0,0005)$ age difference telween of the analysed groups: $G 1$ (28ะ6yrs) and G2 (33 $\pm 7 y$ rs). These two groups differed also with respect to the efficiency of glycemia control ( $\mathrm{G} 1-87 \pm 13 \mathrm{mg} / \mathrm{dl} ; \mathrm{G} 2-109 \pm 28 \mathrm{mg} / \mathrm{dl}$ ) in spite of the comparable HibA. concentrations found at the time of GDM recognition (G1-6.18\%, G2 $-6,85 \%, 0>0,05)$. The mean gestation time was in $G 139$ weeeks \pm 3 days and in group $G 237$ weeks 2 days $(p<0,05)$. Pregnancy induced hypertension was found in $17(12 \%)$ of G1 diabeteics and in $26(31 \%)$ from the G2. 14 patients from G1 group (10\%) and $17(20 \%)$ trom the G2 had to be treated because of imminent preterm delivery.

\begin{tabular}{|c|r|r|c|c|c|}
\hline Fetal outcome & $G 1 \quad \mathrm{~N}=136$ & $\mathrm{G} 2 \mathrm{~N}=85$ & Fetal outcome & $G 1 \mathrm{~N}=136$ & $G 2 \mathrm{~N}=85$ \\
\hline Prematurity & $24(18 \%)$ & $23(27 \%)$ & LGA $>90 \%$ & $17(25 \%)$ & $28(31 \%)$ \\
\hline SGA $<10 \%$ & $6(4,4 \%)$ & $3(3,5 \%)$ & Hypoglycemia & $21(15 \%)$ & $20(24 \%)$ \\
\hline Hiperbilirubin. & $26(19 \%)$ & $26(31 \%)$ & RDS & $4(2,9 \%)$ & $4(4,7 \%)$ \\
\hline Cong.malform & $1(0,7 \%)$ & $3(3,5 \%)$ & Birth trauma & $2(1,5 \%)$ & $3(3,5 \%)$ \\
\hline Ces. section & $57(42 \%)$ & $51(60 \%)$ & Mortality & $1(0,7 \%)$ & $1(1,2 \%)$ \\
\hline
\end{tabular}

78 subjects out of 163 pregnancies delivered between $1990-93$ were analysed under the scope of postgestation carbohydrate metabolism ( 1 year after delivery). According to the $75 \mathrm{~g}$ CIGTT results, $28 \%$ (22) of all patients were diagnosed as diabetics $2(4,5 \%$ ) from the 61 , and 20 $(60 \%)$ from the G2, and $14(18 \%)$ as having IGT $8(18 \%)$ from the G1 group and $6(16 \%)$ from the $\mathrm{G} 2$.

We conclude that GDM is a serious risk factor for maternal and fetal complications during pregnancy as well as for the development of $D M$, especially among women who had to be treated with insulin during pregnancy. 
GESTATIONAL DIABETES AND NITROSOAMINES IN PARENTS INDUCE DISTURBANCES OF REPRODUCTIVE SYSTEM IN MALE OFFSPRING

G. Brizgalova, L. Sergienko, V. Poltorack, J. Lementa and T. Bondarenko. Ukrainian Researsh Institute of Endocrine Diseases Pharmacotherapy, Kharkov, Ukraine

The aim of the study was to estimate the influence of the increased consumption of nitrosoamines (ICN) by parents and of gestational diabetes (GD) in mothers on the functional state of the reproductive system organs (testes, prostate) and reaction of them to streptozotocin (STZ) in male offspring. Parental mice $(\mathrm{CBA} \times \mathrm{C} 57 \mathrm{BL} / 6 \mathrm{~J}) \mathrm{F}_{1}$ received $0.25 \%$ solution of sodium nitrite and amidopyrine in water ad libitum the whole experiment. From the 46th day of the study's beginning female mice were treated with $\mathrm{STZ}(40 \mathrm{mg} / \mathrm{kg} / \mathrm{day} \times 5$ days i.p.) and 15 days after the $1 \mathrm{st}$ STZ injection mice were coupled. The male offspring were killed at 3 months of age. To detect the sensibility to STZ of the testes and prostate the offspring were treated a single injection nondiabetogenic dose of STZ ( $30 \mathrm{mg} / \mathrm{kg} \mathrm{b}$ w i.p.) I month before killing. The mice which mothers received ICN+STZ and fathers-ICN had the inhibition of testicular spermatogenesis, the decrease of Leydig cells and the fall of plasma testosterone levels $(p<0.01$ vs controls). The prostate $5 \alpha$-reductase activity was decreased in offspring from $\mathrm{ICN}+\mathrm{STZ}$ mothers or mothers-ICN+STZ and fathers-ICN ( $<<0.05$ vs controls). Prostates of the same mice had loci of the hypotrophy of secretory epithelium and the connective tissue expansion. We conclude that gestational diabetes, against a background of ICN in parents, predisposes to the development of testes-prostate axis hypofunction in offspring, connected with the disturbances of sex hormones production and metabolism.

\section{PS 41}

\section{Diabetes and Pregnancy}

781

ANOMALIES OF FOETAL DEVELOPMENT IN DIABETIC GK RATS

F. Malaisse-Lagae, C. Vanhoutte, F. Rypens*, S. Louryan* and W.J. Malaisse. Laboratory of Experimental Medicine and *Laboratory of Human Anatomy and Embryology, Brussels Free University, Brussels, Belgitrm.

Adult GK rats are currently used as an animal model of non-insulindependent diabetes. The present study draws attention to anomalies of foetal development in these diabetic rats. Between day 15 and day 20 after identification of a positive sperm plug, the GK rats gained less weight $(26 \pm 3 \mathrm{~g})$ than control animals $(45 \pm 2 \mathrm{~g})$. The number of conceptus in each litter was not significantily different in control animals $(10.6 \pm 0.6)$ and GK rats $(12.5 \pm 0.9)$. The incidence of abortive foetal development, however, averaged $39.7 \pm 9.1 \%$ in GK rats, as distinct from only $5.6 \pm 0.2 \%$ in control animals. The placental weight in living foetuses was slightly lower in GK rats $(351 \pm 8 \mathrm{mg})$ than control rats $(372 \pm 5 \mathrm{mg})$. The crown-rump length was identical in the foetus of control and diabetic mothers $(25.8 \pm 0.3 \mathrm{~mm})$. The number of ossification points in the lumbo-sacral spine, pelvic girdle and anterior and posterior limbs was also significantly lower in foetuses of GK rats, as compared to control animals. These anomalies could not be blamed to a lower plasma insulin concentration in GK than control animals, whether before or during (days 15 and 20) pregnancy. On the contrary, the plasma insulin concentration was higher in GK than control rats, at least before pregnancy. Moreover, in both control and GK rats, the insulinogenic index was increased during pregnancy. These findings indicate that GK rats represent a new model for the study of diabetesrelated foetal anomalies, their pathogeny and prevention.
782

ANTEPARTUM PITUITARY INFARCTION: A RARE CAUSE OF INSULIN HYPERSENSITIVITY IN THE THIRD TRIMESTER TE T WEST AND J A EAVES, PRINCESS ROYAL HOSPITAL, TELFORD TF6 6TF

Antepartum pituitary infarction (API) is a rare complication of pregnancy occurring in the third trimester. Almost all of the small number of cases reported have been women with longstanding Type I diabetes though not necessarily with overt microangiopathy. The condition is to be contrasted with other causes of pregnancyassociated hypopituitarism viz Sheehan's syndrome and lymphocytic hypophysitis both of which occur post partum. We present a patient with API: a 33 year old woman with a 23 year history of Type I diabetes and without micro angiopathy progressed uneventfully in her second pregnancy until 34 weeks. Glycaemic control had been excellent with glycated proteins remaining within normal ranges. Sudden onset of unprecedented and impressive headache suggested the possibility of a subarachnoid haemorrhage but CT brain scan was negative. Over the succeeding three weeks she experienced multiple hypoglycaemic episodes (up to 8 per day), and falling insulin requirements and became extremely lethargic. Investigation showed evidence of ACTH and prolactin deficiencies with normal TSH secretion. She was started on cortisol replacement with dramatic symptomatic improvement and subsequently the insulin requirements rose to their former level. Following successful delivery by caesarean section because of proteinuria and decreased fetal movements she failed to lactate. More detailed endocrine investigation has shown persistent ACTH and PRL deficiency but normal TSH, GH and gonadotrophin secretion with no evidence of DI. MRI of the pituitary has shown no abnormality. All of the small number of reported cases of API have been ACTH deficient and some have also been PRL or GH deficient also. Failure of TSH secretion appears rare API is a rare cause for abruptly declining insulin requirements in third trimester but should be considered especially in women with Type I diabetes of over 20 years duration. 
783

PREGNANCY OUTCOMES IN MOTHERS WITH PREGESTATIONAL DIABETES (PGD): A POPULATION-BASED STUDY IN NORTHRHINE (GERMANY) R. Kimmerle, R. von Kries ${ }^{1}$, M. Berger. Department of metabolic diseases and nutrition, dep. of children's diseases', Heinrich-Heine-University, Düsseldorf With specialized care and normoglycemia throughout pregnancy diabetesrelated poor pregnancy outcomes can be avoided. To achieve "normal" pregnancy outcomes in pregestional diabetic women on a population-basis is one of the goals of the St. Vincent declaration. The aim of the study was a) to estimate the proportion of pregnancies with PGD in Germany; b) to compare the outcome of these pregnancies to those in nondiabetic mothers; $c$ ) to identify risk markers for poor pregnancy outcomes in PDG. Methods: Data of the "Perinatatalerhebung Northrhine", an ongoing quality assurance project of pre- and perinatal care in the South Western part of Northrhine-Westphalia from 1988-1993 were analysed. During the period $93-100 \%$ of all officially registered births (annual birthrate $\approx 100000$ ) were included, i.e. prepregnancy conditions, pregnancy complications and outcome were documented by the obstetrician in a standardized multiple choice questionaire. Glycemic control was not documented. Results: a) There were 2402 births in mothers with PGD $(0.4 \%$ of all births $)$. b) The proportion of poor pregnancy outcomes in women with PGD and their relative risks (RR) compared to nondiabetic mothers was for perinatal mortality $2.7 \%$ (RR 4.4 (95 $\% \mathrm{Cl} 3.4-5.6)$; for prematurity $21.1 \%$ (RR 3.1, $95 \% \mathrm{Cl} 2.8-3.4)$; for perinatal asphyxia $3.1 \%$ (RR: $2.3,95 \% \mathrm{Cl} 1.8-2.9$ ); for macrosomia $27 \%$ (RR 3.8, $95 \%$ Cl 3.5-4.2), for malformations $2.1 \%(\mathrm{RR} 1.4,95 \% \mathrm{Cl} 1.1-1.8)$. The main risk factors for perinatal mortality for children of diabetic mothers was substandard use of the standard gynecological prenatal care system $(<1$ visit/month) and malformations. Conclusions: The prevalence of births from mothers with PGD and their pregnancy outcomes were similar to the figures in other Western countries. The standard for nondiabetic mothers has not been reached yet. Further improvements will be achieved if all diabetic women are convinced to use the standard and specialized prenatal care facilities throughout pregnancy.

\section{5}

INSULIN SENSITIVITY AND INSULIN SECRETION IN LEAN AND OBESE, NORMAL PREGNANT WOMEN.

A.Fagulha ${ }^{1}$, M.Carvalheiro', I.Fagulha' ${ }^{2}$, L.Gomes ${ }^{1}$, S.Paiva ${ }^{4}$, E.Marta ${ }^{2}$, E.Sobral ${ }^{2}$, F.Leitão ${ }^{3}$, M.L.Pinto ${ }^{3}$, M.M.A.Ruas ${ }^{1}$ and T.Buchanan ${ }^{4}{ }^{1}$ Dept of Endocrinology, ${ }^{2}$ Obstetric Clinic and ${ }^{3}$ Clinical Pathology, University Hospital Coimbra, Portugal, "University of Southern California, LoS Angeles, USA

We studied insulin secretion and insulin sensitivity in normal pregnant women in comparison with non-pregnant normal women and the effects of obesity in normal pregnancy. We determined insulin sensitivity (SI), Glucose effectiveness $(\mathrm{Sg})$ and insulin secretion in 26 normal pregnant women (Group I) and in 27 non-pregnant women (Group II) with similar

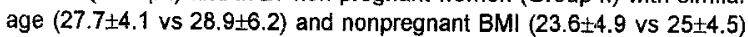
). In Group I there were, 19 lean and 7 obese (BMI $<27$ and $\geq 27$, respectively). The women were submitted in the 3rd trimester of pregnancy or in the luteal phase of the menstrual cycle to an insulin modified IVGTT (300 mg glucose $/ \mathrm{Kg}$, followed in $20 \mathrm{~min}$ by a $5 \mathrm{~min}$ infusion of insulin $6 \mathrm{mU} / \mathrm{Kg} / \mathrm{min}$ and blood was sampled 14 times for $240 \mathrm{~min})$. SI $\left(\times 10^{-4} \mathrm{~min}^{-1}\right.$ per $\left.\mu \mathrm{U} / \mathrm{ml}\right)$ and SG $\left(\mathrm{min}^{-1}\right)$ were estimated by Bergman's minimal model. In Group I, SI was reduced $50 \%$ below that of the group II $(2.41 \pm 2.08$ vs $4.99 \pm 2.41) p<0.05$. Sg was higher in Group I when compared with Group II $(0.023 \pm 0.008$ vs $0.022 \pm 0.009)$ $p<0.05$. In Group I mean $1^{\text {st }}$ phase of insulin secretion was increased more than twofold as compared with Group II $(730+502$ vs $322+165)$ $p<0.05$. Comparing lean and obese pregnant women, the obese had a lower $S 1(1.85 \pm 2.25$ vs $2.65 \pm 2)$, Sg $(0.24 \pm 0.11$ vs $0.022 \pm 0.006)$ and a higher mean $1^{\text {st }}$ phase insulin secretion ( $954 \pm 749$ vs $647 \pm 369$ ) although the difference was not statistically significant. Our data confirm that late pregnancy is a state of physiologic insulin resistance compensated by a compensatory increase of insulin secretion. In this group of pregnant women obesity had no significant influence on insulin sensitivity and on insulin secretion.
784

MODULAR EDUCATION FOR DIABETES AND PREGNANCY: OUTCOME IN 63 PREGNANCIES UNDER FUNCTIONAL INSULIN TREATMENT Ch. Schlusche, K. Howorka, J. Pumprla, A. Feiks*, Ch. Nowotny* and M. Ulm ${ }^{\star}$, Departments of Biomedical Engineering \& Physics and of Prenatal Diagnostics and Therapy, University of Vienna, Austria

Functional insulin treatment (FIT) based on the outpatient education for selective use of insulin for fasting, eating or correction of hyperglycaemia was used between 1985 and 1995 prospectively in 63 pregnancies (in 18 cases after conception) in 52 pregnant diabetic patients. We hypothesized that a near-normalization of glycaemia is possible throughout pregnancy by modular outpatient group education (FIT, pregnancy/delivery preparation and hypertension if necessary), individual counselling and functional insulin treatment. We wanted to investigate to which degree it might eliminate classical diabetes-associated neonatal complications. Patients were taught both the primary adjustment (immediate correction of hyperglycaemia) and the secondary adjustment of the insulin dosages: correction of individual algorithms for insulin use according to daily insulin consumption and mean blood glucose MBG. A target metabolic control (MBG $<100$ and $<90 \mathrm{mg} / \mathrm{d}$ after the 28th week of gestation respectively) was achieved in $80 \%$ of the pregnancies. Severe hypoglycaemia occured in 15 patients $(23 \%)$. The gestational age at delivery was $39,0 \pm 1,6(34-41$; in 3 cases only $(5 \%)<37)$ weeks with an average birth weight of $3305 \pm 519(1950-4450) \mathrm{g}$. The birth weight of only 5 newborns $(8 \%)$ was above the 90th percentile and one below the 10 th percentile for weight of a comparable population. No cases of respiratory distress were observed. Hypoglycaemia was recorded in only 4 newborns $(6 \%)$ what was comparable thus to its prevalence in offsprings in non diabetic women. Malformations were found in two offsprings whose mothers had presented first for diabetes education after conception whereby in one case of meningomyelocele pregnancy was terminated. The cesarean sectio $(n=16 ; 25 \%)$ was due primarily to maternal reasons. Functional insulin treatment prior to conception, modular diabetes group education, continuous outpatient motivation as well as interdisciplinary care allow pregnancy outcome in diabetic patients similar to that in non diabetic women.

\section{6}

SOME FIBRINOLYTIC FACTORS IN TYPE I DIABETICS DURING PREGNANCY AND AFTER DELIVERY

A Bronisz, M Kotschy, D. Rość, A. Graczykowska-Koczorowska; University School of Medical Science, Bydyoszcz, Poland

The aim of the study was to compare the fibrinolytic activity in two groups of IDDM women with and without microangiopathy. We examined 31 pregnants with IDDM aged $29,1 \pm 6,2$ (mean, SD) years divided in two groups: 17 without microangiopathy - $B$ and $C$ classes acc. White's $(B C)$ and 14 with microangiopathy - D class acc. White's (D). All the patients had normoglycaemia $\left(\mathrm{HbA}_{\mathrm{Ic}}\right.$ in $\mathrm{BC}-5,6 \pm 1,4 \%$ and in $\mathrm{D}-5,8 \pm 1,1 \%$ ). The control group (CG) contained 24 age matched healthy pregnants. The blood was collected in the 12th, 24th and 36th weeks of pregnancy, after delivery and after puerperium. The concentrations of tPA antigen ( $\mathrm{PA}: \mathrm{Ag}$ ), PAl-I activity (PAI-1 Act), euglobulin lysis time (ELT), fibrinogen (F) and fibrin degradation products (FDP) were determined. In the third trimester the ELT was longer $200 \pm 51 \mathrm{~min}$. than in controls $148 \pm 48 \mathrm{~min} .(\mathrm{p}<0,01)$ but only in $B C$ group. In the first and second trimester, after delivery and after puerperium no differences in ELT among the groups were observed. The fibrinogen concentrations and FDP level were similar in all groups in different study time. In third trimester in group with microangiopathy the concentration of $\mathrm{PAA}: \mathrm{Ag}$ was 3 times higher $-21 \pm 12 \mathrm{ng} / \mathrm{ml}$ than in controls $7,7 \pm 3,9(p<0,01)$ as well as in group without microangiopathy: $8,4 \pm 6,3$ $\mathrm{ng} / \mathrm{ml}(\mathbf{p}<0,01)$. PAl-1 activity was significant lower only after delivery in diabetics without microangiopathy. Conclusions: in normoglycaemic diabetic pregnants without microangiopathy the fibrinolytic activity was similar as in healthy pregnants. In diabetics with microangiopathy during pregnancy a significant increase of tPA:Ag occurrs. It seems to be related to the damage of vascular endothelium cells. 


\section{7}

MACROSOMIA FOETALIS IN ANTECEDENT DELIVERIES OF TYPE 2 (NONINSULINDEPENDENT) DIABETIC WOMEN

R.Lichiardopol, C. Dumitrescu and R. Perciun . " N. Malaxa " Hospital, Bucharest-Romania.

Macrosomia (MF) foetalis (birth weight $>4000 \mathrm{~g}$ ), as a witness of some glucoregulatory disturbances during pregnancy, may herald the subsequent development of diabetes in the mother. The aim of this study was to learn about the frequency of this event in a group of diabetic women. From 1619 women with newly discovered diabetes mellitus $1149(71 \%)$ gave, at least, one birth before. 225 of them ( $20 \%$ vs. $4 \%$ in general population; $p<0.001)$ gave birth , at least, to one macrosomatic infant. 216 women $(96 \%)$, with type 2 (noninsulindependent) diabetes, had mean (SD) age $56.9(19.6)$ years and $B M I 31.7(4.7) \mathrm{kg} / \mathrm{m}^{2}$. In these, the mean time interval from the index birth to diagnostic was $33.1(10.6)$ years. As compared to puerperal period, $(25.1 \pm 4.7 ; p<0.001)$ their BMl was greater at the time of diagnostic.

The index birth preceded the weight increase. The time interval between birth and diagnostic correlated ( $r=+0.48 ; p<0.001)$ with weights excess duration. This may suggest that the associated weight increase could be a consequence of the cellular defects involved in the pathogenesis of type 2 (noninsulindependent) diabetes. Our data show an over 5 fold increased frequency of macrosomia foetalis in antecedent deliveries of type 2 (noninsulindependent) diabetic women as compared to general population.

\section{8}

Expression of GLUT $4 \mathrm{mRNA}$ in the Skeletal Muscle and Adipose lissue from Rats treated with Progesterone and $17 \beta$-Estradiol A.Sugaya, S.Yanase, X.Shen, H.Minoura, T.Sugiyama and N.Toyoda. Department of Obstetries and Gynecology. Mie University. Tsu, Japar.

Insulin resistance is a well described feature of pregnancy. [t. is reported that the insulin resistance is mediated at a postbinding level. In order to elucidate the mechanism of insulin resistance during pregnancy, we focused on sex steroids which increases markedly during pregnancy, and also glucose transporter 4 (GLUT 4). which is one of the major postbinding effecters of insulin in the insulin-sensitive tissue such as the skeletal muscle and adipose tissue. We examined the changes in the expression of GLUT 4 mRNA in the skeletal muscle and adipose tissue from rats treated with progesterone (P) and $17 \beta$-estradiol (E). Female Sprague-Dawley rats at 6 weeks were ovariectomised and after two weeks the animals were daily injected subcutaneously for 3,7 or 10 days with 10,50 or $250 \mu \mathrm{g}$ of $\mathrm{E}$ (group E) or 1,5 or $25 \mathrm{mg}$ of $\mathrm{P}$ (group P) or $50 \mu \mathrm{g}$ of $E$ plus $5 \mathrm{mg}$ of $\mathrm{P}$ (group $\mathrm{E}+\mathrm{P}$ ). The skeletal muscle and the adipose tissue were removed and total RNA was isolated, and the expression of GLUT $4 \mathrm{mRNA}$ was assessed by ribonuclease protection assay. In the adipose tissue, the GLUT $4 \mathrm{mRNA}$ in group $E$ and $E+P$ was significantly $(p<0.01)$ decreased to about the half of the control, but was not in group P. No change in the GLUT4 mRNA was seen in the skeletal muscle. These findings suggest that the reduction in the expression of GLUT 4 mRNA in the adipose tissue by $\mathrm{E}$ or $\mathrm{E}+\mathrm{P}$ is one of the factors which mediate insulin resistance in late pregnancy.
789

The St Vincent Declaration and Pregnancy - How close are we to achieving its aims?

$R$. Fraser N. Titchiner and S. Heller. Northern General Hospital, Sheffield, UK

The St Vincent declaration includes in its aims "achieving pregnancy outcome in the diabetic woman that approximates to that of the nondiabetic woman". We have completed a 10 year audit in this specialist unit and present the results in comparison with our non-diabetic obstetric population (1986-1995). There were 285 pregnancies in 215 women, $25 \%$ IDDM $7 \%$ NIDDM, 22\% Gestational Diabetes (CDM) and 45\% Impaired Glucose Tolerance (IGT), by the WHO criteria.

\begin{tabular}{l|c|c|c}
\hline Outcomes & $\begin{array}{l}\text { Non- } \\
\text { diabetics }\end{array}$ & $\begin{array}{l}\text { Diabetes } \\
\text { [IDDM/ } \\
\text { NIDDM/ } \\
\text { GDM] }\end{array}$ & IGT \\
\hline Number of pregnancies & 29,114 & 142 & 128 \\
\hline Rate/1000 pregnancies & - & 5 & 4.4 \\
\hline $\begin{array}{l}\text { Maternal Hypoglycaemia requiring } \\
\text { outside assistance }\end{array}$ & $18 \%$ & $0 \%$ \\
\hline Caesarean Section & $10 / 1000$ & $35 / 1000$ & $8 / 1000$ \\
\hline Perinatal Deaths & $10 / 1000$ & $59 / 1000$ & $8 / 1000$ \\
\hline Major Malformation & - & $28.3 \%$ & $42.5 \%$ \\
\hline $\begin{array}{l}\text { Birthweight > 90th Centile for } \\
\text { Gestational age }\end{array}$ & & $6.6 \%$ & $0 \%$ \\
\hline $\begin{array}{l}\text { Respiratory Distress Syndrome } \\
\text { Neonatal Hypoglycaernia (requiring iv } \\
\text { dextrose) }\end{array}$ & & $35.4 \%$ & $6.1 \%$ \\
\hline
\end{tabular}

Recent improvements in self monitoring and ultrasound surveillance do not appear to have reduced the mortality or morbidity associated with maternal diabetes in pregnancy. The achievement of the St Vincent aims will require new diagnostic and therapeutic interventions.

\section{0}

ANTIOXIDANTS BLOCK EMBRYONIC MALDEVELOPMENT IN DIABETIC SERUM IN VITRO

P. Wentzel and U. J. Eriksson. Department of Medical Cell Biology, University of Uppsala, Uppsala, Sweden.

We have previously found antioxidants to block maldevelopment caused by high glucose concentration in vitro. In the present study we aimed to characterize the teratogenicity of serum from rats with different severity of diabetes. We cultured day- 9 rat embryos for $48 \mathrm{~h}$ in serum from nor$\mathrm{mal}$ rats (C) and from rats given either $50 \mathrm{mg} / \mathrm{kg}(50 \mathrm{D})$, or $75 \mathrm{mg} / \mathrm{kg}(75 \mathrm{D})$ of streptozotocin with the glucose concentration adjusted to $30 \mathrm{mmol} / \mathrm{l}$. We found the malformation score (range: $0-10$, where 0 denotes normal morphology, and 10 severely malformed embryo) increased (C: $6.9,50 \mathrm{D}$ : $7.3,75 \mathrm{D}: 10.0$ ), and somite number (C: $23 \pm 1,50 \mathrm{D}: 19 \pm 1$, 75D: $10 \pm 1$ ) decreased in embryos cultured in diabetic serum, also in comparison with control serum culture at $10 \mathrm{mmol} / \mathrm{l}$ glucose (malformation score: 0.3 , somite number $31 \pm 0.4$ ). Addition of the antioxidants superoxide dismutase (S) or N-acetylcysteine $(\mathrm{N})$ to the culture medium partly restored malformation score $(\mathrm{C}+\mathrm{S}: 1.1,50 \mathrm{D}+\mathrm{S}: 3.3$, $75 \mathrm{D}+\mathrm{S}: 8.3, \mathrm{C}+\mathrm{N}: 2.1,50 \mathrm{D}+\mathrm{N}: 2.5,75 \mathrm{D}+\mathrm{N}: 9.8)$, and somite number $(\mathrm{C}+\mathrm{S}: 29 \pm 1,50 \mathrm{D}+\mathrm{S}: 25 \pm 1,75 \mathrm{D}+\mathrm{S}: 17 \pm 1, \mathrm{C}+\mathrm{N}: 28 \pm 1$, $50 \mathrm{D}+\mathrm{N}: 27 \pm 1,75 \mathrm{D}+\mathrm{N}: 17 \pm 1$ ), the prevention was least pronounced in the $75 \mathrm{D}$ serum. The results show that the teratogenicity of diabetic serum in vitro is dependent on other serum factors in addition to high glucose concentration, and suggest that an excess of free oxygen radicals is involved in the teratogenic process. 
791

Expression of Hexokinase-II mRNA and Activity in Rat Skeletal Muscle and Adipose Tissue During Pregnancy and Lactation S.Yanase, A.Sugaya, X.Shen, H.Minoura, T.Sugiyama and N.Toyoda Department of Obstetrics and Gynecology, Mie University, Tsu, Japan.

[nsulin resistance during late pregnancy is a key phenomenon for understanding the mechanism of maternal metabolic adaptation for supplying glucose to the fetus and also for understanding the pathophysiologic characteristics of gestational diabetes mellitus. HexokinaselI (HKII) is predominantly expressed in insulin-sensitive tissues, such as skeletal muscle and adipose tissue. There is evidence that insulin increases the activity of HKII in these tissuse, which is of central importance in insulin-stimulated glucose uptake. The aim of this study was to assess the role of this enzyme in the mechanism of insulin resistance in late pregnancy. We have examined the changes in expression of HKII mRNA by Ribonuclease Protection Assay and measurement of HK enzyme activity by utilizing a glucose-6.phosphate coupled assay in skeletal muscle and adipose tissue of rats during pregnancy and lactation $(n=6-10)$. In the adipose tissue, HKLI mRA was decreased to $55.42 \pm 8.41 \%(\mathrm{p} \times 0.001)$ of the control (nonpregnant rats) at day 20 of pregnancy, and increased again during lactation to the same level as the control. HKII activity was decreased at day 20 of pregnancy $(39.34 \pm 3.05$ vs $27.81 \pm 3.61$. p 0.05 ), which correlates the changes in the HKII mRNA. In contrast, the HKII mRNA and activity did not change in the skeletal muscle. The findings indicate that HKII is regulated in a tissue-specific manner during pregnancy and lactation and suggests that changes in HKII gene expression in adipose tissue contribute to the insulin resistance near the end of pregnancy.

\section{2}

MATERNAL DIABETES IS TERATOGENIC WITHOUT DAMAGING EMBRYONIC MITOCHONDRIAL DNA $\mathrm{X}$. Yang, N. Welsh, M. Welsh, and U. J. Eriksson Department of Medical Cell Biology, University of Uppsala, Uppsala, Sweden.

Altered mitochondrial morphology and function have been demonstrated in embryos exposed to a diabetic environment, which also induces embryonic dysmorphogenesis. Teratogenic concentrations of glucose have recently been shown to both inhibit oxygen consumption and increase superoxide generation in embryonic neural tissue. Increased nuclear DNA damage in embryos of diabetic rats have also been reported, and we therefore aimed to study mitochondrial DNA (mtDNA) in these embryos. DNA was extracted either from whole embryos or from neuroepithelial tissue of embryos from diabetic and non-diabetic rats at gestational day 11. DNA content of the embryos was measured. Southern Blot was performed with a cytochrome $b$ gene probe after digestion with restriction enzymes, Sac I, Bam HI, EcoR I, or Hind III. In addition, the proportion of the mtDNA forms (multimer complex, closed circular, open circular, and linear) was assessed. The average total DNA content was lower in the diabetic group $(27 \pm 2 \mu \mathrm{g}, \mathrm{n}=36)$ than in the non-diabetic group $(52 \pm 2 \mu \mathrm{g}, \mathrm{n}=30)$. DNA/mtDNA was not different between the two groups. Furthermore, no differences could be found in the proportion of the mtDNA forms. The results indicate that maternal diabetes does not preferentially affect mtDNA in the embryos.

\section{3}

EMBRYONIC DYSMORPHOGENESIS AND CATALASE ISOENZYMES IN RAT DIABETIC PREGNANCY

J. G. Cederberg and U. J. Eriksson, Department of Medical Cell Biology, University of Uppsala, Uppsala, Sweden

Previously we have identified two rat strains, $H$ and $U$, with different rates of diabetes-induced embryonic dysmorphogenesis. The $\mathrm{H}$ strain with erythrocyte-catalase type $\mathrm{Cs}_{\mathrm{s}} \mathrm{1}^{\mathrm{b}}$ has no diabetes-induced malformations, whilst the U strain with type $\mathrm{Cs}-1^{a}$ has a high malformation-rate in diabetes. The aim was to evaluate a possible functional difference by measuring catalase activity in embryos of normal and diabetic mothers of these strains on pregnancy day 11. The DNA content was higher in the $\mathrm{H}$ embryos both in the nondiabetic (H: $23.0 \pm 1.0 \mu \mathrm{g}$, U: $17.8 \pm 1.1 \mu \mathrm{g}$ ) and diabetic pregnancies ( $\mathrm{H}: 21.1 \pm 2.1 \mu \mathrm{g}, \mathrm{U}: 12.3 \pm 1.1 \mu \mathrm{g}$ ), indicating a lower susceptibility of the $\mathrm{H}$ embryos to the diabetic milieu. Furthermore, the $\mathrm{H}$ embryos of normal mothers showed higher catalase activity $(1.8 \pm 0.1 \mathrm{U} / \mu \mathrm{g}$ DNA) than did the $\mathrm{U}$ embryos $(1.1 \pm 0.1 \mathrm{U} / \mu \mathrm{g}$ DNA). The difference in catalase activity between the strains increased when the mother was diabetic, the $\mathrm{H}$ embryos showed unchanged activity $(2.1 \pm 0.1 \mathrm{U} / \mu \mathrm{g} \mathrm{DNA})$, whereas the activity decreased in the $\mathrm{U}$ embryos $(0.8 \pm 0.1 \mathrm{U} / \mu \mathrm{g} \mathrm{DNA})$. In conclusion, we have found a functional difference in embryonic catalase with a higher activity in the teratologically resistant $H$ embryos compared to that in the malformation-prone and growth retarded U embryos. This suggests that the catalase isoenzyme may have a functional role in the pathogenesis of congenital malformations in diabetic rat pregnancy.

\section{4}

GLUCOSE DECREASES THE LEVELS OF FREE OXYGEN RADICALS IN RAT EMBRYONIC CELLS IN VITRO.

H. Forsberg, U. J. Eriksson and N. Welsh, Department of Medical Cell Biology, University of Uppsala, Uppsala, Sweden

In previous studies antioxidant treatment has prevented glucose- or ketone body-induced malformations in vitro, and it has been suggested that free oxygen radicals (FOR) mediate the teratogenic effect of maternal diabetes. The aim of this study was to directly assess if high concentrations of glucose or $\beta$-hydroxybutyrate (BHB) in vitro affect FOR levels in dispersed embryonic cells. Trypsinized cells from day 10 or day 12 embryos of normal and diabetic rats where loaded with dichlorofluorescein (DCFH), which emits fluorescent light upon oxidation by FOR. Increased glucose levels reduced DCFH oxidation in cells from all experimental groups in a dose-response manner, an effect also observed when the non-metabolized analogue 2-deoxyglucose was used. The mitochondrial oxidation of glucose was undetectable in day 10 cells and $2.1 \pm 0.6 \mathrm{pmol} / 10^{3}$ cell $\times 90 \mathrm{~min}$ at $5 \mathrm{mM}$ glucose $(n=7)$ in day 12 cells. Addition of BHB resulted in unaffected DCFH oxidation in day 10 cells, and in increased DCFH ox idation in day 12 embryonic cells. Normal day 12 cells oxidized $1.5 \pm$ $0.5 \mathrm{pmol} \mathrm{BHB} / 10^{3}$ cells $\times 90 \mathrm{~min}$ at $15 \mathrm{mM} \mathrm{BHB}(n=5)$, whereas $\mathrm{BHB}$ oxidation in day 10 cells was undetectable. We conclude that glucose, but not $B H B$, reduces $F O R$ levels by acting as a weak free radical scavenger. Moreover, the low mitochondrial activity in day 10 cells may preclude mitochondrial generation of FOR at this developmental stage. After the establishment of the chorioallantoic placenta (day 11 . 12 ), and a concomitant general mitochondrial activation nutrient such as BHB induce FOR production. These results do not support a role for FOR generation in diabetic embryopathy. 


\section{Clinical Diabetes}

795

\section{ELEVATED SERUM FERRITIN LEVELS IN SUBJECTS WITH TYPE II DIABETES MELLITUS}

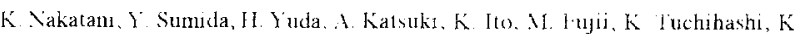

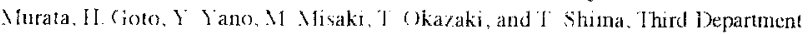

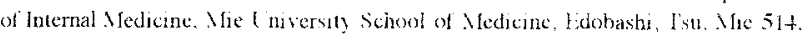
i.tinin

Serum fermun levels were found to be elctatcd in dabeuc patients. To investigate the relationship between ferrtin and diabetes mellitus, the cxpression of ferritun mRNA was examined in rat liver. In diabetse rats. the level of ferritin mRNA was increased to about $150 \%$ of control which suggested that elevaled level of scrum ferritun was caused by increascd expression of mRNA. Moreover, $t o$ clarify the assoctation of lemin with diabetes mellutus in vivo we evaluated the relutionshap between serum terrum levels and glycemk control, insulin scoretion and insulin resistance in diabetic subjects. Ten male patients with lype II diabecs mollitus and hyperferritincmia $(518 \pm 34 \mathrm{ng} / \mathrm{ml})$ and without possible obvious causes of elevated serum ferritin levels were subjected to our study. Six of the ten patients were re-tested after hospitalization for one month. The serum lerritin level was not significantly correlated with insulin secretion nor with fasting plasma glucose levels. A weak correlation was found between serum ferritin and glycosylated hemoglobin levels but it was not statistically significant $(r=0.446, P<$ ().1). Scrum ferritin levels were significantly correlated with the glucose infusion rate (GIR), measured with the hyper-msulinemic euglycemic clamp $(\mathrm{r}=0.570, \mathrm{P}=0,02 \mathrm{l})$. The six patients who were re-tested after a one-month hospitalization had improved GIRs and serum ferritin levels were reduced, reflecting the previously demonstrated correlation with the GIR. It is suggested that the serum ferritin level might be a new marker of insulin resistance in diabetic patients with elevated serum levels of ferntin

\section{6}

SERUM FERITIN AND IRON METABOLISM IN TYPE-2 DIABETIC PATIENTS TREATED WITH DIET, ORAL IYYPOGLYCEMIC AGENTS OR INSULIN. E.Krexner, P.Hopmeier and G.Schernthaner Dept.Medicine I.Rudolfstiftung Mospital Vienna, Austria Recent epidemiologic studies suggest, that high serum ferritin levels constitute an increased risk for coronary heart disease in nondiabetic subjects. Thus, we investigated serun ferritin levels and parameters of iron metabolism (ferritin, iron, transferrin and transferrin-saturation) in 430 patients with type- 2 diabetes (169 men and 261 women; mean age: $62+13$ years) in comparison with control subjects ( $n=50$; mean age: $\overline{6} 2+13$ years).In addition, urinary albumin excretion rate (UAER) was studied in all patients. Ferritin levels above $1000 \mathrm{ng} / \mathrm{ml}$ were detected in only 3 patients. Existance of hemochromatosis could be excluded by NMR technique. Interestingly, serum ferritin levels were highest in dietary-treated patients and lowest in patients on insulin $(\mathrm{p}<0,002)$. By contrast, iron transferrin and transferrin saturation did not significantly differ between type-2 diabetic patients with the 3 different therapeutic strategies. Parameters of iron metabolism and serum ferritin levels were not significantly different in patients with normo-,micro- or macroalbuminuria. We can conclude, that bemochromatosis is not a common cause of diabetes mellitus. The lower ferritin levels in the insulin-treated group with the more advanced stage of type- 2 diabetes and the lack of correlation between ferritin and UAER is not in line with the hypothesis that serum ferritin levels are an important risk factor for coronary heart disease.

\begin{tabular}{|c|c|c|c|c|c|c|}
\hline & $\mathrm{n}$ & Age & $\begin{array}{l}\text { UAER } \\
\mathrm{mg} / 24^{\mathrm{h}}\end{array}$ & Iron & ansferrin & Ferritin \\
\hline Giet & 62 & $62+11$ & $25+90$ & $93+40$ & $289+44$ & $273+290$ \\
\hline & 200 & $62 \pm 11$ & $82 \pm 319$ & $88 \mp 34$ & $30 \overline{4}+49$ & $222+207$ \\
\hline ulin & 168 & $67+9$ & $225+827$ & $83+27$ & $286+40$ & $158+148$ \\
\hline
\end{tabular}

OHA: Oral hypoglycemic agents

\section{7}

SERUM FERRITIN AS AN INDEPENDENT FACTOR FOR HIGHER GLUCOSE AND HbA $A_{1 C}$ LEVELS

W. Ricart, J.M. Fernåndez-Real, E. Arroyo, R. Balançá and D. Cabrero. Department of Endocrinology and Hormonal Laboratory. Hospital "Dr Josep Trueta". Girona. SPAIN.

Objective: Several lines of evidence suggest that iron stores may influence metabolic control and chronic complications in the diabetic patient. As elevated serum ferritin levels have been found in up to $30 \%$ of individuals with noninsulin dependent diabetes mellitus (NIDDM), the identification of this influence may have therapeutical implications. We investigated the influence of iron stores, measured as serum ferritin, on glucose control. Patients and Methods: Seventy six consecutive patients with NIDDM, and fifty subjects with normal basal and mean glucose levels, as estimated by serum glycosylated hemoglobin concentrations, were studied. Serum ferritin, iron, transferrin, total hemoglobin, $\mathrm{HbA}_{1}$ and $\mathrm{HbA}_{1 \mathrm{c}}$ were analyzed in all subjects. Patients who had possible causes of an elevated level of serum ferritin,as chronic liver disease, malignant processes, uremia and inflammatory diseases were excluded. Results: Serum ferritin above $300 \mu \mathrm{g} / \mathrm{L}$ were found in 11 (14\%) NIDDM patients. $\mathrm{HbA}_{1 \mathrm{c}}$ levels (mean $8.6 \pm 2.0 \%$ ) correlated with $\log$ transformed serum ferritin (logfer) $(r=0.25, p=0.03)$, with serum glucose $(r=0.57, p<0.0001)$, and weakiy with age $(r=0.19, p=0.1)$ but not with serum iron or transferrin. This correlation remained significant after controlling for age $(t=0.25, p=0.03)$, and a trend persisted after controlling for body mass index $(\mathrm{r}=0.21, \mathrm{p}=0.08)$. In a Multiple Linear Regression in a stepwise manner, with serum $\mathrm{Hb} \mathrm{A}_{1 c}$ as dependent variable, only serum glucose $(p<0.00001)$ and logfer $(p=0.03)$ independently predicted the value of $\mathrm{HbA}$. Among nondiabetic subjects logfer correlated with serum glucose $(r=0.33$, $\mathrm{p}=0.019)$, age $(\mathrm{r}=0.54, \mathrm{p}<0.0001)$, and serum total hemoglobin $(\mathrm{r}=0.32, \mathrm{p}=0.03)$ but not with serum $\mathrm{HbA}_{\mathrm{Ic}}(\mathrm{r}=0.17, \mathrm{p}=0.25)$. Conclusions: Iron stores may influence glycosylation in NIDDM subjects. The correlation between serum glucose and serum ferritin in the control subjects may explain why ferritin has been proposed as a cardiovascular risk factor.

\section{8}

INTRACELLULAR MAGNESIUM DEPLETION IS RELATED TO INCREASED URINARY MAGNESIUM LOSS IN TYPE 1 DIABETES

\section{ALPER GÜRLEK ${ }^{1}$, M. BAYRAKTAR ${ }^{1}$ and N. ÖZALTIN ${ }^{2}$}

(1):Department of Endocrinology, Haceftepe University Facilty of Medicine, Ankara,TURKEY

(2):Department of Analytical Chemistry, Hacettepe University Faculty of Pharmacy, Ankara, TURKEY

Magnesium ( $\mathrm{Mg}$ ) deficiency has been demonstrated both in the patients with Type 1 and Type 2 diabetes mellitus. Increased urinary $\mathrm{Mg}$ excretion may contribute to reduced $\mathrm{Mg}$ stores in diabetic individuals. To test this hypothesis,we investigated the $\mathrm{Mg}$ levels in plasma, erythrocytes and urine in twelve patients with Type 1 diabetes. Twelve healthy subjects served as the control group. Plasma,erythrocyte and urine $\mathrm{Mg}$ concentrations were determined using a graphite-fumace atomic-absorption spectrophotometer with Zeeman background correction. Mean plasma $\mathrm{Mg}$ concentrations were comparable between diabetic patients and control subjects $(0.90 \pm 0.29 \mathrm{mmo} / \Omega$ vs $1.04 \pm 0.14$ $\mathrm{mmol} / \mathrm{A}$, respectively; $\mathrm{p}=0.16$ ). Mean erythrocyte $\mathrm{Mg}$ concentration was significantly lower in the diabelic group compared with the control group $(1.41 \pm 0.56 \mathrm{mmol} / \mathrm{l}$ vs $2.94 \pm 1.13 \mathrm{mmol} / \mathrm{h}$, respectively; $p<0.0001)$. Mean urine $\mathrm{Mg}$ concentration was significantly elevated in the diabetic group with respect to controls $(4.21 \pm 1.92 \mathrm{mmol} / \mathrm{l}$ vs $2.49 \pm 0.77$ $\mathrm{mmo} / \mathrm{A} ; \mathrm{p}<0.001$ ). As to the diabetic group, erythrocyte $\mathrm{Mg}$ concentration showed inverse correlation with urine $\mathrm{Mg}$ concentration $(r=-0.53)$ which approached statistical level of significance $(p=0.08)$. There was no correlation between urine $\mathrm{Mg}$ concentration and glycosylated hemoglobin or fasting plasma glucose level. The data suggest that intracellular Mg depletion without significant hypomagnesemia is related to increased urinary $\mathrm{Mg}$ loss in patients with Type 1 diabetes. Hypermagnesuria is independent from the degree of metabolic control. 
799

LOW CALCITRIOL LEVELS IN NIDDM ELDERLY SUBJECTS J. M. González-Clemente, *M.J. Martínez-Osaba, M. Borrell, **J.I. Conget ${ }^{*} \mathrm{E}$. Vilardell and J. Camp. CAP Les Ccrts, ${ }^{*}$ Hormonology Unit, **Endocrinology Unit. C. S. Clínic. Barcelona. Spain.

IDDM is associated with osteoporosis and low calcidiol and calcitriol levels. The relationship between NIDDM and osteoporosis remains controversial and little information on calcidiol and calcitriol is available. The aim of this study has been to evaluate whether the presence of NIDDM in elderly subjects is associated with a decrease in calcidiol and calcitriol levels. From November 1995 to March 1996, consecutive elderly patients $(>65 \mathrm{yr})$ were recruited in a primary care setting (exclusion criteria: IDDM, NIDDM requiring insulin, renal failure, liver or malabsorptive disorders, secondary osteoporosis, drugs affecting vitamin $\mathrm{D}$ metabolism). Serum creatinine, phophatase alkaline (PA), total calcium, phosphate, magnesium, $\mathrm{PTH}$, calcitriol, calcidiol, creatinine clearance $(\mathrm{CC})$ and urinary $\mathrm{Ca} / \mathrm{Cr}$ were assessed. One hundred and twenty-four subjects $(74.3 \pm 0.6 \mathrm{yr}, \mathrm{M} / \mathrm{F}: 49 / 75)$ were included, 37 having NIDDM $(75.7 \pm 1.2 \mathrm{yr}, \mathrm{M} / \mathrm{F}$ : 18/19, NIDDM evolution: $7.6 \pm 1.1 \mathrm{yr}, \mathrm{HbA}$ c: $7.2 \pm 0.3 \%$ ), No significant differences were found between subjects with and without NIDDM in relation to age, sex, BMI, total calcium, phosphate, PA, iPTH, calcidiol and urinary $\mathrm{Ca} / \mathrm{Cr}$. However, subjects with NIDDM had lower $\mathrm{CC}$ $(55.9 \pm 2.8$ vs $60.8 \pm 1.5 \mathrm{ml} / \mathrm{min} ; \mathrm{p}=0.05)$, serum magnesium $(2.07 \pm 0.06$ vs $2.2 \pm 0.06 \mathrm{mg} / \mathrm{dl}, \mathrm{p}=0.02)$, and calcitriol levels ( $24.6 \mathrm{vs} 29.0 \mathrm{pg} / \mathrm{ml}$; $\mathrm{p}=0.04)$ and higher serum creatinine $(1.03 \pm 0.02$ vs $0.97 \pm 0.02 \mathrm{mg} / \mathrm{dl}$; $\mathrm{p}=0.02)$. The presence of NIDDM was independently and negatively related to calcitriol levels (stepwise regression analysis, $p=0.00$ ). We conclude that NIDDM not requiring insulin in otherwise healthy elderly subjects is associated with low calcitriol levels.

\section{1}

INPATIENT SCREENING FOR UNDIAGNOSED DIABETES K.P. Shotliff, A. Blight and S. Patel. St George's \& St Helier Hospitals, London, U.K.

Approximately $10 \%$ of people over 65 years of age have diabetes, but about one third will be undiagnosed. A random blood glucose (RBG) assay is commonly used to screen for diabetes. Guidelines for further investigation of a $R B G \geq 7.8 \mathrm{mmol} / 1$ are included in a handbook given to all new doctors in our hospital. We examined our use of these guidelines and our junior doctors' ability to diagnose diabetes.

In this unit a prospective study of 515 consecutive acute admissions (aged $\geq 65$ years) found $7.6 \%$ were already known to have diabetes and $4.7 \%$ were newly diagnosed on the basis of subsequent fasting blood glucose estimations. Nearly 1 in 5 patients were however not screened and no reason for this omission was documented in any case. Using a RBG level $\geq 7.8 \mathrm{mmol} / \mathrm{L}$ nearly 1 in 5 of those screened needed further investigation with 1 in 3 of these having previously undiagnosed diabetes. We therefore assessed how well this recommended investigation plan was known amongst the junior doctors requesting and interpreting these tests, and also their knowledge of the World Health Organisation (WHO) criteria for the diagnosis of diabetes. This involved a questionnaire given by hand and immediately returned to the investigators. Of the 58 doctors asked $55 \%$ did not know the correct level suggested for further investigation of a RBG (19\% used $10.0 \mathrm{mmol} / \mathrm{l}$ and $12 \% \quad 11.1$ $\mathrm{mmol} / \mathrm{l})$. Knowledge of the WHO criteria: $72 \%$ knew the fasting venous plasma level and 52\% the 2 hour post-prandial level. Physicians were not significantly more knowledgable than surgeons in any area.
800

FROM IMPAIRED GLUCOSE TOLERANCE (IGT) TO NONINSULIN DEPENDENT DIABETES MELLITUS (NIDDM). RETROSPECTIVE ANALYSIS OF 632 OBSERVATIONS.

G.Guarneri, T. Lavagnini, A. Maran, A. Tiengo, and S. Del Prato. Diabetes Clinic. University and General Hospital of Padova, Italy. Identification of predictive markers for NIDDM is needed for planning screening and prevention programs. Over the past $15 \mathrm{yrs}$, at our diabetic clinic IGT was diagnosed by OGTT in 632 individuals $(318 \mathrm{M} / 314 \mathrm{~F}$; age $=55.3 \pm 10.5$ yrs, $B M I=27.8 \pm 4.3$, fasting glucose $(F P G)=6.1 \pm i$. $\mathrm{mM} ; \mathrm{HBA}_{\mathrm{Ic}}=5.94 \pm 1.06 \% ; \mathrm{m} \pm \mathrm{SD}$ ). 5 yrs after IGT diagnosis, NIDDM developed in 106 subjects $(16.8 \% ; 54 \mathrm{M} / 52 \mathrm{~F} ; \mathrm{FPG}=10 \pm 1.9$ vs $5.8 \pm 0.9$; $\mathrm{HBA}_{1 \mathrm{c}}=8.3 \pm 1.6$ vs $5.9 \pm 1.4 \%$; both $\mathrm{p}<0.0001$ ). In NIDDM, at time of IGT diagnosis, FPG $(6.3 \pm 1.2$ vs $6.1 \pm 1.01 ; p=0.016)$, and post OGTT glucose $(60 \mathrm{~min}=13.0 \pm 2.0$ vs $12.2 \pm 2.0, \mathrm{p}=0.001 ; 120 \mathrm{~min}=8.9 \pm 1.1$ vs $8.6 \pm 1.2, p=0.006)$, and $\mathrm{HBA}_{1 \mathrm{c}}(6.3 \pm 0.7$ vs $5.8 \pm 1.1 \%, \mathrm{p}<0.0001)$ were higher than those who maintained FPG $<7.8 \mathrm{mM}$. Their BMI was higher at baseline (29.2 25.1 vs $27.5 \pm 4.0)$ and at 5 yrs $(28.8 \pm 4.8$ vs $27.0 \pm 3.6$; both $\mathrm{p}<0.0001)$. There was no difference in baseline age $(53.7 \pm 10.6 \mathrm{vs}$ $55.6 \pm 10.5 \mathrm{yrs})$, total cholesterol $(6.2 \pm 1.2$ vs $6.1 \pm 1.3 \mathrm{mM} ; \mathrm{n}=29$ vs $104)$, triglycerides $(2.5 \pm 2.6$ vs $1.9 \pm 1.4 \mathrm{mM} ; n=27$ vs 99 ), diastolic $(85 \pm 9$ vs $87 \pm 10 \mathrm{mmHg} ; \mathrm{n}=47$ vs 58$)$ and systolic pressure $(150 \pm 15$ vs $150 \pm 16$ ). These parameters also did not differ at 5 yrs (chol $=6.1 \pm 1.3$ vs $6.2 \pm 1.4$; trigl $=2.7 \pm 2.0$ vs $2.0 \pm 1.1$; dias $=88 \pm 8$ vs $88 \pm 12$; sys $\mathrm{BP}=151 \pm 19$ v $148 \pm 16$ ). Prevalence of cardiovascular disease was similar. at baseline ( 2.8 vs $2.5 \%)$ and at 5 yr ( 3.8 vs $3.8 \%$ ). Family history for NIDDM was more frequent in diabetics $(50.5$ vs $38.4 \% ; \mathrm{p}<0.05)$. In the overall IGT population. FPG at 5 yrs was related with BMI $(\mathrm{r}=0.163)$, FPG at baseline $(r=0.169)$, post OGTT $(r=0.169 ; p<0.0001)$ after OGTT, and $\mathrm{HBA}_{1 \mathrm{c}}(\mathrm{r}=0.182 ; \mathrm{p}=0.007)$. In a multivariate analysis both BMI and FPG were associated with development of NIDDM. In conclusion, $17 \%$ of IGT individuals develops NIDDM over 5 yrs, NIDDM development is associated with BMI and FPG at IGT diagnosis with no association with lipid profile and blood pressure.
802

DEHYDROEPIANDROSTERONE SULFATE IN THE DIAGNOSIS AND TREATMENT OF TYPE II DIABETES MELLITUS.

P. Tatti\#, F. Strollo*, R. Guarisco\#, P. Di Mauro\# and G. Strollo*. \# Diabetes Care Unit, Marino Hospital, Roma; "Endocrine Unit, INRCA, Roma, Italy The recent demonstration of low levels of DHEA-s (DS) in the insulinresistant statcs, and in subjects with adverse cardiovascular events makes DS an interesting clinical tool in type II diabetes mellitus (NDDM). We assayed routine parameters and DS in 184 NIDDM patients (DM) in fairly good control on diet alone or on a low dose of sulphonylureas and in 46 matched control (C) subjects (C vs DM: Sex (F:M), 22:24 vs 90:94; Age, $50 \pm 9$ vs $53 \pm 8$ yrs; BMI, $31.6 \pm 6.0$ vs

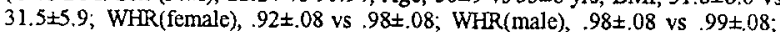
SystBP, $135 \pm 2 \mathrm{I}$ vs $152 \pm 19 \mathrm{mmHg}$; DiastBP $83 \pm 7.0$ vs $86.2+11.2 \mathrm{mmHg}$; Blood Glucose $[\mathrm{BG}$ ], $98 \pm 22$ vs $148 \pm 40 \mathrm{mg} / \mathrm{dl}$; Creatinine $.92 \pm .1 \mathrm{vs} .98 \pm .21 \mathrm{mg} / \mathrm{dl}$; Cholesterol [Chol], $203 \pm 34$ vs $234 \pm 44 \mathrm{mg} / \mathrm{dl}$; Triglycerides, $103 \pm 42 \mathrm{vs} 166 \pm 22$ $\mathrm{mg} / \mathrm{dl}$; HDL-Chol, $54 \pm 16$ vs $55 \pm 17 \mathrm{mg} / \mathrm{dl}$; Insulin $16.0 \pm 7.2$ vs $19.6 \pm 16.5 \mathrm{mU} / \mathrm{l}$ ) We found no significant differences between the two groups, except for Chol $(p<.005)$ and, by definition, BG $(p<.001)$. We used the Homeostatic Model Assessment to calculate the Insulin Sensitivity $(\%)$ and the partial correlation the linear regression and the MLR to evaluate the data. Both $C$ and DM presented a weak but significant linear correlation DS to $\%$ S, yet the $\%$ S accounted for $16 \%$ and $9 \%$ of the variance of DS respectively. Therefore, DS did not prove to be of any practical usefulness in the evaluation of insulin sensitivity in our hands. Within our DM group, DS levels were lower in patients with high blood pressure and dysipidemia than in those without such cardiovascular risk factors $(80 \pm 20$ vs $120 \pm 62 \mu \mathrm{g} / \mathrm{dl}$ ); such difference, however, was not statistically significant. Furthermore, during the first 18 months of follow-up we could not observe any higher prevalence of adverse cardiovascular events among our patients with low DS levels. In summary, circulating DS levels do not seem to add much to either the diagnosis or the therapeutical approach of NIDDM, and have no clinically relevant prognostic significance in terms of cardiovascular risk, at least in the shor run. 
803

EFFECT OF AN ORAL GLUCOSE LOAD ON RENAL DOPAMINE PRODUCTION IN HEALTHY AND TYPE 2 DIABETIC SUBJECTS,

0 . Segers and G. Somers. Department of Internal Medicine, Academic Hospital, Vrije Universiteit Brussel, Brussels, Belgium.

A deficiency of renal dopamine production was suggested to be implicated in the sodium retention of diabetic patients. Indeed, lower urinary dopamine excretions have been evidenced in basal conditions and after dynamic tests in Type 1 and Type 2 diabetes.

The aim of the present study was to evaluate the influence of hyperinsulinemia and/or glucosuria on kidney dopamine (UDA) production in 9 Type 2 diabetic patients on diet treatment only (aged: $51.4 \pm 3.2$ years, fasting glycemia: $115 \pm 7 \mathrm{mg} / \mathrm{dl}, \mathrm{HbA} 1 \mathrm{c}: 7.0 \pm 0.3 \%$ ) and in 13 age-matched healthy controls. In healthy volunteers, an glucose load induced an increase in insulinemia (from $8.8+1.5 \mathrm{mU} / /$ to $70.0 \pm 21.3 \mathrm{mU} / \mathrm{l} ; P<0.01$ ) and a decrease in urinary sodium (UNa) and UDA excretion: to $73.7 \pm 8.6 \%$ and $77.9 \pm 12.6 \%$ of baseline values respectively $(P<0.03$; Wilcoxon matchedpairs signed rank test). In Type 2 diabetic patients, a glucose load induced comparable increases in insulinemia (from $10.6 \pm 1.7 \mathrm{mU} / \mathrm{i}$ to $62.1 \pm 12.8 \mathrm{mU} / \mathrm{i}$ $P<0$ 01) overt glucosuria and an increase in UNa excretion (to $154.1 \pm 21.7$ $\%$ of baseline values; $P<0.01$ ). In contrast, UDA excretion declined to $78.5 \pm 6.3 \%$ of baseline values $(P<0.001)$. A statistically significant negative correlation was present between UDA excretion and peak plasma insulin levels in diabetic and control subjects $(r=0.40 ; P<0.05)$

These findings confirm the deficiency of the renal dopaminergic system in Type 2 diabetes as an increased natriuresis failed to stimulate UDA excretion. Hyperinsulinemia seems to exert an inhibitory effect on renal dopamine production and could thus contribute to the decreased dopamine production in diabetes.

\section{4}

PREDICTORS OF GLYCAEMIC EFFICACY OF FOUR TREATMENT STRATEGIES IN NIDDM PATIENTS WITH SECONDARY FAILURE TO ORAL HYPOGLYCAEMIC AGENTS.

P.S. van der Wal, W. Devillé and R.J. Heine. Academisch Ziekenhuis Vrije Universiteit, department of endocrinology, Amsterdam, The Netheriands.

The aim of this multicentre study was to evaluate predictors of success of four different insulin treatment strategies in NIDDM-patients with secondary failure (SF) to oral hypoglycaemic agents (OHA: $3 \times 5 \mathrm{mg}$. glipizide $+2 \times 850 \mathrm{mg}$. metformin). A total of 134 patients, aged 40 to 75 years (mean 59.3 yrs.(SD 8.2 )) with body mass index (BMI) of $29.0(3.0) \mathrm{kg} / \mathrm{m}^{2}$, defined as SF by fasting blood glucose levels higher than $7.8 \mathrm{mmol} / \mathrm{l}$ on iwo days 6 weeks apart, entered the study. Patients were randomly allocated to treatment with bedtime NPH insulin alone (I), in combination with either glipizide (IG) or metformin (IM) or with glipizide and metformin (IMG). Success was defined as an HbAic level lower than $1.2 \times$ upper limit of the laboratory reference range at 26 weeks. Logistic regression analysis identified the baseline level of $\mathrm{HbA1C}$ as the only predictor of success : Odd's ratio 2.86 per $10 \%$ decrease of $\mathrm{HbA1c}$ $p=0.05$. An ANOVA for the percentage change in HbA1c corrected for baseline value comparing subjects treated with (IM, IMG) and without metformin $(I, I G)$ showed a gender difference for the effect of metformin: in women coninuation of metformin following transfer to bedtime insulin resulted in a decrease of HbA1c by $12 \%$, whereas cessation of metformin resulted in an in crease by $6 \%(p=0.005)$. In males no treatment effect was observed. This could not be explained by differences in waist-to-hip ratio, BMI, age, diabetes duration or fasting C-peptide levels. In conclusion, baseline $\mathrm{HbA1c}$ is the most important predictor of efficacy of combination therapy in NIDDM patients with SF to OHA treatment. This finding suggests that bedtime insulin therapy should be added to the daytime OHA treatment as soon as glycaemic targets cannot be fulfilled. Cessation of metformin administration when initiating bedtime insulin therapy results in an increase of $\mathrm{HbA} 1 \mathrm{C}$ values in women but not in men. Our results suggest that metformin, when combined with insulin at bedtime in women and not necessarily in men, contributes to the efficacy of the insulin treatment strategy.

\section{5}

\section{NOIUPROVENFT IN QUAIITY OF LIFE AFTFR STARTIYG} INSIIN IREATMEVI IN NIDBN PATIENTS

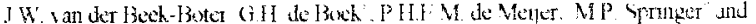

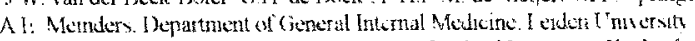

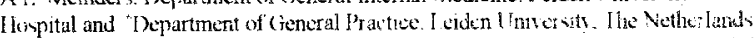

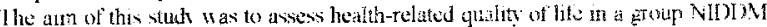

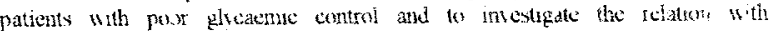
improvement of glveaemic control during misulin treatment incnty nine NIDIOM patients with insufficent glicacme control durng tratment w the of broplyca mic agents were incluled in the studs. During a three month run-in per ice treatmern with det and sulphony lureas was optimized Patients were instructed to pertion home monitoring of blood gluose. When the target valies nert not achicted after three moths, insulin treatment was startid Sexenteen patients (10 female. 7 male. age $60.0 \pm 11.2$ years, diahetes duration $[10.6 \pm 6.6$ years) wore treated w wh msulan. A combination of NPH and regular mstlin was administered twice daly (with disposable insulinpen). Parameters of glveatmic control (t thAle and fasing blood glueose, FB(Y) and quality of life were collected belore and after sis months of insulin treatment. Health related quality of life was asiessed using two instruments firstly, the Dufch version of the SIP (Sickoss Impact Profile), which was developed as a general measure of sickness-related dysfunction and secondly, the DSC. I ype 2 (Type 2 Diabetes Symptom Checklist), a diabetes-specific questionnaire which measures physical and psychological symptoms related to NIDIM and its possible complications. FBG decreased from $13.7 \pm 4.5 \mathrm{mmol} / /$ (mean \pm SD) to $9.9 \pm 3.4$ mmol/ $(p=0.01)$ and $\mathrm{HhAlc}$ from $8.5 \pm 1.3 \%$ to $7.3 \pm 0.9 \%(p<0.0001)$. Total SIP score did not improve: $9.3 \pm 6.0$ before and $9.4 \pm 5.8$ after insulin treatment. None of the 12 dimensions showed a significant change in the scores during insulin treatment. Total ISSC-Type 2 did not improve either: $1.7 \pm 1.7$ and $1.6 \pm 1.7$, respectively, without significant change in the six dimensions of this questionnaire Conclusion. Our results did not show an improvement in health related quality of life during insulin treatment, despite a significant improvement of glycaemic control.

\section{6}

INSUFFICIENT SUSPENSION OF NPH AND MIXED INSULIN BEFORE INJECTION IN IDDM AND NIDDM PATIENTS

PM Jehle* C. Micheler, D. Breitig', Intemal Medicine*, Lotope Division', Liriversity of Ulm, FRG. Intermediate acting NPH insulin [NPH] and mixed insulin [M] preparations contain a predetermined ratio of NPH and solvent or NPH and quick-acting soluble insulin Therefore it is necessary to mix these preparations carefully before injection. Methods: 26 diabetic patients (IDDM vs. NDDM: 13/13; intensified conventional insulin therapy vs. conventional insulin therapy: 16/10) were investigated concerning their accuracy in mixing NPH or MI before injection, their hypoglycemic events and their frequency of daily blood glucose monitoring. The patients routinely visited the hospital where they had absolved a diabetes education program ( 7 centers excluding university hospitals). The currently used insulin cartridges were analyzed with respect to the predetermined ratio of components [PRC] (NPH vs. solvent or NPH vs. soluble insulin). After spontaneous sedimentation for $24 \mathrm{~h}$ NPH appeared as a sharply limited pellet. Using an optical system (Kathetometer, Fuess, Berlin) the PRC was determined with an error of less than 5\%. Results: In control experiments the PRC remained constant over the total cartridge volume if correctly mixed (rolling and tipping over 20 times), whereas the PRC varied considerably when the same experiments were performed with insufficiently mixed cartridges (shaking twice). All patients reported to mix their NPH before injection by rolling $(n=6)$ or shaking $(n=20)$ the cartridges at least 4 times. However, only in 9 of 26 patients the PRC showed a variation of less than $5 \%$. In the other patients the PRC was significantly changed with a higher content of NPH (control $=100 \%$ ) in 7 cases (IDDM: $\mathrm{n}=3: 147 \%, \mathrm{p}<0.05 ;$ NIDDM: $\mathrm{n}=4: 171 \%, \mathrm{p}<0.01$ ) and a lower content in 10 cases (IDDM: $\mathrm{n}=5: 72 \%, \mathrm{p}<0.05 ; \mathrm{NIDDM} ; \mathrm{n}=5: 63 \%, \mathrm{p}<0.01$ ). Linear regression analysis yrelded a significant direct correlation between the error of mixture $[\mathrm{EM}]$ and the frequency of hypoglycemic events $(r=0.6, p<0.01)$. Furthermore, EM was inversely correlated with the number of daily blood glucose self monitoring $(r=-0.4, p<0.05)$. Rolling the NPH cartridge before injection significantly diminished EM $(r=-0.65$ $p<0.01)$, whereas shaking tended to increase EM $(r=0.2)$. Neither the insulin regimen nor the insulin preparation nor the manufacturer did influence EM. No correlation was observed between EM and HbAlc. Conclusions: Although all patients had been educated, only one third of them correctly mixed their insulin preparations. The direct correlation between hypoglycemia and the error of mixture emphasizes the clinical importance of this issue. For the safety of daily practice, however, the mixing procedure of NPH or MI should be facilitated by improving the mixing element within the cartridge. 
807

CAN INSULIN PHARMACOKINETICS BE ACCURATELY ASSESSED USING STORED SERA CONTAINING ANTIINSULIN ANTIBODIES? S E Fineberg, J Woodworth, J A Anderson, S Hufferd, R Gibson, and N S Fineberg, Indiana U. and Lilly Laboratories, Indianapolis, IN USA Despite the desirability of using samples from large scale clinical insulin trials for population pharmacokinetic analyses, it is seldom possible to extract sera for free insulin at the bedside. Since most circulating antibodies are low in insulin affinity, we examined the hypothesis that measurement of insulin concentraions in unextracted sera would accurately reflect the total serum insulin (free and antibody bound). Insulin was measured using a DPC Coat-A-Count ${ }^{\mathrm{TM}}$ assay and \% cross reactive insulin antibody binding (\%CRA-binding inhibited by human insulin or insulin lispro) was assessed using 125 I human insulin and parallel incubation of aliquots of charcoal extracted sera in buffer and antigen excess. The relationship between measurement of antibody binding and insulin in unextracted sera was examined in stored sera known to contain antibodies ( $n=43$, study 1 ) and in clinical trial specimens from studies comparing insulin lispro vs regular human insulin ( $n=3021$ and 3083 , study 2 ). In study $1, \%$ CRA ranged from 4.7 to $58 \%$. Free insulin concentrations were not significantly correlated with total insulin or \%CRA. However, total insulin and unextracted serum insulin were linearly correlated, $r=0.90, p<0.001$. \%binding in unextracted insulin was correlated only in a third order equation, $r=0.74$, $\mathrm{p}<0.01$. In sera from study $2, \%$ binding varied between undetectable to $>80 \%$. There was a quadratic relationship between \%CRA and unextracted serum insulin levels, $r=0.62, p<0.01$. Antibody binding has little if any effect on the measurement of unextracted serum below $40 \%$ CRA binding. Free insulin can not be accurately assessed in antibody containing stored sera whereas total insulin can be accurately assessed using a coated tube assay without antibody removal.

\section{8}

METABOLIC CONTROL IN PATIENTS WITH NON-INSULIN-DEPENDENT DIABETES: VALUE OF EVENING BLOOD GLUCOSE DETERMINATION P-J. Guillausseau

Dep ${ }^{t}$ of Medicine B, Hôpital Lariboisière and Université Paris VII Paris FRANCE

Monitoring of metabolic control in patients with non-insulin-dependent diabetes (NIDDM) is usually based upon blood giucose assay in the morning (after an overnight fast) and in the postprandial state (breakfast or lunch). However, this schedule does not permit the detection of "silent" low blood glucose values, especially in the evening. We have therefore conducted a prospective study of laboratory blood glucose profile determination (8 AM, $9.30 \mathrm{AM}$ after a $35 \mathrm{-g}$ carbohydrate breakfast and in the evening between 5 and $7 \mathrm{PM}$ ). We have included 58 consecutive NIDDM patients regularly followed in our clinic as outpatients $(39$ men, $60 \pm 11.5$ years diabetes duration $8.6 \pm 6.5$ years, BMl $25.5 \pm 3$ ), treated with the sulfonylurea gliclazide (40-320 mg/2.4 h, mean $170 \pm 110 \mathrm{mg}$ ), alone (group $1, n=32$ ) or in combination with metformin (1000-3000 mg/24h, $2400 \pm 620 \mathrm{mg}$ ) (group 2 $n=26$ ). All patients were stable, with no change in dosage for at least 3 months. The patients were in satisfactorly glvcemic control according to the NIDDM Management EASD Consensus (group $1 \mathrm{HbA}_{1 \mathrm{c}}: 6.5 \pm 1.1 \%$, group 2: $6.9 \pm 0.7 \%$ ). Evening blood glucose values were the lowest of the day in 26 patients of group $1(81.3 \%)$ and in 22 of group $2(84.6 \%)$. The lowest observed value was $4.3 \mathrm{mmo} / \mathrm{h}$. Mean evening blood glucose levels were lower $(P=0.001)$ than 8 AM values (group 1: $5.8 \pm 1.4$ vs $6.1 \pm 1.6 \mathrm{mmol} / \mathrm{L}$ group 2: $6.5 \pm 1.8$ vs $6.9+1.9$ ) and than 9.30 AM values (group 1: $7.6 \pm 1.5$ group 2: $12.3 \pm 2.8$ ). $\mathrm{HbA}_{1 \mathrm{c}}$ was strongly correlated $(P=0.002$ to 0.0001 ) in the whole group with 8 AM ( $r=0.39), 9.30$ AM ( $r=0.56)$, and evening blood giucose values $(r=0.42)$. These results indicate that, in patients treated with the sulfonylurea gliclazide, alone or in combination with metformin, the lowest biood glucose values occur in the evening more frequently $(4 / 5)$ than in the morning. Therefore evening blood glucose determination should be performed systematically in the course of the metabolic evaluation of NIDOM patients.

\section{9}

\section{STANDARDISATION OF GLYCATED HAEMOGLOBIN MEASUREMENTS} S.M. Marshall for the Northern Region Biochemistry Audit Group, UK.

The ability to compare $\mathrm{HbA}_{1 \mathrm{c}}$ measurements is essential to aid estimation of an individual's risk of microvascular complications of diabetes and hypoglycaemia and for audit and research between centres. Fourteen laboratories in the Northern Region, UK, using 8 different analytical methods, participated in an attempt to standardise results. Six fresh whole blood samples and 4 lyophilised standards (SKZL, Netherlands) were assayed by participants twice in 2 weeks, and by a referee laboratory using the Diamat HPLC (DCCT laboratory, University of Minnesota, USA). Using DCCT-aligned results for the lyophilised standards, a standardised $\mathrm{HbA}_{1 \mathrm{c}}$ result for each fresh blood sample was calculated and the inter-laboratory distribution of original and calculated results compared. For original results, the mean inter-laboratory coefficient of variation was 17.2 (range 11.1-21.9) $\%$ and for standardised results $8.9(7.2-10.7) \%$. However, despite this reduction in inter-laboratory variation, the lyophilised material did not behave similarly in all methods, being particularly erratic in non-liquid chromatography methods. Further comparison using fresh blood samples was therefore undertaken. Each laboratory sent 4 patient samples to the regional reference laboratory for assignment as calibrators. These values were used for result calculation following participants' analysis of 6 unknown patients samples supplied by the reference laboratory, the derived results being designated standardised $\mathrm{HbA}_{1 c}$. This cycle was repeated on 6 occasions over 3 months. For all 36 unknown samples, inter-laboratory agreement was closer for standardised results (CV 7.9 (4.8-11.0)\%) than for the measured values $(11.9(8.6-18.5) \%)$. Calculated bias against the referee result was improved in 12 of 14 laboratories by standardisation. Thus in the absence of a calibrator preparation suitable for the range of methods in common use for $\mathrm{HbA}_{10}$ analysis, cyclical referee calibration using fresh whole blood represents a useful interim strategy.
810

\section{NEW INTENSIVELY COLOURED BLUE BORONIC ACID DERIVATIVES FOR MONITORATION OF DIABETES.}

E.Dworsky ${ }^{2)}$, F.Franzen ${ }^{1}$, T.Løvli 1), A.L.Faaren ${ }^{1)}$, K.Bernström ${ }^{2)}$, L.Corneliussen ${ }^{2)}$ and E.Romundset ${ }^{2)}$. Axis Biochemicals AS, Oslo, Norway ", R\&D Section, Nycomed Pharma AS,Oslo, Norway").

Monitoring of $\mathrm{HbA}_{1 \mathrm{C}}$ is considered to be important during routine follow up of diabetic patients.A good probe for reflectometric testing of glycohemoglobin must have a) a good binding to Amadori rearranged glycoprotein residues, b) a colour different from the colour of hemoglobin, c) an extinction coefficient at the same order of magnitude as hemoglobin's, and d) a low non-specific binding to protein and filter. We have synthesized a new group of boronic acid conjugates with the triphenyl-methine dye Xylene Cyanole. In order to increase the acidity of the boronic acid residue, a para-phenyl-boronic acid coupling was used. A probe of the formula

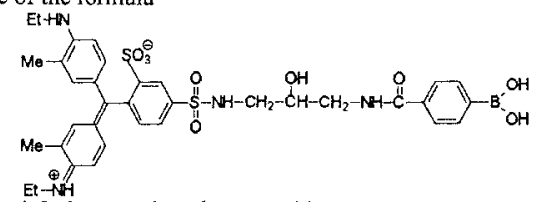

was found to satisfy these needs and was used in a reflectometric filter assay for glycohemoglobin. The reagent also includes agents to lysate erythrocytes and $\mathrm{Zn}^{2+}$ to precipitate all hemoglobin specifically. The filter device is a modified NycoCard containing a porous membrane and an underlying absorbing layer. When an aliquote of blood-reagent mixture is added to the card device, all precipitated hemoglobin remains on top of the filter. The ratio between blue glycated and red total hemoglobin is measured by reflectance. This assay correlates well with $\mathrm{HbA}_{1 \mathrm{C}}$-values obtained from chromatografic (Diamat) and immunometric (Tinaquant and DCA 2000) methods, $r \geq 0,96$. 
Evaluation of two enzyme amplified immunoassays detecting total and intact proinsulin.

C.Dark', D.Lewrey', E.L.Willers', N.M.Wisdom, L.Ashworth', N. Walker' ${ }^{2}$, D. Humphriss' D.L.Bates'. 'DAKO Diagnostics Ltd, Angel Drove, Ely, Cambridgeshire. CB7 4ET, UK. 'Department of Medicine, The Medical School, Newcastle upon Tyne, NE2 4HH, UK. Two proinsulin enzyme immunoassays have been developed by DAKO Diagnostics Ltd. The protocol for both assays is simple and easy to perform. Total assay time is less than 3 hours untll results become availabie. Hands on time is less than 15 minutes. Both assays achieve detection imits less than $0.1 \mathrm{pmol} / \mathrm{L}$. Assay performance was evaluated and gave the following results.

\begin{tabular}{cccccccc} 
& & \multicolumn{3}{c}{ Within assay imprecision } & \multicolumn{3}{c}{ Between assay imprecision } \\
\hline \multirow{5}{*}{ Total Proinsulin } & & Proinsulin(pmo/L) & $n$ & CV\% & Proinsulin(pmoliL) & $\pi$ & CV\% \\
& Pool 1 & 29 & 20 & 4 & 29 & 34 & 4 \\
& Pool 2 & 48 & 20 & 3 & 47 & 34 & 5 \\
& Pool 3 & 121 & 20 & 6 & 115 & 34 & 7 \\
Intact Proinsulin in & Pool 1 & 9 & 20 & 5 & 11 & 58 & 7 \\
& Pool 2 & 26 & 20 & 4 & 29 & 58 & 5 \\
& Pool 3 & 100 & 20 & 5 & 99 & 58 & 5
\end{tabular}

A theoretical detection limit (plus 2.5 sd of zero calibrator) of $0.07 \mathrm{pmol} / \mathrm{L}$ was obtained using an overnight protocol for both assays, whilst a detection limit of $0.15 \mathrm{pmol} / \mathrm{L}$ was obtained for both assays using a three hour protocol. Mean observed recovery after dilution down to 1 in 8 of the original value was $104 \%$ for total proinsulin (range 99 to $110 \%$, and $108 \%$ for intact proinsulin (range 101 to 119\%). Proinsulin was spiked into normal human serum and gave recoveries ranging from 87 to $98 \%$ for both assays over the range 20 to $75 \mathrm{pmol} / \mathrm{L}$. No high sample read back onto the standard curve up to $4000 \mathrm{pmol} / \mathrm{L}$. No interference was seen with Insulin up to $6000 \mathrm{pmol} / \mathrm{L}$ and $\mathrm{C}$-peptide up to $30,000 \mathrm{pmol} / \mathrm{L}$. These assays were used to determine ranges for intact and total proinsulin for 150 normal controls with no family history of diabetes or related disorders over the course of an OGTT. Samples were kindly provided by the Newcastle family study group. Mean fasting anaiyte concentration was $9.6 \mathrm{pmol} / \mathrm{L}$ (range $3.4-27.3 \mathrm{pmo} / \mathrm{L}$ ) for total proinsulin and $4.1 \mathrm{pmo} / \mathrm{L}$ (range 0.95 10.6pmol/L) for intact proinsulin. Both proinsulin assays are ideal for use in the investigation of type II diabetes and related disorders.

\section{PS 43 \\ Devices}

\section{2}

TREATMENT OF DAWN PHENOMENON CONTROLLEI) BY CONIINI OUS TISSUE GLITOSE MONITORING

U. Hoss, M.I. Salgado, F. Stemberg and E.F. Pfeiffer, Institute of I iabetes Technology at the Univ ersity of Ulm, Ulm, Germany

Most diabetic patients have increased insulin requirements in the morning hours. To treat this dawn phenomenon it is recommended to give bed time injection of retard insulin late in the evening for intensively treated patients (IIT) or concerning patients on continuolis subcutaneous insulin infusion (CSII) to rise hasal rate at about $3.00 \mathrm{am}$. The efficacy of this therapies was determined by continuous monitoring of tissue glucose during night. Tissue glucose was recovered by perfusing a microdialysis probe implanted in the abdominal subcutaneous tissue and measured by a portable enzvmatic amperometric glucosensor. Correlation to intermittently taken venous blood glucose reference values was excellent so that we could evaluate continuous glucose profiles during the whole night. We examined 12 patients treated with IIT and 12 patients with CSII. Dinner was given at $6: 00 \mathrm{pm}$ and all patients performed their usual insulin therapy. The quality of control of dawn phenomenon was evaluated by the Fasting Ascending Glycemic Excursion - index (FAGE) representing the difference between fasting glucose and the lowest glucose concentration during night. FAGE of patients treated with insulin pump therapy were significant lower than of IIT patients $(\alpha<0.05)$. Mean values $+/$-standard deviations were calculated to $\mathrm{FAGE}_{\mathrm{II}}=93.0+/-51.5 \mathrm{mg} / \mathrm{dl}, \mathrm{FAGE}_{\mathrm{CsI}}=34.9+/-28.4 \mathrm{mg} / \mathrm{dl}$. Also the range of FAGE index was greater in the IIT group $(\max =198 \mathrm{mg} / \mathrm{dl})$ than in the CSIl (max $=107 \mathrm{mg} / \mathrm{dl}$ ) group. Nevertheless 5 patients with insulin pump therapy had FAGE values greater than the acceptable range (FAGE $>36 \mathrm{mg} / \mathrm{dl}$ ). We conclude that CSII is superior to IIT in treating dawn phenomenon but there is still need for improvement for example by feed back regulation.

\section{3}

ARE CATHETER OCCLUSIONS RELATED TO INSULIN IMMUNOGENICITY DURING TREATMENT BY IMPLANTABLE PUMPS ? P.Belicar*, V. Lassmann-Vague*,MC Rojat-Habib**, JF Pellissier ${ }^{* *}$, C. Alessis* and P. Vague ${ }^{*} .{ }^{*}$ Diabetes Department, **Laboratory of anatomy and pathology. CHU Timone- Marseille - France.

We evaluated the relation between intraperitoneal (IP) catheter occlusions and insulin immunogenicity in IP insulin treated patients. Twenty-four type 1 IDD patients were treated by an implantable pump infusing Hoechst* $21 \mathrm{PH}$ insulin. Cumulative follow-up was 65.4 patients-years. Twenty-six definitive catheter occlusions were diagnosed (incidence : $39.7 \%$ p.y ). Anti-insulin antibodies (AIA) were measured by RIA. Patients were divided in 3 groups. Group 1 consisted of 6 patients who did not experience any catheter occlusion, group 2 included 13 patients who had only 1 catheter occlusion and group 3 consisted of 5 patients who experienced 2 or more catheter blockages. Before implantation, AIA level was $12 \pm 8 \%(\mathrm{~m} \pm \mathrm{SD})$ in group $1,23 \pm 18 \%$ in group2, $29 \pm 11 \%$ in group3. Twenty-four months after implantation, AIA level increased in the 3 groups : $24 \pm 21 \%$ in group $1,35 \pm 29 \%$ in group 2,55 $\pm 12 \%$ in group 3. Pre and post-implantation AIA levels are significantly lower in group 1. No significant increase of AIA level was observed just before and after a catheter occlusion ( $36 \pm 28 \%$ vs $40 \pm 29 \%$ ). The occlusion was : in 8 cases, an encapsulation around the peritoneal catheter tip for which histological analysis revealed anti-insulin amyloid deposits, and in 14 cases, a clot inside the lumen of the catheter. Mean AIA level, was $50 \pm 35 \%$ in patients with catheter encapsulation and $28 \pm 27 \%$ in patients with clot-occluded catheters.In conclusion catheter occlusion per se does not modify anti-insulin immunogenicity. But occurrence of catheter obstructions during IP insulin rreatment is related to AIA level. So a specific immune predisposition could be a risk factor for catheter occlusions. Catheter encapsulation is induced by a peritoneal immune reaction leading to anti-insulin amyloïd deposits,and is associated to a higher systemic immune reaction against insulin. 


\section{4}

\section{PRELIMINARY CLINICAL EVALUATION OF A NEW} SUBCUTANEOUS GLUCOSE SENSOR

J.J. Mastrototaro', W.P. Van Antwerp ${ }^{1}$, and J.H. Mestman' ${ }^{2}$, ${ }^{1}$ MiniMed, Inc., Sylmar, CA, USA, ${ }^{2}$ University of Southern Califomia, Center for Diabetes and Metabolic Diseases, Los Angeles, CA, USA.

Eleven Type I and one Type II volunteers participated in a clinical feasibility study of a subcutaneous glucose sensor [MiniMed Inc.]. The feasibility study evaluated the safety and performance characteristics of the sensor system over a four day period. The commercial product would provide a practical, user-friendly system for continuous glucose monitoring of patients with diabetes. The electroenzymatic sensor is manufactured using integrated circuit fabrication processes on a small flexible substrate for added patient comfort. A one-step, relatively painfree, insertion process is used to position the sensor in the tissue. In some patients, more than one sensor was inserted. Following sensor insertion, patients were asked to follow their typical meal schedule and insulin dosing regimens. An intravenous line was placed in the forearm or hand of the volunteer for periodic venous sampling. The accuracy of the sensor was determined by a parallel comparison to the venous blood glucose concentration determined using a Yellow Springs Instruments 2700 Glucose Analyzer or a HemoCue B-Glucose Analyzer. Sensors were inserted into the subcutaneous tissue of the abdomen $(\mathrm{N}=16)$ and upper arm $(N=10)$. Initial results have shown the sensor to be simple to insert, safe, and non-irritating in the subcutaneous tissue (average implant duration of 49.9 hours). Sensor functional performance following a 1-4 hour equilibration period has been mixed - some sensors (67\%) performing within specification $( \pm 15 \%)$, and others demonstrating intermittent performance. Issues requiring additional study include sensor connection failures, inadequate signal filtering, and potential questions regarding tissue fluid perfusion at the sensor site. Sensor design modifications are being evaluated to address these issues.

\section{5}

NOVEL "MEMBRANE FREE" ENZYMATIC-AMPEROMETRIC GLUCOSENSOR ALLOWS LONG-TERM CONTINUOUS TISSUE GLUCOSE MONITORING WITH MICRODIALYSIS

F. Sternberg, M.I. Salgado, U. Hoss, E.F. Pfeiffer. Institut fuir Diabetes-Technologie an der Universität Ulm, Ulm, Germany

Several types of glucosensors are actually under clinical trial. Most of them face problems such as lack of long-term stability when applied in-vivo. We developed a novel method to on-line continuously monitor the tissue glucose (TG). This technique is based on a "membrane -free" enzymatic-amperometric method combination with microdialysis, i.e glucose oxidase is no longer immobilized in a double layer membrane. It consists of perfusing phosphate buffered saline in a microdialysis probe ( perfusion flow $=0.5-1,0 \mu \mathrm{l} / \mathrm{min}$., in-vivo glucose recovery $>$ $50 \%$ ) inserted in the abdominal s.c. tissue. TG diffuses into the probe and is transported through a tubing system to a flow chamber. In the tubing system glucose is oxidized by fresh supplied glucose oxidase producing $\mathrm{H}_{2} \mathrm{O}_{2}$. and gluconolactone. $\mathrm{H}_{2} \mathrm{O}_{2}$ delivers electrons (proportional to the TG) at a $\mathrm{Ag} / \mathrm{Ag} / \mathrm{Pt}$ electrode system (polarization $=700 \mathrm{mV}$ ). The resulting electric current (sensor signal) is amplified, digitalized, minutely stored in a memory and telemetrically transmitted to a wrist watch which shows the actual glucose value and contains an optic and an acoustic alarm. The method showed to be stable for over a period of two weeks in in-vitro experiments in glucose standards and in plasma. When applied in humans, the glucose monitoring remained stable for more than three days covering a glucose range of $37-445 \mathrm{mg} / \mathrm{dl}$. Due to the high TG recovery, extrinsic factors such as body movement did not affect the excellent quality of the sensor signal. Temperature influence on the sensor signal has been mathematically compensated. A "one-point in vitro, one-point in-vivo" calibration was performed. Our glucosensor allows a long-term stable TG monitoring, may be miniaturized to an acceptable size (now: $15 \times 8 \times 3 \mathrm{~cm} ; 250 \mathrm{~g}$ ), it could be used as hypo/hyperglycemic alarm and for an automated glucose controlled insulin delivery system.

\section{6}

IMPROVED NON-INV ASIVE GLUCOSE MONITORING IN DOGS M. Rebec, K. Black, T. Benson, D. Neal* and A. Cherrington* Vanderbilt University*, Nashville, TN, Bayer Corporation, Elkhart, IN

Several reports and patents have described non-invasive methods for measuring glucose that employ transmission or reflectance of near infrared light. The calibration protocol commonly used was to monitor glucose by an invasive method while collecting spectral data after administration of a glucose load by ingestion or bolus IV. This procedure can cause significant changes in temperature, tonicity and capillary bed flow at the measurement site. The spectral features related to the physiological changes would become incorporated in the calibration model. The calibration model then becomes experiment specific and may not transfer well. By mirimizing the inclusion of non-glucose spectral information, one can develop a more general and robust model. To reach this goal, we used a protocol of up to 7 clamped glucose levels (each held constant for up to two hours). Plasma glucose determinations were by with a Beckman ${ }^{1}$ Glucose Analyzer instrument. Spectral measurements were made after physiological steady state was achieved. The transmission spectra were collected over the range of 1450 to $2400 \mathrm{~nm}$, and we monitored and controlled the temperature and pressure at the interface of the optoid and tissue. The measurement sites were the cutaneous marginal sac of anesthetized dogs' ears. With these improvements, we were able to extend the range of glucose prediction from 1.94 to $24.36 \mathrm{mmol} / \mathrm{l}$. Partial Least Squares and Neural Network analyses were used. The best standard error of prediction (SEP) with cross-validation achieved was $0.29 \mathrm{mmol} / \mathrm{l}$. For 20 studies, the average SEP was $0.57 \mathrm{mmol} / 1$ with 7 fitting factors, and $r^{2}=$ 0.984 for the correlation with plasma glucose values. We conducted several hypoglycemic studies covering glucose concentrations from 1.94 to 13.00 $\mathrm{mmol} / 1$ and obtained standard errors of predictions of $0.50 \mathrm{mmol} / 1$. This combination of a unique experimental and instrument approach provides a solid basis for significant advances in non-invasive glucose measurements.

\section{7}

\author{
ASSESSMENT OF THE "TISS" - GLUCOSE UTLLIZATION \\ MEASUREMENT SYSTEM
}

J.Wójcicki, J.Krzymień*, J.Blachowicz, P.Ładyżyński, P.Pacuła* and A.Czyżyk*, Institute of Biocybernetics \& Biomedical Engineering P.A.S., *Department of Gastroenterology \& Metabolic Diseases M.A., Warsaw, Poland.

Aim of the study was to evaluate TISS - recently constructed computerized system for measurement of the glucose utilization (GU) using euglycemic clamp technique. Technical characteristic : target clamp value $-90 \mathrm{mg} / \mathrm{dl}$, insulinemia level $-100 \mu \mathrm{U} / \mathrm{ml}$; duration of the procedure $-90 \mathrm{~min}$, evaluated period - last 60 min; BG measurements by Bayer Ames Ghucofilm reagent strips - doubled and carried out every 5 minutes. Basic evaluation of the system was performed for 3 groups of patients: heaithy volunteers $(\mathrm{N}=14$ ), patients with newly diagnosed NIDDM $(N=17)$ and patients with acromegaly $(N=14)$. Obtained results of GU measurements are as follow: for the first group $4.11 \pm 1.98 \mathrm{mg} / \mathrm{kg} / \mathrm{min}$, for the second group - $1.9 \pm 1.1 \mathrm{mg} / \mathrm{kg} / \mathrm{min}$ and for the third group $2.45 \pm 0.9 \mathrm{mg} / \mathrm{kg} / \mathrm{min}$. Accuracy of the measurements assessed by the coefficient of the target clamp value variation (c.v.) has been found to be: $6.44 \pm 2.2 \%, 5.1 \pm 2.3 \%$ and $5.74 \pm 1.4 \%$ respectively for all mentioned above groups of patients. An analysis of the mean GU measurements $(\mathrm{mg} / \mathrm{kg} / \mathrm{min})$ in respect to their BMI values led to following results : for healthy volunt. with $\mathrm{BMI}>27-\mathrm{GU}=3.04$ and with $\mathrm{BMI}<27$. $\mathrm{GU}=5.55$, for NIDDM patients with $\mathrm{BMI}>27-\mathrm{GU}=1.61$ and with $\mathrm{BMI}<27$. $\mathrm{GU}=2.85$, for patients with acromegaly with $\mathrm{BMI}>27-\mathrm{GU}=2.13$ and with $\mathrm{BMI}<27-\mathrm{GU}=3,25$. The presented above data has been achieved with following mean values of the coefficient of variation (\%) : for healthy volunt. with $B M I>27$ - c.v. $=5.4$ and with $B M T<27$ - c.v. $=7.8$, for NIDDM patients with BMI $>27$ c.v. $=4.4$ and with $B M \Pi<27-$ c.v $=5.4$, for patients with acromegaly with $B M I>27$ c.v. $=5.4$ and with $B M I<27-c . v .=6.7$. It is seen that the developed system operates with highest accuracy for patients with insulin resistance and obesity (c.v. $\sim 5 \%$ ). For patients with BMI $<27$ the values of c.v. are $\sim 8 \%$. TISS has been found to be easy to handle by one person from the middle medical staff. 
818

A. COMPARATIVE STUDY BETWEEN A NEW PC-BASED INFRARED PHOTOPLETHYSMOGRAPY AND A LASER DOPPLER FLOWMETRY S. Pruna, C. Ionescu-Tirgoviste and I. Mincu, Electrophysiology and Bioelectronics Laboratory, Institute "N. Paulescu", I. Movila Street, 5-7, 79811, Bucharest, Romania. The medical technology to predict diabetes foot complications is becoming increasingly more expensive. In order to address this problem we have developed an inexpensive, PCbased piece of equipment, capable of non-invasive measurement of peripheral nerve function and vascular assessment and automated analysis of the signals for simple, quickly and routinely use in clinical practice and research in diabetes foot care. To qualify the equipment's ability for early detection of the impairment of the skin blood perfusion at the foot level, the new PC-based infrared photoplethismography (PPG) and a laser Doppler flowmetry (LDF) were evaluated by a preliminary comparative study. Blood perfusion in skin was measured in 29 IDDM selected patients (mean age 37.65 t 14.7 yrs., duration of diabetes $15.76 \pm 8.38 \mathrm{yts}$.) both with a laser Doppier flowmeter, emission of $780 \mathrm{~nm}, 0.8 \mathrm{~mW}$ (Periflux System 4001, Perimed, Sweden) and with the new PC-based device, with infrared sensor which has the potential to record and realtime analysing of the signal. The probe assembly uses a spectrally matched near infrared ernitter and sensor. The infrared emitter has $15 \mathrm{mw}$ radiant output power, wavelength $950 \mathrm{~nm}$ and the sensor is a high-speed PIN photodiode with high photosensitivity, housed in a black infra-red transmisive moulding which reduces ambient white light interference. Measurements were made successively at the same anatomical sites to measure blood flow in the same vascular bed: left big toe, right big toe and left index finger at the same local skin temperature. The subjects were supine and in resting condition during the recording session. Two sets of measurements: average PPG amplitude ( $\mathrm{mV}$ ) and LDF perfusion units (PU) were made from each subject, each set lasting 26 seconds, i.e. about $35 \mathrm{HR}$ cycles. There was a significant correlation between the average PPG amplitude, expressed in $\mathrm{mV}$, and LDF expressed in arbitrary perfusion units (PU) at each anatomical site: left big toe $298.7 \pm 232.2 \mathrm{mV}$ for $\mathrm{PPG}, 34.3 \pm 19.8$ $\mathrm{PU}$ for $\mathrm{LDF}$, ( $=0.64, \mathrm{p}<0.002$ ); right big to $517.1 \pm 336,9 \mathrm{mV}$ for $\mathrm{PPG}, 53.9 \pm 41.2 \mathrm{PU}$

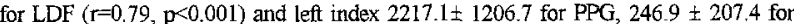
LDF ( $r=0.45, p<0.001$ ). In conclusion, this work has indicated that the PPG and LDF are equally suitable of use in the diagnosis of the skin blood perfusion impairment at foot level in diabetes. However, the PPG technique is cheaper than the LDF technique.
819

\section{EVALUATION OF THE NEW IMPROVED DCA $2000^{\circ}$ ANALYZER FOR RAPID HbA1c MEASUREMENTS}

B. Bürgstein, H.G. Wahl, R. Lehmann, H.-U. Häring and R.-M. Schmüiling, Medizinische Universitätsklinik Abt. IV. 72076 Tübingen, Germany

The DCA 2000 Analyzer (Bayer Diannostics. Germany) was developed for rapid $\mathrm{HbAlc}$ measurements in outpatient settings. The assay is based on a latex immunoagglutination inhibition methodology, Both the concentration of total hemoglobin and HbAlc specifically are measured and the ratio calculated as percent $\mathrm{HbAlc}$. By modifying the buffer solution as well as the absorbance measurements total analysis time was reduced from 9 to 6 minutes. We measured 643 capillary, EDTA and NaF blood samples in our diabetic clinic with the old and the new reagent kits and compared the results to the routine HPLC method (Diamat, Biorad Germany). Data were evaluated by the Passing/Bablock procedures. The intra-assay coefficients of variation in a $\mathrm{HbAl}$ c range from 4.3 to $14.1 \%(n=50)$ were determined as 0.9 to $2.5 \%$ (HPLC), 1.6 to $3.1 \%$ (9min DCA) and 0.6 to $2.4 \%$ (6min DCA). There was no difference observed when venous blood samples with either EDTA or NaF as anticoagulants were used instead of capillary blood samples $(n=74)$. The DCA 2000 assay can be used for a total hemoglobin range from 6 to $20 \mathrm{~g} / \mathrm{dL}(n=42)$. For both DCA test kits there were no interferences observed in blood samples with bilirubin up to $40 \mathrm{mg} / \mathrm{dL}$ $(n=55)$, triglycerides up to $2056 \mathrm{mg} / \mathrm{dL}(n=57)$ and rheumatoid factor up to $551 \mathrm{U} / \mathrm{mL}(\mathrm{n}=41)$. Old blood samples with high triglycerides caused error messages and could not be measured. The correlation with the HPLC method yielded different results $(y=$ HPLC, $x=D C A 2000): y=-0.56+1.16 x$ $(r=0.97)$ for the 9 minutes test kit and $y=-0.70+1.10 x(r=0.98)$ for the 6 minutes test kit. Therefore patient results from the new test kit should not be related directly to those from the old one, especially at higher HbAlc levels. The new improved test kit shows better precision and stronger correlation to the HPLC method and with the immediate availability of $\mathrm{HbAl} \mathrm{c}$ results it is therefore of advantage for use in outpatient settings.

\section{0}

BENEFITS OF CONTINUOUS SUBCUTANEOUS GLUCOSE MONITORING ON THE METABOLIC STATE IN IDDM PATIENTS M.I. Salgado, U. Hoss, F. Stemberg, E.F. Pfeiffer: Institut für DiabetesTechnologie an der Universität Ulm, Ulm, Germany

The aim of the diabetes treatment is to achieve a good metabolic control in order to avoid the late complications. Since many years, the determination of $\mathrm{HbA}_{15}$ has been considered the "golden standard" for the long term evaluation of the metabolic state. However, the daily fluctuations of the blood glucose mav only be determined by its continuous monitoring. In Ulm we developed a glucosensor "Ulmer Zuckeruhr-System", based on the enzymaticamperometric method which is combined with the microdialysis technique We performed two series of $24 \mathrm{~h}$ continuous tissue glucose (TG) monitoring $(n=20)$ in 10 intensified insulin treated IDDM patients, (4 women, 6 men; age $36 \pm 11$ years; BMI $24.2 \pm 1.3 \mathrm{~kg} / \mathrm{m}^{2}$; diabetes duration $20 \pm 13$ years; $\mathrm{Hb} \mathrm{A}_{1 \mathrm{c}}$ $6.7 \pm 0.5 \%$ ) using the Ulmer Zuckeruhr-System, in order to compare ordinary daily glucose profiles with the patients $\mathrm{Hb}_{16}$. The glucose range was between $39-325 \mathrm{mg} / \mathrm{dl}$ (mean $\pm \mathrm{SD}: 140 \pm 26 \mathrm{mg} / \mathrm{d}$ ). The mean daily glucose excursion was $179 \pm 46 \mathrm{mg} / \mathrm{dl}$ (range $=121-286 \mathrm{mg} / \mathrm{di}$ ). Hypoglycemic episodes occurred in 15 experiments and were effectively detected with our glucosensor. In 8 cases glucose values over $250 \mathrm{mg} / \mathrm{dl}$ were observed. The Schlichtkrull "M" value and the "J" index were $17 \pm 7$ and $20 \pm 7$, respectively i.e. the profiles can be assigned to "ideal" and "good". This study shows that although the conventional parameters used to evaluate the metabolic state in our patients were acceptable, a continuous glucose monitoring reflecting the minutely TG changes demonstrated that considerable glucose fluctuations occur during daily profiles. Further studies should evaluate the possible longterm effects of these extreme daily glucose fluctuations. In addition, the conventional methods to evaluate the metabolic state should be reconsidered.

\section{1}

BLOOD GLUCOSE SELF-MONITORING N BLIND DLABETIC PATIENTS R. Windecker, L. Heineraann, M. Seid and P.T. Sawicki, Department of metabolic diseases and nutrition, Heinrich-Heine-University Düsseldorf, Germany

Reliable blood glucose self-monitoring is most important for blind patients with insulin-dependent (type I) diabetes mellitus to improve their metabolic control and to reduce the increased risk of severe hypoglycemia. Therefore, we studied the reliability and handling of blood glucose self-monitoring (BGSM) using the One Touch II talk device (OT II) in 21 type I-diabetic patients (18 legally blind patients (duration of blindness $10 \pm 8$ years), 3 patients with severe vision impairment, 18 on intensified insulin therapy, 3 on conventional insulin therapy, age $43 \pm 13$ years, duration of diabetes $27 \pm 9$ years; meant $\mathrm{SD}$ ) in comparison to their previous $\mathrm{BGSM}$ method. The blood glucose values of the OT II were compared to 101 parallel glucose measurements in venous blood-samples performed by a trained laboratory assistant using the glucose-oxidase-method. (6.8 03.7 (range: $0.8-17.3$ ) vs. $6.9 \pm 3.7$ $(0.9-16.9) \mathrm{mmol} / \mathrm{l}$; mean absolute difference $0.1 \pm 0.7(-1.3-2.1) \mathrm{mmol} / \mathrm{j} ;=0.98)$. After the patients had used the OT II for at least 4 weeks the glucose values of 3 different capillary blood samples measured by every patient were compared again to laboratory control measurements $(9.7 \pm 2.8(3.1-14.9)$ vs. $9.6 \pm 2.9(3.6-14.3)$ $\mathrm{mmol} / /$; mean absolute difference $0.1 \pm 0.8(-1.9-2.8) \mathrm{mmol} / / \mathrm{r}=0.96)$. Additionally, in 11 patients the precision of their previously used BGSM-devices was compared to laboratory control measurements ( 8 different types of glucometers; $11.2 \pm 5.8$ $(5.2-27.8)$ vs. $11.7 \pm 5.7(5.5-27.7) \mathrm{mmol} / /$; mean absolute difference $-0.5 \pm 2.9$ $(-7.7-4.8) ; r=0.88) .76^{\circ} \%$ of these values showed a deviation from the reference value of $>10 \%, 46 \%$ a deviation of $>20 \%$. Compared to this, $12 \%$ of the measurements of the OT II revealed a deviation from the reference values of $>10 \% ; 3 \%$ a deviation of $>20 \%$ (deviation difference between the methods $p<0.0001$ ). In conclusion, the One Touch II talk device used by blind patients showed a higher precision than previously used BGSM-devices. Blind patients with insulin dependent diabetes are able to perform reliable BGSM without help. 
PS 44

\section{Hypoglycaemia}

822

BLOOD GLUCOSE AWARENESS IN PATIENTS WITH REPEATED SEVERE HYPOGLYCEMIA

A. Fritsche, G. Schnauder, M. Stumvoll, W. Renn, H.U. Häring and R.M. Schmülling Medizinische Klinik IV, Universität Tübingen. Germany Diabetic patients with severe hypoglycemia are at high risk to suffer from repeated severe hypoglvcemia (i.v. glucose/glucagon). In order to further characterize these patients, we studied blood glucose (BG) perception and frequency of low BG-levels of 33 unselected type 1 diabetic patients taking part in a structured inpatient group teaching and treatment programme of one week. 11 had repeated severe hypoglycenia (SH), the others served as control (C) Patients with SH were older ( $43 \pm 12$ vs $31 \pm 10$ years) and had a longer duration of diabetes ( $18 \pm 7$ vs $9 \pm 10$ years). To specify hypoglycemia perception they estimated their BG-level every time before measurement for 10 weeks during and after the education. We calculated accuracy of blood glucose perception [error grid analysis, accuracy index reaching from $-100 \%$ (dangerous) to $+100 \%$ (correct)], sensitivity for and prevalence of low BGlevels (BG $<3.9 \mathrm{mmol} / \mathrm{l})$. Patients with $\mathrm{SH}$ had significantly lower accuracy of BG-estimation (SH: $31 \pm 7, \mathrm{C}: 48 \pm 4 \%$, mean \pm SEM) and sensitivity for BG levels $<3.9$ mmol/1 (SH: $40 \pm 6$, C: $58 \pm 5 \%$ ), whereas HBAlc (SH: 8.1 $\pm 0.3, \mathrm{C}: 7.9 \pm 0.4 \%)$ and prevalence of $\mathrm{BG}<3.9 \mathrm{mmol} / \mathrm{l}(\mathrm{SH}: 19 \pm 2, \mathrm{C}: 18$ $\pm 2 \%$ ) were not different. In both groups HBAlc decreased after the treatment programme (SH $-0.7 \pm 0.4 \%, C-1.0 \pm 0.3 \%$ ) with no significant difference between groups. Only SH were able to improve their accuracy of BG perception significantly during the study whereas both groups failed to improve sersitivity for low BG-levels significantly $(p=0.06)$. Changes in prevalence of BG levels $<3.9 \mathrm{mmol} / 1$ did not correlate with changes in sensitivity for low BG levels. We conclude that patients with repeated severe hypoglycemia have poorer awareness of BG but similar metabolic control compared to patients without SH. Despite improved metabolic control patients with severe hypoglycemia are able to improve their awareness of BG after our teaching and treatment programme.

\section{3}

Previous hypoglycemic episodes do not cause permanent cognitive brain dysfunction in IDDM on intensive insulin treatment

L KRAMER, P FASCHING, C MADL, W WALDHÄUSL, K IRSIGLER, $G$ GRIMM Departments of Medicine $N$ and III, Universify of Vienna, 2nd Department of Medicine, Krankenhaus Wien - Lainz; Vienna, Austria

Intensive insulin treatment of insulin-dependent diabetes mellitus (IDDM) may be associated with increased frequency of severe hypoglycemia. While brain function is impaired during acute hypoglycemia, it is not clear whether there are permanent sequelae after recovery. This study addressed the impact of previous severe hypoglycemia on cognitive brain function in patients with IDDM on intensive insulin treatment. A total of 108 IDDM patients - 55 (age, $38 \pm 14$ years, mean $\pm S D$ ) with a history of at least one episode (median 3, range 1 to 35 ) of comatose hypogiycemia and 53 without a history of severe hypoglycemia $(34 \pm 13$ years) were studied by $\mathrm{P} 300$ event-related potentials (an objective electrophysiological measure of cognitive brain function) and psychometric tests (mini-mental state, trailmaking test) during euglycemia at least four weeks after recovery from severe hypoglycemia. Findings were compared to 108 healthy subjects matched by age and sex. No difference was seen in P300 latencies and psychometric tests between patients with a history of severe hypoglycemia and those without (P300 latency at vertex: $344 \mathrm{~ms}$ with severe hypoglycemia versus $342 \mathrm{~ms}$ without; trailmaking test: 31 versus $30 \mathrm{~s}$; mini-mental state score: 29.5 versus 29.6 , respectively). In the cohort of all patients, P300 peak latencies were prolonged ( $344 \mathrm{~ms}$, impaired) as compared to healthy subjects $(332 \mathrm{~ms}, \mathrm{P}<0.001)$. However, mini-mental state $(29.5$ versus 29.6, $P=0.59$ ) and trailmaking test ( 31 versus 28 seconds, $P=0.10$ ) did not differ between patients and healthy subjects. In conclusion, episodes of hypoglycemic coma do not commonly cause permanent impairment of cognitive brain function in patients with IDDM, although their cognitive brain function is subclinically impaired compared to that of healthy subjects.
824

ABNORMALITIES ON THE ECG DURING SPONTANEOUS OVERNIGHT HYPOGLYCAEMIA IN PATIENTS WITH TYPE 1 DIABETES

J.L.B. Marques, N.D. Harris, S. Marlow ${ }^{a}$, C. Bedford ${ }^{a}$, T. Cochrane and S.R. Heller ${ }^{\text {a }}$. Department of Medical Physics, Royal Hallamshire Hospital and ${ }^{a}$ Diabetes Centre, Northern General Hospital, Sheffield S5 7AU - UK.

We have previously shown that experimental hypoglycaemia alters the ECG of non-diabetic, IDDM and NIDDM subjects; changes which are associated with malignant tachy-dysrhythmias in other conditions. However it is unknown whether such changes occur during spontaneous overnight hypoglycaemia ( $\mathrm{SOH}$ ). We studied the ECG of 7 Type 1 diabetic patients (19-50 years, $H_{b A_{1 C}} 5.2-8.3 \%$ ) over 9 nights, using a highresolution ECG system. Patients kept their routine treatment but were admitted for overnight monitoring with hourly measurements of blood glucose (BG) and ECG analysis (QRS complex duration and corrected QT interval $Q T C)$. We calculated the maximum change in $Q R S$ and QTC throughout the night when $B G>5.0 \mathrm{mmol} / 1$. The mean [range] change in QRS was $3.7 \mathrm{~ms}[1.3$ to 6.7$]$ and $14 \mathrm{~ms}[12$ to 18$]$ in QTC interval $(n=5$, a total of 46 hours). SOH occurred on 4 nights with a $B G$ nadir (Mean $\pm S E M$ ) of $2.6 \pm 0.2 \mathrm{mmol} / \mathrm{l}$. On these nights, there were clear alterations of the ECG throughout the period (1-3 hours) when $B G<3.5 \mathrm{mmol} / 1$. On 3 nights, QRS and QTC changed from $92.4 \pm 0.4 \mathrm{~ms}$ and $431 \pm 12 \mathrm{~ms}$ when $B G>5.0 \mathrm{mmol} / \mathrm{l}$, to $100.9 \pm 2 \mathrm{~ms}$ and $487 \pm 33 \mathrm{~ms}$ dusing $\mathrm{SOH}$, respectively. One patient $(B G=2.5 \mathrm{mmol} / \mathrm{l})$ developed and sustained $(>5 \mathrm{~min}$.) an aberrant cardiac rhythm of $33 \mathrm{bpm}$ precluding ECG measurements. This preliminary study has shown that in patients with a normal or high $B G$ the ECG is stable throughout the night whereas $\mathrm{SOH}$ can cause alteration of the ECG (lengthening the QRS and QTC interval), in a similar way to that seen during induced hypoglycaemia. Around one third of all nights were characterised by recurrent SOH episodes. These patients may experience alterations of cardiac repolarisation for relatively lang periods of time during the night which may predispose them to cardiac rhythm disturbances.
825

A COMPARISON OF HYPOGLYCAEMIA INDUCED BY HUMAN SOLUBLE INSULIN AND LYS-PRO (LY275585) INSULIN. RJ MCCRIMMON and BM FRIER

Department of Diabetes, Royal Infirmary, Edinburgh, Scotland. Lys (B28) Pro (B29) (LP) is an analogue of human insulin, characterised by its rapid absorption, and short duration of action. To examine the symptom profile, counterregulatory hormonal response, and the glycaemic threshold for onset of the sympatho-adrenal (autonomic) response to hypoglycaemia induced by LP, 16 patients with insulin-dependent diabetes, aged $32.5(20-45)$ (median (range)) years, disease duration $3.0(0.5-4.5)$ years, and BMI $24(21-26) \mathrm{kg} / \mathrm{m}^{2}$ were enrolled in a randomised, double-blind study comparing LP with human soluble insulin (HS). At baseline subjects were stabilised at normoglycaemia. A slow intravenous infusion of insulin ( $2 \mathrm{rU} / \mathrm{kg} / \mathrm{min}$ ) induced a controlled decline in blood glucose. Measurements of hypoglycaemia symptoms, cognitive function (Digit Symbol Substitution and Trail-Making B tasks) and counterregulatory hormones (adrenaline, noradrenaline, glucagon, $\mathrm{ACTH}$, growth hormone and pancreatic polypeptide) were made at intervals preceding and following the autonomic reaction $(\mathrm{R})$, which was identified by significant increments in sweating, heart rate and systolic blood pressure. Results: At R significant increments occurred in systolic blood pressure $(p<.05)$, heart rate $(p<.05)$, and in autonomic $(p<.05)$ and neuroglycopenic symptom scores $(p<.05)$, while a significant deterioration was observed in cognitive performance $(p<.05)$ for both HS and LP insulins. This coincided with a significant increase from baseline in all of the counterregulatory hormones $(p<.01)$. No significant differences were apparent between HS and LP insulins for any of the variables studied at any of the time points in this study. The glycaemic thresholds (mean $\pm S D$ ) for the autonomic reaction $(R)$ were $2.05( \pm 0.6) \mathrm{mmol} / \mathrm{l}$ for $\mathrm{LP}$ and $1.94( \pm 0.6) \mathrm{mmol} / \mathrm{t}$ for HS, and were not significantly different. Conclusion: The glycaemic threshold for symptoms, and the symptom profile, autonomic, and counterregulatory hormonal responses to hypoglycaemia induced by Lys-Pro were identical to human soluble insulin in insulin-treated diabetic patients. 
826

\section{GIN AND TONIC AND REACTIVE HYPOGLYCAEMIA. WHAT IS IMPORTANT - GIN , TONIC OR BOTH?}

D. Flanagan, ${ }^{*}$ R. Sherwin, J. Murphy, P.Wood and D. Kerr. Royal Bournemouth Hospital, England and * Yale University School of Medicine, New Haven, USA.

Drinking alcohol at lunchtimes can cause reactive hypoglycaemia up to 5 hours later. The aim of this study was to determine whether the combination of alcohol (gin) and mixer (tonic) is more likely to cause hypoglycaemia than either alcohol or the mixer alone. Eight healthy volunteers ( 5 men, aged $20-40 \mathrm{yrs}$ ) drank, using a randomised, double-blind design:

(a) three large gin and tonics $(0.5 \mathrm{~g} / \mathrm{kg}$ of alcohol and $60 \mathrm{~g}$ of carbohydrate, mainly sucrose),

(b) same amount of alcohol with "Slimline" tonic $(0.5 \mathrm{~g}$ of carbohydrate)

(c) tonic containing $60 \mathrm{~g}$ of sucrose without alcohol

Over the next 5 hrs both alcohol studies were associated with inebriation but only gin and regular tonic together caused reactive hypoglycaemia (nadir glucose $3.3 \pm 0.1$ vs $3.9 \pm 0.1 \mathrm{mmol} / 1$ for gin and "Slimline" and $3.9 \pm 0.2 \mathrm{mmol} / \mathrm{l}$ for tonic alone, both $\mathrm{p}<0.02$ ) and greater hyperinsulinaemia ( $40 \pm 9$ vs $6 \pm 2$ and $28 \pm 7 \mu \mathrm{U} / 1$, both $\mathrm{p}<0.05$ ). Both alcohol studies caused suppression of growth hormone release ( $\mathrm{p}<0.02$ vs regular tonic) and all subjects felt

"hypoglycaemic" ( $48 \pm 9$ and $46 \pm 14$ vs $26 \pm 14 \mathrm{~mm}$, p<0.01) unrelated to the prevailing glucose level without any change in cerebral blood flow. Conclusion: The combination of alcohol and a sucrose containing mixer can cause reactive hypoglycaemia and makes the perception of a low blood glucose level difficult.

\section{7}

FEAR OF HYPOGLYCAEMIA VERSLS RISK OF
HYPOGLYCAEMIA IV DIABETIC PERSONS ISING INSULIN

R. B.W Smith and S.F Lee, Department of Medicme, Wellington School of Medicine, Wellington, New Zeaiand.

In clunical practice it would be useful to identify insulin users with mappropriately low or high fear of hypoglycaemia in relation to their risk of hypoglycaemia. Persons with diabetes using insulin for at least one year were recruited from consecutive hospital diabetes clinics. Sixty-five persons completed questionaarres recording their symptoms and frequency of hypoglycaemia, their recent blood glucose results, and the Fear of Hypoglycaemia Scale of Irvine. Cox and Gonder-Frederick. Of various anecdotal measures of apparent hypoglycaemic risk only the recalled frequency of mild hypoglycaemic episodes correlated significantly with the Fear of Hypoglycaenia worry subscale score $(p<0.001)$. We used the Scale's authors' median splits of blood glucose means and blood glucose variability (standard deviation) to create a two by two matrix. The results showed a general trend with the low mean high variability blood glucose group having the higtiest worry score (mean 29.6, standard deviation 10.6, $\mathrm{n}=1 \mathrm{l}$ ), and the high mean low variability group having the lowest worry score ( 242,7 , sd $11.5, \mathrm{n}=10)$; the high mean high variability group (m29.5, sd 10.9, $\mathrm{n}=22)$ and the low mean low variability group ( $m 28.4, s d 13.4, n=22)$. The highest and lowest worry scores were not significantly different $(t=0.93,32<p<42)$. The results of this study suggest that further refinements in the measuring of hypoglycaemic risk will be required before the Fear of Hypoglycaemia worry subscale can be clinically useful in detecting inappropriate fear of hypoglycaemia.

\section{8}

EPINEPHRINE RESPONSE TO HYPOGLYCAEMIA AND AUTOIMMUNTTY TO ADRENAL MEDULLA IN INSULIN-DEPENDENT DIABETES

D.J. Becker. M.M. Zanone*. M Peaknan**. Division of Endocrinology and ${ }^{* *}$ Inmmunogenetics, Children's Hospital, Pittsburgh, USA: Dept of Internal Medicine, University of Torino, Italy.

Inpaired response of the counterregulatory hormones, including epinephrine, in response to hrpoglycaemia is documented in IDDM. Although controversial, this nay be an important cause of erratic control of diabetes. Anti-adrenal medullary antibodies (AMA) have been reported in IDDM as well as mononuclear cell infiltration and fibrosis of the adrcial modulla. The present study aimed to examine the relationship between AMA and epinephrine response to lovpoglycaemia in 36 adolescents with IDDM (mean (SD) age $15.8(2.1)$ years) and 9 age-matched healthy controls. AMA were detected by indirect conplenent-fixation immunofluorescence. Hypoglycaemia was induced either by insulin intravenous bolus $(0.15-0.75 \mathrm{U} / \mathrm{kg})$ or hypoglycacmic clamp. and blood samples collected at 15-30 minute intervals. Plasma epinephrine was measured by HPLC and the difference between the basal and peak values was expressed as percentage of increase from basal. AMA were detected in $3(8 \%)$ patients and not in controls. Epinephrine response was lower in diabetic patients compared to controls $(p=0.009)$. The patients with AMA had significantly lower response (nedian $5 \%$. range 2 - 27) than the patients without antibodies $(122 \%,-10-708)(p=0.03)$. Considering patients with an epinephrine response $<100 \%$ as low responders. there was a trend for AMA to be associated with a low response $(p=0.08)$. Our data suggest an association between a reduced epinephrine response to hypoglycacnia and adrenal autoimununity, and future studies will need to establish whether this reflects primary damage of the adrenal medulla by the immune sisten.
829

DISTINCTIVE CHANGES OF SINGLE COMPONENTS IN COGNITIVE FUNCTIONS DURING HYPOGLYCEMIA IN NORMAL MAN

R. Lobmann', H.G. Smid', B.G. Trümper', K. Wagner', G. Pottag', H.J. Heinze ${ }^{2}$ and $H$. Lehnert'; 'Dept. of Endocrinology and Metabolism, ${ }^{2}$ Dept. of Neurophysiology, Otto-von-Guericke-University, Magdeburg, Germany

Little is known about distinctive and topographically related effects of hypoglycemia on cognitive functions. We employed novel eventrelated potential recording techniques (ERP's) to study single components (stimulus selection, response choice, reaction time) of cognitive function impairement during a stepped hypogiycemic clamp (<3mmol//). Two groups of 12 healthy volunteers were studied. One group performed different cognitive tasks during the following phases: euglycemia-hypoglycemia-euglycemia. The second group had only three euglycemic phases (randomized, single blind). The tasks consisted of recognition memory performance, selective attention and choice motor responses to coloured letters. We also investigated 12 diabetic patients with IDDM and a group of 12 NIIDM patients. The ERP's related to selective identification of coloured letters, to central activation of motor responses in primary motor cortex and reaction times to these letters were delayed during hypoglycemia as compared with the first phase of normoglycemia ( $32 \mathrm{msec}, 72 \mathrm{msec}, 27 \mathrm{msec}$, ps all $<.05$ ).

In particular we found that hypoglycemia impairs recognition memory and primarily affects early components of cognitive responses such as motor cortex activation and selection of input information. Our results suggest that hypoglycemia delays stimulus selection, with consecutively delayed central and motor processing. This effect disappears when normoglycemia is restored. 
830

SEVERE HYPOGLYCAEMIA IN CHILDREN WITH IDDM. A PROSPECTIVE POPULATION STUDY 1994-1995. J. Ludvigsson, S. Norfeldt, Department of Pediatrics, Faculty of Health Sciences, Linköping, Sweden

Multiple insulin therapy (MIT) is said to cause unacceptable incidence of severe hypoglycaemia ( $\mathrm{SH}$ ), especially in children. Can education and self-control keep incidence of SH low in diabetic children even with MIT and low HbAlc?

Patients and Methods: 139 patients, 57 girls and 82 boys, aged 1. -18.4 years (median $(m) 14.0$ ), with age at onset $0.5-17.4$ years (m 6.8) and duration $0.1-16.6$ years $(\mathrm{m} 3.8$ ) registered every $\mathrm{SH}$ and delivered data every 2-4 months. Fasting C-peptide ranged 0.0-0.83 $\mathrm{nmol} / \mathrm{L}(\mathrm{m} \mathrm{0.03})$ and $\mathrm{HbAlc}$ year mean values 4.4-10.3\% (m 6.9; normal range 3.6-5.4). Insulin dose, weight, length etc. was also registered. SH was split into "Not Unconscious (NU) but needing help from another person", and "Unconscious (U)". Corrections were done for missing registration periods ( $<2 \%$ in 1.995$)$.

Results:

\begin{tabular}{|c|c|c|c|c|c|c|c|}
\hline & 90 with & Insulin & $\begin{array}{l}\text { median } \\
\text { HbAlc } \%\end{array}$ & NU/pat & No wit] & Utpat & $\mathrm{Ut}$ \\
\hline 1994 & 95 & 3 & 6.7 & 1.26 & 34 & 0.17 & 16 \\
\hline 1995 & 94 & 4 & 7.0 & 1.21 & 32 & 0.16 & 11 \\
\hline
\end{tabular}

None with HbA1c $>8,2 \%$ had U. Otherwise there was no relation between $U$ and $\mathrm{HbAlc}(\mathrm{r}=0.08$; ns) and no relation between $\mathrm{U}$ or $\mathrm{NU}$ and other factors such as age, duration, weight, insulin dose. Conclusion: MIT and low $\mathrm{HbA} 1 \mathrm{c}$ must not increase incidence of severe hypoglycemia in diabetic children.

\section{1}

ADRENOMEDULLIN LACKS PARALLELISM WITH EPINEPHRIN DURING HYPOGLYCEMIA.

P. Preuss, K. Ehlenz*, C. Loeffler, U. Oltmanns, B. Koch*, R.E. Lang**, H. Lochs, and I.Koop. Depts. Internal Medicine, Univ. Hospital Charité, Berlin and *Marburg, ${ }^{* *}$ Dept. Physiology, Marburg, FRG.

Adrenomedullin is co-localized with epinephrin in adrenal medullary cells. The aim of this study was to investigate whether adrenomedullin is co-secreted with epinephrin during hypoglycemia-induced hormonal counterregulation. Methods: Four healthy volunteers ( $2 \mathrm{~m}, 2 \mathrm{f}$, age:22-25) were studied by means of a stepwise hypoglycemic clamp with steps lasting for $45 \mathrm{~min}$. Plasma catecholamines were determined by HPLC, cortisol was measured by ELISA. Adrenomedullin determination was performed by RIA with prior SEP-PAK extraction Results: As expected, epinephrin, norepinephrin (not shown) and cortisol were significantly elevated at blood glucose concentrations of 3.0 and $2.3 \mathrm{mM}$ compared to $4.9 \mathrm{mM}\left({ }^{*}: \mathrm{p}<0.05\right.$ vs. $\left.4.9 \mathrm{mM}\right)$ Adrenomedullin concentrations did not show significant

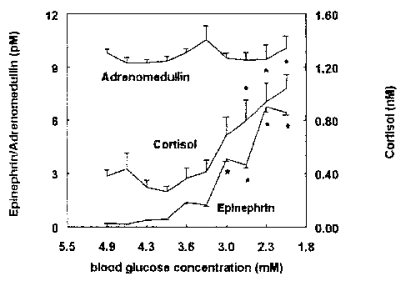
changes during hypoglycemia. Discussion: Adrenomedullin is not involved in hypoglycemia-induced counterregulatory hormone response. Although adrenomedullin is mainly localized in the adrenal medulla and secreted in parallel with epinephrin under in vitro-conditions it lacks co-secretion with epinephrin during hypoglycemia. These preliminary data suggest different stimulatory and possibly intracellular signal transduction pathways during various ,stress conditions“.
832

IN SEARCH OF A HYPOGLYCAEMIC ALARM

N.D. Harris*, J.L.B. Marques*, E. George, C. Bedford and S.R. Heller. "Department of Medical Physics, Royal Hallamshire Hospital and Diabetes Centre, Northern General Hospital, Sheffield S5 7AU - UK

Many patients refuse intensive insulin therapy because of the risks of severe hypoglycaemia, particularly during the night. In the absence of a practical glucose sensor there is urgent need for a means of detecting the onset of hypoglycaemia non-invasively. We have evaluated the potential of integrating several physiological responses to hypoglycaemia by using a pocket computer system to record changes in heart rate, blood flow, skin resistance (sweating) and skin temperature. Measurements were carried out on Type 1 diabetic patients during clamped hypoglycaemia $(6 \mathrm{M} / 1 \mathrm{~F}$, age 19-38 years) and overnight on 11 occasions (4M/6F, age 20-50 years) During overnight monitoring blood glucose (BG) was measured hourly and physiological data recorded for 2 out of every 12 minutes. There were 6 spontaneous hypoglycaemic episodes ( $\mathrm{BG}<3.5 \mathrm{mmol} /$ ). There were comparable changes in the physiological responses during clamped and spontaneous nocturnal hypoglycaemia. A hypoglycaemic response was

\begin{tabular}{|l|l|l|}
\hline \multicolumn{1}{|c|}{ Mean [range] } & $\begin{array}{c}\text { CLAMPED } \\
\text { HYPOGLYCAEMIA }\end{array}$ & $\begin{array}{c}\text { SPONTANEOUS } \\
\text { HYPOGLYCAEMIA }\end{array}$ \\
\hline Blood flow (\%) & $48.9[105$ to -38$]$ & $62.6[162$ to -42$]$ \\
\hline Heart rate (BPM) & $-2.2[16.6$ to -17.7$]$ & $-2.8[10.9$ to -23$]$ \\
\hline Sweating (\%) & $15.2[15.0$ to 30$]$ & $28.3[49.0$ to -3.5$]$ \\
\hline Skin temp. $\left({ }^{\circ} \mathrm{C}\right)$ & $-1.1[1.1$ to -2.3$]$ & $-1.0[-0.5$ to -1.8$]$ \\
\hline
\end{tabular}

defined as a change of greater than $1.5^{*} \mathrm{SD}$ above the cumulative mean baseline values, in 3 out of 4 variables. We were able to detect hypoglycaemia in all the clamp and in $5 / 6$ overnight studies. There were 2 false positive responses during the euglycaemic nights. The system offers promise of detecting hypoglycaemia although more sophisticated processing may be required
833

\section{REDUCED FREQUENCY OF NOCTURNAL HYPOGLYCEMIA BY} BEDTIME CORNSTARCH SUPPLEMENT IN IDDM SUBJECTS M. Axelsen, C. Wesslau, P. Lönnroth and U. Smith.

Lundberg Laboratory for Diabetes Research, Dept. of Internal Medicine. Sahlgrenska University Hospital, Göteborg, Sweden.

The incidence of severe hypoglycemia in intensively treated IDDM subjects is 3 times that observed with conventional treatment and $55 \%$ of all episodes occcur during sleep. In a blind, randomized, cross-over study we have evaluated if a reduced frequency of nocturnal hypoglycemia (blood glucose (BG) levels $<3.0 \mathrm{mmol} / \mathrm{l}$ ) can be achieved, concomitant with maintained glycemic contol as assessed by HbAlc, by bedtime intake of uncooked cornstarch in adult IDDM subjects.

Nine IDDM subjects (age $39.4 \pm 8.7$, HbAlc $6.2 \pm 0.7$, on $\geq 4$ daily insulin injections/day) were randomly assigned to uncooked cornstarch $(0.30 \mathrm{~g} / \mathrm{kg}$ bodyweight) or carbohydrate-free placebo, to be taken for 4 weeks together with the beditime insulin injection. HbAlc was measured before and after each period. The subjects recorded all fasting- and bedtime BG levels, as well as all symtomatic hypoglycemic episodes. In addition, the 3 AM BG level was recorded for $2 \times 7$ days in each period, followed by an overnight investigation at the laboratory.

The BG levels were higher during the cornstarch- than the placebo period, both at $3 \mathrm{AM}(10.5 \pm 0.5$ vs $8.5 \pm 0.5, \mathrm{p}<0.05)$ and in the morning $(9.7 \pm 0.5$ vs $8.3 \pm 0.6, \mathrm{p}<0.05$ ). The laboratory investigation showed that the nocturnal lowest levels occured in most patients at 2-4 AM in the placebo period, but in early night ( 11PM) or early moming ( $\sim 6 \mathrm{AM})$ in the comstarch period. The number of hypoglycemic events at 3 AM in the 9 subjects was significantly reduced during the 14 nights of registration in the cornstarch period $(n=3)$ compared to in the placebo period $(n=9)(p<0.05)$. However, there was no difference in the HbAlc levels.

In conclusion, the results show that the frequency of nocturnal hypoglycemic episodes can be reduced with maintained glycemic control by bedtime cornstarch ingestion. This suggests that cornstarch may be useful to balance the effect of bedtime insulin and, thus, diminish the frequency of nocturnal hypoglycemia in IDDM subject. 


\section{4}

THE INFLUENCE OF OBESITY ON NEUROPHYSIOLOGICAL RESPONSES TO HYPOGLYCEMIA.

T. Veneman, A. Mitrakou, M. Mokan, T. van Haeften, and J. Gerich. University of Utrecht, The Netherlands and University of Rochester, NY, USA.

To assess whether obesity influences hormone responses, autonomic (AS) and neuroglycopenic symptoms (NGS), and cognitive function (CF) to hypoglycemia, 10 lean (L)(BMI $22.2 \pm 0.6 \mathrm{~kg} / \mathrm{m}^{2}$ ) and 10 obese (OB)(BMI 33.0 \pm 1.7 ) subjects were studied during glycemic plateaus of $5.5 \pm 0.1,4.3 \pm 0.2,3.6 \pm 0.2,3.0 \pm 0.1$, and $2.3 \pm 0.1$ $\mathrm{mmol} / \mathrm{l}$. In the $\mathrm{OB}$ group glycemic thresholds for adrenaline and noradrenaline release were lower $(3.6 \pm 0.1$ vs $4.0 \pm 0.1 \mathrm{mmol} / 1$ and $3.6 \pm 0.1$ vs $4.0 \pm 0.1 \mathrm{mmol} / 1, \mathrm{~L}$ vs $\mathrm{OB}$, respectively $(\mathrm{P}<0.05)$ ), but not for growth hormone and cortisol. Maximal plasma adrenaline and noradrenaline responses were higher in the OB group $(880 \pm 111$ vs $1320 \pm 158 \mathrm{pg} / \mathrm{ml}$ and $290 \pm 55$ vs $505 \pm 102 \mathrm{pg} / \mathrm{ml}$, respectively, L vs OB $(P<0.05))$. Maximal growth hormone concentration tended to be lower in the OB group $(26.4 \pm 4.9 \mathrm{vs} 19.8 \pm 4.4 \mathrm{ng} / \mathrm{ml},(0.1<$ $\mathrm{P}<0.05)$ ). Cortisol responses did not differ $(167 \pm 25 \mathrm{vs} 191 \pm 16 \mathrm{ug} / \mathrm{L}$, (NS)). The magnitude of AS, but not NGS was higher in the $L$ subjects $(9.3 \pm 1.6$ vs $6.1 \pm 0.5,(\mathrm{P}<0.05)$ and $6.7 \pm 1.1$ vs $4.9 \pm 0.5$ (N.S.)), respectively in $\mathrm{L}$ vS OB groups). Thresholds for AS and NGS were not different $(3.3 \pm 0.1$ vs $3.4 \pm 0.1 \mathrm{mmol} / 1$, and $2.8 \pm 0.1$ vs $2.7 \pm 0.1$ $\mathrm{mmol} / 1$ in $\mathrm{L}$ vs $\mathrm{OB}$, (both N.S). Thresholds and magnitudes for $\mathrm{CF}$ were not different, $2.7 \pm 0.1$ vs $2.7 \pm 0.1 \mathrm{mmol} / 1$, (Z-scores $8.9 \pm 1.9$ vs $11.3 \pm 3.0, \mathrm{~L}$ vs $\mathrm{OB})$. In conclusion obesity is accompanied by increased catecholamine responses, lower thresholds for catecholamine release, and lower AS responses during insulin-induced hypoglycemia; this suggests a decreased sensitivity to adrenergic stimulation in obesity.

\section{6}

REVERSAL OF MALARIA-INDUCED HYPOGLYCAEMIA K.M.Elased and J.H.L. Playfair. Department of Immunology,

UCLMS, London, UK

Hypoglycaemia is a serious complication in Plasmodium falciparum malaria which conelates with mortality. The mechanism is not clear and usually attributed to the hyperinsulinaemic effect of quinine. The present study was designed to investigate the possible role of insulin in a murine model of malaria. Blood glucose, plasma insulin and parasitaemia were measured in normal and infected mice before and after treatment with diazoxide, adrenaline, sandostatin and quinine. Severe hypoglycaemia ( $<2 \mathrm{mM}$ glucose) developed during nonlethal P.chabaudi and lethal Pyoelii $\quad(\mathrm{P}<0.0001, \mathrm{n}=10-12)$. This hypoglycaemia was associated with hyperinsulinaemia (control, $2.2 \pm$ $0.13 ; \mathrm{PC}, 90 \pm 10 ; \mathrm{LPY}, 180 \pm 11 \mathrm{ng} / \mathrm{ml}$ ). A single injection of diazoxide (25 mg/kg i.p.) or adrenaline $(0.03 \mathrm{mg} \mathrm{s.c.)} \mathrm{significantly}$ lowered insulin concentration in normal and infected mice $(p<0.001)$, reversed the hypoglycaemia in both infections and significantly reduced the hyperinsulinaemia in $P$. chabaudi-infected mice $(\mathrm{p}<0.0001)$. Quinine $(25 \mathrm{mg} / \mathrm{kg}$ i.p.) significantly increased blood glucose in normal and infected mice $(p<0.001)$. Our data suggest that the severe hypoglycaemia seen during murine malaria infection is secondary to hyperinsulinaemia possibly mediated through a parasite-derived molecule(s) acting directly on pancreatic islets, rather than high consumption of glucose by host and parasites or chemotherapy with quinine, and that hypoglycaemia can be reversed by correcting the hyperinsulinaemia.
835

ADAPTATION TO HYPOGLYCAEMAC STRESS DEFNED BY WHOLE BODY ${ }^{18} \mathrm{~F}$ FLUORODEOXYGLUCOSE (FDG) SCINTIGRAPHY

O.S. Hoekstra, ${ }^{*}$ K. Kanc, G.J.I. Teule and *R.J. Heine. Department of Nuclear Medicine, *Department of Endocrinology, 'Vrije Universiteit' Hospital, De Boelelant 1117, 1081 HV Ansterdam, The Netherlands

Cerebral integrity during hypoglycaemia relies on local (perfusion antoregulation. transporter enzymes) and system factors (i.e. redistribution of cardiac output. gluconeogenesis). Positron emission tomograply (PET) offers in vivo quantitation of key variables like regional blood flow, oxygen and glucose consumpion, but PET studies usually focus on a single organ. With whole body scanning (WBS) and a static late FDG image, the observed tissue FDG deposition is proportional to and influx constant and the integral of the blood FDG concentration. To assess the effect of hypoglycaemia on FDG uptake in multiple tissucs simultaneously, we compared FDG distribution (dual head gamma camera) during hyperinsulinaemic, hypoglycaemic clamping (at $2.5 \mathrm{mmol} / 1$ serun glucose, arterialized venous blood) and in hyperglycaemia (at $20.0 \mathrm{mmol} / \mathrm{l}$ ). in 2 adult male with IDDM, using WBS 1.5 and 2.5 hrs after injection of $100 \mathrm{Mbg} F D G$. We found that the two testing conditions had similar effects on FDG handling in both subjects. Throughout 45 min of blood sampling, circulating FDG levels at $20 \mathrm{mmol} / 1$ level were twice those at $2.5 \mathrm{~mol} / \mathrm{l}$. Total body FDG uptake was enhanced in hypoglycaemia, and redistribution towards brain ( $3-5 \mathrm{x}$ higher uptake than at $20 \mathrm{mmol} / \mathrm{l}$ ) and heart $(2 \mathrm{x})$, at the expense of kidneys and urinary excretion ( $1.5 \mathrm{x}$ less). The latter may reflect vasoconstriction during hypoglycaenia and/or less tubular reabsorption during hyperglycaemia. Skeletal tmuscular, hepatic and pulmonary uptake were unchanged. These results correspond with theoretical predictions and PET data. The technique can therefore be used to study the net inpact of the interplay between 'fight or flight' circulatory and the metabolic response on glucose uptake.

\section{7}

MRI OF THE BRAIN IN ADULT PATIENTS WITH IDDM: EFFECTS OF DIABETES AND RECURRENT SEVERE HYPOGLYCAEMIA

P. Perros ${ }^{1}$, I.J. Deary ${ }^{2}$, R.J. Sellar ${ }^{3}$, and B.M. Frier ${ }^{1}$. Departments of lDiabetes, Roval Infirmary of Edinburgh, 2Psychology, University of Edinburgh, ${ }^{3}$ Clinical Neuroradiology, Western General Hospital, Edinburgh, SCOTLAND

A history of recurrent severe hypoglycaemia was associated with modest impairment of cognitive function in a cohort of IDDM patients (1). The aim was to determine whether these IDDM patients had lesions in the brain identifiable by MRI. MRI scans of the brain were performed in 22 patients. Eleven IDDM patients with no history of severe hypoglycemia (group A), were compared with 11 IDDM patients who had a history of 5 or more episodes of severe hypoglycemia (group B). None of the patients had other medical conditions likely to affect cognitive function. The two groups were matched for age, sex, and duration of diabetes. Twelve patients $(54.5 \%)$ had matched for age, sex, and duration of diabetes. Twerve patients (S4.5\%) had brain atrophy in $8(36.4 \%)$ patients. Leukoaraiosis was associated with age 742 years, the presence of microvascular diabetic complications and systolic blood pressure $\geq 130 \mathrm{mmHg}$ ( $<<0.02$ ). Brain atrophy was associated with microvascular complications and diastolic biood pressure $\geq 80 \mathrm{mmH}$. Patients in group $B$ had a higher prevalence of abnormal MRI brain scans than patients in group $\mathrm{A}(63.6 \%$ ys $36.4 \%, \mathrm{p}<0.001)$. In conclusion, brain abnormalities demonstrated by MRI are common in patients with IDDM of long duration suggesting that premature ageing of the brain may occur. IDDM per se may be an important pathogenic tactor, and severe recurrent hypoglycaemia may" exacerbate these pathological changes.

(1) Deary IJ et al (1993). Diabetes 42:341 
838

LOW-DOSE-ASPIRIN DOES NOT LOWER BLOOD GLUCOSE IN NIDDM PATIENTS TREATED WITH GLIBENICLAMIDE

\author{
J. Hasbach, T. Koschinsky, H. Reinauer and F.A. Gries \\ Diabetes-Forschungsinstitut an der Heinrich-Heine-Universität \\ Düsseldorf, Germany
}

It is well known that higher doses of aspirin (>1g) are able to lower the blood glucose level and enhance the effect of sulphonylurea derivates. Meanwhile low-dose-aspirin has become the standard therapy in the secondary prevention of cardial and cerebral ischaemic events, but it is not known whether low-dose-aspirin increases the risk of hypoglycaemia in diabetic patients treated with sulphonylureas. We therefore studied the influence of $100 \mathrm{mg}$ of aspirin on the blood glucose level of NIDDM patients treated with the commonly used sulphonylurea derivate glibenclamide.

On two consecutive days 36 NIDDM patients $(16 \mathrm{f} ., 2.0 \mathrm{~m}$., age $62 \pm 9 \mathrm{yrs}$, diabetes duration $11,3 \pm 7,5 \mathrm{yrs}$, fasting blood glucose $159 \pm 36 \mathrm{mg} / \mathrm{dl}$ ) received glibenclamide and a standardized breakfast. On the first day all patients were given placebo after the breakfast. On the second day 18 patients received aspirin, the other 18 placebo. Both treatments were assigned in a randomized and dobbleblind manner.

The daily blood glucose and insulin profiles of the treatment groups did not differ $(p=0,3$ and 0,14$)$. The lowest mean postprandial blood glucose concentrations of the first day were identical $(161+62 \mathrm{mg} / \mathrm{dl})$. The second day's lowest mean postprandial blood glucose was $152 \pm 60 \mathrm{mg}$ in the aspirin group and $154 \pm 62 \mathrm{mg}$ in the placebo group. Moreover, the changes of the lowest blood glucose concentrations of both days were not significantly different between the two treatment groups $(p=0,73 ; 90 \%$ confidence interval $-12,7 / \pm 8,4 \mathrm{mg} / \mathrm{dl}$ ). Hypoglycaemias did not occur in the aspirin group. We conclude that low-dose-aspirin does not increase the risk of hypoglycaemia in NIDDM patients treated with glibenclamide.

\section{PS 45}

\section{Insulin Analogues}

\section{9}

EFFECT OF BASAL INSULIN ON REDUCED FREQUENCY OF NOCTURNAL HYPOGLYCEMIA IN PATIENTS TREATEJ WITH INSULIN LISPRO

I H Anderson, R L Brunelle, V Arora, J S Phillips, L Vignati, V A Koivisto and M E Trautnann, Lilly Research Laboratories, Indianapolis, IN, USA

The analog insulin lispro has a rapid onsel and short duration of activity. To compare the freguency and timing of hypoglycemia between insulin lispro (IL) and regular human insulin (HI) during intensive insulin treatment, 6 month crossover studies were performed. One thousand eight patients with type I diabetes and 722 patients with type II diabetes were ranuomized into two treatment sequences. Patients treated with IL had significantly reduced postprandial glucose excursions with equivalent HbAle compared to HI. Each hypoglycemic event (any sign or symptom or blood glucose $<3.5 \mathrm{mmol} / \mathrm{L}$ ) was documented by recording symptoms, severity and treatment. A total of 18632 episodes during IL and 20994 during $\mathrm{HI}$ therapy were detected in patients with type I diabetes and 5655 episodes during IL and 6178 during HI therapy were detected in patients with type II diabetes. An analysis by quartile of day revealed significantly less hypoglycemia in the IL treated group from midnight to 0600 hrs in patients with iype I and type II diabetes (type I: $1.3 \pm 2.2$ IL ws. $2.2 \pm 3.4 \mathrm{HI}, \mathrm{p}<.001$ and type II: $0.5 \pm 1.3 \mathrm{IL}$ vs. $0.7 \pm 1.9 \mathrm{HI}, \mathrm{p}=.004)$. In patients with type [, hut not type II diatbetes, treated with IL there wats also less hypoglycemia from 1200 to 1800 hrs $(p<.001)$ and from 1800 to $2400 \mathrm{hrs}$ $(\mathrm{p}<.001)$. There was an interaction between study treatment and basal insulin $(p=.005)$ and berween study treament and the number of basal insulin injections $(p<.001)$ for the mean frequency of noctumal hypoglycemic episodes. In both cases the mean frequency was higher during HI therapy especially with the use of human ultralente and with two doses of the bassal insulin. Type of basal insulin or number of injections did not have an effect on nocturnat hypoglycernia in patients with type II diabetes. In patients with type I or II diabetes treated with intensive insulin therapy, insulin lispro therapy is "wsisociated with significantly less nocturnal hypoglycemiat.

\section{0}

COMPARISON OF PRE-MEAL THERAPY WITH INSULIN LISPRO AND REGULAR INSULIN IN PATIENTS WITH IDDM

H. Schmitt', S.M. Symanowski 'F. Holleman ', A. Rees ${ }^{3}$ R. Rottiers "and J.H. Anderson' for the Benelux-UK LISPRO study group, Lilly Laboratories', Indianapolis, USA, Diakonessenhuis ', Utrecht, The Netherlands, Universit?' Hospital Wales ", Cardiff, U.K., Universitair Ziekenhuis Gent ${ }^{2}$, Belgium.

This open, randomized, 6 month crossover study compared insulin lispro (Humalog ${ }^{\circledR}$ ) with regular insulin (Actrapid ${ }^{\Phi}$ ) for improvements in glycemic control measured by home blood glucose monitoring, $\mathrm{HbAlc}$, rate and timing of hypoglycemias and quality of life. There were 199 patients with IDDM. Mean (SD) HbAlc was 7.3 (1.1) \%, duration of diabetes : 13.1 (9.1) years, age : 35.4 (9.6) years, BMI : $24.9(3.1) \mathrm{kg} / \mathrm{m}^{2}$. HbAlc was equivalent for both groups throughout the study. Post prandial (pp) glycemias and 2-hr excursions were lower with lispro. The average daily blood glucose and the within-day variability were lower with lispro $(p<0.01)$. Pre-supper $(\mathrm{pm})$ glycemia was lower with regular insulin.

Table : 7-point blood glucose profile ( $\mathrm{mmol} / \mathrm{L}$ ) at endpoint. Mean (SD). \begin{tabular}{|l|l|l|l|l|l|l|l|}
\hline Treatment & am fbg & 2 -hr pp & noon fbg & 2-hr pp & pm fbg & 2-hr pp & Bedtime \\
\hline
\end{tabular} \begin{tabular}{|l|l|l|ll|l|l|l|l|l|}
\hline Humalog $^{\text {e }}$ & $9.3(2.6)$ & $7.7(2.6)$ & $72(2.4)$ & $7.5(23)$ & $8.7(2.8)$ & $7.7(2.6)$ & $9.1(2.7)$ \\
\hline
\end{tabular} \begin{tabular}{|l|l|l|l|l|l|l|l|}
\hline Actrapid $^{6}$ & $8.9(2.7)$ & $9.7(32)$ & $73(2.5)$ & $8.6(2.5)$ & $7.5(2.6)$ & $8.9(3.1)$ & $8.7(3.0)$ \\
\hline p-value & 0.083 & $<0.001$ & 0.583 & $<0.001$ & $<0.001$ & $<0.001$ & 0.215 \\
\hline
\end{tabular} \begin{tabular}{|l|c|c|c|c|c|c|c|}
\hline p-value & 0.083 & $<0.001$ & 0.583 & $<0.001$ & $<0.001$ & $<0.001$ & 0.215 \\
\hline
\end{tabular}

The total symptomatic and asymptomatic hypoglycemic episodes did not differ between the treatments. Severe hypoglycemias were less for patients receiving lispro (36 out of 2249) than regular insulin ( 58 out of 2344), $(\mathrm{p}<0.05$ ). The total hypoglycemic episodes from midnight to 6 am were less for patients receiving lispro $(176$ vs. $312, p<0.01)$. Hypoglycemia occurred $1 \mathrm{hr}$ earlier after a meal for lispro than regular insulin ( 4.3 vs. $5.2 \mathrm{hr}$ ). Patients expressed more satisfaction for lispro treatment, felt a better quality of glycemic control and greater flexibility of life (all, $p<0.01$ ). In well controlled type I diabetic patients, lispro (Humalog") allowed better postprandial glycemic control, less severe and less night hypoglycemias, and increased quality of life compared with regular insulin (Actrapid"). 
841

COMPARISON OF METABOLIC CONTROL IN IDNM WTTH TWO DIFFERENT INTENSIVE REGIMENS OF [LYS(B28), PRO(B29)]- HUMAN INSULIN (LISPRO) PLUS NPH INSULIN

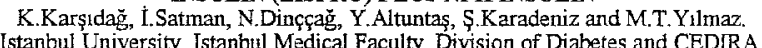
Institute for Experimental Medicine, TURKEY. The aim of this open-label stucty was to compare the effects of once vs twice a day
NPH on metabolic control in 9 iDDM patients in addition to intensive lispro insulin NPH on metabolic control in 9 DDM patients in addition to intensive lispro insulin
(LP) therapy. The age of patients was $21.8 \pm 6.2$ years and duration of diabetes was 3.1 122.1 years. The study was conducted in 3 phase. After a 3 month lead-in period with regular human insulin given 30 minutes before each main meal and NPH at beditime (Phase $\mathrm{I}$, patients were switched to a LP insulin immediately before each main meal and NPH at bedtime for 3 months (Phase II). Then for the following 3 months, NPH insulin was given 2 times (breakfast NPH+LP, lunch LP, dinner LP, bedtime NPH) (Phase III).

\begin{tabular}{|c|c|c|c|c|}
\hline Parameters & Phase I & Phase II & Phase III & p value \\
\hline $\begin{array}{l}\text { quirement } \\
\left.y^{-1}\right)\end{array}$ & $0.48 \pm 0.18$ & $0.49 \pm 0.18$ & $0.49 \pm 0.18$ & NS \\
\hline $\mathrm{HbA}_{\mathrm{Ic}}(\%)$ & $8.6 \pm 0.9$ & $8.9 \pm 1.6$ & $6.9 \pm 1.7$ & $\begin{array}{cc}\text { I vs II } & \text { NS } \\
\text { I vs III } & 0.04 \\
\text { II vs III } & 0.01\end{array}$ \\
\hline $\begin{array}{l}\text { Hypogly } \\
\text { episode }\end{array}$ & $1.2 \pm 0.9$ & $1.3 \pm 1.2$ & $1.4 \pm 1.2$ & NS \\
\hline $\begin{array}{l}\text { Anti-insulin antibody } \\
\text { (AIA) }(\% \text { binding) }\end{array}$ & 35 & & 12 & $\begin{array}{lr}\text { I vs II } & \text { NS } \\
\text { I vs III } & \mathbf{0 . 0 3} \\
\end{array}$ \\
\hline
\end{tabular}

HbA1c and AIA was significantly decreased after phase III treatment. Although there were no change in BMI and frequency of hypoglycaemic episodes, patients reported that there was a tendency to an immediately started hypoglycaemic episodes with lesser-pronounced adrenergic symptoms. The level of human insulin specific antibodies was lowered during LP therapy ( $p>0.05$ phase I vs II, and $\mathrm{p}<0.05$ phase I vs III). In conclusion covering 24 hour basal insulinemia with twice daily NPH in intensive LP therapy without changing the dose of insulin is twice daily NPH in intensive LP therapy without changing the dose of insulin is more effective than once daily NPH. Moreover, LP insulin seems to be a suitable
alternative to regular human insulin, particularly in patients with higher insulin antibody levels.

\section{3}

USE OF INSULIN LISPRO IN PEDIATRIC PATIENTS WITH IDDM Pfützner A., Gudat U., Trautmann M.E. and Holcombe J., Lilly Deutschland GmbH, Bad Homburg, Germany \& Lilly Res. Laboratories, Indianapolis, USA

Intensive insulin treatment with the short acting insulin analogue lispro has been shown to improve postprandial blood glucose control and to reduce the frequency of hypoglycemic episodes in adult patients with IDDM. In Germany, 46 pediatric patients (17 male, mean age: $15 \pm 1.7 \mathrm{yrs}$. (range: 11 to $18 \mathrm{yrs}$.), 29 female, mean age: $15 \pm 1.6$ yrs., (12 to 17 yrs.)) participated in an eight-month, randomized crossover trial to evaluate the efficacy and safety of insulin lispro (LP) in comparison to regular human insulin ( $\mathrm{HI})$. Insulin lispro injected directly before each meal, while an injection-to-meal interval of 30 to 45 min was used with regular human insulin. Each treatment was given for four months. Measurements included daily blood gluose profiles, hemoglobin A1c, frequency of hypoglycemic episodes, and the number and type of adverse events. The premeal (e.g. breakfast) glucose values (mean \pm SEM) for the LP and HI groups were $185 \pm 8 \mathrm{mg} / \mathrm{dl}$ and $164 \pm 6 \mathrm{mg} / \mathrm{dl}$, respectively. Treatment with LP reduced the $2 \mathrm{~h}$ postprandial blood glucose excursions (e.g. breakfast: LP: $-37 \pm 8 \mathrm{mg} / \mathrm{dl}, \mathrm{Hl}:-4 \pm 7.7 \mathrm{mg} / \mathrm{dl}, \quad p<0.005)$ while hemoglobin $A t c$ remained unchanged (LP: $8,0 \pm 0.2 \%, H I: 8,1 \pm 0.3 \%$, n.s.). The frequencies of hypoglycemic episodes in the LP and the $\mathrm{HI}$ groups were $5.0 \pm 3.9$ episodes/month and $6.0 \pm 3.9$ episodes $/$ month, respectively $(p=0.112)$. The types and frequencies of adverse events did not differ between the treatment groups. This study with adolescent patients has shown that compared with regular insulin, insulin lispro improves postprandial blood glucose values and provides comparable long-term glucose control without increase of hypoglycemia. The ability to give insulin lispro immediately before the meal may allow greater flexibility of lifestyle and greater compliance in pediatric diabetic patients.
842

COMPARISON OF LYS ${ }^{1328}$,PRO ${ }^{1329}$-HUMAN INSULIN ANALOG AND HUMAN REGULAR INSULIN IN THE CORRECTION OF INCIDENTAL HYPERGLYCEMIA.

F. Holleman, J.J.G. van den Brand, R.A.R.A. Hoven, J.M. van der Linden, I. van der Tweel, D.W. Erkelens and J.B.L. Hockstra. Diakonessenhuis and University Hospital Utrecht, The Netherlands.

This randomized, non-clamped, double-blind cross-over study compared the

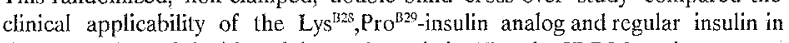
the correction of incidental hypersfycemia in 27 male IDDM patients. Hyperglycemia was induced by witholding the normal evening dose of insulin; the next morning patients fasted and received study insulin according to a BMI and initial glucose-value-related nomogram.

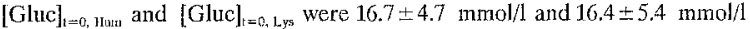
respectively. [Gluc $]_{1=2 \neq 0 \text {. H.m }}$ was $8.7 \pm 2.3 \mathrm{mmol} / \mathrm{l}$ and $[\mathrm{Gluc}]_{1=260 \text {. Lys }}$ was $8.5 \pm 2.5 \mathrm{mmol} / \mathrm{l}$ (means $\pm \mathrm{SD}$ ). The lime courses of blood glucose and $\mathrm{G}_{\mathrm{Ii}}$ (a measure of glucose corrected for differences in administered insulin dose; $G_{R}$ - Glucose*B Body Mass Index / insulin dose) during the 4 study hours were significantly different after regular insulin in comparison with Lyspro (MANOVA, $\mathrm{p}<0.001$ ). At $\mathrm{t}=120 \mathrm{~min}$. glucose had decreased $1.4 \mathrm{mnol} / 1$ more $(95 \% \mathrm{CI} 0.6$ to $2.3, \mathrm{p}=0.002)$ and $\mathrm{G}_{\mathrm{R}}$ had decreased $4.4 \mathrm{~mol}^{*} \mathrm{~kg} /(\mathrm{I}-$ $\left.\mathrm{U}^{*} \mathrm{~m}^{5}\right)$ more $(95 \% \mathrm{Cl} 2.6$ to $6.2, \mathrm{p}<0.001)$ with Lyspro than with regular insulin. At $\mathrm{t}=\mathbf{1 8 0}$ min. Lyspro had exerted $98 \%$ of its effect and regular insulin less than $84 \%$. Overall difference in glucose values was 0.87 mmol/l (Lyspro $<$ regular insulin, MANOVA, $p=0.036$ ) and overall difference in $\mathrm{G}_{\mathrm{R}}$ values was $1,96 \mathrm{~mol}^{1 / \mathrm{kg}} /\left(\mathrm{IU}^{*} \mathrm{~m}^{5}\right)($ Lyspro $<$ regular insulin, $\mathrm{p}=$ ns).

Conclusion: The more rapid action of Lyspro is an advantage in the correction of hyperglycemia even though actual differences in glucoselevels are smaller than suggested by previous, clamped, studies.

\section{4}

INSULIN LISPRO PROTAMINE SUSPENSION (NPL): NIGHT-TIME INSULIN KINETICS AND GLYCEMIC CONTROL IN INSULIN-DEPENDENT DIABETES MELLITUS (IDDM) PATIENTS

M.M.J Janssen ${ }^{1}$ S Casteleiin ${ }^{1}$ C. Popp-Snijders ${ }^{1}$ W. Devillé, $P$. Roach ${ }^{2}$ and R.J. Heine'. 'Academisch Ziekenhuis Vrije Universiteit, Amsterdam, The Netherlands, ${ }^{2}$ Eli Lilly Research Laboratories, Indianapolis, U.S.A.

Insulin lispro protamine suspension (NPL) is a new intermediate-acting insulin preparation composed of protamine and the human insulin analogue Lys(B28) Pro(B29) (lispro). In order to judge the clinical effectiveness of NPL in controlling overnight glycemia we studied insulin kinetics and fasting blood glucose levels following subcutaneous evening administration of NPH and NPL. The study had a double-blind, randomized cross-over design. We studied 11 IDDM patients (10 men, 1 women, age (average) 36.3 (SD: 8.4) years, duration of diabetes $12.5(4.6)$ years, HbA1C $7.3(0.7) \%)$. During a lead-in period of 10 to 14 days patients' evening dose of regular insulin was replaced with lispro insulin. After the lead-in period 2 experiments were carried out, with an interval of 2 to 7 days. During these experiments, which started at 5 p.m. and lasted until $11 \mathrm{a} . \mathrm{m}$. the next morning, blood samples for determination of glucose and insulin concentrations were collected at 30 minute intervals. At 6 p.m., after injecting the evening dose of lispro insulin, patients consumed a standard evening meal. At 9 a.m. patients administered lispro insulin in the usual pre-breakfast dosage of regular insulin and consumed a standard breakfast. At 10 p.m. NPH or NPL was administered. No statistically significant differences between NPH and NPL were detected for the following parameters: insulin peak $(271.2$ (S.D. $=155.9) \mathrm{pmol} / \mathrm{L}$ versus $284.5(132.7) \mathrm{pmol} / \mathrm{L}, \mathrm{p}=0.7)$ (NPH versus NPL), time to peak $(158.2(68.5)$ minutes versus 120.0 (86.9) minutes, $p=0.1)$, insulin area under the curve $(174282.3(92169.8) \mathrm{pmol} / \mathrm{L}$. min. versus $182199.3(100128.2)$ pmol/L.min., $p=0.8$ ), fasting insulin concentration (159.1 (84.9) pmol/L versus $156.6(103.0) \mathrm{pmol} / \mathrm{L}, \mathrm{p}=0.9)$ and fasting glucose concentration ( 7.3 (2.9) $\mathrm{mmol} / \mathrm{L}$ versus 7.9 (3.3) $\mathrm{mmol} / \mathrm{L}, \mathrm{p}=0.7$ ). For extended-action insulin preparations the total duration of insulin effect is an important determinant of their suitability for a basal-bolus regimen, controlling overnight glycemic excursions. In this respect fasting insulin and glucose concentrations suggest no differences between NPH and NPL in clinical effectiveness, despite a tendancy to a shorter time to peak for NPL $(p=0.1)$. 


\section{5}

\section{MEAL COMPOSITION AT BREAKFAST AND TREATMENT WITH} INSULIN LISPRO

K. Bergis, S. Schimers, A. Kuhn-Röpke, U. Gudat, M.E. Trautmann and A Pfützner, Diabetesklinik Bad Mergentheim und Lilly Deutschland GmbH, Bad Homburg, Germany

A randomized crossover pilot study was conducted to compare the effect of varying breakfast compositions on subsequent glycemia in patients treated with insulin lispro directly prior to the meal. Eight patients with type 1 diabetes ( 3 female, 5 male; mean age ( \pm SD): $35 \pm 8.9$ yrs.; mean disease duration: $15 \pm 6.6$ yrs.; [Hemoglobin Atc: $10.0 \pm 1.1 \%$;] BMl: $25 \pm 2.5 \mathrm{~kg} / \mathrm{m}^{2}$ ) participated in the study. On four consecutive days, patients ate either a standard breakfast $(40-45 \%$ carbohydrate(C) $35-40 \%$ fat $(F) / 20 \%$ protein $(P)$, or meals with a high fibre (C: $48 \% / F: 35 \% / \mathrm{P}: 16 \%$ and $6,8 \mathrm{~g}$ fibre), low fibre (C: 46 $\% / \mathrm{F}: 39 \% / \mathrm{P}: 14 \%$ and $3,6 \mathrm{~g}$ fibre), lipid enriched (C: $28 \% / \mathrm{F}: 62 \% / \mathrm{P}$ $11 \%$ and $4,2 \mathrm{~g}$ fibre) or low fat composition (C: $67 \% / \mathrm{F}: 25 \% / \mathrm{P}: 3 \%$ and $3,6 \mathrm{~g}$ fibre). Breakfasts were assigned in randomised order. Basal insulin treatment remained unaltered from day to day. The effect of varying breakfast compositions was assessed by glucose excursions $1 \mathrm{~h}$ and $2 \mathrm{~h}$ after the meal (mean \pm SEN $)$ :

\begin{tabular}{|l|c|c|}
\hline Composition & $\begin{array}{c}1 \mathrm{~h} \text { pp blood glucose } \\
\text { excursion [mg/dl] }\end{array}$ & $\begin{array}{c}2 \mathrm{~h} \text { pp blood glucose } \\
\text { excursion [mg/dl] }\end{array}$ \\
\hline Standard & $17 \pm 12$ & $3 \pm 9$ \\
\hline High fibre & $-22 \pm 4$ & $-3 \pm 4$ \\
\hline Low fibre & $-2 \pm 5$ & $-38 \pm 9$ \\
\hline High fat & $-11 \pm 7$ & $-14 \pm 4$ \\
\hline Low fat & $-19 \pm 7$ & $-29 \pm 13$ \\
\hline
\end{tabular}

As expected, meal content has an influence on postprandial blood glucose excursions (pp BGE) in patients treated with insulin lispro. In order to optimize postprandial blood glucose control it may be appropriate to consider meal composition in addition to preprandial glucose levels
846

MIMICRY OF 1st-PHASE INSULIN SECRETION BY INSULIN LISPRO IN NIDDM; EFFECT ON GLYCEMIA AND THERMIC EFFECT OF THE MEAL. I.K. Daskalova, R.E.Ratner, R.Robbins, J.Holcombe, J. Varshavsky, D.C. Robbins. Lilly Research Laboratories, Indianapolis IN 46285 and Medlantic Research Institute, Washington, D.C. 20010 U.S.A.

Deficient first-phase insulin secretion (FPIS) in NIDDM is associated with impaired glucose tolerance (GT) and diminished thermic effect of a meal (TEM). We hypothesized that "restoration" of FPIS with injection of rapid-onset insulin lispro would decrease post-meal GT and TEM. Nine NIDDMs without FPIS $\{1+3 \mathrm{~min}<20 \mu \mathrm{U} / \mathrm{ml})$ were given lispro Humulin-R or placebo injections $(0.1 \mathrm{U} / \mathrm{kg}$ ) before identical liquid meals $(t=0 \mathrm{~min}$ ). Frequent blood samples were taken ( $t=-10$ to $t=300 \mathrm{~min}$ ) for glucose and insulin. TEM was measured by continuous indirect calorimetry.

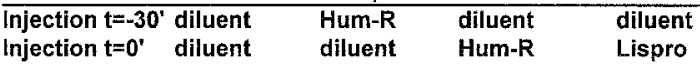

Insulin pM [Values as mean (S.D.)]

Peak value $\quad 310(189) \ddagger \quad 354(148) \ddagger 385(153)^{*} \quad 469(157)$

$\log \Sigma \Delta$ baseline $\quad 4.72(4.45) \quad 4.59(4.21) \quad 4.65(4.23) \quad 4.70(3.45)$

glucose $m M$

Peak value $\quad 15.3(4.1) \quad 13.8(3.7) \quad 15.3(4.1) \quad 14.7(1.0)$

Peak time (min) $95(28) \quad 80(26)^{*} \quad 74(38)^{*} \quad 55(18)$

AUC $\triangle$ baseline $515(303)^{*} \quad 49(412)^{*} \quad-129(556) \ddagger \quad-389(704)$

TEM (Kcal)

kcal 0-300 min $369(64) \quad 361(59) \quad 373(62) \quad 370(56)$

ANOVA, significantly different from Lispro ${ }^{*} p<0.05, t 0<0.01, \pm P<0.001$

Insulin lispro given just before a meal produced significantly higher peak insulin levels and less postprandial glycemia than Humulin- $R$ at $t=0, t=-30$ or placebo. Lispro had no discernable effect on TEM. Thus, in NIDDMs Lispro insulin given just before the meal is effective and results in less postprandial glycemia than regular insulin given at $t=0^{\prime}$ or $t=-30 \mathrm{~min}$.

\section{7}

CATHETER DISCONNECTION IN TYPE I-DIABETIC PATIENTS TREATED WITH CSII: COMPARISON OF INSULIN LISPRO ANO HUMAN REGULAR INSULIN $M$ Pein ${ }^{1}, \mathrm{C}$ Hinselmann ${ }^{1}, \mathrm{~A}$. Pfüzner ${ }^{2}$, and $M$. Drever ${ }^{1}, 1$

Krankenhaus Bethanien. Hamburg and ${ }^{2}$ Bad Homburg. Germany

Diabetic ketoacidosis is one of the risks of continuus subcutaneous insulin infusion therapy (CSII). This study compared the deterioration of glycemic control following interruption of insulin infusion between administration of insuiln Iispro (LP) and human regular insulin (HR) during CSII. Seven patients with type I diabetes (2 male. 5 female, age $33 \pm 7$ years (mean $\pm S D$ ), mean disease duration $18 \pm 7$ years) participated in an eight month crossover trial comparing L $P$ - and HRtreatment by CSI. In each of both treatment periods, the HR $L P$ supply was intentionally interrupted on one morning Deterioration of glycemic control was observed by measuring blood glucose every 15 minutes and ketonuria every 30 minutes. The following cut-of criterla were defined incremental increase in blood glucose greater than 40 $\mathrm{mg} / \mathrm{d} / / 15 \mathrm{minutes}$ rise in blood glucose $150 \mathrm{mg} / \mathrm{dl}$ above baseline. appearance of ketonuria or clinical symptoms of ketoacidosis Mean rollow up times differed between HR (386 \pm 107 minutes (mean + SD) range 135 to 510 minutes $)$ and $L P(251 \pm 111$ minutes, range 45 to 380 minutes, $p<0,05$ ). Despite of its rapid time-action profile the mean follow up time with insulin lispro exceeded four hours in conclusion. for both. numan regular insulin and instilin lispro. substantial deterioration of metabolic control atter interruption of infusion is delayed considerably long eriough to be suitable for use in CSII.

\section{8}

THE USE OF LISPRO INSULIN IN SUBCUTANEOUS INSULIN PUMPS: COUNTERREGULATORY HORMONE RESPONSES.

E. Tsui, , J.L. Chiasson, H. Tildesley, A. Barnie, S. Simkins, X.J. Gao and B. Zinman. Toronto/Montreal/Vancouver, Canada

Insulin Lispro (LP) is a human insulin analog which has advantageous pharmacokinetics in relationship to meal glycemic control. The aim of this study is to determine whether $\mathrm{LP}$ affects the counterregulatory response to hypoglycemia. Ten patients (Age range: 26-51 years: Male:Female 9:1: BMl: 24.9 \pm 0.48 ; mean HbAlc: $7.84 \pm 0.25 \%$ ) with Type 1 diabetes treated with continuous subcutaneous insulin infusion (CSII) (Disetronic H-TRON V $100^{\mathrm{TM}}$ ) were studied using a double-blind, cross over desiun. Patients were randomized to LP or human regular insulin (HR) for 3 monihs, and then crossed over to the other insulin for an additional 3 months. All meal boluses were given $0-15 \mathrm{~min}$. before breakfast, lunch and dinner. The counterregulatory hormone responses to a stepped hypoglycemic clamp (consecutive glucose levels in $\mathrm{mmol} / \mathrm{L}: 4.2 ; 3.5 ; 2.8$, each for 1 hour) were evaluated at the end of each treatment period. HbAlc was significantly lower with LP vs. HR: $7.47 \pm 0.28^{\circ} \%$ vs. $7.9 \pm 0.26 \%(P=0.04)$. The incidence of hypoglycemia per 30 days (capillary blood glucose $<3.0 \mathrm{mmol} / \mathrm{L}$ and/or symptoms) was comparable in each group: 10.6(LP) vs. 11.3(HR). There was no episode of severe hypoglycemia or diabelic ketoacidosis. The peak hormone response to hypoglycemia after each treatment period were not significantly different :

\begin{tabular}{llccc}
$\begin{array}{c}\text { Growth Hormone } \\
(\mathrm{ug} / \mathrm{L})\end{array}$ & $\begin{array}{c}\text { Cortisol } \\
(\mathrm{nmol} / \mathrm{L})\end{array}$ & $\begin{array}{c}\text { Geak level } \pm \text { SEM } \\
(\mathrm{ng} / \mathrm{L})\end{array}$ & $\begin{array}{c}\text { Epinephrine } \\
(\mathrm{nmol} / \mathrm{L})\end{array}$ \\
\hline $\mathrm{HR}$ & $40.7 \pm 5.5$ & $403 \pm 35$ & $75.6 \pm 6.8$ & $0.90 \pm 0.31$ \\
$\mathrm{HP}$ & $39.1 \pm 5.1$ & $430 \pm 28$ & $79.6 \pm 5.6$ & $1.18 \pm 0.34$
\end{tabular}

We conclude that the use of LP in CSII results in improved glycemic control without increasing the rate of hypoglycemia or adversely affecting counterregulatory response to hypoglycemia. 
849

NEUTRAL PROTAMINE LISPRO: ACTIVITY PROFILE OF S.C. ADMINISTRATION WITH AND WITHOUT ADMIXTURE OF SOLUBLE LISPRO J.R. Radziuk, B. Bradley, L. Welsh, M.R. De Fellipis and P. Roach, Otrawa Civic Hospital, Otawa Canada, Eli Lilly Company. Indianapolis, USA An intermediate-acting formulation (neutral protanine suspension [NPL]) of insulin lispro (Lys ${ }^{\mathrm{B} 28} \mathrm{Pro}^{\mathrm{B} 29}$ human insulin) was developed. NPL was compared to NPH in a cross-over study in 8 normal subjects using a euglycemic clamp to maintain fasting glycemia for $16 \mathrm{~h}$ after subcutaneous (s.c.) injection of either formulation $(0.4 \mathrm{U} / \mathrm{kg})$. The biveffectiveness of biphasic mixtures of soluble lispro and NPL wals assessed in ten 10h-fasted normal subjects where lispro and NPL (total dose: $0.3 \mathrm{U} / \mathrm{kg}$ ) were given s.c. in 5 combinations (lispro/NPL: $100 / 0 \%, 75 / 25 \%, 50 / 50 \%, 25 / 75 \%, 0 / 100 \%$. Fasting glycemia was maintained with a $20 \%$ glucose infusion. NPL and NPH reached similar $\mathrm{R}_{\max }$ (maximal glucose infusion rates, $G I R)$ of $3.69 \pm 0.51$ and $3.03 \pm 0.47 \mathrm{mg} / \mathrm{kg}$-min $(p=0.36)$. NPL demonstrated a more rapid onset of action with differences in GIR for $75<t<180 \mathrm{~min}(p<0.05)$. There was no difference in GIR at $t=960 \mathrm{~min}$. In the 3 mixtures of lispro and NPL $(25 \%, 50 \%$, and $75 \%$ lispro), lispro demonstrated a mean profile which approximated a square-wave pattern. Initial GIR plateaued between 50 and $240 \mathrm{~min}$ followed by a rapid decrease over $\sim 60 \mathrm{~min}$ and maintenance until $t=20 \mathrm{~h}$, only gradually tapering to $0.7 \pm 0.3,0.95 \pm 0.3$, $0.96 \pm 0.3 \mathrm{mg} / \mathrm{kg}$-min for the 3 mixtures $(25 \%, 50 \%$, and $75 \%$ lispro) resp. For the 5 groups $\left(100 \%\right.$ to $0 \%$ lispro), maximum insulin concentrations $\left(\mathrm{C}_{\text {trax }}\right)$ were $6.6 \pm 1.0,5.3 \pm 0.7,2.6 \pm 0.4,1.9 \pm 0.3,1.3 \pm 0.3 \mathrm{ng} / \mathrm{ml}$ at $\left(T_{m a x}\right) 71 \pm 15,65 \pm 9$, $83 \pm 9,110 \pm 11$ and $163 \pm 40 \mathrm{~min}$. $\mathrm{R}_{\max }$ was $11.0 \pm 1.4,8.7 \pm 1.1,8.4 \pm 0.7,6.2 \pm 0.7$ and $3.7 \pm 0.6 \mathrm{mg} / \mathrm{kg}-\min$ at $T_{\max }=104 \pm 23,78 \pm 9,135 \pm 5,157 \pm 32$ and $311 \pm 73$ $\min . \mathrm{C}_{\max }, \mathrm{R}_{\mathrm{max}}$, and fractional dose of lispro were highly correlated $(\mathrm{r}=0.80$ $0.75,0.72, \mathrm{p}<0.0001$ ). In the presence of lispro, $\mathrm{T}_{\max }$ and $\mathrm{T}_{\mathrm{Rmax}}$ were not different. These data indicate that NPL demonstrates an extended activity similar to NPH and that mixtures of NPL and lispro maintain the rapid onset of action previously seen with lispro.
850

\section{THE TIME-ACTION OF ACYLATED INSULINS DEPENDS} ON THE ANIMAL MODEL

Svend Havelund, P. Kurtzhals, I. Jonassen, $U$. Ribel and $J$. Markussen,

Insulin Research, Novo Nordisk A/S, DK-2880 Bagsvaerd, Denmark.

There is a tremendous need for a long-acting insulin that results in lower day-to-day variation and fewer hypoglycemic episodes than the NPH and Lente type insulin suspensions. NN304 (Lys ${ }^{\mathrm{B} 29}$-tetradecanoyl des(B30) insulin) is a novel insulin analog that might fulfil these criteria. Together with the side-chain and carboxylate group of Lys ${ }^{\mathrm{B} 22}$, the fatty acid attached to the $\epsilon$-amino group of $L y s^{629}$ provides an albumin binding ligand, giving the acylated insulin an affinity of $1.0 \times 10^{5} \mathrm{M}^{-1}$ for human serum albumin. The binding to albumin in subcutis, serum and target tissues renders NN304 long-acting after subcutaneous injection.

Due to the correlation between albumin binding and time-action of the acylated insulins, preclinical experiments with these analogs must be interpreted considering their affinity for albumin in the applied animal model. The amino acid sequences of albumins from various species show a considerable degree of variation, which is reflected in differences in ligand affinities. In the present study, it is shown that the affinity of fatty acid acylated insulin for albumin varies more than $\mathbf{5 0}$-fold among species. The relative affinities of acylated insulins for albumin in human, pig and rabbit serum are determined to about $1: 1.5: 35$. It is shown that the several fold higher binding affinity in rabbit serum than in pig serum is reflected in a relatively more protracted effect after s.c. injection in rabbits than in pigs. Due to the similar binding affinities in pig serum and human serum, the pig model should provide a useful estimate of the degree of protraction of NN304 in humans. More generally the results show that species differences in ligand binding can be of major importance in the preclinical pharmacokinetic and-dynamic evaluation of albumin bound drugs.
851

IN VIVO FATE OF THE LONG-ACTING INSULIN ANALOG NN304 EXAMINED BY RAT SCINTIGRAPHY

U. Ribel, S. Havelund, I. Jonassen, U.D. Larsen, J. Markussen, and P. Kurtzhals, Novo Nordisk A/S, DK-2880 Bagsvaerd

The prolonged effect of the new basal insulin analog NN304 $\left(\mathrm{Lys}{ }^{\mathrm{B} 2 \mathrm{~g}}\right.$-tetradecanoyl des(B3O) insulin) is ascribed to its high affinity for serum albumin. In order to examine how the albumin binding affects the pharmacokinetics of NN304, we injected ${ }^{123}$ l-labeled NN304 i.v. into rats and followed the distribution of radioactivity by whole-body scintigraphy. NN304 disappeared from the heart window, which represents the plasma compartment, with a $t_{1 / 2}$ of $6.8 \pm 0.7 \mathrm{~min}(n=5), 5-6$ times more slowly than human insulin. After injection of NN304, the liver radioactivity increased slowly and reached a maximum after $15 \mathrm{~min}$, whereas maximal liver radioactivity was seen $4 \mathrm{~min}$ after injection of human insulin. The cumulated radioactivity in the kidneys was $1.6 \pm 0.6$ $\%(n=5)$ and $12.8 \pm 0.7 \%(n=13)$ after injection of NN304 and human insulin, respectively. To explain the marked difference in the kinetic fate of NN304 and human insulin, we performed a series of receptor- and albumin binding experiments. Firstly, we found that NN304 cannot simuitaneously bind to albumin and to the insulin receptor. Consequently, only the unbound fraction of the total amount of NN304 present in the bloodstream may at any given time-point undergo receptor-mediated clearance. Secondly, we found that NN304 dissociates from serum albumin with a half-time in the order of seconds, which is similar to the sinusoidal transit time. Hence, the dissociation of NN304 from albumin may be the rate-limiting step in the hepatic uptake of the anaiog. We conclude that the tight binding of NN304 to serum albumin has a major influence on the pharmacokinetics of the analog after $i . v$. injection. In contrast, it has previously been shown that differences in the kinetic fate of non-albumin bound insulins can be explained entirely by differences in their relative receptor binding affinities. 
PS 46

New Pharmacological Approaches

\section{2}

EFFECTS OF CYCLIC AMP PHOSPHODIESTERASE INHIBITORS ON INSULIN SECRETION AND GLYCEMIA

Janice C. Parker, Maria A. VanVolkenburg, Nancy A. Nardone and Kim M. Andrews. Pfizer Inc, Central Research Division, Groton, CT, USA.

Since inhibitors of cyclic AMP phosphodiesterase (PDE) have been demonstrated to stimulate insulin secretion both in vitro and in vivo, we have attempted to evaluate their potential as agents for the treatment of diabetes. The contribution of various isozymes of PDE to the modulation of insulin secretion in vitro was estimated using isozyme-selective inhibitors, and the effect of these agents on insulin secretion and plasma glucose levels evaluated in vivo in an animal model of diabetes, the ob/ab mouse. Selective inhibition of FDE III by milrinone stimulated insulin secretion from isolated rat islets, but inhibition of PDE IV by rolipram had no effect. Oral administration of the PDE III-selective inhibitor milrinone $(10 \mathrm{mg} / \mathrm{kg})$ stimulated plasma insulin levels from $1060 \pm 150 \mathrm{pM}$ to 3690 $\pm 215 \mathrm{pM}(\mathrm{p}<0.001)$, in obese diabetic ob/ob mice 90 min. after dosing. However, glucose tolerance did not improve; $90 \mathrm{~min}$. after receiving $1 \mathrm{gm} / \mathrm{kg}$ glucose, plasma glucose levels were $27.8 \pm 3.2 \mathrm{mM}$ in untreated $o b / 0 b$ mice, but rose to $60.4 \pm 3.4 \mathrm{mM}(p<0.001)$ in $a b / a b$ mice that had received $10 \mathrm{mg} / \mathrm{kg}$ milrinone concurrently with the glucose load. This elevation in plasma glucose was probably a consequence of inhibition of the PDE III activity expressed in liver and adipose tissue, with resulting activation of glycogenolysis and lipolysis. Incubation of isolated rat hepatocytes with $50 \mathrm{uM}$ milrinone increased glucose release from $1.93 \pm 0.01$ to $2.00 \pm 0.01 \mathrm{mg} / 10^{6}$ cells $/ \mathrm{hr}(p<0.001)$. Milirinone stimulation $(50 \mu \mathrm{M})$ of isolated rat adipocytes increased glycerol release from 8.4 \pm 1.5 to $35.2+0.7 \mu \mathrm{g} / 10^{6}$ cells/hr $(\mathrm{p}<0.001)$. We conclude that while selective inhibitors of PDE III are effective insulin secretagogues, their therapeutic utility is likely to be limited by their concurrent stimulation of lipolysis and hepatic glucose output.

\section{3}

THE EFFECT OF MAGNESIUM REPLACEMENT IN INCREASING DOSES ON THE CONTROL OF NON-INSULIN-DEPENDENT DIABETES MELLITUS

T.Cnuz. J.Pousada, K.Barbosa and M.L.Lima. Federal University of Bahia Brazil. Hypomagncsemia occurs in 25 to $38 \%$ of patients with NIDDM. Screral studics have suggested an association betwcen magnesium( $(\mathrm{Mg})$ depletion and insulin resistance and/or reduction of insulin sccretion in these cases. Aiming to evaluate if $\mathrm{Mg}$ replacement (as $\mathrm{MgO}$ ) would improve metabolic control in NIDDM. 128 patients (32 male, 96 femalc aged 30-69) treated by diet or diet plus oral antidiabetic drugs were studied in the Bahia Federal University Hospital. Brazil. Cases in risk for hyponagnescmia or with reduced renal function were excluded. The study was a clinical randomised double-blind placebo-controlled assay. Patients either reccived placebo. $\mathrm{MgO} 20.7 \mathrm{mmol}$ or $\mathrm{MgO} 41.4 \mathrm{mmol} /$ day (clementary Mg) for 30 davs. $\mathrm{Mg}$ concentrations were measured in plasma, in mononuclear cells and in $24 \mathrm{~h}$ urine samples. Fasting blood sugar. HbAlc and fructosamine were used as parameters of metabolic control. Low Mg levels in plasma and mononuclear cells were found in 47.7\% and $31.1 \%$ respectively. Intracellular $\mathrm{Mg}$ in diabetic patients was significantly

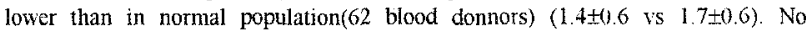
correlations were seen between plasma and intracellular $\mathrm{Mg}$ concentrations $(\mathrm{r}=-0.179 ; \mathrm{p}=0.15)$ and between $\mathrm{Mg}$ levels and glycemic control $(\mathrm{r}=-0.165$ $\mathrm{p}-0.12)$. In the diabetic patients with peripheral neuropathy. intracellular $\mathrm{Mg}$ levels were lower than in those without ( $1.2 \pm 0.5$ vs $1.5 \pm 0.6 \mathrm{\mu g} / \mathrm{mg}$ of protein). The same was observed in those with coronary disease ( $(1.0 \pm 0.5 \mathrm{vs} 1.5 \pm 0.6 \mu \mathrm{g} / \mathrm{mg}$ of protein). In placebo and in $\mathrm{Mg} 20.7$ mmol groups neither a change in plasma and intracellular levels nor improvement in glycemic control were observed. Replacement with $\mathrm{Mg} 41.4 \mathrm{mmol}$ increased plasma . cellular and urine $\mathrm{Mg}$ and caused a significant fall $(4.1 \pm 0.8$ to $3.8 \pm 0.7 \mathrm{mmol} / 1$ in fructosamine (normal 1.9 2.9). $\mathrm{Mg}$ depletion is common in NIDDM patients, especially in those with neuropathy and with coronary disease. More prolonged use of $\mathrm{Mg}$ in doses higher then usually is needed in order to establish its routine or selective administration in NIDDM either for improving its control or preventing chronic complications.

\section{4}

ENDOCRINE AND METABOLIC EFFECTS OF DEXFENFLURAMINE IN OBESE WOMEN WITH POLYCYSTIC OVARY SYNDROME.

Ö. Açbay and S. Gündoğdu. Department of Internal Medicine, Division of Endocrinology, Cerrahpaşa Medical Faculty of istanbul University, İstanbul, Turkey.

Insulin resistance (IR) seems to be a contributing factor of gonadotropin and sex steroid abnormalities in polycystic ovary syndrome (PCOS). It has been shown that weight loss induced by a low calorie diet reduces these hormonal abnormalities in obese women with PCOS. Dextenfluramine (dF) not only aids weight loss in obese subjects but also reduces IR in Type 2 diabetic subjects, independently of weight loss. Therefore, to determine the hormonal and metabolic effects of $\mathrm{dF}$ in PCOS, thirty insulin-resistant obese women with PCOS were enrolled in a single-blind, placebo-controlled study. Sixteen of the 30 patients were given $\mathrm{dF}$ ( $15 \mathrm{mg}$ twice daily) and the remaining 14 were given placebo $(\mathrm{pl})$ in addition to a low calorie diel for 1 year. Both groups were homogenous with regard to age, height, weight, waist/hip ratio (WHR), insulin sensitivity (estimated with an i.v. insulin tolerance tesi), serum levels of lipids and androgens. After 6 months, body weight, WHA, serum levels of glucose, insulin, total cholesterol, LDLcholesterol, triglyceride, androgens, luteinizing hormone/folliclestimulating hormone ratio and the areas under the curve for plasma glucose and insulin during an oral glucose tolerance test decreased significantly more $(\mathrm{p}<0.01$ for $\mathrm{dF} / \mathrm{pl}$, in all) whereas insulin sensitivity and HDL-cholesterol level increased significantly more $(p<0.01$ for $\mathrm{dF} / \mathrm{pl}$, in both) with $\mathrm{dF}$ than with placebo. After 12 months, the differences in the changes in these parameters between the two groups increased further. We conclude that dF-plus-diet is more efficient than diet alone in the reduction of hormonal and metabolic abnormalities in obese women with PCOS.
855

Superoxide scavanging effects of free aminoacids. The Diabetic Angiopathy Research Group: S. Habersack-Wallner, E. Kreuz, W.F. Graier*, B. Bahadori, H. Toplak, T.C. Wascher. Dept. Internal Medicine and *Inst. of Medical Biochemistry, Univ. Graz, Austria

The effect of aminoguanidine on the formation of advanced glycation endproducts is, at least in part, thought to be based on radical scavanging properties. Since aminoguanidine chemically resembles to aminoacids, the aim of the present investigation was to assess the effects of various aminoacids on superoxide driven autooxidation of pyrogallol in comparison to aminoguanidine. Pyrogallol autooxidation was monitored photometrically as the change of absorption at $320 \mathrm{~nm}$. The probes $(840 \mu \mathrm{l}$ water, $100 \mu \mathrm{l}$ tris-HCl-EDTA buffer, $40 \mu 1$ test substance) were placed in the photometer $\left(25^{\circ} \mathrm{C}\right)$, reaction was started by addition of $20 \mu \mathrm{l}$ pyrogallol (10mM) and monitored over 3 minutes. Results of 4 to 6 experiments in each group are given as change of absorption per minute (meantSD) and were compared by ANOVA. Pyrogallol autooxidation induced a change of absorption of $0.172 \pm 0.010 / \mathrm{min}$. Aminoguanidine dose dependently reduced pyrogallol autooxidation $(0.119 \pm 0.007 / \mathrm{min}$ at $4 \mathrm{mM}, \mathrm{p}<0.001)$. The most effective aminoacid tested was L-cysteine $(0.025 \pm 0.004 / \mathrm{min}$ at $4 \mathrm{mM}, \mathrm{p}<0.001$ vs. aminoguanidine). L-ornithine and glycine were significantly $(\mathrm{p}<0.05)$ more potent than aminoguanidine. L-lysine, $\mathrm{L}$-arginine, $\mathrm{L}$-leucine and $\mathrm{L}$-serine showed superoxide scavanging effects comparable to aminoguanidine. L-citrulline and Hydroxy-Lproline on the other hand did not affect pyrogallol autooxidation In conclusion, our results demonstrate superoxide scavanging properties of several aminoacids, comparable or even stronger than aminoguanidine. These effects obesrved might be involved in the control of oxidative stress in glycoxidation and diabetic vascular disease. 
856

EFFECTS OF DOXAZOSN AND ENALAPRIL ON BLOOD FLOW TO LEG MUSCLES

H. Lithell and P-E. Andersson, Department of Geriatrics,

Samariterhemmets Hospital, Uppsala, Sweden

The aim of this double-blind, parallel group study was to investigate the effects of doxazosin or enalapril treatment on the insulin-induced increase in skeletal muscle blood flow in hypertensive, hypertriglyceridaemic patients. Treatment duration was 6 months. Evaluations included systolic blood pressure (SBP) and ultrasonic measurements (Doppler) of femoral artery blood flow performed in connection with a euglycemic hyperinsulinaemic clamp procedure. At baseline, there was a significant inverse relationship between sitting SBP and insulin-induced change in femoral artery blood flow ( $r=-$ $0.32, p<0.05, n=41$ ). This finding suggests that patients who are most hypertensive are also most insulin resistant. We previously reported that doxazosin, in contrast to enalapril, significantly increased insulin sensitivity, but neither treatment significantly increased femoral artery blood flow, in this study. After 6 months' treatment, there was a significant positive relationship between change in sitting SBP and change in femoral artery blood flow with doxazosin ( $r=0.49, p<0.05$, $\mathrm{n}=16$ ). In contrast, patients treated with enalapril showed a significant inverse relationship between change in sitting SBP and change in femoral artery blood flow $(r=-0.46, p<0.04, n=21)$. In conclusion, while it is known that doxazosin reduces blood pressure without compensatory increase in cardiac output, and it is speculated that doxazosin may increase transit time for blood over the muscle bed by capillary recruitment. These factors may explain the increase in insulin sensitivity, although not necessarily leading to increased skeletal muscle blood flow.

\section{7}

EFFECT OF PHENSUCCINAL ON NEPHROPATHY

DEVELOPMENT IN RABBITS WITH DIABETES

V. Poltorack, V. Natarov, N. Gorbenko, A. Gladkich,

L. Pivovarevich, V. Lipson, O. Borodina and V. Petruk.

Ukrainian Scientific Research Institute of Endocrine Diseases

Pharmacotherapy, Kharkov, Ukraine

We previously reported antidiabetogenic effect of the new lowtoxic succinic acid derivative phensuccinal $(\mathrm{Ph})$ due to stimulation of pancreatic beta-cells regeneration and antioxidative properties. The aim of the study was to assess the impact of $\mathrm{Ph}$ on nephropathy development and metabolic state in rabbits with long-term absolute insulin insufficiency. Male chinchilla rabbits were made diabetic by i.v. injection of dithizone $(35 \mathrm{mg} / \mathrm{kg} \mathrm{bw})$ and were subsequently ( 2 months after the induction of diabetes) randomized into a control diabetic group ( $\mathrm{C}$-group, $\mathrm{n}=6$ ) and a $\mathrm{Ph}$-treated group ( $\mathrm{Ph}$-group, $\mathrm{n}=6$ ). $\mathrm{Ph}$-group was given $25 \mathrm{mg} / \mathrm{kg}$ bw $\mathrm{Ph}$ per os for 2 months. At the end of the study metabolic parameters were better in Ph-group compared to C-group (the decrease of basal plasma glucose $-8.5 \pm 1.1 \mathrm{mmol} / 1$ and $14.9 \pm 2.4$ $\mathrm{mmol} / \mathrm{l}, \mathrm{p}<0.01$, the improvement of glucose tolerance and the lowering of plasma diene conjugates, $p<0.01$ ). After Ph-treatment it was revealed partial restoration of pancreatic islets architectural pattern, significant $(p<0.01)$ reduction of the total kidney weight, albuminuria and glomerular basement membrane thickening $(440 \pm 50 \mathrm{~nm}$ and $780 \pm 30 \mathrm{~nm}, \mathrm{p}<0.01$, respectively in $\mathrm{Ph}$ - and C-groups compared to $380 \pm 40 \mathrm{~nm}$ in intact controls) as well as protection from mesangial expansion. Our study suggests that $\mathrm{Ph}$ inhibits the development of diabetic nephropathy in rabbits with dithizone-induced diabetes due to improvement of metabolic characteristics including suppression of oxidative stress.

\section{8}

EFFECT OF SEROTONIN-4 RECEPTOR AGONIST CISAPRIDE ON ALDOSTERONE SECRETION IN DIABETIC PATIENTS

Y.Y. Huang, B.R.S. Hsu and J.S. Tsai, Department of Endocrinology and Metabolism, Chang-Gung Medical Center. Taipei, Taiwan

Serotonin-4 (5-HT4) receptor agonist has been shown to involve in regulating aldosterone secretion in normal subjects. The kinetic study indicated that $10 \mathrm{mg}$ of Cisapride, a $5-\mathrm{HT} 4$ receptor agonist, given orally to the normal subjects raise the plasma aldosterone about two-folds and attains a plateau at 120 minutes in the supine position. Its action is not mediated by a change of the plasma renin activity. Hypoaldosteronism is a complication of some of the diabetic patients. To examine the aldosterone secreting activity in the diabetic patients, forty patients with adult onset diabetes mellitus and eleven healthy volunteers were enrolled in this study. Diabetic patients were further subcategorized into group A (normotensive, not nephropathic, $n=26$ ) and group $B$ (mild renal impairment, $n=14$ )

\begin{tabular}{|lcrc|}
\hline Group & Control & \multicolumn{1}{c|}{ A } & \multicolumn{1}{c|}{ B } \\
\hline Age $(\mathrm{yr})$ & $50.4 \pm 3.2$ & $56.9 \pm 2.2$ & $63.9 \pm 1.7$ \\
Gr $(\mathrm{mg} / \mathrm{dL})$ & $0.9 \pm 0.1$ & $1.1 \pm 0.0$ & $1.8 \pm 0.1$ \\
$\mathrm{~K}(\mathrm{mEq} / \mathrm{L})$ & $4.2 \pm 0.1$ & $4.2 \pm 0.1$ & $4.5 \pm 0.1$ \\
O' $^{\prime}$ Aldosterone $(\mathrm{pg} / \mathrm{mL})$ & $108.2 \pm 15.2$ & $118.1 \pm 14.9$ & $108.2 \pm 27.3$ \\
$\Delta$ Aldo $(\%)$ & $180.7 \pm 26.0$ & $175.2 \pm 38.2$ & $157.6 \pm 38.0$ \\
\hline
\end{tabular}
$\triangle$ Aldo $(\%) \quad 180.7 \pm 26.0 \quad 175.2 \pm 38.2$

Data were expressed as mean \pm SEM.

These results suggest that both non-complicated and mild nephropathic diabetic patients have normal aldosterone responsiveness to the 5-HT4 receptor agonist. Our data indicates that the oral cisapride is useful in studying the aldosterone secretion among the diabetic patients. This pharmacological agent has been recommended to treat diabetic gastropathic patients. The implication of chronic stimulation of aldosterone secretion by this agent on those patients needs to be further evaluated.

\section{9}

LIPOIC ACID IMPROVES GLUCOSE MEDIATED GLUCOSE UPTAKE IN PATIENTS WITH NIDDM

T.Konrad P.Vicini ${ }^{1}$ C Cobelli' K Kusterer. O.Frankenberger and K.H. Usadel

Department of Medicine I. University of Frankfurt. Gormany. Department of Electronics and Infonnatics. University of Padova. Italy

The modified frequently sampled intravenous glucose tolerance tost (FSIGTT) allows to investigate the contribution of the mass action of glucose itself (glucose effectiveness. SG) and insulin sensitivity (SI) on glucose tolerance. SG and insulin sensitivity (SI) are reduced in patients with non-insulindependent-diabetes mellitus (NIDDM. In this study 10 lean (group A) and 10) obese patients with NIDDM were treated orally with lipoic acid. $600 \mathrm{mg}$. twice a day. Before the treatment started. the patients undervent an oral glucose tolerance test (OGTT. $75 \mathrm{~g}$ ) and the modified protocol for FSIGTT $(0.05 \mathrm{IE} / \mathrm{kg}$. Insulin. bolus at $20 \mathrm{~min})$. Further. pyruvate acid. lactate and $\beta$ hydoxybutyrate acid were determined during OGTT and FSIGT. The analysis of the FSIGT data was based on Bergman's Minimal Model of glucose disappearance. Glucose and insulin profiles were analyzed by using the progam SAAM II. The initial increased pyruvate acid concentrations during oral (A: $88.8 \pm 21 \mu \mathrm{mmol} / \mathrm{l}: \mathrm{B}: 103.1 \pm 23 \mu \mathrm{mol} / \mathrm{l})$ and intravenous glucose load dropped significantly under oral treatment with lipoic acid after 4 wecks A:47士 $21 \mu \mathrm{mol} / \mathrm{l}$ : B: $54.7 \pm 12 \mu \mathrm{mol} / \mathrm{l}: \mathrm{p}<0.05$ ). Fasting plasma glucose levels and the glucose concentraion after 120 min OGTT were significantly lower under lipoic acid treatment in group A $(\mathrm{p}<0.05)$. In group B the basal glucose was significantly decreased after treament. Further. SG increased from $1.81 \pm 0.054$ to $2.32 \pm 0.13(\mathrm{CV}<24 \% ; \mathrm{p}<0.05)$ in group A. from $1.23 \pm 0.08$ to $1.66 \pm 0.09$ $10^{-2} \mathrm{~min}^{-1} \quad(\mathrm{CV}<28 \%)$ in group $\mathrm{B}$. The decreasing pyruvate concentrations and decreased fasting plasma glucose levels under lipoic acid treatment in diabetic patients indicate an improved intracellular glucose mctabolism of the muscle and of the liver. This findings are confirmed by increased SG values which reflect an improved glucose uptake in muscle tissue and inhibition of hepatic glucose output by glucose itself at basal insulin concentrations. 
THE EFFECT OF UBIKINON (COENZYME O10) ON GLYCAEMIC CONTROL AND WELL-BEING IN IDDM PATIENTS.

J.E. Henriksen, C.B. Andersen, O. Hother-Nielsen, A. Vaag and H. BeckNielsen. Diabetes Research Centre, Odense University, Denmark.

Several IDDM patients in our outpatient clinic reported independently that they had observed accumulated hypoglycaemic episodes after initiating treatment with Ubikinon (COQ10). Thus, the aim of our study was to investigate the effect of CoQ10 on glycaemic control and insulin requirement. Thirty-five IDDM patients were included in a randomised double blinded placebo con-trolled study with a run-in period of four weeks and an intervention period of 12 weeks. Patients received either $\mathrm{CoQ} 10,100 \mathrm{mg}$ daily or placebo. Insulin doses were adjusted based on patients measurements of home blood glucose concentrations. At randomisation no differences existed between the $\mathrm{C} 0 \mathrm{Q} 10$ and the placebo groups: Age ( $35.5 \pm 2.0$ vs $35.3 \pm 2.4$ yr), BMI ( $23.5 \pm 0.7$ vs $\left.24.0 \pm 0.6 \mathrm{~kg} / \mathrm{m}^{2}\right)$, HbA1c $(8.04 \pm 0.19$ vs $8.02 \pm 0.2 \%)$, daily insulin dose $(52.1 \pm 3.1$ vs $52.4 \pm 5.0 \mathrm{U})$ or mean daily blood glucose concentration ( $8.90 \pm 0.42$ vs $8.96 \pm 0.42 \mathrm{mM})$. Serum $\mathrm{COQ} 10$ concentration increased in the CoQ10 group $(0.9 \pm 0.1$ vs $2.0 \pm 0.24$, $\mathrm{p}<0.005)$ whereas no changes were observed in the placebo group $(0.9 \pm 0.1$ vs $0.9 \pm 0.1$. Following intervention no differences existed between the CoQ10 and the placebo groups regarding $\mathrm{HbA1c}(7.86 \pm 0.21$ vs $7.84 \pm 0.20 \%)$, mean daily blood glucose concen-trations $(8.06 \pm 0.45$ vs $8,53 \pm 0.46)$. mean insulin dose $(52.4 \pm 3.1$ vs $52.6 \pm 5.3 \mathrm{U}$ ), hypoglycaemic episodes $(2.0 \pm 0.4$ vs $2.5 \pm 0.5$ episodes/week), or cholesterol concentrations $(4.81 \pm 0.22$ vs $4.78 \pm 0.26 \mathrm{mM})$. Furthermore no differences existed in the well-being of the patients reported from a visual analog scale (physical: $0.67 \pm 0.05$ vs $0.69 \pm 0.05$, mental: $0.70 \pm 0.06$ vs $0.73 \pm 0.06$ ). In conclusion $\mathrm{CoQ} 10$ does not improve glycaemic control or reduce insulin requirement and can be taken freely by IDDM patients. However, this study does not indicate that $\mathrm{CoQ} 10$ has any beneficial effect on the well-being of the diabetic patient.

\section{PS 47}

\section{Sulfonylureas and Metformin}

\section{1}

THE EFFECTS OF METFORMIN ON GLUCOSE DESENSITIZATION IN ISOLATED HUMAN ISLETS

P. Marchetti, R. Lupi, R. Giannarelli, A. Coppelli, C. Tellini, S. Del Guerra, M. Lorenzetti, M. Carmellini, F. Mosca, R. Navalesi. Cattedra Malattie Metabolismo, Istituto Clinica Medica II, Università di Pisa, Italy

Glucose $(G)$ desensitization is a temporary, readily induced and reversible state of beta-cell refractoriness to $G$ itself, due to exposure of the beta cells to hyperglycemias of varying degrees and durations. In the present study we evaluated whether the oral of varying degrees and durations. In the present study we evaluated whether the oral
antidiabetic agent metformin affects $G$ desensitization in isolated, human islets. After antidiabetic agent metformin affects $\mathrm{G}$ desensitization in isolated, human islets. After
the isolation of human islets from the pancreas of two multicrgan donors, batches of 10 islet equivalents ( $150 \mu \mathrm{m}$ diameter sized islets) were incubated for 24h in M199 culture medium, supplemented with antibiotics and serum, and containing either 100 (group A), 200 (group B) or 400 (group C) $\mathrm{mg} / \mathrm{dl} \mathrm{G}$, with $(\mathrm{M}+$ ) or without (M-) therapeutical concentration $(2.4 \mu \mathrm{g} / \mathrm{ml})$ of metformin. Then, the islets were washed, and challenged with 60 and $300 \mathrm{mg} / \mathrm{dl}$ glucose during static incubation (at least 6 replicates per each experimental point). Insulin release from group A, M-was $6.1 \pm$ $0.4 \mu \mathrm{U} / \mathrm{ml}$ (mean $\pm \mathrm{SE}$ ) at $60 \mathrm{mg} / \mathrm{dl} \mathrm{G}$, and increased to $15.7 \pm 4.2 \mu \mathrm{U} / \mathrm{ml}$ at $300 \mathrm{mg} / \mathrm{dl}$ $\mathrm{G}(\mathrm{p}<0.05)$. Insulin secretion at 60 and $300 \mathrm{mg} / \mathrm{dl} \mathrm{G}$ from group $\mathrm{B}, \mathrm{M}$ - was $8.6 \pm 1.4$ and $12.9 \pm 1.3$ (NS vs $60 \mathrm{mg} / \mathrm{dl} \mathrm{G}) \mu \mathrm{U} / \mathrm{ml}$, respectively, and that from group $\mathrm{C}, \mathrm{M}$ was $10.1 \pm 1.8$ and $14.7 \pm 4.0(\mathrm{NS}$ vs $60 \mathrm{mg} / \mathrm{dl}) \mu \mathrm{U} / \mathrm{ml}$. Thus, when the islets had been cultured for $24 \mathrm{~h}$ with 200 or $400 \mathrm{mg} / \mathrm{dl} \mathrm{G}$, the release of insulin at $300 \mathrm{mg} / \mathrm{dl} \mathrm{G}$ was not significantly higher than at $60 \mathrm{mg} / \mathrm{dl} \mathrm{G}$, showing islet desensitization. When we tested the isiets that had been previously exposed to both glucose and metformin, the following results were obtained upon challenge of the islets with 60 and $300 \mathrm{mg} / \mathrm{dl} G$ group $\mathrm{A}, \mathrm{M}+: 6.6 \pm 0.1$ and $17.2 \pm 4.6 \mu \mathrm{U} / \mathrm{ml}(\mathrm{p}<0.05)$; group $\mathrm{B}, \mathrm{M}+: 6.3 \pm 0.3$ and $14.7 \pm 2.6 \mu \mathrm{U} / \mathrm{ml}(\mathrm{p}<0.05)$; group $\mathrm{C}, \mathrm{M}+: 9.5 \pm 1.0$ and $18.9 \pm 2.9 \mu \mathrm{U} / \mathrm{ml}(\mathrm{p}<0.05)$ Thus, islets exposed to metformin maintained their glucose sensitivify, despite concomitant incubation at high glucose levels. These in-vitro results suggest a possible protective role of metformin on human islets exposed to hyperglycemia.

\section{2}

METFORMIN ENHANCES THE ACTION OF INSULIN IN ISOLATED PERFUSED HINDLIMB

Z. Zhang, N. Wiernsperger, and J. Radziuk, Ottawa Civic Hospital, Ottawa, Canada and Groupe Lipha, Lyon, France.

In order to determine possible actions of metformin at the level of peripheral tissue, an isolated rat hindlimb preparation was perfused with an oxygenated medium consisting of 4 parts human erythrocytes and 6 parts Krebs-Ringer bicarbonate buffer with $3 \%$ bovine serum albumin. Prior to perfusion, rats were fasted for $20 \mathrm{~h}$. Four groups were studied: (I) control and hindlimb with initial perfusate concentrations of: (II) $90 \mu \mathrm{g} / \mathrm{ml}$ metformin or (III) $0.17 \mathrm{pmol} / \mathrm{ml}$ insulin or (IV) $90 \mu \mathrm{g} / \mathrm{ml}$ metformin $+0.17 \mathrm{pmol} / \mathrm{ml}$ insulin. In III and IV insulin was also infused at $0.28 \mathrm{pmol} / \mathrm{min}$. Perfusate glucose levels were monitored and clamped at approximately $5.6 \mathrm{mmol} / \mathrm{L}$. Perfusion was continued for $2 \mathrm{~h}$ and samples were taken at the inflow to, and outflow from the system at $15 \mathrm{~min}$ intervals. The uptake of glucose by the hindlimb was calculated from the inflowoutflow concentration difference and the perfusate flow rates. Total uptake over the perfusion period increased from $46.2 \pm 2.6 \mathrm{mg}$ (I) to $60.6 \pm 2.2$ (II) with metformin $(p<0.01)$ but showed no increase $(49.6 \pm 1.6 \mathrm{mg}, p>0.05)$ with insulin. However, insulin in the presence of metformin increased glucose uptake to $81.0 \pm 1.3 \mathrm{mg}$ (IV, $\mathrm{p}<0.01$ relative to insulin alone, III). There was a significant interaction between insulin and metformin $(p<0.05)$. A mechanism for this interaction became apparent since insulin concentrations were $0.22 \pm 0.03$ $\mathrm{pmol} / \mathrm{ml}$ with insulin (III) and $0.32 \pm 0.02 \mathrm{pmol} / \mathrm{ml}$ with the addition of metformin (IV) $(p<0.02)$. Insulin extraction by the hindlimb was reduced from $15.1 \pm 3.4 \%$ to $5.5 \pm 0.5 \%(p<0.02)$ explaining the increased levels. In conclusion, these data demonstrate a possible action of metformin on muscle glucose uptake - both independently and enhancing the action of insulin. A decrease in insulin removal by muscle likely contributes to the latter effect. 


\section{3}

COMBINED GLIBENCLAMIDE PLUS METFORMIN IMPROVES INSULIN SENSITIVITY IN NON OBESE NIDDM PATIENTS ${ }^{1} \mathrm{~L}$. Pastore, ${ }^{1} \mathrm{~L}$. Morviducci, ${ }^{2} \mathrm{~A}$. Giaccari, ${ }^{1} \mathrm{G}$. Tamburrano.

'Endocrinology, Clin. Med. 2, "La Sapienza" University and 2Department of Endocrinology, Catholic University; Rome, Italy.

Mefformin improves insulin resistance in several conditions, and is widely used in obese NIDDM patients. However, insulin resistance is involved also in the pathogenesis of non-obese NIDDM; aim of this study is to evaluate the effects of metformin on insulin sensitivity in non-obese NIDDM. 12 non-obese (BMI $<30 \mathrm{~kg} \cdot \mathrm{m}^{-2}$ ) patients (age $56.1 \pm 2$ ) treated with OHA (range 1-2 tablet(s) per day) were studied. All patients, in good glycaemic control, were treated for at least one month before the study with glibenclamide alone to standardize all characteristics. Patients were then randomly divided into 3 groups: metformin $(M)$, glibenclamide $(G)$ or metformin + glibenclamide $(G+M)$. Patients' clinical data were similar in the three groups (ANOVA): BMl: $27.1 \pm 0.6 \mathrm{~kg} \cdot \mathrm{m}^{2} ; \mathrm{HbA}_{1 \mathrm{c}}: 7.2 \pm 0.5 \%$; fasting glycaemia: $8.8 \pm 0.8 \mathrm{mM}$; post-prandial glycaemia: $10.9 \pm 1.1 \mathrm{mM}$. Insulin sensitivity (Steady State Plasma Glucose: SSPG) was evaluated before and after one

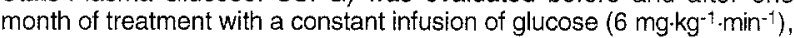
insulin $\left(1 \mathrm{mU} \cdot \mathrm{kg}^{-1} \cdot \mathrm{min}^{-1}\right)$ and octreotide $\left(100 \mathrm{ng} \cdot \mathrm{kg}^{-1} \cdot \mathrm{min}^{-1}\right)$. At the end of the study, BMI remained unchanged in the three groups; fasting glycaemia was significantly increased in $M(9.4 \pm 0.4 \mathrm{mM})$ and decreased in $\mathrm{G}+\mathrm{M}(7.7 \pm 0.5 \mathrm{mM})$. SSPG, similar before the study (G: $9.18 \pm 0.52 ; M: 9.26 \pm 0.28 ; S+M: 9.36 \pm 0.37$ ), significantly improved in $G+M(7.56 \pm 0.42, p=0.0025)$, worsened in $M(11.9 \pm 0.56 ; p<0.05)$ while no changes were observed in $G$. On this results we conclude: 1) Metformin alone might not be sufficient in non-obese NIDDM patients 2) Glibenclamide + metformin ameliorates the peripheral insulin sensitivity, as evaluated with SSPG. Taking into account the pivotal role of insulin resistance in NIDDM, a therapeutic protocol of association (glibenclamide + metformin) can be suggested as first choice also in non-obese patients.
864

RATE OF FAILURE OF TREATMENT WTTH SILFONYLUREA PLUS METFORMIN IN NDDM PATENTS WITH PREVIOUS SULFONYLUREA FAIIURE

Vázquez JA; Santamaria J; Pérez B; Izuzquiza A. Endocrine Unit. Hospital de Cruces. Baracaldo, Vizcaya. Spain.

Backoround and aim. It has been shown that short term Metformin treatment added to sulphonylureas improves glucose control in Type II (NIDDM) diabetic patients. However, long term improvement and failure rate to this combination have not been established. Patients and methods. Three hundred and six patients, 177 females and 129 males, with NIDDM were restrospectively studied. Their mean age was $60.8+1-$ $8,2 \mathrm{yr}$ old and mean body mass index (B.M.I.) was of $27,9+/-4,1 \mathrm{~kg} / \mathrm{m} 2$. Time from diagnosis of diabetes to metformin treatment was $8.7+1-6,1 \mathrm{gr}$. Patients were on maximal doses of sulfonylurea treatment, but their control was inadequate (fasting plasma glucose $207+\% 34.5 \mathrm{mg} / \mathrm{dl}$ and $\mathrm{HbAlc} 9.2+/-1.4 \%$ ). After adding metformin BMI, fasting plasma glucose and HbAlc were recorded along the study. Mcan of patients follow up was $57,2+/-39,3$ months. Insulinitation rate analysis was performed according to the method of Kaplan - Meier, and differences among subgroups were compared by long rank test. Cox model was used in the multivariate analysis. Results. The effects of adding metformin is illustrated in next table:

\begin{tabular}{|c|l|l|l|l|l|}
\hline Basal & 6 months & $1 \mathrm{yr}$ & $3 \mathrm{yr}$ & $5 \mathrm{yr}$ & $10 \mathrm{yr}$ \\
\hline
\end{tabular}

\begin{tabular}{|l|l|l|l|l|l|l|}
\hline Patient & 006 & 306 & 289 & 210 & 136 & 27 \\
\hline
\end{tabular}

\begin{tabular}{|l|l|l|l|l|l|l|}
\hline B.M.I. & $27,9+1-1,1$ & $28.0+/-4.3$ & $27.7+/-4.3$ & $27.7+/-3.9$ & $27.5+/-3.9$ & $26.3+/-2,3$ \\
\hline
\end{tabular} \begin{tabular}{|l|l|l|l|l|l|l|l|}
\hline Glucose & $207 / 2136$ & $169+/-49$ & $172+/-45$ & $163+/ 42$ & $167+/-41$ & $187+/-43$ \\
\hline
\end{tabular} \begin{tabular}{|l|l|l|l|l|l|l|}
\hline HbA1c & $9,2+/ 4 / 5$ & $8.4+/-1.7$ & $8.1+/-1.4$ & $8+/-1.5$ & $8.3+/-1.7$ & $8.6+/-1.6$ \\
\hline
\end{tabular} Seventy six patients ( $25 \%$ ) were transfered to insulin therapy. Insulinitation rate was $4 \%, 11 \%, 20 \%$ and $52 \%$ at $1,3,5$ and $10 \mathrm{yr}$, respectivily. Sex, age, BMI and duration of diabetes were not predictive factors for insulinitation; basal values of fasting plasma glucose and HbAlc were the only ones significantly higher $(p<0.05)$ in patients who needed insulin. Conclusions. 1) Metormin added to sulfonylurea is long term effective in patients with secondary failure to sulfonylurea. 2) Cummulative annual rate of failure to combined treatment is $5 \%$ 3) Initial improvement doesn't predict long term efficacy. 4) Patients with the highest $\mathrm{HbAlc}$ and fasting plasma glucose levels requiered insulin therapy more frequently.
865

METFORMIN IMPROVES ENDOTHELIUM-DEPENDENT VASCULAR RESPONSES IN NIDDM PATIENTS.

L.Coppola, G.Verrazzo, R.Marfella, R.Acampora, R.Giunta, M.A.Caccavale, F.D'Onofrio, D.Giugliano, Department of Geriatrics and Metabolic Diseases, Naples, Italy

L-arginine, the natural precursor of nitric oxide, induces vasodilation and inhibits platelet activity in man. We studied the effects of metformin $(1700 \mathrm{mg} /$ day for 8 weeks) on hemodynamic and rheologic responses to L-arginine $(1 \mathrm{~g} / \mathrm{min})$ in 10 newly-diagnosed NIDDM patients with mild fasting hyperglycemia (7.5+0.3 mol/1, m+SE). Before metformin, L-arginine caused significan decrements of systolic (SBP, $-8+1 \mathrm{mmHg}$ ) and diastolic (DBP, $-4+1 \mathrm{mmHg}, p<0.01$ ) blood pressure, platelet aggregation and blood viscosity $(-10 \%, p<0.05)$. Metformin treatment was associated with reduced fasting plasma glucose $(-0.5 \pm 0.3 \mathrm{mmol} / 1)$ and HbAlc $(-0.3 \pm 0.1 \%)$ levels and platelet aggregation $(-9+4 \%)$. In addition, the decrease of SBP $(-12+2 \mathrm{mmHg})$ and DBP $(-9+2 \mathrm{mmHg})$, platelet aggregation $(-18+4 \%)$ and blood viscosity $(-20 \%)$ following L-arginine was significantly greater after metformin ( $p<0.05-0.01$ vs pretreatment); heart rate, plasma norepinephrine and blood filterability showed significant improvement during L-arginine infusion. Iarginine produces beneficial vascular changes in NIDDM patients without vascular complications; these effects are amplified by a 8 -week treatment with metformin.

\section{6}

POSSIBLE EFFECT OF METFORMIN ON SERUM HOMOCYSTEINE LEVEI IN NON-INSULIN-DEPENDENT DLABETES MELLITUS

E.K. Hoogeveen', R.J. Heine ${ }^{1,2}$, P.J. Kostense ${ }^{1}$, C. Jakobs ${ }^{3}$, L.M. Bouter ${ }^{1}$ and C.D.A. Stehouwer ${ }^{1,2}$. Institute for Research in Extramural Medicine, ${ }^{2}$ Department of Internal Medicine and ${ }^{3}$ Clinical Chemistry, Vrije Universiteit Amsterdam Elevated total serum homocysteine level is an important risk factor for atherosclerosis. Low serum vitamin B12 may increase the serum homocysteine level. Metformin may decrease serum vitamin B12 levels by up to 30 percent through inducing vitamin B12 malabsorption. The aim of the study was to estimate the effect of metformin on the homocysteine level in Caucasian NIDDM patients. Fasting serum homocysteine was measured in 40 patients ( 21 men and 19 women; age $64 \pm 8$ years [mean $\pm S D$ ]; median diabetes duration 8 years, range 1 to 28 years, $\mathrm{HbA} 1 \mathrm{c} 7.4 \pm 1.3 \mathrm{mmol} / \mathrm{l}$ ) who had received treatment with metformin (500-2550 mg/day) for at least six months (range 6 to more than 24 months) and in 71 NIDDM patients not treated with metformin but matched for sex, age $( \pm 5$ years), serum creatinine $( \pm 5 \mu \mathrm{mol} / \mathrm{l})$ and current smoking habits. Except in nine individuals who were matched with one, each 'exposed' patient was matched with two 'non-exposed' patients. Patients using vitamin B or folic acid supplements or medications which interfere with folic acid metabolism, and those with impaired renal function (serum creatinine $>120 \mu \mathrm{mol} / 1$ ) or malignancies were excluded. A two-way analysis of variance was performed. The weighted mean (range) serum total homocysteine level was $11.5 \mu \mathrm{mol} / \mathrm{A}(7.0$ to $19.2 \mu \mathrm{mol} / \mathrm{l}$ ) in the metformin-exposed and $10.6 \mu \mathrm{mol} / \mathrm{l}(4.9$ to $20.8 \mu \mathrm{mol} / \mathrm{l})$ in the non-exposed. Thus, the metformin-exposed patients had slightly higher homocysteine levels (difference $0.8 \mu \mathrm{mol} / 1,95 \%$-confidence interval, -0.4 to $2.0 \mu \mathrm{mol} / 1$ ). There was no interaction between sex and metformin. Finally, no effect of cumulative exposure to metformin on the homocysteine level could be demonstrated.

We conclude that the effect of metformin on serum homocysteine in NIDDM patients, if any, is likely to be small. The significance of small rises in serum homocysteine in diabetic patients is unknown, however, and requires further study. 
867

INTRACELLULAR TOLBUTAMIDE STIMULATES CALCIUM-DEPENDENT EXOCYTOSIS IN PANCREATIC B-CELLS

Erik Renström and Patrik Rorsman. Islet Cell Physiology, Novo Nordisk A/S, Fruebjergvej 3, DK-2100 Copenhagen, Denmark

Sulphonylureas stimulate insulin secretion by closing K-ATP channels. This leads to membrane depolarization and acceleration of $\mathrm{Ca}^{2+}$ dependent secretion. They also stimulate exocytosis at a site distal to elevation of $\mathrm{Ca}^{2+}$. This study was undertaken to further investigate the latter action of the sulphonylureas. Secretion was monitored in the standard whole-cell configuration using capacitance measurements and carbon fibre amperometry. When the internal $\mathrm{Ca}^{2--}$ concentration was clamped at $170 \mathrm{nM}$, inclusion of $0.1 \mathrm{mM}$ tolbutamide in the cytoplasmic medium resulted in $>10$-fold acceleration of exocytosis: from $1.61 \pm 1.9 \mathrm{fF} / \mathrm{s}(\mathrm{n}=13)$ to $18.0 \pm 3.8 \mathrm{fF} / \mathrm{s}(\mathrm{n}=17 ; \mathrm{P}<0.001)$. It was verified that this increase in cell capacitance reflects exocytosis using amperometry and amperometric current spikes (reflecting the discharge of individual granules) were only seen in the presence of tolbutamide. The stimulatory action of tolbutamide on exocytosis was antagonized by diazoxide and the rate of capacitance increase fell from $19.7 \pm 2.4 \mathrm{fF} / \mathrm{s}(\mathrm{n}=6)$ in the presence of tolbutamide alone to $3.5 \pm 2.1 \mathrm{fF} / \mathrm{s}(\mathrm{n}=5 ; \mathrm{p}<0.001)$ in the presence of both tolbutamide and diazoxide. Tolbutamide was ineffective when applied in the complete absence of $\mathrm{Ca}^{2+}(<10 \mathrm{nM})$ and lacked additional stimulatory action when when exocytosis was stimulated by $2 \mu \mathrm{M}$ internal $\mathrm{Ca}^{2+}$ and 0.1 mM cyclic AMP. Thus, the stimulatory action is most easily observed when exocytosis is sub-maximally stimulated. These observations suggest that the sulphonylureas in addition to inhibiting K-ATP channels also stimulate $\mathrm{Ca}^{2+}$-dependent exocytosis by an intracellular mechanism. We speculate that the latter effect is raediated by binding to sulphonylurea receptors in the granular membranes.

\section{8}

TOLBUTAMIDE STIMULATED INSULIN SECRETION IS ACHIEVED BY AMPLIFICATION OF THE MASS OF INSULIN SECRETORY BURSTS. N. Pørksen, S. Munn, J. Steers, O. Schmitz, J.D. Veldhuis and P.C. Butler; Endocrine Research Unit, and Division of Transplant Surgery; Mayo Clinic, MN, USA, Medical Department M, Aarhus Kommunehospital, Aarhus, Denmark, and Deparment of Endocrinology, University of Virginia, Charlottesville, VA, USA. In the fasting basal state (BAS) $70 \%$ of insulin secretion is derived from the pulsatile mode of insulin secretion. While it is known that sulphonylurea stimulate insulin secretion, it is unknown if this is achieved by modulation of the pulsatile or non-pulsatile component of insulin secretion and, if the former, through stimulation of frequency and/or mass of insulin secretory bursts. We employed a recently validated canine model employing deconvolution of portal vein insulin concentration profiles obtained for 40 minutes before (BAS) and 40 minutes during (TOL) a tolbutamide infusion $(0.2 \mathrm{mg} / \mathrm{kg} / \mathrm{min})$ in $7 \mathrm{dogs}$, the plasma glucose concentration being clamped at euglycemia by a glucose infusion. The mean arterial $(85 \pm 12$ to $325 \pm 66$ $\mathrm{pmol} / \mathrm{l}, \mathrm{BAS}$ to TOL), and portal vein $(345 \pm 55$ to $1288 \pm 230 \mathrm{pmol} / 1, \mathrm{BAS}$ to TOL) insulin concentrations increased during tolbutamide. This increase was due to an increase in insulin secretion rate from $2.4 \pm 0.3$ to $10.0 \pm 2.0$ $\mathrm{pmol} / \mathrm{kg} / \mathrm{min} \mathrm{p}<0.01)$ with no changes in insulin clearance $(0.8 \pm 0.1$ vs $0.8 \pm 0.1 \mathrm{t} / \mathrm{min}$ ). The percentage of insulin derived from pulsatile insulin secretion before or during tolbutamide was unchanged at $\sim 70 \%$. Thus, the predominant increase in insulin secretion with TOL was derived by increased pulsatile insulin secretion which was achieved by a $220 \%$ increase in pulse mass (266 \pm 64 vs $817 \pm 144 \mathrm{pmol} / \mathrm{pulse}$, BAS vs TOL, $\mathrm{p}<0.005)$, but no change in pulse frequency $(10.1 \pm 0.6$ vs $11.1 \pm 0.8$ pulses/hour, BAS vs TOL, $\mathrm{p}=0.33$ ). We conclude that sulphonylurea mediated insulin secretion is mediated by amplification of insulin secretory burst mass.

\section{9}

TOLBUTAMIDE ENHANCES GLUCOSE EFFECTIVENESS FOLLOWING ACUTE ADMINISTRATION IN NON-DIABETIC SUBJECTS.

A Rostami-Hodjegan, SA Peacy, SR Heller and GT Tucker, Diabetic Centre and University Dept. of Medicine, Northern General Hospita: ; University Dept. of Medicine and Pharmacology, Royal Hallamshire Hospital, Sheffield, UK

Tolbutamide (TB) is believed to have both insulinergic and extra-pancreatic effects (EXPEF). The use of TB-injection as part of an improved protocol for frequently sampled i.v. glucose tolerance tests (FSIGT) has led to inconsistent results, emphasising the need to assess EXPEF of TB. In a series of glucose clamp studies 15 subjects (12Male; BMI: $23.8 \mathrm{~kg} \mathrm{~m}^{-2}(\mathrm{CV}=11 \%)$ ) with normal fasting blood glucose (4.46 mmol 1-1 $(5 \%)$ ) received either TB or insulin (INS) on 2.5 occasions. Clamps consisted of euglycaemic (0-70, 0-120, 0-20min) and hypoglycaemic (90-110, 20-50) periods. Bedside glucose measurement was performed every $5 \mathrm{~min}$ and dextrose infusion adjusted accordingly. These data together with serum INS were entered into an adapted "Minimal Model". Using a population PK-PD program (P-Pharm version 1.3e), $\mathrm{Si}$ (a function of insulin sensitivity, ISEN), $\mathrm{Sg}$ (a function of glucose effectiveness, GEF) and $K_{L y m 0}$ (a constant for INS exit from lymph) were calculated (Table). The $\log$ transformed population parameters were compared by $Z$-test. The results indicated that TB significantly increased $\mathrm{Sg}(\mathrm{p}<0.05, \mathrm{p}<0.001$ for INS1 vs $\mathrm{TB} 1$ and INS2 is TB2, respectively) and decreased $\mathrm{K}_{\mathrm{Lym0}}$ ( $\mathrm{p}<0.001$ for INS1 vs TB1 and INS2 vs TB2). Mean Si was higher in TB arms compared to respective INS arms but not significantly. These observations confirm EXPEF of TB after acute administration and explain inconsistancy between TB-injection and INS-injection FSIGTs. These also cast doubt on using the former to assess ISEN and GEF. Table. Model parameters describing insulin sensitivity and glucose effectiveness

\begin{tabular}{|c|c|c|c|c|c|}
\hline \multirow[b]{2}{*}{ Study } & \multicolumn{3}{|c|}{ Model Parameters * } & \multicolumn{2}{|c|}{ FSIGT Equivalents** } \\
\hline & $\begin{array}{c}K_{\mathrm{L}, \mathrm{ymo}} \\
\left(\mathrm{min}^{-1} \quad 10^{-3}\right)\end{array}$ & $\frac{\mathrm{Sg}}{\left(\mathrm{dil} \mathrm{min}^{-1}\right)}$ & $\begin{array}{c}\mathrm{Si} \\
\left.\text { (dI } \mathrm{min}^{-1}(\mathrm{mU} /)^{-1}\right)\end{array}$ & $\begin{array}{c}S^{\prime} \mathrm{g} \\
\left(\mathrm{min}^{-1}\right] \\
\left.10^{-3}\right)\end{array}$ & $\begin{array}{c}\mathrm{Si} \\
\left(\min ^{-1}(\mathrm{xuU} / 2)^{-1}\right)\end{array}$ \\
\hline $\operatorname{NNS} 1(n=8)$ & 50 & 1.0 & 0.12 & 8.8 & $7.05 \times 10^{-4}$ \\
\hline $\operatorname{NS} 2(n=10)$ & 226 & 1.1 & 0.36 & 9.0 & $20.5 \times 10^{-4}$ \\
\hline $\operatorname{TBI}(\mathrm{n}=8)$ & 33 & 1.2 & 0.18 & 10.2 & $10.03 \times 10^{-4}$ \\
\hline $\operatorname{TB} 2(n=10)$ & 28 & 9.3 & 0.41 & 79.2 & $23.44 \times 10^{-4}$ \\
\hline
\end{tabular}

* mean population values from $\log$ transformed clistribution of parameters

** conversions made assuming that the central glucose space is $158 \mathrm{ml} \mathrm{kg}-1$

\section{0}

FASTING INSULIN PREDICTS THE ACUTE INSULINERGIC EFFECT OF TOLBUTAMIDE, WHICH IS NOT RELATED TO HYPOGLYCAEMIC EFFECT

SR Heller, A Rostami-Hodjegan, SA Peacy and GT Tucker, Diabetic Centre and University Dept. of Medicine, Northern General Hospital ; University Dept. of Medicine and Pharmacology, Royal Hallamshire Hospital, Sheffield, UK

Tolbutamide (TB) has a widely variable hypoglycaemic effect (HPOGEF) in nondiabetic stibjects. Whether this variability is related to variable drug kinetics (PK) or variation in dynamics (PD) is not known. Moreover, it is not clear that variability in $\mathrm{PD}$ is related to inter-individual differences in insulinergic response (NNSRSP), in a possible extrapancreatic effect (EXPEF) of the drug or simply variation in the HPOGEF of insulin (INS) itself. We studied some of these aspects. In a series of glucose clamp studies 15 subjects (12Male; BMI: $23.8 \mathrm{~kg} \mathrm{~m}^{-2}$ $(\mathrm{CV}=11 \%)$ ) with normal fasting blood glucose (FBG: $4.46 \mathrm{mmol}^{-1}(5 \%)$ ) received up to $2 \mathrm{~g}$ TB ( $1.7 \mathrm{iv}$ followed by $0.130 \mathrm{~g} \mathrm{~h}^{-1}$ iv infusion from $10 \mathrm{~min}$ ) in $1-3$ occasions. Serum TB, INS, c-peptide and net effect on glucose economy (dextrose infusion) were measured. The difference in serum TB between individuals varied less than two fold. However, a six fold difference was observed in the HPOGEF. ANOVA showed much lower inter-subject variability in the PK parameters of TB compared to those of INSRSP (Table). The $\operatorname{AUC}_{\text {INS }}(0-60)$ was correlated significantly with fasting serum insulin $(T=0.83, \mathrm{p}<0.001)$. However, the level of INS did not predict the HPOGEF ( $1=0.065, p=0.75)$. It appears that impaired INS sensitivity, compensated by higher INS secretion, increases INSRSP to TB while the HPOGEF of a high INSRSP is minimal due to INS resistance of these subjects. It is likely that the EXPEF rather than INSRSP or PK is responsible for the major part of variability in HPOGEF. This may complicate interpretation of frequently sampled i.v. glucose tolerance tests (FSIGT) after TB-injection.

Table. PK-PD parameters of TB and their inter- and intra-subject variability

\begin{tabular}{|c|c|c|c|c|}
\hline & \multicolumn{3}{|c|}{$\mathrm{PK}$} & \multirow{2}{*}{ 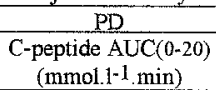 } \\
\hline & $\begin{array}{c}\mathrm{CL} \\
\left(\mathrm{ml} \mathrm{min}^{-1}\right)\end{array}$ & $\begin{array}{l}\mathrm{V} \\
\text { (1) }\end{array}$ & $\begin{array}{c}\mathrm{fu} \\
(\%)\end{array}$ & \\
\hline Average & 27.1 & 6.9 & 8.4 & 43 \\
\hline CV (inter-individual) & $44 \%$ & $23 \%$ & $34 \%$ & $92 \%$ \\
\hline CV (intra-individual) & $26 \%$ & $13 \%$ & $14 \%$ & $34 \%$ \\
\hline
\end{tabular}


871

COSTS OF HYPOGLYCAEMIA IN TYPE || DIABETICS TREATED WITH ORAL HYPOGLYCAEMIC AGENTS

SJ O'Shea*, AC Lloyd**, ML Keech**, BR Rowe*, SC Bain*, AH Barnett", *Heartlands Hospital, Birmingham, UK and ${ }^{* *}$ Glaxo Wellcome, Greenford, UK. Objective: To estimate the incidence and measure the resource use associated with hypoglycaemia in type II diabetics treated with oral hypoglycaemic agents (OHAs). Patients and assessments: 923 Caucasian patients with type II diabetes were screened for hypoglycaemia. Of patients receiving OHAs without insulin, 9.0\% recalled an episode of hypoglycaemia in the previous five years $(7.3 \%$ if receiving sulphonylureas [SUs] alone, $6.3 \%$ biguanides alone, $14.6 \% \mathrm{SU}$ and biguanide in combination). Twenty-eight patients who had experienced hypoglycaemia were interviewed and data collected on resource use, symptoms associated with episode(s), demographic and clinical background and diabetic medications. The severity of each episode was graded I-IV by an experienced diabetes nurse. Unit costs were collected from Heartlands Hospital. Results: Information was gathered on 100 hypoglycaemic episodes (mean episodes per patient 3.6 , range 1-17) Ninety-two episodes were minor (Grade $1-11$ ) and 8 were serious (Grade III-IV). The total cost of health care resource used was £6.136. Fourteen episodes resulted in patients taking time off work, 13 resulted in patients reporting reduced effectiveness while working and 35 required a caregiver to miss normal activities to manage the episode. Using UK average wages to value productivity gives an estimated total indirect cost of $£ 1,793$. The total economic burden of these episodes was $£ 7,928$, equal to $£ 79.28$ per episode or £283.16 per patient affected. Total cost per episode varied from $£ 15.05$ for Grade I to $£ 1.154 .29$ for Grade IV. $84 \%$ of the direct cost and $35 \%$ of the indirect cost were associated with the 8 serious episodes. Conclusions: The majority of hypoglycaemic episodes in type II diabetics treated with OHAs. although unpleasant and distressing, are self-managed. However, a minority of events entail substantial resource use and lost productivity

\section{2}

Absorption of sulfonylurea drugs in patients with Type 2 Diabetes mellitus

H.P. Kopp, *A. Melander, *W. Krugluger, *P. Hopmeier \& G.Schernthaner Dept.Med. 1 \& *Clin.Chem., Rudolfstiftung Hospital, A-1030 Vienna; **NEP! Foundation Malmö, Sweden

An impaired absorption of sulfonylurea (SU) drugs could be responsible for secondary failure, since SU absorption is reduced, when hyperglycaemia is experimentally induced in healthy subjects. Thus we analyzed absorption of glipizide in 10 NIDDM patients with fasting hyperglycaemia $(>220 \mathrm{mg} / \mathrm{dl})$, despite maximal SU treatment, in the hyperglycaemic and normoclycaemic state, in a randomized placebo-controlled cross-over trial. Normoglycaemia was induced by overnight infusion of insulin. Blood-glucose, insulin, C-peptide and glipizide levels were measured following a standard meal and $10 \mathrm{mg}$ glipizide or placebo. Absorption of glipizide was significantly higher under normoglycaemic compared with hyperglycaemic conditions (table).

$\begin{array}{llll}\text { glipizide } & \text { hyperglycaemia } & \text { normoglycaemia } & \text { p-value } \\ \text { Tmax (min) } & 180 \pm 106 & 101 \pm 70 & \text { n.s. } \\ \text { Cmax }(\mathrm{ng} / \mathrm{mi}) & 846 \pm 233 & 1377 \pm 511 & 0.01 \\ \text { AUC } & 21050 \pm 6875 & 33200 \pm 12908 & 0.01\end{array}$

The higher absorption rate did not lead to augmented insulin secretion and lowering of blood glucose. In normoglycaemia the AUC of insulin levels under administration of glipizide $(740 \pm 326)$ was higher than under placebo $(592 \pm 294 ; p<0.01)$. In summary, absorption of $S U$ is significantly influenced by the level of glycaemia in NIDDM patients confirming experimental absorption of glipizide in healthy subjects. However, the increased absorption of glipizide did not improve $B$-cell function and glucose control, suggesting that insulin resistance might be prevailing.

\section{3}

EFFICACY OF 24-WEEK MONOTHERAPY WITH ACARBOSE, METFORMIN OR PLACEBO IN NIDDM PATIENTS WITH DIETARY FAILURE

J. Hoffmann and M. Spengler, Clinical Research Collaboration Study Group, Essen, and Bayer AG, PH-D Medical Department, Leverkusen, Germany

OBJECTIVE - To compare the therapeutic potentials of acarbose, metformin or placebo as first line treatment in patients with NIDDM. RESEARCH DESIGN AND METHODS - 96 NIDDM patients ( $35-70$ years of age, BMI $\leq 35$, insufficiently treated with diet alone, $\mathrm{HbA}_{1 \mathrm{C}} 7-11 \%$ ) were randomized into three groups and treated for 24 weeks with $3 \times 100 \mathrm{mg}$ acarbose or $2 \times 850 \mathrm{mg}$ metformin per day or placebo. Efficacy, based on $\mathrm{HbA}_{1 \mathrm{C}}$ (primary efficacy criterion), fasting $\mathrm{BG}$ and insulin, $1 \mathrm{~h} \mathrm{pp}$ (standard meal test) BG and insulin, pp insulin increase, and plasma lipid profile; and tolerability, based on subjective symptoms and laboratory values, were investigated. ANCOVA was done for endvalues with adjustment on baseline values. RESULTS - Baseline adjusted means at endpoint were as follows: BG fasting and $1 \mathrm{~h}$ pp 9.2 and $10.9 \mathrm{mM}$ with placebo (PI), 7.6 and $8.7 \mathrm{mM}$ with acarbose (A), and 7.8 and $9.0 \mathrm{mM}$ with metformin $(\mathrm{M}), \mathrm{HbA}_{1 C}$ was $9.8 \%$ with placebo, $8.5 \%$ with acarbose, and $8.7 \%$ with metformin. Comparisons $A$ vs $\mathrm{Pl}$ and $\mathrm{M}$ vs $\mathrm{Pl}$ were statistically significant, but not A vs $\mathrm{M}$. No effect on fasting insulin could be observed. Relative pp insulin increase was 1.90 with placebo, 1.09 with acarbose and 1.03 with metformin. Comparisons A vs Pl and $\mathrm{M}$ vs Pl were statistically significant, but not A vs M. With respect to lipid profile, acarbose was superior to metformin. LDL/HDL cholesterol ratio increased by $14.4 \%$ with placebo, was unchanged with metformin, but decreased by $26.7 \%$ with acarbose. Comparisons A vs Pl and A vs M were statistically significant, but not $\mathrm{M}$ vs Pl. Slight body weight changes were observed under acarbose $(-0.8 \mathrm{~kg})$ and metformin $(-0.5 \mathrm{~kg})$, but not under placebo treatment. Acarbose led to mild or moderate intestinal symptoms in $50 \%$ of the patients within the first 4 weeks, but in only $13.8 \%$ of the patients within the last 4 weeks. CONCLUSIONS - Acarbose and metformin are effective drugs for the monotherapy of NIDDM patients if diet alone fails. With respect to plasma lipid profile, especially HDL, LDL and LDL/HDL cholesteroi ratio, acarbose may be superior to metformin.

\section{4}

EFFECTS OF GLIBORNURID ON PLASMA ATRIAL NATRIURETIC FACTOR LEVELS IN PATIENTS WITH TYPE II DIABETES MELLITUS G.Ayvaz,B.Çakur,N.CCakır,M.Kitapç,I,Y.Yetkin and M.Arslan. Gazi University Medical School, Endocrinology and Metabolism Division, Ankara, Turkey

The inhibitory effect of glubornurid on the plasma atrial natriuretic factor (ANF) secretion was studied in 10 ( 5 male and 5 female) newly diagnosed Type II diabetic patients (36-63 years old) and in 10 ( 5 male and 5 female) age and sex matched healthy controls (35-63 years old).Basal urine samples were taken for urine $\mathrm{Na}^{+}$and $\mathrm{K}^{-}$. After normalizing the glycemia by the means of euglycemic clamp, blood samples were taken for basal ANF from both diabetics and controls. Following one hour intravenous $0,9 \%$ saline infusion, blood samples for ANF and urine samples for $\mathrm{Na}^{+}$and $\mathrm{K}^{+}$were taken again. 24 hour later, same procedure was performed to the patient group before and 30 minutes after giving $50 \mathrm{mg}$ glibornurid orally. Mean ANF levels increased significantly following $0,9 \%$ saline infusion in either control group (from $11.13 \pm 0.85$ $\mathrm{pg} / \mathrm{ml}$ to $20.18 \pm 2.48 \mathrm{pg} / \mathrm{ml})(\mathrm{p}<0,05)$ or diabetic group (from $15.05 \pm 2.32 \mathrm{pg} / \mathrm{ml}$ to $28.89 \pm 4.72 \mathrm{pg} / \mathrm{ml})(\mathrm{p}<0,05)$. The rate of increament in diabetics was same with the controls $(p>0,05)$. Basal urine $\mathrm{Na}^{+}$and $\mathrm{K}^{+}$excretions in diabetic group weren' $\mathrm{t}$ different from control group $(p>0,05)$. In both control and diabetic groups, following the $0,9 \%$ saline infiusion, mean urine $\mathrm{Na}^{+}$excretion (from $11.73 \pm 1.14$ to $33.63 \pm 2.73$ and from $10.54 \pm 1.24$ to $44.99 \pm 7.47$ respectively) and mean urine $\mathrm{K}^{+}$excretion (from $3.33 \pm 0.62$ to $10.51 \pm 1.61$ and from $2.70 \pm 0.24$ to $12.40 \pm 1.69$ respectively) increased significantly $(p<0,05)$. However, diabetic subjects didn't give any response to saline loading after glibornurid and ANF levels stayed unchanged (from $15.99 \pm 2.26 \mathrm{pg} / \mathrm{ml}$ to $15.13 \pm 1.00$ $\mathrm{pg} / \mathrm{ml})(\mathrm{p}>0,05)$. Although ANF levels didn't changed after saline infusion, mean urine $\mathrm{Na}^{+}$excretion increased $(47.78 \pm 8.18)(\mathrm{p}<0,05)$ but $\mathrm{K}^{+}$excretion stayed at the same level $(3.34 \pm 0.32)(p>0,05)$ in diabetic group. These results suggested us that, glibomuride may has an inhibitory effect on the ANF secretion and may provide a nonkaliuric natriuresis response to the volume loading by blocking the renal ATP sensitive $\mathrm{K}^{+}$ channels. This inhibitory effect may help to revert or prevent the hyperfiltration; that is seen in the begining of diabetes is thought to be caused by the high ANF levels and leads to diabetic nephropathy. 
875

INSULIN SENSITIVITY IS IMPROVED AFTER GLIPLZIDE GITS MONOTHERAPY AND IN COMBINATION WITH METFORMIN.

W.T. Cefalu, A.D. Bell-Farrow, Z.Q. Wang, D. McBride, and C. Cobelli. Bowman Gray School of Medicine. Winston-Salem, N.C., U.S.A., and Univ. of Padova, Padova, Italy.

The pathophysiology of non-insulin dependent diabetes mellitus (NIDDM) is associated with abnormalities in pancreatic insulin secretion and insulin resistance. Glipizide in a once daily formulation utilizing the Gastrointestinal Therapeutic System (GTS) has been shown in preliminary studies to improve insulin sensitivity as assessed with meal tolerance testing. Glipizide GITS was evaluated in it's ability to improve insulin sensitivity in vivo in a double-blind, placebo-controlled 13 week trial of 40 NIDDM subjects randomized to either Glipizide GITS (Smg-1st month; 10mg-2nd month) or placebo. A six month open label phase followed the double blind trial. Insulin Sensitivity $\left(S_{1}\right)$ and Glucose Effectiveness $\left(S_{C}\right)$ were determined with a 4-hour insulin modified frequently sampled intravenous tolerance test. Insulin Sensitivity $\left[\mathrm{S}_{1}\left(\mathrm{x} 10^{-}\right.\right.$ ${ }^{4} \mathrm{~min}^{-1} \mu \mathrm{U}^{-1} \mathrm{ml}$ ) ] showed a significant improvement with Glipizide GITS (baseline 1.4, 2 month value $=2.5,5$ month value $=2.1,8$ month value $=2.8, p<.001$ ). There was no effect of placebo on $S_{1}$ (baseline 9,2 month value=1.0, $p=n s$ ), but a significant improvement was noted with Glipizide GITS treatment during the open label phase (5 month value $=1.7,8$ month value $=1.8, \mathrm{p}<001)$. A significant dec:ease for both glycated hemoglobin $(\mathrm{GHb})$ and fructosamine (FA) was observed. Patients with high levels of intra-abdominal fat as assessed by MRI scans $(n=12)$ were hyperinsulinemic when compared with those with lower amounts of intra-abdominal fat (fasting insulin $=186 \pm 24$ pmol/ vs $90+12.6 \mathrm{mmol} / \mathrm{h}, 001$ respectively) despite average blood glucose of 7.6 mmol/L in all patients on monotherapy. In the hyperinsulinemic NDDM patients, Metformin (2-2.5 grams t.i.d.) was added to Glipizide GITS monotherapy ( $5-10 \mathrm{mg} q$ d). There was a significant decrease in fasting blood glucose $(\Delta=-3.6 \mathrm{mmol} / \mathrm{L})$, fasting insulin $(\Delta=-48 \mathrm{pmol} / \mathrm{L})$, and a significant improvement in insulin sensitivity $(\Delta=+1.8$ $\left.\mathrm{x} 10^{-4} \mathrm{~min}^{-1} \mu \mathrm{U}^{-1} \mathrm{ml}\right)$ in all patients. Conclusion: Combination Glipizide GITS/Metformin normalizes glucose and further improves insulin sensitivity when compared to Glipizide GITS monotherapy.

\section{6}

NO HYPOGLYCEMIA AFTER FASTING PLUS EXERCISE DURING TREATMENT OF NIDDM WITH SUSTAINED-RELEASE GLIPIZIDE.

M.C. Riddle and P.A. MCDaniel, Oregon Health Sciences University, Portland, Oregon, USA

Hypoglycemia is the main unwanted effect of sulfonylureas. Fear of it may limit use of these agents in combination with calorie-restriction and exercise in NIDDM. Glipizide-GITS (GG), a new sustained-release form of glipizide, provides a low and nearly constant 24-hour blood level of the agent even when given as a single morning dose. We postulated that GG may have little tendency to cause hypoglycemia accompanying changes of daily routine. We tested the effect of a fasting and exercise protocol during chronic use of GG $(5-20 \mathrm{mg} / \mathrm{d})$ versus placebo $(P)$. The subjects were 10 women and 15 men taking part in a larger dose-titration trial, with mean age $\pm \mathrm{SE} 60 \pm 2 \mathrm{yr}$, diabetes for $4.2 \pm 0.5 \mathrm{yr}$, and $\mathrm{BMI} 30.7 \pm 0.8$. Their fasting plasma glucose (FPG) was $11.7 \pm 0.6 \mathrm{mM}$ off hypoglycemic agents 2 weeks. They were assigned randomly and double masked to $G G(n=17)$ or $P(n=8)$. After 13 weeks FPG at 0800 was lower on $G G$ than $P$ $18.7 \mathrm{mM} \pm 0.7 \mathrm{mM}$ vs $13.3 \pm 1.2 \mathrm{mM}, \mathrm{p}<0.001)$. On the morning of the protocol the study drug was given, breakfast was witheld, and the subjects walked at 2.5 miles per hour from 0800 to 0930 with two 5 -minute rest periods. The decline of glucose from baseline was small and the same with each treatment: $-1,1 \pm 0,3 \mathrm{mM}$ on $G \mathrm{G}$ and $-1,2 \pm 0.7$ on $P$ at 0930 , and $-1.6 \pm 0.3$ vs $-1.5 \pm 0.5$ at 1130 . Serum insulin $(159 \pm 23$ vs $84 \pm 11 \mathrm{pM}$, $p<0.05)$ and C-peptide $(1419 \pm 153$ vs $846 \pm 57 p M, \quad p<0.02)$ concentrations were higher at 0800 with $G G$ than with $P$, but unchanged up to 1130 on both treatments. No subject had hypoglycemic symptoms with exercise. Summary: Missing breakfast and mildly exercising causes no more decline of plasma glucose with ongoing use of $G G$ before breakfast than with placebo. Conclusion: Glipizide-GITS and lifestyle changes can safely be used together for NIDDM, without undue risk of hypoglycemia.
877

Glipizide GITS is Effective and Safe in a Wide Range of NIDDM Patients: Results of a DoubleBlind Placebo-Controlled Efficacy and Safety Trial

L. Blonde, R.D. Guthrie, Jr., L. Tive, C. Fischette and the Glipiride Gits Efficacy and Safety Trial Study Group, New Orleans, LA and New York, NY

This 16 week, multicenter, randonized, double-blind, placebo-controlled trial was conducted to assess the safety and efficacy of glipizide GITS in a broad spectrum of NIDDM patients. Patients (N=594) were randomized to glipizide GITS $(\mathrm{N}=393)$ or placebo $(\mathrm{N}=201)$ at 62 sites. There was a one week washout phase; a 3-week single-blind placebo (PBO) phase; an 8-week double-blind, dose-titration phase; and an 8-week, double-blind efficacy phase. Efficacy and safety parameters were evaluated in the total population as well as in sub-populations stratified by age, sex, race. BMI, duration of NIDDM, total population as well as in sub-populations stratified by age, sex, race. BMI, duration of NTDDM, blood pressure, renal function, previous antidiabetic therapy and degree of glycenic control at baseline.
Glipizide GTTS decreased HbA $\mathrm{A}_{\mathrm{c}}$ and FPG concentrations significantly more than PBO in the total Glipizde GITS decreased $\mathrm{HbA}_{1 \mathrm{c}}$ and FPG concentrations significantly more than PBO in the total population and in the sub-populations examined. Over $78 \%$ of the patients in the glipizide GrTS group had FPG leveis less than $200 \mathrm{mg} / \mathrm{dL}$ at endpoint, compared with $37 \%$ of patients in the PBO group. Neither the Glipizide GITS nor the PBO group showed significant increases in fasting insulin or Cpeptide levels or any evidence of hyperinsulinemia from baseline to endpoint. Total cholesterol decreased more in the Glipizide GITS group and LDL cholesterol decreased more in the PBO group. Body weight decreased in the PBO group, but did not change in the Glipizide GITS group. There werc no significant differences between treatment groups with regard to hemstology, serum chemistry, vital signs, or electrocardiogram results. There were no documented episodes of hypaglycemia in the study and infrequent discontinuations due to symptomatic hypoglycemia $(6 / 393 ; 1.5 \%$ in the Glipizide GrTS group and $1 / 201 ; 0.5 \%$ in the PBO group). Changes from baseline to endpoint in some of the primary efficacy and safety parameters are listed below:

\begin{tabular}{llll} 
& & \\
HbAlc (\%) & PLACEBO & GLIPIZIDE GITS & Betw. Tx \\
FPG (mg/dL) & +0.57 & -0.87 & $\mathrm{p}<0.0001$ \\
Fasting Insulin (wU/mL) & -0.80 & -56.8 & $\mathrm{p}<0.0001$ \\
Fasting C-peptide (ng/mL) & +2.6 & +0.7 & NS \\
Body Weght (pounds) & $-\mathbf{5 . 6 1}$ & -0.82 & NS \\
Total Cholesterol (mg/dL) & +1.2 & -0.03 & $\mathrm{p}<0.0001$ \\
\hline
\end{tabular}

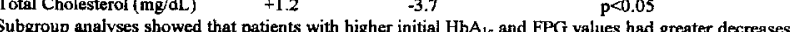
in these values than patients with lower initial values, patients who had been previously maintained on immediate reiease glipizide had significantly lower HbA $A_{1 e}$ levels when trented with glipizide GrTS and the efficacy and safety of Glipizide GITS was not effected by age, degree of obesity or mild to moderate renal impairment. CONCLUSION: Glipizide GITS effectively improved glycemia in a broad range of NIDDM patients. Glipizide GITS was effective even in patients with FPG $>250 \mathrm{mg} / \mathrm{dl}$, decreased renal function, significant obesity and in the elderly. Successful treatment was not associated with weight gain, hypoglycemia, hyperinsulinemia or deleterious effects on lipids as thas been reported with some other sulfonylurea agents.
878

BENEFICIAL EFFECTS OF IMPROVED GLYCEMIC CONTROL WITH GLIPIZIDE GITS ON QUALITY OF LIFE AND SYMPTOMS IN NIDDM.

M.A. Testa and D.C. Simonson. Boston, Massachusetts, USA.

The potential benefits of either short- or long-term strict glycemic control on quality of life (QOL), cognition, or palliation of hyperglycemic (HYPER) symptoms have generally been reported as minimal or absent. Conversely, the risks of acute hypoglycemia (HYPO) from intensive therapy (particularly in IDDM) are well documented. To examine this issue in NIDDM, we studied 569 patients (age $\left.=58 \pm 12 \mathrm{yrs}, \mathrm{FPG}=9.4 \pm 2.7 \mathrm{mmol}^{2} \mathrm{~L}, \mathrm{HbA}_{\text {lc }}=8.5 \pm 1.4 \%\right)$ in a prospective, randomized, double-blind dose-titration trial of $5-20 \mathrm{mg}$ glipizide GITS $(n=377)$ vs placebo $(n=192)$. Assessments (glucose levels, symptoms, and QOL) were made in clinic at baseline, 4,8 , and 12 weeks of therapy, and at home by daily self-administered diaries. After 12 weeks, $\mathrm{HbA}_{10}$ and FPG were lower with glipizide GITS vs placebo $(7.5+1.2$ vs $9.3 \pm 1.9 \%$, and $7.0 \pm 1.8$ vs $9.3 \pm 2.9 \mathrm{mmol} / \mathrm{L}$, respectively; $\mathrm{p}<0.001)$. HYPO symptoms were reported by $4.8 \%$ of patients on placebo vs $6.0 \%$ on glipizide GITS $(p=N S)$, but no patient in either group had a corresponding blood glúcose level $<3.0 \mathrm{mmol} / \mathrm{L}$. Patients on glipizide GITS had consistently more favorable measures of analogue health rating (overall, physical, emotional, petsonal, work/daily role) $(p=0.04)$, cognitive function (acuity, disorientation, detachment) $(\mathrm{p}=0.005$ ), perceived health status (general health, sleep, vitality, sexual dysfunction) $(p=0.004)$, and symptom distress (diabetes-specific and general) $(\mathrm{p}<0.001)$. The favorable outcomes for glipizide GITS were largely due to a reduction in symptoms of HYPER (e.g,, polyuria, dry mouth, thirst, blurred vision, fatigue, weakness) with no increase in symptoms of HYPO (e.g., trembling, fast pulse, palpitations). A global QOL change from baseline score showed a strong relationship with change in $\mathrm{HbA}_{1 \mathrm{c}}(\mathrm{r}=0.95 ; \mathrm{p}<0.001)$ such that decreases in $\mathrm{HbA}_{1 \mathrm{c}}$ of $0.5 \%$ or greater were associated with clinically significant improvements in QOL. QOL improved more in patients on glipizide GITS vs placebo, even after controlling for worsening or improvement in $\mathrm{HbA}_{\text {lc }}(\mathrm{p}=0.03)$. Conclusion: Improved glycemic control in NIDDM with glipizide GITS consistently and significantly improves QOL across all major domains, due in part to a reduction in adverse symptoms of HYPER with no increase in adverse symptoms or frequency of HYPO. 
EFFECTS OF GLICLAZIDE ON MICROVASCULAR REACTIVITY AND PERMEABILITY IN DIABETIC HAMSTERS

E. Bouskela, F.Z.G.A. Cyrino, C.M.S, Conde and A.A. Garcia. Laboratório de Pesquisas em Microcirculação, State University of Rio de Janeiro, RJ, Brazil

The aims of our study were to determine, using the cheek pouch preparation, the effects of gliclazide, a 2nd generation sulphonylurea oral hypoglycemic agent, on (1) macromolecular permeability increase induced by histamine and $30 \mathrm{~min}$ ischemiareperfusion (I/R) obtained with a cuff placed around the neck of the everted pouch (topical application, $1.2 \times 10^{-6} \mathrm{M}$ to $1.2 \times 10^{-4} \mathrm{M}$ ) and (2) microvascular reactivity after $90 \mathrm{~min} \mathrm{I/R}$ (Chronic oral treatment for 28 days, $10 \mathrm{mg} / \mathrm{kg} / \mathrm{day}$ ) in diabetic male hamsters (glycemia $>240 \mathrm{mg} / \mathrm{dl}$ ). Diabetes was induced by 3 i.p. injections of streptozotocin $(50 \mathrm{mg} / \mathrm{kg} /$ day $)$ given over 3 days. For permeability studies, FITClabeled dextran 150 was given i.v. and quantified by UV-light microscopy. Application of gliclazide for (1) $15 \mathrm{~min}, 10 \mathrm{~min}$ prior to histamine, 2 uM for $5 \mathrm{~min}$ and (2) $30 \mathrm{~min}, 10 \mathrm{~min}$ prior the onset of reperfusion, dose-dependently decreased the macromolecular permeability increase [for histamine: $228 \pm 8$ leaks $/ \mathrm{cm}^{2}$ versus $262 \pm 3\left(1.2 \times 10^{-6} \mathrm{M}\right) ; 1.80 \pm 4$ versus $241 \pm 6\left(1.2 \times 10^{-5} \mathrm{M}\right)$ and $150 \pm 8$ versus $226 \pm 5$ $\left(1.2 \times 10^{-4} \mathrm{M}\right)$ and for $\mathrm{I} / \mathrm{R}: 131 \pm 6$ leaks $/ \mathrm{cm}^{2}$ (placebo), $84 \pm 3\left(1.2 \times 10^{-6} \mathrm{M}\right), 64 \pm 6$ $\left(1.2 \times 10^{-5} \mathrm{M}\right)$ and $\left.61 \pm 3\left(1.2 \times 10^{-4} \mathrm{M}\right)\right]$. For microvascular reactivity, the cheek pouch was placed under an intravital microscope coupled to a closed circuit TV system. Arteriolar and venular internal diameters, red blood cell velocity and functional capillary density [ $[$ FCD), number of red-cell-perfused capillaries per observation field] were measured. The microvascular volume flow (VF) was calculated using an analogue circuit. At the onset of reperfusion, VF and FCD were significantly higher in animals treated with gliclazide compared with placebo-treated ones (VF: $3.5 \pm 0.2$ $\mathrm{nl} / \mathrm{min}$ versus $1.1 \pm 0.1$ in arterioles, $3.0 \pm 0.2$ versus $1.2 \pm 0.1$ in venules and FCD: $183 \pm 9 / 4 \mathrm{~mm}^{2}$ versus $105 \pm 6$ ). No changes in insulinemia or glycemia could be observed after topical and chronic administration of gliclazide in any of the groups studied. This indicates that the observed improvement in the microcirculation was independent of the hypoglycemic properties of the drug.

\section{PS 48}

\section{New Antidiabetic Drugs}

\section{0}

LACK OF PHARMACOKINETIC INTERACTION BETWEEN TROGLITAZONE AND GLIBENCLAMIDE IN NIDDM PATIENTS

K. Püchler, K. Sasahara, P.U. Witte and P. Wasserhess. Sankyo Europe GmbH, Düsseldorf, Germany; Sankyo Co Ltd, Tokyo, Japan; IMFORM GmbH, Görlitz. Germany.

The objective of this study was to evaluate any pharmacokinetic interaction between troglitazone $(T)$ and glibenclamide $(G)$ following coadministration at steady state. Twenty six male and female Caucasian NIDDM patients (age 35-65 years) received daily oral doses of $200 \mathrm{mg} \mathrm{T}$ and $2 \times 1.75 \mathrm{mg} \mathrm{G}$ alone or in combination, each for 12 days, in a randomised, double blind, double dummy, 3 way cross-over study. Blood samples were drawn predose during the treatment period and up to $48 \mathrm{~h}$ after the last administration of each treatment period. Concentrations of $T$, its conjugated sulphate metabolite (Ml) and $\mathrm{G}$ were determined in plasma by validated liquid chromatography methods. Trough concentrations indicated that steady state conditions were reached on day 12 of each treatment period. The mean values for $\mathrm{AUC}_{0.24}(\mu \mathrm{g} \cdot \mathrm{h} / \mathrm{ml}), \mathrm{C}_{\max }(\mu \mathrm{g} / \mathrm{mJ})$, $\mathrm{T}$ (h, melian) and $\mathrm{T}^{1} / 2(\mathrm{~h}$, melian), respectively, comparing $\mathrm{T}$ and $(\mathrm{T}+\mathrm{G})$ were for $T$ : $7.5 / 7.2,0.97 / 0.85,2.0 / 1.0,22.6 / 27.3$ and for $M 1: 62.5 / 57.8,4.22 / 3.79$, $3.0 / 2.0,30.4 / 30.6$. Comparing $G$ and $(T+G)$ these parameters were determined to be for $G: 0.6 / 0.57,0.22 / 0.2,1.0 / 1.5,2.3 / 3.0$. The unbound fraction of both $\mathrm{T}$ and $\mathrm{G}$ in plasma was $<1 \%$. The $90 \%$ confidence intervals $(\mathrm{Cl})$ equivalent to a two one-sicled tests procedure (with $\alpha=5 \%$ for the one-sided tests) were calculated for the ratios $(\mathrm{T}+\mathrm{G}) / \mathrm{G}$ and $(\mathrm{T}+\mathrm{G}) / \mathrm{T}$ (based on $\mathrm{T}$ or $\mathrm{MI})$. All $\mathrm{CI}$ for $A U C_{023}$ and $C$ were within the commonly used acceptance ranges of 80-125\% and $70-143 \%$, respectively. In conclusion, equivalent pharmacokinetics of $T$ and $\mathrm{G}$ were denonstrated when administered alone or in combination, i.e. no interaction was shown. All treatments were well tolerated.

\section{1}

CLINICAL EVALUATION OF A NEW ORAL HYPOGLYCEMIC AGENT CS-045 IN COMBINATION WITH INSULIN

Y. Akanuma, K. Kosaka, T. Toyoda, T. Kuzuya, Y. Shigeta, T. Kaneko and M.Shichiri. CS-045 Study group, Tokyo, Japan.

CS-045 (Troglitazone) is a new oral hypoglycemic agent which acts through improving insulin resistance and reducing hepatic gluconeogenesis. In a randomized Jouble-blind placebo-controlled trial, the hypoglycemic effect and safety of CS-045 in combination with insulin were studied in 146 non-insulin dependent diabetes (NIDDM) and 44 insulin dependent diabetes (IDDM) subjects, whose glycemic control was unsatisfied $(\mathrm{HbA} 1 \mathrm{c} \geq 8 \%$ ) in spite of previous insulin therapy for more than 12 weeks. Patients randomly allocated to either troglitazone $200 \mathrm{mg}$ twice daily group ( $n=96$ ) or to placebo group $(n=94)$. The insulin regimen present a baseline was continued and the test drug was added for 16 weeks. No significant difference was observed between two groups in patients' baseline characteristics. A significant improvement of glycemic control was observed only in the CS-045 group, but not in the placebo group. In the CS-045 group, $\mathrm{HbAl}$ c was redwed from $10.1 \pm 1.0 \%$ to $9.4 \pm 1.4 \%(\mathrm{p}<0.001)$. However, in the placebo group, HbAlc was in the range from $10.2+1.4 \%$ to $10.1+1.4 \%$ (NS). The reduction of insulin dose was greater in the CS-045 group. Body weight increased slightly only in the CS-045 group. There were no differences in changes in blood pressure, c-peptide level and frequency of hypoglycemic sign between the two groups. No life-threatening adverse events were observed and all adverse events were reversible in the CS-045 group. In conclusion, these results indicate that CS-045 gives a new treatment strategy in combination with insulin 
882

SIMILAR PHARMACOKINETICS OF TROGLITAZCNE IN YOUNG AND ELDERLY SUB.JECTS

M A Young, $\mathrm{Z} \vee$ Williams and $\mathrm{R}$ Eastmond, Glaxo Wellcome Research and Development Lid, Greenford, Middlesex, UB6 OHE, U.K.

Troglitazone (TR), an insulin action enhancer, is under development for treatment of NIDDM. The pharmacokinetics of TR in young (18-40yrs) and elderly (70-85yrs) heal thy subjects (Study 1) and NIDDM patients (Study 2) at doses up to $800 \mathrm{mg}$ daily have been studied. Complete pharmacokinetic profiles were taken on days 1 and 14 in normals and trough samples were taken in NIDDM patients at clinical visits after weeks $1,2,4,6,8$ and 12. Pharmacokinetic samples were measured for TR and its main circulating metabolites (M1-a sulphate conjugate and M3-an oxidative quinone product). Geometric mean parameters and $95 \%$ confidence intervals for TR aro shown below followed by median and range plasma trough concentrations of TR and its metabolites following $400 \mathrm{mg}$ bd of TR (nd = not detected):

\begin{tabular}{|c|c|c|c|}
\hline STUDY 1 & $\begin{array}{c}\text { Young Normals } \\
(800 \mathrm{mg})(\mathrm{n}=12)\end{array}$ & $\begin{array}{c}\text { Elderly Nornals } \\
(800 \mathrm{mg})(\mathrm{n}=11)\end{array}$ & p-value \\
\hline AUC $(\mu \mathrm{g} . \mathrm{h} / \mathrm{mL})$ & $12.5(10.3,15.1)$ & $15.6(12.7,19.1)$ & 0.111 \\
Cmax $(\mu \mathrm{g} / \mathrm{mL})$ & $2.40(1.90,3.02)$ & $2.43(1.91,3.10)$ & 0.931 \\
Half-1ife $(\mathrm{h})$ & $13.9(9.3,20.9)$ & $14.7(9.6,22.4)$ & 0.848 \\
\hline
\end{tabular}

\begin{tabular}{|c|c|c|}
\hline & $\begin{array}{c}\text { Elderly Normals (Study 1) } \\
(800 \mathrm{mg})(\mathrm{n}=11)\end{array}$ & $\begin{array}{c}\text { Elderly NIDDM (Study 2) } \\
(800 \mathrm{mg})(\mathrm{n}=40)\end{array}$ \\
\hline Trough TR $(\mu \mathrm{g} / \mathrm{mL})$ & $0.57(0.35-1.19)$ & $0.60(\mathrm{nd}-1.88)$ \\
Trough M1 $(\mu \mathrm{g} / \mathrm{mL})$ & $5.3(3.8-11.4)$ & $7.5(\mathrm{nd}-33.6)$ \\
Trough M3 $(\mu \mathrm{g} / \mathrm{mL})$ & $0.76(0.49-1.48)$ & $0.88(\mathrm{nd}-3.39)$ \\
\hline
\end{tabular}

At a daily dose of $800 \mathrm{mg}$ (top of dose range) there was no evidence of accumulation in the elderly populations, indicating that advancing age alone does not necessitate a dosage adjustment for troglitazone based on pharmacokinetic considerations.

\section{3}

TROGLITAZONE IMPROVWS GLYCAEMIA BUT DOES NOT INCREASE THE RISK OF HYPOGLYCAEMIA FOLLOWING ALCOHOL IN DIET-TREATED NIDDM SUBJECTS DJ.A. Eckland, Z.V. Williams and E.A. Fool. GlaxoWellcome Research \& Development Ltd, Greenford Road, Greenford Middlasex. UB6 0HE. UK

Drinking alcohol is a common social event and is associated witl a recognised risk (1) hypoglycaemia. This double-blind, placebo-controlled, parallel group study was designed to determine whether alcohol taken with the evening meal alters the glucoregulatory response to troglitazone (TR), an insulin action enhancer, in NIDDM subjects to increase the risk of hypoglycaemia. Twenty-three, dict-treated, NIDDM subjects were randomised to received either TR $200 \mathrm{hng}$ od $(n=I 1)$ or placebo (PL) $(n=12)$ for 45 days. Al the end of treatment subjects were given, on separate days, an alcohol challenge $(\mathrm{AC}), 0.6 \mathrm{mg} / \mathrm{kg}$ ethanol in orange juice, and a control challenge (CC), orange jujce alone, with the evening meal. Serm glucose, insulin, intact and les 31,32 pro-insulin, C-peptide, non-esteritied falty acids, $\beta$-hydroxybutryrate and triglycerides were measured at intervals during the study, for the 4 hours following each challenge and a single fasting measurement the following morning. The overnight urine cortisolereatinine ratio (an index of hypoglycaemia) was also deiermined. Analysis was performed on log transfomed data. After 6 weeks fasting serum glucose was decreased compared to placebo by $19 \%, p=0.003$ (adjusted yeometric mean: $9.0 \mathrm{mmol} / \mathrm{l}$ for PL and $7.3 \mathrm{mmol} / 1$ for TR. Following the AC fasting serum glucose the following morning (adjusted geometric mean: $6.8 \mathrm{mmol} / \mathrm{L}$ for $\mathrm{CC}$; $6.7 \mathrm{mmnol} / \mathrm{L}$ for $\mathrm{AC}$ ) and weighted mean were not statistically significant different compared to $\mathrm{CC}$. Mean trough glucose for TR after the evening meal was $5.7 \mathrm{mmol} / \mathrm{l}$ following both the $\mathrm{AC}$ and $\mathrm{CC}$. Analysis of the other parameters showed no symptomatic or pharmacodynamic evidence of an acute interaction between TR and alcohol. Conclusions: these results suggest: (1) NrDDM subjects treated by diet alone would derive additional glycaemic control hy taking TR and (2) moderate alcohol consumption with meals (approximately 4 grlasses of wine or equivalent) will not increase the risk of hypoglycaemia in TR traltcd subjects.

\section{4}

TROGLITAZONE IMPROVES INSULIN SENSITIVITY DESPITE NO INFLUENCE ON PYRUVATE DEHYDROGENASE Or 3-HYDROXYACYL-COA DEHYDROGENÁSE Y. Oshida, M.Kako, L. Li, X. -C. Hu, N. Nakai, J. Sato, Y. Sa to Nagoya University, Nagoya, Japan

It has recently been known that troglitazone has the capacity to improve insulin resistance. The present study was undertaken to determine the effect of troglitazone on in vivo insulin action and the activities of pyruvate dehydrogenase ( $\mathrm{PDH}$ ) complex and 3-hydroxyacyl-COA dehydrogenase (AADH) in obese Zucker rats. obese male Zucker rats were fed normal chow diet with and without troglitazone as a food admixture (0.3\%). In vivo insulin action was measured by the sequential euglycemic clamp technique at two different insulin infusion rates, 6.0 (low-dose) and 30.0 (high-dose) $\mathrm{m} U / \mathrm{kg} \cdot m i n$. Plasma insulin concentrations during the low-dose and the high-dose insulin infusion were $100-150 \mu \mathrm{U} / \mathrm{ml}$ and $1,000-1,400 \mu \mathrm{V} / \mathrm{ml}$, respectively and blood glucose was clamped at fasting levels (7.2-7.8mnol/1) by periodic adjustment of the i.v. glucose infusion rate. Af ter the clamp studies, the activi ties of PDHcomplex and HADH in red gastrocnenius muscles and heart were deternined. Fasting plasma insulin concentrations in troglitazone-treated obese rats were nearly identical to those in control obese rats. Troglitazone treatment produced a significant rise in glucose disposa]. rate (GDR) during the low-dose insul in clamp study $(14.7 \pm 2.2$ vs $7.1 \pm 1.1 \mathrm{mg} / \mathrm{kg} \cdot \mathrm{min}, \mathrm{P}<0.05)$. In the high-dose insulin clamp study, no significant difference in GDR was found between both groups. The activities of PDHcomplex and HADH were similar in both groups.

Conclusion : Troglitazone improves in vivo insalin action in physiological insulin levels with no influence of PDH or HADH activities.

\section{5}

TROGLITAZONE INCREASES THE RESISTANCE OF LDL TO OXIDATION IN HEALTHY VOLUNTEERS

L. Cominacini, U. Garbin, A. Fratta Pasini, M. Campagnola, M.M.R. Young* and A. Capriati*. Istituto di Semeiotica e Nefrologia Medica, University of Verona, Italy, and GlaxoWellcome Research and Development Ltd., Greenford, UK.

The oxidative modification of low density lipoprotein (LDL) is of importance in atherogenesis. Antioxidant supplementation has been shown, in published work, to increase LDL resistance to oxidation in both healthy subjects and diabetics; in animal studies a contemporary reduction in atherogenesis has been demonstrated. Troglitazone (TR) is in development for the treatment of non-insulin dependent diabetes meilitus and has similarities in structure with vitamin $E$. In vitro studies indicated that the compound possessed antioxidant activity. The present study assessed the effect of TR $400 \mathrm{mg}$ bd for 2 weeks on the resistance of LDL to oxidation in healthy male subjects. Ten subjects received TR and ten received placebo in a randomised, placebo-controlled, parallel-group design. The lag phase (i.e. resistance of $L D L$ to oxidation) was determined by measurement of fluorescence during copper-catalysed oxidative modification of $L D L$. The lag phase was increased by $27 \%(p<0.001)$ at week 1 and by $24 \%(p<0.001)$ at week 2 in the TR treated group compared with the placebo group. 15-hydroxyeicosatetraenoic acid (15-HETE) (an expression of LDL hydroperoxide content) was measured by using a radioimmunoassay method. The increase in the lag phase was parallelled by a significant decrease of 15 -HETE $(p<0.001)$. A number of variables known to influence the resistance of $L D L$ to oxidation were also measured. They included $\alpha$ tocopherol and $\beta$-carotene levels in LDL, mono and polyunsaturated fatty acids, LDL particle size, body weight and macronutrient consumption. The lack of any significant change in these parameters suggested that the increase in the lag phase was due to the activity of TR as an antioxidant and was not secondary to changes in the intrinsic properties of LDL. 
ANALYSIS OF THIAZOLIDINEDIONE, BIGUANIDE AND RETINOID EFFECTS ON ADIPOGENESIS AND THE NUCLEAR RECEPTORS PPARY AND RXR,

J. M. Lenhard, L. Hamacher, M. Paulik, K. Beck, S. Kliewer, J. Lehmann, J. Cobb, B. Henke, T.Wilson, D. Parks and S. G. Blanchard, Durham, NC.

Troglitazone and metformin are antidiabetic agents that are members of the thiazolidinedione and biguanide families of compounds, respectively. These agents enhance glucose disposal and insulin sensitivity in both animal models and patients with NIDDM. To determine if the molecular mechanism of action for these agents are distinct from each other we made a direct comparison of these agents using several in vitro assays. Recently, we showed that thiazolidinediones stimulate preadipocyte differentiation in vitro and are high affinity ligands for the nuclear receptor peroxisome proliferator activated receptor gamma (PPAR $\gamma$ ). Using a number of thiazolidinediones with varying potency, we show that the in vivo antihyperglycemic activity correlates ( $r>0.9$ ) with (i) stimulation of adipogenesis, (ii) activation of PPARy in a transient transfection assay, and (iii) binding to pure PPAR $\gamma$. Troglitazone was found to have an EC50 of $480 \mathrm{nM}$ for stimulation of adipogenesis, $430 \mathrm{nM}$ for trans-activation of PPAR $\gamma$, and 3 $\mathrm{mM}$ for binding to pure PPAR $\gamma$. In contrast, $\leq 50 \mathrm{mM}$ of the bigaunides metformin and phenformin had no effect on adipogenesis, trans-activation of PPAR $\gamma$, or binding to pure PPAR $\gamma$. Further, we report that synthetic retinoids and insulin potentiate the effects of thiazolidinediones on adipogenesis but have no effect on adipogenesis in the presence of biguanides. Synthetic retinoids also had no effect on the binding of thiazolidinediones to pure PPARy in the presence of its heterodimer partner retinoid $X$ receptor. Taken together, these observations suggest that (1) the synergistic ability of thiazolidinediones and retinoids to promote adipogenesis is not due to an increase in binding affinity to their respective receptors and (2) thiazolidinediones and bigaunides exert their antidiabetic effects through two distinct molecular mechanisms.
COULD 4-HYDROXYISOLEUCINE BE USED AS A HYPOGLYCAEMIC AGENT IN THE TREATMENT OF TYPE 2 DIABETES MELLITUS? 1.Fernández-Alvarez. Y.Sauvaire', P.Petit ${ }^{2}$ R.Casamitjana ${ }^{3}$, G.R.hes and R.Gomis Endocrinology Unit ${ }^{3}$ Hormonal Laboratory.Hospital Clínic, Barcelona. Spain. ${ }^{1} L$. Recherehe $/{ }^{2} \mathrm{~L}$. Phar macologie. University Montpellier. France. ${ }^{4}$ L. Pharmacologie, CNRS, University Montpellier.

The 4-hydroxyisoleucine (4-HIL) is an aminoacid from Trigonella foenum graecum which increases the insulin secretion "in vitro" and "in vivo" in different animal models. In these models, 4-HIL increases insulin-secretion in the presence of glucose and is effective at concentrations lower than leucine and isoleucine. However.until now, we don't have any infomation abrut its effect in human pancreatic islets. The aim of study is investivate the effect of $4-\mathrm{HIL}$ in human islets. Methodology: Human islets were obtaned from cadaveric donors by a modifiation of Ricordi": automatic digestion tehnique. Insulin reledse and content were meastured in the presence of different concentrations of $\mathrm{D}-\mathrm{gl}$ lucose and 4-HIL by RIA. Results:

\begin{tabular}{lll}
\hline $\begin{array}{l}\text { Experimental } \\
\text { Condition }\end{array}$ & $\begin{array}{l}\text { Insulin Secretion } \\
\text { (pmol/8 islets) }\end{array}$ & $\begin{array}{l}\text { Insulin Content } \\
\text { (pmol/8 islets) }\end{array}$ \\
\hline Glucose $8.3 \mathrm{mM}$ & $1.3 \pm 0.3(15)$ & $46.2 \pm 4.6(15)$ \\
Glucose $16.7 \mathrm{mM}$ & $2.6 \pm 0.6(16)$ & $54.3 \pm 5.1(16)$ \\
Glucose $8.3 \mathrm{mM}+0.1 \mathrm{mM}$ & $2.6 \pm 0.5(16)^{*}$ & $53.8 \pm 5.9(16)$ \\
Glucose $8.3 \mathrm{mM} \div 0.2 \mathrm{mM}$ & $2.8 \pm 0.5(16)^{*}$ & $59.1 \pm 5.5(16)$ \\
Glucose $8.3 \mathrm{mM}+0.5 \mathrm{mM}$ & $2.9 \pm 0.4(16)^{* *}$ & $51.4 \pm 5.4(16)$ \\
Glucose $8.3 \mathrm{mM}+1 \mathrm{mM}$ & $2.2 \pm 0.3(15)^{*}$ & $47.8 \pm 5.4(16)$ \\
\end{tabular}

$* \mathrm{p}<0.05 ; * \mathrm{p}<0.005$ v.s. control $8.3 \mathrm{mM}$ Glucose

Conclusions: 4-HIL increases insulin secretion in human islets at muromolar concentrations similar to that observed in the different animal models. These results suggest the possibility that 4-HIL could be used as a hypoglycaemic agent in type 2 Diabetes mellitus.

\section{8}

STRUCTURE ACTIVITY ANALYSIS OF DIFFERENT ANALOGUES OF THE NEW INSULINOTROPIC AGENT 4-HYDROXYISOLEUCINE

G. Ribes ${ }^{1}$, C. Broca ${ }^{1,2}$, P. Petit ${ }^{2}$, M. Jacob ${ }^{3}$, Y. Baissac ${ }^{4}$, M. Manteghetti ${ }^{1}, M$. Roye $^{1}$ and Y. Sauvaire ${ }^{4}$. 'Fac. Med., UMR 9921 CNRS; 'Lab. Pharmacol. (EA 1677), Fac. Med., University Montpellier I; ${ }^{3}$ URA CNRS 0468; ${ }^{4}$ Lab. Rech Subst. Nat. Végét. (EA 1677), University Montpellier II, FRANCE.

We have previously reported that 4-hydroxyisoleucine (4-OH Ile), an original amino acid extracted from fenugreek seeds (Trigonella foenumgraecum L.) stimulates insulin secretion at micromolar concentrations and only in the presence of moderate to high glucose concentrations. Recently, we have purified from the seeds two different isomers: $2 \mathrm{~S}, 3 \mathrm{R}, 4 \mathrm{~S}$ as major component $(97 \%)$ and $2 \mathrm{R}, 3 \mathrm{R}, 4 \mathrm{~S}$ as minor component $(3 \%)$. The present study was designed to determine which one of these two isomers is more efficient in inducing insulin secretion and also to test the effects of different synthetic structural analogues (monomethylated derivatives) on insulin secretion. The experiments were performed in vitro in isolated perfused rat pancreas as well as in isolated islets in the presence of $8.3 \mathrm{mmol} / 1$ glucose. In isolated pancreas, all substances were perfused at $200 \mu \mathrm{mol} / \mathrm{l}$. Only the major isomer of 4-OH Ile elicited a clear biphasic insulin response (peak at $2 \mathrm{~min}+$ $210 \%, \mathrm{p}<0.01$ ); the other compounds were completely ineffective. In incubated islets, we compared the insulinotropic action of these different substances with that of the amino acids, leucine and isoleucine. The concentrations tested were $200,500 \mu \mathrm{mol} / 1$ and $1,5,10 \mathrm{mmol} / 1$. The threshold for a significant increase in insulin release $(p<0.01)$ was $200 \mu \mathrm{mol} / 1$ for the major isomer, $1 \mathrm{mmol} / \mathrm{l}$ for the minor isomer and monomethylated analogues; leucine and isoleucine stimulated insulin secretion only from 5 and $10 \mathrm{mmol} / \mathrm{l}$ respectively. We conclude that the major isomer of $4-\mathrm{OH}$ Ile is the most potent of all analogues tested, increasing insulin secretion at micromolar concentrations. However, the other analogues are more potent than leucine and isoleucine. Thus, 4-OH Ile and derivatives may be considered as new insulinotropic agents of potential interest in the treatment of NIDDM

\section{9}

BETA-1,3-D POEYGLUCOSE IMPROVES WOUND HEALING IN DIABETES MELLITUS

M.Berdal, R.Seljelid and T.G.Jenssen. Univergity of Tromso, Norway.

Healing of diabetic wounds is delayed not only by reuropatric changes and local ischemia, but also by way reuropathic changes and local ischemia, but also by way of altored cellular mechanisme in the healing wound. The healing depends partly on the infiltraton of macrophages generating the inflammator response with cytokines and growth factors. This process seems to be impaired in patients with diabetes mellitus. The preaent study was undertaken to see if beta-1,3-D polyglucose, a substance improving macrophage chemotaxis and secretory activity, would improve wound healing in diabetes. Cutaneous wounds were established on the back diabetes. of either diabetic (db/db) ox non-diabetic $(\mathrm{db} / \mathrm{m}$ mice. Four groups of animals were studied: 1 . Db/db mice with placebo wound treatment $(n=10$, average blood glucoge $27.5 \pm 2.7 \mathrm{mM}), 2$. Db/db mice treated with insulin $(\mathrm{n}=6$. average blood glucose $4.8 \pm 3.1 \mathrm{mM} / 3$. Db/do mice treated locally with beta-1,3-D polyglucose only $i n=6$, average blood glucose $27.9 \pm 1.6 \mathrm{mM} /, 4$. Db/ $+\mathrm{m}$ non-diabetic mice without specific wound treatment $(\mathrm{n}=10$, average blood glucose $4.7 \pm 0.4 \mathrm{~mm})$. Biopsies from untreated diabetic wounds showed decreased infilt tation of macrophages. and we peritoneal macrophages was pha (TNF-alpha) Erom isolated peritoneal macrophages was only $1 / 8$ of that from non-diabetic animals. The percentage reduction in wound area after 10 days was for group 1-4: $20 \pm 4, \quad 32 \pm 6,44 \pm 6$ and $57 \pm 5 \%$ (meantSEM, p<0.05). The corresponding results after 16 days were: $5 \varepsilon_{ \pm 10}, 63 \pm 7$, $94 \pm 6$ and $93 \pm 58$ (p<0.05) Conclusion: Macrophage function, as judged by TNF-alpha release, is impaired in diabetes. Stimulation of macrophages with beta-1,3-D polygiucose 
890

EFFECTS OF IMIDAZOLINE DERIVATIVE RX871024 ON INSULIN, GLUCAGON AND SOMATOSTATIN SECRETION FROM ISOLATED PERFUSED RAT PANCREAS.

I.B. Efanova, S.V. Zaitsev, A.M. Efanov, C.-G. Östenson, P.-O. Berggren and S. Efendic; The Rolf Luft Center for Diabetes Research, Department of Molecular Medicine, Karolinska Institute, Karolinska Hospital, 17176 Stockholm, Sweden

Compounds with imidazoline structure are known as potent stimulators of insulin secretion. They also exert antidiabetogenic effects in men. So far, the effects of the imidazoline compounds on secretion of other pancreatic hormones have not been studied. Therefore, we investigated the effects of the imidazoline compound RX871024 on arginine-induced insulin, glucagon and somatostatin secretion in the perfused rat pancreas. Arginine was infused for 20 $\mathrm{min}$ at $20 \mathrm{mM}$ concentration in Krebs-Ringer buffe: supplemented

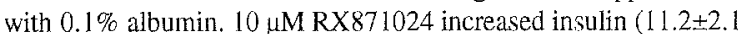
$\mathrm{mU} / 20 \mathrm{~min}$ vs. $3.8 \pm 0.4 \mathrm{mU} / 20 \mathrm{~min}, \mathrm{n}=6$ ) and somatostatin release ( $970 \pm 90 \mathrm{pg} / 20 \mathrm{~min}$ vs. $170 \pm 10 \mathrm{pg} / 20 \mathrm{~min}, \mathrm{n}=6$ ). In contrast, glucagon secretion was markedly inhibited by $10 \mu \mathrm{M}$ imidazoline $(31.2 \pm 2.0 \mathrm{ng} / 20 \mathrm{~min}$ vs. $86.6 \pm 12.7 \mathrm{ng} / 20 \mathrm{~min}, \mathrm{n}=6)$. In conclusion, RX871024 exerts a complex effect on endocrine pancreas comprising of the stimulation of insulin and somatostatin release and the inhibition of glucagon release. These hormone effects probably constitute the main mechanism of the antidiabetogenic effect of the imidazolines.

\section{1}

NEPHRECTOMY SLOWS CLEARANCE OF PRAMLINTIDE AND RAT AMYLIN IN RATS.

W. Vine, P. Smith, R. LaChappell, E. Blase, R. Lawler and A. Young. Amylin Pharmaceuticals, Inc., San Diego, CA 92121, USA

Amylin is a 37 amino acid peptide hormone co-secreted with insulin from the pancreatic $\beta$-cell. Pramlintide is an amylin analog currently in Phase III clinical trials for the treatment of insulin requiring patients with diabetes. To investigate the potential role of the kidney in amylin and pramlintide clearance, plasma peptide concentrations were compared following S.c. injections of synthetic rat amylin $(100 \mu \mathrm{g})$ or of pramlintide $(10 \mu \mathrm{g})$ in fasted, halothane anesthetized Sprague Dawley rats in which renal vessels were ligated (functional nephrectomy) or ligatures were placed loosely around the vessels (sham controls). Peptide concentrations were determined by a monoclonal immuno-enzymometric assay with fluorescent detection. Data are means \pm SEM. Compared to sham controls, functional nephrectomy resulted in $\sim 3.5$-fold greater incremental area-under-the-curve 0-120 minutes after injection for each peptide (rat amylin $6122 \pm 850 \mathrm{pM}$ hr vs $1707 \pm 175 \mathrm{pM}$ hr, $P<0.001, \mathrm{n}=6,6$; pramlintide $1933 \pm 199 \mathrm{pM}$ hr vs $579 \pm 58 \mathrm{pM}$ hr, $P<0.001, n=7,6)$. Nephrectomy increased elimination half life of rat amylin to $25.8 \pm 2.3 \mathrm{~min}$ from $17.4 \pm 0.9 \mathrm{~min}$ in the sham group $(P<0.01, n=6)$. Nephrectomy had similar effects on the metabolism of pramlintide; half-life was increased to $35 \pm 2 \mathrm{~min}$ from $28 \pm 2 \mathrm{~min}$ in the sham group $(P<0.05)$. These data suggest that, in rats, the majority of administered rat amylin and pramlintide is cleared at the kidney.

\section{2}

EFFECT OF DIFFERENT SULPHONYLUREA DRUGS ON ARRHYTHMIAS INDUCED BY ISCHAEMIA AND REPERFUSION G.Pogátsa, M.Z.Koltai, I.Posa and E.Kocsis, National Institute of Cardiology, Budapest. Influence of glimepiride (GM), glibenclamide (GB), gliclazide (GC) and tolbutamide (TB) was analyzed on incidence of ventricular ectopic beats (VEB) and on duration of ventricular fibrillations (VF) induced by coronary clamping (CC) or reperfusion (CR). VEB and VF were registered in 140 healthy (H) and in 140 streptozotocyn-diabetic (D) rats during $30 \mathrm{~min} C \mathrm{C}$, as well as in $230 \mathrm{H}$ and $300 \mathrm{D}$ rats during $50 \mathrm{~min} C R$ following a $10 \mathrm{~min} \mathrm{CC.} \mathrm{Drugs}$ $(0.1-1000 \mu \mathrm{mol} / \mathrm{kg})$ were administered i.p. $30 \mathrm{~min}$ before $C C$. During $C C$ only $G B$ decreased these parameters (VEB: $y=-400 \log x+635 ; \mathrm{VF}: \mathrm{y}=-45 \log \mathrm{x}+97$ ) in $\mathrm{H}$ rats, whereas GC (VEB: $y=732 \log x+1350 ; \mathrm{VF}: y=174 \log x$ $+179)$, GM (VEB: $y=573 \log x+739 ; V F: y=24 \log x+82)$ and TB (VEB: $y=626 \log x+1635 ; \mathrm{VF}: y=285 \log x+260)$ increased them. During $C R$ only $G M$ decreased these variables (VEB: $y=-6 \log x+13 ; V F: y=-5 \log x+13$ ), while GB (VEB : $y=20 \log x+32 ; \quad V F: y=3 \log x+22), G C(V E B: y=9 \log x+46$; $\mathrm{VE}: \mathrm{y}=3 \log \mathrm{x}+22)$ and $T B(\mathrm{VEB}: \mathrm{y}=19 \log \mathrm{x}+46 ; \mathrm{VF}: \mathrm{y}=8 \log \mathrm{x}$ +42 ) enhanced them. In D condition similar effects could be registered except of those induced by GM. Namely, GM decreased these parameters (VEB: $y=-269$ $\log x+425 ; \mathrm{VP}: \mathrm{y}=-146 \log \mathrm{x}+251$ ) in $\mathrm{D}$ rats during $\mathrm{CC}$. Suggesting that GM has only a week effect on cardiac ATP-sensitive potassium channels. Consequently, only GM should be preferred in oral antidiabetic treatment of type-2 diabetic patients suffering from ischaemic events or during interventions (i.e. streptokinase, PTCA) inducing reperfusion.

\section{3}

ALPHA ENDOSULFINE IS A NOVEL MOLECULE, STRUCTURALLY RELATED TO A FAMILY OF PHOSPHOPROTEINS

K. Peyrollier, L. Héron, A. Le Cam and D. Bataille. INSERM U 376, CHU Arnaud-de-Villeneuve, 34295 Montpellier Cedex 05, France

Endosulfine, endogeneous ligand for the sulfonylurea receptor, exists under two molecular forms, $\alpha$ and $B$, in both the pancreas and the central nervous system. The $B$ form is a low molecular weight peptide which possesses the biological properties of sulfonylureas. The $\alpha$ form, recently isolated from porcine brain, has a molecular mass close to $13 \mathrm{KDa}$, as determined by mass spectrometry. From several internal amino acid sequences, it has been shown that $\alpha$ endosulfine presents strong similarities with a 12-13 KDa protein, called ARPP-19, a substrate for CAMP-dependent protein kinase previously isolated from bovine brain. To determine whether the differences observed between the two protein sequences reflects species differences, we have designed degenerated probes for cioning the bovine brain $\alpha$ endosulfine $c D V A$ using a reverse transcriptase-polymerase chain reaction (RT-PCR) approach. A 260 bp fragment, representing 70-80\% of the full length mRNA was obtained, using several sets of probes. We deduce that, in spite of similarities already observed at the protein level and confirmed by the cDNA sequence, bovine $x$ endosulfine is different from ARPP-19 within the same animal species. In particular, N-terminal moieties of a endosulfine and ARPP-19 are quite different. These data clearly indicate that the two proteins are distinct molecular entities belonging to the same protein family. Cloning the human alpha endosulfine cDNA will aliow to analyze possible mulations in the translated part of the alpha endosulfine gene in type II diabetic patients. 


\section{4}

EFFECT OF L-CARNITINE ON ACTIVITY OF LIVER ALCOHOL DEHYDROGENASE IN DIABETIC RATS

M. Yıldınıkaya, O. Yıldız* M. Özata** K. Yılmaz, A. Çorakç̧**, T Kutluay and M.A. Gündoğan ${ }^{* *}$. Department of Biochemistry, Medical Pharmacology* and Endocrinology and Metabolism**, Gülhane School of Medicine, 06018, Ankara, Turkey

Supplementary L-carnitine attenuates ethanol metabolism in rats and thus offers protection to aninals against ethanol-induced lipid abnormalities by decreasing the load of ethanol-derived metabolites on the liver Experimental diabetes increases ethanol metabolism in rats by affecting the activity of liver alcohol dehydrogenase (ADH). L-carnitine has been used for treatment of diabetic neuropathy in humans. We have therefore evaluated the effect of L-carnitine on activity of liver ADH in Wistar diabetic male rats. Two groups $(\mathrm{n}=5)$ of rats were rendered diabetic with alloxan $(75 \mathrm{mg} / \mathrm{kg}$ ) and $\mathrm{L}$-camitine (200 $\mathrm{mg} / \mathrm{kg}$ day, i.p.) was given to diabetic rats starting from the $3 \mathrm{rd}$ week after the induction of diabetes and lasting for 4 weeks. A third group of rats $(n=5)$ which was not diabetic and was not supplemented with L- carnitine served as controls. The activity of $\mathrm{ADH}$ was determined by enzymatic method. Liver ADH activity in diabetic rats $(4.5 \pm 0.3 \mathrm{U} / \mathrm{g}$ liver) was significantly higher $(\mathrm{p}<0.05)$ by about $30 \%$ as compared with L-camitine supplemented diabetic group $(3.5+0.3 \mathrm{U} / \mathrm{g}$ liver) or control group $(3.2 \pm 0.2 \mathrm{U} / \mathrm{g}$ liver). Since the activity of $\mathrm{ADH}$ may be affected by hormonal status, we measured the serum levels of testosterone, growth hormone and thyroxine. A significant decrease in thyroxine and testosterone levels ( $\mathrm{p}<0.01$, respectively), but not in growth hormone levels was observed in all diabetic rats. However, there were no correlations between ADH activity and hormone levels studied. We concluded that supplementary L-carnitine decreases the diabetes-induced activity of ADH and it may therefore have beneficial effects against ethanol-induced lipid-abnormalities in liver.

\section{5}

EFFECTS OF GYMNEMA SYLVESTRE AND MOMORDICA CHARANTIA ON SERUM LIPID LEVELS OF IDDM AND NIDDM MODEL RATS

S. Murshed', S. Sarkar', B. Rokeya', N.G. Banik', L. Ali', A.K. Azad Khan', N. Nahar ${ }^{2}$ and M. Mosihuzzaman'2. ${ }^{1}$ Research Division, BIRDEM; ${ }^{2}$ Dept of Chemistry, University of Dhaka, Bangladesh.

Changes in serum lipids contribute cardiovascular complications in diabetes mellitus. Therefore, effects on the lipid metabolism by a therapeutic agent need to be investigated. It has been shown that methanol extract of $G$ sylvestre leaves and $M$ charantia fruit pulp have got hypoglycemic effect on IDDM and NIDDM model rats, respectively. In the present study, these extracts have been tested to evaluate their effect on the lipid profile of the respective rat models. The $G$ sylvestre and $M$ charantia were fed daily by mouth to IDDM and NIDDM model rats, respectively. The control rats were fed with water only. Blood was collected by decapitation of rats of the $G$ sylvestre group after 14 days and for $M$ charantia group after 28 days. As compared to control, $G$ sylvestre group showed a significantly lower value of serum TG $(1.11 \pm 0.05$ vs $0.69 \pm 0.09 \mathrm{mmol} / \mathrm{l}, M \pm S E M, p<0.001)$, total cholesterol $(6.05 \pm 0.13$ vs $5.37 \pm 0.26 \mathrm{mmol} / \mathrm{M}, \mathrm{SEM}, p<0.04)$ and $L D L$ cholesterol $(4.68 \pm 0.23$ vs $3.68 \pm 0.24 \mathrm{mmol} / \mathrm{M} \pm \mathrm{SEM}, \mathrm{p}<0.03$ ) in IDDM model rats. No significant change was observed in the HDL cholesterol level. The $M$ charantia extract showed a similar change in the NIDDM model rats; TG, $1.15 \pm 0.04$ vs $0.70 \pm 0.08 \mathrm{mmol} / / \mathrm{M} \pm S E M, p<0.001$, total cholesterol, $1.58+0.09$ vs $1.32+0.07 \mathrm{mmol} / \mathrm{M}+\mathrm{SEM}, \mathrm{p}<0.04$ and LDL cholesterol $0.45 \pm 0.05$ vs $0.25 \pm 0.03 \mathrm{mmol} / \mathrm{p}, \mathrm{p}<0.004$. The results suggest that $G$ sylvestre and $M$ charantia may help prevent the dyslipidemia in diabetes mellitus.

\section{6}

EFFECTS OF VARIOUS EXTRACTS FROM FOUR PLANTS ON SERUM GLUCOSE OF NONDIABETIC AND DIABETIC MODEL RATS.

M. Mosihuzzaman'1, N. Nahar1, A.K. Azad Khan², B. Rokeya ${ }^{2}$, L. Ali ${ }^{2}$, N.S. Chowdhury ${ }^{2}$, J.M.A. Hannan'. 'Department of Chemistry, University of Dhaka; ${ }^{2}$ Research Division, BIRDEM, Dhaka, Bangladesh

Gymnema sylvestre, Coccinia indica, Pterospermum acerifolium and Spirulina platensis are used in traditional medicine in the treatment of diabetes mellitus. Although the first two of these plants have been reported to have hypoglycemic properties, the active principle(s) of the plants and the mechanism(s) of the hypoglycemic effects are not established. These plants or extracts thereof, suspended/dissolved in water were tested on nondiabetic and diabetic model rats at various prandial states. $S$ platensis powder and $C$ indica juice had significant effect $(p<0.05-0.02)$ on the fasting as well as postprandial serum glucose levels of the nondiabetic rats. In NIDDM model rats all the plants showed significant hypoglycemic effect $(p<0.005-0.001)$ but only when fed simultaneously with glucose. The water soluble extracts of $S$ platensis, $G$ sylvestre and $P$ acerifolium showed significant effect in IDDM model rats when the extracts were fed simultaneously with glucose $(p<0.003-0.001)$ and the $C$ indica extract was effective in this model only when fed 30 minutes before the oral glucose load. The completely water-immiscible lipophilic chloroform extract of the plant materials were made water soluble with the help of PVP (polyvinyl-pyrolidine). The chloroform extracts were fed against water and PVP controls. The chloroform extracts of $S$ platensis and $P$ acerifolium significantly lowered the serum glucose levels of NIDDM model rats $(p<0,05)$, but those of $G$ sylvestre and $C$ indica had no significant effect. The results showed that these plants contain hypoglycemic agents which may act through different target tissues. 
PS 49

\section{Coagulation Endothelium}

\section{7}

RELATIONSHIP BETWEEN LIPOPROTEIN(a) PHENOTYPES AND FIBRINOLYSIS IN DIABETES MELLITUS.

R.Simó, C.Hernández, P.Chacón*, L.García-Pascual\#, R.Burgos and J.Mesa. Depts. of Endocrinology and *Biochemistry. Hospital Vall d'Hebron. Barcelona.\#Hospital Mutua de Terrassa. Spain.

It has been demonstrated in vitro that lipoprotein(a) [Lp(a)] increases the endothelial synthesis of PAI-1(plasminogen activator inhibitor type 1). This effect has not been observed in vivo and the relationship between PAI-1 and Lp(a) phenotypes has not been established. The aim of the study was to determine the influence of $L p(a)$ and its phenotypes on PAI-1 serum concentrations. For this purpose serum $L p($ a) and PAI-1 antigen were assessed by ELISA in 62 diabetic patients ( 31 consecutive IDDM and 31 consecutive NIDDM.Lp(a) phenotypes were determined in 55 diabetic patients by SDS-PAGE followed by immunoblotting and grouped according size in small (F,B,S1,S2), big (S3,S4) and null. Statistics: Pearson linear correlation coefficient and ANOVA. Lp(a) concentration was $\log$ transformed.A linear correlation between Lp(a) and PAI-1 was not observed either as a whole of diabetic patients were considered or when IDDM and NIDDM were analyzed separately. However, significant differences were detected in PAI-1 levels when Lp(a) phenotypes were considered (small: $\mathrm{n}=23, \mathrm{X}=42.1 \pm \mathrm{SD} 31.8 \mathrm{ng} / \mathrm{ml} ;$ big: $\mathrm{n}=23$, $\mathrm{X}=37.2 \pm \mathrm{SD} 26.1 \mathrm{ng} / \mathrm{ml} ;$ null: $\mathrm{n}=9, \quad \mathrm{X}=14.4 \pm \mathrm{SD} 14.5 \mathrm{ng} / \mathrm{ml}$; $\mathrm{p}<0.05$ ). The low concentrations of PAI-1 detected in patients with null phenotype were observed both in type 1 and in type 2 diabetic patients (Type 1:small $\mathrm{X}=22.8 \pm 18.7$, big $\mathrm{X}=21.7 \pm 15.6$, null $\mathrm{X}=10.1 \pm 6.2$; Type 2: small $X=57 \pm 32.3$, big $X=54.2 \pm 25.1$, null $X=29.5 \pm 29.6$ ). The lowest PAI- 1 concentration associated to diabetic patients with $\mathrm{Lp}(\mathrm{a})$ null phenotype suggests that fibrinolytic activity is preserved in these patients. Furthermore, it could be speculated that diabetic patients with null phenotype should be prone to low cardiovascular risk.

\section{9}

TISSUE FACTOR PATHWAY INHIBITOR (TFPI) IN NORMAL, IGT, AND NIDDM SUBJECTS

B.H.R.Wolffenbuttel ${ }^{\prime}$, R.P.Stolk ${ }^{2}$, R.van Oerle', K. Hamulyak', D.E.Grobbee ${ }^{2}$ P.B.Leurs'. Dept. of Internal Medicine, University Hospital Maastricht', Dept. of Epidemiology \& Biostatistics, Erasmus University Medical School, Rotterdam², The Netherlands.

NIDDM and impaired glucose tolerance (IGT) are associated with cardiovascular complications. Endothelial damage is one of the main initiating events in the development of atherosclerosis. Until now, von Willebrand factor (vWF) t-PA development of atherosclerosis. Until now, von Willebrand factor ( $V W F$, t-PA, PAI-I and thrombomodulin (TM), have been identified as markers of endothelial dysfunction in diabetes. TFPI, a coagulation inhibitor, is produced by and mainly bound to the vascular endothelium, and may therefore serve as a marker of endothelial dysfunction. Recently, we demonstrated that TFPI activity (TFPI-act) is increased in IDDM patients, and is correlated with $\mathrm{HbA}_{1 c}$ and albuminuria (ALB). In a cross-sectional population-based study (Rotterdam Study), we examined TFPI-act, measured chromogenically, in normal (group I; 18M:33F), JGT (group II; 23M:25F) and NIDDM (group III; 29M:18F) subjects, in relation to $\mathrm{vWF}, \mathrm{t}-$ PA, PAI-1, TM, and ALB. The results were as follows.

Age (years) I: normals $25 \pm 6$ $\mathrm{HbA}$ ic $(\%)$ Il: IGT $69 \pm 6$
$28.0 \pm 3.5^{+}$ $\frac{28.0 \pm 3.5}{6.0 \pm 0.5}$ $5.7 \pm 0.5$ $5.4(1.3-261)$ $111 \pm 16$ $10.7 \pm 3.5$ $13.7-26$ $6.5(1.4-105)$ $113 \pm 15$ $140 \pm 44$ $39.7+10.3$ $14.0 \pm 4.2^{2}$ $39.7 \pm 10.3 \quad 44.9 \pm 12.8 \quad 41.8 \pm 15.7$

III: NIDDM $69 \pm 6^{\circ}$
$27.3 \pm 3.3^{*}$ $7.2 \pm 1.3^{3}$ $7.3 \frac{1}{(2.1-685)}$ $116 \pm 16$ $168 \pm 63$. $15.4 \pm 58^{\circ}$ $39.7 \pm 10.3 \quad 44.9 \pm 12.8 \quad 41.8 \pm 15.7$ PAI-I $(\mathrm{AU} / \mathrm{ml})$ TM $(\mathrm{n} g / \mathrm{ml})$ (Data are means \pm SD or medians (ranges). $* p<0.001$ vs $1, \# p<0.0001$ vs $\mathrm{I}+\mathrm{II}$; ANOVA). TFPl-act was increased in all three grouns, and was correlated with age $(\mathrm{r}=0.18, p<0.05), \mathrm{HbA}_{1 \mathrm{c}}(\mathrm{r}=0.21, \mathrm{p}<0.01)$, fasting blood glucose $(r=0.16, p<0.05)$, albuminuriat $(r=0.17, p<0.05), v W F(r=0.17, p<0.05)$ and PAI-1 $(r=0.15, p<0.05)$. In the NIDDM group, a significant correlation was found between TFPI-act and vWF $(r=0.28, p<0.05)$, PAI-1 $(r=0.50, p<0.001)$, and albuminuria $(r=0.28, p<0.05)$. Conclusions. 1. TFPI-act is increasing with age, glucose intolerance and $\mathrm{HbA}_{1 \mathrm{c}}$. 2. In NIDDM, TFPI-act is related to $\mathrm{WWF}$ PAI- 1 and albuminuria. Therefore, TFPI-act may reflect endothelial damage.
898

INCREASED TISSUE FACTOR PATHWAY INHIBITOR (TFPI) ACTIVITY AND COAGULATION IN IDDM PATIENTS.

P.B.Leurs, R.van Oerle, B.H.R. Wolffenbuttel, K.Hanulyak. Dept, of Internal Medicine, University Hospital Maastricht, The Netherlands.

Diabetes is characterized by the occurrence of premature cardiovascular events. A prethrombotic state could thereby piay an important role. Recently, we found an increase in tissue factor pathway inhibitor (TFPI) activity (TFPl-act) in patients increase in tissue factor pathway inhibitor (TFPI) activity (TFPl-act) in patients with insulin-dependent diahetes mellitus (IDDM). TFPI is a coagulation inhibitor,
bound to the endothelium by glycosaminoglycans (GAGs). The increase in TFPIbound to the endothelium by glycosaminoglycans (GAGs). The ncrease in TFPIact could therefore be a reaction on an increased thrombin formation and/or altered
binding to GAGs. We therefore examined TFPI-act in relation to coagulation, measured by prothrombin $F_{1+2}$ fragments and endogenous thrombin potential (ETP) according to Hemker, in 46 patients with IDDM with or without complications and 18 age and sex-matched healthy controls. Because of their effect on thrombin generation, prothrombin and antithrombin were also measured. Thrombomodulin, an inhibitor of thrombin and a marker of endothelial dysfunction, was determined in the diabetic patients. TFPl-act was medsured chromogenically. ANOVA was performed. In IDDM patients. TFP I-act was sisnificantly increased $(112+11 \%$ ys $99+18 \%, p<0.01) . F_{1+3}$ levels were also hisher in the diabetic

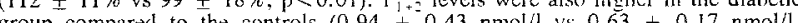
group compared to the controls $(0.94 \pm 0.43 \mathrm{nmol} / \mathrm{vs} 0.63 \pm 0.17 \mathrm{nmol} / 1$, $\mathrm{p}<0.001)$. On the other hand, a lower ETP $(339+57 \mathrm{nM}$. min vs $365+52$ $\mathrm{nM} \cdot \mathrm{min}, \mathrm{p}<0.05)$, level of prothrombin antigen $(97 \pm 13 \%$ vs $116 \pm 12 \%$, $\mathrm{p}<0.001)$ and antithrombin activity $(91 \pm 11 \%$ vs $99 \pm 7 \%, p<0.001$ ) were
found in comparison with the control group. In IDDM patients with microalbumifound in comparison with the control group. In IDDM patients with microalbumi-
nuria $(n=9)$, a manifestation of generalized angiopathy, TFPI-act, $F_{1}$, levels, and thrombomodulin levels were higher than in patients with only retinopathy $(n=18)$ or without complications $(n=19)$. No correlation hetween TFPI-det and $F_{1+2}$ levels was found, while TFPl act was negatively correlated with ETP $(r-0.27, p<0.05)$. Microalbuminuria was positively correlated with TFPI-act $(r=0.46, p<0.001)$, $F_{1: 2}(r=0.56, p<0.001)$, and thrombomodulin $(r=0.52, p<0.001)$. In TFPI. depleted plasma, ETP increased, indicating that ETP is alfected by TFPI. In conclusion, the absence of correlation betwe increse in TFPI-act in IDDM patients may not be increase in TFPI-act in IDDM patients may not be considered to be a reaction on a buminuria, it is hypothesized that vascular damage, leading (o) alterations in GAGs, is in part responsible for the changes in TFPI-act, $F_{1-2}$ levels and ETP.

\section{0}

Immunocrtochemical Evaluation of Anticoagulant, Hemostatic, and Fibrinolytic Pathways Within Allografts of Diabetic Recipients. A Pantaleo, RJ Torry, CA Labarrere, DR Nelson, WP Faulk. Methodist Hospital, Indianapolis, IN 46202.

Diabetes mellitus (DM) is known to induce microvascular alterations and plasma hemostatic abnormalities. However its effect on microvascular regulation of hemostasis is not known. We used immunocytochemical techniques to assess changes in vascular anticoagulant (antithrombin, AT), hemostatic (fibrin); and fibrinolytic (tissue plasminogen activator, tPA) pathways in biopsies from renal allografts of type I diabetic recipients $(n=30)$ and serial biopsies $(n=15.8 \pm 0.5 / p t)$ from cardiac allografts of type II diabetic recipients $(n=13)$. The vascular status of these biopsies was compared to biopsies from non-diabetic renal $(n=103)$ and cardiac $(n=93)$ recipients matched for follow-up time as well as to normal donor kidneys $(\mathrm{n}=36)$ and hearts $(\mathrm{n}=106)$ obtained prior to transplantation. Biopsies from normal donor kidneys and hearts demonstrated AT reactivity in arteries and veins tPA reactivity was confined to arterial/arteriolar smooth muscle cells, and no fibrin deposition was identified. Semiquantitative assessment of these pathways demonstrated no significant differences in renal biopsies from diabetics and non-diabetics taken $2.0 \pm 0.3$ months or $45 \pm 4.7$ months after transplantation. Similarly, there was no significant difference in vascular AT, tPA, and fibrin deposition between cardiac biopsies of type II diabetic and non-diabetic cardiac recipients followed for $41 \pm 2$ months after transplantation. The incidence and severity of transplantinduced coronary artery disease, evaluated by serial angiography, was similar between groups. There was no significant difference in the degree of cellular infiltration or renal/cardiac allograft survival between groups. These findings suggest that DM does not induce changes in microvascular anticoagulant, hemostatic, or fibrinolytic pathways within allografts of diabetic recipients compared to allografts of non-diabetic recipients. 
901

PLATELET ACTIVITIES AND THROMBOXANE SYNTHESIS DURING EXTERNAL INSULIN PUMP TREATMENT

Z.Turk, I.Flego, G. Kerum and Ž. Metelko.

"Vuk Vrhovac" Institute, Zagreb, Croatia

The effect of metabolic control on platelet activities was studied in 11 IDDM patients during 6 months of continuous subcutaneous insulin infusion (CSil) treatment in comparison to 3 months of conventional therapy. Patients chosen for the study were free of microvascular disease. The effectiveness of intensive therapy in lowering blood glucose was reflected in a significant $\mathrm{HbA} 1 \mathrm{c}$ reduction $(8.26 \pm 1.78$ vs $6.16 \pm 0.46 \%$ $. p<0.001$ ). The accompanying improvement in ex vivo aggregation of platelets in response to two doses of agonists was only observed with the achievement of glycemia control (ADP: $20.84 \pm 4.61$ vs $14.84 \pm 3.03 \%$, $p<0.001$; arachidonic acid: $22.04 \pm 4.26$ vs $16.0 \pm 3.15 \%, p<0.01$ ). The synthesis of thromboxane- $B_{2}$ as a response to arachidonate, was lower during the CSII period $\left(\mathrm{TXB}_{2}: 415 \pm 51\right.$ vs $\left.382 \pm 46 \mu \mathrm{g} / \mathrm{l} \times 10^{6} \mathrm{plts}\right)$, but with no statistical significance. Inspite of the fact that in all patients, lipoproteins were of normal values both before and after intensified therapy (HDL-C: $1.48+0.5$ vs $1.52 \pm 0.4 \mathrm{mmol} / /$, NS: LDL-c: $3.2 \pm 1.3$ vs $2.78 \pm 1.0 \mathrm{mmol} / /, \mathrm{NS}$ ), a significant correlation was observed between the atherogenic lipoproteins and proaggregatory thromboxane $B_{2}$. Thus, in all patients, LDL-cholesterol before and during CSII showed $a$ significant correlation with $\mathrm{TXB}_{2}$ aeneration $(r=0.75, p<0.001)$, whereas at the same time its correlation with the corresponding values of HDL-cholesterol $(r=-0.77$, $p<0.001)$ was negative. Along with normal fibrinogen level pre- and post-CSII $(346 \pm 79$ vs $307 \pm 56 \mathrm{~g} / \mathrm{l})$ we found a significant association between fibrinogen and ADP-induced aggregation in pretreatment period $(=0.63, p<0.03)$, but during CSII treatment this association was not abserved. In the present study plasma fibrinogen significantly correlated with the level of glycemia $(r=0.61, p<0.002)$. Results suggested that intensive insulin treatment reduced platelet aggregation in patients without microvascular disease when strict glycemia control was maintained and indicated that changes in platelet aggregation could directly result from changes in plasma glucose concentrations

\section{2}

IN VIVO PRODUCTION OF INTERLEUKIN-6 BY HUMAN

JS Yudkin, V Mohamed-Ali, S Stanner. S Goodrick, and SW Coppack. University College, London.

Multiple cytokines induce a number of alterations in lipid metabolism which can produce hyperlipidaemia. These metabolic derangemants involve cytokines such as TNF $\alpha$, IL-I and IL-6. TNF $\alpha$ and IL- 6 have been shown to inhibit LPL activity and decrease its production in murine adipocyte cell-lines. In order to examine abdominal adipose tissue cytokine production in vivo arteriovenous balance was determined in 6 caucasian men (BMI Mean $=27.9 \pm 6.8 \mathrm{Kg} \cdot \mathrm{m}-2$; Age $47.0 \pm 2.2$ years). Blood samples were taken simultaneously from an abdominal vein, draining subcutaneous adipose tissue, and a radial artery. Abdominal vein IL-6 concentrations (Mean $8.6 \pm 4.7$; range $2.1-13.4 \mathrm{pg} . \mathrm{ml}^{-1}$ ) were consistantly greater than arterial values (Mean $1.6 \pm 0.7$; range $\left.0.8-2.6 \mathrm{pg} . \mathrm{ml}^{-1}\right) \mathrm{p}=0.01$. The levels of TNF $\alpha$ and PAI-1 activity or antigen were not significantly different between the venous and arterial samples. Levels of IL-6 was significantly correlated to BMI $(r=0.80 ; p=0.05)$, both venous $(r=0.87$; $\mathrm{p}=0.025)$ and arterial $(\mathrm{r}=0.84 ; \mathrm{p}=0.035) \mathrm{PAI}-1$ antigen, while arterial IL-6 correlated with arterial PAI-I activity $(\mathrm{r}=0.88$; $p=0.022)$ and antigen $(r=0.92 ; p=0.01)$. In conclusion, $11-6$ is produced by human adipose tissue. its levels are related to adiposity and it correlates with concentrations of PAI-1. Cytokines produced by adipose tissue may mediate metabolic effects of obesity.

\section{3}

Procoagulant profile of thrombin activation markers and low PAI-1 activity in IDDM patients

E. Kozek, J. Sieradzki; Department of Metabolic Diseases, Jagellonian University, Kraków, Poland

In diabetic patients increased activity of coagulation activators, decreased activity of coagulation inhibitors and reduced fibrinolytic activity increase the risk of hypercoagulation, which triggers a release of sensitive thrombinogenesis markers in the bloodstream. Aim of the study. The purpose of the present study was to assess thrombin activation markers, antithrombin III (AT III) and tissue plasminogen activator inhibitor (PAI-1) in patients with IDDM. Material and Methods. In 47 persons -32 diabetics $(18 \mathrm{M}, 14 \mathrm{~F}$, mean duration of IDDM $11.6+1-6.6$ years, mean age $35.28+/-9.2$ years) and 15 controls $(11 \mathrm{M}, 4 \mathrm{~F})$ we determined thrombin- antithrombin III complex (TAT), prothrombin fragments $1+2(F 1+2)$, D-Dimer with the ELISA method whereas Chromo Time System Beringwerke was used to analyze ATIII and PAl-1 activities. Results. Diabetic patients compared with controls had significantly higher concentrations of $F 1+2(\times 2.20+1-1.44$ vs. $0.77+1-0.30$ $n$ mol $/ L, p<0.001)$, and significantly lower AT III activity ( $x$ 101.54+/-20.69 vs. $123.38+/-23.58 \%, p<0.01)$. PAl activity in diabetics was significantly lower ( $x 3.12+/-0.22$ vs. $3.24+/-0.11 \%, p<0.05$ ). A significant positive correlation was found between urinary albumin excretion rate (AER) and D-Dimer concentration ( $r=0.58, p<0.001)$ and albumin/creatinine ratio and D-Dimer concentration $(r=0.48, p<0.01)$. No significant differences were seen in coagulation markers concentrations, AT III and PAI activities with respect to the presence of retinopathy, peripheral neuropathy and cardiovascular autonomic neuropathy.

Conclusions. The increased $\mathrm{F} 1+2$ concentration and the relationship between D-Dimer and AER may indicate activation of thrombinogenesis in IDDM patients and its role in the development of diabetic nephropathy. The reduced PAI-1 activity indicates that in IDDM patients fibrinolytic disonders result from changes in the activity of other than PAl elements of the fibrinolytic system.

\section{4}

FIBRINOLYTIC PARAMETERS IN RELATION TO C-PEPTIDE AND ENDOGENOUS DIGITALIS-ITE SUESTANCES IN DIABETIC SUBJECTS.

M.Mokan, E.Martinka, P.Galajda, P.Kubisz. Department of Internal Medicine $I$ and Department of Hematology Jesenıus Medical School, Martin, SLOVAKIA. INTRODUCTION: The clinical coincidence of diabetes mellitus (DM) with hypertension and hypofibrinolysis is well-known. The base of this coincidence, however, is not clear. Several recent, works have suggested importance of an increased intraceliular calcium $\left(\mathrm{Ca}^{2+}\right)$ as a possible Iink of these abnormalities. Endogenous dicitalis-like substances (EDLS) represent recently identified substances which are close to cardiac glycosides. These substances via inhibition of $\mathrm{Na}^{+}-\mathrm{K}^{+}-\mathrm{ATP}$ ase increase $\mathrm{Ca}^{2+}$ i concentrations and nay modulate various $\mathrm{Ca}^{2+}$-dependent processess (such as vasoconstriction, insuin secretion, etc.). EDIS has been found increased in DM. AIM OE STUDY: In this pilot study we were interested if there are some relationships of EDLS to fibrinolytic paraneters. SUBJECTS AND METHODS: 17 type-2 diabetic subjects (NIDDM), 7 type-1 diabetics (IDDM) and 11 healthy controls (HC). Following parameters has been measured: fasting plasma qlucose, C-peptide, immunoreactive insulin (IRI), cholesterol, triglycerides, tissue plasminogen activator (tPA), plasminogen activator inhibitor 1 (PAII), blood pressure and BDLS (which has been measured as a digoxin-like immunoactivity (DLIA)). RESULTS: Plasma levels of of $\mathrm{PAA}$ $(9.93 \pm 5.70 \mathrm{ng} / \mathrm{ml})$ and PAI-1 (45.84 $23.02 \mathrm{ng} / \mathrm{ml})$ in NIDDM subjects were significantly higher than in $\mathrm{BC}(t P A=5.34 \pm 4.42 \mathrm{ng} / \mathrm{ml} ; \mathrm{PAI}=15.92 \pm 19.44 \mathrm{ng} / \mathrm{mL})$ and IDDM $(t P A=4.75 \pm 2.55 \mathrm{ng} / \mathrm{ml} ; \quad$ PAIl $=11.31 \pm 12.11 \mathrm{ng} / \mathrm{ml})$. Both $\operatorname{tPA} \quad(\mathrm{r}=0.61, \mathrm{p}=0.00038)$ and PAI- $\quad(r=0.630 ; \quad n=0.00032)$ correlated with C-peotide. EDLS(DLIA) plasma levels in NICCM $(0.18+0.15 \mathrm{mmol} / 1)$ were significantly hiaher than in IDDM $(0.04 \pm 0.04 \mathrm{nmol} / 1)$ and non-significantly higher than in $\mathrm{HC}(0.10 \pm 0.07 \mathrm{nmol} / 1)$. In all subjects EDLS(DLIA) correlated both with C-peptide levels $(r=0.46$; $\mathrm{p}=0.0015)$, tPA $(\mathrm{r}=0.364 ; \mathrm{p}=0.049)$ and PRI1 $(\mathrm{r}=0.387 ; \mathrm{r}=0.041)$. EDLS(DIIA) did not correlate with other measured parameters: plasma glucose, blood pressure, triglycerides, cholesterol and IRI. CONCLUS10N: Our preliminary findings suggest possibility of relation of EDLS to tPA and PAI-1 in diabetic subjects. The suggest possible (contributive/modulating) role of EDLS in mechanisnis involved in athero-thrombogenesis. This hypothesis need however further investigation. 
905

ALTERED PROPERTIES OF FIBRIN GEL STRUCTURE IN PATIENTS WITH IDDM

G. Jörneskog, N. Egberg, B. Fagrell, K. Fatah, B. Hessel,

H. Johnsson, K. Brismar and M. Blomback. Department of Internal Medicine, Department of laboratory Medicine, Division of Clinical Chemistry and Blood Coagulation, and Department of Endocrinology och Diabetology, Karolinska Hospital, Stockholm, Sweden.

High plasma fibrinogen levels are associated with valscular complications in the general population. Fibrin, the structural element in a clot, is derived from fibrinogen by activation of thrombin. An abnormal fibrin network has been demonstrated in patients with myocardial infarction and in diabetic patients during poor metabolic control. In the present study the properties of fibrin gel structure were investigated in 20 patients with IDDM: 10 patients without (age: $30 \pm 8$; diabetes duration: $7 \pm 6$ years), and 10 patients (age: $44 \pm 7$; diabetes duration: $27 \pm 9$ years) with microangiopathy. 15 healthy subjects served as controls (age: 4()$\pm 8$ years). The glucosylated hemoglobin level (HbAlc) was elevated $(p<0.001)$ in the patients: $6.5 \pm 1.5 \%$ in diabetic patients without, and $7.1 \pm 1.0 \%$ in diabetic patients with microangiopathy. C-reacrive protein and plasma fibrinogen were similar, as compared to healthy controls. The properties of the fibrin gel structure, i e the permeability coefficient $(\mathrm{Ks})$ and the fiber mass length ratio $(\mu)$ formed in recalcified plasma on addition of thrombin were investigated. $\mathrm{K} s$ was decreased in the diabetic patients, with $\left(6.5 \pm 2.0 \mathrm{~cm}^{2} ; \mathrm{p}<0.01\right)$ and without microangiopathy $\left(6.5 \pm 2.7 \mathrm{~cm}^{2} ; \mathrm{p}<0.05\right)$, as compared to healthy controls $(10.0 \pm 3.4$ $\left.\mathrm{cm}^{2}\right)$, while $\mu$ was not significantly $(p=0.14)$ altered. The results indicate a lower fibrin gel porosity in patients with IDDM, despite normal plasma fibrinogen and irrespective of microangiopathy. The abnormal fibrin gel structure may be due to long-tern hyperglycemia and may be of importance for the development of angiopathy in diabetic patients.

\section{6}

EFFECT OF HYPERINSULINEMIC CLAMP ON RENAL FUNCTION AND $\mathrm{NO}_{2}^{-} / \mathrm{NO}_{3}{ }^{-}$EXCRETION IN NIDDM.

R. Komers, T. Pelikánová and L. Kazdová Institute for Clinical and Experimental Medicine, Prague, Czech Republic

To explore the role of nitric oxide (NO) in renal changes induced by hyperinsulinemia. the glomerular filtration rate (GFR). renal sodium handling and plasma and urinary $\mathrm{NO}_{2}-\mathrm{NO}_{3}$ - levels $\left(\mathrm{NO}_{\mathrm{X}}\right)$, a marker of systemic and renal activity of NO, were assessed before and during euglycaemic hyperinsulinemic clamp (EHC) in 8 male normotensive patients with NIDDM (D: age $47 \pm 1$ years: BMI $26 \pm 1$ ) and 10 healthy male volunteers (C: average age $34 \pm 2$ years; BMI $24 \pm 1$ ). After completion of basal measurements an infusion of insulin at a rate of $1 \mathrm{mU} / \mathrm{kg}$ bwt $\mathrm{min}$ was started with a variable amount of $15 \%$ glucose to achieve constant blood glucose levels $4.5 \mathrm{mmol} / 1 \pm 5 \%$. The urine was collected throughout timed study periods $(-120$ minutes) with blood samples being withdrawn at midtime of each period. Mean arterial blood pressure (MAP) was monitored throughout the study, GFR was assessed as a creatinine clearance and $\mathrm{NO}_{\mathrm{x}}$ levels were measured using Griesse method. Basal values vs data for the 1 st clamp period are displayed. MAP remained unchanged in both groups during the EHC when compared to basal values (C: $73 \pm 3$ vs $73 \pm 3 \mathrm{mmHg}$; n.s., D: $82 \pm 4$ vs $79 \pm 4 \mathrm{mmHg}$; n.s.). Basal GFR was similar in both groups. An increase in GFR in response to the EHC was observed in $C(110 \pm 9$ vs $157 \pm 8 \mathrm{ml} / \mathrm{min} ; \mathrm{p}<0.01)$ but not in the $D$ group ( $112 \pm 16$ vs $116 \pm 10 \mathrm{~m} / \mathrm{min}$; n.s. vs basal, $\mathrm{p}<0.01$ vs C). Plasma NOr values during the $\mathrm{EHC}$ did not change in both groups. however. basal and EHC plasma NO $x$ were significantly lower in the $D$ group than in C (basal: $18 \pm 3$ vs $37 \pm 4 \mu \mathrm{mol} / 1 ; \mathrm{p}<0.05$, EHC: $18 \pm 2$ vs $35 \pm 4 \mu \mathrm{mol} / 1: \mathrm{p}<0.01)$. EHC resulted in a similar rise in urinary $\mathrm{NO}_{\mathrm{X}}$ both in $\mathrm{C} 10.10 \pm 0.02$ vs $0.43 \pm 0.12 \mu \mathrm{mol} / \mathrm{min}: \mathrm{p}<0.05)$ and D subjects $(0.14 \pm 0.04$ vs $0.51 \pm 0.15$ $\mu \mathrm{mol} / \mathrm{min} ; \mathrm{p}<0.05$ ). Unlike to D. C subjects responded to the EHC with marked natriuresis $(p<0.05)$. Present data show that normogiycaemic hyperinsulinemia is in healthy subjects associated with increases in GFR. natriuresis and excretion of NO metabolites. Despite similar rise in urinary excretion of $\mathrm{NO}_{\mathrm{x}}$. the $\mathrm{D}$ patients demonstrated blunted renal response to the EHC. This suggests that NIDDM is a state of decreased renal responsiveness to the renal vasodilatory and natriuretic actions of NO.

\section{7}

REVERSAL BY L-ARGININE, BUT NOT D-ARGININE, OF THE ACUTE VASCULAR EFFECTS OF HYPERGLYCEMIA IN MAN.

R.Marfella, R.Acampora, G.Verrazzo, J.Coppola, P. Ziccardi, F.Nappo, R.Giunta, D.Giugliano, Department of Geriatrics, Second University of Naples, Italy

Acute hypergiycemia increases vascular tone in normal subjects. To evaluate whether the vascular effects of hyperglycemia are related to reduced nitric oxide availability, acute hyperglycemia $(15 \mathrm{mmol} / \mathrm{l})$ was induced in 12 young healthy subjects with the aid of an artificial pancreas. Systolic $(+8+3 \mathrm{mmHg}$, meantSD) and diastolic $(+5+2 \mathrm{mmHg})$ blood pressure, heart rate $(+6+3$ $\mathrm{b} / \mathrm{min}$ ) and plasma catecholamines (norepinephrine: $+29+12$ $\mathrm{ng} / 1$ ) showed significant increases $(\mathrm{p}<0.05-0.01)$ during the clamp, while. leg blood flow decreased $(-91+40$ $\mathrm{mI} / \mathrm{min}, \mathrm{p}<0.05)$. Platelet aggregation $(+9+4 \%)$ and blood viscosity $(+0.7+0.2 \mathrm{cp})$ also showed significant increments. The infusion of L-arginine ( $1 \mathrm{~g} / \mathrm{min})$, the natural precursor of nitric oxide, but not D-arginine, in the last $30 \mathrm{~min}$ of the clamp completely reversed all vascular changes brought about by hyperglycemia. Infusion of $\mathrm{N}^{\mathrm{G}}$-monomethyl-L-arginine (L-NMMA), an inhibitor of nitric oxide synthesis, produced vascular changes (increase of blood pressure, blood viscosity and platelet aggregation) similar to those occurring during hyperglycemia; heart rate and catecholamines decreased during L-NMMA, presumably as a consequence of baroreflex activation. Acute hyperglycemia may reduce nitric oxide availability in normal man.

\section{8}

IS A GLOBAL ENDOTHELIAL DYSFUNCTION PRESENT IN INSULIN DEPENDENT DIABETES MELLITUS (IDDM)?

Huvers FC. de Haan CHA, Houben AJHM, Hamulyak K, Leurs P, Wolffenbuttel BHR, Schaper NC; Dept. of Int. Medicine, University Hospital Maastricht, The Netherlands.

Introduction: Various studies reported an impaired production of endothelial derived nitric oxide (EDNO) and an increased tissue factor pathway inhibitor (TFPI) activity in IDDM, suggesting a "global" endothelial dysfunction. The aim of the present study was to determine the basal and stimulated EDNO production in relation to TFPI activity in 35 normotensive IDDM patients with a minimal diabetes duration of 10 yrs. Patients and methods: 11 patients without complications (C-), 8M/3F, age 32 (25/45, median and interquartile ranges); 11 patients with (pre)proliferative retinopathy $(\mathbf{R}+), 10 \mathrm{M} / 1 \mathrm{~F}$, age $37(26 / 43) ; 13$ patients with microalbuminuria $(\mathbf{M}+), 10 \mathrm{M} / 3 \mathrm{~F}$, age $38(28 / 40)$ yrs. Also were studied 12 healthy volunteers (HV), 10M/2F, age 31 (33/47) yrs. The effects on forearm blood flow of the following drugs, stepwise infused into the brachial artery after an overnight fast under euglycemic conditions were measured by strain gauge plethysmography: sodium nitroprusside (SNIP) $1,3,10 \mu \mathrm{g} / \mathrm{min}$, acetylcholine $(\mathrm{ACh}) 3,10,30 \mu \mathrm{g} / \mathrm{min}$ and on a separate day L-NMMA $1,2,8$ $\mu \mathrm{mol} / \mathrm{min}$. TFPI-activity was measured by a chromogenic substrate assay (Sandset). Results: No differences were observed in the response to ACh, SNIP, L-NMMA between any group. Due to lack of space only the reduction of the FBF-index (FBF infused/FBF control arm) to L-NMMA, $8 \mu \mathrm{mol} / \mathrm{min}$, is shown C-: $26(21-52) \%, \mathrm{R}+:$ 29(15-37)\%, M+: 43(32-57)\%, HV: 39(20-53)\%. TFPI was, in comparisson to HV, increased in all patient groups $(\mathrm{p}=0.04), \mathrm{C}$-: 1 $13(108-125) \%, \mathrm{R}+:$ 109(105-122)\%, M+: 118(114-127)\%, HV: $102(97$ $107) \%$. Conclusions: in contrast to earlier studies basal and stimulated EDNO production were not decreased in long-term IDDM patients with microvascular complications. However, TFPI-activity was increased relative to controls. These data suggest that during euglycemic conditions no global endothelial dysfunction is present in IDDM patients with and without microvascular complications. 
909

METAL CHELATOR AND FREE RADICAL SCAVENGER TREATMENTS PREVENT CHRONIC AORTA RELAXATION DEFECTS IN DIABETIC RATS. A. Keegan, M.A. Cotter, V. Archibald and N.E. Cameron. Biomedical Sciences, University of Aberdeen, Scotland UK

Defective nitric oxide (NO) mediated endothelium-derived relaxation contributes to impaired perfusion in tissues such as peripheral nerve in diabetic rats. NO action may be impaired by the elevated oxygen free radical activity in diabetes. Abnormal endothelium-dependent relaxation is prevented by aldose reductase inhibition, probably by improving glutathione redox cycle free radical scavenging. Glucose autoxidation, catalyzed by transition metal ions, is a potentially important source of free radicals in diabetes. The aim was to determine whether treatment with the transition metal chelator, trientine, (TRI, $20 \mathrm{mg} \mathrm{kg}^{-1}$ day $^{-1}$ ), the lipophilic free radical scavenger, butylated hydroxytoluene (BHT, 1\% diet), or the hydrophilic glutathione precursor N-acetyl-L-cysteine (ACYS, $250 \mathrm{mg} \mathrm{kg}^{-1}$ day $^{-1}$ ) prevented chronic endothelial dysfunction in streptozotocin-diabetic rat aortas. Two months of diabetes caused a $24.1 \pm 4.6 \%$ ( \pm SEM; $p<0.01$ ) reduction in endotheliumdependent relaxation to acetylcholine for aortas precontracted by phenylephrine in vitro. This was unaffected by the concentration of glucose in the bathing fluid ( 5.5 or $40 \mathrm{mM}$ ) or by 30 min preincubation with the NO substrate, L-arginine $(1 \mathrm{mM})$, suggesting a chronic defect. The relaxation abnormality was prevented $(\mathrm{p}<0.01)$ by chronic (but not acute) treatment with BHT, ACYS and TRI, nonsignificant deficits of $5.7 \pm 4.5 \%, 6.2 \pm 3.4 \%$, and $7.1 \pm 3.2 \%$ respectively remaining. Diabetes and treatment did not affect endothelium-independent relaxation by nitroglycerine. Thus, free radical activity resulting from autoxidative glycosylation / glycoxidation reactions is a probable cause of chronic endothelial dysfunction in experimental diabetes.

\section{1}

\section{ELEVATED CIRCULATING P-SELECTIN IN INSULIN DEPENDENT} DIABETES MELLITUS

Peter Fasching ${ }^{2}$, Bernd Jilma ${ }^{1}$, Christine Ruthner ${ }^{1}$, Anna Rumplmayr ${ }^{1}$, Sabine Ruzicka ${ }^{2}$, Stylianos Kapiotis ${ }^{3}$, Oswald F. Wagner ${ }^{3}$, Hans-Georg Eichler ${ }^{1}$

Departments of ${ }^{1}$ Clinical Pharmacology, ${ }^{2}$ Internal Medicine III, Div. of Endocrinology and Metabolism, ${ }^{3}$ Clinical Institute of Medical and Chemical Laboratory Diagnostics

Based on findings showing increased P-selectin expression on platelets and choroidal microvessels of patients with insulin dependent diabetes mellitus (IDDM), we hypothesized that plasma concentrations of circulating (c) Pselectin may be increased in these patients. To alanyse that hypothesis plasma levels of $\mathrm{CP}$-selectin were compared between non-smoking IDDM's on an intensitied insulin therapy, and healthy control subjects, $N=42$ each. The study was designed prospectivly and analyst-blinded with subjects beeing matched individually for sex, age and body mass index. Plasma levels of $\mathrm{CP}$-selectin and of von Willebrand antigen ( $\mathrm{VWf}-\mathrm{Ag}$ ) were determined by enzyme linked immunoassays. Pairs available for intergroup comparison displayed. Median plasma concentrations of $\mathrm{cP}$-selectin in IDDM's (285ng/mL; interquartile range: 233-372) averaging $21 \%$ above those of health controls subjects $(236 \mathrm{ng} / \mathrm{mL}$; interquartile range: $175-296$; $p=0.004)$. In parallel, median plasma levels of $\mathrm{WWF}$-Ag were $10 \%$ higher in IDDM's (96 U/dL; interquartile range: $82-127)$ than in controls $(87 \mathrm{ng} / \mathrm{mL}$; interquartile range: $70-104 ; p=0.025$ ). No correlation was observed between plasma concentrations of CP-selectin and VWF-Ag levels in either group $(\mathrm{p}>0.05)$.

In conclusion, the obtained results of increased $\mathrm{cP}$-selectin levels in patients with IDDM are consistent with a role of P-selectin in the pathogenesis of IDDM associated makrovascular complications.
910

INCREASE IN SERUM LEVELS OF ADHESION GLYCOPROTEINS IN NIDDM EFFECT OF INTENSIVE INSULIN TREATMENT.

J.P Albertini, P. Valensi, B. Lormeau, J.R Attali, S. Gronowski, F. Ferrière and L. Gattegno. Cell Biology, Avicenne Hospital, Bobigny and Diabetology, Jean Verdier Hospital, Bondy. France.

An increase in the serum levels of adhesion glycoproteins has been reported in patients with NIDDM. The aim was to determine the serum levels of selectin E, ICAM-1 (Inter Cellular Adhesion Molecule-1) and VCAM-1 (Vascular Cell Adhesion Molecule-1) in eight poorly controlled patients with NIDDM and the effects of 15 days of continuous subcutaneous insulin treatment. Before insulin treatment (day 0 ), selectin $\mathrm{E}$ and VCAM-1 were significantly higher than in the controls ( $73 \pm 29 \mathrm{vs}$ $46 \pm 19 \mathrm{ng} / \mathrm{ml}, \mathrm{p}<0.01$ and $1086 \pm 660$ vs $527 \pm 126 \mathrm{ng} / \mathrm{ml}, \mathrm{p}<$ 0.007 , respectively), whereas ICAM-1 was not significantly different $(266 \pm 61$ vs $236 \pm 66 \mathrm{ng} / \mathrm{ml})$. Within a few days of insulin treatment glycemia improved sharply. After 15 davs of treatment resulting in normoglycemia, selectin $E$ levels returned to normal $(51 \pm 17 \mathrm{ng} / \mathrm{ml})$ and VCAM-1 levels decreased to 668 $\pm 180 \mathrm{ng} / \mathrm{ml}$. In the entire series of blood samples obtained at days 0,8 and 15 , selectin $E$ correlated significantly with triglycerides $(r=0.51, p<0.01)$ and lipoparticules $\mathrm{A} 1(\mathrm{r}=0.41$, $\mathrm{p}<0.04)$ but not with glycemia or serum $\mathrm{C}$ peptide. These data suggest that in poorly-controlled diabetic patients 1) serum levels of selectin $\mathbf{E}$ and VCAM-1, are increased and may return to normal or be strongly improved after short-term improvement of glycemic control, possibly owing to an improvement in the lipid parameters, 2) assaying selectin $E$ makes it possible to detect endothelium activation and; to follow its reversal with euglycemia.

\section{2}

DETERMINANTS OF BLOOD VISCOSITY AFTER PANCREAS AND KIDNEY TRANSPLANTATION

A.J Reininger 1 A.C. Mellinghoff 2 KD. Hepp 2 and R Landgraf 3

${ }^{1}$ Dep. of Anatomy. Technische Universität, Biedersteinerstr. 29, 80802 München, 23 rd Med. Dep. and Diabetes Center Bogenhausen, ${ }^{3}$ Med. Klinik Innenstadt, University of Munich, Munich, Germany

Simultaneous pancreas and kidney transplantation (PKT) is associated with a deterioration of hemorheology. We investigated the determinants of plasma and whole blood viscosity after PKT $(n=49)$, in IDDM $(n=26)$ and in healthy controls $(n=24)$. Patients after PKT were subdivided due to their graft function (intact pancreas and kidney graft, $n=26$ : pancreas rejected, intact kidney graft, $n=23$ ). We examined the correlations of total serum protein albumin, fibrinogen, $\alpha_{2}$-macroglobulin, total cholesterol, LDL-cholesterol, HDL-cholesterol and triglycerides with plasma and whole blood viscosity measured at a continuous shear range of $600-0.2 \mathrm{sec}^{-1}$ with a rotational viscometer (Haake, Germany). Kruscal analysis was used for regression analysis, the level of significance was set to $p<0.05$. Total protein was strongly associated with plasma viscosity in all examined groups $(r>0.5$, $p<0.03$ ), it determined whole blood viscosity over the whole shear range in IDDM, but only at high shear rates after PKT $\left(\geq 100 \mathrm{sec}^{-1}\right)$. The strong association of albumin and whole blood viscosity in IDDM and in healthy controls (shear rates $\geq 10 \mathrm{sec}^{-1}$ ) was not found after PKT. Fibrinogen correlated with plasma and whole blood viscosity $\left(\geq 25 \mathrm{sec}^{-1}\right)$ after PKT $(p<0.03)$. $\alpha$-macroglobulin correlated with plasma and high shear blood viscosity after PKT only after pancreas rejection, no correlation was found after successful PKT. Total cholesterol and low shear blood viscosity correlated positively in successfully transplanted patients $(r>0.44)$, but negatively after pancreas rejection ( $>-0.44$ ). No correlation was found in IDDM, a positive association was found in healthy controls. Likewise LDL-cholesterol correlated negatively (after pancreas rejection) or positively (healthy controls) with low shear blood viscosity $(p<0.03)$. HDL-cholesterol was negatively associated with high shear blood viscosity in all groups $(p<0.05)$, except after successful PKT where no association was found. Triglycerides did not contribute significantly to blood viscosity in the examined groups. The metabolic alterations after PKT influence plasma proteins, lipids and corpuscular elements of blood with regard to their effect on rheology 


\section{3}

\section{ADVANCED GLYCOSYLATION END PRODUCTS (AGE) HAVE NO INFLUENCE ON PLASMA VISCOSITY}

A.C. Mellinghoff ${ }^{1}$, B. Liesenfeld ${ }^{1}$, A.J. Reininger ${ }^{2}$, R. Landgraf ${ }^{3}, R$ Renner ${ }^{1}$ and K.D. Hepp ${ }^{1}{ }^{1}$ Third Med. Dep. and Diabetes Center Bogenhausen, Englschalkinger Straße 77, 81925 München, ${ }^{2}$ Dep. of Anatomy, Technische Universität, München, ${ }^{3}$ Med. Klinik Innenstadt, Universität München, Germany

Plasma viscosity is mainly determined by large non-spherical proteins. In IDDM plasma viscosity increases with deterioration of diabetic control. Since protein glycation and formation of AGEs alters the structural and functional properties of proteins, AGEs might influence the rheological properties of plasma proteins. Therefore, we investigated the influence of plasma-AGE on plasma viscosity in 34 normoalbuminuric diabetic patients (17 IDDM, 17 NIDDM). In an additional experiment $6 \mathrm{ml}$ plasma of nine healthy volunteers were incubated under sterile conditions for 14 days at $37^{\circ} \mathrm{C}$ in the presence of 5.2 and $32.9 \mathrm{mmol} / /$ glucose. Plasma viscosity was measured at $250 \mathrm{sec}^{-1}$ with a rotational viscometer (Haake, Germany), plasma-AGE was analysed in competitive format with monoclonal 4G9 after digestion with proteinase $\mathrm{K}$ in a final dilution of 1:30 (Alteon inc., Ramsey, New Jersey, USA). Pearson correlation for two-sided tests and t-test for paired samples were used. In diabetic patients plasma-AGE was not correlated with plasma viscosity. Plasma-AGE levels in healthy controls $(246 \pm 37 \mathrm{U} / \mathrm{ml}$, mean $\pm \mathrm{SD})$ were raised significantly $(p<0.001)$ after incubation at $37^{\circ} \mathrm{C}(392 \pm 57 \mathrm{U} / \mathrm{ml}$ and $552 \pm 58 \mathrm{U} / \mathrm{ml}$, resp.), no difference, however, was found in plasmas viscosity (pre-incubation: $1.25 \pm 0.04$ mPas, post-incubation: $1.23 \pm 0.03$ and $1.24 \pm 0.03$, resp.). There is no influence of plasma-AGE on plasma viscosity.

\section{4}

RHEOLOGIC AND METABOLIC FACTORS MAY INFLUENCE THE VASCULAR RESPONSE TO SYMPATHETIC STIMULI IN NIDDM. O. Smagghue, P. Valensi, P. Velayoudon, J. Pariès, and J.R Attali. Dept. of Diabetology, Jean Verdier Hospital, Bondy. France.

We have previously shown the importance of the peripheral vasoconstrictive response measured by laser doppler in the evaluation of sympathetic activity. The present work was designed to study the factors affecting microcirculatory cutaneous blood flow (CBF) both at rest and during three tests which activate the sympathetic system (deep breathing, lying to standing and Valsalva), in particular the influence of rheologic and metabolic disorders. Forty-two NIDD's and 14 age-matched controls were investigated. The standard deviation of $\mathrm{CBF}$ at rest correlated significantly with the decrease in CBF during the lying to standing and Valsalva tests in the controls and during the three tests in the NIDD's. In the latter, mean resting CBF correlated negatively with the erythrocyte rigidity index (measured by hemorheometer) and positively with $\mathrm{HbA1c}$ (multivariate analysis : adjusted $\mathrm{r}^{2}=0.488$ ). During the deep breathing test the downward slope of $\mathrm{CBF}$ correlated negatively with the erythrocyte rigidity index $(p=0.023$ ) and during the lying to standing and Valsalva tests it correlated positively with plasma cholesterol $(\mathrm{p}=0.050)$ and triglycerides $(\mathrm{p}$ $=0.015)$ respectively. Neither basal CBF nor its response during the three tests correlated with microangiopathic complications or with peripheral or cardiac autonomic neuropathy. This study suggests that in NIDD's 1) spontaneous changes in CBF at rest are under sympathetic control, 2) rheologic and metabolic factors may influence CBF at rest and the amplitude of vasomotor reflexes.

\section{5}

Yoninvasive Determination Of Peripheral Endothelial Dysfunetion In Diabetic Patients. Is There A Difference Between IDDM And NIDDM?

Y.-D. Enderle. M.Pfohl. J. Günert. K. Lilienfein. G. Demirci. N. Benda, R, $-\mathrm{M}$ Schmülling and H.-U. Häring. Dept. IV of Internal Medicine. Univ. of Tuebingen. Otfried-Mueller Straße 10. D-72076 Tuebingen. Germany

Endothelial dystunction (ED) is thought to be an early step in the development of atherosclerosis. To study ED non-invasively we adapted the method described by Celermajer (1992) using high resolution uitrasound $413 \mathrm{MHZ}$ linear aाTay, axial resolution of $0.12 \mathrm{~mm}$ ). Changes in the lumen diameter (LD) of the brachial artery were measured under the following conditions: $L D 1=$ rest. $L D 2=$ postocclusive (endothelial dependent vasodilatation), LD3 $=400 \mathrm{~kg}$ nitroglvcerine p.o. (endothelial independent vasodilatation). We examined 29 NIDDM patients: sex $12 \mathrm{~m}, 17 \mathrm{f}$ : age $0.2 \pm 8.9 \mathrm{y}:$ BMI $30.0 \pm 7.6 \mathrm{~kg} / \mathrm{m}^{2} ; \mathrm{HbAl}_{\mathrm{c}} 8.5 \pm 1.6 \%$; duration of diabetes $8.9 \pm 7.4 \mathrm{y}$; bloodglucose $152 \pm 63 \mathrm{mg} / \mathrm{dl}$. 10 IDDM patients: sex $4 \mathrm{~m}$. $6 \mathrm{f}$; age $+1.0 \pm 14.2 \mathrm{y}$; BMI $25.5 \pm 5.5 \mathrm{~kg} / \mathrm{m}^{2}$ : duration of diabetes $21.3 \pm 6.2 \mathrm{y} ;$ bloodglucose $225 \pm 35 \mathrm{mg} / \mathrm{dl} ; \mathrm{HbAl}$ $8.1 \pm 1.2 \%$ and compared them with 51 healthy subjects: sex $24 \mathrm{~m} .27 \mathrm{f}$; age $28.7 \pm 8.9 \mathrm{y}$; BMI $21.9 \pm 2.5 \mathrm{~kg} / \mathrm{m}^{2}$; no history of cardiovascular risk factcrs. Values are given as means \pm SEM. significance was tested by means of Tukey-Kramer Test $(*), \alpha=0.05$ :

\begin{tabular}{|c|c|c|c|c|c|c|}
\hline & LD1 (mm) & LD2 (mm) & LD3 $(\mathrm{mm})$ & $\mathrm{LD}(2-1)(\mathrm{mm})$ & LD $(3-1)(\mathrm{mm}$ & \\
\hline NDDM & $3.93 \pm 0.15$ & $4.05 \pm 0.14$ & $4.43 \pm 0.15$ & $0.12 \pm 0.04$ & $0.47 \pm 0.05-$ & \\
\hline DODM & $3.43 \pm 0.15$ & $3.75 \pm 0 ! 1$ & $4.04 \div 0.21$ & $0.32+0.067$ & $0.61 \pm 0.09$ & \% \\
\hline Healthy & $3.60 \pm 0.10$ & $3.90 \pm 0.10$ & $4.29 \pm 0.10$ & $0.31 \pm 0.03$ & $0.71 \pm 0.03$ & \\
\hline
\end{tabular}

\begin{tabular}{l|l|l|l|l|l|l} 
Endothelial dependent vasodilatation is almost lost in NIDDM, while IDDM patients & $0.71 \pm 0.03$ \\
\hline End
\end{tabular} do not show any impaiment of endothelial dependent or independent vasodilatation. This indicates that the endothelial dysfinction in the NIDDM patients is at least in parts due to other factors besides hyperglycemia.

\section{6}

INSULIN PUMP TREATMENT (CSII) IMPROVE THE METABOLIC CONTROL, HEMOSTATIC AND FIBRINOLYTIC SYSTEM IN IDDM I. Schulze, T. Lindner, W. Leonhardt, H. Rietzsch, G. Siegert and M. Hanefeld. Medical Clinic III, Inst. for Metabolic Research and Inst. of Clinical Chemistry, Medical Faculty of TU Dresden. Fetscherstr. 74, 01307 Dresden, Germany Diabetes mellitus is a complex metabolic disorder and leads to typical long-term complications by microcirculatory disturbances. Does a near physiological insulin application by Continous Subcutaneous Insulin Infusion (CSII) improve the metabolic control, lipid metabolism, microcirculation, hemostatic and fibrinolytic system in comparison with an optimal Intensified Conventional Insulin Therapy (ICT)? methods: insulin therapy of 19 male IDDM was changed from ICT to CSII, investigations on 3 days (before, after 4 and after 12 weeks CSII), at 3 times $(8$ a. m., 2 p. m., 9 p. m. $)$ microcirculation, carbohydrate and lipid metabolism, hemostatic and fibrinolytic system, bloodflow parameters in the retina by scanning-laser ophtalmography, selected results: decrease of $\mathrm{HbA}_{1 \mathrm{C}}(8,2 \pm 1,4 \%$ before and $6,9 \pm 1,1 \%$ after 12 weeks CSII), insulin/d (from 61,8 19,1 I. U. to $42,2 \pm 9,7$ I. U.), plasma viscosity (from $1,24 \pm 0,08 \mathrm{mPa}^{*} \mathrm{~s}$ to $1,21 \pm 0,05 \mathrm{mPa}^{*} \mathrm{~s}$ ), fibrinogen (from $2,8 \pm 0,7 \mathrm{~g} / \mathrm{l}$ to $2,5 \pm 0,6 \mathrm{~g} / \mathrm{l}$ ), WWFr: Ag (from $136,6 \pm 41,5 \%$ to $129,3 \pm 33,4 \%$ ). PAI-1 (from $102,4 \pm 82,2 \mathrm{ng} / \mathrm{ml}$ to $59,9 \pm 32,8 \mathrm{ng} / \mathrm{ml}$ ), VLDL-cholesterol (from $0,58 \pm 0,56$ $\mathrm{mmol} / \mathrm{l}$ to $0,33 \pm 0,29 \mathrm{mmol} / \mathrm{l}$ ), triglycerides (from $1,18 \pm 0,46 \mathrm{mmol} / \mathrm{l}$ to $0,85 \pm 0,35 \mathrm{mmol} / \mathrm{l}$ ); increase of $\mathrm{HDL}$-cholesterol (from 1,08 $\pm 0,21 \mathrm{mmol} / \mathrm{l}$ to $1,26 \pm 0,30 \mathrm{mmol} / \mathrm{l}$ ); erythrocyte deformability, antiplasmines, tPA-ag, tPAactivity, LDL-cholesterol, other parameters remained unchanged conclusions: in comparison with an optimal ICT the CSII improves the metabolic control with less insulin. This and other facts suggest the hypothesis that there is an exogeninduced insulin resistance under ICT. The CSII decreases or eliminates the resistance. Improvements in rheologic, coagulation and lipid parameters showed the tendency of an improvement in microcirculation. Die CSII decreases the prethrombotic state of the diabetic. Long-term benefits in prevention of late complications may follow from these results. 


\section{Eye Complications}

\section{7}

HbAIC AND THE RISK OF PROGRESSION OF RETINOPATHY $N$ PATIENTS WITH IDDM. TEN YEARS RETROSPECTIVE ANALYSIS K. Irsigler, C. Willinger, C. Gurdet, D. Rabensteiner and "T. Waldhör L.Boltzmann Inst. for Metab. Dis. and Nutrition, City Hospital Vienna-Lainz \& Dept. of Epidemiology, University Cancer Res. Inst., Vienna, Austria. HoAlc predicts the incidence and progression of diabetic retinopathy (DRP) Current treatment strategies do not allow full normalization of HbAlc values. The evaluation of a threshold value for $\mathrm{HbAlc}$ in patients with IDDM above which the risk of retinopathy increases greatly would be helpful for definition of targets for patients and clinicians. From a group of more than 600 IDDM patients who are in continuous surveillance in our outpatient department fundoscopic examinations (FK ${ }_{50}$ ZEISS) were done at least in yearly intervals since 1984 by the same observer, one to four HbAlc determinations (HPLC method) were documented since 1984 per year. DRP was documented according to a modified Arliehouse classification. Three categories were used for evaluation: mild DRP (none ore only microaneurysms + retinal hemorrhages), moderate DRP (background DRP + hard and soft exsudates, venous loops and intraretinal microvascular abnormalities) proliferative DRP. For statistical analysis means of all annual $\mathrm{HbAlc}$ values were used. diabetes duration was calculated from the tenth year of life (postpubertal duration of diabetes). Evaluation is completed now for 470 patients, for presentation more than 600 patients will be evaluated. Patients were subdivided into tertiles based on $\mathrm{HbAlc}$ values in $1984-90(-7.5 \%,-8.3 \%,-12.5 \%)$. Tertiles of diabetes duration (DD) were $(-21,-31,-53$ years). To control for the effect of duration of diabetes and degree of hyperglycemia odds ratios for progression to moderate and/or proliferative DRP were calculated. Patients who had diabetes 21 years or less with only mild DRP were used as a reference point for calculating the odds ratios for other combinations of $\mathrm{HbA} 1 \mathrm{C}$ values and $\mathrm{DD}$.

\begin{tabular}{|c|c|c|c|}
\hline$D D / H b / I c \longrightarrow$ & $-7.5 \%$ & $-8.3 \%$ & $-12.5 \%$ \\
\hline-21 years & $1(n=57)$ & $0.8(n=31)$ & $3.4(n=51)$ \\
\hline -3lyears & $2.0(n=52)$ & $3.8(n=64)$ & $14.3(n=55)$ \\
\hline-53 years & $6.4(n=43)$ & $6.2(n=61)$ & $6.2(n=55)$ \\
\hline
\end{tabular}

The relative risk for progression from mild to any more severe form of DRP is obviously increased with $\mathrm{HbAlc}$ exceeding $8.3 \%$ with $\mathrm{DD}$ less than 31 years

\section{9}

RISK FACTORS FOR THE PROGRESSION OF DIABETIC RETINOPATHY AMONG INSULIN-DEPENDENT DIABETIC PATIENTS: A 5-YEAR FOLLOW-UP V. PANKIV. Central Regional Hospital, Kolomyja, Ukraine

The aim of the study was to assess the incidence and progression of diabetic retinopathy and explore risk factors associated with them among insulin-dependent diabetes mellitus (IDDM) patients. A total of 942 IDDM subjects aged $<40$ were recruited from 21 primary health care centres of Ukrainian Carpathians in 1990 and followed up for 5 years. Their ocular fundi were clearly visible by ophthalmoscopy and the status of diabetic retinopathy could be graded. A structured questionnaire interview was conducted to collect basic data. Overnight fasting venous blood was collected every year to measure the levels of glucose, glycosylated haemoglobin $\left(\mathrm{HbA}_{10}\right)$, cholesterol and high density lipoprotein cholesterol. Among the 753 subjects who had no retinopathy initially, 138 subjects developed retinopathy 5 years later giving a 5-year cumulative incidence of $18.3 \%$. Of the 178 subjects initially with background or preproliferative retinopathy, evidence of deterioration developed in 44 subjects. The cumulative incidence of progression was $24.7 \% .11$ subjects developed proliferative retinopathy giving a cumulative incidence of progression to proliferative retiropathy of $6.2 \%$. The univariate analysis disclosed that the development of retinopathy was correlated with mean fasting blood glucose and $\mathrm{HbA}_{10}$, diabetes duration and residential area. The progression of retinopathy correlated with mean fasting blood glucose and proteinuria, and the progression to proliferative retinopathy correlated with mean fasting blood glucose. Stepwise logistic regression analysis revealed that mean fasting blood glucose and $\mathrm{HbA}_{1 \mathrm{~g}}$ were the significant risk factors related to the development of retinopathy. Diabetic control assessed by mean fasting blood glucose or $\mathrm{HbA}_{10}$ was the significant risk factor correlated with the incidence and progression of retinopathy.

\section{8}

PROSPECTIVE EPIDEMIOLOGICAL MONITORING OF RETINOPATHY IN THE COHORT OF NIDDM FROM DEFINED POPULATION

P. Luźniak, A. Czech and J. Tatoń. Department of Internal Medicine and Diabetology, Warsaw Medical School, 8 Kondratowicza Str., Warsaw, Poland.

Monitoring of epidemiological determinants of retinopathy (RD) constitutes the essential component of any RD control programme. It applies particularly to the NIDDM - condition very often less strictly controlled. For this reason the epidemiological monitoring indices were studied in the cohort of 1334 NIDDM representing all subjects registered in regional diabetes care center and living in the well defined city population of 160000 . During the 3-years study period prevalence and incidence of different types of $\mathrm{RD}$, identification of specific for RD risk profile in NIDDM and correlation between the prevalence of RD and risk factors under study were determined. The following risk factors of RD were under study: age, sex, NIDDM duration, BMI, plasma cholesterol, triglyceride and creatinine levels. fasting and postprandial glycemia, proteinuria, blood pressure and type of hypglycemic treatment. Total prevalence of any type of RD was $31.4 \%$, of proliferative type was $1.3 \%$ of NIDDM subjects. It equals 262 cases of RD per 100000 of general population. The incidence of all RD types was $3.9 \%$ and of proliferative $\mathrm{RD} 0.6 \%$ annually. It equals annually 67 new cases of RD per 100000 of general population. The differences in NIDDM duration, fasting and postprandial glycemia and 24-hour proteinuria between subjects with $\mathrm{RD}$ and without RD were significant. The differences in other examined parameters were not statistically significant. In the logistic analysis risk of any RD was increased by the duration of NIDDM $>15$ years, $>0.3 \mathrm{~g} 24$-hour proteinuria, fasting glycemia $>7.8 \mathrm{mmol} / \mathrm{l}$ and male sex. After including these risk factors the influence of other parameters under study were not significant. The annual changes in the RD incidence and prevalence calculated both in the cohort of NIDDM and in the general, defined population are sufficiently sensitive to be applied for epidemiological monitoring. Correlation between these indices and risk factors may serve on the base for local intervention programmes.

\section{0}

MICROVASCULAR AND MACROVASCULAR DISEASE IN SUBJECTS WITH INCREASED BUT NOT DIABETIC FASTING GLUCOSE LEVELS S Karunakaran, R J Morris, R C Turner and R R Holman Diabetes Research Laboratories, University of Oxford, UK.

227 subjects (41\% male), thought to be at increased risk of non-insulin dependent diabetes, with increased fasting glucose (IFG) i.e. fasting plasma glucose (ipg) 5.5 to $7.7 \mathrm{mmol} \mathrm{f}^{-1}$ inclusive on two consectitive occasions, were recruited into the Fasting Hyperglycaemia Study (FHS). Exclusions were known diabetes, significant macrovascular discase, impaircd renal function, uncontrolled hypertension, life threatening illnesses or drugs that aftect glucose metabolism. Mean (SD) fpg was $6.1(0.7) \mathrm{mmol}^{-1}$, age 50 (9) years, body mass index (BMI) $29.2(4.8) \mathrm{kg}$. A $75 \mathrm{~g}$ two hour oral glucose tolerance test (OGTT) classified $37 \%$ as normal glucose tolerance, $38 \%$ as impaired glucose tolerance and $26 \%$ as diabetes mellitus. Microvascular disease was seen in $18 \%$ of 148 subjects with retinal photographs who had retinopathy $15 \%$ micro-aneurysms in one or both eyes, $3 \%$ (all male) with haenorrhages or exudates in addition). Only $3 \%$ of all subjects had microalbuminuria on a spot sample $\left(>50 \mathrm{mg} \mathrm{l}^{-1}\right) .10 \%$ had 2 or more absent lower limb reflexes and $12 \%$ of males reported impotence. Macrovascular discase was less prominent with $11 \%$ of subjects having Minnesota coded ST segment or T-wave abnormalities or myocardial infarction on digital electrocardiography. $22 \%$ were hypertensive $(\geq 150 / 90 \mathrm{~mm} \mathrm{Hg}$ or laking antihypertensive therapy). No significant trends were seen with any measure of microvascular or macrovascular disease and OGTT status. Multiple logistic regression analysis for presence of retinopathy or absent reflexes showed no significant associations with fpg, fasting plasma insulin, HOMA assessed beta cell function, BMI, systolic blood pressure or gender.

Conclusion: Retinopathy was present in $18 \%$ of this selected group of subjects with IFG, most of whom had glycaemic levels below the standard criteria for diabetes. 
921

\section{LONGITUDINAL STUDY OF DETERMINANTS OF RETINOPATHY IN TYPE II (NON-INSULIN-DEPENDENT) DIABETES MELLITUS}

M. Virally, P. Massin, M.A. Charles, H. Allaguy, Z. Meric, D. Tielmans, M. Assayag,

C. Ajzenberg, A. Wamet, J. Lubetzki, and P.J. Guillausseau Hôpital Lariboisière 75010) Paris DNSERM U 2194807 Villejuif (FRANCE)

Relationships between glycemic control, hypertension, and development of microangiopathy have been well documented in insulin-dependent (IDDM) but not in non-insulin-dependent diabetes (NIDDM). Therefore, we have investigated these relationships in a cohort of 64 NIDDM patients free of retinopathy (angiofluorography) at inclusion and regularly followed for 7 to 14 years (median: 9.5) at the Lariboisière Diabetes Center as outpatients. Glycemic control was assessed by 2 to $5 \mathrm{HbA}_{1}$ determinations per year in our laboratory $\left(\mathrm{N}^{\mathrm{ai}} 6.1 \pm 2.4 \%\right.$ ). Retinal status was monitored by annual angiofluorography. Nonproliferative retinopathy developed in 14 patients (13 years-cumulative incidence: $29.8 \%$ ). In multivariate analysis (Cox model). mean $\mathrm{HbA}_{1}$ during follow-up $(\mathrm{P}<0.001$ ), and hypertension at inclusion $(\mathrm{P}=0.09)$ were associated with the development of retinopathy, but age, sex. BMI, diabetes duration. smoking, and fasting blood glucose were not. The relative risk for developing retinopathy (RR) was 7.2 (IC 95\%: 1.61-32.4) in patients with mean $\mathrm{HbA}_{1}$ during follow-up above compared to below the median value of the population ( $8.3 \%$ ), 2.5 (IC $0.8-8$ ) in patients with $\mathrm{HbA}_{1}$ at inclusion above compared to below the median value $(8.4 \%$ ), and 3 (IC $0.9-10$ ) in patients treated for hypertension at inclusion compared to the others. A six fold increase in retinopathy prevalence was observed between patients with mean $\mathrm{HbA}_{1}$ in the highest $(6 / 16)$ and lowest (1/16) quartile of mean $\mathrm{HbA}_{1}$ distribution during follow-up. This longitudinal study indicates that long-term glycemic control is the major determinant of the development of diabetic retinopathy in NIDDM as well as in IDDM patients.

\section{3}

\section{SERUM TOTAL RENIN, AN INDEPENDENT NEW MARKER OF ACTIVE} DIABETIC RETINOPATHY

P Summanen, S Mäkimattila, I Matinlauri, M Mäntysaari and H Yki-Järvinen. Universities of Helsinki and Turku, Central Military Hospital, Finland. Recent studies have demonstrated that prorenin is locally expressed in the eye, especially in retina, and that a marked concentration gradient exist between blood and ocular tissues. We determined whether serum total renin, which mostly consists of prorenin, might be a marker of the severity of retinal changes independent of other diabetic complications. Total renin concentrations were measured with a time resolved immunofluorometric assay in 32 patients with IDDM (age $38 \pm 1$ years, duration of disease $24 \pm 1$ years, serum creatinine $100 \pm 4 \mu \mathrm{mol} / \mathrm{L}$, urinary albumin excretion rate (UAER) $392 \pm 172 \mu \mathrm{g} / \mathrm{min}, \mathrm{HbA}$ $8.4 \pm 0.2 \%$ ) and in 13 matched normal subjects. All subjects were carefully characterized with respect to the presence of retinopathy (Early Treatment of Diabetic Retinopathy Study, ETDRS score), renal function (UAER) and neuropathy using 7 different tests of autononic neuropathy. Serum total renin was on the average 5 -fold higher in IDDM $(1102 \pm 300 \mathrm{ng} / \mathrm{L})$ than in normal subjects $(201 \pm 24 \mathrm{ng} / \mathrm{L})$. Serunt total renin was 3 -fold increased in patients with active proliferative or preproliferative retinopathy $(n=8$, serum total renin $2583 \pm 1045 \mathrm{ng} / \mathrm{L}$ ) compared to those with inactive laser treated proliferative retinopathy $(\mathrm{n}=8,845 \pm 202 \mathrm{ng} / \mathrm{L}, \mathrm{p}<0.01$ ), and 5 -fold conipared to those with minimal to moderately severe non-proliferative retinopathy $(n=16,490 \pm 90 \mathrm{ng} / \mathrm{L}$, $\mathrm{p}<0.001)$ and 13-fold compared to normal subjects $(\mathrm{n}=13,201 \pm 24 \mathrm{ng} / \mathrm{L}$, $p<0.001)$. In multiple linear regression analysis the ETDRS score $(p<0.005)$, but not UAER or any index of autononic neuropathy was independent determinant of serum total renin, and explained $54 \%$ of its variation $(\mathrm{R}=0.73$ for model, p<0.001). We conclude that serum total renin concentrations are markedly increased in patients with active retinopathy requiring laser therapy, independent of renal function. Serum total renin might be a tool to identify and follow such patients in populations where photoscreening is not available.

\section{2}

RETINOPATHY AND BLOOD PRESSURE IN TYPE I (INSULINDEPENDENT) DIABETES: AN INDEPENDENTT RELATIONSHIP. R. Miccoli, G. Penno, R. Cionini, M. Nannipieri, L. Rizzo, A. Bertolotto, A. Rapuano, S. Bandinelli, S. Viti and R. Navalesi. Cattedra di Malattie Metaboliche e del Ricambio - Pisa, Italy.

The significance of blood pressure in the progression of nephropathy in type 1 diabetes has been well established. Whether its role is as much important in retinopathy is not clear.

Staging of diabetic retinopathy (DR: absent, background, proliferative) was assessed by 2 -field per eye fundus photography, albumin excretion rate (AER) was evaluated by immunoturbidimetric method on timed 24hour urines, seated blood pressure (BP) was measured twice by a random zero sphygmomanometer, and HbAlc was assayed by HPLC in 129 type 1 diabetics $30.3 \pm 8.8$ years old, whose disease duration was $13.3 \pm 8.0$ years and whose previous 2 year $\mathrm{HbAlc}$ was $8.0 \pm 1.6 \%$.

Increasing systolic $(113 \pm 12,124 \pm 21,125 \pm 14 \mathrm{mmHg}, \mathrm{p}=0.0006)$ and diastolic blood pressure were associated with the severity of retinopathy $(69 \pm 8,74 \pm 10,82 \pm 11 \mathrm{mmHg}, \mathrm{p}=0.0001)$ in subjects with no DR ( $\mathrm{n}$. 79), background DR (n. 31) and proliferative DR (n. 13). The association with systolic BP (step 2) persists after adjustment for the mean previous 2-year HbAlc levels and AER (step 1; stepwise regression, multiple $r=0.46$ ), but not after adjustment for diabetes duration (multiple $\mathrm{r}=0.62$ ). The association with diastolic BP (step 3) persists even after adjustment for AER (step 2) and diabetes duration (step 1 ; multiple $\mathrm{r}=0.70$ ).

In conclusion, in type 1 diabetic patients retinopathy shows a positive association with blood pressure. This relationship is independent from diabetes duration and long-term glicaemic control and only in part reflects the coexistence of other microvascular complications, i.e. increased $\mathrm{AER}$.

\section{4}

EARLY DEFECTS IN PEROXIDATION, NA/K ATPASE ACTIVITY AND TAURINE UPTAKE IN THE RETINA OF STREPTOZOTOCIN-INDUCED DIABETIC RAT.

M.A.S. Di Leo ${ }^{1}$, S.A. Santini ${ }^{2}$, D. Lepore ${ }^{3}$, S. Caputo $^{1}$, S. Cercone ${ }^{1}$, G. Portale ${ }^{1}$, L. Antico 1 , A.V. Greco ${ }^{1}$ and G. Ghirlanda 1 . Institutes of ${ }^{1}$ Internal Medicine, ${ }^{2}$ Biochemistry and ${ }^{3}$ Ophthalmology, Catholic University, Rome, Italy.

To study the susceptibility of diabetic retinas to oxidative stress, we evaluated the roles of the metabolism of lipid peroxides, the activity of membrane-bound enzymes such as $(\mathrm{Na}, \mathrm{K})$-ATPase and the Nadependent uptake of taurine (TAU) in retinas from male Wistar adult non-diabetic and streptozotocin-induced diabetic (STZ-D) rats. After induction of diabetes, (STZ-D) rats were sacrificed at 1,2 and 4 weeks and their retinas removed. (Na,K)-ATPase activity and conjugated dienes (CD) were measured spectrophotometrically and expressed as $\mu \mathrm{mol} \mathrm{ATP} / \mathrm{h} / \mathrm{mg}$ protein and $\mu \mathrm{mol}$, respectively; lipid hydroperoxides (HP) were calculated with a calorimetric assay and expressed as $\mathrm{nmol} / \mathrm{LOOH} / \mathrm{mg}$ protein. The uptake was measured by TAU $0.1 \mathrm{mmol} / \mathrm{h}$, containing $3 \mathrm{H}-\mathrm{TAU}$ and $\mathrm{Vmax}$ was expressed as nmol/mg/min. Results are given as means $\pm S D$. The level of $H P$ and $C D$ were $0.8 \pm 0.07$ and $3.7 \pm 0.3$ in control retinas, respectively. We found a significantly increase $(\mathrm{p}<0.05)$ of HP and CD ( 1 week $=$ HP: $2 \pm 0.2 ; \mathrm{CD}: 5.9 \pm 0.3)$; ( 2 week $=$ HP: $5.2 \pm 0.3 ; \mathrm{CD}: 6 \pm 0.4) ;(4$ week $=$ HP: $5.3 \pm 0.2 ; \mathrm{CD}: 8$ \pm 0.3 in (STZ-D) rat retinas. $(\mathrm{Na}, \mathrm{K})$-ATPase activity was reduced at 2 weeks $(72.1 \pm 19.5 ; p=0.02)$ with respect to that of control rats $(100.3 \pm$ 18 ) and after 4 weeks $(42.1 \pm 13.1 ; \mathrm{p}<0.0001)$. TAU V $\max$ which was $0.25 \pm 0.008$ in control rats showed a significantly reducion in (STZ-D) rats after 2 weeks $(0.19 \pm 0.001 ; p<0.0001)$ and 4 weeks $(0.14 \pm 0.001$; $p<0.0001$ ) but not after 1 week. Our results suggest that increased HP and $C D$ in diabetic retinas produce an oxidative damage causing a dysfunction of membrane-dependent $(\mathrm{Na}, \mathrm{K})$-ATPase. TAU uptake reduction which is involved in osmotic alterations may be related to the disruption of $(\mathrm{Na}, \mathrm{K})$ pump activity. 
925

GAD65-ANTIBODY LEVELS AND HLA-DR/DO IN RELATION TO SEVERE RETINOPATHY IN IDDM PATIENTS

D. Agardh, E. Agardh, M. Landin-Olsson, L.K. Gaur, C.-D. Agardh and A. Lernmark. Dpt of Medicine and Ophthalmology, University Hospital, Lund, Sweden.

A minority of IDDM patients are affected by a malignant form of retinopathy. Putative risk factors such as IDDM duration. hypertension, poor metabolic control. nephropathy and growth hormone levels do not seem to completely explain the rapid progress of retinopathy in these patients. The aim of the present study, was to find out if certain HLA-DR/DQ types and GAD65-antibody levels were associated with rapid progressive severe retinopathy. One group with severe non-proliferative or proliferative retinopathy $(n=28)$ were compared with another group, which in spite of long diabetes duration had no or mild signs of retinopathy $(n=28)$. Subtyping of HI.ADR/DQ was done by PCR and hybridisation with oligospecific alleles. GAD65-antibodies were analysed with radioimmunoassay using human GAD65. A GAD65-index was calculated in relation to a positive and a negative reference sample. The freguency of $D Q B 1 * 0201 / 0302$, was higher in patients developing severe retinopathy $(57 \%$ vs $25 \% ; p<0.0001)$. GAD6.5-index was lower in patients with than without severe retinopathy $(0.09 \pm 0.17$ vs $0.26 \pm 0.39 ; p<0.04$ ). GAD65-antibody levels were lower in patients with severe retinopathy positive for DRB $1 * 0.301 \quad(0.08 \pm 0.15 \mathrm{vs}$ $0.30+0.45 ; p<0.05)$. DRB $1 * 040) \quad(0.08+0.18$ vs $0.28 \pm 0.36$; $\mathrm{p}<0.05), \mathrm{DQA} \mid * 050 \mathrm{l}(0.09 \pm 0.15$ vs $0.30 \pm 0.45 ; \mathrm{p}<0.05), \mathrm{DQA} \mid * 0.3$ $(0.08+0.18$ vs $0.30+0.40 ; \bar{p}<0.03)$, and $\overline{D Q B 1} 1^{*} 0.302(0.10 \pm 0.18$ vs $0.31+0.40 ; \mathrm{p}<0.03)$. In conclusion. the high risk genotype for IDDM. DQBI*0201/0302, do also confer high risk for severe retinopathy. The higher GAD65-antibody levels in patients without severe retinopathy maybe due to some remaining b-cell function giving these patients better metabolic control.

\section{7}

ALDOSE REDUCTASE LEVEL IS ASSOCIATED WITH THE PROGRESS OF DIABETIC RETINOPATHY

Y. Takahashi, S. Takayama, T. Ito, T. Tachikawa* and Y. Omori, Diabetes center, Tokyo Women's Medical College and * Mitsubishi Gas Chemical Co., Tokyo, Japan

Prolonged hyperglycemia is thought to be the primary cause of diabetic complications, however, it is well known that some diabetic patients have severe retinopathy early on diagnosis of diabetes, while others develop no or mild retinopathy even after many years of poorly controlled diabetes. To investigate other causes on the progress of diabetic retinopathy besides hyperglycemia, erythrocyte aldose reductase level was determined in 76 NIDDM patients by a two-site ELISA using recombinant human aldose reductase. In 25 patients who had proliferative retinopathy within 5 years of diagnosis, aldose reductase level was significantly higher than 51 patients who had no or mild retinopathy with poorly controlled diabetes for more than 8 years $(12.2 \pm 5.1$ vs. $8.7 \pm 1.8 \mathrm{ng} / \mathrm{mg} \mathrm{Hb} p<.0001)$. Age at examination was younger and maximum body mass index in the past was higher in patients with severe diabetic retinopathy than those without ( $49 \pm 9$ vs. $59 \pm 9$ y/o and $29.7 \pm 5$ vs. $25.8 \pm 4 \mathrm{~kg} / \mathrm{m}^{2}$, respectively), while no difference in the rate of sex, the level of glycemic control and the stage of other diabetic complications was observed between the two groups. These results indicate that the level of erythrocyte aldose reductase in NIDDM patients is associated with the progress of diabetic retinopathy.

\section{6}

OBLITERATION OF A PHYSIOLOGIC NIGHTDAY DIFFERENCE IN GROWTH HORMONE SECRETION IN DIABETIC RETINOPATHY. JKrassowski, P.Soszyniski, W.Jeske and S.Zgliczynski. Department of Endocrinology, Medical Centre of Postgraduate Education, Warsaw, Poland.

We have earlier found that some diabetic patients have higher growth hormone (GH) levels during the day than during the night. Therefore we have compared day and night $\mathrm{GH}$ secretion in 45 type I diabetics (28-without retinopthy, 17 -with retinopathy) and in 14 healthy subjects matched for age(mean 29 years). GH was determined hourly during 24 hours and analyzed with Pulsar program. The whole 24-hour period was divided into two 12-hour periods lasting from $9 \mathrm{AM}$ to $8 \mathrm{PM}$ (day) and from 9 PM to 8 AM (night). Diabetic patients $(n=45)$ had. higher $\mathrm{GH}$ levels than healthy controls: 6.10 vs $2.18 \mathrm{ng} / \mathrm{m} 1, \mathrm{p}<0.0001$ and higher mean peak amplitude: 14.34 vs $5.97 \mathrm{ng} / \mathrm{ml}, \mathrm{p}<0.001$, Mean $\mathrm{GH}$, mean peak amplitude and number of pulses were not different in patients with and without retinopathy. The comparison of day and night secretion revealed higher nighttime $\mathrm{GH}$ levels in healthy subjects ( $3.16 \mathrm{vs} 1.20$ $\mathrm{ng} / \mathrm{ml}, \mathrm{p}<0.001)$ and in diabetic patients without retinopathy $(7.54 \mathrm{vs}$ $5.05 \mathrm{ng} / \mathrm{ml}, \mathrm{p}<0.01$ ). In patients with retinopathy the difference was not significant: 6.48 vs $5.24 \mathrm{ng} / \mathrm{ml}$. Diabetic subjects had higher number of pulses during the day than the controls $(3.2$ vs $2.3, \mathrm{p}<0.01)$ but the difference was not significant for the whole 24-h period. GH levels did not correlate with metabolic control of diabetes $(\mathrm{HbAl})$. We conclude that in patients with retinopathy a physiological night/day difference in GH secretion is obliterated, while in patients without retinopathy this difference is preserved as it healthy subjects

\section{8}

EARLY AND LATE INTERVENTION THERAPY FOR DIABETIC RETINOPATHY WITH AN ALDOSE REDUCTASE INHIBITOR

T.C. Hohman, ${ }^{1}$ J.L. Jacot, ${ }^{2}$ J.P. Glover, ${ }^{2}$ M.D. Basso, ${ }^{1}$ and W.G. Robison, Ir. ${ }^{2}$ Wyeth-Ayerst Research, ${ }^{1}$ Princeton, NJ; National Eye Institute, ${ }^{2}$ NIH, Bethesda, $\mathrm{MD}$

Galactose-fed rats develop retinal microangiopathies similar to those of diabetic patients. In this model aldose reductase inhibitor (ARI-509) treatment can prevent retinal damage. This study compared the ability of ARI intervention and galactose withdrawal to interrupt the progression of early retinal changes. Weanling S-D rats were divided into 8 weight-matched groups with 10 animals per group and were fed for up to 24 months Purina \#5001 laboratory chow plus: 50\% starch-CONTROL (CON); $50 \%$ d-galactose-GALACTOSE (GAL); 50\% d-galactose with ARI-509 at either 10 or $25 \mathrm{mg} / \mathrm{kg} / \mathrm{day}-L O W$ DOSE PREVENTION $(L D P)$ and HIGH DOSE PREVENTION (HDP); $50 \%$ d-galactose for 4 or 8 months and then intervention by addition of ARI-509 (25 mg/kg/day)-4-MONTH INTERVENTION (4IN) and \&MONTH INTERVENTION $(8 I N)$; or galactose withdrawal by replacing the galactose diet with the $50 \%$ starch diet at either 4 or 8 months-4-month GALACTOSE WITHDRAWAL (4GW) and 8-month GALACTOSE WITHDRAWAL $(8 \mathrm{GW})$. Electron micrographs were analyzed by computer morphometry for retinal capillary basement membrane thickening (RCBMT) in the outer plexiform layer at $4,8,16$, and 24 -month time points. RCBMT progressed most rapidly in GAL rats, becoming 2 -fold greater than in CON rats by 24 months. RCBMT was not significantly different from CON rats in either the LDP or the HDP groups at any time point. In contrast, all intervention groups showed RCBMTs intermediate between those of the GAL vs the CON, LDP, or HDP rats at 24 months. Therefore treatment with ARI-509, at doses that inhibit retinal polyol formation by $95 \%$, prevents RCBMT if initiated at the onset of galactosemia, whereas intervention by either ARI-509 treatment or galactose withdrawal at later times fails to arrest increased RCBMT. This suggests that irreversible hexoseinduced capillary alterations occur early, and that treatments for diabetic patients may be more effective if initiated before elinical signs of diabetic retinopathy. 
929

DECREASE OF TRANSCAPILLARY ESCAPE RATE OF ALBUMIN DURING TREATMENT WITH VITAMIN C IN IDDM PATIENTS WITH RETINOPATHY B. Juhl, F. Klein and J.S. Christiansen. Medical Department M, Endocrinology \& Diabetes, Aarhus Kommunehospital, DK-8000 Aarhus C, Denrnark.

Marginal ascorbic acid (AA) deficiency induced tissue changes have resemblance to the widespread vascular disease reflected in elevated transcapillary escape rate (TER), von Willebrand factor (vWf) and increased capillary fragility (CF) found in diabetic microangiopathy. We aimed to investigate the possible impact of low plasma level of AA observed in insulin-dependent diabetes mellitus (IDDM). Twenty normoalbuminuric IDDM patients with retinopathy were included in a randomized double-blind, parallel, placebo-controlled study to either 6 months treatment with AA ( 6 $\mathrm{g} /$ day $)(\mathrm{n}=10$, mean $\pm \mathrm{SD}$, age $35.3 \pm 8.5$ years, diabetes duration $17.2 \pm 3.3$ years) or placebo (tartaric acid (TA)) ( $n=10$, age $37.8 \pm 6.6$ years, diabetes duration $18.4 \pm 1.7$ years). Paired and unpaired t-tests were used analysing results. TER (125 -labelled human albumin) was normalized in the AA group (6.8 (1.0) to $5.4(1.5) \%$ /hour, $\mathrm{p}=0.018)$ and significantly reduced from baseline compared to the TA group $(p=0.031)$ which was unchanged $(5.6$ (1.5) to $5.9(1.1) \%$ /hour, $\mathrm{p}=0.64)$. CF decreased in the AA treated group compared to the placebo group $(p=0.028)$. Von $W f$, fibrinogen and fibronectin were unchanged during treatment in both groups. An inverse correlation between plasma AA and TER was found $(r=-0.66, p=0.002)$. Multiple regression analysis revealed the level of plastaa AA to be the only significant independent predictor for the degree of elevation of TER also when $\mathrm{HbAlc}$, blood pressure and diabetes duration were taken into account $(\mathrm{p}=0.002)$. In conclusion: Low plasma AA predicts an increased TER reflecting widespread vascular disease in normoalbuminuric IDDM patients with retinopathy. AA treatment resulted in a normalization of TER and a decrease in CF. Abnormal AA metabolism could be involved in development of diabetic microangiopathy.
930

\section{MORTALITY IN BLIND SUBJECTS WITH DIABETES COMPARED TO THE GENERAL POPULATION}

A. Icks ${ }^{1}$, C. Trautner ${ }^{1}$, B. Haastert ${ }^{1}$, F. Plum ${ }^{2}$, M. Berger ${ }^{3}$, and G. Giani ${ }^{1}$. ${ }^{1}$ Diabetes Research Institute at Düsseldorf University, Germany Department of Biometrics and Epidemiology ${ }^{2}$ Department of Ophthalmology, Krefeld City Hospital, Germany ${ }^{3}$ Department of Metabolic Diseases and Nutrition, Düsseldorf University

Objective: There is only little information about survival in newly blind persons. It was the aim of this study to compare mortality in this cohort with the mortality in the general population.

Methods: Using a register of blindness allowance recipients, a closed cohort of all blind persons newly registered in 1990-1993 ( $\mathrm{n}=2680$, mean age 70.4 (SD 22.1) years, 1803 of them women) in the district of WürttembergHohenzollern, Germany, was followed up to 48 months. Mortality was compared to that of the general population by estimating comparative mortality figures (CMF).

Results: 582 of the subjects died before February 1, 1994. 772 had diabetes, 226 of them died. The overall mortality rate was 12,179 per 100,000 person years. Compared with the entire population, mortality was increased in the blind cohort (CMF $4.79, \mathrm{CI} 3.96 ; 5.79$ ), especially in the subjects with diahetes (CMF 6.55, Cl 4.71; 9.11). The relative risk decreased with increasing age.

Conclusions: The study population may be considered a nearly complete sample of incident cases of blindness in the study district. Compared with the general population, mortality was considerably increased. even more than in previous studies on the mortality in the blind. Mortality was particularly high in the subjects with diabetes. The relative mortality risk in the diabetic blind was higher than the approximately two-fold mortality risk of individuals with type 2 diahetes. compared with the general population, as it had been described previously.

\section{1}

INCREASED LENS AUTOFLUORESCENCE IN OFFSPRINGS OF NIDDM PATIENTS. P. Koefoed Theil', T. Hansen ${ }^{2}$, S.A. Urhammer ${ }^{2}$, M. Larsen ${ }^{1}$ O. B. Pedersen ${ }^{2}$ H. LundAndersen ${ }^{1}$

Dept. of Ophthalmology, Herlev Hospita, University of Copenhagen, Denmark ${ }^{1}$.Steno Diabetes Center, Gentofte, Denmark ${ }^{2}$

Background: Lens autofluorescence represents a cumulative index of lifelong glycemia due to non-enzymatic glycosylation of lens proteins. This leads to an increase in lens fluorescence with age and it has been shown to be increased in newly diagnosed NIDDM patients compared to healthy, age-matched control subjects. Aim: To test whether normal glucose tolerant offsprings of NIDDM patients have higher lens fluorescence levels than an age-matched, normoglycemic control group. Method: Quantitative determination of lens and cornael fluorescence was carried out on a fluorometer lexcitation $430-490 \mathrm{~nm}$, emission $530-630 \mathrm{~nm}$ ) on 100 offsprings of NIDDM patients and 34 age.matched, normoglycemic control subjects with no family history of diabetes (all with a normal oral glucose tolerance test); age $36,6 \pm 7,6 \mathrm{yrs}$ (mean $\pm \mathrm{SD}$ ) versus age $33,8 \pm 7,8 \mathrm{yrs}$; NS. Results: Lens fluorescence was significantly higher among the offsprings of NIDDM patients $(272,5 \pm 83,2 \mathrm{ng} / \mathrm{ml}$ versus $232,7 \pm 77,8 \mathrm{ng} / \mathrm{ml} ; \mathfrak{p}=0,016)$. Also the $2 \mathrm{~h}$ plasma glucose was significantly higher among the offsprings $(5,7 \pm 1,2$ mmolij) versus $5,0 \pm 1,2$ mmolil; $p=0,002)$. No significant difference was found for corneal fluorescence $(15,2 \pm 3,4$ $\mathrm{ng} / \mathrm{ml}$ versus $14,0 \pm 3,0 \mathrm{ng} / \mathrm{m})$ or for $\mathrm{HbA} 1 \mathrm{c}(5,41 \pm 0,46 \%$ versus $5,5 \neq 0,38 \%)$. Lens fluosescence was significantly corretated to the $2 \mathrm{~h}$ plasma glucose $\{p=0,004\}$. Conclusion: Lens autofluorescence levels were significantly higher among offsprings of NIDDM patients compared to controls. As iens fluorescence represents the cumulated glucose exposure, it strongly indicates an impaired glucose metabolism in the group of offsprings and could therefore be of importance as a predictor of later developement of NIDOM in this high rise group. 


\section{Experimental Neuropathy}

932

INMINOREACTIVITY PARVALBUMIN REDUCTION OF THE NEURONS AT VISCERAL SENSORY CORTEX IN THE DIABETIC RATS.

A. Odriozola, T. Rivalta *, X. Navarro** and R. Gomis. Endocrinology and Diabetes Unit and Department of Pathology*. Hospital Clinic Barcelona. Department of Cellular Biology and Fisiology**. University Autonoma of Barcelona, Spain.

There is littie data on the development of diabetic cerebral disorders and autonomic neuropathy. Gamma-aminobutitic acid (GABA) is the main intibitory neurotransmiter in the cerebral visceral cortex and Parvalbumin (PALB) has been used as selective neuronal inarkets. Aim: to investigate the impact of chronic hiperglycaemia on the GABA-rgic nentons at the viscersi strisury attonomic control area of the neocortex in diabetic rats with autonomic nelujopathy. Method: Wistar rats were made diabetic by pancreatectomy of $95 \%(\mathrm{PX})$ and received daily pancreatic enzymes. Divided into 3 groups: A. (n=6) PX, diabetic; group $\mathrm{B}(\mathrm{n}=6) \mathrm{PX}$, diabetic and insulin therapy; group $C(n=5)$ sham controls. We meas ufed blond glucose weekly duringhine months, after this time were examined the autonomic innervation at bladder and sweat glands by inmunohistochemistry with antiboties to protein gene product 9.5 and the abdominal vagus branche by sectort tnicrosenjy. Nissl staith frecly floating mnunohistoohumistry PAL.B was caned out in suctions of bratin cingulate areas 1,2 and 3 . The number of neurons cells were counted $(700-j / \mathrm{m}$-kitde strips) with ocular micrometer. Results: glucose tevels (mmolli) were higher in group A: (mean +- SD) $25.2+3.2$, group B: $13.4+5.8$, group C 0.2 tw 1.0 . A vs B $(p<0.01)$, A vs. C (pe0.001); B vis. C? $0.01 \%$ Microscopy degenerative autonomic lesions were observed in ail jats in gxoup A and isolated ultrastnetural alteratons in group $B$ PALB munbers cellsmm2 (mein + SD) was lower in group A: 87.3 +- 59; group B:

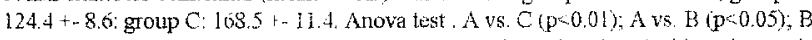
vs. $C$ (p 0.05$)$. Conclusions: These data suggesi that the chronic hiperglycaemia associated with autonomine neuropathy produce a significative inmunoreactivity reduction of GABA- urgic inhibitory neurons at the sensory visceral autonomic control area of the cerbbral cortux

\section{4}

INCREASED NERVE BLOOD FLOW IN ACUTELY HYPERGLYCEMIC RATS IS PREVENTED BY SUPEROXIDE DISMUTASE MIMIC

J.R. Williamson, K. Chang, W. LeJeune, and $Y$. Ido, Washington University, St. Louis, MO, USA.

Several different lines of evidence support the likelihood that increased retinal and sciatic nerve blood flow in animal models of diabetes is mediated by increased production of both superoxide and nitric oxide. The objective of the present experiments was to assess the effects of a low molecular weight ( $M W=341$ ) manganese-based superoxide dismutase mimic on increased (sciatic nerve) endoneurial blood flow induced by acute hyperglycemia in nondiabetic rats. 18 hours after indwelling catheters were placed in the femoral vein, male Sprague-Dawley rats were infused for $5 \mathrm{~h}$ with: 1) $5 \mathrm{mM}$ glucose in saline (16 ml/kg bwt $/ \mathrm{h} ; n=7$ ) \pm SOD mimic ( $35 \mu \mathrm{mole} / \mathrm{kg}$ bwt $/ \mathrm{h} ; \mathrm{n}=4$ ), or 2) $50 \%$ glucose $(44.4$ mmole glucose $/ \mathrm{kg}$ bwt $/ \mathrm{h} ; \mathrm{n}=6) \pm$ SOD mimic $(35 \mu \mathrm{mole} / \mathrm{kg}$ bwt/h; n=7). Rats were then anesthetized with $100 \mathrm{mg} / \mathrm{kg}$ Inactin and blood flow was assessed with $10 \mu \mathrm{m} 46 \mathrm{Sc}$ microspheres. Final plasma glucose levels were $6.4 \pm 1.9 \mathrm{mM}(x \pm S D), 6.4 \pm 2.4,21.7 \pm 1.6 \quad(p<0.05$ vs controls), and $21.6 \pm 3.6$ ( $p<0.05$ vs controls), respectively. Blood flow $(\mu \mathrm{l} / \mathrm{g}$ wet $\mathrm{wt} / \mathrm{min})$ was increased $(p<0.01)$ in endoneurium of hyperglycemic vs control rats $(149+12$ vs $98+10)$, but not in brain $(587 \pm 50$ vs $615 \pm 55)$. The SOD mimic had no effect on endoneurial blood flow in saline-infused control rats $(114 \pm 10)$ but prevented the increased blood flow in hyperglycemic rats $(105 \pm 14,(p<0.01)$. These observations are consistent with other evidence which suggests that hypoxia-like reductive stress (induced by elevated glucose levels) increases superoxide production which increases intracellular calcium levels resulting in activation of the constitutive isoform of nitric oxide synthase

\section{3}

ENERGY METABOLISM AND MITOCHONDRIAL REDOX STATE OF NADCOUPLES IN SCIATIC NERVE IN RATS WITH SHORT-TERM DIABETES

I.G.Obrosova1, M.F.Lou 2, and J.R.Williamson 1, Washington University,St.Louis, MO, and University of Nebraska, Lincoln, NE,USA

The study was aimed at evaluating the mitochondrial redox state of NAD-couples, parameters of energy metabolism, and glutathione (GSH) levels in sciatic nerve in rats with short-term (10 d) diabetes. Experiments were performed on male STZ diabetic (D) rats treated with/without the aldose reductase inhibitor, tolrestat, at a dose of $100 \mathrm{mg} / \mathrm{kg}$ bwt/day. The levels of $\beta$-hydroxybutyrate $(\beta-\mathrm{HB})$, acetoacetate, dose of $100 \mathrm{mg} / \mathrm{kg}$ bwt/day. The levels of $\beta$-hydroxybutyrate(B-HB), acetoacetate,
glutamate(G), 2-oxolutarate, ammonia, GA3P, 3-PG, pyruvate, lactate, ATP, ADP. phosphocreatine $(\mathrm{PCr})$, and creatine $(\mathrm{Cr})$ were assayed in individual nerves spectrofluorometrically by enzymatic methods; sorbitol and myo-inositol were quantified by GCMS. GSH was measured by Ellman's procedure. Free mitochondrial $\mathrm{NAD}+\mathrm{NADH}$ (for cristae and matrix) and cytosolic ATP/ADP* $\mathrm{P}_{i}$ were calculated from the dehydrogenase (DH) systems ( $\beta-\mathrm{HBDH}, \mathrm{GDH}$, and GA3PDH / 3-PG kinase, respectively).Sorbitol levels increased to $1.75 \pm 0.35$ in $\mathrm{D}$ (vs $0.120 \pm 0.021 \mu \mathrm{mol} / \mathrm{g}$ in controls(C)) while myo-inositol levels decreased. ATP levels and ATP/ADP ratio decreased to $0.551 \pm 0.088$ and $1.01 \pm 0.18$ (vs $0.696 \pm 0.193 \mu \mathrm{mol} / \mathrm{g}$ and $1.53 \pm$ 0.45 in $\mathrm{C}, \mathrm{p}<0.01$ and $<0.001$ ); $\mathrm{PCr}$ levels and $\mathrm{PCr} / \mathrm{Cr}$ ratio were unchanged. Free cytosolic ATP/ADP*P, decreased while GSH levels as well as both mitochondrial $\mathrm{NAD}^{+} / \mathrm{NADH}$ ratios (which are decreased in ischemic and hypoxic tissues) were indistinguishable from those in C. ARI decreased sorbitol levels to $0.014 \pm 0.007$ and increased myo-inositol, $\mathrm{PCr}$ levels, $\mathrm{PCr} / \mathrm{Cr}$ and free cytosolic ATP/ADP*P ratios. ATP and GSH levels as well as ATP/ADP and mitochondrial NAD+/NADH ratios in both cristae and matrix were not affected In conclusion, 1) nerve energy metabolism is impaired at early stages in D,2) hypoxia (if present) is insufficient to decrease NADH oxidation by respiratory chain, and, therefore, does not contribute to energy deficiency, 3) preventive effects of ARIs on nerve energy metabolism in shortterm $D$ are not mediated via an increase in oxygen supply, 4) nerve dysfunction in short-term D can not be attributed to GSH depletion-linked oxidative stress.

\section{5}

EFFECT OF ELEVATED EXTRACELLULAR D-GLUCOSE ON SCHWANN CELL PROLIFERATION IN VITRO. P. Magnani, $P$. Tilli, P. Cotroneo, A. Manto, G. Marra, L. Mancini, A.V. Greco and G. Ghirlanda, Medicina Interna e Geriatria, Univ. Cattolica, Roma, Italy.

Schwann cells surround the axon in its entirety and support it from a mechanical and trophic point of view. Since segmental demyelination and remyelination are prominent features of diabetic neuropathy, a role of Schwann cells has been hypothesized in the pathogenesis of such complication of diabetes. The aim of our work was to study the effect of different extracellular levels of D-glucose on Schwann cell growth. Primary Schwann cell culture was obtained through dissection and digestion with collagenase and trypsin of sciatic nerves from neonatal rats. Cultured cells were characterized by indirect immunofluorescence using anti S-100 (a protein specific for Schwann cells) antibody. Cell proliferation was measured in cells exposed for 2, 6 and 10 days to increasing D-glucose concentrations (5 $\mathrm{mM}, 15 \mathrm{mM}$ and $30 \mathrm{mM}$ ) using MTT tetrazolium salt colorimetric assay and was expressed in terms of optical density (O.D.). The three different D-glucose concentrations do not modify significantly cell growth after 2 and 6 days, respectively: $(5 \mathrm{mM}$ O.D. $=0.152 \pm 0.035 ; 15 \mathrm{mM}$ O.D. $=0,198 \pm 0.039, p=n . s ; 30 \mathrm{mM}$ O.D. $=0.158 \pm 0.067, p=$ n.s. and $5 \mathrm{mM}=0$. D. $0.238 \pm 0.075 ; 15 \mathrm{mM}$ O.D. $=0.299 \pm 0.078, p=n . s$.; $30 \mathrm{mM} \mathrm{O.D.}=0.264 \pm 0.055, p=n . s$. After 10 days the growth rate of cells cultured in the presence of $5 \mathrm{mM}$ D-glucose is higher, though not significantly, than that of cells grown in the presence of $15 \mathrm{~mm} D$-glucose $(O . D .=0.369 \pm 0.097$ vs O.D. $=0.351 \pm 0.019 ; p=n . s$.), while it is significantly superior to that of cells grown in the presence of $30 \mathrm{mM} \mathrm{D}$-glucose $(O . D=0.278 \pm 0.093 ; p<0.0001)$. These results suggest that Schwann cell proliferation is impaired in the presence of elevated levels of extracellular D-glucose: this might be a mechanism through which hyperglycaemia induces alterations of peripheral nerve fibers in the course of diabetic neuropathy. 
936

INCREASED VASODILATORY VERSUS CONSTRICTOR EICOSANOIDS IN SCIATIC NERVE OF DIABETIC RATS

P.A. Stoltje and J.R. Williamson. Washington University School of Medicine, St. Louis, MO USA.

The aim of these studies was to examine the effects of diabetes on vasoactive cyclooxygenase products which have been implicated in mediating peripheral nerve blood flow changes in diabetic rats. Extracts from freshly isolated sciatic nerves of male Sprague-Dawley rats with streptozotocin diabetes (D) of 2, 4, 6, and 12 weeks duration were analyzed for eicosanoids by ELISA assays ( $\mathrm{pg} / \mathrm{mg}$ nerve protein, mean \pm SD). Vasodilator eicosanoids measured were prostaglandin $\mathbb{E} 2$ (PGE2) and 6-ketoprostaglandin $F 1 \alpha(6 \mathrm{kPGF})$, the stable metabolite of prostacyclin. The yasoconstrictive eicosanoid thromboxane $\mathrm{A} 2$ was assessed as its stable metabolite, thromboxane B2 (TxB2). PGE2, 6kPGF, and TxB2 levels in control (C) rats did not differ at 2 and 12 wks $(27 \pm 13,32 \pm 17$, and $20 \pm 7$ at 2 wks, respectively, $n=28$; $29 \pm 12,32 \pm 16$, and $22 \pm 7$ at $12 \mathrm{wks}, \mathrm{n}=14)$. All 3 eicosanoids were increased in $D$ at 2 wks $(44 \pm 17,43 \pm 15$, and $26 \pm 7 ; p<0.02$ vs. 2 wk $C$ for each, $n=26)$, but each decreased progressively (especially TxB2) to levels at 12 wks of $19 \pm 15$ and $30 \pm 18$ for PGE2 and 6kPGF ( $\mathrm{p}<0.05$ vs. 2 wk $\mathrm{D}$ for both), and $10: \pm 8$ for TxB2 ( $\mathrm{p}<0.001$ vs. 2 wk D and 12 wk $C, n=13$ ). Vasodilator to vasoconstictor ratios (PGE2/TxB2 and $6 \mathrm{kPGF} / \mathrm{TxB} 2$ ) in $\mathrm{C}$ did not differ between 2 and 12 wks. Likewise, the PGE2/TxB2 ratio in D did not vary with duration of diabetes; however, in $12 \mathrm{wk} D$ the ratio was almost twice that in $12 \mathrm{wk} \mathrm{C}(2.2 \pm 0.6$ vs. $1.3 \pm 0.3, \mathrm{p}<0.001)$. In contrast, the $6 \mathrm{kPGF} / \mathrm{TxB} 2$ ratio in $\mathrm{D}$ was greater than $\mathrm{C}$ at $2,4,6$, and 12 wks and increased progressively with longer duration of diabetes so that the ratio in $12 \mathrm{wk} D$ was 2.5 times that in 12 wk $C(3.5 \pm 0.05$ vs. $1.4 \pm 0.5, p<0.001)$ and 1.9 times that in 2 wk $D(1.9 \pm 0.5, p<0.005)$. Thus, the ratio of vasodilatory to vasoconstrictor eicosanoids in sciatic nerve of diabetic rats increased with longer duration of disease. To the extent that these changes in eicosanoids are associated with changes in nerve blood flow, they predict increased rather than decreased flow. Decreased flow would imply imbalances involving other vasoactive agents.

\section{7}

IGF-I PREVENTS GLUCOTOXICITY IN NEUROGLIAL CELLS E.L. Feldman, D. A. Greene ${ }^{I}$, M. Stevens ${ }^{1}$ and P Killen ${ }^{2}$ Departments of Neurology, Internal Medicine ${ }^{1}$ and Pathology ${ }^{2}$, University of Michigan, Ann Arbor, MI 48109, USA.

Insulin-like growth factor-I (IGF-I) is a polypeptide with both growth promoting and insulin-like actions. In vitro, IGFs enhance Schwann cell proliferation and myelination. In the current study, we examined the effect of high glucose $(G)$ on transfected MT4H1 rat Schwann cells (SC). These cells provide a model system in which to study not only IGF-I's effects but the potential mechanisms which underlie glucotoxicity of SC, as seen in diabetic neuropathy. SC were plated at $1 \times 10^{5} \mathrm{cells} / \mathrm{cm}^{2}$, grown $2 \mathrm{~d}$ in $10 \%$ calf serum, serumdeprived for $24 \mathrm{~h}$, then exposed to $25-100 \mathrm{mM} \mathrm{G}+10 \mathrm{nM}$ IGF-I for $0-72 \mathrm{~h} .100 \mathrm{mM} \mathrm{G}$ decreased cell number by $50 \%$ while addition of IGF-I restored cell number to normal. SC were then plated directly into serum-free defined media $\pm 100 \mathrm{mM} \mathrm{G}$ and increasing concentrations $(0.1,1$ and $3 \mathrm{nM})$ IGF-I \pm the blocking antibody to the IGF-I receptor, $\alpha$-IR 3 . IGF-I rescued SC from $\mathrm{G}$-induced growth arrest in a dose-dependent fashion and this effect was blocked by $\alpha$ IR3. Programmed cell death (PCD) is an active process which occurs when cells are exposed to a noxious stimulus. SC underwent significant PCD with $\mathrm{G}$ treatment. By $72 \mathrm{~h}, 80 \%$ of cells had undergone $\mathrm{PCD}$, measured by flow cytometry and DNA fragmentation gels. IGF-I addition decreased PCD to less than $25 \%$ $(p<0.001)$. Coincident with its glucoprotective effects, IGF-I augmented high $\mathrm{G}$ induced increases in SC aldose reductase and sodium-myoinositol transporter gene expression. These data imply that high G induces SC injury which can lead to PCD. IGF-I prevents SC injury and subsequent PCD by facilitating the expression of the osmoregulatory proteins, aldose reductase and the sodiummyoinositol transporter. Collectively our results suggest IGF-I can protect SC from glucotoxicity which may have therapeutic implications in the treatment of diabetic neuropathy. Supported by NIH grant R29 NS32843and grants from the American Diabetes Association and the Juvenille Diabetes Foundation.

\section{8}

ALPHA-LIPOIC ACID AND GLUTATHIONE TREATMENT IN DIABETIC RATS: NERVE BLOOD FLOW, NERVE FUNCTION AND ANTIOXIDANT CAPACITY. P.S. van Dam ${ }^{1,2}$, B. Bravenboer ${ }^{2}$, B.S. van Asbeck, G. Biessels ${ }^{2}$, J.F.L.M van Oirschot', W.H. Gispen ${ }^{2}$ and J.J.M. Marx'. 'Department of Internal Medicine and Rudolf Magnus Institute for Neurosciences, Utrecht University, Utrecht, The Netherlands,

It has been demonstrated that antioxidant treatment can prevent the development of nerve dysfunction in experimental diabetes. The aim of this study was to determine the effect of treatment with the antioxidants reduced glutathione (GSH) and $\alpha$-lipoic acid (LA) on sciatic nerve blood flow, nerve conduction velocity and oxidative stress in diabetic rats. We studied streptozotocin-diabetic male Wistar rats treated with GSH ( 400 $\mathrm{mg} / \mathrm{kg} /$ day i.p.), LA (30 mg/kg/day i.p.) or placebo, and non-diabetic control animals. Sciatic nerve blood flow (NBF) and vascular resistance (VR) were determined after 12 weeks of treatment using Laser Doppler flowmetry and arterial blood pressure after catheterization of the carotid artery. Lipid peroxidation and antioxidant capacity were measured in sciatic nerve, plasma and red blood cells. Sciatic motor and sensory nerve conduction velocity were assessed every four weeks. NBF was decreased and VR increased in the placebo-treated diabetic animals compared :o non-diabetic controls (NBF $37.6 \pm 3.5$ vs. $55.3 \pm 1.1 \mathrm{U}, P<0.01$. VR. $3.1 \pm 0.2$ vs. $1.6 \pm 0.2 \mathrm{mmHg} \mathrm{U} ; P<0.001$; mean \pm SEM). NBF was significantly increased by LA, but not GSH treatment (LA $52.4 \pm 7.1 \mathrm{U}, P<0.05 ; \mathrm{GSH} 38.7 \pm 1.6 \mathrm{U}$ ). VR was partly corrected by both drugs, but the effect was significant in the $\mathrm{LA}$ treated group only $(P<0.05)$. The findings were accompanied by a marked decrease in plasma lipid peroxidation in both treatment groups (malonyldialdehyde controls $1.55 \pm 0.09$, placebo $2.43 \pm 0.30$, GSH $2.03 \pm 0.19$ and LA $2.00 \pm 0.26$ umol/l; controls vs. placebo $P<0.01$ ). An increase in erythrocyte total glutathione levels after LA, but not after GSH treatment was observed (controls $8.41 \pm 0.44$, placebo $7.01 \pm 0.38$, GSH $7.39 \pm 0.36$ and LA $8.89 \pm 0.63 \mu \mathrm{mol} / \mathrm{gHb}$; controls vs. placebo $P<0.05$ ). No treatment effect could be observed on sciatic nerve antioxidant capacity, lipid peroxidation or nerve conduction. The present data show that antioxidant treatment with either LA or GSH can be beneficial in the prevention of reduced nerve microcirculation, probably by protecting against the increased systemic oxidative stress that is usually observed in diabetes.

\section{9}

DOSE-DEPENDENT INHIBITION OF LEUKOCYTE ADHERENCE BY $\alpha$-LIPOIC ACID AFTER ISCHEMIA-REPERFUSION INJURY

K. Kusterer, G. Bayer, T. Konrad, M. Enghofer, A. Oldenburg, H.J. Tritschler* and K.H. Usadel, Dept. Int. Med. I. J.W.-Goethe University \& *ASTA Medica, Frankfurt, Germany

$\alpha$-Lipoic acid is widely used in the treatment of human diabetic polyneuropathy. Animal experiments revealed that endoneural ischemia with free radical production is an important pathophenomenon in diabetes mellitus. Treatment of rats with $\alpha$-lipoic acid in the polyneuropathy model of streptozotocin-induced diabetes improves the reduced nerve blood flow and nerve conduction velocity. Ischemia-reperfusion is associated with increased leukocyte adherence to the endothelium with subsequent extravasation of the leukocytes and release of toxic mediators including free radicals. We investigated, whether $\alpha$-lipoic acid inhibits leukocyte adherence in an ischemia-reperfusion model in normal and diabetic rats to further characterize its protective effect in experimental polyneuropathy. Male Sprague-Dawley rats were anesthetized with pentobarbital $(60 \mathrm{mg} / \mathrm{kg})$. After laparotomy mesenteric veins were exposed under a microscope attached to a CCD-video camera and a video recorder. Ischemia was induced for $30 \mathrm{~min}$ with a stamp. Leukocyte adherence was recorded during $30 \mathrm{~min}$ of reperfusion. Pretreatment with $\alpha$-lipoic acid $(0,25,50,100 \mathrm{mg} / \mathrm{kg}$ i.v. $)$ reduced leukocyte adherence dose-dependently from $13719 \pm 1294 \mathrm{cells} / \mathrm{mm}^{2}$ vein cross section to $13369 \pm 1280(25 \mathrm{mg} / \mathrm{kg}), 5546 \pm 561(\mathrm{P}=0.0002 ; 50$ $\mathrm{mg} / \mathrm{kg}), 3574 \pm 722(\mathrm{P}=0.00004 ; 100 \mathrm{mg} / \mathrm{kg})$ respectively. Four groups were made diabetic with a single i.p. injection of streptozotocin $(100 \mathrm{ng} / \mathrm{kg})$. Two groups were treated with daily i.p. injections of vehicle and the other witholipoic acid $(100 \mathrm{mg} / \mathrm{kg} / \mathrm{d}$ i.p. for 4 weeks). In the vehicle treated groups, pretreatment with $\alpha$-lipoic acid $(100 \mathrm{gm} / \mathrm{kg}$ i.v.) did not reduce ischemiareperfusion-induced leukocyte adhesion. In the $\alpha$-lipoic acid $(100 \mathrm{mg} / \mathrm{kg} / \mathrm{d}$ i.p. for 4 weeks) treated groups ischemia-reperfusion-induced leukocyte adhesion was reduced from $7550 \pm 1073$ to $1774 \pm 840(\mathrm{P}=0.0048)$ by $\alpha-$ lipoic acid $(100 \mathrm{mg} / \mathrm{kg}$ i.v. $) \alpha$-lipoic acid protects against ischemiareperfusion injury. In diabetic rats protection can only be achieved by continuous treatment with $\alpha$-lipoic acid. 
940

DIABETIC NEUROPATHY IN OTSUKA LONG-EVANS TOKUSHIMA FATTY RATS: EFFECT OF AN ANTICOAGULANT. N. Koh 1 , F. Sakakibara ${ }^{1)}$, J. Nakamura ${ }^{1}$, Y. Hamada 1 ), T. Hara ${ }^{1}$, H. Sasaki1), E. Nakashima 1), K. Naruse1), M. Kamijo ${ }^{2)}$ and N' Hotta ${ }^{1)}$, Nagoya ${ }^{1}$ and Hirosaki ${ }^{2)}$, Japan.

Hemorrheological abnormalities have recently received considerable attention as one of the pathogenesis of diabetic neuropathy. Otsuka Long-Evans Tokushima Fatty (OLETF) rats have been recognized as an animai model of human NIDDM. However, diabetic neuropathy in this model have not been studied well. In the present study, sucrose (S) was administered to OLETF rats for 6 months to establish diabetic neuropathy, and the effect of an anticoagulant, cilostazol (C) on it was investigated by measuring motor nerve conduction velocity (MNCV) $(\mathrm{m} / \mathrm{sec})$ and $\mathrm{Na}^{+} / \mathrm{K}^{+}$-ATPase activity $(\mu \mathrm{mol} \mathrm{Pi} / \mathrm{mg}$ protein $/ h$ ) in sciatic nerves. Morphological assessments including fiber size (FS) $\left(\mathrm{mm}^{2}\right)$ of sural nerves were also conducted. Sucrose-fed OLETF rats showed remarkable hyperglycemia compared with OLETF control rats of which blood glucose levels were not significantly different from those of their non-diabetic litter mates. Results are as follows ${ }^{*} p<0.05$ vs other experimental groups):

\begin{tabular}{lccc}
\hline & $\mathrm{MNCV}$ & $\mathrm{Na}^{+} / \mathrm{K}^{+}$-ATPase & $\mathrm{FS}$ \\
\hline OLETF & $44.5 \pm 0.7$ & $3.78 \pm 0.39$ & $52.9 \pm 0.7$ \\
OLETF $+\mathrm{S}$ & $32.3 \pm 1.0^{*}$ & $2.10 \pm 0.20^{*}$ & $42.5 \pm 1.2^{*}$ \\
OLETF $+\mathrm{C}$ & $43.2 \pm 0.7$ & $3.54 \pm 0.36$ & $48.7 \pm 1.3$ \\
OLETF + S + C & $43.5 \pm 0.6$ & $3.25 \pm 0.32$ & $49.1 \pm 2.0$ \\
\hline
\end{tabular}

Sucrose-fed OLETF rats also demonstrated a reduced sciatic nerve blood flow and an increase in ADP-induced platelet aggregation. These defects were ameliorated by treatment with cilostazol. 'These observations suggest that the sucrose-fed OLETF rat is an useful animal model for studying the pathogenesis of diabetic neuropathy in NIDDM from the viewpoint of vascular factors, and that cilostazol has an inhibitory effect on the development of this neuropathy.

\section{1}

ARI-TREATMENT INCREASES NGF-RECEPTOR (NGF-R) EXPRESSION IN REGENERATING DIABETIC NERVE.

\author{
A.A.F. Sima, A.C. Merry and 1. Levitan, Wayne State \\ University, Detroit, MI, USA
}

ARl-treatment promotes nerve fiber regeneration in human and murine diabetic neuropathy. To explore the mechanism underlying this treatment effect, the expression of NGF and its low affinity receptor (NGF-R) were examined in control and diabetic BB/W rats. Untreated and ARI-treated $(3 \mathrm{mg} / \mathrm{kg}$ WAY 509) control and 6 wk diabetic BB/W-rats underwent sciatic nerve axotomy. Non-axotomized, proximal and distal stumps of axotomized nerve were examined for expression of NGF by RTPCR and low affinity NGR-R expression by RNase protection assay in 5 animals each at 3,7 , and $14 \mathrm{~d}$ postaxotomy. No differences in NGF or NGF-R were found between nonaxotomized non-treated control and diabetic rats, whereas nonaxotomized ARI-treated control and diabetic rats showed increased NGF-R $(p<0.05)$ but no change in NGF expression. ARI-treatment significantly lowered NGF expression in control and diabetic distal stumps $3 \mathrm{~d}$ postaxotomy $(p<0.05)$ but no longer at $14 \mathrm{~d}$. NGF-R expression was increased 3-6-fold in ARItreated distal stumps 7 and $14 \mathrm{~d}$ postaxotomy in control and diabetic rats $(p<0.01)$. Long term $(8 \mathrm{mo})$ treatment with $A R \mid$ is associated with increased fiber regeneration in both diabetic and control BB/W-rats. We conclude that increased nerve fiber regeneration following ARI-treatment is in part due to upregulation of the NGF receptor.

\section{2}

EFFECTS OF SORBITOL DEHYDROGENASE INHIBITION ON TISSUE POLYOLS AND NERVE FUNCTION IN DIABETIC RATS

M.A. Cotter', M. Basso², T.C. Hohman'², N.E. Cameron'. 'Biomedical Sciences, Aberdeen University, Scotland, UK and 'Wyeth-Ayerst, Princeton, NJ, USA.

Polyol pathway activity is implicated in diabetic complications although the importance of sorbitol to fructose conversion by sorbitol dehydrogenase is uncertain. We aimed to assess the biochemical effects of 2 weeks treatment with a sorbitol dehdrogenase inhibitor (SDI), WAY135706 (250 mg kg' day $\left.{ }^{-1}\right)$ in 8 week streptozotocin-diabetic rats, compared with an aldose reductase inhibitor (ARI), WAY $121509\left(10 \mathrm{mg} \mathrm{kg}^{-1}\right.$ day') Sciatic nerve conduction and blood flow were also chosen as functional end points. Diabetes increased $(\rho<0.01)$ sorbitol levels (mear \pm SEM fold) in lens by $32.0 \pm 1.0$, sciatic nerve $12.6 \pm 0.8$, retina $9.2 \pm 0.6$, renal cortex $3.4 \pm 0.2$, renal medulla $3.1 \pm 0.4$ and red blood cells $3.4 \pm 0.3$. This was completely corrected $(p<0.01)$ by the ARI whereas SDI treatment further increased $(p<0.01)$ sorbitol in lens $(52.7 \pm 1.1$ fold vs nondiabetic

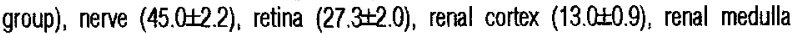
$(5.2 \pm 0.5)$ and red cells $(26.6 \pm 2.0)$. In nondiabetic rats, SDI treatment caused sorbitol elevations $(p<0.01)$ approximating those of diabetes in lens $(17.2 \pm 1.3)$, nerve $(11.7 \pm 0.3)$, retina $(6.8 \pm 0.5)$, renal cortex $(2.9 \pm 0.3)$, renal medulla $(2.6 \pm 0.6)$ and red cells $(6.4 \pm 0.5)$. Fructose concentrations were elevated by diabetes in all tissues $(p<$ 0.05 ), ranging from $12.0 \pm 0.7$ fold for retina to $1.6 \pm 0.2$ fold for red cells, and were reduced by both SDI and ARI treatments $(p<0.05$ ). Myo-inositol inversely correlated ( $p$ $<0.01)$ with sorbitol content for lens, nerve and renal cortex, in line with the complimentary osmolyte hypothesis, but this was not noted for retina, renal medulla or red cells. Sciatic nerve motor conduction velocity, $20.2 \pm 0.7 \%$ reduced by diabetes $(p<$ $0.01)$, was corrected by ARI $(p<0.01)$ whereas SDI was completely ineffective. A $48.6 \pm 2.9 \%(p<0.01)$ diabetic endoneurial nutritive blood flow deficit was $78 \pm 9.6 \%(p<$ 0.01 ) corrected by ARI but unaffected by SDI treatment. The increase in tissue sorbitol content with SDI treatment in nondiabetic and diabetic rats is potentially deleterious where osmotic stress has a role in dysfunction, for example lens. However, the data also stress the importance of the first half of the polyol pathway for nerve dystunction.

\section{3}

INHIBITORS OF SORBITOL DEHYDROGENASE AND ALDOSE REDUCTASE PREVENT IMPAIRED NERVE CONDUCTION IN DIABETIC RATS.

Y. Ido, P.J. Oates, B.L. Mylari, J. Burgan, M. Bache, A.P. Mizisin, and J.R. Williamson, Univiversity of California, San Diego, CA, Pfizer Inc., Groton, CT and Washington University, St. Louis, MO, USA.

The aim of this study was to compare the effects of inhibitors of sorbitol dehydrogenase (SDI; CP-166,572) and aldose reductase (ARI; Zopolrestat) on tibial motor nerve conduction velocity (MNCV), metabolites, and morphology in rats with streptozotocin diabetes (induced by iv injection of $50 \mathrm{mg} / \mathrm{kg}$ ) of 26 weeks duration. 5 groups of male Sprague-Dawley rats $(-300 \mathrm{~g}$ bwt) were prepared: $\mathrm{C}=\mathrm{controls}$, C+SDI, D=untreated diabetics, D+SDI, and D+ARI (12-14 rats each group). SDI $(250 \mathrm{mg} / \mathrm{kg} / \mathrm{day})$ and ARI $(100 \mathrm{mg} / \mathrm{kg} / \mathrm{day})$ were given in drinking water or ground chow, respectively. MNCV was measured every 6 weeks. Diabetes decreased weight gain $(603 \pm 39 \mathrm{~g}$ (mean $\pm \mathrm{SD})$ in $\mathrm{C}$ at 26 weeks vs $396 \pm 39$ in $\mathrm{D}, \mathrm{p}<0.0001$ ) and increased plasma glucose levels $(6.4 \pm 0.6 \mathrm{mM}$ in $\mathrm{C}$ vs $36.1 \pm 4.1$ in $\mathrm{D}, \mathrm{p}<0.0001)$. Neither SDI nor ARI affected weight gain or plasma glucose levels. At 26 weeks MNCVs were $62.5 \pm 4.6$ in $\mathrm{C}, 63.1 \pm 6.9$ in C+SDI, $50.9 \pm 4.0$ in $\mathrm{D}$ ( $\mathrm{p}<0.0001$ vs $\mathrm{C}$ ), $57.9+4.1$ in $\mathrm{D}+\mathrm{SDI}$ ( $\mathrm{p}<0.001$ vs $\mathrm{D}$ ), and $57.4 \pm 5.6$ in $\mathrm{D}+\mathrm{ARI}$ ( $\mathrm{p}<0.001$ vs $\mathrm{D}$ ). SDI increased sorbitol levels in $C$ and $D(118 \pm 36 \mathrm{nmol} / \mathrm{g}$ in $\mathrm{C}$ vs $1337 \pm 393$ in C+SDI, $\mathrm{p}<0.0001 ; 1223 \pm 284$ in $\mathrm{D}$ vs $4842 \pm 1052$ in $\mathrm{D}+\mathrm{SDI}, \mathrm{p}<0.0001$ ), while ARI reduced sorbitol $(51 \pm 25, \mathrm{p}<0.0001$ vs $\mathrm{C}$ or $\mathrm{D})$. myo-Inositol decreased slightly in $\mathrm{D}$ $(1525 \pm 321 \mathrm{nmol} / \mathrm{g})$ vs $\mathrm{C}(1937 \pm 468, \mathrm{p}=0.001)$, was unaffected by SDI in $\mathrm{C}$ $(2010 \pm 239)$ or D $(1387 \pm 367)$, and tended to increase slightly with ARI $(1738 \pm 322$ $\mathrm{p}=0.059$ vs D). Morphometry revealed no differences in axonal area $\left(12.5 \pm 1.79 \mathrm{\mu m}^{2}\right.$ in C) or diameter $(3.75 \pm 0.28 \mu \mathrm{m})$ among the groups. In conclusion, both ARI and SDI attenuated decreased MNCV in diabetic rats despite discordant effects on sorbitol levels. The findings that MNCV was not impaired and myo-inositol levels were not decreased in C+SDI, even though sorbitol levels were as high as in D, indicate that impaired MNCV in diabetic rats is linked more closely to increased oxidation of sorbitol to fructose than to changes in sorbitol and/or $m y o$-Inositol levels per se. 
944

THERAPEUTIC EFFECT OF BRLP-42 ON PERIPHERAL DIABETIC NEUROPATHY IN RATS

K. Bíró, A. Jednákovits, *T. Kukorelli, L. Korányi

BIOREX Res. \& Dev. Co., Veszprém, *Univ. Eötvös ¿oránd, Dept. of Comparative Physiology, Budapest, Hungary

Our aim was to determine whether therapeutic treatment with BRLP-42 (newly developed drug against diabetic complications; BIOREX, Hungary) could correct the deficit in nerve conduction velocity (NCV) and ischemic resistance (RICF) caused by streptozotocin diabetes. Diabetes was induced by STZ, $45 \mathrm{mg} / \mathrm{kg}$ i.v. in Wistar rats $(8$ groups, 8 animals in each group). Before and after one or two months duration of diabetes electrophysiological exaninations were done and normal saline or BRLP-42 treatment was introduced by $20 \mathrm{mg} / \mathrm{kg} /$ day p.o. or by $50 \mathrm{mg} / \mathrm{kg} /$ day p.o. for one or two months. Untreated diabetic groups of $1-, 2$, and 4-month duration as well as non-diabetic aged-matched control were also investigated. The therapeutic treatment with BRLP-42 was effective, irrespective of the duration of diabetes ( 1 or 2 months). There was no significant difference between the effects of the two doses applied. As a consequence of diabetic neuropathy in untreated animals MNCV and SNCV decreased by $24 \%$ and $26 \%$, resp. $20 \mathrm{mg} / \mathrm{kg} \mathrm{BRLP}-42$ reversed the motor and sensory deficits by $67.9 \%$ and $74.0 \%$, resp., $(\mathrm{p}<0.01)$ in 2-month diabetes. Giving the same dose over 2 month after 2 -month of untreated diabetes, similar inprovements $(61.1 \%$ and $61.0 \%$ $p<0.01$ ) of NCVs were obtained. Time for $90 \%$ nerve action potential reduction (T90) revealed about $82.0 \%$ or $61.0 \%(\mathrm{p}<0.01)$ increase in 2 or 4 -montl diabetes, resp., when sciatic nerve was exposed to ischemia. $20 \mathrm{mg} / \mathrm{kg}$ BRLP-42 slowed down the progression of RICF by $92.3 \%$ and $67.8 \%$, resp., $(\mathrm{p}<0.01)$. It is concluded that the therapeutic treatment was as effective as the preventive one against peripheral nerve dysfunction.

\section{6}

CORRECTION OF NERVE CONDUCTION VELOCTTY SLOWING BUT NOT NERVE BLOOD FLOW DEFICTT BY ACETYL-L-CARNTTINE IN STREPTOZOTOCIN-DIABETES. R.Pop-Busui, C.Van Huysen,L.Beyer,R.Towns,D.A.Greene and M.J.Stevens, Univ.of Michigan,Ann Arbor,MI,USA.

Reduced nerve blood flow (NBF), decreased nerve myo-inositol (MI) content and altered fatty acid (FA) metabolism have been implicated in nerve conduction velocity (NCV) slowing in diabetes (D).Therapeutic interventions aimed at correcting defects in FA metabolism in D improve NBF and NCV in part through a cyclo-oxygen-ase (COX) mechanism. Nerve acetyl-L-carnitine (ALC) depletion in D has been implicated in long chain FA accumulation and altered nerve energy metabolism and NCV. Nerve ALC repletion corrects Na,K-ATPase activity, transiently restores nerve MI levels and prevents NCV slowing in a dose-dependent fashion. This study evaluated whether these beneficial effects of ALC involved a COX-mediated vascular component. Sciatic motor NCV, NBF (by laser Doppler flowmetry), systolic blood pressure (BP) and Na,K-ATPase and MI levels were measured after 28 days in control rats (C), C rats given the COX-inhibitor Flurbiprofen (F) (6rng/kg/d) (C+F), C rats given F plus ALC ( $50 \mathrm{mg} / \mathrm{kg} / \mathrm{d})(\mathrm{C}+\mathrm{F}+\mathrm{ALC})$, D rats (D), D rats given ALC $(\mathrm{D}+\mathrm{ALC})$, $\mathrm{D}$ rats given $\mathrm{F}(\mathrm{D}+\mathrm{F}), \mathrm{D}$ rats given $\mathrm{F}$ plus $\mathrm{ALC}(\mathrm{D}+\mathrm{F}+\mathrm{ALC})$. BP did not differ among groups.

\begin{tabular}{lllcc} 
Group $(\mathrm{N} \geq 7)$ & $\mathrm{NCV}$ & $\mathrm{NBF}$ & ATPase activity & Myo-inositol \\
\hline C & $56 \pm 1$ & $201 \pm 9$ & $142 \pm 5$. & $4.6 \pm 0.1$ \\
$\mathrm{C}+\mathrm{F}$ & $49 \pm 2 * \dagger$ & $147 \pm 11^{*} \dagger$ & $64 \pm 5^{*}$ & $3.5 \pm 0.2^{*}$ \\
$\mathrm{C}+\mathrm{F}+\mathrm{ALC}$ & $54 \pm 11^{*} \dagger$ & $160 \pm 5^{*} \dagger$ & $143 \pm 1^{* *} \dagger$ & $3.7 \pm 0.1^{*}$ \\
D & $45 \pm 7^{*}$ & $139 \pm 9^{*} \dagger$ & $101 \pm 4^{*}$ & $2.8 \pm 0.1^{* \dagger}$ \\
D+ALC & $56 \pm 6^{* *} \dagger$ & $129 \pm 5^{*}$ & $130 \pm 4^{* * \dagger}$ & $2.8 \pm 0.2^{*} \dagger$ \\
D+F & $42 \pm 1^{*}$ & $115 \pm 7^{*}$ & $65 \pm 8^{*}$ & $1.8 \pm 0.1^{*}$ \\
D+F+ALC & $51 \pm 5^{* *+}$ & $121+9^{*}$ & $119 \pm 3^{*+}$ & $2.4 \pm .2^{*} \dagger$
\end{tabular}

${ }^{*} \mathrm{p}<0.05 \mathrm{vs} . \mathrm{C},{ }^{* *} \mathrm{p}<0.05 \mathrm{vs} . \mathrm{D}, \dagger \mathrm{p}<0.05 \mathrm{vs} . \mathrm{D}+\mathrm{F}, \dagger \dagger \mathrm{p}<0.05 \mathrm{vs} . \mathrm{C}+\mathrm{F}$.Data=mean \pm SEM $F$ decreased NCV,MI,Na,K-ATPase and NBF in C and lowered MI, Na,K-ATPase and NBF in D. In D, ALC corrected NCV and improved Na,K-ATPase without affecting NBF or neve MI.In D, F failed to block the effects of ALC on NCV, despite further deterioration in NBF. In D, ALC overcame the effect of F to cecrease Na,K-ATPase and MI levels. Therefore, the beneficial effects of ALC on NCV and Na,K-ATPase are not COX-mediated and are independent of NBF. Regulaton of nerve MI levels however involves a COX-dependent mechanism. NCV slowing in experimental D is not an inevitable consequence of reduced NBF.

\section{5}

PREVENTION OF NEUROPATHIC CIRCULATORY DYSFUNCTION BY BRLP-42 IN DIABETIC DOGS

László Jaszlits, Violctta Kćkesi* and Alcxander Juhász-`agy

Biorex Co., Veszprem and Dept. of Cardiovase. Surgery, Semmelweis Univ. Budapest

The effect of BRLP-42 (Biorex $\mathrm{Co}$.) on diabctic neurovegetative circulatory disturbances was studied in dogs. Diabetes was induced by alloxan $(300-400$ $\mu m 0 / / \mathrm{kg})$. BRLP-42 treatment started on the 7 th day after alloxan injection. Dogs were divided into four groups (n=6-7): control (C, healthy) untreated diabetic (D) and treated diabetic dogs (TI. $2 \times 2.5$ and TII $2 \times 5$ mg/kg/day BRLP-42 p.o.) After four wecks, all dogs werc anasthetized for acute testings. In group D total peripheral resistance (TPR) was moderately reduced compared to the other threc gronps, but basic homodynamic variables did not exhibit physiologically relevant differences among the four groups. The baroreflex sensitivity determined by the Oxford mothod ( $R-R$ intervals regressed against systolic pressure), was charactcrized by a significantly less stecp regression in the $D$ than in the other groups (C: $9.04 \pm 1.09$, D: $4.04 \pm 0.89$, TI: $7.85 \pm 0.51$, TII: $6.81 \pm 0.76$ Amsec/ $\triangle m m \mathrm{Hg} . \mathrm{p}<0.05$ ). The increases of TPR during the dircelly elicitcd carotid sinus hypertensive reflex were significantly smaller in the D group than in all other groups (C: $+24.6 \pm 5.3, \mathrm{D}:+9.4 \pm 3.7, \mathrm{TI}:+28.4 \pm 5.6$, TII: $+30.2 \pm 5.8 \Delta \%$ TPR, $\quad \mathrm{p}<0.05$ ). BRLP-42 treatment allgmented the diabetically reduced bradycardic component of the Bczold-Jarisch reflex (stimulation of cardiopulmonary afferents by veratrine) and that of the direct peripheral vagal stimulation. Systemic circulatory adaptation to a marked volume expansion (infusion of $20 \mathrm{ml}$ dextran) revealcd similar cardiac output changes $(\geq 200 \%)$ and similar summits of stroke work $\left(\geq 2.0 \mathrm{mkg}^{-1}\right)$ in all groups, but the drop in TPR was half in size in untreated diabctics than in other groups, (C: $-47.8 \pm 2.0, D$ : $-25.3 \pm 4.3, \mathrm{TI}:-45.3 \pm 3.7, \mathrm{TII}:-45.7 \pm 3.0 \Delta \%)$ which meant a significantly different $(\mathrm{p}<0.02)$ responsiveness of the $\mathrm{D}$ group compared to the healthy $(\mathrm{C})$ and treated (TI, TII) groups. Indices of coronary adaptation wcre similarly modificd. In conclusion, although diabctic cardiovascular dysfunction scems to be not very apparent at the carliest stage of the discase, nervous regulatory mechanisms for the acute adjustments of peripheral vessel tone and heart rate become considerably restricted. These neuropathic disturbances could be prevented by BRLP-42 


\section{Autonomic Neuropathy}

\section{7}

DIABETIC SMALL FIBRE NEUROPATHY; A SELECTIVE AND SPECIFIC DISORDER CAUSING SYMPTOMATIC AUTONOMIC NEUROPATHY

Ejskjaer $N^{\text {th }}$. Watkins $P J^{y}$. 'King's Diabetes Centre. King's College Hospital. London. United Kingdom. ${ }^{~ M e d i c a l ~ D e p a r i m e n t ~} M$ Andocrinologv \& Diabutes). Aarhus University Hospital. Denmark

Nerve function studies on five long standing insulin-dependent diabetes mellitus patients (age range 27 - 44 years, duration of diabetes $15-39$ years, all females) with severe symptomatic autonomic neuropathy (gustatory sweating 4 of 5 , gastroparesis 3 of 5 , diarthoea 4 of 5 , bladder paresis 2 of 5 , postural hypotension 3 of 5) and peripheral sensory neuropathy, show a most striking dissociation of peripheral sensory modalities, indicating the existence of a distinctive subgroup of the diabetic neuropathies, almost exclusively affecting small fibres. INVESTIGATIONS and RESULTS Cutaneous pressure perception (normal $<1 \mathrm{~g}$ ) was completely preserved for all patients and vibration perception thresholds relatively preserved: 13,2 $\pm 3,9$ (Mean \pm SD) volts, (normal < 12 volts), both of them large fibre modalities, - in comparison to the grossly abnormal small fibre modalities: All patients were anaesthetic to thermal sensory perception, being unable to detect any temperature change within the limits of the apparatus $\left(15^{\circ}-45^{\circ} \mathrm{C}\right)$. Autonomic function tests based on cardiovascular reflex responses were grossly abnormal; HR-variation on deep breathing, 3,20 $\pm 1,92$ (Mean \pm SD) beats $/ \mathrm{min}$, (normal $>10$ beats $/ \mathrm{min}$ ), HR increase on standing at 15 seconds; 7,4 44,93 (Mean \pm SD) beats $/ \mathrm{min}$, (normal > 12 beats $/ \mathrm{min}$ ) and postural BP - drop on standing: $22 \pm 10,2$ (Mean \pm SD) $\mathrm{mm} \mathrm{Hg}$, (normal $<10 \mathrm{~mm} \mathrm{Hg}$ ) IMPLICATIONS Clinical observations indicate an association between this distinctive smail fibre neuropathy and the development of symptomatic autonomic neuropathy

\section{8}

TIME DOMAIN ANALYSIS OF 24 H HEART RATE VARIABILITY IN NDDDM PATIENTS COMPARED TO HEALTHY CONTROLS

M.Brimnes Damholt,T.Melchior,J.Tof, V.Rasmussen and J.Hilsted Dept. of Endocrinology and Dept. of Cardiology,Hvidovre Hospital,Denmark. Patients with NIDDM have decreased bed-side tests of heart rate variability. The ain was to examine if time domaine analysis of $24 \mathrm{~h}$ heart rate variability is also reduced in NDDM patients compared to bealthy controls. During the period 1/10 1991 to 1/9 1993 all diabetic patients in our out-patient clinic were offered examination. This material comprises the first 139 patients examined.Patients with secondary diabetes were excluded. Of the remaining recordings 95 were of NIDDM patients, age $58 \pm$ $11($ mean $\pm \mathrm{SD}), 55$ men and 40 women. A subgroup of the "Copenhagen City Heart Study " comprising 130 healthy controls (age $57 \pm 15$ ), 83 men and 47 women, were also studied.The tapes were analysed using commercial systems. Reproducibility of the analysis was estimated in controls: Coefficient of variation was $1,7 \%$; in BlandAltmann plot $4 \%$ was outside $\pm 2 \mathrm{SD}$ - limits for interagreement difference. The tapes were analysed for time domain parameters. There was no significant difference in age between the groups. MEANN (mean value of RR intervals between normal beats) was $752 \pm 106 \mathrm{~ms}$ versus $828 \pm 110 \mathrm{~ms}$ in NIDDM versus controls (mean $\pm \mathrm{SD}$ ) ( $<<0,000005)$, SDNN(Standard deviation of the RR interval between normal beats) $111 \pm 38 \mathrm{~ms}$ versus $139 \pm 35 \mathrm{~ms}(\mathrm{p}<0,0000005$ ), SDNNIDX (Average of standard deviation of RR intervals between normal beats in all 5 min periods of the monitoring time) $40 \pm 14 \mathrm{~ms}$ versus $54 \pm 19 \mathrm{~ms}$ ( $\mathrm{p}<0,00000005$ ), SDANN(Standard deviation of the mean value of RR intervals between normal beats measured in all $5 \mathrm{~min}$ period of the monitoring time) $103 \pm 38 \mathrm{~ms}$ versus $125 \pm 33 \mathrm{~ms}(\mathrm{p}<0,000005)$, RMSSD (Root mean square of successive differences) $27 \pm 15 \mathrm{~ms}$ versus $32 \pm 21 \mathrm{~ms}(\mathrm{p}=0,055)$ and $\mathrm{pNN} 50$ (per cent RR intervals between normal beats that differ $50 \mathrm{~ms}$ or more from the preor succeding RR interval) $5,5 \pm 6,6 \%$ versus $7,8 \pm 8,8 \%(\mathrm{p}<0,05)$.In conclusion type 2 diabetes is associated with decreased $24 \mathrm{~h}$ heart rate variability which most likely is due to impaired autonomic nervous function including depressed vagal tone. This may contribute to the well known increase in mortality in these patients.

\section{9}

BREATHING AT 15 CYCLES/MIN TO DETECT VAGAL IMPAIRMENT IN DIABETES: SUPERIOR TO DEEP BREATHING AT 6 CYCLES/MIN? J Gerritsen ${ }^{1.2}$, JND de Neeling ${ }^{2}$, BJ TenVoorde', LM Bouter ${ }^{2}$ and RM Heethaar'. 'Dept. of Medical Physics and Informatics, ${ }^{2}$ EMGO Institute, Faculty of Medicine, Vrije Universiteit, Amsterdam, The Netherlands Breathing tests are used to assess autonomic dysfunction in diabetes mellitus (DM). Recent findings suggest that the Inspiration-Expiration RR-interval difference (IE-value, in ms) during deep breathing at 6 cycles/min reflects both vagal and sympathetic function, whereas a IE-value obtained at 15 cycles/min exclusively measures vagal function. As early autonomic dysfunction in diabetes is primarily parasympathetic, we hypothesized that in a general population a $15 / \mathrm{min}$ breathing test would show a stronger association with the degree of glucose tolerance than a $6 / \mathrm{min}$ test. Subjects $(n=514)$ were sampled from a 50-74 yr-old population and categorized as normal (NGT, $n=241$ ), impaired (IGT, $n=138$ ) glucose tolerant, newly-diagnosed (NDM, $n=76$ ) or known DM (KDM, $n=59$ ). IE-values based on 1 min of breathing in supine position were computed using the 'classical' time domain method and a new spectral analysis. $6 / \mathrm{min}$ IE-values computed classically and spectrally were highly correlated $(r=0.97) ; 6 / \mathrm{min}$ and $15 / \mathrm{min}$ IE-values correlated weakly but significantly $(r=0.56)$. Contrary to our expectations, regression analysis showed that, adjusting for potential confounders. $6 / \mathrm{min}$ IE-values were most strongly associated with glucose tolerance (see table $p$-values).

\begin{tabular}{llll}
\hline $\begin{array}{l}\text { Glucose } \\
\text { tolerance }\end{array}$ & IE 6/min & IE $6 / \mathrm{min}$ & IE $15 / \mathrm{min}$ \\
\hline classical & spectral & spectral \\
NDM vs. NGT & 0.060 & 0.049 & 0.445 \\
KDM vs. NGT & 0.004 & 0.011 & 0.293 \\
\hline W & 0.017 & 0.009 & 0.065 \\
\hline
\end{tabular}

We conclude that either, the $15 / \mathrm{min}$ IE-value has a less favourable signal-tonoise ratio with respect to detection of vagal impairment than the $6 / \mathrm{min}$ IEvalue, which is possibly due to smaller tidal volumes. or early diabetesrelated autonomic dysfunction is not as exclusively vagal as is commonly thought, and includes also sympathetic vasomotor components.

\section{0}

POWER SPECTRAL ANALYSIS (PSA) OF HEART RATE VARIABILITY (HRV AND FINGER-BLOOD PRESSURE VARIABILITY (FBPV) DURING HEAD UP TILT IN TYPE II DIABETICS WITH CARDIAC AUTONOMIC NEUROPATHY A. Mölle, J.Tank, K. Matthies, K. Plowka and M. Weck, Clinic Bavaria Kreischa, FRG

The aim of the study was to evaluate selective parameters for early diagnosis of sympathetic damage in type II diabetics (D) with cardiac autonomic neuropathy (CAN) in comparison with healthy volunteers (H). $7 \mathrm{D}(3 \mathrm{~m} / 4 \mathrm{f}$, age $54.7+7.8 \mathrm{yr}$, duration of diabetes $8.5 \pm 1.7 \mathrm{yr}, \mathrm{HbA} / \mathrm{c} 7.7 \pm 0.4 \%)$ and $12 \mathrm{H}(5 \mathrm{~m} / 7 \mathrm{f}$, age $58.2 \pm 6.4 \mathrm{y}$ $\mathrm{HbA}_{\mathrm{Ic}} 5.7 \pm 0.4 \%$ ) were investigated. Inclusion criterion for $\mathrm{D}$ was proven $\mathrm{CAN}$ (Ziegler score < 4/7). Methods: During head up tilt HRV and FBPV (PENAZvolume clamp technique) were investigated in the time and frequency domain Resuits (mean \pm SD): $* p<0.05$ H vs.D $\S p<0.05$ sup vs. upr

\begin{tabular}{|c|c|c|c|c|c|}
\hline HRV & H & D & $\begin{array}{c}\text { FBPV } \\
\text { (diastolic) }\end{array}$ & H & $\bar{D}$ \\
\hline \begin{tabular}{|l|} 
CV sup \\
.
\end{tabular} & $3.45 \pm 1.25$ & $1.90 \pm 0.73^{*}$ & CV sup & $4.71 \pm 2.11$ & $6.26 \pm 2.33$ \\
\hline LF sup $\%$ & $37.5 \pm 14.1$ & $53.7 \pm 11.3^{*}$ & LF sup $\%$ & $46.1 \pm 14.9$ & $67.1 \pm 13.2^{*}$ \\
\hline upr $\%$ & $37.8 \pm 16.4$ & $62.4 \pm 11.3^{*}$ & upr\% & $28.8 \pm 16.6 \S$ & $59.8 \pm 12.5^{*}$ \\
\hline MFsup $\%$ & $39.5 \pm 13.4$ & $18.9 \pm 6.3^{*}$ & MFsup $\%$ & $40.8 \pm 13.9$ & $20.4 \pm 7.3^{*}$ \\
\hline upr $\%$ & $43.9 \pm 14.5$ & $21.4 \pm 10.4^{*}$ & upr $\%$ & $58.1 \pm 14.7 \S$ & $24.1 \pm 11.8^{*}$ \\
\hline HF sup $\%$ & $23.0 \pm 11.0$ & $27.5 \pm 17.7$ & LFF sup $\%$ & $13.1 \pm 16.1$ & $12.5 \pm 7.3$ \\
\hline upr $\%$ & $18.2 \pm 11.7$ & $16.1 \pm 6.6$ & upr $\%$ & $13.1 \pm 11.2$ & $16.1 \pm 12.6$ \\
\hline
\end{tabular}

LF ... 0.015-0.05 Hz, MF ... 0.05-0.15 Hz, $\mathrm{HFF} \ldots 0.15-0.5 \mathrm{~Hz}$

sup ... supine, upr ... upright

Compared to $\mathrm{H}$ DM showed a reduced modulation of $\mathrm{HR}$ and FBP in the MFfrequency band. Conclusion: By PSA of FBPV during head up tilt an early determination of sympathetic damage is possible in D with CAN, whereas the reduced MF power during HRV might be due to combined parasympathetic and svmpathetic damage. 
951

PARASYMPATHETIC NEUROPATHY ASSOCIATED WITH CARDIAC DIASTOLIC DYSFUNCTION

G. Sundkvist, H. Nilsson, L. Öberg. B. Lilja, and R. Willenheimer. Departments of Endocrinology, Cardiology, and Clirical Physiology, Malmö University Hospital, Malmö, Sweden

The reason for the increased morbidity and mortality in cardiac complications in diabetic patients has to be clarified. The aim of the current study was to examine whether autonomic neuropathy was associated with cardiac dysfunction. To accomplish this, we evaluated 34 IDDM patients (age 40 years [15]; duration 21 years [14]) (median [inter-quartile range]) with regard to autonomic nerve function (heart rate variation during deep breathing: $E / I$ ratio = a sign of parasympathetic neuropathy; the immediate heart rate reaction to tilt: acceleration $[\mathrm{AI}]$ and brake indices $[\mathrm{BI}]=$ signs of sympathetic function) and cardiac function (echocardiography: systolic and diastolic [E/Amax and $\mathrm{E} / \mathrm{A}$ integral] ventricular functions). Eleven patients had signs of parasympathetic neuropathy (abnormal $E / \mathrm{I}$ ratio with and without abnormal AI and/or BI), 10 patients had sympathetic nerve dysfunction (abnormal AI and/or BI but normal $\mathrm{E} / \mathrm{l}$ ), and 13 were without signs of autonomic neuropathy. Patients with parasympathetic neuropathy showed significantly lower left ventricular $E / A_{\max }(1.1[0.2]$ vs. $1.4[0.7] ; p=0.022)$ and $E / A$ integral $(1.7[0.3]$ vs. $2.3[1.2] ; p=0.006$ ) than patients without autonomic neuropathy. Moreover, left ventricular E/A integral was significantly lower in patients with parasympathetic neuropathy compared with those with sympathetic neuropathy $(2.2[0.7] ; \mathrm{p}=0.009)$. In addition, right ventricular $\mathrm{E} / \mathrm{A}$ max was significantly lower in patients with parasympathic neuropativy than in those with sympathetic neuropathy (1.3[0.3] vs. $1.8[0.3] ; p=0.0001)$. In conclusion, parasympathetic neuropathy was associated with cardiac diastolic dysfunction. Autonomic neuropathy may contribute to cardiac dysfunction in diabetic patients.

\section{2}

HEART RATE VARIABILITY DISTURBANCES IN MICROAL BUMINURIC TYPE I DIABETIC PATIENTS.

M.Rigla. X.Garcia-Moll* X.Viñolas*. L.Dumaresq*, M.Garcia Moll* and J.M.Pou Endocrinology and Cardiology* Departments. H.Sant Pau. Barcelona. Spain.

To investigate the relationship between microalbuminuric state and the cardiac autonomic function, we studied 41 normotensive type I diabetic patients $(26 \mathrm{men}$, 15 women, mean age $33.6 \pm 8$ years. time from diagnosis $19.6 \pm 8$ years. HbAlc $7.5 \pm 1.4 \%$. blood pressure $124.8 \pm 16 / 76.4 \pm 10 \mathrm{mmHg}$ ). Twenty-one paticnts were microalbuminuric (MA) and 20 had normal values of albumin excretion rate (NA). mean albuminuria $149.8 \pm 44$ and $9.28 \pm 6 \mu \mathrm{g} / \mathrm{min}$ respectivelv. Both groups were similar in age. time from diagnosis. sex. \% of smokers. BMI. HbAlc. mean heart rate and Ewing test results. 24-hour Holter recordings were studied and normally corrected using commercially available Del Mar Software. RR interial oscillations were analysed by means of 1) statistical descriptions of $R R$ variation (SDNN index: standard deriation of normal RR internals; pNN50: \% of consecutive RR differing $>50 \mathrm{msec}$ ) and 2) measurements of the different frequency components in the $R R$ interval oscillations (LF: low-frequency component. mainly related to sympathoragal interaction: and HF: high-frecucncy component. a pure vagal signal modulated by the frequency of breathing). Range of frequency was $0.04-0.15 \mathrm{~Hz}$ and $0.15-0.40 \mathrm{~Hz}$ for $\mathrm{LF}$ and $\mathrm{HF}$ respectively. RESULTS: We have found a correlation between SDNN index and LFP $(p<0,001)$. Besides. pNN50 was related to HFP $(p<0,05)$. SDNN index. but not $\mathrm{pNN} 50$. was negatively associated with microalbuminuria $\left(\mathrm{R}^{2}=0.4 \mathrm{p}=0.001\right)$ even after adjusting for other related variables such as age and evolution time. MA patients had reduced SDNN index $(\mathrm{p}=0.003)$ and $\mathrm{LF}(\mathrm{p}<0.05)$. but no differences were observed in pNN50 or HF between both groups. CONCLUSION: Microalbuminuric type 1 diabetic patiens have impaired sympathoragal balance measured as SDNN index and LF, compated to normoalbuminutic ones
953

EARLY DETECTION OF AUTONOMIC DYSFUNCTION BY SPECTRAL ANALYSIS OF HEART RATE AND BLOOD PRESSURE VARIABILITY. PJ Westun. MA James, RB Panerai, PG MeNally, JF Potter, H Thurstun. Dept of Medicine and Therapeutics, Clinical Sciences Bulding, Leicester Royal Infirmary LEISWW.

The aim of this study was to assess heart rate and blood pressure variability in IDDM patients and compare this with traditional tests of autonomic function. A group of 30 patients with insulin dependent diabetes mellitus and 30 age, sex and $\mathrm{BP}$ matched control subjects underwent traditional tests of autonomic function. In addition. we assessed heart rate and systolic blood pressure variability using power spectral anaivsis. Data were collected non-invasively nsing the Finapres beat-tobeat blowd pressure recording system. Baroreceptor-cardiac reflex sensitivity was assessed from the resting hean rate and blood pressure data. Standard tests of autonomic function revealed no differences between IDDM patients and controls, but reductions in baroreceptor-cardiac reflex sensitivity were detected in IDDM patients. BRS in the diabetic group was $9.7 \pm 0.7 \mathrm{~ms} / \mathrm{mmHg}$ compared to $18.5 \pm$ $1.7 \mathrm{~ms} / \mathrm{mmHg}$ for the control group (mean $\pm \mathrm{SEM}$ ). Using a bandwidth of $0.05-$ $0.15 \mathrm{~Hz}$ as mid-frequency and $0.2-0.35 \mathrm{~Hz}$ as high frequency total spectral power of R-R interval was significantly reduced in the IDDM group for both mid. frequency $\left(473 \pm 62.8\right.$ vs $\left.746.6 \pm 77.6 \mathrm{~ms}^{2} \mathrm{p}=0.002\right)$ and high frequency bands $125.2 \pm 12.9$ vs $459.3 \pm 89.8 \mathrm{~ms}^{2} \mathrm{p}<0.0001$. No differences were found between the groups for spectral powers of systolic blowd pressure. Thus, spectral analysis of heart rate variability detects autonomic dysfunction more frepuently in IDDM patients than conventional tests. and is suggestive of an absormaliry of parasympathetic function. The abnormality of baroreceptor-cardiac reflex sensitivity could be explained by this impaimnent of parasympathetic finction.

\section{4}

TIME DOMAIN ANALYSIS OF BARORECEPTOR-CARDIAC REFLEX SENSITIVITY IN INSULIN-DEPENDENT DIABETES MELLITUS

I.G. Lawrence, P.J. Weston, R.D. Panerai, P.G. McNally, J.F. Potter, H. Thurston.

Department of Medicine and Therapeutics, Clinical Sciences Building, Leicester Royal Infirmary, Leicester. United Kingdom.

Sudden, unexpected death is well recognised in insulin-dependent diabetes mellitus (IDDM), buts its pathogenesis remains uncertain. The aim of the current study was to investigate the baroreflex sensitivity (BRS) in a group of IDDM patients with documented normal conventional tests of autonomic function and thus determine whether early abnormalities can be detected. Autonomic function had been assessed using the standard bedside cardiovascular reflex tests in 30 healthy IDDM patients and 30 age, sex and BP matched control subjects. BRS was assessed using time domain analysis of systolic blood pressure (SBP) and heart rate interval data, being recorded non-invasively using the Finapres system and a surface electrocardiogram. 'Up BRS' sequences (increases in SBP associated with lengthening of R-R interval) and 'down BRS' sequences (decreases in SBP associated with shortening of $R-R$ interval) were identified. However, BRS was impaired in IDDM patients independent of position: 'Up BRS' when supine was $11.2 \pm$ 6.5 vs. $20.4 \pm 14.3 \mathrm{~ms} / \mathrm{mmHg}$ (mean $\pm \mathrm{SD}, \mathrm{p}<0.003$ ) and standing $4.1 \pm 1.9$ vs. $7.6 \pm 3.7 \mathrm{~ms} / \mathrm{mmHg}(\mathrm{p}<0.001)$. 'Down BRS' when supine was $11.5 \pm$ 5.3 vs. $22 \pm 12.5 \mathrm{~ms} / \mathrm{mmHg}(\mathrm{p}<0.001)$ and standing $4.4 \pm 1.9$ vs. $7.3 \pm 3.6$ $\mathrm{ms} / \mathrm{mmHg}(\mathrm{p}<0.003)$. There were significant correlations between impairment of BRS and duration of diabetes $(p<0.001)$ and $\mathrm{HbA}_{1}$ $(p<0.001)$. Thus apparently uncomplicated IDDM patients demonstrate impaired baroreceptor-cardiac reflex sensitivity, which may in turn contribute towards the 'dead in bed syndrome' 


\section{5}

QTC PERIOD MEASURED BY A 24-HOUR HOLTER RECORDING SYSTEM IN DIABETIC AUTONOMIC NEUROPATHY.

N. Watzinger, M. Schumacher, B. Möstl, B. Semlitsch, B. Eber, F. Fruhwald, R. Zweiker, R. Pokan, W. Klein and T. Pieber. Dep. of Internal Medicine, Karl-Franzens-University, Auenbruggerplatz 15, 8036 Graz, Austria

Prolonged QT period and dispersion in a 12-lead surface ECG seems to be associated with the appearence of cardiac autonomic neuropathy.

We analyzed simultaneously QT-, QTc-values and different time-domain indices of heart rate variability in a 24-hour Holter ECG in 42 consecutive patients (19 males, 23 females; mean age: $38 \pm 12$ years, range: $20-70$ ) with type I diabetes meliitus. Duration of diabetes ranged from 1 week to 47 years. The Holter recording system was a Reynolds Pathfinder 700 version 4.02 . QT-time was measured in every recognizable single beat and corrected by the Bazett's formula. Mean values for every hour and for the complete recording time were calculated. Heart rate variability indices were SDNN, SDNNi, SDANN, St. George's index (TINN) for sympathetic and parasympathetic activities and sNN50, sNN6\% and RMSSD for parasympathetic activity. Mean values for every hour and for the complete recording time were available.

Mean QT-time was associated with mean hearte rate $\left(r_{s}=0.61, p<0.001\right)$, but with none of the heart rate variability indices. Mean QTc-time was weakly correlated with mean heart rate $\left(r_{s}=-0.37, p<0.02\right)$ and correlated with SDNN $\left(r_{s}=-0.48, p<0.002\right)$, SDNNi $\left(r_{5}=-055, p<0.001\right)$, SDANN $\left(r_{s}=-0.44, p<0.004\right)$ and TINN $\left(r_{s}=-0.44, p<0.004\right)$. The strongest correlation was seen with the parasympathetic indices sNN50 $\left(r_{5}=-0.59, p<0.001\right), \quad s N N 6 \% \quad\left(r_{5}=0.59\right.$, $\mathrm{p}<0.001)$ and $\operatorname{RMSSD}\left(\mathrm{r}_{\mathrm{s}}=-0.59, \mathrm{p}<0.001\right)$

We, therefore, conclude that QTc-time reflects parasympathetic disturbances rather than sympathetic abnormalities.
956

THE COLD PRESSURE TEST IN THE ASSESSMENT OF SYMPATHOPARASYMPATHETIC BALANCE IN DIABETIC PATIENTS

J. SIERADZKI, D.GALICKA-LATALA, A. SURDACKI*, J. DUBIEL*

Depts. of Metabolic Diseases \& ${ }^{*}$ Cardiology, Jagellonian Universiy, Cracow, Poland.

In 96 insulin dependent patients (mean: age 33.4 years, diabetes duration10.54 years! who received insulin as the only medication observations were carried out in 5 minutes periods: at rest $(R \mid$, after a 90-s cold pressure test (CPT). The following shortuterm heart rate variability (HRV) measures were assessed by a computer assisted technique (ProSciCard, Linden, Germany) standard deviation (SDR, SD-CPT), coefficient of variation (CVR, CV-CPT), roo mean square successive difference of RR intervals (RMSSD-R, RMSSD-CPT) expiration inspiration ratio $\{E / \mid$, mean circulant resultant $(M C R)$, Valsalva ratio (VR), max/min RR ratio on standing. Absolute and relative $|\%|$ spectral HRV power in 3 standard frequency bands [very low [(VLF) $\{0.01-0.05 \mathrm{~Hz}]$, low [ [LF 10.05-0.15Hz: high [HF $[0.15-0.5 \mathrm{~Hz}]$ was assessed by Fast Fourier Transform. The following groups were compared: without retinopathy (RO), with nonproliferative retinopathy $\{R \mid\}$, with proliferative retinopathy $\{[R 2\}$, as well as without $[\mathrm{NO})$ and with peripheral neuropathy $\{\mathrm{N} 1\}$ :

Results: The following parameters had the highest strength of discrimination between groups:

Ro vs. R1: VLF-CPT $(0.008)$, LFE-CPT(0.01), VLF-CPT/LF-CPT (0.08), HFCPT/LF-CPT 10.01$)$, total power-CPT 10.07 , LF-CPT/total power-CPT 10.002 R1 vs. R2: HR-CPT 10.09), HF-CPT/LF-CPT 10.001):

RO vs. R2: VLF-CPT $(0.008)$, LF-CPT 10.00005$)$, LF-CPT 10.011 , VLF-CPF/LF-CPT

$\{0.00005)$, HF-CPT/LF-CPT $(0.005)$, total power CPT $[0.002)$, LF-CPT/total-CPT 10.000005 , HR-CPT 0.000001$)$, RMSSD-CPT $(0.005) ;$

No vs. N1: HR-CPT 10.0002$)$, SD-CPT 10.0003$)$, VLF-CPT 10.002$)$, LF-CPT $10.0004)$ VL-CPT/LF-CPT 10.0005$)$ HF-CPT/LF-CPT 10.031 , total power $R$ [0.00003\}, total power CPT 10.003 ]. LF-CPT/total-CPT 10.002 , RMSSD-CPT $\{0.001\}$.

Conclusion: HRE after sympathetic stimulation may be helpful in screening for diabetic complications.

\section{7}

\section{CARDIOVASCULAR AUTONOMIC DYSFUNCTION IN INSULIN} DEPENDENT DIABETIC PATIENTS

Galickz-Latata, A Surdacki* 」. Dubiel* J. Sieradzki, Depts of Metabolic Diseases and Clinic of Cardiology, Jagellonian University, Cracow, Poland

Cardiovascular autonomic dysfunction (CAD) in diabetic patients results in various magnitude of decrease in spectral power in very low $\{0.01-0.05 \mathrm{~Hz}$. VLF]. low $(0.05-0.15 \mathrm{~Hz})$ and high $\{0.15-0.5 \mathrm{~Hz}\}(\mathrm{HF})$ frequency bands of hear rate variability (HRV). In 96 insulin dependent patients, who received insulin as the only medication observations were carried out in 5 minutes periods: at rest $(R)$, after 2 min of paced deep breathing $(A)$, at standing $0-5$ min (S) , 5-10 min (S2), $10.15 \mathrm{~min}$ (S3). The following short term HRV measures were assessed by a computer assisted technicue (ProsciCard, Linden, Germany): standard deviation (SD), coefficient of variation (CV), root mean square successive difference of RR intervals (RMSSD), expirationinspiration ratio (E/l, mean circulant resultant (MCR), Valsava ratio (VR) max/min ration on standing. Absolute and relative $(\%)$ spectral HRV power in 3 frequency bands were assessed by Fast Fourier Transform. The following groups were compared: without retinopathy [RO, $49 \mathrm{pts}$, mean: age -30.2 years, diabetes duration - 6.0 years), with nonproliferative [R], 24 pts, mean age -355 years, diabetes duration -13 . I yearsl, with proliferative retinopathy IR2 23 pts mean: age 38.2 years, diabetes duration - 17.5 years] as well as without peripheral neuropathy iNO, 63 pts, mean: age -30.3 years, diabetes duration - 7.66 years, and with peripheral neuropathy (N1, 33 pts, mean: age - 39.3 years, diabetes duration - 16.1 years!. Results: The following paramaters had the highest strength of discrimination between groups: Ro vs. R1: VLFS1 10.0005$)$. LFS 10.0004$)$, LFS2 10.004$)$, LFS3 $\{0.001\}$, HFS 1 $0.0003)$, HFS2 (0.0005), Total power S1 $(0.0001)$,R1 vs. R2: Total power $A$ $0.001\}$. VLA $\{0.005\}, \operatorname{LFR}\{0.007\}$, RMSSD-R $\{0.002\}$. RMSSD-A $\{0.003)$, Ro vs. R2. VLFR $(0.001)$ VLFA 10.00007$)$ VEF51 10.00008$)$, LFA 10.0002$)$. LFSI 0.0004 VLFALFA 10.0001$)$ VLFSI/LFS1 10.000051 . HFS $2 / L F S 2$ 0.00001 No vs. N1: LnVLFR $\mid 0.00001\}$, LnLFR 10.00001$)$. LnHFR 10.00001$)$ LnVLFA 10.00001 , LnLFA $10.00001 \%$ LnHFA 10.00001$)$, total power $R$ 10.00031 SDR 10.000011 SDA 10.000011 , SDS1 10.000011 , HRR 10.00001 . Development of both retinopathy and peripheral neuropathy is associated with marker changes in frequency domain HRV.
958

AUTONOMIC NEUROPATHY AND QTC-LENGTHENING IN IDDM AND IN NEWLY DIAGNOSED IDDM: THERE IS A RELATIONSHIP

P. Kempler, A. Marton, É. Kádár, K. Keresztes, Zs. Hermányi, P. Vargha and Gy. Tamas. 1st Dept. of Medicine, Semmelweis University, $\mathrm{H}-1083$ Korányi S. u. 2/a, Budapest, Hungary

Sudden death is not rare in IDDM patients with autonomic neuropathy (AN) in partly due to major arrhythmias and prolongation of the QT-interval is thought to be important in this respect. Aim of our study was to evaluate the relationship between AN and corrected QT-interval (QTCl). We examined 162 patients with IDDM (14-57 years of age, mean of 33,2 years, mean diabetes duration of 13 years) and 27 patients with newly diagnosed (ND)-DDM (14-38 years of age, mean of 23,4 years). QTcl has not been examined previously in patients with ND-IDDM. We evaluated heart rate responses to deep breathing, standing and Valsalva maneouvre as well as biood pressure responses to standing and sustained handgrip. QTcl was determined with Bazett's formula. $107 / 162$ patients with IDDM and 7/27 with ND-NIDDM had AN. Abnormal QTCl (>440 msec) was seen significantly more often in patients with AN compared to patients without AN ( $<<0,001$ in IDDM, $p<0,01$ in ND-NIDDM). Significant linear regression was found between severity of AN - based on the number of abnormal reflex tests on patient - and QTc-lengthening ( $p<0,001$ in both groups). Analysing the relationship between $\mathrm{QTCl}$ and the five reflex parameters separately, a significant negative correlation was found in the IDDM group between QTcl and heart rate response to deep breathing, 30/15 ratio, Valsalva ratio. sustained handgrip test (alt $p<0,001$ ) and blood pressure response to standing $(p<0,01)$; indicating that beside the previously established role of sympathetic imbalance even parasympathetic neuropathy contributes to the development of QTc-lengthening. Conclusions: autonomic neuropathy is associated with prolonged QTCl even in newly diagnosed IDDM. Follow up studies may reveal whether QTCl prolongation in these patients reflects a functional, reversible alteration or it is associated with increased cardiovascular risk. 
959

HEART RATE VARIABILTTY IN DIABETIC CHILDREN A. Üüvar ${ }^{*}$, N. Saka*, H. Kudat**,A. Tutar"*, and F. Korkut. ** * Department of Pediatric Endocrinology and ${ }^{\star *}$ Department of Cardiovascular Research Center, Istanbul Facuity of Medicine,Istanbul University,Istanbul, Turkey

In this study, holter monitoring were applied to 28 children $(14 \mathrm{M}, 14 \mathrm{~F})$ with insulin dependent diabetes mellitus to investigate the frequency of diabetic autonomic neuropathy(DAN).Mean age of the patients was $12.0 \pm 3.7$ years (range 5.5-17.8), duration of diabetes $4.0 \pm 3.6$ years(range 3 weeks-12 yrs), HbA1 $9.8+1.6 \%$ (range 7.4\%-13\%). The subjects were subdivided into three groups according to the duration of diabetes ( $<$ 1year, 1-5 yrs, and $>5 \mathrm{yrs}$ ), Nine $(5 \mathrm{M}, 4 \mathrm{~F})$ healthy, age-matched controls were also studied.Autonomic integrity was assessed with a technique of measuring heart rate variability(HRV) for $24 \mathrm{~h}$ ambulatory ECG recording by using two channel Marquette series 8000 Laser Holter SXP analysis system. HRV was measured by calculating six time-domain(normal cycle interval[NN] standard deviation of RR intervals [SDNN], mean of the standard deviations of RR intervals for all 5-minute segments[SDANN], mean of the standard deviations of successive 5 minute RR intervals [SD],percent of difference between adjacent $R R$ intervals that are greater than $50 \mathrm{~ms}$ [pNN50], root mean square of the differences of successive RR intervals[rMSSD]) and four frequency-domain (low frequency[LF], high frequency $[\mathrm{HF}$, total heart rate power spectra and LF/HF power ratio) indices. In diabetic children, parameters mainly indicative of parasympathetic disease including SD, rMSSD, pNN50, total heart rate power spectra, HF values were significantly lower $(p<0.05)$ and LF/HF ratio was significantly higher $(p<0.05)$ than the control group.No significant correlation was found between HRV and the duration of diabetes or HbA1 levels. The HRV values of three groups of diabetic children were also evaluated according to the \pm 2 SD values of the control group.Although DAN was more frequent in children with duration of diabetes $>5 \mathrm{yrs}$, even the newly diagnosed patients (duration of diabetes < 1year) showed pathologic results for some of the holter parameters.In conclusion. HRV measurements by using holter monitoring is easily performed in the early diagnosis of DAN.

\section{0}

Mortality in Diabetes Mellitus:

Effects of QT-Interval Prolongation

P. Diem, S.L. Suter, L. Zanchin, A. Teuscher

Bern, Zurich and Langenthal, Switzerland.

It has been suggested that diabetic autonomic neuropathy (DAN) resulting in QT-interval prolongation predisposes to cardiac arrhythmia and sudden death, and as a consequence leads to increased mortality. This hypothesis was tested in a subset of the Swiss Cohort of the WHO Multinational Study of Vascular Disease in Diabetes.

The first 100 patients recruited in the Swiss Cohort of the WHO study were studied. Baseline investigations were carried out in 1974/5. QT-intervals were measured on the baseline EKGs using a digitiser. QT was corrected for heart rate using Bazett's formula (QTc). Life/death status was assessed on January 1 1988. Three patients were lost for follow up. The underlying cause of each death was determined from a copy of the death certificate, autopsy and terminal illness reports, and other medical records. The underlying cause of death was coded according to the International Classification of Disease (ICD-9). Mortality overall was $26.8 \%$. from cardiovascular disease (CVD) $11.3 \%$ and from ischemic heart disease (IHD) $7.2 \%$. In the Cox proportional hazards model QTo was not an independent predictor of mortality from IHD ( $\mathrm{p}=0.87$ ). from CVD $(p=0.97)$ or overall $(p=0.16)$. However, when analysing patients with proteinuria at study entry separately, QTc was an independent predictor of overall mortality $(\mathrm{p}=0.02)$.

These data, obtained in a limited number of patients, indicate that DAN resulting in QT-interval prolongation is associated with increased mortality overall in patients with coexistent proteinuria. In patients without proteinuria QT interval prolongation is not associated with increased mortality.

\section{1}

Q-TC INTERVAL IN RELATION TO THE SEVERITY OF AUTONOMIC DIABETIC NEUROPATHY

N.Katsilambros, N. Tendolouris, F.Filippides, G.Papazahos, D.Papadogiannis, E.Stamboulis, D.Mitropoulos and K. Papageorgiou. 1st Department of Propaedeutic Medicine, Laikon Hospital and Department of Neurology, Athens University Medical School, 17 Aghiou Thoma str., GR-11527 Athens, Greece

Aim of the present study was to investigate to which extent the existence of objective signs of diabetic autonomic neuropathy (DAN) affects the corrected Q-T interval. Therefore 91 diabetic subjects (type 1: $n=45 ;$ type $2: n=46$ ) were examined using a battery of five autonomic function standardized tesis (Valsalva test deep breathing test heart rate and blood pressure responses from lyingto-standing and handgrip test). The studied subjects were classified in two groups according to the degree of abnormality of the autonomic function tests Igroup A: subjects with normal tests or borderline DAN (score 0-4) and group B: subjects with definite or severe DAN (score 5-10)]. The corrected Q-T interval $(Q-T c)$ was determined at rest with the Bazett's formula. Mean $( \pm S D)$ Q-Tc intervals were $390(+29) \mathrm{msec}$ and $412(+22) \mathrm{msec}$ for the groups $A$ and $B$ respectively $(p=0.002$ ). In adition, subjects with abnormal autonomic function tests (score $\geq 2$ ) presented a high rate $(16 \%)$ of pathologically prolonged Q-Tc interval ( $>433 \mathrm{msec}$ ). The respective rate in subjects with no signs of DAN was $0 \%$ $\left(x^{2}=15.28, p=0.001\right)$. Furthermore, there was no any significant relation between the duration of $Q-T c$ and type of diabetes as well as the other parameters

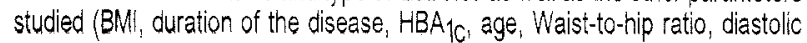
blood pressure). There was however a slight but significant positive relation between $Q$ - Tc interval and the systolic blood pressure $(t=2.51, p=0.013)$.

Conclusion: Q-Tc interval is prolonged only in diabetic subjects with relatively severe DAN.

\section{2}

POSTPRANIOIAL VARIATION OI: THII: QTC-INTIIRVAL IN INSUTIN-

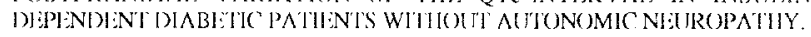
M. Iried, D.li. Schoors. M. De Smet, P'. Block and G. Somers. Departments of Intermal Medicine and Cardiology. Free Intiversily of Brussels, Belgium,

Diurnal variations of QT- and QTe-intervals, with lowest values during daytime but

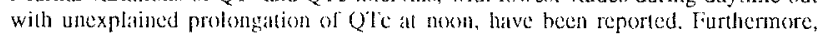
prolongation of O'Ie wils found in patlients with insulin-dependent diabete's molitus (IDDM) Cven in the absence of autonomic neurepathy (ANP) and was associated with a higher mortality risk. Present study aims it investigating the effect of a cooked lunch (1000) keali on RR-, QT- and QTe-intervall. Twelve lead ISCGis were laken in 20 IDDM patients (atge: $29.3 \pm 1.4$ yrs, diaheles duration: $14.9 \pm 1.5 \mathrm{yrs}, \mathrm{lb} \wedge$ Ic: $12.3 \pm 4 \%$ of the upper normal valuc: meitl $\pm S I M M$ ), without $A N P$ (according to the Ewing/Clarke crilerial) and in 33 anc-matched normal controls (NC). Resting ECGs were done from $1 \mathrm{~h}$ pre- 10,3 h postprandietly, every 30 min. QT-interval was correcled with Bayclt's (QTC) and verified by the tinear (Franningham) correction formula. Baseline RR- and QT-intervals were significintly (Mann-Whitncy U-test, $\mathrm{p}<0.01)$ lower in $\mathrm{ID}$ ) $\mathrm{M}$ patticnts $(871+22 \mathrm{msec}$ and $372+5 \mathrm{msec})$ then in $\mathrm{NC}$ (993 26 msec and $387 \pm 3$ msec). Pestprandially RR - and OT-interval decreatsed similarly in NC: and pallicuts. Ifowever, return to basethe wats significantly earlier in diabcles. In NC and palticuts. Q'Te increalsed $(+3.2 \pm 0.9 \%$ and $+3.4 \pm 0.6 \%$ respectively, Wilcoxon, $p<0.03)$ to a similar extent after the meal. In IDDM patients QTe renained clevaled for 120 min, whereas in NC it resurned to bascline after 60 min. In patients, these posiprandial changes were neiber influenced by duration of disease, nor by pose frandial glyeemia. Ifowever, the duration of QTe prodongation was

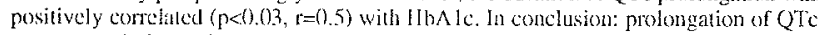
at mon and shortening of QT-and RR- intervals result from the ingestion of the meat. Such changes also occur in II)|MM palicuts withou ANP and the phenomenon is prokonged. This linding correlates pesilively with poor metabolic control. Whether these observations refect early atlomonic dystunction remains to be investigated 
963

MORBIDITY IN IDDM PATIENTS RELATED TO SYMPTOMS ON AUTONOMIC NEUROPATHY.

Elsebeth Hansen Duun, Kirsten Norgaard and Philip Hougaard* Steno Diabetes Center and Novo Nordic Statistic*, Denmark.

To study morbidity related to symptoms on autonomic neuropathy (AN) in a large unselected population of IDDM patients. A questionnaire was mailed to all IDDM patients attending the outpatient diabetic clinic at Steno Diabetes Center. All IDDM patients $(n=2247$ ). age 18-65 years with diabetes onset before age 36.1887 patients returned the questionnaire (ascertainment $=84 \%$ ). Mean age $39.6 \pm 11.5 \mathrm{yrs}$, diabetes duration $21.7 \pm 11.5$ years, mean $H D A 1 C$ C $8.5 \pm 1.3 \%$, sex-ratio $1.1(\mathrm{M} / \mathrm{F})$. The patients who did not answer were comparable regarding these data. Morbidity was scored as the number of hospital admissions during a period of 5 yrs, employment during the last year and reported days lost from work through sickness. Symptoms on AN was reported as palpitation without physical activity, orthostatic hypotension, abnormal sweating, gustatoric sweating and sweating localized to head and upper part of thorax. Symptoms on AN were listed for each patient as number of symptoms present.

\begin{tabular}{llll}
$\begin{array}{l}\text { Symptoms } \\
\text { on AN } \\
\text { (no) }\end{array}$ & Employment* & $\begin{array}{l}\text { Lost days } \\
\text { through } \\
\text { sickness } \\
11-30 \text { days } \\
\text { (per year) }\end{array}$ & $\begin{array}{l}\text { Hospital* } \\
\text { admissions(H.A) } \\
>3 \text { H.A }\end{array}$ \\
\hline 0.2 & $89.2 \%$ & $39.2 \%$ & $7.4 \%$ \\
$3-5$ & $10.4 \%$ & $26.9 \%$ & $20.0 \%$ \\
\hline
\end{tabular}

" $p<0.00001$ between groups

Conclusion: A significant increase in morbidity related to increasing symptoms on AN is demonstrated. Incidence and degree of hypoglycemia will be analyzed.

\section{5}

MICROCIRCULATION AFTER CONTRALATERAL COOLING IN PATIENTS WITH DIABETS MELLITUS WITHOUT NEUROPATHY

E. Haak, T. Haak, Y. Grözinger, G. Krebs, K. Kusterer, and K. H. Usadel, Medical Department I, University-Hospital Frankfurt/Main, Germany

In healthy volunteers cooling of the contralateral hand results in a rapid impairment of the ipsilateral capillary perfusion. In contrast, these findings are lacking in patients with diabetic neuropathy. In order to evaluate whether these changes in the reflex circuit might also be present in diabetic patients without neuropathy 12 patients with diabetes mellitus ( 6 female, 6 male, age $45.9 \pm 4.5$ Jahre, $\mathrm{HbA}_{10}$ $5.4 \pm 0.1 \%, 3$ smokers, 9 non-smokers) were studied. Twelve healthy volunteers $(6$ female, 6 male, age $37.5 \pm 3.1$ years, 2 smokers, 10 non-smokers, age $36.8 \pm 3.1$ years, $\mathrm{HbA}_{1 \mathrm{c}} 4.8 \pm 0.2 \%$ ) served as controls. Capillary blood cell velocity [CBCV] was measured continously by nailfold videocapillaroscopy over $2 \mathrm{~min}$ before and during the following $20 \mathrm{~min}$ after the contralateral hand has been immersed into a thermoregulated bath of $15^{\circ} \mathrm{C}$ for 3 minutes. Blood pressure and heart rate were monitored in 5-minute intervals throughout the investigation. $\mathrm{CBCV}$ at rest did not differ between patients and controls $(0.44+0.05 \mathrm{~mm} / \mathrm{s}$ vs $0.42+0.05 \mathrm{~mm} / \mathrm{s})$. While contralateral cooling resulted in a decrease in $\mathrm{CBCV}$ in controls $(0.29 \pm 0.05$ $\mathrm{mm} / \mathrm{s}, \mathrm{p}<0.03$ vs before cooling, $p<0.03$ vs patients) $C B \bar{C} V$ remained unchanged in patients. Blood pressure and heart rate did not alter during the investigation both in patients and controls. These results demonstrate that also in diabetic patients without clinical signs of diabetic neuropathy nerval reflex circuits are impaired. A long-term follow-up in a larger amount of patients is required to evaluate whether these findings might serve as a very early diagnostic tool for the diagnosis of developing diabetic neuropathy.

\section{4}

SILENT MYOCARDIAL ISCHEMIA OR AUTONOMIC NEUROP.ATHY AS THE CAUSE OF QTC DISPERSION IN NIDDM

L Walasik. J. Lcowski. J. Taton. Z. Szczeklik-Kumala. M. Chmielewski. A. Mancarz and JKuch. Department of Internal Diseases and Diabetology. Departinent of Cardiology. Warsars Medical School. Poland The study was aimed at elucidation which correlate: 1) silent myocardial 1schemua (SMl) or 2) autonomic neuropathy (AN) has the pathogenetic influence on QT dispersion in NIDDM.

For the investigation a) 56 male NIDDM patients. aged 40-64 ( $\mathrm{x} \pm \mathrm{SD}$ $54.9=6.1)$ years and b) 39 healthy control subjects. males aged 40-68 ( $\mathrm{x} \pm$ SD $-53.2 \pm 6.2)$ years vere qualified. All of them were hospitalized and subjected to the following studies: clinical examination. parameters of metabolic compensation ( $\mathrm{HbAlc}$. glvcenia and lipids profiles. serum electrolytes). resting ecg. exercise treadmill testing (ET) accordingly to Bruce protocol. tests for $\mathrm{AV}$ based on cardiovascular reflexes accordingly to Clark protocol. 12 leads ECGs were recorded at $25 \mathrm{~mm} / \mathrm{sec}$. RR and QT intervals were measured manually. QTc as mean from 12 leads was calculated by Bazett's formula. Dispersion of QTc was defined in ms. as the maximal difference between QTc in any of 2 leads. In comparison with the control group in NIDDM the statistically significant increment of QTC was observed: $x \pm$ SD $-41 \pm 21$ is $23 \pm 14: \mathrm{p}<0.0000$ l. although the QTc ralues remained similar: $x \pm$ SD $-418 \pm 17$ is $414 \pm 15$. In the NIDDM subgroup with SMI abnormalities of QTc were not encountered while in subgroup with $\mathrm{AN}$ the significantly higher values of QTe dispersion were tipicalls present: $x \pm S D-53 \pm 18$ is $38 \pm 20: p<0.05$

It could be concluded that in NIDDM without symptoms of coronary heart disease AN but not SMI impairs ventricular repolarization and increases QTc dispersion.

\section{6}

IS THERE AN ASSOCIATION BETWEEN AUTONOMIC NEUROPATHY AND CAI CIFICATION OF THE VAS DEFERENS AND ARTERIES?

T.S. Purewal, M.E. Edmonds, and P.J. Watkins. Diabetic Department, Kings College Hospital, LONDON SE5 9RS. In diabetes, tramline calcification of the arteries and vas deferens are well described. Arterial calcification is associated with diabetic autonomic neuropathy, but this has not been noted in the vas deferens. This study investigated a possible correlation between the two sites of calcification, which if present would point to a common aetiopathogenesis. Standard pelvic and foot radiographs were compared in 35 IDDM patients with severe autonomic neuropathy (DAN) and 19 longstanding IDDM controls (DC) free of all complications (DAN vs DC: age; $54 \pm 11$ vs $44.9 \pm 12.9$ years, $\mathrm{p}=0.02$, duration IDDM; $32.2 \pm 8.6$ vs $25.9 \pm 9.1$ years, $\mathrm{p}=0.03$, $\mathrm{HbA}_{1 \mathrm{c}} 8.1 \pm 1.6$ vs $7.2 \pm 0.9 \%, \mathrm{p}=0.04$, mean $\pm \mathrm{SD}$ ). Serum creatinine and smoking frequency were similar in the two groups (creatinine: DAN 89.0 \pm 20.4 vs DC $81.7 \pm 11.6$ moll $^{-1}$, normal $(N)<120$, $p=0.2$, smoking: DAN 7 of 35 : DC 5 of $19, p>0.5)$. Vibration threshold (DAN $26.7 \pm 10.8$ vs DC $-6.53 \pm 2.2, \mathrm{~N}<15.0$ Volts, $p<0.0001$ ), Marstock thermal threshold (DAN 19.2 \pm 4.4 vs DC $6.8 \pm 2.0, \mathrm{~N}<10^{\circ} \mathrm{C}, \mathrm{p}<0.0001$ ), and presence of retinopathy (DAN 20 of $35, D C O$ of 19) were higher, whilst heart rate variation to deep breathing (DAN $5.4 \pm 3.2$ vs DC $22.4 \pm 5.6, \quad N<15$ beatsmin ${ }^{-1}$, $p<0.0001$, and heart rate rise on standing were lower in the neuropaths (DAN $10.6 \pm 4.8$ vS DC $25.3 \pm 8.0, \quad N<15$ beatsmin ${ }^{-1}$, $P<0.0001)$. Vas deferens calcification was found in 3 DAN and $i$ control, foot arterial calcification in 17 DAN and 2 controls, whilst pelvic arterial calcification was evident in 17 DAN and 5 controls. Using the Chi squared test no significant associations were found for vas deferens calcification. We conclude that there is no association between arterial and vas deferens calcification that would help support a common aetiopathogenesis. 
967

DIASTOLIC DYSFUNCTION AND HEART RATE VARIABILITY IN PATIENTS WITH DIABETES MELLITUS TYPE $I$.

M. Schumacher, B. Möstl, B. Semlitsch, F. Fruhwald, R. Pokan, R. Zweiker, N. Watzinger, B. Eber, W. Klein and T. Pieber. Dep. of Internal Medicine, Karl-Franzens-University, Auenbruggerplatz 15, 8036 Graz, Austria

Diastolic dysfunction and a decreased heart rate variability are well known disorders in patients with diabetes mellitus type I. No data are available about the association of these two complications.

We analyzed echacardiographic estimates of systolic and diastolic function and ventricular dimension and different time-domain indices of heart rate variability in a 24 -hour Holter ECG in 42 consecutive patients (19 males, 23 females; mean age: $38+12$ years, range: $20-70$ ) with type I diabetes mellitus. Duration of diabetes ranged from 1 week to 47 years. The Holter recording system was a Reynolds Pathfinder 700 version 4.02. Heart rate variability indices were SDNN, SDNNi, SDANN, St. George's index (TINN) for sympathetic and parasympathetic activities and $\mathrm{sNN} 50, \mathrm{sNN} 6 \%$ and RMSSD for parasympathetic activity. Echocardiographic data were obtained by a Vingmed 800 (Diasonics).

A significant correlation was observed between the peak A-wave and heart rate variability indices and the ratio between peak early and late mitral filling velocities (E/A-ratio) and heart rate variability values. No correlation was found between heart rate variability indices and ejection fraction, fractional shortening, left ventricular dimensions and left ventricular mass index.

\begin{tabular}{|c||c|c|c|c|c|}
\hline & sNN50 & sNN6\% & RMSSD & SDANN & TINN \\
\hline \hline E/A ratio & & & & & \\
$\mathrm{r}_{\mathrm{s}}$ & 0.57 & 0.58 & 0.58 & 0.345 & 0.31 \\
p-value & $<0.001$ & $<0.001$ & $<0.001$ & $<0.03$ & $<0.05$ \\
\hline
\end{tabular}

Sex, duration of diabetes and age did not influence the correlation of parasympathetic indices and diastolic function in a multiple regression model We conclude that diastolic dysfunction is strongly correlated to abnormalities of the parasympathetic activities.

\section{8}

PLASMA PROINSULIN IS RELATED TO IMPAIRED AUTONOMIC FUNCTION EVALUATED BY POWER SPECTRAL ANALYSIS IN NIDDM JP Töyry, LK Niskanen, EA Länsimies, SM Haffner, HJJ Miettinen and MIJ Uusitupa. Kuopio University Hospital, Kuopio, Finland and University of Texas Health Science Center at San Antonio, Texas, U.S.A.

Immunoreactive insulin could predict the autonomic neuropathy, but the pathogenetic impact of its precursors is obscured. Therefore, 75 ( 36 men) NIDDM patients (age $66.8 \pm 5.8 \mathrm{yr}$, mean $\pm \mathrm{SD}$ ) and 108 (53 men) control subjects $(65.3 \pm 5.3 \mathrm{yr}$ ) were examined and the relation of specific insulin and proinsulin to impaired autonomic function at the 10-year study was examined. Quiet breathing test in the supine position was performed after 5 min resting period. The frequency domain analysis of heart rate variability was determined with spectral analysis from stationary regions of registrations. Total power in the frequency range from $0 \mathrm{~Hz}$ to $0.5 \times$ frequency equal to mean $R-R$ interval $(\mathrm{Hz})$ was divided into three frequency bands: low frequency band at $0-0.07 \mathrm{~Hz}$, medium frequency band at 0.07 . $0.15 \mathrm{~Hz}$ and high frequency band at $0.15-0.50 \times$ frequency equal to mean $R$ $\mathrm{R}$ interval $(\mathrm{Hz})$. Signal powers in low. medium and high frequency bands (LFP, MFP and HFP, respectively) were expressed in absolute units $\left(\mathrm{ms}^{2}\right)$. Also, MFP/HFP ratio (\%) (i.e. sympathovagal balance) was calculated. In NIDDM patients fasting proinsulin was inversely related to MFP/HFP ratio $(r=-0.324, p=0.014)$ and the same was true with control subjects $(r=-0.286$, $\mathrm{p}=0.003$ ). In control subjects fasting specific insulin was inversely related to MFP/HFP ratio $(r=-0.256, p=0.008)$ but this association was not significant in NIDDM patients $(r=-0.219, p=0.101)$. In NIDDM patients $(r=-0.291$, $p=0.028)$ and control subjects $(r=-0.228 . p=0.017$ ) fasting total immunoreactive insulin was inversely related to MFP/HFP ratio. For proinsulin the results did not change after controlling for the effects of age, body mass index and fasting glucose. To conclude, proinsulin markedly modulated the sympathovagal balance possibly by decreasing the sympathetic component of autonomic nervous function.

\section{9}

ROLE OF AUTONOMIC NEUROPATHY IN DEVELOPMENT OF NEPHROPATHY AND HYPERTENSION IN IDDM PATIENTS. A LONGITUDINAL STUDY.

V. Spallone, M.R. Maiello, E. Cicconetti, A. Barini, S. Gambardella, and G. Menzinger. Endocrinolgy, Tor Vergata University; Biochemistry, UCSC, Rome, Italy

To evaluate a possible role of autonomic neuropathy (AN) in the development of nephropathy and hypertension in IDDM, we studied 16 normotensive non-proteinuric IDDM patients: 6 with cardiovascular $\mathrm{AN}\left(\mathrm{AN}^{+}\right)$ (age: $33 \pm 11$, duration of disease: $21 \pm 4 \mathrm{yr}, \mathrm{HbA}_{1 \mathrm{c}}: 8 \pm 1.8 \%$ ), and 10 without AN (AN) (age: $39 \pm 11$, duration: $18 \pm 12 \mathrm{yr}, \mathrm{HbA}_{1 \mathrm{c}} 7 \pm 1.6 \%$ ). 24h ambulatory blood pressure (BP) and urinary albumin excretion rate (UAE) on urine collections timed day and overnight were measured simultaneously at baseline and after 4 years. Although baseline BP and UAE were similar in the two groups, after 4 years $24 \mathrm{~h}$ systolic BP, day, night and $24 \mathrm{~h}$ diastolic BP, night and 24h UAE were significantly higher in $\mathrm{AN}^{+}$than in $\mathrm{AN}^{-}$patients $24 \mathrm{~h}$ BP: $130 \pm 18 / 81 \pm 6$ vs $122 \pm 7 / 71 \pm 5 \mathrm{mmHg}$. $P<0.05$ : night UAE: $102(4-210)$ vs $3(1-123), 24 \mathrm{~h}$ UAE: $114(6-216)$ vs $9(2-118) \mu \mathrm{g} / \mathrm{min}, \mathrm{p}<0.05)$. Only $\mathrm{AN}^{+}$ patients showed a significant follow-up increase in $24 \mathrm{~h} \mathrm{BP}(116 \pm 11 / 75 \pm 5$ vs $130 \pm 18 / 81 \pm 6 \mathrm{mmHg}, p<0.05)$. Hypertension developed in 4 patients and was associated with baseline presence of microalbuminuria $\left(x^{2}=5.7, p<0.02\right)$. Follow-up increase in $24 \mathrm{~h}$ UAE was related to increase in $24 \mathrm{~h}$ diastolic $B P$ $(r=0.6, p<0.01)$. We performed a stepwise regression analysis including baseline values of age, diabetes duration, $\mathrm{HbA}_{1 \mathrm{c}}$, retinopathy, autonomic score (index of cardiovascular tests impairment), 24h UAE, and 24h BP as independent variables, and follow-up values of $24 \mathrm{~h} \mathrm{BP}$ or $24 \mathrm{~h} U A E$ as dependent variables. Using this model, we found that baseline autonomic score, $24 \mathrm{~h}$ systolic BP, and age were significant predictors of follow-up $24 \mathrm{~h}$ systolic BP; baseline autonomic score and retinopathy were predictors of follow-up 24h diastolic BP; baseline 24h UAE and autonomic score were predictors of follow-up 24h UAE. In conclusion, although microalbuminuria antedates the development of hypertension, and baseline UAE is the major determinant of final UAE, the independent relation of follow-up BP increase, final $B P$, and final UAE to AN, supports a contributing role of $A N$ in the development of hypertension and nephropathy in IDDM.

\section{0}

PLASMA ENDOTHELIN-1 LEVEL IS USEFUL FOR ASSESSING OF MYOCARDIAL AUTONOMIC NEUROPATHY IN DIABETES.

K. Murata, Y. Sumida, S. Murashima, K. Matsumura, H. Takeda, and T. Shima Third Department of Internal Medicine, Mie University School of Medicine, Bdobasti, T'su, Mie 514, Japan

The relationship between the degree of sympathetic denervation measured through ${ }^{123}$ I-MIBG Myocardial Scintigraphy and plasma endothelin-1 levels was investigated in order to evaluate their potential application for the assessment of myocardial autonomic neuropathy in patients with diahetes mellitus. This study comprised 20 Type 2 diabetic patients without coronary vascular disease and arterial hypertension. Plasma cndothelin- 1 levels werc measured using an immunoassay kit in supinc position. The myocardial uptake of ${ }^{123} 1-\mathrm{MIBG}$ was measured by using the single photon emission computed tomography (SPECT) and the early and delayed inages were recorded. Scoring of the ${ }^{123}$ I-MIBG uptake of seven cardiac segments from $0-3$ was performed and the total uptake was calculated. Plasma endothelin-1 levels were not significantly higher in diabetic patients $(186+-0.60 \mathrm{pg}, \mathrm{ml})$ than in a health group of subjects $(1.50--0.52 \mathrm{pg} \mathrm{ml})(\mathrm{n}=38)$ Plasma candothelin-1 levels obtained in the group with diahetic cardiac autononic neuropathy $D(x)$ Ni were significanty higher as compared to those of the $\mathrm{DC}$ AN $(-)]$ gromp $(2.14+0.39$

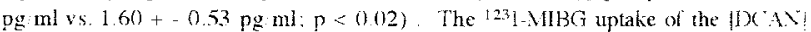
group was significantly lower as compared to those of the [DX A $(-)]$ group (I8.1 $3.56 \times 11.30+-3.77, p<0.12)$. The $[D C A N(+)]$ group was further separated intis two groups, one group with and another withour orthostatic hypotension IOHI] Plasma endothelin-1 levels were significantly higher in the $\mathrm{FOH}(+)]$ group than in the IOH(-)] group $(2.31+-0.60 \mathrm{pg}$ ml vs. $1.62+-015 \mathrm{pg} \mathrm{mL}: \mathrm{p}<0.05)$ The ${ }^{123 \mathrm{I}}$ VIBG uptake of the $[O H(+)]$ group was significantly lower than that of the $|0|(-) \mid$ group $(9+3+-3.60$ vs $16.5+-3.76 ; \mathrm{p}<0.001)$ There was a significant inverse correlation between plasma endothelin- 1 levels and the ${ }^{12}$ II $_{I-\mathrm{M}} \mathrm{MB}(\mathrm{r}$ uptake $(\mathrm{r}=-0.63, \mathrm{n}$ $<0.001)$. These results suggest that plasma endothelin-1 levels might be a useful marker for the assessment of myocardial autonomic neuropathy in patients with diabetes mellitus. 
971

CHANGES IN CARDIAC AUTONOMIC FUNCTION TESTS AND GLYCEMIC CONTROL

P. Valensi, O. Verier-Mine, S. Idriss, J. Pariès, E. Bertin, P. Fossati, M. Leutenegger and J.R Attali. Dept. of Diabetology, CHU, Bondy ; Lille and Reims. France.

The aim was to examine the changes in cardiac parasympathetic tests and the influence of glycemic control in a prospective study of 119 diabetic patients. Deep breathing (DB), lying to standing (LS) and Valsalva ( $\mathrm{V}$ ) tests were performed on these patients at baseline (T0) and after $29.0 \pm 8.9$ months (T1), and compared with a control group. At T0 early, confirmed or severe, cardiac autonomic neuropathy (CAN), defined by 1, 2 or 3 abnormal tests, was found in $33.9 \%, 9.8 \%$ and $4.5 \%$ of the patients respectively. Among the 45 patients free of CAN at T0, early or confirmed CAN appeared at T1 in 12 patients. Among the 27 patients with early CAN at T0, CAN was not found again at T1 in 11 patients whose $\mathrm{HbAlc}$ at $\mathrm{T} 1$ was significantly lower than in the 16 others $(\mathrm{p}=$ 0.038 ). In the 7 patients with confirmed $\mathrm{CAN}$ and the 4 with severe CAN at T0, the same CAN stage was found at T1. In the 12 patients with an abnormal $\mathrm{V}$ test at both $\mathrm{T} 0$ and $\mathrm{T} 1$ or whose $\mathrm{V}$ test became abnormal at T1, post-prandial glycemia at T0 was higher than in the 79 patients with a normal $V$ test at both $\mathrm{T} 0$ and $\mathrm{T} 1$ or whose $V$ test returned to normal at T1 $(p=0.06)$, and fructosamine was significantly higher at $\mathrm{T} 1(\mathrm{p}=0.031)$. In 25 patients, the LS test was.abnormal at both $\mathrm{T} 0$ and $\mathrm{T} 1$ or became abnormal at T1. Their fasting glycemia at T1 was higher than in the 80 patients with a normal LS test at both T0 and T1 or whose LS test returned to normal at $\mathrm{T} 1(\mathrm{p}=0.05)$. These data indeed suggest that poor glycemic control may participate in the deterioration of the cardiac autonomic function tests.

\section{2}

\section{EFFECT OF TOLRESTAT ON HEART RATE VARIABILITY IN} PATIENTS WITH DIABETIC AUTONOMIC NEUROPATHY.

\section{Didangelos, V. Athyros, A. Kontopoulos, A. Papageorgiou,} G. Kourtoglou, M. Avramidis, D. Karamitsos.

Divisions of Diabetology and Cardiology, 2nd Prop. Department of Internal Medicine, Aristotelian University, Hippocration Hospital, Thessaloniki, Greece. Background: Heart rate variability (HRV) time and frequency domain indexes are strong predictors of malignant arrhythmias and sudden cardiac death. Patients (pts) with diabetic autonomic neuropathy (DAN) have increased incidence of sudden cardiaa death compared with diabetics without DAN. Methods: The present double$b$ ind, randomised and placebo controlled study analysed the effect of toirestat $(T)$ on HRV time and frequency domain indices in pts with DAN. 30 pts (13 men and 17 women) of mean age 53 (range 19-68) years, free of coronary artery disease and arterial hypertension, were randomised into $T(n=15)$ or placebo $(n=15)$. HRV was assessed at months 0 and 3 . The parameters measured were: SDNN/24h = standard deviation of all $24 \mathrm{~h} \mathrm{R}-\mathrm{R}$ intervals, SDNN/5min $=$ mean of all 5 -min standard deviations of R-R intervals, rmsSD = root-mean-square of the differences of successive $R-R$ intervals, pNN50 = percentage of the R-R intervals differing more than $50 \mathrm{~ms}$, TP = total power, HFP = high frequency power, LFP = low frequency power VLFP $=$ very low frequency power. The $30 \mathrm{pts}$ were compared with 15 matched diabetics, of analogous glycemic control, without DAN and 15 healthy controls. Results : At baseline, HRV time and frequency domain indexes were lower than the ones of controls. At the 3rd treatment month, $T$ increased pNN50 and rmsSD $(p<0.05)$ and decreased the LFP. / HFP ratio $(p<0.05)$ in $9 / 15$ pts with mild DAN, in comparison to placebo. Despite the beneficial effect of T on parasympathetic variables of HRV, these remained significantly lower than the ones of diabetics without DAN and of healthy controls. Conclusion : Tolrestat significantly increases parasympathetic activity in pts with mild DAN 3 months after treatment initiation. This might contribute to the reduction of the risk for malignant ventricular arthythmias in these pts. Early diagnosis of DAN is essential for its successful management.
974

CAN A SPECIFIC NERVOUS OR VASCULAR IMPAIRMENT EXIST IN DIABETIC PATIENTS WITH ERECTILE DISORDERS EVALUA. TED BY RIGISCAN?

Marin N., Bax G., Fagherazzi C., Piarulli F., Cospite A. Grandis U., Fedele D. Dpt of Intemal Medicine, Metabolic Disease, Padova University (Italy)

Patients with diabetes have often erectile disorders. The pathogenesis of these disorders are complex and at present the exact mechanisms are not known. We evaluated in patients with diabetes if the nocturnal tumescence of penis (NPT) evaluated by Rigiscan is linked to autonomic and somatic nervous system or to vascular impairment. 26 diabetic patients (mean age $54 \pm 8.7 \mathrm{yrs}$ ) and with discrete metabolic control (mean $\mathrm{HbA} 1 \mathrm{c}=7.3 \pm 0.8 \%$ ) affected by erectile disorders were studied. We evaluated a) NPT with Rigiscan for 3 nights; b) diabetic autonomic test (LS;SL1, SL2, EI, VR, Squat sympatetic and parasympatetic test); c) Vibration Perception threshold at the base of penis (VPTBP) and the tip of penis (VPTTP); d) Brachial penis index basal (BPI) and after exercise (BPIAE). After NPT test we divided the patients into two groups: Group $A$ (13 diabetic patients mean age $55 \pm 8 \mathrm{yrs}$ and NPT at the base of the penis $<30 \mathrm{~mm}$ of circonference and duration of erection greater than 10 minutes): Groups B (13 diabetic patients mean age $53 \pm 1.9$ yrs and NPT greater than $30 \mathrm{~mm}$ and duration of erection $>10$ minutes). With the "Student't test" we found statistical difference between group $A$ and $B$ in LS $(1.0 \pm 0.95$ vs $1.2 \pm 0.16 ; p<0.02)$ SL1 $(1.08 \pm 0.08$ vs $1.2 \pm 0.2$; $\mathrm{p}<0.04)$ Squat vagal test $(1.06 \pm 0.24$ vs $0.8 \pm 0.2 ; \mathrm{p}<0.03)$ and autonomic score (7.6 \pm 5 vs $3.6 \pm 3 ; \mathrm{p}<0.03)$. There are no differences in VPTBP $(7.5 \pm 4 \mathrm{~V}$ vs $7.6 \pm 2$ V) and VPTTP (11.2 $\pm 7 \mathrm{~V} \mathrm{vs} 8.3 \pm 3 \mathrm{~V}), \mathrm{PBI}(1.04 \pm 0.11$ vs $1.01 \pm 0.17)$ and PBIAE $(0.86 \pm 0.24$ vs $0.94 \pm 0.16)$. In conclusion in diabetic patients the impairment of erectile function is linked only to autonomic nervous system disorders. The values of NPT $<30 \mathrm{~mm}$ and duration of erection less than 10 minutes, evaluated by Rigiscan,can be considered a marker of parasimpathetic impairment in the penile district. 


\section{5}

EMBOLIZATION OF DEEP DORSAL PENILE VEIN - WAY OF TREATMENT OF VASCULAR IMPOTENCE.

C.Oblak and M.Ravnik-Oblak. University Medical Centre Ljubljana, Slovenia.

Venous occlusive disease (VOD) is a frequent underlying condition in the organically caused male impotence, especially in diabetic patients. The aim of the study was to compare efficacy of embolisation of deep dorsal penile vein (method A) and use of the vacuum apparatus (mehod B) in diabetics (D) and nondiabetics (ND). Patients and methods: 40 patients with absent erection due to VOD were included. The four groups (Group AD: $n=10$, method A - D: Group AND : $n=10$, method A - ND Group BD $n=10$, method B - D; Group BND: $n=10$, method B ND) did not significantly differ as to the age of patients (AD: $39+/-12, \mathbf{A N D}$ : 40+/-12, BD: $37+/-11$, BND: $41+/-11$ yrs) and duration of impotence (AD: $1.9+/-$ 1.3, AND: $2.1+/-1.3$, BD: $1.5+/-0.8$, BND: $1.8+/-0.9$ yrs). The patients were followed up for 9 months. We did not advise them as to the frequency of intercourse, and patients AD and AND could use intracavernous injections of a vasoactive drug (IIVD). Patients described their present satisfaction with sexual life (questionnaire: points $0-6 ; 0$ : no satisfaction, 6 : full satisfaction) and so made their subjective evaluation of the success of the treatment. Results: $45 \%$ of patients treated with method $A$ had spontaneous erections, and $65 \%$ in case they used IIVD. There was no statistically significant difference between D and ND. All the patients using the vacuum apparatus had erection. The average frequency of intercourse did not vary in different groups (AD: 2, AND: 2.1, BD: 2.3, BND: 2.2 coitus/14days). D and ND were equally happy with their sexual life (AD: 4.8 , AND: 4.9 , BD: 5.2 , BND: 4.9 points). Conclusions: According to the results of the study, the success of method $A$ is comparable in $\mathbf{D}$ and ND. Method A is an effective way of treating VOD. In combination with IIVD, the results are even better. Patients reported equal satisfaction with their sexual life regardiess of the method of treatment. There was no difference between diabetics and nondiabetics. The age of the patients had no influence on the stccess of method $A$. No significant side-effects were noticed with either method. The decision on the method of treatment is made according to the patient's preference.

\section{PS 53}

\section{Somatic Neuropathy}

\section{6}

SUBCLINICAL PERIPHERAL NEUROPATHY MAY BE PRESENT AT ONSET OF IDDM IN HIGH RISK PATIENTS.

D.V. Coppini and P.H. Sönksen, Division of Medicine, St. Thomas' Hospital, London, U.K.

The aim of the study is to validate the usefulness of vibration perception thresholds (VPT) performed at time of diagnosis in identifying insulin dependent diabetic patients at risk of future neuropathy. Vibration perception thresholds were measured at the pulp of the great toes using a Bio-thesiometer in 37 insulin dependent diabetic patients at the time of diagnosis (1982-85), We have defined insulin dependent diabetes mellitus as ketosis at presentation or insulin requirement within 6 months of diagnosis. All patients (mean age at diagnosis $30.2 \pm$ 8.3 years; mean duration of diabetes at review $11.6 \pm 1.0$ years) have been reviewed in 1995 and vibration perception thresholds repeated. We have defined peripheral neuropathy as an abnormal toe VPT measurement (i.e. $>2$ s.d. above mean measurement for age.) Using this criterion, 1 patient $(2.6 \%)$ at time of diagnosis and 6 patients $(15.8 \%)$ at review had an abnormal VPT measurement. All but 1 patient (absent ankle jerks ) had a completely normal examination of the lower limbs at presentation. Patients who eventually developed peripheral neuropathy had a significantly higher VPT measurement at time of diagnosis (13.0 $\pm 5 .+$ volts is $7.5 \pm 2.4$ volts; $p=0.01$ ) and 1 patient developed end stage neuropathy (ulceration and charcot ankle joints.) There was no significant differcnce in mean age or height between patients in both groups. We conclude that although most IDDM patients $(97.4 \%)$ have a VPT within the normal range at the time of diagnosis, those who subsequently develop overt peripheral neuropathy have a higher VPT measurement at presentation. We conclude that subclinical peripheral neuropathy may be present at a very early stage in course of diabetes and vibration perception thresholds are useful in identifying patients at risk.

\section{7}

CLINICAL SIGNIFICANCE OF STANDARDIZED NEUROPATHY QUESTIONING.

A.M.L. Tjon-A-Tsien, S.M.A. Groenen, P.A. Grootenhuis, H.H.P.J. Lemkes, Leiden University Hospital, and I.E.M.R., Amsterdam, The Netherlands.

The diagnostic value of neuropathic complaints was assessed in patients with diabetes mellitus. Neuropathic complaints were evaluated with a questionnaire of 10 neuropathy specific questions, subdivided into 2 categories: Nsens (sensibility) and Npain. Clinical testing of neuropathy consisted of a modified neurological deficit score (NDS, score 0-38) and of measuring the vibration perception threshold with the Biothesiometer (BM). Results of the NDS were dichotomised on the basis of the results in non-neuropathic subjects (NDS $\leq 4=0,>4=1$ ) and results of BM-testing were dichotomised after age-correction. 89 patients with longstanding diabetes $\left(\right.$ age $=48.5 \pm 15.9 \mathrm{yrs}$, duration $\left.19.3 \pm 11.7 \mathrm{yrs}, \mathrm{HbA}_{\mathrm{cc}} 8.5 \pm 1.3 \%, 503^{3}, 61 \mathrm{IDDM}\right)$ were studied. $61.8 \%$ of the patients reported sensibility complaints and $42.7 \%$ pain. Most frequently reported complainis were tingling in the lower legs and feet $(37.1 \%)$, shooting pains in the legs $(33.7 \%)$ and numb feet $(32.6 \%)$. Clinical neuropathy testing revealed that $55.1 \%$ of the patients had an abnormal NDS-score and $31.5 \%$ an abnormal BM-score. Sensibility complaints significantly correlated $(\mathrm{p}<0.005)$ with clinical neuropathy (NDS 0.58 , BM 0.52). Painful symptoms les strongly cotrelated with clinical neuropathy (NDS 0.38, BM 0.39). In the following table the diagnostic values of this study are represented.

\begin{tabular}{llllllll}
\hline \multicolumn{1}{r}{ NDS: } & Nsens & Npain & Ntor & BM: & Nsens & Npain & Ntot \\
\hline Sensitivity & 65.3 & 30.0 & 63.3 & & 25.0 & 17.9 & 25.0 \\
Specificity & 80.0 & 70.0 & 77.5 & & 91.8 & 95.1 & 95.1 \\
LR $^{+}$ & 3.27 & 1.70 & 2.81 & & 3.0 & 5.63 & 5.08 \\
Odds ratio & 7.53 & 2.43 & 5.93 & & 3.73 & 4.20 & 6.44 \\
Accuracy & 71.9 & 59.6 & 69.7 & & 70.8 & 70.8 & 73.0
\end{tabular}

Conclusion: The frequency of neuropathic complaints in this population was much higher than expected on the basis of neurological examination and biothesiometry. Use of a standardized questionnaire is a simple tool in screening for diabetic neuropathy. 


\section{8}

SENSITIVITY AND SPECIFICITY OF SIGNS, SYMPTOMS AND VIBRATORY PERCEPTION: MULTICENTRE STUDY ON DIABETIC NEUROPATHY

S. Gentile and S. Turco for the Southern Italy Multicentre Study on Diabetic Neuropathy, Naples, Italy

BACKGROUND: The criteria so far proposed for the diagnosis of diabetic peripheral neuropathy (DPN) and based on both clinical and instrumental procedures attain an accurate diagnosis but are rather complex and require the use of cumbersome and time-consuming methodologies. In addition, the confirmation of the diagnosis by electroneurography (ENG) is often unsuitable for the majority of diabetic peaple. AlM of the study was to evaluate the sensitivity (ST) and the specificity (SP) of signs (SI) symptoms (SY) and vibratory sensations (VPT) of simmetrical DPN in comparison with ENG. PATIENTS: we studied 204 type 2 diabetics (89M, $115 \mathrm{~F}$ ), age $58 \pm 9$ years, diabetes duration $9 \pm 7 y$, BMI $27.3 \pm 4.4$, fasting glycemia at the moment of the testing (FG) $154 \pm 14.5 \mathrm{mg} / \mathrm{dl}$, HbA1c $8.1 \pm 1.8 \%$; patients with DN depending on other causes, under drugs influencing DPN, or with $F G>200 \mathrm{mg} / \mathrm{d}$ l during tests were excluded. DPN was diagnosed by ENG (Medelc Sapphire 1500) according to S Antonio Consensu's Conference. METHODS, we evaluated: 1) SI by the questionnaire (Q) proposed by the Study Group of the Italian Soc. Diabetol. (SID) in $1983 ; 2$ ) SY by the neurological Symptom Score and the Neurologiacal Disability Score according to Dyck in 1988 (NE); 3) VPT by Biothesiometer VPT3. A single neurologist performed all tests to avoiding intra-observers variability, In 26 patients $(P, 15$ with and 9 without $D N)$ and 15 comparable controls (C) the variability coefficient (VC) was evaluated. RESULTS, the VCs were: for $Q(C=15.5 \%, P=21.21 .6 \%), N E(C=3.3 \%$ $P=5 \%$ ) and VPT (allux: $C=12.5 \%, P=16.5 \%$; maleolus: $C=14.4 \%$, $P=18.6 \%$ ). DN was diagnosed by ENG in $47 / 204$ patients $(14.3 \%$ were ssymptomatic). ST and SP were $87 \%$ and $59.8 \%$ for Q $89.3 \%$ and $92.7 \%$ for NE, and $97.4 \%$ for VPT, respectively. CONCLUSON, based on these results, the scored NE seems to be an useful screening method for the simmetrical DNP, that is considred the most common form of DPN, whwreas $Q$ and VPT exhibited a too large VC and an unsatisfactory senitivity and specificity vs ENG
979

Comparison of different clinical methods for diagnosis of diabetic peripheral neuropathy (DPN) with 768 hospitalised patients

B. Mertes, U. A. Müller and K. Höffken. Klinik für Innere Medizin II, Friedrich-Schiller-University, Jena, Germany

Introduction: DPN is a common diabetes related complication. One reliable and economical method for diagnosis is Young and Boulton's neuropathy symptom (NSS) and disability score (NDS) [Diabetologia 1993, 36: 15]. However, it is a time consuming test compared with conventional clinical tests. The aim of this study is to compare the result of conventional clinical tests for DPN diagnosis with the results of the NSS and NDS. Patients and Methods: $A$ tota of 768 patients were studied consecutively (age $54,3+/ .18,3$ years: duration of diabeles $10,4+/-9$ years, IDDM $28,6 \%$, NIDDM 71,4 $\%)$. The diagnosis DPN was made, when the following symptoms or signs were found: $\mathbf{A}$ : presence of paresthesia, absence of ankle reflex and C 64 tuning fork score (TFS;) smaller than $6 / 8$ at the great toe; B: like A, but Liniger and Assal's age-related TFS; C: absence of ankle reflex and TFS; smaller than 6/8 at the great toe only; D: Iike C, but Liniger and Assal's age-related TFS; E: experience of pain or discomfort (NSS) and examination of ankle reflex, vibration pin-pick and temperature (NDS). Results: The prevalence of DPN varied strongly depending fom the applied method $(E-37,6 \% ; C$ $30,1 \% ; A 19,1 \% ; D 16 \%$ and $B 11,1 \%$ ). In comparison to method $E$, the results of methods $A$ to $D$ are seldom false positive (C $2,9 \%$. $A 0,1 \% B$ and $D 0 \%$ ), however, fals negativ results are frequent ( $B$ $26.6 \%$; D 21,6\%; A 18,6\%; C 10,4\%). Conclusion: Since fals negativ results are frequent, conventional methods for diagnosis of DPN are not suitable. By using NSS and NDS it is possible to improve the diagnosis of DPN. This would help to reduce the high incidence of foot uncerations and amputations in diabetic subjects.

\section{0}

THE SIGNIFICANCE OF THE DURATION OF CLINICAL SYMPTOMS FOR CARDIAC AUTONOMIC AND PERIPHERAL SENSORY NEUROPATHY IN NEWLY-DIAGNOSED IDDM

G. Krob, O. Schnell, A. Ruf, E. Standl and M. Haslbeck. Diabetes Research Institute and Schwabing City Hospital, Munich, Germany

Diabetic neuropathy has been observed even at diagnosis of insulindependent diabetes mellitus (IDDM). The aim of the study was to evaluate the presence of cardiac autonomic and peripheral sensory neuropathy in newly-diagnosed IDDM with regard to different duration of preceeding typical symptoms of manifestation (SOM) in three groups of diabetic patients: A) SOM 0 - 2 weeks $(n=33 ; H b A 1 c 12,7 \pm 2,5 \%$; age $27 \pm 7,5$ years; BMI $22 \pm 3,3$ $\tilde{x} \pm S D$ ) B) SOM $3-6$ weeks ( $n=26$; HbAlc $11,8 \pm 2,5 \%$ : age $29 \pm 7,5$ years:

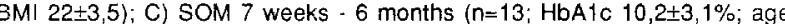
$27 \pm 6,6$ years; $B M I 22 \pm 3,4$ ). Five cardiac reflex tests were used for evaluation of cardiac autonomic neuropathy (CAN): Heart rate variation at rest and during deep breathing (index: coefficient of variation (CV)), Valsalva maneouvre, 30/15 ratio and systolic blood pressure response (age-related normal ranges). Peripheral sensory neuropathy was assessed with quantitative vibration- and thermal-thresholds (age-related normal ranges) Neurological examinations were performed within the first 2 weeks of intensive insulin therapy. CAN (2 or more abnormal reflex tests) were obeserved as follows: group A $1 / 33(3 \%)$, group B $2 / 26(8 \%)$ and group C $2 / 13(15 \%)$. In the entire group of diabetic patients $7 \%(5 / 72)$ were found to have CAN whereas none of the patients demonstrated peripheral sensory neuropathy. All newly diagnosed IDDM patients with CAN had an abnormal CV of heart rate variation at rest. $3 / 5$ patients with CAN demonstrated an abnormal CV during deep breathing. The presence of CAN was riot associated with $\mathrm{HBA} 1 \mathrm{C}, \mathrm{BMI}$, age or sex. The results suggest that the prevalence of CAN in newly diagnosed IDDM is higher in patients with a longer duration of preceeding clinical symptoms of manifestation. Whether the observed changes in cardiac autonomic function are reversible with improved metabolic control is to be determined by follow-up investigations.

\section{1}

RELATIONSHIP BETWEEN DIABETIC NEUROPATHY, Na/K ATPASE ACTIVITY AND ATP1 A1 GENE POLYMORPHISM.

P. Vague, D. Dufayet, T. Coste and D. Raccah. CHU Timone, Marseille, France.

The diabetes induced impairment of $\mathrm{Na} / \mathrm{K}$ ATPase activity in men and animals may play a role in development of diabetic neuropathy. A genetic susceptibility could explain inter individual variations in predisposition to neuropathy. $\mathrm{Na} / \mathrm{K}$ ATPase possess three isoforms. Alpha 1 isoform, predominant in nervous tissue and red cells is encoded by the ATP1 Al gene. A RFLP is detected by Bgl II restriction enzyme on the first intron. In a series of 71 insulin dependent patients with more than 10 years of diabetes(m \pm sem $=21 \pm 3$ ), red cell $\mathrm{Na} / \mathrm{K}$ ATPase activity, ATP1 A1 polymorphism were studied in relation to diabetic complications. 43 patients were homozygote for the non restricted allele, 1 for the restricted and 27 heterozygote (allele frequency $0.80 / 0.20$ ). Red cell ATPase activity which was lower in IDD patients than in 25 controls $(283 \pm 8$ vs $410 \pm 15 \mathrm{nmol} \mathrm{Pi} / \mathrm{mg}$ prot/h $\mathrm{p}<10^{-4}$ ) was not related to sex, age nor $\mathrm{HbAlc}$ value. A genotype phenotype relationship was observed, ATPase activity being lower in the 28 IDD patients bearing the restricted allele that in those without $(237 \pm 11$ vs $313 \pm 9 \mathrm{p}<10^{-4}$ ). Neuropathy was absent in 41 patients, mild in 12 , severe in 18. In these 3 groups, mean ATPase activity was $311 \pm 10,263 \pm 14,227$ \pm 15 respectively, $\left(\mathrm{p}<10^{-3}\right)$ and the prevalence of the restriction site 7,75 and $89 \%$. The relative risk conferred by the presence of the restriction site was 7.7 (95\% CI, 5.4-11). ATPase activity and RFLP were not associated to nephropathy. A borderline association was observed with retinopathy and disappeared after controlling for neuropathy. A genetic polymorphism of the $\mathrm{ATP} 1 \mathrm{~A} 1$ gene is associated with variation in red cell $\mathrm{Na} / \mathrm{K}$ ATPase activity. Presence of the restriction site detected by $\mathrm{Bgl} \mathrm{II}$ enzyme on the first intron is a major risk factor of neuropathy in IDD patients. 
982

IS THERE CONNECTION BETWEEN LOWER LIMB BLOOD FLOW, AUTONOMIC AND SENSORY NEUROPATHY, SERUM ENDOTHELIN ACTIVITY AND OSTEOPOROSIS IN DIABETIC PATIENTS?

A. Marton, K. Keresztes, Zs. Hermányi, P. Lakatos, Cs. Horvath, L. Tamási, A. Fazakas, M. Zempléni and P. Kempler, Ist. Dept. of Medicine, Semmelweis University, Budapest, Hungary

We suppose that as a consequence of arteriovenous shunting, autonomic imbalance may cause distal perfusion disturbance of the lower extremities, that leads to distally predominant lower osteodensity. Higher serum endothelin level has been observed to be connected to vasoconstriction, neuropathy and osteoporosis in previous studies. The aim of our study was to investigate the connection between autonomic and peripheral neuropathy, lower limb blood flow, osteodensity and serum endothelin activity in diabetic patients. We examined 33 patients (mean age: 54,7, range: $23-81$ years, mean diabetes duration: 12.0 range: $0-26$ years, NIDDM: 22, IDDM: 11). Autonomic neuropathy was characterized with the five standard cardiovascular reflex tests, peripheral nerve function was studied by measurement of the current perception threshold (CPT) using Neurometer $\otimes$ (Neurotron Inc., Baltimore, MD.), lower extremity blood flow was evaluated with arterial Doppler examination, osteodensity was examined with dual fotonabsorptiometry and ultrasound osteodensitometry, serum endothelin activity was determined with radioimmunoassay. Decreased lower limb blood flow correlated significantly with parasympathetic $(p=0.004)$ and sympathetic parameiers $(p=0.007)$, CPT results $(p=0.029-0.004)$, and serum endothelin leve $(p=0.03)$. The severity of parasympathetic neuropathy correlated significantly with some parameters of osteodensity $(p=0.021-0.006)$, just as the serum endothelin level with sensory neuropathy $(p=0.026)$ Conclusion: Our results appear to support our hypothesis: decreased lower extremity blood flow, lower osteodensity, autonomic and peripheral neuropathy and higher endothelin level seem to be associated with each other and may play a role in díabetic foot syndrome.

\section{4}

\section{MOTOR ULNAR NEUROPATHY IN DIABETES}

BB Abuaisha, W Schady and AJM Boulton. Manchester Royal Infirmary Hospital, University of Manchester, UK

It is well known that mononeuropathies can occur in patients with diabetes, sometimes in the absence of a clinically significant background polyneuropathy. Though the ulnar nerve is often mentioned as potentially vulnerable, there are surprisingly few detailed descriptions of ulnar neuropathy in diabetic patients. which is thought to be as a result of compression in the cubital tunnel. We have identified 20 diabetic patients with the clinical features of a motor ulnar neuropathy ( 16 men, aged 39-77, 7 insulin-dependent, mean duration of diabetes 22 years). All but one had long term complications, mostly severe: 10 were amputees, 4 had a renal transplant and 2 were blind. They all had a high Neurological Disability Score. The onset of hand weakness and wasting was sudden in 5 , often after an operative procedure. All patients had a classical "ulnar hand" (bilaterial in 15) but forearm muscles were little affected. Sensory loss was prominent in only one half. On nerve conduction studies, ulnar compound muscle action potentials (CMAP) were markedly reduced (mean 1.2 $\mathrm{mV}$ vs $7.4 \mathrm{mV}$ in controls) as were ulnar/median CMAP ratios. Motor conduction was disproportionately slowed across the elbows, with or without conduction block, in $8 / 34$ affected ulnar nerves. Five of these patients had a habit of learning on their elbows and/or a Tinel's sign. Median sensory action potentials were record able in 12 patients but ulnar SAPs were absent in 30/34 affected nerves. Electromyography revealed advanced denervation of ulnarsupplied hand muscles. We conclude that motor unar neuropathy is not uncommon in diabetes, especially in those with long term complications. Nerve entrapment at the elbows may occur in some, but in most cases the lesion is axonal and probably ischaemic and ulnar nerve decompression is not indicated in most cases.
983

EFFECTS OF PANCREAS GRAFT FAILURE ON EVOLUTION OF DIABETIC POLINEUROPAHY

S. Martinenghi, G. Comi, G. Galardi, N. Canal, V. Di Carlo, G. Ferrari, R. Castoldi, L. Beretta, G. Pozza and A. Secchi

San Raffaele Scientific Institute, University of Milan, Italy.

Whether pancreas transplantation could positively affect clinical course of diabetic polineuropathy is still matter of debate, the main confounding factor being the effect of the simultaneously transplanted kidney. The aim of our study was to evaluate the effects of pancreas transplantation 'per se' on diabetic neuropathy. A particular subset of patients submitted to kidney/pancreas $(K / P)$ transplantation $(t x)$ were selected: these patients lost the pancreas graft few years after transplantation, whereas the kidney graft was still functioning. Nerve conduction velocity was studied in these patients before and after successful kidney/pancreas transplantation (at least two years follow-up). The patients were studied again after having lost pancreas function (at least two years follow-up). 5 patients were studied, they lost pancreas function $5 \pm 2$ years after transplantation (range $2-6 \mathrm{yrs}$ ), whereas $\mathrm{K} t \mathrm{t}$ was perfectly functioning. Sensory nerve conduction velocity (NCV) was recorded in the sural and in the proximal and distal median nerves, and motor NCV in the peroneal and ulnar nerves, before transplantation and yearly thereafter. NCV was expressed as a cumulative index for each patient, where the difference between patients' NCV and controls' $\mathrm{NCV}$, divided by controls' SD for each nerve, was corrected for the number of nerves recorded in each patient. The normal value was 0.89 . Metabolic control, which improved after successful pancreas transplantation (HbA1c at 2 year: $6.5 \pm 1.1 \%$ ), worsened after pancreas failure ( 2 yrs $\mathrm{HbA1c}: 7.9 \pm 0.4 \%$ ). Serum creatinine levels remained stable before and after pancreas graft failure $(1.1 \pm 0.1$ vs $1.5 \pm 0.9 \mathrm{mg} / \mathrm{dl})$. NCV values improved after KP tx $(3.8 \pm 0.7$ vs $2.6 \pm 0.9$, $\mathrm{P}=0.0019)$. Two years after pancreas failure $\mathrm{NCV}$ values returned to pre-tx values $(3.6 \pm 1.0, \mathrm{P}$ vs post $t \mathrm{x}$ values: 0.034$)$. In conclusion these data show that pancreas transplantation exerts a positive effect 'per se' on clinical course of diabetic polineuropathy.
985

\author{
Diagnostic and prognostic importance of stabilometric test in diabetic \\ neuropathy \\ M.A Comaschi.L.Barat10*.P.Morasso*.R.Capra*.G. Spada*.P.Ubaldi. \\ L.Car allo.G.Carboni.M Pizorn.M. P Marino.C. Salani.F. Meno $z i$ \\ Dpt. of Int Med. - Diabetes Linit "La Collettz"Hospital Genoa Italy \\ *Center of Rchabulhtation Bioengineering - University of Genoa
}

Evaluation of postural stability performed with stabilometric platform in diabetic patients with neuropathy, shows abnormal increased values of sway area and center of pression (c.o.p.) velocity. Classical method of analisys (sway path, sway area) are not capable to identify neither the physiopathologic meaning of such data nor the difference of peripheric neurologic disease. The atm of this study was to proceed to analitic analysis (S. Antonio Consensis Conference) on a selected group of neuropathic diabetic subjects ( $\mathrm{DN}=15$ ), and two control groups made by not neuropathic diabetic patients $(\mathrm{DNN}=14)$ and healthy subjects $(N=64)$

The tume evolving trajectory of c.op. during a test, performed for $20 \mathrm{sec}$. at open eyes and 20 sec at closed eyes shows a systein organization based on centers of equilibrium evidenced by position and velocity density histograms. Furthermore instantaneous plot of velocity shows a significant increase of mean velocity valizes ( $N \quad 23.56 \pm 9.77$, DNN $3833 \pm 28.11, \mathrm{DN}: 55.78 \pm 33.23$ ) (ANOVA $p=00001$ ) and a peak development of velocity plot not present in controls with significant difference $(p=0.05)$ also in the percentual time of permanence under the threshol 1 of mean $(\mathrm{N}: 64.34 \pm 6.80$, DNN: $63.57 \pm 5.85$, DN: $6788 \pm 568$ ). Such data suggest that diabetic patient could loss postural statility, both in consequence of sensitive and motor deficit. Alterated values evidenced by this method also in patients without clinical and electrophysiological detected neuropathy suggest an important value of early diagnosis in afferent way defictt. 


\section{6}

SENSORY AND VESTIBULAR INTERACTION DEFICITS AS ASSESSED BY DYNAMIC POSTUROGRAPHY IN IDDM. S. Cercone 1 , M.A.S. Di Leo 1, W. Di Nardo 3 , A. Ciervo ${ }^{2}, M$ LoMonaco $^{2}$, V. Di Lazzaro 2 , G. Briglia ${ }^{3}$, A. Cosenza ${ }^{3}$, A.V. Greco ${ }^{1}$, G. Paludetti 3 and G. Ghirlanda 1 . Institutes of ${ }^{1}$ Internal Medicine, ${ }^{2}$ Neurology, ${ }^{3}$ Otorhinolaryngology, Catholic University, Rome, Italy. We used dynamic posturography (DP) to identify functional performance deficits underlying balance and posture impairment in $30 \mathrm{IDDM}$ patients with and without peripheral neuropathy and/or a central motor pathway dysfunction. The patients had no signs and/or symptoms of neuropathy. By using a moveable platform and moveable visual surroundings, the sensory inputs can be altered and the correcting motor responses measured. Nerve conduction velocities (NCVs) of peroneal and sural nerves and central motor evoked potentials (MEPs) latencies after cortical and after spinal stimulation were measured. Twelve IDDM patients with reduced NCVs showed a significant reduction in the following equilibrium performance score ( 100 and 0 represent no body sway and maximum sway, respectively) in comparison with control subjects: test 1 (eyes open, fixed platform): mean \pm SD: $92.5 \pm 1.7$ vs $94.6 \pm 1 \%$; ANOVA $\mathrm{F}=6.4, \mathrm{p}=0.004$; test 2 (eyes closed, fixed platform) $90.6 \pm 4.1$ vs $93.3 \pm 1.5 \% ; \mathrm{F}=3.4, \mathrm{p}=0.04$; test 6 (sway-referenced platform and surroundings) $56.4 \pm 12.2$ vs $67.4 \pm 8 \% ; F=4 ; p=0.02$ ). No significant difference between control subject and IDDM patients without neuropathy was shown. IDDM patients with neuropathy $(148.6 \pm 17.6$ $\mathrm{ms}$ ) showed longer muscle response latencies (control subjects: $134 \pm$ $16.5 \mathrm{~ms} ; \mathrm{F}=3.5, \mathrm{p}=0.04$ ) for correction of posture when the platform was moved forward. We found correlations between test 1 scores of DP and spinal MEP latencies $(r=0.2 ; p=0.01)$ and between sural NCV $s$ and muscle response latencies $(r=0.26 ; p<0.01)$. Our results suggest that a nonuniform somatosensory-vestibular and visual deficit may affect IDDM patients with neuropathy probably due to an impairment of somatosensory function rather than corticospinal function.

\section{7}

POSTURAL CONTROL IN IDDM PATIENTS WITH SUBCLINICAI NEUROPATY

L.Uccioli, P.Giacomini, P. Boccasena, E. Calandriello, G.Monticone, E.Bruno, S.Di Girolamo, P. Ferrigno, P. Pasqualetti, L. Parisi e G. Menzinger Roma, Italy

Diabetic patients with peripheral neuropathy show an impaired postural control revealed by posturography. Aim of our study is to evaluate whether such abnormality is present also in patients with electrophysiologic nerve impairments (EI), but without clinical signs of peripheral neuropathy. Three groups of age matched patients (30yrs) were evaluated: Controls ( $\mathrm{C}, \mathrm{n}=21$ ), IDDM patients without $(D, n=9)$ and with EI (DN, $n=16)$. The following parameters were recorded: Static posturography: Trace Surface (TS) and Lenght (TL); Motor (MCV) and Sensitive (SCV) nerve conduction velocity $(\mathrm{m} / \mathrm{sec})$; Sensitive Evoked Potentials (SEP) (msec), Motor Evoked Potentials (MEP) (msec).

$\begin{array}{lcccccc} & \text { TS } & \text { TL } & \text { SVC } & \text { MVC } & \text { SEP } & \text { MEP } \\ \text { C } & 204 \pm 28 & 367 \pm 21 & 48 \pm 1 & 49 \pm 2 & 39 \pm 1 & 13.5 \pm .8 \\ \text { D } & 238 \pm 27 & 384 \pm 41 & 48 \pm 2 & 46 \pm 1 & 38 \pm 1 & 13.6 \pm 1 \\ \text { DN } & 274 \pm 110 & 394 \pm 75 & 40 \pm 2 * & 40 \pm 1 * & 45 \pm 2^{*} & 15.1 \pm 3\end{array}$

In conclusion diabetic patients with EI but without clinical neuropathy demonstrate a normal ability in maintaining postural control. Electrophysiological methods are more sensitive than posturcgraphy in detecting subclinical peripheral neuropathy.

\section{8}

NERVE FUNCTION IN NIDDM PATIENTS WITH SUBCLINICAL NEUROPATHY ON AND OFF TOLRESTAT TREATMENT.

R.Acampora, R.Marfella, A.Quatraro, N.De Rosa, L.De Angelis, G.Bencivenga, F.Saccomano, D. Giugliano, Department of Geriatrics, Naples, Italy

The aim of this study was to find out whether, in NIDDM patients with subclinical neuropathy, aldosereductase inhibition with tolrestat improves nerve function and whether this improvement is reversible. Of the initial 60 patients, 57 completed a double-blind, randomized study of 12 months which demonstrated a significant effect of tolrestat $(n=29)$ over placebo $(n=28)$ an peripheral and autonomic nerve function. Fifty-three patients completed the second year during which they received a 12 -month treatment with tolrestat with significant improvement of both vibration perception threshold (VPT) or reflex tests (former placebo group) or stabilization of nerve function (former tolrestat group). During the third year, all patients were switched to placebo. Compared with 24month values, at 36 -months the patients $(n=49)$ presented significant impairment of VPT $(-3.2+1.7$ volts $)$ and reflex tests (squatting ratios deteriorated by 0.03 , $0.015-0.046$, CI $95 \%, \mathrm{p}<0.05)$. Twelve patients showed a progression of urinary albumin excretion (from $<15 \mathrm{mg}$ to $20 \pm 3 \mathrm{mg} / 24 \mathrm{~h}, \mathrm{p}<0.05)$. Lipid peroxidation, as measured by serum malondialdehyde, increased by $40 \%(5.2 \pm 1.8$ vs $3.7 \pm 1.4 \mathrm{ug} / \mathrm{d} 1, \mathrm{p}<0.05)$. The beneficial effects of tolrestat on nerve function are reversible after stopping treatment.

\section{9}

MEXILETIN TREATMENT IN PAINFUL DIABETIC POLYNEUROPATHY M.Reljanović, M.Pibernik, Ž.Beer, M.Koselj, V.Profozić, J.Limmer, Ž.Metelko, Z.Škrabalo.

Vuk Vhovac Institute, Zagreb, Croatia

Based on positive clinical experience, $450 \mathrm{mg} /$ day Mexiletin hydrochloride effectiveness in diminishing pain and unpleasant perceptual sensations caused by painful diabetic polyneuropathy was analysed. A prospective six-week double-blind, cross over design was used. 60 patients with the average age of 54.1 , diabetes duration of 13.1 years and painful symptomatology with average duration of 3.1 years were included in the study. Subjective pain evaluation consisted of numerical rating scales referring to overall pain, pain symptoms (burning, pricking, formication, stabbing, inadequate perception of heat, cramps) and the Zung pain and distress scale (PAD) were used as primary efficacy parameters. Daily pain evaluation by visual analogue scales, physician's assessment of drug efficacy and paracetamol consumption were considered secondary end-points. Wilcoxon matched-pairs signed ranks test was used to determine the differences between the two treatment periods. The results obtained in 55 patients, having completed the study, indicated that there were no differences between the verum and placebo periods with respect to subjective evaluation of overall pain $(z=-.4713 \quad p=6374)$, pain symptoms (all $p>150$ ) and the subjective well-being as measured by PAD $(z=-1.2694 \quad p=.2043)$. There were also no differences between the two treatment periods with regard to the secondary efficacy variables. Mexiletin applied in $450 \mathrm{mg} /$ day doses administered was not shown to be effective in reducing subjective neuropathic pain symptoms. The possible cause of inefficacy could be too low dose administered, which is in accordance with the results of dose response study carried out recently. 
990

THE EFFECT OF $\alpha$-LIPOIC ACID ON MICROCIRCULATION IN PATIENTS WITH DIABETIC POLYNEUROPATHY

T. Haak, E. Haak, R. Frommeyer, K. Kusterer, and K. H. Usadel, Med. Department I, University-Hospital, Frankfurt/Main, Germany

Although the mechanisms by which $\alpha$-lipoic acio [ALP] exerts ist beneficial effects are unknown to date, a variety of studies have proved that ALP is capable to improve the clinical signs of diabetic neuropathy. In order to study the effect of high-dose ALP on microcirculation, 8 patients with diabetic polyneuropathy ( 4 female, 4 male, age $59,0 \pm 3,0$ years, $H b A_{1 c} 6,3 \pm 0,3 \%, 2$ smokers, 6 nonsmokers) were studied before and after a 6 -week treatment with $1200 \mathrm{mg}$ ALP daily per os using nailfold videocapillaroscopy. Capillary blood cell velocity was measured at rest anc after a 2-minute ischemia as provocation test. In addition, signs of peripheral and autonomic neuropathy (temperature discrimination, vibration sensitivity, variation in heart rate at rest and during deep inspiration and subjective estimation of symptoms) were determined before and after treatment. The treatment with ALP resulted in a significant decrease in the time to peak capillary blood cell velocity after reactive hyperemia $(12,6 \pm 3,0$ vs $35,4 \pm 10,9 \mathrm{~s}, \mathrm{p}<0.01)$. Blood pressure, heart rate and heart rate variation remained unchanged. Subjective symptoms of peripheral neuropathy were slightly improved by the treatment with ALP. Temperature discrimination $(6.8 \pm 0.2$ vs $7.4 \pm$ $\left.0.2^{\circ} \mathrm{C}, p<0.001\right)$ and vibration sensitivity $(5.8+0.4 \mathrm{vs} 4.6+0.3[\mathrm{I}$ $\left.8^{-1}\right], p<0.005$ ) were also improved. Thus, these results demonstrate that ALP has a positive effect on microcirculation in patients with diabetic neuropathy indicating that this might also be present in nerval perfusion. It is tempting to speculate that this effect could represent one mechanism of ALP action.

\section{1}

A COMPARISON OF CALCITONIN AND ACETYL-L-CARNITINE IN SYMPTOMATIC PERIPHERAL DIABETIC NEUROPATHY.

A. Quatraro, P. Roca, M. Consoli, G. Moraglia, R, Marfella* and D. Giugliano*. Diabetic Clinic, Santa Rita, Taranto; *Department of Geriatric Medicine and Metabolic Diseases, Second University of Naples, Italy.

The purpose of the study was to examine and compare the efficacy of calcitonin and acetyl-L-carnitine (ALC) in NIDDM patients whit symptomatic peripheral neuropathy. Forty insulin treated NIDDM patients (28 women and 12 men, age $59 \pm 2$ years, mean $\pm S D$, duration of disease $16 \pm 1$ years) were asked to stop any previous drug they took for relief of symptoms for at least 2 weeks (only aspirin was allowed) before entering a single-blind randomized trial for parallel groups. The patients were assigned to receive either calcitonin nasal spray (group A, $n=20,100 \mathrm{UI} / \mathrm{day}$ ) or ALC (group B $n=20$, $500 \mathrm{mg} / \mathrm{im}$ b.i.d.) for 4 consecutive weeks. The baseline characteristics of the group were comparable. All patients were asked to grade the severity of neuropathic symptoms (pain/paresthesia) with a visual scale graded 0 to 10 $(0=$ no symptoms, $10=$ very severe). In group $A$ the mean basal value of symptom score was $7.6 \pm 0.6$ and decreased to $5.1 \pm 0.4$ after treatment $(\mathrm{p}<0.01)$. Eight patients were considered non-responders (change less than $10 \%$ of basal). In responders, symptoms reappeared 4-6 days after stopping calcitonin. In group B, the symptom score decreased from a basal value of $7.3 \pm 0.6$ to $3.2 \pm 0.4(\mathbf{p}<0.001)$ after ALC. Only 4 patients were considered as non-responders, in responders the benefits lasted for at least 4 weeks. Both treatments produced no significant changes of vibratory perception threshold (biothesiometry ) and cardiovascular reflex tests (squatting and deep breathing). In conclusion, both calcitonin and ALC can improve symptoms of peripheral neuropathy in a significant proportion of diabetic patients; the benefits seem greater with ALC, but more studies are needed.

\section{PS 54 \\ Diabetic Foot}

\section{2}

A SURVEY OF LOWER-EXTREMITY AMPUTATIONS IN DIABETIC AND NON-DIABETIC PtS IN MADRID (AREA 7). A.L. Calle-Pascual, M.A. Martinez Salinas, M.J. Redondo, M.D. Ballesteros, M. Jimenez, J. Serrano and J.P. Marañes. Servicio de Endocrinologia. Hospital Universitario S. Carlos. Madrid. Spain. In order to know the incidence of lower-extremity amputations (LEA) in Madrid (Area 7), a retrospective study (1989-1993) and a prospective study (1994-1995) were performed. Patients were identified by the Servicio de Cirugia Vascular records. Population of our area was 569307 (M/F:261529/307778). RETROSPECTIVE STUDY (19891993): Incidence $/ 10^{5}$ population in risk per year (cases) was for nondiabetic patients 3.47 (106), male 5.17 (79) and female 1.76 (27) and for diabetic patients 69.74 (136), male 92.90 (86) and female 54.05 (50). PROSPECTIVE STUDY (1994-1995): Incidence $/ 10^{5}$ population in risk per year (cases) was for nondiabetic patients 1.7 (19), male 2.6 (13) and female $1.0(6)$, and for diabetic patients 52.6 (36), male 76.4 (24) and female 32.4 (12). Diabetic patients had a 29 fold higher risk for LEA than nondiabetic people. Minor amputations was markedly higher in diabetic patients than did nondiabetic people. These data show that the incidence of IEA in diabetic patients is lower than those reported by other European countries, but are compatible with the view that management of the diabetic foot remains suboptimal in our area.

\section{3}

NON-TRAUMATIC LOWER EXTREMITY AMPUTATIONS IN DIABETIC PATIENTS IN UMBRIA, ITALY

L. Scionti, R. Norgiolini, G. Luca, A. Nicolucci and M. Massi Benedetti on behalf of Coopcrative Study Group of "Progetto Umbria Diabete", Department of Internal Medicine, Endocrine and Metabolic Sciences, University of Perugia, Italy A survey of all non-traumatic lower-extremity amputations (LEAs) carried out in Umbria Italy (811.887 inhabitants) during 1991 and 1992 was made by the Cooperative Study Group (CSG) of the "Progetto Umbria Diabete" to measure the prevalence and incidence of LEAs in diabetic patients in our region. An cxamination of the registrics of all surgical wards in Umbria was firstly performed to quantify LEAs and identify the subjects, whereas the surgical records of amputees were used to obtain information about the patients. Diabetic patients were recognized by history, or pharmacological hypoglycemic therapy, or fasting plasma glucose $>140 \mathrm{mg} / \mathrm{dl}$. In 1991 and 1992,323 patiets underwent LEA and $48 \%$ of them were diabetics. The median age at ampuation was simlar between diabetic (75 yrs, range $38-96$ ) and non diabetic (75 yrs, rangc 44-97) subjects, but the scx ratio ( $\mathrm{m} / \mathrm{f})$ within the groups was markedly different ( 1.0 in diabetics and 2.5 in non diabetics; $\chi 2=14.361, p<0.0002$ ). More than $59 \%$ of diabetics suffered from a below-knee LEA, wheras this figure was $40 \%$ in non diabetics $(\chi 2=14.682, p<0.0001)$. The incidence of new amputees was $30.6 / 10.000 / \mathrm{yr}$ in diabetic patients and $1.6 / 10.000 / \mathrm{yr}$ in non diabetic subjects. The incidence increased with age in both groups and was significantly higher in diabetic patients at all the considered ages; however, the incidence ratios between the groups decreased with age. In 1991 and 1992, 59 patients $(18.2 \%)$ underwent more than one LEA and $61 \%$ of them were diabetics $(\chi 2=4.965, p<0.02)$. This study shows that in Umbria, Italy, diabetic patients had a 18.7-fold higher risk for LEAs. A comprehensive program was developed by the CSG of "Progetto Unbria Diabete" to reduce LEAs in diabctic patients. 
994

Amputations in newly diagnosed diabetes - A substantial problem.

JP New, D McDowell and RJ Young.

Diabetes Centre, Hope Hospital, Manchester, UK.

A reduction of $50 \%$ or more in diabetes-related amputations is a primary target of the St Vincent Declaration. This target is known to be achievable because both primary and secondary preventative health care strategies are effective in reducing the incidence of diabetic foot ulceration and progression to amputation. Unfortunately there is a group who can not benefit from preventative health care - newly diagnosed diabetic patients with already established severe complications. We investigated the contribution this group make to the number of diabetic amputations. In our urban district of 230,000 people, $2.1 \%$ of the population are known to have diabetes, 821 (16.3\%) insulin dependent diabetes (IDDM) and 4145 (83.7\%) non-insulin dependent diabetes (NIDDM). From our annually updated population based register, established in 1992, the incidence of diabetes related major (below or above knee) amputation was related to age, duration and type of diabetes. All amputation data was independently verified using hospital podiatry records.

Duration (years)

\begin{tabular}{lllllll} 
& \multicolumn{3}{c}{ IDDM } & \multicolumn{3}{c}{ NIDDM } \\
Age $(y)$ & $0-1$ & $2-15$ & $>15$ & $0-1$ & $2-15$ & $>15$ \\
$<45$ & 0 & 1 & 1 & 2 & 0 & 0 \\
$46-65$ & 1 & 2 & 6 & 10 & 12 & 5 \\
$>66$ & 1 & 2 & 4 & 8 & 15 & 7 \\
Totals & 2 & 5 & 11 & 20 & 27 & 12
\end{tabular}

Thus $29 \%$ of all amputations, and $34 \%$ of amputations in NIDDM people occurred within the first year of diabetes being diagnosed. Cigarette smoking (Chi squared $p$ $<0.001)$ and male sex $(75 \%$ male) were associated with an increased risk of amputation within the first year. These amputations, which are not amenable to preventative health care strategies, are a major challenge to achieving the goals of the St Vincent Declaration.

\section{5}

FOOT AMPUTATIONS DURING UP TO 25 YEARS OF IDDM IN THE ERFURT DISTRICT

U.J.W. Schaver and A. Preuss, Department of Internal Medicine, Erfurt Hospital, Erfurt, Germany

The aim of this study was to find out the incidence of foot amputations and associated factors in IDDM within a geographically defined population. The centralized diabetes care system in the former GDR had been the basis for the almost complete registration of all diabetic patients. Amputations (foot, lower leg, thigh) were analyzed by checking the original charts up to 1990 of all the 1132 patients with IDDM onset until 40 years of age in the Erfurt district between 1966 and 1988. Cumulative incidence of foot amputations after 25 years of IDDM was $6.08+0.04 \%$ (men 9.90 , women $0.79 \%$ ). Amputations were performed after $16 \pm 4$ years of IDDM at an age of $46+6$ years. In comparison $(p<0.05)$ to patients with similar age and duration of IDOM at the end of follow up $(n=181)$ patients with amputations $(n=8)$ had higher nonfasting blood glucose during the first year of IDDM (13.6 vs 10.7 mmol/1) despite similar weight related insulin dose, a higher BMI at IDDM onset (26.2 vs $\left.23.2 \mathrm{~kg} / \mathrm{m}^{2}\right)$, suffered more often (25 vs $4 \%$ ) and earlier (after 8 vs 15 years of IDDM) from myocardial infarction and developed more frequently hypertension ( 75 vs $45 \%$ ), proliferative retinopathy ( 25 vs $5 \%$ ), proteinuria ( 75 vs $23 \%$ ) and terminal renal failure (13 vs $1 \%$ ) during the fallow up period. In conclusion in the Erfurt district foot amputations in IDDM clustered in patients with higher body weight at IDDM onset, less well controlled by usual insulin doses, and with high risk of hypertension, early myocardial infarction, nephropathy and severe retinopathy, resembling in part the metabolic syndrome of NIDDM.

\section{6}

EVALUATION OF FOOT CARE AND AMPUTATIONS IN ELDERLY PATIENTS TREATED WITH INSULIN

M. Spraul. A.M. Schönbach. I. Mühlhauser, and M. Berger

Heinrich-Heine-University. Düsseldorf. Germany

Little is known about the longterm outcome of diabetes care in elderly patients treated with insulin. In this tertiary care centre based study, 94 consecutively admitted diabetic patients, living within $50 \mathrm{~km}$ of our hospital, (age $\geq 60 \mathrm{yrs}$; 56 females) were re-evaluated 10 years after participation in a 5 -day insulin treatment and teaching programme for improvement of metabolic control. A high rate of complications $(68 \%$ peripheral neuropathy. $20 \%$ arterial vascular disease) was already present in these patients at the time of participation in the programme. Information about deceased patients was obtained through family members. physicians, and hospital reports. During the 10 year follow-up period $60(64 \%)$ patients had died. Among the remaining 34 patients 33 were evaluated (age $77 \pm 5 \mathrm{yrs}$. diabetes duration $21 \pm 6 \mathrm{yrs}$ ). All patients being alive had no acute foot complications or amputations: $26 \%$ attended our diabetesfoot-clinic for foot care. In another $26 \%$ of the patients the feet were never inspected by their family doctor. In 55 of the 60 deceased patients information was available about previous amputations: 19 patients had 20 above knee amputations and 2 a forefoot amputation. All amputations were performed in city hospitals in the region. None of the patients were amputated in the university hospital and no patient was referred to our diabetes-foot-clinic for a second opinion. In conclusion. in these elderly patients treated with insulin an unacceptable high number of above knee amputation was performed, despite having participated in education sessions about proper foot care and the handling of minor foot lesions. These elderly patients require special education about their high amputation risk and need to be trained about possible options (e.g. second opinion) when a major amputation is suggested. Surgical departments should perform quality control. Unless deceased patients are included in follow-up complication studies amputation rates will be grossly underestimated.
997

THE PREVALENCE OF THE CHARCOT FOOT IN AT RISK

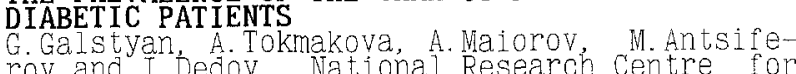
rov and i. Dedov. Nationa Res

The pathogenesis of diabetic neuropathic osteThe pathogenesis of diabetic neuropathic oste-
oarthropathy is complex. with peripheral neurooarthropathy is complex, with peripheral neuro-
pathy, increased olood flow to bone, secondary to autonomic neuropathy. We screened 596 diabetic patients with various foot lesions co estimate the prevalence of the charcot foot in risk mate une prevalence of the charco $=045 \mathrm{c}$ cases group patients with foot syndrome. In 536 cases
diabetic neuropathy was registered. using standiabetic neuropathy was registered using stan-
dardized neuropathic score. Among this neuropathic pationts 4 ( 8 , 8\%) had advanced arthropathic patients 4 (8, $8 \%$ had advanced arthropathic changes wi patients (age $18-62$ yrs) had long-standing IDDM patients (age $18-62$ yrs) had ong-standing
diabetes - mean 22 yrs (10-15yrs - 2. 15-20 -
18 , >20-16), while 11 NTDDM patients with mean diabetes duration - 12 yrs $5-10$ yrs -6 , >10 yrs - 2 . The neuroarthropathy was bilateral in 38 cases $(80,8 \%), 15$ subjects (32\%) had spontaileuos fractures, none of them
develops pain, $15(31,9 \%)$ had toe amputation develops pain, $15(31,9 \%)$ had toe amputation
previous to osteoarthropathy formation, 42 Previous to osteoarthropathy formation, 42
$(89,3 \%)$ patients had infected ulceration with previous history of longstanding unhealed ulcers. Conclusion: The charcot foot occured in 8. $8 \%$ of patients with diabetic neuropathy more diabetes compare to NIDDM patients. osteoartdiabetes compare were particularly high in pahropathic changes were particularly high in pa-
tients with previous unhealed ulcers. Long tients with previous unhealed ulcers. song
standing infected ulceration may accelerate standing infected ulceration may accelerate
charcot foot formation. Patients with toe ampttation causing alteration of the foot biomechanics and resulting of microfractures. 
998

A RETROSPECTIVE STUDY OF EIGHT PATIENTS WITH DIABETIC OSTEOARTHROPATHY TO DETERMINE THE INFLUENCE OF NEUROPATHIC FRACTURES.

C. Créton, I. Got, R. Aussedat, P. Drouin, O. Ziegler. Nancy, FRANCE.

This study was done to assess the pathogenic factors which might explain a negative evolution to Charcot's foot after a neuropathic fracture considered as a causative factor. Eight diabetic patients, aged 27-50 years, having among them 13 feet showing the characteristic Charcot changes were selected for study $X$ rays showed that all the patients had tarsal fractures which preceded the development of diabetic osteoarthropathy (DOAP). These fractures were bilateral for 3 patients, ending in 11 fractures ( 7 of the navicular bone, 2 of the tibia, 1 cuneiforme boen, 1 calcaneum). Minor trauma was reported in 5 cases only. The time between trauma and the fracture diagnosis was 0-60 days. Blood flow abnormalities were found in 5 patients with a continuous diastolic flow determined by Doppler. Venous occlusion plethysmography has also demonstrated increased blood flow in 6 patients. Severe vasomotor disturbances were proved by skeletal scintigraphy in 5 of them. In 7 patients, evolution led to a typical Charcot's arthropathy. Several factos seemed to be involved in this pejorative outcome. First, the delay in diagnosing the fracture observed in 9 cases on 11 , and the persistent weight-bearing on diseased bones and joints probably played a crucial negative role. Secondly, the presence of vasomotor disturbances should not be neglected and must be investigated. Lastly, all these patients had a diabetes of long duration (mean : $15 \pm 6$ years), with several complications and poor metabolic control ( $\mathrm{HbAlc}$ $>10 \% \mathrm{~N}: 4-6 \%$ ). In conclusion: Preventive measures are strongly advised in order to avoid this outcome. The first is to identify the patients at high risk of DOAP. The second, is to educate these patients as to the need to consult at the least trauma, or vasomotor disturbance to permit early diagnosis.
999

PREVALENCE AND DETERMINANTS OF FOOT ULCERATION IN TYPE II DIABETIC PATIENTS IN PRIMARY HEALTH CARE. TYPE II DIABETIC PATIENTS IN PRIMARY HEALTH CARE. J.J.J. de Sonnaville, C. Blok, D. Wi jkel and R.J. Heine. Research Center
Primary/Secondary Health Care, Laboratory for General Practitioners Primary/Secondary Health Care, Laboratory for General Practitioners
(ATAL) and Department of Endocrinology, Free University Hospital, P.O (ATAL) and Department of Endocrinology, Free U
Box 7057, $1007 \mathrm{MB}$ Amsterdam, The Netherlands.

The purpose of the study was to assess the prevalence of foot (preulcers and their determinants in type II diabetic patients in a primary health care setting. Six hundred and nine patients $(246$ men, mean age 64.8 (range 40-94) years, diabetes duration 4.3 (044.9) years, mean HbAlc 7.8 (sd 2.1) \% from 22 general practices attended a regional shared care project in Amsterdam. Hypoglycaemic therapy consisted of insulin injections, oral agents and diet only in 2, 58 and $40 \%$ respectively. At first visit blood was drawn for metabolic control and all patients were examined by a podiatrist. This included antropometry and assessment of peripheral vascular disease (dorsal pedal artery absent in $6 \%$ of the patients; resting ankle/brachial ischemic index of dorsal pedal and posterior artery (ABIpt) $<0.9$ in 23 and $18 \%$ respectively) and neuropathy (absent achilles and knee tendon reflexes in 56 and $26 \% ; 128 \mathrm{~Hz}$ vibration sense disturbed in $28 \%$; Semmes-Weinstein monofilament 5.07 (SWM) not felt in 15\%; dry non-sweating feet present in 38\%). Amputations, active foot ulcers (Wagner stage 1 or 2) and preulcers (Wagner stage 0 ) were recorded in $0,11(1.8 \%)$ and 79 patients $(12.9 \%)$ respectively. In multivariate logistic regression analysis, af ter adjusting for age and gender, diabetes duration $(>5$ versus $\leq 5$ years; Odds Ratio $2.09 ; p=0.0061$ ), cigarette smoking (yes/no; OR 2.31; $\mathrm{p}=0.0021$ ), peripheral vascular disease assessed by ABIpt $(</<0.9$; OR 3.50; $\mathrm{p}=0.0000)$, sensory neuropathy with SWM $5.07(3.18 ; \mathrm{p}=0.0002)$, dry feet $(\mathrm{OR} 2.03 ; \mathrm{p}=0.0063)$ and severe hammer toes with clawing (OR $1.97 ; \mathrm{p}=0.0404$ ) were independently and significantly associated with foot (pre-)ulceration. In conclusion, 1 of every 7 type II diabetic patients in primary health care has a foot (pre-)ulcer. Patients at risk for foot ulceration can easily be identified by inspection and the use of simple instruments.
1000

THE PREVALENCE OF FOOT DEFORMITIES AND NEUROPATHY IN PEOPLE WITH DIABETES: A POPULATION BASED STUDY C.Vermigli, A.CarrIngton and A.J.M. Boulton. Manchester Royal Infirmary UK Peripheral sensory neuropathy and peripheral vascular disease play a major role in the incidence of foot ulceration in diabetes. However the contribution of foot deformities (FD) has not been elucidated. The purpose of this study was to investigate the prevalence of $F D$, neuropathy and peripheral vascular disease (PVD) in people with diabetes. Consecutive people with diabetes $(n=666)$ were assessed for: FD using a foot deformity score (FDS) detected by the presence of small muscle wasting, hammer/claw toes, bony prominences, prominent metatarsal heads, Charcot loot and limited joint mobility; peripheral neuropathy using the neurological disability score (NDS) derived from examination of the ankle reflex, vibration, pain and temperature sensation; plantar cutaneous pressure perception (PPP) using Semmens Weinstein monofilaments; PVD assessed by pulse palpation (dorsalis pedis and posterior tibial pulse on both feet). Abnormality was assigned with a standard system. The prevalence of FD, neuropathy assessed by NDS and PPP and PVD of the whole population was respectively $61.7 \%, 27.3 \%, 23.9 \%$ and $35.7 \%$. To as sess the relationship between these abnormalities and ulceration, this group of patients was divided into two groups: diabetic patients without ulceration ( $D: n=626$, mean age $+S D=60.6+16.2$, sex ratio $M / F=322 / 304$ ) and those with past or present ulcers $(U: n=40$, mean age $+S D=63.9+14.2 ; M / F=22 / 18)$. Abnormal FDS was similar in both groups (D:U, $61.0 \% \vee 72.5 \% p>0.05-\mathrm{Chi}$ square test-). Neuropathy assessed by both NDS and PPP was more prevalent in the ulcer group (D:U NDS, $25.2 \%$ v $60.0 \%$ p $<0.05$, PPP, $21.9 \% \vee 55.0 \% p<0.05$ ). There was no significant difference in palpable puises between both groups ( $35.9 \% \vee 32.5 \% \quad p<0.05$ ). FD assessed using this method are very common in the general diabetes popufation, but unrelated to ulceration, however neuropathy is directly associated with foot ulceration.

\section{1}

PREVALENCE OF ULCERATIVE RISK FACTORS IN DIABETIC PATIENTS AT FIRST FOOT INSPECTION

D.Bloise, M.Ceci, A.M.Gargano, F.Fallucca and A.Maldonato. Diabetes Unit, CIMS, Inst. Cl. Medica 2, La Sapienza University, Rome, Ilaly.

It has heen observed for a long time that lower limb amputations in diabetic patients are preventahle by daily foot care. In order to detect patients at risk and help them prevent font lesions, we evaluated the specific knowledge and carefully examined the feet in 61 patients - first attending our clinic over the last 6 months - who did not complain of foot problems nor had ever heen foot-inspecied hefore. Inclusion criteria were: for type 1, diabetes duration $>10 \mathrm{yr}$; for type 2 , age $>55 \mathrm{yr}$ or disease duration $>10 \mathrm{yr}$. We evaluated: vibratory sense (Rydel-Seiffert tuning fork, TF) and thermal sensation (Thermocross): dorsalis pedis and posterior tibial pulses (DP); foot deformities; presence of dry skin, hyperkeratotic tissue and fungal infection; nail care and personal hygiene; adequacy of shoes. Knowledge was evaluated by a 52 -item questionnaire. Mean age was $58 \pm 20$ yr and diahetes duration $18 \pm 9$ yr (11 type 1 and 50 type 2). Arcording to TF and DP, we divided the patients into 4 groups: Gr.0 (33\%) with TF $>4$ and DP $>2$; Gr.V (24.5\%) with vascular disease (DP $\leq 2$ ); Gr.N (13\%) with neuropathy (TF $\leq 4$ ); Gr.V+N $(29.5 \%)$ with DP $\leq 2$ and $T F \leq 4$. The share of other risk factors was as follows:

\begin{tabular}{|c|c|c|c|c|c|c|c|c|c|}
\hline $\begin{array}{r}\% \\
\text { Groups }\end{array}$ & $\mathbf{n}$ & $\begin{array}{c}\text { Thermal } \\
\text { insensit. }\end{array}$ & Deformity & $\begin{array}{c}\text { Dry } \\
\text { skin }\end{array}$ & $\begin{array}{c}\text { Corns } \\
\text { calli }\end{array}$ & $\begin{array}{c}\text { Fungal } \\
\text { infect. }\end{array}$ & $\begin{array}{c}\text { Poor } \\
\text { hygiene }\end{array}$ & $\begin{array}{c}\text { Bad } \\
\text { shoes }\end{array}$ & $\begin{array}{c}\text { lgnored } \\
\text { lesions }\end{array}$ \\
\hline Gr. 0 & 20 & 10 & 45 & 20 & 80 & 15 & 15 & 40 & 5 \\
\hline Gr.V & 15 & 33 & 50 & 53 & 86 & 6 & 20 & 46 & 0 \\
\hline Gr. N & 8 & 62.5 & 87 & 50 & 50 & 25 & 25 & 37 & 12.5 \\
\hline Gr.V+N & 18 & 50 & 55 & 78 & 72 & 5.5 & 44 & 55 & 5.5 \\
\hline
\end{tabular}

The answers to the questionnarie about diahetic foot were: $60 \%$ right, $14 \%$ "don't know", $26 \%$ wrong. Twentythree patients ( $38 \%$ ), of whom 21 were at risk, answered they had never heen informed about foot complications. These data confirm the need - often stated but seidom put into practice - for a systematic routine foot examination, as the only starting point in order to develop appropriate educational interventions for preventing amputations. 
1002

RECURRENT NEUROPATHIC FOOT ULCER DISEASE IN DIABETES MELLITUS.

T.Tanudjaja, E.Chantelau. Diabetic Foot Clinic University of Düsseldorf/FRG.

Background: The rate of appearance of the first foot ulcer in neuropathic diabetic patients is about $0.1 /$ patient year. The rate of ulcer recurrences is unknown. Aim: To assess the incidence rate and related conditions of repetitive foot ulceration in diabetic patients with at least 2 consecutively documented lesions. Patients and methods: 53 patients, $28 \%$ females, $19 \%$ with IDDM, $81 \%$ with NIDDM, mean(SD) duration of diabetes 19 (SD 14)years, were followed prospectively for 3.2 (SD 2.4) years. $75 \%$ of the feet were purely neuropathic, and 25\% were neuroischaemic.

Results: In a total of 170 patient years, the mean ulcer recurrence rate was 1.02 /patient year. yearly ulcer recurrences were $<1$ in $47 \%, 1-2$ in $30 \%$, and $>2$ in $23 \%$ of patients, respectively, and were correlated inversely with diabetes duration $(\mathrm{p}<0.05)$. Recurrences were due to mismatch between foot and shoe $(77 \%$ of ulcers in patients using normal, and $37 \%$ of ulcers in patients using special shoes), to insufficient callus removal (17\%), and to acute foot trauma (12\%). In $30 \%$ of recurrences, the cause could not be identified. Conclusions:1) The rate of foot ulcer recurrence is about 10 fold higher than the rate of appearance of the first ulcer. 2) Recurrences are related to inappropriate footwear, foot care and trauma prevention. 3) The longer the duration of diabetes, the better the patients may learn to cope with their foot ulcer disease.

\section{3}

BETWEEN-TOE PRESSURE IN PATIENTS WITH LIMITED JOINT MOBILITY (LUM): THE EFFECT OF FOOTWEAR. E.Chantelau, O.Schröer, T.Tanudjaja. Diabetic Foot clinic, University of Düsseldorf/FRG Background:interdigital neuropathic ulcers and calluses indicate increased horizontal pressure exerted by footwear; exact pressure monitoring, however, has never been reported. Methods: the medial and lateral parts of a toe cap belonging to an industrial workers' safety-shoe were cut and fixed vise-like to a frame and a screw. Thus, the cap diameter could be varied. Under 4 conditions; the forefoot was placed between both parts of the toe cap: metatarsophalangeal joints (MPJs) extended vs.20-45 dorsiflexed, with vs. without slight compression (cap diameter 95\% vs.100\% of forefoot diameter). Between the 4 th and 5 th toe, pressure was recorded by F-Scan transducer. 19 diabetic patients with previous neuropathic ulcer and IJM of the MPJs, and 19 controls were studied.Results: interdigital pressure $\left(\mathrm{g} / \mathrm{cm}^{2}\right.$, medians with $95 \% \mathrm{CI} ; * \mathrm{p}<0.05$ vs controls) is summarized in the Table:

Metatarsophalageal joints (MPJs) patients * $\begin{array}{ll}\text { extended } & \text { dorsiflexed compressed } \\ 356(190-634) & 732(295-1150) \text { no }\end{array}$ $855(323-1780) 1980(800-3240)$ yes controls $0(0-0)$ no $42(0-253) \quad 347(126-484)$ yes Conclusion: in diabetic patients with LJM and previous neuropathic foot ulcer, between-toe pressure is higher $(\mathrm{p}<0.05)$ than in controls. Toe caps increase interdigital pressure during normal walking movements, and more so if narrow.

\section{4}

PLANTAR PRESSURE IS INDEPENDENT OF MOTOR NEUROPATHY IN NIDDM PATIENTS

T Kästenbauer. G. Sokol, S. Stary and K. Irsigler. Ludwig Boltzmann Institute for Metabolic Diseases and Nutrition, Hospital Lainz, 1130 Vienna, Austria

The aim was to investigate whether peripheral motor neuropathy causing atrophy and clawing of the toes or foot deformity or limited joint mobility (LJM) influences plantar pressure in diabetic patients. We investigated 157 patients with longstanding NIDDM. divided into two subgroups concerning the mean peak plantar pressure (PPP) of the whole study population at the right and left hallux, the first MTH $\mid$ ) and the second/third metatarsal head (MTH 2/3). If PPP at one out of these SIx regrions was above the mean $+1 \mathrm{SD}$ for this particular region, the patient was apportioned to group $B$ (high PPP, $n=82$ ). All other subjects were apportioned to yroup A (low PPP, $n=75$ ). We used pedograply for measuring PPP, notor conduction velocity of the peroneal nerve (mNCV), biothesiometry (VPT) and cardiorespiratory reflex tests (cANP) for diagnosis of neuropathy, blue-prints (foot deformity). and clinical evaluation of the ankle jerk (LJM). Statistical analyses: Student t- chi-square- and Kolmogorov-Smirnov-test. There were no significant differences between group $A$ and $B$ concerning age $(60,2 \pm 6,9$ vs. $59,1 \pm 7,8$ vears $)$ duration of diabetes ( $11,5 \pm 5.9$ vs. $12,5 \pm 7,1$ years), body mass index $(29,1 \pm 4,6$ vs. $29.2 \pm 4,1)$ and $\mathrm{HbA}_{1 \mathrm{c}}(9,6 \pm 1,3$ vs. $9,7 \pm 1,6 \%$ ). cANP, ankle-arm-indices, prevalence of atrophy and of clawing toes as mNCV (A $42,5 \pm 4,6$ vs. B $42,7 \pm 4,5 \mathrm{~m}^{*} \mathrm{sec}^{-1}$ ) were not different. Patients of group $B$ had significant higher PPP values at the forefoot. especially at the right MTH $2 / 3$ median $68,5 \mathrm{~N}^{*} \mathrm{~cm}^{-2}$ (36/98,5 lower/upper quartile) vs. group A $39(30 / 47,5) p<0,001$, and at the left MTH $2 / 3: 68,5(38 / 110)$ vs. 42,5 $(31 / 56) \mathrm{p}<0.001$. Furthermore, VPT at the hallux was significant higher in group B $21,2 \pm 11,8$ Volt vs. $17,2 \pm 9,0(p=0,02)$ as were the prevalence of splayfoot $(p<0,001)$ and of limited mobility of the ankle jerk $(\mathrm{p}=0,04)$. The data suggest that mNCV has no influence on PPP in NIDDM patients. We conclude that one has to consider foot deformity and linited joint mobility for risk evaluation of high plantar pressure in NIDDM patients.

\section{5}

COMPARING A NEW SIMPLE PLANTAR PRESSSURE MEASURING DEVICE WITH PEDOBAROGRAPHICALLY MEASURED PRESSURES.

van Schie C.H.M. ${ }^{\text {a }}$, Vileikyte L. a, Abbott C.A. ${ }^{\text {, }}$, Shaw J.E. ${ }^{a}$, Hollis S. ${ }^{\text {b }}$ and Boulton A.J.M. ${ }^{\mathrm{a}}$ a Department of Medicine, Manchester Royal Infirmary, Oxford Road, Manchester M13 9WL and bedical Statistics Unit., Lancaster University, Lancaster, UK.

The Podotrack is a new semi-quantitative footprint mat which measures peak pressures by comparing the darkness of the footprint with a calibration card containing 7 different ranges of pressure. Aim of the study was to test the accuracy and reliability of the Podotrack. The Podotrack was tested by placing it on top of the optical pedobarograph, and pressures from both systems were compared. Plantar pressures were measured in 24 subjects: 5 controls, 9 diabetes without neuropathy, 4 with neuropathy and 6 with neuropathy and previous ulceration. Mean VPT was $8.3 \pm 4.4$ (sd) $V$ for first two groups. and $30.7 \pm 8.9 \mathrm{~V}$ for the neuropathic group. A total of 164 footprints were assessed. Three independent observers, blinded to the subjects' status, quantified the plantar pressure in different areas. Sensitivity of Podotrack to identify areas of high pressure $\left(9-15 \mathrm{~kg} / \mathrm{cm}^{2}\right)$ was $69.5,32.4$ and $36.2 \%$ (observer $\mathrm{A}, \mathrm{B}$ and $\mathrm{C}$ ), and specificity was $92.9,98.8$ and $98.4 \%$. Observers $A$ and $C$ scored $46 \%$ of all scores identically, and $96.5 \%$ within one range. Thus although variability existed between observers, and the Podotrack did not identify all high pressure areas, not many falsely high areas were reported. The main difficulty was choosing between adjacent pressure ranges. $A$ s the Podotrack costs 26 pence per mat whereas the pedobarograph costs $>\mathfrak{f} 10$, $(0)()$, with adequate training, the Podotrack could be used to identify high risk patients for ulceration in clinical practice. 
1006

PEAK FOOT PRESSURES INFLUENCE HEALING TIME OF DIABETIC FOOT ULCERS TREATED IN TOTAL CONTACT CASTS

D.G. ARMSTRONG †े, L.A.. LAVERY $\uparrow$, AND T.R. BUSHMAN $\dagger$; †UNIVERSITY OF TEXAS HEALTH SCIENCE CENTER AT SAN ANTONIO, $\ddagger$ NASHVILLE TENNESSEE

The aim of this study was to describe the progression of healing of neuropathic ulcers using the total contact cast and to examine factors that influence wound healing. Design: 25 consecutive diabetic subjects with grade I (Meggitt-Wagner) plantar ulcers had toe-brachial index, vibration perception threshold and maximum plantar pressure recorded prior to initiation of total contact casting. All subjects received weekly cast changes with wound and skin temperature assessment. Following healing, patients were fitted with prescription shoe gear and were followed with skin temperature monitoring for a minimum of nine weeks.

Population: 25 diabetic patients (4 type I, 21 type II), 17 male, 8 female, with an average age of $52.4 \pm 11.6$ years, and an average $13.8 \pm 7.8$ years' diabetes duration. Results: Mean duration of casting until healing was $38.8 \pm 21.3$ days. Those with maximum pressures over $99 \mathrm{~N} / \mathrm{cm}^{2}$ took longer to heal $(33.1 \pm 13.0 \mathrm{vs}$. $53.4 \pm 31.4$ days, $P=0.05$ ) and had significantly longer mean ulcer duration prior to treatment $(52.7 \pm 37.2$ vs. $180.7 \pm 145.0$ days, $P=0.02)$. Men took longer to heal than did women $(43.8 \pm 22.9$ vs. $28.1 \pm 12.9$ days,$P=0.03)$. Patients with wound size greater than $8 \mathrm{~cm}^{2}$ took longer to heal $(50.2 \pm 26.2$ vs. $29.9 \pm 10.6$ days, $\mathrm{P}=0.02$ ). Skin temperature was higher on the ulcerated foot than on the corresponding site contralaterally on initial presentation $\left(91.1\right.$ vs. $84.2^{\circ} \mathrm{F}, \mathrm{t}=8.9$, $\mathrm{P}<0.0001,95 \% \mathrm{CI} 5.3$ to 8.5 ). Following healing, there was no significant contralateral difference in skin temperature. Conclusions:High plantar pressures, wound size greater than $8 \mathrm{~cm}^{2}$ and male gender were associated with significanly longer healing times.
1007

ASSESSING THE VALUE OF NON-INVASIVE EXAMINATION OF ANGIOPATHY AND NEUROPATHY IN PATIENTS AT RISK OF THE DIABETIC FOOT IN A MODEL AREA

A.Jirkovská, V.Wosková, V.Bartoš, and J.Skibová. Institute for Clinical and Experimental Medicine, Prague, Czech Republic

The aim of the study was to compare non-invasive examinations of angiopathy and neuropathy with regular diabetes clinic-based diagnosis in diabetic patients in 6 diabetes clinics in a model area, and to characterize those at risk of peripheral arterial disease (PAD) based on their Doppler examination. Over a one-year period, specially trained nurses examined 300 consecutive diabetic patients (130 men, 170 women) over 30 years of age (mean $66 \pm 10$ ) with diabetes duration longer than 5 years (mean $13 \pm 7$ ). Doppler examination identified 180 patients $(60 \%)$ at risk of angiopathy (ankle/arm index below 1.0 ); PAD was diagnosed in the diabetes clinics in only $37 \%$ of these patients. Clinical signs of PAD were not very frequent, $30 \%$ of these patients reported claudications, $15 \%$ had femoral artery bruit, only $5 \%$ had non-palpable peripheral pulsations. Patients with a risk Doppler index did not differ significantly in age, duration of diabetes, HbA1c and triglycerides from other patients in the study group, but they had significantly higher cholesterol $(6.3 \pm 1.2$ vs $5.97 \pm 1.2$ mmol $/, \mathrm{p}<0.05)$, creatinine ( $99 \pm 35$ vs $90 \pm 17 \mu \mathrm{mol} / \mathrm{p}, \mathrm{p}<0.01)$ and vibration perception threshold (VPT) $(29.9 \pm 13$ vs $25.2 \pm 12 \mathrm{~V}, \mathrm{p}<0.01) .150$ patients $(50 \%)$ were at risk of the diabetic foot as determined by a biothesiometer (VPT over 30V), and 129 patients (43\%) were at the same risk as established by $10 \mathrm{~g}$ mono-filament, polyneuropathy was diagnosed, in diabetes clinics, in $68 \%$ of these patients. 162 patients (54\%) were at high risk of the diabetic foot as identified by non-invasive testing (Doppler index below 0.8 and/or VPT over $30 \mathrm{~V}$ or foot insensitive to mono-filament). The results of the study suggest the need for non-invasive angiopathy and neuropathy examination in diabetes clinics for identification of patients at high risk for the diabetic foot.

1009

\section{IDENTIFYING AND EVALUATING DIABETIC PATIENTS AT RISK OF} FUOT ILCERATION

Ch.Manes, N.Papazoglou, E.Sossidou, D.Milarakis, A.Satsoglou and A. Sakallerou. «ST.PALL» General Hospital, Diab. Unit. Thessaloniki, Greece Identificaion of patients at potential risk of foot ulceration (FU) in clinical practice and further evaluation of this risk are essential. if we are to succeed in reducing the incidence of this disorder. This population based study was conducted to identify the independent predictors of FL and assess the individual's risk giving attention to multiple risk factor intervention. Eight hundred and twenty diabetic patients $\left(80^{\circ} \circ\right.$ of the known diabetic population of a region), 304 males. 780 type 2 . were studied. Mean age and diabetes duration were $59,5 \pm 7,46$ and $7,6 \pm 6,9$ y ts respectively. Smokers were $27,5^{\circ}$ o. Neuropathy (DN) was defined and evaluated (NDS) by a standard examination according to Neurodiab criteria (abnormalities in symptoms. sensory and motor signs using score techniques and the estimated vibration perception thresholds-VPT) Peripheral vascular disease (PVD) was established clinically. The prevalence of D $\backslash$ was $33.5^{\circ} \circ\left(95^{\circ} \circ \mathrm{CI}, 30,3-36,7^{\circ} \mathrm{o}\right)$ of PVD $12,7^{\circ} \circ$ (C.I. $10,7-14,7^{\circ} 0$ ) and of FU $4.75^{\circ}$ o (C.I. $3.3-6,2^{\circ}{ }^{\circ}$ ). Patients with FU had more severe DN (NDS $11,6 \pm 5.26)$ and higher VPT $(40 \pm 13,3)$ than those without (NDS $6,92 \pm 2,83 . V P T ~ 30 \pm 13,8)(p=0,001$ in both cases). Duration $(8.28 \pm 0,25$ is $15 \pm 1,+8, p<0,05), D N, P V D\left(13,5^{\circ} \circ\right.$ is $3,5^{\circ} 0, v P T \geq 25$ and smoking $\left(7,96^{\circ}\right.$ is $\left.3,54^{\circ}\right)$, were significant predictors for $F U$ in bivariate analysis (chi-square $p<(), 05$ for the last three), although in stepwise multiple regression analysis oniy DN. PVD (p<0,05 for both) as significant and smoking $(p=0.69)$ remained in the model from the above predictors. Conclusion: Aroiding of smoking may exert a favourable effect on the development of $\mathrm{F} \mathrm{C}$. Whatever once identified patients smokers with DN and PVD should be considered as the highest risk group of FU requiring more intensive care. 
1010

10 g SEMMES-WEINSTEIN MONOFILAMENT: A SIMPLE AND ACCURATE SCREENING INSTRUMENT TO PREVENT COMPLICATIONS OF THE DIABETIC FOOT

A. Staudenmeyer, R. Zick, M. Eslage

Peripheral neuropathy is the leading cause of most diabetic foot lesions and produces nearly $25 \%$ of all diabetes patient ${ }^{\prime}$ costs in the hospital. The most important effect of peripheral neuropathy on the diabetic foot is the loss of protective sensation. Semmes-Weinstein monofilaments seem to be an accurate, simple and inexpensive instrument of testing touch and pressure sensation. Therefore we tested these monofilaments in comparison to the "Gold standard" of thermal and vibratory sensation. We studied 37 diabetes patients with known neuropathy and tested them with the $10 \mathrm{~g}$ monofilament. The testing site was the plantar side of the foot. Cour control group based on 25 diabetes patients without neuropathy and 20 healthy patients. All 45 patients of our control group could feel the pressure caused by the $10 \mathrm{~g}$ monofilament. In contrast to this 19 of 22 patients with peripheral neuropathy did not perceive the touch of the $10 \mathrm{~g}$ monofilament and none of the neuropathic ulcer group felt the pressure caused by the bending of the $10 \mathrm{~g}$ monofilament.

Our study shows that the sensory threshold measurement with the $10 \mathrm{~g}$ monofilament is a highly accurate, inexspensive and simple screening method for detecting diabetic patients at risk for peripheral neuropathy and foot ulcers.

\section{1}

STRUCTURAL DIFFERENCES IN SKIN BETWEEN THE ARM AND FOOT IN NORMAL SUBJECTS AND DIABETIC PATIENTS

D. Walker ${ }^{1}$, R.A.Malik ${ }^{1}$, A.J.M.Boulton ${ }^{1}$, G.Rayman ${ }^{2}$. Dept. Medicine, Manchester Royal Infirmary ${ }^{1}$, Ipswich Hospital ${ }^{2}$, UK.

Investigators have commonly assessed functional and structural abnormalities of the skin in diabetic patients in the upper limb and have extrapolated their findings to the diabetic foot. To assess the validity of this approach we have employed detailed light and electronmicroscopy in punch skin biopsies taken sinultaneously from the forearm (A) and the dorsum of the foot (F) in control subjects $(n=13),(C)$ and patients with NIDDM (D) $(<5$ yrs diagnosis, no complications) $(n=11)$. Both controls and diabetic patients displayed a significantly greater Epidermal thickness (um) in the foot $(C-35.5 \pm 4.4, D-35.0 \pm 4.3)$ compared to the arm ( C- $28.2 \pm 3.8, \mathrm{D}-28.8$ $\pm 4.2)(\mathrm{P}<0.002)$. There was no significant difference for capillary density in the foot (C- $31.9 \pm 16.7, D-31.6 \pm 19.9)$ v arm $(C-30.8 \pm 17.3$, D- $26.6 \pm 13.9)$ or large vessel density in the foot $(\mathrm{C}-30.9+17.0, \mathrm{D}-34.3 \pm 12.4) \mathrm{v}$ arm $(\mathrm{C}-26.4 \pm 12.2$, D $26.4 \pm 9.9)$. Detailed microvascular abnormalities were assessed in a randomly selected subgroup of control subjects $(n=6)$, and NIDDM patients $(n=6)$. Capillary Basement membrane area $\left(\mu \mathrm{m}^{2}\right)$ was increased in the foot $(64.8 \pm 8.9) \mathrm{v}$ arm $(46.4$ $\pm 12.9)(\mathrm{p}<0.04)$ in the control subjects but not in diabetic patients $(\mathrm{F}-69.9 \pm 19.9 \mathrm{v}$ A- $69.5+12.1)$. Luminal area, endothelial cell area and capillary area failed to differ between control subjects and diabetic patients and between the forearm and foot. The greater epidermal thickness in the foot should be accounted for as it may attenuate functional abnormalities such as laser Doppler blood flow and oxygen tension. Intravascular pressure may modulate the expression of basement membrane thickening as demonstrated by its increase in the capillaries of the foot compared to the arm in control subjects. In diabetic patients at an early stage without complications there is no difference from control subjects in capillaries from the foot. However a difference is observed in the forearm $(\mathrm{P}<0.03)$ between control subjects and diabetic patients suggesting that this site may be more sensitive for detecting early basement membrane thickening attributable to non pressure related factors.

\section{2}

MICROVASCULAR RESPONSE TO TRANSCUTANEOUS ELECTRICAL NERVE STIMULATION AND POSTOCCLUSIVE ISCHAEMIA IN THE DIABETIC FOOT

E. Küstner, T. Forst, A. Pfützner, R. Bauersachs, B. Bach, H. Biehlmaier, J. Beyer

III Med Clinic: Dept Internal Medicine / Endorinology: University Mainz

Neurogenic inflammation, mediated by unmyelinated $\mathrm{C}$-nerve-fibres, is part of the acute neurovascular response to injury. Laser doppler flowmetry was used to measure the flare response to transcutaneous electrical nerve stimulation (TENS) and postischaemic hyperaemia in the skin of the foot in diabetic and non-diabetic subjects. The effect of TENS and postocclusive ischaemia on skin microcirculation was measured at the dorsum of the foot and the pulp of the hallux in 57 diabetic patients and in 21 control subjects. Fourteen of the diabetic patients were free from diabetic complications (NP-/RP-): 14 patients suffered from neuropathy without evidence of retinopathy (NP+/RP-); 8 patients suffered from retinopathy without neuropathy $(\mathrm{NP}-/ \mathrm{RP}+)$ and 21 patients suffered from neuropathy and retinopathy $(\mathrm{NP}+/ \mathrm{RP}+)$. All participants were free from peripheral macrovascular disease. Following TENS an increase in skin blood flow was found at the dorsum of the foot without any significant difference between the groups. Compared to the control group. axon reflex vasodilatation was significantly reduced in the $\mathrm{NP}+/ \mathrm{RP}$ - group and the NP+/RP+ group at the pulp of the hallux ( $61 \pm 15$ vs. $-6 \pm 16$; vs. $23 \pm 5$; $p<0.05$ respectively). All investigated groups exhibited a significant increase in skin blood flow after arterial occlusion without any significant difference between the groups. A good association was observed between postocclusive and TENS-induced hyperaemia at the dorsum of the foot $(r=0.39 ; p=0.0002)$ but only a weak association was found at toe $(r=0.24 ; p=0.03)$. TENS- induced hyperaemia was associated with a diminished sweat response $(p=0.03)$, but not with pathological cardiovascular function tests $(p=0.07)$. In diabetic neuropathy, axon reflex vasodilatation is reduced at the pulp of the hallux reflecting the need for an intact nerve supply for the neurovascular regulation in areas rich with arteriovenous anastomoses. The diminished neurovascular response is independent of vascular alterations or endothelial dysfunction.

\section{3}

INFRARED DERMAL THERMOMETRY IN THE HIGH RISK DIABETIC FOOT

A Lavery, DG Armstrong, PJ Liswood, and WF Todd The University of Texas Health Science Center at San Antonio, 7703 Folyd Curl Drive, San Antonio, Texas $78284-7776$

The aim of this study was to compare skin temperatures using an infrared skin thermometer in feet with peripheral neuropathy, neuropathic ulcers and Charco fractures in relation to contralateral foot temperatures.

Research Design and Methods: One hundred forty three consecutive diabetic subjects presenting for care between 1993-1995 were divided into three groups for purposes of evaluation. These groups included subjects that had 1) asymptomatic peripheral sensory neuropathy $(n=78), 2)$ neuropathic foot ulcerations $(n=44)$, and 3 ) neuropathic (Charcot) fractures $(n=21)$. Subjects were excluded if they had Charcot's joint a concomitant ulceration, peripheral arterial occlusive disease, or active infection. We evaluated skin temperatures with an infrared skin temperature probe. Each subject's contralateral extremity served as their own control. The anatomic site of ulceration or Charcot fracture was matched on the contralateral extremity. Three anatomic sites were compared in the group without pathology. Results: There was a significant differences in skin temperature between both the Charcot $\left(8.30^{\circ} \mathrm{F}, \mathrm{t}=14.027, \mathrm{P}<0.0001\right)$ and Ulcer groups $\left(5.60^{\circ} \mathrm{F}, \mathrm{t}=14.219, \mathrm{P}<0.0001\right)$ and the region overlying the site of pathology on the corresponding contralateral limb. There was not a significant difference between feet in the group without pathology $(\mathrm{P}>0.05)$, with mean temperature differences never exceeding $0.35^{\circ} \mathrm{F}$ at any one site compared with the same site contralateraliy. Conclusions: The data suggest that monitoring of the corresponding contralateral foot site may provide objective, clinical information to evaluate high risk diabetic foot complications. Patients with high skin temperatures unilaterally may be at risk for ulceration or fracture. 


\section{4}

INCREASED CAPILLARY FRAGILITY IN THE DIABETIC FOOT.

P.D. Brash and J.E. Tooke. Dept. of Vascular Medicine, Diabetes Research, PGMS, Exeter, United Kingdom.

The long term complications of diabetes are associated with increased capillary fragility. Haemorrhage into callus beneath the diabetic foot is clinically well recognised, and evidence of microscopic haemorrhage in plantar soft tissues of diabetic patients with a history of ulceration has been described. It is unknown whether these haemorrhages are due to increased vertical or shear forces or a primary microvascular deficit. This study examines the hypothesis that capillary fragility may be associated with diabetic neuropathic foot ulceration. Neuropathic patients with prior ulceration, without prior ulceration and nonneuropathic controls; 12 per group, matched for age, sex and diabetes duration were studied. The neuropathic groups were matched for degree of neuropathy; mean VST \pm S.D. (volts) was $33.5 \pm 4.2 \mathrm{~V}$ (Previous uicer) $v 31.0 \pm 6.9 \mathrm{~V}$ (No ulcer). Resistance of capillaries to rupture was examined with the suction cup method on the dorsum of the foot. The least suction applied for one minute, producing one or more petechiae was the value of capillary fragility. The tests were interpreted by a second "blinded" observer. The resistance of capillaries to rupture in the foot was significantly reduced in the group with previous neuropathic ulceration $(29.8 \pm 5.2 \mathrm{kPa})$ compared to neuropathic subjects without prior ulceration $(40.0 \pm 5.2 \mathrm{kPa}$, $\mathrm{p}<0.001)$ and non-neuropathic controls $(35.5 \pm 5.3 \mathrm{kPa}, \mathrm{p}<0.03)$. This study shows increased capillary fragility in the feet of diabetic subjects with previous neuropathic foot ulceration and may suggest a role in the pathophysiology of neuropathic ulceration.

\section{6}

AUGMENTED EXPRESSION OF LOCALLY SYNTHESIZED ENDOTHELIN 1 IS DETECTABLE IN DIABETIC FEET DUE TO MACROVASCULAR DISEASE BUT NOT TO NEUROPATHY.

M. Derwahl, J. Hammer, M. Bröcker and H. Schatz

Medizinische Universitätsklinik Bergmannsheil, Bochum. Germany.

We have recently reported on the augmented expression of endothclin I (ET I) in small and large vessels of patients with a diabetic foot. Since hyperinsulinemia leads to increased ET I synthesis. but increascd ET I expression is also found in non-diabetics with artherosclerotic lcsions. wo addressed the question whether augmented local secretion of $E T 1$ is restricted to patients with a diabetic foot or is also detectable in pattents with a neuropathic foot. We investigated systemic and local ET I plasma concentrations of diabetics with a diabetic foot due to neuropathy, but without clinically apparent arterosclerosis $(n=10)$, due to macrovascular disease $(n=9)$ and as control, of 6 healthy volunteers. Blood samples were obtained from a subcutaneous vein in the forearm and additionally from a vein of the foot. Samples of 10 healthy volunteers taken from both locations were used as the control. In addition. ET 1 levels were measured in 101 diabetics (27 type I, 74 type II) without diabetic foot. Plasma samples were extracted by Sep-Pak C18 cartridges and endothelin was measured by a RIA kit. In comparison to the controls without clinically apparent arterosclerosis (ET concentrations in a forearm vein $4.0 \mathrm{pg} / \mathrm{ml}$ and in a foot vein $3.8 \mathrm{pg} / \mathrm{ml}$ ). patients with macrovascular disease showed 2.5 (forearm) and 3.4 (foot) times higher ET concentration, while in patients with neuropathic foot no significant difference was found between both local and systemic ET levels. The importance of macrovascular lesions as a major cause of increased ET concentration was supported by our findings in whole group of diabetics: In diabetics with clinically apparent arterosclerosis $(\mathrm{n}=52)$ significantly higher ET level $(\mathrm{p}<$ 0.0001 ) were detectable than in diabetics without macrovascular disease whereas neuropathy did not show any effect. From these data we conciude that due ro its growth-stimulating and vasoconstrictive effect $E T 1$ is involved in the pathogenesis of angiopathic, but not of neuropathic foot.

\section{5}

Comparative study of microvascular blood flow disturbances in the diabetic foot lesions .

I.V.Gourieva,I.V.Mironova,I.A.Strokov and M.M.Kositchkin.

Diabetic Foot Centre, International Diabetes Program, Institute of Woking Ability and Rehabilitation, Moskow, Russia.

Both neuropathy and large vessels disease exhibit the impact on microvascular function playing one of the key roles in the development of diabetic foot. Aim of the study was to analyse microvascular blood flow lesions and evaluate a correlation between microangiopathy and neuropathy disturbances. 17 healthy controls (C) and 82 IDDM and NIDDM patient participated in the study: 15 without neuropathy $(\mathbb{N}-), 16$ with neuropathy $(\mathrm{N}+), 28$ with pure neuropathic ulcers (PNU) 9 with Charcot foot (Ch) and 14 with neuroichemic ulcers (NIU) (ankle/brachial index 0,65 $\pm 0,18$ (mean \pm SD)), Neuropathy was evaluated using Neuropathy Disability Score (NDS) and Autonomic Disability Score (ADS) according to Ewing. Blood flow response to local heating (1), three minute arterial occlusion (2) and active postural probe (3) was measured on the toe using a laser Doppler fluxmetry (LDF). The index of blood flow (IBF) to analyse the maximum blood flow response was calculated: $\mathrm{IBF}=(\max \mathrm{BF}$. basal BF) / basal BF) $\times 100 \%$. IBF correlates to NDS $\left(\mathrm{r}_{1}=0,44, \mathrm{p}<0,05 ; \mathrm{r}_{2}=0,84\right.$ $\left.p<0,01 ; r_{3}=0,69, p<0,05\right)$ and $\operatorname{ADS}\left(r_{1}=0.54, p<0,05 ; r_{2}=0,94 ; r_{3}=0,78, p<0,01\right)$.

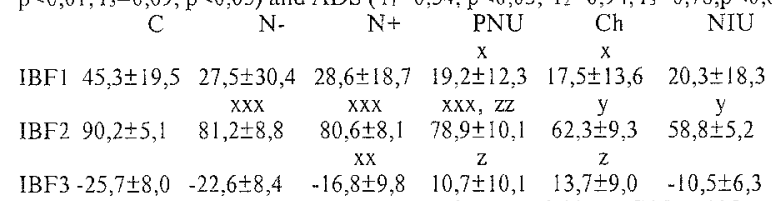
$x-p<0,05 ; x x-p<0,01 ; x x x-p<0,001$ vs $C . y-p<0,001$ vs $C, N$ and $N+$. $z-$ $\mathrm{p}<0,001$ vs NIU, $\mathrm{zZ}-\mathrm{p}<0,001$ vs $\mathrm{Ch}$ and NIU.

Conclusions: PNU and $\mathrm{Ch}$ groups exhibit the highest lack of response to thermal and posture tests. NIU exibit the most important lack of postocclusive hyperemia response. The results might be explain the different pathways of foot lesions in diabetes.

\section{7}

\section{FOOT INFECTION IN DIABETICS}

V.Urbančič-Rovan, M.Gubina*. University Medical Centre, Dept. of Endocrinology, ${ }^{*}$ Institute for Microbiology, Medical Faculty, Ljubljana, Slovenia.

Clinical and microbiological characteristics of 130 diabetic foot ulcers, treated at the Out-Patient Diabetic Foot Clinic, were analyzed. The majority of the observed group belonged to the group of spontaneously developed neuropathic ulcers, grade 2 by Wagner classification. The mean leukocyte count in the observed group was 8.22, with the values above normal $(\mathrm{L}>10.0)$ in $17.1 \%$ of cases. On the contrary, the mean ESR was 35.88 , with the values above 15 in $82.9 \%$ of cases. Wound surface area was below 2.0 $\mathrm{cm}^{2}$ in $56.2 \%$ of cases, $2.0-10.0 \mathrm{~cm}^{2}$ in $36.2 \%$ and over $10.0 \mathrm{~cm}^{2}$ in $7.7 \%$. No association between wound surface area and leukocyte count could be shown, on the other hand, there was positive linear association between wound surface area and $\operatorname{ESR}(P=0.0175)$. Superficial swabs were taken simoultaneously from the wound base and from the surrounding skin and cultivated in aerobic and anaerobic conditions. Wound swabs yielded on average 2.065 bacteria per sample and skin swabs 2.074 . Linear association between the number of bacteria in the wound and on the skin could be shown $(P=0.0001)$. The most frequent isolates were Staphylococci $(47.1 \%)$, Streptococci $(23.5 \%)$ and Gram-negative non-fermentative bacilli (19.5\%). Anaerobes represented only $5.4 \%$ of the isolates from the wounds. The isolates from the surrounding skin were essentially similar. $89.2 \%$ of the isolates were susceptible to ciprofloxacin and $86.4 \%$ to amoxicillint clavulanate. We conclude that in grade 2 diabetic foot ulcers leukocytosis is usually absent, while ESR as a more sensitive but less specific parameter, is elevated. Mixed flora, similar to that on the surrounding skin, can be isolated, with anaerobes being found only rarely. Good in vitro sensitivity to amoxicillin - clavulanate and to ciprofloxacin was shown in our group. 
1018

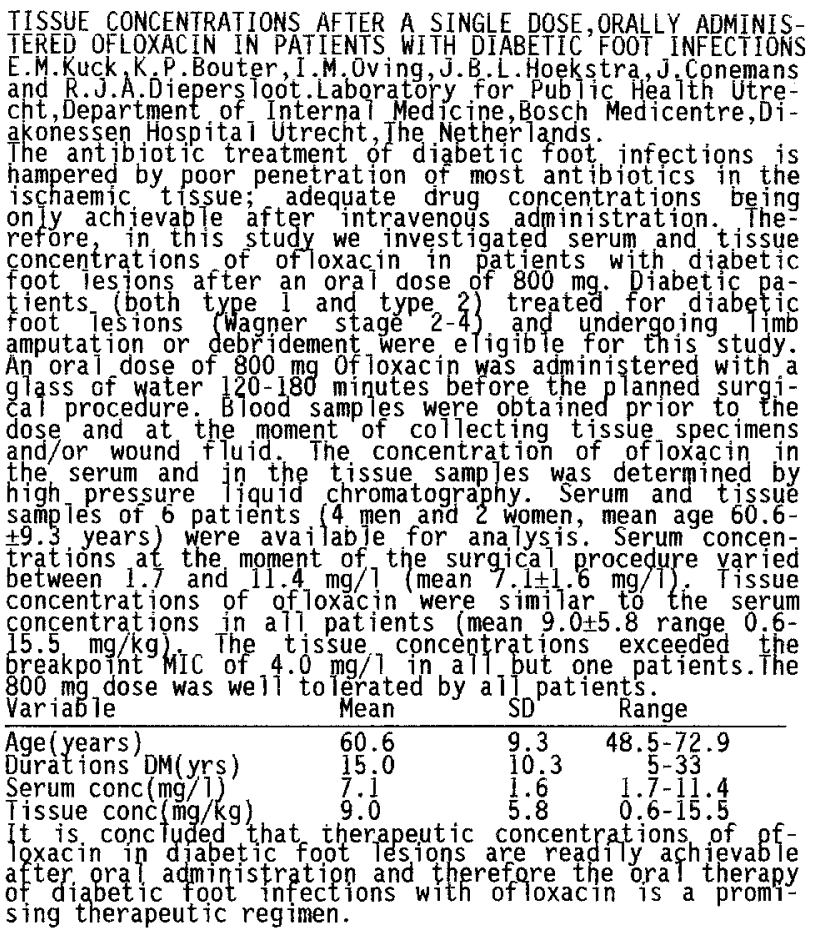

1019

a Clinical evaluation of a Collagen-Alginate topical WOUND DRESSING IN THE MANAGEMENT OF DIABETIC FOOT ULCERS. V.M. Donaghue, J.S. Chrzan, B.I. Rosenblum, J.M. Giurini, G.M. Habershaw and A. Veves. Deaconess-Joslin Foot Center, Boston, USA As the optimal local treatment for diabetic foot ulcers is not known we have compared the efficacy and safety of a collagen-alginate topical wound dressing (FIBRACOLTM, Johnson\&Johnson, Arlington, TX) and a regular gauze moistened in normal saline. Seventy-five patients with foot ulceration were randomly assigned in a 2:1 mode to Fibracol or gauze treatment groups. The two groups were matched for age, sex, weight, type and duration of diabetes, complications of diabetes, the size $(2.2 \pm$ $0.5 \mathrm{~cm}^{2}$ (mean \pm SE) vS. $3.3 \pm 0.8, p=N S$ ) and severity of the foot ulceration. Patients were followed until they healed or for a maximum period of 8 weeks. Forty-four ( $88 \%$ ) patients in the Fibracol and $17(68 \%)$ in the gauze group finished the study $(p<0.05)$. At the end of the study the mean percent reduction of wound area was $80.6 \% \pm 0.1$ in the Fibracol and $61.1 \% \pm 0.3$ in the gauze group $(p=N S)$. The survival analysis results showed that $43(98 \%)$ Fibracol treated patients reached $50 \%$ wound area reduction compared to $15(88 \%)$ gauze patients while the mean time required to $\geq 50 \%$ wound healing was $2.4 \pm 0.3$ weeks for the Fibracol and $2.5 \pm 0.7$ for the gauze group $(\mathrm{p}=\mathrm{NS})$. Complete healing was achieved in $24(55 \%)$ Fibracol and $9(53 \%)$ gauze ulcers ( $p=N S$ ) and the mean time required for this was $5.9 \pm 0.4$ vs. $5.3 \pm 0.6$ weeks $(p=N S)$. Patients' assessment of test dressing at study exit differed significantly in favor of Fibracol. Compared to previous treatment the dressing was reported to be easier to remove by 30 out of $39(77 \%)$ Fibracol and 1 out of $13(8 \%)$ gauze patient $(p<0.0001)$ while redressing was found to be easier by 34 out of $39(87 \%)$ and 0 out of 12 patients respectively (p $<0.0001)$. There were no difference in the number and severity of the reported adverse reactions, including infection, between the two groups. We conclude that Fibracol is equally effective and safe as the currently used treatment and that it may be more suitable for patient self. treatment.

\section{0}

DIFFERENCES IN THE OUTCOME OF FOOT ULCER TREATMENT IN TWO SEPARATE DIABETES CENTERS.

R.G. Frokburg. A. Piaggesib. V.M. Donaghue, E. Schipani ${ }^{\mathrm{b}}$, G.M. Habershaw". R. Navalesi ${ }^{\text {h }}$ and A Veves. ${ }^{a}$ Boston, USA and ${ }^{b}$ Pisa, Italy

Primary car: of the diabetic patient with foot ulcer can be provided by medically or surgically trained practitioners. We have prospectively followed 90 sequential patients with newly developed foot ulcers from two major centers: one in the USA where the primary doctor was a podiatrist and one in Europe with a diabetologist. Thirty-four patients from Boston and 56 from Pisa (mean age (range) 55.6 (26-75) years vs. $66.5(35-94), p<0.001$ ), matched for sex, weight, type, duration of diabetes, renal impairment and retinopathy took part. Boston patients had more severe neuropathy, assessed with clinical examination utilizing a neuropathy disability score $[16 \pm 6$ (mean \pm sd) vs. $6 \pm 3, p<0.001]$ and vibration perception threshold (46 \pm 8 Volts vs. $35 \pm 12 . p<0.001$ ) while no difference existed in the number of paticnts with clinical infection, a history of lower extremity by-pass operation $(6(18 \%)$ vs. $3(5 \%), p=N S)$ and in the size and the severity of the ulcer, according to the Wagner classification. Initial treatment was similar in both centcrs with emphasis on outpatient ulcer debridement, pressure relieving foot-wear and topical wound care. Hospitalization was needed in 5 $(15 \%)$ Boston and $12(21 \%)$ Pisa patients $(\mathrm{p}=\mathrm{NS})$ while surgery was performed on $5(15 \%)$ Boston and $16(29 \%)$ Pisa patients ( $\mathrm{p}=\mathrm{NS})$. The inhospital stay was similar in both centers (1.4 days \pm 4.4 vs. $2.1 \pm 5.9$, $\mathrm{p}=\mathrm{NS}$ ). The most common operations in both centers were incision, drainage and bone debridement. Ulcers healed in all patients but the amount of healing time was shorter in Boston patients $(6.7 \pm 4.2$ weeks vs. $10.5 \pm 6.5$, $p<0.02$ ). We conclude that despite the differences in the two systems similar success rates were achieved in the two centers while a more surgically oriented strategy may have resulted in a slightly shorter healing time.

\section{1}

TOTAL CONTACT CASTS : PRESSURE REDUCTION AT ULCER SITES AND THE EFFECT ON THE CONTRALATERAL FOOT

SA Vela, TL Quebedeaux, LA Lavery, and DC Lavery University of Texas Health Science Center, San Antonio, Texas 7703 Floyd Curl Drive Our aim was to compare the effectiveness of total contact casts with a cast boot, total contact casts with a cast heel, and commercially available therapeutic shoes to reduce mean peak planter foot pressures at the site of diabetic neuropathic ulcers and to determine if TCC's increase foot pressures on the contralateral foot. We used a repeat measures design with replications to compared peak pressures at ulcer sites under the 1st metatarsal (IMET $n=10$ ), and $2 n d-5$ th metatarsals (2-5MET $\mathrm{n}=10$ ) using three treatments: total contact casts with a cast bout (TCCB), total contact casts with a cast heel (TCCH), and P.W.Minor Xtra Depth® shoes (EDS).EDS were worn on the contralateral foot in each TCC treatment group. A rubber sole canvas oxford was used to establish baseline pressure values as a "worse case scenario". Pressure measurements at ulcer sites and on the contralateral foot were measured with the EMED Pedar in-shoe pressure measurement system and data for 40 steps were collected for each treatment. We used ANOVA and Tukey's Studentized Range Test for simultaneous multiple comparisons to compare treatments. TCCH reduced pressure better than TCCB for IMET and 2-5MET ulcers $(p<0.05)$. Both TCC methods reduced pressures better than EDS $(p<0.05)$, and neither TCC method increased contralateral foot pressures compared to EDS. Pressures in $\mathrm{N} / \mathrm{cm}^{2} \pm \mathrm{SD}$

$\begin{array}{lllll}\text { Ulcer Site } & \text { Baseline } & \begin{array}{l}\text { Xtra Depth } \\ \text { Shoes }\end{array} & \text { TCC-Boot } & \text { TCC-Heel } \\ \text { 1MET } & 44.6 \pm 17.6 & 39.5 \pm 14.9 & 7.0 \pm 5.4 & 6.0 \pm 2.6 \\ \text { 2-5MET } & 51.5 \pm 16.1 & 38.3 \pm 15.1 & 8.5 \pm 5.1 & 3.9 \pm 1.7 \\ \text { Contralateral Foot } & & & & \\ \text { 1MET } & 41.6 \pm 10.6 & 32.7 \pm 9.1 & 31.2 \pm 13.5 & 31.8 \pm 14.6 \\ \text { 2-5MET } & 42.6 \pm 15.6 & 32.0 \pm 12.1 & 28.3 \pm 10.3 & 31.1 \pm 11.7\end{array}$




\section{PS 55}

\section{Lipids}

1022

\section{THE EFFECT OF CHOLESTEROL ON POST- PRANDIAL LIPOPROTEINS}

G.H. Tomkin, C. Taggart J. Gibney D, Owens A. Johnson, P.Collins The Adelaide Hospital Dublin, Department of Biochemistry, The Royal College of Surgeons in Ireland and Department of Clinical Medicine, Trinity College Dublin.

We have previously demonstrated an increase in apo B48-containing lipoproteins post-prandially in NIDDM patients. This study examines the effect of dietary cholesterol on post-prandial lipoproteins in NIDDM. Ten poorly controlled NIDDM and ten control subjects - matched for age and BMI - were examined on two separate occasions one week apart. Subjects were given, in random order, a $1300 \mathrm{kcal}$ cholesterol-free breakfast with or without $1 \mathrm{~g}$ of cholesterol. Blood was taken fasting and post-prandially every 2 hours for up to $8 \mathrm{~h}$. Chylomicron and VLDL fractions were isolated by sequential ultracentrifugation and apo B 48 and apo B 100 were measured by gradient gel electrophoresis and quantified by densitometric scanning using an apo B100 standard. There was no significant difference in chylomicron apo B48 in the control subjects with either meal, but the cholesterol meal caused a significant reduction in apo B48 area under curve (AUC) in the chylomicron fraction in NIDDM patients (181.9 \pm 5.2 vs $153.9 \pm 2.8 \mathrm{P}<0.001)$. In the VLDL fraction from the diabetic patients VLDL apo B48 AUC increased from $16.8 \pm 1.1$ to $49 \pm 1.3(p<0.001)$ compared to controls which increased from $11.9 \pm 1$ to $21.5 \pm 1.5$ ( $p<0.001)$. Two subjects in each group had apo E phenotype other than $3 / 3$. Exclusion of these subjects did not alter the statistical significance of the results. In conclusion, in diabetes dietary cholesterol significantly increases intestinally-derived chylomicron remnants which are thought to be particularly atherogenic.

\section{4}

CTOLESTERYL ESTER TRANSFER IN THE PLASMA OF NIDDM PATIENTS

T Murakami. H Kawamura. T Yoshida. Y Yamazaki, T Kashiwa. Y Mizushima. S Iino, G Gianfranceschi (*) .C R Sirtori $(*)$, G Franceschini (*). Institute of Medical Sciences. St. Marianna University School of Medicine, Japan. University of Milan, Italy (*).

The aim of our study was to evaluate the mechanism of decreased cholesteryl ester transfer rate (CETR) in NIDDM patients. Subjects were 30 NIDDM patients (DM) treated by diet alone and compared to 30 age-, sex-, body mass index-matched non DM subjects ( $N-D M)$. CETP was evaluated by measuring the net mass transfer of CE from HDL (High density lipoprotein) to lower density lipoproteins. Plasama total cholesterol (TC) (210 \pm 32 in DM vs $218 \pm 40 \mathrm{in} \mathrm{N-IN.} \mathrm{mg/dl.} \mathrm{mean} \pm S D)$ and triglycerides (TG) $(185 \pm 101$ in DM vs $220 \pm 42$ in $\mathrm{N-DM}$. $\mathrm{mg} / \mathrm{dl})$ were comparable in the two groups. CETR was significantly lower in DM patients compared to $\mathrm{N}-\mathrm{DM}$ subjects $(42 \pm 21$ vs $70 \pm 31) \mathrm{nmol} / \mathrm{mi} / \mathrm{h}, \mathrm{p}<0.01)$. VLDL and HDL core weight ratio (CWR) were significantly higher in $D M$ patients $(0.921 \pm 0.040$ vs $0.780 \pm 0.051, \mathrm{p}<0.01 .0 .325 \pm 0.142$ vs $0.214 \pm$ $0.091, p<0.01$. respectively). In DM patients, CETR correlated positively with plasma VLDL-TC $(r=0.476, p<0.01)$ and apo $B(r=0.446$. $\mathrm{p}<0.02)$. inversely with $\mathrm{HDL}-\mathrm{TC} / \mathrm{TG}$ ratio $(\mathrm{r}=-0.46 \ldots, \mathrm{p}<0.02)$. apo A I $/$ AII ratio $(r=-0.651, p<0.001)$ and 1ipoprotein 1ipase (LPL) masses $(r=-0.448, p<0.02)$, but did not correlate with LPL or hepatic triglycerides lipase (HTGL) activity. Furthermore LPL masses were inversely correlated with $\mathrm{HDL}-\mathrm{CWR}(\mathrm{r}=-0.476, \mathrm{p}<0.01)$ and the $24 / \mathrm{h}$ urinary $C$-peptide excretion $(r=-0.425 . p<0.02)$. In conclusion. increased LPL masses, together with 1ipid compositions of acceptor or donor lipoprotein. was closely associated with decreased CETR in NIDDM. Further studies will have to assess the relation between the role of insul in resistance and decreased CETR in diabetic patients.

\section{3}

HIGH TRIGLYCERDE RESPONSE AFTER AN ORAL METABOLIC TOLERANCE TEST IN SONS WITH PARENTAL HISTORY OF THE METABOLIC SYNDROME

W.Stürmer and J.Schrezenmeir Dep. of Internal Med., Univ. of Würzburg, Germany Previous studies in 113 healthy young male volunteers have shown that during a standardized oral metabolic tolerance test (OMTT) $15 \%$ were characterized by a high triglyceride response (HR). A link to the metabolic syndrome was assumed because this distinct phenomenon was associated with higher p.p. blood glucose and insulin levels, increased proinsulin/insulin ratio, higher mass of abdominal adipose tissue and increased p.p. energy expenditure. The study presented here assessed apparently healthy sons whose father or mother suffered from a complete metabolic syndrome. During an OMTT (500ml liquid diet containing $58 \mathrm{~g}$ fat, $75 \mathrm{~g} \mathrm{CHO}, 10 \mathrm{~g}$ ethanol and $30 \mathrm{~g}$ protein) 13 out of 28 tested sons (46\%) showed fasting TG above $1.65 \mathrm{mmol} / 1$ and $19(69 \%)$ a high triglyceride response (p.p. $\left.\mathrm{TG}_{\max }<2.65 \mathrm{mmol} / \mathrm{l}\right)$. These HR-sons were compared with normal responding (NR) controls matched for age and BMI but without family history of NDDM, hypertension and hypertriglyceridemia.

\begin{tabular}{|c|c|c|c|c|c|c|c|}
\hline mean & $\mathbf{n}$ & $\begin{array}{c}\text { age } \\
\text { (years }\end{array}$ & $\begin{array}{l}\text { BMI } \\
\mathrm{kg} / \mathrm{m}^{2}\end{array}$ & WHR & $\begin{array}{l}\text { fast.TG } \\
(\mathrm{mmol} / \mathrm{)})\end{array}$ & $\begin{array}{l}\max \text { TG } \\
(\operatorname{mm} 0 / /)\end{array}$ & $\begin{array}{l}\text { fast. IRI } \\
(\mu \mathrm{U} / \mathrm{ml})\end{array}$ \\
\hline HR-sons & 19 & 28.9 & 26,1 & 0,93 & 2.30 & 4,74 & 15,0 \\
\hline controls & 19 & 27,9 & 25,9 & 0,90 & 1,05 & 2,46 & 10,5 \\
\hline p. value & & n.s. & n.s. & $<0,01$ & $<0,01$ & $<0,001$ & n.s \\
\hline \multicolumn{8}{|c|}{ WHR: waist to hip ratio; TG: triglycerides; OGTT: oral glucose tolerance test (75g) } \\
\hline mean & \multicolumn{2}{|c|}{$\begin{array}{l}\text { fast BG } \\
(\mathrm{mmol} / \mathrm{l}) \\
\end{array}$} & $\begin{array}{l}\text { BP syst. } \\
\text { (mmHg) }\end{array}$ & $\begin{array}{l}\text { BP diast. } \\
(\mathrm{mmHg})\end{array}$ & $\begin{array}{c}\mathbf{H b A}_{1 \mathrm{c}} \\
(\%)\end{array}$ & \multicolumn{2}{|c|}{$\begin{array}{c}\text { AUC-BG-OGTT } \\
(\mathrm{mmol} / / \times 180 \mathrm{~min})\end{array}$} \\
\hline HR-sons & \multicolumn{2}{|c|}{4,55} & 132 & 82 & 5,2 & \multicolumn{2}{|c|}{1463} \\
\hline controls & \multicolumn{2}{|c|}{4,38} & 130 & 80 & 5,1 & \multicolumn{2}{|c|}{1096} \\
\hline $\mathrm{p}$-value & \multicolumn{2}{|c|}{ n.s. } & n.s. & n.s. & n.s. & \multicolumn{2}{|c|}{ n.s. (no case of IGT) } \\
\hline
\end{tabular}

Conclusion: The penomenon of high TG response after OMTT might be a simple approach to screen apparently healthy (normotensive, glucosetolerant) young adults with family history of NIDDM for their individual metabolic risk.
1025

Insulin resistance and increased cholesteril ester transfer protein (CETP) activity in offspring of patients with the metabulic syndrome

D. Kopf, *A. Ambrosch, 1. Mühlen, *S. Westphal, B. Nestler. I. Sendzik, *C. Luley, and H. Lehnert. Klinik für Endokrinologie und Stoffwechsel, *Institut fuir Klinische Chemie, Uniklinik Magdeburg, Leipziger Str. 44, 39120 Magdeburg, Germany.

In this study, wc investigated insulin sensitivity and the effect of non esterified fatty acids NEFAs on the extent of CETP activation in healthy subiects with and without a family history of the metaholic syndrome. Probands: 9 healthy, young (mean age $24 \pm 5$ years) relatives from families with hypertension. diaheles and / or hyperlipidemia (group 1) were compared with 15 controls (group 2). Body mass index and waist to hip ratio were comparable. Methods: All probands obtained an intravenous heparin bolus (100 $\mathrm{iU} / \mathrm{kg} \mathrm{KG)} \mathrm{after} \mathrm{an}$ overnight fast. Blood samples were collected hefore and $30 \mathrm{~min}$ after heparin. and concentrations of NEFAs were determined. Extent of CETP activation was expressed as difference of cholesterolester transfer aetivity $(\triangle C E T)$ between basal values $\left(\mathrm{CET}_{\text {basa }}\right)$ and after heparin bolus $\left(\mathrm{CET}_{\text {basal }}\right)$ ( $\triangle \mathrm{CET}=$ $C E T_{\text {pessheparin }}-\mathrm{CET}_{\text {basal }}$ ). Insulin sensitivity was determined hy hyperinsulinemic cuglycemic clamp test. Results: Probands with a positive family history had higher fasting triglycerides and a significantly lower glucose utilisation rate $(39,3 \pm 10.1 \mathrm{mg} / \mathrm{kg} \mathrm{KG})$ than subjects without a negative family history $(49,4 \pm 8,6 \mathrm{mg} / \mathrm{kg} \mathrm{KG}, \mathrm{p}=0,04)$. They also had a greater fatty acid excess after heparin holus and higher extent of CETP activation ( $\triangle$ CET group 1: 23,2 $\pm 8,5$ $\mathrm{pmol} / \mathrm{ml} / 3 \mathrm{~h} ;$ grour 2; $6,9 \pm 6,7 \mathrm{pmol} / \mathrm{ml} / 3 \mathrm{~h} ; \mathrm{p}=0,0016)$. $\Delta$ CET correlated well with the NEFA excess $(r=0,785 ; p<0,001)$. Conclusion: Healthy offspring of families with a history of metaholic syndrome had higher trigylceride levels, a consequently greater excess of NEFAs after heparin and a lower insulin sensitivity. As the extend of CETP activation correlated well with the NEFA excess, they also showed a higher degree of CETP activation. 
1026

CETP POLYMORPHISM AND MODULATION OF LIPID PARAMETERS IN TYPE 2 DIABETES.

A. Durlach ${ }^{1}$, C. Clavel ${ }^{1}$, V. Durlach ${ }^{2}, P$. Birembaut ${ }^{1}$ and $M$. Leutenegger ${ }^{2},{ }^{1}$ Pol Bouin Institute, ${ }^{2}$ Medical Clinic, Reims, France.

Cholesterol Ester Transfer Protein (CETP) is a key regulating factor of lipid metabolism and the polymorphism of its gene may be a candidate for modulating the lipid parameters in NIDDM subjects. In a group of 384 NIDDM patients aged $59.4 \pm 10.9$ years, with a BMI of $28.7 \pm 5.4$ $\mathrm{kg} / \mathrm{m} 2$ and $\mathrm{HbA} / \mathrm{c}=8.3 \pm 9 \%$, we studied the $\mathrm{B}$ polymorphism at the CETP locus detectable with the restriction enzyme TaqI. Patients were separated in 220 male $(74 \mathrm{~B} 1 \mathrm{~B} 1,103 \mathrm{~B} 1 \mathrm{~B} 2,43 \mathrm{~B} 2 \mathrm{~B} 2)$ and 164 female (45 B1B1, $87 \mathrm{~B} 1 \mathrm{~B} 232 \mathrm{~B} 2 \mathrm{~B} 2$ ) and compared on the basis of their lipid parameters (total cholesterol, triglycerides, HDL-cholesterol, ApoA1 ApoB LDL-cholesterol, Lp(a)). The only significant differences were found in the male group which showed higher HDL-cholesterol and apoA1 serum levels in the $\mathrm{B} 2 \mathrm{~B} 2$ group compared to B1B2 (respectjvely $1.32 \pm 0.43$ vs $1.20 \pm 0.60 \mathrm{mmol} / \mathrm{l}, \mathrm{p}<0.05$, and $1,33 \pm 0.21$ vs $1.22 \pm 0.20 \mathrm{~g} / \mathrm{l}$ $\mathrm{p}<0.05$ ). No significant difference was observed in the female group. Thus CETP polymorphism seems to exert a modulating role only on HDL-cholesterol and apoA1 in male NIDDM subjects which is not observed in females and may be of importance in the perspective of macroangiopathic complications.

\section{8}

AUTOANTIBODIES AGAINST MODIFIED LDL ARE ASSOCIATED WITH INSULIN RESISTANCE IN DYSLIPIDEMIA

$K$. Malminiemi, $O$. Malminiemi and T. Lehtimäki Tampere University Hospital and Tampere University Medical School, Tampere, Finland

We studied the reversibility of LDL-autoantibodies and their association with other metabolic risk factors in 148 dyslipidemic, non-diabetic patients. The criteria for dyslipidemia was LDL $>4$ $\mathrm{mmol} / \mathrm{l}$ and/or HDL-ratio $<0.2$. An oral glucose $(1.2 \mathrm{~g} / \mathrm{kg})$ rolerance test (OGTT) with serum insulin and lipid measurements was carried out on the pre-study therapy and after 6 and 12 months on a different antihypertensive monotherapy. Autoantibody (Ab) titers of oxidized LDL were measured using an enzyme-linked immunosorbent assay. EDTA plasma samples were incubated in wells coated with antigens: native $L D L$ protected against oxidation (nat-LDL), or oxidized LDL (tot-LDL, copper-facilitated). Color was produced with a peroxidase-mediated reaction. Difference in absorbance at $492 \mathrm{~nm}$ between tot-LDL $A b$ and nat-LDL $A b$ represents specific autoantibody against oxidized LDL (ox-LDL $A b$ ).

$\mathrm{Ox}-\mathrm{LDL} \mathrm{Ab}$ was statistically significantly associated with fasting serum insulin $(r=.27, p=.003\}$ and cumulative $(0-2 h)$ insulin secretion in OGTT $(r=.46, p<.0001)$. Association with glucose parameters was weaker and not significant; eg. with cumulative serum glucose during $0.2 \mathrm{~h}$ in OGTT $r=.12(p=.066)$. Ox-LDL Ab did not correlate with serum total cholesterol, LDL, HDL. triglycerides or blood pressure. Weak associations were seen with smoking $(r=.22, p=.04)$ and body mass index $(r=.16, p=.07)$. These results indicate that autoantibodies against oxidized LDL are independently associated with hyperinsulinemia and decreased insulin sensitivity. Ox-LDL Ab titer may prove to be a clinically useful indicator of metabolic risk in atherosclerotic diseases.
1027

CHOLESTEROL SYNTHESIS IN TYPE II DIABETIC PATIENTS.

A. Scoppola ${ }^{* \wedge}$, G. Testa, D. Caccese ${ }^{\circ}$, A. Mancini, G. Menzinger*, A. Lala*.

Cattedre di *Endocrinologia e ${ }^{\circ}$ Biochimica Clinica, Università di Roma "Tor Vergata"; NIstituto Dermopatico dell'Immacolata, IRCS, Roma; Servizio di Diabetologia, Osp. S Camillo, Roma.

We have previously demonstrated in a selected group of normolipidemic type II diabetic patients an increased cholesterogenesis that was normalized after insulin therapy. In this study, we evaluated the cholesterol synthesis in a group of 64 type II diabetic outpatients $(33 \mathrm{M}, 31 \mathrm{~F}$; aged $58 \pm 7.5$ years; BMI $28.5 \pm 4.5 \mathrm{Kg} / \mathrm{m}^{2}$ ). The 24 -h urinary mevalonate excretion (MVA) an index of whole body cholesterol synthesis, was evaluated by $\left.\mathrm{GC}_{[} \mathrm{Cl}^{-}\right] \mathrm{MS}$. MVA excretion ranged from 0.5 to $5.0 \mu \mathrm{mol} / 24 \mathrm{~h}$ with a mean value of $1.7+0.1 \mu \mathrm{mol} / 24 \mathrm{~h}$ (mean $\pm E S M)$. The $\mathrm{HbAlc}$ ranged from 4.1 to $14.6 \%$ with a mean value of $8.6 \pm 0.3 \%$. The patients with $\mathrm{HbAlc}<8 \%(\mathrm{n}=26)$ had a MVA excretion of $1.3 \pm 0.1 \mu \mathrm{mol} / 24 \mathrm{~h}$, while those having $\mathrm{HbAlc}$ $>8 \%(\mathrm{n}=38)$ had a MVA excretion of $2.1 \pm 0.2 \mu \mathrm{mol} / 24 \mathrm{~h} \quad(\mathrm{p}<0.001)$ A multiple regression analysis demostrated a direct relationship between MVA excretion and HbAlc $(r=0.27$ $\mathrm{p}<0.004$ ) while no significant relationships were found with BMI, total cholesterol, LDL-cholesterol and triglycerides. Finally MVA values were $1.9 \pm 0.2 \mu \mathrm{mol} / 24 \mathrm{~h}$ in patients under OHA $(n=30)$ and $1.6 \pm 0.2 \mu \mathrm{mol} / 24 \mathrm{~h}$ in those under insulin treatment $(n=24)$. In conclusion, the present study demonstrates that cholesterogeresis has wide variations in type II diabetic patients. Among the possible factors involved, the degree of metabolic control seems to be the major determinant.
1029

FATTY ACID COMPOSITION OF LDL SUBFRACTIONS IN NIDDM

D. Owens, J. Prescott P.Collins, A. Johnson, amd GH. Tomkin. Department of Clinical Medicine Trinity College Dublin, The Royal College of Surgeons in Ireland and The Adelaide Hospital Dublin, Ireland

Small dense LDL (LDL3) which is associated with atherogenesis, is more frequently found in diabetes. We have previously demonstrated the dependence of LDL oxidisability on LDL fatty acid composition. This study examines fatty acid composition in large buoyant LDLI and small dense LDL3. Ten well-controlled and 10 poorly-controlled NIDDM patients and 10 control subjects were examined. LDL subfractions were isolated by density gradient ultracentrifugation and LDL fatty acids measured by GLC. Ten of the diabetic patients could clearly be defined as having a small dense LDL 3 and 5 had large LDL1 phenotypic pattern. NIDDM patients had significantly more fatty acids in LDL compared to controls ( $985 \mu \mathrm{g} / \mathrm{mg}$ LDL protein vs $568 \mu \mathrm{g} / \mathrm{mg}$ LDL protein $p<0.01$ ) The poorly controlled NIDDM patients had similar LDL1 fatty acids as well controlled subjects but double the amount of LDL 3 fatty acids $(294.8 \mathrm{vs} 144.8 \mu \mathrm{g} / \mathrm{mg}$ LDL protein $p<0.05)$. The only significant difference in LDLI fatty acids was a lower oleic acid in the poorly-controlled NIDDM patients compared to control and wellcontrolled subjects $(49 \pm 8$ vs $89 \pm 10$ and $87 \pm 14 \mu \mathrm{g} / \mathrm{mg}$ LDL protein $p<0.01$. Linoleic acid was significantly higher in LDL 3 from poorlycontrolled NIDDM compared to both well-controlled and control subjects $(75 \pm 7$ vs $33.1 \pm 11.3$ and $32.5 \pm 6 \mu \mathrm{g} / \mathrm{mg} \mathrm{p}<0.05)$ as were palmitic ( $143.7 \pm 20$ vs $45.7 \pm 10$ and $38.4 \pm 7.5 \mu \mathrm{g} / \mathrm{mg} \quad p<0.002$ ) and palmitoleic acid ( $32.0 \pm 7$ vs $6.1 \pm 1.5$ and $13.8 \pm 3.3 \mu \mathrm{g} / \mathrm{mg} \quad \mathrm{p}<0.002)$. Comparison of those diabetic patients with small dense LDL phenotype with those with large LDL phenotype revealed significantly greater \% linoleic acid in LDL $3(83 \pm 10$ vs $53 \pm 9 \mathrm{p}<0.05)$. The study suggests that in poorly-controlled NIDDM patients with small dense LDL phenotype the linoleic acid content may be responsible for the increased oxidisability of LDL3. 
1030

\section{APOLIPOPROTEIN H LEVELS IN NORMAL AND DIABETIC SUBJECTS.}

R. Gambino, G. Ruiu, M. Cassader, F. Veglia* and G.Pagano. Dipartimento di Medicina Interna, C so Dogliotti 14, 10126 Torino, Italy. * Unita' di Epidemiologia - Istituto Scientifico H S.Raffaele - Milano Italy.

One of the most common alterations in non-insulin-dependent diabetes mellitus (NIDDM) involves the triglyceride-rich lipoproteins. Quantitative and qualitative apolipoprotein variations appear to figure among the several factors responsible. Of the several apolipoproteins that modulate $\mathrm{Tg}$ metabolism. apolipoprotein $\mathrm{H}(\mathrm{Apo} \mathrm{H}$ ) may be involved. In order to assess the role of Apo $\mathrm{H}$ on lipid metabolism in diabetes mellitus we bave examined the correlation between its plasma concentration and the main plasma lipid levels in 127 non-insulin-dependent and 118 insulindependent (IDDM) patients. The data are compared with those in 286 nor-diabetics. Our data show a significant increase in plasma Apo $\mathrm{H}$ in

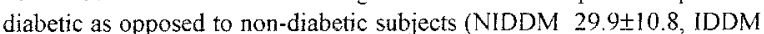
31.3 \pm 9.9 , controls $22.5 \pm 7.7 \mathrm{mg} / \mathrm{dl}, \mathrm{F}=53.3, \mathrm{p}=0.0001$ ). The relation between plasma lipids and Apo $\mathrm{H}$ was simultaneously evaluated in the three groups with inclusion of diabetes, sex, BMI and age as covariates in the model. This analysis revealed a strong positive correlation $(\mathrm{p}=0.0009)$ between apolipoprotein $\mathrm{H}$ and total cholesterol, a weaker positive correlation with triglycerides $(p=0.016)$. The correlation between the levels of Apo $H$ and those of $\mathrm{HbA}_{\mathrm{Ic}}$ in diabetics $(\mathrm{p}=0.03)$ highlights the importance of glycaemic control toward plasma levels of this apoprotein which is highly glycated. Though the role of Apo $\mathrm{H}$ in lipid metabolism is still uncertain. the recent investigations on the possible relation between plasma Apo $\mathrm{H}$ levels and increased plasma lipids and the thrombotic risk could explain the increased atherosclerotic risk in diabetic patients.

1032

POSTPRANDIAL FAT INTOLERANCE IS NOT MODIFIED BY PRESENCE OF APOLIPOPROTEIN $€ 4$ ALLELE IN NIDDM SUB.JECTS N. Mero, R Malmström and M.-R. Taskinen, Department of Mcdicine, Helsinki University, Haartmaninkatu 4, 00290 Helsinki, Finland

Apolipoprotein $\epsilon 4$-allele has been associated with increased risk for CAD and delayed clearance of postprandial triglyceride-rich lipoproteins (TRL). Since fat intolerance is a feature of diabetic dyslipidemia, the aim of this study was to investigate does the presence of $\in 4$-allele aggravate postprandial lipemia in NIDDM subjects. We compared 16 subjects with apo E $3 / 3$ phenotype with 9 subjects with apo $E 3 / 4(n=7)$ and $E 4 / 4(n=2)$ phenotypes. The two groups had similar age (mean $\pm \mathrm{SE} ; 55.8 \pm 1.6$ vs. $60.8 \pm 0.6$ years), BMI ( $30.1 \pm 0.6$ vs. $31.1 \pm 0.89 \mathrm{~kg}$ ) and glycemic control $\left(\mathrm{GhBA}_{1 \mathrm{C}} 8.0 \pm 0.2 \mathrm{vs.} 7.4 \pm 0.4 \%\right)$. Likewise, fasting TG ( $2.9 \pm 0.2$ vs. $2.5 \pm 0.3 \mathrm{mmol} / \mathrm{l})$ and HDL-cholesterol ( 1.12 \pm 0.03 vs. $1.10 \pm 0.05 \mathrm{mmol} / \mathrm{l}$ ) were comparable. The subjects were given a liquid fat tolerance test (FTT) consisting of soybean oil $\left(50 \mathrm{~g} / \mathrm{mi}^{2}\right)$ and $150000 \mathrm{IU}$ of Vitamin A. Blood samples were obtained before and 4, 6 and 8 hrs after the FTT. Lipoproteins were subfractionated with gradient UCF into $S \mathrm{~S}>400$, Sf $60-400, \mathrm{Sf}$ 20-60 and Sf 12-20. Apolipoprotein B-48 (apo B-48) as a marker of intestinal TRL and apolipoprotein B-100 (apo B-100) were measured with SDS-PAGE from these fractions. After the FTT the maximum concentrations of TG, apo B48 and apo B-100 were reached at the same time and postprandial responses (area under the curve and incremental areas) in each fraction were similar in the two groups. Responses of TG and apo B-48 in S $>400$ and Sf $60-400$ were aggravated, and the return to the baseline delayed similarly in the two NIDDM groups as compared to the normal range. At $8 \mathrm{hrs}$ the $\mathrm{Sf}>400$ apo B-48 concentration was $0.478 \pm 0.075 \mathrm{mg} / \mathrm{l}$ (vs. $0.046 \pm 0.019 \mathrm{mg} / \mathrm{l}$ at baseline, $p<0.001)$ in apo $E 3 / 3$ subjects and $0.470 \pm 0.100 \mathrm{mg} / 1(0.065 \pm 0.025 \mathrm{mg} / \mathrm{l}$ at baseline, $p<0.001)$ in $\in 4$-allele subjects. We conclude that the delayed clearance of chylomicron remnants is an inherent feature of diabetic dyslipidemia and this is not further aggravated by the presence of $€ 4$-allele.

\section{1}

LONG-TERM EFFECTS OF FISH-OIL ON LIPOPROTEIN SUBFRACTION DISTRIBUTION AND LDL SIZE IN NIDDM PATIENTS.

A. Maffettone, C. Iovine, L. Patti, L. Di Marino, G. Annuzzi, M. G. Ferrara, G. Riccardi and A. A. Rivellese. Institute of Internal Medicine and Metabolic Diseases, Medical School of Medicine, Federico Il University, Via S. Pansini, 5, 80131 Naples, Italy

The effects of fish-oil on the composition and distribution of lipoprotein subfractions as well as on LDL size in NIDDM hypertriglyceridemic patients are unknown. To elucidate this point, 16 NIDDM hypertriglyceridemic (TG $>2.25<5.65$ $\mathrm{mmol} /$ ) patients, after a 4-week wash-out period and a 3-week run-in period, were randomly assigned to a six month period with either fish-oil (EPA+DHA $2.7 \mathrm{~g} /$ day for 2 months, then $1.7 \mathrm{~g} /$ day for 4 months) $(n=8)$ or placebo ( $3 \mathrm{~g} /$ day olive oil) $(\mathrm{n}=8)$. Diet and hypoglycemic treatment remained unchanged throughout the experiment. At baseline and after six months plasma lipids, VLDL, LDL, HDL lipoproteins (preparative ultracentrifugation+precipitation method) and LDL size (gradient gel electrophoresis) were evaluated. Moreover, three VLDL and LDL subfractions (density gradient ultracentrifugation) were evaluated only at the end of the two periods. Cholesterol (CHOL), triglycerides (TG) and phospholipids (PL) were determined on each lipoprotein and subfraction. Fish-oil treatment induced:1) a reduction of all lipids in VLDL (TG $2.35 \pm 0.24$ vs $4.25 \pm 0.66 \mathrm{mmol} / \mathrm{l}, \mathrm{p}<0.01$; CHOL $1.47 \pm 0.13$ vs $2.77 \pm 0.76 \mathrm{mmol} / \mathrm{p}$, $<0.05:$ PL $0.82 \pm 0.07$ vs $1.42 \pm 0.31 \mathrm{mmol} / \mathrm{l}$, NS) (M \pm SEM) without changes in the composition; 2) a significant increase in LDL CHOL ( $3.29 \pm 0.49$ vs $2.88 \pm 0.20 \mathrm{mmo} / / \mathrm{l}, \mathrm{p}<0.01)$ with a subsequent enrichment in their CHOL content (CHOL/TG $10.8 \pm 1.13$ vs $8.45 \pm 1.15$, p $<0.04$ ); 3) no significant change in LDL size (255.4+2.2 vs $254.2 \pm 1.7 \AA) ; 4)$ no effect on the concentration and composition of HDL. There was no change in all these parameters in the placebo group. Moreover, the percentage distribution of VLDL subfractions (large $38 \pm 3$ vs $38 \pm 3 \%$, intermediate $28 \pm 1$ vs $31 \pm 2$, small $35 \pm 4$ vs $31 \pm 2 \%$ ) and LDI subfractions (large $17 \pm 3$ vs $17 \pm 2 \%$; intermediate $62 \pm 3$ vs $65 \pm 3 \%$; small $21 \pm 3$ vs $18 \pm 2 \%$ ) was very similar at the end of fish-oil and placebo. In conclusion, long-term fish oil treatment induces: 1) a significant reduction of VLDL without changes in the composition and distribution of VLDL subfractions; 2) a cholesterol enrichment of $L D L$ without changes in the size of LDL and distribution of their subfractions.

\section{3}

APOLIPOPROTEIN A-IV PHENOTYPE AND PREVALENCE OF MACROVASCULAR DISEASE IN CAUCASIAN NIDDM PATIENTS. B.Vergès, L.Lagrost, G.Vaillant, J.M. Petit, M.Cohen, P.Gambert and J.M.Brun University Hospital, Dijon, France.

Apolipoprotein A-IV (apoA-IV), which might play an important role in lipoprotein metabolism, is characterized by genetic polymorphism with apoA-IV 1-1 and apoAIV 1-2 as the two most frequent phenotypes in humans. Several studies have pointed out structural and functional differences between the 2 major isoproteins apoA-IV-1 and apoA-IV-2. Recently, the apoA-IV 1-2 phenotype has been shown to be associated with increased atherosclerosis in Hispanic NIDDM patients from a Colorado community. The aim of the present study was to look for an association between the apoA-IV phenotype and prevalence of macrovascular disease in Caucasian NIDDM patients. One hundred and thirty six NIDDM patients (71 men 65 women) of Caucasian origin were studicd. ApoA-IV phenotyping was performed by isoelectric focusing ( $\mathrm{pH}$ 5-6) and immunoblotting. Macrovascular disease was assessed, in each patient, by a standardized questionnaire, physical examination, resting ECG and continuous wave Doppler velocimetry. Moreover, patients without any history of coronary heart disease and showing a normal resting ECG underwent a bicycle exercise test or a dipyridamole thallium scintigraphy in order to detect possible silent myocardial ischemia. The apoA-IV 1-1 phenotype was found in $83 \%$ (113) of the NIDDM patients and the apoA-IV 1-2 phenotype in $27 \%$ (23).No significant differences between the 2 apoA-IV phenotypes were observed for age, sex distribution, hypertension, total cholesterol, triglycerides, HDL-cholesterol, fasting glycemia. HbAlc, fasting insulin and fasting C-peptide. Mean Body Mass Index was significantly slightly higher in the apoA-IV 1-2 phenotype ( $p=0.03$ ). However, prevalence of macrovascular disease was not significantly different between the 2 phenotypes ( $45 / 113$ vs 11/23). The influence of different factors (age, BMI, cigarette smoking, hypertension, total cholesterol, triglycerides, HDLcholesterol, fasting glycemia, fasting C-peptide, microalbuminuria and apoA-IV phenotype) on the prevalence of macrovascular disease was analyzed with a logistic regression model. In the multivariate analysis, only age $(p=0,016)$, triglycerides $(\mathrm{p}=0.019)$ and hypertension $(\mathrm{p}=0.045)$ were positively associated with macrovascular disease when apoA-IV phenotype was not. In conclusion: The apoAIV phenotype is not associated with prevalence of macrovascular disease in Caucasian NIDDM patients. 
1034

LACK OF ASSOCIATION OF LIPOPROTEIN (a) WITH CORONARY HEART DISEASE IN SPANIARD TYPE 2 DIABETIC PATIENTS

F. Relimpio, A. Pumar, F. Losada, F. Morales, D. Acosta, R. Astorga

Servicio de Endocrinologia. Hospital Universitario Virgen del Rocio. Seville. Spain

We aim to elucidate if there is a relationship between lipoprotein (a) levels and coronary heart disease and to assess the presence of lipoprotein (a) covariates in NIDDM. We have selected 41 type 2 diabetic patients harboring coronary heart disease and 82 type 2 diabetic patients free from cardiovascular disease adjusted for age, sex and duration of diabetes. Routine chemistries have been measured by standard procedures, $\mathrm{HbA}_{10}$ by HPLC and lipoprotein (a) and urinary albumin excretion rate by immunonephelometry. No difference has been found in lipoprotein (a) levels between both groups of study $(18[144.25] \mathrm{mg} / \mathrm{dl}$ in cases vs. 23 [197.25] in controls (median [range]), Mann-Whitney $U$ test, $\mathrm{p}>0.1$ ). No association has been found between coronary heart disease and a lipoprotein (a) level greater than $30 \mathrm{mg} / \mathrm{dl}$ (Pearson's chi-square, $p>0.1$ ). Significant and independent linear relationships have been found between the square root of lipoprotein (a) levels and serum creatinine and total cholesterol (multiple r-square: $0.15, \mathrm{p}<0.001$ ). Patients submitted to insulin therapy had greater square root of lipoprotein (a) levels, even after adjusting for serum creatinine and total cholesterol $(5.87 \pm 0.35$ vs. $4.76 \pm 0.36$ (mean $\pm \mathrm{SE}$ ) ANCOVA, $\quad \mathrm{<}<0.05$ ). These data fail to find an association between symptomatic $\mathrm{CHD}$ and $\mathrm{l}(\mathrm{a}$ (a) in NIDDM. Significant and independent relationships have been found between this variable and serum creatinine, total cholesterol and insulin therapy.
1035

FREE FATTY ACIS IN PLASMA: DIABETES INDEPENDENT CAUSE FOR THE ACCELERATED ATHEROGENESIS IN CAPD?

C.Holler $^{1}$, F.Ulberth ${ }^{2}$, M.Auinger ${ }^{1}$, and K. Irsigler ${ }^{1}$. 1) L. Boltzmann Institute for Metabolic Diseases and Nutrition, City Hospital Lainz, Vienna 2) Department of Dairy Research and Bacteriology, Agricultural University Vienna, Austria.

In patients, undergoing continuous ambulatory peritoneal dialysis (CAPD) various lipid abnormalities are apparent: hypertriglyceridemia, hypercholesterolemia and dysapoproteinemia. Less data are available concening free fatty acids (FFA) as another cause for the accelerated atherogenesis in CAPD. The aim of this study was to determine the FFAs in plasma by capillary gaschromatography after direct transesterification in patients undergoing CAPD without diabetes $(n=8)$, with insulin dependent diabetes $(n=10)$ and non insulin dependent diabetes (NDDM, $n=9)$ in comparison to healthy controls ( $\mathrm{n}=41$ ), matched by sex, duration of $\mathrm{CAPD}$, age and body mass index except NIDDM. Statistical analysis was performed using ANOVA to determine differences among groups. The results showed significantly higher levels of FFA in the CAPD groups in comparison to the control group with no significant differences within the CAPD groups (Mean \pm SD; FFA $(\mu \mathrm{g} / \mathrm{ml})$ control $2736 \pm 878$ vs. CAPD-all $4070 \pm 1103$; $\mathrm{p}<0,001$ ). Looking at the fatty acids in detail, most of them were significantly higher in the CAPD groups, especially the atherogenic saturated-and trans fatty acids, only the dihomo- $\gamma$-linoleic acid and eicosapentaenoic acid were significantly lower, independent of the diabetic state. Thus in conclusion we may say that all CAPD patients are at higher risk for atherogenesis, independent of concomitant diabetes. The main causes of the accelerated atherosclerotic risk were the high concentrations of saturated-and trans fatty acids and the cisproportion of arachidonic acid to eicosapentaenoic acid, leading to a higher formation rate of potentially proatherogenic metabolites as thromboxane $\mathrm{A}_{2}$.

HIGH HDL-CHOLESTEROL LEVELS DO NOT PROTECT AGAINST ATHEROSCLEROSIS IN INSULIN DEPENDENT DIAHETES MELLTUS (IDDM)

${ }^{1} P . C$. Eskildsen, ${ }^{1} E$. Funder and ${ }^{2} S$. Stender

${ }^{\mathrm{I}}$ Medical Department and Dept. of Clinical Chemestry, Køge County Hospital, DK4600 Denmark, ${ }^{2}$ Clinical Institute, University of Odense, DK-5000, Denmark.

Based upon the observation of high levels of HDL-cholesterol (HDL-C: $>2.0$ mmol/1) in $20 \%$ of the 500 patients with IDDM, controlled in our out-patient clinic, we examined if the presence of high HDL-C was connected with a low incidence of complications of atherosclerotic origin. All the patients were separated in 3 groups according to their HDL-C level: Groun $1,0.9-1.5 \mathrm{mmol} / 1(\mathrm{n}=220)$, Group 2, 1.6-2.0 mmol/1 $(\mathrm{n}=188)$ and Groun 3, $2.1-4.4 \mathrm{mmol} / \mathrm{l}(\mathrm{n}=99)$. The groups were comparable with respect to age (48-50 years), duration of diabetes (1621 years), BMI (25-27), glycemic control (HbAlc 7.8-8.0\%), levels of total cholesterol $(5.2-5.8 \mathrm{mmol} / \mathrm{l})$, while that of triglyceride was somewhat lower in Group $3(0.9$ (mean) mmol/l) than in $1(1.5 \mathrm{mmol} / \mathrm{l})$ and $2(1.2 \mathrm{mmol} / \mathrm{l})$. The number of patients (in \%) with simplex retinopathy, sensoric neuropathy and nephropathy (U-albumin excr. $>30 \mathrm{mg} / 24 \mathrm{t}$ ) was almost similar (about $30 \%$ ) in the 3 groups, like the frequency of myocardial infarction (4-5\%) and ischemia of the legs $(5 \%)$. The occurrence of hypertension (BP > 145/85 mmHg), ishemic heart disease, stroke, proliferative retinopathy and autonomic neuropathy showed some discrepancy between the groups, but was in no case expecially low in Group 3, in contrast often showing a higher incidence of complications than Group 1 and 2. We suggest that high levels of HDL-C do not protect against atherosclerotic lesions and complications in patients with IDDM. 
1038

LDL SUBFRACTION PHENOTYPES IN TYPE I AND TYPE II DIABETES MELLITUS EFFECT OF GLYCEMIC CONTROL

A Pérez, A Caixàs, C Otal ${ }^{*}$, G.Carreras,J Ordóñez* A deLeiva. Endocrinology and *Biochemistry Depts. H.Sant Pau. Barcelona. Spain.

We evaluated the effect of optimizing glycemic control on LDL subfraction phenotypes in 70 diabetic subjects (37 IDDM and 33 NIDDM) and we compared them to 58 non-diabetic subjects of similar age, sex and BMl The study was performed in poor glycemic control (HbAlc $9.5 \pm 2.9 \%$ in IDDM and $9.8+2 \%$ in NIDDM) and after 3 months of improvement (HbAIc $5.8 \pm 1.3 \%$ and $5.9 \pm 1.0 \%$ respectively). Six LDL subfractions were isolated by gradient ultracentrifugation and the phenotypes were classified according to the quotient $\mathrm{LDL}_{1}-\mathrm{LDL}_{3} / \mathrm{LDL}_{4}-\mathrm{LDL}_{4}[\mathrm{~A}>1.8, \mathrm{~B}<1.1$, and $\mathrm{AB}$ 1.8-1.1 ]. Compared to the control group, diabetic patients $(\mathrm{p}=0.05)$ tended to a higher frequency of non-A phenotype ( $34 \%$ vs $19 \%)$ and a decrease to $20 \%(p<0.05)$ after improving glycemic control. IDDM patients showed a similar phenotype distribution $(81 \% \mathrm{~A}$ and $19 \%$ non-A) to that of their control group ( $88 \% \mathrm{~A}$ and $12 \%$ non-A), and it was not modified by the optimization of glycemic control. However, in NIDDM patients, the frequency of non-A phenotype was higher than that of their control group $(51 \%$ vs $28 \%)$ and was normalized $(30 \%, \mathrm{p}<0.05)$ after improving glycemic control. We conclude that 1 - Glycemic control is an important factor for the distribution of LDL subfraction phenotypes in NIDDM. 2- The apparent non-influence of glycemic control effect on LDL subfraction phenotype ditribution in IDDM could be attributed to the low expression of non-A phenotype, in this group of patients.

\section{9}

LDI MODIFICATIONS IN TYPE II DIABETES MELLITUS (NIDDM) . EFFECT OF GLYCAEMIC CONTROL . A. Caixàs, A. Pérez, JL. Sánchez*, A. Payés, J . OrdóñezLlanos*, A.de Leiva. Endocrinology and *Biochemistry Dpts. H.Sant Pau. UAB. Barcelona. Spain.

We evaluated the effect of optimizing glycaemic control on LDL phenotypes, the percentage of the electronegative LDL subform (LDL-) and LDL susceptibility to oxidation, in 25 NIDDM and in 25 age-sex-BMI matched control subjects. LDI susceptibility to oxidation was measured by dienes formation induced by $\mathrm{CuSO}_{4}$ (Lag phase). LDL- was isolated by anion exchange in a Fast protein Liquid Chromatography Device. LDL phenotypes were determined by density gradient ultracentrifugation and classified as the quotient $\mathrm{LDL}_{1}-\mathrm{LDL}_{3} / \mathrm{LDL}_{4}-\mathrm{LDL}_{5}$ (phenotype $A>1.8, A B$ 1.1-1.8, B 1.1 ). Differences were assessed by student's $t$ test. Fisher exact test and McNemar test. Compared to the control group, NIDDM patients at baseline showed higher proportion of non-A phenotype ( $52 \%$ vS 28\%) and LDI- $(19.0 \pm 8.5 \%$ vs $14.3 \pm 5.5 \%)$, while Lag phase was similar ( $47.5 \pm 6.1$ vs $49.7 \pm 7.6 \mathrm{~min})$. After 3 month of optimized glycaemic control (HbAlc decreased from $10.3 \pm 1.7 \%$ to $5.8 \pm 0.9 \%$, $\mathrm{p}<0.0005)$, non-A phenotype decreased to $28 \%$ $(p<0.05)$, while LDL- $(18.1 \pm 8.1 \%)$ and the Lag phase $(46.6 \pm 6.5 \mathrm{~min})$ were not modified. We conclude that, in NIDDM patients 1 -Small dense IDL but not LDL- are highly dependent on glycaemic control. 2The increase in small dense LDL particles and LDLproportion in NIDDM is not associated to an enhanced LDL susceptibility to oxidation.

\section{0}

\section{EFFECT OF INSULIN ON LOW-DENSITY LIPOPROTEIN OXODABILITY IN HEALTHY MEN}

T. Pelikánová, L. Kazdová, A. Zák and E. Tvrzická, Postgraduate Medical School, Institute for Clinical and Experimental Medicine and Ist Medical Faculty of Charles University, Prague, Czech Republic.

Recent evidence has shown that hyperinsulinaemia and insulin resistance are involved in the pathogenesis of atherosclerosis, although the underlying mechanism remains unclear. The aim of our study was to evaluate the effect of acutely induced hyperinsulinaemia on an oxidative modification of lowdensity lipoprotein (LDL) and on fatty acid composition (FA) of LDL phosphatidylcholine. Euglycaemic hyperinsulinaemic clamp $(75 \mu \mathrm{U} / \mathrm{m})$ lasting 10 hours and a time-controlled study with saline infusion were performed in 11 healthy men. Acute hyperinsulinaemia enhanced LDL susceptibility to in vitro $\mathrm{Cu}^{2+}$ induced oxidative modification as shown by a) a decrease in the lag time ( 74 vs. $61 \mathrm{~min} ; \mathrm{p}<0.01$ ), although the elevation of diene production did not reach the statistical significance, b) an increase in thiobarbituric acid reactive substances ( 45.5 vs. $66 \mathrm{nmol} / \mathrm{mg}$ of LDL protein; $p<0.001$ ), and c) a rise in oxidative modification of protein moiety measured by the emission fluorescence spectra at $430 \mathrm{~nm}$ with excitation at $355 \mathrm{~nm}(+51 \% ; \mathrm{p}<0.01)$. On the other hand, no significant changes were seen in the FA composition of LDL phosphatidylcholine during insulin infusion. No significant changes in the above parameters were seen during timecontrolled saline infusion. Furthermore, no significant correlations between insulin-induced changes in LDL oxidability and insulin stimulated glucose disposal during clamp were found. We conclude that acutely induced hyperinsulinaemia increases LDL, susceptibility to oxidative modification, which is not mediated by changes in the FA composition of LDL. Alterations of LDL oxidability may be one of the mechanisms involved in the pathogenesis of accelerated atherosclerosis mediated by insulin.

\section{1}

POSTPRANDIAL TRIGLYCERIDE LEVELS IN NON-INSULIN-DEPENDENT DIABETES MELLITUS ARE INFLUENCED BY INSULIN SENSITIVITY CE. Friedberg, W DevillE, T Teerlink C Popp-Sniiders and R I Heine, Depts of Irternal Medicine, Endocrinology, Clinical Chemistry, Biostatistics and Epidemiology. Free University Hospital, Amsterdam.

Insulin resistance may contribute to abnormal postprandial lipoprotein metabolism which may lead to cardiovascular disease This is highly prevalent in non-insulin-dependent diabetes mellitus. We assessed the contribution of insulin resistance in a group of 24 , well controlled, non-insulin-dependent diabetes patients $(58 \pm 9$ years, $20 \mathrm{~m} / 4 \mathrm{f}$. HbA1C $7.0 \pm 1 \%$ ). Fasting lipid concentrations were (median [range] mmol/l): Tcholesterol 5.5 [41 86]. LDL cholesterol $3.75[1.37,5.57]$, HDL2 cholesterol $0.16[0.01,0.65], \mathrm{HDL} 3$ cholesterol $0.82[0.72 .0 .92]$. Triglycerides $1.6[0.55,6.75]$. After 4 months of prestudy diet patients consumed, after a twelve hour fast, a linoleic enriched test meal (P/S 1.0, $791 \mathrm{Kcal}$, fat $57 \mathrm{en} \%)$ with added vit A (27.000 IE/m2 bsa). Lipid and glycemic parameters were followed for twelve hours. A week later, these subjects underwent a hyperinsulinemic euglycemic clamp (M) values: 0.03 [0.01-0.09] $\mathrm{mmol} / \mathrm{kg} / \mathrm{min}$ ). Backwards multiple linear regression analysis showed that $86 \%$ of the variability of the log transformed postprandiai triglyceride levels could be explained by three factors: fasting trigtyceride leveis ( $B: 0.24$, confidence interval $[0.160 .32], p=0.000$ ), $M$ value $(B:-4.2[-9.14,0.74] p=0.091)$ and HDL2 cholesterol area under the curve $(B$ : $-0.09,[-014,-0.04], p=0.0014$ ). Fasting triglyceride levels explained $77.6 \%$, HDL2 cholesterol area under the curve an additional $6.9 \%$ and the $M$ value $1.5 \%$ of this variability. Bivariate analysis showed a negative Spearman correlation coefficient of $-0.47(p=0.018)$ between the $M$ value and $\mathrm{Log}$ Triglyceride area under the curve. Our data show that insulin resistance possibly has a small, inverse effect on the postprandial increase in triglyceride concentrations. Fasting triglyceride levels are however the most important contributor 
DOXAZOSIN IMPROVISS PIASMA IIIJID ABNOORMAITIJSS IN DIABHZILC RATS

A.S. Reddi, G.N. Jyothirmayi and Indira Alluru, Department of Medicine, University of Medicine and Dentistry of New Jersey, New Jersey Medical School, Newark, New Jersey 07103, US

We previously demonstrated that doxazosin, an $\alpha_{1}$-adrenergic blocker, prevents albuminuria, preserves glomerular synthesis of heparan sulphate, and favourably improves glycated $\mathrm{HbA}_{3}$ and plasma glucose in diabetic rats. We now present data on the effects of doxazosin on plasma lipids and lipoproteins in diabetic rats. Diabetes was induced in male Wistar rats by streptozotocin. One week after persistent hyperglycaemia, diabetic rats $(n=10)$ were given doxazosin $(75 \mathrm{mg} / \mathrm{l})$ in drinking water for 20 weeks. Control groups of diabetic rats were treated with insulin to maintain good glycaemic control $(n=8)$ or given water $(n=8)$. Normal control rats received doxazosin $(n=10)$ or water $(n=10)$. Compared with normal rats, diabetic rats showed significant elevations in plasma total cholesterol (4.48 vs $2.24 \mathrm{mmol} / 1, \mathrm{p}<0.001$ ), triglycerides (7.26 vs $1.23 \mathrm{mmol} / 1, \mathrm{p}<0.001$ ), VLDL cholesterol ( 1.79 vs $0.11 \mathrm{mmol} / 1, p<0.001)$ and LDL cholesterol ( 2.09 vs $0.16 \mathrm{mmol} / \mathrm{l}, \mathrm{p}<0.001$ ), and a reduction in HDL cholesterol $(0.60$ vs $1.97 \mathrm{mmol} / \mathrm{l})$. These abnormalities in plasma lipids were normalised by good glycaemic control. Doxazosin improved plasma total cholesterol (2.65 mmol/l, $p<0.005)$, triglycerides ( $1.63 \mathrm{mmol} / 1, \mathrm{p}<0.005)$, VLDL cholesterol $(0.50 \mathrm{mmol} / \mathrm{h}$, $p<0.005)$, LDL cholesterol $(0.51 \mathrm{mmol} / \mathrm{l}, \mathrm{p}<0.005)$ and HDL cholesterol $(1.64 \mathrm{mmol} / \mathrm{l}, \mathrm{p}<0.005)$ in diabetic rats. These results indicate that doxazosin has a beneficial effect on lipid levels in diabetic rats.
Influence of hypolipidemic treatment with fenofibrate on hemostatic factors blood pressure and insulin levels in patients with polymetabolic syndrome. B. Idzior-Waluś. J. Siendzki. A. Zdzienicka. W. Roziworowski. Department of Ifotabolic Diseases ond Departntent of Biochemical Diagnostics, Jagiellonian Linuerstu, Sirokow Poland

The nost important findings of the PMS are hyperlipidemia (HLP), increased throubogenicity. hypertension (HT) and central obesity (CO). The reduced insulin sensitivity and hyerinsulinemia has been considered as the hallnark of these associations. The aim of the study was to assess the potential effects of fenofibrite on fibrinogen and factor VIl. strong predictors of ischemic hear discase. on blood pressure and serum insulin fasting. and during oral gheose tolenatue test (OGTT) Material included 37 males with PMS (HLP and 2 of the following (O. HT, itnpuired glucose tolerance). (reated wilh micronised fenolibrile $2(\mathrm{~h}) \mathrm{hg}$ daily. after 4 weeks of wash-out and 4 weeks of diclary treatment only. Cholesterol lowering dict was maintained throughout the whole study period Coagulation factors were evaluated using chronometric assay, scrum lipids and glucose by enzymatic methods. insulin by radioimmunoassay. blood pressure (BP) in the sittung position. after at least 10 min. rest with increur splivgnomanonter. After 12 wecks of treatment significant decrease of total. LDL-cholesterol and triglycerides and increase of HDL-cholesterol were obseried $(p<.001$ to $p<.01)$ Fibrinogen decreased from $2.27+0.6$ to $1.84 \pm 0.4$ $g / 1(p<01)$ : factor VI from $111.9+45.5$ to $91.5+36.2 \%$ ( $p<02)$ : sistolic BP from $1450+15.1$ 10 $129.8+179$ mmltg and diastolic BP from 90.3+ 10.7 to 80.4 +78 mmHe ( $p-001)$. Fasting insulin and insulin area under the cure (AUC) during OGTT by $28.6 \%$ and $18.7 \%$ respectively $(\mathrm{p}<.05)$. while fasting and AUC glucose during OGTT did not change. Clanges in insulin AUC during OGTT correlated with the degree of sistolic BP drop $(r=-36 . p<05)$ There did not appear to be any correlation between magnitude of lipid reduction and the degre of BP drop. hemostatic factors or insulin changes

The results indicate that fenofibrate treatment affects favourabis not only serum lipids. but also powerful predictors of coronary hear disease fibrinogen and faclor Vif. lowers blood pressure values and also insulin levels. without itcriase of glucose concentrations. What is relevant in the treatment of patients with PMS to reduce corolan risk.

\section{PS 56}

\section{Macroangiopathy Risk Factors}

\section{4}

FIBRINOGEN AND ALBUMINURIA ARE RELATED TO THE PRESENCE AND SEVERITY OF PERIPHERAL ARTERY DISEASE IN WOMEN WITH TYPE 2 DIABETES MELLITUS

I. Tkac, A. Molcanyiova and R. Tkacova, Department of Medicine IV, Department of Clinical Biochemistry, Department of Medicine I, The Faculty Hospitals, Safarik University, Kosice, Slovakia

The relationship between the ultrasonographically determined presence and severity of peripheral artery disease (PAD) and cardiovascular risk factors was studied in 30 postmenopausal, non-smoking women with Type 2 diabetes mellitus. PAD was established on the basis of decreased ankle/arm index (AAI) of $<0.9$ in 15 patients. The control group included 15 Type 2 diabetic females with $\mathrm{AAI}>1.0$. There were no differences with respect to fasting blood glucose, $\mathrm{HbA}_{1}$, creatinine, and systolic blood pressure between females with $\mathrm{PAD}$ and the control group. The patients with PAD had higher levels of total cholesterol, LDL-cholesterol, triglycerides, apolipoprotein B, and lipoprotein(a), as well as lower levels of HDL-cholesterol and apolipoprotein A, than patients in the control group, but the differences were not significant. Only mean fibrinogen level (mean \pm SEM: $4.75 \pm 0.35$ vs. $3.53 \pm 0.36 \mathrm{~g} /$; $\mathrm{p}<0.01)$ and albuminuria (UAE) values $(893 \pm 501$ vs. $57 \pm 24 \mathrm{mg} /$ day; $p<0.05)$ were significantly increased in the patients with PAD in comparison to the controls. In the linear regression, $\log$ UAE $(r=-0.55$, $p<0.01)$, fibrinogen $(r=-0.49, p<0.01)$, creatinine $(r=-0.42, p<0.02)$ and age $(r=-0.39, p<0.05)$ correlated significantly with the severity of PAD expressed by AAI. However, in the multiple stepwise regression analysis with AAI as a dependent variable, only $\log \mathrm{UAE}(\mathrm{p}=0.029)$ and fibrinogen $(p=0.033$ ) were included in the best model. In conclusion, in non-smoking females with Type 2 diabetes mellitus fibrinogen and albuminuria were the only risk factors associated with both the presence and severity of peripheral artery disease.

\section{5}

LEFT VENTRICULAR HYPERTROPHY IS ASSOCIATED WITHMICROALBUMINURIA INDEPENDENTLY OF BLOOD PRESSURE IN TYPE II DIABETES M.W. Spring, A.E. Raptis, E.M. Murphy, J. Chambers* and G.C. Viberti - Units for Metabolic Medicine and Cardiology*, UMDS, Guy's Hospital, London, UK

Microalbuminuria is a powerful risk factor for cardiovascular morbidity and mortality in non-insulin-dependent diabetes mellitus (NIDDM). Increased left ventricular mass $(\mathrm{LVM})$, a predictor of coronary artery disease $(\mathrm{CAD})$, may mediate this effect but whether this occurs through a concomitant elevation of blood pressure (BP) is unclear. This study examined the relationship between microalbuminuria (albumin excretion rate, AER, 20-200 $\mu \mathrm{g} / \mathrm{min}$ ), LVM and BP in 63 (44 men) European non-proteinuric NIDDM patients. They were divided into 3 groups: 25 with microalbuminuria and hypertension (BP>140/90 $\mathrm{mmHg}$ without antihypertensives, group A), 25 with microalbuminuria and normotension (group $B$ ) and 13 with normoalbuminuria and normotension (group C). No patient had clinical or ECG evidence of CAD and the 3 groups were matched for age $(62 \pm 1.5$ vs $58 \pm 1$ vs $61 \pm 2 \mathrm{yrs}$ ) and duration of diabetes $(8 \pm 1$ vs $8 \pm 1$ vs $7 \pm 1 \mathrm{yrs})$. LVM was evaluated by two-dimensional echocardiography and the LVM index (LVMI) calculated as the ratio of LVM and body surface area. AER was the median of 3 successive timed overnight urine collections. BP was the mean of 4 separate measurements sitting without antihypertensives. By selection $\mathrm{BP}$ was higher in group A (mean \pm SEM, systolic-SBP: $151 \pm 2$; diastolic-DBP: $80 \pm 2 \mathrm{mmHg}$ ) but was similar in groups B and C (SBP: $126 \pm 2$ vs $131 \pm 2$; DBP: $75 \pm 2$ vs $74 \pm 2 \mathrm{mmHg}$ ). LVMI was similar in groups $A$ and $B$ which were both higher than group $C$

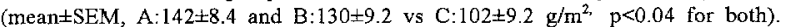
There was a significant correlation between LVMI and AER $(\mathrm{r}=0.3, \mathrm{p}=0.02)$ but no correlation between LVMI and either SBP or DBP in the combined groups. These results suggest that increased LVM in NIDDM patients with microalbuminuria is unrelated to $\mathrm{BP}$ and may independently contribute to the poor cardiovascular prognosis of this group. 
1046

THROMBOCYTOPENIA PROTECTS AGAINST MACROANGIOPATHY IN NON-INSULIN DEPENDENT DIABETICS WITH HEPATOPATHY.

T. Salvatore, D. Cozzolino, F. Cacciatore, M. Marino, FC. Sasso, M. Natale and R. Torella. Internal Medicine and Hepatology Unit, II University of Naples, Italy.

Age, hypertension, smoking, obesity, hyperlipidemia, and hyperfibrinogenemia are well known risk factors underlying accelerated atherosclerosis in NIDDM. Chronic liver disease (CLD), a condition associated per se with reduction of lipids and hemostatic factors, is frequently seen in NIDDM patients. We aim at investigating if CLD may modify the risk for macrovascular disease (MVD) in NIDDM. 491 consecutively hospitalized NIDDM patients were studied (age $=61.7 \pm 11.1$ yrs). 103 of these suffered from biopsy-proven CLD. Evidence for MVD (coronary artery disease, and/or peripheral vascular disease, and/or cerebrovascular disease) was present in $46.9 \%$ of non-hepatopatic and $18.4 \%$ of hepatopatic patients $(p<0.0001)$. In multiple logistic regression analysis with MVD as dependent variable, CLD appeared to be negatively related to the presence of MVD (OR= $0.31 ; 95 \% \mathrm{Cl}=0.13-0.42$ ) independently of age, sex, BMI, smoking, known duration of diabetes, and hypertension in such model only age, diabetes duration, smoking and hypertension were independent risk factors for MVD. In a second model the relationship of MVD with CLD, lipids and hemostatic factors was evaluated. The inverse association betwen MVD and CLD was independent of fibrinogen, triglycerides, total cholesterol. PT and PTT, Addition of blood platelet count makes no longer significant the association between MVD and CLD (OR $=0.56 ; 95 \% \mathrm{Cl}=0.40-1.01)$. Nevertheless, platelet count was significantly related to the presence of MVD $(O R=1.37: 95 \% \mathrm{Cl}=1.21-1.57)$ The prevalence of MVD is significantly reduced in NILIDM patients suffering from CLD comparing to non-hepatopatic ones. Thrombocytopenia seems to play the most important role in this protective effect.
1047

RISK FACTORS FOR CORONARY HEART DISEASE IN NONINSULIN DEPENDENT DIABETIC SUBJECTS

R. C. Turner, H. Millns, I. M. Stratton, H.A.W. Neil and R. R. Holman for UK Prospective Diabetes Study Group, Radcliffe Infirmary, Oxford, OX2 6HE, UK

A total of 3055 white Caucasian newly diagnosed non-insulin dependent diabetic subjects, with median follow up 7.9 years, were studied after three months diet therapy, to assess risk factors for subsequent coronary heart disease (myocardial infarction or angina), that developed in 335 subjects. A multivariate Cox proportional hazards model was fitted, with censoring at 10 years. A stepwise selection technique, adjusting for age and gender, indicated which risk factors were included in the model. The estimated hazard ratios ( $95 \%$ confidence intervals) for the middle and upper tertiles of these risk factors, relative to the lower tertile were, for LDL cholesterol $(p=0.00001)$ $1.41(1.05,1.90)$ and $2.26(1.70,3.00)$; for $\mathrm{HDL}$ cholesterol $(\mathrm{p}=0.000 \mathrm{I})$ $0.90(0.71,1.15)$ and $0.55(0.41,0.73)$; for haemoglobin Alc $(p=0.002)$ $1.47(1.12,1.95)$ and $1.52(1.15,2.01)$; and for systolic blood pressure $(p=0.007) 1.52(1.12,2.06)$ and $1.82(1.34,2.47)$. They wcre $1.03(0.77,1.37)$ for ex-smokers and $1.41(1.06,1.88)$ for current smokers, relative to neversmokers $(p=0.06)$. After adjustment for these risk factors, body mass index; waist-hip ratio, exercise, triglyceride and insulin were not statistically significant. Microalbuminuria and retinopathy were also not significant when added to the multivariate model. When fitting a model for only fatal myocardial infarction, raised blood pressure was a major risk factor. In summary, in non-insulin dependent diabetic subjects, lipid status, glycaemia, blood pressure and smoking are the main risk factors for coronary heart disease.

\section{8}

ATHEROSCLEROSIS IN PATIENTS WITH AND WITHOLT DIABETES MELLITUS:HOSPITALIZATION ANI) MORTALITY IN THE NETHERLANDS IN 1989 AND $10 \% 4$.

F.L.J.Visseren, K. P. Bouter, R.R.M.de (iront. D).W. Erkelems and R.J.A.Dienerskot. Department of Microhiokegy. Diakonessen Hospital Utrecht. Department of Internal Medicine.

Bosch Medicentre and University Hospital Utresht.

The Netherlands.

Introduction: Macrovascular complications contrihute to high morhidity and mortality rates in patients with diahetes mellitus. The burden on hospitalization is not exactly known. This register-hased study was designed to evaluate the hospital use and in-hospital mortality of macrovascular complications of all patients with and without diahetes mellitus admitted to all hospitals in the Netherlands comparing 1989 with 1994.

Methods: Datis on hospitalizations were obtained from the Dutch National Medical Registration. Results: In the Dulch diabetes mopulation $\% 955(2.27 \%)$ patients and $9222(2.31 \%)$ were admitted for coronary heart disedse in 1989 and 1994 respectively. For non-diabeties these higures 94+67 $(0.63 \%)$ and $11073(0.74 \%)$ patients. The increase in dumissions in the non-diabetics was inainly due to the female population $118.3 \%$ increase), whereas the prevalence of admissiuns for diahetio patients was stable over this 5-year-time-periok. For Cerehrovascular Accidents a similar nattern was ubsersed. Total numher of admitted patients with diabetes in 1989 and 1901 were respectively $29 \pm 8(0.74 \%)$ and $2990(0.75 \%)$. For non-diathetics this was $22151(0.15 \%)$ and $24891(0.17 \%)$ respectively. Conclusion:Although the relative incidence cexpressed as perentage of each population of admission due to macrovascular diseasses is highter in diahetic patients, a stahilization in adtuission incidence is seen in this patient group comnared th the mon-diabetic patients oherved over the time period 19891994.

\section{9}

Serum sialic acid, a new cardiovascular risk factor, is higher in NIDDM women than men.

H M Mather, G A Roberts, A M Kehely, C Pasapula, $G D$ Chusney and $J C$ Eickup. Ealing Hospital, Southall, Middlesex \& Guy's Hospital, London, UK.

Serum sialic acid concentration [SA] is a potent cardiovascular risk factor, and is elevated in NIDDM. To further examine its associations, we estimated [SA] in 411 consecutive NIDDM patients attending a diabetic clinic. MeantSD [SA] were significantly higher in women than men $(2.69 \pm 0.44$ $\mathrm{v} 2.57 \pm 0.58 \mathrm{mmol} / 1, \mathrm{p}<0.05)$. Mean [SA] were also significantly elevated in patients with a history of ischaemic heart disease (IHD) ( $2.71 \pm 0.56 \mathrm{~V}$ $2.61 \pm 0.52 \mathrm{~mol} / 1, \quad p<0.05)$ and clinical proteinuria $(2.74 \pm 0.52$ v $2.61 \pm 0.52 \mathrm{mmol} / \mathrm{l}$, $\mathrm{p}<0.05)$. Significant correlations were found with plasma urea $(r=0.20, p<0.001)$ and creatinine $(r=0.16, p=0.001)$. The elevated mean [SA] in women could not be explained by other variables. Mean [SA] in 90 women and 93 men with no retinopathy, proteinuria or history of IHD and plasma creatinine $<120 \mu \mathrm{mol} / 1$, were $2.71 \pm 0.45$ and $2.54 \pm 0.53 \mathrm{mmol} / \mathrm{l}$ respectively $(p=0.002)$. We conclude that mean [SA] is elevated in NIDDM women compared with men. This is previously undescribed, and could contribute to the increased impact of diabetes on IHD prevalence in women. 
1050

ELEVATED LEVELS OF HAEMIOSTATIC RISK MARKERS IN FIRSTDEGREE RELATIVES OF NON-INSULIN DIABETIC PATIENTS.

M.W. Mansfield, D.M. Heywood and P.J. Grant. Diabetes and

Thrombosis Research Group, Research School of Medicine,

Lniversity of Leeds, Leeds, UK

Elevated circulating levels of haemostatic factors which predict coronary events may contribute to the increased vascular risk of firstdegree relatives of non-insulirı-dependent diabetic (NIDDM) patients, possibly through association with insulin resistance (IR). The aim of the study was to examine the hypotheses that elevated levels of factor VII , fibrinogen and von Willebrand factor (VWF) occur in first-degree relatives of NIDDM patients and in association with features of IR. Fasting blood samples were taken from 132 first-degree relatives of NIDDM patients and 151 controls of similar age for measurement of factor VII:C (FVII:C), fibrinogen, VWF, insulin, total and HDLcholesterol, triglyceride and glucose. Levels of FVII:C (130\% v $122 \%$, $p<0.02)$ and fibrinogen $(3.0 \mathrm{~g} / 1 \times 2.7 \mathrm{~g} / 1, p=0.002)$ were higher in relatives than controls respectively and there was no significant difference in levels of $\mathrm{VWF}$ (relatives: $098 \mathrm{IU} / \mathrm{ml}$ vs controls: 0.95 $[U / \mathrm{ml})$. When the groups were combined there was a graded association with features of $[R$, for example the correlation with triglyceride level, being strongest for FVII $C(r=0 . \$ 6, p<0.0005)$, weaker for fibrinogen $(r=024, p<0.0005)$ and weakest for $\mathrm{vWF}$ $(r=0.06$, not significant). When adjusted for the features of $I R$, levels of FVIL and fibrinogen were similar in relatives and controls. These results suggest that elevated levels of factor VII and fibrinogen, may contribute to cardiovascular risk in relatives of patients with NIDDM, possibly through an association with insulin resistance

1052

ANGIOTENSIN I-CONVERTING ENZYME GENE POLYMORPHISM IN GENERAL MOSCOW POPULATION AND IN NIDDM PATIENTS WITH MYOCARDIAL INFARCTION

E.Anokhin ${ }^{1}$, I.Dedov', L.Demurov ${ }^{2}$, Y.Kondratiev ${ }^{1,2}$, M.Balabolkin', G.Mamaeva', and V.Nosikov', 'Endocrinology Research Centre and "National Research Centre "GNII Genetika", Moscow, Russia

Insertion (I)/deletion (D) polymorphism in gene for angiotensin l-converting enzyme (ACE) is now recognised to associate with myocardial infarction (MI) in different European populations. Ethnic/racial differences in both incidence of pathology and distribution of ID/ACE alleles have also been demonstrated through over the world, excluding Russia. Therefore, we studied distribution of PCR (polymerase chain reaction)-detected ID/ACE alleles/genotypes in general Moscow populations (118 diabetes-unrelated subjects) and in 21 NIDDM patients with one or more Ml occasion(s) registered and treated earlier. The highest of known in the European populations prevalence of Ml susceptibility risk markers ( $D$ allele and DD genotype), has been found in general Moscow population: 67.4 and $50.8 \%$, respectively, whereas prevalence of markers of resistance (I allele and II genotype) was the lowest: 32.6 and $16.1 \%$, respectively. Due to small sample size studied, we could not find statistically significant difference in ID/ACE allele/genotype distribution (Fisher's exact test), thereby confirming an association of ID/ACE polymorphism with MI in Russian NIDDM patients. Nevertheless, a tendency to accumulation of susceptibility and depletion of resistance markers has been observed: in patients, the prevalence of $D$ allele and DD genotype was 76.2 and $57.1 \%$, and the prevalence of I allele and II genotype was 23.8 and $4.8 \%$, respectively. Further study is going on.

Conclusion: despite a preliminary character, our findings demonstrate, on the one hand, unexpectedly high prevalence of $\mathrm{Ml}$ risk allele and genotype of ACE gene in general Moscow population and, on the other hand, re-distribution of susceptible and protective ID/ACE alleles/genotypes between NIDDM patients with MI and healthy control from the same population towards accumulation of predisposing and depletion of protecting markers in Ml affected individuals.
1051

THIS ABSTRACT HAS BEEN . WITHORAWN BY THE AUTHOR.
1053

PREVALENCE OF CORONARY HEART DISEASE AND TIS RELATION TO VARTOUS RISK FACTORS IN A POPULATION SAMPLE OF TYPE 2 DIABETIC PERSONS P. Tsapogas, Z. Alexiou, M. Arvanitis, N. Tritos, D. Papadogiannis, G. Papazachos, C. Richardson, and N. Katsilambros. 1st Department of Propedeutic Medicine, Laikon Hospital, Athens University Medical School. Ag. Thoma 17, GR 11528 Athens, Greece.

Studies in a hospital have the disadvantage of selecting patients with the most serious complications of their disease. Therefore, studies of general population may be preferred. We evaluated the prevalence of coronary heart disease ( $\mathrm{CHD}$ ) on the basis of electrocardiographic findings of ischaemia (Minnesota code) and/or clinical history of myocardial infarction or angina in a random urban population sample of 198 type 2 diabetic persons. We also investigated the relation of $\mathrm{CHD}$ to various risk factors. Results: a) Prevalence of $\mathrm{CHD}$ was $41.9 \%$ b) $\mathrm{CHD}$ was more frequently present in hypertensive diabetic patients $(51.0 \%)$ than nonhypertensive $\left(31.9 \%, x^{2}=6.60, p=0.010\right)$, more frequent in albuminuric patients $\left(54.2 \%\right.$ vs $36.2 \%$ in nonalbuminuric patients $x^{2}{ }_{1}=3.92$, $\mathrm{p}=0.048$ ) and amongst the males, in patients, with peripheral vascular disease (PVD) $\left(61.5 \%\right.$ vs $36.5 \%$ CHD without PVD; $\left.x^{2}=4.63, p=0.031\right)$. In addition. major ECG ischaemic findings (MECG) were more frequent in hypertensive, than nonhypertensive patients $\left(20.2 \%\right.$ vs $\left.9.6 \%, x_{1}^{2}=3.54, p=0.06\right)$. Higher body mass index (BMI) among the male subjects $(p=0.051)$ and elevated triglyceride levels among the females $(\mathrm{p}=0.026)$ were associated with $\mathrm{CHD}$. No significant bivariate correlations were found between, on the one hand, CHD or MECG, and on the other, age, duration of diabetes, waist-to-hip ratio, percent body fat, smoking, fasting blood sugar, $\mathrm{HbA}_{1}$, total cholesterol, $\mathrm{HDL}$ - and LDLcholesterol levels, signs of retinopathy, neuropathy, smoking habits, and mode of treatment. Likelyhood ratio tests in logistic regression analyses showed that CHD was significanily associated with male sex $(p=0.0003)$, hyprtension, $(\mathrm{p}=0.029)$, and BMI $(\mathrm{p}=0.044)$, while MECG was associated with male sex $(p=0.013)$ and duration of diabetes $(p=0.031)$. The data clearly support the hypothesis that $\mathrm{CHD}$, although a frequent finding in type 2 diabetic persons, is not necessarily related to the whole spectrum of the classical risk factors of atherosclerosis. Apparently, diabetes per se is the major atherosclerotic factor. 
1054

PROGNOSTIC VALUE OF HYPERGLYCEMIA IN ACUTE MYOCARDIAL INFRACTION. B. Dimopoulos, S. Bousboulas, S. kraklianou, Em. Lebidakis, S. Foussas, A. Melidonis Cardiology Clinic and Diabetology Department of Tzanio Hospial, Piraeus, Greece.

AlM: Diabetus mellitus is a major risk factor in coronary heart disease (CHD). In our study we observed the progression of acute myocardial infraction and its complications in patients with hyperglycemia.

PATIENTS - METHODS: Random perspective study of 168 patients who were hospitalized with acute myocardlal infraction during the year 1995 at Tzanio State Hospital 50 pattents $(20.76 \%)$ with diabetes (Group A). 15 patients $(8.92 \%)$ presented diabetes during hospitalization (OIGT) (Group B) 103 patients without diabete: (Group C). Mean age Group A: 70.3 yrs - Group B: 69.7 yrs, Group C: 64.5 yrs. We estimated Alc (glycated hemoglubin) blood glycose levels upon entrace (Levels fasting and after meals) we also located the position of the infract and complications during hospitilization CONCLUSIONS:

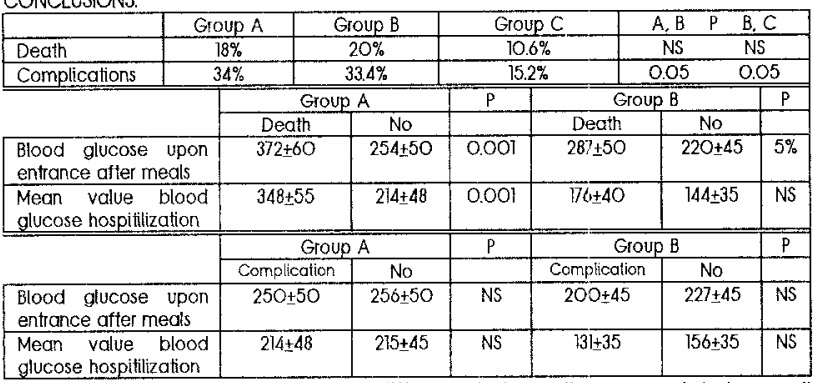

The location of infraction did not prove difference between the groups of study as well as complications.

RESULTS: 1. Blood glycose upon entrance and during hospitilization is a high death risk factor for patients hospitilized with acute myocardial infraction, where as blood glycose upon entrance has a significant statisfic relationship with outcome of acute myocardial infraction in $\lg T$ group. 2. Complications during hospitilization appear frequency $(p<0.005)$ in diabetic patients and $\mathrm{g}^{\top}$ compared to non diabetics. 3. Complications during hospitilization were not affected by blood glycose.

\section{5}

ELEVATED PROINSULIN CONCENTRATIONS IN CORONARY HEART DISEASE H.J. Lüddeke, S. Stowasser, R. Renner and K.D. Hepp Klinikum München Bogenhausen I. Dept. Englschalkingerstr. 77, 81925 Munich, Germany

The role of hyperinsulinaemia in the pathogenesis of coronary heart disease (CHD) has been challenged by several recently puplished studies. An association of $\mathrm{CHD}$ to proinsulin concentrations has been suggested, but data for caucasian subjects are missing. Patients:35 caucasian men with significant $\mathrm{CHD}$ as shown by coronary angiography. 26 of them normoglycaemic (Age $57 \pm 10.6$ y, BMI $28.1 \pm 3.5$, HbA1c $5.46 \pm 0.7 \%$ ), 9 with impaired glucose tolerance (Age $55 \pm 3.7$ y. BMI 28.3 \pm 4.1 , HbA.1c $5.69 \pm 0.7 \%$ ). Methods:oGTT according WHO. Blood glucose measurement in intervals of $30 \mathrm{~min}$, serum insulin and proinsulin at $0,30,120 \mathrm{~min}$. Serum insulin was determined by a commercial sandwich two step monoclonal ELISA (Abott, cross reactivities proinsulin<0.005\%, 64/65 des $5 \%, 31 / 32$ des $<1 \%$ ), serum proinsulin by a commercial RIA (Linco, cross reactivities insulin $<0.1 \%, 64 / 65$ des $<0.1 \%, 31 / 32$ des $95 \%)$. Blood glucose: YSI-2300, HbA1c: HPLC. Statistics : SPSS ( all concentrations logtransformed). Data presented as backtransformed means and SD adjusted for $\mathrm{BMl}$ and age. Insulin and proinsulin expressed as pmol/t. Results: normoglycaemic $0 \mathrm{~min} \quad 30 \mathrm{~min} \quad 120 \mathrm{~min}$ insulin $\quad 62(43 / 90) \quad 409(359 / 477) \quad 455(336 / 617)$ proinsulin $\quad 16(15 / 18) \quad 34(31 / 38) \quad 71(63 / 81)$ $\begin{array}{llll}\text { ratio }(\mathrm{P} / \%) & 26 & 8.4 & 15.6\end{array}$ IGT insulin $36(18 / 71)$ proinsulin $13(11 / 15)$ ratio(P/1\%) 35

$818(458 / 1462)$ $\begin{array}{ll}22(16 / 29) & 56 \\ 7.3 & 6.9\end{array}$

Conclusion: in absoiute and relative terms proinsulin concentrations in these patients with CHD are elevated, even after adjusting for age and BMI. In a recently published study Kahn et al. found in Japanese-American men an adjusted $P / \mid$ baseline ratio of $12 \%$ for normoglycaemic and $12.6 \%$ for $1 \mathrm{GT}$ patients with $\mathrm{CHD}$. Our results for caucasian subjects with $26 \%$ for normoglycaemic and $35 \%$ for IGT-patients show a more than doubled range

\section{6}

PROSPECTIVE STUDIES OF THE ISCHEMIC HEART DISEASE IN THE COHORT OF NIDDM FROM DEFINED POPULATION

A. Czech, P. Luźniak and J. Tatoń. Department of Internal Medicine and Diabetology, Warsaw Medical School, Warsaw Poland

The pathogenetic influence of NIDDM on the ischemic heart disease (IHD) including myocardial infarction (MI) seems to be related to intrinsic clinical form of NIDDM and external environmental factors - defined population. For gaining the insight into this problem the 3-years, prospective study of the cohort of 1334 NIDDM subjects was organized. It has recruted all NIDDM patients living within the 160000 city general population and registered in the regional diabetes care center. The charactestics of the cohort participants was investigated using preprogrammed records and consultations. IHD and MI diagnosis was based on clinical symptoms and/or ECG tracing analysed accordingly to Minnesota code. The IHD risk factors under investigations were: age, sex, BMI, NIDDM duration, plasma cholesterol triglyceride and creatinine levels, glycemia proteinuria and blood pressure. After 3-years of the cohort observation the following results were established: the total prevalence of IHD was $35.6 \%$ of all NIDDM subjects, among them $47.6 \%$ had the signs of MI. As much as $39.6 \%$ of IHD subjects showed only silent, ECG tracing positive IHD. The IHD prevalence in woman was $37.7 \%$ and in men $33.0 \%$ (difference not significant). Logistic analysis has indicated that the nsk of IHD in the cohort was significantly related to the age ( $>65$ years by $100 \%)$ NIDDM duration ( $>10$ years by $75 \%$ ), arterial hypertension (by $33 \%$ ), plasma creatinine level $(>88.5 \mu \mathrm{mol} / 1$ by $48 \%)$ and postprandial glycemia $>10 \mathrm{mmol} / 1$ by $15 \%$ ). In comparison with the general population of the city the NIDDM cohort was 2.5 fold prone to symptomatic IHD and 5.1 fold more prone to silent ischemia. NIDDM constituted the subpopulation of the very high risk for [HD and MI both in the symptomatic and ever more in the silent forms. It is mostly due to the risk factors, which are coexisting with hypoglycemia. Group of NIDDM under study influenced the total incidence of MI in general population of the city on the level 1 fifth

\section{7}

INSULINRESISTANCE/HYPERINSULINAEMIA, A MAJOR BIOCHEMICAI FEATURE IN PATIENTS WITH CEREBROVASCULAR DAMAGE

C. Ionescu-Tirgoviste ${ }^{1}, O$. Bajenaru ${ }^{2}, Z$. Mirodon ${ }^{1}, C$. Panea ${ }^{2}$ and I. Ferariu 1- Institute "N. Paulescu" and 2- Municipal University Hospital, Neurological Department, Bucharest, ROMANIA

The aim of our study was to analyze the plasma glucose and serum immunereactive insulin (IRI): $\left(\mathrm{I}_{0}, \mathrm{I}_{1}\right.$ and $\mathrm{I}_{2}$ the values in $\mu \mathrm{U} / \mathrm{ml}$ recorded before, at $\mathrm{Ih}$ and at $2 \mathrm{~h}$ respectively) during a $75 \mathrm{~g}$ OGTT carried oul on 31 patients, previously undiagnosed as diabetics, hospitalized for a neuro-vascular disorder (stroke, transient ischaemic attack). The investigation was carried out in the survivors, 3 months after the acute events. In terms of plasma glucose values (WHO criteria) the patients were divided in three groups: (A) - diabetes mellitus - 11 cases (6M/5F; mean age $\pm \mathrm{SD}, 55.9 \pm 11.1$; body mass index $-\mathrm{BMI}-\mathrm{kg} / \mathrm{m}^{2}, 26.4 \pm$ $\left.4.05, \mathrm{I}_{0}=20.9 \pm 10.4, \mathrm{I}_{1}=90.3 \pm 68.7, \mathrm{I}_{2}=68.1 \pm 66\right) ;(\mathrm{B})$ - impaired giucose tolerance (IGT) - 7 cases (4M/3F; mean age $54.9 \pm 16.8 \mathrm{yrs}$; BMI $-25.9 \pm 3.2$, $=23.3 \pm 13.1, I_{1}=99 \pm 40, I_{2}=69 \pm 25.6$ ); (C) - non-diabetics - 13 cases (3M/10F; mean age $55.5 \pm 17.1$ yrs.; $\mathrm{BMI}-23.5 \pm 3.1, \mathrm{~L}_{0}=17.9 \pm 8.9, \mathrm{I}_{1}=63.1$ $\pm 3.5, I_{2}=41.8 \pm 29.1$ ). Serum insulin values recorded in these 3 groups were compared, performing a Student test, with those found in group D comprised of 24 apparemly healthy subjects (10M/14F; mean age $53.2 \pm 6.2 \mathrm{yrs}$; $\mathrm{BMl}-25.6$ $2.5, \mathrm{I}_{0}=14.3 \pm 5.1, \mathrm{I}_{1}=53.4 \pm 17.2, \mathrm{I}_{2}=26.5 \pm 18.6$ ) with plasma glucose values during OGTT in normal range. Significantly higher serum insulin levels were found in group $B$, fasting at $\mathrm{lh}$ and at $2 \mathrm{~h}(\mathrm{t}=17.29 ; 9.79$ and respectively 14.77 ; $p<0.001)$, in group $A(t=12.77 ; 10.37$, respectively $9.47 ; p<0.01)$ and nonsignificantly higher in group $\mathrm{C}(\mathrm{t}=1.56 ; 1.1$, respectively 1.95$)$ compared to group D. A dysfunction in insulin secretion (hyperinsulinemia and/or a dissociation between plasma glucose and serum insulin curves) was recorded in $90.3 \%$ of cases with neuro-vascular disorders.

Conclusion: Insulinresistance and hyperinsulinism may be the fundamental underlying metabolic defect in patients with major neuro-vascular events, irrespective of the presence and degree of glucose intolerance. 
1058

INSULIN RESISTANCE IS ASSOCIATED WITH MORE ATHEROGENIC CARDIOVASCULAR RISK FACTORS IN DIABETES R. D'Agostino, Jr. and S. Haffner. The University of Texas Health Science Center at San Antonio TX, USA

Insulin resistance (IR) is strongly associated with increased cardiovascular risk factors (CVRF) in non-diabetic subjects. However, there is less data on associations of IR with CVRF in the non-insulin dependent diabetes mellitus (NIDDM) population. We examined the relationship of IR (as determined by the frequently sampled intravenous glucose tolerance test with insulin injection) to CVRF and intimal medial wall thickness (IMT) in 479 NIDDM subjects from the Insulin Resistance Atherosclerosis Study (IRAS), a four center study in African Americans, non-Hispanic whites and Hispanics in the USA. We defined the proportion of insulin sensitive (IS) subjects as $\mathrm{S},\left(\mathrm{min}^{-1} / \mu \mathrm{U} / \mathrm{ml}\right) \geq 1.61$ (above the median for non-diabetic subjects in the IRAS study). Results (mean \pm se) (adjusted for ethnicity, center, age and gender) are shown below:

$\begin{array}{lcll} & \text { Resistant } & \text { Sensitive } & \text { p-value } \\ \text { Common Carotid IMT }(\mu \mathrm{M}) & 879 \pm 13 & 827 \pm 40 & 0.480 \\ \text { Internal Carotid IMT }(\mu \mathrm{M}) & 949 \pm 24 & 870 \pm 74 & 0.272 \\ \text { Fibrinogen }(\mathrm{mg} / \mathrm{dl}) & 293 \pm 3 & 270 \pm 10 & 0.011 \\ \text { PAI (ng/ml) } & 33.6 \pm 1.2 & 21.7 \pm 3.8 & <0.001 \\ \text { LDL Cholesterol }(\mathrm{mg} / \mathrm{dl}) & 140 \pm 2.0 & 146 \pm 6 & 0.610 \\ \text { HDL Cholesterol }(\mathrm{mg} / \mathrm{dl}) & 39.5 \pm 0.5 & 45.3 \pm 1.7 & 0.005 \\ \text { Triglycerides }(\mathrm{mg} / \mathrm{dl}) & 204 \pm 8 & 159 \pm 25 & 0.027 \\ \text { Systolic BP }(\mathrm{mmH}) & 130 \pm 1 & 129 \pm 3 & 0.814\end{array}$

Insulin sensitive subjects $(\mathbf{n}=37)$ had significantly lower triglyceride, fibrinogen and PAI and higher HDL cholesterol than insulin resistant subjects $(n=442)$ There was a tendency towards thinner carotid wall thickness in insulin sensitive subjects but these differences were not statistically significant. We conclude that relatively few NIDDM subjects are insulin sensitive but IR is associated with increased CVRF (especially dyslipidemia and impaired fibrinolysis).

\section{0}

GLUCOSE TOLERANCE STATUS AND SEVERITY OF ATHEROSCLEROSIS IN CORONARY ANGIOGRAMS - A RELATIONSHIP?

C. Sloth, M Seibæk, H Burchardt S. Urhammer ${ }^{1} T$. Hansen ${ }^{1}$ C. TorpPedersen, O. Pedersen and P. Hildebrandt ${ }^{2}$. Gentofte University Hospital, Copenhagen, Denmark, 'Steno Diabetes Center, Copenhagen, Denmark, ${ }^{2}$ Frederiksberg Hospital, Copenhagen, Denmark

Several studies have indicated a more intense and diffuse atherosclerosis in coronary angiograms in patients with diabetes compared to non-diabetics. We studied the relation between glucose tolerance and severity of atherosclerosis in coronary angiograms. The study population was patients with coronary artery disease (CAD) referred for coronary arteriography; 28 consecutively selected men with known NIDDM and 99 randomly selected men without a history of diabetes. Patients without a history of diabetes underwent a standard OGTT. Angiograms were evaluated using a 14 segment model of the coronary tree. Two indices were created; a quantitative index as the sum of all stenoses in percentage divided by the number of segments seen and a qualitative index as the sum of all segments with stenoses divided by the number of segments seen.

\begin{tabular}{|c|c|c|c|c|}
\hline $\begin{array}{l}\text { essels with significant } \\
\text { enoses: }\end{array}$ & normal & $\begin{array}{l}\text { Impaired } \\
\text { glucose } \\
\text { tolerance }\end{array}$ & $\begin{array}{c}\text { NIDDM, } \\
\text { newly } \\
\text { diagnosed }\end{array}$ & $\begin{array}{l}\text { NIDDM, } \\
\text { known }\end{array}$ \\
\hline $\begin{array}{l}\text { vessel disease, } \%(n) \\
\text { essels with diffuse } \\
\text { herosclerosis: }\end{array}$ & $14,5(9)^{2}$ & $23,8(5)$ & $25,0(4)$ & $25,0(7)$ \\
\hline - & 34,4 & 40 & $37,5(6)$ & $57,7(15)^{W}$ \\
\hline & & 28 & $( \pm 2,5)$ & \\
\hline Qualitative index & & & $0,46(+0,04)$ & $0,52( \pm 0,04)^{* *}$ \\
\hline \multicolumn{5}{|c|}{$\begin{array}{l}p=0,2 \quad p=0,04{ }^{*} p=0,06{ }^{* *} p=0,07 \text {. indices are mean( }( \pm S E M) \text {. } \\
\text { By analysis of variance no statistically significant differences were found in } \\
\text { severity or distribution of CAD. However, in comparing CAD patients with } \\
\text { normal glucose tolerance and patients with known NIDDM a trend was seen } \\
\text { towards more intense and diffuse disease in patients with NIDDM. }\end{array}$} \\
\hline
\end{tabular}

\section{9}

ABNORMAL GLUCOSE TOLERANCE AND HYPERINSULINEMIA IN PATIENTS WITH CORONARY ARTERY DISEASE

M. Seibæk, L. Vallebo, H. Burchardt, S. Urhammer ${ }^{1}$, T. Hansen ${ }^{1}$, C. TorpPedersen, O. Pedersen ${ }^{1}$, P. Hildebrandt ${ }^{2}$. Gentofte University Hospital, Copenhagen, Denmark, ${ }^{1}$ Steno Diabetes Center, Copenhagen, Denmark, ${ }^{2}$ Frederiksberg Hospital, Copenhagen Denmark

Disturbances in glucose metabolism including NIDDM, IGT and insulin resistance are important risk factors for the development of coronary artery disease(CAD). We studied the prevalence of NIDDM and IGT and the levels of plasma insulin following an OGTT. The study population were 99 randomly selected male patients with $C A D$ and no history of diabetes and 116 randomly selected men from the general population with similar age, gender and BMI and without a history of diabetes

\begin{tabular}{|c|c|c|c|}
\hline & $\begin{array}{c}\text { Prevalence } \\
\%(n)\end{array}$ & $\begin{array}{c}\text { Plasma Insulin } \\
\text { o hour }\end{array}$ & $\begin{array}{c}\text { Plasma insulin } \\
2 \text { hours }\end{array}$ \\
\hline \multicolumn{4}{|c|}{ CAD patients $(n=99)$} \\
\hline normal OGTT & $62,6(62)$ & $55,7( \pm 5,9)^{*}$ & $338,8( \pm 37,6)^{\star *}$ \\
\hline IGT & $21,2(21)$ & $58,0( \pm 6,3)$ & $764,6( \pm 94,6)$ \\
\hline NIDDM & $16,2(16)$ & $141,2( \pm 48,7)$ & $745,9( \pm 176,3)$ \\
\hline \multicolumn{4}{|c|}{ Controls $(n=116)$} \\
\hline normal OGTT & $81,9(95)$ & $43,3( \pm 2,4)^{\star}$ & $180,4( \pm 15,5)^{\star \star}$ \\
\hline IGT & $12,9(15)$ & $72,2( \pm 12,6)$ & $502,1( \pm 98,0)$ \\
\hline NIDDM & $5,2(6)$ & $58,3( \pm 8,4)$ & $403,7( \pm 96,7)$ \\
\hline
\end{tabular}

Values are mean $( \pm S E M)$. Insulin data are given in $\mathrm{pmol} / \mathrm{h}$.

Twice as many CAD patients had an abnormal OGTT compared to the controls $(37,4 \%$ versus $18,1 \%)$.CAD patients with normal glucose tolerance had significantly higher levels of fasting and stimulated plasma insulin compared to controls $\left({ }^{*} p=0,06,{ }^{* *} p=<0,01\right)$. The hyperinsulinemia in CAD patients with normal glucose tolerance is indicating resistance to insulin mediated glucose uptake in spite of normal glucose tolerance

\section{1}

HYPERGIYCEMIA AS A PROGNOSTIC FACTOR FOR PATIENTS WITH ACUTE MYOCARDIAL NFARCTION AMI)

$X-Y$ Lin, Z-O Lin, Z Lin Department of pathology, Military Medicine School of Fuzhou and Department of Dongfang Hospital, Fuzhou China

Paticnts with AMI of ten have hyperglycemia.Stress caused by AMI may be restonsible for hyperglycemia, but a certain portion of patients with AMI may preexist diabetes mellitus which was not diagnosed by the time of onset of AMI. The aim of this study was, by measuring HbAlc concentration, to differentiate between the two conditions and to evaluate the prognostic significance of hyperglycemia in mottality in patients with AMI. Seventy eight pat 54 patients who survived AMI and without a prexisting known diabetes, glucosc tolctance tests were thout prexistisutions on fasting and performed by 12 paticnts had known diabctes and 24 patients, not previously known to have momill. Scren of them had clcvated Hodece illustrating preexisting survived AMl had abnormal glucose tolerance ites and of undiagnosed diabetcs. The prevalence of precxisting diabetes and of undagnosed diabetes were $15.3 \%$ and $10.6 \%$ respectively. The remaining patients with blood glucose concentrations of 7 . Smmol/ $\mathrm{L}$ and HbAle concentrations of $>7.0 \%$ were evaluated, two months later, for glucose tolerance test, which showed normal. This indicated that stress, not precxisting diabctes, was responsible for hyperglycemia in these patients. Twelve patients(15.3\%)died during hospitalization. Among them fout diabetics (21, $1 \%$ ) and eight non diabetics $(13.5 \%)$. On admission.died patients had morc pronounced hyperglyccmia than that in survivorsidiabetics $16.6 \pm 5.4$ vs $12.6 \pm 4.3 \quad 0<0.07$ and non diabetics $9.9 \pm$ 1.7 vs $7.8 \pm 1.0 \mathrm{p}(0.05)$. In conclusion, our study revealed that $36.1 \mathrm{~W}$ of paticnts who wetc not previously known to have diabetes had blood paticnts who wete nalence of undiagnosed diabetes in patients with aMl in this group was 10 . 6\%. It is the diabetes in particte factor in AMI indicating poor prognosis. 
1062

ASSOCIATION BETWEENCARDIOVASCULAR RISK FACTORS AND "CORNELL VOLTAGE" INDIABETES MELLITUS.

S. Vigili de Kreutzenterg, A. Avogaro, A. Tiengo and S. Del Prato. Cattedra di Malattie del Metabolisma dell'Università di Padova, Italia.

High mortality rate in diabetes mellitus is due to increased cardiovascular disease (CVD). An increased left ventricular mass (LVM) is an independent risk factor for CVD in diabetic population and has been associated with insulin resistance. Estimation of LVM is best determined by echocardiography. However, this procedure is money and time-consuming, hence poorly suitable for population studies. In this study, an ECGraphic parameter, the "Cornell voltage" ( $C V=R$ wave in aVL + S wave in V3) was employed to assess LVM in a diabetic population. Several clinical and metabolic parameters were also determined to assess the clustering among these parameters and CV. in 100 subjects (NIDOM=n 47; sex $M / F=22 / 25 ;$ age $=58 \pm 2 y r s ; \quad B M l=27.3 \pm 0.8 \mathrm{~kg} / \mathrm{m}^{2} ; \quad I D D M=n \quad 38 ; \quad M / F=14 / 24 ; \quad$ age $=36 \pm 2 ;$ $B M=228+0.4$ controls (C) $=n$ 15: MF $=4111 ;$ age $=46 \pm 5 ; B M l=23.4 \pm 1$ ) a resting $E C G$ for $C V$ and blood pressure (BP) measurements were performed; blood and urine samples for the determination of glycated haemoglobin ( $\mathrm{HBA} 1 \mathrm{C}$ ), C-peptide (IRCP), total cholesterol (TC), HDL-cholesterol (HDL-CT), triglyceride (TG), fibrinogen (F), uric acid (UA) and microalbuminuria $(\mu A)$ were collected. CV was significantly higher in NIDDM $(13,0 \pm 0.7 \mathrm{~mm})$ than in IDDM $(9.3 \pm 0.8)$ and $C(8.7 \pm 1.0$; both $p<0.005)$. When all subjects were pooled together, $C V$ was correlated with $B M l(r=0.54 ; p<0.001)$, mean $B P(r=0.44 ; p<0.001)$ and TG $\{r=0.22 ; p<0,03)$. No significant correlation between $\mathrm{CV}$ and the other parameters was found in $C$ and in IDDM. In NIDDM, $C V$ was correlated with $B M I(r=0.40 ; p<0.01)$, mean $B P$ $\{r=0.36 ; p<0.018)$, UA $(r=0.28 ; 0<0.05)$, IRCP $(r=0.34 ; p<0.05)$, and with $T G(r=0.25$; $\mathrm{p}<0.07$ ). No correlation was found with the duration of diabetes, HDA1C, F, CT, HDL-CT and $\mu \mathrm{A}$. In order to evaluate whether high values of $\mathrm{CV}$ were discriminant for association with CVD risk factors, NIDDM patients with a CV $<25^{\text {th }}$ percentile $(<9 \mathrm{~mm} ; \mathrm{n}=12$ ) and those with a $\mathrm{CV}>75^{\text {th }}$ ( $\left.>17 ; \mathrm{n}=13\right)$ were compared. Despite similar BP values $(105 \pm 2$ vs $101 \pm 4), \mathrm{CV}$ was associated with increase in BMl ( $30.8 \pm 1.9$ vs $25.8 \pm 1.8), \mathrm{TG}(4.6 \pm 1.6$ vs $2.7 \pm 0.5 \mathrm{mmol} / \mathrm{A})$ and UA $(0.36 \pm 0.05$ vs $0.31 \pm 0.04$ mmol/1). In conclusion: 1$) \mathrm{CV}$ is a simple $E C G$ index for $L V M ; 2)$ in general population it is influenced by BM! and BP; 3) in NHDDM CV is correlated with metabolic CVD risk factors, independently of BP value, metabolic control and duration of diabetes.

\section{3}

CORONARY HEART DISEASE IN NON-DNSUNN-DEPENDENT DIABETES WEIGHT LOSS REDUCES PLASMINOGEN ACTTVATOR RHHIBTOR-1LEVELS K.Lalié, P.B.Djordjevic, W.MLalis, D.Boškovic, M.Zamaklar, M.N.Iite, A.Jotic,

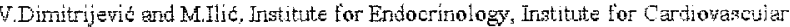
Diseases and Institute for Biochemistry. Belgrade, Yuzozlayia

It has keen postulated that increased plasminogen activator inhibitor-1 (PAi-1) levels was an important factor for accelerated coronary heart disease (CHD) it tron-insulin-dependerit diabetes mellituz (NDDM) patients and in nondiabetics, and that weight lose might be beneficial for reducing the risk for $\mathrm{CHD}$. However the influence of weight loss on PAI-1 aetivity in NIDDM patients with CHD has not yet been clarified. The atm of this study was to evaluate the effect of a moderate weight reduction diet in 32 obese NDDM patients with angiographically verified CHD on PAI-1 activity as well as on other related risk factors for CHD The dietary regimen 1000 kcal/day, $55 \%$ carbohydrates, $30 \%$ tat and $15 \%$ vroteins) was applied for 3 months. Inmediately before and at the end of its appication, we deternined PAY-1 levels by plastrinogen/chromogenie plasmin substrate assay, total body mass by body mass index (BMD, body fat distribution by waistoto-hip ratio (WHR), glycosylated hemogiotin (FHAlc) by chromatography, plasma insulin levels by RIA total cholesterol (Ch). HDI with,

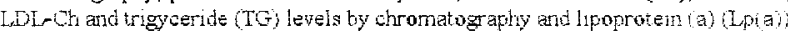
by nephelometry. We found that $P$ RI- iactivity was significantly decreased atter

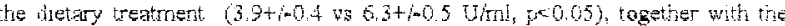
decrease in BMI $(26.8+1-1.3$ vis $29.7+1-1.8 \mathrm{~kg} / \mathrm{m} 2$, peo.05), and WHR both in male and ferrale patients (m:0.94+1-0.11 vs $1.09+1-0.21$, $10.86+1-0.11$ us $0.55+1-0.06$,

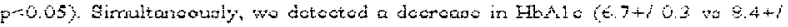

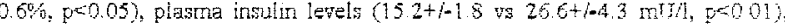

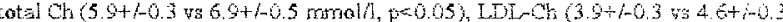

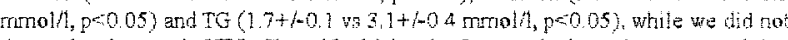
detect the charges in HDL-Ch and Lp(a) levels. Cur results have dernonistrated that the weight loss in obese NDDM patuents with CHD was astociated with a remarkable decrease in PAI-1 activity. These results imply that the reduction of PAT-1 levels could be an important determinant which contributes, together with the charges in insulin and lipoprotein levels, to the secondary prevention of CED in these NDDNM patients

\section{PS 57}

\section{Macroangiopathy Pathogenesis}

\section{4}

EFFECTS OF INSULIN-GLUCOSE INFUSION ON LEFT VENTRICLLAR FUNCTION IN HEALTHY AND NIDDM SUBJECTS.

F. C. Sasso, T. Salvatore, A. Cantillo. D. Cozzolino, F. De Gregorio*, L. Mansi*, T Sessa and R. Torella. Unit of Intemal Medicine *Unit of Nuclear Medicine Faculty of Medicine, $2^{\text {nd }}$ University of Naples, Italy.

The role of insulin on cardiac function, both in rest and in stress conditions. has to be investigated yet. The effects of normal saline (test $A$ ) and insulin-glucose (insulin= $1.7 \mathrm{mU} \cdot \mathrm{kg}^{-1} \cdot \mathrm{min}^{-1}$; glucose $=6 \mathrm{mg} \mathrm{kg} \cdot \mathrm{min}^{-1}$ ) (test B) infusions on left ventricular ejection fraction (LEVF) at rest and during dynamic exercise were examined by radionuclide ventriculography. Six non insulin-dependent diabetic patients and 6 sex- age- and BMI-matched healthy subjects were investigated. Diabetic patients were relatively young $(40.3 \pm 2.9$ years, $\mathrm{M} \pm \mathrm{DS})$ and had short duration ( $3.8 \pm 1.5$ years) and good metabolic control of disease ( $\mathrm{HbA}, \mathrm{c}: 6.9 \pm 0.4 \%$ ). Coronary artery disease microangiopathy, neuropathy and any cardiovascular abnormalities detectable by non-invasive technique were preliminarly excluded in all sutjects. Both uroups had normal scintigraphic parameters at rest and during dynamic exercise. Rest- and stress-LVEF were signiffcantly $(\mathrm{p}<0.001)$ lower in diabetic than healthy subjects. both in test $\mathrm{A}$ and $\mathrm{B}$. Rest- $\mathrm{LVEF}$ was significantly higher during test $\mathrm{B}$ than test $\mathrm{A}$ only in diabetic subjects $(p<0.01)$. Stress-LVEF was significantly higher $(p<0.05$ or less) during test $\mathrm{B}$ than $\mathrm{A}$, both in diabetic and healthy group. A significant lineat correlation between rest- and stress-LEVF and the index of insulin sensitivity (M/RR ratio) was found in diabetic patients ( $r=0.90$ or more; $p<0.05$ or less). Insulin induces both in normal and diabetic humans a very important rise in LVEF in response to a submaximal work. In diabetic subjects insulin increases LEVF at rest too, even if this parameter permains lower than in non-diabeic patients. Due to the strict exclusion criteria, it is reasonable to speculate that only diabetic disease could have conditioned this depressed, albeit normal, systolic function in diabetic group.

\section{5}

ACUTE HYPERINSLLINEMIA DECREASES PLASMA FIBRINOLYTIC ACTIVITY IN THE RAT

A. Pandolfi, A. Giaccari* , L. Morviducci ${ }^{*}$, E. Vitacolonna, F. Spitaleri and A. Consoli. Universita' D'Annunzio, Chicti and 'Universita' Cattolica, Roma, Italy

Hypofibrinolysis might be one of the factors responsible for increased atherotrombotic risk in diabetes. In population studies, plasma insulin levels, often increased in type II diabetes, have been shown to correlate directly with plasma Plasminogen Inhibitor Type 1 (PAI-1) levels and inversely with plasma fibrinolytic activity. However, it is not known whether insulin can directly affect plasma fibrynolisis in vivo. Aim of this study was to determine the effect of acutc, short-term hyperinsulinemia on plasma fibrinolysis in the rat. To this end, we performed a $4 \mathrm{hrs}$ eugiycemic insulin clamp (insulin infusion $4.0 \mu \mathrm{U} / \mathrm{Kg} / \mathrm{min}$, steady state plasma insulin levels $\sim 100 \mu \mathrm{U} / \mathrm{Kg} / \mathrm{min}$ ) in 8 Sprague Dowley rats (age 9 weeks, weight $203 \pm 10$ g) fasted for $24 \mathrm{hrs}$. Before and during the last $5 \mathrm{~min}$ of the insulin infusion, plasma samples were obtained for determination of plasma fibrinolytic activity (lysis of fibrin plates), free t-PA activity and t-PA/PAI-1 complex levels (fibrin autography analysis). Plasma fibrinolytic activity was sharply reduced by hyperinsulinemia (mean lysis areas on the fibrin platc $=210+18 \mathrm{~mm}^{2}$ before vs $41 \pm 8 \mathrm{~mm}^{2}$ after clamp. $\mathrm{p}<0.001$ ). As compared to plasma samples bcfore insulin infusion. fibrin autography analysis showed a marked reduction of the band corresponding to free t-PA and a significant increase of the band corresponding to the t-PA/PAI-1 complex in plasma samples obtained after insulin infusion. We conclude that in this animal model acute hyperinsulinemia sharply reduces plasma fibrinolytic potential and that this is likely due to increased plasma PAI-1 levels. Therefore, insulin can directly affect plasma fibrinolytic potential and hyperinsulinemia might contribute in determining a hypofibrinolitic statc. 
TISSUE PLASMRNOGEN ACTNATOR AND ITS INHIBTTOR DN TYPE \& DIABETES WITH AND WTTHOUT MACROANGIOPATHY

D.Rosć, W. Drewniak, M. Rewakowicz, B.Kowalewska, A GraczykowskaKoczorowrska, M.Kotschy. Departments: Pathophysiology, Endocrinology and Clinical Diabetology and General and Vascular Surgery, University School of Medical Sciences, Bydgoszcz, Poland

Haemostatic disturbances seem to be important in the pathogenesis of vascular diabetic complications. Laboratory investigations show hypercoagulability and decreased fibrinolytic activity in the blood of patients with type 2 diabetes. The ain of study was to compare tissue plasminogen activator it-PAl and plasminogen astiqator intibitor (PAI-1) in blood of type 2 diatetics with and without macroangiopathy. The study groups consisted of 23 patients aged $34-6,5$ years with macroangiopathy and of 36 aged $46-60$ years wathout macroangiopathy. Ali studied patients were orally treated with sulfonylcarbamide derivates. Control group contained 10 age-matched healthy pernons, In the blood the concentrationis of $t-P A$ and PAI-1 antigen were determined using ELISA, t-PA and PAI- 1 activity with chromogenic substrates (Biopool, Sweder). Results are given below:

\begin{tabular}{|c|c|c|c|c|}
\hline irameter & & $\begin{array}{c}\text { Contro: } \\
n=10\end{array}$ & $\begin{array}{c}\text { Macroangiopathy } \\
n=23\end{array}$ & $\begin{array}{c}\text { No macroangiopathy } \\
\mathrm{n}=35\end{array}$ \\
\hline & & $9,57 \pm 5,05$ & $15,02 \pm 11,93$ & $15,43 \pm 3,55$ \\
\hline & & $0,84 \pm 0,20$ & $1,50 \pm 0,93 *$ & $0,44 \pm 0,35$ \\
\hline & & $9,05 \pm 1,08$ & $33,3 \pm 26,5=$ & $43,78 \pm 11,78$ \\
\hline $1 \mathrm{Act}$ & $\mathrm{mi}$ & 6.9 & $=7,0 \%$ & $16,6 \pm \$, 5$ \\
\hline
\end{tabular}

$6,9 \pm 2,0$

* $p<0,05$ diabetics versus controls

In diabetics without macroangiopathy $t-P A$ Ag was stgnificantly higher than $n$ controls but t-PA activity was significantly higher in macroangopathic patients than in controls and in diabetics without macroanglopathy. PAI-1 antigen and activity in both groups of diabetics were significantly higher in comparison to controls. Independently of macroanglopathic complications high PAI- I level is the nost important factor diminishing fibrinolytic activity in type 2 drabetes.
AUTOANTIBODIES AGAINST OXIDISED LOW DENSITY LIPOPROTEINS IN PATIENTS WITH TYPE 1 DIABETES MELLITUS E. J. Menzel*, A. Festa,, H. P. Kopp and G. Schernthaner; I. Medical Dept., Rudolfstiftung Hospital, Vienna, Austria and *Dept. of Immunology, University of Vienna, Austria

Patients with diabetes mellitus are exposed to an increased risk of premature atherosclerosis. The oxidation of low density lipoproteins (LDL) is considered a key event in the initiation and progression of atherosclerosis. To investigate LDL oxidation in vivo we measured autoantibodies against oxidised LDL (ox-LDL) in 94 patients with type 1 diabetes mellitus without clinical evidence of atherosclerotic complications, compared to 27 age-matched, non-diabetic controls. Patients and controls were screened for autoantibodies against ox-LDL using a solid phase ELISA. Wells were coated either with ox-LDL or with native LDL (n-LDL). LDL was isolated from blood of healthy individuals by sequential ultracentrifugation. Oxidation of LDL was performed with $5 \mu \mathrm{mol} / \mathrm{l} \mathrm{Cu}$ + (kindly donated by H. Esterbauer). Dilution of sera was 1:50. As second antibody, antihuman Ig from rabbits was used in peroxidase labeled form. Since some sera showed nonspecific binding of Ig to the ox-LDL coated solid phase, we compared binding of ox-LDL with that obtained in n-LDL wells. The ELISA result was defined as positive, if the binding ratio ox-LDL/n-LDL exceeded the value 1,3 . In patients with type 1 diabetes the oxLDL/nLDL ratio was significantly higher than in controls $(2,2 \pm 0,3$ SEM vs. $1,17 \pm 0,2, \mathrm{p}<0,02) .48 \%$ of the patients $(n=45)$ showed a positive antibody ratio, compared to $19 \%(n=5)$ of controls $(p=0,01)$. Patients with a positive ratio had a shorter duration of diabetes $(10 \pm 7 \mathrm{SD}$ vs. $13,6 \pm 10$ years; $p<0,05)$, whereas no difference could be detected with respect to age $(30 \pm 8 \mathrm{SD}$ vs. $34 \pm 1$ lyears $)$ and metabolic long-term control (mean of repeated $\mathrm{HbA}_{\mathrm{lc}}$ measurements: $7,8 \pm 1,3 \mathrm{SD}$ vs. $8,3 \pm 1,4 \%$ ). The presence of incipient nephropathy or retinopathy was not related to the ox-LDL antibody status. Our data demonstrate, that enhanced oxidation of LDL occurs in vivo in patients with type 1 diabetes mellitus without clinical evidence of atherosclerosis. The absence of a relationship of LDL oxidation with metabolic long-term control underlines the importance of additional factors - other than hyperglycemia - in the pathogenesis of atherosclerosis in these patients.

\section{8}

INDUCTION OF MACROPHAGE GROWTH BY OXIDIZED LOW DENSITY LIPOPROTEIN IN NIDDM

T. Matsumura, S. Kobori, M. Sakai, Y. Sato, S. Horiuchi* and M. Shichiri, Departments of Metabolic Medicine and

*Brechemistry, Sehexol of Medicine, Kumamto, Japan

It is w cll kmoun that oxidized low density lipoprotein (Ox-LDL) plays screral kinds of role to the development and progression of atheroscleresis. Considering many reports that the Ox-LDL may bc incrcascd in NIDDM, this is an important issue in a view of diabetic macrisanglopathy. Wc recently demonstrated that murine macrophage grow th is induced by Ox-LDL. In the present study, the mechanisms fir macrophage growth induced by Ox-LDL were examined using ${ }^{3} \mathrm{H}$-thy midine incorporation and cell-counting assays. Whereas the mitogenic activity of acetylated LDL (AcLDL.) for murine resident macrophage was negligible, treatment of Ac-LDL with phospholipase $\mathrm{A}_{2}$ led to an increase in lysophosphatidy lcholine (LPC), and a concominant increase in its mitogenic effect, indicating an essential role of LPC in macrophage grow th. The role of the scavenger receptor was also examined. Malevlated borine scrum albumin (M-BSA), an effective ligand for the scavenger receptor, did not induce macrophage growth even in the presence of LPC. Moreover, Ox-LDL-induced macrophage growth was inhibited by $70 \%$ by the presence of M-BSA. M-BSA competitively inhibited the scavenger receptor-mediated endocytic uptake of Ox-LDL, thus reducing a concominant uptake of LPC, whereas non-specific direct transfer of LPC from Ox-LDL to cell surface was not affected. These results suggest that internalization of LPC through the scavenger receptor may lead to generation of an intracellular signal for macrophage growth.

\section{9}

MYOCARDIAL DYSFUNCTION IN PATIENTS MITH DIABETES MELLITUS AND BRAIN NATRIURETIC PEPTIDE

H.Himei, J.C.Fang, T. Matsuoka, T.Ka.ji tani and Y.Kawanishi, 0kayama Red Cross General Hospjtal, Okayama -700 . Japan

We measured brain natriuretic peptide (BNP), which is secreted from the myocardium and is considered to specifically reflect ventricular dysfunction from an early stage and studied the clinical usefulness of this parameter for the early detection of asymptoma tic myocardial infarction in patients ith diabetes mellitus. BNP was measured by the IRMA method in 496 diabetic outpatients and in 157 non-diabetic individuals undergoing health checks. More of the diabetics had high BNP levels than the non-diabetics $(21.7 \%$ vs. $12.6 \%)$. In the non-diabetics, the BNP level showed no age-related differences. In the diabetics, however, the BNP level was $15.3 \mathrm{pg} / \mathrm{ml}$ for those aged $\leq 64$ years and $33.7 \mathrm{pg} / \mathrm{ml}$ in those aged $\geq$ 65 years $(p<0.0001)$. The cardiothoracic ratio and duration of disease were also significantly greater in diabetics aged $\geqq 65$ years $(P<0.0001)$. Two of the diabetics with high BNP levels were found lo have asymptomatic myocardial infarction. The incidence of arrhythmia on Holter electrocardiograms and hyperventilatory electrocardiograms was definitely higher in patients with high BNP levels $(p<0.001)$. suggesting that there was a correlation with the incidence of arrhythmia. Measurement of BNP allows the evaluation of myocardial function by a simple blood test and is considered to be useful for the detection of asymptomatic myocardial infarction and the prevention of sudren death in diabetic patients. 
1070

PROGRESSION OF CAROTID ATHEROSCLEROSIS AND THE ROLE OF ENDOTHELIN IN DIABETIC PATIENTS

I.N. Migdalis, K. Kalogeropoulou, V. Iliopoulou, P. Triantafilou, C. Nounopoulos and A. Bouloukos. Dept. of Diabetes, NIMTS Hospital and Dept of Biochemistry, University of Athens, Athens, Greece

An increased thickness of the carotid artery wall is thought to be a sign of early atherosclerosis: Since vascular endothelium is the site of formation of several substances, we have investigated the rate of progression of carotid atherosclerosis and the contribution of endothelin (ET), lipid peroxides (measured as thiobarbituric acid reacting species (TBARS)) and prostacyclin levels $\left(\mathrm{PGI}_{2}\right.$ ), at baseline and after 30 months. Fifty NIDDM patients without evidence of macroangiopathy, hypertension, proteinuria or proliferative retinopathy, and 27 non-diabetic subjects were studied. Arterial wall thickness was measured as the mean of the maximum intimal-medial thickness (IMT) in 16 carotid segments by B-mode ultrasound. The IMT value was significantly increased in diabetic subjects (at baseline: $1.11 \pm 0,31$, after 30 months: $1.26 \pm 0,28 \mathrm{~mm}, \mathrm{p}<0.01$ ), but not in control subjects $(1.10 \pm 0.28,1.20 \pm 0,29 \mathrm{~mm})$. At baseline time both groups had similar levels of ET, TBARS and $\mathrm{PGI}_{2}$. In 30 months follow-up, the ET level $8.0 \mathrm{pmol} / 1$ (5.8-10.7, 95\% confidence limits) was significantly elevated in diabetic subjects, compared with the level at baseline time $7.3 \mathrm{pmol} / \mathrm{l}(4.8-10.9), \mathrm{p}<0.01$. No significant differences were found in other examined parameters in the studied groups. In conclusion, endothelin is the main associate of the change of IMT value over 30 months in NIDDM patients, in whom the extent of atherosclerosis was significantly greater than in control subjects.

\section{1}

INCREASED PLATELET EXPRESSION OF CONSTITUTIVE ICAM-2 IN NIDDM (TYPE-II) PATIENTS.

D. Tschoepe, S.Hesse, U.Rauch, B.Schwippert, Diabetes Research Institute at the Heinrich Heine University, Duesseldorf, Germany

Increased interaction between $<p \mid>$ atelets and $<\mid$ eu $>$ kocytes has been shown in diabetic patients with vascular complications. ICAM-2 which is constitutively expressed on peripheral $<p \mid>$ independent of activation could be potentially involved as a <leu> CD11/ CD18 ligand. Here, we investigated the $<\mathrm{p}|>| \mathrm{CAM}-2$ expression in a cohort of 17 NIDDM patients (gender $\mathrm{f} / \mathrm{m}$ 9/8, median age 64.0<y>ears, body mass index <bmi> $30.4 \mathrm{~kg} / \mathrm{m}^{2}$, HbA1 $12.7 \%$, mean blood glucose $<\mathrm{mbg}>170.0 \mathrm{mg} / \mathrm{dl}$, $<$ chol>esteral $234.0 \mathrm{mg} / \mathrm{dl}$, LDL $156.0 \mathrm{mg} / \mathrm{dl}$, HDL $41.0 \mathrm{mg} / \mathrm{dl}$, <tri >qlycerides $179.0 \mathrm{mg} / \mathrm{dl}$, , <fib>rinogen $309.0 \mathrm{mg} / \mathrm{dl},<\mathrm{pl}>228.0 \times 10^{3} / \mu \mathrm{l},<\mid \mathrm{eu}>6.1 \times 10^{3} / \mathrm{ul}$, $<a l b>$ uminuria $9 \mu \mathrm{g} / \mathrm{min}$ ) compared to 18 healthy controls (gender $\mathrm{f} / \mathrm{m} 14 / 4$, median age $54.0<\mathrm{y}>,<\mathrm{bmi}>24.0 \mathrm{~kg} / \mathrm{m}^{2}, \mathrm{HbA} 16.8 \%$, <chol $>233.0 \mathrm{mg} / \mathrm{dl}$, LDL $152.5 \mathrm{mg} / \mathrm{d}$, HDL $55.5 \mathrm{mg} / \mathrm{dl}$, <tri $>117.0 \mathrm{mg} / \mathrm{dl},<\mathrm{fib}>274.5 \mathrm{mg} / \mathrm{dl}$, $\langle\mathrm{pl}\rangle$ $262.5 \times 10^{3} / \mu \mathrm{l}$, <leu> $\left.6.0 \times 10^{3} / \mu \mathrm{l}\right)$. Washed sample platelets were stained with a monocional anti- Icam-2 antibody (cbr-jC2/2) and then evaluated by the DIII flow cytometry assay which provides an expression index by multiplication of the percentage $\langle \%\rangle$ of positive sample platelets with the mean fluorescence intensity (arbitrary units $\langle a u\rangle$ ). Both values were separately increased with the platelets from NIDDM patients: $69.6 \pm 11.0<\%>111.1 \pm 1.9<\mathrm{AU}\rangle$ vs $56.5 \pm 14.3\langle \%\rangle / 9.6 \pm 2.3\langle\mathrm{AU}\rangle$ with the index value being $786.0 \pm 240.1$ vs $566.4 \pm 270.3, p<0.01$ for all comparisons. The correlation matrix with the documentation parameters proved significance exclusively with the HDL: $-0.54, p<0.01$. We conclude that platelets from NIDDM patients constitutively express more ICAM-2 ("diabetic thrombocytopathy") favouring CD11/CD18 mediated coaggregation with <leu>. HDL dyslipoproteinemia rather than glycemia appears to interfere with the regulatory transcriptional apparatus of the bone marrow precursor megakaryocytes which is lacking in peripheral platelets.

\section{2}

IMPAIRED ENDOTHELIAL FUNCTION OF CONDUIT ARTERIES IN TYPE 1 DIABETIC PATIENTS WITH GOOD METABOLIC CONTROL. M Alevizaki, J Lekakis, C Papamichael, H Anastasiou, A Souvatzoglou and DA Koutras. Dept Medical Therapeutics and $1^{\text {st }}$ Endocrine Unit \& Diabetes Centre, ALEXANDRA University Hospital, 11528 Athens, GR.

Endothelial dysfunction is a well recognised complication in type 1 diabetes mellitus (DM1), most reports referring to small resistance vessels. The aim of the present study was to evaluate endothelia function of conduit arteries in well controlled patients (pts) with DM1 without microalbuminuria. Nineteen diabetic pts (age 30 $\pm 8.5 \mathrm{yrs}$, 7 male/12female, duration of diabetes $9.4 \pm 7.4 \mathrm{yrs}$, mean daily insulin dose $42 \pm 16 \mathrm{U}, \mathrm{HbA1c} 6.2 \pm 1.9 \%$ ) were examined by high resolution ultrasound imaging. None had dyslipidaemia or hypertension. Nineteen healthy individuals matched for age and sex served as controls. Brachial artery diameter was measured at rest during reactive hyperaemic flow (endothelium dependent dilatation), as well as after sublingual nitroglycerin administration (endothelium independent response). The thickness of carotid intima media (IMT) was also measured. Brachial artery diameter at rest did not differ between pts and controls $(3.4 \pm 0.5,3.5 \pm 0.5 \mathrm{~mm})$, nor did reactive hyperaemia (pts vs controls, $452 \pm 220,530 \pm 233 \%$ ). Flow-mediated dilatation (FMD) was lower in DM1 pts $(6.9 \pm 7.9,11 \pm 7 \%, p=0.038)$ indicating impaired endothelial function. Nitroglycerin-induced dilatation did not differ between pts and controls $(23 \pm 16,26 \pm 9 \%)$ showing normal vascular smooth muscle response. No differences were observed in IMT either $(0.54 \pm 0.1,0.55 \pm 0.1 \mathrm{~mm})$. No correlation between FMD and either age, duration of diabetes, $\mathrm{HbA} 1 \mathrm{c}$, total cholesterol, triglycerides, or mean insulin dose was observed in the DM1 group. In conclusion, endothelial dysfunction of conduit arteries can be detected in type 1 diabetic patients with good metabolic control, probably contributing to the high prevalence of atherosclerosis in these patients.

\section{3}

PLATELETS OF LEAN NIDDM SUBJECTS SHOW A NORMAL SENSITIVITY TO INSULIN.

G. Anfossi, E. Mularoni, S. Burzacca, M. C. Ponziani, P. Massucco, L. Mattiello, V. Piretto, F. Cavalot, and M. Trovati. Diabetes Unit, Dept of Clinical and Biological Sciences of the University of Turin, Turin, Italy.

Insulin plays a platelet anti-aggregating effect in heaithy subjects, attributable to the activation of soluble guanylate cyclase; this effect is blunted in obese subjects and in obese NIDDM patients, suggesting that platelet insulin resistance plays a role in the increased cardiovascular risk of the insulin-resistance syndrome. To verify whether also lean NIDDM patients present a reduced sensitivity to the anti-aggregating effect of insulin, we studied 11 lean normotensive NIDDM patients ( $8 \mathrm{M} / 3 \mathrm{~F}$; age: $53.4 \pm 1.9$ yrs, BMI: $23.6 \pm 0.4$, fasting blood glucose $7.4 \pm 0.3 \mathrm{mmol} / \mathrm{l}$; fasting plasma insulin $35.8 \pm 8.3 \mathrm{pmol} / \mathrm{l}$ ), submitting their platelet-rich plasma to a 3 -min incubation with human recombinant insulin at the concentrations of $0,240,480,960$ and $1920 \mathrm{pmol} / \mathrm{l}$ and determining the insulin effects on: a) ADP-induced aggregation, evaluated by Born's method; b) platelet cyclic guanosine monophosphate (cGMP). Results: a) insulin dose-dependently decreased platelet sensitivity to ADP (ANOVA for repeated measures, $\mathrm{p}<0.0001$ ), ADP ED50 with insulin $1920 \mathrm{pmol} / 1$ being $141.5 \pm 6.4 \%$ $(\mathrm{m} \pm \mathrm{SEM})$ of the value without insulin; $\mathrm{b})$ insulin dose-dependently increased platelet cGMP (ANOVA for repeated measures $p<0.0001$ ), from the basal value of $7.5 \pm 0.2$ to $22.9 \pm 3.7 \mathrm{pmol} / 10^{\circ}$ plts with insulin $1920 \mathrm{pmol} / 1$. These results were comparable to those obtained in ageand sex-matched healthy volunteers. In conclusion, lean NIDDM patients show a normal sensitivity to the anti-aggregating effect of insulin, suggesting that obesity plays a major role in the blunted platelet response to insulin observed in obese NIDDM patients. 
1074

MIGHT HYPERHOMOCYSTEINEMIA CONTRIBUTE TO THE ACCELERATED ATHEROSCLEROSIS OF DIABETICS PATIENTS WITH NEPHROPATHY? A.Chico, A.Córdoba**, F. Blanco*, M.Rigla, R.Arcelus*, C.Carrascosa*; J.Ordóñez*, A.Pérez. Endocrinology and Biochemistry*, Hospital Sant Pau. UAB Barcelona. Spain. Univ. of Antioquía**. Medellín. Colombia. Increased incidence of cardiovascular disease in diabetic patients is only partially explained by classical risk factors. Plasma homocysteine (Hcy) has been identified as a risk factor for atherosclerosis. We determined the plasma Hcy leveis in 70 diabetic patients ( 33 type 1 and 37 type 11 ; age of $47.5 \pm 16.7$ years; diabetes duration of $12.2 \pm 17$ years) and in 50 nondiabetic controls. Both groups were comparable for age, sex, BMI, creatinine and lipid levels. Total plasma Hcy was isolated by HPLC and measured by fluorimetric detection. Mean plasma Hcy was higher in diabetic subjects than in controls $(8.45 \pm 4.3$ vs $6.82 \pm 2.45 \mu \mathrm{mol} / \mathrm{L}$. $\mathrm{p}<0.05)$ without differences between type I and type II diabetic patients Hyperhomocisteinemia was present in $16 \%$ of patients $(58 \%$ of patients with nephropathy) and in $4 \%$ of controls. Hcy levels positively correlated with serum creatinine and albumin excretion rate $(p<0.01)$, but not with fructosamine and $\mathrm{HbAlc}$ levels. In addition, plasma $\mathrm{Hcy}$ concentration was higher in diabetic patients with macroangiopathy $(\mathrm{N}=10 ; 11 \pm 3.2$ vs $7.7 \pm$ $4.3 \mu \mathrm{mol} / \mathrm{L} ; \mathrm{p}<0.05)$ and proteinuria $>300 \mathrm{mg} /$ day $(\mathrm{N}=12 ; 12.6 \pm 3.6$ vs 7.6 $\pm 4.3 \mu \mathrm{mol} / \mathrm{L} ; \mathrm{p}<0.001)$. In conclusion, these results suggest that hyperhomocisteinemia might contribute to the accelerated atherosclerosis of diabetic patients with nephropathy

\section{5}

PLASMA HOMOCYSTEINE CONCENTRATIONS AS PREDICTOR OF MORTALITY IN NON-INSULIN-DEPENDENT DIABETES MELLITUS M.-A. Gall', C.D.A. Stehouwer', P. Hougaard ${ }^{3}$, C. Jakobs ${ }^{2}$ and H.H. Parving ${ }^{1}$. ${ }^{1}$ Steno Diabetes Center, Gentofte, Denmark, 2Free University Hospital, Amsterdam, The Netherlands, ${ }^{3}$ Novo Nordisk A/S, Bagsvaerd, Denmark.

Previous studies in non-diabetic populations have revealed a link between high total homocysteine in plasma (tHcy) and cardiovascular disease (CVD). As NIDDM patients suffer increased morbidity and mortality from CVD, we performed a prospective study to evaluate the impact of tHcy concentrations on mortality in 211 Caucasian NIDDM patients followed for a median of 3.5 (range $0.2-4.1$ ) years. Normoalbuminuria (urinary albumin excretion (UAE) $<30$ $\mathrm{mg} / 24 \mathrm{~h}$ ) was present in $106(56 \mathrm{M} / 50 \mathrm{~F}$ ), microalbuminuria (UAE 30-299 $\mathrm{mg} / 24 \mathrm{~h}$ ) in $61(34 \mathrm{M} / 27 \mathrm{~F})$, and macroalbuminuria ( $\geq 300 \mathrm{mg} / 24 \mathrm{~h}$ ) in 44 (37 M/7 F) patients at baseline. Micro- and macroalbuminuric patients were older than normoalbuminuric patients, being 58 (8) (mean (SD)), 59 (7), and 55 (9) years, respectively $(p<0.05)$. The level of $s$-creatinine increased with increasing levels of albuminuria being 71 (43-143) (median (range)), 79 (48-170), and 90 (54-539) $\mathrm{umol} / \mathrm{l}$, in normo- micro-, and macroalbuminuric patients, respectively $(p<0.001)$. thcy levels increased with increasing levels of albuminuria, being 6.4 (2.7-14.4) (median (range)), 7.0 (2.4-16.5), and 9.7 (3.7-46.7) $\mu \mathrm{mo} / \mathrm{I}$, in normomicro-, and macroalbuminuric patients, respectively $(p<0.001)$. Seven percent $(n=7)$ of patients with normoalbuminuria, $13 \%(n=8)$ with microalbuminuria, and $30 \%(n=13)$ with macroalbuminuria died during follow up; 14 from CVD, 2 from uraemia, 8 from other causes, and 4 from unknown causes. Cox regression analysis revealed age-adjusted tHcy concentration measured at baseline to predict all-cause mortality: thcy (1 $\mu$ mol/l), $1.12(1.07-1.18)$ (relative risk $(95 \%$ Cl)), $(\mathrm{p}<0.001)$. The level of tHcy did not predict CVD mortality: thcy $(1 \mu \mathrm{mo} / / \mathrm{l})$ $1.05(0.90-1.22),(p=0.5)$, which could be due to the relative small number of cardiovascular deaths. We suggest that the elevation of thcy predicts all-cause mortality in NIDDM patients.

\section{6}

EXPRESSION OF ANGIOPATHY-SPECIFIC PROTEINS IN HUMAN ARTERY-ENDOTHELIAL CELLS:

A. Haslinger. H.K. Müller and R. Landgraf, Medizinische Klinik Klinikum Innenstadt, LMU-Murich

Aim of this study is to investigate the role of the PKC-system in endothelial cells and to delcrmine the involvement in the expression of ICAM-1. To investigate the mechanism of diabetic vascular complications endothelial cells from arteria femuralis were ultivated in glucose-free EGM (PromoCell) up to confluence. After incubation in $0 \mathrm{mM}$ to $30 \mathrm{mM}$ D-glucose expression of PKC-isoforms was shown. Endothelial cells were not more passaged than four times and for Western-Blotting cells were lysated at $4^{\prime \prime} \mathrm{C}$ by addition of $0.5 \%$ SDS (including $0,4 \mathrm{mM}$ PMSF). $30-50 \mu \mathrm{g}$ were applied on $75 \%$ and $10 \%$ SDS-PAGE Detection of $\mathrm{PKC}$-isoforms was performed with monospecific polyclonal alkaline-phosphatase conjugated antibodies, ICAM-1 was detected with a monoclonal antibody. B-actin antibody was coincubated as a glucoseindependent control. Quantification of western-blot signals was performed by densitomerric scanning. In contrast to HUVEC's growth of artery endothelial cells took two times longer. Growth to primary culture took two weeks after supplementing glucose-free EGM with $20 \%$ of FCS. After the first passage cells were grown in EGM without FCS and D-glucose. Viability of cells was controlled by staining with trypanblue and MTT, endothelial cells were identified by detection of von-Willebrand factor. Four of nine PKC-isoforms were found in artery endothelial cells. After 12h incubation with $15 \mathrm{mM}$ D-glucose PKC-delta was identified as main isoform and an expression increase versus $0 \mathrm{mM}$ of $2.0 \pm 0.3$ was found. For PKC beta II an expression increase of $1.6+0.2$ for PKC epsilon $1.45+0.1$ and for PKC zeta $1.35 \pm 0.2$ was found. By incubation of endothelial cells with $10 \mu \mathrm{g} / \mathrm{ml}$ Phorbolester involve-ment of $\mathrm{PKC}$ in ICAM- 1 expression could be shown. Results show, that expression of $\mathrm{PKC}$-isoforms in veins and arteries seems to be very similar and that $\mathrm{PKC}$ plays an important role in the increased expression of ICAM-1.

\section{7}

PLASMA TOTAI RADICAL-TRAPPING ANTIOXIDANT PARAMETER AND THROMBOSIS IN DIABETES MELLITUS

A. Ceriello ${ }_{2}$ N. Bortolotti, A. Crescentini, R. Giacomello, G. Stel, L. Tonutti, E. Motz, S. Lizzic, A. Russo, C. Taboga. Chair of Internal Medicine and Chair of Clinical Pathology University of Udine, Diabetology Unit, A.O. S.M.M. Udine, Italy

The existence of an oxidative stress in diabetes mellitus is still debated, as well as its possible contribution to the activation of thrombosis. The Total Radical-trapping Antioxidant Parameter (TRAP) has very recently been proposed as a tool to explore the antioxidant power of a plasma sample. In this study we evaluated in 46 NIDDM patients and in 47 normal controls, all non-smokers, matched for age $(58.2+1.5$ v's $55.1 \pm 1.3$ years, $11-5 \mathrm{SF})$, sel (27M and $19 \mathrm{~F}$ ys $30 \mathrm{M}$ and $17 \mathrm{~F}$ ) and diet habit, TRAP, protein-bound-SH $(\mathrm{SH})$, uric acid (U), vitamin $\mathrm{E}(\mathrm{E})$ and vitamin $\mathrm{C}(\mathrm{C})$, as well as the markers of thrombosis fibrinogen $(F)$, prothrombin fragments $1+2(\mathrm{~F} 1+2)$ and D-dimer (D-D). TRAP $(677.1 \pm 15.8$ vs $950.2 \pm 16.0 \mathrm{umol} / 1, \mathrm{p}<0.001)$, SH $(490.8 \pm 8.0$ vs $547.6 \pm 10.1 \mu \mathrm{mol} / 1, \mathrm{p}<0.001)$ and $\mathrm{L}(4.7 \pm 0.2$ vs $5.2 \pm 0.1 \mathrm{mg} / \mathrm{dl}$, $\mathrm{p}<0.01)$, were significantly reduced in diabetic patients, while $\mathrm{E}(10.4 \pm 0.5$ vs $8.9 \pm 0.3 \mathrm{mg} / \mathrm{l}, \mathrm{p}<0.005$ ) was significantly increased, even when corrected for serum cholesterol $(5 \pm 0.1 \mathrm{vs} 4 \pm 0.1 \mathrm{~F} \mathrm{mg} / \mathrm{l} /$ cholesterol $\mathrm{mg} / \mathrm{ml}, p<0.005)$. $\mathrm{C}(3.1 \pm 0.3$ vs $3.4=0.2 \mathrm{mg} / \mathrm{l})$ was not different. $\mathrm{F}(346.8 \pm 13.4$ vs $288.6 \pm 8.5$ $\mathrm{mg} / \mathrm{dl}, \mathrm{p}<0.001), \mathrm{F} 1+2(1.32+0.00$ vs $0.74+0.05 \mathrm{kmol} / \mathrm{l}, \mathrm{p}<0.001)$ and $\mathrm{D}) \mathrm{D}$ (26.7 2 2.9 vs $14.2+1.7 \mathrm{mg} / \mathrm{dI}, \mathrm{p}<0.001$ ) were increased in diabetic patients. TRAP, but no other antioxidant, was strongly associated and predictor, by step-wise analysis of variance, of $F(r=0.32, p<0.03 ; F$ to enter 5.1$)$, $F 1+2(r=0.61, p<0.001 ; F$ to enter 26.5) and D-D $(r=51, p<0.001 ; F$ to enter 15.2) in diabetic patients. These data show decreased TRAP levels in NIDDM patients, suggesting the existence of an oxidative stress in diabetes. Moreover, this study supports the hypothesis that lotal antioxidant capacity, more than the reduction of a single antioxidant. significantly conditiones thrombosis in diabetes mellitus. 
1078

\section{GLUCOSE-INDUCED OXIDATIVE STRESS IN VASCULAR SMOOTH MUSCLE CELLS}

P.C. Sharpe, K.K.M. Yue, M.A. Catherwood, D. McMaster* and E.R. Trimble. Department of Clinical Biochemistry and 'Medicine, Queen's University of Belfast, Belfast, UK.

Increased oxidative stress is believed to be important in the pathogenesis of diabetic vasculopathy. The aim of this study was to investigate direct effects of glucose-induced oxidative stress on endogenous antioxidant defences of vascular smooth muscle cells (VSMCs). To address this, intracellular maiondialdehyde (MDA), glutathione and the activities and gene expression (Northern analysis) of the key aritioxidant enzymes superoxide dismutase (SOD), catalase and glutathione peroxidase (GPX) were measured in porcine VSMCs cultured in $5 \mathrm{mM}$ glucose, $25 \mathrm{mM}$ giucose or $5 \mathrm{mM}$ glucose $+20 \mathrm{mM}$ 3-O-methyl glucose (OMG) for 10 days. CDNA probes were produced by reverse-transcriptase PCR (RT-PCR). MDA concentrations were increased in cells cultured in $25 \mathrm{vs} 5 \mathrm{mM}$ glucose ( $1.88 \pm 0.24$ vs $1.34=0.11 \mathrm{nmol} / \mathrm{mg}$ protein, mean $\pm \mathrm{sem} ; n=15, p<0.01$ ) but not in OMG $(1.08 \pm 0.20 \mathrm{nmol} / \mathrm{mg}$ protein). Total glutathione was markedly reduced in $25 \mathrm{mM}$ glucose compared to $5 \mathrm{mM}(0.69 \pm 0.12$ vs $1.40 \pm 0.13 \mathrm{nmol} / \mathrm{mg}$ protein, $p<0.0001)$ but not in OMG $(1.95 \pm 0.40$ $\mathrm{nmol} / \mathrm{mg}$ protein). There were no significant differences in the activities of CUZnSOD. MnSOD, catalase or GPX but $25 \mathrm{mM}$ glucose increased the gene expression of CuZnSOD by $60 \% . \mathrm{N}$-acetyicysteine $(0.5,1.0$ and $5 \mathrm{mM}$ ) restored glutathione in VSMCs grown in $25 \mathrm{mM}$ glucose in a dosedependent fashion but MDA was only partially reduced ( $N S, n=3$ ). In conclusion, these results demonstrate (1) endothelium- and glycationindependent glucose-induced oxidative stress in VSNCs with effects on the gene expression of CuZnSOD; (2) the inability of glutathione repletion to normalise MDA concentrations highlights antioxidant specificity and suggests multiple free radical species induction by glucose

\section{9}

CARDIAC FUNCTION AND ERYTHROCYTE Na+LI+ CT ACTIVITY IN NORMOTENSIVE, NON-PROTEINURIC INSULIN DEPENDENT DIABETIC PATIENTS

P.S. Kontessis, S. Toumanidis, A. Paraskevopoulos, A. Zaga, R. Charitopoulou, L. Sarika, H. Anastassiou and A. Souvatzoglou. Renal Unit and 1st Endocrine Section, "Alexandra" Hospital, Athens, Greece.

in order to assess the relationship between erythrocyte $\mathrm{Na}+/ \mathrm{Li}+\mathrm{CT}$ activity and cardiac anatomy-function in normotensive, non-proteinuric IDDM patients, we designed a cross-sectional study of IDDM patients with high and low erythrocyte $\mathrm{Na}+/ \mathrm{Li}+\mathrm{CT}$ activity in an ouptatient clinic. On the basis of Na+/Li+ CT activity 9 normotensive, non proteinuric IDDM subjects $(5 \mathrm{M} / 4 \mathrm{~F}$, age $31 \pm 10$ yrs, diabetes duration $14 \pm 6$ yrs, $x \pm S D$ ) with Nat/Li+ CT activity $>0.260 \mathrm{mmo} / \mathrm{L} \quad \mathrm{RBC} / \mathrm{hr}$ $(0.320 \pm 0.04 \mathrm{mmol} / \mathrm{L} \mathrm{RBC} / \mathrm{hr}$ ) (Group 1) and 11 age-sex-blood pressure-BMl matched IDDM subjects (3M/8F, age $33 \pm 8$ yrs, diabetes duration $13 \pm 8 \mathrm{yrs}$ ) with $\mathrm{Na} a+\mathrm{Li}+\mathrm{CT}$ activity $<0.260 \mathrm{mmol} / \mathrm{L} \quad \mathrm{RBC} / \mathrm{hr}(0.170 \pm 0.06 \mathrm{mmo} / \mathrm{L} \mathrm{RBC} / \mathrm{hr}$ ) (Group 2), were examined. M-mode and 2D rest and stress (with dipyridamole) echocardiograms with Doppler analysis were performed in all subjects. Erythrocyte $\mathrm{Na}+/ \mathrm{Li}+\mathrm{CT}$ activity (Canessa's method) and plasma lipids were measured. The left ventricular end diastolic dimension (LVEDD) ( $5.2 \pm 0.44$ vs $4.70 \pm 0.40 \mathrm{~cm} ; \mathrm{p}=0.019$ ), the left ventricular end diastolic volume (LVEDV) (131 $\pm 26.2 \mathrm{vs} 103 \pm 21.2 \mathrm{ml} ; \mathrm{p}=0.018$ ) and the stroke volume (SV) $(92.2 \pm 15.7 \mathrm{vs} 71.6 \pm 15.1 \mathrm{ml} ; \mathrm{p}=0.019)$ were significantly higher in Group 1 compared to Group 2. After the stress echo test with dipyridamole no significant difference was found in left ventricular wall motion index (LWWMM) between the two groups. Plasma level of HDL-cholesterol was lower in Group 1 ( $1.35 \pm 0.4$ vs $1.86 \pm 0.3 \mathrm{mmol} / \mathrm{L} ; p=0.008$ ). In all subjects $\mathrm{Na}+/ \mathrm{Li}+\mathrm{CT}$ activity was correlated to LVEDD ( $r=0.476, p=0.017)$ to LVEDV $(r=0.46, p=0.021)$ and to SV $(r=0.528, p=0.008)$. In stepwise multiple regression analysis with $\mathrm{Na} a+i \mathrm{~L}+\mathrm{CT}$ activity as dependent variable, LVEDD emerged as a significant and independen determinant of left ventricular function $(F=7.75 ; p=0.027)$. Our findings suggest that in normotensive, non-proteinuric IDDM patients increased $\mathrm{Na}+/ \mathrm{Li}+\mathrm{CT}$ activity is correlated to left ventricular dysfunction and lipids abnormalities.

\section{PS 58}

\section{Macroangiopathy Diagnosis and Treatment}

\section{0}

\section{MYOCARDIAL INFARCTION IN DIABETIC PATIENTS: PRESENTATION, RESIDUAL SYSTOLIC FUNCTION AND HEART FAILURE.}

P. Kaiser-Nielsen,P. Hildebrandt, M.Seibak, L Kober, C. Torp-Petersen and the TRACE Study Group. Department of cardiology, Frederiksberg Hospital, Denmark.

Morbidity and mortality from ischemic heart disease are increased in patients with diabetes mellitus, with an unknown underlying mechanism. In 5960 patients surviving the initial $2-6$ days of a myocardial infarction (MI), we studied pre-hospital history, clinical presentation and complications including heart failure (CHF). Residual systolic function expressed as left ventricular ejection fraction (LVEF), was measured by echocardiography. Methods. Analysis were made in patients without diabetes and 3 groups of patients with diabetes, according to preadmission history of diabetes treatment.

Results:

$\begin{array}{lllll} & \text { Non-diabetics } & \text { Diet } & \text { Oral } & \text { Insulin } \\ \text { Number } & 5367 & 173 & 319 & 125 \\ \text { Age (year) } & 66.5 & 66.8 & 70.3 & 66.0 \\ \text { \% female } & 30.3 & 35.3 & 37.4 & 49.0 \\ \text { History of MI (\%) } & 22.5 & 28.4 & 35.4 & 32.0 \\ \text { History of CHF (\%) } & 14.6 & 30.1 & 34.6 & 35.5 \\ \text { CHF at screening(\%) } & 49.1 & 68.2 & 64.0 & 70.4\end{array}$

By multivariate analysis, including age, sex, LVEF, history of hypertension and MI, diabetes was found to be an independent predictor of CHF. Conclusions: Regardless of type of diabetes treatment, patients with diabetes and $\mathrm{MI}$ are characterized by a larger prevalence of $\mathrm{CHF}$, not due to previous infarctions, and not explained by a more severe reduction in residual systolic function.

\section{1}

MORTALITY RATE AFTER MYOCARDIAL INFARCTION EQUALLY INCREASED IN DIABETIC PATIENTS TREATED WITH ORAL ANTI- DIABETIC AGENTS AND INSULIN. P. Hildebrandt, M. Seibæk, C. Torp-Pedersen, L. Kober and the TRACE Study Group. Department of cardiology, Frederiksberg Hospital, Denmark. Patients with diabetes have a poor prognosis after myocardial infarction (MI). However, the relation to different types of diabetic treatment, MI complications and residual systolic function is uncertain. The aim of the present study was a further evaluation of these relationships. Methods: 5960 Patients with confirmed MI, surviving 2-6 days after admission were included in the study. Pre-admission medical history, including type of diabetic treatment, complications during hospital stay and echocardiograpic evaluation of left ventricular wall motion index (WMI) were obtained, as well as 3 year mortality data.

Results: \% mortality

$\begin{array}{lrlll} & \text { Non-diabetics } & \text { Diet } & \text { Tablets } & \text { Insulin } \\ \text { Number } & 5367 & 173 & 319 & 125 \\ \text { 1 year } & 17,0 & 24,3 & 29,2 & 29,6 \\ 2 \text { year } & 23,2 & 34,3 & 42,8 & 41,7 \\ 3 \text { year } & 28,8 & 41,7 & 53,7 & 51,6\end{array}$

By multivariate analysis including age, sex, WMI, history of hypertension and previous MI, diabetes was found to be an independent predictor of mortality (odds ratio 1,488 ( $95 \% \mathrm{CI}: 1,221-1,814)$. Conclusion: patients with diabetes have a poor prognosis after $\mathrm{MI}$, with a significant increased mortality rate, regardless of other risk factors. This increased mortality is similar in patients treated with insulin and oral anti diabetics agents. 


\section{2}

SILENT MYOCARDIAL ISCHEMIA. IN NDDDM - CLINICAL IMPLICATIONS J.Leowski, J.Tatoń, Z.Szczeklik, A.Mamcarz, W.Braksator, J.Król, J.Kuch. Dpt of Internal Dis. and Diabetology, Dpt of Cardiology, Warsaw Medical School, Poland Both symptomatic and silent myocardial ischemia (SMI) appears more frequently and worsen prognosis in diabetics. The aim of the study was to asses the frequency of SMI in non-insulin dependent diabetic (NIDDM) patients and to evaluate prospectively its influence on exercise tolerance and left ventricular function. 67 males aged $54 \pm 0.8$ years with NIDDM (mean duration $6.3 \pm 0.6$ years) and 40 healthy male controls aged $52.8 \pm 1.0$ were studied. Patients with history of angina pectoris and rest ecg abnormalities indicative for coronary heart disease were excluded. Clinical examination, laboratory work-up, exercise treadmill test (ET), Holter ecg and echocardiography were performed initially and then after one year SMI diagnosis was based on typical ETT ischemic criteria (SMI type I according to Cohn). SMI was found in 21 out of $67(31 \%)$ diabetics and in 4 out of $40(10 \%)$ control subjects $(\mathrm{p}<0.01)$. In comparison with controls NIDDM patients demonstrated lower exercise tolerance $(8,98 \pm 0.22$ vs $9.93 \pm .31$ METS; $p<0.05)$ and inferior left ventricle diastolic function as determined by $E / A$ index $(0,86 \pm 0,02$ is $1.10 \pm 0.05 ; \mathrm{p}<0.05$ ). The major clinical predictors of SMI in diabetics were disease duration and insulin treatment. SMI appeared also somehow more frequently (but not significantly so) in diabetics with autonomic neuropathy. On initial examination there were no differences in exercise tolerance and left ventricle function between diabetics with SMI (SMI+) and without SMI (SMI-). When diabetics were reexamined after one year SMI+ (but not SMI-) patients showed deterioration in ETT duration time (464 vs $420 \mathrm{sec} ; \mathrm{p}<0.05$ ) and ETT workload (8.8 vs 7.9 METS; $p<0.05$ ). SMI had no influence on systolic and diastolic left ventricle function. The results of the study indicate that: 1) SMI appears 3 times more frequently in NIDDM patients, 2) diabetics with SMI show progressive deterioration of exercise tolerance, 3) SMI appears not to be a predictor of poor lefi ventricle diastolic function that is characteristic for NIDDM males without clinical coronary heart disease symptoms, 4) SMI detected in NIDDM patients is an indication for antiischemic therapy.

\section{3}

\section{ASYMPTOMATIC ISCHAEMIC HEART DISEASE IN PATIENTS} WITH NON-INSULIN-DEPENDENT DIABETIC

Rius F, Castells I, Murillo J, Pereferrer D, Luque T, Romero R, Sanmarti A Endocrinology, Nephrology, Cardiology and Nuclear Medicine Services. Hospital Germans Trias i Pujol, Badalona (Barcelona), Spain

Aim: To study the prevalence of asymptomatic ischaemic heart disease (IHD) in a group of patients with non-insulin-dependent diabetes mellitus (NIDDM). Patients and methods: Sixty-two NIDDM patients from our outpatient clinic were enrolled. The exclusion criteria were previous heart history and/or pathologic EKG register. A scintyghraphic thallium-dipiridamole test with physical exercise was performed with a bicycle ergometer. EKG monitoring was obtained throughout the test. All scintygraphic data were evaluated by the same physician. Familial heart disease history, smoking habit, body mass index, evolution and treatment of diabetes, chronic complications of diabetes, hypertension, analytical parameters of diabetic control, serum lipids and renal function were collected for all patients. Comparisons were made using Chisquare test and Mann-Whitney $U$ - test as appropriate.

Results: $41.9 \%$ (26) patients showed ischaemic scintygraphic images and $19.4 \%$ (12) had a pathologic EKG register during the exercise. Together, up to $48.4 \%(30 / 62)$ of the patients had findings suggesting ischaemic heart disease. No differences were observed in any clinical or analytical parameter between these patients and those with normal scintygragphy or EKG registers. However, it should be pointed out that the sample size of smokers (12) and proteinuric (8) patients was very small

Conclusions: A large proportion of our patients had scintygraphic or EKG changes suggesting asimptomatic IHD. There was no relation between asimptomatic IFD and the clinical and analytical factors considered Significance and prognosis of this findings are unknown and need further assessment.

\section{4}

ASYMPTOMATIC HEART DISEASE IN NEPHROPATHIC IDDM PATIENTSTHE USE OF DOBUTAMINE STRESS ECHOCARDIOGRAPHY MEGriffin,KNikookam, THennessy,MMTeh,HM MCann,NO'Meara \&RGFirth Depts of Diabetes \& Cardiology,Mater Misericordiae Hospital, Dublin.

Ischaemic Heart Disease (IHD) is the leading cause of death in IDDM. When nephropathy coexists with IDDM this risk increases dramatically. In these patients IHD may be asymptomatic and present with sudden death. Deciding on a screening test which is sensitive and specific is difficult. Therefore the aim of this study was to assess the frequency of asymptomatic IHD in a group of young patients (<45years) with IDDM and nephropathy using dobutamine stress echocardiography (DSE). DSE was performed in $18(13 \mathrm{M} / 5 \mathrm{~F})$ subjects with confirmed nephropathy (present $>5$ years). DSE is a newly available tool in diagnostic cardiology and detects wall motion changes secondary to ischaemia when the myocardial oxygen consumption is increased by dobutamine. In nondiabetic subjects it has a specificity and sensitivity of $85-95 \%$. Standard cardiac risk factors were also assessed. Results: Mean age was $37.8 \pm 3.4$ years, mean duration of IDDM was $23.7 \pm 1.2$ years and mean duration of nephropathy was $10.9 \pm 1.3$ years. 7 subjects had a positive DSE. $(6 \mathrm{M} / 1 \mathrm{~F}) .6$ of these patients subsequently underwent angiography. 1 patient had triple vessel disease, 1 patient had minimal coronary artery narrowing while 4 of 6 patients with positive DSE had negative coronary angiography. Conclusion: Though very useful in the investigation of IHD in non-diabetic subjects DSE was found, in this study, to be a non-specific investigative tool in the evaluation of asymptomatic IHD in subjects with IDDM and nephropathy. The high rate of false positive testing $(66 \%)$ may be due to intrinsic wall motion changes due to a diabetic cardiomyopathic process. DSE testing is not of benefit in the evaluation of the high risk patient with IDDM.

\section{5}

THALLIUM MYOCARDIAL SCINTIGRAPHY AND SILENT HEART ISCHEMIA : AN ATTEMPT TO EXPLAIN FALSE POSITIVE RESULTS. F. Paycha, P. Valensi, B. Buyukoglu, A. Ramadan, R,N Sachs and J.R Attali. Diabetology, Verdier Hosp, Bondy. Nuclear Medicine, Mourier Hosp, Colombes. France.

Assessment of the positive predictive value of thallium myocardial scintigraphy (TMS) for the detection of silent myocardial ischemia in diabetic patients has varied in the literature. The aim was to reexamine a new series of 132 consecutive patients (124 NIDDM and 8 IDDM), aged > 37 years, free of cardiac symptoms, with diabetes duration $=5-40$ years, 1 risk factor, and a normal 12-lead ECG. An exercise TMS or a dipyridamole TMS was performed. In 14 patients with a positive TMS (out of 20) and 10 with a litigious one (out of 17), a coronary angiography was performed and significant stenoses were found in 8 cases; the positive predictive value of TMS was thus $8 / 22=36.4 \%$. In the 14 other cases, the coronary angiogram was normal or subnormal and the false positive or litigious results of TMS corresponded to defects in mostly obese patients or to an anteroseptal defect in two nonobese subjects. An echocardiogram was performed in 10 of these 14 patients and revealed left ventricle hypertrophy in 2 cases. In the patients with a positive exercise-TMS scanning the specificity of the simultancous ECG recording for the detection of coronary stenoses was equal to $90 \%$. In conclusion 1) we confirm the low positive predictive value of TMS for the detection of silent coronary stenoses ; 2) left ventricle hypertrophy is not involved in the false positive result ; 3) most of the fixation defects result from attenuatior artefacts, but a reduction in coronary reserve may be involved ; 4) When the scintigraphy imaging is positive, the good specificity of the exercise ECG test coulc be useful for the indication of a coronary angiography. 
1086

ANGIOGRAPHICAL STUDY OF CORONARY ARTERY DISEASE IN TYPE \| DIABETES MELLITUS.

A. Melidonis, B.D. Dimopoulos, St. Garoufallis, A. Tsatsoulis, D. Goudevenos, S. Foussas.

Cardiology Clinic and Diabetology Department of Tzanio Hospital, Piraeus, Greece

Diabetes mellitus (DM) causes coronary artery disease (CAD) of severe form. However it is not yet well known the effect of D.M. in the distribution of CAD, in the developement of collateral circulation and in the differantiation of the lumen diameter at the coronary arteries as well For we retrospectively evaluated 463 diabetic patients with $C A D$ (pts) (Group A) and compared them with 210 pts with CAD but without D.M. (Group B). Both groups were similar as regards the age, gender and the rest of risk factors.

RESULTS: Group A suffers more often of multivessel (3A) disease $(47.1 \%$ vs $27.6 \%{ }^{* \star}$ ) and of worse function of the left ventricle (IV) as well (EF: $44.6 \%$ vs $53.8 \%{ }^{*}$ ) Men diabelics suffer more of severe form of CAD compared with women diabetics of same age $\left(37.3 \%\right.$ vs $21.7 \%{ }^{*}$ ). Among women, CAD of same severity appears in diabetics earller (47.8 6 vs $51,3 \pm 5+$ ). Right coronary artery (RCA) is the vessel more often damaged in diabetics ( $73.8 \%$ vs $56.6 \%{ }^{*}$ ). When or 2 vessel disease (VD) no differences were found as regards the segmenta effect of CAD, though in 3 V.D. the distal segment of left anterior descending (LAD). Suffers more often in diabetics. Total occlusions are more often in Group $A$ in the proximal segment of RCA and of $L A D$ in men $<55$ years.

Collateral circulation with full tilling of epicardial artery (RENTROP lii) is more often in Group A (17.3\% vs $3.7 \%$ ) though there is no effect in the diameter of the vessel lumen. This D.M. causes severe form of CAD diminishes the function of the L.V. attacts more the RCA and causes more occlusions in proximal RCA and $L A D$. Collateral circulation is better developed. while the vessel lumen is not influenced.

$+p<0.05, \quad p<0.01, * 0<0.001$

\section{8}

CAROTID INTIMA-MEDIA THICKNESS IN HYPERLIPIDEMIC TYPE 1 AND TYPE 2 DIABETIC PATIENTS

S.D.J.M. Kanters, A. Algra* and J.D. Banga; Dept of Internal Medicine and

*Dept. of Clinical Epidemiology, University Hospital Utrecht, The Netherlands

The first clinical manifestations of cardiovascular disease often arise in a stage of well-advanced atherosclerosis. Ultrasonographic measurements of the combined thickness of the carotid intima and media can be used to examine early vessel wall changes, in subjects at high risk for vascular disease. Intimamedia thickness (IMT) was quantified in type 1 and type 2 diabetic patients with hyperlipidemia and relationships between various risk factors and IMT were investigated. Thirty-one patients with type 1 and 56 with type 2 diabetes were examined. They were all included in a pilot study of intensive lipid lowering treatment. Inclusion-criteria for this study were a plasma level of LDLcholesterol of $>2.6 \mathrm{mmol} / \mathrm{l}$ and/or triglycerides of $>1.7 \mathrm{mmol} / \mathrm{l}$ and/or $\mathrm{HDL}$ cholesterol of $<0.9 \mathrm{mmol} / \mathrm{l}$ for men or $<1.1 \mathrm{mmol} / \mathrm{l}$ for women. IMT was measured on-line, over a length of $1 \mathrm{~cm}$ in the common carotid artery. Lateron, IMT for each subject was calculated from the average or the maximum of six measurements (left and right side, both in three directions). Mean IMT was $0.63 \pm 0.18$ ( $\pm \mathrm{SD}$ ) $\mathrm{mm}$ for type 1 diabetes and $0.80 \pm 0.31 \mathrm{~mm}$ for type 2 diabetes. The $95 \%$ confidence interval for this difference of $0.17 \mathrm{~mm}$ was 0.05 $-0.29 \mathrm{~mm}$. Adjusted for the difference in age between type 1 and type 2 diabetic patients the difference was $0.06 \mathrm{~mm}$, with a $95 \%$ confidence interval of $-0.08-0.20 \mathrm{~mm}$. In multivariate regression analysis, age over 50 years and higher $\mathrm{HbA}_{1 \mathrm{c}}$ were independently associated with an increase in mean IMT in type 1 diabetes. In type 2 diabetes none of the variables reached a significance level of 0.10 or less. Results for maximum IMT were essentially the same. In conclusion, mean and maximum values of carotid IMT are larger in type 2 diabetes than in type 1 diabetes. This can partly be explained by the difference in age between both groups. Age over 50 years and $\mathrm{HbA}_{1 \mathrm{c}}$ are strong independent determinants for carotid wall thickening in type 1 diabetic patients.
1087

\section{CORONARY FLOW RESERVE IS REDUCED IN YOUNG ADULT IDDM PATIENTS}

O.-P. Pitkänen, P. Nuutila, O.T. Raitakari, H. Laine, T. Takala, H. lida, J. Vikari, T. Rönnemaa and J. Knuuti. Turku, Finland and Akita, Japan.

The prevalence and incidence of coronary heart disease (CHD) are increased in IDDM. To investigate whether coronary vascular reactivity is impaired in young IDDM patients without diabetic complications myocardial blood flow and flow reserve were measured using positron emission tomography and ${ }^{15} \mathrm{O}$-labelled water. We studied 12 nonsmoking male IDDM subjects (age $30.1 \pm 6.6 \mathrm{y}$ ) and 12 healthy matched volunteers (CTRL). Subjects were similar with respect to blood pressure and serum lipid concentrations (Total, LDL- and HDLcholesterol, trigycerides, Apo $A-1, A p o B$ and $L p(a)$ ) (ns. IDDM vs. CTRL). IDDM subjects were without microvascular complications and had normal exercise echocardiography. Myocardial blood flow was measured during euglycemic hyperinsulinemia (insulin infusion $1 \mathrm{mU} / \mathrm{kg} / \mathrm{min}$ ) at baseline and after dipyridamole infusion. The baseline myocardial blood flow was similar in both groups $(0.88 \pm 0.25$ vs. $0.84 \pm 0.18 \mathrm{~mL} \cdot \mathrm{g}^{1} \cdot \mathrm{min}^{1}$, CTRL vs. IDDM, ns.). The flow during hyperemia was lower in IDDM $\left(3.2 \pm 1.6\right.$ vs. $\left.4.5 \pm 1.4 \mathrm{~mL} \cdot \mathrm{g}^{-1} \cdot \mathrm{min}^{-1}, \mathrm{p}=0.04\right)$. Consequently, coronary flow reserve (the ratio of flow during hyperemia and at baseline) was lower in IDDM than in CTRL $(3.76 \pm 1.69$ vs. $5.31 \pm 1.86, p=0.04)$ and the total coronary resistance during hyperemia was higher in IDDM $\left(53.7 \pm 31.5\right.$ vs. $31.4 \pm 11.6 \mathrm{mmHg} \cdot \mathrm{min} \cdot \mathrm{g} \cdot \mathrm{mL}^{-1}$ $p=0.03$ ). In conclusion, coronary vascular reactivity is impaired in IDDM without microvascular complications. This abnormality can not be explained by other known risk factors for CHD. The results imply early impairment of coronary vasomotion in IDDM patients which may represent an early precursor of future $\mathrm{CHD}$.
1089

EARLY DETECTION OF CAROTID AND PERIPHERAL VASCULAR DISEASE BY COLOR DOPPLER IN 52 DIABETIC PATIENTS

M.H. Bouin-Pineau*, F. Carrié, T. Kharitonnoff, F. Torremocha, A. Muller. D. Herpin**, T. Rosenberg*, F. Bégon*, R. Maréchaud. Dept of Diabetology, *Dept of Nuclear Medicine, and **Dept of Cardiology, CHU La Milétrie, Poitiers, France.

The purpose of this prospective study was to evaluate the frequency of asymptomatic carotid and peripheral atherosclerosis in diabetic patients aged from 41 to 65 years old, without clinical signs of coronary artery disease. 15 insulindependent diabetes mellitus (IDDM) patients and 37 non-insulin-dependent diabetes mellitus patients, 32 women and 20 males, with a mean age of 54 years, were studied. Duration of disease was of 10 years or more in IDDM subjects. Every patient had one or more cardiovascular risk factors associated with diabetes mellitus. Questionnaire, physical examination and resting electrocardiogram were negative for macrovascular disease. Ultrasonography study of carotid arteries, abdominal aorta and lower limbs arteries was done by the same operator with a color doppler (Acuson 128XP 10). Asymptomatic carotid stenosis of $601075 \%$ was found in two patients. A successfull endarterectomy was performed in both cases 6 months later because of progression of stenosis to $80 \%$. A stenosis lower than $60 \%$ was found in 6 patients. Intimal-medial thickness was $1 \mathrm{~mm}$ or more in 8 cases, including 6 patients without carotid stenosis. Ultrasonography of the lower limbs demonstrated ilio-femoral stenosis of $50 \%$ in 4 patients, distal stenosis in 7 patients, and atheromatous deposits without stenosis in 10 other patients. Ultrasonography examination was normal in 31 patients. Coexistence of mediacalcinosis has been shown in 20 patients. Abdominal aortic ultrasonography showed some atheromatous deposits without hemodynamic repercussion in 14 cases. In this study, an asymptomatic carotid and/or peripheral macroangiopathy from mild to more severe importance was present in $54 \%$ of the cases. These data suggest that non invasive detection of asymptomatic extra-coronary macroangiopathy is justified in diabetic patients older than 40 if diabetes mellitus coexists with other cardiovascular risk factors. Therefore, an appropriate follow-up should be scheduled and the opportunity of revascularisation surgery, especially for carotid arteries, should be considered. 
1090

ARTERIAL COMPLIANCE IN IDDM - AN INDICATOR AND A PREDICTOR OF CARDIOVASCULAR DISEASE ?

O. Snorgaard, J. Faber, N. Winberg and J. Mehlsen. Department of Endocrinology and Clinical Physiology, Frederiksberg Hospital, Denmark.

Arterial compliance is an estimate of the structural and functional components of the arterial wall. We studied arterial compliance in the upper arm and the calf in 67 consecutive IDDM patients with a noninvasive volume-oscillometric method. Compliance $(\mu \mathrm{l} / \mathrm{mmHg} / 10 \mathrm{~cm})$ was measured at a transmural pressure of zero (C0) and $100 \mathrm{mmHg}$ (C100), normal values ${ }_{\text {calf }} 20 \pm 7$ and $5.9 \pm 2.4$. The mean age of the 38 male and 29 female subjects were $41 \pm 12$ yrs, with a disease duration of $16 \pm 12$ yrs. Compliance estimates from the calf were inversely correlated to age and disease duration. However, in a multiple regression analysis age was eliminated from the model, leaving disease duration to account for $21 \%\left(\mathrm{R}^{2}\right)$ of the variation in $\mathrm{CO}_{\text {calf }}(\mathrm{p}<0.0001)$ and $24 \%$ of the variation in $\mathrm{C} 100_{\text {calf }}(\mathrm{p}<0.005)$. HbA1c, blood glucose and blood pressure (arm and calf) were of no or minor $(\leq 10 \%)$ importance. $\mathrm{CO}_{\text {calf }}$ and $\mathrm{C} 100_{\text {calf }}$ were lower with the severity of retinopathy ( $p=0.11$ and $p<0.05$ (one-way ANOVA) ) and the severity of renal disease $(\mathrm{p}<0.01$ and $\mathrm{p}<0.005) . \mathrm{C}_{\text {cadf }}$ was $21.0 \pm 5.8$ in subjects without signs of cardiovascular disease against $11.2 \pm 3.1$ in subjects with cardiovascular disease $(\mathrm{N}=7), \mathrm{p}<0.0001$. The respective $\mathrm{C} 100_{\text {calt }}$ values were $7.4 \pm 2.1$ against $3.8 \pm 1.2, \mathrm{p}<0.0001$. A lower compliance in the upper arm tended to be related to only nefropathy and the use of antihypertensive drugs. We conclude, that arterial compliance of the calf is an indicator and probably a predictor of arterial complication in IDDM.

\section{1}

STRUCTURAL AND FUNCTIONAL ALTERATIONS OF RADIAL AND CAROTID ARTERIES IN UNCOMPLICATED IDDM.

F. Paleari, PL. Gamba, M. Failla, AA. Mangoni, S. Carugo, M. Pozzi, D. Maglia, G. Marelli, G. Torchio, A. Piperno, C. Giannattasio and G. Mancia. Div.ne di Medicina Generale 1, Ospedale S. Gerardo, Monza - Università degli Studi di Milano; Istituto di Fisiologia Clinica ed Ipertensione, Centro Auxologico Italiano, Milano, Italy.

IDDM is frequently associated with circulatory abnormalities. Whether IDDM alters medium and large arteries structure and function at an early stage is unknown. We evaluated carotid and radial artery compliance $(\mathrm{C})$ and thickness $(\mathrm{T})$ in 50 normotensive patients $(\mathrm{P}$, age $=$ $34 \pm 1$ ys, mean \pm SE), affected by IDDM since $14 \pm 1$ ys, insulintreated $(42 \pm 1 \mathrm{U} / \mathrm{die})$ and in fair-to-good metabolic control (mean blood glucose $=141 \pm 9 \mathrm{mg} / \mathrm{dl} ; \mathrm{HbAlc}=8 \pm 0.2 \%$ ). 50 healthy sexage- and BMI-matched subjects served as controls. No $P$ had clinical macroangiopathy, retinopathy or microalbuminuria. Carotid $(\mathrm{Ca})$ and radial artery $(\mathrm{Ra})$ diameters were evaluated by echotracking devices while blood pressure (BP) was simultaneously registered by a Finapres and Dinamap device. $\mathrm{CaC}$ was obtained by Reneman formula and $\mathrm{RaC}$ by Langewouters formula. CaT was measured by M-mode echodoppler while RaT by the echotracking device. BP was similar in the two groups. In IDDM however $\mathrm{CaC}$ and $\mathrm{RaC}$ were significantly less then in controls $(-23 \%$ and $-15 \%$ respectively, $\mathrm{p}<0.01)$; conversely $\mathrm{CaT}$ and RaT were both greater in IDDM than in controls $(+32 \%$ and $+90 \%$ respectively, $\mathrm{p}<0.001$ ). Thus IDDM is accompanied by clearcut functional and structural alterations of medium and large size arteries. These alterations can be detected in absence of BP increase and clinical diabetes-related complications.

\section{2}

TWO NOVEL METHODS USING PULSE OXIMETRY FOR MEASUREMENT OF ARTERIAL TOE PRESSURE

P Samuelsson, G Blohmé, J Fowelin and J W Eriksson, Diabetes Unit, Dept of Medicine, Sahlgrenska University Hospital, S-413 45 Göteborg, Sweden

Occlusive arterial disease in the lower extremities is a common and serious problem in diabetes patients. Systolic ankle pressures are often misleading due to medial sclerosis, and arterial toe pressure (ATP) assessed with the strain gauge technique is a cumbersome method. We evaluated a novel, simple, noninvasive approach to assess ATP by monitoring pulsatile toe blood flow with a regular pulse uximeter (POX). It was empolyed in 70 subjects, of which 44 had suspected or established lower extremity arterial disease (LEAD) and 26 did not. 55 of the subjects had diabetes and 15 were non-diabetic. The POX sensor was placed on the tip of the great toe. A small blood pressure cuff was placed around the proximal part of the toe and connected to a sphygmomanometer. Systolic pressure was estimated as the cuff pressure at which pulsatile blood flow ceased during cuff inflation. In parallell, ATP was obtained using the established strain gauge (SG) plethysmographic technique. There was a good concordance between the two methods (linear regression: $\mathrm{r}=0.93$, $\left.\operatorname{ATP}_{\mathrm{POX}}=1.1^{*} \mathrm{ATP}_{S \mathrm{SG}}-6.4(\mathrm{mmHg}), \mathrm{p}<0.01\right)$. The POX method was reproducible and $\mathrm{CV}$ for duplicate measurements was $3.7 \%$. However, patients with systolic toe pressures $<20 \mathrm{~mm} \mathrm{Hg}$ could not be reliably assessed with this method. We also evaluated a modified so-called pole test to estimate ATP in patients with threatening/critical ischemia. The POX sensor but no cuff was used. The leg of the supine patient was gradually elevated, and the vertical distance above the heart level, where pulse signals ceased, was used as an estimation of ATP (cm divided by 1.3 to adjust for the densities of blood and $\mathrm{Hg})$. This method also correlated fairly well with $\mathrm{ATP}_{\mathrm{SG}}(\mathrm{r}=0.69, \mathrm{p}<0.05)$. Conclusion: The two novel methods employing pulse oximetry for the measurement of ATP seem promising for rapid, simple, cost-effective and reliable estimation of the functional degree of LEAD in diabetes patients.

\section{3}

CYCLIC GMP PRODUCTION IN CAROTID ARTERIES OF DIABETIC RATS. CHRONIC EFFECTS OF AMINOGUANIDINE

F.R.L. Crijns*, J.G.R. De Mey, P.M.H. Schiffers, G.M.J. Janssen, H.A.J. Struijker Boudier and B.H.R. Wolffenbuttel". Depts. of "Internal Medicine and Pharmacology, Univ. of Limburg, Maastricht, The Netherlands.

Impaired endothelium-dependent and endothelium-independent relaxation may play a role in the development of vascular complications in diabetes mellitus. In experimental diabetes, aminoguanidine treatment prevents or reduces several of these complications. We investigated basal and stimulated cyclic GMP (cGMP) production in intact and denuded carotid arteries of control and diabetic rats, with or without aminoguanidine (AG) treatment. Diabetes was induced in Wistar Rp rats by streptozotocin (STZ; D, $n=6$ ). Control rats $(C, n=7)$ received vehicle only. AG was injected daily $(50 \mathrm{mg} / \mathrm{kg}$ s.c.) (CAG, $n=7$; DAG, $n=8$ ). Three months later, both carotid arteries were isolated from each animal. One artery was mechanically denuded of endothelium. Each vessel was divided into three segments, which were pre-incubated for $60 \mathrm{~min}$ in modified Krebs-Henseleit buffer, after which they were exposed for $10 \mathrm{~min}$ to 3-isobutyl-1-methylxanthine with or without further addition of $10^{-5} \mathrm{M}$ acetylcholine (ACh) or $10^{-6} \mathrm{M}$ sodium-nitroprusside (SNP) during the last $2 \mathrm{~min}$. The vessel segments were then processed for determination of cGMP (RIA) and protein content. Results are expressed as mean cGMP per protein content $\pm \mathrm{SE}$. In carotid arteries with endothelium, basal cGMP was comparable in all groups and higher than in denuded preparations. ACh caused a marked increase of cGMP in the intact segments, that tended to be smaller in D than in C (139 $16 \mathrm{fmol} / \mu \mathrm{g}$ vs $222 \pm 76 ; p=0.34)$; SNP stimulated cGMP production comparably in all groups. In denuded arteries, however, SNP elevated cGMP content to a lesser extent in D and CAG than in C $(91 \pm 32$ $\mathrm{fmol} / \mathrm{ug}$ and $98 \pm 25$ vs $283 \pm 58 ; \mathrm{p}<0.05$ ). AG had no effect on cGMP production in diabetic rats. These results suggest that cGMP production is shifted from arterial smooth muscle to the endothelium in diabetic rats. AG does not prevent this. but rather induces the same disturbance in control animals. 


\section{4}

AN ALDOSE REDUCTASE INHIBITOR PREVENTS GALACTOSEINDUCED MACROANGIOPATHY IN BEAGLE DOGS

N. Hotta 1), Y. Kasuya ${ }^{1)}$, N. Koh ${ }^{1)}$, F. Sakakibara ${ }^{1)}$, J. Nakamura ${ }^{1)}$, Y. Hamada1), T. Hara 1 ), H. Sasaki 1), E. Nakashima1), K. Naruse ${ }^{1}$ ) K. Kato and M. Itoh, Nagoya 1 ), Japan.

Increasedpolyol pathway activity has been invoked in the pathogenesis of diabetic microangiopathy including retinopathy, nephropathy and neuropathy. However, the relationship between polyol pathway hyperactivity and diabetic macroangiopathy has not been studied well. In the present study, the effects of galactose feeding and an aldose reductase inhibitor (ARI), epalrestat on morphological changes in coronary artery of beagle dogs. The dogs were divided into four groups as follows: (a) normal control group, (b) $30 \%$ galactose-fed group, (c) $30 \%$ galactose + low dose ARI (20 $\mathrm{mg} / \mathrm{kg} /$ day), (d) $30 \%$ galactose + high dose ARI ( $50 \mathrm{mg} / \mathrm{kg} /$ day) After 44 months feeding, dog hearts were transversely dissected at the bifurcation of coronary artery and stained with Elastica Wangison for light microscopic observations. Intimal area ratio (intimal area/ intimal area + medial area: IA) and maximal intimal thickness ratio (MIT) were measured as the indicators of atherosclerosis by Imaging System (Compix Inc., USA). Galactitol content in red blood cell (RBC) ( $\mu \mathrm{g} / \mathrm{gHb}$ ) was also measured by gas chromatography. Results are as follows ${ }^{*},{ }^{* *} p<0.05$ vs Control and Galactose, respectively):

\begin{tabular}{lllc}
\hline & \multicolumn{1}{c}{ IA (\%) } & MIT (\%) & RBC galactitol \\
\hline Control & $29.9 \pm 2.2$ & $35.6 \pm 2.2$ & ND \\
Galactose & $40.9 \pm 1.5^{*}$ & $47.0 \pm 1.6^{*}$ & $470 \pm 46$ \\
Galactose + low ARI & $35.9 \pm 1.4$ & $43.3 \pm 1.0^{* *}$ & $387 \pm 25$ \\
Galactose + high ARI & $32.9 \pm 1.0^{* *}$ & $38.5 \pm 1.0^{* *}$ & $293 \pm 30^{* *}$ \\
\hline
\end{tabular}

These observations suggest that increased polyol pathway activity is one of the pathogenic factors of diabetic macroangiopathy.

\section{PS 59}

\section{Hypertension}

\section{5}

BLOOD PRESSURE CONTROL IN A NON INSULIN-DEPENDENT DIABETIC POPULATION: PRIMARY CARE VS. DIABETES CLINIC

F.J. Martínez, P.L. de Pablos, V. Sánchez, C. Santana, I. Garuîa, M. P. Martínez and A. Macías, Endocrinology Dpt, Hospital N. S. del Pino, Las Palmas, Spain.

In order to compare the degree of blood-pressure control in a population of NIDDM hypertensive patients who were attended only by their family physicians with those referred to our diabetes clinic, we have estimated cross-sectionally the prevalence of hypertension (HBP) and diabetic nephropathy (DN), and obtained the actual values of BP in a group of NIDDM patients representative of the Gran Canaria population. Clinical, anthropometric and metabolic data were obtained from 205 patients $(56 \%$ women, age $61.5 \pm 8.2$ ) with NIDDM in the primary care setting (PC), and 153 patients (46\% women, age $56.5 \pm 10.2$ ) with NIDDM in the diabetes clinic (DC). In PC, $71 \%$ of the patients were hypertensives, of which $79 \%$ were not sufficiently controlled; $76 \%$ maintained systolic BP $>135$ and $39 \%$ diastolic $\mathrm{BP}>85.39 .5 \%$ of them did not receive antihypertensive drugs $(87 \%$ of them not controlled), and $60.5 \%$ received diverse drugs, most often ACE inhibitors followed by calcium chamel blockers ( $72 \%$ not controlled). In the DC, $72 \%$ were hypertensives (pNS vs. $\mathrm{PC}$ ), of which $61 \%$ were not sufficiently controlled ( $\mathrm{p}<0.05$ vs. PC), with $58 \%$ of uncontrolled systolic BP and $31 \%$ of uncontrolled diastolic BP. Only $11 \%$ did not receive antihypertensive drugs ( $\mathrm{P}<0.01 \mathrm{vs}$. $\mathrm{PC}$ ), but the treatment distribution was similar. In PC. $13 \%$ of the patients had normal weight, $37 \%$ were overweight and $50 \%$ were obese; in DC they were respectively $26 \%, 40 \%$ and $34 \%(p<0.01$ vs. PC). In PC, $69 \%$ were normoalbuminuric, $27.4 \%$ microalbuminuric and $3.6 \%$ macrodbuminuric; in DC they were respectively $57 \%, 22 \%$ and $21 \%(\mathrm{p}<0.001 \mathrm{vs}$. PC). Plasma creatinine and $\mathrm{HbA}_{1 \mathrm{C}}$ were in $\mathrm{PC} 97.1 \pm 43.8 \mathrm{mM} / \mathrm{L}$ and $7.7 \pm 1.9 \%$; in DC were $91.9 \pm 74.5 \mathrm{mM} / \mathrm{L}$ and $7.6 \pm 1.8 \%(\mathrm{pNS})$. In conclusion, hypertensive DMNID in DC had a better BP control than in PC, though both were clearly unsatisfactory; in DC the use of drugs was more frequent, though the types used were similar; the patients were younger and less obese, with similar glycemic control and more advanced DN. Detection of DN appears to be a main motive of referral to DC.

\section{6}

HYPERTENSION IN BLACK AFRICAN DIABETICS

M. Ducorps, B. Bauduceau, J.M. Poirier. E. Cosson, G. Belmejdoub and $H$. Mayaudon. Hôpital d"Instruction des Armées Bégin, 94160 Saint Mandé. France.

It has been established that hypertension prevalence was higher in american Blacks than Whites or Mexicans. In other respect hypertension is more frequent in diabetics. The present study reports the frequence of hypertension in a black african population of 550 diabetic patients attending to Central Hospital of Yaounde (Cameroon) who were screened for hypertension according to the 1993 WHO criteria (systolic blood pressure (SBP) $\geq 140$ or diastolic blood pressure (DBP) $\geq 90 \mathrm{~mm} \mathrm{Hg}$ ). Patients being previously treated with anthypertensive drug have also been considered to have hypertension. For the whole study population, characteristics were : age (inclusion) $54.2 \pm 12.8$ yrs: 341 men and 209 women (sex ratio 1.63:1); duration of diabetes $5.7 \pm 5.6 \mathrm{yrs}$; Body Mass Index (BMI) $24.4 \pm 4.8 \mathrm{~kg} / \mathrm{m}^{2}$; classification 136 IDDM, 405 NIDDM and 9 other types. In normotensive patients, blood pressure levels were : SBP $117 \pm 11$ and DBP $75 \pm 8 \mathrm{~mm} \mathrm{Hg}$ while in hypertensive : SBP $156 \pm 23$ and DBP $95 \pm 13 \mathrm{~mm} \mathrm{Hg}$. Characteristics of hypertensive group were : age $57 \pm 11.2 \mathrm{yrs}, 229$ men and 136 women (sex ratio 1.68:1), BMI $24.9 \pm 4.8 \mathrm{~kg} / \mathrm{m}^{2}, 63$ IDDM, 297 NIDDM and 5 other types. According to recent WHO criteria (140/90) 365 subjects 1550 were found hypertensive, thus overall prevalence of hypertension was $66.4 \%$ in the study population. Using former definition (160/95) the prevalence was $42.2 \%$. No significant difference was found for hypertension prevalence between male $(67.2 \%)$ and female $(65.1 \%)$ but difference is significant between IDDM (46.3\%) and NIDDM (73.3\%) (p<0.001). Hypertension prevalence studies in Aftica have shown varying results (2.5$30 \%$ ), with higher rates in urban than rural population. In african studies hypertension prevalence rates in diabetes were reported in the range 13$44 \%$. The result of the present study is very near the high limit of known data in Africa. Such a prevalence rate of $66.4 \%$ in this cameroonian diabetic population appears to be high, compared to previous westem or african studies. These considerations bring to light the question of sensitiveness to hypertension of african Blacks when exposed to a number of risk factors as overfeeding, salt or diabetes. 


\section{7}

AMBULATORY BLOOD PRESSURE MEASUREMENT IN RELATION TO CLINIC BASED MEASUREMENT IN DIABETIC PATIENTS

E. Ebbehæj, K.W. Hansen, P.L. Poulsen and C.E. Mogensen. Medical Department M, Diabetes \& Endocrinology, Aarhus Kommunehospital, DK-8000 Aarhus C, Denmark

Aim: To quantify differences between clinic and ambulatory blood pressure (AMBP) recordings in diabetic patients with suspected hypertension, and to telate the clinicAMBP differences to renal function, gender, age and antihypertensive treatment. Methods and Patients: If elevated blood pressure (BP) was suspected from three measurements obtained in the usual out-patient clinical setting, patients were referred to AMBP monitoring. AMBP was measured using Spacelab apparatus (model 90202) considering day-time values. We studied 67 consecutively referred patients 43 males, 24 females). 41 had insulin dependent diabetes, 26 non-insulin dependen diabetes. The mean age for the whole population was 46.9 years (21.8-71.8 years), 27 patients received antihypertensive treatment. Thirty-two patients were normoalbuminuric (albumin/creatinine ratio below $2.5 \mathrm{mg} / \mathrm{mmol}$ for males and below 3.5 for females), 35 patients had microalbuminuria or nephropathy. Among the 32 normoalbuminuric patients 10 received antihypertensiva prior to the AMBP recording. Results: BP measurements recorded in the clinic was higher compared with day-time AMBP: for the systolic BP in average $16.0 \mathrm{mmHg}$ ( 5 and $95 \%$ percentiles: -12.6 and $46.1 \mathrm{mmHg}$ ) and for the diastolic $\mathrm{BP}$ in average $8.7 \mathrm{mmHg}$ ( 5 and $95 \%$ percentiles: -11.0 and $29.2 \mathrm{mmHg}$ ). The differences between the BP measurements were not related to the level of clinic BP recordings, gender, age, diabetes duration, type of diabetes, UAE, or to antihypertensive treatment. In consequence of the AMBP measurement only 4 patients among 32 normoalbuminuric patients started antihypertensive treatment, in another 5 patients ongoing treatment was intensified. In conclusion: 1) The difference between clinic and ambulatory day-time BP measurements in diabetic patients are in accordance with other studies of non-diabetics. 2) The difference between clinic and AMBP recording varies considerably among patients and is not predictable. 3) AMBP recording may be relevant especially with high clinic $\mathrm{BP}$ recordings in normoalbuminuric diabetic patients, since unnecessary trearment may be avoided.

\section{9}

GLUT1 GLUCOSE TRANSPORTER GENE IN NIDDM: ASSOCIATION TO DIASTOUC HYPERTENSION IN A MEDITERRANEAN POPULATION

Gutierrez C, Broch M, Llor C, Pastor R, Vendrell J, Simón I, Richart C. Endocrinology Unit and Internal Medicine Department. Hospital Universitari Joan XXIII. Tarragona. Spain.

INTRODUCTION: HepG2 erythrocyte glucose transporter (GLUT1) is a member of the facilitative glucose transporter family. The association between the GLUT1 gene and Non-Insulin-Dependent Diabetes Mellitus (NIDDM) has been studied in several groups being the results still inconclusive. We have analyzed a NIDDM diabetic mediterranean population in order to evaluate the role of this glucose transporter in the susceptibility to diabetes and its long-term complications. MATERIAL AND METHODS: 194 NIDDM patients aged $59.5 \pm 10.2$ and 90 healthy subjects aged $45.4 \pm 12.6$ were included. Plasma cholesterol, $\mathrm{cHDL}$, triglycerides, glycated haemoglobin $(\mathrm{HbA1c})$ and microalbuminuria were quantified. DNA was studied through PCR amplification of the human GLUT1 gene detected by Xbal restriction enzyme polymorphism. RESULTS: We did not find any statistical significant difference neither in genotypic nor allelic distributions between controls and NIDDM patients (X1: $40.5 \%$ vs $38.6 \%$ and $X 2$ : $59.5 \%$ vs $61.4 \%$, respectively). We have found a significant association between the $\mathrm{X} 1 \mathrm{X} 1$ genotype and high diastolic blood pressure in diabetic patients $(p<0.01)$. There were not any association between retinopathy, microalbuminuria and lipidic profile and this genotype. CONCLUSIONS: The finding of an association between GLUT1 transporter and high diastolic blood pressure suggests that arterial hypertension in NIDDM patients could be partially genetically determined.

\section{8}

RENIN-ANGIOTENSIN SYSTEM GENES AND LONG-TERM COMPUCATIONS IN NIDDM PATIENTS

Richart C, Aguilar C, Broch M, Gutiérrez C, Llor C, Vendrell J.

Endocrinology Unit and Internal Medicine Department. Hospital Universitari Joan XXIII. Tarragona. Spain.

INTRODUCTION: Non-Insulin-Dependent Diabetes Mellitus (NIDDM) is a disease frequently associated to long-term complications such as retinopathy and nephropathy. Microalbuminuria, a first step in nephropathy, is generally associated to arterial hypertension. Angiotensin I Converting Enzyme (ACE) and Angiotensinogen (AGT) genes have been implicated as candidate genes of high blood pressure. We have analyzed whether the ACE and AGT genotypes could be associated to long-term complications in a NIDDM population. MATERIAL AND METHODS: 194 NIDDM patients aged $59.5 \pm 10.2$ and 90 healthy subjects aged $45.4 \pm 12.6$ were included. A routine biochemistry analysis including lipidic profile, glycated haemoglobin, microalbuminuria and evaluation of retinopathy was performed. DNA was studied through a PCR reaction at the ACE gene and at two variants of the AGT gene, AGT M235T and T174M, using Aspl or Ncol restriction enzymes respectively for the former genes. RESULTS: We did not find any statistically significant differences neither in the genetic or allelic distributions between controls and diabetic patients (ACE gene: I allele $42.2 \%$ vs $39.4 \%$ and $D$ allele $57.8 \%$ vs $60.6 \%$, respectively; and AGT gene M235T variant: T allele $55.6 \%$ vs $57.9 \%$, C allele $44.4 \%$ vs $42.1 \%$ and $\mathrm{T} 174 \mathrm{M}$ variant: T allele $14.2 \%$ vs $12.2 \%$, C allele $85.8 \%$ vs $87.8 \%$, respectively). There were not any differences in the NIDDM group when we studied the presence of hypertension, retinopathy or microalbuminuria according to ACE or AGT genotypes. CONCLUSIONS: Neither the ACE nor the AGT genotypes were associated to hypertension nor to any of the long-term complications of NIDDM in our population.

\section{0}

'THE INFLUENCE OF HYPERTENSION ON PROOXIDANT - ANTIOXIDANT IMBALANCE IN PATIENTS WTTH TYPE I DIAPETES MELJTIUS

B.Lacka, W.Grzeszczak, K.Strojek, K.Twardowska, J.Froehlich

Department of Internal and Occupational Diseases, Silesian School of Medicine, Zabrze, Poland

Pro-oxidant - antioxidant imbalance has been found in diabetes mellitus. The influence of hypertension on free radical generation and antioxidant activity in diabetic patients has been studied very scarcely. The aim of the study was to assess the influence of hypertension $(\mathrm{HT})$ on pro-oxidant and antioxidant activity in type 1 diatbetic patients. Superoxide mion $\left(\mathrm{O}_{2}{ }^{-1}\right)$ generation by polvmorphonuclear leukocytes plasme hydroperoxide (HPO) level and malondialdehyde (MDA) concentration, erythrocyte activities of superoxide dismutase (CwZn-SOD), ghtathione reductase (CSSG-Red) were assessed in 16 patients with IDDM and uncontrolled HT (mean age $36.4 \pm 3.7$ years, duration of diabetes $7.4 \pm 2.4$ years, HbAlc $7.7 \pm 0.2 \%$ UAE $30 \mathrm{mg} / 24$ hours) in comparison with 18 normotensive patients with DDM (mean age $34.4 \pm 2.9$ years. duration of diabetes $7.9 \pm 2.7$ years, $\mathrm{HbAlc} 7.4 \pm 0.3 \% \mathrm{UAE}<30 \mathrm{mg} / 24$ hoursi and 20 healthy controls (mean age $33.9 \pm 4.4$ years).

\begin{tabular}{|l|c|c|c|}
\hline Paraneter & IDDM+HT & IDDM & Controls \\
\hline $\mathrm{O}_{2}^{-}\left(\mathrm{nmol} / \mathrm{min} / 10^{6}\right.$ cells) & $6.26 \pm 0.15$ & $4.58 \pm 0.39$ & $2.16 \pm 0.31$ \\
\hline $\mathrm{MDA}(\mathrm{nmol} / \mathrm{ml})$ & $2.67 \pm 0.36$ & $1.25 \pm 0.14$ & $0.33 \pm 0.24$ \\
\hline $\mathrm{HPO}(\mu \mathrm{M})$ & $4.25 \pm 0.18$ & $3.64 \pm 0.33$ & $103 \pm 0.08$ \\
\hline $\mathrm{Cu}-\mathrm{ZnSOD}(\mathrm{U} / \mathrm{mg} \mathrm{Hb})$ & $0.73 \pm 0.06$ & $0.96 \pm 0.06$ & $1.22 \pm 0.07$ \\
\hline $\mathrm{GSSG}-\mathrm{Red}(\mathrm{U} / \mathrm{g} \mathrm{Hb})$ & $2.10 \pm 0.03$ & $2.18 \pm 0.08$ & $2.33 \pm 0.14$ \\
\hline $\mathrm{GSH}-\mathrm{Px}(\mathrm{U} / \mathrm{g} \mathrm{Hb})$ & $21.19 \pm 1.87$ & $27.67 \pm 2.66$ & $33.17 \pm 3.38$ \\
\hline $\mathrm{Zn}(\mu \mathrm{mol} / \mathrm{h})$ & $136.69 \pm 10.40$ & $181.72 \pm 9.72$ & $199.67 \pm 13.20$ \\
\hline $\mathrm{Cu}(\mu \mathrm{mol} / \mathrm{l})$ & $19.33 \pm 3.96$ & $19.11 \pm 3.80$ & $18.10 \pm 4.40$ \\
\hline $\mathrm{Se}(\mu \mathrm{mol} / \mathrm{h})$ & $0.113 \pm 0.004$ & $0.139 \pm 0.005$ & $0.180 \pm 0.009$ \\
\hline
\end{tabular}

Statistically significantly increased $\mathrm{O}_{2}^{-}$generation, MDA and HPO concentration, and on the other hand, decreased antioxidant enzymes activities have been found in patients with IDDM and HT in comparison with normotensive diabetics, and in normotensive diabetic when compared to controls. Conclusions: 1.Pro-oxidant - antioxidant imbalance is found in nomotensive insulin-dependent diabetic patients. 2.Mypertension increases pro-oxidant - antioxidant imbalance in insulin-dependent diabetics. 
1101

HEMODYNAMIC AND METABOLIC EFFECTS OE TRANSDERMAL CLONIDINE IN NIDDM PATIENTS WITH HYPERTENSION.

D.Giugliano, R.Acampora, C.La Marca, M.Marfella, M.A. Caccavale, A.Palmieri, F.Turano, F.D'Onofrio, Departinent of Geriatrics, 2nd University of Naples, J.taly

The aim of this study was to evaluate the effects of transdermal clonidine on hemodynamic and metabolic parameters in NIDDM patients with hypertension. After a 2-week run-in placebo period, twenty patients ( $10 \mathrm{M} / 10 \mathrm{~F}$, age $55 \pm 7$ yrs, $m+S 1$, BMI $27.5 \pm 3$ ) underwent a singleblind, randomized, placebo-controlled, cross-over study of 4-week treatment with clonidine (transdermal patch 5 $\mathrm{mg} /$ week) or placebo (inactive patch). Compared with placebo, clonidine significantly reduced systolic (153+6 vs $163 \pm 8 \mathrm{mming})$ and diastolic $(88+2$ vs $93 \pm 3.5 \mathrm{mnH}$, $\mathrm{p}=0.001)$ blood pressure, left ventricular mass $(94 \pm 11$ vs $\left.99 \pm 12 \mathrm{~g} / \mathrm{m}^{2}, \mathrm{p}<0.01\right)$ and fasting glucose levels $(-0.8 \pm 0.5$ vs $0.1+0.6 \mathrm{mmol} / 1, p<0.01)$. Total glicose disposal (euglycemic clamp) was $6.5 \pm 1.5$ with placebo and $7.1 \pm 1.6$ $\mathrm{mg} / \mathrm{kg} / \mathrm{min}$ with clonidine $(p<0.01)$. Oxidative glucose disposal (indirect calorimetry) was also greater after clonidine. Plasma glucose, insulin and C-peptide responses following oral glucose $(75$ g) were significantly lower after clonidine, as well as urinary albumin excretion $(p=0.05-0.01)$. Transdermal clonidine is effective in reducing blood pressure in hypertensive NIDDM patients and is well tolerated. It may be useful to reduce the cardiovascular impact of hypertension in NIDDM.

\section{2}

CELIPROLOL IMPROVES INSULIN SENSITIVITY AND DECREASES SERLM CHOLESTEROL LEVELS IN HY PERTENSIVE NIDDM PATIENTS. S.V. Gelding, I. Wadsworth*, K. Ali, R. Niththyananthan, A.D. Hendersen and D. (3. Johnston. Linit el Metabx)lic Medicine and *Department of Epidemiologe and Public Health, St Mary s Fiespital Mcdical School, London W2, UK

VIDDM and hyperiension are buth associuted with insulin resistance and

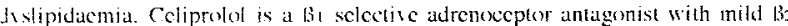
agemist and vatsodidutor properties ussed for treatment of mild to moderate aypertension. To inestigate the clifects of ecliprolol on glucose and lipid

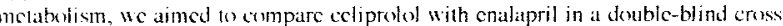
mer stufy in 15 stable hivpertensive NIDDN patients treated with dict or oral agents.

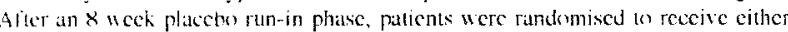

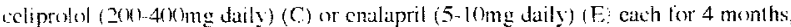

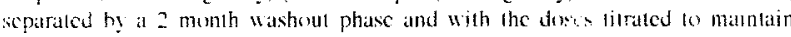
dustulic blond pressure $<90 \mathrm{mmHg}$. Before and after each unament period insulin

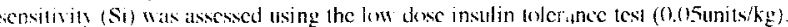
lasting werum lipid and fibrinngen let els were measured and overnight urine collected for allhumin cecretien ratc (AER), A significant carry=-(ner effect was demonstratch, whechy initial tecalment with $C$ reduced the changes in $\mathrm{Si}$ and lipids during Ireat ment with E. Therefore, results are presented fir the first treatment period oml? Thres patients withdren lrom the study helore completion, feaving 7 in group $C$ and 5 in greup $\mathrm{E}$. Bith treatments lowered systolic and diastolic blong pressures by a similur event. Body weipht remained stable during the cours: of the study. Si

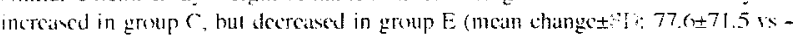

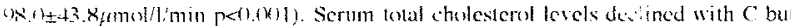

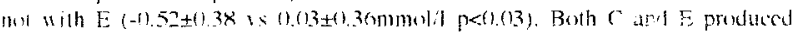

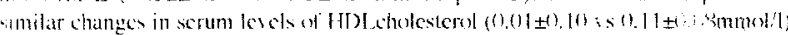

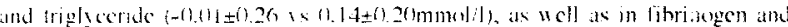

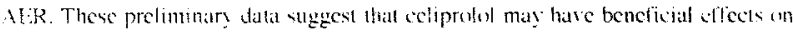

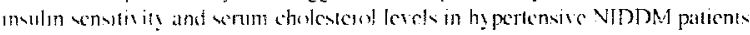

\section{4}

DIABETIC-HYPERTENSIVE BB.SA RATS ARE NOW AVAILABLE FOR THE STUDY OF DIABETIC HYPERTENSION

Klöting, I. Berg, S., B. Voigt, and L.Vogt „Gerhardt Katsch" Institute of Diabetes, University of Greifswald, Karlsburg

Suitable animal models to study the well-knowri association of diabetes and hypertension are currently not available. This prompted us to establish a congenic BB.Sa rat line which is genetically identical with $\mathrm{BB}$ rats except a single chromosome segment containing the blood pressure locus $S_{a}$ of the spontaneously hypertensive SHR rat. This Sa gene is highly expressed in the kidney and is involved in the development of hypertension. To characterize this newly established line. 15 BB.Sa $(8 \mathrm{M}, 7 \mathrm{~F})$ and $16 \mathrm{BB}(8 \mathrm{M}, 8 \mathrm{~F})$ rats were longitudinally studied for body weight gain, glucose tolerance, serum and urine constituents (24-h excretion) up to an age of 28 weeks. Systolic (SBP, $\mathrm{mmHg}$ ) and diastolic blood pressure (DBP. $\mathrm{mmHg}$ ). hear rate (HR) and motoric activity (MA) were measured telemetrically for 4 consecutive days at an age of 14. 18.22 and 26 weeks. 5 out of 15 BB.Sa and 6 out of 16 BB rats were diabetic with $15 \pm 2$ and $17 \pm 3$ weeks, respectively. Comparing nondiabetic $B B$ and BB.Sa rats as well as their diabetic equivalents, the Sa segment of the SHR rat caused not only significant differences in body weight gain or glucose area in the i.p.GTT but also in $24 \mathrm{~h}$ urine excretion of total protein, albumin and minerals (Na. $\mathrm{K}, \mathrm{Ca} . \mathrm{P}$ ). Most interesting findings were seen in blood pressure regulation. Although the mean SBP was comparable between nondiabetic BB and BB.Sa rats $(125 \pm 10$ vs. $127 \pm 9)$, the mean $D B P$ was significantly higher in $B B$. Sa than $B B$ rats (110 10 vs. 95 56 ). Furthermore, the changes from maxima to minima within minutes in SBP. DBP and also in HR were the highest and occur more frequently in BB.Sa rats (max$\min =40 \mathrm{mmHg})$, a phenomenon never observed in $\mathrm{BB}(15-20 \mathrm{mmHg})$ or hypertensive SHR rats $(20-25 \mathrm{mmHg})$. In contrast to $\mathrm{BB}$ rats in which the blood pressure is significantly higher in the diabetic state after a diabetes duration of at least 14 weeks, the SBP and DBP increased immediately after manifestation and was already significantly increased 3 weeks later $(+8 \mathrm{mmHg})$ in diabetic BB.Sa rats. Interestingly. the increase of blood pressure was connected with a moderate decrease of the HR and MA. These findings demonstrate the power of congenic BB.SHR rat lines to study the effect of genes involved in the development of hypertension and their interaction in the diabetic as well as nondiabetic state. 


\section{5}

NO INFLUENCE OF ENALAPRIL AND NITRENDIPINE ON INSULIN SENSITIVITY IN PATIENTS WITH ESSENTIAL HYPERTENSION T. Heise, L. Heinemann, K. Kristahn and P.T. Sawicki. Dep. of Metabolic Diseases and Nutrition, Heinrich-Heine-University Düsseldorf, Germany Hypertension has been repeatedly associated with reduced insulin sensitivity. An improvement of insulin resistance with some antihypertensive agents has been postulated which is of particular interest in hypertensive diahetic patients. However, to date, no studies adressing this issue and using a two-step euglycemic glucose clamp are availabie. We studied therefore the effect of Enalapril (ENA, $10 \mathrm{mg}$ o.d.) and Nitrendipine (NIT, $20 \mathrm{mg}$ o.d.) on insulin sensitivity ( $\mathrm{S}_{\mathrm{I}}$ ) in a randomized double blind cross-over study. Twenty patients with essential hypertension ( $35 \pm 12$ years (mean $\pm S D$ ), BMI $31.9 \pm 5.0 \mathrm{~kg} / \mathrm{m}^{2}$, initial blood pressure $152 \pm 10 / 99 \pm 6 \mathrm{~mm} \mathrm{Hg}$ ) received each treatment for 4 weeks. respectively. with a wash-out period of 3 weeks. $S_{3}$ was measured at the beginning and at the end of each drug period. In order to avoid an activation of the renin-angiotensin system during the two-step euglycaemic glucose clamp (insulin infusion 0.25 and $1.0 \mathrm{mU} / \mathrm{kg} \times \mathrm{min}$ ) serum potassium concentrations were kept constant by variable infusion of $\mathrm{KCl}$. Both drugs induced a comparable decline in systolic and diastolic bload pressure (ENA: $-15 \pm 9 /-13 \pm 8 \mathrm{~mm} \mathrm{Hg}$. NIT: $-16 \pm 8 /-12 \pm 6 \mathrm{~mm}$ $\mathrm{Hg}$ ). No significant change in body weight occured with both treatments (ENA $-0.4 \pm 2.0$. NIT: $0.6 \pm 1.1 \mathrm{~kg}$ ). $\mathrm{S}_{1}$ did not change significantly with the reduction of blood pressure and was not significantly different between the drugs (ENA $5.2 \pm 2.0$ (basal $\mathrm{S}_{1} 5.1 \pm 2.2$ ). NIT: $5.8 \pm 3.0$ (basal $\mathrm{S}_{\mathrm{I}} 5.1 \pm 2.4$ ) $\mathrm{mL} / \mathrm{min} \times \mathrm{m}^{2} /$ $\mu \mathrm{U} / \mathrm{mL}$ ). The observed overall difference in $S_{1}$ between both treatments was $0.6 \pm 2.1 \mathrm{~mL} / \mathrm{min} \times \mathrm{m}^{2} / \mu \mathrm{U} / \mathrm{mL}$. The study had a power of $80 \%$ to detect a difference between both treatments in $S_{1}$ of $1.5 \mathrm{~mL} / \mathrm{min} \times \mathrm{m}^{2} / \mu \mathrm{U} / \mathrm{mL}$. Reduction of blood pressure had no effect on insulin sensitivity and no differences between ENA and NIT were observed. Previously reported changes in insulin sensitivity with angiotensin-converting-enzyme inhibitors or calcium-channel blockers might have been due to interferences in potassium homeostasis and body weight or the kind of insulin sensitivity measurement method.

\section{7}

24-H BLOOD PRESSURE MONITORING IN KIDNEY TRANSPLANT RECIPIENTS WITH POSTTRANSPLANT DIABETES MELLITUS

L. Gerö, I., Barna, K. Földes, N. Spitzer, J. Jansen, Á. Remport, J. Járay and F. Perner, 1st Department of Medicine, Department of Transplantation and Surgery, Semmelweis University, Budapest, Hungary

Ambulatory blood pressure was monitored for 24 hours in 30 kidney transplant recipients with posttransplant diabetes mellitus (PTDM) to determine the characteristics of their cyrcadian rhythm. All patients (22 men, 8 women, ages 23-59 yrs, onset of diabetes 6-36 months after transplantation) had normal kidney function and were on standard steroid+cyclosporine therapy. Diurnal indices were registered in each case and results were compared with those of 197 normotensive controls (NC) and 419 patients with essential hypertension (EH). Mean diurnal systolic/diastolic indices (i.e. the percentage decrease of blood pressure during the night) were $12 \pm 6.5 / 16 \pm 8.4 \%$ for $N C$ $8 \pm 8.8 / 13 \pm 10.4 \%$ for $E H$ and $0 \pm 10.3 / 3 \pm 9.5 \%$ for PTDM, resp. $(p<0.01$ with analysis of variance). The prevalence of reversed diurnal indices (i.e. higher systolic and/or diastolic blood pressure during the night) was $2 / 2 \%$ in NC, $14 / 8 \%$ in EH and $52 / 35 \%$ in PTDM, resp., $p<<0.001$ with chi-square test. No significant correlation was found between the decrease in diurnal indices and the absolute blood pressure values, magnitude of microalbuminuria or degree of autonomic parasympathic lesions. Results show that elevated blood pressure during the night occurs with a significantly increased frequency in PTDM patients. This may contribute to the high cardiovascular mortality which has been well documented in patients with organ transplants.

\section{6}

ENHANCEMENT OF OPIOID AGONISM HAS HYYPERGLICAEMIC EFFECTS IN PATIENTS WITH ESSENTIAL HYPERTENSION

D. Cozzolino, F.C. Sasso, G. Di Giacomo Russo, T. Salvatore, M. Fratta*, M. Cioffi* and R. Torella. Department of Geriatics and Metabolic Diseases, *Institute of Oncology, 2nd University of Naples, Italy

Essential hypertension (EH), obesity, and type-2 diabetes mellitus (NIDDM) are associated to abnormalities of glucose metabolism in humans. High plasma levels of $\beta$-endorphin (BE) have been reported in these diseases: many evidences have suggested some role for $\mathrm{BE}$ in glucose homeostasis both in obesity and NIDDM. In order to determine whether opioid peptides exert glucometabolic effects in EH, $8(4 \mathrm{M} / 4 \mathrm{~F}$; age: $40+6$ yrs mean $+\mathrm{DS}$; BMI: $24.3 \pm 1.4 \mathrm{Kg} / \mathrm{m}^{2}$ ) subjects (P) affected by EH (SBP/DBP: $170 \pm 9 / 103$ $+4 \mathrm{mmHg})$ underwent in the morning to 1-hour-infusion of BE $(500 \mu \mathrm{g})$, and in another occasion (after a week interval) to the same opioid infusion protocol immediately preceded by a i.v. bolus ( $5 \mathrm{mg}$ ) of naloxone, an opioid receptor antagonist. $8(4 \mathrm{M} / 4 \mathrm{~F})$, normotensive (SBP/DBP: $136 \pm 10 / 78 \pm 6$ $\mathrm{mmHg}$ ), healthy, age-, and BMI-matched subjects (C) served as controls. None of the subjects investigated had taken any drug during the 3 weeks preceding the experiments, or was affected by other major diseases. BE alone provoked: in $\mathrm{P}$, a significant $(\mathrm{p}<0.05$ at least) increase of plasma glucose and glucagon and a contemporary inhibition of plasma insulin and $\mathrm{C}$-peptide; in $\mathrm{C}$, a slight increment of plasma glucose and a significant ( $<<0.05$ al least) arising of plasma glucagon, insulin, and C-peptide. BE preceded by naloxone provoked both in $\mathrm{P}$ and $\mathrm{C}$ significant $(\mathrm{p}<0.001$ and 0.05 , respectively) increments of plasma glucagon, without appreciable variations in plasma glucose, insulin and C-peptide. In conclusion, hyperglycaemic response induced by $\beta$-endorphin in hypertensive patients leads to suggest that opioid agonism might contribute to alterations of glucose metabolism often found in essential hypertension.

\section{8}

INSLILIN ACTION IS INDEPENDENT OF INSULIN SECRETORY PULSE FREOLENCY IN ESSENTIAL HYPERTENSION

Mi Wiggam. SI Hunter. C Ennis. B Shoridan. AB Atkinson and PM Bell. Metabolic Unit and Endocrinc Laborators. Royal Victoria Hospital. Belfast.

It non-itisuliu dependent diabetes mellitus (NIDDM) and normat man. there is a strong negative corrclation between peripheral insulin action the frequency of itsulin secreton pulses. Howerer. it remains unclear whether abnormal pulsatile insulin secretion in NIDDM is a prinar or secondar defect and it is not known whether this relationship between insulin pulsatility and insulin action cxists in other insulin resistant states. The aim of this study was to exmme this relationship in essential hypertension (EHT). Twelve subjects with EHT (duration $5+1$ years) were studied at least 6 weeks after withdrawal of antily pertensive medication (BP $15+ \pm 2 / 100 \pm 1 \mathrm{mmHg}$ ). Insulin action was ussessed using the hyerinsulinacmic glucose clamp technique ( $2 \mathrm{~h}$ infusion at $2 \mathrm{mU} / \mathrm{k} \mathrm{g} / \mathrm{min}$ ) combincd with isolope dilution methodology. Insulin pulsatility was assessed by sampling ever. 2 minutes for $9(\%$ minutes after an overnight fast. Puises were identified using the computer program Pulsar. Glucose infusion rates (GIR) required to maintain euglycaenia ranged from 17.210 $53.2 \mu \mathrm{mol} / \mathrm{kg} / \mathrm{min}$ (mean $34.0 \pm 3.1 \mu \mathrm{mol} / \mathrm{kg} / \mathrm{min}$ ). Insulin pulse frequency rangod from $101016 \mathrm{pulscs} / 90 \mathrm{~min}$ (mean $13.1 \pm 0.6 \mathrm{pulses} / 90 \mathrm{~min}$ ). No correlation between GIR and insulin pulse frequency was found $(r=-0.21$. $\mathrm{p}=(0.5)$. In addition there was no association between GIR and pulse amplitude $(r=-() .3(), p=0.35)$ or pulse arca $(r=0.11 . p=0.74)$. Similarly. none of these pulse parameters were related to either basal or suppressed endogenous glucose production. In conclusion. insulin action was not related to frequency of insulin secretor pulses in essential hypertension. in contrast to published findings in normal man and NIDDM. This suggests a role for other factors as determinants of insulin action in essential hypertension. Our resuits do not favour the hypothesis that increased frequency of insulin secretory pulses is a non-specific secondary response to insulin resistance. 


\section{Clinical Nephropathy}

\section{9}

SCREENING FOR EARLY RENAL IMPAIRMENT OF DIABETIC PATIENTS IN PRIMARY HEALTH CARE SYSTEM IN HUNGARY K.Farkas, É.Nol1 and G.Jermendy. 3rd Med.Dept.? Bajcsy-Zsilinszky Hospital, Budapest, Hungary.

In order to detect early renal impairment, screening procedure for microalbuminuria was carried out in diabetic patients registered in primary health care system in Hungary. A total of 1016 diabetic subjects was investigated and microalbuminuria was verified by results of albumin to creatinine ratio (ACR) in first morning urinary samples (immunturbidimetric method, abnormal value $\geqslant 2.5 \mathrm{mg} / \mathrm{mmol}$ in men, $\geqslant 3.5 \mathrm{mg} / \mathrm{mmol}$ in women). After applying exclusion criteria data of 933 diabetic patients (424 men, 509 women; 129 IDDM, 804 NIDDM) were analysed. Abnormal ACR was found in 315 patients $(33.8 \%$ ) with a predominance of men (IDDM: $41.3 \%$ vs $28.8 \%$; NIDDM: $38.0 \%$ vs $30.0 \%$; men vs women, respectively). The prevalence of macroalbuminuria (ACR > 20.0 $\mathrm{mg} / \mathrm{mmol})$ was very similar in men (40/424=9.4\%) and women $(42 / 509=8.3 \%)$. Significant difference was found between diabetic patients with $(n=315)$ and without $(n=618)$ abnormal ACR values regarding age $(64.3+0.7$ vs $61.4-0.5$ years, p $\left.<0_{+} 001\right)$, duration of diabetes (10.3-0.5 vs $7.9-0,3$ years, pro.001), serum creatinine ( $99+2$ vs $88-7$, plmol/1), systolic blood pressure ( $151_{+}^{+} 1$ vs $146-1 \mathrm{mmHg}, \mathrm{p}: 0.01)$ and blood glucose (10.4-0.2 vs $9.4-0.1 \mathrm{mmo} 1 / 1$, p 0.001 ; $x$-SEM). Because of renal impairment, one third of diabetic patients should be carefully controlled in primary health care system in Hungary.

\section{1}

INCREASE IN SERUM AGES ("ADVANCED GLYCOSYLATION END-PRODUCTS") IN IDDM PATIENTS WHO SMOKE

\footnotetext{
${ }^{1} \mathrm{H}$.-J. Baumgartl, ${ }^{1} \mathrm{E}$. Standl, ${ }^{2} \mathrm{H}$. Vlassara and ${ }^{2} \mathrm{R}$. Bucala

${ }^{1}$ Diabetes Research Institute and City Hospital Munich-Schwabing, FRG

${ }^{2}$ The Picower Institute for Medical Research, Manhasset, N.Y., USA
}

Recent studies indicate that IDDM patients who smoke more often manifest a microalbuminuria and as a consequence a diabetic nephropathy than non-smokers with IDDM (Diab.Med. 11: 666$669,1994)$. "Advanced glycosylation end-products" (AGEs) are irreversible products consisting of glucose-modified proteins and lipids, are found more frequently in diabetics due to chronic persisting hyperglycemia, and are considered to be one of the possible causes for diabetic secondary complications e.g. diabetic nephropathy. In order to examine the potential influence of smoking on AGE levels in serum, we measured by means of a competitive AGE-ELISA 20 long-term IDDM patients with an active smoker's history and 25 non-smoking IDDM patients as controls. The duration of the diabetes was similiar in both groups $(19+/-3$ years) and there was no significant difference in glucose control (HbA1c 8,7 \%+/-1.9). The serum AGEs proved to be significantly higher in the patients with smoking histories compared to the non-smokers ( 16.9 vs $11.4 \mathrm{U} / \mathrm{ml}), p<0.01)$. In a preliminary study, we measured serum AGE levels serially in one non-diabetic individual after smoking two cigarettes. Serum AGE levels increased from $5 \mathrm{U} / \mathrm{ml}$ to $10 \mathrm{U} / \mathrm{ml}$ after smoking and remained elevated for at least 90 minutes thereafter. These results indicate that smoking could have an influence on serum AGE levels leading to a more rapid development of a diabetic complications including nephropathy.

\section{0}

LOW BONE MINERAL DENSITY IN DIABETIC PATIENTS WITH MILD TO MODERATE RENAL FAILURE.

M. Rix, H. Andreassen, Bente Langdahl* \& P. Eskildsen; Med. Dept.,Køge County Hospital, DK ; ${ }^{*}$ Dept Endocrinology, Aarhus Amtssygehus, DK. objective: To asses the bone mineral density and the biochemical markers of bone metabolism in a group of diabetic patients with mild to moderate renal failure. Patients \& Methods: 27 patients (18 males, 9 females) with diabetes (13 IDDM, 14 NIDDM) and mild to moderate renal failure defined as creatinine clearance of $<65 \mathrm{~m} / / \mathrm{min}$ and not on dialysis, were examined in a cross-sectional study. Patients were recruited from the out-patient clinics of 3 Danish County hospitals. Mean (range) age 55 years(25-81), bodymass index 25.9 (17.9-39.1),duration of diabetes 21years (5-46), creatinine clearance $32 \mathrm{ml} / \mathrm{min}(6-65), \mathrm{s}-\mathrm{Ca}++1.15 \mathrm{mmol} / \mathrm{l}$ $0.71-1.27)$ s- PO4- $1.49 \mathrm{mmol} /(0.8-2.9)$ and s-PTH $212.5 \mathrm{pg} / \mathrm{ml}(14-$ 1329). Measurements: Primary outcome meașure was Bone Mineral Density (BMD) measured by Dual Energy X-Ray Absorptiometry of the lumbar spine, the femoral neck and the distal forearm. Secondary outcome measures were s- PTH, biochemical markers of bone formation ( $\mathrm{s}$ Osteocalcin,s-Bone Alkaline Phosphatase) and of bone resorption ( $u$ Hydroxyproline, u-Pyridino line(Pyr) \& u-Deoxypyridinoline (DPyr)).

Results: BMD, expressed as Z-scores (BMD normalized to age- and sex matched mean) mean (SEM ; $p$ value), was significantly reduced at all sites compared to normals : Lumbar spine - $0.86(0.28 ; p<0.005)$, femoral neck $1.12(0.24 ; p<0.0001)$, distal forearm $-1.47(0.29 ; p<0.0001)$. Of the biochemical markers s-PTH, s-osteocalcin, u-Pyr \& u-Dpyr $(p<0.001)$ were significantly increased compared to normal controls.

Conclusion: In a group of diabetic patients with mild to moderate renal failure low BMD compared to age- and sex matched normal controls was found. Increased values of some of the biochemical markers of bone metabolism may indicate increased bone-turnover in this group.
1112

SERUM URIC ACID AND URINARY TRANSFERRIN IN TYPE 2 DIABETIC PATIENTS

M. Shinada. Department of Medicine, Atsuta National Health Insurance Hospital, Atsuta, Japan.

Serum uric acid has been reported to decrease in some diabetic patients because of a disturbance in reabsorption or an increase of uric acid excretion at the proximal tubule in the kidney. To determine the enhancer of urinary transferrin elevation in the stage of normoalbuminuria in type 2 diabetics, we examined the relationship of serum uric acid, $\mathrm{HbA1c}$ and other factors concerning urinary transferrin. The subjects consisted of 113 patients ( 63 men, age: 58 years \pm 12 ) with normoalbuminuria (albumin excretion rate $20 \mathrm{cg} / \mathrm{min}$ ) without urinary tract infections, history of renal diseases, use of uric acid synthesis inhibitors or uric acid excretion promoters. Overnight urine samples were analyzed by immunoturbidity for transferrin and albumin. An elevation of urinary transferrin $(>1.0 \mathrm{mg} / \mathrm{g} . \mathrm{cr}$ ) was detected in 35 patients $(31 \%)$. Serum uric acid level was $5.0 \pm 1.2 \mathrm{mg} / \mathrm{d} 1$ (F:4.5 $\pm 1.2, \mathrm{M}: 5.3 \pm 1.2)$ in the normal transferrin group and $3.9 \pm 1.5 \mathrm{mg} / \mathrm{dl}(\mathrm{F}: 3.8 \pm 1.5, \mathrm{M}: 4.0$ \pm 1.6 ) in the elevated transferrin group. Multivariate analysis using multiple regression revealed that urinary transferrin had a significant correlation with serum uric acid $(p=0.0021)$ and $\mathrm{HbA1c}(\mathrm{p}=0.0108)$. Transferrin had no significant correlation with age, sex, body weight, systolic or diastolic blood pressure. The increase of urinary transferrin was strongly related to the decrease of serum uric acid. This suggests that the urinary transferrin elevation during normoalbuminuria in type 2 diabetics may be associated with the excretion of uric acid. 
1113

IOHEXOL PLASMA CLEARANCE. AN ACCURATE METHOD TO EVALUATE GFR IN DIABETIC PATIENTS.

G. Penno, S. Bandinelli, L. Pucci, M. Nannipieri, A. Bertacca, L. Rizzo, A. Rapuano and R. Navalesi. Malattie Metaboliche - Pisa, Italy.

The plasma clearance $(\mathrm{pCl})$ of iohexol has recently been proposed as a new method for estimating GFR. Iohexol-pCl was compared with $51-\mathrm{Cr}$ EDTA-pCl in 30 diabetics (13 IDDM, 17 NIDDM; age 23-70; diabetes duration 1-35 years) with normal to impaired renal function (serum creatinine: $0.9-6.4 \mathrm{mg} / \mathrm{dl})$. Bolus i.v. injection of $51-\mathrm{Cr}-\mathrm{EDTA}(1 \mu \mathrm{Ci} / \mathrm{kg})$ was followed by $5 \mathrm{ml}$ slow (0.5-1 min) i.v. injection of Omnipaque (Nycomed. Oslo). Samples for radioactivity and iohexol analysis were drawn at $0,5,10,15,30,60,90,120,150,180,210,240,270,300 \mathrm{~min}$ $(+360$ and 420 min if serum creatinine $>2.0 ;+1440 \mathrm{~min}$ if $>5.0 \mathrm{mg} / \mathrm{dl})$. For iohexol assay, plasma samples were deproteinized by adding 4 vol $5 \%$ perchloric acid and centrifugating. Samples were stored at $-20^{\circ} \mathrm{C}$ up to 3 months and centrifuged for $5 \mathrm{~min}$ at $14000 \mathrm{rpm}$ before injection. Supernates were assayed in double by HPLC with the detector set at 254 $\mathrm{nm}$ and throughout a Nova-Pak C18 column (Waters-Millipore, USA). Elution was obtained by a $97: 3$ water/acetonitrile mix $(\mathrm{pH} 2.6)$ pumped at 1.4 $\mathrm{ml} / \mathrm{min}$ rate. Only the second peak obtained during elution of iohexol (about $4.5 \mathrm{~min}$ ) was used for calculation. Repeated dilution tests show highly linear regressions for concentrations between $3.25-650 \mu \mathrm{g} / \mathrm{ml}\left(\mathrm{r}^{2}\right.$ $>0.98$ ). Imprecision of iohexol assay (the whole procedure from deproteinization to chromatography) was: intra-assay: $1.4 \pm 1.5 \%, \mathrm{~m} \pm \mathrm{sd}$ (95\% Cl: $1.0-1.8 \%$ ); inter-assay: $3.0 \pm 2.7 \%(1.4-4.6 \%)$. pCl for both $51-\mathrm{Cr}-$ EDTA and iohexol were calculated as dose/AUC. Iohexol-pCl ranged between 12.9 and $150.9 \mathrm{ml} / \mathrm{min}$, while 51 - $\mathrm{Cr}$-EDTA-pCl between 11.9 and $149.8 \mathrm{ml} / \mathrm{min}$ with excellent correlation (iohexol= 0.98 51-Cr-EDTA + $2.45 ; r^{2}=0.99$ ). Mean $\mathrm{CV}$ between the two methods was $1.7 \%$ (range $0-$ $4.9 \%)$ with a significant negative correlation $(\mathrm{r}=0.51, \mathrm{p}=0.007)$ with the GFR levels. In diabetics, GFR measured by iohexol shows an excellent agreement with tracers clearance throughout a wide range of renal function. Iohexol provides an alternative accurate method for measuring GFR

\section{4}

EARLY RENAL CHANGES IN NEWLY DIAGNOSED DIABETIC SUBJECTS UNDER THIRTY YEARS OF AGE

L. Ali', M.R. Alam², Z. Hassan', A.K. Azad Khan', H.U. Rashid', N.S. Chowdhury', S.K. Siddique ${ }^{3}$ Research Oivision, BIRDEM; ${ }^{2}$ Department of Nephrology, IPGMR, Dhaka: 3NM, IPGMR, Dhaka

To help in understarding the early nephropathic changes in malnutition related diabetes melitus (MRDM) subjects glomerular filtration rate (GFR), kidney size, uninary albumin excretion (UAE) and urinary transferin excretion (UTRF) were determined in 68 newly diagnosed nonproteinuric diabetic subjects under 30 years of age in a Bangladesh population. Following the criteria of the WHO Study Group 40 patients belonged to NIDDM and 28 belonged to MRDM type of which 19 were of PDDM (protein-deficient diabetes mellitus) variety and 9 were of FCPD (fibrocalculus pancreatic diabetes) variety. Eighteen age-matched healthy subjects with no family history of diabetes were included as Controls GFR was measured by 99mTCDTPA two sample whole plasma clearance technique, and urinary albumin and transferrin were measured by immunoturbidimetric method. Albumincreatinine ratio $(A C R)$ and transferin-creatinine ratio $(T C R)$ from ambulatory samples were taken as substitutes for UAE and UTRF in overnight samples. GFR values were found to be similar in the three diabetic groups but they were higher compared to Control (Duncan test, $p$ 0.05). The percentages of patients with increased GFR $\left(143 \mathrm{~m} / 1 / .73 \mathrm{~m}^{2}\right.$ body surface) were 0 in the Control, but $38 \%, 58 \%$ and $38 \%$ in the NIDDM, FCPD and PDDM groups respectively. Kidney sizes were similar in all groups. Both the ACR and TCR values were significantly higher in the 3 diabetic groups in comparison to Control (Mann-Whitney test, $p 0.001-0.001$ ). FCPD and PDDM showed higher values of ACR and TCR compared to NIDDM. The differences were more marked in case of TCR than in case of ACR. The rise in median ACR values were 1.4, 1.6 and 1.9 times in NIDDM, FCPD and PDDM respectively compared to Contral; the corresponding rise in median TCR values were 2.16 3.24 and 5.80 times. The results show that early renal hemodynamic changes are similar in all the diabetic groups in this young population, but early microvascular changes seerns to be pronounced in MRDM patients. The results also indicate that microtransferrinuria may be an earlier and more sensitive marker of incipient nephropathy than microalbuminuria.

\section{5}

INFUSION OF ATRIAL NATRIURETIC FACTOR AND ALBUMINURIA IN HEALTHY VOLUNTEERS: MINICKING DIABETIC NEPHROPATHY?

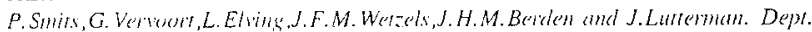

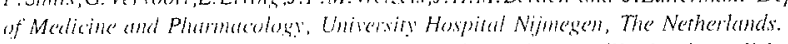
Objective: About $30 \%$ of type 1 diabetic patients will develop diabetic nephropathy (DNP). A hemodynamic hasis for DNP is suggested by the occurence of hyperfiltration and glomerular hypertension found in experimental models. The Atrial Natriuretio Factor (ANF) is a natriuretic and vasodilating hormone. The most striking hemodynamic renal effect of ANF is the increase in glomerular fiftration fraction (FF) due to preglomerular vasodilation and postglomerular arteriolat contriction. In IDDM increased levels of ANF have been found. Intusion of ANF in low dose calses diuresis, natriulesis and increase of FF. We hypothesize that infuxion of low tose ANF will increase FF in humans and may induce (microlathuminuria without an intluenee on systemic blood pressure. Design and methods: In 49 healiny voluneers we investigated the effects of ANF intusion (dose steph 0.01 and $0.02 \mu \mathrm{g} / \mathrm{kg} / \mathrm{min}$ ) on glomerulas tiltration rate. effective renal plasina tlow (ERPF). FF. albuminuma and blood pressure. GFR and ERPF were measured by inuline and PAH elearance periods of 20 mir. Albuminuria was meatudel by an ELISA. Blood pressure was medsured

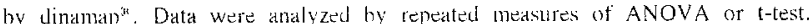
Results are expressed as mein + SEM. Results: GFR was $107 \pm 2 \mathrm{ml} / \mathrm{min} / \mathrm{1} .73 \mathrm{~m}^{2}$ at baseline and increased dose related duting ANF infusion to $111 \pm 2$ and $116 \pm 2$ $(P<0.001)$. ERPF was $525+13 \mathrm{~m} / / \mathrm{min} / 1.73 \mathrm{~m}^{\circ}$ and decreased to $491+12$ and $474+12$ renpetively during $A N F$ intioion $(\mathrm{P}<0.001)$. FF increated from $20.7 \pm 0.5$ \& $23.4 \pm 0.6$ and $25.0 \pm 0.5 \%(p<0.001)$. Mean arterial pressure did not change $\left(90+1\right.$ at batseline to $90 \pm 1$ and $90 \pm 1 m \mathrm{mH}_{ \pm}$respectively). Basal albumin excretion was $5.2+0.4$ and increated to $11.3+1.9 \mathrm{and} 38.2+7.6 \mu \mathrm{g} / \mathrm{min}$ respectively $(\mathrm{P}<0.001)$. During the 1 st ANF Jose $(0.01 \mu \mathrm{g} / \mathrm{kg} / \mathrm{min})$ albuminuria Was $>20 \mu \mathrm{g} / \mathrm{min}$ in 5 nut of 45 volunter's whereas this was 22 out of 44 volunters during the 2nd ANF-dose $(0.02 \mu u / k g / m i n)$. Conclusions: ANF intusion in low dose increases ghomerulat eapiliary pressure and induces (micro)alhuminuria in healtly volunters without a signiticant influence on systemic blood pressure. The hemolynamic changes indued by ANF infusion mimic the functsonal shanges seen in dialsetic patientw with microathuminuria.

\section{6}

TUBULAR FUNCTION IN RELATION TO RENAL STRUCTURE IN TYPE 2 DIABETIC PATIENTS.

M. Velussi, P. Fioretto, M. Mauer, E. Brocco, I. Barzon, A. Sfriso, A. Carraro, F. Frigato M. Trevisan, B. Muollo, M. Sambataro and R. Nosadini. C.N.R. Center for the Study of Aging, Universities of Padova and Minnesota, U.S.A. and Italy.

We have recently described by light microscopy an heterogeneity in renal structure in microalbuminuric type 2 diabetic patients (pts). More particularly we have found that $29 \%$ have normal or near normal renal structure (Cl); $29 \%$ have changes typical of diabetic nephropathy (glomerular, arteriolar hyalinosis and tubulo-interstitial changes occurring in parallel) (C2) and 42\% have important tubulo-interstitial and/or arteriolar hyalinosis and/or glomerular sclerosis with mild glomerular abnomnalitics (C3). Aim of the present study was to investigate tubular function, using the rate of urinary excretion of alpha-1 microglobulin, in NIDDM patients with micromacroalbuminuria (MA). We studied 45 patients with albumin excretion rate (AER) $<20 \mu \mathrm{g} / \mathrm{min}$ (NA) and 34 with MA (AER: 20-1368 $\mu \mathrm{g} / \mathrm{min}$ range, median 45 $\mu \mathrm{g} / \mathrm{min})$. Means $\pm \mathrm{S}$. D. of age (yrs), duration of diabetes $(\mathrm{yrs}), \mathrm{HbA}, \mathrm{C}(\%)$ and GFR $\left(\mathrm{m} / / \mathrm{min} / 1.73 \mathrm{~m}^{2}\right)$ were in $\mathrm{C} 154 \pm 9,8 \pm 3,7.5 \pm 0.8,111 \pm 20$, in $\mathrm{C} 2 \quad 60 \pm 6$, n.s., $14 \pm 6, \mathrm{p}<0.05$ vs $\mathrm{C} 1,9.6 \pm 1.8, \mathrm{p}<0.005$ vs $\mathrm{C} 1,91 \pm 36$, n.s., and in $\mathrm{C} 361 \pm 6$, n.s., $10 \pm 8$, n.s., $8.5 \pm 1.3$, p $<0.05$ vs $\mathrm{C} 1,101 \pm 21$, n.s. Among NA pts $53 \%$ were $\mathrm{Cl}, 9 \%$ $\mathrm{C} 2$ and $38 \% \mathrm{C} 3$. Among MA $20 \%$ were $\mathrm{C} 1$ ( $p<0.05$ vs NA). $32 \% \mathrm{C} 2$ ( $p<0.01$ is NA) and $47 \%$ C3 (n.s.vs NA). The excretion rate of alpha-1 microglobulin in category $\mathrm{C} 2$ was altered both in $\mathrm{NA}(14.3 \pm 1.7 \mathrm{mg} / 24 \mathrm{hrs}$ mean $\pm \mathrm{S}$.E.. $\mathrm{p}<0.01)$ and in MA $(13.7+3.1, \mathrm{p}<0.05$ ) (normal values $3.4 \pm 0.8$ ). Both NA and MA in category C1 had normal rates of alpha-l microglobulin excretion $(4.2 \pm 1.0$ and $6.2 \pm 2.2$ respectively). Only MA but not NA in category C3 had slightly increased rates of alpha-1 microglobulin excretion $(7.3 \pm 1.7, p<0.05$ and $5.4 \pm 1.7$, n.s. respectively). Abnormalities in alpha-1 microglobulin excretion rate, a marker of tubular function, are present in NIDDM patients with the tipical lesions of diabetic glomerulopathy, irrespective of microalbuminuria, but not in those with near normal glomerular structure, even when tubulo-interstitial fibrosis is found. It is suggested that alpha- 1 microglobulin urinary excretion is allered when the overall glomerular-tubular complex is damaged. 


\section{7}

INSULIN-LIKE GROWTH FACTOR BINDING PROTEIN-3 AND TRANSFORMNG GROWTH FACTOR- 3 , IN KIDNEY ENDOTHELIAL CELLS S. Giannini. B. Cresci. L.Pala. G. Galli and C. M. Rotella. Department of Clinical Physiopathology. Endocrinology Unit. Section of Metabolic Diseases and Diabctes, Zniversity of Florence - Viale Pieraccini. 6 - 50134 - Florence

The Transforning Growth Factor $-\beta_{1}\left(\right.$ TGF- $\left.\beta_{1}\right)$ is a widespread growth factor involved in differen biological effects and it las been demonstrated play an important role in the pathogenesis of diabetic nephropatiy. together with other urowth factors such as Insulin-like Gronth Factors (IGFs) and their six carrier IGF Binding Proteins (IGFBPs). The aim of our study is to investigate the relationship between TGF- $\beta_{1}$ and the IGFs in diabetic nephropathy. addressing our attention to the possible modulation of TGF $\beta_{1}$ on IGFBPs release by human kidney endothelial vells (GEN). Methods: GEN have been characterized based on positivity to the low density lipoprotein uptake and to the factor Vill antigen. Conditioned medium (CM) from GEN was harvested affer 48 h senum-free culture and electrophoresed on SDS-

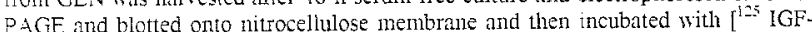
II]. The IGFBPs released br GEN in their CM were then characterized by a Western Innmunoblor analysis using policlonal antibodies against JGFBP-1 to -5 . Restits: Ligand Blot analysis revealed the presence of four different BP bands: a major doubler band $43-45 \mathrm{~kg}$ ) another strong lower band $(2+\mathrm{kDa})$ and two intermediate weaker bands of 34 and $30 \mathrm{kDa}$ were detected and then identified with the Immunoblot analysis as BP-3. BP-4 and BP-2. respectively. The last $30 \mathrm{kDa}$ band terealed no positive signal. According to its MW. this bard could represent either BP- 6 or a glvcosilated form of BP-4. To further characterize this band we used the enzr me $N$-glycanase which blunted the level of this band suggesting that the $30 \mathrm{kDa}$ band could represent the glycosilated form of BP-t. Treatment of GEN with TGF- $\beta_{1}$ resulted in a decreased production of BP-3. The dose-response curve indicated that this effect was significant at $1 \mathrm{llM}$ TGF- $\beta_{3}$. A specific proteasic activity has been reported for BP-3. Howerer. the addition of protease inhibitors, such as aprotinin, EDTA and PMSF did not block the effect of TGF- $\beta_{1}$ and the incubation of human fibroblast CM. which contains high levels of BP-3, with GEN CM previously treated with TGF- $3_{1}$ did not show any decrease of the BP-3 band. We can concinde that TGF- $\beta$. in GEN could be involved in the regulation of IGF availability, with autacrine or paracrine purposes, through a non-enzrmatic control of BP-3 level which represents the rost important reservoir of IGFs in biological fluids.

\section{9}

STABILITY OF RED CELL SODIUM-LITHIIUM COUNTERTRANSPORT ACTIVITY AND AMBULATORY BLOOOD PRESSURE MONITORING IN TYPE I DIABETIC CIIILREN

A. Kömer, V. Pataki, M. Dobos, L. Madácsy, (i. Reusz, J. Kélemen, M. Miltényi and T. Tulassay; First Department of Pacdietrics, Semmelweis Medical Univerisity

Aim: To study the stablitiy of sodium-lithium countertransport (SLC.T) acitivity and ambulatory blood pressure monitoring (ABPM) in type I diabetic children over time, in order to define their significance as markers of susceptibility for diabetic renal disease. Subjects and methods: Repeated measurements of SLCT acitvity and ABPM were performed in II recent onset diabetic children (age: 14.03, range: $10.8-16.5$ years) and in 11 patients with longer duration of diabetes (age: 16.04, range: 1 1.6-19.98 years). Results: Excluding recent onset diabetic patinents with apparently elevated SLCT activity presumably due to altered lipid and hormonal protile, a significant correlation in SLCT activity between the first and second study was observed $(r=0.69, p<0.05)$. SLCT significantly correlated with intravellular sodium concentration $(r=0.44, p<0.03)$ and $\mathrm{HbAlc}(r=0.47, \mathrm{p}<0.04)$, but not with duration of diabetes. Noctumal blood pressure significantly rose in the originally recent onset diabetic patients $2-3$ years after the first study resulting in a decrease in , dipper"/,non-dipper" ratio. Diurnal systolic (S) and diastolic (D) BP were comparable in both time points withing the same group of diabetic

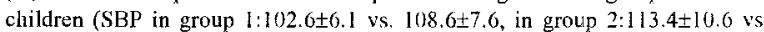

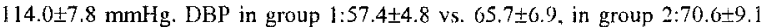
vs. $68.5 \pm 5.3 \mathrm{mmHg}$.) Moreover, there was a significant correlation in thoth diurnal $(\mathrm{r}=0.5, \mathrm{p}<0.05)$ and nocturnal $(\mathrm{r}=0.58, \mathrm{p}<0.02)$ systolic $\mathrm{BP}$ between the tirst and second study in the whole diabetic population. Conclusion: Both SLCT activity and BP values obtained by ABPM are stable. reproducible markers in type 1 diabetic children over time. Their interaction and role in the development of diabetic nephropathy remains to be further elucidated.

\section{8}

ELEVATED LEVELS OF URINARY TRANSFORMING GROWTH FACTOR- $\beta 1$ IN YOUNG IDDM PATIENTS

E. Korpinen, A.-M. Teppo, P.-H. Groop, J. Fagerudd, H.K. Ảkerblom, and

O. Vaarala The Children's Hospital and the Department of Medicine, Division of Nephrology, University of Helsinki, Helsinki, and the Department of Biochemistry, National Public Health Institute, Helsinki, Finland.

Transforming growth factor $-\beta$ (TGF- $\beta$ ) is a $25 \mathrm{kD}$ polypeptide with three isoforms. TGF- $\beta 1$ increases matrix production in kidney mesangial cells. Elevated levels of mRNA for TGF- $\beta 1$ have been found in human diabetic kidneys. In patients with focal glomerulosclerosis urinary TGF- $\beta 1$ levels were reported to be elevated. We analysed the urinary TGF- $\beta 1$ concentrations before the manifestation of incipient diabetic nephropathy in children and adolescents with IDDM. 39 IDDM patients with disease duration at least 3 years, and 15 healthy subjects were studied. 37 patients had 3 timed overnight urine collecions to measure AER, 2 patients had 2 collections. The controls had one timed overnight urine collection. All AERs, except a single control subject, were below $20 \mu \mathrm{g} / \mathrm{min}$. TGF- $\beta 1$ was measured from one timed overnight urine sample in all subjects with double antibody EIA. The results were related to urinary creatinine $(\mathrm{Cr}$ ) concentration. Clinical characteristics and results (mean \pm SEM) are shown below. IDDM $(n=39) \quad$ Control $(n=15)$ $\begin{array}{lll}\text { Age (yrs) } & 12.8 \pm 0.5 & 12.9 \pm 0.5\end{array}$ Duration of IDDM (yrs) $\quad 6.4 \pm 0.3$

$\mathrm{HbAlc}(\mathrm{mmol} / \mathrm{l})$

$\operatorname{AER}(\mu \mathrm{g} / \mathrm{min})$

TGF- $\beta$ l (ng/mg Cr)

$5 \pm 0.4$

$9.7 \pm 3.9$

$9 \pm 3.4$

$0.5 \pm 0.2$

The urinary TGF- $\beta 1$ levels were higher in patients with IDDM than in controls $(\mathrm{p}=0.04$, Mann-Whitney $U$ test). No correlation was found between the TGF- $\beta 1$ levels and HbAlc or duration of the disease, $r=0.19 \quad(p=0.24)$ and $r=-0.251$ $(\mathrm{p}=0.12$, Spearman test), respectively. We conclude that elevated levels of urinary TGF- $\beta 1$ are found in patients with IDDM before elevated AER.

1120

\begin{tabular}{|c|c|c|c|c|}
\hline $\begin{array}{l}\text { VASOACTIVE } \\
\qquad \mathrm{M} \text {. } \\
\text { Endothelium is } \\
\text { mellitus. Endo } \\
\text { relaxing facto } \\
\text { constricting fa } \\
\text { intrarenal hael } \\
\text { studied the pro } \\
\text { tromboxane A } \\
\text { (AER < } 30 \mathrm{mg} \\
\text { with hyperfiltr } \\
\text { assessed by } \\
\text { platelet guanyla }\end{array}$ & $\begin{array}{l}\text { OF DIA } \\
\text { hestakova, } \\
\text { tonal Endo } \\
\text { the first ta } \\
\text { helial dysf } \\
\text { s - nitric } \\
\text { tors - endo } \\
\text { odynamics } \\
\text { duction of } \\
\text { (TXA } A_{2} \text { ) in } \\
\text { day). } 9 \text { p-ts } \\
\text { tion (GFR } \\
\text { timulatory } \\
\text { t-cyclase (G }\end{array}$ & $\begin{array}{l}\text { ATIC NEPHR } \\
\text { rinology Cente } \\
\text { get tissue fo } \\
\text { nction can } \\
\text { xide (NO) a } \\
\text { helin- } 1 \text { (ET-1 } \\
\text { and initiate t } \\
0 \text { and plasm } \\
21 \text { IDDM p } \\
\text { with DN (AE } \\
140 \text { mlimin. } \\
\text { fect of L-argi } \\
\text { ) activity. Re }\end{array}$ & $\begin{array}{l}\text { OPATHY (DN } \\
\text { Severina and } \\
\text { er Moscow. } \\
\text { r hyperglycen } \\
\text { lead to ov } \\
\text { and prostacyo } \\
\text { : which may } \\
\text { he progressio } \\
\text { la levels of } P \\
\text { atients. } 7 \text { p-t } \\
: R>100 \text { mg/ } \\
1.73 m^{2} \text { ) NO } \\
\text { inine (NO-pre } \\
\text { sults M M SEM }\end{array}$ & $\begin{array}{l}\text { 1. Dedor } \\
\text { Russia } \\
\text { hia in diabetes } \\
\text { erproduction of } \\
\text { clin (PG| or } \\
\text { influence the } \\
\text { on of DN. We } \\
G l_{2} \text {. ET-1 and } \\
\text { s- without DN } \\
\text { day, and } 5 \text { p-ts } \\
\text { release was } \\
\text { cursor, on the } \\
\text { t. }\end{array}$ \\
\hline Parameters & $\begin{array}{c}\text { Controls } \\
n=18\end{array}$ & $\begin{array}{c}\text { AER }<30 \\
n=7\end{array}$ & $\begin{array}{c}A E R>100 \\
n=9\end{array}$ & $\begin{aligned} \text { GFR } & >140 \\
n & =5\end{aligned}$ \\
\hline $\begin{array}{c}\text { GC-activation } \\
\text { (n-fold) }\end{array}$ & $1.0=0.1$ & $0.8=0.1$ & $1.4=0.1^{*}$ & $1.8=0.3^{*}$ \\
\hline$\left.\mathrm{PG}\right|_{2}(\mathrm{pg} / \mathrm{ml})$ & $313=101$ & $208=75$ & $585=105^{*}$ & $676=98^{*}$ \\
\hline ET-1 $(\mathrm{pg} / \mathrm{ml})$ & $1.3=0.2$ & $1.2=0.1$ & $1.9=0.1^{*}$ & $1.5=0.2$ \\
\hline$T X A_{2}(\mathrm{pg} / \mathrm{ml})$ & $364=95$ & $729=102^{\star}$ & $1302=139^{*}$ & $804=107^{\star}$ \\
\hline $\begin{array}{l}-p<0.05 \text { con } \\
n p \text {-ts without } D\end{array}$ & $\begin{array}{l}\text { ared to co } \\
\text { only plas }\end{array}$ & $\begin{array}{l}\text { ols and } p \text {-ts } \\
T X A_{2} \text { was } e\end{array}$ & $\begin{array}{l}\text { Without DN } \\
\text { evated. The } \\
\text { ad in p-ts with } \\
\text { ctors ( } E T-1\end{array}$ & $\begin{array}{l}\text { laximal activity } \\
\text { hyperfiltration. } \\
\left.\text { nd } T \times A_{2}\right)=\text { in } \\
\text { theliai dilating } \\
\text { ntribute :o the } \\
\text { tors s moved } \\
\text { orogression of } \\
\text { ant role in the }\end{array}$ \\
\hline
\end{tabular}


1121

GLOMERULAR STRUCTCRE IN NON-PROTEINURIC TYPE 2 DIABETIC PATIENTS: ELECTRON MICROSCOPY.

P. Fioretto, M. Mauer, G. Re, M. Velussi, F. Frigato, B. Muollo, E. Brocco, B. Baggio, G. Crepaldi and R. Nosadini. CNR Center for the Study of Aging of Padova, Universities of Minnesota, Padova and Sassari, Italy and LSA

Diabetic glomerulopathy, characterized by mesangial expansion and thickening of the glomerular basement membrane (GBM) is present in type 1 diabetic patients (pts) with increased albumin excretion rate (AER). In caucasian type 2 diabetic (D) pts morphometric studies have been performed only in a group of proteinuric pts. Airn of this study was to evaluate glomerular structure in non-proteinuric D pts. Kidney biopsy and renal function studies were performed in 41 unselected D pts: 21 had AER $<20 \mu \mathrm{g} / \mathrm{min}$ (NA) and $20 \mathrm{had}$ AER of $20-200 \mu \mathrm{g} / \mathrm{min}$ (MA). 27 normal kidney donors ( $14 \mathrm{M}$, age $56 \pm 10$ yrs) served as controls (C). These studies were approved by the Ethical Committee and pts gave written informed consent. AER was measured on three $24 \mathrm{hr}$ urine collections. Glomerular filtration rate (GFR) was assessed by plasma clearance of 51 [Cr]-EDTA. Electron microscopic morphomeric analysis was used to estimate GBM width and mesangial and mesangial matrix volume fraction [Vv(mes/glom) and $V v(m a l / g l o m\}$. Age and known $D$ duration were similar in $\mathrm{NA}$ and MA pts ( $58 \pm 7$ vs $56+8$ yrs. $8 \pm 5$ vc $12 \pm 6$ vs) as were HbAlc ( $8.1-1.6$ v $8.3 \pm 1.6 \%)$ and GFR $\left(96 \pm 23 \times 593 \pm 25 \mathrm{ml} / \mathrm{min} / 1.73 \mathrm{~m}^{2}\right)$. Although several pts had glomerular structure in the normal range, considered as a group. all structural parameters were abnormal in NA pts [GBM width: $393 \pm 68 \mathrm{~nm}$ (C: $310=38$. $\mathrm{p}<.001), \mathrm{Vv}(\mathrm{mes} / \mathrm{glom}): .24 \pm .03$ (C: $.19 \pm .03, \mathrm{p}<.001) . \mathrm{Vv}(\mathrm{mat} / \mathrm{glom}) .11 \pm .02$ (C) $.08 \pm .02, \mathrm{p}<.001)]$. MA compared to NA pis had higher $V v(m e s / g l o m): .28 \pm .06$ $\mathrm{p}<.02$ and $\mathrm{V} v(\mathrm{mat} / \mathrm{glom}): .14 \pm .04$. $\mathrm{p}<.005$. GBM width also tended to be higher $(444 \pm 96 \mathrm{~nm}, \mathrm{p}<.06)$. NA. D pts as a group, have early changes of diabetic glomerulopathy: more advanced glomerular lesions, especially expansion of mesangial matrix. are present in MA vs NA D pts. although some MA pts have normal glomerular structure. Thus, glomerular changes in type 2 are similar to those in lype 1 diabetes. However, on average lesions are less advanced in type 2 than in type 1 pts in comparable renal functional caregories and there is much more overlap in glomerular parameters between C. NA and MA prs.

\section{MECHANICAL STRETCH INDUCES VASCULAR PERMEABILITY FACTOR GENE EXPRESSION AND PROTEIN SECRETION IN HUMAN MESANGIAL CELLS.}

G.Gruden, S.Thomas, S.Sacks, D.Burt, G.Chusney and GC.Viberti Unit for Metabolic Medicine, UMDS, Guy's Hospital, London U.K. Diabetic and other nephropathies are characterised by increased glomerular permeability, which may be related to haemodynamic abnormalities. Vascular Permeability Factor (VPF) is a powerful promoter of vascular permeability and is secreted by mesangial cells. We studied VPF gene expression and protein secretion in human mesangial cells in response to mechanical stretch. Human mesangial cells were cultured in $5 \mathrm{mM}$ glucose on flexible bottom plates, serum deprived for 48 hours and subjected to cyclical stretching (pressure: $8 \mathrm{KPa}$, frequency: 60 cycles/min) for 1-2-4-6-12 and 24 hours Control cells were grown in non deformable but otherwise identical plates in parallel. Total RNA was extracted and VPF gene expression quantitated by competitive RT-PCR. VPF protein level was determined by a in-house immunoenzymometric assay (range: 1-40 pM, intra-assay C.V.: 5.8). VPF gene expression increased after 6 hours of stretch (percentage increase vs control at 6 hours: $80.86 \%$, 12 hours: $31.34 \% \mathrm{p}=0.043$ ) and returned to baseline by 24 hours. VPF protein level in the supernatant was significantly greater at 12 hours in cells exposed to mechanical stretch compared to cells in a mechanically stable environment $[20.05(17.2-22.2)$ vs 8.89 (7.7 13.4) $\mathrm{pM}$, median (IQ), $\mathrm{n}=5 \mathrm{p}=0.043$ ). VPF gene expression and protein secretion is stimulated by mechanical stretch in human mesangial cells. Stretch-induced VPF may play a role in the increased glomerular permeability of diabetic and other glomerulopathies.

\section{2}

NO DIFFERENCE IN RENAL HAEMODYNAMIC AND ALBUMNURIC RESPONSE TO NORADRENALINE WN IDDM AND HEALTHY SUBJECTS K. Hoogenberg', W.J. Sluiter, T.W van Haclen ${ }^{3}$, A.J. Stmi ${ }^{2}$, W.D. Reitsma and R.P.F. Dullaart 'Divisions of Endocrinology' andd lnternal Medicinc ${ }^{2}$, State University Hospital, Groningen, The Netherlands.

Exaggerated renal sensitivity to noradrenaline (NA) may lead to intraglomerular hypertension and elevated urinary albumin excretion rate (Ualb.V) in Type 1 (insulindependent) diabetes mellitus (IDDM). Therefore, we mesured renal haemodynamic and albuminuric response to stepvise exogenous $\mathrm{NA}$ (at individually deternined threshold (cmean arterial pressure (MAP) $=0 \mathrm{mmHy}$, period T), $20 \%$ prossor $(\mathrm{MAP}=4 \mathrm{mmHg}$ period $20 \% \mathrm{P}$ ) and pressor dose ( $\mathrm{MAP}=3(\mathrm{mmm} \mathrm{Hor}$ period $\mathrm{P})$ ) in 8 matched normoalbuminuric IDDM (D1), 8 microalbuminuric IDDM (D2) and 8 healhy subjects (C) in a randornized, placebo controlled fashion during a euglyciomic clamp. The NA dose response curve on MAP was shifted lelwards in D1 and D2 compared to $C(P<0.05)$ and so the systemic responsiveness was higher in boh IDDM groups. Baseline effective tenal plasma flow (ERPF), glomerular filiraton rate (GFR) and filtration fraction (FF) were not different bewcen the group: (ERPF: $458 \pm 2+$ (C). $451 \pm 22$ (D1), 442 440 (D2) $\left(\mathrm{ml} / \mathrm{min} / 1.73 \mathrm{~m}^{2}\right)$; GFR: $101 \pm 6$ (C), $105 \pm 5$ (D) $104 \pm 8$ (D2) $\left(\mathrm{ml} / \mathrm{min} / 1.73 \mathrm{~m}^{2}\right)$; FF : $22 \pm 1$ (C), $23 \pm 1$ (D), $24 \pm 1$ (D2) (mean+SEM). Durng NA blood pressure rose to target levels. ERPF decreased gradually a each in fusion siep in all groups (T: $-12 \pm 3(\mathrm{C}),-9 \pm 2$ (D1), $-8 \pm 1$ (D2); 20\%P: $-19 \pm 3$ (C) $-15 \pm 3$ (D),$-13 \pm 1$ (D2); P: $-33 \pm 3$ (C),$-28 \pm 2$ (D1), $-29 \pm 3$ (D2) (\%change in mean $\pm S E M, P<0.05$ 10 $P<0.001)$ ). No belween group difference in ERPF decline was obverved alier idjustment for NA dose. GFR did nol change significantly during the $3 \mathrm{NA}$ inf usion steps in either group. Consequently, FF rose in all groups. Baseline Ualh V ( o mini) was $46(3.28 .5)$ (C) $3.6(2.6-8.8)$ (D1) and 44.5 (16.5-237.7) (D2) (medim and range). Ualb. $V$ remained unchanged in each group at $\mathrm{T}$ and $20 \% \mathrm{P}$. During $\mathrm{P}, \mathrm{U}$ alb.V rose significuttly by $54 \%$ (14\% to $491 \%$ ) (median and rango) in C, by $81 \%(-4 \% 10294 \%)$ in Di and by $63 \%(5 \%$ to $377 \%)$ in D2 (Ualb. V $P<0.05$ for all), no betwecm group Uifferences $(P>0.20)$. In conclusion, exogenous NA infusion causes a dose dependent renal vasoconstriction in humans. NA increases albuminuria only at doses that gives rise to a large increase in systemic bloodpressure suggesting that $N A_{1}$ does not increase intraglomerular pressure independently from its systemic effecl. Our data do not provide evidence for the hypothesis that altered renal sensitivity to $\mathrm{NA}$ is atr important determinant of the imbalance of renal haemodynamics and clcvaled Ualb. $Y$ seen in IDDM

\section{4}

ANTIOXIDANT PROTECTION AND CELL RESPONSE TO OXIDATIVE STRESS IN SIBLINGS OF IDDM PROBANDS.

E. Matteucci, C. Bertoni, E. Iazzolino, A. Troilo and O. Giampietro. Ist. Clinica Medica II, Università di Pisa, Italy.

Oxidative stress has a role in the genesis and progression of tissue damage in IDDM. Since we observed kidney and metabolic abnormalities in non-diabetic siblings of type 1 diabetics (heavier if the proband had diabetic nephropathy), we sought evidence of impaired endogenous defences against oxidants. Eighteen siblings of diabetic probands ( 8 with and 10 without nephropathy) and 13 healthy controls were studied. Were measured: erythrocyte glutathione content and cell response to oxygen radicals (xanthine+xanthine oxidase for $3 \mathrm{~h}$ ); serum albumin, lipids, electrolytes, iron, transferrin, ferritin, copper, ceruloplasmin, uric acid, bilirubin, SGGT, SGOT, SGPT: urinary outputs of glucose, uric acid, albumin, electrolytes, enzymes (UGGT, UNAG, UAAP). Siblings vs controls had lower serum HDL cholesterol and apoA1 $(\mathrm{p}<0.05)$ as well as urine glucose $(\mathrm{p}<0.01)$ and uric acid $(p<0.05)$ outputs. Siblings of nephropathic probands vs siblings of non-nephropathic had higher serum total cholesterol and apoB $(\mathrm{p}<0.01)$, GOT $(\mathrm{p}<0.01)$, ceruloplasmin $(\mathrm{p}<0.05)$ and urine GGT output $(\mathrm{p}<0.05)$; serum HDL cholesterol and urine glucose excretion were lower $(\mathrm{p}<0.05)$; red cell methemoglobin content after oxidative stress was higher $(p<0.05)$. Urinary GGT output was related to red cell methemoglobin content after stress. glycemia and total cholesterol $(\mathrm{p}<0.001)$; serum ceruloplasmin with $3 \mathrm{~h}$-hemolysis, methemoglobin content, triglycerides, apoB, SGGT, copper $(\mathrm{p}<0.001)$; serum apoB with hepatic enzymes $(\mathrm{p}<0.001)$; urine sodium excretion rate with glucose and uric acid outputs $(\mathrm{p}<0.001)$. Our findings suggest that: 1$)$ non-diabetic siblings of nephropathic diabetics are more susceptible to oxidative stress; 2) indices of cell damage correlate with indices of oxidation, ceruloplasmin and low-density lipoproteins; 3) renal handling of molecules (glucose, uric acid) and ions (natrium) is abnormal; its supposed association with insulin-resistance could explain the observed dyslipidaemia in otherwise healthy subjects. 
1125

PLATELET CONTENT OF REDUCED GLUTATHIONE, ITS RELATED ENZYMES, CATALASE AND SUPEROXIDE DISMUT'ASE IN INSULINDEPENDENT DIABETES MELLITUS : RELATION TO METABOLIC CONTROL AND TO MICROVASCULAR COMPLICATIONS.

G.Seghieri, L.A. De Giorgio, R. Lecis, F. Orlandini, S.Carro, R.Anichini, A. Fazzini, F.Franconi, E.Lupis, and P. Di Simplicio. Diabetes Unit, Spedali Riuniti, Pistoia, Medical Dpt. and Diabetes Unit, Ospedale S.Andrea, La Spezia, Dpt. of Biology ,Univ. of Siena, and Inst. of Biochemistry, Univ. of Sassari, Italy. The role of free radicals in the pathogenesis of microvascular disease in insulindependent diabetes mellitus (IDDM) is controversial. It is equally matter of debate the role played by intracellular enzymatic scavengers such as reduced glutathione (GSH) and related enzymes (GSH-peroxidase: GSH-Px, GSH-reductase: GSH-Red, GSH-transferase: GSH-Tr, glucose-6-P-dehydrogenase: G6PDH) as well as thioltransferase (TT), catalase (CAT) and superoxide dismutase (SOD). The aims of this study were therefore a) to measure the platelet content of GSH, its related enzymes, TT, CAT and SOD in a group of 35 IDDM patients (mean age $23 \pm 3$ (SD) years), as compared with 10 control subjects age- and sex- matched, and b) to relate these enzymatic activities to metabolic control and to the presence of retinopathy (diagnosed by fluoroangiography) and nephropathy (by an urinary albumin excretion rate (AER) repeatedly $>20 \mathrm{\mu} / \mathrm{min}$ ). As compared to controls diabetic patients had a slight, non significant increase in the content $\times 10^{-9}$ platelets of GSH (3.7 72.4 vs $2.8 \pm 1.9 \mathrm{mmol}), \mathrm{GSH}-\mathrm{Tr}(75.2 \pm 1$ vs $59.9 \pm 1.53 \mathrm{mU})$, GSH-Red $(33.1+1.5 v s 28.5 \pm 1.3 \mathrm{mU})$, GSH-Px $(8.5 \pm 2.4 v s 4.8 \pm 2.3 \mathrm{mU}), \mathrm{G} 6 \mathrm{PDH}(69.4 \pm 1.7 v s$ $63.4 \pm 1.8 \mathrm{mU})$, SOD $(5.7 \pm 1.4$ vs $4.9 \pm 1.5 \mu \mathrm{g})$, CAT $\quad(37400.2 \pm 170 \quad$ vs $28430 \pm 150 \mathrm{mU})$, and a decrease in TT $(6.7 \pm 1.9 \mathrm{vs} 8.5 \pm 2.3 \mathrm{mU})$. Either GSH or all other enzymes were unrelated to glycated haemoglobin (mean value:8.6 $\pm 1.2 \%$ ). When compared to the normoalbuminuric group patients with increased AER $(n=12)$ had a significant decrease ( $\left(x 10^{-9}\right.$ platelets) in SOD $(4.2 \pm 1.2$ vs $6.4 \pm 1.4 \mu \mathrm{g}$, $\mathrm{p}=0.003)$, as well as in TT ( $3.5 \pm 1.8$ vs $8.2 \pm 1.8 \mathrm{mU} \mathrm{p}=0.0002$ ), while retinopathy was not associated to modifications in the enzymatic pattern. In summary a) platelet GSH and related enzymes are not modified in IDDM and seetn marginally influenced by metabolic control, b) nephropathy is assosiated to a selective decrease in platelet content of SOD and TT

\section{7}

ABNORMAL REGULATION OF CELL MEMBRANE FLUIDITY IN DIABETIC NEPHROPATHY

S.C. Jones, S.M. Marshall \& T.H. Thomas. Department of Medicine, University of Newcastle Upon Tyne.

It has been suggested that albuminuria in diabetic nephropathy (DN) is part of a generalised cell membrane abnormality linking hypertension, insulin resistance, cardiovascular disease and cell membrane transporter abnormalities. One such transporter, sodium-lithium countertransport (Na-Li CT), a putative marker of nephropathy, is modulated by protein thiol group inhibition using nethylmaleimide (NEM). Cell membrane fluidity is related to Na-Li CT and could link the abnormalities observed in DN. We measured cell membrane fluidity at baseline and after thiol group inhibition. 11 cases with IDDM and nephropathy, 9 diabetic and 15 non-diabetic controls had mean age of $45.8(31$ - 61$)$ : 49.4 (35 - 64) : $41.2(28$ - 59) years, diabetes duration of $23.8(10-35): 29.6(20$ - 49) years and sex (M:F) 7:4, 6:3, 8:7. Serum creatinine was $<150$ umol $I^{-1}$ to avoid confounding effects of uraemia. Fluidity was assessed by measuring the fluorescence anisotropy, which is inversely related to fluidity, in whole red blood cells. DPH and TMA-DPH were used to study the deep and superficial regions of the cell membrane,. Analysis of variance revealed that at baseline anisotropy was similar in all three groups using TMA-DPH $(0.278 \pm 0.019$ vs $0.273 \pm 0.005$ vs $0.274 \pm 0.011$ nephropaths: IDDM controls: normal controls) and DPH ( 0.225 \pm 0.018 vs $0.229 \pm 0.017$ vs $0.220 \pm 0.2$. After thiol group inhibition by NEM there was a significant and similar decrease in DPH anisotropy in both IDDM and normal controls which was not observed in the nephropaths (nephropaths $\Delta$ $+0.008 \pm 0.015$, IDDM controls $\Delta-0.014 \pm 0.008$, normal controls $\Delta-\mathbf{- 0 . 0 1 6} \pm$ $0.014, p<0.001$ ). Na-Li CT kinetics show a similar abnornal response to thiol blockade in DN. This suggests that a cell membrane controlling protein, with key thiol groups, is abnormal in nephropathy and its effects on cell membrane function could provide the link between the abnormalities commonly observed in this condition.
1126

NON-INVASIVE HEMODYNAMIC MONITORING AND ASSESSMENT OF DIASTOLIC FUNCTION IN IDDM PATIENTS WITH NEPHROPATHY

A.Holler, G.Gratze, J.Fortin, P.Kotanko and F.Skrabal. Department of Internal Medicine, Krankenhaus der Barmherzigen Brüder, Teaching Hospital of the Karl-Franzens University, A-8020 Graz, Austria

Objective: To assess hemodynamic contributions to the development of diabetic nephropathy. Material and Methods: Using a newly developed technique for the real-time beat-to-beat monitoring of stroke volume (impedance cardiography) and blood pressure (Finapres ${ }^{\mathrm{TM}}$ corrected by Dinamap $(3)$ ) we have calculated beat-to-beat variation of total peripheral resistance index (TPRI) and cardiac index (CI) in 8 IDDM patients without nephropathy, 9 IDDM patients with nephropathy and 12 healthy control persons during supine bed rest and after $60^{\circ}$ passive head-up-tilt. Duration of diabetes mellitus and metabolic control were comparable in the two IDDM patient groups. Furthermore the diastolic left-ventricular function was assessed by echocardiography. Results: IDDM patients with nephropathy showed an increased mean arterial blood pressure $(p<0.05)$, increased TPRI $(p<0.05)$, decreased stroke index $(\mathrm{SI})(p<0.05)$ and decreased $\mathrm{CI}$ $(p<0.05)$. After passive head-up-tilt subjects with nephropathy showed a reduced fall of SI and $\mathrm{CI}(\mathrm{p}<0.05)$. IDDM patients with nephropathy showed impaired diastolic function compared to the control groups ( $p<0.001$ ). Discussion: In IDDM patients with nephropathy increased blood pressure is caused by elevated peripheral resistance and impaired diastolic function of the left ventricle probably leads to a reduced cardiac output, which is not able to counteract the effect of TPRI on blood pressure. The reduced fall of stroke index after passive head-up-tilt may be due to reduced vascular compliance

\section{8}

POOR GLYCAEMIC CONTROL CAUSES BY IT SELF INCREASIES BLOOD PRESSURE IN IDDM ?

M Bojestig*, H J Arnquist*, B E Karlberg*, and I Ludvigsson** Department of *Intemal Medicine and **Pediatrics, University Fospial, Linkoping, Sweden.

In a population study we assessed trends in blood pressure between those who developed nephropathy or did not. We studicd all 213 patients in whom IDJ)M was diagnosed before the age of $15 y$ ears between 1961 and 1980 in a district in southeastern Sweden. Ninety-two percent of the patients were followed fron the unset of diabetes to 1991 or to death. Patients with persistent albuminuriat

(nositive Albustix test) were considered to have nephropathy $(\mathrm{N})$.

Vicroabuminuria (M) was defined as $20 \cdot 200 \mu \mathrm{g} / \mathrm{min}$ in 2 of 3 timed overnight sanples between 1990-91. Blood pressure was measured from onset to diagnowis of $\mathrm{N}$ or to the end of study. Glycosylated hemoglobin $\left(\mathrm{HbA}_{1}\right.$ ) was measured regulary begining in 1980 . The patients were grouped according to degree of glycaemic control (mear value of $\mathrm{HbA} / \mathrm{c}$ ), before the complication, Fair $[n=152]$ ) and Poor $(\geq 1,4 *$ nomal $\mathrm{HbA}$ 1c value $[\mathrm{n}=27]$ ). Statistical analysis was performed by an unnaired (-1est. Results: (Values are mean) * Sign $\mathrm{p} \leq 0.01 \mathrm{us}$

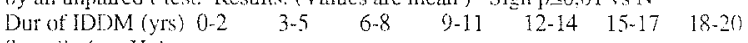
Systolic (mmlig)

Norn-fatir $\quad 109 * 110 \% \quad 115 \% 118 \% 122 * 123 \% \quad 123 \%$ $\begin{array}{llllllll}\text { Nom-nom } & 116 & 110 * & 115 * & 118 * & 122^{*} & 123 * & 123 *\end{array}$ $\begin{array}{llllllll}\text { Nom-por } & 116 & 118 & 122 & 125 & 126 & 123 * & 123 *\end{array}$ $\begin{array}{llllllll}\mathrm{M} & 110 * & 114 & 118 & 122 * & 124 * & 124 * & 128 \\ \mathrm{~V} & 115 & 120 & 122 & 129 & 132 & 136 & 140\end{array}$ Diastolic (minllg) Norm-frim

Norm-porm $\mathrm{M}$ N

$\begin{array}{lllllll}71^{*} & 70^{*} & 74 & 75 * & 77^{*} & 77^{*} & 78 * \\ 74 & 72 & 78 & 75 & 84 & 83 & 81 \\ 72 & 70^{*} & 75 & 75 & 78 & 77 * & 80 * \\ 76 & 80 & 76 & 80 & 82 & 85 & 88\end{array}$

The Norm patients with poor control had higher systolic pressure during the first 12 yeass then the monn patients with fair control. 120 is 113 p<0.00]. Not only patients with later $\mathrm{N}$ but also nom patients with poor control have increased blood pressure already after short duration of IDDM. These observations suggest wat poor glycaemic condul canes increased blond pressure which further increases when renal changes develop. 
1129

INSLLIN RESISTANCE IN PATIENTS WITH CHRONIC KIDNEY DISEASE

R. Toll, T. Doñate*, J.M. Mallafré** M.A. Ortiz, J A Arroyo and J.M. Pou. Depts of Endocrinology. Hospital Sant Pau, *Nephrology, F. Puigvert and ${ }^{*}$ Nephrology, Hospital Creu Roja. Autonomous University of Barcelona.

In previous studies we observed insulin resistance in non diabetic patients on dialysis. The aim of this work was investigated the relationship between uremic status and insulin resistance. We studied the insulin resistance in 3 groups of non diabetic patients with chronic kidney disease. Group 1: creatinine clearance 40-65 $\mathrm{ml} /$ minute, 12 patients, group 2: $20-40 \mathrm{ml} /$ minute, 8 patients, and group: $3:<20 \mathrm{ml} /$ minute, 8 patients. No differences could be seen in age. BMI and sex distribution between groups. We calculated insulin response (IRG), Glucose metabolization (M), sensitivity index of insulin (SI) after 75 gr of oral glucose administration (Cederholm and Wibell method) and $F I R I\left(G_{0} \times Y_{0} / 25\right)$. Insulin was measured by RIA and PTHi by luminescence method

Results: It was observed a progressive deterioration of insulin sensitivity in group 2 and 3 [(FIRI, p<0.05 groups 1 and 2 vs controls and $p<0.05$ group 3 vs. 2) and (SI $p<0.04$ yroups 2 and 3 vs. group 1)] and deterioration of glucose metabolization $[p<0.02$ vs. group 1]. An inverse correlation between PTHi levels and insulin sensitivity was found $(1=72, \mathrm{p}<0.02)$ with a moderate increase of insulin secretion (IRG)

Conclusions: Patients with chronic kidney disease displayed progressive insulin resistance which was lirked to uremic status and suggest some relationship to changes in calcium metabolism

\section{PS 61}

\section{Genetics of Nephropathy}

\section{0}

ACE GENE POLYMORPHISM AND INSULIN SENSITIVITY IN THE PARENTS OF INSULIN DEPENDENT DIABETIC PATIENTS WITH AND WITHOUT ALBUMINURIA

S. De Cosmo, S. Bacci, G. Placentino, GP. Piras*, M. Cignarelli ${ }^{\wedge}$, M. Margaglione ${ }^{\circ}$, G. Di Miinno $0^{\circ}$, R. Giorgino ${ }^{\wedge}$, A. Liuzzi and GC. Viberti

Division of Endocrinology and Metabolism and Unit for Thrombosis and Atherosclerosis, IRCCS "Casa Sollievo della Sofferenza" San Giovanni Rotondo, *Unit for Metabolic Medicine, Ospedale San Michele, Cagliari, "Clinica Medica, Policlinico, Bari, Italy **Unit for Metabolic Medicine,UMDS Guy's Hospital London UK

Reduced insulin sensitivity is associated with cardiovascular disease in the general population and the ACE gene D/D polymorphism is related to coronary genert disese in non insulin-dependent diabetic patients. Insulin dependent heart disease in non insulin-dependent diabetic patients. Insulin dependent cardiovascular disease, increased insulin resistance and a familial predisposition to cardiovascular disease. Insulin sensitivity, by the Insulin Tolerance Test (ITT) and the Insertion/Deletion polymorphism of the ACE gene, by PCR, were measured in $59(M 26, F$ 33) of 92 living parents of 62 IDOM patients. Fifty percent of the patients had albuminuria. The parents $(n=25)$ of the IDDM patients with albuminuria were more insulin resistant (Kitt $3.7=1.5 \%$ per min) than the parents $(n=34)$ of the IDDM patients without albuminuria (Kitt $4.6 \pm 1.4 \%$ per min $p<0.05$ ) after adjustment for age and body mass index. ACE genotype distribution in the parents was $27 \mathrm{D} / \mathrm{D}, 27 \mathrm{~V} / \mathrm{D}$ and $5 \mathrm{~V} / \mathrm{l}$. The three groups of parents with different genotypes were similar for age ( $D / D$ 62 \pm 7 vs $1 / D$ $63 \pm 5$ vs $1 / 168 \pm 5$ yrs) body mass index $(28 \pm 4$ vs $30 \pm 6$ vs $31 \pm 5 \mathrm{Kg} / \mathrm{m} 2)$ and prevalence of arterial hypertension or hypelypidaemia, but insulin sensitivity was significantly reduced in the $\mathrm{D} / \mathrm{D}$ group compared to the $\mathrm{I} / \mathrm{D}$ and $\mathrm{I} / \mathrm{l}$ groups (Kitt $30 \pm 17$ vs $4,4=16$ vs $63=25 \%$ per min : $P=0.03$ by ANOVA) Seventyseven percent of parents with the $D$ allele had a Kitt value equal or below the median of $4 \%$ per min while 41 percent of parents with the I allele had a Kitt value above the median (chi $2=3.85 \quad p<0.05$ ). In conclusion the ACE $D / D$ genotype and $D$ allele are associated with insulin resistance in the parents of IDDM patients. Parents of IDDM patients with albuminuria are less insulin sensitive. These familial traits may contribute to the elevated cardiovascular mortality and morbidity in IDDM patients with nephropathy.

\section{1}

POLYMORPHISM OF ANGIOTENSIN I-CONVERTING ENZYME GENE AND DIABETIC NEPHROPATHY: RESISTANCE VERSUS SUSCEPTIBILITY?

Y.Kondratiev'1,2, L.Demurov' ${ }^{2}$, L.Chugunova', M.Shamkhalova', E.Anokhin', M.Shestakova', V.Nosikov', and I.Dedov', 'Endocrinology Research Centre and ${ }^{2}$ National Research Centre "GNII Genetika", Moscow, Russia

Unlike coronary heart disease, findings of association of diabetic nephropathy (DN) with insertion/deletion (IDD) polymorphism of angiotensin I-converting enzyme (ACE) gene remain still debated. Both ethnic/racial variations in distribution of ID/ACE markers and probable predominance and masking effects of metabolic risk factors (e.g., hyperglycaemia and diabetes duration) in aetiology of multifactorial DN might be responsible for such a discrepancy. In order to minimise a contribution of non-genetic risk factors to the DN phenotype formation in Russian population of insulin-dependent diabetic (IDDM) patients, we studied ID/ACE allele/genotype distribution in three groups of individuals using the following selection criteria for patients: 1) "case" (DN+) - IDDM duration $\leq 15$ years and albumin excretion rate (AER) $\geq 300 \mathrm{mg} / 24 \mathrm{~h}(\mathrm{n}=7) ; 2)$ "control" (DN-) IDDM duration $\geq 20$ years and AER $\leq 200 \mathrm{mg} / 24 \mathrm{~h}(\mathrm{n}=13) ; 3$ ) diabetes-unrelated subjects from general Moscow population ( $\mathrm{n}=118$ ),

Distribution of metabolic and genetic risk factors $(95 \% \mathrm{CI})$

\begin{tabular}{|c|c|c|c|c|c|c|}
\hline Parameter & 1 & 2 & 3 & $P_{1-2}$ & $P_{1-3}$ & $P_{2-3}$ \\
\hline Age at DM onset (yr) & $9.9 \pm 4.3$ & $11.5 \pm 5.5$ & & NS & & \\
\hline DM duration (yr) & $11.4 \pm 2.4$ & $27.2 \pm 8.9$ & & 0.0004 & & \\
\hline $\mathrm{Hb} \mathrm{A}_{1}(\%)$ & $13.1 \pm 2.6$ & $11.3 \pm 2.4$ & & NS & & \\
\hline \multicolumn{7}{|l|}{ Prevalence $(\%)$ of: } \\
\hline I allele & 28.6 & 61.5 & 32.6 & 0.048 & NS & 0.004 \\
\hline D allele & 71.4 & 38.5 & 67.4 & 0.048 & NS & 0.004 \\
\hline II genotype & 0 & 30.8 & 16.1 & NS & NS & NS \\
\hline ID genotype & 57.1 & 61.5 & 33.1 & NS & NS & 0.042 \\
\hline DD genotype & 42.9 & 7.7 & 50.8 & NS & NS & 0.002 \\
\hline
\end{tabular}

In conclusion, our preliminary data indicate that: (i) genetic risk factor in multifactorial aetiology of DN could be "unmasked" and really isolated; and (ii) this risk factor could be attributed to rather protection than susceptibility. 
1132

ROLE OF ANGIOTENSIN-CONVERTING ENZYME (ACE) GENE POLYMORPHISM AND ANTIPROTEINURIC EFFECT OF ACE INHIBITION IN DIABETIC NEPHROPATHY

K. Rossing P. Jacobsen, L. Tarnow, P. Rossing and H.-H. Parving, Steno Diabetes Center, Gentofte, Denmark

The antiproteinuric effect of ACE inhibition in IDDM patients with diabetic nephropathy varies considerably. Therefore, we tested the potential role of an insertion (1)/deletion (D) polymorphism of the ACE gene on this antiproteinuric responsiveness. Sixty (DD, $n=21 ; I D, n=26$ and $I I, n=13$ ) young hypertensive IDDM patients suffering from diabetic nephropathy were investigated during 3 months before and for the initial 6 months during ACE inhibition (captopril 50 (SE 3) mg/24 h, no differences in drug dose between groups). Blood pressure (MABP) and albuminuria (ELISA) were measured 3 (1-6) before and $3(1-13)$ during ACE inhibition. At baseline the groups had comparable MABP ( $\mathrm{mm} \mathrm{Hg}$ ) 114(SE 2)/108(2)/113(3), alijuminuria (geometric mean (antilog SE) $1261(\mathrm{x} / \mathrm{-1.2}) / 1176(\mathrm{x} / \mathrm{-1.2})$ and $1394(\mathrm{x} / \mathrm{-1.3}) \mathrm{mg} / 24 \mathrm{~h}$, and GFR (mean (SE)) 82(5)/96(6)/91(6) $\mathrm{ml} / \mathrm{min} / 1.73 \mathrm{~m}^{2}$, respectively. ACE inhibition induced a significant reduction in MABP and albuminuria in all 3 groups (DD/ID/II): MABP (mean (SE)) $8(2) / 5(1) 12(2) \mathrm{mm} \mathrm{Hg}$ (ANOVA p $=0.02)$ and albuminuria (mean (95\% Cl)) 31(13-46)/22(3-37)/61(34-77)\%,ANOVA $p<0.01$ ), respectively. A multiple linear regression analysis revealed that the $\mathrm{ACE} / \mathrm{D}$ polymorphism, albuminuria and MABP at baseline independently influences the initial decline in albuminuria during $A C E$ inhibition $R^{2}=0.21$ $(p<0.01)$. Linear regression analysis between changes in MABP and albuminuria showed, $R^{2}=0.16, \quad(p<0.01)$. Our data show that hypertensive proteinuric IDDM pateints with the II/ACE genotype are particularly susceptible to commonly advocated renoprotective treatment.

\section{4}

SERUM ACE ACTIVITY IN NORMOFILTERING 'AND HYPERFILTERING DIABETIC PATIENTS.

A. Manto, P.Cotroneo, C.Saponara, P. Magnani, L. Todaro, A.V Greco and G.Ghirlanda.. Medicina Interna e Gerontologia, Università Cattolica Sacro Cuore, Roma - Italy.

Increased activity of serum angiotensin-converting enzyme (ACE) has been demonstrated in type I diabetic patients with microangiopathic complications. ACE transforms active kinins into inactive peptides and angiotensin I into angiotensin II. We have studied 121 IDDM patients 65 men/56 women, mean age $33.3 \pm 9.2$ yrs, mean duration of diabetes $14.4 \pm 9.3$ yrs )and 52 control subjects matched for age and sex; IDDM patients were divided into two groups consisting of 74 normofiltering and 47 hy. perfiltering patients. All diabetic patients were normotensive, while microalbuminuria $(20-200 \mathrm{mg} / \mathrm{ml} / \mathrm{min})$ was present in 8 normofiltering In all groups $\mathrm{HbA}_{1 \mathrm{c}}$, albumin excretion rate (AER), glomeruar filtration rate (GFR) and ACE activity were evaluated.. GFR was assayed by radioisotopic techniques, ACE activity by spectrophotometric assay and AER by radioimmunoassay. Mean $\mathrm{HbA1}$ c values were similar in both groups. ACE activity in hyperfiltering $(31.1 \pm 17.2 \mathrm{U} / \mathrm{L})$ was significantly higher than in normofiltering $(26.2 \pm 11 \mathrm{U} / \mathrm{L}, \mathrm{p}<0,05)$ and in control subjects $(18.8 \pm 7.8$ $\mathrm{UI} / \mathrm{L}, \mathrm{p}<.0 .0001)$. The group of diabetic patients taken as a whole showed a significant increase of $\AA \mathrm{CE}$ activity $(28.4 \pm 14.4 \mathrm{UU} / \mathrm{L})$ compared with control subjects $(18.8 \pm 7.8 \mathrm{U} / \mathrm{L}, \mathrm{p}<0.0001)$. In the 8 microalbuminuric patients ACE activity was slight increased with respect to the other diabetic patients ( $32.2 \pm 8.6 \mathrm{U} / \mathrm{L}$ vs $27.8 \pm 14.2 \mathrm{U} / \mathrm{L}, \mathrm{p}=\mathrm{ns}$ ) There is a significant correlation between GFR and FPR, $(p<0.0001)$ and between serum ACE activity and FF $(\mathrm{p}<0.05)$ in all diabetic patients. ACE activity was not correlated with $\mathrm{HbAl}_{\mathrm{C}}$ levels, or duration of diabetes, or stages of retinopathy. Since ACE is synthesized by glomerular endothelial cells, the increase of ACE activity in hyperfiltering patients could be explained by an alteration in the process of secretion and/or inactivation of the enzyme itself. Another hypothesis is related by an imbalance of the renal kallikrein-kinin system which contributes to hyperfiltration. Furthemore ACE enhances the production of angiotensin II, which is involved in the pathogenetic mechanisms of hyperfiltration in diabetes. In conclusion altered ACE activity may be one of the mechanisms involved in the pathogenesis of hyperfiltration.
1133

DELETERIOUS EFFECT OF A DELETION POLYMORPHISM IN ANGIOTENSIN-CONVERTING ENZYME GENE ON PROGRESSION OF DIABETIC NEPHROPATHY DURING ANGIOTENSIN-CONVERTING ENZYME INHIBITION

P.Jacobsen ${ }^{*}$,L.Tarnow ${ }^{*}$, P.Rossing ${ }^{*}$, F.Cambien ${ }^{\star \wedge}$, L. Lecerf $f^{\star \wedge}$, O.Poirier ${ }^{* \wedge}$ and H-H.Parving.Steno Diabetes Center, Gentofte,Denmark. AINSERM,Paris, France.

We tested the concept that a deletion polymorphism (DD) of the ACE gene predicts the therapeutic efficacy of ACE inhibition on progression of kidney function in diabetic nephropathy. Thirty five (DD, $n=11$, group $A$ vs $\mid D+11$, $\mathrm{n}=24$, group $B$ ) previously untreated young (mean age 33 (SD 7) years) hypertensive IDDM patients suffering from diabetic nephropathy, were investigated before and during ACEI for 8(3-9) and 7(3-9) years, respectively. GFR $\left({ }^{51} \mathrm{Cr}\right.$-EDTA plasma clearance), albuminuria (UAE), glycemic control $\left(\mathrm{HbA}_{1 \mathrm{c}}\right)$ and blood pressure (MABP) were measured $15(4-15)$ vs $9(3-16)$ times in group $A$ and $B$ respectively. In addition to captopril (mg/day) (A:75(37.5-100) and $B: 50(6.25-150)$. NS) $73 \%$ and $83 \%$ of the patients received a diuretic, respectively. At baseline the groups had comparable GFR's (m//min/1.73m²)(97(75-145) vs $98(26-146)$, respectively). UAE $(\mu \mathrm{g} / \mathrm{min})(530(95-2351)$ vs $713(179-3547)$, respectively), MABP $(\mathrm{mmHg})\left(111\right.$ (SD 7) vs 108(11), respectively) and $\mathrm{HbA}_{10}$ (\%) 9.7(SD 1.8) vs 9.0(1.2), respectively)(all NS). During ACEl A and B had UAE ( $\mu \mathrm{g} / \mathrm{min}$ ) of $484(118-6939)$ vs $546(49-2733)$, respectively, MABP $(\mathrm{mmHg})$ of 100 (SD 4) vs $99(9)$, respectively, and $\mathrm{HbA}_{1 \mathrm{c}}$ of 9.7 (SD 1.3) vs $9.5(1.2)$, respectively, (all NS). The sustained rate of decline in GFR (linear regression of all GFR measurements during $A C E I, \mathrm{ml} / \mathrm{min} / \mathrm{yr}$ ) was significantly steeper in $A$ than $B$ $4.7(13$ to 0.6$)$ vs $2.3(9.2$ to -2.5$)$, respectively $(p=0.01)$. A multiple linear regression analysis revealed that the $H_{b A_{10}} U A E$ and $D D$ genotype independently influences the sustained rate of decline in GFR, $R^{2}$ (adjusted) $=0.51$. Our study suggests that the deletion polymorphism in the angiotensinconverting enzyme gene reduces the long-term beneficial effect of angiotensin-converting enzyme inhibition on progression of diabetic nephropathy in insulin-dependent diabetic patients.

\section{5}

ANGIOTENSIN CONVERTING ENZYME POLYMORPHISM AND MICROVASCULAR COMPLICATIONS IN TYPE 1 DIABETES.

A. Bertacca, L. Pucci, G. Penno, M. Nannipieri, L. Rizzo, A. Rapuano, S. Bandinelli and R. Navalesi. Malattie Metaboliche - Pisa. Italy.

The occurrence of diabetic nephropathy (DN) in family clusters suggests that genetic factors may contribute to susceptibility. The relationship between DN and an insertion/deletion (VD) polymorphism in intron 16 of the ACE gene is still under debate.

That relationship has been examined in 177 type 1 diabetics. Microalbuminuria/nephropathy (n. 43/15) has been diagnosed when previous year median albumin to creatinine ratio was above 2.5 $\mathrm{mg} / \mathrm{mmol}$ in males and 3.5 in females. Hypertension was defined as SBP $\geq 140$ and/or DBP $\geq 90 \mathrm{mmHg}$ from mean values of the previous year of when patients were on anti-hypertensives. Retinopathy was classified as absent, background and proliferative. The mean value of $\mathrm{HbA} 1 \mathrm{c}$ measured bimonthly during the previous 3 years was used to evaluate glycaemic control. PCR was performed according to Rigat et al (with and without DMSO 5\%). To avoid misclassification of ID genotype as DD, each DD sample was subjected to a second, independent PCR with an insertion-specific primer-pair. Between patients with nephropathy (n. 58) and normo-albuminuric subjects (n. 119) there were no differences in age ( $36 \pm 9$ vs $36 \pm 10$ years), mean HbAlc levels $(8.2 \pm 1.5 \%$ vs $8.0 \pm 1.4 \%)$ and BMI. Disease duration was longer $(21 \pm 8$ vs $16 \pm 9$ years, $\mathrm{p}<0.001)$ and SBP and DBP higher (128/81 $\pm 20 / 15$ vs $118 / 74 \pm 14 / 9 \mathrm{mmH}$, $\mathrm{p}<0.005$ ) in subjects with DN. There was a significant difference in the distribution of ACE genotypes (DD/1D/II) between normoalbuminuric and nephropatic patients [46 (39\%)/ $49(41 \%) / 24(20 \%)$ vs $27(47 \%) / 28$ $(48 \%) / 3(5 \%) ; \mathrm{X} 2=6.79, \mathrm{p}=0.033$ ]. Marginally significant differences in genotypes distribution persist when only normoalbuminuric patients with diabetes duration longer than 15 years $(n .56)$ were included $\left(X^{2}=5.7\right.$, $\mathrm{p}=0.057$ ). There was no significant association between ACE genotype and both retinopathy and hypertension. 


\section{6}

POLYMORPHISMS IN THE INTERLEUKIN-I GENE CLUSTER AND DIABETIC NEPHROPATHY L.Tarnow, F.Pociot, P.M.Hansen, P.Rossing, F.S.Nielsen, B.V.Hansen, H.H.Parving. Steno Diabetes Center, Gentofte, Denmark.

Interleukin-1 (II-1) stimulates mesangial cell proliferation and extracellular matrix production, abnormalities characteristically found in diabetic glomerulosclerosis. The natural inhibitor of IL-1 (IL-1Ra) is a competitive inhibitor of the IL-1 receptor (IL-1RI). A penta-allelic insertion polymorphism in the IL-1Ra gene has been suggested as a risk marker for diabetic nephropathy. Polymorphisms in the genes coding for IL-1B and IL-1Ra is involved in regulation of the function of IL-1. The aim of the study was to examine the relationship between polymorphisms in the genes coding for IL-1B, IL-1Ra and IL-1R and diabetic nephropathy. We studied IDDM patients with diabetic nephropathy (122M/77F, age $40.9 \pm 10$ years, diabetes duration $27 \pm 8$ years) and with normoalbuminuria $(118 \mathrm{M} / 74 \mathrm{~F}$, age $42.7 \pm 10$ years, diabetes duration $26 \pm 8$ years

\begin{tabular}{|c|c|c|c|c|}
\hline \multicolumn{5}{|c|}{ Distribution of genotypes or alleles - data are n } \\
\hline & & NEPHROPATHY & NORMOALBUMINURIA & $\mathrm{p}$-value \\
\hline IL-1B & $\begin{array}{l}\mathrm{B}^{* 1} 1, \mathrm{~B}^{*} 1 \\
\mathrm{~B}^{* 1} 1 \mathrm{~B}^{*} 2 \\
\mathrm{~B}^{* 2} 2, \mathrm{~B}^{*} 2\end{array}$ & $\begin{array}{r}5 \\
86 \\
105 \\
\end{array}$ & $\begin{array}{r}13 \\
75 \\
104 \\
\end{array}$ & NS \\
\hline IL-1RI & $\begin{array}{l}3.2,3.2 \\
3.2,1.2 \\
1.2,1.2 \\
\end{array}$ & $\begin{array}{l}11 \\
93 \\
93 \\
\end{array}$ & $\begin{array}{l}18 \\
94 \\
80 \\
\end{array}$ & NS \\
\hline IL-1Ra & $\begin{array}{l}A_{1} \text {-allele } \\
A_{2} \text {-allele } \\
A_{3} \text {-allele } \\
A_{4} \text {-alle } \\
A_{5} \text {-allele }\end{array}$ & $\begin{array}{r}300 \\
84 \\
\mathrm{~g} \\
1 \\
0 \\
\end{array}$ & $\begin{array}{r}285 \\
88 \\
10 \\
1 \\
0 \\
\end{array}$ & NS \\
\hline
\end{tabular}

Our study does not support previous findings of an association between the $1 \mathrm{~L}-1 \mathrm{Ra}$ polymorphism and diabetic nephropathy, nor does polymorphisms in the IL-1B and IL-1RI genes contribute to the genetic susceptibility to diabetic nephropathy in Caucasian IDDM patients.
1137

Dde I MELTING POLYMORPHISM OF THE ANGIOTENSIN ICONVERTING ENZYME [ACE] LOCUS IS ASSOCIATED WITH THE DEVELOPMENT NEPHROPATHY IN NON-INSULIN-DEPENDENT DIABETES MELLITUS (NIDDM).

W. Grzeszczak, E. Żukowska-Szczechowska, K. Strojek

Department and Clinic of Internal Medicine and Occupational Diseases Silesian Academy of Medicine, 41-800 Zabrze, Poland

Diabetic nephropathy is the one of the major determinant of premature morbility and mortality in NIDDM patients. The identities of the genetic factors are unknown, but several pieces of evidence point to $\mathrm{ACE}$ as a candidate gen. In search of genetic determinants of susceptibility to diabetic nephropathy, we examined the association between DNA sequence differences at the locus of $\mathrm{ACE}$ and renal complications in 111 NIDDM patients with a diabetes duration over 10 years. This nested case control study included 76 nommoalbuminuric control subjects (albumin excretion rate $<20 \mu \mathrm{g} / \mathrm{min}$ ) (group I) and 35 cases with evidence of nephropathy ranging from microalbuminuria to overt proteinuria (group II). DNA was extracted from peripheral blood leukocytes. PCR was performed using genomic DNA. Dde I RFMP genotypes were determined by the DGGE. Some obtained results are presented in the table : $\left(X^{2}=9,242505 ; \mathrm{df}=2 ; \quad\right.$ pearson $\left.=0,00985\right)$

\begin{tabular}{|l|c|c|c|c|}
\hline \multicolumn{1}{|c|}{ Parameter } & \multicolumn{5}{|c|}{ Dde I alleles } \\
Group & - & \multicolumn{1}{c|}{+} & Sum \\
\hline $\begin{array}{l}\text { Group I } \\
\text { without nephropathy }\end{array}$ & $76(50 \%)$ & $8(5,3 \%)$ & $68(44,7 \%)$ & $152(100 \%)$ \\
\hline $\begin{array}{l}\text { Group II } \\
\text { with nephropathy }\end{array}$ & $26(37,1 \%)$ & $12(17,1 \%)$ & $32(45,71 \%)$ & $70(100 \%)$ \\
\hline
\end{tabular}

Conclusions: 1 . The "=" allele was significantly more frequent in NIDDM patients with nephropathy than in normoalbuminuric controls. 2. NIDDM patients with susceptible Dde I haplotypes ("=", "+") had a 1.69 increase in the risk of nephropathy compared to NIDDM individuals with other genotypes $(95 \% \mathrm{Cl}: 0,95$ $-3,02)$.

\section{8}

CLINICAL MANIFESTATION OF HEREDITARY PREDISPOSITION TO NEPHROPATHY IN TYPE II DIABETES

K.Strojek, W. Grzeszczak, E. Morawin, M. Adamski, B. Lacka, H. Rudzki, "CK Keller, "S.Schmidt and"E.' Ritz

Dept. of internal and Occupational Diseases, Silesian Med. Academy, Zabrze, Poland and "Dept of internal Medicine, Ruperto Carola University, Heidelberg, Germany.

Life expectancy, and quality of life, of patients with type II diabetes is limited by late complications. Family studies point to an important genetic element in the genesis of diabetic nephropathy, but is not known whether renal abnormalities are present prior to the onset of diabetes. To clarify this issue we examined an 425 consecutive type ll diabetic patients and examined all available normotensive, normoglycemic, non smoking offspring ( $n=56 ; 30$ of whom without parental history of nephropathy- $\mathrm{nDN}$ and 26 with- DN and compared them to 30 healthy offspring of non diabetic parents (controls). All groups had similar age and BMI. Measurements in offspring and controls included: night-time AER (median, range); AER after protein load; AER after submaximal physical exercise and ambulatory BP (mean \pm SD). Results:

\begin{tabular}{|c|c|c|c|}
\hline & Offspring no DN & Offspring DN & Controls \\
\hline AER night ( $\mu \mathrm{g} / \mathrm{min})$ & $4.8(0.3-18)^{a}$ & $7.8(1-19)^{a}$ & $4.4(0.1-18)$ \\
\hline AER after protein load & $24.5(4-227)^{a, b}$ & $40.2(11-431)^{a a}$ & $11.1(23-108)$ \\
\hline AER after exercise & $26.8(0.5-605)^{\mathrm{b}}$ & $90.2(4-1242)^{a a}$ & $17.5(2.4-241)$ \\
\hline Systolic BP $(\mathrm{mmHg})$ & $114 \pm 10.3$ & $121 \pm 16.7^{\mathrm{a}}$ & $112 \pm 10.3$ \\
\hline
\end{tabular}

a-p $<0.05$ vs controls; aa- $p<0.001$ vs controls; $b-p<0.05$ offspring no $D N$ vs offspring DN

The data document higher AER, basal and particularly post-exercise, as well as high $B P$ in offspring of diabetic patients with $D N$

\section{9}

CLUSTERING OF ABNORMALITIES IN ALBUMIN EXCRETION RATE (AER) IN FAMILIES OF TYPE 2 DIABETIC PATIENTS. R. Nosadini, E. Brocco, P. Faronato, A. Siriso, B. Muollo, F. Frigato, M. Vclussi, G.C. Tonolo, S, Inchiostro, M. Maioli. V. Donadon, A. Bueti, F. Santeusanio and S. Crepaldi. Italian NIDDM Nephropathy Study Group.

Clustering of diabetic nephropathy is observed in families of IDDM patients. Increased albumin cxerction rate (AER) is reported in first degree relatives of NIDDM The sib-pair model allows to study whether genetic predisposition plays a role in determining AER abnormalities in NIDDM, without hypothesis as to the mode of iransmission of this disorder or its penetranec and it accomodates genetic heterogencity. We examined: 1) the concordance rates for nicro-macroalbuminuria $1>30 \mu \mathrm{gr} / \mathrm{min})(\mathrm{MA})$ in two sets of families in which both probands and siblings had VIDDM." In one sel the probands had MA and in the other set no (NA). 2) the concordance rates for AER in two sets of families in which probands, but not siblings, had NIDDM. 233 sib-pairs concordant for NIDDM (onsci $>40$ ycars of age. no insulin treatment in the first three ycars of diseasc. the proband was the first to derelop NIDDM) and 140 discordant for NIDDM were identified. NIDDM probands were divided in two groups. MA or NA. The prevalence of MA in the overall VIDDM population (322 patients) was $36 \%$. The concordance for MA was $56 \%$ in 113 NIDDM sib-pars with one proband with MA $(p<0.01$ is $N A)$ and $32 \%$ in 120 NIDDM sib-pairs with one proband without MA. Duration of diabetes, prevalence of hypertension and HbA I c levels were similat in NIDDM siblings of probands with or without MA. The non diabetic siblings of the 140 sib-pairs discordant for NIDDM werc divided in two groups: A) 66 w'ith NIDDM probands with and B) 74 without MA. AER was $12 \mu \mathrm{g} / \mathrm{min}$ in $\mathrm{A}$ ) and 7 in B) (median, $p<0.056)$. Bloced pressure was similar in A) and B). Conclusions: 1) The concordance for MA in NDDM sib-pairs is higher when the proband has MA. 2) AER is higher in non diabetic siblings of VIDDM probands with MA, albeit within normal ranges. These findings support the vicu that familial, possibly genetic, factors contribute to determine abnormalitics in AER in NIDDM, at least partially independent of NIDDM and hypertension. 


\section{0}

\section{GLLICOSE INTOLERANCE IN PARENTS OF IDDM-PATIENTS} WITH DIABETIC NEPHROPATHY.

I. Fagcrudd, P-H. Groop and C. Grönhagen-Riska. Department of Mcdicine, Division of Nephrology, Helsinki, Finland.

(ienetic factors seem to play a role in the development of diabetic renal discase. Our aim was to study whether parental glucose intolerance is rciated to diabetic nephropathy in IDDM. We therelore studied parents of 21 normoalbuminuric (normo) and 26 proteinuric (prot) IDDMpatients of comparable gender, age, duration, BMI and smoking habits. An oral glucose tolerance test was performed in 27 parents ( 9 male/ 18 femalc) of normo and $36(15 \mathrm{M} / 21 \mathrm{~F})$ of prot. Parents of prot werc vounger ( $64 \pm 1$ vs $68 \pm 1$ years; $\mathrm{P}<05)$ and had a higher BMI $(29.2 \pm 0.7$ is $26.6 \pm 0.6 \mathrm{~kg} / \mathrm{m}^{2} ; \mathrm{P}<05$ ). Abnormal glucose tolerance was more common in parents of prot $(50 \%$ vs $26 \% ; \mathrm{P}=05)$ due to paternal excess of glucose intolerance ( $67 \%$ vs $22 \% ; P<.05$ ). Furthermore, fathers of prot tended to have higher $w /$ h-ratio $(0.98 \pm 0.02$ vs $0.92 \pm 0.02)$, higher HbA $1 \mathrm{c}(6.7 \pm 0.5$ vs $5.6 \pm 0.2 \%)$ and higher s-triglycerides $(2.4[0.8$ $5.5]$ vs $1.3[0.6-2.1] \mathrm{mmol} / 1)$. Parents of prot had higher systolic blood pressure ( $144 \pm 3$ vs $132 \pm 4 \mathrm{mmHg} ; \mathrm{P}<.05)$ and tended to have higher diastolic blood pressure $(80 \pm 2$ vs $75 \pm 2)$. We conclude that parents of IDDM-patients with diabetic nephropathy are glucose intolerant and, lurthermore, exhibit other features of the metabolic syndrome. Whether this finding is related to the development of diabetic nephropathy in IDDM remains to be elucidated.

\section{1}

INFLUENCE OF LIPOPROTEIN(a) PHENOTYPES ON ALBUMIN EXCRETION RATE IN DIABETIC PATIENTS C.Hernández ${ }^{1}$, R.Simó ${ }^{1}$, P.Chacón ${ }^{2}$, A.Segarra ${ }^{3}$, L. Garcia-Pascual ${ }^{4}$ and J.Mesa ${ }^{1}$. Depts. of ${ }^{1}$ Endocrinology, ${ }^{2}$ Biochemistry and ${ }^{3}$ Nephrology. Hospital Vall d'Hebron. Barcelona. ${ }^{4}$ Hospital Mutua de Terrassa. Spain. The aim of the study was to evaluate the relationship between serum lipoprotein(a) $[\mathrm{LP}(\mathrm{a})]$ concentrations and its phenotypes with albumin excretion rate (AER) in diabetes. We included 191 consecutive diabetic patients: 69 IDDM and 122 NIDDM.Lp(a) was determined by ELISA and its phenotypes by SDS-PAGE under reducing conditions followed by immunoblotting. Lp(a) phenotypes were grouped by size in small $(\mathrm{F}, \mathrm{B}, \mathrm{S} 1, \mathrm{~S} 2)$, big $(\mathrm{S} 3, \mathrm{~S} 4)$ and null. AER was assessed by RIA and expressed as the mean of three urine samples on 24 hour collections obtained in the last 9 months. Statistics: ANOVA and multiple regression analysis taking $L p(a)$ as a dependent variable and age, body mass index, AER, total cholesterol, cholesterol-LDL, cholesterol-HDL, tryglicerides, apo-B100, apo- $\mathrm{A}_{1}, \mathrm{HbA}_{1 \mathrm{c}}$ as independent variables. AER rate was independently correlated with serum $L p(a)$ concentrations $(R=0.31 ; p=0.001)$. This relationship remains at significant level when IDDM and NIDDM were analyzed separately. We observed differences in the AER when Lp(a)phenotypes were considered (small: X 55.2 \pm SD $122.5 \mu \mathrm{g} / \mathrm{min}$, median=4.9; big: X $58.5 \pm \mathrm{SD} 122.7 \mu \mathrm{g} / \mathrm{min}$, median $=5.7 ;$ null: X $3.2 \pm$ SD $2.4 \mu \mathrm{g} / \mathrm{min}$, median $=2.3 ; \mathrm{p}=0.019$. Low AER was observed in all patients with null Lp(a) phenotype. These patients did not differ in duration of diabetes in comparison with the other $L p(a)$ phenotype groups. Our results suggest that the elevated cardiovascular risk observed in diabetic patients with high AER might be associated to $\mathrm{Lp}$ (a) concentrations. Furthermore, patients with null Lp(a) phenotype could be considered as a group at low risk for the development of diabetic nephropathy.

\section{2}

APOLIPOPROTEIN E GENES IN DIABETIC NEPHROPATHY. TA Chowdhury, S Kumar, SCL Gough, AH Barnett and SC Bain. Department of Medicine, University of Birmingham and Birmingham Heartlands Hospital, Birmingham, UK.

IDDM patients with diabetic nephropathy have a dramatically increased risk of vascular disease. Apolipoprotein $E$ (apo $E$ ) is a unajor constituent of lipoproteins, playing an important role in lipoprotein metabolism by its interaction with receptors. Three alleles of the apolipoprotein $E$ gene are observed: $\varepsilon 2, \varepsilon 3$ and $\varepsilon 4$; presence of the $: 2$ allele is associated with reduced catabolism of triglyceride rich remnants and hence predisposes to atherogenesis. The aim of this study was to determine the role of the apolipoprotein $\varepsilon$ polymorphism as a risk factor in diabetic nephropathy. Apo $E$ genolypes were determined by RFLP analysis of polymerase chain reaction amplified genomic DNA. Genotypes in 233 IDDM patients with nephropathy (presence of retinopathy, hypertension and persistent proteinuria in the ahsence of other (auses) were compared to 196 recently diagnosed IDDM subjects (background IDDM population) and 138 IDDM patients with long duration of disease $(>20$ yearsi) and no nephropathy. All IDDM subjects were white Caucasian. Frequency of the $e 2 / 2,2 / 3$ and $2 / 4$ genotypes were signifitantly higher in the nephropathy group compared to the recently diagnosed group $(p<0.01)$ and the long duration no nephropathy group ( $\mathrm{P}<0.05)$. A po 82 allele frequency was significantly higher in the nephropathy group $(10.5 \%)$ compared to the recently diagnosed IDDM grour $(3.8 \%)(p<0.01)$ and the long duration no nephropathy group $(4.3 \%)(p<0.01)$. Presence of the Apo 82 allele may be a risk factor for the development of nephropathy in IDDM subjects.

\section{3}

\section{HEREDITARY RISK DETERMINANTS OF MICRO- AND MACROALBUMINURIA IN YOUNG IDDM PATIENTS \\ EL Stattin, S Rudberg, G Dahlquist. \\ Department of Pediatrics. Umeả University, Umeå and Department of Women and Child Health, Karolinska Institute, Stockholm.}

The aim of the study was to investigate in a population based study the influence of familial cardiovascular disease (CVD), hypertension, diabetic nephropathy and non-insulin-dependent diabetes mellitus on the risk to develop diabetic nephropathy. Seventyfive insulin-dependent diabetes mellitus (DDM) patients with albumin excretion rate (AER) $>15 \mu \mathrm{g} /$ minute in at least two consecutive overnight samples were compared with three normo-albuminuric controls per case matched for diabetic duration. In the univariate analysis CVD among relatives (three generations) and hypertension (with medication) among siblings and parents were associated with significant risk increases $O R=1.96(p=0.02)$ and $\mathrm{OR}=3.78(\mathrm{p}=0.005)$ respectively. However, diabetic nephropathy and NDDM in three generations were not. Female/male sex ratio, current age, BMI, current blood pressure as well as prevalence of retinopathy were significantly higher among cases than controls. Using conditional logistic regression analysis it was shown that familial cardiovascular disease and familial hypertension were independent risk determinants for micro- and macroalbuminuria. When adjusting for current HbAIC, current blood pressure, BMI, age and sex, familial hypertension only remained independently significant. In conclusion, this large population based nested case-control study showed that familial hypertension and cardiovascular disease were associated with an increased risk for micro- and macroalbuminuria independently of each other. This indicates that they may both be risk determinants for diabetic nephropathy. 


\section{Microalbuminuria and Hyperfiltration}

1144

HYPERFILTRATION IN TYPE I DIABETIC PATIENTS

L.Todaro, P.Cotroneo, A.Manto, C.Saponara, P.Magnani, M.Garganese*, G.D'Errico*, AV.Greco, G.Ghirlanda. Medicina Interna e Gerontologia, * Medicina Nucleare, Università Cattolica del S.Cuore, Roma, Italy.

The increase in glomerular filtration rate (GFR) is a necessary but not sufficent prerequisite for the development of diabetic nephropathy. An 8 year-follow-up study showed that hyperfiltration has a positive predictive value for incipient/overt nephropathy. The aim of our study was to evaluate the prevalence of hyperfiltration in 159 normotensive IDDM patients (mean age $33 \pm 10 \mathrm{yrs}$, duration of disease $15 \pm 10 \mathrm{yrs}$ ) in comparison with 58 control subjects (mean age $29 \pm 6 \mathrm{yrs}$ ). GFR and renal plasma flow (ERPF) were evaluated by single injection technique for $\mathrm{Cr}{ }^{51}$ EDTA and $\mathrm{I}^{125}$ Hippuran with 11 point sampling, $\mathrm{HbA} 1 \mathrm{c}$ levels by HPLC method, albumin excretion rate (AER) by radioimmunoassay. Mean value of GFR in controls was $120 \pm 15 \mathrm{ml} / \mathrm{min} / \mathrm{m}^{2}$ : patients with GFR $>150 \mathrm{ml} / \mathrm{min} / \mathrm{m}^{2}$, corresponding to the mean $\pm 2 \mathrm{SD}$ of controls, were defined as hyperfiltering. 58 diabetic patients were hyperfiltering: HBA 1 c $7.66 \pm 1 \%$, GFR $171 \pm 20 \mathrm{ml} / \mathrm{min} / \mathrm{m}^{2}$, ERPF $707 \pm 157 \mathrm{ml} / \mathrm{min} / \mathrm{m}^{2}$ and Filtration Fraction (FF) $0.254 \pm 0.070$; while 105 diabetics were normofiltering: $\mathrm{HBA}_{1} \mathrm{c} 7.45 \pm 1.2 \%$, GFR $126 \pm 19 \mathrm{ml} / \mathrm{min} / \mathrm{m}^{2}, \mathrm{p}<0.0601$; ERPF $601 \pm 139 \mathrm{ml} / \mathrm{min} / \mathrm{m}^{2}, \mathrm{p}<0.0001$ and FF $0.220 \pm 0.052, \mathrm{p}<0.001$. In our population we found a prevalence of hyperfiltration of $34 \%$. There was a positive correlation between GFR and ERPF ( $p<0.0001)$, a negative correlation between GFR and the age of patients $(p=0.002)$ and between GFR and duration of disease $(p=0.043)$. Hypérfiltration was not correlated to poor metabolic control $(\mathrm{HbA} 1 \mathrm{c})$ or to high protein intake. The increase in GFR was strictly related to augmented ERPF, suggesting that hyperfiltration is mainly linked to vasodilation of afferent arterioles. This may be due to hyporesponsiveness to vasoconstrictor stimuli (angiotensin II, catecholamines) or to prevalence of vasodilatory peptides (kinins, prostaglandins). In hyperfiltering patients the vasodilation of afferent arterioles seems to be associated

\section{6}

DETERMINANTS OF GLOMERULAR HYPERFILTRATION IN JUVENILE IDDM SUBJECTS

B. BOUHANICK, M MARRE, Y GALLOIS, J J LE LEUNE, V P.OHMER and J.M. LIMAL. ANGERS - FRANCE.

Kidney damage may be predicted by glomerular hyperfiltration in IDDM subjects. In a cross-sectional study of 139 juvenile (age $<35$ years) IDDM subjects ( $\mathrm{M} / \mathrm{F}$ ), we studied the relationship between Glomerular Filtration Rate (GFR; ${ }^{51} \mathrm{Cr}$ EDTA plasma disappearance: hyper $\geq 135$, normo $134-100$, hypo $\leq 100 \mathrm{ml} / \mathrm{min} / 1.73 \mathrm{~m}^{2}$ ) and age, sex, age at IDDM onset, IDDM duration, HbAlc, normo- micromacroalbuminuria $(\mathrm{N} / \mu / \mathrm{M})$, and angiotensin I converting enzyme $\mathrm{I} / \mathrm{D}$ polymorphism (DD/ID/II). Results are median (range) or mean \pm SD.

$\begin{array}{lllll}\text { GFR } & \text { Hyper } & \text { Normo } & \text { Hypo } & \mathrm{p} \\ \mathrm{n} & 37 & 89 & 13 & \mathrm{NS} \\ \text { Sex (M/F) } & 19 / 18 & 46 / 43 & 8 / 5 & .0077 \\ \text { age (years) } & 20(9-35) & 25(12-35) & 29(19-35) & .02 \\ \text { age at IDDM onset } & 11(1-27) & 14(0-33) & 10(3-19) & .02 \\ \text { (years) } & & & & \\ \text { IDDM duration (years) } & 9(2-23) & 9(1-30) & 19(5-24) & .059 \\ \text { HbAlc (\%) } & 9.3 \pm 1.7 & 9.1 \pm 1.9 & 9.2 \pm 1.6 & \mathrm{NS} \\ \text { N/ } / \mathrm{M} & 28 / 9 / 0 & 70 / 14 / 5 & 7 / 1 / 5 & .001 \\ \text { DD/ID/II } & 11 / 21 / 5 & 32 / 44 / 13 & 4 / 8 / 1 & \mathrm{NS}\end{array}$

Subjects with hyper GFR were younger $(\mathrm{p}=.0132)$ and younger at IDDM onset $(\mathrm{p}=02)$ than those with normo GFR. In a stepwise multiple regression analysis including age, IDDM duration, $\mathrm{HbA} / \mathrm{c}$ and urinary albumin, age was the only independent determinant of GFR $(F=12.4)$; this persisted after exclusion of subjects with hypoGFR $(\mathrm{F}=7.6)$. Among the subjects aged $<20$ years, $41 \%$ displaved nyperGFR. In this cross-sectional study, age seems the only determinant of glomerular hyperfiltration in juvenile IDDM subjects.

\section{5}

GLOMERULAR HYYERFILTRATION IN INSULIN-DEPENDENT DIABETES MELLITUS: RENAL COLOR DOPPLER ULTRASOUND FINDINGS

M.A.Marzano, P. Cotroneo, F. De Luca, C. Saponara, C. Cellerino, A. Manto, M. Pompili, G.L.Rapaccini and G. Ghirlanda.

Institute of Internal Medicine, Catholic University of Sacred Heart, Rome, Italy.

Diabetic nephropathy is a common cause of renal dysfunction and one of the main complications observed in long term diabetes. Glomenular hyperfiltration represents the first prealbuminuric stage of renal involvement. It is well known in literature that Doppler ultrasound (US) adds to the anatomic information provided by simple US imaging physiopathologic data about the charateristics of intrarenal flow and it is useful in the study of a variety of renal pathologies such as acute prerenal failure, acute tubular necrosis and non obstructive dilation of the collecting system. Aim of this study was to compare Doppler US with radioisotopic glomerular filtration rate (GFR) assay and laboratory tests in the detection of renal involvement. 76 normotensive patients with IDDM and 19 normal subjects (average age $30 \pm 6$ yrs) underwent duplex-color Doppler US blind examination. The IDDM patients were divided into 3 groups according to GFR and albumin urinary escretion. Group I: 34 normoalbuminuric patients (average age $31 \pm 6$ ) with hypeffiltration; gxoup II: 33 patients (average age $35 \pm 10$ ) with normal GFR and normal urinary albumin excretion; group III: 9 normofiltering microproteinuric patients (mean age $38+14$ yrs). We measured the volume of the kidneys and the renal interlobar artery Resistivity Index [RI=(peak systolic value-end diastolic value)/ peak systolic valuc ]. The RI value was $0.55+0.06$ in group I; $0.58+0.05$ in group II and $0.59 \pm 0.08$ in group III and $0.57 \pm 0.04$ in normal subjects. Mean renal volume resulted $354 \pm 62,319$ $\pm 57,323 \pm 62$ and $335 \pm 66 \mathrm{ml} / 1.73 \mathrm{~m}^{2}$ respectively. Statystical analysis was worked out by the ANOVA test, considering significant $p$ values of 0.05 or less and showed a significant RI difference between group I and group IL. Despite a positive trend, no statistical difference was observed in renal volume. Taking 0.5 as the lower normal limit, a RI $<0.5$ showed, in the detection of renal hyperfiltration in IDDM, a sensitivity of $29 \%$, a specificity of $95 \%$, a positive predictive value of $73 \%$ and a predictive negative value of $76 \%$. This study suggests a promising rolc of Doppler US as a safe, non invasive method in the study of early renal involvment in IDDM, although the low sensitivity of the test does not permit its introduction as a screening test.

\section{7}

THIS ABSTRACT HAS BEEN WITHDRAWN BY THE AUTHOR. 
1148

CORRELATION OF INSULIN-LIKE GROWTH FACTOR-2 (BUT NOT IGF-1) WITH GFR IN IDDM PATIENTS WITH HYPERFILTRATION. Soper C P R, and Hyer S L. St Helier Renal Unit \& Diabetic Centre, Carshalton, Surrey, UK.

Early haemodynamic abnormalities in IDDM contribute to renal injury in animal models, and may be significant in man. IGF-2 receptors are upregulated in nephrectomy models and in streptozotocin-induced diabetes prior to hypertrophy. We examined GFR in 29 IDDM patients [16 male, aged 18-43 yrs, duration of diabetes 4-8 yrs]. GFR was determined by inulin clearance, by a urineless method. Protein intake was kept strictly below $10 \mathrm{~g}$, no caffeine, or aicohol ingested, and smoking forbidden for 12 hours before testing. PAH clearance, HbAlc fructosamine, glucose at the end of the infusion, IGF $-1,-2$, and IGF Binding Protein (IGFBP) -1 and 3 were determined. Results were analysed by paired sample Student $t$ testing and correlations by linear regression. The influence of two other covariates was then analysed by ANOVA.

GFR was significantly correlated with IGF-2 levels, $r=0.39, p=0.036$. No single data point was essential to the significance of this correlation. No significant correlation was identified between GFR and IGF-1 or glycaemic control (HbAlc, Fructosamine, or glucose). IGF-2 did not show any correlation with glycaemic control. Analysis of variance between GFR and IGF-2, using IGFBP-1 and IGFBP-3 as additional covariates weakened the correlation slightly. ERPF and filtration fraction showed no correlation with IGF 1 or 2 .

Since IGF-2 is renally excreted, an inverse correlation with GFR would be expected, rendering the results more striking. These findings suggest a significant association between diabetic hyperfiltration and IGF-2, which merits further study.
1149

THE IMPORTANCE OF AGE IN DEFINING GLOMERULAR HYPERFILTRATION IN NON INSULIN DEPENDENT DIABETES. K. de Abrew, L. George, F. Dunstan, S. Luzio, W. Evans, A. Burch, J. Peters, J. Vora and D. Owens, Cardiff, UK.

The study was to investigate the influence of age on glomerular filtration rate (GFR) and the effect of age adjustment of GFR on the prevalence of hyperfiltration in newly diagnosed non-insulin dependent diabetes mellitus (NIDDM) subjects. 157 previously untreated normotensive non-proteinuric NIDDM patients, mean (SD) age $51.7(10.7)$ years and 82 heal thy controls were studicd. GFR and effective renal plasma flow (ERPF) were determined simultaneously by using $\mathrm{Cr}^{\text {si }}$ EDTA and $I^{125}$ Iodohippurate respectively. The supine blood pressures for the NIDDM subjects and controls were $131.5(18.1) / 82.5(9.8)$ vs $119.5(13.9) / 76.6(9.0) \mathrm{mmHg}$, with fasting plasma glucose $12.4(3.3)$ vs $5.3(0.5) \mathrm{mmol} / \mathrm{H}, \mathrm{HbAl} 11.0(2.3)$ vs $6.2(0.9) \%$ and total triglycerides $2.7(2.3)$ vs $1.5(1.0) \mathrm{mmol} / 1$ respectively. BMI was higher in the NIDDMs with mean $28.9(4.9)$ vs $26.5(4.8) \mathrm{kg} / \mathrm{m}^{2}$. Mean GFR was $119(22.4)$ in NIDDM compared with $101.8(20.2) \mathrm{ml} / \mathrm{min} / 1.73 \mathrm{~m}^{2}$ in the controls. GFR decreased linearly with age in both NIDDM and controls. The regression equations were mean GFR $=153-0.99 \mathrm{x}$ age for the controls and mean GFR $=179$. $1.16 \mathrm{x}$ age for NIDDM. The difference in slopes was not significant but there was a significant difference between the intercepts $(\mathrm{P}=0.03)$, showing that the age dependence was similar but the values were higher on average in NIDDM. Age dependent reference limits were derived from the controls, using Altman's method; those NIDDMs with GFR higher than the upper limit $(188-0.99 \mathrm{x}$ age $)$ were classed as hyperfiltrators, those below the lower limit (118-0.99 $\mathrm{x}$ age) as hypofiltrators. These classifications were not the same as those obtained ignoring age, that is defining normofiltrators to be within 2 SDs of the mean, (65.9-137.8), with hyperfiltrators above $137.8 \mathrm{ml} / \mathrm{min} / 1.73 \mathrm{~m}^{2}$ and hypofiltrators below 65.9 $\mathrm{ml} / \mathrm{min} / 1.73 \mathrm{~m}^{2} .27 \%$ were now classed as hyperfiltrators, as opposed to $18.4 \%$ using constant reference limits. 16 subjects who would not be classed as hyperfiltrators using the constant limits were now regarded as hyperfiltrators.

\section{0}

PREDICTORS OF RENAL MORPHOLOGICAL CHANGES IN THE TRANSITIONAL STAGE OF MICROALBUMINURIA IN IDDM ADOLESCENTS

S Rudberg, R Østerby, G Dahlquist, G Nyberg and B Persson. Department of Women and Child Health, Karolinska Institute, Stockholm; Department of pediatrics, Umeå University Hospital, Umeå; Transplant Unit, Sahlgrenska Hospital, Göteborg, Sweden and Electron Microscopy Lab, Institute of Pathology, Arhus University, Árhus, Denmark

The aim of the present longitudinal study was to investigate the influence of putative clinical risk determinants on the degree of renal morphometric changes in early glomerulopathy. Fifteen adolescents ( 7 boys/ 8 girls), 18.6 (15.4-23.3) years old (mean, 95\% confidence interval), with $10.9(6.0-16.0)$ years of insulindependent diabetes, and with low-grade microalbuminuria $(15-30 \mu \mathrm{g} / \mathrm{min})$ participated All patients had a prepubertal onset of diabetes. Seventeen living kidney donors served as healthy controls. 5-year mean $\mathrm{HbAlc}$, systolic- and diastolic blood pressure, 3-year mean albumin excretion rate, glomerular filtration rate-, cholesterol-, and triglycerides $2-5$ years prior to renal biopsy, age, duration of diabetes were investigated and related to basement membrane thickness, matrix and mesangial volumne fractions and the overall glomerulopattiy index [(basement membrane thickness/10+matrix volumne fraction, \%) +matrix stal volumnel. Basement membrane thickness and the overall diabetic glomerulopathy were increasead in diabetic patients as compared to controls ( $p<0.001$ respectively), whereas matrix volumne fraction tendend to be increased $(p=0,11)$. Mesangial volumne fraction did not differ between groups. In multivariate analysis, basement membrane thickness was predicted by 5 -year mean $\mathrm{HbAlc}$ diabetes duration and previous glomerular filtration rate $\left(\mathrm{R}^{2}=0.71, p=0.003\right)$. With matrix volumne fraction as the dependent variable, basement membrane thickness and diabetes duration were the only significant determinants $\left(\mathrm{R}^{2}=0.63, \mathfrak{p}=0.003\right)$. Diabetes duration, 5-year mean HbAlo and glomerular filtration rate were the variables with an inclependent influence on the overall diabetic glomerulopathy index $\left(\mathrm{R}^{2}=0.72, \mathrm{p}=0.003\right)$. Conclusion: In the transitional stage of microalbuminuria, a high percentage of the variation in basement membrane thickness and overall severity of glomerulopathy is explained by prolonged hyperglycemia, diabetes duration, and previous glomerular hyperfiltration. Preceding blood pressure- and lipid levels or age do not seem to have an independent on these morhpometric changes in our series of adolescents.

\section{1}

ELEVATED BLOOD PRESSURE IN MICROALBUMINURIC IDDM PATIENTS IS ASSOCIATED WITH LOW RENAL PLASMA FLOW P.L. Poulsen, E. Ebbehøj, and C.E. Mogensen, Medical Department M (Diabetes \& Endoctinology), Aarhus Kommunehospital, DK-8000 Aarhus C, Denmark. Background: The pathophysiological background for the concomitant rise in urinary albumin excretion (UAE) and blood pressure remains to be elucidated. New ultrastructural findings indicate abnormalities in renal arterioles of microalbuminuric patients, possibly affecting renal function and blood pressure. Objective: To evaluate possible associations between renal function, ambulatory blood pressure (AMBP) and glycemic control in microalbuminuric IDDM patients compared to normoalbuminuric patients. Methods: Nineteen male patients (age $33 \pm 6$ y) with slight microalbuminuria (UAE $20-70 \mu \mathrm{g} / \mathrm{min}$ ), compared to 19 normoalbuminuric (UAE $<15 \mu \mathrm{g} / \mathrm{min}$ ) age matched (age $33 \pm 6 \mathrm{y}$ ), male patients. Using constant infusion technique, ${ }^{225}$-iothalamate marked glomenlar filtration rate (GFR) and ${ }^{131}$ hippuran effective renal plasma flow (RPF). AMBP was measured by oscillometric technique (Spacelabs 90202). Results: The microalbuminuric group had higher daytime systolic AMBP $(132 \pm 11$ compared to $125 \pm 7 \mathrm{mmHg}, \mathrm{p}<0.05)$ and a poorer glycemic control $\left(\mathrm{HbA}_{\mathrm{ic}} 9.5 \% \pm 1.5\right.$ compared to $\left.8.2 \% \pm 1.3, \mathrm{p}<0.01\right)$. GFR $(135 \pm 22$ and $135 \pm 17 \mathrm{ml} / \mathrm{min})$ and $R P F(598 \pm 112$ and $542 \pm 98 \mathrm{ml} / \mathrm{min})$ were similar in the two groups. In the microalbuminuric group daytime systolic AMBP was inversely correlated to both RPF $(\mathrm{r}=-0.77, \mathrm{p}<0.005)$ and GFR $(\mathrm{r}=-0.53$, $\mathrm{p}=0.02) . \mathrm{HbA}_{1 \mathrm{c}}$ and GFR correlated positively in the microalbuminuric group $(r=0.47, p<0.04)$. In contrast, the nomoalbuminuric patients exhibited no such associations. Conclusions: The presence of elevated AMBP and the strong negative association with RPF in low grade microalbuminuric IDDM patients leaves several possibilities of interpretation: Primary blood pressure increase (of unknown origin) may induce morphological changes leading to reduction in renal function. Alternatively, blood pressure increase early in the course of incipient nephropathy may represent a compensatory mechanism, initially aiming at preservation of renal function, but later becoming maladaptive. 


\section{2}

SALT SENSITIVITY OF BLOOD PRESSURE AND INSULIN RESISTANCE ARE ASSOCIATED IN MICROALBUMINURIC INSULIN-DEPENDENT DIABETIC PATIENTS.

D. Bruttomesso, R. Trevisan, M. Vedovato, A. Semplicini, E. lori, C. Marescotti, A. Pianta, G. Guarnieri, A. Tiengo, S. Del Prato. Department of Clinical and Experimental Medicine, Padova, Italy

Clustering of insulin resistance and increased blood pressure occurs in microalbuminuric insulin-dependent diabetic patients (IDDs). Moreover exchangeable $\mathrm{Na}^{+}$is increased and correlates with bload pressure in IDDs Since a relationship between increase in blood pressure in response to $\mathrm{Na}$ intake (salt sensitivity) and insulin action was suggested, we assessed blood pressure, body weight, renal hemodynamics and insulin-sensitivity (euglycemic $100 \mu \mathrm{U} / \mathrm{ml}$ insulin clamp) in 8 normoalbuminuric (MA-) and 5 microalbuminuric $(\mathrm{MA}+)$ IDDs after 6 days on a low $(20 \mathrm{mEq}) \mathrm{Na}^{+}$diet and again after 6 days on a high $(250 \mathrm{mEq}) \mathrm{Na}^{+}$diet. in MA-, low or high $\mathrm{Na}^{+}$ diets did not affect mean blood pressure (MBP) $(84 \pm 3 \mathrm{mmHg}$ [mean \pm S.D. vs $87 \pm 4$, but it increased significantly in $\mathrm{MA}+(94 \pm 3 \mathrm{mmHg}$ vs $103 \pm 3$; $\mathrm{p}$ < $0.01)$. After high $\mathrm{Na}^{+}$diet, $\mathrm{MA}+$ gained more weight $(+3.3 \pm 1 \mathrm{Kg})$ than $\mathrm{MA}$ $(+19+1 ; p<005)$ and in response to angiotensin II ( $3 \mathrm{ng} / \mathrm{Kg} / \mathrm{min})$, they had greater MBP increase $(+15 \pm 3 \mathrm{mmHg})$ and smaller decrement in renal plasma flow $\left(-180 \pm 23 \mathrm{ml} / \mathrm{min} / 1.73 \mathrm{~m}^{2}\right)$ than in MA- $(+7 \pm 4$ and $-236 \pm 15$ respectively: $p<0.05$ for both). Insulin-mediated glusose disposal was lower in MA+ $(6.5 \pm 1.5 \mathrm{mg} / \mathrm{Kg} / \mathrm{min})$ than in MA- $(10.2+2.5 ; p<0.05)$. In conclusion, in IDDs microalbuminuria is associated with 1 . an increased responsiveness of blood pressure to salt intake and angiotensin II, 2. impaired modulation of renal blood flow, and 3 . insulin resistance. Therefore, salt sensitivity in IDDs clusters with other factors which are likely to play an important role in the pathogenesis of diabetic nephropathy and cardiovascular complications.

\section{3}

MICROALBUMINURIA IN RELATION TO INSULIN RESISTANCE IN A GENERAL CAUCASIAN POPULATION: THE HOORN STUDY A. Jager', J.N.D. de Neeling', C.D.A. Stehouwer' and R.J. Heine' ${ }^{12}$. 'EMGO Institute, 2Department of Internal Medicine, Vrije Universiteit, Amsterdam, NL. l'o assess whether microalbuminuria (MA) in elderly Caucasian subjects is associated with insulin resistance (IR)-related variables, we studied an age-, sex- and glucose tolerance- stratified sample from a 50-74 year-old general population $(n=680)$. The urinary albumin concentration was measured in a first morning urine sample upon arising by radio-immunodiffusion, with a threshold of $6.2 \mathrm{mg} /$. A valid albumin /creatinine ratio (ACR) could be obtained from 220 subjects with normal (NGT), 137 with impaired (IGT) glucose tolerance, 75 with newly-detected (NDM) and 41 with known (KDM) diabetes. Insulin and proinsulin wese measured by a highly specific double antibody RIA in plasma two hours after an oral glucose load, and included in the analysis only if the fasting glucose was $<8.0 \mathrm{mmol} / \mathrm{K}$ KDM was associated wilth a 1.7-fold increase in the median ACR $(0.60 * 3.04 p=0.011)$. The presence of hypertension (WHO-criteria) was associated with a 1.5 -fold increase in the median ACR $(0.53$ vs $0.82 p=0.000$ ), aiso after adjusting for $\mathrm{KDM}$. After exclusion of the $\mathrm{KDM}$ subjects, all IR-related variables (see p-values in the table) showed a significant relationship with $\mathrm{ACR}$, except for HDL-cholesterol and triglycerides. However, after adjusting for age, the associations with (pro)insulin were not significant anymore When systolic blood pressure (SBP) was also added to the regression model, the statistically significant associations with post load glucose and waist to hip ratio (WHR) disappeared as well.

\begin{tabular}{llllllll}
\hline & $\begin{array}{l}\text { Glucose } \\
\text { post-load }\end{array}$ & $\begin{array}{l}\text { Insulin } \\
\text { post-load }\end{array}$ & $\begin{array}{l}\text { Proinsulin } \\
\text { post-load }\end{array}$ & WHR $^{1}$ & $\begin{array}{l}\text { HDL- } \\
\text { chol. }\end{array}$ & $\begin{array}{l}\text { Trigly- } \\
\text { cerides }\end{array}$ & SBP $^{1}$ \\
\hline univ. $^{2}$ & 0.006 & $\mathbf{0 . 0 4}$ & $\mathbf{0 . 0 1}$ & $\mathbf{0 . 0 0 5}$ & 1.00 & 0.17 & $\mathbf{0 . 0 0 0}$ \\
+ age & 0.04 & 0.36 & 0.12 & $\mathbf{0 . 0 3}$ & 0.75 & 0.11 & $\mathbf{0 . 0 0 0}$ \\
+ SBP & 0.29 & 0.96 & 0.36 & 0.08 & 0.88 & 0.23 & - \\
\hline
\end{tabular}

stronger predictors than BM and diastolic blood pressure, respectively, ${ }^{2}$ univariate associations We conclude that in this Caucasian population, microalbuminuria is strongly related to known diabetes and blood pressure, but doesn't show a clear association with any other insulin restistance-related variable

\section{4}

WHAT OF WSLLAN ON VASCULAR REACTIVITY MICRO- AND YOR YO YLBLAINE RIC PATIENTS VERSES NON-DIABETIC CONTROLS

WW Salvage", A.B. Walker". P.Chartington". M. Wallymaheed", and G. Williams"

"Roval Liverpool Iniversity Hospital, Prescot St, LIVER̄POOL L7 8XP 1) Dapanent af Medicine Eniversity of Liverpool, PO Box 169. LIVERPOOL L69 WX: and Diabeles Centre. Walton Hospital Rice Lane LIVERPOOL, UK

Diabetes is a condition that is characterised by abnomal vascular reactivity Microalbuminuric $(20-200 \mathrm{~g} / \mathrm{min})$ diabetic subjects may have a vasculature that is

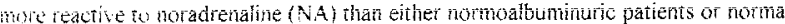
non-diabetic controls There may be a pathogenic role for insulin. We present a study of small resistance arteries in non-diabetic controls $(n=11$, age $47.2[4.11] \mathrm{yr}$; BMI $25.41 .22] \mathrm{kg} / \mathrm{m}^{2}$ : mean BP $98.6[2.75] \mathrm{mmHg}$ vessel diameter $\left.223.9[2 ! 13] \mathrm{km}\right)$, as

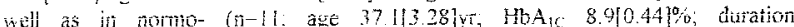

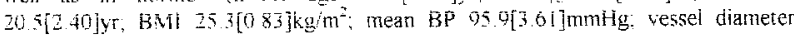
$232.2[22.06] \mathrm{mm})$, and microalbuminuric diabetic subjects $(\mathrm{n}=11$; age $35.1[3.74 \mathrm{fr}$

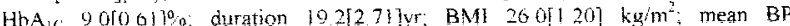

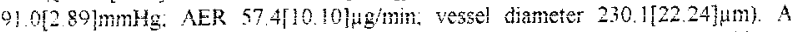
sinall biopsy of skin was removed under local anaesthesia Two arteries per biopsy were studied in a myouraph. If the vessels generated $100 \mathrm{mmH}$. NA dosc-response curves wert constructed. $9.44 \times 10^{-9}$ to $7.2 \times 10^{-6}$ molh Vessels were then exposed to insulin for 20 minutes at low and medium-high physiological concentrations 15 and $50 \mu \mathrm{l} / \mathrm{ml}$ ). The $\mathrm{NA}$ drise-response cuives were repeated at the two insulin levels Analysis of the $\mathrm{NA}$ dose-response showed that there were no differences between them, $\mathrm{F}=0.848_{126,}, \mathrm{p}=0.429$. Furthermore there was no effect of insulin in any group and there was no difference in mavimal force generated before and atier insulin There was increased mean force senerated in the ressels from microalbuminuric subjects, but this did not reach significance, $F=2.144_{12.32,}, p=0.135$. These data neither support the hypothesis that microalbuminuric vessels are more reactive, nor the hypothesis that insulin has a vasodilator action in resistance arteries

\section{5}

PREVALENCE AND RISK FACTORS FOR MICROALBUMINURIA IN ELDERLY PATIENTS WITH NIDDM

Erbas T, Gürlek A, Gedik O. Hacettepe University, Faculty of Medicine, Ankara, Turkey

It has been suggested that microalbuminuria in elderly diabetic patients may be caused not only by diabetic glomerular changes but also by glomerular changes secondary to aging and hypertension. The aim of this study was to determine the prevalence of microalbuminuria in middle-aged and elderly diabetic patients and their relationship with some known risk factors. Totally 169 diabetic patients were studied They were 79 middle aged patients less than 65 years $(57.6 \pm 4.6,35 \mathrm{M} / 44 \mathrm{~F})$ and 90 elderly patients aged 65 or more $(70 \pm 49,34 \mathrm{M} / 56 \mathrm{~F})$. Fasting plasma gilicose HbA1c, cholesterol, triglyceride. HDL, LDL, VLDL, blood pressure and BM! showed no significant difference between two groups. The levels of BUN and creatinine, the duration of diabetes and hypertension were higher in elderly patients. The prevalence of microalbuminuria was almost identical in elderly $(22.2 \%)$ and middle-aged $(13.9 \%)$ patients. The prevalence of coronary heart disease ( 52.3 vs $27.9 \% \quad p=0.004$ ), cerebrovascular disease (18.9 vs $3.8 \% \mathrm{p}=0.002$ ) cataract ( 576 vs $14.5 \% \mathrm{p}=0.0001$ ), peripheral neuropathy $(72.2$ vs $48.1 \% \mathrm{p}=0.001)$ and autonomic neuropathy (18.2\% vs $65 \% \mathrm{p}=0.02)$ were significantly higher in elderly patients. A significant but weak, correlation was observed between microalbuminuria tevel and systolic blood pressure in elderly patients $(r=0.36 \quad p=0.0001)$ in elderly microalbuminuric patients, OFs $(95 \% \mathrm{Cl})$ for retinopathy, autonomic neuropathy and peripheral neuropathy were $7.36(2.23-24.31$ ), 5.9 (f 8 $19.3)$ and 10.0 (1.2-79.9), respectively. In multiple logistic regression variables independently related to microalbuminuria in elderly patients were retinopathy $(O R=7.53 p=0.01)$ and autonomic neuropathy $(O R=10.41$ $\mathrm{p}=0.017$ ). The results of this study showed that prevalence of microalbuminuria did not differ between elderly diabetics and middle-aged diabetics. Elderly patients with microalbuminuria had higher retinopathy and neuropathy prevalence than patients without microalbuminuria 
1156

PREVALENCE AND RISK FACTORS FOR MICRO- AND MACROALBUMINURIA IN NIDDM PATIENTS

A. Colorado, A. Becenra, V. Lechón, O. González, G. Piédrola and N. Palacios. Hospital Ramón y Cajal, Madrid, Spain.

Microalbuminuria (MI) has been extensively studied in IDDM. Far less recognition and dedication, however, has been awarded to its study in NiDDM unti very recently. In NIDDM, MI is not only an independent predictor of progressive renal disease but also an important marker of athercscterotic disease and premature death. The aim of this cross-sectional study was to assess prevalence of Ml and macroalbuminuria (MA), and their relationship with some known risk factors in two groups of NIDDM patients: (A) 126 with hypertension, defined as systolic (SBP) $>140$ or diastolic blood pressure (DBP) $>90 \mathrm{mmHtg}$ or both ( $63 \mathrm{~W} / 63 \mathrm{M}$, age $62 \pm 9$ years [mean $\pm \mathrm{SD}$ ], range $38-74$, diabetes duration [DD] $8 \pm 7 \mathrm{y} / 5$ ), and (B) 62 without hypertension $(24 \mathrm{~W} / 38 \mathrm{M}$, age $60 \pm 9$ years, range $4 \uparrow-71, \mathrm{DD} 8 \pm 9 \mathrm{yIs})$. Uninary albumin excretion (UAE) in 24 h unine samples by radioimmunoassay, SBP, DBP, body mass index (BMI), fasting plasma glucose (FPG), HbA1c, and serum levels of total cholesterd (TC), triglycerides (TG) and high-density lipoprotein cholesterol (HDL) were measured in both groups. The respective prevalences of MI (UAE $20-200 \mu \mathrm{g} / \mathrm{min}$ ) and MA (UAE $>200 \mu g /$ min) were significantly different ( $p<0.01$; chi-squared test) in A group (54 and $27 \%$ ) compared with those of $B$ group (61 and $8 \%)$. Mean values of UAE and BMI were higher in $A$ than in $B(135.2+107.3$ vs $79.8+119.2 \mu g / \mathrm{min}, p<0.01$; and $29.8 \pm 4.2$ vs $25.6 \pm 2.4$ $\mathrm{kg} \cdot \mathrm{m}^{2}, p<0.05$, respectively; Student's t-test). Levels of FPG, HbA1c, TC, TG and HDL were similar in both groups. In all patients, UAE was significantly correlated to $D D(r=0.50$; $p<0.01$ : Pearson's coefficient), to SBP $(r=0.54 ; p<0.05)$ and to TC $(r=0.62 ; p<0.01)$. In conclusion, hypertension is the more important risk factor for Ml and MA in NIDDM subjects. In the group of our hypertensive patients the prevalences of Ml and MA were higher than those obtained on patients attending diabetic clinics. UAE was associated with duration of diabetes, blood pressure and serum cholesterol; however, there was no significant relationship with glycemic control. From a clinical point of view, in NIDDM patients identification and treatment of some known risk factors might be more effective in slowing the progression of diabetic nephropathy than improving glycemic control alone.

\section{7}

MICROALBUMINURIA AS AN EARLY MARKER OF RENAL OSTEODYSTROPHY IN INSULIN DEPENDENT DLABETIC PATIENTS. P. Clausen (1), B. Feldt-Rasmussen (2), P. Jacobsen (1), K. Rossing (1), H-H. Parving (1), P.K. Nielsen (2), U. Feldt-Rasmussen (2) and K. Olgaard (2). From (1) Steno Diabetes Center, Gentofte, Denmark and (2) Department of Medicine P. Division of Nephrology and Endocrinology, Rigshospitalet, University of Copenhagen, Denmark

A reduction of the bone mineral densiiy, i.e. diabetic osteopenia has been observed in insulin dependent diabetic patients. The aim of this study was to examine the relationship between bone mineral density (BMD) and the severity of diabetic nephropathy. BMD of total body, lumbar spine, non-dominant forearm, femoral neck, trochanter major and Wards triangel were measured using dual energy x-ray absorptiometry in 59 diabetic patients and 25 healthy controls. The 59 patients had a mean duration of diabetes of 26 years. They were subdivided in groups according to the urinary albumin excretion rate (UAER). Group 1) UAER <30 mg/24h, group 2) $30-300 \mathrm{mg} / 24 \mathrm{~h}$, group 3) $>300 \mathrm{mg} / 24 \mathrm{~h}$ and 4) $>300 \mathrm{mg} / 24 \mathrm{~h}$ and plasma creatinine elevation to $120-350$ $u \mathrm{~mol} / \mathrm{l}$. Normal BMD was found in the long-term diabetic patients with normal UAER, whereas bone mineral densities of femoral neck, trochanter major and Wards triangel were significantly reduced in all patient groups with increased UAER. BMD in femoral neck were (mean $\pm \mathrm{SD}, \mathrm{g} / \mathrm{cm} 2)$ : Controls) 0.90 $\pm 0,13$, Group 1) $0.98 \pm 0.13,2) 0.84 \pm 0.16$, 3) $0.81 \pm 0.12$, 4) $0.80 \pm 0.11$ $(\mathrm{p}=0.001$-ANOVA). A significant stepwise increase in mean serum parathyroid hormone levels were observed in these patients. The lowest levels of PTH were found in group 1 and the highest levels in group 4 (mean $\pm \mathrm{SD}, \mathrm{pg} / \mathrm{ml}$ ). Group l) $25 \pm 11$ 2) $32 \pm 193) 43 \pm 23$ 4) $81 \pm 55$ ( $p=0,0002$-Kruskal-Wallis). In conclusion: BMD is normal in long-term insulin dependent diabetic patients with normal UAER. The first signs of renal osteodystrophy are demonstrated already in microalbuminuric insulin dependent diabetic patients with normal kidney function.

\section{8}

ENDOTHELIAL FUNCTION IN RELATION TO RENAL STRUCTURE IN MICROALBUMINURIC TYPE 2 DIABETIC PATIENTS.

E. Brocco, C. Stehouwer, V. van Hinsbergh, P. Fioretto, M. Mauer, M. Trevisan, A. Sfriso, M. Bruseghin, G. Crepaldi and R. Nosadini. CNR Center for the Study of Aging, University of Padova, Free University of Amsterdam and University of Minnesota, Italy, The Netherlands and USA.

We have recently described by light microscopy an heterogeneity in renal structure in microalbuminuric (MA) type 2 diabetic (NIDDM) patients (pts): in fact $29 \%$ have normal or near normal renal structure (C1); $29 \%$ have changes typical of diabetic nephropathy (glomerular, arteriolar hyalinosis and tubulo-interstitial changes occurring in parallel) (C2) and $42 \%$ have important tubulo-interstitial and/or arteriolar hyalinosis and/or glomerular sclerosis with mild glomerular abnormalities (C3). In addition, microalbuminuria has been shown to predict cardiovascular disease only when levels of von Willebrand factor ( $v W F$ ), a possible marker of endothelial function, are elevated. Aim of this study was to evaluate endothelial function, by measuring vWF plasma levels and to relate it to the different patterns of renal injury observed in MA NIDDM pts. In 33 MA pts (albumin excretion rate: 20-200 $\mu \mathrm{g} / \mathrm{min}$ ) we performed kidney biopsy and measured vWF (normal values: 50-150\%). Based on light microscopic evaluation $11 \mathrm{MA}$ pts were classified as $\mathrm{Cl}$ and their vWF levels were $113 \pm 11$ (Mean $t$ SE), 12 were classified as C2 and vWF levels were $193 \pm 12$ and 10 were classified as $\mathrm{C} 3$ and vWF levels were $169+10$ ( $\mathrm{C} 2$ and $\mathrm{C} 3$ vs $\mathrm{Cl}$ : $\mathrm{p}<0.001$ and $\mathrm{p}<0.01$, respectively). Means $\pm S . \mathrm{D}$. of age (yrs), duration of diabetes (yrs), $\mathrm{HbA}_{1} \mathrm{C}$ $(\%)$ and GFR $\left(\mathrm{ml} / \mathrm{min} / 1.73 \mathrm{~m}^{2}\right)$ were in $\mathrm{Cl} 52.4 \pm 7.7,9.0 \pm 4.0,8.1 \pm 1.6$, $124.5 \pm 28.0$, in $\mathrm{C} 259.4 \pm 4.6, \mathrm{p}<0.05$ vs $\mathrm{C} 1,14.1 \pm 5.3$, n.s., $9.6 \pm 2.3$, n.s., $92.8+33.3$ n.s. and in C3 $58.0 \pm 9.3$, n.s., $12.4 \pm 6.1$, n.s. $82 \pm 2.0$, n.s., $96.8 \pm 25.3$, n.s. Proliferative retinopathy was more frequently found in $\mathrm{C} 2$ (5 out 12 ). These findings support the existence of two types of microalbuminuria in NIDDM: 1) MA associated with significant renal damage (both typical and atypical patterns of diabetic renal injury) and "generalized endothelial dysfunction" (with high vWF), and 2) MA with nomal renal structure and normal endothelial function. The mechanisms responsible for MA in this latter group of patients, as well as the predictive value of the two types of MA for cardiovascular disease and overt nephropathy, need to be further investigated. 


\section{Experimental Nephropathy}

\section{9}

EFFECT OF LOW MOLECULAR WEIGHT HEPARIN ON PORE SIZE ON GLOMERULAR BASEMENT MEMBRANE IN DIABETIC RATS

S. Isogai, T. Inokuchi, K. Shin, K. Mogami and G. Yoshino. Dept. of Med. Toho Univ. Sch. of Med,, Tokyo, Japan.

To clarify whether low molecular weight heparin ( $L M W H, M W<5000$ ) with lower risk of bleeding than conventional heparin prevents from the disruption of the size barrier of glomerular basement membrane (GBM) of streptozotocin (STZ)-diabetic rats, pore sizes of the meshwork in ultrastructure of the GBM were measured by a newlydeviced tissue negative staining method after LMWH treatment. Simultaneously, the number of anionic site (AS) on GBM and urinary excretion of albumin (UAE) were evaluated in STZ rats after treatment. Thirty international units $/ \mathrm{kg}$ of LMWH was injected subcutaneously to STZ-diabetic rats twice daily for 6 weeks. The short diameter and long dimension of the pores of STZ-rats increased from $1.2 \pm 0.4$ and 1.9 \pm 0.5 to $2.4 \pm 0.5$ and $2.8 \pm 0.5 \mathrm{~nm}$ (mean $\pm \mathrm{SD}, \mathrm{p}<0.001$, respectively) after induction of diabetes, with a significant decrease in AS and an increase in UAE. On the other hand, LMWH treated diabetic rats had significantly smaller pore sizes $(1.7 \pm 0.4$ and 2.3 $\pm 0.4 \mathrm{~nm}, p<0.01$, respectively) compared to diabetic rats without treatment in spite of an absence of recovery from hyperglycemia. Neither decrease in AS number nor increase in UAE was found in this group. In conclusion, LMWH treatment prevented from the enlargement of pore size on the GBM in STZ-diabetic rats, irrespective of their bloodglucose control. Although the precise mechanisms for the filtration barrier are still uncertain, LMWH therapy seems to be a useful intervention for the prevention of diabetic nephropathy.
1160

HEPARANSULPHATE SYNTHESIS BY GLOMERULAR EPITHELIAL CELLS CULTURED IN HIGH GLUCOSE.

L. Guidobaldi, M. D'Erme*, R. Cipriani, F. Medici, A. Gabriele, M. Sensi, D Andreani, U.Di Mario** and S. Morano. Endoctinology, Clinica Medica 2 and *Department of Biochemistry University La Sapienza, Rome; **Department of Experimental Medicine, University of Catanzaro - Italy

Alterations in proteoglycan metabolism bave been involved in the pathogenesis of proteinuria and of other aspects of diabetic nephropathy. To evaluate the effects of different glucose concentrations on the proteoglycan production, mouse glomerular epithelial cells in culture have been incubated in media containing plysiological (5mM), elovated glucose (30 $\mathrm{mM})$, and isoosmolar (30 nMM) mannitol concentrations. After a week sodium 35-Sulphate was added to the cell cultures. The synthesis of 35-SO4-labeled molecules and the amounc of proteoglycans have been deternined by DEAE-Sephacel chromatograplyy. Two main peaks of 35-S labeled proteoglycans were separated. An increase of $52 \%$ in total cellmayer proteoglycan synthesis was induced by 30 mM gheose. Similarly an increase of about $100 \%$ it mediun associated proleoglycan syndesis was observed in $30 \mathrm{mM}$ glucose culured cells compared to control. On the basis of radiolabel, fractions were pooled and separated according to size on Sepharose CL6B columm afier digestion with condroitinase ABC. Heparaisulphate accounted for about $80 \%$ of the total $35-5$ labeled macromolecules in cell layer and mediun of nomm gilucose cultured ceils. Of lhese, a latge mul ichain heparmsulphate proteoglycans (HSPG-I) accounted for aboul 33\% (Kav 61\} 0.03). A scoend population of multiclain protcosfycans (ISSP-II) acculuted for about $30 \%$ (Kav $6 B 0.24$ ). A third population (20\%) (IS-GAG, Kay 0.4-0.8), characlerized by sitigle cliain glycosaminoglycain, was found exclusively in the cell layer compartment. In epithelial cells cultured in high glucose HSPG-I accounted for about $11 \%$, HSPG-II for about $20 \%$ and $1] S-G \wedge G$ for about $12 \%$, respectively. These results indicate that glomerular eqithelial cells play a central role in the proteoglycan synthesis and that high giucose influence the different species production of these macromolecules.

\section{1}

\section{DIFFERENT INFLUENCE OF NICOTINAMIDE ON} ALTERATIONS OF KIDNEY AND OXIDATIVE STATE IN RATS WITH NIDDM

V. Natarov, V. Poltorack, N. Gorbenko, A. Gladkich,

O. Borodina and V. Petruk. Ukrainian Scientific Research Institute of Endocrine Diseases Pharmacotherapy, Kharkov, Ukraine The aim of the study was to evaluate the effect of treatment with nicotinamide (NA) on glomerular disease development and oxidative status in rats with NIDDM. The newborn (2-3 days of age) rats were injected streptozotocin (STZ, $100 \mathrm{mg} / \mathrm{kg}$ bw i.p.) and then were randomized into a control group (C-group, $n=5$ ) and a NA-treated group (NA-group, $n=5$ ). NA-group was given $25 \mathrm{mg} / \mathrm{kg}$ bw/day NA in the diet for 3 months. Antioxidant effect of NA was assessed in liver homogenate by spectrophotometric determination of malonic dialdehyde (MDA), vitamins $\mathrm{A}, \mathrm{E}$ and $\beta$-carotenoids. Treatment with NA did not significant influenced the basal hyperglycaemia and the impaired glucose tolerance, albuminuria, the increased total kidney weight and glomerular capillary basement membrane thickening, body weight and vitamin $E$ concentration in liver. However, after the 3 months treatment with NA it was reduced accumulation of MDA in liver homogenate in the presence of ascorbate and NADPH $(7.03 \pm 0.52$ $\mathrm{nmol} / \mathrm{mg}$ protein and $9.43 \pm 0.73 \mathrm{nmol} / \mathrm{mg}$ protein, $7.81 \pm 0.63$ $\mathrm{nmol} / \mathrm{mg}$ protein and $10.94 \pm 0.71 \mathrm{nmol} / \mathrm{mg}$ protein, $\mathrm{p}<0.01$, respectively). NA-treatment increased vitamins $A$ and $\beta$-carotenoids content, respectively $0.031 \pm 0.002 \mathrm{~mol} / \mathrm{g}$ and $0.91 \pm 0.05$ $\mathrm{mol} / \mathrm{g}$ vs $0.024 \pm 0.003 \mathrm{~mol} / \mathrm{g}$ and $0.72 \pm 0.02 \mathrm{~mol} / \mathrm{g}(\mathrm{p}<0.01)$ in $\mathrm{C}$ group. Thus NA possesing the antioxidative properties did not influenced glucose metabolism disturbances and diabetic alterations of kidney in rats with neonatally STZ-induced NIDDM.
1162

ALBUMINURIA IN 24 WEEK EXPERIMENTAL DIABETES: ROLE OF ANGIOTENSIN II AND BRADYKININ

TJ Allen, UL Hulthen, C Zemin, J Rumble, ME Cooper. Dept of Medicine, Austin and Repatriation Medical Centre, Heidelberg, 3084 Vic, Australia

ACE inhibitors, which inhibit angiotensin II (AII) formation and block bradykinin (Bk) degradation, prevent the development of albuminuria in diabetic rats. The aim of the study was to determine the effect of AII blockade as opposed to Bk accumulation on albuminuria (AER), and blood pressure (SBP) in diabetic rats. The ACET ramipril (R) was given with and without the $\mathrm{Bk} \mathrm{B}_{2}$-receptor antagonist $\mathrm{HOE} 140(\mathrm{H})$ and was compared to the AII-receptor blocker valsartan (V), and calcium channel blocker, lacidipine (L). 8 week old Sprague-Dawley rats were allocated to 7 groups and studied for 24 weeks: control (C), streptozotocin-diabetic rats (D), D rats given R, 3mg/l in the drinking water (DR), D rats given $\mathrm{H}, 0.5 \mathrm{ng} / \mathrm{kg} / \mathrm{day}$ by miniosmotic pump (DH), $D$ rats given $\mathrm{R}+\mathrm{H}$ (DRH), D rats given $\mathrm{V} 30 \mathrm{mg} / \mathrm{kg}$ /day by gavage (DV) and $\mathrm{D}$ given $2.8 \mathrm{mg} / 1$ of $\mathrm{L}$ (DL) in the drinking water. Mean \pm SEM shown for wk24, SBP=mean of wk4-24, AER=geometric mean $\mathrm{x} / \div$ tolerance factors

\begin{tabular}{|c|c|c|c|c|c|}
\hline & $n$ & $\begin{array}{c}\mathrm{HbA1c} \\
(\%)\end{array}$ & $\begin{array}{l}\mathrm{BW} \\
(\mathrm{g})\end{array}$ & $\begin{array}{c}\text { SBP } \\
(\mathrm{mmHg})\end{array}$ & $\begin{array}{c}\mathrm{AER} \\
(\mathrm{mg} / 24 \mathrm{hrs}) \\
\end{array}$ \\
\hline $\mathrm{C}$ & 14 & $2.3 \pm .0 .1$ & $617 \pm 24$ & $135 \pm 3$ & $1.1 \times / \div 0.1$ \\
\hline $\mathrm{D}$ & 19 & $4.0 \pm 0.1$ & $421 \pm 20$ & $137 \pm 2$ & $4.2 x / \div 0.11$ \\
\hline $\mathrm{DH}$ & 6 & $4.1 \pm 0.2$ & $427 \pm 21$ & $129 \pm 9$ & $5.5 x / \div 0.21$ \\
\hline DR & 11 & $3.9 \pm 0.2$ & $452 \pm 12$ & $125 \pm 6^{*}$ & $1.0 \times / \div 0.1^{* * *}$ \\
\hline DRH & 6 & $3.7 \pm 0.3$ & $345 \pm 13$ & $115 \pm 4+* *$ & $0.4 \times / \div 0.1 * *$ \\
\hline $\mathrm{DL}$ & 7 & $4.1 \pm 0.2$ & $379 \pm 18$ & $135 \pm 3$ & $4.3 x / \div 0.2 \dagger$ \\
\hline DV & 9 & $3.7 \pm 0.3$ & $385 \pm 24$ & $126 \pm 3 *$ & $0.7 x / \div 0.1^{* * *}$ \\
\hline
\end{tabular}

tp<0.01 vs $C ;{ }^{*} p<0.05$,** $^{*}<0.01$ vs $D$

No drug therapy significantly influenced glycaemic control or body weight (BW). HOE 140 did not reduce the hypotensive effect of ramipril. Both ramipiril and valsartan prevented the increase in albuminuria at week 24 . H did not influence the albumin lowering effect of $\mathrm{R}$. L results were not different to untreated diabetic rats. The effects of ACE inhibition on albuminuria in diabetic rats appears to depend primarily on AII blockade and is not significantly effected by changes in BK action. These findings have potential implications for human diabetes with the recent release of AII receptor antagonists in to clinical practice. 
1163

HYPERGLYCEMIA AND HIGH PROTEIN DIET INDUCE SIMILAR FUNCTIONAL ALTERATIONS IN THE RAT KIDNEY

S. Berg, A. Dunger, and I. Klöting, Gerhardt Katsch Institute of Diabetes Karlsburg, Germany

The aim of this study was to compare the effect of long-term diabetes with that of a long-term high protein diet in vivo on the kidney function and in vitro on cellular parameters of isolated glomeruli of BB rats. Four groups of rats were investigated: group $1=$ normoglycemic rats $>250$ days old; group $2=$ agematched diabetic rats with a diabetes duration of more than 150 days; group3 $=\mathrm{BB}$ rats which were fed a protein diet of $8 \%$ (low protein $=\mathrm{LP}$ ) and group 4 $=$ rats which received a high protein (HP) diet $(32 \%)$ for more than 80 weeks. Every fourth week the animals were placed in metabolic cages for the determination of excretion and metabolic parameters. At the end of the study the total kidney weight was determined and glomeruli were isolated for in vitro experiments. Hyperglycemic and HP fed rats were characterized by a significantly increased excretion of albumin, total protein and urea compared to normoglycemic or $\mathrm{LP}$ fed animals. Creatinine excretion was neither affected by hyperglycemia nor HP diet. Hyperglycemia and HP caused a significant

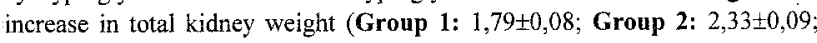
Group 3: $2,26 \pm 0,18$; Group 4: $3,28 \pm 0,46 \mathrm{~g}$ ). The increased kidney weight of diabetic and $\mathrm{HP}$ rats correlated well with a significantly enhanced DNA content of the isolated glomeruli (Group 1: $3,41 \pm 0,15$; Group 2: 4,45 $\pm 0,19$; Group 3: 4,18 $\pm 0,35$; Group 4: $6,40 \pm 0,62 \mu \mathrm{g} / 1000$ glomeruli). In addition, glomeruli abtained from either hyperglycemic or HP fed BB rats incorporated significantly more ${ }^{3} \mathrm{H}$-thymidine into their DNA indicating an elevated rate of DNA synthesis. In summary, HP diet resulted in markedly altered kidney function and induced cellular changes of glomeruli which are interpreted as enhanced proliferative processes. These alterations are comparable to those associated with diabetes mellitus. Therefore, high protein diet is already a risk factor for the development of functional and structural impairments of the kidney and should be avaided in dietary regimes of diabetics.

\section{4}

EARLY - BUT NOT ADVANCED - GLOMERULOPATHY IN EXPERIMENTAL DIABETIC RATS IS REVERSED BY PANCREATIC ISLET TRANSPLANTS

G. Pugliese ${ }^{1}$, F. Pricci ${ }^{1}$, G. Romeo ${ }^{1}$, M. Sensi ${ }^{1}$, S. Morano ${ }^{1}$, C. Pesce ${ }^{2}$, E Lenti ${ }^{2}$ V. Caltabiano ${ }^{3}$, M. Vetri ${ }^{3}$, F. Purrello ${ }^{3}$, and U. Di Mario ${ }^{4}$

Univ. of ${ }^{1}$ Rome "La Sapienza", ${ }^{2}$ Genoa, ${ }^{3}$ Catania and ${ }^{4}$ RC-Catanzaro, Italy In this study, we assessed whether (a) restoration of "euglycemia" by pancreatic islet transplants prevents and/or reverses abnormalities in renal function and structure in experimental diabetes, and (b) changes in glamerular extracellular matrix (ECM) and cell turnover and biochemical abnormalities associated with hyperglycemia correlate with the renal outcome after transplantation. Male inbred Lewis rats, rendered diabetic by streptozotocin, were subjected to homologous islet transplants via the portal vein 2 weeks (Study A) 4 months (Study B) and 8 months (Study C) after diabetes induction, and were killed 12 months, in Study $A$, and 4 months, in Studies $B$ and $C$, after transplantation. Age-matched nondiabetic (ND) and untreated diabetic (D) rats were used as controls, and studied at 4,8 and 12 months. In $D$ animals the glomerular morphological lesions and the increases vs. ND rats in erythrocyte polyol accumulation (5.1x), tail tendon collagen content of advanced glycation endproducts (AGE, 2.9x), proteinuria (5.4x), albuminuria (4.1x), kidney weight (by $10 \%$ ) and mean glomerular volume (by $17 \%$ ) were associated with enhanced glomerular mRNA expression for the ECM components fibronectin $(2.1 \mathrm{x}\rangle$ and collagen IV $\langle 2.8 \mathrm{x}\rangle$ and for transforming growth factor- $\beta$ (TGF- $\beta)(2.3 x)$, whereas cell proliferation was unaffected by diabetes. Islet transplantation was followed by restoration and long-term maintenance of "euglycemia" in all 3 studies. Diabetes-induced biochemical, functional and structural changes were completely prevented, in Study $A$, and virtually reversed, in Study $B$, by is let transplants. By contrast, in Study $C$, islet transplantation did not affect significantly the course of renal changes observed in $D$ rats, and was associated with persistent upregulation of glomerular ECM and TGF- $\beta$ gene expression (2.0x ND values) and increased tail tendon collagen AGE levels ( $2.3 \times$ ND values). These experiments show that restoration of "euglycemia" by islet transplants can prevent experimental diabetic glomerulopathy as well as reverse early lesions. In a later phase, when glomerular matrix gene expression appears to be independent of hyperglycemia, metabolic control is not capable of reversing renal changes

\section{5}

DI:VELOPMENT OF RENAL IMPAIRMENT IN EXPERIMENTAL DIABETES IFFECTS OF CHRONIC CALCIUM ANTAGONISM AND/OR ACE INHIBITION M. Kirchengast, S. Hergenröder, K. Münter, and S. Schult. Knoll AG, Research and Development, Preclinical Cardiology, 67008 Ludwigshafen, Germany

Aim: To characterise the effect of long term oral treatment with either the $\mathrm{Ca}^{2+}$ antagonist verapamil, or the ACE inhibitor trandolapril, or their combination in the model of streptozotocin (STZ) induced type I diabetes in spontaneously hypertensive rats (SHR).

Design and Methods: Male SHR were unilateraly nephrectomized and received a single iv application of $S T Z(30 \mathrm{mg} / \mathrm{kg})$. Rats showing a glucose excretion above 500 $\mathrm{mg} / \mathrm{d}]$ were randomized to receive verapamil $(46.4 \mathrm{mg} / \mathrm{kg} / \mathrm{d})$, trandolapril $(1.0$ $\mathrm{mg} / \mathrm{kg} / \mathrm{d}$ ), or their combination orally with the feed over 12 weeks. Systolic blood pressure and albuminuria were determined every 4 weeks.

Results: At the end of the 12 week treatment period non-diabetic control SHR had a mean systolic blood pressure of $263 \pm 7.9 \mathrm{mmHg}$. This was not significantly different from either the STZ group (282 $\pm 7.4 \mathrm{mmHg}$ ) or the STZ + verapamil group (261 \pm $9.4 \mathrm{mmHg}$ ). Trandolapril alone ( $198 \pm 7.9 \mathrm{~mm} \mathrm{Hg}$ ) or in combination with verapamil $(204 \pm 7.2 \mathrm{mmHg}$ ) significantly reduced hypertension. At the end of 12 weeks SHR controls excreted $27 \pm 3.7 \mathrm{mg} / \mathrm{kg} / \mathrm{d}$ albumin. In diabetic untreated animals this was dramatically increased to $236 \pm 95 \mathrm{mg} / \mathrm{kg} / \mathrm{d}$. Verapamil treatment, despite having no effect on blood pressure in the dose used in this study, reduced albuminuria significantly to $141 \pm 43 \mathrm{mg} / \mathrm{kg} / \mathrm{g}$. Trandolapril alone further reduced albuminuria (51 I $17 \mathrm{mg} / \mathrm{kg} / \mathrm{d}$ ), however, the combination was able to lower albumin excretion to 24 $\pm 3.8 \mathrm{mg} / \mathrm{kg} / \mathrm{d}$, a value seen in the hypertensive controls and thus no longer diabetes related. Glucose excretion remained high in all groups; thus an antidiabetic effect of the two drugs tested can be excluded

Conclusions: In the present study treatment with either verapamil or with trandolapril or, most pronouncedly, a verapamil-trandolapril combination reduced urinary albumin excretion in hypertensive diabetic rats. The combination of the two drugs normalised renal function despite persistent diabetes.

\section{6}

OCULAR AND RENAL EFFECTS OF AMINOGUANIDINE, PERINDOPRIL AND LACIDIPINE ON VASCULAR PERMEABILITY IN EXPERIMENTAL DIABETES. S Panagiotopoulos*, G Jerums*, T LimJoon ${ }^{\dagger}, \mathrm{V}$ Lee ${ }^{*}, \mathrm{~T} \mathrm{Gin}^{\dagger}, \mathrm{N}$ Cohen ${ }^{*}, \mathrm{~T}$ Soulis ${ }^{*}$ and $\mathrm{H}$ Taylor ${ }^{\dagger}$, Endocrine Unit, A\&RMC, MUDO, University of Melbourne, $\mathrm{RVEEH}^{\dagger}$, Australia.

To study the relative contributions of advanced glycation, calcium influx and angiotensin converting enzyme (ACE) in experimental diabetes, we examined the effects of aminoguanidine $(A)$, perindopril $(P)$ and lacidipine (L) on ocular and renal cortical vascular permeability and aibuminuria (AER) in the streptozotocin (STZ) diabetic rat. Male Sprague Dawley rats were randomized to control (C) or diabetic groups: untreated (D) or treated with aminoguanidine $(1 \mathrm{~g} / \mathrm{L}$ drinking water, $[\mathrm{D}+\mathrm{A}])]$ perindopri] $(2 \mathrm{mg} / \mathrm{L}$ drinking water $[D+P])$ or lacidipine $(0.5 \mathrm{mg} / \mathrm{kg} /$ day by gavage $[\mathrm{D}+\mathrm{L}])$. All diabetic groups had equivalent glycaemic control (mmol/1). Systolic blood pressure $(\mathrm{SBP}, \mathrm{mmHg})$ was lowered by $\mathrm{P}(\mathrm{p}<0.01)$ but not altered by $\mathrm{L}$ or A. Vascular permeability to albumin was assessed by the tissue to blood isotope ratio (TBIR) after 16 weeks. Retinal TBIR was increased approximately two-fold in D compared with $C$ rats and the diabetes-related increase was completely prevented by all three treatments. Similar increases in anterior and posterior uveal TBIR in diabetic rats were also prevented by all three interventions. Renal cortical TBIR was increased in $D$ vs $C$ rats $(p<0.01)$, and the diabetes-related increase was prevented by $P$ and $A$ but not by L. AER ( $\mathrm{mg} / 24 \mathrm{hr}$ ) increased in D compared to $\mathrm{C}$ tats and the diabetes-related increase was prevented by $\mathrm{P}(\mathrm{p}<0.05)$ but not by $\mathrm{A}$ or $\mathrm{L}$. These results suggest that the relative contribution of advanced glycation, calcium influx and ACE in the pathogenesis of diabetic vasculopathy mav differ in the eye and kidney.

\begin{tabular}{lccccc}
\multicolumn{7}{c}{ vasculopathy may differ in the eye and kidney. } \\
\hline \multicolumn{7}{c}{$\mathbf{C ( 1 0 )}$} & $\mathrm{D}(12)$ & $\mathrm{D}+\mathrm{A}(7)$ & $\mathrm{D}+\mathrm{L}(\mathbf{6})$ & $\mathrm{D}+\mathrm{P}^{(6)}$ \\
Glucose & $7.8 \pm 0.5$ & $34.4 \pm 2.3$ & $34.5 \pm 1.1$ & $40.2 \pm 4.7$ & $27.7 \pm 3.3$ \\
SBP & $148 \pm 3$ & $168 \pm 6$ & $178 \pm 7$ & $170 \pm 7$ & $124 \pm 8^{* *}$ \\
T retina & $1.26 \pm 0.05$ & $2.56 \pm 0.37$ & $1.44 \pm 0.06^{* *}$ & $1.47 \pm 0.12^{*}$ & $1.49 \pm 0.13^{* *}$ \\
T kidney & $2.68 \pm 0.43$ & $6.79 \pm 0.97$ & $2.49 \pm 0.54^{* *}$ & $5.11 \pm 0.85$ & $2.46 \pm 0.33^{* *}$ \\
AER & $0.53[1.41]$ & $3.80[1.45]$ & $3.47[1.91]$ & $3.39[1.62]$ & $1.12[1.23]^{*}$ \\
\hline AER
\end{tabular}

AER: geometric mean [tolerance factor] Other: mean \pm SEM, T $=$ TBIR (n per group). ${ }^{*}=p<0.05,{ }^{* *}=p<0.01$ versus $D$ 


\section{Natural History and Treatment of Nephropathy}

1167

RENAL COMPLICATIONS AT FOLLOW UP OF WOMEN WITH PREVIOUS GESTATIONAL DIABETES MELLITUS

Gy.Bibok, Gy.Tamás, Á.Nádasdi ${ }^{*}$ and Zs.Kerényi* , Semmelweis University and Szent Imre Hospital*, Budapest, Hungary

Our aim was to study the occurrence of late diabetic renal complications later in life in 66 women with previous gestational diabetes (GDM) insulinized during pregnancy after reclassifying their carbohydrate metabolism (GDM-I, mean age: 38 [range:24-52]yrs, time elapsed since diagnosing GDM: 6.8 [3-14]yrs). As controls served $23 \mathrm{GDM}$ on diet during pregnancy (GDM-D: age 35 [26-42/yis, follow up. $6.3[4-10] \mathrm{yrs}$ ) and 28 pregestational NIDDM first insulinized during gestation (NIDDM-I: age 38 [26-46]yrs, follow up 5.9 [216lyrs, duration of diabetes: 8.8 [2-16yrs]. Serum creatinine, beta-2-microglobulin [B2M; beta-2-micro RIA, Pharmacia, Sweder; normal value $<2.3 \mathrm{mg} /$ l] urinary microalbumin [MAU; immunoturbidimetric method for Hitachi, Boehringer Mannheim, Germany; 30$300 \mathrm{mg} /$ day, 24 hour timed collection] and B2M [normal value $<0.3 \mathrm{mg} /$ ] were measured to evaluate renal function Neither overt proteinuria / macroalbuminuria nor elevated serum creatinine (mean [range]: GDM-I: 82 [54-112] [moll;; GDM-D: 82 [70-93]; NIDDM-I 81 [65-113]) were detected in any groups. Microalbuminuria was found in 11 GDM-I cases: in $6 / 38$ of verified diabetic patients $(15.8 \%$ [33-158 mg/day]) in 1/6 IGT [51 $\mathrm{mg} / \mathrm{day}]$ and $4 / 22$ (18.1\% [31-85 mg/day]) in non-diabetic persons, in 2 GDM-D cases ( $1 / 5$ diabetes, $1 / 5$ [GT; 81 and $34 \mathrm{mg} / \mathrm{day}$ ) and in $6 / 23 \mathrm{NIDDM}-\mathrm{I}$ women (26.1\% [32-251 $\mathrm{mg} / \mathrm{day}]$ ). Elevated serum B2M were observed 4 GDM-I (diabetes:2, IGT: 1, healthy: 1; mean of the group $1.37 \mathrm{mg} / \mathrm{l}$-pathological: 2.33-2.85 mg/t); 1 GDM-D (diabetes; $2.5 \mathrm{mg} /$ ); 2 NIDDM$(2.65,3.27 \mathrm{mg} /$ ). Elevated urinary $\mathrm{B} 2 \mathrm{M}$ was measured only in $1 \mathrm{GDM}-\mathrm{I}$ patient (diabetes; $0.398 \mathrm{mg} /$ ). In 6 GDM-I patients with diabetes and microalbuminuria a higher mean HoAlc (9.8 [range: 6-12.57\%) was found compared to the mean of all GDM-I patients with diabetes (mean: $7.6[4.6-13.5] \%$ ) similarly to the 6 NDDDM-I with diabetes and nicroalbuminuria compared to the mean value of the NIDDM-I group (HbAlc 10.6 [7.5$15.4] \%$ vs. 8.0[4.9-15.47\%). The relatively high prevalence of microalbuminuria at follow up with elevated $\mathrm{HbAlc}$ underlines the importance of regular care of women with previous GDM to treat them early enough to avoid late diabetic renal complications.

\section{9}

RESIDUAL $\beta$-CELL FUNCTION AND THE DEVELOPMENT OF DIABETIC NEPHROPATHY IN IDDM

M. Yokota, K. Nakanishi, T. Kobayashi, T. Murase and K. Kosaka. Dept.of Endocrinology and Metabolism, Toranomon Hospital, Tokyo. Japan.

The aim of this study is to elucidate the contribution of residual $\beta$-cell function to the development of diabetic nephropathy in IDDM. We studied the relationship among residual $\beta$-cell function. HLA-antigen, long-term glycemic control and development of diabetic nephroparhy in 71 IDDM patients. Residual $\beta$-cell function was assessed by $C$-peptide immunoreactivity response $(\triangle C P R$ ) $10100 \mathrm{~g}$ oral glucose using a sensitivity RIA. The patients were stratified into two groups; thove with $\triangle \mathrm{CPR}<0.033 \mathrm{nM}$ (group 1) and $\geqq 0.033 \mathrm{nM}$ (group 2). The presence of micro- al ubuminuria $\geqq 100 \mathrm{mg} / \mathrm{gCre}$ at consecutive determinations was defined as the development of diabetic nephropathy. The mean HbAic values and fasting blood glucose (FBG) values were regarded as the indexes of long-term glycemic control. The cumulative incidence of diabetic nephropathy differed significantly berween the two groups $(\mathrm{P}=0.0011)$. Incidence rates of diabetic nephropathy were $1.06 / 100$ patient-years in group 1 and $0.48 / 100$ patient-years in group 2 . The mean $\mathrm{HbAlo}$ value in group $1(10.4 \pm 0.2 \%)$ was higher than in group $2(9.3 \pm 0.2 \%, P=0.01)$ and the mean FBG levels in group $1(10.85 \pm 0.36 \mathrm{mM})$ was higher than in group 2 $(9.15 \pm 0.33 \mathrm{mg} / \mathrm{dl}, \mathrm{P}<0.0001)$. In group $1,92.6 \%$ (25/27) patients had HLA A24, whereas 43.2\% (19/44) patients had this antigen in group $2(P<0.0001)$ There was no difference between two groups in terms of sex, age at onset, BMI. insulin dose and frequency of hypertension. The independent contribution of the variables was analyzed using Cox's proportional hazard model. Only mean $\mathrm{HbA} A \mathrm{c}$ value was identified as independent risk factor for development of diabetic nephropathy (hazard ratio: $2.85 / 1 \%$ mean HbAic change, $\mathrm{P}=0.0278$ ). Both residual $\beta$-cell function and the age at the were just short of significance ( $P=0.086$, $\mathrm{P}=0.087$, respectively). These findings indicate that complete loss of $\beta$-cel! function, which is associated with HLA-A24, leads to the higher incidence of diabetic nephropathy through the long-term glycemic control.

\section{8}

LONG-TERM FOLLOW UP OF GLOMERULAR FILTRATION RATE IN NON-INSULIN-DEPENDENT DIABETES MELLITUS

Salinas I, Rius F, Pizarro E, Lucas A, Castells I, Romero R, Sanmarti A. Endocrinology and Nephrology Services, Hospital Germans Trias i Pujol, Badalona (Barcelona), Spain

Background: Glomerular filtration rate is the main measure of renal function. Its evolution and the factors that influence it in non-insulin-dependent diabetes mellitus are not completely understood.

Patients and methods: Seventy-two NTDM patients without proteinuria (mean age $54.3 \pm 9.3$ ) were included in this study. Glomerular filtration rate (GFR) was measured at least twice in all them and the minimum follow-up was of 3 years. Forty patients had a follow-up of four years and 25 of five years. Presence of hypertension and microalbuminuria (UAER $>30 \mathrm{mg} / 24 \mathrm{~h}$ ) was recorded. The initial and mean values of glucose, glicacted haemoglobin, lipid profile, urinary albumin excretion rate (UAER) and blood pressure were also recorded.

Results: There was a wide variability in the evolution of GFR. The mean descent at three years was of $1.1 \pm 23 . \mathrm{m} 1 / \mathrm{min} / 1.73 \mathrm{~m}^{2}$ and at five years of 5.7 $\pm 26.7 \mathrm{ml} / \mathrm{min} / 1.73 \mathrm{~m}^{2}$. There was a trend for a higher descent in patients with hypertension and with microalbuminuria, but without statistical significance. A negative correlation with baseline GFR $(r=-0.26, p<0.05$ at 3 years; $r=-$ $0.39, p=0.05$ at 5 years $)$ and FDL-cholesterol $(r=-0.26, p<0.05$ at 3 years; at 5 years non-significant). There was also a trend for mean systolic pressure and mean UAER $(\log )$, that did not reach statistical significance either.

Discussion: There is not an important descent of GFR in our follow-up of NIDDM patients without proteinuria. Patients with a lower baseline GFR have a higher rate of descent. The wide range of variation precludes the identification of factors influencing the evolution of GFR, although an influence of systolic pressure, UAER and HDL-cholesterol is likely to become clear with a longer follow-up.

\section{0}

PROSPECTIVE VARIABILITY OF Na-Li COUNTERTRANSPORT A 6-YEAR FOLLOW-UP STUDY IN IDDM

R. Mangili, R. Ghelardi, G. Meregalli, G. Zerbini, M.R. Pastore, D. Gabellini and G. Pozza. Istituto Scientifico San Raffaele, Università di Milano, Italy.

Elevated erythrocyte sodium-lithium countertransport activity (SLC) is an intermediate phenotype of essential hypertension that may also characterize nephropathy in IDDM. Susceptibility to change over time may unfavorably affect the viability of a candidate marker, and the long-term intra-individual variability of SLC in diabetes under the ordinary variations of outpatient glycaemic control is poorly known. Forty-two normoalbuminuric patients with IDDM $(22 \mathrm{M}, 20 \mathrm{~F})$ were studied between 1988 and 1990 , and were re-evaluated after $4.8-6.6$ years. Albumin excretion rates higher than $20 \mu \mathrm{g} / \mathrm{min}$ had developed in three patients. Mean HbAIc was unchanged ( $8.6 \pm 0.4$ vs $8.6 \pm 0.3 \%$ ), but there was no relationship between base-line and follow-up measurements $\left(\mathrm{r}^{2}=0.009, \mathrm{p}=0.6\right)$ because of wide absolute intraindividual changes that paralleled changes in fasting glycemia as expected $\left(r^{2}=0.29, p<0.001\right)$. Conversely, initial SLC rates remained strongly related with follow-up remeasurements $\left(\mathrm{r}^{2}=0.45, \mathrm{p}<0.00001\right)$, and were positively associated with follow-up systolic $(\mathrm{p}<0.03)$, and possibly diastolic $(0.05<p<0.1)$ blood pressure, independent of age, sex and BMI. Residual variability accounted for a small but significant increase in mean SLC by $82 \mu \mathrm{mol} \cdot \mathrm{IRBC}_{\mathrm{RC}}{ }^{-1} \cdot \mathrm{hr}^{-1}$, but arithmetical differences in SLC between base-line and follow-up observations were not paralleled by variations in glycemic control, major blood lipids, insulin dose, albumin excretion or somatometrics. We suggest that SLC appears to be little affected by ordinary changes of glycemic control and may remain a practicable test variable in prospective studies of the predisposition to nephropathy in IDDM. 
THE NATURAL COURSE OF ALBUMIN EXCRETION IN LOW GRADE MICROALBUMINURIC IDDM-SUBJECTS WITH DISTINCTIVE MORPHOLOGICAL CHANGES IN THE KIDNEYS

H.J. Bangstad, R. Østerby, K. Dahl-Jorgensen, A. Hartmann, T. J. Berg and K.F. Hanssen, Aker Diabetes Research Centre, Aker University Hopital, Medical Dept. B, Rikshospitalet, Oslo, Norway and University Institute of Pathology, Arhus Kommunehospital, Denmark.

In a prospective study we investigated the natural history of AER during 5 years and the relationship to morphological changes in patients with IDDM and low grade MA and not on ACE-inhibitors. 18 subjects (median and range) age: 19 (18-29) yrs, duration of diabetes 12 (7-18) yrs, mean $\mathrm{HbAl}_{\mathrm{c}} 10.3$ (7.9-12.6) \% and mean AER 31 (15-194) ug/min the preceding year, took part in a prospective study. Renal biopsies were taken at 0 and 2,5 years, $\mathrm{HbAl}_{\mathrm{c}}$ and AER 3-4 times a year. Glomerular structures were assessed by stereological methods. AER (geometric mean $+95 \mathrm{CI})$ decreased during the study, from $34(24-48)$ to $22(10-46)$ $\mu \mathrm{g} / \mathrm{min}$ without reaching statistical significance $(2 \mathrm{p}=0.07) . \mathrm{HbAl}$ (mean $+95 \mathrm{CI}$ ) was reduced from $10.1(9.2-11.1)$ to $9.1(8.6-9.6) \%$. There was a significant correlation $(\mathrm{p}<0.05)$ between AER at 5 years and BMT ( $r$ $=0.55)$, matrix/glomerular volume fraction $(r=0.51)$ and matix-thickness $(\mathrm{r}=0.48) 2.5$ years before. However, only matrix-thickness at baseline was correlated to AER 5 years later $(r=0.50)$. No association was found between mean $\mathrm{HbAl}_{\mathrm{C}}$ during the study and change in AER. In conlusion: in this group of patients with $\mathrm{HbAl}_{c}<10 \%$, morphological changes were more predictive than mean $\mathrm{HbA} 1_{0}$ with respect to $\mathrm{AER}$. No increase was found in AER in the total group as has been shown by other investigators. This questions whether ACE-inhibitors are required for all patients with low grade AER.
PROGRESSION OF ADVANCED MTCROALBUMNURIA IN NORMOTENSTVE TYPE-2 DIABETIC PATEENTS DESPITE IMPKOOVED METABOLIC CONTROL. N.Eibl, Ch.Schnack, M.Frank and G. Schernthaner. Department of Medicine 1, Rudolfstiftung Hospital, Vienna, Austria

Persistent increased urinary albumin excretion rate (UARR) is associated with increased cardiovascular mortality in type-2 diabetes, however there ane no concivsive data atout the progression of advanced UAER ir these palients. We have investigated the effect of melabolic intervention on the progression of JAER in relations to initial JAER leveis. 18 patients with type-2 diabetes and secondary failwre to sulfonylurea were observed duriag one year (Age 60-70 yrs; $\mathrm{HbH}_{7 \mathrm{C}} 10,8+1,2 \%$, duration of diabetes $16+10 \mathrm{yrs}$ ) and divided into 2 groups: Group $A(n=9$; VAER $30-100 \mathrm{mg} / 24 \mathrm{~h}$; group $B$ ( $\mathrm{n}=9$; UARR $100-300 \mathrm{mg} / 24 \mathrm{a}$ ). Despite a significant iuprovement of metabolic control by insulin treatment in both groups ( $\mathrm{HbA}_{10}:$ : Troup $A: 10,4 \%$ vs $8,4 \%$ gromp $B: 10,9$ vs $9,1 \%, \mathrm{p}<0,001$ ), 3 progression of UAER was observed in group $B$ (232 vs $692 \mathrm{mg} / 24 \mathrm{~h} ; \mathrm{p}<0,02)$, but not in group A (52 vs $56 \mathrm{mg} / 24 \mathrm{~h})$. Six patients iri group \& developed macroproteinuria $(>300 / 24 \mathrm{~h})$, although serum creatinine levels remained normal during the observation period. The 24 hours blood pressure (RR) values in the 2 groups remained normal throughout the study (group A: RR syst.131vs136 mm Ho; $R$ R diast $81 v s 81$ mm Hg; mean arterial pressure (ihAD) 99 vs 101 ma Eg; group B: RR syst. 139 vs $134 \mathrm{man}$ Fg; $R R$ diast. 78 vs $75 \mathrm{~mm}$ Hg: MAD 103vs $98 \mathrm{~min} \mathrm{Hg}$ ). Our data show that in type-2 diabetic patients with normotensive blood pressune values the initial urinary albumin excretion levels determine the progression of UARR. When metabolic control is improved incipient UAER remains constant, but advanced UAER shows progression. Our data indicate the importance of early recognition of increased wrinary allowion excretion rate.

\section{3}

THE NATURAL HISTORY OF URINARY ALBUMIN EXCRETION IN INSULIN TREATED NON-INSULIN DEPENDENT DIAEBETES MELLITUS

V.J. Parfit, S.J. Twyman, P. Mansell R.A. Leatherdale and D.J.F.Rowe. Diabetes and Endocrinology Unit. Royal South Hants Hospital Southampton Dept. Chemicai Pathology, Southampton General hospital, Southampton Dept. Medicine, Salisbury District Hospital, Salisbury Great Britain. The Wessex Diabetic Nephropathy Project investigated the prevaience and progression of diabetic nephropathy in insulin-treated non-insulin dependent patients. Patients were classified on the basis of their albumin:creatinine ratio in at least 2 of 3 consecutive random urine samples thus:- Group 1 (normal) $=<3$, Group 2(microalb.) $=3.30$, Group 3 (proteinuria) $>30$ $\mathrm{mg} / \mathrm{mmol}^{-1}, 210$ adult patient (baseline age range 44-87 yrs, duration of diabetes range 1.36 yrs) were followed for 4.7 yrs (range 1.6-7.6yrs). At baseline $129(61.4 \%)$ were in Group1, 72(34.3\%) were in Group 2 , and 9 (4.3\%) were in Group 3. After the follow up period, of patients in Group1, $107(83 \%)$ remained, $20(15.5 \%)$ went to Group 2 , and $2(1.6 \%)$ to Group 3 . Of patients in Group 2, 39(54.2\%) remained, 10 $(13.9 \%)$ reverted to Group1 and $23(31.9 \%)$ went to Group 3 . Of patients in Group $3,6(66.7 \%)$ remained and $3(33.3 \%)$ reverted to Group2. Subgroups classified as $11,12,13,21$, $22,23,32,33$ (where $11=$ patients at group1 at start and end, $12=$ group1 at start but group 2 at end of follow up period etc). Mean(SD) HbA1 $=10.1(1.2)$ $10.3(1.2), 9.9(1.6), 9.1(1.5), 10.6(1.5), 10.8(1.8), 9.4(0.1), 11.1(2.4)$. Diastolic $\mathrm{BP}=82(7.5), 77(19.1), 79(4.2), 80(8.7), 82(8.5), 87(6.3), 93(5.6), 86(6.3)$. Systolic BP=147(15.9), 147(15.6),148(15.8), 137(46.2),156(19.6),171(20.3). The progression regression of urinary albumin excretion (UAE), was not associated with age or duration of diabetes at baseline, or changes in $\mathrm{HbA}$ t blood pressure (systolic, diastolic, mean arterial, pulse pressure) body mass index, or insulin dose during the follow up period. The natural history of UAE in this group is variable, but appears to include regression as well as progression, as has been demonstrated in other subgroups of diabetes.

\section{4}

LONG TERM IMPACT OF LISINOPRIL AND ATENOLOL ON KIDNEY FUNCTION IN NIDDM PATIENTS WITH DIABETIC NEPHROPATHY.

F. S. Nielsen, P. Rossing, M.A. Gall, P. Skgtt, U. M. Smidt and H.H. Parving, Steno Diabetes Center, Gentofte, Denmark.

The aim of our study was to evaluate whether inhibition of angiotensin converting enzyme (ACE) (lisinopril $10-20 \mathrm{mg} / d a y$ ) can reduce the rate of decline in kidney function more than reducing blood pressure with conventional antihypertensive treatment (atenolol 50-100 mg/day), usually in combination with a diuretic. We performed a prospective, randomized, parallel study for 42 months, double blind for the first 12 months hereafter single blind. Forty three (21 lisinopril and 22 atenolol) hypertensive NIDDM patients with diabetic nephropathy were enroled. Data from 35 (16 lisinopril and 19 atenolol, age 60 (SD, 7 years, 27 males) who completed at least 12 months study period are presented. At baseline the two groups were comparable regarding GFR ${ }^{55} \mathrm{Cr}$ EDTA plasma clearance) 75 (SE, 6) and $74(8) \mathrm{m} / \mathrm{min} / 1.73 \mathrm{~m}^{2}$, mean 24hour ambulatory blood pressure (A\&D TM2420) (ABP) 110 (SE, 3) and 114 (2) $\mathrm{mmHg}$ and 24-hour urinary albumin excretion rate (UAE) 961 (range, 331 to 5727) and 1578 (476 to 5806) $\mathrm{mg} / 24$-hour in the lisinopril and atenolol group, respectively. The mean follow up time was similar 37 and 35 months in the lisinopril and atenolol group, respectively. Mean ABP was equally reduced in the two groups 12 (SE, 2) and 10 (2) $\mathrm{mmHg}$ in the lisinopril and atenolol group, respectively. GFR declined in a biphasic manner with a faster initial (0 to 6 months) 1.0 (range -3.5 to 6 ) and $0.8(-1.0$ to 3.5$) \mathrm{m} / \mathrm{month}$ followed by a slower sustained decline in GFR $0.4(0.1$ to 1.4$)$ and $0.3(0.1$ to 1.9$)$ milmonth in the lisinopril and atenolol group, respectively. No significant differences were observed in either initial or sustained decline in GFR between the two groups. UAE was reduced (\% reduction of baseline) more in the lisinepril as compared to the atenolol group $55(95 \%$ confidence interval 29 to 72$)$ and $15(-13$ to 34$) \%$, respectively $p=$ 0.01 ). In conclusion: the relentless decline in kidney function characteristically found in hypertensive NIDDM patients with diabetic nephropathy can equaliy effective be reduced by antihypertensive treatment with either the B.blocker atenolol or the ACE-inhibitor lisinoprii. 


\section{5}

ANTI-HYPERTENSIVE TREATMENT AND MICROALBUMINURIA IN PATIENTS WITH ABNORMAL DIURNAL BLOOD PRESSURE VARIATION. E. Andrysiak-Mamos, H. Fuchs, S. Pynka, P. Moleda and S. Czekalski, University School of Medicine, Szczecin, Poland

A direct relationship between diurnal blood pressure (BP) abnormalities in patients with insulin-dependent diabetes and. microalbuminuria was suggested in earlier siudies. The aim of this study was to compare the effect of treatment with enalapril (E) or lisinopril (L) on microalbuminuria in patients with insulin-dependent diabetes and normal (dippers, $n=15$ ) or abnormal (non-dippers, the mean decline in systolic (s) and diastolic (d) BP from day to night $<5 \%, n=9$ ) diurnal BP variation, Twenty four hour ambulatory BP was measured with Spacelabs equipment and microalbuminuria (RIA mean of three 24 h urine samples) was evaluated before and after 18 months lasting treatment with $\mathrm{E}$ or $\mathrm{L}$ which permitted to stabilise $\mathrm{BP}$ below $95 \mathrm{mmHg}$ during follow-up. Mean BP reduction to $89.2 \pm 8.7 \mathrm{mmHg}$ in dippers and to $88.3 \pm 10.2 \mathrm{mmHg}$ in non-dippers at the end of follow-up was not alssociated with the change of night/day sBP and $\mathrm{dBP}$ ratio in dippers (from $0.89=$ 0.06 to $0.89 \pm 0.05$ and from $0.86 \pm 0.03$ to $0.85 \pm 0,05$ before and at the end of follow up, respectively) and in non-dippers (from $0.99 \pm 0.07$ to $0.98 \pm 0.07$ and from 0.99 0.05 to $0.95 \pm 0.05$, respectively). Effect of anti-hypertensive treatment was markedly

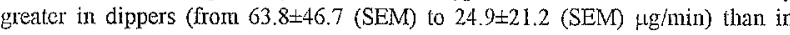

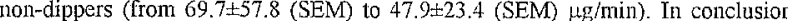
our study suggest that abnormal diurnal BP variation in patients with incipien diabetic nephropathy is associated with relative resistance of microabuminuria to treatment with angiotensin converting enzyme inhibitors.

\section{7}

INTENSIVE MULTIFACTORIAL INTERVENTION IN NIDDM PATIENTS WITH PERSISTENT MICROALBUMINURIA

P. Vedel, P. Gæde, J. Obel, H.-H. Parving, O. Pedersen, Steno Diabetes Center Copenhagen, Denmark

Aim: To assess the effect of intensified multifactorial intervention on diabetic microangiopathy and increased macrovascular morbidity and mortality characteristically found in NIDDM patients with persistent microalbuminuria. Protocol: 160 patients were randomized (age 45-65 yr, gender 117 $0 / 41 \%$, albuminuria $30-300 \mathrm{mg} / 24 \mathrm{~h}, \mathrm{HbA} \mathrm{Ac}_{1 \mathrm{c}} 8.6 \%$ (range 5.6-12.4), systolic BP $148 \pm 20 \mathrm{~mm}$ $\mathrm{Hg}$, diastolic BP $85 \pm 11 \mathrm{~mm} \mathrm{Hg}$ ) to a control group ( $N=80$ ) treated conventionally at their GP's and a group $(\mathrm{N}=80)$ undergoing aggressive intervention focusing on metabolic control, hypertension, dyslipidemia and lifestyle modification. In this interim report $2 \mathrm{yr}$ results are presented. A separation of $1.3 \%$ in $\mathrm{HbA}_{1 \mathrm{c}}$ was obtained without weight gain ( 8.0 (SE 0.2$)$ vs $9.3(0.2), p<0.05)$. In the intensively treated group a near normalisation of blood pressure was achieved, mainly by $A C E$ inhibition, $135(2) / 77(2) \mathrm{mm} \mathrm{Hg}$ vs $144(2) / 81(2) \mathrm{mm} \mathrm{Hg}, \mathrm{p}<0.05$. No differences in serum lipids were observed; s-cholesterol $4.9(0.1) \mathrm{mmol} / \mathrm{h} 5.5(0.2) \mathrm{mmo} / / \mathrm{l}$, HDLcholesterol was $1.0(0.05) \mathrm{mmol} / \mathrm{l}$ in both groups, s-triglycerides $1.4(0.1) \mathrm{mmol} / \mathrm{l}$ vs $3.3 \mathrm{mmol} / \mathrm{l}, \mathrm{p}=\mathrm{NS}$. The number of smokers declined from $38 \%$ to $19 \%$ in the intervention group and remained unchanged $40 \%$ in the control group. During the study urinary albumin excretion increased from 69 (32 to 292) to 99 (11 to 832 ) $\mathrm{mg} / 24 \mathrm{~h}$ in the control group (NS), while it declined from $77(32$ to 264$)$ to 50 (5 to $656) \mathrm{mg}$ in the intensively treated group $(p<0.01)$. The difference between the changes in urinary albumin excretion during the study period was highly significant, $p<0.0001$. Nine control patients and 6 intensively treated patients developed overt nephropathy (albuminuria $>300 \mathrm{mg} / 24 \mathrm{~h}$ in 2 out of 3 urine collections). Glomerular filtration rate $\left(\mathrm{ml} / \mathrm{min} / 1.73 \mathrm{~m}^{2}\right)$ showed a slight, insignificant decline in both groups: $118(3)$ vs $111(3)$ and $116(3)$ vs $109(3)$ in the control group vs intensively treated group, respectively. Cardiovascular events (intensive vs control group): Myocardial infarction 3 vs 4 , coronary by-pass 2 vs 3 , strokes 3 vs 5 , limb amputations 2 vs 2 , deaths 3 vs 1 . Our study indicates that multifactorial intervention had beneficial effects on micro- and macroangiopathic risk factors and progression of microalbuminuria.

\section{6}

IMPACT OF NISOLDIPINE AND LISINOPRIL ON KIDNEY FUNCTION IN HYPERTENSIVE IDDM SUBJECTS WITH DIABETIC NEPHROPATHY

P.Rossing, L Tarnow, S Boelskifte, B.R. Jensen, F.S. Nielsen, H.-H. Parving. Steno Diabetes Center, Gentofte, Denmark

The aim of our study was to compare the effect of antihypertensive treatment with a long-acting calcium antagonist (nisoldipine) with an angiotensin converting enzyme inhibitor (lisinopril) on kidney function in hypertensive IDDM patients with diabetic nephropathy. We performed a one year, double blind, randomised, controlled study comparing nisoldipine (20. $40 \mathrm{mg} / \mathrm{day})$ with lisinopril $(10-20 \mathrm{mg} / \mathrm{day})$. Fiftytwo patients were randomised (26 nisoldipine, 26 lisinopril). Three patients dropped out and results for the remaining 49 (25 nisoldipine, 24 lisinoprill) are presented. Diuretics were required in 10 of 25 nisoldipine and 8 of 24 lisinopril patients. Every 3 months $24 \mathrm{~h}$ ambulatory blood pressure was measured with the A\&D TM2420 device and albuminuria in $3 \times 24 \mathrm{~h}$ samples was measured with enzyme immunoassay, every six months glomerular fittration rate (GFR) was measured with ${ }^{51} \mathrm{Cr}$-EDTA plasma clearance. Results: during the 12 months study, 24h mean arterial blood pressure was reduced from (mean(SE) 108 (3) at baseline to 101 (2) in average during treatment in the lisinopril group and from 105 (2) to 103 (2) in the nisoldipine group ( $p=0.06$ comparing changes in the two groups). Albuminuria was on average reduced $47 \%$ $(95 \%$ CI 21 to 65$)$ in the lisinopril vs an increase of $11 \%(-3$ to 27$)$ in the nisoldipine group $(p-0.001$ between groups). Fractional albumin clearance decreased $37 \%(95 \% \mathrm{Cl}$ 4 to $59 \%$ ) in the lisinopril vs an increase of $35 \%$ (95\% Cl 8 to $69 \%$ ) in the nisoldipine group ( $\mathrm{p}<0.01$ between groups). GFR decreased from 85 (5) $\mathrm{m} / 1 \mathrm{~min} / 1.73 \mathrm{~m}^{2}$ (mean (SE)) to 73 (5) in the lisinopril group vs a decline from 84 (6) to 80 (7) in the nisoldipine group ip $<0.05$ between groups|. The effect of study medication on albuminuria and GFR was independent of the change in blood pressure during the study and baseline variables. In conclusion: Lisinopril reduced albuminuria, and GFR, to a greater extend than nisoldipine, and these effects are independent of systemic blood pressure changes. Longer follow-up is required to clarify whether these drugs have different renoprotective effects. 
PS 65

\section{Other Complications}

\section{8}

N-DEACETYLASE MRNA EXPRESSION IN DIABETIC RATS

P.S.Oturai, B.Rolin, T.Jensen, and A.Kofoed-Enevoldsen. Steno Diabetes Center and Novo Nordisk Research, Gentofte and Bagsværd, Denmark. Heparan sulphate (HS) changes in the extracellular matrix play a central role in diabetic nephropathy. Diabetes causes decreased activity of $\mathrm{N}$-deacetylase (ND), the key enzyme in the synthesis of HS. The mechanism by which diabetes affects ND activity has not been clarified, we therefore aimed to investigate the expression of ND mRNA in experimental diabetes. Diabetes was induced by a single i.v. injection of streptozotocin $(55 \mathrm{mg} / \mathrm{kg})$ in male SpragueDawley rats. After 10 weeks and 8 months treatment blood was sampled for glucose measuremient and liver tissue was quick frozen. Hepatic ND MRNA was quantified after RNA extraction, reverse transcription and amplification by PCR. Cyclophilin was $\mathrm{CO}$-amplified and served as an internal standard. Hepatic ND activity was determined by measuring the amounts of acetate released from a $\mathrm{N}-{ }^{3} \mathrm{H}$ ]acetyl labelled polysaccharide substrate. Diabetic rats were all severely hyperglycaemic, and diabetes caused a significant decrease in ND activity. The relative amount of ND mRNA was not altered significantiy in the diabetic rats compared to the non-diabetic control rats. However, a positive correlation between ND activity and mRNA expression was seen, $p<0.03, r=0.33, n=46$. This correlation was even more pronounced when only diabetic rats were analyzed, $p<0.01, r=0.5, n=31$.

\begin{tabular}{lcccc}
\hline Mean (SD) & $\begin{array}{c}\text { Normal } \\
(10 \text { wks })\end{array}$ & $\begin{array}{c}\text { Diabetic } \\
(10 \text { wks })\end{array}$ & $\begin{array}{c}\text { Normal } \\
(8 \text { months })\end{array}$ & $\begin{array}{c}\text { Diabetic } \\
(8 \text { months })\end{array}$ \\
\hline $\begin{array}{l}\text { ND-activity } \\
\text { (cpm) }\end{array}$ & $205(19)^{*}$ & $148(25)$ & $206(30) *$ & $141(28)$ \\
ND-mRNA a & $0.32(0.16)$ & $0.27(0.1)$ & $0.26(0.07)$ & $0.23(0.11)$ \\
\hline
\end{tabular}

${ }^{*}$ Different from diabetic groups $(p<0.001)$. ${ }^{a} \mathrm{ND} / c y c i o p h i l i n$ ratio.

In conclusion, a positive correlation between ND activity and ND mRNA expression indicates that diabetes, at least partly, inhibits ND activity by reducing the ND mRNA expression.

\section{0}

THE PREVALENCE OF REACTIVE GASTRITIS IN DIABETES MELLITUS. M.Małecki, A. I. Bień, J.Stachura, D.Galicka-Latała, J. Sieradzki; Department of Metabolic Diseases, Jagellonian University, Kraków, Poland

There is very few data on the prevalence of reactive gastritis ( $R G$ ) in diabetes mellitus (DM). The typical histological findings in RG are foveolar hyperplasia, oedema with apparent diminution in glands, teleangiectasia of lamina propria, a paucity of inflammatory cells. RG has been connected so far with a long term use of non-steroidal anti-inflammatory drugs (NSAIDs) or bile reflux, particullary after cholecystectomy. RG is regarded as less sensitive to the Helicobacter pylori $(\mathrm{Hp})$ infection and being precancerous condition. The aim of the study was to define: 1 . The prevalence of RG in $D M$ as compared with the control group. 2. The prevalence of $\mathrm{Hp}$ infection in RG as compared with chronic active gastritis ( CAG) in DM. 3. The association of these types of gastritis with autonomic neuropathy (AN). Study design: Gastroscopy was performed in 152 patients with symptoms related to upper alimentary tract disorders - 52 with DM ( 13 with IDDM and 39 with NIDDM ) and 100 without DM. The presence of RG, CAG and HP infection were analysed in 3 biopsies. AN was evaluated in 35 diabetics in 4 cardiovascular tests using Proscicard. Results: RG was significantly more frequent in DM than in the control group ( $10 / 52,19,2 \%$ vs. $3 / 100,3 \%$, respectively, $p<0,01$ ). This difference was preserved after excluding all patients with NSAIDs use or being after cholecystectomy ( $8 / 36,22,2 \%$ vs. $2 / 81,2,5 \%$, respectively, $p<0.001$ ). Hp infection in diabetics with RG was significantly lower than diabetics with CAG $(2 / 10,20 \%$ vs. $16 / 23,69,5 \%$, respectively, $p<0,05)$. AN was more frequent in diabetics with RG than with CAG ( $7 / 10,70 \%$ vs. $6 / 15,40 \%$, respectively, ), the difference was not significant with this sample size. Conclusions: 1 . RG seems to be more frequent in DM than in general population. 2. Hp infection is less frequent in $R G$ than in CAG in DM. 3. The higher prevalence of RG in DM may result from impaired motility and reflux of bile caused by AN

\section{9}

FREE RADICAL STATUS OF HIGH FRUCTOSE DIET INSULIN RESISTANT RATS : EFFECTS OF METFORMIN.

P. Faure, E. Rossini, P.Y. Benhamou and S. Halimi.

GREPO, Université J.Fourier \& CHU Grenoble -38700- France. In NIDDM patients an exagerated free radical activity and lipid peroxidation accompanied insulin resistance. Such enhanced oxidative stress could play a role in the development of diabetic complications. Metformine has an effecet on insulin sensitivity and endothelial cells.Aim: to investigate in rats fed with a high fructose diet $(58 \%$ of $\mathrm{CHO}$ ), the effects of Metformin ( $200 \mathrm{mg} /$ day) on insulin sensitivity (glucose uptake $=$ Rd) by an euglycemic hyperinsulinemic clamp ( $2 \mathrm{mU} / \mathrm{min} / \mathrm{rat})$ in conscious animals peroxidation parameters (MDA, $\mathrm{SH}, \mathrm{CO}$ ) and protective enzymes against oxidative stress (SOD and GPX).

\begin{tabular}{|c|c|c|c|c|}
\hline $\begin{array}{l}\text { Groups } \\
\mathrm{Rd} \mathrm{mg/min} / \mathrm{kg}\end{array}$ & $\begin{array}{c}\text { Control } \\
n=6 \\
31.7 \pm 1.6\end{array}$ & $\left\{\begin{array}{l}\text { Fructose } \\
\mathrm{n}=9 \\
14.5 \pm 1.3\end{array}\right.$ & $\begin{array}{l}F+\text { Fetf } \\
h=8 \\
24 \pm 4.8\end{array}$ & $\begin{array}{c}\mathrm{p}= \\
\text { rus } \mathrm{F}+\mathrm{M} \\
\mathrm{p}<0.05\end{array}$ \\
\hline$\overline{\mathrm{SOI}}$ & $1.44 \pm 0.34$ & $0.87 \pm 0.21$ & $1.59 \pm 0.3$ & $(p<0.005)$ \\
\hline & $3.03 \pm 0.34$ & $1.74 \pm 0.24$ & $2.66 \pm 0.57$ & $(p<0.005$ \\
\hline SH $\mathrm{pm} / \mathrm{g}$ prot & $8.18 \pm 0.63$ & $4.6 \pm 0.8$ & $4.1 \pm 1.0$ & NS \\
\hline & $0.34 \pm 0.13$ & $0.29 \pm 0.10$ & $0.39 \pm 0.10$ & NS \\
\hline & $2.31 \pm$ & 0.39 & $\$ 12$ & $\mathrm{~N}$ \\
\hline & & & & \\
\hline
\end{tabular}

In this animal model, Metformin improves insulin sensitivity and parallely corrects the abnormaly low levels of protective enzymes against radical attacks (SOD GPX) and reduces triglycerides concentrations, indicating its potential effects on the vascular risk of insulin resitant states in addition to the well known effects on glucose homeostasis and lipid profile.

\section{1}

THE IMPACT OF NON-INSULIN DEPENDENT DIABETES MELLITUS AND OBESITY ON BONE TISSUE METABOLISM.

A. Zonenberg, B. Telejko, I. Borejszo, I. Kinalska. Department of Endocrinology, Medical School, Bialystok, Poland

The study was designed to investigate the possible influence of obesity, NIDDM and its complications on bone tissue metabolism. The study was carried out in 25 type II diabetics aged $55.4 \pm 8.3$ ys and 38 controls aged $54.5 \pm 13.2$ with no history of osteoporosis. All participants were divided into 2 subgroups according to BMI $\left(\mathrm{kg} / \mathrm{m}^{2}\right)$; below 28 and above 28 . Biochemical markers of bone tissue remodelling: alkaline phosphatase activity, serum total and ionized calcium $(\mathrm{Ca})$, serum magnesium $(\mathrm{Mg})$ and osteocalcin (GLAprotein, by RIA method) were determined. The index of calcium to creatinine in the morning portion of urine was calculated (Cau/ Cru); normal value < 0.21 ). Glycemia and $\mathrm{HbAlc}$ were also estimated. The level of serum total calcium was similar in all groups, however the level of ionized calcium was lower in subjects with type II diabetes, especially with BMI $>28(p<0.05)$ in comparison to non diabetics. Similarly, the level of $\mathrm{Mg}$ in the blood serum of diabetics was lower in comparison to the controls $(p<0.01)$ and moreover in subjects with BMI $>28(p<0.05)$. Cau/Cru index was slightly higher in persons with $\mathrm{BMI}<28$, but the difference was not statistically significant. Osteocalcin level was lower in diabetic patients $(20.02 \pm 13.44 \mathrm{ng} / \mathrm{ml})$ in comparison to the control group $(39.91 \pm 25.89 \mathrm{ng} / \mathrm{ml}, \mathrm{p}<0.05)$ and among healthy subjects - in the obese $(31.98 \pm 15.30 \mathrm{ng} / \mathrm{ml}$ vs $42.32 \pm 28.17 \mathrm{ng} / \mathrm{ml})$. In diabetic subjects osteocalcin concentration was independent of BMI, HbAlc or duration of the disease. Osteocalcin levels were also lower in men than in women, both - healthy and diabetic. But extremely low concentrations were found in patients with symptoms of neuropathy $(13.86 \pm 8.74 \mathrm{ng} / \mathrm{ml}$, p $<0.01)$ and retinopathy $(11.38 \pm 9.49 \mathrm{ng} / \mathrm{ml}, \mathrm{p}<0.05)$. Our results may confirm the role of neurotrophic factors and microvascular damage in the disturbances of bone metabolism and osteoporosis. 
1182

FFFFC OF GLYCEMIC CONTROI ON THF TUPAIRED PHAGOCYTIC FINCTION IN DIABETTC POLYMORPHONUCI.FAR LEUJKOCYTFS A. Kuzuyama, K. Ohohashi, T. Ogasawara ' R. Takashima, H. Nar ihara, H. Mor i, H. Park, K. Kashima ${ }^{2}$ and H. Senmaru ${ }^{3}{ }^{1}$ Department of Internal Medicine,0tsu Municipal Hospital, ${ }^{2}$ The $3 r d$ Department of Internal Medicine, Kyota Pref. liniv. of Medicine and ${ }^{3}$ Rakuwakaj-Marutamachi Hospital, Japan

Phagocytic function of polymorphonuclear leukocytes (PMNs) was studied by the rapid technique using Micrococous lysodeiktios (M. 1ys) in 83 diabetics and 60 controls. Hepariorized blood was inculated with M.1ys in saline ontaining $6 \%$ hydroxyethyl starch up to 10 mins. Samples were taken at 1 min intervals for the quantitative determination of phagocytosis. In 60 controls $100 \%$ of plagocytosis was alserved within 7 min after the incubation and therefore percentage phagueytosis reaching to less than $100 \%$ at $7 \mathrm{~min}$ was assessed as impaired phagocytosis. Impaired phagocytosis was found in $42 \%$ of diabetics. Hbatc level was significantly elevated in diabetics with impaired phagocitosis compared to those with normal. In 19 porrly controlled diabetics phagncytic function of PMNs was studied before and after ylycemic control. After treatmerts for 3-4 weeks in the lospitalization, FPG and HbA 1 levels were significantly decreased $213 \pm 93$ vs $11 ! \pm 19 \mathrm{mg} / \mathrm{d} 1,10.6$ \pm 2.7 us $8.7 \pm 1.4 \%, M \pm S D$, and percentage phagncytosis at 7 min was significantly improved $38.9 \pm 25.4$ vs $63.4 \pm 28.2 \%, M \pm S D$. These results suggest that glycemic control is important factor to improve the impared phagocytic function of PMN in diabeties.

\section{4}

Left ventricular diastolic function during isometric exercise in hypertensive diabetics with albuminuria A-M. Salmasi, C. Kirrollos. A. Grentell. E. Jepson, P. Moore, S. Purwaha and M. Dancy. Central Middlesex Hospital, London. U.K.

Left ventricular ( $\mathrm{LV}$ ) diastolic dysfunction during isometric exercise (IME) is common in patients with angina pectoris and documented coronary artery disease (CAD). In view of the high incidence of $\mathrm{CAD}$ in diabetics, $\mathrm{LV}$ diastolic function in response to IME was studied by assessing transmitral flow (TMF) using combined echo and pulsed-wave Doppler cardiography in 21 hypertensive diabetics with macroalbuminuria (Mac) and 11 with ninicroalbuminuria (Mic). For comparison 17 diabetics without complications (DM) and 20 non-diabetic hypertensive subjects (HP) were studied. All patients were free from cardiac symptoms and history. TMF was assessed both at rest and at peak standardized IME using handgrip. The peak velocity (Vp) of the early (E) and atrial (A) components of the TMF was derived. LV ejection fraction (LVEF) was also measured. Each patient underwent an exercise ECG test (ET). As previously described $V p$ E/A<1 was taken to indicate LV diastolic dysfunction.

\begin{tabular}{|c|c|c|c|c|}
\hline & $\operatorname{Mac}(\%)$ & $\operatorname{Mic}(\%)$ & $\mathrm{DM}(\%)$ & $\mathrm{HP}(\%)$ \\
\hline Number of patients & $21(100)$ & $11(100)$ & $17(100)$ & $20(100)$ \\
\hline$V_{p} E / A<1$ at rest & $9(+3)$ & $1(9)$ & $1(6)$ & $9(+5)$ \\
\hline $\mathrm{Vp} E / A<1 \mathrm{IME}$ & $16(76)$ & $10(91)$ & $9(53)$ & $16(80)$ \\
\hline positive ET & $4(19)$ & $3(27)$ & $7(41)$ & $1(5)$ \\
\hline$\vee p E / A<$ with negative & ET $13(62)$ & $7(64)$ & $6(35)$ & $16(80)$ \\
\hline LVEF $<50 \%$ & $5(24)$ & $3(27)$ & $1(6)$ & $1(5)$ \\
\hline $\begin{array}{l}\text { concluded that } \\
\text { ics develop LV }\end{array}$ & & & & \\
\hline
\end{tabular}

1183

\section{DIFFERENT PATTERNS OF RESPIRATORY RESPONSE} DEMONSTRATED BY pCO2 IN DIABETIC KETOACIDOSIS

M. Auinger and K. Irsigler, $3^{\text {rd }}$ Med.Dep., Hospital Lainz, Vienna, Austria. Aim of our study was to evaluate the compensatory ventilatory response (measured by $\mathrm{pCO} 2$ ) to acidosis in diabetic ketoacidosis (DKA) according to Winter's formula ( $\mathrm{pCO} 2=1,5 \times$ [bicarbonate] $+8 \pm 2$ ), 121 episodes of DKA with a pH 7,1 to 7,25 (limits of the formula) and a mean blood glucose of $598 \pm 207$ $\mathrm{mg} / \mathrm{dl}$ at hospitalization were studied. Arterial $\mathrm{pCO} 2$ computed from bicarbonate was adequate to actually measured $\mathrm{pCO} 2$ in 42 patients $(34,7 \%)$ : $19 \pm 4$ versus $19 \pm 5 \mathrm{mmHg}$ (group 1). The computed $\mathrm{pCO} 2$ was lower than measured (superimposed respiratory acidosis) in 44 patients (36.3\%): $21 \pm 4$ versus $28 \pm 6 \mathrm{~mm} \mathrm{Hg}$ (group 2). And furthermore, a higher computed pCO2 than actually measured (superimposed respiratory alkalosis) was found in 35 patients $(28,9 \%$ ): $19 \pm 4$ versus $15 \pm 4 \mathrm{mmHg}$ (group 3). There were no differences in age, sex, diabetes duration, blood glucose, $\mathrm{pH}$, anion gap, osmolality between the three groups of patients. pO2 $(\mathrm{mmHg})$ was highest in group $3(115 \pm 15)$, lowest in group $2(103 \pm 17)$ and $111 \pm 15$ in group 1 , according to the degree of ventilation. Final outcome: there were 2 deaths in group 1, 4 deaths in group 2 and 1 death in group 3. This implicates the potential danger of superimposed respiratory acidosis in the prognosis of DKA. The reasons for this different behavior of ventilation at same degrees of $\mathrm{pH}$ and bicarbonate are not completely clear. Despite potential different time intervalls allowing for respiratory compensation in DKA, factors like ventilatory restriction, circulatory problems, central nervous depression and exhaustion of respiratory muscles must be ruled out in superimposed ventilatory acidosis, factors stimulating disproportional ventilation like sepsis, lactic acidosis or salycilate intoxication should be looked for in superimposed respiratory alkalosis. In conclusion, only one third of patients with DKA shows adequate ventilation according to an established formula. Therefore, computing of $\mathrm{pCO} 2$ can be helpful in evaluating comorbid conditions in DKA

\section{5}

HOW BIG ARE DIARRHOEA AND CONSTIPATION PROBLEMS IN DLABETICS ? AF Celik. Z Ersanli.T Damci, S Beyhan, H Savas, H Ilkova, U Gorpe,M Ozyazar and N Bagriacik. Lniversity of Istanbul, Cerrahpasa Med Fac, Dept of Int Med,Turkey. To uncover the actual prevalance of constipation and diarthoea in diabetics. 250 diabetic (DM) outpatients (159 Females, 91 Males, mean diabetes age: 10.6+7.6) with good diabetic control ( $\mathrm{HbAlc}<8 \%$ ) were compared with a group of age and sex matched 250 non-diabetic (NDM) outpatients (161 Females, 89 Males) with respec to the presence constipation and diarthea (if both have been existent for more than six munths). People who had had abdominal operation or those with organic gastrointestinal. metabolic or hormonal diseases were excluded. As subjective constipation criteriae: 2 different types of questionnaires were used; simple questionnaire* which is answered as either 'yes' or 'no' and a visual scale analogue** to be marked between 0 and 10 and values $>3$ were considered constipation positive. Objective constipation criteriae were accepted as less than 3 stooliweek + pellety or hard stool consistency + straining more than $25 \%$ of the time and objective diarrhoae criteriae were watery stool consistency or more than 3 normal or soft stool/day.

\begin{tabular}{|c|c|c|c|}
\hline & $\frac{(\mathrm{DM})}{250}$ & $\frac{(N D M)}{n: 250}$ & \\
\hline & Mean age: $53+13$ & Mean age: $50+13$ & $p$ value \\
\hline Subjective Constipation ${ }^{*}$ & $150(60 \%)$ & $110(44 \%)$ & $p<0.05$ \\
\hline Subjective Constipation** & $1: 2(45 \%)$ & $93(37 \%)$ & $\mathrm{p}<0.05$ \\
\hline Objective Constipation & $50(20 \%)$ & $26(10 \%)$ & $p<0.05$ \\
\hline Objective Diarthea & $20(8 \%)$ & $12(5 \%)$ & $p>0.05$ \\
\hline Alternate Const'Diarl freq & $55(22 \%)$ & $35(14 \%)$ & $\mathrm{p}<0.05$ \\
\hline Bowel opening (freq/day) & $0.6 \pm 0.1$ & $0.9+0.1$ & $p<0.05$ \\
\hline Laxative use & $38(15 \%)$ & $19(8 \%)$ & $p<0.05$ \\
\hline
\end{tabular}

Our results confirm that constipation is seen in $60 \%$ of diabetics when only simple questionnaire is used. However. using the visual scale analogue which may give more accurate information, the frequency of constipation is less than it is found out by the simple questionnaires $(45 \%)$. But when the term constipation is regarded as a function of more than one criteriae to be more objective, actual frequency is much less $(20 \%)$. Constipation is found to be more prevalent in diabetics than in non diabetics whilst diarrhoae is not. In conclusion consripation seems to be a widespread problem in diabetics whereas diarrhoae is not different from the background population. 